\title{
Initial Performance Assessment of the Disposal of Spent Nuclear Fuel and High-Level Waste Stored at Idaho National Engineering Laboratory
}

\section{Volume 1: Methodology and Results}

\author{
Rob P. Rechard, Editor \\ Performance Assessment Department \\ Sandia National Laboratories \\ Albuquerque, New Mexico 87185
}

\begin{abstract}
This performance assessment characterized plausible treatment options conceived by the Idaho National Engineering Laboratory (INEL) for its spent fuel and high-level radioactive waste and then modeled the performance of the resulting waste forms in two hypothetical, deep, geologic repositories: one in bedded salt and the other in granite. The results of the performance assessment are intended to help guide INEL in its study of how to prepare wastes and spent fuel for eventual permanent disposal. This assessment was part of the Waste Management Technology Development Program designed to help the U.S. Department of Energy develop and demonstrate the capability to dispose of its nuclear waste, as mandated by the Nuclear Waste Policy Act of 1982.
\end{abstract}

The waste forms comprised about 700 metric tons of initial heavy metal (or equivalent units) stored at the INEL: graphite spent fuel, experimental low enriched and highly enriched spent fuel, and high-level waste generated during reprocessing of some spent fuel. Five different waste treatment options were studied; in the analysis, the options and resulting waste forms were analyzed separately and in combination as five waste disposal groups. When the waste forms were studied in combination, the repository was assumed to also contain vitrified high-level waste from three DOE sites for a common basis of comparison and to simulate the impact of the INEL waste forms on a moderate-sized repository. The performance of the waste form was assessed within the context of a whole disposal system, using the U.S. Environmental Protection Agency's Environmental Radiation Protection Standards for Management and Disposal of Spent Nuclear Fuel, High-Level and Transuranic Radioactive Wastes, 40 CFR 191, promulgated in 1985.

Although numerous caveats must be placed on the results, the general findings were as follows: Though the waste form behavior depended upon the repository type, all current and proposed waste forms provided acceptable behavior in the salt and granite repositories. In the salt repository, the behavior was acceptable even without a moderately corrosion-resistant canister or chemically-adsorptive backfill. Furthermore, in

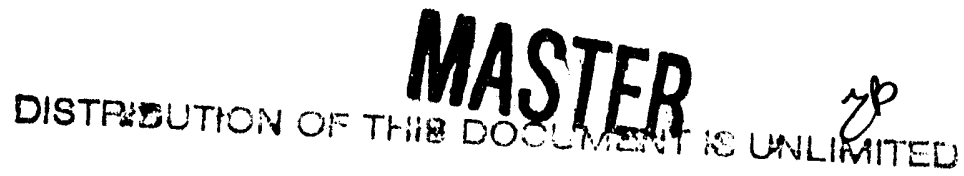


the salt repository, differences among the individual waste forms were less pronounced than in the granite repository and the differences nearly disappeared when the waste forms were combined as a disposal group with vitrified high-level waste. In cortrast, in the granite repository, the differences among waste forms were greater and these differences, though small, remained for the waste forms when combined as a disposal group. These findings suggest that treatment of the relatively small mass of INEL fuel and waste cannot substantially improve the performance of a moderate- or large-sized repository. Although waste treatment generally improved performance, an exception occurred for the graphite spent fuel from Fort St. Vrain, which was assumed to contain intact fuel particles. Moderate treatment showed degraded performance in comparison to whole block disposal; only extensi $v$ treatment improved performance.

Observations on other factors important to the performance assessment are that the diffusive transport of radionuclides from the waste parcel holes into the drifts and then the advective transport through the drifts were very important phenomena to model-their importance was comparable to the effect of the waste treatment options. Also, because of the requirements of 40 CFR 191, direct release of radionuclides to the ground surface from exploratory drilling into the repository dominated the releases from the salt repository.

An important assumption for the analysis was that critical conditions were to be avoided in the repository when a waste parcel completely degraded; thus only a small amount of fissionable uranium was permitted in each container. The result of this assumption was that a large number of containers was required (especially for the highly enriched spent fuel), which, because of the cost implications, suggested that future work should attempt to demonstrate that critical noncatastrophic conditions in a repository would be technically acceptable. Furthermore, other performance criteria such as dose to an individual (pending promulgation of new regulations on required repository performance) need to be examined to substantiate the initial guidance from this performance assessment, which indicates that the current waste forms are acceptable for long-term environmental compliance. Finally, the second phase of the performance assessment should be pursued and should examine the behavior of the waste forms in an unsaturated tuff repository. 


\section{Preface}

This document reports on an initial performance assessment for a U.S. Department of Energy (DOE) program, "Idaho National Engineering Laboratory/Idaho Chemical Processing Plant Spent Fuel and Waste Management Technology Development Program." The program is designed to help the U.S. Department of Energy develop and demonstrate the capability to dispose of its nuclear waste, as stated in the Nuclear Waste Policy Act (NWPA) of 1982. Specifically, this performance assessment characterized five different, plausible, treatment options developed by the Idaho National Engineering Laboratory for spent fuel and high-level waste and then studied the effects of these treatment options on the performance of the waste and spent fuel in a repository setting. The results of the performance assessment are intended to be used as guidance to the Waste Management Technology Development Program about how to prepare wastes and spent fuel for eventual permanent disposal.

\section{Description of Participants}

The U.S. Department of Energy (DOE), through the Environmental Restoration Program Undersecretary, is investigating options for the conditioning and disposing of spent nuclear fuel and high-level waste (HLW) including currently stored spent nuclear fuel from military and experimental reactors and high-level waste that is generated when spent nuclear fuel is reprocessed. The primary coordinator for the study is the Westinghouse Idaho Nuclear Company (WINCO), one of two major operating contractors at the Idaho National Engineering Laboratory. The Performance Assessment Department at Sandia National Laboratories, a major team member for this program, is providing expertise in performance assessment methodology and is the primary author of this report.

\section{Organization of Report}

The executive summary reviews the scope and method of analyses, but its emphasis is on the important ideas and conclusions of this initial performance assessment. The introductory chapter describes the purpose of the report, briefly describes the regulatory setting, sets the stage for the data and concepts that are presented in later chapters, and highlights some of the analysis decisions made during the assessment: Chapter. 2 provides details on the U.S. Environmental Protection Agency (EPA) Standard, Environmental Radiation Protection Standards for Management and Disposal of Spent Nuclear Fuel, High-Level and Transuranic Radioactive Wastes, 40 CFR 191, and Chapter 3 gives an overview of the performance assessment process. The remaining chapters cover the tasks of the performance assessment in the order in which they are described in the Introduction, i.e., disposal system characterization (Chapters 4, 5 and 6); scenario development (Chapter 7); scoping calculations (Chapter 8); probability modeling (Chapter 9); consequence modeling (Chapters 10 through 15); and results (Chapter 16). Because of the extensive amount of information that must be described for each major task of a performance assessment, the chapters primarily summarize or highlight data that are critical or important to the performance assessment. Detailed data necessary to provide a complete picture of the disposal system are provided in the appendices (Volume 2 of this report).

\section{Suggested Use of Report}

- For a summary of the results, refer to the Executive Summary.

- For an overview of the entire performance assessment process, refer to Chapter 1, Introduction.

- For specific details pertaining to performance assessment tasks, consult the individual chapters.

- For background information pertaining to specific performance assessment tasks, consult the appendices. 


\section{Related Documents}

In the early 1980s, Sandia National Laboratories developed computational tools and procedures for assessing the safety of high-level waste disposal in hypothetical deep geologic repositories (both bedded salt and basalt) for the Nuclear Regulatory Commission (NRC) (Campbell and Cranwell, 1988; Cranwell et al., 1987; Bonano et al., 1988). These performance assessments simulated a steady-state groundwater flow field, evaluated a particle pathway, and then calculated radionuclide transport along this pathway from a simple source.

At the same time, Sandia also was involved with assessing the feasibility of high-level waste disposal in the subseabeds of the deep oceans for the U.S. Department of Energy (DOE) and the International Nuclear Energy Agency of the Organization for Economic Co-operation and Development, Paris (e.g., de Marsily et al., 1988). The approach used in these performance assessments was to start with a repository model that contained detailed physical/chemical processes (Hickerson et al., 1988; Lanza, 1988) and continue with both transient fluid flow and transport through successive barriers (Shephard et al., 1988; Marietta and Simmons, 1988; Pentreath et al., 1988; Brush, 1988), culminating in a dose assessment.

The computational tools developed for these performance assessments were often exploratory because they were used to examine hypothetical repositories. Sandia has since expanded these concepts and developed new application tools in its assessment of actual sites such as the Yucca Mountain Project (YMP) (e.g., Barnard et al., 1992; Gauthier et al., 1992; Dudley et al., 1988) and the Waste Isolation Pilot Plant (WIPP) Project. Although the performance assessment methodology is similar for both projects, the computational tools are different. The reasons for the differences between the two projects are that (1) each project is in a different stage of study, (2) YMP must adhere to an additional NRC regulation (Disposal of High-Level Radioactive Wastes in Geologic Repositories, 10 CFR 60), and (3) the understanding of unsaturated flow phenomenon is less well developed at YMP. The work reported here most closely resembles the studies of the WIPP and so primarily the computational tools and methodology developed for that project are used; however, material from the YMP is also used. Several reports from the WIPP Project, which are frequently cited, are listed below:

WIPP PA (Performance Assessment) Department. 1992. Preliminary Performance Assessment for the Waste Isolation Pilot Plant, December 1992. Volume 1: Third Comparison with 40 CFR 191, Subpart B. SAND92-0700/1. Albuquerque, NM: Sandia National Laboratories.

WIPP PA (Performance Assessment) Department. 1992. Preliminary Performance Assessment for the Waste Isolation Pilot Plant, December 1992. Volume 2: Technical Basis. SAND92-0700/2. Albuquerque, NM: Sandia National Laboratories.

Sandia WIPP Project. 1992. Preliminary Performance Assessment for the Waste Isolation Pilot Plant, December 1992. Volume 3: Model Parameters. SAND92-0700/3. Albuquerque, NM: Sandia National Laboratories.

WIPP PA (Performance Assessment) Department. 1993. Preliminary Performance Assessment for the Waste Isolation Pilot Plant, December 1992. Volume 4: Uncertainty and Sensitivity Analysis for 40 CFR 191, Subpart B. SAND92-0700/4. Albuquerque, NM: Sandia National Laboratories.

\section{Analysis Rigor}

The work reported herein (software, analysis, and data) is, for the most part, experimental or preliminary. In general, most initial performance assessments are carried out at this level because information about the disposal system under study is not completely known; analyst judgment must supplement the available information. In terms of quality assurance, the class of software, analysis, and data used in this study is Level X (experimental). Although this level of quality assurance is not a reflection of the intrinsic value of the work, it does indicate that an ability to trace back to adjudicated sources for the data currently cannot be guaranteed. A complete description of the quality assurance levels, which were originally developed for the Waste Isolation Pilot Plant Project, and the assurance that 
each provides regarding traceability, repeatability, peer review, and other issues, can be found in the following reports:

Rechard, R.I., P.J. Roache, R.L. Blaine, A.P. Gilkey, and D.K. Rudeen. 1991. Quality Assurance Procedures for Computer Software Supporting Performance Assessments of the Waste Isolation Pilot Plant. SAND90-1240. Albuquerque, NM: Sandia National Laboratories.

Rechard, R.P., D.K. Rudeen, and P.J. Ruache. 1992. Quality Assurance Procedures for Analyses and Report Reviews Supporting Performance Assessments of the Waste Isolation Pilot Plant. SAND91-0428. Albuquerque, NM: Sandia National Laboratories.

Rechard, R.P., K.M. Trauth, and R.V. Guzowski. 1992. Quality Assurance Procedures for Parameter Selection and Use of Expert Judgment Panels Supporting Performance Assessments of the Waste Isolation Pilot Plant. SAND91-0429. Albuquerque, NM: Sandia National Laboratories.

When future performance assessments examine related areas and concepts, with more accurate data and a better understanding of the important phenomena to study, the findings of this study may be modified. Therefore, decisions based on this performance assessment should encompass the knowledge that the findings may be adjusted in the future and, therefore, that impacted decisions might also be re-examined.

\section{Acknowledgments}

The authors of this report appreciate the review of the performance assessment approach and the guidance provided by the Program Review Panel, established by WINCO and Sandia, which includes the following individuals:

C. G. Whipple, ICF Kaiser Engineers, Oakland, CA

J. J. Jicha, Jr., U.S. Department of Energy, Office of Environmental Restoration and Waste Management, EM-37

L. Wade, U.S. Department of Energy, Office of Environmental Restoration and Waste Management, EM-323

C. Russomanno, U.S. Department of Energy, Office of Civilian Radioactive Waste Management, RW-22

K. Svinicki, U.S. Department of Energy, Idaho Operations Office

J. M. Boak, U.S. Department of Energy, Office of Geologic Disposal

and the technical review panel, established by Sandia, which includes the following individuals:

G. Ross Heath, Chairman, College of Ocean and Fishery Sciences, Seattle, WA

Jane C. S. Long, Lawrence Berkeley Laboratory, Berkeley, CA

Thomas McLaughlin, Los Alamos National Laboratory, Los Alamos, NM

Donald W. Peaceman, Independent Consuitant, Houston, TX

Thomas H. Pigford, University of California, Berkeley, CA

Richard Westerman, Battelle Pacific Northwest Laboratories, Richland, WA

The authors also appreciate the time and suggestions provided by the final technical reviewers of this document, K.M. Trauth (6342), and R.C. Dykhuizen (1513). Finally, the editorial help on the text and illustrations provided by J. Chapman and D. Pulliam, respectively, of Tech Reps, Inc., Albuquerque, NM, and the original illustrations by D. Duncan, of Mac Tec, greatly improved the report.

A performance assessment of a complex system using stochastic simulation requires a tremendous volume of long running calculations; thus, the reliability of the computer system is very important. During this fiscal year, new computers were acquired and the entire Nuclear Waste Management Center moved, which placed a great burden on personnel providing computer services. The help of M. McNeil and J. Davis of the Computational Support Department during this hectic time is acknowledged. 
A performance assessment is a multidisciplinary task and requires the expertise of numerous individuals. The individuals involved are noted in the organization chart that follows.

\section{Managers}

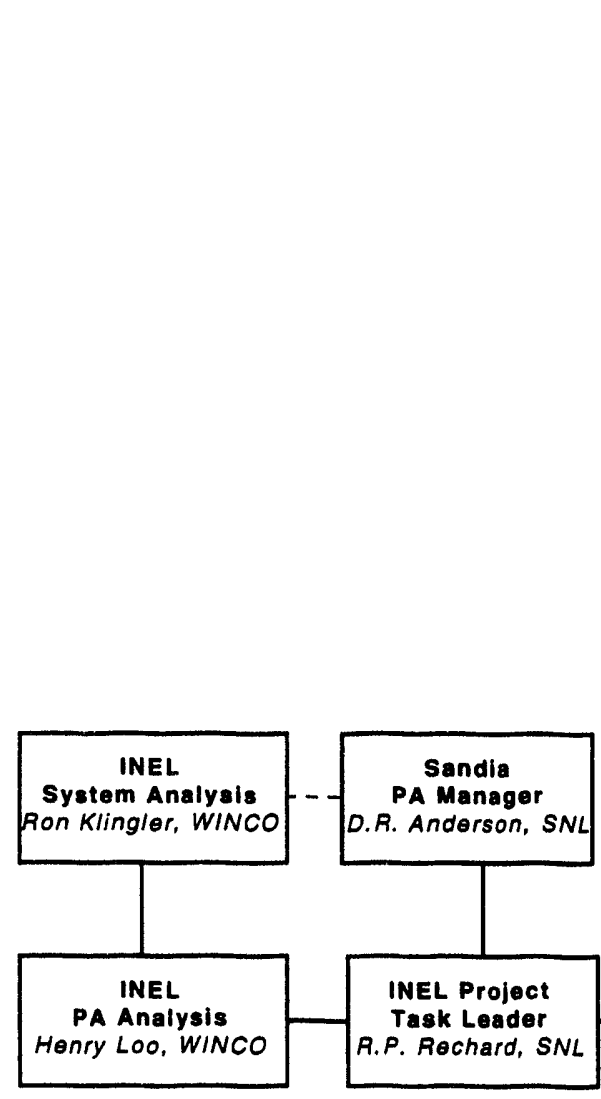

Activities and Subtask Leaders

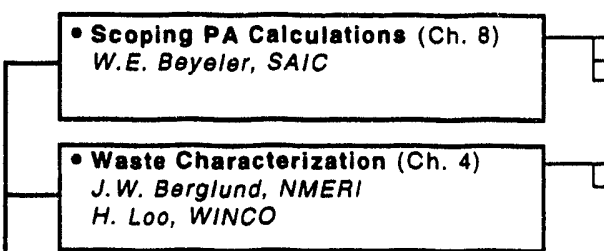

B. Palmer, WINCO

J. L. Ramsey, API

C.T. Stockion, SAIC - WINCO Personnal

- Repository Design (Ch. 6)

- Cuttings Modeling (Ch. 10)

J.W. Berglund, NMERI

- Geologic Barriers (Ch. 5)

- Secondary Data Base

K.F. Brinster, SAIC

D.K. Duncan, MAC A. Johnson, SAIC - A. LOChOl, NMERI R. LOChOI, NMERI
H.J. IUZZOlino, GOOC.

- Scenario Development (Ch. 7) R. V. Guzowski, SAlC M.S. Tierney, SNL

- Room Chemistry (Ch. 11) R. LBChol, NMERI C.T. Stockman, SAIC

\section{P.}

- Complex Repos. Models (Ch. 12/13) _ M.E. Fowell, SNL - Model Verification

- Respository Modeling

- M.E. Lord, API

E D. Stoolzel, GeOC

- Simple PA Models (Ch. 14\& 15)

C.T. Stockman, SAIC

Flow a Transport

W.E. Beyeler, SAIC

API - Applied Physics Incorporated

GeoC - Geo-Centers Incorporated

MAC - MACTEC

NMERI - New Mexico Engineering Research Institute

SAIC - Scientific Applications International Corporation

SNL - Sandia National Laboratories

TRI - Tech. Reps., Incorporated

WINCO - Westinghouse Idaho Nuclear Company

\section{Organization Chart for} INEL Project (September, 1993)

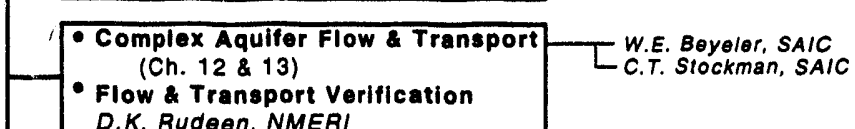

D.K.Rudeen. NMERI

- PA Overview (Ch. 3)

- Probability Model (Ch. 9)

M.S. Tierney, SNL

- Sensitivity Analysis (Ch. 16) W.E. Beyeler, SAIC

L. Smith, SAIC

- Compliance lseves (Ch. 2) S. Bigger, SNL

- Review Panels $\quad$ F F.T. Mondonhall, SNL

M. Gruebel, TRI

E s. Tullar. TAI

- Analysis Toolbox Enhancement H.J. Iuzzolino, GooC J.S. Rath, NMERI

\section{- Document Support}

D D. Pulliam, TRI

J. Chapman, TRI $\quad$ L. Tartaglia, TRI

- QA Coordination G. Pullon, SNL

- Application Software

L G.K. Duncan, MAC 


\section{Contents}

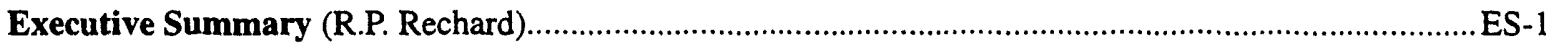

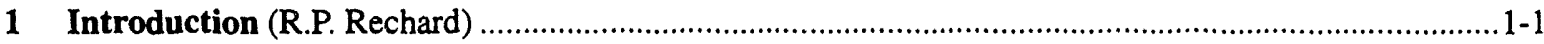

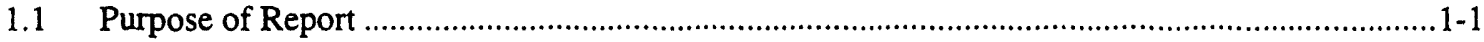

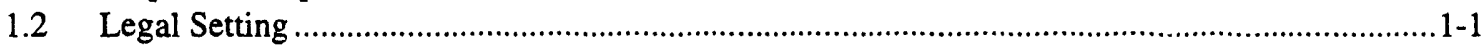

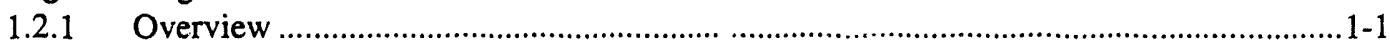

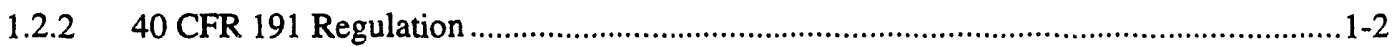

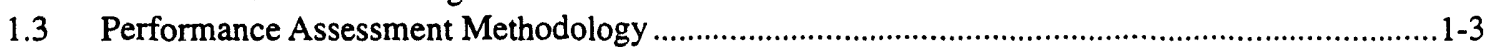

1.3.1 Theoretical Foundation of the Performance Assessment ...............................................1-3

1.3.2 Organization and Mechanics of the Performance Assessment.......................................1-4

1.3.3 Sandia Analysis System Developed for Performance Assessment.................................1-4

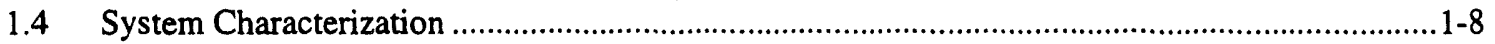

1.4.1 Waste Containment System ....................................................................................1-8

1.4.2 Waste Parcel Description ......................................................................................1-8

1.4.3 Geologic Barrier Characterization ........................................................................1-14

1.4.4 Repository Design ...........................................................................................1-15

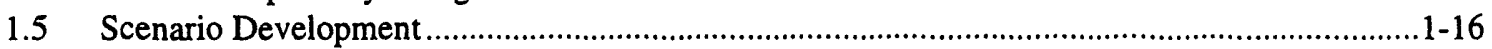

1.5.1 Selected Features, Events, and Processes ................................................................1-17

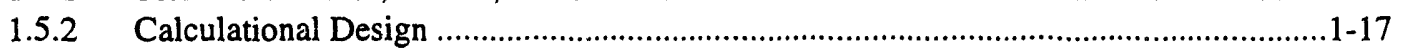

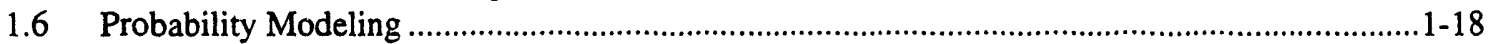

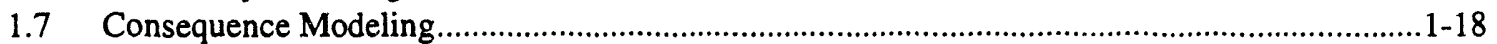

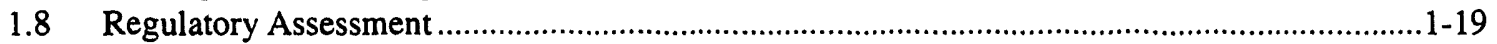

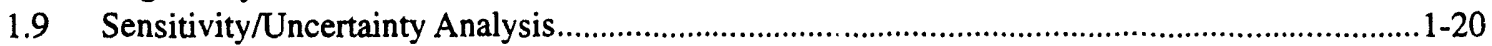

2 Regulatory Criteria for Radioactive-Waste Repositories (M.M. Gruebel) ........................................2-1

2.1 Summary of Regulatory Setting for This Assessment (R.P. Rechard) ...........................................2-1

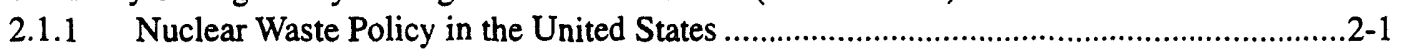

2.1.2 EPA's Standards for Radioactive-Waste Disposal ....................................................2-2

2.1.3 NRC's Standards for Radioactive-Waste Disposal..........................................................2-3

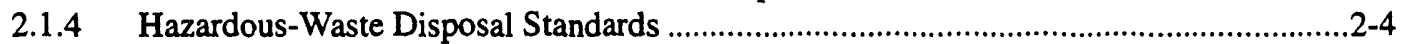

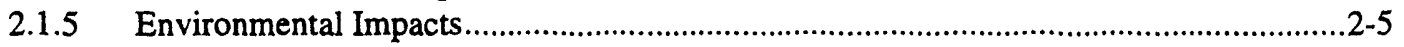

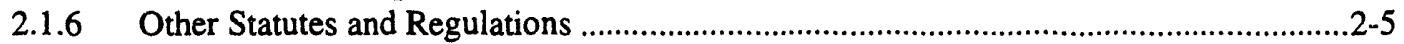

2.2 International Criteria for Radioactive-Waste Disposal ................................................................ $2-6$

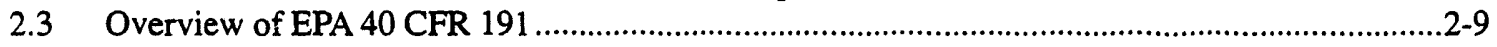

2.3.1 Containment Requirements ..................................................................................2-10

2.3.2 Assurance Requirements ............................................................................... $2-16$

2.3.3 Individual Protection Requirements ...........................................................................

2.3.4 Groundwater Protection Requirements ................................................................

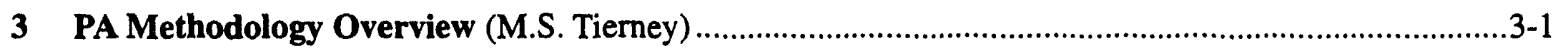

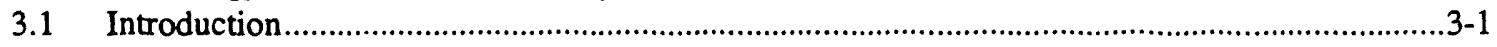

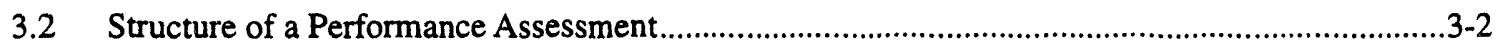

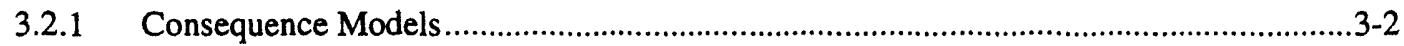

3.2.2 Parameter Space ……................................................................................................

3.2.3 Probability Models ...................................................................................................

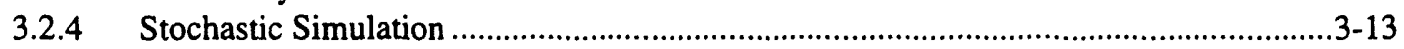

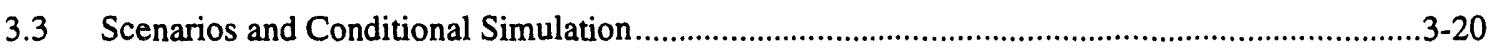

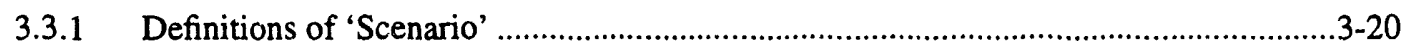


3.3.2 Scenarios as Subsets of Parameter Space ......................................................................

3.3.3 Probability of a Scenario, Conditional Simulation.......................................................2-26

3.3.4 Construction of CCDFs in the WIPP PA ……........................................................

4 Description of Waste and Waste Parcels (J.W. Berglund and J.S. Rath) …........................................4-1

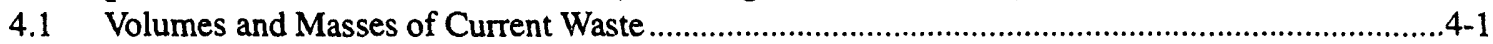

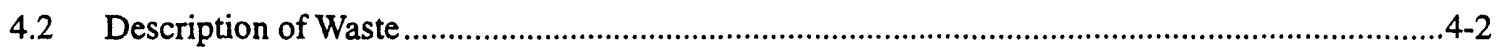

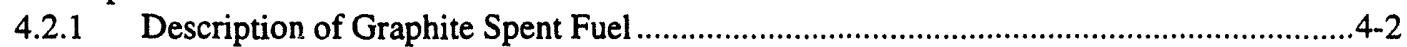

4.2.2 Description of Special Spent Fuel ................................................................................

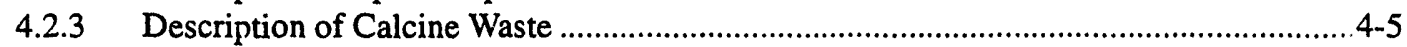

4.2.4 Descripion of Borosilicate Glass from Other DOE Sites ...............................................4-8

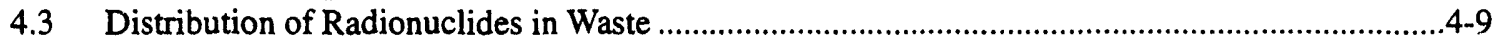

4.3.1 Radionuclide Inventory ..........................................................................................4-9

4.3.2 Radionuclide Chains and Half-Lives............................................................................ 4-11

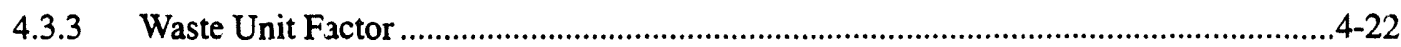

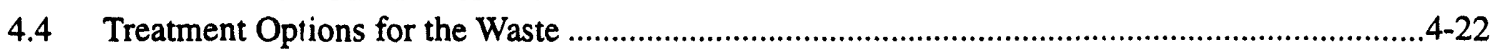

4.4.1 Processing Options for Graphite Spent Fuel ...........................................................4-23

4.4.2 Processing Options for Special Spent Fuel ..............................................................23

4.4.3 Processing Options for Calcine Waste.....................................................................27

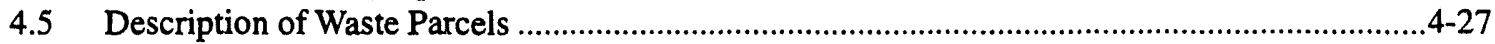

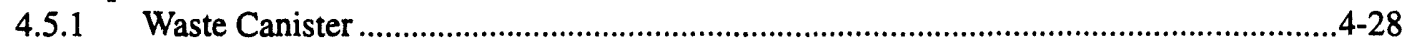

4.5.2 Internal Backfill .................................................................................................. 4-28

4.5.3 Optional Canister Overpack ………………................................................................... 4-28

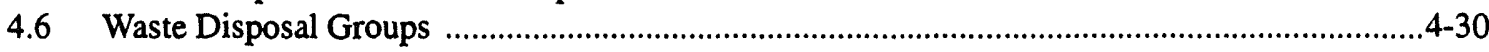

4.6.1 Waste Disposal Group 1 ..................................................................................

4.6.2 Waste Disposal Group 2 ...............................................................................................4-41

4.6.3 Waste Disposal Group 3 …………………..................................................................4-46

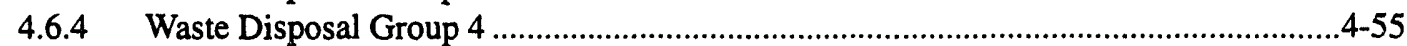

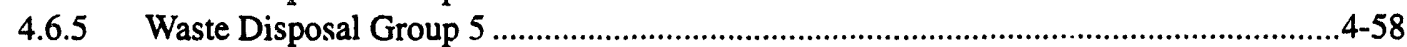

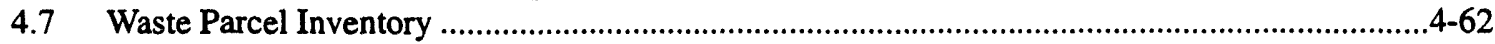

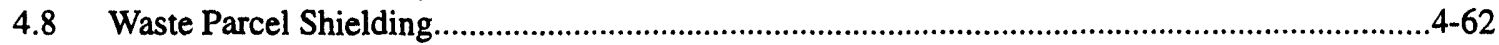

4.9 Summary of Modeling Assumptions for Waste Forms and Waste Parcels ..................................4-62

5 Hypothetical Geologic Barrier (K.F. Brinster and R.P. Rechard) ....................................................5-1

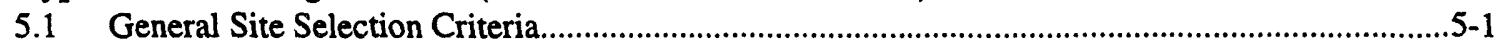

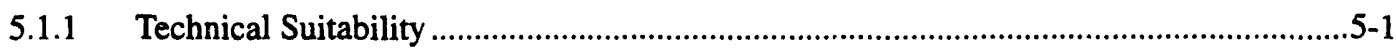

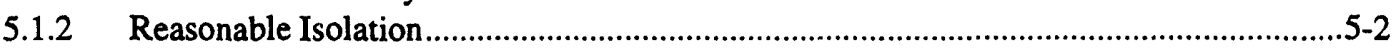

5.1.3 Salt Deposits as Geologic Barrier.......................................................................... $5-2$

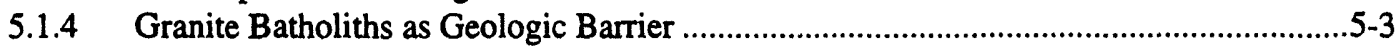

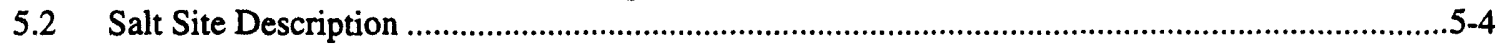

5.2.1 Origin and Occurrence of Salt Deposits ......................................................................

5.2.2 Hypothetical Salt Stratigraphy ................................................................................5-5

5.2.3 Modeling Assumptions for Geologic Barrier in Salt .......................................................5-9

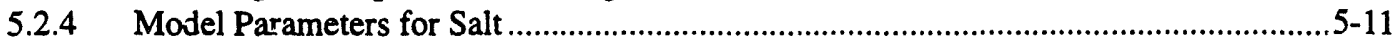

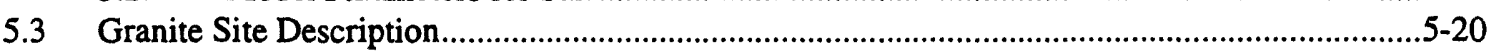

5.3.1 Origin and Occurrence of Granite Batholiths.......................................................

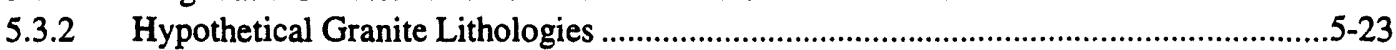

5.3.3 Modeling Assumptions for Geologic Barrier in Granite ..........................................5-23

5.3.4 Model Parameters for Granite .................................................................................. $5-26$ 


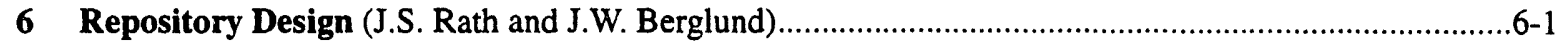

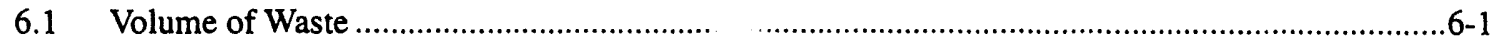

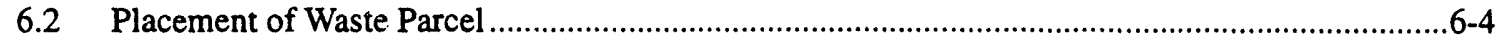

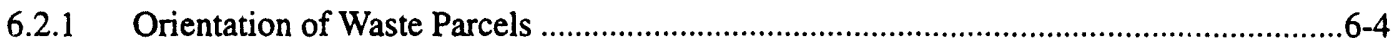

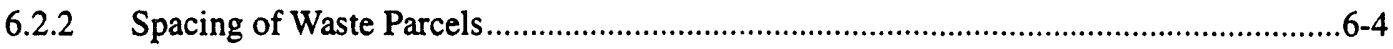

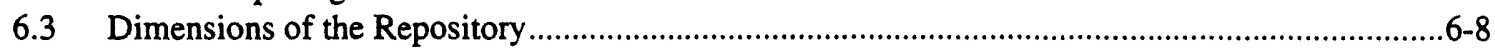

6.3.1 Waste Disposal Region .............................................................................................6-10

6.3.2 Access Drifts and Shafts ........................................................................................6-11

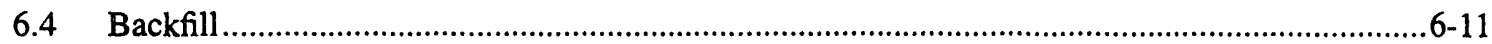

6.4.1 Backfill-Buffer around Waste Parcel ......................................................................12

6.4.2 General Backfill in Rooms, Access Drifts, and Shaft................................................6-14

6.5 Modeling Assumptions for Repository ...................................................................................6-18

7 Scenario Development (M.S. Tierney, R.V. Guzowski, and R.P. Rechard) ......................................... 7-1

7.1 Purpose and Components of Scenario Development ....................................................................

7.2 Identification and Selection of Features, Events, and Processes ...............................................

7.2.1 Step 1: Identifying the 'Universe' of Features, Events, and Processes ...........................7-3

7.2.2 Step 2: Classifying Features, Events, and Processes...................................................... 7-3

7.2.3 Step 3: Providing Rough Estimates of Probabilities and Consequences of Features, Events, and Processes ................................................................................. 7-3

7.2.4 Step 4: Screening Features, Events, and Processes to Establish History Space............... 7-6

7.3 Optionally Grouping Features, Events, and Processes ..........................................................

7.3.1 Step 1: Combining Features, Events, and Processes into Summary Scenarios..............7-11

7.3.2 Step 2: Grouping Summary Scenarios Having Similar Consequences ...........................7-14

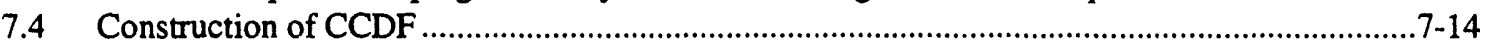

7.4.1 Advantages of Monte Carlo Method ...................................................................... $7-14$

7.4.2 Construction of CCDF without Scenarios (without Importance Sampling) ....................7-15

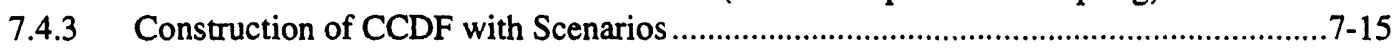

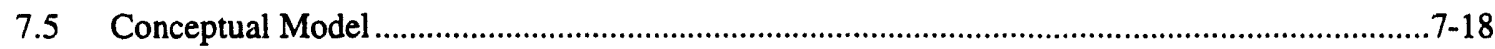

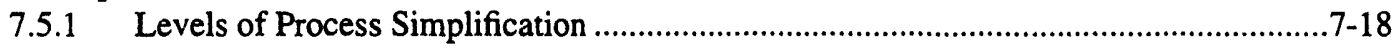

7.5.2 Scenario Selected for Study in Calculations ……...................................................... 7-18

7.5.3 Conceptual Model for Disposal System in Salt............................................................... 7-19

7.5.4 Conceptual Model for Disposal System in Granite .......................................................... 7-19

7.6 Computational Submodels and Calculation Design ................................................................. 7-22

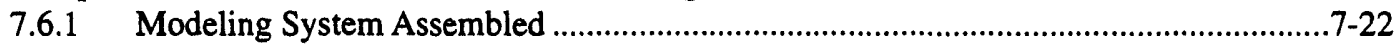

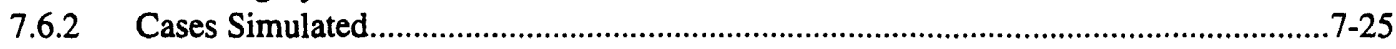

8 Modeling Conclusions Drawn from Scoping Calculations (R.P. Rechard) ...................................... $8-1$

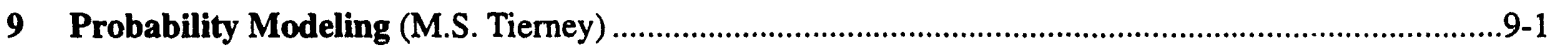

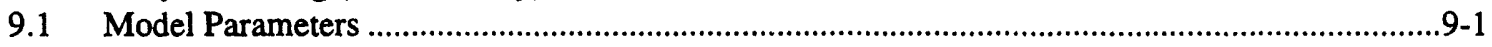

9.1.1 Characterizing Parameter Uncertainty ............................................................................9-1

9.1.2 Some Limitations on Distributions.............................................................................9-3

9.1.3 Parameters Selected for Sampling .................................................................................9-9

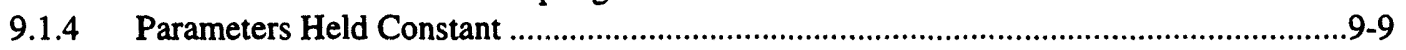

9.2 Poisson Model for Probability of Human Intrusion.................................................................9.9.

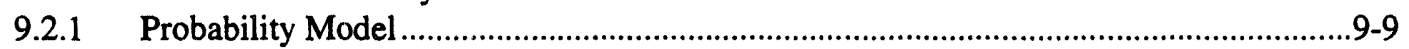

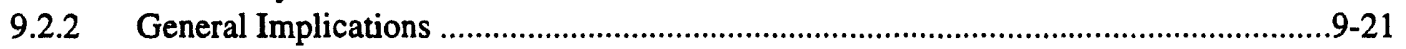

9.2.3 Area Ratios for Probability of Hitting Different Waste Parcels ......................................9-21

9.3 Probability of Exploratory Drillhole Intersecting a Brine Reservoir .............................................9-21

9.4 Summary of Modeling Assumptions for Human Intrusion.........................................................9-21 
10 Modeling Drilling into Repository - CUTTINGS (J.W. Berglund) .............................................10-1

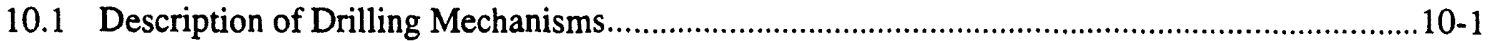

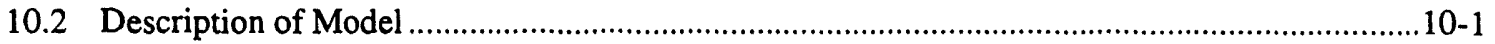

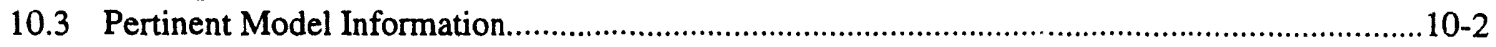

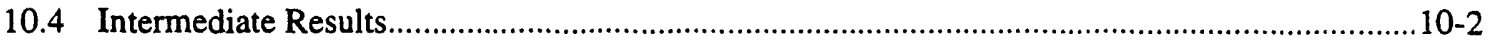

10.5 Summary of Modeling Assumptions for Cuttings Removal .....................................................10-2

11 Gas Generation and Source Term Submodels (C.T. Stockman) .................................................11-1

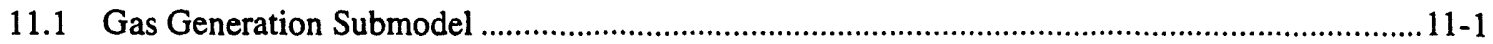

11.1.1 Types of Corrosion in a Waste Parcel ..........................................................................11-1

11.1.2 Conceptual Model of Corrosion Process .......................................................................11-3

11.1.3 Mathematical/Computational Model .........................................................................11-5

11.1.4 Interface of GASSNF with BRAGFLO_T ..............................................................11-8

11.1.5 Interface of GASSNF with SALFLOW and GRFLOW .........................................11-12

11.1.6 Modeling Assumptions for Gas Generation Submodel (GASSNF) ...........................11-12

11.2 Source Term Submodel of Waste Emplacement Package ........................................................11-13

11.2.1 Conceptual Model of Location of Radionuclides.......................................................11-14

11.2.2 Mathematical/Computational Model ......................................................................11-17

11.2.3 Interface of CONCSNF with STAFF2D ............................................................11-19

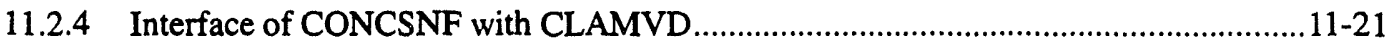

11.2.5 Summary of Modeling Assumptions ..................................................................11-22

11.3 Modeling Parameters for Gas Generation and Source Term Submodels...................................11-22

11.3.1 Corrosion Parameters .........................................................................................11-22

11.3.2 Early Breach Fraction ....................................................................................11-47

11.3.3 Matrix Alteration Parameters .................................................................................11-47

11.3.4 Fraction of Metal Carbide Fuel Particles that Breach in 10,000 yr .............................11-48

12 Complex Consequence Modeling for Salt Disposal System (J.L. Ramsey, M.E. Lord,

D.K. Rudeen, and D. M. Stoelzel).

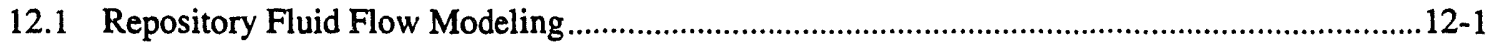

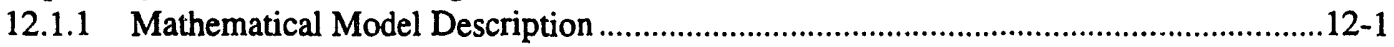

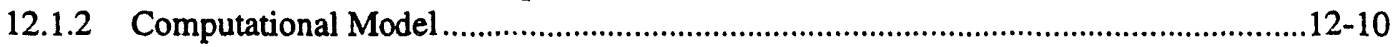

12.1.3 Applied Model ............................................................................................12-12

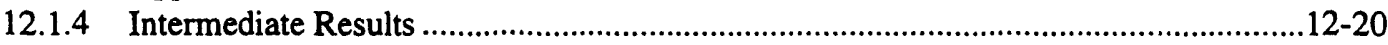

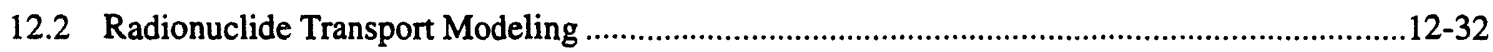

12.2.1 Mathematical Model Description ......................................................................12-33

12.2.2 Mathematical Model Implementation (Computational Model) ...................................12-35

12.2.3 Applied Model...............................................................................................12-36

12.2.4 Initial and Boundary Conditions ......................................................................12-36

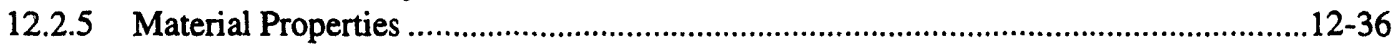

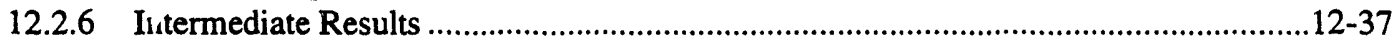

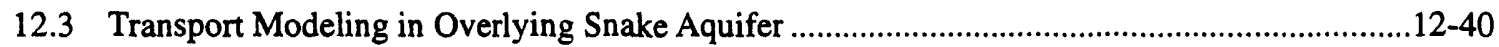

12.3.1 Mathematical and Computational Model Description .................................................12-41

12.3.2 Applied Model ........................................................................................................12-43

12.3.3 Initial and Boundary Conditions ……..................................................................12-44

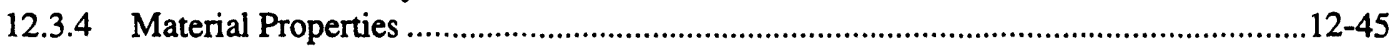

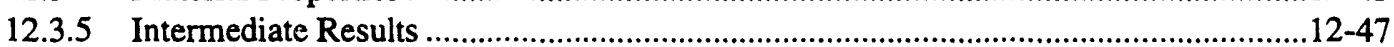

13 Complex Consequence Modeling for Granite Disposal System (J.L. Ramsey, D.K. Rudeen, and D. M. Stoelzel)

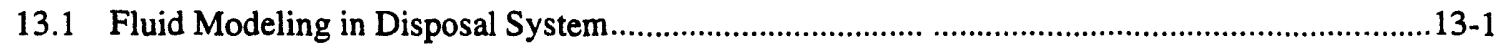

13.1.1 Mathematical and Computational Model Descrif tion .................................................13-1 
13.1.2 Applied Model .......................................................................................................

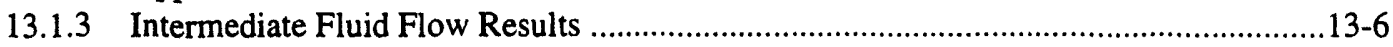

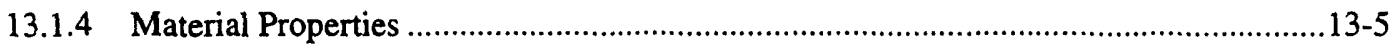

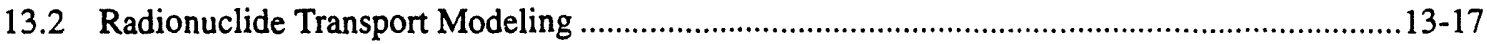

13.2.1 Mathematical and Computational Model Description ..............................................13-17

13.2.2 Applied Model .......................................................................................................13-17

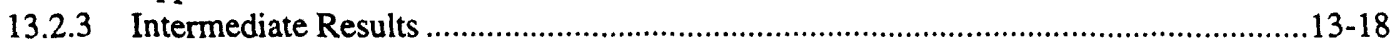

14 Simplified Consequence Modeling for Salt Repository (W.E. Beyeler) .........................................14-1

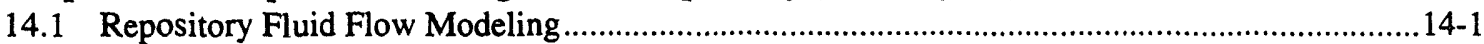

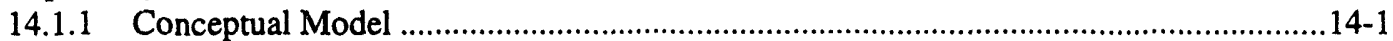

14.1.2 Mathematical Model ......................................................................................................

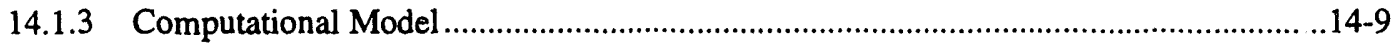

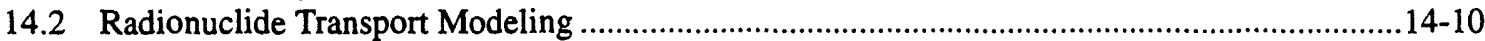

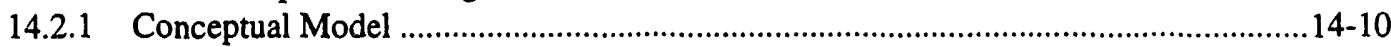

14.2.2 Mathematical and Computational Model of Advection/Dispersion in Room/Drifts .......................................................................................................14-11

14.2.3 Mathematical and Computational Model of Diffusion in Waste Emplacement

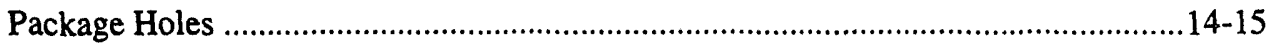

15 Simplified Consequerce Modeling for Granite Repository (W.E. Beyeler) …….............................15-1

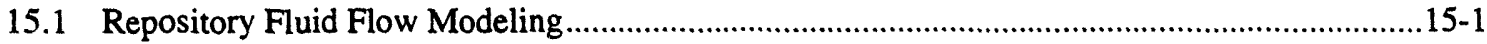

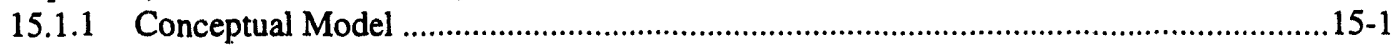

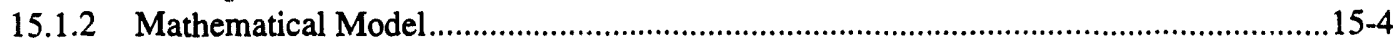

15.1.3 Computational Model .................................................................................................

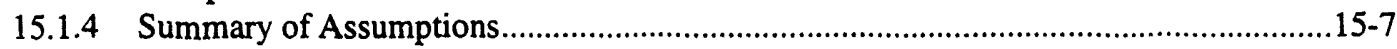

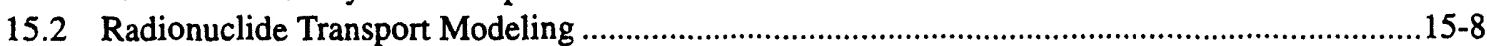

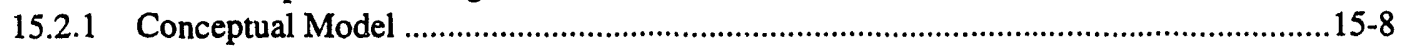

15.2.2 Mathematical and Computational Model ..................................................................

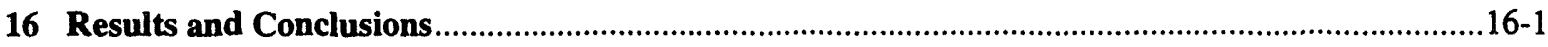

16.1 Performance Metrics and Analysis Techniques (R. P. Rechard) .................................................16-1

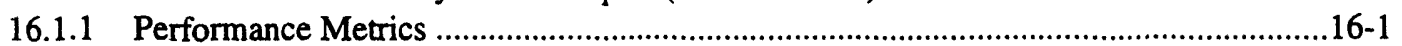

16.1.2 Description of Terms and Graphical Presentation of Results .......................................16-1

16.1.3 Uncertainty Analysis Techniques ................................................................................16-3

16.2 Complex PA Results for Disposal in Bedded Salt ..................................................................16-5

16.2.1 Release from the Disposal System (R. P. Rechard and H. J. Iuzzolino) ........................16-5

16.2.2 Percentage of Radionuclides Leaving the Waste Parcel (D. K. Rudeen) ........................16-5

16.2.3 Normalized Activity Leaving Repository (D. K. Rudeen) ...........................................16-8

16.2.4 Normalized Activity Leaving the Disposal System in the Snake Aquifer

(D. K. Rudeen) .

16.3 Complex PA Results for Disposal in Granite Batholith ........................................................16-18

16.3.1 Release from the Disposal System (R. P. Rechard and H. J. Iuzzolino) ......................16-18

16.3.2 Percentage of Radionuclides Leaving the Waste Parcel (D. K. Rudeen) ......................16-19

16.3.3 Normalized Activity Leaving Repository (D. K. Rudeen) …………...........................16-19

16.4 Simple PA Results for Disposal in Bedded Salt....................................................................16-29

16.4.1 Fluid Flow through Salt Repository (L. Smith).....................................................16-29

16.4.2 Percentage of Radionuclides Leaving the Waste Parcel (W. E. Beyeler and C. T. Stockman) ......................................................................................................

16.4.3 Normalized Activity Leaving Repository (W. E. Beyeler and C. T. Stockman) ...........16-38

16.5 Simple PA Results for Disposal in Granite Batholith .............................................................16-45

16.5.1 Fluid Flow through Granite Repository (L. Smith) ...................................................16-45 
16.5.2 Percentage of Radionuclides Leaving the Waste Parcel (W. E. Beyeler and C. T. Stockman)

16.5.3 Normalized Activity Leaving Repository (W. E. Beyeler and C. T. Stockman) ..........16-54

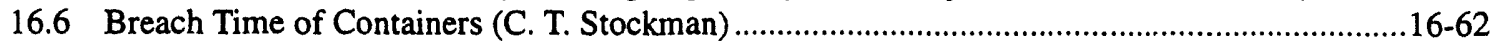

16.7 Calculations Using Corrosion-Resistant Waste Parcel (C. T. Stockman) ..................................16-71

16.8 Tracer and Other Radionuclide Transport in the Salt and Granite Disposal Systems

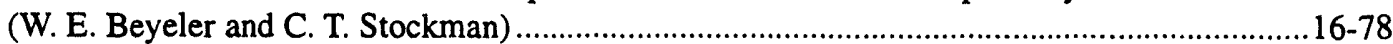

16.8.1 Tracer Transport ........................................................................................16-18

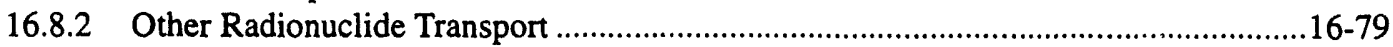

16.9 Comparison of Simple and Complex Consequence Models (D. K. Rudeen) .............................16-84

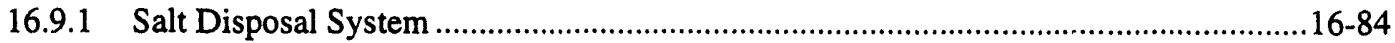

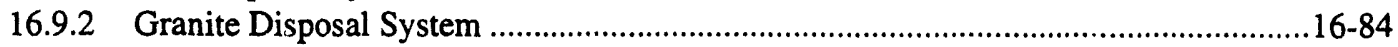

16.9.3 Comparison of Waste Disposal Group 1 .................................................................

16.10 Direct Release to Ground Surface During Drilling (J. W. Berglund, J. S. Rath, and H. J. Iuzzolino).

16.11 Summary of Findings and Program Guidance as Related to 40 CFR 191 (R. P. Rechard) .........16-93

16.11.1 Summary of Findings about the Disposal System..................................................16-93

16.11.2 General Findings about the Performance Assessment Modeling ...............................16-93

16.11.3 Program Guidance ....................................................................................................16-99

References

Glossary Glossary-1

Volume 2

Appendix A: Description of Spent Nuclear Fuel and High Level Waste at INEL (H. Loo, L. Taylor and D. Evans)

Appendix B: Development of EPA 40 CFR 191 (M. M. Gruebel) ......................................................... B-1

Appendix C: Scoping Calculations (R.P. Rechard, W.E. Beyeler, C.T. Stockman, B. Palmer, J.L. Ramsey, and J.W. Berglund)

Appendix D: Waste Parcel Design and Contents (J.W. Berglund) .......................................................

Appendix E: Demonstration Calculation for Dose to Humans (R. D. McCurley) ................................. E-1

Appendix F: Disposition of Events and Processes as a Result of Screening for a Disposal Facility in Bedded Salt and Saturated Granite (R.V. Guzowski) ................................... F-1

Appendix G: CAMCON Software Used in Performance Assessment (R.P. Rechard) ............................1 Appendix H: Summary of Code Enhancements (M.E. Lord, J.W. Berglund, and D.K. Rudeen)...............H-1 


\section{Figures}

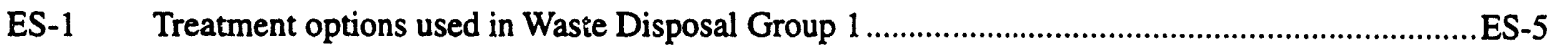

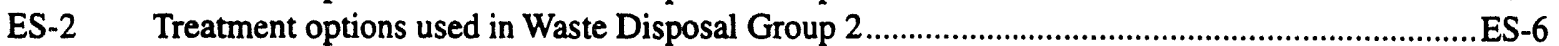

ES-3 Treatment options used in Waste Disposal Group 3 …................................................................

ES-4 Treatment options used in Waste Disposal Group 4 …..............................................................ES-8

ES-5 Treatment options used in Waste Disposal Group 5 ...................................................................

1-1 Sandia's method for conducting INEL Performance Assessment calculations involves

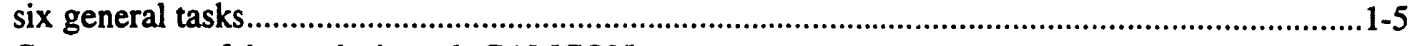

1-2 Components of the analysis tool, CAMCON

1-3 Subsystems and components of a radioactive waste containment system ......................................

2-1 ThePA's 40 CFR 191 - Environmental Standards for the Management and Disposal of Spent Nuclear Fuel, High-Level and Transuranic Radioactive Wastes .......................................2-10

2-2 Artist's concept of a mined geologic disposal system portraying terminology used in

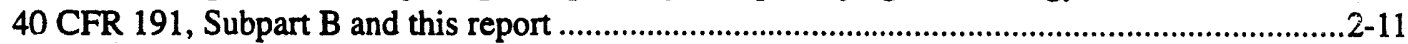

2-3 Complementary cumulative distribution function (CCDF) illustrating compliance with

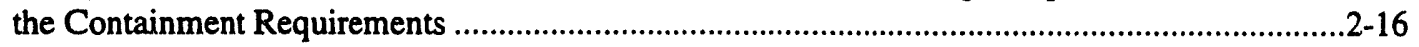

3-1 Submodels used in 1992 WIPP performance assessment.........................................................

3-2 Distribution of historical drill bit diameter .................................................................................

3-3 Measured distribution for tortuosity of Culebra matrix .............................................................

3-4 Typical comparison between predicted and experimental CDF for the parameter, $\varepsilon_{\mathrm{s}}$, at

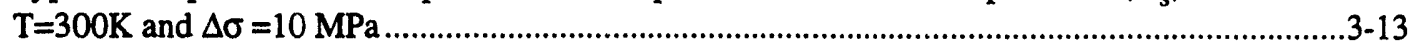

3-5 An informal five-step procedure used to construct cumulative distribution functions for the 1990 WIPP performance simulations .................................................................................... $3-14$

3-6 Constructed distribution for gas production rates from corrosion under inundated conditions ......3-14

3-7 Constructed distribution for solubility of plutonium .................................................................15

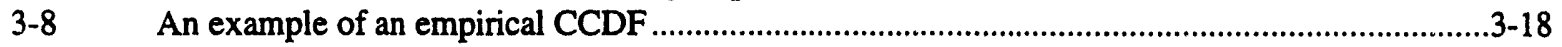

3-9 Example family of CCDFs generated by Latin hypercube sampling for comparison with the

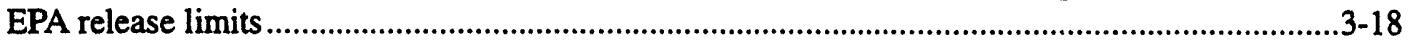

3-10 Mean and percentile curves for the family of CCDFs shown in Figure 3-9 ................................ 3-19

3-11 The cyclical nature of mathematical modeling ............................................................................. 3-21

3-12 Logic diagram for the construction of summary scenarios .................................................. 3-?

3-13 Schematic illustrating partitioning of parameter space into summary scenarios and

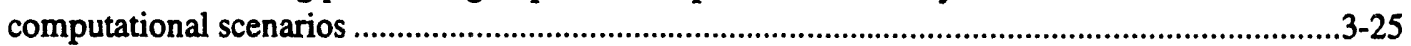

4-1 Fort St. Vrain whole block element ...............................................................................................

4-2 Peach Bottom fuel element.....................................................................................................

4-3 Representative low enriched uranium fuel assembly ...................................................................4-6

4-4 Representative highly enriched uranium fuel assembly ................................................................4-7

4-5 Initial (year 2030) activity of 49 radionuclides considered in the performance assessment...........4-12

4-6 Normalized activity decay history of individual radionuclides that exceed a normalized release of (a) 0.1 i d (b) 0.01 , based on 40 CFR 191 criterion....................................................4-16

4-7 Radionuclide decay chains considered for spent fuel and high-level waste for complementary cumulative distribution function development .........................................................................4-19

4-8 Treatment options used for graphite spent fuel ........................................................................24

4-9 Treatment options used for high and low enriched spent fuel......................................................4-25

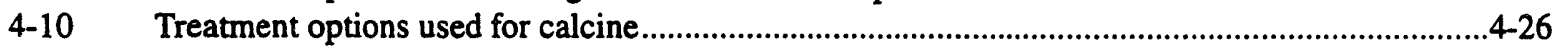

4-11 Schematic of cross-section of waste parcel, consisting of waste form, waste containers, and internal backfill .....................................................................................................................4-27

4-12 Relative sizes of 10 different canisters considered in this performance assessment........................4-29

4-13 Waste Parcel 1 in Waste Disposal Group 1..............................................................................4-32 


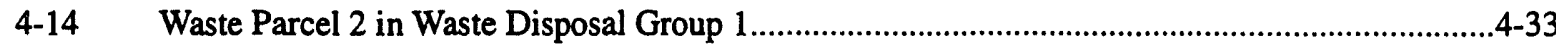

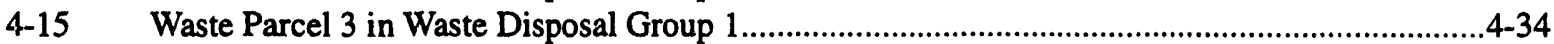

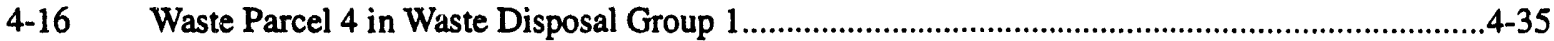

4-17 Waste Parcel 5 in Waste Disposal Group 1 ......................................................................................

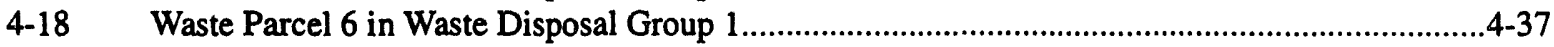

4-19 Waste Parcel 7 in all waste disposal groups ................................................................................4-38

4-20 Waste Parcel 8 in all waste disposal groups .......................................................................................4-39

4-21 Waste Parcel 9 in all waste disposal groups ....................................................................................4-40

4-22 Waste Parcel 10 in Waste Disposal Group 2 ..............................................................................4-42

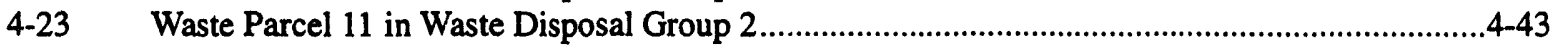

4-24 Waste Parcel 12 in Waste Disposal Group 2 ............................................................................4-44

4-25 Waste Parcel 13 in Waste Disposal Group 2 ..........................................................................45

4-26 Waste Parcel 14 in Waste Disposal Group 3 ...........................................................................4-47

4-27 Waste Parcel 15 in Waste Disposal Group 3 ..........................................................................4-48

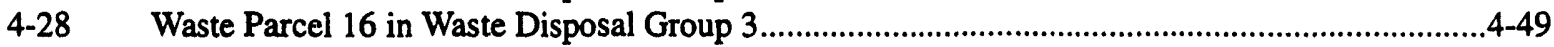

4-29 Waste Parcel 17 in Waste Disposal Group 3 .....................................................................................4-50

4-30 Waste Parcel 18 in Waste Disposal Group 3 ......................................................................................4-51

4-31 Waste Parcel 19 in Waste Disposal Group 3 ......................................................................................5-52

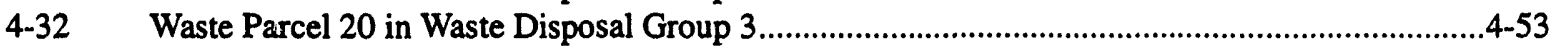

4-33 Waste Parcel 21 in Waste Disposal Group 3...............................................................................4-54

4-34 Waste Parcel 22 in Waste Disposal Group 4..........................................................................56

4-35 Waste Parcel 23 in Waste Disposal Group 4..........................................................................5-57

4-36 Waste Parcel 24 in Waste Disposal Group 5......................................................................................4-59

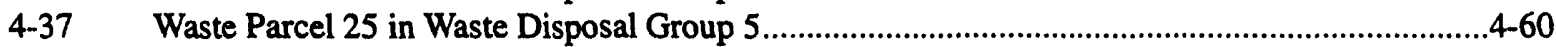

4-38 Waste Parcel 26 in Waste Disposal Group 5..................................................................................4-61

4-39 Curie content of waste parcels based on full inventory of 177 radionuclides and on

reduced inventory of 49 radionuclides at time of emplacement....................................................4-62

4-40 Heat rate of waste parcels at time of emplacement accounting for all 177 radionuclides ..............4-63

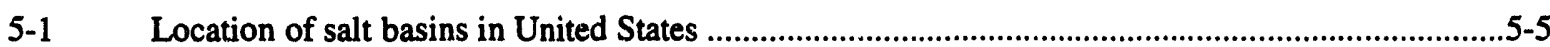

5-2 Stratigraphic layers of geoiogic barrier for bedded salt repository ................................................5-7

5-3 Stratigraphy in Sodium Springs Formation near repository .....................................................5-8

5-4 Detailed lithology of hypothetical Cattle River Formation..............................................................5-10

5-5 Generalized locations of surface and subsurface stocks and batholiths in the United States.........5-20

5-6 Geologic barrier for granite repository .....................................................................................

5-7 Seven dominant fracture zones used in conceptual model at Stripa mine in Sweden....................5-25

6-1 Disposal pattern within rooms for waste parcels.............................................................................6-5

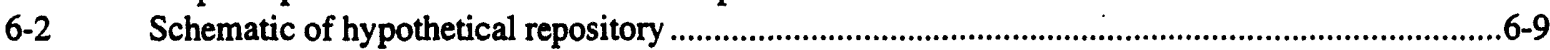

6-3 Schematic of panel in disposal region of repository ................................................................ $6-11$

7-1 Scenario development, in conjunction with system characterization, establishes how the real world will be represented in the applied model.................................................................... 7-2

7-2 Identification and selection of features, events, and processes to model ........................................7-4

7-3 Grouping of features, events, and processes that survive initial screening into summary

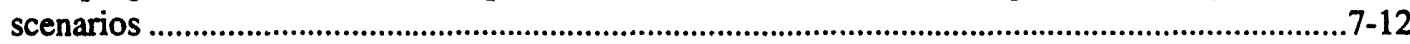

7-4 Demonstration logic diagram for the construction of summary scenarios....................................7-13

7-5 Method of calculating CCDF depends on whether sample space is discretized .............................7-16

7-6 General conceptual model for salt disposal system....................................................................20

7-7 General conceptual model for granite disposal system .............................................................. 7-22

7-8 The modeling sysiem assembled for the Complex PA consists of a suite of support codes plus the computational modules of BRAGFLO_T, STAFF2D, CUTTINGS, and CCDFPERM. 
7-9 The modeling system assembled for the Simple PA consists of a suite of support codes plus the computational modules of SALFLOW, GRFLOW, CLAMVD, CUTTINGS, and CCDFPERM

9-1 Probability distributions of fraction of silicon-carbide coatings that have failed in the waste parcels containing graphite spent nuclear fuel in both salt and granite repositories

9-2 Uniform probability distributions of alteration rates for layers and matrices of the waste parcel in both salt and granite.

9-3 Uniform probability distributions of alteration rates for aluminum and stainless steel in salt and granite.

9-4 Probability distributions of fraction of mass removed at perforation of lead and stainless steel in salt and granite repositories.

9-5 Probability distributions of solubility of americium, plutonium, thorium, and uranium radionuclides in brine in salt repository

9-6 Probability distributions of solubility of americium, plutonium, thorium, and uranium radionuclides in fresh water in granite repository

9-7 Probability distributions for repository and geologic barrier model parameters in salt ...................9-18

9-8 Probability distributions for repository and geologic barrier model parameters in granite ...........9-19

9-9 Probability of a human-intrusion estimated by Poisson analytic probability model with constant $\lambda$.

10-1 Total curie content at six intrusion times for (a) WP01 to WP09, (b) WP10 to WP18, and (c) WP19 to WP26

11-1 Corrosion and breach of waste container and waste form layers ..................................................11-4

$11-2$ Interface of GASSNF with BRAGFLO_T

11-5 General interface between source term submodel and transport model .......................................11-18

12-1 Sample capillary pressure and relative permeability diagrams

12-7 Plan view of conceptual model near borehole for salt repository centered in an element of side leg of rooms in panels 3 and 8

12-8 Integrated net brine flows at $10,000 \mathrm{yr}$ for the five waste disposal groups at each of the monitoring points: (a) the shaft, (b) borehole 1, (c) borehole 2, (d) brine reservoir, and (e) surrounding halite

12-9 Integrated net gas flows at $10,000 \mathrm{yr}$ for the five waste disposal groups at each of the monitoring points: (a) the shaft, (b) borehole 1 , (c) borehole 2 , and (d) halite.

12-10 Total brine flow into salt repository by $10,000 \mathrm{yr}$ at the five monitoring points for all 20 simulations in Waste Disposal Group 1

12-11 Brine inflow from the brine reservoir is only slightly greater than brine outflow through Borehole 2 indicating a slight loss of fluid to the repository in Waste Disposal Group 1.

12-12 Brine inflow from the surrounding halite is highly dependent upon the sampled halite permeability 
12-13 Total brine flow out the salt repository after $10,000 \mathrm{yr}$ for all 20 simulations in Waste Disposal Group 1

12-14 Total brine flow out Borehole 2 is highly dependent on the permeability of the upper segment of Borehole 2 .

12-15 Total gas inflow for Waste Disposal Group 1 occurs primarily from the gas-saturated shaft, or from gas that initially moved into the salt but later flowed back into the repository .....12-27

12-16 The only significant gas outflow occurs into the surrounding liquid-saturated salt

12-17 Cumulative hydrogen gas generated by container corrosion at $10,000 \mathrm{yr}$ in all 20 sample sets for all waste disposal groups in the salt repository

12-18 Cumulative hydrogen gas production correlates well with the stainless steel corrosion rate up to $3.5 \times 10^{-10} \mathrm{~kg} / \mathrm{m}^{2} \cdot \mathrm{s}$, at which point the availability of reactants possibly controls the process

12-19 Salt repository average (a) brine saturation, (b) brine pressure, and (c) gas pressure for all 20 sample sets of Waste Disposal Group 1

12-20 . Final (a) brine pressure and (b) gas pressure attained in the salt repository for all five waste disposal groups.

12-21 Maximum temperature observed throughout the salt repository for all five waste disposal groups

12-22 Contours of mass fraction of contaminant ( $\mathrm{kg} / \mathrm{kg}$ fluid) for salt repository, Waste Disposal Group 1, Run 17, at (a) $1000 \mathrm{yr}$ and (b) $10,000 \mathrm{yr}$.

12-23 Contours of mass of contaminant per element volume $\left(\mathrm{kg} / \mathrm{m}^{3}\right.$ element) for salt repository, Waste Disposal Group 1, Run 17, at (a) 1000 yr and (b) 10,000 yr .

12-24 Darcy velocity vector plot representative of the flow field after intrusion and resaturation, salt repository Waste Disposal Group 1, Run 17.

12-25 Conceptual hydrologic model of the Snake Dolomite Member... $12-42$

12-26 Example of regional and local grids used for distributed fluid flow and transport calculations

12-27 Particle paths for neutrally buoyant particle released at intrusion borehole centered in repository for 20 flow fields used in Snake Aquifer transport simulations

12-28 Travel times to $2.4-\mathrm{km}$ boundary of Snake Aquifer

12-29 Concentration $\left(\mathrm{kg} / \mathrm{m}^{3}\right)$ contours for ${ }^{234} \mathrm{U}$ in Snake Aquifer at (a) $1000 \mathrm{yr}$ and (b) $10,000 \mathrm{yr}$ for Waste Disposal Group 1, salt disposal system.

13-1 Conceptual model of the granite repository and surrounding host rock for Complex PA:

(a) vertical cross-section ( $x-z$ plane) and (b) plan view ( $x-y$ plane)...

13-2 Plan view of borehole for granite repository centered in an element of the main legs of rooms in panels 3 and 8

13-3 Two-dimensional logical grid of granite repository and surrounding geologic barrier...................13-4

13-4 Schematic of granite disposal system depicting the area increase of elements to account for area increase in radial direction

13-5 Net water flows through the granite repository are the same for each waste disposal group at each of the five monitoring points: (a) shaft, (b) top fracture, (c) bottom fracture, (d) borehole top, and (e) borehole bottom

13-6 Total water flowing into granite repository by $10,000 \mathrm{yr}$ through five monitoring points for all 20 simulations using Waste Disposal Group 1.

13-7 Water inflow into the granite repository when the connecting fracture permeability is (a) low and (b) high

13-8 Total water outflow for all 20 runs, Waste Disposal Group 1, granite repository ........................13-12

13-9 Total gas flow in the granite repository for all 20 simulations of Waste Disposal Group 1.........13-13

13-10 Net gas flow through the borehole top in the granite repository for all five waste disposal groups...

13-11 Cumulative hydrogen gas generated by container corrosion by $10,000 \mathrm{yr}$ in all 20 simulations for all waste disposal groups at the granite repository

13-12 Scatterplot of volume of hydrogen gas generated vs. corrosion rate 13-15 
13-13 Granite repository average (a) water saturation, (b) water pressure, and (c) gas pressure

for all 20 sample sets of Waste Disposal Group 1 .

13-14 Maximum temperatures observed throughout granite repository for all five waste disposal groups.

13-15 Contours of mass fraction $(\mathrm{kg} / \mathrm{kg}$ ) liquid of contaminant for Waste Disposal Group 1 ,

Run 17, in granite repository, at (a) $1000 \mathrm{yr}$ and (b) $10,000 \mathrm{yr}$

13-16 Contours of mass of contaminant per element volume $\left(\mathrm{kg} / \mathrm{m}^{3}\right.$ element) for Waste Disposal Group 1, Run 17, in granite repository at (a) $1000 \mathrm{yr}$ and (b) 10,000 yr

13-17 Darcy velocity vector plots representative of the flow field after intrusion and resaturation, granite repository, Waste Disposal Group 1, Run 17.

14-1 General conceptual model and one-dimensional mathematical model of salt repository and nearby geologic barrier in Simple PA.

14-2 Relationship between control volumes in flow model of salt disposal system used in mathematical model.

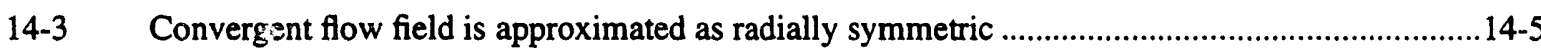

14-4 Schematic showing repository/borehole intersection control volume.........................................14-8

14-5 Canisters emplaced in floor are isolated to some extent from drift flow ..................................14-10

14-6 General repository flow network for repository control volume .............................................14-11

14-7 Specific flow network for control volume of salt repository ................................................14-12

14-8 Storage can occur within the leg in the flow model ..............................................................14-12

14-9 Each fluid flow leg in the repository is divided into a number of transport cells of equal size

14-10 Mathematical one-dimensional transport model for repository

Waste emplacement package hole concentration is a function of time and elevation from the bottom of the hole

14-12 Approximation of source rate and drift concentration

15-1 General conceptual model and one-dimensional mathematical model of granite repository and nearby geologic barrier in Simple PA

15-2 Representation of flow through granite disposal system

15-3 Relative permeability of a connection to the fracture zones in the granite repository is a function of saturation of the repository

16.2-1 Mean composite complementary cumulative distribution functions (CCDFs) for Waste Disposal Group 1 salt evaluated at 2.4-km disposal system boundary in Snake Dolomite, assuming six intrusion times

16.2-2 Mean composite CCDF of normalized integrated activity of only groundwater releases from repository boundary of salt disposal system for all five waste disposal groups

16.2-3 CCDF, conditional on intrusion of fraction of initial, total waste disposal group inventory discharge into backfill-buffer for waste parcels in salt disposal system for all five waste disposal groups

16.2-4 Frequency with which waste parcel activity fraction for each waste disposal group in the salt disposal system was ranked showing, in general, a ranking of $4,5,2,3$, and 1 .

16.2-5 CCDF, conditional on intrusion, of summed normalized activity from the waste disposal groups into the pore space of the backfill-buffer surrounding the waste parcels (curves on right) and summed normalized discharges from repository (up the intrusion boreholes) (curves on left) for the salt disposal system

16.2-6 Frequency with which summed normalized activity from the repository for each disposal group in the salt disposal system was ranked showing, in general, a ranking of $4,5,2,3$, and 1

16.2-7 Contributions of individual isotopes to summed normalized discharges from repository (up the intrusion borehole) for salt repository, Waste Disposal Group 1. 
16.2-8 Contributions of individual isotopes to summed normalized discharges from repository (up

the intrusion borehole) for salt repository, Waste Disposal Group 2.

16.2-9 Contributions of 11 individual isotopes in Complex PA to summed normalized discharges

from repository (up the intrusion borehole) for salt repository, Waste Disposal Group 3 ...........16-13

16.2-10 Contributions of individual isotopes to summed normalized discharges from repository (up

the intrusion borehole) for salt repository, Waste Disposal Group 4...........................................16-14

16.2-11 Contributions of individual isotopes to summed normalized discharges from repository (up the intrusion borehole) for salt repository, Waste Disposal Group 5..........................................16-15

16.2-12 Scatterplot of activity passing repository boundary normalized by EPA release limits vs.

(a) log permeability of the upper intrusion borehole, and (b) log permeability of surrounding

Sodium Springs Formation for salt disposal system, Waste Disposal Group 1............................16-16

16.2-13 Comparison of CCDFs, conditional on intrusion of summed normalized release to the accessible environment in the Snake Dolomite aquifer of the salt disposal system and summed normalized activity discharge from the repository (injected into aquifer),

Waste Disposal Group 1

16.3-1 Mean composite CCDF of normalized integrated activity of only groundwater releases

from the repository boundary of granite disposal system for all five waste disposal groups........16-18

16.3-2 Comparison between salt and granite mean composite CCDFs of summed normalized activity of groundwater releases at the respective repository boundaries for Waste Disposal Groups 1 and 4.

16.3-3 CCDF, conditional on intrusion of fraction of initial waste disposal group inventory discharge into backfill-buffer. granite disposal system, for all five waste disposal groups

16.3-4 Frequency with which waste parcel activity fraction into backfill-buffer for each waste disposal group in the granite repository was ranked showing, in general, a ranking of 4 , $5,2,3$, and 1 .

16.3-5 CCDF, conditional on intrusion, of summed normalized activity discharges from the waste parcels into the pore space of the backfill-buffer surrounding the waste parcels (right curves) and from the repository at the horizontal fracture zones (left curves) from the granite disposal system

16.3-6 Frequency with which summed normalized activity discharge from the repository boundary for each disposal group in the granite repository was ranked showing, in general, a ranking of $5,4,3,1$, and 2 .

16.3-7 Contributions of 11 individual isotopes in Complex PA to summed normalized discharges

at horizontal fracture zones for granite disposal system, Waste Disposal Group 1

16.3-8 Contributions of 11 individual isotopes in Complex PA to summed normalized discharges

at horizontal fracture zones for granite disposal system, Waste Disposal Group 2 .

16.3-9 Contributions of individual isotopes in Complex PA to summed normalized discharges at horizontal fracture zones for granite disposal system, Waste Disposal Group 3.

16.3-10 Contributions of individual isotopes in Complex PA to summed normalized discharges at horizontal fracture zones for granite disposal system, Waste Disposal Group $4 .$.

16.3-11 Contributions of individual isotopes in Complex PA to summed normalized discharges at horizontal fracture zones for granite disposal system, Waste Disposal Group 5 ..

16.3-12 Scatterplot of (a) summed normalized discharge at horizontal fracture zones vs. stainless steel corrosion rate and (b) summed normalized discharge of ${ }^{234} U$ vs. its solubility, showing some correlation for granite disposal system, Waste Disposal Group 1

16.4-1 Representative scatterplot of cumulative mass of brine discharged from repository vs. logarithm of undisturbed permeability of the anhydrite marker bed for Waste Parcel 6 in salt disposal system

16.4-2 Scatterplot of cumulative mass of gas generated vs. the corrosion rate of type 304L stainless steel for Waste Parcel 6 in salt disposal system 
16.4-3 CCDF, conditional on two intrusions, of number of waste parcel inventory equivalents discharged into backfill-buffer for high-level waste in borosilicate glass from DOE sites (WPs 7, 8, and 9), salt disposal system

16.4-4 CCDF, conditional on two intrusions, of number of waste parcel inventory equivalents discharged into backfill-buffer for graphite spent fuel (WPs 1, 10,14, 22, and 24), salt disposal system.....

16.4-5 CCDF, conditional on two intrusions, of number of waste parcel inventory equivalents discharged into backfill-buffer for highly enriched uranium spent fuel (WPs 4, 12, 16, 23, and 25), salt disposal system.

16.4-6 CCDF, conditional on two intrusions, of number of waste parcel inventory equivalents discharged into backfill-buffer for low enriched uranium spent fuel (WPs 5, 13, 17, 23 , and 25), salt disposal system ....

16.4-7 CCDF, conditional on two intrusions, fraction of initial waste parcel inventory by one waste parcel into backfill-buffer for calcine (WPs 6, 11, 18, and 26), salt disposal system ........16-34

16.4-8 Frequency with which waste parcel fractional release for graphite spent fuel (WPs 1, 10, 14,22 , and 24) in salt disposal system was ranked with the least (rank 1) or most (rank 5) discharge.

16.4-9 Frequency with which waste parcel fractional release for highly enriched uranium spent fuel (WPs 4, 12, 16, 23, and 25) in salt disposal system was ranked with the least (rank 1) or most (rank 5) discharge.

16.4-10 Frequency with which waste parcel fractional release for low enriched uranium spent fuel (WPs $5,13,17,23$, and 25) in salt disposal system was ranked with the least (rank 1) or most (rank 5) discharge

16.4-11 Frequency with which waste parcel fractional release for calcine (WPs 6, 11, 18, and 26)

in salt disposal system was ranked with the least (rank 1) or most (rank 5) discharge.

16.4-12 CCDF, conditional on two intrusions, of normalized activity discharge at repository boundary for high-level waste in borosilicate glass from DOE sites (WPs 7, 8, and 9), salt disposal system.

16.4-13 CCDF, conditional on two intrusions, of normalized activity discharge at repository boundary for graphite spent fuel (WPs 1, 10,14, 22, and 24), salt disposal system...

16.4-14 CCDF, conditional on two intrusions, of normalized activity discharge at repository boundary for highly enriched uranium spent fuel (WPs $4,12,16,23$, and 25), salt disposal system......

16.4-15 CCDF, conditional on two intrusions, of normalized activity discharge at repository boundary for low enriched uranium spent fuel (WPs 5, 13, 17,23, and 25), salt disposal system...

16.4-16 CCDF, conditional on two intrusions, of normalized activity discharge at repository boundary for calcine (WPs $6,11,18$, and 26), salt disposal system .

16.4-17 Frequency with which waste parcel fractional release at repository boundary for graphite spent fuel (WPs 1, 10,14, 22, and 24) in salt disposal system was ranked with the least (rank 1) or most (rank 5) discharge .

16.4-18 Frequency with which waste parcel fractional release at repository boundary for highly enriched uranium spent fuel (WPs 4,12,16,23, and 25) in salt disposal system was ranked with the least (rank 1) or most (rank 5) discharge .....

16.4-19 Frequency with which waste parcel fractional release at repository boundary for low enriched uranium spent fuel (WPs 5,13,17,23, and 25) in salt disposal system was ranked with the least (rank 1) or most (rank 5) discharge. $16-42$

16.4-20 Frequency with which waste parcel fractional release at repository boundary for calcine (WPs 6, 11, 18, and 26) in salt disposal system was ranked with the least (rank 1) or most (rank 5) discharge.

16.5-1 Representative scatterplot of fracture flow rate into repository for WP 6 in granite disposal system. $.16-46$

16.5-2 Scatterplot of the stainless steel corrosion rate for WP 11 in granite disposal system $. .16-46$ 
16.5-3 CCDF, conditional on one intrusion, of number of waste parcel inventory equivalents discharged into backfill-buffer for high-level waste in borosilicate glass from DOE sites (WPs 7, 8, and 9), granite disposal system

16.5-4 CCDF, conditional on one intrusion, of number of waste parcel inventory equivalents discharged into backfill-buffer for graphite spent fuel (WPs 2, 3, 10, 15, 22, and 24), granite disposal system.

16.5-5 CCDF, conditional on one intrusion, of number of waste parcel inventory equivalents discharged into backfill-buffer for highly enriched uranium spent fuel (WPs 4, 12, 19, 23 , and 25), granite disposal system

16.5-6 CCDF, conditional on one intrusion, of number of waste parcel inventory equivalents discharged into backfill-buffer for low enriched uranium spent fuel (WPs 5, 13, 20, 23, and 25), granite disposal system

16.5-7 CCDF, conditional on one intrusion, of number of waste parcel inventory equivalents discharged into backfill-buffer for calcine (WPs 6, 11, 21 and 26), granite disposal system ......16-50

16.5-8 Frequency with which waste parcel fractional release for graphite spent fuel (WPs 2, 3, 10 , 15,22 , and 24) in granite disposal system was ranked with the least (rank 1) or most (rank 5) discharge

16.5-9 Frequency with which waste parcel fractional release for highly enriched uranium spent fuel (WPs 4, 12,19,23, and 25) in granite disposal system was ranked with the least (rank 1) or most (rank 5) discharge

16.5-10 Frequency with which waste parcel fractional release for low enriched uranium spent fuel (WPs 5, 13, 20, 23, and 25) in granite disposal system was ranked with the least (rank 1) or most (rank 5) discharge....

16.5-11 Frequency with which waste parcel fractional release for calcine (WPs 6, 11, 21, and 26) in granite disposal system was ranked with the least (rank 1) or most (rank 5) discharge ..........16-52

16.5-12 Scatterplot of the alteration rate for borosilicate glass and total activity discharge from the repository boundary for WP 24 in granite disposal system...

16.5-13 CCDF, conditional on one intrusion, of normalized activity discharge at repository boundary for high-level waste in borosilicate glass from DOE sites (WPs 7, 8, and 9), granite disposal system.

16.5-14 CCDF, conditional on one intrusion, of normalized activity discharge at repository boundary for graphite spent fuel (WPs 2, 3, 10, 15, 22, and 24), granite disposal system

16.5-15 CCDF, conditional on one intrusion, of normalized activity discharge at repository boundary for highly enriched uranium spent fuel (WPs 4,12,19,23, and 25), granite disposal system.

16.5-16 CCDF, cunditional on one intrusion, of normalized activity discharge at repository bou idary for low enriched uranium spent fuel (WPs 5, 13, 20, 23, and 25), granite disposal system.

16.5-17 CCDF, conditional on one intrusion, of normalized activity discharge at repository boundary for calcine (WPs 6,11,21, and 26), granite disposal system.

16.5-18 Frequency with which waste parcel fractional release at repository boundary for graphite spent fuel (WPs 2, 3, 10,15, 22, and 24) in granite disposal system was ranked with the least (rank 1) or most (rank 5) discharge.

16.5-19 Frequency with which waste parcel fractional release at repository boundary for highly enriched uranium spent fuel (WPs 4,12,19,23, and 25) in granite disposal system was ranked with the least (rank 1) or most (rank 5) discharge ...

16.5-20 Frequency with which waste parcel fractional release at repository boundary for low enriched uranium spent fuel (WPs 5, 13,20,23, and 25) in granite disposal system was ranked with the least (rank 1) or most (rank 5) discharge ...

16.5-21 Frequency with which waste parcel fractional release at repository boundary for calcine (WPs 6, 11, 21, and 26) in granite disposal system was ranked with t... least (rank 1) or most (rank 5) discharge 
16.6-1 Mean time of perforation (breach) for all protective layers in all waste parcels in repository of Waste Disposal Group 1, in the Complex PA (BRAGFLO_T), salt disposal system...

16.6-2 Mean time of perforation (breach) for all protective layers in all waste parcels in repository of Waste Disposal Group 1, in the Simple PA (SALFLOW), salt disposal system.

16.6-3 Mean time of perforation (breach) for all protective layers in all waste parcels in repository of Waste Disposal Group 1, in the Complex PA (BRAGFLO_T), granite disposal system

16.6-4 Mean time of perforation (breach) for all protective layers in all waste parcels in repository of Waste Disposal Group 1, in the Simple PA (GRFLOW), granite dispos:i: system

16.6-5 Exposure times in vertical cross-section ( $\mathrm{i}=11$ ) of Waste Disposal Group 1, in the Complex PA, for the salt disposal system

16.6-6 Breach times for containers in Waste Disposal Group 1 in salt disposal system using Complex PA consequence models

16.6-7 Breach times for containers in Waste Disposal Group 1 in granite disposal system using Complex PA consequence models

16.6-8 Rank of penetration rate assuming fully saturated conditions for stainless steel and lead for the salt disposal system.

16.6-9 Rank of penetration rate assuming fully saturated conditions for stainless steel and lead for the granite disposal system

16.6-10 Exposure times for Waste Disposal Group 5, run 9, in the Complex PA (BRAGFLO_T) for (a) the salt disposal system and (b) the granite disposal system.

16.7-1 Mean breach times of all mesh elements for containers for waste parcels in Waste Disposal Group 1 in salt disposal system using Complex PA consequence models

16.7-2 Mean breach times of all mesh elements for containers that have defects (median of $0.5 \%$ ) for waste parcels in Waste Disposal Group 1 in salt disposal system using Complex PA consequence models

16.7-3 Mean breach times of all mesh elements for containers for waste parcels in Waste Disposal Group 1 in granite disposal system using Complex PA consequence model:s.

16.7-4 Mean breach times of all mesh elements for containers that have defects (median of $0.5 \%$ ) for waste parcels in Waste Disposal Group 1 in granite disposal system using Complex PA consequence models.

16.7-5 CCDFs, conditional on two intrusions, of summed normalized activity discharge at repository boundary for Waste Disposal Group 1 in reference containers (refer also to 16.2-5) and corrosion-resistant containers for the salt disposal system

16.7-6 CCDFs, conditional on two intrusions, of summed normalized activity discharge at repository boundary for Waste Disposal Group 1 in reference containers (refer also to 16.3-5) and corrosion-resistant containers for the granite disposal system.

16.8-1 Cumulative distribution function (CDF) of fraction of initial tracer mass released. $16-78$

16.8-2 Comparison of CCDF, conditional on two intrusions, for transport of an extended list of radionuclides and the standard list of radionuclides in the Simple PA consequence models of the salt disposal system for (a) waste parcel equivalents discharged from waste parcels and (b) waste parcel equivalents discharged from repository boundary ....

16.8-3 Comparison of CCDF, conditional on one intrusion, for transport of an extended list of radionuclides and the standard list of radionuclides in the Simple PA consequence models of the granite disposal system for (a) waste parcel equivalents discharged from waste parcels and (b) waste parcel equivalents discharged from repository boundary. 
16.9-1 Conditional CCDFs comparing simple and complex consequence model results for total EPA normalized for the five waste disposal groups, in salt disposal system, (a) activity discharge from waste parcels and (b) defined discharge from repository boundary

16.9-2 Conditional CCDFs comparing simple and complex consequence model results for total EPA normalized for the five waste disposal groups, in granite disposal system, (a) activity discharge from waste parcels and (b) defined discharge from repository boundary

16.9-3 Comparison of conditional CCDFs of complex consequence model with and without waste in panel intersected by lower vertical secondary fracture, Waste Disposal Group 1, in granite disposal system.

16.9-4 Comparison of conditional CCDFs of simple and modified complex consequence models for Waste Disposal Group 1, in granite disposal system .

16.10-1 Composite CCDF of summed normalized releases from cuttings/cavings from the salt repository $\left(\lambda^{*}=30\right.$ borehole $\left./ \mathrm{km}^{2} / 10,000 \mathrm{yr}\right)$ for all waste disposal groups

16.10-2 Composite CCDF of summed normalized releases from cuttings/cavings from the granite repository $\left(\lambda^{*}=3\right.$ borehole $\left./ \mathrm{km}^{2} / 10,000 \mathrm{yr}\right)$ for all waste disposal groups... 


\section{Tables}

ES-1 Treatment Options Used in the INEL Performance Assessment .....................................................

ES-2 Summary of Information Gathered about Waste Form ............................................................

ES-3 Summary of Information Gathered about Waste Containers ....................................................12

ES-4 Summary of Information Gathered about the Repository ........................................................13

ES-5 Summary of Information Gathered about the Geologic Barrier..........................................ES-14

ES-6 Summary of Information Gathered about the Agents Acting on the Disposal System ..............ES-15

ES-7 Recommendations for Future Analysis and Waste Treatment Program Tasks

1-1 Selected Statutes and Regulations Affecting the Disposal of Radioactive Waste in

Geologic Repositories

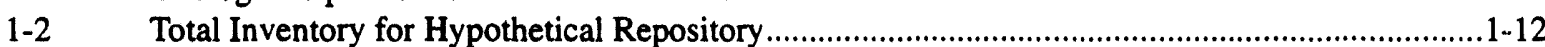

2-1 Regulatory Waste-Disposal Objectives/Criteria at the International Level and in Individual Countries

Event-and-Process and Probability-Model Parameters of Consequence Models Used in 1991 WIPP PA Exercises.

Data Summary for Waste from Savannah River, Hanford, and West Valley.................................4-9

Radionuclides Considered in Performance Assessment ..........................................................4-10

Comparison of Radionuclides Selected for Transport with Inventory Exceeding a

Normalized Value of 0.1 and 0.01 during the Period of $1000 \mathrm{yr}$ in the $10,000 \mathrm{yr}$

Regulatory Period.

4-7 Units of Waste for the INEL PA, Based on 40 CFR 191, Appendix A....................................4-22

4-8 Waste Parcels Found in Waste Disposal Groups 1 through 5 .............................................4-30

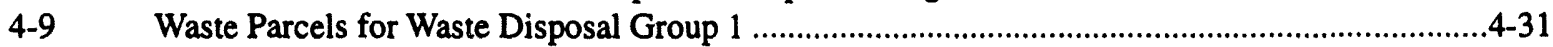

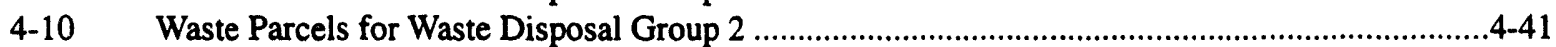

4-11 Waste Parcels for Waste Disposal Group 3 ........................................................................4-46

4-12 Waste Parcels for Waste Disposal Group 4 ..............................................................................55

4-13 Waste Parcels for Waste Disposal Group 5 ...................................................................4-58

5-1 Summary of Salt Deposits in Coterminous United States...........................................................5-6

5-2 Parameter Values for Halite and Polyhalite within Sodium Springs Formation near

Repository...

5-3 Parameter Values for Anhydrite Marker Bed within Sodium Springs Formation near

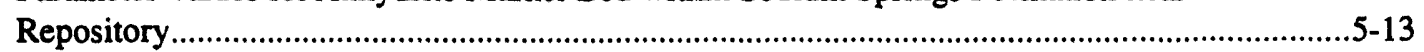

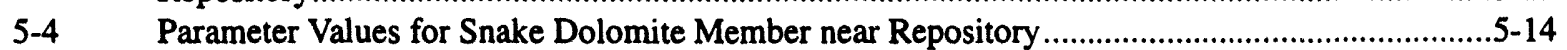

5-5 Parameter Values for Tower Formation beneath Repository ..................................................5-15

5-6 Parameter Values for Global Materials and Agents on the Salt Disposal System ........................5-16

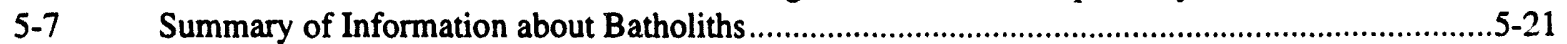

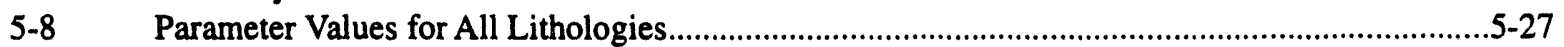

5-9 Parameter Values for Massive Gray Granite ...................................................................5-28

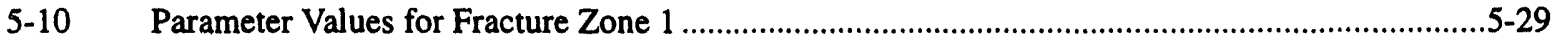

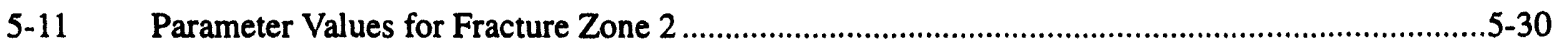

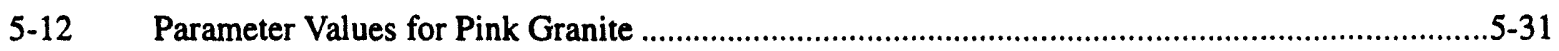


5-14 Parameter Values for Global Materials and Agents Acting on the Granite Disposal

System

6-1 Total Number of Waste Parcels and Rooms Required for Five Waste Disposal Groups in

Salt and Granite Repositories

Backfill-Buffer Between Waste Parcel and Host Rock (Granite).

Backfill in Drift and Panels (Salt)

10-1 Contents (in Curies) of 49 Radionuclides of Waste Parcels at Time of Emplacement (year 2030) and after $1000 \mathrm{yr}$

11-1 Corrosion Resistance in Waste Parcel Layer Materials

12-1 Analysis Monitoring Points for Salt Disposal System …......................................................12-20

13-1 Analysis Monitoring Points for Granite Disposal System ....................................................13-7

16.4-1 Number of Discharges from Waste Parcel (out of a Total of 20) Showing Significant Correlation Between the Ranks of Independent and Dependent Flow Variables in Salt Disposal System for the Simple PA.

16.4-2 Number of Waste Parcel Designs with Discharges from Repository having Significant

Correlations with Sampled Flow Parameters in Bedded Salt for Simple PA.

16.4-3 Number of Waste Parcel Designs with Discharges from Repository having Significant

Correlations with Sampled Transport Parameters in Bedded Salt for Simple PA

16.5-1 Number of Waste Parcel Designs with Discharge from Waste Parcel (Out of a Total of 21) showing Significant Correlation Between the Independent and Dependent Flow Variables in the Granite Disposal System for the Simple PA.

16.5-2 Number of Waste Parcel Designs with Discharges from Repository having Significant Correlations with Sampled Transport Variables in Granite for the Simple PA 
16.5-3 Number of Waste Parcel Designs with Discharges from Repository having Significant

Correlations with Sampled Transport Variables in Granite for the Simple PA $16-61$

16.7-1 Materials Used in Layers for Individual Corrosion-Resistant and Reference Waste Parcels.......16-72

16.8-1 Radionuclides Included in Extended Simple PA, Simple PA, and Complex PA.........................16-82

16.10-1 Waste Group Release Ranking Based on CUTTINGS Only Conditional on Hitting the Repository.

16.11-1 Summary of Information Gathered about Waste Form

16.11-2 Summary of Information Gathered about Waste Containers ....................................................16-96

16.11-3 Summary of Information Gathered about the Repository

16.11-4 Summary of Information Gathered about the Geologic Barrier.

16.11-5 Summary of Information Gathered about the Agents Acting on the Disposal System

16.11-6 Recommended Tasks of the Waste Treatment Program 


\title{
Executive Summary
}

\author{
R. P. Rechard
}

The purpose of this report is to assist the Department of Energy (DOE) in evaluating the potential value of various waste treatment options on disposal of nuclear materials in a deep geologic repository. Questions that were addressed included what decisions about waste treatment could be made before a repository site had been identified and what decisions should be made only after a repository site was known. This evaluation may also lead to the development of minimum acceptance criteria for disposal of the waste in a generic, deep geologic repository and aid the DOE in developing and demonstrating the capability for nuclear waste disposal. The waste-treatment options must ensure that disposal will meet long-term environmental requirements (performance metrics). The single environmental requirement considered in this performance assessment is the Environmental Protection Agency (EPA) standard, Environmental Radiation Protection Standards for Management and Disposal of Spent Nuclear Fuel, HighLevel and Transuranic Radioactive Wastes, 40 CFR 191, as promulgated in 1985. Although the U.S. Court of Appeals vacated sections of Subpart B of 40 CFR 191 and remanded them to the EPA for revision, this report treats 40 CFR 191 as if all parts of the standard were still in effect. Current information indicates that the repromulgation of 40 CFR 191 will likely extend the limits on the Individual Protection Requirements to 10,000 $\mathrm{yr}$ (the same regulatory period as the Containment Requirements) and, thereby, possibly increase the importance of dose calculations. 40 CFR 191 was chosen here because it is expected to be applied fairly soon in a modified form at the Waste Isolation Pilot Plant, which is a repository in bedded salt for waste contaminated with transuranic radionuclides from the DOE's nuclear weapons complex.

In this study, the placement of containers of various radioactive materials in two liquid-saturated generic sites was examined for compliance with 40 CFR 191. The sites considered were bedded salt and igneous rock (granite). These geologic media were thought different enough to highlight various aspects of the 40 CFR 191 regulation (e.g., the salt site has releases solely by human intrusion, but the granite site has the possibility of some releases even when the site is undisturbed), yet similar enough (e.g., both fully saturated) to be analyzed within the time and budget given. $^{\dagger}$ The materials considered for disposal were graphite spent nuclear fuel, experimental ("special") spent nuclear fuel, and some high-level waste (HLW) that was generated during processing of fuel stored at the Idaho National Engineering Laboratory (INEL). These materials are described more fully in the following sections. The wastes were evaluated individually in an analysis that used ordinary differential equations in a mathematical model and is referred to here as the "Simple PA." They were also evaluated in combination in five disposal groups to more realistically simulate the impact of the waste forms on repository response. This analyses used partial differential equations in a mathematical model and is referred to here as the "Complex PA." For the Complex PA, the repository was assumed to also contain defense high-level radioactive waste immobilized in borosilicate glass from the Savannah River site, the Hanford Reservation, and the West Valley Demonstration Plant to study the effect of INEL waste forms in a reasonably sized repository. The waste from the DOE sites was selected as a reference for comparison with the high-level waste and spent fuel from INEL even though the DOE waste has been tentatively designated for Yucca Mountain, the proposed repository in Nevada for spent fuel from public utilities.

This summary emphasizes the general findings of the performance assessment and the results of those findings in the form of guidance for the Spent Nuclear Fuel and High-Level Waste Management Technology Development Program at INEL. The findings are presented in Section ES.1 of this summary. The general recommendations for the future performance assessments and guidance for the technology development program are provided in Section ES.2. As background, a brief discussion of the methodology used in the performance assessment is presented in Section ES.3, and the caveats and assumptions in the evaluation are underscored in Section ES.4.

\footnotetext{
- At this time, it is not clear what the environmental requirements will be for an actual geologic repository for high-level waste and spent fuel. Currently, the National Academy of Sciences, at the request of the U.S. Environmental Protection Agency and based on requirements in the Energy Policy Act of 1992 (EPA of 1992), is exploring possibilities for the development of a new standard to replace 40 CFR 191 for the highlevel waste and spent nuclear fuel repository at Yucca Mountain, NV. This action also affects the NRC regulation, 10 CFR 60 . See Chapter 2 for more discussion

$\dagger$ At present, the only site in the United States for a deep geologic repository for high-level waste and spent fuel is at Yucca Mountain, NV, in unsaturated tuff. Analysis of this site was not pursued in this initial performance assessment because of the technical challenges presented by unsaturated tuff and because DOE had not yet designated the spent fuel from INEL for Yucca Mountain. Therefore, analysis of a site in unsaturated tuff was delayed until the second year of this 2- to 3-yr effort.
} 


\section{ES.1 Analysis Approach}

The focus of this analysis, as mentioned previously, is to help guide the DOE in its decisions about how to prepare high-level wastes and spent fuel stored at INEL for eventual permanent disposal. Specific goals were to (1) evaluate the potential impacts of various waste treatment options on a compliance determination for materials disposed of in a deep geoiogic repository (Section ES.3.1), (2) provide information that could be used in developing minimum waste acceptance criteria (Section ES.3.2), and (3) provide information that could be used to guide technology development programs (Section ES.3.3). The approach taken in designing an analysis to meet these goals was to (a) consider five possible waste-treatment options, (b) use the methodology developed by Sandia National Laboratories for assessing the long-term performance of the resulting waste forms in a deep geologic disposal system, (c) examine several levels of simplification in the consequence models, and (d) propose several iterations of the analysis process. The first aspect of the analysis approach-evaluating the impacts of the five treatment options-is discussed in Section ES.2. A discussion of the other three aspects follows.

The primary means of determining whether waste is acceptable for geologic disposal is through a process called a performance assessment. In general, a performance assessment is an analysis that assesses whether a system meets a set of long-term performance criteria. For the analysis documented in this report, the system is actually a composite mathematical model that represents a deep geologic repository disposal system for spent nuclear fuel and high-level waste, and the performance criteria are various long-term (10,000-yr) metrics (not short-term operational safety issues) specified by U.S. government regulations (e.g., 40 CFR 191). In essence, the performance assessment can be viewed as the complex process that estimates various performance metrics (including their uncertainties) for comparison with the applicable regulations. The methodology developed by Sandia National Laboratories for assessing a disposal system resembles a probabilistic risk assessment (PRA) in which the performance measure is a radioactive release specified in probabilistic terms, specifically, as a complementary cumulative distribution function (CCDF).

In an engineering analysis, physical phenomena (e.g., processes in the repository and the host rock) are simplified. Although the level of simplification that is appropriate cannot always be known a priori, evaluating the apprnpriate level is an important task. Two distinct levels of simplification of the consequence models of the disposal system (or categories of calculations) were performed for this report: (1) calculations to assess disposal system performance using sophisticated, lumped-parameter models (Simple PA), and (2) calculations to assess disposal system performance using a model based on spatially distributed parameters (Complex PA). Also, scoping calculations were performed prior to the Simple and Complex PAs (which were performed in parallel) to evaluate important features, events, and processes. The general findings from all three categories of calculations were presented in this summary.

Furthermore, in this performance assessment, the major emphasis was on evaluating the potential impact of various waste treatment options on a compliance determination for materials disposed of in a deep geologic repository. This evaluation may also leac to development of minimum acceptance criteria for disposal of the spent nuclear fuel and high-level waste. Because only one set of performance criteria, 40 CFR 191, was examined in this performance assessment, neither focus can be accomplished through this report alone; studies examining other environmental performance criteria are necessary. ${ }^{\ddagger}$

The process of performing a probabilistic risk assessment or performance assessment is preferably iterative (i.e., in general, the assessments are performed periodically, each covering different areas or exploring new concepts while reevaluating results of previous assessments). In this way, the system under study becomes better understood. Because this performance assessment of the waste treatment options is the first iteration, neither the data underlying the model parameters nor the model form is fixed; all aspects of this study are subject to review and subsequent change in future iterations. This report provides an opportunity for inturested parties to consider some of the options that the DOE may explore in the future and to give constructive input for future performance assessments.

\footnotetext{
\# As noted car uer in this summary, the environmental regulations for disposal sites in the United States are currently undergoing revision, and so
} perhaps only the internationally recognized criterion of individual doses can be easily identified at this time. 


\section{ES.2 General Findings from the Analysis}

The focus of the performance assessment documented in this report was to investigate the total system behavior of possible treatment options for spent fuel and high-level waste in a deep geologic repository.

This performance assessment uncovered many pieces of information about the waste form and its interaction with the disposal system itself. In many cases, the performance assessment analysis identified important parameters (i.e., data that are critical for determining how the system will perform over the long term). In some cases, the analysis pointed to data that are considered valuable but which are either limited or not currently available. The acquired information and modeling issues are categorized into five areas: conclusions about the waste form, the waste containers, the repository, the geologic barrier, and the agents acting on the disposal system, and are described in the following sections. These conclusions are presented here as a summary of Chapter 16, "Results and Conclusions," which contains information supporting these findings.

\section{ES.2.1 Waste Form}

The waste form is the type of waste (e.g., graphite spent fuel or special spent fuel) in combination with a specific treatment option (i.e., the form of the waste just before placement in a canister). The type of waste materials considered for disposal were graphite spent nuclear fuel, experimental ("special") spent nuclear fuel, and some high-level radioactive : iste (HLW) that was generated during processing of fuel stored at the Idaho National Engineering Laboratory (INEL). The unprocessed spent fuel is similar in some aspects to public utility commercial spent fuel; however, the concentration of enriched uranium, ${ }^{235} \mathrm{U}$, is much greater and the degree of burnup much less in some of the unprocessed spent fuel. The graphite spent fuei, special spent fuel, and high-level waste represent about 700 metric tons of heavy metal (MTHM). ${ }^{* *}$ Although each waste form was studied individually, the waste forms were also combined into five waste disposal groups. When the forms were studied in combination, the repository was assumed also to contain radioactive waste produced and/or stored at the Savannah River site, the Hanford Reservation, and the West Valley Demonstration Plant. The high-level waste from these DOE sites was added to the hypothetical repository to illustrate a more realistic mix of waste in the repository and to simulate the impact of the INEL waste forms in such a repository.

Five treatment options that applied to each of the three general waste types (i.e., graphite spent fuel [sometimes specified as Fort St. Vrain fuel and Peach Bottom fuel], experimental or "special" spent fuel [sometimes grouped as low enriched uranium and highly enriched uranium], and calcine high-level waste) were considered in this performance assessment. ${ }^{\dagger \dagger}$ Although many combinations of treatments could be constructed, the five waste treatment options selected ranged from incorporating currently existing spent fuel and high-level waste directly into waste parcels to encapsulating or processing spent fuel and high-level waste into a glass-ceramic matrix. These five waste treatment options roughly correspond to one low, two moderate, and two high levels of stabilization or processing of the spent fuel and high-level waste. Also, in some options waste types were comingled. When more than one waste form was studied together, the forms were combined into five "waste-disposal groups" from the 26 individual waste parcels. (In this report, the waste parcel is defined as the waste form, internal backfill, and waste canister.) The five Waste Disposal Groups are briefly described in Table ES-1. Figures ES-1 through ES-5 show the 26 waste parcels that make up each waste disposal group. Each waste disposal group includes the high-level waste immobilized in borosilicate glass from the Savannah River site, the Hanford reservation, and the West Vailey Demonstration Project.

\footnotetext{
- Another initialism, MTIHM (metric tons of initial heavy metal), is used by some authors who wish to emphasize that the measurement is the initial mass of heavy metai rather than the current mass of heavy metal. In this report, we use the designation, MTHM, not only because it is found more frequently in the literature but also because 40 CFR 191 defines "heavy metal" as "... all uranium, plutonium, or thorium placed into a reactor ..." (40 CFR 191.12; emphasis added). Thus, the use of MTIHM, while useful in calling attention to this fact, is not necessary.

${ }^{7 t}$ The treatment options considered and the results are relevant not only to the waste stored at the INEL but also to the DOE sites as a whole because the spent fuel and high-level waste stored at the INEL are similar to spent fueis and waste stored at other DOE sites.
} 
Table ES-1. Treatment Options Used in the INEL Performance Assessment

\begin{tabular}{|c|c|c|}
\hline $\begin{array}{c}\text { Treatment } \\
\text { Level }\end{array}$ & $\begin{array}{l}\text { Waste } \\
\text { Disposal } \\
\text { Group }\end{array}$ & Description of Treatment Option \\
\hline Low & 1 & $\begin{array}{l}\text { Existing spent fuel and high-level waste is placed directly in containers (Figure ES- } \\
\text { 1). For graphite spent fuel, the graphite blocks and graphite rods are placed in the } \\
\text { waste parcel (whole block disposal). Special spent fuel is placed directly in a canis- } \\
\text { ter, and the void space is filled with lead backfill (special spent fuel disposal). Liquid } \\
\text { high-level waste is calcined, and this new waste and existing calcined waste are } \\
\text { placed directly in containers (granular, calcined disposal). }\end{array}$ \\
\hline Moderate & 2 & $\begin{array}{l}\text { Graphite spent fuel is processed by separating the spent fuel compacts from the } \\
\text { graphite block and rods (rod/block separation) (Figure ES-2). The graphite block } \\
\text { and rods are disposed of separately, possibly at a low-level waste site. Liquid high- } \\
\text { level waste is calcined and combined with existing calcine, then heated and com- } \\
\text { pressed into a glass-ceramic matrix (Hot Isostatic Press [HIP]) (140 MPa pressure, } \\
1323 \mathrm{~K} \text { temperature) (spent fuel/calcine glass-ceramic). The spent fuel is placed in } \\
\text { the center of the containers and the gap filled with HIPs. Any remaining HIPs are } \\
\text { placed alone in containers. }\end{array}$ \\
\hline Moderate & 3 & $\begin{array}{l}\text { Graphite is burned, and the fuel particles are encapsulated in a glass-ceramic } \\
\text { matrix (Figure ES-3). Liquid high-level waste is calcined. Special spent fuel and } \\
\text { calcined waste are packaged together, but the calcined waste is immobilized in a } \\
\text { borosilicate glass matrix. }\end{array}$ \\
\hline High & 4 & $\begin{array}{l}\text { Enriched uranium and plutonium are recycled from the graphite and special spent } \\
\text { fuels as a valuable resource, and the remaining waste is mixed in a glass-ceramic } \\
\text { matrix (spent fuel recycling) (Figure ES-4). High-level liquid waste is calcined, then } \\
\text { compressed into a glass-ceramic matrix (HIP) but is packaged separately from the } \\
\text { processed spent fuel. }\end{array}$ \\
\hline High & 5 & $\begin{array}{l}\text { Graphite is burned and special spent fuels are dissolved (Figure ES-5). Depleted } \\
\text { uranium is also dissolved and added at the average ratio of about } 6: 1 \text { (however, the } \\
\text { ratio may be as high as } 140: 1 \text { for graphite fuel or } 200: 1 \text { for the highly enriched ura- } \\
\text { nium)," to give a }{ }^{235} \mathrm{U} \text { content of the waste equivalent to naturally occurring ura- } \\
\text { nium. The resulting liquid waste is then calcined and immobilized in borosilicate } \\
\text { glass. High-level waste is calcined and immobilized in a borosilicate glass but is } \\
\text { packaged separately from the processed spent fuel. }\end{array}$ \\
\hline
\end{tabular}

- Constraints on the moderate and high waste treatment options, such as development time required or costs, have not yet been evaluated for their conformance with desired timetables or economic realism. If this report had indicated a strong need for moderate or high treatment options, then the evaluation of these constraints would have begun upon the completion of both phases (years) of this initial performance assessment.

** In this performance assessment, the activity of depleted uranium is not added to the waste unit factor for the repository normalization factor. If it were, the performance of this treatment option would be significantly improved beyond its already good performance. 

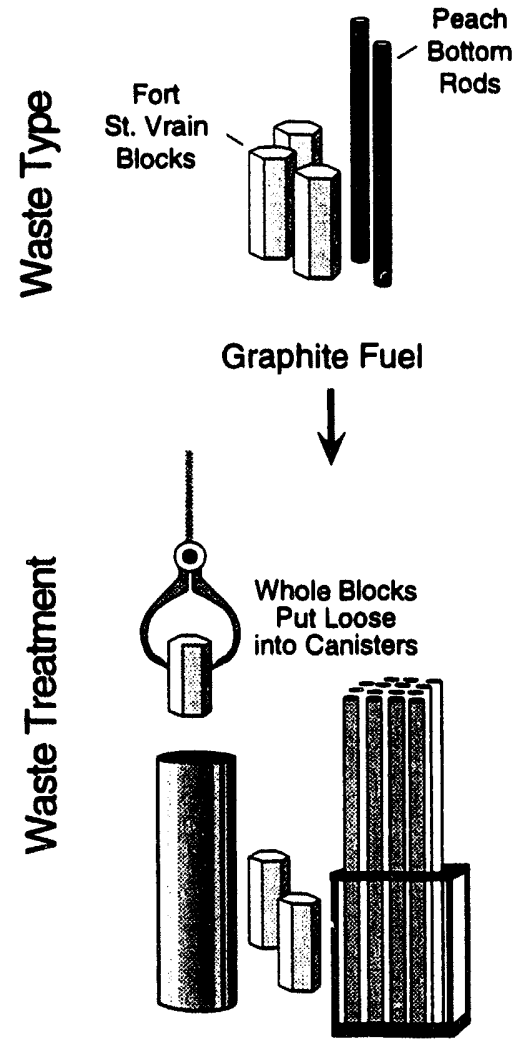

Whole Blocks and Rods

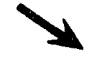

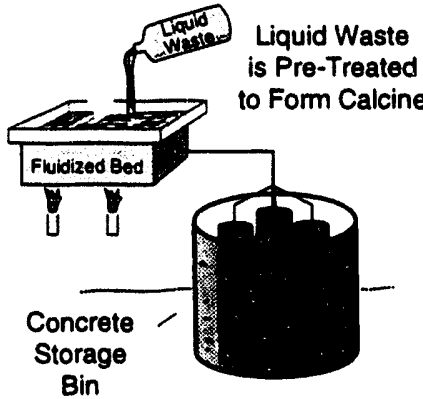

Calcine HLW
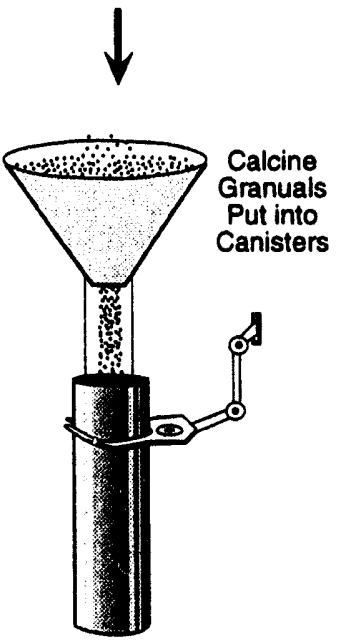

Calcine Granules

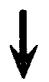

INEL Waste

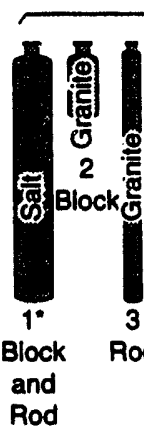

- Number Identifies Waste

Parcel for Modeling

Drawing Not to Scale

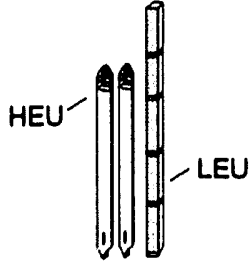

High and Low Enriched Fuel<smiles>C[14CH2]C</smiles>

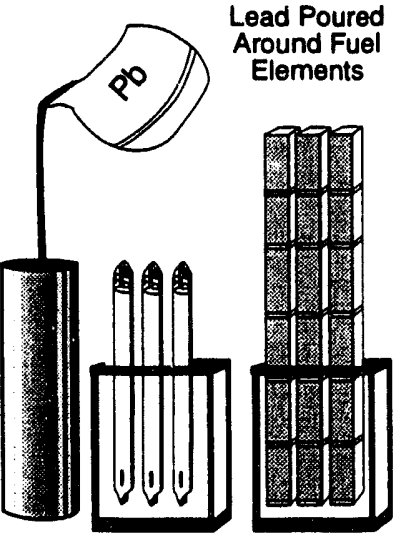

Fuel Elements in Lead

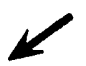

$$
\text { DOE }
$$

Borositicate Glass
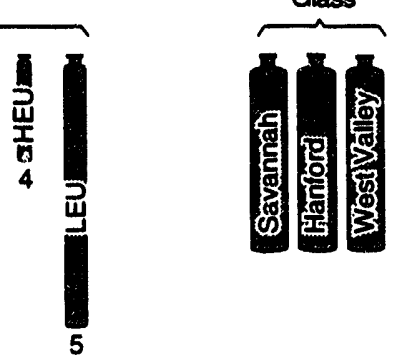

TRI-6342-3711-0

Figure ES-1. Treatment options used in Waste Disposal Group 1. The treatment options include placing currently existing spent fuel and high-level waste directly into waste parcels. The waste parcel numbers shown on this figure identify specific waste parcel containers and contents; these are described in Chapter 4. 

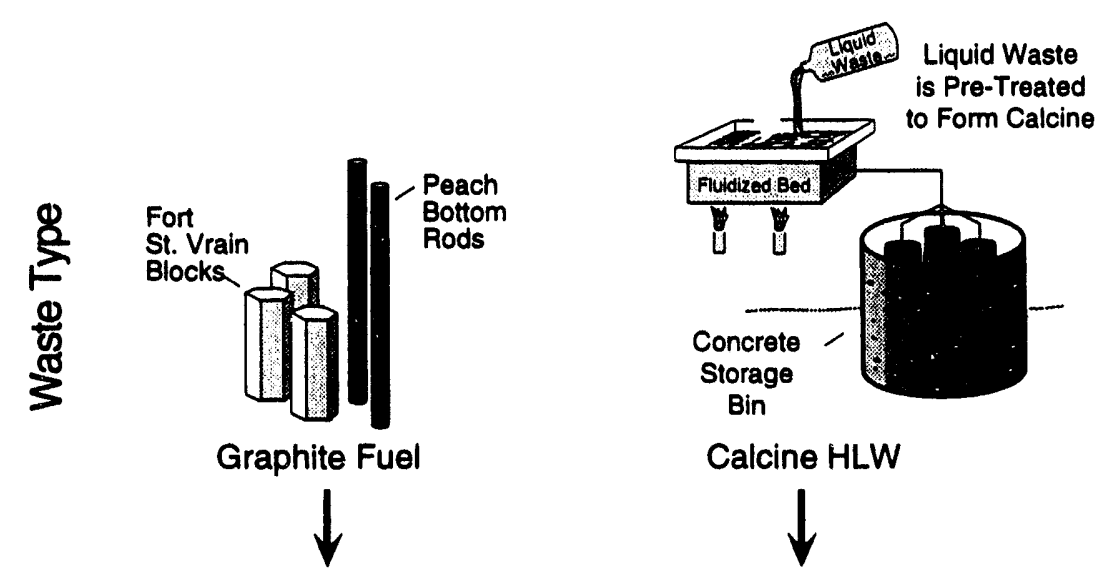

Calcine HLW<smiles>C1CCC1</smiles>
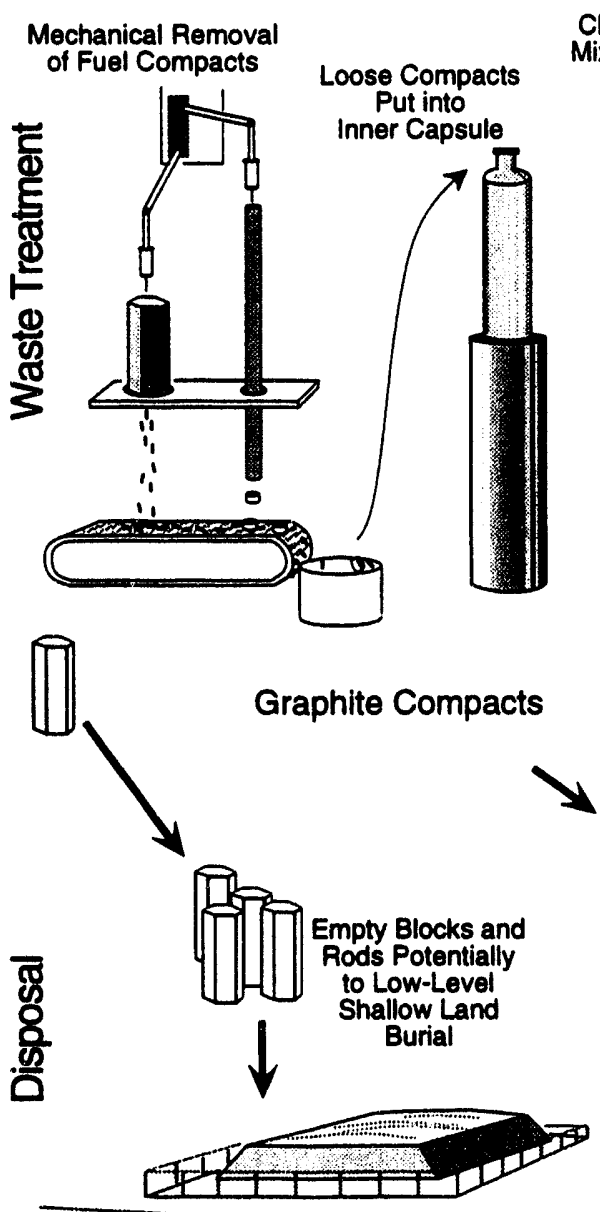

"Number Identifies Waste

Parcel for Modeling

Drawing Not to Scale

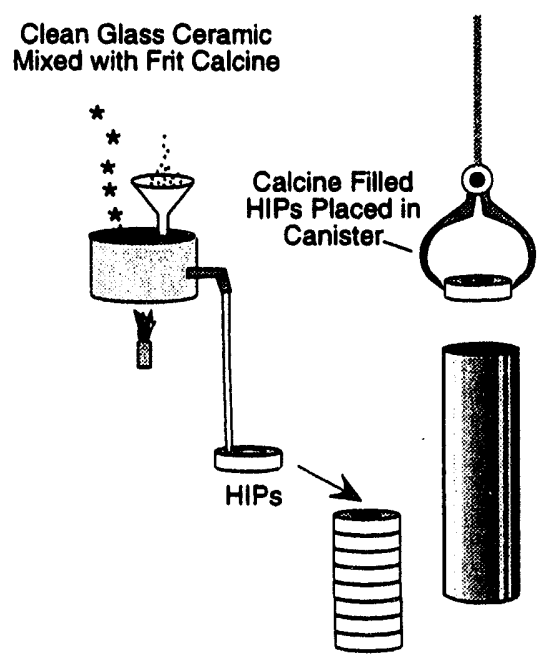

Calcine HIPs

INEL Waste

$\downarrow$

To Salt or Granite

Repository
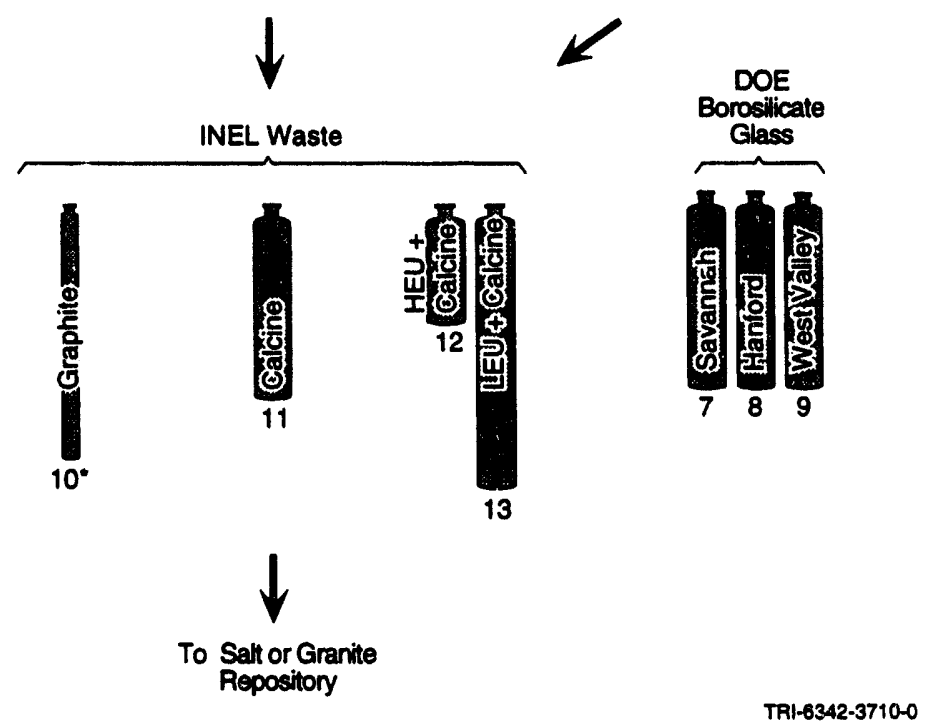

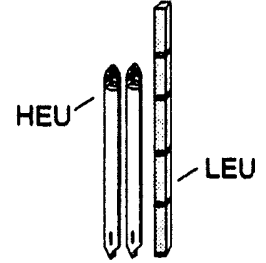

High and Low Enriched Fuel<smiles>C[14CH2]</smiles>

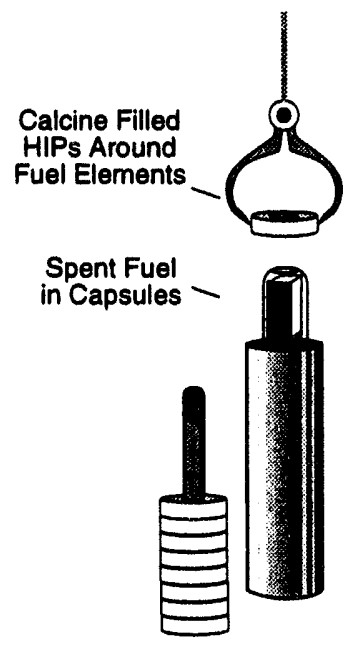

Fuel Elements and HIPs

Figure ES-2. Treatment options used in Waste Disposal Group 2. For the graphite spent fuel, the option considered is rodblock separation. For the special spent fuel and the high-level waste, the option is to package the special spent fuel and calcine high-level waste together, with the calcine waste emplaced in compressed glass ceramic. 

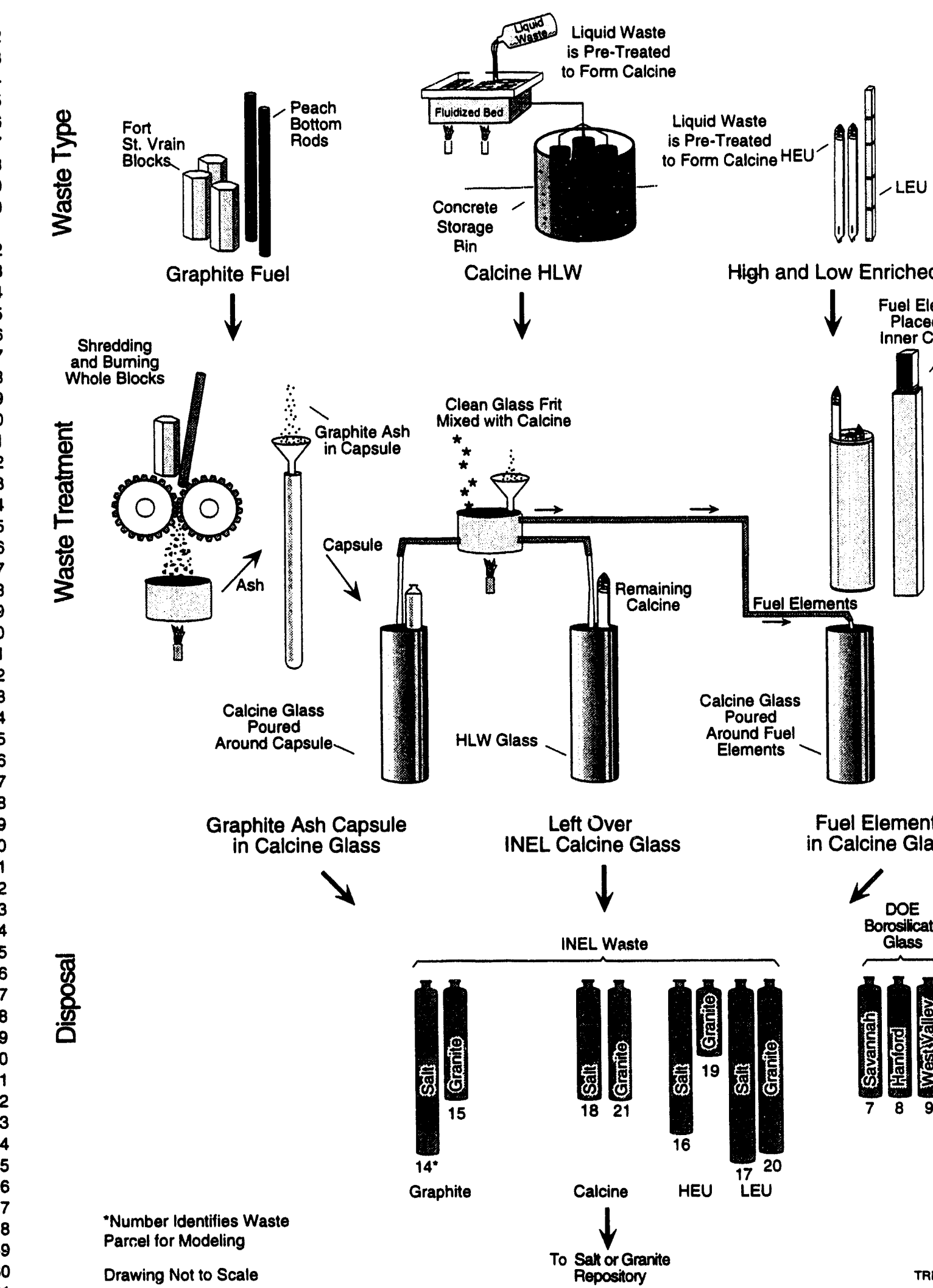

High and Low Enriched Fuel

Clean Glass Frit Mixed with Calcine
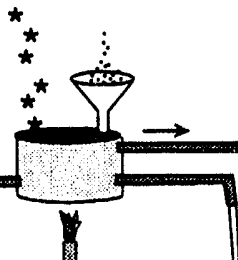

HLW Glass

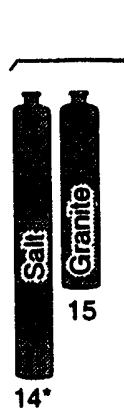

INEL Waste

Graphite
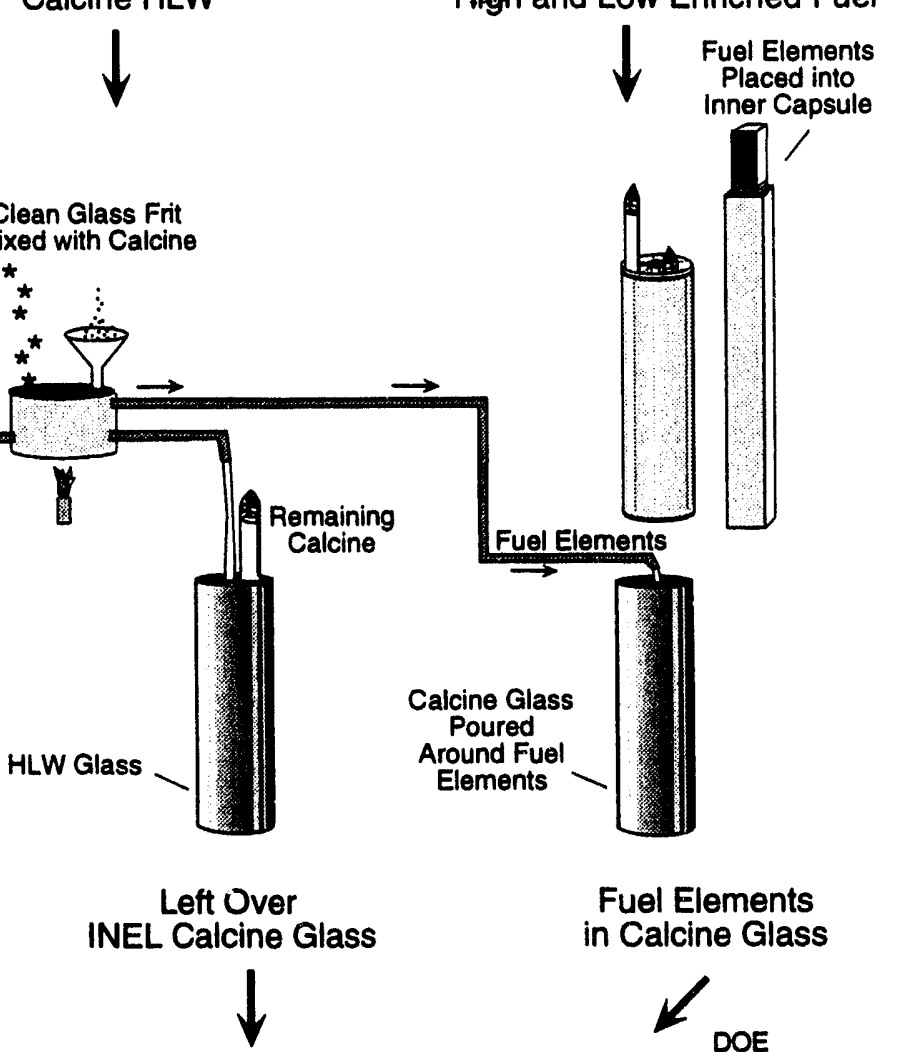

Figure ES-3. Treatment options used in Waste Disposal Group 3. For the graphite spent fuel, the option is graphite burning, with the fuel particles encapsulated in glass-ceramic. The spent fuel and calcine high-level waste are packaged together, with the calcine waste mixed in borosilicate glass. 


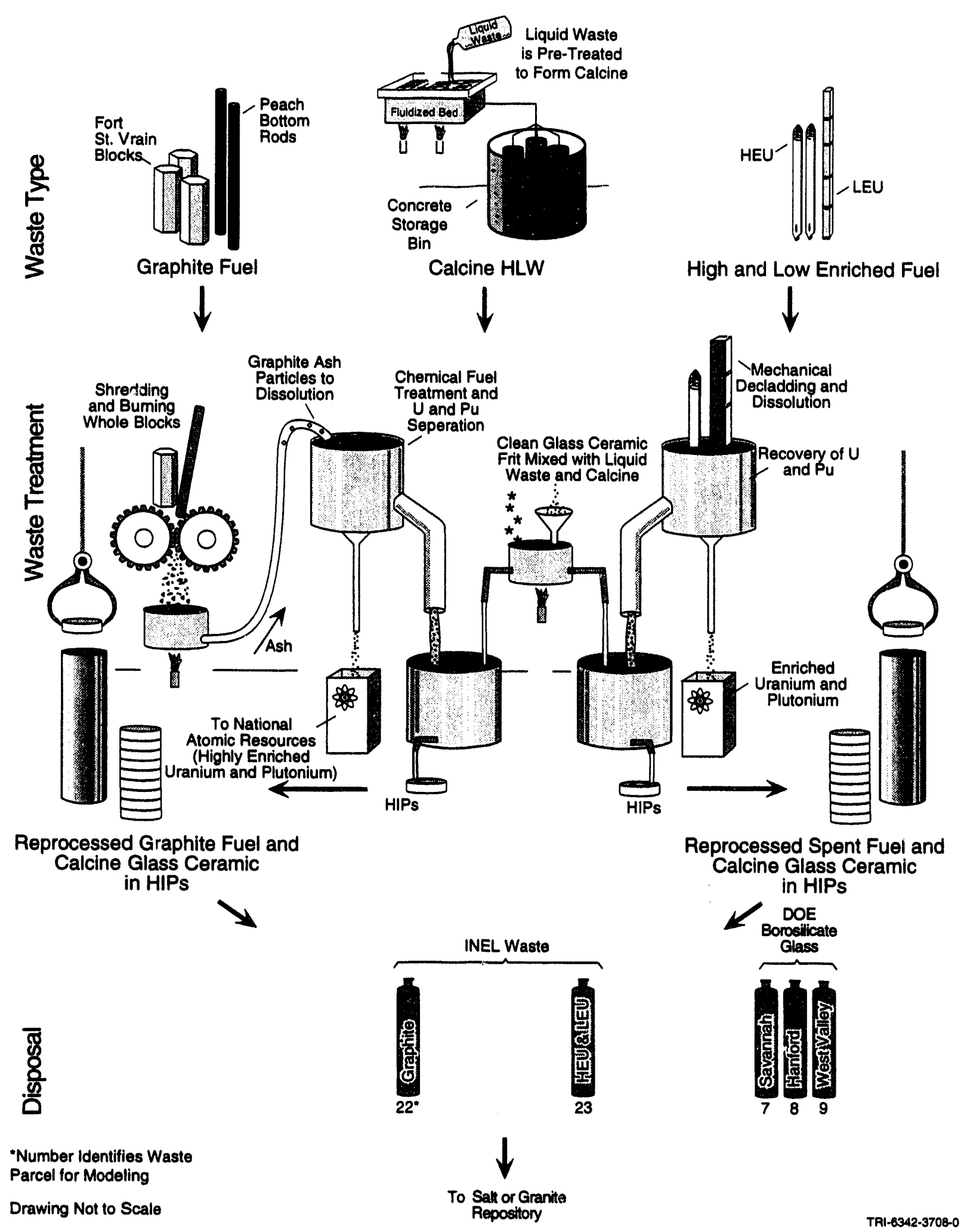

Figure ES-4. Treatment options used in Waste Disposal Group 4. For the graphite and special spent fuels, the option is to recycle the spent fuels and mix the remaining waste with borosilicate glass or glassceramic. Like option 2, the high-level waste is mixed in compressed glass ceramic but is packaged separately from the processed spent fuels. 


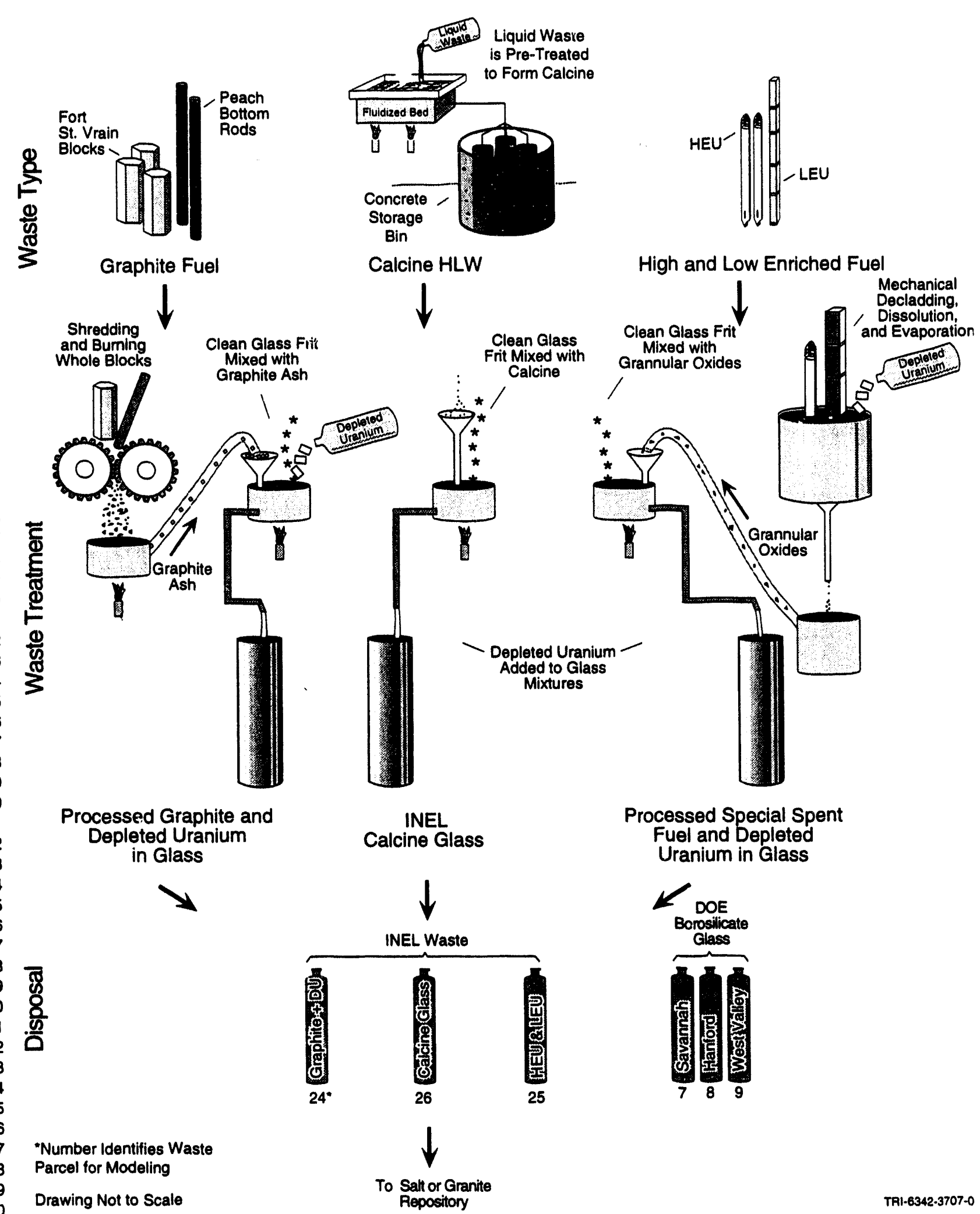

Figure ES-5. Treatment options used in Waste Disposal Group 5. For the graphite and special spent fuels, the option includes oxidation and dilution with depleted uranium. Like option 3, the high-level waste is mixed in borosilicate glass but then is packaged separately from the processed spent fuels. 
Table ES-2 is a summary of the information acquired about the waste form. Because all proposed waste forms were acceptable for compliance with 40 CFR 191, the findings that related to improvement of the treatment options are not presented here. (They are provided, however, in Chapter 16.)

Table ES-2. Summary of Information Gathered about Waste Form

1. All proposed waste forms are acceptable for compliance with 40 CFR 191 in salt and granite disposal systems. Specifically,

- In salt, all waste forms are acceptable without either a container or adsorptive, clay backfill.

- In granite, all waste forms are acceptable with a stainless steel container and clay backfill to adsorb radionuclides, but could possibly require at least a moderately corrosion-resistant container.* (Note that acceptability is highly dependent on the modeling parameters for the granite site, see Section ES.4.)

2. In general, higher levels of waste treatment monotonically improved performance. However,

- When waste forms were analyzed as a group (Complex PA), extensive treatment, as represented by Waste Disposal Groups 4 and 5, was required to conclusively see improved disposal system performance.

- An exception occurred for graphite spent fuel, which was composed of fuel particles that were assumed to be generally intact. For graphite spent fuel, moderate treatment led to degraded performance in comparison to low treatment, represented here by disposal of the entire block or rod. Only extensive treatment improved performance. Hence,

- The durability of the graphite spent fuel can be an effective barrier against radionuclide release (e.g., silicon carbide coating).

- The fraction of particles with failed silicon carbide coatings is an important modeling parameter for the graphite spent fuel.

3. Waste Disposal Groups 4 and 5 , and the corresponding individual waste parcels, have the best performance, followed by Waste Disposal Groups 1,2, and 3, and the corresponding individual waste parcels.

- For salt, the improvement with the more extensive treatment options was primarily caused by the increased radionuclide inventory per waste parcel, which lowered the fractional release since many of the radionuclides were solubility limited.

4. The radionuclides that contribute the most to the summed normalized activity discharged (abscissa of complementary cumulative distribution function [CCDF]), at the boundary of the repository differ between (a) the salt and granite disposal systems, (b) the level of simplification of the consequence models (Complex PA versus Simple PA), and (c) Waste Disposal Groups 4 and 5 (since Waste Disposal Group 4 eliminates radionuclides and Group 5 adds a radionuclide).**

- Complex PA:

- In salt, the top contributing radionuclides are ${ }^{234} \mathrm{U},{ }^{235} \mathrm{U},{ }^{226} \mathrm{Ra}$, and ${ }^{230} \mathrm{Th}$.

- In granite, the top contributing radionuclides are ${ }^{14} \mathrm{C}$ (not in Waste Disposal Groups 4 and 5 ), ${ }^{241} \mathrm{Am}$, ${ }^{240} \mathrm{Pu},{ }^{239} \mathrm{Pu}$, and ${ }^{230} \mathrm{Th}$.

- Simple PA:

- In salt, the top contributing radionuclides are ${ }^{241} \mathrm{Am},{ }^{234} \mathrm{U},{ }^{230} \mathrm{Th},{ }^{243} \mathrm{Am}$ (not tracked in the Complex PA), and ${ }^{226} \mathrm{Ra}$.

- In granite, the top contributing radionuclides are ${ }^{14} \mathrm{C}$ (not in Waste Disposal Groups 4 and 5 ), ${ }^{230} \mathrm{Th}$, ${ }^{241} \mathrm{Am},{ }^{239} \mathrm{Pu}$, and ${ }^{237} \mathrm{~Np}$.

5. Element solubility in the assumed chemical environment of the repository is an important parameter affecting release from the repository and to the surrounding accessible environment.

- Stainless steel is not considered a corrosion-resistant material for disposal of spent fuel and high-level waste because of the long time scale involved and stainless steel's susceptibility to several forms of failure besides generalized corrosion.

- The contributing radionuclides are also sensitive to the regulatory period, the performance metric, and the location at which the metric is measured. For instance, individual dose evaluated beyond $10,000 \mathrm{yr}$ at a $5-\mathrm{km}$ boundary of the disposal system will show different radionuclides contributing to the performance metric (human dose) than the normalized integrated activity specified in 40 CFR 191. This sensitivity to and uncertainty as to the form of the final regulation makes it difficult to identify radionuclides that should be immobilized or removed at this time. 

performance (according to the Containment Requirement measure of 40 CFR 191) of a moderately sized disposal system. Thus other considerations (e.g., other regulations or economics) can guide the selection of the treatment option. Specifically,

- In the salt disposal system, the horizontal separation between the CCDF in the most extensive treatment options (Options 4 and 5) and the low or moderate treatment options (Options 1, 2, and 3) was, at most, three orders of magnitude when individual waste forms were compared. When the waste forms are combined as a disposal group with waste from other DOE sites (vitrifled high-level waste from Savannah River, Hanford, and West Valley), the CCDF usually varies less than one order of magnitude.

- In the granite disposal system, separation in CCDFs along individual waste forms are usually relatively large, varying by as much as seven orders or magnitude. When combined as a disposal group with waste from other DOE sites, they vary by at most $21 / 2$ orders of magnitude. 


\section{ES.2.2 Waste Container}

Because the focus of this performance assessment was on the waste forms themselves and the possible treatment options, the majority of calculations modeled containers that were not corrosion resistant but were adequate for transport and handling of the waste. The absence of corrosion resistance quickly allowed direct contact of the waste form with available groundwater. Specifically, a thin-walled, stainless-steel canister was assumed for both the spent nuclear fuel from INEL and the high-level waste from INEL and the three DOE sites. (Stainless steel is subject to localized corrosion and thus is not considered to be corrosion resistant over the 10,000-yr regulatory period.) One set of calculations did study the robustness of a highly corrosion-resistant 3-layer container in the presumed environment of the hypothetical repositories. This latter waste parcel consisted of two types of container: a canister and an overpack. The waste canister encloses the waste form; the overpack is a container surrounding the canister that provides corrosion resistance. For calculations in salt, the canister material was a nickel alloy (Inconel 625) and the overpack material was titanium (Ti-Grade 12); for calculations in granite, both the canister and overpack were Inconel 625. A lead filler was used between the overpack and the canister. Table ES-3 is a summary of the information acquired about the waste containers.

Table ES-3. Summary of : Iformation Gathered about Waste Containers

1. The amount of liquid in contact with the waste parcel differs in the two disposal systems resulting in different breach times and waste parcel corrosion profiles. Specifically,

- In salt, brine is often limited except near the base of the waste parcels. Consequently, the waste parcels corrode faster near the base than at the top and hence, breach relatively quickly at the bottom."

- In granite, water fills the repository faster than in a salt repository. As a result, the entire waste parcel corrodes more uniformly at the fully saturated, inundated rate.

2. The gas generated from corrosion in the granite repository over the 10,000 -yr regulatory period is considerably less than that generated in the salt repository because the corrosion rate of stainless steel is slower in water than in brine.

3. For graphite spent fuel, corrosion-resistant containers are not essential for compliance with the Containment Requirements of 40 CFR 191 in either salt or granite.

4. A corrosion-resistant container of titanium and nickel-based alloys remains intact for periods of time greater than the 10,000-yr regulatory period. This finding also determined that corrosion-resistant containers would not be used in most calculations for evaluating the waste forms.

5. When the corrosion rate is fast enough to cause a breach within the 10,000 -yr regulatory period, the corrosion rate is an important parameter for determining releases for special (highly enriched and low enriched uranium) spent nuclear fuel and calcine.

6. Variation of heat loading has little influence on time of complete corrosion of the container."**

- Wicking of fluid to the container from lowered capillary pressure is also partially modeled in the detailed modeling of consequences of the disposal group (Complex PA).

- Some caveats exist. Although this condition was observed in this study, it may have occurred because (1) the low backfill permeabilities around the canisters prevent convection cells from forming, (2) vaporization was not modeled, (3) pH changes due to evaporation and precipitation were not explicitly modeled, and (4) time constraints and a lack of data for some materials forced their activation energies to be set to zero (corrosion rate independent of temperature); however, an expanded range for the corrosion rate between $293 \mathrm{~K}$ and $373 \mathrm{~K}$ was estimated subjectively. 


\section{ES.2.3 Repository}

The repository is the portion of a waste disposal facility that includes the access drifts, waste panels, and access shafts. The repository does not include either the above-ground facilities or the undisturbed host rock. Table ES-4 is a summary of the information acquired about the repository.

Table ES-4. Summary of Information Gathered about the Repository

1. The diffusive transport of radionuclides from the waste parcel holes into the rooms and access drifts, followed by diffusive transport (salt repository) or advective transport (granite repository) through the rooms and drifts, were very important phenomena to model; their importance to the position of the CCDF relative to the EPA release limits was comparable to the effect of the waste treatment options.

2. The waste form had little influence on the flow of liquid (which is the primary means of radionuclide escape other than direct release through cuttings), in either the salt or granite disposal system.

3. Gas generation and thus gas flow was influenced by the waste form but the small volumes of gas did not, in turn, greatly influence liquid flow.

4. Heat generating capacity of the waste form was included in the detailed analysis (Complex PA) of the disposal systems and resulted in a different maximum temperature, but the heat had little influence on the overall fluid flow.

5. For the Complex PA of the salt disposal system, the existence of the shaft had little influence. Therefore, it was appropriately neglected in the Simple PA calculations.

6. In the granite repository, the shaft was an important source of liquid filling the repository.

7. In the granite disposal system, the chemical adsorption (retardation) provided by the backfill-buffer that surrounds the waste parcel in the borehole and the backfill in the rooms and drifts was an important parameter influencing releases at the repository boundary.

8. The relative difference in the threshold pressure (a property of the rock used in the two-phase fluid model for the Complex PA) between the backfill-butfer and the room backfill can influence the availability of liquid around the waste parcel and consequently the time of complete corrosion of the waste container.

9. In the granite disposal system (for which the permeability of the host rock was zero), the final permeability of backfill for the rooms and drifts influenced the liquid inflow and thus the time of complete corrosion of the containers. 


\section{ES.2.4 Geologic Barrier}

The geologic barrier of the disposal system comprises the sequence of host rock that isolates the repository from the accessible environment. For the performance assessment, two different geologic barriers were studied: salt and granite. Specific information about the geologic barrier is summarized in Table ES-5.

Table ES-5. Summary of Information Gathered about the Geologic Barrier

1. Assuming that the salt behaves as a porous medium, the permeability of the salt is an important parameter controlling the quantity of brine flowing into the repository.

2. In salt, the permeability of the intrusion borehole was an important parameter influencing radionuclide releases.

3. In salt, the initial pore pressure in the salt formation was an important model parameter influencing radionuclide releases.

4. For the parameters assumed in granite, the repository often filled rapidly (after which the chosen horizontal and vertical gradients assumed for the region determined the direction of water flow through the repository).

5. In granite, the permeability of the postulated connections between the repository and the fracture zones was an important model parameter that influenced the amount of water flowing into and out of the repository and, thus, radionuclide releases.

6. A noticeable difference occurred in the isolating capability for a nondecaying, nonabsorbing tracer in the inventory of the salt and granite disposal systems.

- In salt, a maximum of $12 \%$ of the contents of the tracer in a panel is removed.

- In granite, $33 \%$ of the simulations removed the entire contents of the tracer in the repository. 


\section{ES.2.5 Agents Acting on the Disposal System}

Agents that act on the disposal system include items such as drillirg frequency, drilling methods, and climate. Specific information gathered about the agents acting on the disposal system is shown in Table ES-6.

Table ES-6. Summary of Information Gathered about the Agents Acting on the Disposal System

1. Direct release of contaminants to the ground surface from exploratory drilling using present-day drilling technology dominates the release for the salt repositories. Hence,

- The direct release to the surface, as evaluated by EPA normalization factors, is very important.*

- Drilling frequency from an inadvertent human intrusion (for which DOE cannot take credit for preventing after $100 \mathrm{yr}$, and/or deterring, except through passive controls) is the most important parameter influencing radionuclide releases.

- The permeability of the material that eventually fills the intrusion borehole is an important parameter for regulatory compliance per Appendix B of 40 CFR 191. The permeability controls brine flow out of a borehole that intersects a brine reservoir under the repository (which was a feature selected for inclusion in the salt disposal system).

- Direct releases were sensitive to the waste form only because of the different radionuclide inventories that were assumed for the waste parcels; in this assessment, the entire contents of a waste parcel were assumed to have been removed if it was struck by an exploratory drill bit.

2. In the salt disposal system, crushed salt filled the rooms and drifts and was assumed to compress to a low but variable permeability, usually sufficient to limit circulation of brine between intrusion boreholes. Specifically,

- In only 1 case out of 10 was the permeability high enough to allow flow through the repository and up the other intrusion borehole; however, in this case, the volume of brine discharged was very small.

- Calculated doses from the direct release route, however, and possible adverse affects are likely much lower because the EPA normalized factors were originally based on drinking contaminated water (ingestion).

Also, it is conceivable that the domination of direct release would diminish for regulatory periods longer than $10,000 \mathrm{yr}$, especially if exploratory drilling were assumed to stop after sorne period of time. But this diminishing influence at longer periods is by no means certain since exploratory drilling is required to initiate any releases other than diffusion through salt. 


\section{ES.3 Guidance to the Project}

\section{ES.3.1 General Guidance for Waste Treatment Program}

The findings listed in the previous section have been used to provide the DOE with specific information about the potential impact of various waste treatment options on spent nuclear fuel and high-level waste to be disposed of in two saturated generic repositories: bedded salt and igneous rock (granite). In answer to the question of what decisions can be made by DOE about treatment of the waste stored at INEL before a repository site or type is identified, the findings suggest that most decisions should wait until a repository type is known. The findings differ somewhat, however, between high-level waste and spent nuclear fuel. For high-level waste, it would seem that only those decisions related to temporary storage (as calcine) should be made before a repository site is known; this recommendation is based not only on the results of this performance assessment, which indicate that the currently proposed waste forms are acceptable for meeting the Containment Requirements of 40 CFR 191, but also in light of possible changes to the regulations. For example, policies may change from specifying generic regulatory criteria to developing site-specific criteria (e.g., as suggested in the Energy Policy Act of 1992, Section 801). For spent fuel, the same recommendations as the high-level waste apply as long as critical conditions are avoided in the containe:. To be sure, this is an important assumption that must be carefully studied before general conclusions about the spent nuclear fuel waste forms can be drawn.

\section{ES.3.2 Guidance for Waste Acceptance Criteria}

The general findings have also been analyzed to provide the DOE with information about minimum acceptance criteria for waste to be placed in a generic, deep geologic repository.

Using the total system analysis approach consistent with 40 CFR 191, the results of the preliminary performance assessment indicate that the acceptability of the waste form shows a strong sensitivity to the specific geologic medium (i.e., host rock) of the chosen repository. Therefore, any preliminary waste acceptance criteria must clearly show the degree to which acceptance depends upon the geologic media of the repusitory.

- For a repository in bedded salt, all the proposed waste forms (e.g., whole graphite block and rod spent nuclear fuel, low and highly enriched uranium spent nuclear fuel, and calcined high-level waste) can be placed directly in the disposal region as a group or individually, without being placed in a corrosion-resistant container, or without using a backfill buffer such as bentonite clay. Thus, little treatment is expected to be necessary for compliance with 40 CFR 191 . To be consistent with the extremely conservative assumptions of the $\mathrm{PA}$ analysis, up to $10 \mathrm{~kg}$ of ${ }^{235} \mathrm{U}$ can be placed in each container.

- For a repository in granite, when disposed of as a group or individually, all currently existing waste types can be placed in a repository without conditioning but do require a moderately corrosion-resistant container and a bentonite clay backfill-buffer around the waste and some bentonite clay in the rooms and drifts to adsorb radionuclides. To be consistent with the conservative assumptions of the PA analysis for preventing critical conditions, only $0.7 \mathrm{~kg}$ of ${ }^{235} \mathrm{U}$ can be placed in each container (i.e., cut up to keep the ${ }^{235} \mathrm{U}$ content at or below $0.7 \mathrm{~kg}$, if necessary).

\section{ES.3.3 Recommendations for Future Analysis and Data Acquisition}

The Nuclear Waste Policy Act (NWPA) of 1982 mandates that the DOE develop a permanent disposal option. Although this option may not have to be fully implemented, its feasibility should be demonstrated. 
A performance assessment is a valuable and low-cost tool (relative to implementing waste treatment and disposal) that responds to the spirit of the NWPA by developing and demonstrating the capability to dispose of spent nuclear fuel stored at the INEL. The performance assessment is also the primary means by which the DOE will demonstrate compliance with long-term environmental regulations, and, therefore, is an essential component of any development program concerned with conditioning spent fuel and radioactive waste for temporary storage and eventual permanent disposal.

Because performance assessments are iterative, an initial performance assessment serves to inform the interdisciplinary team performing the study about (a) the scientific information and data that must be gathered (either from the literature or investigative studies) and (b) the resolution required for converting available information and data into forms suitable for use in the model selected for the performance assessment. The performance assessment presented in this report was no exception. In addition to providing guidance on the waste treatment options available for the waste stored at the INEL, the study identified future goals for data collection and modeling development efforts. This information, which represents a valuable benefit to the program as a whole, is presented below (Table ES-7). The recommendations are grouped into two categories: those that can be treated through the performance assessment portion of the waste treatment program, and those that may require input from throughout the DOE complex, possibly with consensus building.

Recommended Tasks for Future Performance Assessments. In the performance assessment reported here, the results hint that the waste form shows a strong sensitivity to the specific geology of the repository. In response to this finding and the mandate of the NWPA Amendments of 1987, which require an examination of unsaturated volcanic tuff, a performance assessment of a repository in unsaturated tuff should be pursued. This type of calculation should continue the practice of using several models to evaluate consequences (possibly at different levels of simplification) in order to provide checks on each other and thereby develop a deeper understanding of the disposal system, or an understanding that can be quickly achieved. This recommendation does not mean to imply that a performance assessment in unsaturated tuff is not being done (see, for example, Barnard et al., 1992), but rather is suggesting an assessment similar to the one performed here that focuses on INEL wastes and their incremental effects on a moderately sized repository. Nor does this recommendation imply that we view the examination of the salt and granite disposal systems as complete; on the contrary, we have spent much time and money developing tools to examine these disposal systems, which should be used to reexamine the salt and granite disposal systems with more defensible data and, just as important, more defensible conceptual and computational models (e.g., improved discretization) to provide more defensible understanding and thus possibly more confident predictions.

Although most model results were compared between the Simple and Complex PAs, the gas generation and source term models were nearly identical. Because of the demonstrated benefit of model-to-model comparisons, we need to review gas generation and source term models developed elsewhere in other high-level waste repositories and compare them with the models used in this performance assessment.

Other performance criteria need to be examined, besides 40 CFR 191, to substantiate the initial guidance from this performance assessment, which indicates that the current waste forms are acceptable for long-term environmental compliance. Because of changes contemplated for regulations on required repository performance, the internationally recognized criterion of individual doses may be the only other criterion easily identified at this time for this purpose. (It is also the criterion stipulated by the Energy Policy Act of 1992 for Yucca Mountain.)

Because modeling analyses are conditional on what was modeled, phenomena not currently modeled should be examined in future work. Some phenomena were omitted because they were thought unimportant; this assumption may need to be substantiated. More important, some phenomena were omitted because of a lack of time and resources available for this initial performance assessment, and these should be explored. ${ }^{\ddagger \ddagger}$ For example, critical conditions in the repository were not examined in the initial performance assessment because the containers were limited to a mass of $10 \mathrm{~kg}$ and $0.7 \mathrm{~kg}$ of ${ }^{235} \mathrm{U}$ in the salt and granite repositories, respectively. The results of this assumption was that a large number of containers (especially for the highly enriched fuel) were required which, because of cost implications, suggest that future work should attempt to determine whether nuclear critical

\footnotetext{
$\$$ Many of these tasks would not require entirely new performance assessment calculations, but instead would entail a more thorough examination of existing results, a search for information from other radionuclide waste disposal programs, or performing supporting side calculations.
} 
Table ES-7. Recommendations for Future Analysis and Waste Treatment Program Tasks

\section{Tasks for PA}

1. Carry out a performance assessment of a repository containing spent nuclear fuel and high-level waste from INEL in unsaturated volcanic tuff. Also,

- Continue the practice of using several models to evaluate consequences.

2. Reexamine the salt and granite disposal options after the data, conceptual models, and computational models are improved so that more defensible (and possibly more confident) predictions can be made.

3. Review gas generation and source term models developed in other high-level waste repositories and compare with the models used in this performance assessment.

4. Examine performance criteria other than 40 CFR 191.

5. Examine phenomena not currently modeled:

- Determine whether nuclear critical conditions in a repository are noncatastrophic and would be technically acceptable.

- Explore the possibility of reaching critical mass from migration of radionuclides to a central location.

- Attempt to model diffusion of contaminants through the failed layers of the containers.

- Perform calculations of detailed waste package models to determine the most effective annular thickness of the backfill-buffer, including a disturbed rock zone around the waste package in the granite disposal system.

- Perform scoping calculations to determine how long it takes to consume $\mathrm{O}_{2}$ in the repository since different corrosion rates could occur.

6. Develop versions of a corrosion submodel and source term submodel that can be run separate from the fluid flow and transport codes.

\section{Tasks for Waste Treatment Program}

1. Seek to confirm alteration rates used in the performance assessment." For example, perform a more thorough literature search to obtain corrosion data. Specifically,

- For the gas generation submodel, generalized corrosion rates more specific to conditions expected in the salt and granite repositories are needed for container materials.

- Temperature-dependent corrosion data between $293 \mathrm{~K}$ and $373 \mathrm{~K}$ are needed.

- For the source term submodel, more reliable information about susceptibility and data on failure modes for the container materials are needed.

2. Seek data on alteration rate of a preferred glass-ceramic matrix and percentage of radionuclides trapped in glass and ceramic phases.

3. Pursue better characterization of the current fuel types. Specifically,

- Data on the fraction of particles with failed silicon carbide coatings and cladding on special nuclear fuel.

- A better understanding of element solubility.

- Estimates of the likelihood of the failure of silicon carbide coatings in graphite fuel in the environment of the repository during the 10,000-yr regulatory period.

4. Select a preferred (e.g., "base case" or "reference design") type of waste parcel container and filler to use for the performance assessment calculations.

- INEL may already possess a means of gathering information on alteration rates since some radionuclides have been detected in the basins used to store the fuel, which indicates that the protective layers on some fuel have failed. 
conditions in a repository are noncatastrophic and thus technically acceptable. Furthermore, a situation in which ${ }^{235} \mathrm{U}$ migrates from several containers to a central location was not simulated. Although this possibility is remote, a future performance assessment should perform supporting calculations to explore the possibility of reaching critical mass from migration of radionuclides to a central location.

Moreover, because movement of radionuclides at the waste package is almost exclusively by diffusion and will likely cause substantial delay, attempts should be made to model diffusion of radionuclides through failed layers of the containers. Finally, if emplacement within drillholes remains a preferred base case option to consider, then optimization of annular thickness of the backfill-buffer should be pursued and inclusion of a disturbed rock zone around the waste package when modeling the granite disposal system.

Scoping calculations using appropriate oxic corrosion rates need to be performed to determine how long it would take for the $\mathrm{O}_{2}$ in the repository to be consumed. If the time period for consuming all the $\mathrm{O}_{2}$ is not small compared to the regulatory period, the gas generation model should be modified to include two sets of stoichiometries and corrosion rates: one set for oxic conditions and one set for anoxic conditions.

Many of the results reported herein are directly related to the output of the gas generation and source term submodels, which presently run with the fluid flow and transport codes. To respond rapidly to future "what if" questions about the waste forms, it could be useful to develop a corrosion submodel (not necessarily the gas generation submodel) and source term submodel that can be run separate from the fluid flow and transport codes.

Recommended Tasks for Waste Treatment Program. In general, an extensive need exists for defensible data for future iterations of evaluating the performance of INEL spent fuel and radioactive wastes in a geologic repository. Several specific data needs are mentioned below.

A more therough literature search is needed to find corrosion data; specifically, temperature-dependent corrosion data between $293 \mathrm{~K}$ and $373 \mathrm{~K}$ are needed; for the gas generation submodel, generalized corrosion rates for container materials such as lead, stainless steel $304 \mathrm{~L}$, and Inconel 625 nickel-based alloy are needed at conditions expected in the salt and granite repositories. For the source term submodel (and, to some extent, the gas generation submodel), information on susceptibility and data on failure modes for the container materials are needed on stress-corrosion cracking, pitting, crevice corrosion, delayed hydrogen-induced failure, and aging reactions ("localized" corrosion) to adequately assess the longevity of the barrier capabilities of the container.

In this analysis, information was only available on experimental glass-ceramic; specific alteration rates for calcined waste immobilized in a glass-ceramic or borosilicate glass matrix were not available (except for the $\sim 70 \%$ radionuclide loading possible with glass-ceramic, as opposed to $\sim 30 \%$ for glass). To distinguish these two waste treatment options, data on the alteration rate for a preferred glass-ceramic matrix and percentage of nuclides trapped in glass and ceramic phases are needed. For this need, the alteration rate refers to any change from one phase to another that liberates radionuclides.

In addition, scientifically agreed-upon characterization of many aspects of the current waste form is needed for the DOE to rely on many of the findings discussed here, for example, the general finding that the current graphite spent fuel (Waste Parcel 1) in a borehole already provides an effective engineered barrier. Specific information needed includes data on the fraction of particles with failed silicon carbide coatings (an important parameter for the graphite spent fuel) and cladding on special spent nuclear fuel. Also, estimates are needed of the likelihood of failure of silicon carbide coatings of the graphite fuel in the environment of the repository during the regulatory period. (As mentioned before, the regulatory period for 40 CFR 191 is $10,000 \mathrm{yr}$, but this time period could change with new regulations.)

Even accounting for numerous metal layers in spent nuclear fuel, the ultimate limit to release from the source term submodel is elemental solubility. Although direct measurement may be infeasible because (1) equilibrium conditions are difficult to reach in short lab experiments and (2) the experiments are extremely costly and time consuming because of environmental, safety, and health issues, an understanding of elemental solubility in proposed repositories is needed, if uncertainties in the predicted releases are to be reduced (or at least corroborated). As 
pointed out earlier, the particular radionuclides that are a significant percentage of the releases change with the performance metric and the location at which the metric is measured; hence, listing the most important solubilities to determine is difficult.

The Waste Treatment Program should select a preferred type (e.g., "base case" or "reference design") of the waste parcel container to use for the performance assessment calculations. Some containers (for example, selfshielding), use significant amounts of metal that can affect the amount of gas produced through corrosion. Furthermore, highly corrosion-resistant containers can provide a redundant barrier for the waste form in addition to deep geologic disposal. Thus, policy decisions related to container barriers versus geologic barriers may need to be carefully defined. Another decision that would be useful is specification of the type of filler (e.g., sand or metal) in the container to use for the performance assessment calculations. Ideally, the process would be as follows: (a) establish the requirements for the container system, (b) select container configuration and material of construction, (c) determine whether a filler material is necessary, and (d) select the filler material, if it is necessary. Although this thorough process may be difficult to implement before the next phase of this initial performance assessment, it could be implemented over the long term. Furthermore, the first steps for establishing the minimum containment requirements that corrosion-resistant containers must meet could be discovered by further analysis of the results of this performance assessment.

\section{ES.4 Analysis Caveats and Consequence Modeling Assumptions}

This report was commissioned to provide information to the DOE concerning general decisions on program direction. In this situation, even preliminary findings from a performance assessment using preliminary data, such as described herein, are extremely pertinent and helpful in making reasonable choices. Hence, the general results are presented in this executive summary with the understanding that the reader appreciates that the findings are conditional on the models and data used to generate them. The models of the disposal system must be judged in combination with the results to set priorities for additional data acquisition, model development, and other decisions on general program direction. To make these judgments, details concerning the models and the data must be known. These detailed assumptions should be examined before actions are taken on the findings and guidance presented in this summary.

The detailed assumptions cannot be summarized here but the main report attempts to list clearly those assumptions about the disposal system's major subsystems and components that were used. In fact, the model description and assumptions comprise a major portion of the document. However, five general caveats regarding the analysis are as follows.

First, only one set of performance criteria, 40 CFR 191 (and primarily only the Containment Requirements [40 CFR 191.13]), is examined in this performance assessment. sion.

Second, the environmental regulations for disposal sites in the United States are currently undergoing revi-

Third, the assessment of the disposal system is performed on a model of the disposal system, not the system itself. Although the U.S. regulatory criteria were developed using simplified models of generic disposal systems, and so making comparisons with the criteria is appropriate, the generally accepted style (e.g., levels of modeling detail expected and accepted assumptions on events such as human intrusion) of these type of compliance calculations continues to evolve over time.

Fourth, critical conditions near each container are avoided by limiting the amount of fissionable uranium permitted in each container.

Fifth, this is an initial performance assessment in which information is being gathered to understand the behavior of these complex disposal systems and the underlying data are preliminary. 
Consequently, it is very difficult to predict precisely which waste form will be acceptable in the future and thus none of the three goals mentioned in the previous section can be definitively accomplished through this report alone. Periodic iterative studies, perhaps with different stylizations of the disposal system and examinations of other environmental performance criteria, will be necessary in the future. 


\title{
1. Introduction
}

\author{
R. P. Rechard
}

\subsection{Purpose of Report}

The purpose of this report is to assist the U.S. Department of Energy (DOE) in evaluating the potential impact of various waste treatment options for specific nuclear materials on a compliance determination for a deep, geologic repository. The waste that DOE is considering for geologic disposal is spent fuel either that is currently stored at defense-related nuclear facilities or that may be generated in the future. The materials under study in this report include graphite spent nuclear fuel (SNF) from the Fort St. Vrain and Peach Bottom 1 (reactor Cores I and II), over 90 experimental "special" spent fuels, and high-level waste (HLW) from reprocessing of spent fuels. The materials are currently stored at the Idaho National Engineering Laboratory (INEL)." This report examines only one set of performance criteria, 40 CFR 191, Subpart B (as promulgated in 1985), and two generic saturated geologic repositories: bedded salt and partially fractured granitic rock. Other performance criteria and geologic repositories will need to be examined in future iterations of the performance assessment methodology.

Although there is an international consensus that deep geological disposal is the best option for disposing of high-level (HLW) and transuranic (TRU) (atomic number greater than 92, emitting alpha radiation) radioactive waste (BRWM, 1992, p.1), there are many different approaches to waste treatment and processing of spent fuel. The number of waste-treatment approaches is related to the value placed on the spent fuel and the wide range of geologic media being considered for eventual disposal (NEA, 1991, p. 20). This performance assessment is part of a general effort by DOE to examine options for conditioning spent fuel and high-level waste before its eventual disposal in a deep, geologic repository; the overall program's focus is on the development of disposal criteria in order to provide input on the selection of an optimal waste-treatment option, not the selection of a repository site. The DOE program effort is intended to examine whether waste treatment options can be acceptable in meeting the requirements of 40 CFR 191. If so, the results can be used to recommend not only a waste-treatment plan that can be implemented but also to provide direction for necessary research programs.

This chapter outlines important concepts in a performance assessment and highlights decisions that affect how the disposal system was modeled. Chapters 2 through 16 expand on the concepts and assumptions presented here; appendices offer even more detailed information about the waste, geology, repository, waste parcel design, scenario development and modeling systems. Readers should turn to related chapters and appendices for detailed information about areas of interest.

\subsection{Legal Setting}

One of the primary criteria that DOE will use in selecting the treatment options for spent fuel and high-level waste is the acceptability of the waste type for disposal under currently applicable federal regulations. This section provides a listing of applicable statutes, briefly describes 40 CFR 191, and notes potential changes to the regulations.

\subsubsection{Overview}

Before a deep, geologic site can open as a repository for spent fuel, high-level waste, and/or transuranic waste (which is potentially mixed with some hazardous chemicals), DOE must demonstrate that the site complies with a

This performance assessment examines the influence of waste-treatment options on some, but not all, types of spent fuel and high-level waste currently stored at INEL. Besides supporting several other missions, the INEL reservation near Idaho Falls, Idaho, serves as a storage facility for the waste mentioned above, and spent fuel from naval propulsion reactors, non-govemment research reactors, and failed spent fuel from the Three Mile Island reactor. 
number of environmental regulations. These regulations determine the short-term and long-term performance metrics of a repository with which specific repository performance modeling can be compared. Numerous statutes and regulations affect and establish performance criteria for the management, transportation, and disposal of radioactive waste. Many of these statutes and regulations apply to hazardous waste as well. Several regulations are of primary importance to the disposal of radioactive waste in geologic repositories (Table 1-1). A brief description of these statutes and regulations is provided in Chapter 2. Note that the Energy Policy Act of 1992 (passed in October, 1992, after work began on this project) strongly suggests the use of maximum annual effective doses to individuals (which also implies that the period of time under consideration may be extended), as a possible criterion to be studied by the National Academy of Sciences. This change in the regulatory setting could have a profound influence on the issues studied in this report.

\section{Table 1-1. Selected Statutes and Regulations Affecting the Disposal of Radioactive Waste in Geologic} Repositories

\begin{tabular}{|c|c|}
\hline Policy Act or Regulation & $\begin{array}{l}\text { Influence on Repository } \\
\text { and/or Waste Treatment }\end{array}$ \\
\hline $\begin{array}{l}\text { - Nuclear Waste Policy Act of } 1982 \\
\text { - Nuclear Waste Policy Amendments Act of } 1987\end{array}$ & $\begin{array}{l}\text { Sets national policy on nuclear waste disposal (primary } \\
\text { importance) }\end{array}$ \\
\hline - Energy Policy Act of 1992 (EPA) & $\begin{array}{l}\text { Asks the National Academy of Sciences to recommend } \\
\text { to the Environmental Protection Agency (EPA) stan- } \\
\text { dards for the Yucca Mountain repository site and } \\
\text { requires the Environmental Protection Agency and the } \\
\text { Nuclear Regulatory Commission to make subsequent } \\
\text { revisions in their regulations for this specific site. The } \\
\text { law strongly suggests the use of maximum annual effec- } \\
\text { tive doses to individuals. }\end{array}$ \\
\hline $\begin{array}{l}\text { Environmental Radiation Protection Standards for } \\
\text { Management and Disposal of Spent Nuclear Fuel, } \\
\text { High-Level and Transuranic Wastes, } 40 \text { CFR } 191 \\
\text { - Disposal of High-Level Radioactive Wastes in Geo- } \\
\text { logic Repositories, } 10 \text { CFR } 60\end{array}$ & $\begin{array}{l}\text { Establishes long-term performance criteria for geologic } \\
\text { repositories (primary importance) - repromulgation of } \\
40 \text { CFR } 191 \text { will likely extend individual dose calcula- } \\
\text { tions to } 10,000 \mathrm{yr} \text {. }\end{array}$ \\
\hline $\begin{array}{l}\text { Resource, Conservation, and Recovery Act of } 1976 \\
\text { (RCRA) and Hazardous and Solid Waste Amend- } \\
\text { ments of } 1984 \text { (implemented in } 40 \text { CFR } 124 \text { through } \\
40 \text { CFR 181) } \\
\text { - National Environmental Policy Act of } 1969 \text { (NEPA) }\end{array}$ & $\begin{array}{l}\text { Potentially important in the establishment of long-term } \\
\text { performance criteria }\end{array}$ \\
\hline
\end{tabular}

\subsubsection{CFR 191 Regulation}

In September 1985, the U.S. Environmental Protection Agency (EPA) promulgated 40 CFR Part 191, Environmental Standards for the Management and Disposal of Spent Nuclear Fuel, High-Level and Transuranic Radioactive Wastes. The regulation's preamble states that the intent of 40 CFR 191 is (1) to provide an achievable level of protection, given the existing disposal alternatives, and (2) to reduce the risk from nuclear waste to future generations to "acceptably" low levels. The latter purpose is accomplished by isolating the wastes for is significantly long time such that the risk is no greater than if the source of the waste, uranium ore, had not been mined (50 FR 38066) where $10,000 \mathrm{yr}$ was used as a sufficiently "long time." The EPA's stated rational for the 10,000-yr regulatory period is described in Chapter 2. Foreign geologic disposal programs often use a longer time period (e.g., 1 million yr), but the corresponding calculations incorporate compensating assumptions such as assuming no change in geologic stability of the site or human behavior. 
The U.S. Court of Appeals for the First Circuit vacated Subpart B of 40 CFR 191 in July 1987 (NRDC $v$. US EPA, 824 F. 2d 1258 [1st circuit 1987]) and remanded it to the EPA for further consideration. The points of major contention were the Individual Protection Requirements, the 1000-yr time periods for the Individual Protection Requirements; and the Groundwater Protection Requirements. The Waste Isolation Pilot Plant Land Withdrawal Act of 1992 (Public Law 102-579) reinstated those portions of Subpart B that were not specifically cited for reconsideration and mandated timely revision of 40 CFR 191 . Those revisions will be promulgated by early 1994 and will likely extend the time frame for Individual and Groundwater Protection to $10,000 \mathrm{yr}$, which, for some repository settings, may be more limiting than the containment requirements. Hence a unique feature of the original US standard-limits based on cumulative release - appears to be moving toward limits on individual doses, which is similar to the style of compliance calculation used in other geologic disposal programs throughout the world (see Chapter 2). References in this report to 40 CFR 191 are to the portions of the 1985 version unaffected by the remand (including the Containment Requirements, the supplementary information, and the nonbinding guidance) (refer to Appendix B of this report).

Although 40 CFR 191 does not require that risk be evaluated, its containment requirements ( $\$ 191.13)$ are riskbased. The containment requirements and Appendix A of 40 CFR 191 establish radionuclide releases and a probability for their occurrence that may not be exceeded. Sandia's approach for assessing the disposal system follows the nonbinding guidance provided in Appendix B of 40 CFR 191. Consequently, the Sandia Performance Assessment (PA) Department's approach to evaluating repository compliance resembles a probabilistic risk assessment (PRA) in which the performance metric, a radioactive release specified in probabilistic terms, is presented as a complementary cumulative distribution function (CCDF). The Sandia procedure was developed simultaneously with early drafts of the EPA Standard and was used to evaluate many early options for the EPA and the Nuclear Regulatory Commission (NRC) (e.g., Chu et al., 1983; Pepping et al., 1983b). EPA's 40 CFR 191 is described in more detail in Chapter 2.

The EPA's probabilistic requirements in Subpart B of 40 CFR 191 (191.13) are computationally demanding. An advantage, however, is that with some modifications, the stochastic computations can be used to assess compliance with other environmental regulations and laws concerned with long-term release of contaminants, which tend to require deterministic calculations (e.g., 10 CFR 60, the National Environmental Policy Act [NEPA] [1970], and the Resource, Conservation, and Recovery Act of 1976 [RCRA] [1976]). Therefore, the PA methodoiogy, which was originally developed to respond to the requirements of 40 CFR 191, can also be used for these other regulations in the future.

\subsection{Performance Assessment Methodology}

The primary means of determining whether a waste is acceptable for inclusion as part of a geologic disposal system is a performance assessment. The term performance assessment is defined in 40 CFR 191.12(q) as: "an analysis that (1) identifies the processes and events that might affect the disposal system, (2) examines the effects of these processes and events on the performance of the disposal system, and (3) estimates the cumulative releases of radionuclides, considering the associated uncertainties, caused by all sig:ificant processes and events." In essence, the performance assessment can be viewed as the complex process that estimates these various performance metrics. As used herein, the term refers to only long-term performance metrics, not short-term operational safety issues.

\subsubsection{Theoretical Foundation of the Performance Assessment}

The theoretical foundation used to construct the complementary cumulative distribution function (CCDF) in accord with 40 CFR 191 and other regulations that pertain to disposal of waste highly contaminated with radionuclides and possibly co-contaminated with some hazardous waste constituents is described in Chapter 3, "PA Methodology Overview." What follows is a brief synopsis (see also Chapter 3 of WIPP PA Division, 1991a, Vol. 1).

Although risk is often defined as consequence times probability to make risk comparisons, viewing risk as a set of ordered triples is a convenient way to organize a performance assessment and leads to presenting the results as a CCDF (Kaplan and Garrick, 1981; Helton et al., 1991; Helton, 1991). The first element of the triple describes things 
that may happen to the disposal system in the future (called scenarios, $S(x)$, dependent on model parameters, $x$ ). The second element describes how likely these scenarios are to happen (the probability of a scenario, $P[S(x), x])$. The third element of a triple describes the consequences of the scenario occurring, $\mathrm{C}[\mathrm{S}(\mathbf{x}), \mathbf{x}]$. The CCDF displays the second and third elements (scenario probability and consequence) of the ordered triple.

The uncertainty in the calculation (prediction) of the CCDF (disposal system performance) can arise from any of the three elements (scenarios, probability, consequence). Quantitative evaluation of the uncertainty in the predictions is part of the PA methodology described below (Helton, 1991, p. 3-5; Rechard, 1989, p. 39). (Evaluation of the uncertainty that cannot be easily described quantitatively [e.g., through a parameter] must be qualitatively evaluated through peer review [see, for example, Rechard et al., 1992a, p. 18; 1992b, p. 31].)

\subsubsection{Organization and Mechanics of the Performance Assessment}

The organization and mechanics of the Sandia process for assessing the compliance of the disposal system with 40 CFR 191 (191.13) and other long-term (10,000 yr in U.S.) regulations consists of six general tasks (Figure 1-1) (Rechard, 1989). The six tasks are

1. Collection of data on spent fuel and high-level waste, site, regional geology, hydrology, and facility design to characterize the disposal system and regional area (system characterization); first phase in conceptual model development.

2. Identification of the continuous phenomena (sometimes called processes), short-term phenomena (events), and features of the disposal system whereby radionuclides might be released to the accessible environment, and selection of what to model (from known features, events, and processes) (scenario development); second phase in conceptual model development.

3. Development and execution of probability models to predict the likelihood of the scenarios (probability modeling),

4. Development and execution of consequence models to predict the amount of radionuclide release, including evaluating the uncertainties associated with the predictions (consequence modeling),

5. Comparison of the predicted releases and doses with government regulations (regulatory compliance assessment),

6. Evaluation of the important variables that influence the results (sensitivity/uncertainty analysis).

The remainder of this Introduction and the report itself are organized according to these general tasks.

\subsubsection{Sandia Analysis System Developed for Performance Assessment}

Requirements for PA Software. Based on the performance assessment process just described, software used in PA calculations must meet several requirements. Generally, the requirements can be grouped into two categories: built-in flexibility and built-in quality assurance (QA) features. Specifically, the software must

- Accurately link several distinct model components with little analyst intervention to eliminate tedious preparation of parameters (or previous results from the chained analysis) by the codes (QA),

- Trace the calculations so that they can be repeated $(\mathrm{QA})$, in addition to software $\mathrm{QA}$ features on individual modules, 


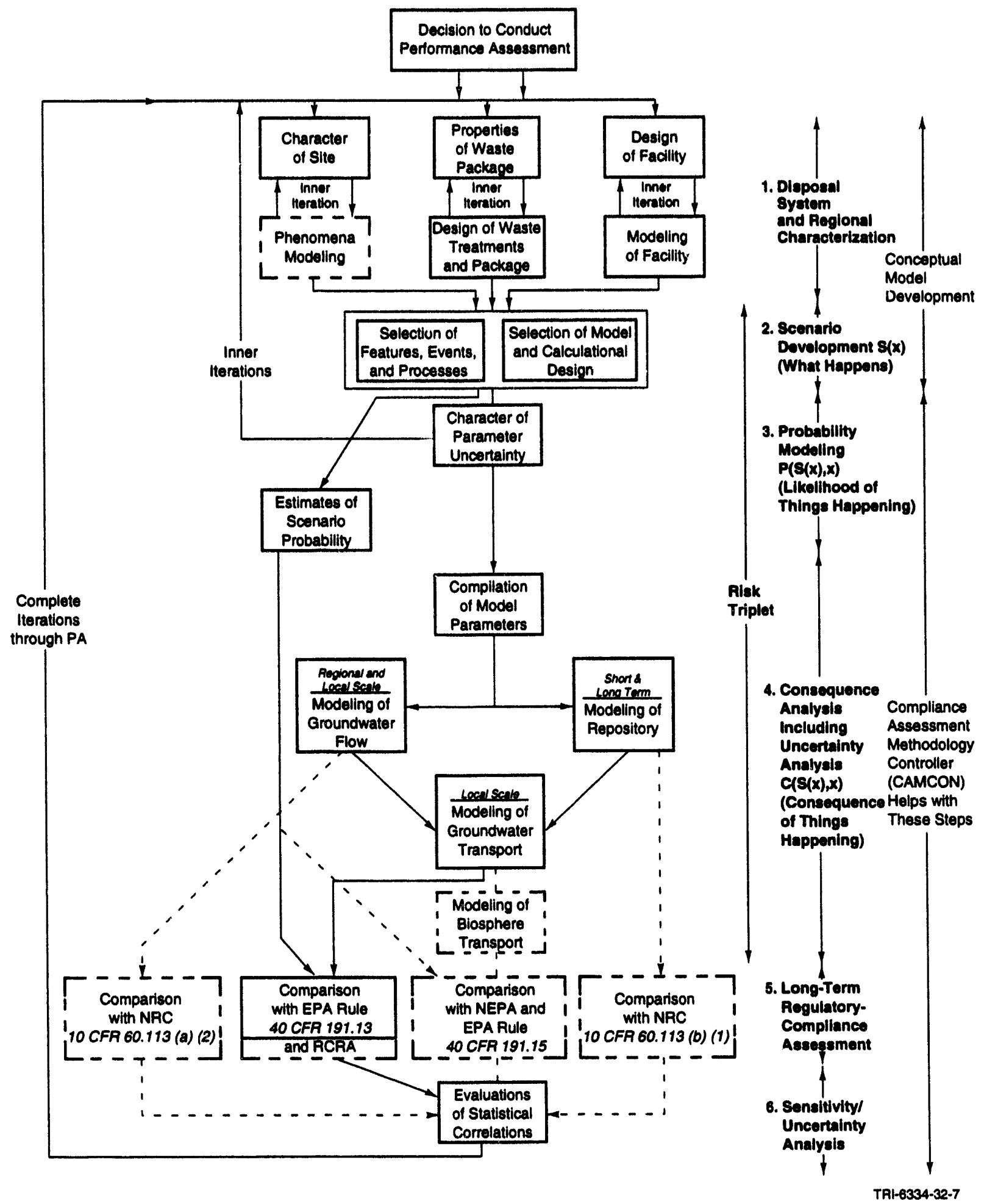

Figure 1-1. Sandia's method for conducting INEL Performance Assessment calculations involves six general tasks (after Rechard, 1989, Figure 3.1). This report is organized around these tasks. Components of the tasks shown with dashed lines were not performed in this initial performance assessment. (Although not within the original scope of work, a partial analysis of "Modeling Biosphere Transport" is provided in Appendix E.) Note that only a few of the possible inner iterative refinements are shown. 
- Propagate uncertainty (by Monte Carlo analysis in this performance assessment), uniquely identify calculations to avoid misinterpretation (QA and flexibility),

- Permit an analyst to examine a large number of intermediate and final results (QA and flexibility),

- Allow interpolation between modeling scales (flexibility),

- Permit iteration between computer codes (flexibility),

- Aid in exploring phenomena modeled by codes (e.g., of the repository model) (flexibility),

- Be flexible in switching between codes to aid in scenario screening, model/model comparisons, sensitivity analysis, and fine-tuning of the system for final compliance assessment calculations (flexibility),

- Be able to track the computational results and software used.

In other words, the variety of scenarios analyzed, the varying complexity of models used, and the need to switch codes when cycling through the procedure are characteristics of compliance assessments that require a flexible and versatile implementation. Furthermore, the number of repetitive computer runs, the need to properly identify runs, and the need to recreate runs are characteristics that require adequate quality assurance implementation.

CAMCON, Compliance Assessment Methodology Controller. Several software analysis systems have been built to meet the above-mentioned general requirements of a performance assessment. One approach is to build one code with numerous submodels (see, for example, VANDAL [Unsworth, 1989], SYVAC [Unsworth, 1989], or LISA [Saltelli et al., 1984; Saltelli et al., 1990; Bertozzi and Saltelli, 1985]) or couple one analysis code with data preparation, Monte Carlo sampling, and results display (for example NEFTRAN-S [Campbell et al., 1991]).

The Sandia Performance Assessment Department chose to build an analysis "tool box" (not one tool) by linking separate codes (either complex numerical or simple analytic codes) together. This approach

- Directly makes use of the interdisciplinary expertise represented in already existing codes as opposed to indirectly incorporating these concepts into one code, and,

- Allows the same tools to be used for both the detailed examination of components as well as the overall disposal system (with the possible exception of substituting fast-running modules or modeling subdomains in the later analysis when necessary).

Both points are especially important for nuclear waste repositories where the calculations are under intense scrutiny and elaborate procedures are sometimes necessary to justify that all steps taken in the analysis are defensible. For example, common engineering judgment used in developing a simplified model, although frequently correct, is not always rigorously defensible; instead, detailed modeling must often precede simplification. Hence, a PA system that is capable of handling both detailed and simplified models is important.

The CAMCON system analysis "tool box" for performing an assessment consists of six major components (Rechard, Ed., 1992, Ch. 2) (Figure 1-2):

1. Directory structure and protocols for storing codes for rudimentary configuration control,

2. A collection of frequently used subroutines in FORTRAN object libraries (e.g., plot libraries),

3. The Computational Data Base, or CAMDAT, for storing output data during the modeling task and the Secondary Data Base for storing site geometry and parameter distributions,

4. On-line documentation (help files), 


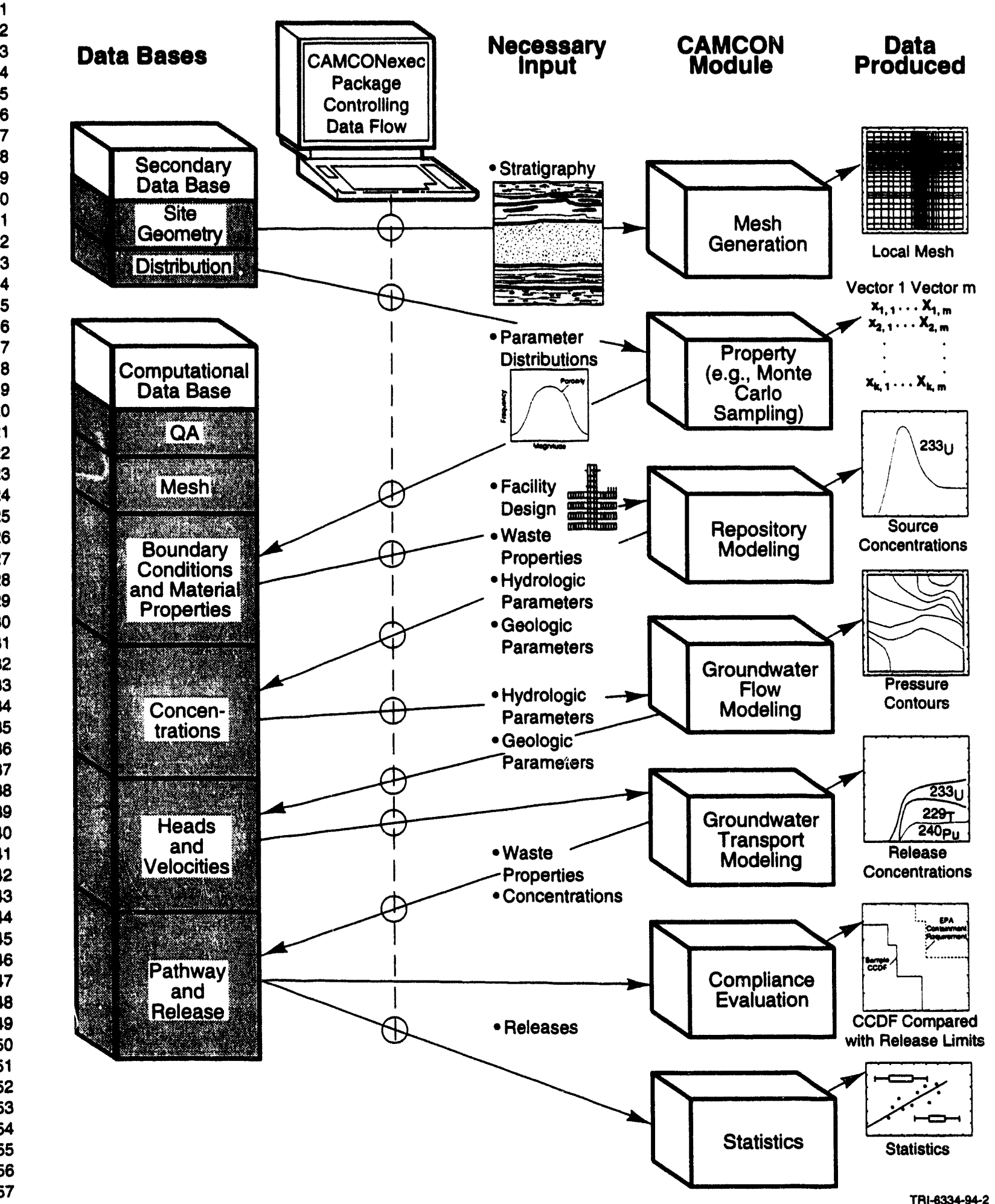

Figure 1-2. Components of the analysis tool, CAMCON. The CAMCON system consists of (1) directory structure and protocols for storing codes (not shown); (2) software libraries (not shown); (3) a secondary data base and computational data base; (4) on-line documentation (not shown); (5) an executive package to access models, and (6) code models. One pass through this system with one set of model parameters (some authors use the term "vector") represents one simulation or "run." 
1. Introduction

5. CAMCONexec (a suite [or executive package] of procedural files, logicals, and symbols to set up the computer environment) for ready access and execution (either batch or interactively) of the computational and support modules,

6. Ten code modules (e.g., computational, support, and utilities modules) containing the various codes used for an analysis.

Software Used within CAMCON. Section 7.6, "Computational Submodels and Calculational Design" and Appendix G, "CAMCON Software Used in Performance Assessment," list the codes for this performance assessment used to produce the CCDF, the primary performance metric used in this report. Appendix $G$ also lists the version number of the software used.

\subsection{System Characterization}

System characterization is the first phase in conceptual model development. In this phase, the physical systems such as the waste, its treatment options, the geologic repositories, and the backfill and seals, are defined and represented in the model.

\subsubsection{Waste Containment System}

A radioactive waste containment system includes three major subsystems (institutional controls, geologic barriers, and engineered barriers) and their corresponding major components (Figure 1-3). The first subsystem, institutional controls, consists of components such as legal ownership of the land and resources by the U.S. Government, fencing and signs around the property, permanent markers, public records and archives, and other methods of preserving knowledge about the disposal system (Trauth et al., 1993; Rechard et al., 1993).

The second subsystem, geologic barriers, is limited to the lithosphere up to the ground surface and no more than $5 \mathrm{~km}(\sim 3 \mathrm{mi})$ from the outer boundary of waste-emplacement rooms and drifts.

The physical features of the repository (e.g., design of the repository, waste form, waste parcel, and backfill) are components of the third subsystem, engineered barriers. For purposes of discussion, the components of the engineered barrier system are grouped into two subsystems: the waste parcel and the repository. (In this report, the waste parcel is defined as the waste form, internal backfill, waste canister, and optional overpack. The term waste emplacement package signifies the waste parcel and the backfill-buffer placed in the drillhole between the waste parcel and the host rock.) (As discussed later, this performance assessment assumes waste parcels are placed in vertically bored holes in the floors of the disposal rooms.)

The disposal system, as defined by 40 CFR 191, includes only the geologic and engineered barrier subsystems. As part of the performance assessment, analysts investigate how the disposal system behaves, assuming specific situations (i.e., combinations of features, events, and processes), that represent possible future conditions at the repository. Depending on the situation, different parts of the engineered and geologic barrier subsystems are assembled into a system that can be modeled. These modeling systems are described below in Section 1.7, "Consequence Modeling," and in Chapters 9 through 14.

\subsubsection{Waste Parcel Description}

Because this performance assessment studies the effect of various treatment options for spent nuclear fuel and high-level waste, several waste characteristics are considered. (Herein, waste refers to the contents of a nuclear waste canister such as spent nuclear fuel rods, brackets, hardware, except any internal backfill packed inside the waste canister.) First, the types of waste being modeled are described. Second, the inventory of radionuclides and hazardous 


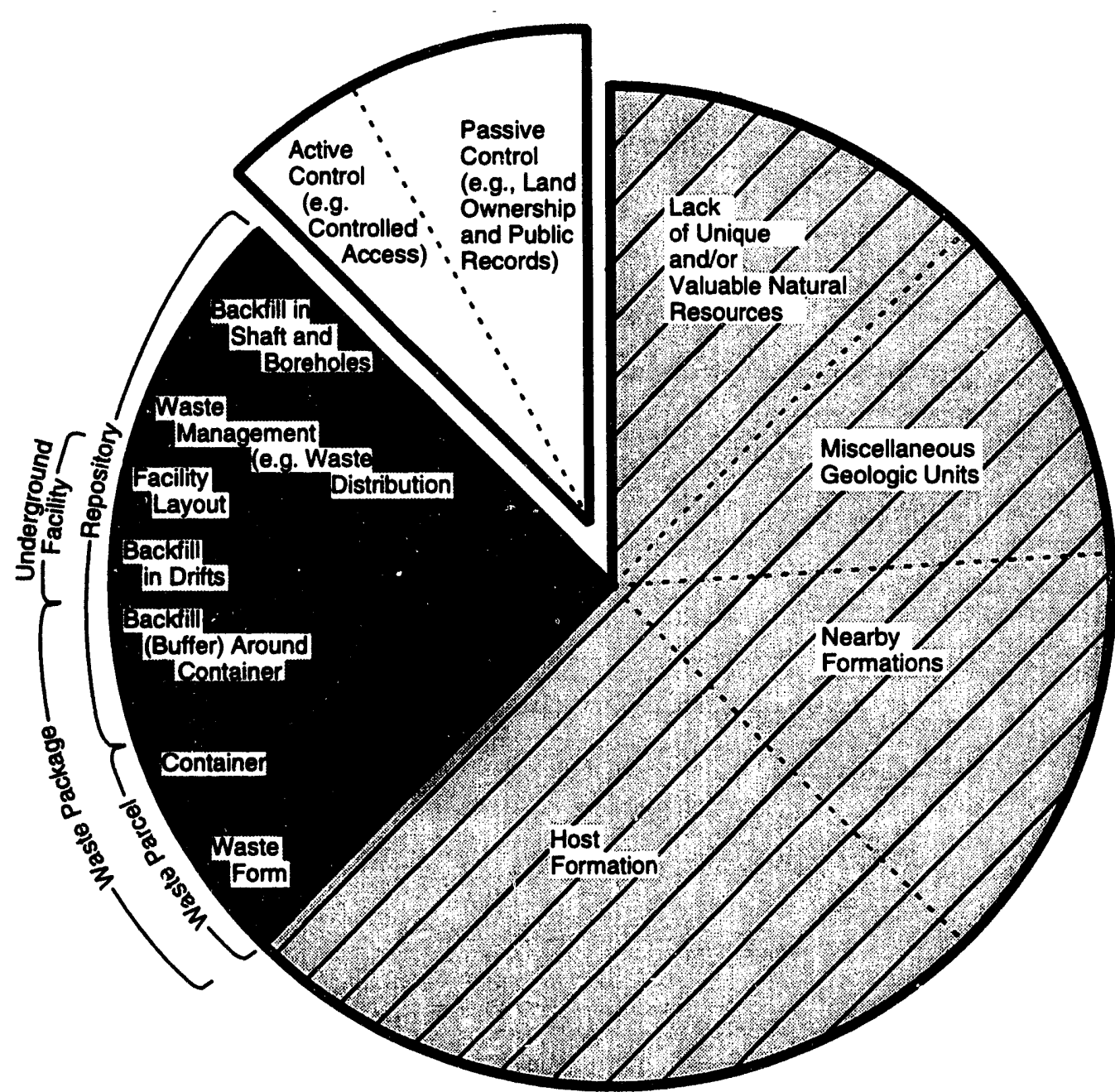

Note: Because importance changes with scenarios and regulations, the areas do not represent the relative worth of the subsystems and components.

Engineered Barrier Subsystem

10 CFR 60 omits shaft and borehole backfill and waste management from engineered barrier system.

Institutional Control Subsystem

40 CFR 191 does not allow the DOE to depend upon the enforcement of active institutional controls after $100 \mathrm{yr}$, for performance assessments.

Goologic Barrier Subsystem

(Maximum $5 \mathrm{~km}$ Distance Around Waste Disposal Area)

The requirement in 40 CFR 191 to consider human intrusion implies that parts or all of the geologic barrier can be effectively by-passed.

Various groupings of the engineered barrier -. "waste parcel" and "repository" are primarily used in this report.

TRI-6334-250-4

Figure 1-3. Subsystems and components of a radioactive waste containment system. The disposal system, as defined in 40 CFR 191, comprises the geologic and engineered subsystems of the waste containment system (after Rechard et al., 1990, Figure 1-10). Herein, the engineered barrier subsystem is discussed as a waste parcel and a repository. 
constituents found in the waste is described. Third, the options for treating the waste before disposal are defined. Finally, the physical characteristics of the waste containers are detailed.

Current Waste Types. This performance assessment considers five main types of material:

Spent Nuclear Fuel:

1. Graphite spent nuclear fuel from

- Fort St. Vrain reactor in Colorado,

- Cores I and II from Peach Bottom reactor 1 in Pennsylvania.

2. Special spent nuclear fuel (over 90 types of "experimental" spent fuel rods) categorized as

- Highly enriched uranium (HEU), i.e., spent fuel with over $20 \%$ by mass fissile $\left({ }^{235} U\right)$ material

- Low enriched uranium (LEU), i.e., spent fuel with less than $20 \%$ by mass fissile $\left({ }^{235} U\right.$ ) material

High-Level Waste:

3. Calcine high-level waste, from oxidation of liquid high-level waste, created when uranium and plutonium are extracted during the reprocessing of some spent fuel.

4. Glass-vitrified liquid high-level waste from three DOE sites: Defense Waste Processing Facility at Savannah River Plant, near Aiken, Georgia; Hanford Waste Vitrification Plant, Hanford, Washington; and West Valley Demonstration Project, West Valley, New York. ${ }^{\dagger}$

5. "Sodium-bearing" liquid waste $e^{\ddagger}$, which is difficult to calcine directly.

The waste types of primary interest for this performance assessment originate with spent fuel that has been accepted for interim storage and reprocessing since 1953 by DOE at the Idaho Chemical Processing Plant (referred to herein as the Chem Plant) on the INEL reservation. Before 1992, the major activity of the Chem Plant was the recycling of spent fuel to recover uranium, krypton, and other resources. During this process, both high-level and lowlevel liquid wastes were produced. In the past, the high-level liquid waste was heated to form oxides, collectively called calcine. Reprocessing has produced about $3800 \mathrm{~m}^{3}$ of calcine high-level waste." Sodium-bearing liquid waste from decontamination of facilities was also produced; about $5700 \mathrm{~m}^{3}$ of liquid waste exists. Before 1992, this waste was blended with the processed waste to form calcine because it is difficult to calcine the sodium-bearing liquid waste directly.

Currently 157 metric tons (MT) ${ }^{* *}$ of graphite spent fuel and $474 \mathrm{MT}$ of special spent fuel of the waste under study are in interim storage at the Chem Plant. In addition, a total of $186 \mathrm{MT}$ of graphite spent fuel is stored at the Fort St. Vrain reactor in Colorado and is likely to be shipped to INEL. Although spent fuel can be considered an important national resource, world events have diminished the immediate need to recycle spent fuel, and since April 1992, DOE has discontinued recycling spent fuel. However, the need to properly dispose of new and remaining spent fuel and the high-level waste in interim storage remains a high priority.

The graphite spent fuel from the Fort St. Vrain reactor consists of $0.360 \times 0.793 \mathrm{~m}$ hexagonal blocks of dense graphite with bored holes. The bored holes are filled with rods formed from uranium and thorium carbide pellets in a loose graphite matrix. The uranium and thorium pellets or microspheres in the Fort St. Vrain reactor and Peach Bottom Core $\mathrm{II}$ are coated with a tough silicon-carbide $(\mathrm{SiC})$ layer. The graphite spent fuel assemblies from Cores $\mathrm{I}$ and II of the Peach Bottom reactor are 0.089-m-dia $\times 3.66-\mathrm{m}$-long rods.

Although this waste is designated for Yucca Mountain, it was selected for disposal in the hypothetical repository to serve as a basis for comparison with the INEL waste and fuel and also to bring the waste inventory for the hypothetical repository to a more realistic level. As of 1988, the Yucca Mountain Project had mission plans to accept high-level waste immobilized in borosilicate glass from DOE, much of which would come from Savannah River, Hanford, and West Valley (U.S. DOE, 1988b; Barnard, in prep.).

* Although the sodium-besring waste is not legally considered high-level waste, it may contain enough radionuclides to have an activity greater than Class $\mathrm{Clow}$-level waste. In this performance assessment, the sodium-bearing waste is grouped and treated with the high-level waste. We also assume that it is pretreated by calcination, so we include it in the calcine waste from INEL when we refer to INEL's three waste types.

*. From a regulatory standpoint, a more useful unit of measure of the waste than either volume or general mass is metric tons of heavy metal (MTHM) (mass of all isotopes of uranium, plutonium, and thorium in the fuel before irradiation in a reactor, not including the mass of the cladding, fuel assembly, etc., surrounding the fuel). The MTHM mass measure is used throughout this report, except when trying to convey a general concept of what the reader would actually see or lift wher sserving the waste. 
The special spent fuel is divided into two categories: highly enriched uranium (HEU) fuel $\left(>20 \%{ }^{235} U\right)$ and low enriched uranium (LEU) fuel $\left(<20 \%{ }^{235} U\right)$. Much of the LEU special spent fuel is similar in shape to commercial reactor spent fuel but includes several types of cladding surrounding the fuel and percentages of fissile uranium $\left({ }^{235} \mathrm{U}\right)$. For HEU special spent fuel, the initial percentage of ${ }^{235} \mathrm{U}$ can be quite high and the degree of reaction ("burnup") quite low, unless the HEU spent fuel is recycled (by the Chem Plant process described below). For unrecycled HEU special spent fuel, the potential for closely packed waste reaching critical mass and generating a substantial amount of heat is much greater than for "normal" fuel from commercial reactors.

As noted earlier, reprocessing of some spent fuel produces both high- and low-level liquid wastes. When the high-level liquid waste is heated to form oxides, collectively called calcine, the volatile fission products, such as ${ }^{3} \mathrm{H}$ ${ }^{14} \mathrm{C}$, and ${ }^{129} \mathrm{I}$, are released to the atmosphere. The calcine granular solid flows easily and is currently stored in partially buried, stainless-steel silos in a cylindrical concrete vault at the Chem Plant.

Inventory. In 1992, the activity of the spent fuel at INEL totaled about $145 \times 10^{6}$ curies (Ci). (Recall that the inventory changes with time as the radioactive isotopes undergo decay to form new isotopes both radioactive and stable.) The activity of high-level waste totals $47 \times 10^{6} \mathrm{Ci}$. The total activity of $192 \times 10^{6} \mathrm{Ci}$ of waste in this study was produced from about 666 metric tons of heavy metal (MTHM) ${ }^{\dagger \dagger}$ (or equivalent waste units) (mass of all isotopes of uranium, plutonium, and thorium before irradiation in a reactor).

Each waste type from INEL was evaluated individually in this performance assessment and also studied in combination as one of five waste disposal groups. The purpose of grouping the wastes was to more realistically evaluate the behavior of the waste in a moderate-sized repository. Since it is clearly not cost effective to build a repository for just the fuel and waste stored at Idaho, when the waste types were studied in combination, high-level waste from three DOE sites was added to the hypothetical repository to illustrate what a more realistic repository would contain. The inventory from the DOE sites includes high-level waste immobilized in borosilicate glass from (1) the Savannah River Plant (SRP) (produced at the Defense Waste Processing Facility [DWPF]), (2) Hanford Waste Vitrification Plant (HWVP), and (3) West Valley Demonstration Project (WVDP) (formally a demonstration plant for reprocessing commercial spent fuel). Together, the INEL fuel and waste and the DOE site waste total 9934 MTHM (Table 1-2) This total inventory (about 10,000 MTHM) does not represent all of the DOE high-level waste and spent fuel, e.g., nuclear reactor spent fuel at Hanford and future decontamination and site cleanup at DOE facilities will result in more spent fuel and high-level waste. However, the 10,000 MTHM is the amount of spent fuel and high-level waste that was clearly identified and for which early plans for disposal were developed (e.g., U.S. DOE, 1985b).

The combined volume and MTHM equivalent of the Idaho fuel and waste as summarized from Chapter 4 are $49 \%$ and $6.7 \%$, respectively, of the total for the hypothetical repository (Table 1-2). The addition of commercial waste (as per NWPA, 1983) would further decrease this percentage and reduce the influence of INEL waste, making it more difficult to identify the better waste treatment options, described below. The influence of high-level waste and spent fuel from the DOE defense complex on the overall commercial repository performance can be addressed in later reports. The jistribution of radionuclides differs for each site and is described more thoroughly in Chapter 4 and Appendices A anu D.

Waste Treatment Options. Before the waste is placed into a container for disposal, waste treatment options are considered with the intent of making the waste type more resistant to transport through the geologic media and eventual release. The treatments differ for each type of spent fuel and high-level waste because characteristics vary among types (e.g., how susceptible the material is to reaching critical mass in certain hypothetical situations). For disposal in a granite repository, no more than $0.7 \mathrm{~kg}$ of ${ }^{235} \mathrm{U}$ is placed in a waste package to conservatively eliminate concerns of criticality; this requirement means that some spent fuel may be physically cut up into smaller pieces or chemically diluted with depleted uranium. Because of salt's low final porosity (which means that only a small amount of water is available to slow neutrons and thus help promote a nuclear reaction), two waste treatment options

\footnotetext{
Another initialism, MTIHM (metric tons of initial heavy metal), is used by some authors who wish to emphasize that the measurement is the initial mass of heavy metal rather than the current mass of heavy metal. In this report, we use the designation, MTHM, not only because it is found more frequently in the literature but also because 40 CFR 191 defines "heavy metal" as "... all uranium, plutonium, or thorium placed into a reactor ..." (40 CFR 191.12; emphasis added). Thus, the use of MTIHM, while useful in calling attention to this fact, does not seem necessary.
} 
Table 1-2. Total Inventory for Hypothetical Repository

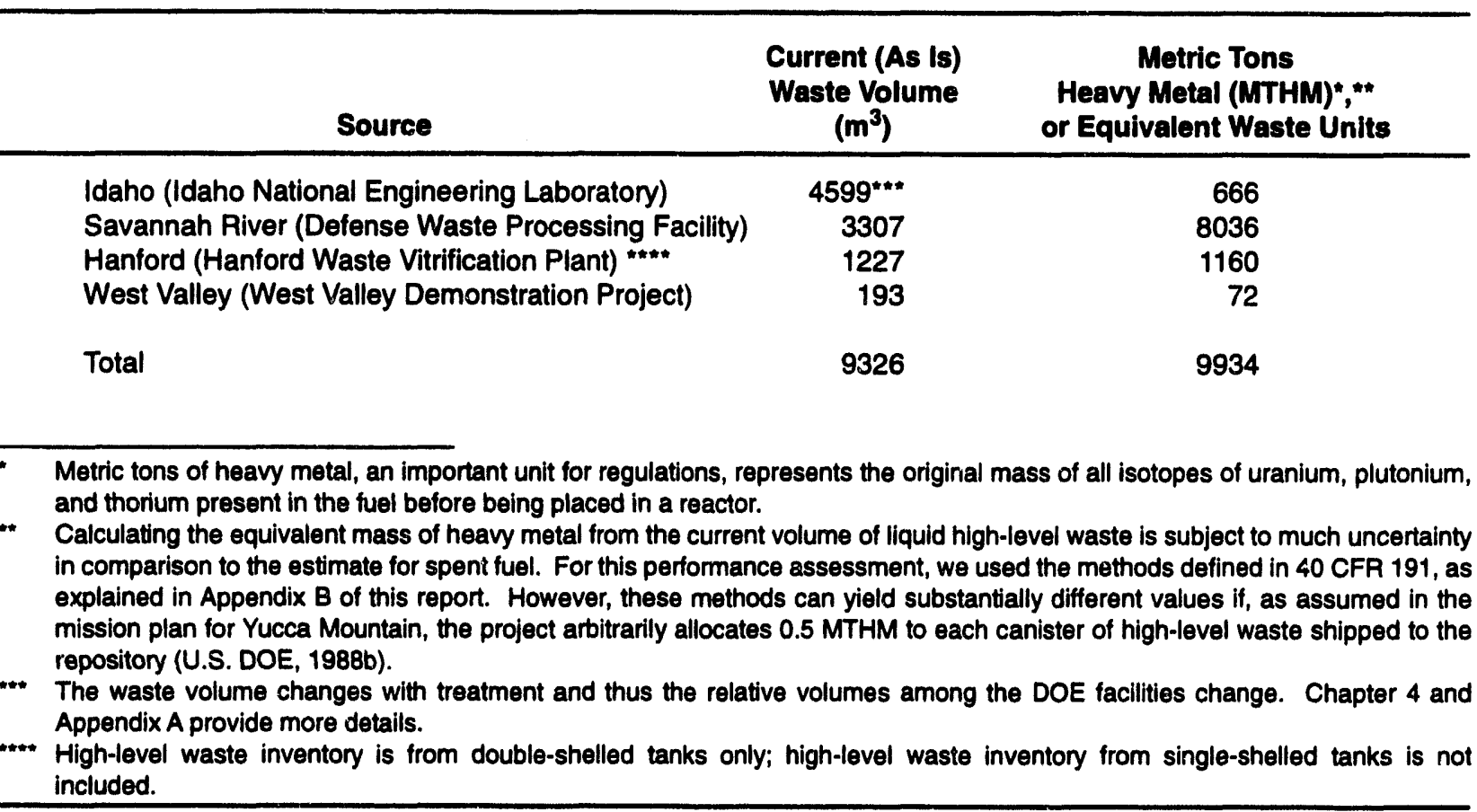

for the salt repository permit up to $10 \mathrm{~kg}$ of ${ }^{235} \mathrm{U}$. In all waste treatment options, the amount of heat-generating isotopes was low enough to keep the surface temperature of the waste parcel below $398 \mathrm{~K}$ and the surface temperature of the waste emplacement package below $373 \mathrm{~K}$.

In this performance assessment, five waste treatment options are considered for the spent fuel and high-level waste types stored at INEL. Although many combinations of treatments could be constructed, the waste treatment options selected range from placing currently existing spent fuel and high-level waste directly into waste parcels to dissolving spent fuel and high-level waste into a glass-ceramic matrix. These waste-treatment options roughly correspond to one low (direct disposal), two moderate (mechanical disassembly and glass-ceramic encapsulation or grinding and glass encapsulation), and two high levels of stabilization or conditioning of the spent fuel and high-level waste (chemical separation or dilution with depleted uranium). The moderate and high levels of stabilization reduce the volume of high-level waste but potentially create low-level waste. Also, in some waste forms resulting from treatment, waste types may be comingled. As used in this report, the waste form is the form of the waste after treatment and before placement in the container. The feasibility and costs of the treatments are not entirely known at this time; at present, the plausibility of the treatment options is only qualitatively based on current experience with highlevel waste and spent fuel.

The five treatment options apply to the two spent nuclear fuel waste types and the high-level waste and were studied individually and as a group in separate performance assessments. When the waste is studied as a group, each waste treatment option is considered in a group, e.g., option 1 for graphite spent fuel, special spent fuel, and calcine high-level waste is considered as Waste Disposal Group 1. For graphite spent fuel, the five options considered are (1) whole or physically cut block disposal, (2) rod/block separation, (3) graphite burning, (4) national resource and spent fuel recycling, and (5) oxidation, and then dilution with depleted uranium. For special spent fuel, the five options considered are (1) spent fuel/lead disposal, (2) spent fuel/glo ss-ceramic calcine, (3) spent fuel/borosilicate calcine disposal, (4) national resource and spent fuel recycling, and (5) dilution with depleted uranium, and then oxidation of mixture. For the calcine, the five options considered are (1) granular calcine disposal, (2) spent fuel/glass-ceramic calcine, (3) spent fuel/borosilicate calcine, (4) glass-ceramic calcine, and (5) borosilicate calcine. 
For the high-level waste (liquid, slurries, and solid) stored at Savannah River, Hanford, and West Valley, only one option was considered: immobilization in a borosilicate glass.

Chapter 4, "Waste Parcel Description," and Appendices A and D provide details on such major characteristics as radionuclide inventory, volume solubility, and leachability for the minimally stabilized waste and the four other treatment options.

Characteristics of Waste Container. The waste container holds the waste for transportation and handling after treatment, and it can also be designed as an important component of the engineered barrier. Because a simple waste container design is assumed for this performance assessment (as described below), it is most often referred to as simply the container. However, this container serves as the handling and transportation canister (i.e., the first container around the waste, which is used for handling, transportation, and structural support) and in one case has an overpack (i.e., an outer container placed around the canister, which serves to provide a specialized function such as corrosion resistance). The waste form, the internal backfill and the container(s) (canister and possibly overpack) are referred to as an emplacement unit, the waste parcel. Once emplaced in the repository with a backfill-buffer, the modeling unit of waste parcel and backfill-buffer is referred to as the waste emplacement package (refer back to Figure 1-3).

The container assumed in this study is a $9.5-\mathrm{mm}$ (3/8-in.) thin-shelled unshielded container of austenitic AISI 304L stainless steel. This type of container is similar to the Yucca Mountain Project container for defense high-level waste but does not have an overpack of corrosion-allowance or corrosion-resistant material. The container is assumed to be filled with waste and filler, and welded shut. After being transported to the site, the container is placed in the floor of the repository. The size of the stainless steel container varies based on the size of the waste form; however, its range is between 0.1 and $0.7 \mathrm{~m}$ in diameter and 1.8 and $4.3 \mathrm{~m}$ long. For comparison, the nominal size of the high-level waste "reference" container for the Yucca Mountain Project is $0.66 \times 4.76 \mathrm{~m}(26 \times 188$ in.). The filler inside the canister is assumed to be castable metal (e.g., lead) to transfer structural and heat loads (Molecke et al., 1981 , p. 30; Goodwin and Westerman, 1992). (Lead is being considered in several waste programs throughout the world [e.g., United States (U.S. DOE, 1989), Swedish, German, Swiss, Argentinian, and Canadian nuclear waste disposal programs]; however it is considered to be a hazardous chemical according to RCRA [1976], thus possibly adding a licensing complication for disposal in the United States.)

Although a single thin-walled stainless steel container without a protective overpack is not being actively considered by the geologic disposal program in the United States, it was chosen here because (1) it is a handling canister being considered for the high-level waste from defense programs and (2) the waste form itself remains the focus of the study rather than the container because the container is allowed to rapidly fail, even from generalized corrosion. ${ }^{\ddagger \ddagger}$

This report does include one calculation using long-lived containers to explore the effects of using more corrosion-resistant containers. In this one calculation, it is assumed that an Inconel- 625 container is emplaced in an overpack container; the overpack is 6.4-mm (1/4-in.) Titanium Grade 12 or Inconel 625 for the salt and granite repositories, respectively. (Inconel-625, a high nickel-based alloy, was chosen because INEL supports a metal recycling program that could supply large quantities of 300 series stainless steel, already contaminated with a small amount of radionuclides, to blend with nickel from the U.S. defense stockpile to make the high-nickel alloy, Inconel.)

Note that although the thin-shelled stainless-steel container is sufficient for evaluating compliance with 40 CFR 191 (the regulation considered for this performance assessment), 10 CFR 60 currently requires a 300- to 1000-yr waste package (i.e., waste form, internal backfill, container, and backfill-buffer around the container) that the thinshelled stainless-steel container may not meet. However, recall that this study is an initial performance assessment; other performance assessments may explore long-lived containers in detail. Currently, numerous cypes of containers and fillers are being considered in the U.S. and abroad. For filler, the Finnish disposal program is considering

$\$$ In our model, gas within the repository did not influence the results (see Chapters 12,13, and 16), and the influence of diffusion through the stress corrosion crack was not modeled. Therefore, if stress corrosion is the primary mode of failure, as in a salt repository, then a thick-walled container would also behave the way the thin-walled container behaved in our model, which is described in Chapter 11, "Gas Generation and Source Term Submodels," as long as significantly more gas (than that generated by the thin-walled container) was not generated by the thickwalled container. 


\section{Introduction}

granular material such as sand, glass beads, or lead shot (Vieno et al., 1992). For containers, Sweden (Vogt, 1993) and Finland (Aikas, 1993) are actively pursuing steel containers for structural strength surrounded by a copper overpack. Spain (Nilsson et al., 1993) is considering the use of thick carbon steel and using the extensive material property data base available for this material. In the U.S., eight conceptual options are being considered (see Appendix D).

\subsubsection{Geologic Barrier Characterization}

The geologic barrier of the disposal system comprises the sequence of sedimentary, metamorphic, or igneous rocks isolating the repository from the accessible environment. The primary technical criteria for site selection are an ability to isolate radionuclides from the biosphere and to provide physical and chemical stability. Because this performance assessment is focused on evaluating waste treatment options rather than sites, two generic sites were modeled for the study: one in bedded salt and the other in a saturated, partially fractured igneous (granite) rock formation. (Other potential rock formations in the United States that could be considered in future performance assessments, if deemed appropriate, include domal salt, basalt flows, and saturated and unsaturated tuff. The latter site will be considered in 1994.)

Salt was selected as one of the generic sites because of its favorable properties (e.g., entombment of the waste through creep) and the availability of data from an actual site, which is necessary to firmly establish the plausibility of the results of the performance assessment As early as 1957, the National Academy of Sciences (NAS) proposed bedded salt for nuclear waste disposal (Carter, 1987, p. 54). The Waste Isolation Pilot Plant (WIPP), a potential repository for transuranic waste from nuclear weapon construction, has been under study since the mid-1970s; most of the data for the hypothetical bedded salt site come from the WIPP, which is located in southeastern New Mexico near the city of Carlsbad (WIPP PA Division, 1991c). Important geologic features at the WIPP, such as an overlying brine aquifer, an anhydrite marker bed slightly above the repository horizon, and the possibility of a pressurized brine reservoir beneath the repository, were retained for the generic site in this study (WIPP PA Division, 1991b). The level of the hypothetical salt repository is deeper than the existing repository, however, to take advantage of a thick sequence of halite, which is better suited for disposal of high-level waste (Weart, 1979).

The second generic repository was assumed to be crystalline rock. Tuff, basalt, and granite-all crystalline rocks that have been considered as potential repositories for radioactive waste-were initially examined. Early in the project, however, tuff was eliminated from consideration by the Waste Treatment Program at INEL until the second year of this 2- to 3-yr effort, because of the technical challenges presented by unsaturated tuff and because the waste and spent fuel under study had not yet been designated to be disposed of in a tuff repository (i.e., the Yucca Mountain Project) (Barnard et al., 1992). Granite was finally selected, over basalt, because, (1) it is tectonically and geochemically stable, (2) the geologic media was thought different enough to highlight various aspects of the 40 CFR 191 regulation (e.g., the salt site releases are solely from human intrusion, but the granite site has the possibility of some releases even when the site is undisturbed), and (3) site characterization data were available from current international studies, such as the nuclear waste management programs in Canada, Sweden, Finland, and Switzerland. Much of the data about the granite site comes from the fairly mature Canadian program in a granite batholith with few fractures, with other supplemental data from the Swedish program at the Stripa mine, which has ubiquitous fractures (Neretnieks, 1986; Everitt and Read, 1989).

For both repositories, uncertainties in the actual properties of the geologic barrier may be large relative to the uncertainties in the engineered barriers ${ }^{* * *}$ (Section 1.4.4) because the geologic barrier is characterized, not designed, and thus the uncertainties have an important influence on the result (i.e., CCDF). However, for this performance assessment, examination of these uncertainties (expressed as distributions of model parameters) was not stressed in the analysis so that the focus would remain on evaluating many waste forms.

On the other hand, uncertainty in the behavior of an engineered barrier and the behavior of a geologic barrier over geologic time may not be so very different. 
The two generic repositories are more thoroughly described in Chapter 5, "Hypothetical Geologic Barrier," and Appendices B and C.

\subsubsection{Repository Design}

The engineered barriers consist of design elements that are intended to isolate the waste from the accessible environment. Components of the engineered barriers that are specific to the repository include (1) the subsurface facility layout, (2) the design for waste emplacement, and (3) specially placed backfill (a subset of which is often called a "seal") in the drifts, shafts, and directly around the waste parcel. These concepts are more thoroughly described in Chapter 6, "Repository Design." Two other design components are the waste form itself and the containers; these were discussed in Section 1.4.2, "Waste Parcel Description."

The mine design is a room and pillar configuration in which 10 rooms are grouped into a panel, with the entire repository consisting of 10 panels connected by access drifts to the shaft. The enclosed area of the disposal region is about $6.55 \times 10^{5} \mathrm{~m}^{2}$ (excluding access drifts from the shafts to the panels). (The terminology for the subsurface facilities, such as access drifts, is shown graphically in Chapter 6, "Repository Design.") Because many of the precise details of the repository - such as the number of shafts and their dimensions, number of passageways (drifts) and their dimensions (e.g., spacing and shapes of drifts), and the size of the operational area-depend more on the operational phase of the repository than behavior after closure, Chapter 6 highlights only the dimensions considered pertinent to post-closure behavior, such as the minimum spacing of canisters.

Subsurface Facilities. The disposal region of the repository was sized to accommodate Waste Disposal Group 1 (base case), which includes about 10,000 MTHM based on the number of waste parcels from Idaho, Savannah River, Hanford, and West Valley (Section 1.4.2). ${ }^{\dagger \dagger}$ The repository was fixed at 10 panels so that the other waste disposal groups and individual waste parcels could be fairly evaluated, i.e., the effects of changes in repository size on observed waste form behavior would be eliminated. Because the volume changed per waste disposal group and individual waste parcels, the volume and mass was proportionally increased for these latter analyses.

Although the salt repository is symmetrical, the granite repository must adapt to local conditions (either favorable or adverse) encountered while mining, and hence the repository is not necessarily symmetrical nor is its final shape precisely known. The design used for the granite repository is similar to the salt repository but the hypothetical granite batholith does include two small fracture regions that were avoided when mining the disposal area.

Waste Placement in Repository. The conceptual model must consider how the waste is placed in the repository, the amount of spacing between waste parcels, and the types of waste in each room.

Waste Orientation. For this performance assessment, the orientation of waste parcels in the repository is vertical placement in bored holes so that gravity assists in drilling and placement. Tunnel, or drift disposal, was also considered (see Section E.3.1). However, note that no final decisions have been made on a preferred orientation for unshielded waste parcels in the United States. A reason for horizontal emplacement (i.e., boring holes in the "walls" of the drifts, rather than the "ffloor") is the opportunity to thoroughly examine and characterize horizontal strata before emplacement, which may be an advantage for a salt repository in meeting the strict characterizations requirements of 10 CFR 60 (the advantage would diminish in a presumably homogeneous mass of granite).

Waste Spacing. The spacing of waste parcels depends primarily upon three factors: (1) thermal limits of the waste form, backfill-buffer, and adjacent geology, (2) considerations of waste criticality, and (3) limits on constructability (including waste parcel diameter, backfill thickness, and boring equipment access). For this performance assessment, the driving factor was thermal limits. Limits of $100^{\circ} \mathrm{C}$ at the backfill-buffer/host-rock interface were used to ensure that groundwater would not vaporize in the granite repository. The low heat loading of the stored DDE

\footnotetext{
tt+ By comparison, the proposed size of the Swedish repository is about 7800 MTHM (SKB, 1992a, p. iv); the amount of waste stored in dry casks or pools at the 111 reactors in the U.S. in 1990 was about 21,500 MTHM (BRWM, 1992, p. 4); and the entire Yucca Mountain repository may store no more than 70,000 MTHM until a second repository is licensed (NWPA, 1983, Section 114).
} 
waste permitted a spacing of $2.6 \mathrm{~m}(8.5 \mathrm{ft})$ center-to-center in a compact hexagonal pattern per room for both the salt and granite repositories. The spacing calculated for criticality using conservative assumptions fell within this range (see Appendix E). Although constructability was not examined in great detail, the 2.6-m center-to-center spacing appeared to be adequate (see Appendix E).

Waste Management. For the consequence model, a uniform mix of the waste within each disposal group was assumed for each room.

Backfill. As used herein, backfill is the material that fills the excavated openings of the repository. The backfill material, which differed between the two repositories, is intended to prevent undue host rock disturbance from drift collapse, impede water movement, and possibly adsorb radionuclides. Special categories of backfill include (a) seals (e.g., concrete) for providing immediate, sometimes short-term, sealing of the shafts and access drifts and (b) backfill-buffer, which is backfill placed around the waste parcel, and thus becomes part of the waste package. ${ }^{\ddagger \ddagger}$ The salt repository used compacted salt backfill in the access drifts and rooms, and 0.3-m-thick crushed-salt backfill around the waste parcels. Based on the experimental program for the WIPP Project, the crushed and compacted salt is assumed to consolidate because of the lithostatic pressure and spent fuel heat loading to within $95 \%$ of the original rock salt density and a corresponding low permeability within $100 \mathrm{yr}$. The granite repository used crushed-rock backfill augmented with bentonite clays in the drifts and rooms, and 0.3-m-thick pure bentonite clay backfill-buffer around the waste parcels. As argued in Chapter 6, "Repository Design," and Appendix C, "Scoping Calculations," the 0.3-m thickness of clay backfill-buffer in the granite repository kept the temperature at the waste parcel/backfill-buffer interface below $125^{\circ} \mathrm{C}$, which is well below $170^{\circ} \mathrm{C}$, the temperature at which the clay changes from montmorillonite to illite crystalline structure.

\subsection{Scenario ${ }^{* * * *}$ Development}

Scenario development is considered the second phase of conceptual model development (the first phase being system characterization, Section 1.4). Generally speaking, scenario development is the process of deciding what may happen to the disposal system in the future and how to model it. Although scenario development is not always a formal step of conceptual model development, it is useful to formalize the process for a large analysis such as a performance assessment. The process entails selecting features (e.g., a brine reservoir under, or an undetected fracture in, the repository), events (e.g., humans drilling into the repository), and processes (e.g., generation of gas in the repository after disposal of the waste).

\section{Scenario development consists of several steps: ${ }^{\dagger \dagger \dagger \dagger}$}

1. Identifying the full scope (i.e., "universe") of features, events, and processes of the disposal system. (Events are also referred to as short-term phenomena; processes, as long-term phenomena.)

2. Selecting those features, events, and processes that might contribute to contaminants being released to the accessible environment.

3. Incorporating those features, events, and processes into the performance assessment calculations.

4. Creating conceptual models based on (a) the disposal system characterization and (b) the selected features, events, and processes.

\footnotetext{
抹 Because some emplacement designs envision using the backfill material to moderate the release of contaminants to the host rock, the backfill for the waste package hole is referred to as "backfill-buffer." This term is used in this report for the crushed-salt backfill, although it does not technically provide a buffering function in the chemical sense.

.... The various meanings of "scenario" are discussed in Chapter 3, "Overview of Performance Assessment"; the reader is advised to peruse at least Section 3.3.1 in order to understand the contexts in which "scenario" is used here.

${ }^{+++\dagger}$ In other documentation, the term scenario development is often synonymous with steps 1 and 2 only, because these two steps initially attracted greater attention than steps 3 and 4 . In this report, however, scenario development includes not only the selection of the features, events, and processes, but also the incorporation of those components into the computational model.
} 
During the scenario development phase, many decisions are made about what to include in the model. However, the decisions themselves are more conveniently discussed in the chapters that describe either system characterization or consequence models. For example, features are discussed in the chapter on geologic barrier (Chapter 5); processes and assumptions are discussed with the consequence models (Chapters 11 through 15). A set of calculations were done in support of the scenario development step and are termed scoping calculations. They include a preliminary performance assessment, criticality safety calculations, and a detailed analysis of a single waste parcel. The scoping calculations are described in Appendix C.

\subsubsection{Selected Features, Events, and Processes}

As part of scenario development, the performance assessment analysts used Sandia's formalized selection procedure (Cranwell et al., 1990) with modifications to identify several features, events, and processes as candidates for modeling at both the granite and salt sites. Note that although this procedure is both extensive and thorough, it still must be viewed simply as a starting point for the analysis. Because so much of the disposal system is characterized, not designed, adding to the initial "universe" remains a likely possibility.

To keep the focus of this performance assessment on the comparison of waste treatment options, not all combiriations of the features, events, and processes were used (e.g., glaciation). The basic features, events, and processes selected for modeling in this performance assessment are described below.

Features. The basic features explored in this report differ for the two generic repositories. For the bedded salt repository, an overlying fractured brine aquifer, an anhydrite marker bed slightly above the repository horizon, and a possible underlying brine reservoir were considered. For the granite repository, fractures in the rock batholith to the accessible environment were considered but modeled as porous media conduits (which is feasible, provided some latitude is permitted in selecting a porosity that maintains appropriate travel times and extensive fracture density, isotropic orientation, and hydraulic connectivity are assumed).

Event. The basic event explored in this report is human intrusion.

Processes. The basic processes (i.e., continuous phenomena) explored in this performance assessment are heat conduction, gas generation within the waste from corrosion of the canister, subsequent two-phase Darcy flow in the repository and transport through fractures. The waste parcel/backfill-buffer interface was assumed to remain below $125^{\circ} \mathrm{C}$, and the backfill-buffer/host-rock interface was assumed to remain below $100^{\circ} \mathrm{C}$ to preclude steam generation (at elevated temperatures); colloid transport was considered only through large ranges of solubility; and increased permeability from additional fractures being formed by gas was not considered. This latter assumption was partially justified by the low gas pressure that developed in the repository over time as described in Chapters 12 and 13 . Also, the possibility of the highly enriched uranium spent fuel reaching critical mass was not included in the total-system performance assessment, although scoping calculations were performed (see Appendix C, "Scoping Calculations). Because phase changes could not be modeled at this time with the computational tools assembled for this performance assessment, the effects of higher temperatures (e.g., from closer waste parcel spacing or critical conditions in the repository) were not modeled.

\subsubsection{Calculational Design}

Besides the selection of features, events, and processes to model, other decisions must be made in the design of the calculation: (1) whether to create broad classes of scenarios called summary scenarios, (2) whether to further discretize the summary scenarios to obtain adequate coverage of various categories of parameters (e.g., time of intrusion), (3) how much simplification is acceptable in the calculations for the features, events, and processes, (4) how much spatial and temporal detail is acceptable to run the calculations, and (5) how many parameters exist for which the ranges are not only sufficiently large to likely influence the results but also the sensitivity of the results to these parameters is of interest. 


\section{Introduction}

How these issues are addressed depends primarily on the purpose of the analysis and the means (time, funds) provided for the work. For this initial performance assessment, the first two issues were addressed as follows (the third and fourth items are discussed under consequence modeling; the fifth item is discussed under probability modeling): In the formal performance assessment for the salt disposal system, one summary scenario was selected and then the permeability of one borehole plug was varied to simulate a range of behavior (see Chapter 5). For the human intrusion event in granite, one summary scenario was also selected and the permeabilities of the connections were varied. Note that this method differs from that used in the scoping calculations, where the human intrusion event for salt was partitioned into three broad classes (summary scenarios of one and two intrusions into the repository, and one intrusion into the brine reservoir) and further discretized into calculational scenarios so that attention could be focused on select subsets of the parameter space (i.e., to highlight situations where flow is forced through the repository, termed an E1E2 situation) (see Appendix C).

The scenario development task-specifically identification of features, events, and processes-is described in Chapter 7, "Scenario Development." Scoping calculations that were performed in support of scenario development (i.e., deciding what to model) are described in Chapter 8, "Scoping Calculations," and Appendix C.

\subsection{Probability Modeling}

For a performance assessment, two types of probability models are used. The first estimates the likelihood of uncertain parameter values by constructing distributions for imprecisely known model parameters. The second estimates the likelihood of the broad classes of the parameter space, called summary scenarios.

The first probability model is used for imprecisely known parameters. The parameter probability model is usually subjective, but a probability model nonetheless. In the case of an assessment of the performance of a repository that has actually been proposed, the subjective generation of the probability distributions from the system characterization data is a significant and time-consuming process. However, in a small integrated program, parameter probability modeling can occur simultaneously with conceptual model development (i.e., system characterization and scenario development), which is the case for this initial performance assessment. Because the purpose of this performance assessment is to examine treatment options, the primary criteria used to select which parameters to vary was the parameter's potential influence on waste treatments; thus, most parameters for the geologic barrier model were kept constant.

The second type of probability model is used to evaluate the probabilities of the scenarios. The probability of the selected scenario event-inadvertent intrusion into the repository by exploratory drilling-was evaluated with a homogenous Poisson probability model.

The probability models for the parameters and the human intrusion event for the salt and granite disposal systems are discussed in Chapter 9, "Probability Modeling."

\subsection{Consequence Modeling}

A major portion of the PA methodology consists of simulating the features, events, and processes to estimate the amount of contaminants released to the accessible environment, including evaluating the uncertainties associated with the predictions. This step in the performance assessment process is referred to as consequence modeling. The model used for consequence modeling is actually a composite function of several models.

To examine the acceptability of various waste forms (e.g., whether little or no treatment of the waste type is necessary, thus relying on the engineered characteristics of the high-level waste or spent fuel), a substantial effort was made to model phenomena related to the transport of radionuclides out of the repository. The two categories of consequence modeling systems for the disposal system are (1) repository, and (2) groundwater flow and transport. The repository model, which evaluates the amount of radionuclides that potentially leaves the repository, was composed 
of two subcomponents: (a) removal of radionuclides through drilling cuttings/cavings and (b) fluid flow and transport. Details of the drilling module are described in Chapter 10, "Release from Drilling into Repository-CUTTINGS." The fluid flow and transport models are discussed in Chapters 12 through 15. Their submodels, gas generation and source term, are discussed in Chapter 11. The fluid flow and transport portion of the repository model is, in turn, a source of input for the groundwater flow and transport consequence modeling system, which evaluates transport of radionuclides from the repository to the accessible environment.

In this performance assessment, two sets of modeling simplifications were made so that the results could be compared and to explore the level of simplification possible for rapid response in the future to questions on alternative waste-treatment options. These two levels of simplification were carried out in parallel so that the performance assessment could be accomplished in the time allotted. The first modeling approach is referred to as the "Simple PA" and the second as the "Complex PA."

In the "Simple PA," a sophisticated description of the phenomena was constructed using ordinary differential equations. The primary goal was to use a level of simplification that allowed simulations to be quickly set up and be geared toward fast run times, relative to the Complex PA described below. Thus, a large number of waste parcels could be studied individually. For this modeling approach, only one waste type (e.g., graphite spent fuel) was placed in the repository at a time. Because of waste parcel design and criticality issues, 20 single waste parcels were studied in the salt repository and 21 single waste parcels in the granite repository. The Complex PA used partial differential equations on a coarse numerical grid. In this approach, the waste disposal groups were uniformly distributed in the repositories. The Complex PA consequence models primarily were used to study the waste parcels in five groups (called waste disposal groups) to examine the influence of the INEL waste on the overall performance of a moderately sized repository. Several separate, complete codes were used to simulate the selected physical components of the disposal systems. The models used in these two approaches are discussed in Chapters 12 through 15. Appendix $G$ lists all the codes used for the performance assessment. In addition, the enhancements made to several codes specifically for this study are summarized in Appendix $\mathrm{H}$.

Because of imprecisely known parameters, uncertainty is incorporated into the performance assessment through a Monte Carlo uncertainty analysis. Integration of mathematical equations through random sampling is discussed in Chapter 3, "PA Methodology Overview." Following the assignment of distributions and identification of the important model parameters to vary, (described under Probability Modeling, Section 1.6), sample elements are generated from the selected parameter distributions. In the analysis reported herein, Latin hypercube sampling (LHS) (McKay et al., 1979; Iman and Conover, 1980) is used to minimize the number of samples needed to adequately capture variability in the parameters and thereby the number of simulations to run through the consequence models. From the consequence results using Monte Carlo analysis, the final two tasks (regulatory assessment and sensitivity analysis) naturally follow.

\subsection{Regulatory Assessment}

In evaluating compliance with 40 CFR 191 (191.13), estimated releases from the consequence modeling are combined with estimated probabilities of each scenario to produce the probability of exceeding certain values (exceedance probability curve or complementary cumulative distribution function [CCDF]). Although results concerning exceedance are of great interest for actual sites, the emphasis here is on the spent nuclear fuel and high-level waste forms. Thus, the results most important to this analysis are (1) the large, ${ }^{\ddagger \neq \neq \ddagger}$ relative changes between CCDFs as the spent fuel and high-level waste form changes, ${ }^{* * * *}$ and (2) the relative importance of various model parameters in determining the position of the curve (from sensitivity analysis). The regulatory assessment is discussed in Chapter 16. As pointed out earlier and in the next chapter, the regulatory setting is rapidly changing in the United States for geologic repositories. This portion of the performance assessment can accommodate these changes; for example,

\footnotetext{
\$¥¥ Although the position of the mean for any one waste form is fairly stable, small changes in the position of a waste form are probably insignificant.

"Because the model parameters of the geologic barrier were usually not varied (as described in Section 1.6, "Probability Modeling"), the absolute position of the CCDF relative to the containment requirements in 40 CFR 191.13 is more uncertain than the relative position.
} 
1. Introduction switching from CCDFs to maximum individual dose would require the addition of only one computational model
(see Figure 1-1).

\subsection{Sensitivity/Uncertainty Analysis}

Sensitivity analysis involves determining the effect of varying parameters of a model on the consequences, $\mathrm{C}$. Uncertainty analysis involves determining the contribution (importance) of an individual parameter $\left(x_{n}\right)$ to the associated uncertainty of the consequences (distribution of C) (Morgan et al., 1990, p. 172). The latter, uncertainty analysis, is discussed in more detail in this report. Both analyses are strongly influenced by the parameter range, but uncertainty analysis also includes the likelihood that the parameter will reach the endpoints of its range. Although the techniques used are more thoroughly discussed in Chapter 16, the uncertainty analysis reported herein basically includes creating scatterplots and developing regression models between the parameters and the various results (see, for example, Helton et al., 1992) and using the absolute values of standardized regression coefficients (or the mathematically related partial correlation coefficients) from regression models.

Four important purposes of the sensitivity/uncertainty analysis are (1) to gain understanding and insight about the disposal system, (2) to help verify the correctness of the calculations, (3) to evaluate the influence of various options for spent fuel and high-level waste forms on the results, and (4) to provide input so that decision makers can allocate resources for collecting data about those parameters that most influence the results, based on what is already known about the site or spent fuel and high-level waste. Because uncertainty and sensitivity analyses are inherently conditional on the models, data distributions, and techniques used to generate them, they cannot provide insight about parameters not sampled, conceptual and computational models not used in the analysis in question, or processes that have been oversimplified in the analysis. Hence, qualitative judgment about the modeling system must be used in combination with the results of sensitivity/uncertainty analyses to set priorities for additional data acquisition and model development. The sensitivity/uncertainty analyses are discussed along with other results in Chapter 16. 


\title{
2. Regulatory Criteria for Radioactive-Waste Repositories
}

\author{
M. M. Gruebel
}

Federal regulations governing radioactive-waste disposal are the direct result of U.S. policy decisions beginning in the 1950s. However, only since the late 1970s have international organizations produced guidelines for the world community that helped in creating radiological standards. The United States was one of the first countries to promulgate radioactive-waste disposal standards when the Environmental Protection Agency (EPA) published 40 CFR 191 in 1985 .

\subsection{Summary of Regulatory Setting for This Assessment (R. P. Rechard)}

Radioactive-waste repositories must comply with the requirements of applicable federal and state regulations. A primary regulation for radioactive-waste disposal is 40 CFR Part 191: Environmental Standards for the Management and Disposal of Spent Nuclear Fuel, High-Level and Transuranic Radioactive Wastes (EPA, 1985). The regulation specifies radioactive-waste disposal based on a total system approach. The results of the performance assessment documented in this report have been compared primarily with 40 CFR 191 because the main interest at this time is a comparison of geologic repositories based on overall long-term performance. Additional requirements and regulations pertaining to both radioactive- and hazardous-waste disposal must also be considered at some point. The Nuclear Regulatory Commission's (NRC) requirements in 10 CFR 60 were based on 40 CFR 191 but also include specifications that regulate engineered- and geologic-barrier subsystems. The Resource Conservation and Recovery Act of 1976 (RCRA) and associated regulations provide for the management of waste contaminated with hazardous chemicals.

\subsubsection{Nuclear Waste Policy in the United States}

The Nuclear Waste Policy Act (NWPA) of 1982 (NWPA, 1983) and subsequent amendments of 1987 (NWPAA, 1987) provide a national policy for the interim storage, monitored retrievable storage, and eventual disposal of radioactive waste. Thus, while not establishing any performance metrics, the act does specify the approach to nuclear waste disposal to be followed in the United States based on the decision that the current generation has an obligation to bear the political and financial costs of developing a permanent disposal option." In general, the NWPA established four features: (1) a detailed schedule for all major decisions related to developing a spent nuclear fuel (SNF) and high-level waste (HLW) disposal option; (2) extensive procedures for involving states and the public in all major decisions, while directing the EPA to issue standards and the Nuclear Regulatory Commission (NRC) to issue regulations to enforce those standards; (3) a Nuclear Waste Fund, paid by a tax on electricity from nuclear reactors to fund development of one repository (two prior to the 1987 amendments) to accept nuclear waste from reactors; and (4) a separate office within the DOE, the Office of Civilian Radioactive Waste Management (OCRWM), to implement the requirements of the NWPA.

Two important policy requirements from this act are pertinent to the disposal of INEL waste. First, the NWPA of 1982 clearly states that a repository for federal defense nuclear waste will comply with all requirements of the NRC (i.e., 10 CFR 60) (NWPA, 1983, Section 8). Defense transuranic waste was exempted earlier by the WIPP Project legislation (Public Law 96-164). Second, it assumed that defense waste would be disposed of with commercial waste unless the President recommended to Congre $i$ that defense wastes be disposed of separately. In 1985, the DOE recommended to the President that defense nuclear waste be disposed of in the same repository as commercial spent fuel (U.S. DOE, 1985a); President Reagan subsequently made that recommendation.

\footnotetext{
- The word "option" is emphasized because, although the initial impulse of all stakeholders impacted by the NWPA was to simultaneously develop an option and fully implement it, current thinking is to develop an option and demonstrate it with a licensed facility but not necessarily fully implement it to give future generations a real choice; there are few reasons to fully implement immediately because the waste can remain safely at the reactors for $-100 \mathrm{yr}$ and immediate implementation could foreclose other options such as recycling that future generations may wish to exercise (Isaacs et al., 1993).
} 


\section{Regulatory Criteria for Radioactive-Waste Repositories}

Because of the policy decisions in the NWPA and the limited scope of this report, the conclusions cannot be used to justify the final decision on the best option for treatment of INEL spent fuel and high-level waste. However, the value of this report is that its very broad system analyses of the nuclear waste disposal issue (similar to the work performed by the European community (BRWM, 1992, p. 58-62]) will provide results that can be used in subsequent iterations of this performance assessment to demonstrate the impact of specific regulations and policy decisions.

The radioactive-waste regulations (40 CFR 191 and 10 CFR 60) are currently in transition. Considerable thought is being given to the supportability and usefulness of the current regulations, especially regarding the diversity of sites that generic standards would regulate. At the present time, only two mined geologic repositories for containerized radioactive waste in the United States are being considered: the Waste Isolation Pilot Plant (WIPP) in New Mexico for transuranic (TRU) waste and the Yucca Mountain Project in Nevada for high-level waste (HLW).

Subpart B of 40 CFR 191, promulgated in 1985, was remanded to the EPA for further consideration in 1987. The EPA has yet to repromulgate the revised regulation, although repromulgation is expected soon. Based on the provisions of the WIPP Land Withdrawal Act of 1992 (WIPP LWA, 1992) and the Energy Policy Act of 1992 (Energy Policy Act, 1992), 40 CFR 191 will apply only to the WIPP, a repository planned for disposal of TRU waste in bedded salt. The EPA is also required by the WIPP Land Withdrawal Act to promulgate criteria for implementing 40 CFR 191 for the WIPP.

The Energy Policy Act of 1992 requires the EPA to formulate a new regulation for the proposed Yucca Mountain repository for disposal of high-level waste in a unsaturated tuff formation. The legislation requires the new regulation to be based on a study by the National Academy of Sciences (NAS) and to consider the feasibility of using a healthbased standard based on doses to individual members of the public; using a system for post-closure oversight based on active institutional controls; and making scientifically supportable predictions of the probability of breaching the repository's engineered or geologic barriers as a result of human intrusion. Subsequent to promulgation of the new EPA regulation for the Yucca Mountain Project, the NRC is required to modify its technical requirements and criteria to be consistent with the new regulation. A specific assumption to be made by the NRC is that, to the extent consistent with the time period recommended by the NAS study, following repository closure the inclusion of engineered barriers and DOE oversight will be sufficient to prevent any activity that poses an unreasonable risk of breaching the engineered or geologic barriers. An additional assumption is that these measures will also prevent any increase in exposure of individual members of the public to radiation beyond allowable limits.

These potential changes to the radioactive-waste regulations make it difficult to determine what criteria might be used to regulate future, as yet unspecified repositories. All or parts of these current and proposed criteria could form the basis for regulating additional repositories. Analysts providing performance assessments of generic repositories should remain aware of the requirements and criteria that might be applied to future repositories.

\subsubsection{EPA's Standards for Radioactive-Waste Disposal}

The 1985 EPA regulation for radioactive-waste disposal (40 CFR 191) is divided into two subparts. Subpart A limits annual radiation doses to the public during the operational period of the facility.

The 1985 version of Subpart B provides performance measures for the entire disposal system, not individual components. Subpart B establishes Assurance Requirements (design and control philosophy that includes a multiplebarrier concept), post-closure Individual Protection Requirements (maximum annual radiation dose to the public for 1000 years), post-closure Groundwater Protection Requirements (maximum radionuclide concentrations in certain sources of water for 1000 years), and post-closure Containment Requirements (probabilistic release limits for 10,000 years). The long-term performance requirements of 40 CFR 191 are discussed in more detail in Section 2.3.

Subpart B of 40 CFR 191 was vacated and remanded to the EPA by the United States Court of Appeals for the First Circuit in July 1987 (NRDC v. US EPA, 1987). The Court found that the EPA had neither reconciled the Individual Protection Requirements with Part C of the Safe Drinking Water Act nor explained the divergence between the two sets of criteria. Furthermore, the C'Jurt found that the EPA had not explained the basis for the 1000 -year design 
criterion in the Individual Protection Requirements. The Court also found that the Groundwater Protection Requirements were promulgated without proper notice and comment (NRDC v. US EPA, 1987, p. 1258). Several working drafts of a revised regulation have been prepared by the EPA since the 1987 remand. Congress had required repromulgation of the regulation before the Test Phase for the Waste Isolation Pilot Plant could begin (WIPP LWA, 1992). However, the Test Phase for the WIPP using radioactive waste has been cancelled, so the regulation now must be in place before disposal begins at the WIPP.

A recent version of the proposed rule was published in the Federal Register in 1993 (EPA, 1993a). The final rule has not yet been published, although repromulgation is expected in the near future. A change that is expected in the new 40 CFR 191 is the lengthening of the time limits for consideration in the Individual Protection Requirements from 1000 years to 10,000 years. In addition, the Groundwater Protection Requirements may be provided as a separate subpart. The time limit for consideration for groundwater protection is expected to be lengthened to 10,000 years, and the requirements are expected to be tied more closely to regulations promulgated as a result of the Safe Drinking Water Act.

Closely related to 40 CFR 191 are proposed criteria for certification of compliance. In February 1993 the EPA issued an Advanced Notice of Proposed Rulemaking for 40 CFR 194: Criteria for the Certification of Compliance with Environmental Radiation Protection Standards for the Management and Disposal of Spent Nuclear Fuel, HighLevel and Transuranic Radioactive Wastes (EPA, 1993b). These criteria will be developed pursuant to the EPA's authority under the WIPP Land Withdrawal Act (WIPP LWA, 1992), which specifies that the EPA issue proposed criteria by October 30,1993, and final criteria by October 30,1994. The EPA intends to specify requirements for implementing 40 CFR 191 and to clarify compliance-related ambiguities that may exist. The criteria will focus on several areas within 40 CFR 191:

- Appropriate analytical "degree of confidence" for determining compliance with the long-term numerical standards. The EPA is considering the relative benefits of requiring a specific statistical test for determining compliance versus specifying a more general "level of confidence" test.

- Methods and quality assurance measures for assuring and confirming accuracy and/or adequacy of estimates of radionuclide inventories.

- Types of models to be used to demonstrate compliance and methods for determining that those models are adequate.

- Methods or procedures to be employed to assure quality and completeness of data used in determining compliance.

- Guidance or criteria for reducing uncertainty or undue speculation that may be associated with performance assessments.

- Types of criteria to be used in assessing compliance with the Assurance Requirements.

- Feasibility of adopting into 40 CFR 194, with or without modification, the procedures, criteria, terms, and conditions for licensing used in the NRC's 10 CFR 60.

Two meetings of an EPA advisory group have been held to discuss proposed criteria for certification of the WIPP. A draft of the proposed criteria is expected to be available in the near future.

\subsubsection{NRC's Standards for Radioactive-Waste Disposal}

The EPA regulations establish standards for the disposal system as a whole to protect the accessible environment. However, the NRC is responsible for ensuring that a DOE disposal system for commercially generated spent fuel and high-level waste meets the requirements in EPA's 40 CFR 191. In June 1983, the NRC promulgated 10 CFR 60- 
Disposal of High Level Radioactive Wastes in Geologic Repositories (NRC, 1983). The NRC anticipated the promulgation of the EPA standard and "implemented" it by incorporating it into their regulations. Yet the NRC also established minimum requirements for the engineered and geologic barriers subsystems of the disposal system (treating the disposal system similar to a nuclear reactor) that required compliance calculations (BRWM, 1992, p. 7). The requirements were stringent: a minimum period for substantially complete containment ( $300 \mathrm{yr})$, a maximum engineered barrier release rate $\left(1 \times 10^{-5} / \mathrm{yr}\right.$ after $1000 \mathrm{yr}$ for any radionuclide released at a rate greater than $0.1 \%$ of the calculated total release rate limit), and a minimum groundwater travel time (1000 yr) for the geologic barrier. Thus, the requirements potentially required the DOE to have a much more thorough understanding of the engineered subsystems than for 40 CFR 191 to show compliance with 10 CFR 60. They also diminished the advantage of some sites because 10 CFR 60 required a robust canister and waste form regardless of the geologic media of the site. For example, according to 40 CFR 191, the favorable characteristic of halite in creeping around a waste container and entombing it could mean that the DOE could place less reliance on the waste canister or waste form; however, according to 10 CFR 60, credit could not be taken for this characteristic in performance assessments.

Other requirements of 10 CFR 60 included detailed site characterization plans (e.g., the 6300-page plan for the proposed repository at Yucca Mountain, Nevada [U.S. DOE, 1988a]) and design of the operations area of the facility "...such that any or all of the emplaced waste could be retrieved on a reasonable schedule starting at any time up to 50 years after emplacement..." where "reasonable schedule" meant in the same time devoted to originally constructing the facility and emplacing the waste.

As discussed in Section 2.1.1, the Energy Policy Act of 1992 (Energy Policy Act, 1992) requires the NRC to revise 10 CFR 60 based on a new EPA regulation specific to the Yucca Mountain Project and on an NAS study with Congressionally specified objectives. The Energy Policy Act of 1992 also specifies that the NAS study should be available by year-end 1993. Promulgation of the EPA regulation for the Yucca Mountain Project is scheduled by year-end 1994. Based on this schedule, revision of 10 CFR 60 probably will not occur until at least 1995.

\subsubsection{Hazardous-Waste Disposal Standards}

The Resource, Conservation, and Recovery Act of 1976 (RCRA) and subsequent amendments (e.g., Hazardous and Solid Waste Amendments of 1984 [HSWA]) provide for the management of waste contaminated with hazardous chemicals. The EPA regulations implementing these acts are contained in 40 CFR Part 224 through 40 CFR Part 281. The statute and EPA implementing regulations are complex; how their provisions will influence fuel- and waste-treatment options and eventual disposal is uncertain at present. Furthermore, a more important fundamental issue is whether consideration of requirements in the RCRA would increase protection to human health and the environment beyond requirements in the two primary regulations for long-term disposal of radioactive waste, 40 CFR 191 and 10 CFR 60. Some points for consideration are presented below, but no final conclusions are presented.

Spent Nuclear Fuel Classification. Spent nuclear fuel has not yet been formally classified as waste by the DOE; thus, the EPA waste management regulations have not been applied to this material. However, spent fuel has been included in the "180-day report" mandated by the Federal Facility Compliance Act of 1992 (FFCA, 1992) as part of the DOE's mixed waste inventory, and spent fuel would likely be declared as waste prior to disposal in a repository.

RCRA Hazardous Waste Characteristics. Although hazardous-waste characteristics are usually not thought to be present in spent nuclear fuel and solidified liquid high-level waste, the RCRA hazardous-waste characteristics potentially exhibited by spent fuel and high-level waste include reactivity, toxicity for metals, and corrosivity.

In some spent fuel, sodium metal was used to bind fuel to the cladding. This fuel might be considered reactive. Also, although a very slow reaction, the silicon carbide coating of the graphite fuels can react with water to form acetylene gas. Finally, the uranium and thorium carbide microsphere, once the silicon carbide coating fails, could react quickly to form acetylene gas. ${ }^{\dagger}$

Similarly, recall that all the gas produced from anoxic corrosion of the stainless steel canisters is hydrogen gas. 
Certain spent fuel contains toxic metals listed in 40 CFR 261.24, including (1) chromium in stainless steel in cladding and assembly components; (2) stable fission products such as barium, cadmium, selenium, and silver; and (3) metals in failed fuel such as the control rods fused with the fuel from the Three Mile Island reactor. Because of low concentrations, the first two sources probably would not fail tests specified by the EPA for evaluating the amount of hazardous material that may leach out if disposed of directly, while the third source (not included in this report) might. ${ }^{\ddagger}$

Liquid high-level waste stored at INEL is acidic and thus corrosive."*

RCRA-Listed Materials. In the past, chemicals currently classified as hazardous were possibly used in the labs handling radioactive material ("hot labs") at the chemical plants. Waste from these labs was routinely placed in stainless steel tanks storing the liquid radioactive waste. Thus, very small quantities of RCRA-listed material can be present in the liquid and possibly solidified high-level waste.

Compliance Strategy. For a repository, the current approach for responding to the RCRA requirements would be to follow the example of the DOE's proposed TRU-waste repository (the Waste Isolation Pilot Plant in New Mexico). The approach is to petition for a "no-migration" variance to the land-disposal restrictions ( 40 CFR 268.6). This petition would allow hazardous chemicals to be disposed of in the repository for the maximum length of time allowed in the permit, with renewals as necessary (WEC, 1989). This approach is taken in lieu of using the "best demonstrated available technology" (BDAT) currently defined by the EPA for radioactive waste co-contaminated with hazardous chemicals listed in EPA 40 CFR 261. The petition for the variance requires monitoring and predictive calculations, but the rigor and the stochastic nature required of the calculations is currently being negotiated.

In contrast, any treatment plant that conditioned spent fuel or high-level waste would need to consider the RCRA requirements. For example, the state of Idaho has already issued a Notice of Noncompliance under RCRA for the sodium-bearing waste stored at INEL, and DOE has entered into a consent order requiring removal of the waste from the stainless-steel tanks by 2015 .

\subsubsection{Environmental Impacts}

The National Environmental Policy Act (NEPA) (NEPA, 1970, as amended) is enforced by regulations that are not specific regulatory guidelines but contain a mandate for evaluating the environmental consequences of all signifcant aspects of a project (EPA, 1978). The DOE has prepared several environmental impact statements (EISs) that have addressed the predicted experimental, operational, and long-term behavior of potential repositories (U.S. DOE, 1979, 1980, 1990). However, the NEPA's impact on the waste-treatment options themseives will be only indirect because its focus is primarily on the requirements for the siting of processing plants and deep geologic repositories. Although not explicitly required for the EIS, the DOE may require evaluation of individual doses to the public to support any decisions of minimal and acceptable environmental consequences.

\subsubsection{Other Statutes and Regulations}

Numerous acts not specifically discussed that have a tertiary effect on the performance criteria of a geologic repository include the Clean Air Act and subsequent amendments, and the Toxic Substances Control Act. Regulations related to transportation of radioactive material also have a tertiary effect on the performance criteria, such as the NRC's 10 CFR 71 for packaging and transportation of radioactive materials.

\footnotetext{
\# Inclusion of lead as an internal backfill and/or container layer might also cause difficulties with RCRA, depending on EPA's interpretation of its use as related to disposal.

** High-level liquid wastes stored at INEL are unique within the DOE's weapons complex because they are stored in an acidic form. Although this type of storage requires stainless steel storage tanks enclosed in concrete vaults, the decision to store the waste in acidic form was based on the desire to (1) maintain radionuclides in solution (as opposed to neutralized sludge at the bottom of less expensive, carbon steel tanks), and (2) greatly reduce the likelihood of precipitates of highly enriched uranium.
} 


\subsection{International Criteria for Radioactive-Waste Disposal}

Radiation protection principles published by international organizations, especially those of the International Commission on Radiological Protection (ICRP), have provided the basis for regulations in countries considering methods for disposal of radioactive waste (Table 2-1). In 1984, the Nuclear Energy Agency (NEA) published an expert group report that introduced the concept of a basic individual risk criterion for radioactive-waste disposal (NEA, 1984). The report recommended that protection of the individual should be expressed in terms of risk, where risk was defined as the product of the probability of exposure and the probability that doses received will give rise to deleterious health effects. Individual risk limits were to correspond to the risk associated with the current dose recommendations of the ICRP (NEA, 1984).

The ICRP's recommendations in Report 46 (ICRP, 1985) complemented the 1984 NEA report. In addition, a risk-based criterion was established that accounted for the probability of the dose being received as well as the probability of serious health effects from the dose. More recently, the International Atomic Energy Agency (IAEA) published safety principles and technical criteria for underground disposal sites that use doses as the performance measure (IAEA, 1989).

The approaches to application of the general radiation protection principles are largely similar in most countries. Their translation into quantitative safety criteria for radioactive-waste disposal, however, may differ somewhat among the countries. Such differences concern (NEA, 1991)

- the explicit use of limits expressed in terms of dose or in terms of risk,

- the use of individual and/or collective dose/risk limits,

- the level of detail of the criteria (e.g., use of subsystem criteria and special criteria for certain scenarios),

- the period for which compliance has to be shown.

All existing regulations use either dose or risk (or both) as a fundamental measure of performance (NEA, 1991). The NEA, ICRP, and IAEA principles (NEA, 1984; ICRP, 1985; IAEA, 1989) express the idea that assessments of radioactive-waste disposal should consider not only the most likely scenario and related expected releases, but also a set of scenarios that are uncertain or even unlikely to happen. The ICRP recommendations use the risk concept to propose a constraint on the annual individual risk of severe health effects at a level of about $10^{-5}$ (consistent with the risk implied by the annual dose limit recommended for individual members of the public). Several national regulations (e.g., the United Kingdom and Canada) explicitly follow this recommendation but set the risk limit at $10^{-6}$ for radioactive-waste disposal practices based on the belief that waste disposal is only one of several potential sources for exposure. Some countries have also chosen to use dose limits for high-probability scenarios and to consider lowprobability disruptive scenarios separately. Thus, several countries (e.g., Switzerland and Germany) have regulations using dose limits and make no explicit use of risk criteria. However, using dose limits does not necessarily mean that these countries do not recognize the need to consider the risks associated with probabilistic events and the need to try to estimate them when appropriate (NEA, 1991).

Only limited use has been made of collective dose or risk in safety criteria and assessments (NEA, 1991). Use of collective dose/risk requires assumptions not only about future critical groups of humans and their habits, but also about the whole society (e.g., number of people and their living conditions and habits). Collective dose calculations are associated with large uncertainties, and differences between disposal options could be masked by the uncertainty ranges affecting such calculations. Most countries generally regard individual dose or risk limits as more appropriate for determining long-term acceptability of high-level waste disposal practices, with collective dose or risk limits used mainly as a comparison tool for the discussion of repository design alternatives (NEA, 1991). 
Table 2-1. Regulatory Waste-Disposal Objectives/Criteria at the International Level and in Individual Countries (adapted from NEA, 1991)

\begin{tabular}{|c|c|}
\hline $\begin{array}{l}\text { Organization/ } \\
\text { Country }\end{array}$ & Main Objective/Criteria \\
\hline NEA (NEA, 1984) & $\begin{array}{l}\text { For HLW, maximum individ- } \\
\text { ual risk } 10-5 / \mathrm{yr} \text { (all sources) }\end{array}$ \\
\hline $\begin{array}{l}\text { ICRP Publication } \\
46 \text { (ICRP, 1985) }\end{array}$ & $\begin{array}{l}\text { For HLW, for individuals (all } \\
\text { sources): } \\
1 \mathrm{msV} / \mathrm{yr} \text { (normal evolution } \\
\text { scenarios); } 10^{-5} / \mathrm{yr} \text { (probabi- } \\
\text { listic scenarios) }\end{array}$ \\
\hline $\begin{array}{l}\text { IAEA Safety } \\
\text { Series } 99 \text { (IAEA, } \\
\text { 1989) }\end{array}$ & $\begin{array}{l}\text { ICRP Publication } 46 \text { (ICRP, } \\
\text { 1985) }\end{array}$ \\
\hline $\begin{array}{l}\text { CANADA (AECB, } \\
\text { 1987) }\end{array}$ & $\begin{array}{l}\text { For HLW, maximum individ- } \\
\text { ual risk objective } 10^{-6} / \mathrm{yr}\end{array}$ \\
\hline
\end{tabular}

FINLAND (Finnish Centre for Radiation and Nuclear Safety, 1991)

FRANCE (Basic Safety Rule of 1992)

\section{GERMANY (Sec-} tion 45, para. 1 of Radiation Protection Ordinance, 1989)

THE NETHERLANDS

For low and medium level waste, maximum individual dose $<0.1 \mathrm{msv} / \mathrm{yr}$, with maximum individual dose $<5 \mathrm{mSv} / \mathrm{yr}$ from accident conditions caused by possible natural events or human actions

For both low- and high-level waste, in normal evolution scenario, maximum individual dose $<0.25 \mathrm{mSv} / \mathrm{yr}$

For HLW, maximum individual dose $<0.3 \mathrm{mSv} / \mathrm{yr}$ for all reasonable scenarios

Not yet developed

Other Main Features

Comments

Individual risk/dose best criterion to judge long-term acceptability

Both probability and doses should be taken into account in ALARA

Period of time for demonstrating compliance: $10^{4} \mathrm{yr}$

No sudden and dramatic increase for times $>10^{4} \mathrm{yr}$
Analysis of emissions beyond 1 million years generally not meaningful.
Calculation of individual doses limited to $10^{4} \mathrm{yr}$ but isolation potential beyond $10^{4}$ yr may be assessed
No consensus on ALARA* (as low as reasonably achievable)/optimization

ALARA useful, notably to compare alternatives, but may not be the most important siting factor

Also includes qualitative technical criteria on disposal system features and role of safety analysis and quality assurance

Additional qualitative, nonprescriptive requirements and guidelines in regulatory documents

For spent fuel or HLW, proposed criterion for maximum individual dose $<0.1 \mathrm{mSv} / \mathrm{yr}$

Technical criteria for siting established in 1987

Additional qualitative technical criteria in guidelines and regulatory documents

Recent assessments used dose criteria from other countries, with risk criteria for scenarios with a high dose

" ALARA - As low as reasonably achievable. 
Table 2-1. Regulatory Waste-Disposal Objectives/Criteria at the International Level and in Individual
Countries (adapted from NEA, 1991) (Continued)

\section{NORDIC COUN-}

TRIES (Basic Cri-

teria Document of 1993)

\section{SWITZERLAND \\ (Regulatory Docu- \\ ment R-21, 1980) \\ For HLW, maximum individ- ual dose $<0.1 \mathrm{mSv} / \mathrm{yr}$ at any time for reasonably probable scenarios}

UNITED KING-
DOM

\section{DOM}

\section{UNITED STATES}

(EPA 40 CFR Part 191, 1985)

(NRC 10 CFR Part

\section{0, 1983)}

46

47

48

49

50

51

52

53

54

56

57
For HLW, maximum individual dose $<0.1 \mathrm{mSv} / \mathrm{yr}$ (normal scenarios); maximum individual risk $<10^{-6} / \mathrm{yr}$ (disruptive events)

No specific criteria for HLW, but likely application of principles similar to existing objectives for low/intermediate level waste (LILW): < $10^{-6} / \mathrm{yr}$ target for individual risk from a single facility

For HLW, SNF, and TRU waste, limits on projected radionuclide releases to the accessible environment for $10^{4} \mathrm{yr}$, based on objective to limit serious health effects to less than 10 in the first $10^{4}$ yr after disposal for each HLW disposed of

For HLW, minimum levels of performance specified for:

Waste package ("substantially complete" containment for 300-1000 yr)

Engineered barrier system (releases $<10^{-5} \mathrm{yr}^{-1}$ of the inventory at $1000 \mathrm{yr}$ after repository closure)

Pre-waste-emplacement groundwater travel time between "disturbed zone" and "accessible environment" > $1000 \mathrm{yr}$ 1000 metric ton of SNF or

- ALARA - As low as reasonably achievable.

\section{Main Objective/Criteria Other Main Features Comments}

Additional criterion on "total activity inflow" limiting releases to biosphere, based on inflow of natural alpha radionuclides

Repository must be designed in such a way that it can at any time be sealed within a few years without the need for institutional control

No time frame for quantitative assessments specified

Individual dose (over 1000 yr) $<0.25 \mathrm{mSv} / \mathrm{yr}$

Other requirements on drinking water contamina. tion

Siting criteria for avoidance of foreseeable adverse human activity; separate consideration of anticipated processes and events (naturally occurring) and unanticipated processes and events (naturally occurring or human induced)
Under revision following broad consultation

Includes other qualitative criteria

Revision of Regulatory Document R-21 underway

ALARA to be used to the extent practical and reasonable

1985 EPA standards were remanded and are being revised for application to the Waste Isolation Pilot Plant; U.S. Congress mandated a new regulation to apply to the Yucca Mountain Project

NRC subsystem requirements are intended to help achieve compliance with the EPA standards; U.S. Congress recently mandated consideration of revisions to the regulation specifically for the proposed Yucca Mountain site in Nevada 
Few countries use specific quantitative criteria for regulating the performance of subsystems within a disposal system. Any derived criteria usually are expressed in qualitative terms so that the regulator can provide guidance without specifying required subsystem performance (NEA, 1991). The philosophy for using only qualitative criteria for subsystems is that detailed quantitative regulations for subsystems might be premature or even counterproductive to a flexible and gradual process of developing the repository system and establishing the optimal balance between different barrier functions (NEA, 1991). As discussed in the previous section, in the United States the NRC has included specific quantitative criteria for subsystem performance that serve as default specifications for achieving a required level of performance. However, the NRC regulations explicitly provide for approval of alternative subsystem criteria, if appropriate (10 CFR 60.113[b]).

All countries examine potential radiological impacts over long time frames (NEA, 1991). Formal requirements for quantitative assessment up to at least 10,000 years are included in some regulations (e.g., Canada, Germany, and the United States). Other countries do not use such "cut-off" times and consider quantitative assessments for one million years (e.g., Finland) or even longer. However, most of the regulations also indicate the recognition of two factors that influence the type of approach required to demonstrate and judge compliance with basic protection principles: (1) uncertainties associated with assessment results increase with time; and (2) radioactivity of the waste decreases with time. These two trends suggest that detailed quantitative assessments become less meaningful over increasingly longer time periods. Thus, some regulators have indicated that detailed quantitative assessments, including dose calculations, are justified for shorter periods, while a less detailed, more qualitative approach should be used for times far into the future (NEA, 1991).

In the United States, the main criterion for radioactive-waste disposal, 40 CFR 191, is quantitative and probabilistic. The standard is based on cumulative releases for 10,000 years of various radionuclides to the accessible environment. These release limits were determined using relatively simple models for the biosphere with the objective of limiting global collective dose so that disposal would result in less than 1000 severe health effects in 10,000 years. This type of criterion is unique to the United States, and similar criteria are neither used nor under consideration in other countries (NEA, 1991).

\subsection{Overview of EPA 40 CFR 191}

The radioactive-waste disposal standards promulgated by the EPA in 1985-40 CFR Part 191: Environmental Standards for the Management and Disposal of Spent Nuclear Fuel, High-Level and Transuranic Radioactive Wastes (EPA, 1985)-are divided into two subparts (Figure 2-1). Subpart A applies to a disposal facility prior to decommissioning. Subpart B applies after decommissioning and contains quantitative and qualitative requirements for longterm performance of a repository:

- The Containment Requirements (\$191.13) set probabilistic limits on cumulative releases of radionuclides beyond the "controlled area" (defined in the regulation as a surface location that encompasses no more than $100 \mathrm{~km}^{2}$ and extends horizentally no more than a $5-\mathrm{km}$ radius from the outer boundary of a radioactive-waste disposal system, plus the underlying subsurface [\$191.12(g)][see Figure 2-2]) for 10,000 years after disposal. The regulation specifies a reasonable expectation that the limits will be met.

- The Individual Protection Requirements $(\$ 191.15)$ set limits on radiation doses to members of the public in the accessible environment (the environment beyond the controlled area [\$191.12(k)]) for 1000 years of undisturbed performance after disposal. The regulation specifies a reasonable expectation that the limits will be met.

- The Groundwater Protection Requirements ( $\$ 191.16)$ set limits on radioactive contamination of certain sources of groundwater within or near the controlled area for 1000 years of undisturbed performance after disposal. The regulation specifies a reasonable expectation that the limits will be met. 


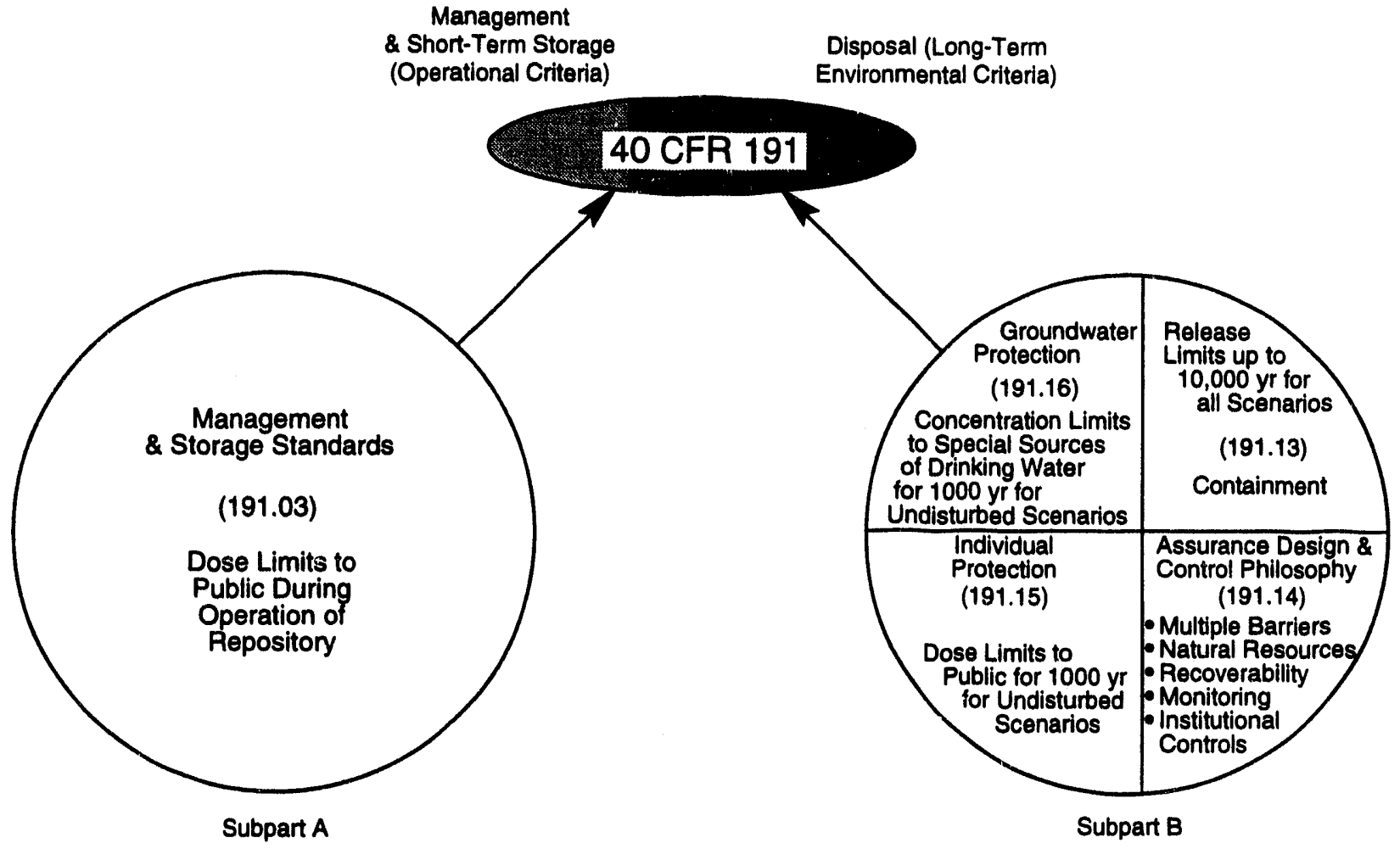

TA1-6342-3479-2

Figure 2-1. The EPA's 40 CFR 191-Environmental Standards for the Management and Disposal of Spent Nuclear Fuel, High-Level and Transuranic Radioactive Wastes (EPA, 1985).

- The Assurance Requirements (\$191.14) are qualitative requirements that specify actions and procedures to increase confidence "because of the inherent uncertainties in selecting and designing disposal systems that must be very effective for more than 10,000 years" (EPA, 1985, p. 38069).

The DOE must provide evaluations showing a reasonable expectation that a repository will comply with the quantitative requirements of Subpart B of 40 CFR 191. Appendix A of 40 CFR 191 specifies how to determine release limits for the Containment Requirements; Appendix B of 40 CFR 191 provides nonmandatory guidance for implementing Subpart B.

Appendix B is based on the analytical assumptions the EPA used to develop the technical basis for the numerical disposal standards. The EPA prepared Appendix B because the agency believed that it was important that the assumptions used by implementing agencies be compatible with those used by the EPA in developing the rule (EPA, 1985, p. 38074). Appendix B of this report contains the text of 40 CFR 191 and provides background information on the development of the regulation.

The following sections summarize the long-term performance requirements of 40 CFR 191, Subpart B, based both on the requirements as stated and on the guidance provided in Appendix B of the rule.

\subsubsection{Containment Requirements}

The primary objective of Subpart B is "to isolate most of the wastes from man's environment by limiting longterm releases and the associated risks to populations" (EPA, 1985, p. 38070). This objective is reflected quantitatively in the Containment Requirements (\$191.13): 
The "Repository Site" or "Site" is the General Location of the

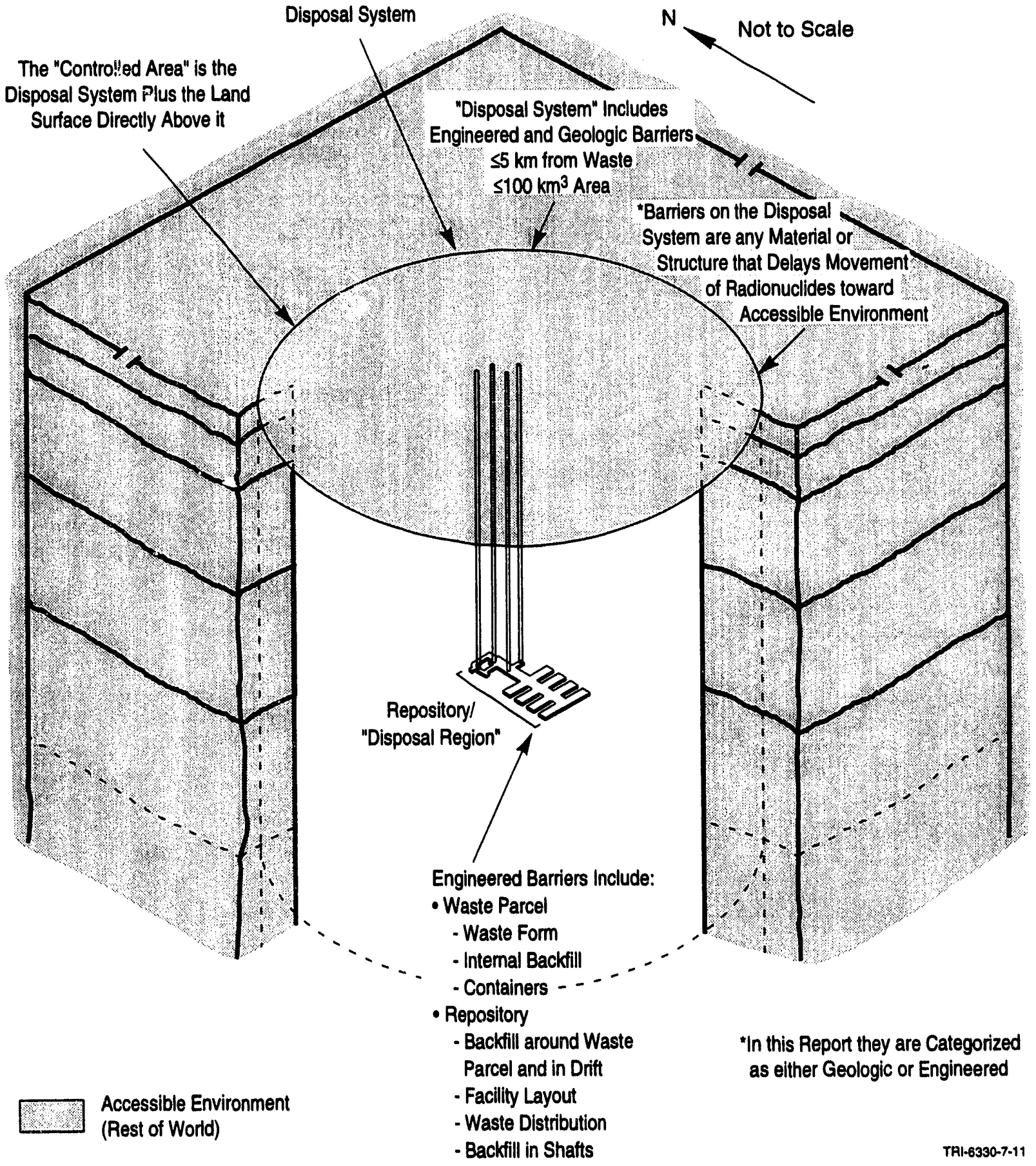

Figure 2-2. Artist's concept of a mined geologic disposal system portraying terminology used in 40 CFR 191, Subpart $B$ and this report (modified from Bertram-Howery and Hunter, Eds., 1989, Figure 1I-1). 
"Disposal systems for spent nuclear fuel or high-level or transuranic radioactive wastes shall be designed to provide a reasonable expectation, based on performance assessments, that the cumulative releases of radionuclides to the accessible environment for 10,000 years after disposal from all significant processes and events that may affect the disposal system shall:

1. Have a likelihood of less than one chance in 10 of exceeding the quantities calculated according to Table 1 (Appendix A) [of 40 CFR 191], and

2. Have a likelihood of less than one chance in 1,000 of exceeding ten times the quantities calculated according to Table 1 (Appendix A)."

The Containment Requirements are explicitly quantitative and probabilistic; they place numerical limits not on cumulative releases but on likelihoods that cumulative releases of radioactivity to the environment over a 10,000-yr period will exceed certain values of release computable from formulae given in Appendix A of 40 CFR 191.

Disposal System. "Disposal system" is defined in 40 CFR 191 to mean "any combination of engineered and natural barriers that isolate...radioactive waste after disposal" (\$191.12). Additionally,

“" [b]arrier' means any material or structure that prevents or substantially delays movement of water or radionuclides toward the accessible environment. For example, a barrier may be a geologic structure, a canister, a waste form with physical and chemical characteristics that significantly decrease the mobility of radionuclides, or a material placed over and around waste, provided that the material or structure substantially delays movement of water or radionuclides" (\$191.12[a]).

The disposal system is the combination of the engineered barriers of the repository/shaft system and the natural barriers of the disposal site that isolate the wastes from the accessible environment. Engineered barriers could be seals and backfill in the emplacement facilities, and plugs in boreholes. Engineered modifications to the repository design could include making the waste itself form a barrier. Natural barriers are the subsurface geologic and hydrologic systems within the controlled area that inhibit release and migration of hazardous materials. Barriers are not limited to the examples given in the regulation's definition, nor are those examples mandatory. As recommended by the EPA in Appendix B, “...reasonable projections for the protection expected from all of the engineered and natural barriers...will be considered" (EPA, 1985, p. 38088).

Performance Assessment. Quantitatively evaluating compliance with the Containment Requirements requires a performance assessment, which has specific meaning within 40 CFR 191:

"Performance assessment' means an analysis that: (1) identifies the processes and events that might affect the disposal system; (2) examines the effects of these processes and events on the performance of the disposal system; and (3) estimates the cumulative releases of radionuclides, considering the associated uncertainties, caused by all significant processes and events. These estimates shall be incorporated into an overall probability distribution of cumulative release to the extent practicable" (\$191.12[q]).

The EPA evidently expects that implementing agencies will make extensive use of mathematical models in the course of a performance assessment:

"The [EPA] believes that the implementing agencies must determine compliance with $\S \S 191.13,191.15$, and 191.16 of Subpart B by evaluating long-term predictions of disposal system performance....In making these various predictions, it will be appropriate for the implementing agencies to make use of rather complex computational models, analytical theories, and prevalent expert judgment relevant to the numerical predictions....In fact, sole reliance on these numerical predictions to determine compliance may not be appropriate; the implementing agencies may choose to supplement such predictions with qualitative judgments as well" (EPA, 1985, p. 38088).

In other words, predictions made with numerical models are to be used as only part of the implementing agency's argument that the disposal system meets 40 CFR 191 . However, the EPA clearly intends that these predictions should 
be more than simple "point estimates" of releases of radioactivity by the disposal system. In the Containment Requirements there is frequent mention of the need to account for all uncertainties that may arise in the modeling of the system's behavior, and a way of quantitatively presenting those uncertainties is proposed:

"...whenever practicable, the implementing agency will assemble all of the results of the performance assessments to determine compliance with $\$ 191.13$ into a 'complementary cumulative distribution function' that indicates the probability of exceeding various levels of cumulative release. When the uncertainties in parameters are considered in a performance assessment, the effects of the uncertainties considered can be incorporated into a single such distribution function for each disposal system considered" (EPA, 1985, p. 38088).

Inclusion of the expression "all significant processes and events" in the Containment Requirements indicates that performance must be determined for both undisturbed and possible disturbed future states of the disposal system. The necessity of identifying the possible future states is the justification for including scenario development in performance assessments. For human intrusion only inadvertent, not purposeful, intrusion need be considered. The EPA reasoned that only future states that helped to distinguish between sites were necessary to consider.

With the minor modification of adding a water withdrawal well to provide a potential pathway for radionuclides to reach humans, the performance-assessment methodology developed for the Containment Requirements can be used to assess compliance with undisturbed performance for the Individual Protection Requirements (see Section 2.3.3).

Human Intrusion. Determining compliance with 40 CFR 191 requires performance assessments that include the probabilities and consequences of disruptive events. Appendix B of 40 CFR 191 indicates that "inadvertent and intermittent intrusion by exploratory drilling for resources...can be the most severe intrusion scenario assumed by the implementing agencies" (EPA, 1985, p. 38089). The EPA gives specific guidance in Appendix B of 40 CFR 191 for considering inadvertent human intrusion.

The EPA specifies in Appendix B that "the likelihood of such inadvertent and intermittent drilling need not be taken to be greater than 30 boreholes per square kilometer of repository area per 10,000 years for geologic repositories in proximity to sedimentary rock formations, or more than 3 boreholes per square kilometer per 10,000 years for repositories in other geologic formations" (EPA, 1985, p. 38089). In addition, the implementing agency can assume that the consequences of inadvertent drilling are no more severe than (1) direct release to the land surface of all groundwater in the repository that would promptly flow through the newly created borehole due to natural lithostatic pressure, and (2) creation of a groundwater flow path with permeability typical of a borehole filled by soil or gravel that would normally settle into an open hole over time (ibid.).

Reliance on future institutional control is limited in that "performance assessments...shall not consider any contributions from active institutional controls for more than 100 years after disposal" ( $\$ 191.14[a]$ ). An additional requirement, however, is that "disposal sites shall be designated by the most permanent markers, records, and other passive institutional controls practicable to indicate the dangers of the wastes and their location" (\$191.14[c]). The possibility of inadvertent human intrusion must be considered, and the use of passive institutional controls to deter intrusion should be "taken into account" in performance assessments. The EPA assumes that passive institutional controls should "...reduce the chance of inadvertent intrusion compared to the likelihood if no markers and records were in place" (EPA, 1985, p. 38080).

The EPA indicates in Appendix B of 40 CFR 191 that, as long as passive institutional controls "endure and are understood," they can be assumed to deter "systematic or persistent exploitation" of the disposal site, and furthermore, "can reduce the likelihood of inadvertent, intermittent human intrusion" to a degree to be determined by the implementing agency, although the possibility of inadvertent intrusion cannot be eliminated (EPA, 1985, p. 38088).

Uncertainties. The EPA recognizes in the preamble to 40 CFR 191 that "standards must be implemented in the design phase for...disposal systems because active surveillance cannot be relied upon" over the long time of interest. The EPA further notes that "standards must accommodate large uncertainties, including uncertainties in our current knowledge about disposal-system behavior and the inherent uncertainties regarding the distant future" (EPA, 1985, 


\section{Regulatory Criteria for Radioactive-Waste Repositories}

p. 38070). The definition of performance assessment in 40 CFR 191 requires "considering the associated uncertainties" (\$191.12).

"Uncertainties in parameters" are the only source of uncertainty specifically identified in 40 CFR 191 (EPA, 1985, p. 38088). Uncertainty in material-property parameters (parameters specifying magnitudes of physical or chemical properties of materials) used in predictive models may result from several sources, including incomplete data, intrinsic spatial variability of the property in question, measurement uncertainty, and uncertainty resulting from differences in scale between data acquisition and model application. In many aspects of data collection, the professional judgment of an analyst with expertise in the area of investigation often enters into the scientific process. For example, selection of methods to collect data, interpretation of data, development of conceptual models, and selection of model parameters all require professional analysis and judgment. The analyst's final data set is based on available data, the intended use of the parameter in the computational model, behavior of analogous systems, and the analyst's own expert judgment.

Additional parameter uncertainty may arise from event-and-process parameters and from probability-model parameters. Event-and-process parameters are the time-domain counterparts of material-property parameters and usually include the number of external events of a given kind that act upon the system during a given interval on a time grid (e.g., the number of exploratory boreholes that penetrate a waste disposal compartment between 2000 and 3000 years after closure). These parameters also include the intensities of external processes that affect the system during each interval of a time grid (e.g., mean precipitation and temperature as functions of time in a region under changed climatic conditions). Probability-model parameters are parameters for the probability models used to assign likelihoods to the ranges of the values taken by uncertain members of material-property and event-and-process parameters sets (e.g., means, variances, and correlation coefficients in analytic probability distributions for values of material-property parameters and mean rates of events of a given kind appearing in probability distributions for event-and-process parameters).

Formal expert judgment may be considered for some parameters or models identified as being important in cases where significant uncertainty exists in the available data and conceptual models, and directly applicable experimental or field data cannot be practicably obtained (Trauth et al., 1992; 1993). In these instances, formal elicitations could provide probability distributions for model parameters. These distributions could be used to provide guidance until experimental or field data become available, or, in those cases where direct acquisition of data is impossible or unrealistic, the elicited distributions could form part of the basis for compliance evaluation. Expert panels may also be used to provide independent evaluation.

Quality assurance (QA) procedures may be used for reducing uncertainty in various aspects of performance assessments. Procedures for assuring the quality of analysis results could be provided for data, software, analysis, elicitation of judgments from expert panels, and documentation (references).

Release Limits. Appendix A of 40 CFR 191 establishes release limits for all regulated radionuclides. Table 1 in that appendix gives the limit for cumulative releases to the accessible environment for 10,000 years after disposal for each radionuclide per unit of waste. Note 6 of Table 1 in 40 CFR 191's Appendix A describes the manner in which the release limits are to be used to determine compliance with the Containment Requirements. For each radionuclide released, the ratio of the estimated cumulative release to the release limit for that radionuclide must be determined; ratios for all radionuclides are then summed for comparison to the requirements of $\$ 191.13(a)$ :

$$
R=\sum_{i=1}^{n R}\left(\frac{Q_{i}}{L_{i}}\right)
$$

where

$\mathbf{R}=$ the summed normalized release to the accessible environment

$\mathrm{nR}=$ the number of radionuclides used in the analysis 
$Q_{i}=$ the cumulative release (in $\mathrm{Ci}$ ) of radionuclide $i$ to the accessible environment during the $10,000-\mathrm{yr}$ period following closure of the repository

and

$L_{i}=$ the release limit (in $\mathrm{Ci}$ ) for radionuclide $\mathrm{i}$ given in Table 1, Appendix A of 40 CFR 191.

Thus, the quantity of a radionuclide that may be released depends on the quantities of all other radionuclides projected to be released but cannot exceed its own release limit. The summed normalized release cannot exceed 1 for probabilities greater than 0.1 , and cannot exceed 10 for probabilities greater than 0.001 but less than 0.1 . Potential releases estimated to have probabilities less than 0.001 are not limited ( $\$ 191.13[\mathrm{a}])$.

An important aspect of the release limits is the waste unit factor $\left(f_{w}\right)$ for limits $\left(L_{i}\right)$. As more waste activity (in $\left.C_{i}\right)$ is placed in the repository, more radionuclides can be legally released, corresponding to the idea that more statistical deaths occur from unmined uranium ore as the volume included in the theoretical calculations increases. Thus, placing more waste into a repository can help a repository meet the release limits provided the waste has physical characteristics that reduce the overall release rate, such as better waste packaging or waste form. However, a difficulty occurs from the ratioing of permitted releases to the amount of activity in the repository. It is difficult to assess a priori the influence of adding a new or processed waste type to a repository if the added activity is significant and the mix of radionuclides is substantially different from the average waste type; the full release calculation must be redone.

Compliance Assessment. The results of the performance assessment for $\$ 191.13(\mathrm{a})$ are expected to be incorporated, to the extent practicable, into an overall probability distribution of cumulative release. In Appendix B of 40 CFR 191, the EPA assumes that, whenever practicable, results can be assembled into a single complementary cumulative distribution function (CCDF) that indicates the probability of exceeding various levels of summed normalized cumulative releases (EPA, 1985, p. 38088) (Figure 2-3).

In the Containment Requirements, only two limit points are specified (i.e., the probabilities of exceeding 1 and 10 are required to be less than 0.1 and 0.001 , respectively); however, to improve readability, on most graphs that compare a calculated CCDF to the limiting points, limiting lines connect these points (Figure 2-3).

Descriptions of a procedure for performance assessment based on the construction of a CCDF are available (Pepping et al., 1983a; Hunter et al., 1986; Cranwell et al., 1987, 1990; Campbell and Cranwell, 1988; Rechard, 1989; Helton, 1993). The construction of CCDFs follows from the development of scenario probabilities and the calculation of scenario consequences. Further, the effects of different types of uncertainties can be shown by constructing families of CCDFs and then reducing each family to a single CCDF.

"Reasonable Expectation" of Compliance. As stated in \$191.13(b),

"Performance assessments need not provide complete assurance that the requirements of $[\$ 191.13(\mathrm{a})]$ will be met. Because of the long time period involved and the nature of the events and processes of interest, there will inevitably be substantial uncertainties in projecting disposal system performance. Proof of the future performance of a disposal system is not to be had in the ordinary sense of the word in situations that deal with much shorter time frames."

For \$191.13(a), the EPA assumes that a single CCDF will incorporate all uncertainty (EPA, 1985, p. 38088). The Containment Requirements ( $\$ 191.13[a])$ state that, based upon performance assessments, releases shall have probabilities not exceeding specified limits. Appendix B of 40 CFR 191 states that "the [EPA] assumes that a disposal system can be considered to be in compliance with $\$ 191.13$ if this single distribution function meets the requirements of $\$ 191.13(\mathrm{a})$ " (EPA, 1985, p. 38088). Additional wording in $\$ 191.13$ (a) is that "...what is required is a reasonable expectation, on the basis of the record before the implementing agency, that compliance with 191.13(a) will be achieved." 


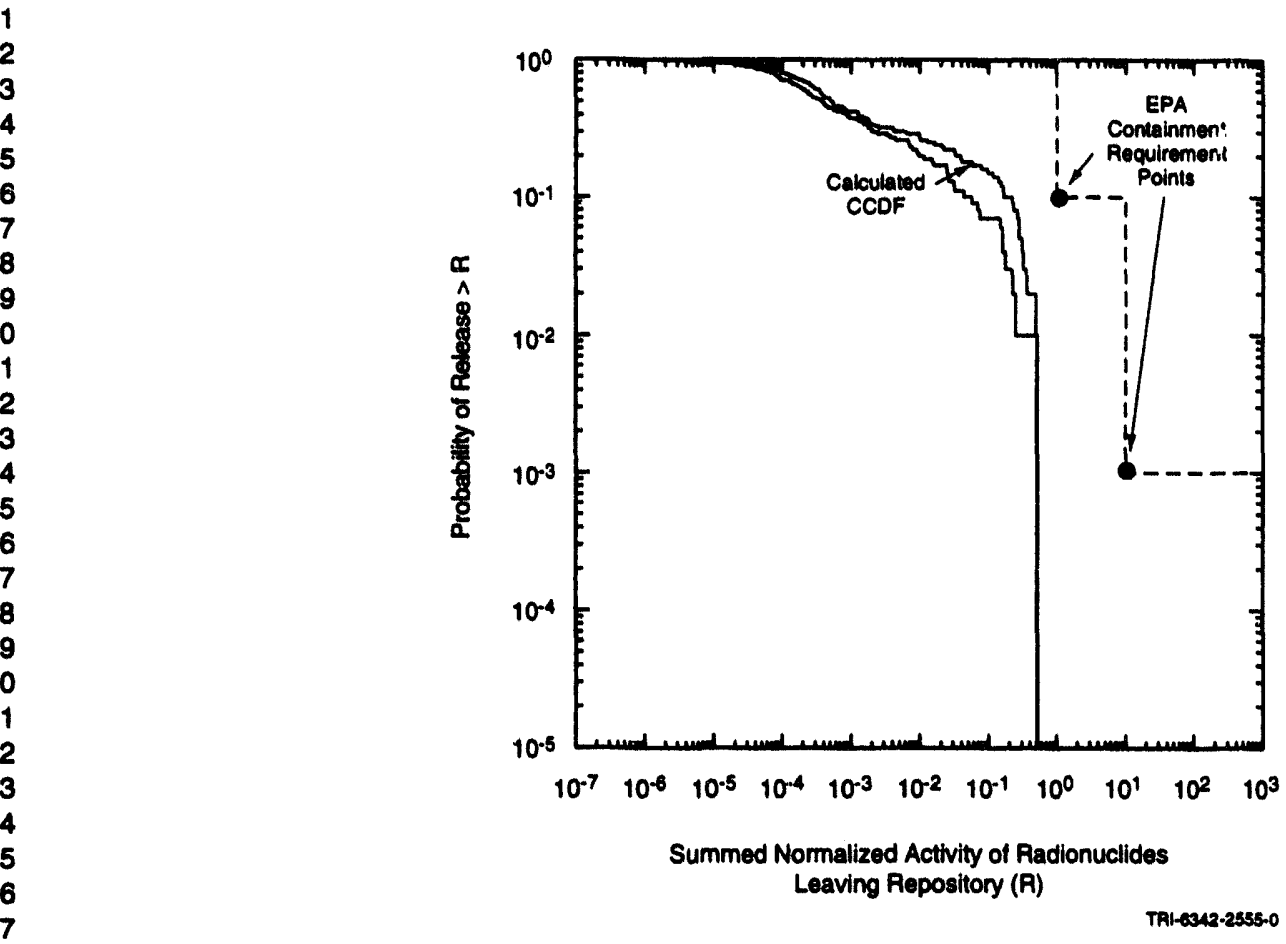

Figure 2-3. Complementary cumulative distribution function (CCDF) illustrating compliance with the Containment Requirements.

\subsubsection{Assurance Requirements}

The EPA included Assurance Requirements ( $\$ 191.14)$ to provide confidence the agency believes is needed for long-term compliance with the Containment Requirements. These requirements apply only to disposal systems not regulated by the NRC, because comparable provisions exist in NRC regulations. The Assurance Requirements are designed to complement the Containment Requirements because of the uncertainties involved in predicting long-term performance of disposal systems (EPA, 1985, p. 38072). They specify the design philosophy to use for disposal systems.

Each Assurance Requirement applies to some aspect of uncertainty about long-term containment:

- Limiting reliance on active institutional controls to 100 years precludes relying on future generations to maintain surveillance;

- Carefully planned monitoring will reduce the likelihood of unexpectedly poor system performance going undetected;

- Using passive institutional controls such as markers and records will reduce the chances of inadvertent or systematic intrusion;

- Including multiple barriers, both engineered and natural, will reduce the risk should one type of barrier not perform as expected;

- Considering future resource potential and demonstrating that the favorable characteristics of the disposal site compensate for the likelihood of disturbance will add to the confidence that the chosen site is appropriate; 
- Selecting a disposal system that permits possible future recovery of most of the wastes for a reasonable period of time after disposal will allow future generations the option of relocating the wastes should new developments warrant such recovery. In promulgating 40 CFR 191, the EPA stated that "the intent of this provision was not to make recovery of waste easy or cheap, but merely possible...because the [EPA] believes that future generations should have options to correct any mistakes that this generation might unintentionally make" (EPA, 1985, p. 38082). The EPA also stated that "any current concept for a mined geologic repository meets this requirement without any additional procedures or design features" (EPA, 1985, p. 38082, emphasis in original).

\subsubsection{Individual Protection Requirements}

The Individual Protection Requirements (\$191.15) require predicting potential doses to humans resulting from releases to the accessible environment for undisturbed performance during the first 1000 years after decommissioning of the repository, in the event that performance assessments predict such releases. Challenges to this requirement contributed to the remand of Subpart B to the EPA.

The Individual Protection Requirements state that "for 1,000 years after disposal, undisturbed performance of the disposal system shall not cause the annual dose equivalent from the disposal system to any member of the public in the accessible environment to exceed 25 millirems to the whole body or 75 millirems to any critical organ" $(\S 191.15[a])$.

The methodology developed for assessing compliance with the Containment Requirements can be used to estimate doses as specified by the Individual Protection Requirements. One of the products of scenario development for the Containment Requirements is a base-case scenario that describes undisturbed conditions. The undisturbed performance of the repository is its design-basis behavior, including variations in that behavior resulting from "ncertainties in the 1000-year performance of natural and engineered barriers, as defined in $\$ 191.12(p)$ :

"'Undisturbed performance' means the predicter' ' vior of a disposal system, including consideration of the uncertainties in predicted behavior, if the disp. of unlikely natural events."

$\imath$ is not disrupted by human intrusion or the occurrence

Undisturbed performance can be understood to mean that uncertainties in such repository features as engineered barriers (seals and plugs) must be specifically included in the analysis of the predicted behavior. Human intrusion means any human activity other than those directly related to repository characterization, construction, operation, or monitoring. The effects of intrusion are specifically excluded from the undisturbed-performance analysis.

The EPA assumes in Appendix B of 40 CFR 191 that compliance with the Individual Protection Requirements "can be determined based upon best estimate predictions" rather than a probabilistic analysis. Thus, according to the EPA, when uncertainties are considered, only "the mean or median of the appropriate distribution, whichever is higher," need fall below the limits (EPA, 1985, p. 38088).

\subsubsection{Groundwater Protection Requirements}

Special sources of groundwater are protected by the Groundwater Protection Requirements (\$191.16) from contamination at levels greater than certain limits:

"Disposal systems...shall be designed to provide a reasonable expectation that, for 1,000 years after disposal, undisturbed performance of the disposal system shall not cause the radionuclide concentrations averaged over any year in water withdrawn from any portion of a special scurce of ground water to exceed:

1. 5 picocuries per liter of radium-226 and radium-228; 
2. 15 picocuries per liter of alpha-emitting radionuclides (including radium-226 and radium-228 but excluding radon); or

3. The combined corcentrations of radionuclides that emit either beta or gamma radiation that would produce an annual dose equivalent to the total body or any internal organ greater than 4 millirems per year if an individual consumed 2 liters per day of drinking water from such a source of ground water."

"Special sources of groundwater" are defined as

"those Class I ground waters identified in accordance with the [EPA's] Ground-Water Protection Strategy published in August 1984 that: (1) Are within the controlled area encompassing a disposal system or are less than five kilometers beyond the controlled area; (2) are supplying drinking water for thousands of persons as of the date that the [DOE] chooses a location within that area for detailed characterization as a potential site for a disposal system (e.g., in accordance with Section 112(b)(1)(B) of the NWPA); and (3) are irreplaceable in that no reasonable alternative source of drinking water is available to that population" (\$191.12[0]).

Class I groundwaters are defined as certain groundwater resources that are in need of special protective measures and that are either (1) an irreplaceable source of drinking water, or (2) ecologically vital (EPA, 1984). 


\title{
3. PA Methodology Overview
}

\author{
M. S. Tierney
}

\subsection{Introduction}

The U.S. Environmental Protection Agency's (EPA's) guidance and intentions concerning the nature and purpose of performance assessment (see Chapter 2) suggests that PA is ideally a stochastic simulation (Section 3.2.4) of possible long-term behaviors of a real system with a computer-implemented mathematical model of that system. In this respect, performance assessment is similar to other large-scale simulations (e.g., the Reactor Safety Study [Rasmussen, 1975]) that have been used by federal agencies in the past to explore policy options and develop regulations. Performance assessments of systems for the disposal of radioactive and toxic wastes may nevertheless differ from most simulations with "regulatory models" (Morgan et al., 1990, p. 292) in two significant and related ways: the way results of a performance assessment are to be used; and the nature of the real system that is being simulated.

In contrast with past simulations with federally sponsored models, PA results are ultimately used to test compliance of a real system with an existing environmental standard, not merely to gain insight into the behavior of the system with the purpose of discovering a rational basis for new standards. Furthermore, a quantitative treatment of uncertainty in PA calculations is not merely good analytical practice but is mandated by the explicitly probabilistic statement of the Standard in which all results are to be combined in an "overall probability distribution" whenever practicable. As will be seen later, such a quantitative treatment of uncertainty forces the development of two categories of models of the same system: the usual, deterministic phenomenological models (called "consequence" models in PA; Section 3.2.1), and probability models (Section 3.2.3) that can provide quantitative estimates of the degree of uncertainty associated with parameters that specify the possible configurations of the consequence models.

The problems arising from direct use of PA results in testing compliance and a mandated quantitative treatment of uncertainty are compounded by the nature of the focal system in PA: geologic waste-disposal systems are built up largely from natural components (as opposed, for example, to the man-made components of a nuclear reactor), the physical and chemical characteristics of which are usually inhomogeneous and distributed in space and time. There is little published work on efficient methods for condecting sensitivity and uncertainty analyses of such distributedparameter systems; objective methods for the assignment of the parametric uncertainty associated with such systems are also lacking. Indeed, in building methodologies for PA, investigators have in the past drawn heavily upon ideas and analytical techniques developed primarily for the study of "lumped parameter" systems and have largely ignored the special needs of PA that arise from the problems mentioned above.

With the hope that a firm understanding of the structure of a PA will help in meeting PA's special needs, this overview offers a description of current PA methodologies in terms and concepts taken from the broader context in which they are imbedded, i.e., the general theory of stochastic simulation, as it is applied to the study of natural systems that may change or "fail" over long periods of time. To minimize misunderstanding among investigators with diverse backgrounds, terminology developed for PA will be maintained throughout the overview, but PA terms will be matched with their mathematical counterparts whenever possible. Discussion of each topic proceeds from generalities to specifics, and from theory to practical applications. The structure of a PA is summarized in Section 3.2, where emphasis is upon the correspondence of PA concepts such as consequence models, probability models, and performance measures, with concepts from the general theory of stochastic simulation. Section 3.3 is a technical treatment of certain topics of special interest to PA practitioners; in particular, the confusing topic of "scenarios" is treated. Applications and examples are taken from the initial performance assessment described in this report and from an assessment of the performance of the Waste Isolation Pilot Plant (WIPP), a research and development faciliiy located in southeastern New Mexico for the purpose of demonstrating safe disposal of defense-generated transuranic wastes (WIPP PA Division, 1991a, 1991b; WIPP PA Department, 1992b). 


\subsection{Structure of a Performance Assessment}

Simulation can be defined as the study of a system or phenomenon using a model of that system or phenomenon. Indeed, the ideas of simulation and modeling are intimately linked since a "model" can be defined as a tentative ideational structure that is used as a testing device. Models used in simulations can take different forms: pictorial, mechanical, verbal, and mathematical. Since the advent of high-speed digital computers in the 1950 s, the use of computer-implemented mathematical models for simulations of natural and engineered phenomena has become routine. In contrast with the other forms of models, a mathematical model is relatively inexpensive and has the advantages of unequivocal and testable structure and numerical output. Nevertheless, good modeling practice dictates that pictorial and verbal models be constructed prior to construction of the mathematical model; PA analysts have called these prior pictorial/verbal models "conceptual models."

The operations of a computer-implemented mathematical model can be viewed as the evaluation of a function, i.e., a correspondence between sets of numbers that has the form $y=f(x)$, where: $x=\left(x_{1}, x_{2}, x_{3}, \ldots, x_{N}\right)$ is a point in a subset of $\mathrm{N}$-dimensional Euclidean space called the domain, $\mathrm{D}_{\mathrm{N}}$ [or parameter space in PA terminology, (Section 3.2.2)]; $\mathbf{y}=\left(\mathrm{y}_{1}, \mathrm{y}_{2}, \mathrm{y}_{3}, \ldots, \mathrm{y}_{\mathrm{M}}\right)$ is a point in a subset of $\mathrm{M}$-dimensional Euclidean space called the range, $\mathbf{R}_{\mathbf{M}}$, (or the "consequences" in PA terminology); $e n d$ the notation $f(\circ)$ stands for the operations of the mathematical model [called a consequence model in PA terminology, [3.2.1]) that transforms parameters $\mathbf{x}$ into consequences $\mathbf{y}$. Since the operations implicit in a function $f(\bullet)$ are nowadays almost always carried out by coded programs for a digital computer, no distinction will hereinafter be made between "function" and "computer code."

\subsubsection{Consequence Models}

In this overview, it is assumed that all functions representing consequence models are deterministic; roughly speaking, this means that for each vector of input parameters, $\mathbf{x}$, there is a unique vector of consequences, $\mathbf{y}$. Note that not all mathematical models of the types commonly used in PA (i.e., models formulated in terms of difference, ordinary, or partial differential equations) can be expected to be deterministic. Because of extreme sensitivity of solutions to initial values or certain problem parameters, some nonlinear models used in PA work could yield different values of $\mathbf{y}$ for consecutive calculations that started with single value of $\mathbf{x}$. Glimm (1991) calls this apparently stochastic behavior "spontaneous chaos" and distinguishes it from the case of "external" or "forced chaos" that arises when some or all of the input parameters $\mathrm{x}_{1}, \mathrm{x}_{2}, \mathrm{x}_{3}, \ldots, \mathrm{x}_{\mathrm{N}}$ are treated intentionally as random variables.

There has been little or no investigation of the effects of spontaneously chaotic models on the probabilistic performance measures of interest in PA. In contrast, PA studies almost exclusively employ forced chaos in the following way: the user of a deterministic model forces it to exhibit stochastic behavior among the results of consecutive runs of the model by randomly sampling the parameters for each run from predetermined probability distributions, i.e., by randomly choosing different "realizations" of the parameter sets for each consecutive run. The collection of outcomes of the consecutive runs can then be treated as a random sample (in the statistical sense) of some quantitative measures of system behavior. This procedure is called Monte Carlo simulation (see Hammersley and Handscomb, 1964; Rubenstein, 1981; Ross, 1985), which is a variant of stochastic simulation (3.2.4; see also Ripley, 1987). The construction of probability distributions suitable for sampling uncertain model parameters in a stochastic simulation can be called probability modeling (Section 3.2.3).

The reasons for introducing forced stochasticity in a PA consequence model are to facilitate the study of the sensitivity of model consequences to changes in the model's parameters (sensitivity analysis; see Helton et al., 1991) and to map parametric uncertainty onto uncertainty in the model's results (uncertainty analyses). In particular, uncertainty analyses can be used to form estimates of probabilistic performance measures (Section 3.2.4) such as the one that is to be compared with EPA's probabilistic standard. 
A mathematical model of a system can be called "complete" or "total" if the model captures all effects or phenomena of concern to the users of the model. Total-system models are usually formed by linking submodels that capture specific effects or phenomena in a network under the control of a master ("driver") routine. The deterministic function that represents the consequences of a total-system model,

$$
\mathbf{c}=\mathbf{f}(\mathbf{x})
$$

can be built up by consecutive application of several functions that individually represent the outputs of models of selected system components (here called "submodels"). For $I \geq 3$ components, a calculation of a consequence $c \varepsilon R_{M}$ might proceed as follows:

$$
\begin{gathered}
y_{1}=f_{1}\left(x_{1}\right), y_{2}=f_{2}\left(y_{1}, x_{2}\right), y_{3}=f_{3}\left(y_{2}, x_{3}\right), \ldots \\
c=y_{I}=f_{I}\left(y_{I-1}, x_{I}\right)
\end{gathered}
$$

In this expression, $x_{i}(i=1,2,3, \ldots, I)$ stands for the vector of free parameters that appear in the ith submodel and $y_{i}(i=$ $1,2,3, \ldots, I)$ stands for the vector of dependent variables produced by the ith submodel. The symbol $f_{i},(i=1,2,3, \ldots, I)$ stands for the function (or computer code) representing the transformations performed by the ith submodel on its input variables and free parameters to produce an output, $\mathbf{y}_{\mathbf{i}}$.

Excepting $\mathbf{y}_{\mathrm{I}}$, which is identified with the output or consequences $\mathbf{c}$, of the total-system model, the dependent variables $y_{i}$ should here be regarded simply as a set of intermediate variables that are generated by the ith submodel and passed as input to the $(i+1)$ st submodel. Though some of these intermediate dependent variables may be of interest to users of the model (in PA analyses, all are usually recorded during the course of a calculation), they play no direct role in determining the dimension and structure of the total-system model's parameter space $D_{N}$ or consequence space $R_{M}$. The crucial role played by the submodels' free-parameter vectors, $\left\{\mathbf{x}_{\mathbf{i}}\right\}$, in determining the dimension and structure of $D_{N}$ is discussed in the next section (Section 3.2.2).

In PA work, the function $f_{i}$, representing the transformations performed by the ith submodel on its input variables and free parameters, is usually a numerical solution of one or a combination of the following kinds of equations:

a. Partial differential equations - which model dynamics and statics of continuous media and are reduced to a set of algebraic equations or ordinary differential equations in order to effect a numerical solution by finite-difference or finite-element methods.

b. Ordinary differential equations - which may result from the reduction of a system of partial differential equations or may directly model the dynamics of a system with discrete components: e.g., a model of a pressurized brine reservoir, or the dissolution of a finite volume of waste material.

c. Algebraic equations of the form

$$
F\left(x_{1}, x_{2}, x_{3}, \ldots, x_{n} ; y\right)=0
$$

which may arise indirectly from equilibrium solutions of orcinary differential equations (i.e., solutions in which time $\rightarrow \infty$ ), or may directly express some physical relationship between parameters $\left(\mathrm{x}_{1}, \mathrm{x}_{2}, \mathrm{x}_{3}, \ldots, \mathrm{x}_{\mathrm{n}}\right)$ and dependent variables $\mathbf{y}$.

Numerical solutions of these types of equations on digital computers require discretization of independent variables specifying spatial coordinates and time. Accordingly, quantities intended to represent spatially continuous model parameters or spatially and temporally continuous solutions are necessarily specified at a finite number of points on a discrete space-time grid. This fact makes reasonable an assumption implicitly made in the foregoing paragraphs: that all parameters of the ith submodel can be represented as a finite-dimensional vector $\mathbf{x}_{\mathbf{i}}$, and that all outputs from the ith submodel can be represented as a finite-dimensional vector $\mathbf{y}_{\mathbf{i}}$. 


\section{PA Methodology Overview}

The composite nature of total-system consequence models distilled by Expression 3-2 is here illustrated with an example taken from a WIPP preliminary performance assessment (WIPP PA Department, 1992b). Figure 3-1 shows eight submodels, named in bold capitals by the names of their implementing computer codes (Table 3-1), that WIPP project investigators have constructed and used to estimate the consequences of inadvertent drilling into the WIPP repository for radioactive wastes (labelled by "Panel" in Figure 3-1). Other important features in Figure 3-1 are the accessible-environment boundary (marked by the line supporting the drilling rig and withdrawal well plus the vertical dotted line on the right) and the brine reservoir at the bottom. Here, consequences are measured in two ways: (a) by the cumulative amount of radioactivity crossing the accessible-environment boundaries in a 10,000-yr period following closure of the repository; and (b) by the radiation dose incurred by humans who use water from the withdrawal well and stock pond.

Several distinct sequences of submodels (or code linkages) are shown in Figure 3-1; the sequence used in this example is one that calculates the groundwater-pathway contribution to type (a) consequences during realization of the following summary scenario (Section 3.3.1):

...one or more boreholes penetrate through a waste-filled room or drift and continue into or through a brine reservoir in the underlying Castile Formation ... radionuclides may reach the subsurface boundary of the accessible environment following long-term groundwater transport up the borehole and laterally down a potentiometric gradient in the Culebra Dolomite Member of the Rustler Formation (WIPP PA Department, 1992b).

The code linkage for this scenario takes the following form:

$$
\begin{aligned}
(\mathrm{SANCHO} \Rightarrow \mathrm{BRAGFLO}) & \Rightarrow \mathrm{PANEL} \Rightarrow(\mathrm{GRASP}-\mathrm{INV} \Rightarrow \mathrm{SECO} 2 \mathrm{D} \Rightarrow \mathrm{SECO} / \mathrm{TP}) \\
& \Rightarrow \text { [summation of type (a) consequences], }
\end{aligned}
$$

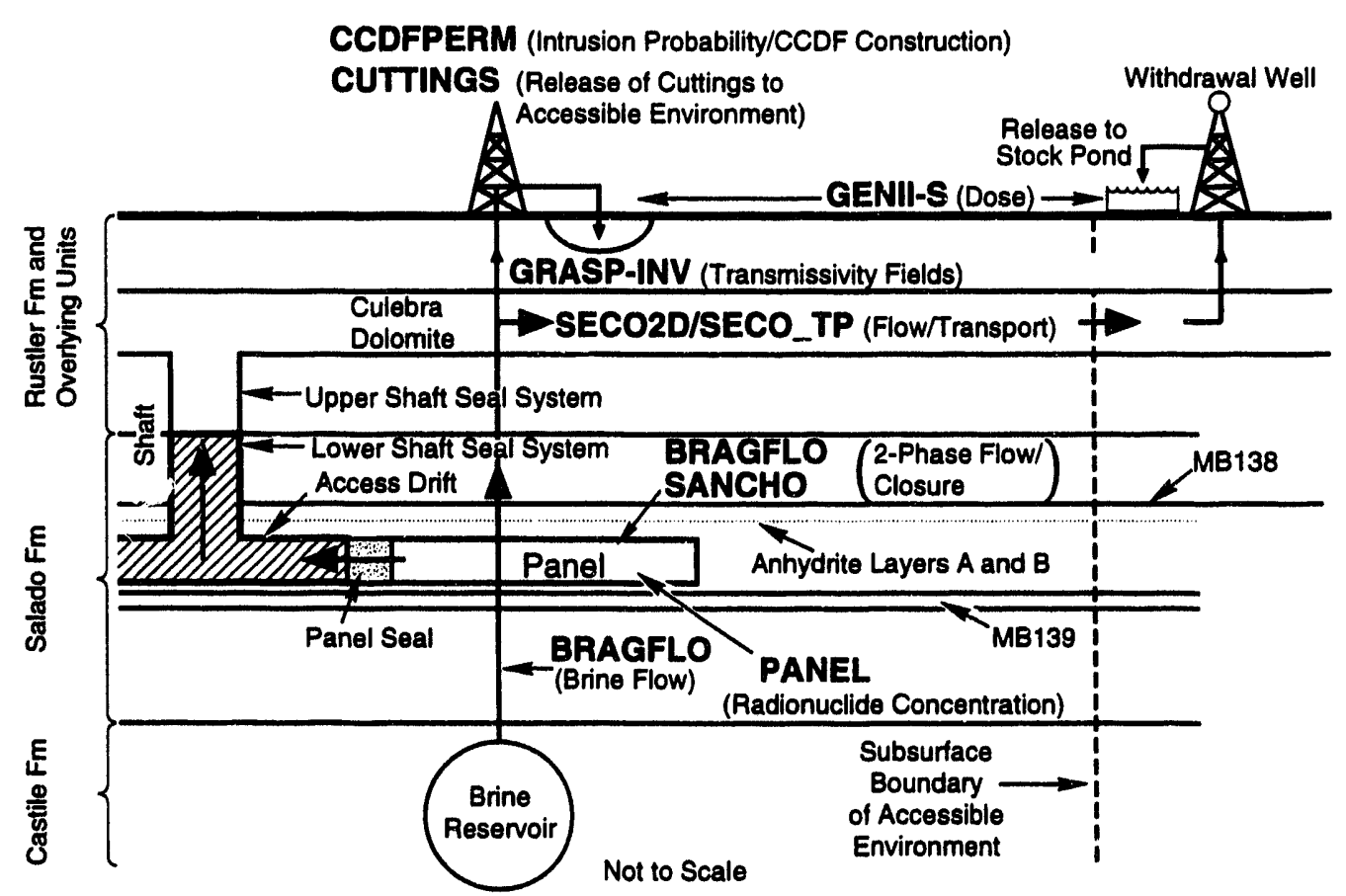

TRI-6342-3401-0

Figure 3-1. Submodels used in 1992 WIPP performance assessment. (adapted from Fig. 3.6, WIPP PA Department, 1992b) 
Table 3-1. Selected Computer Models Used in 1992 WIPP Performance Assessment*

\begin{abstract}
Model
Description

CUTTINGS Calculates the quantity of radioactive material (in curies) brought to the surface as cuttings and cavings generated by an exploratory drilling operation that penetrates a WIPP waste panel.

BRAGFLO Simulates multiphase flow of gas and brine through a porous, heterogeneous reservoir (a collapsed WIPP waste panel); includes gas generation by corrosion of metals and microbial action; requires that porosity of panel as a function of time be given by SANCHO.

GRASP-INV Generates realizations of transmissivity fields in the Culebra Dolomite Member of the Rustler Formation for use in SECO2D.

PANEL Using brine discharge from BRAGFLO, calculates discharge and integrated discharge of radionuclide mass from a collapsed and punctured WIPP waste panel through an intrusion borehole; accounts for nuclide decay chains.

SECO2D Simulates Darcy velocity as a two-dimensional field in the Culebra Dolomite Member of the Rustler Formation; requires transmissivity fields simulated by GRASP-INV.

SECOTP Simulates transport of dissolved radionuclides in the groundwater of the Culebra Dolomite Member of the Rustler Formation which is treated as a fractured, porous, chemically-reacting medium; requires Darcy velocity field simulated by SECO2D.

SANCHO Simulates dynamics of closure of a WIPP waste panel as the quasistatic, large deformation, inelastic response of a two-dimensional, porous solid; accounts for gas pressure in pore space. Used in 1992 to determine average porosity of panel as a function of time and the amount of gas generated by corrosion and microbial action.
\end{abstract}

* From WIPP PA Department, 1992b.

where an arrow $(\Rightarrow)$ denotes passage of dependent, auxiliary variables between submodels, and groupings of dependent submodels are enclosed in parentheses; e.g., SANCHO furnishes variables to BRAGFLO, and the combination SANCHO + BRAGFLO passes variables to PANEL. The function representing the total-system consequences (for the particular scenario described above) may therefore be developed from three functions, each of which represents a grouping of dependent submodels:

$$
\begin{aligned}
& y_{1}=f_{1}\left(x_{1}\right), \quad y_{2}=f_{2}\left(y_{1}, x_{2}\right), \quad y_{3}=f_{3}\left(y_{2}, x_{3}\right), \\
& y_{3} \Rightarrow \text { [summation of type (a) consequences]. }
\end{aligned}
$$

The vectors $x_{1}, x_{2}, x_{3}$ represent arrays of the free parameters appearing respectively in the groups (SANCHO + BRAGFLO), PANEL, and (GRASP-INV + SECO2D + SECO/TP). 
Another example of the composite nature of total-system models can be taken from the general conceptual model for the granite repository analyzed in this report (see Section 7.6.1 and Figure 7-6). Calculation of consequences of the scenario for a breached granite repository requires five submodels:

STAFF2D - Complex model of flow and solute transport,

CLAMVD - Simplified model of transport in repository,

QEDTP - Simplified model of fracture flow and solute transport,

BRAGFLO_T - Complex model of 2-phase flow,

GRFLOW - Simplified model of 2-phase flow.

The code linkages for calculating consequences of a breached granite repository take the following forms: For complex consequence modeling,

$$
\text { (BRAGFLO_T } \Rightarrow \text { STAFF2D) } \Rightarrow \text { STAFF2D } \Rightarrow \text { [summation of type (a) consequences]; }
$$

and for simplified consequence modeling,

$$
\text { (GRFLOW } \Rightarrow \text { CLAMVD) } \Rightarrow \text { QEDTP } \Rightarrow \text { [summation of type (a) consequences]. }
$$

\subsubsection{Parameter Space}

In Section 3.2.1 it was stated without proof that the operations of a mathematical model of a system can be regarded as the evaluation of a function of the form $\mathbf{c}=\mathbf{f}(\mathbf{x})$, where $\mathbf{x}$ is a vector whose elements are the free parameters of the model and $\mathbf{c}$ is a vector whose elements are "consequences", i.e., quantities of interest to users of the model. The ideas of consequences and consequence models were treated briefly in Section 3.2.1; the focus in the present section is upon the meaning and structure of the space of free parameters appearing in a model of the complete (or total) system.

The free parameters of a total-system model are all of the numbers that must be supplied by the user of a model in order to make it run (of course, some of these numbers may be "built into" the implementing computer codes; they are nevertheless free in the sense that they are not calculated numbers and may be easily changed). These numbers may also be called the problem input or the problem's independent variables. In Section 3.2.1, the free parameters for a system comprised of I submodels were arranged as elements of I vectors, $x_{1}, x_{2}, x_{3}, \ldots, x_{1}$, of possibly different dimensions. A typical vector from this collection, say the ith one, can be written as the array

$$
x_{i}=\left[x_{i 1}, x_{i 2}, x_{i 3}, \ldots, x_{i m(i)}\right],
$$

showing the individual free parameters appearing in a submodel with $\mathrm{m}(\mathrm{i})$ independent parameters. Each element in such an array of numbers stands for a single parameter that has a definite meaning in the context of at least one of the submodels: for example, a certain element might stand for the value of a certain material property in a specific cell of the model's spatial grid; another element might represent a dimension of some physical feature being modeled.

Note that the same parameter may be required by more than one submodel and may therefore be a common element of several of the vectors specifying the submodel parameters. These common elements are counted only once in forming the elements of the vector of free parameters of the total-system model: assuming that there are $\mathrm{N}$ distinct parameters, the vector of free parameters of the total system will hereinafter be displayed as

$$
x=\left(x_{1}, x_{2}, x_{3}, \ldots, x_{N}\right)
$$

Although the order in which the elements $x_{n}, n=1,2,3, \ldots, N$, appear in array (3-3) is unimportant for purposes of theoretical description, order is obviously important when numerical values are assigned to elements and so a fixed order for the $\mathrm{N}$ elements in vectors like Expression 3-3 is assumed ior the remainder of this overview. Given an ordering of elements and the fact that the $x_{n} s$ are real numbers or integers, it is seen that $\mathbf{x}$ represents a point in $\mathrm{N}$-dimensional 
Euclidean space. Since the $x_{n} s$ are intended to represent real quantities that have meaning in science or engineering, there will be restrictions on the ranges of numbers that may be assigned to them; by allowing each element to sweep over its natural range, the vector $\mathbf{x}$ will sweep over a subset of $\mathrm{N}$-dimensional Euclidean space that is here called parameter space and denoted by $D_{N}$. A vector $\mathbf{x} \varepsilon D_{N}$ is called a parameter set.

If the reader is to fully appreciate the simplicity and power of stochastic simulation, he/she should first appreciate the broad range of possibilities for the types of quantities that can make up the elements of a parameter set. Roughly following Morgan et al. (1990, p. 50), the types of quantities that can appear as elements of a parameter set fall in at least six categories:

1. Model constants, e.g., precisely known constants such as Planck's constant $=6.6256 \times 10^{-27} \mathrm{erg} \mathrm{s}$, or $\pi=$ 3.14159 .

2. Model-domain parameters, e.g., coordinates of nodes on spatial and temporal grids; locations and dimensions of engineered features of the system.

3. Model control parameters, e.g., any parameter assigned by the user to control timing and accuracy of computations: number of dynamic time steps, numbers giving convergence criteria, cutoff values for certain dependent variables.

4. Material-property parameters. Parameters specifying magnitudes of physical or chemical properties of materials that occupy cells of a spatial grid or parts of system features; this category may also include initial and boundary conditions on submodel dependent variables.

5. Event-and-process parameters. This category is the time-domain counterpart of material-property parameters; it includes the number of external events of a given kind that act upon the system during a given interval on the time grid (e.g., the number of exploratory boreholes that penetrate a waste disposal compartment between 2000 and $3000 \mathrm{yr}$ after closure) and the intensities of external processes that affect the system during each interval of the time grid (e.g., mean precipitation and temperature as functions of time in a region under changed climatic conditions).

6. Indices for alternative models. In many cases, there are several acceptable submodels of a phenomenon of interest to PA that nevertheless may differ in their influence on the results of a calculation with the total-system model. To test the influence of each alternative model, the alternatives are numbered, runs are made of the total-system model incorporating each alternative, and results are compared. Though seemingly arbitrary, the indices of the alternative models can be sensitive elements of the parameter set.

A seventh category can be added to the list above when, for purposes of uncertainty analyses, probability models (Section 3.2.3) are used to assign likelihoods to ranges of the values taken by uncertain members of the parameter set that belong to categories 4,5 , and 6 .

7. Probability-model parameters, e.g., means, variances and correlation coefficients in analytic probability distributions for values of material-property parameters; mean rates of occurrence of events of a given kind (appearing in probability distributions for event-and-process parameters); weights assigned to the indices of alternative forms of a submodel.

Note on uncertain parameters:

An uncertain (or imprecisely known) parameter is one that cannot be assigned a single, precise value because: (a) measurements of quantities from which the parameter can be calculated are dispersed and too few in number to form a sufficiently precise estimate of the parameter using classical methods of statistics; or (b) the parameter characterizes some aspect of a hypothetical event, process, or natural feature (e.g., the number of boreholes that penetrate an underground waste repository in the 10,000-yr period following closure). Parameters in categories 1 through 3 are seldom treated as uncertain quantities; model-domain parameters are almost always chosen by the user to reflect 


\section{PA Methodology Overview}

specifications of engineered components; model control parameters may be varied at will by the user, usually within well-documented limits set by the model's creators.

On the other hand, some degree of uncertainty is always associated with material-property parameters, particularly those derived from properties of inhomogeneous, hard-to-characterize natural (as opposed to engineered) materials. Spatial inhomogeneity of geologic materials and structures, the impracticality of making a statistically significant number of measurements of most geologic quantities, and difficulties in scaling up measured properties to reference volumes appropriate for a mathematical model all dictate that subjective (Bayesian) methods be adopted for the quantitative treatment of uncertainty in category-4 parameters. The need for a Bayesian approach in quantifying the uncertainty of parameters in categories 5 and 6 is even stronger: apart from placing known, common-sense bounds on their ranges, these event-and-process parameters are not amenable to estimation by classical statistical techniques and should be regarded as truly stochastic quantities.

The usual Bayesian approach to modeling uncertainty in event-and-process parameters is to construct a probability model (Section 3.2.3) that the analyst believes will adequately represent that uncertainty, given knowledge of a few category-7 parameters. In simple terms, probability models for event-and-process parameters are invariably conditional probability distributions (see Feller, 1966). Treatment of uncertainty in the category-7 or probability-model parameters then requires adoption of a second level of probability models, and so on; but in practice this hierarchy of probability models is usually closed at the first level by assuming the probability-model parameters to be constants.

In more refined Bayesian methodologies (Martz and Waller, 1982), uncertainty in a parameter is quantified by first postulating a probability model (usually expressed as an analytic formula) that provides a quantitative measure of uncertainty, called the prior distribution, in the parameter's values in terms of a few auxiliary constants-here called category 7 , or probability-model parameters; these auxiliary parameters are then fit initially with available empirical data or, in most cases, chosen by subjective judgment. In principle, further empirical or subjective information can be used to "update" the probability model and/or its constants by application of Bayes' Rule to form posterior distributions, which presumably reflect a better estimate of the range of uncertainty. It is an unfortunate feature of PA work that the high cost of obtaining "further empirical or subjective information" often precludes use of the more-refined Bayesian methods.

The difference between uncertain and fixed parameters in the parameter set of a total-system model can be emphasized by partitioning the parameter space, $D_{N}$, into the space of uncertain parameters, $D_{\mathrm{Nu}}$, and the space of fixed parameters, $D_{\mathrm{Nf}}$, i.e.,

$$
D_{N}=D_{N u} \cup D_{N f}
$$

and splitting the parameter set accordingly: $\mathbf{x}=\left(\mathbf{x}_{\mathrm{u}}, \mathbf{x}_{\mathrm{f}}\right)$. The space of uncertain parameters, $\mathrm{D}_{\mathrm{Nu}}$, is of primary interest in sensitivity analyses and stochastic simulation of system performance (Section 3.2.4); the space of fixed parameters, $\mathrm{D}_{\mathrm{Nf}}$, is of primary interest in sensitivity analyses and in the validation and verification of computational models of system components. Validation of a computational model means finding that the model predicts well-understood phenomena with accuracies deemed sufficient by the user (but note that it may not be practicable to validate PA consequence models intended to predict poorly understood or hypothetical phenomena). Verification of a computational model usually means finding that the model reliably performs its operations or transformations in precisely the ways intended by its creators. Both validation and verification may involve the users' "fine tuning" of the model's fixed parameters.

For purposes of this overview, there is no loss in generality if the space of fixed parameters is ignored and the total parameter space is restricted to the space of uncertain parameters. Thus, the notation $x \in D_{N}$ will hereinafter mean that elements of $x_{u}$ are chosen from the space $D_{N_{u}}$, and that elements of $x_{f}$ remain fixed at the values assigned by the user.

Referring ahead to Section 9.1, the reader will see that 650 to 700 distinct parameters are input to the consequence and probability models used to analyze the salt and granite repositories of this report, but only 23 of these parameters are treated as uncertain quantities. If consequence models are complex and computations with them are 
expensive of time, it becomes impracticable to investigate the sensitivity of uncertain-parameter sets having dimensions much in excess of 50 .

The idea of uncertain parameters is further illustrated by an example from the 1991 WIPF' PA (WIPP PA Division, 1991a). Consequence models used for the 1991 series of calculations employed more than 120 distinct parameters in the five categories mentioned above (including several spatially varying quantities such as formation transmissivity). A total of 45 of these parameters were deemed sufficiently uncertain to warrant the assignment of probability models; of these, 40 were material-property parameters (Table 3-2), and 4 could be classified as eventand-process parameters (Table 3-3). Not mentioned in these tables are five event-and-process parameters specifying the number of accidental penetrations of waste-disposal areas by exploratory drilling during each of five 2000-yr time intervals spanning the 10,000 -yr period following closure of the site. The probability that any one of these five parameters could take values $0,1,2, \ldots$, was calculated with a probability model based on the assumption that accidental drilling follows a homogeneous Poisson process (WIPP PA Division, 1991a, p. 2-7). The single probability-model parameter specifying the Poisson process, the rate constant, is mentioned in Table 3-3.

\subsubsection{Probability Models}

The class of uncertain model parameters introduced in Section 3.2.2 was distinguished by the model users' inability to assign to any one of its members a single, precise value for reasons of imperfect (or total lack of) information concerning the parameter's location within its natural range of values. The very nature of this kind of uncertainty, called parametric uncertainty, suggests that uncertain parameters be regarded as random variables (see Feller, 1966) and that parametric uncertainty be quantified by forming probability distributions for the random variables associated with each uncertain parameter. But even when there are extensive empirical data concerning ranges of values taken by a parameter and the methods of classical statistics can be employed in estimating its distribution, construction of a probability distribution for that parameter will always involve some subjective assumptions. Thus, constructing a

Table 3-2. Uncertain Material-Property Parameters of Consequence Models Used in 1991 WIPP PA Exercises (adopted from Tables 6.0-1 and 6.0-2 of WIPP PA Division, 1991b).

Material

Properties

Halite within Salado Formation

Anhydrite layers within

Salado Formation

Castile Formation Brine

Culebra Dolomite Member

of Rustler Formation

Unmodified waste form
Undisturbed permeability and pore pressure.

Undisturbed permeability, porosity, pore pressure; threshold displacement pressure.

Initial pressure; bulk reservoir storativity.

Longitudinal dispersivity; matrix and fracture porosity; fracture spacing; matrix and fracture partition coefficients for elements Am, Np, $\mathrm{Pu}, \mathrm{Th}$ and $\mathrm{U}$; transmissivity field (60 equally likely realizations).

Gas generation rates for corrosion and microbial action under inundated and humid conditions; stoichiometric coefficients for corrosive and microbial action; solubilities for nuclide charge states Am3+, Np4+, Np5+, Pu4+, Pu5+, Th4t, U4+, U6+; Eh-pH conditions; volume fractions of metals/glass and combustibles; initial waste saturation. 
Table 3-3. Event-and-Process and Probability-Model Parameters of Consequence Models Used in 1991 WIPP PA Exercises (adopted from Table 6.0-3 of WIPP PA Division, 1991b).

Event-and-Process Parameters:

1. Intrusion torehole diameter

2. Intrusion borehole permeability

3. Recharge amplitude factor under climate change

4. Area of pressurized brine reservoir underlying WIPP site

Probability-Model Parameter:

5. Rate constant in Poisson Drilling Model

probability distribution is really an exercise in mathematical modeling and the product of that exercise may be validly called a probability model.

In the present and following sections, the random variable associated with an uncertain parameter, say $x$, will be denoted by its counterpart expressed in capital letters, say $X$. A probability distribution for a scalar random variable $X$ can be defined as a nonnegative, nondecreasing function $F(x)$ defined on the range of the parameter $x$ with the following properties:

$$
\begin{aligned}
& F(x)=\text { probability that } X \text { is less than or equal to } x ; \\
& F(x) \text { tends to zero as } x \text { tends towards its lower limit; }
\end{aligned}
$$

$F(x)$ tends to one (1) as $x$ tends towards its upper limit.

Such a function, called a cumulative distribution function (or CDF), measures the probability that the random variable $X$ will lie in an interval $[a, b], b \geq a$, of its range:

$$
\text { probability that }\{a \leq X \leq b\}=F(b)-F(a) \geq 0 \text {. }
$$

The CDF for an $\mathrm{N}$-dimensional vector of uncertain parameters, say $\mathbf{X}$, can be expressed in a similar fashion:

$$
F(x)=\text { probability that }\{X \leq x\}
$$

where $\{\mathbf{X} \leq \mathbf{X}\}$ means that

$$
X_{1} \leq x_{1}, x_{2} \leq x_{2}, X_{3} \leq x_{3}, \ldots, X_{N} \leq x_{N}
$$

Expression 3-4 is called a joint cumulative distribution function for the set of random variables (i.e., uncertain parameters) $X$. If $[\mathbf{a}, \mathbf{b}]$ denotes an $\mathrm{N}$-dimensional interval in parameter space, $D_{N}$, such that

$$
a_{1} \leq b_{1}, a_{2} \leq b_{2}, a_{3} \leq b_{3}, \ldots, a_{N} \leq b_{N} \text {, }
$$


probability that $\{\mathbf{X} \varepsilon[\mathbf{a}, \mathbf{b}]\}=\mathbf{F}(\mathbf{b})-\mathbf{F}(\mathbf{a})$.

As will be seen later, subsets of $D_{N}$ like the $N$-dimensional interval $[a, b]$ can be construed as quantitative representations of scenarios (Section 3.3.2).

The function $F(x)$ displayed in Expression 3-4 is intended to be a general expression of a probability model for uncertain parameters appearing in a total-system consequence model (Expression 3-1); the function need not be continuous in all elements of the parameter set, and its arguments, the uncertain parameters, could be partially correlated with one another. Correlations among a model's uncertain parameters may arise from the fact that there are natural correlations between measured quantities that determine their form (e.g., the local values of porosity and permeability of a real rock formation could be empirically correlated); or correlations of uncertain parameters may be implicit in the idealizations made in constructing the mathematical models in which they are used (e.g., local values of fracture porosity are automatically correlated with fracture permeability in some models of fluid flow through fractured rock).

Detecting natural correlations between measured quantities is usually difficult, particularly for measured quantities in the earth sciences, and it is often computationally inconvenient to incorporate known model-derived correlations among uncertain parameters of numerical models of the total system. Consequently, after eliminating those parameters that are known to be functionally dependent upon other problem variables, a model maker might simply assume that the remaining uncertain parameters of a total-system model are statistically independent (see, for instance, WIPP PA Division, 1991b, 1-12). This assumption permits the probability model for the total system to be expressed, in an approximate but computationally convenient form, as the product of the marginal distributions of the joint CDF (expression 3-4):

$$
F(x)=F_{1}\left(x_{1}\right) \cdot F_{2}\left(x_{2}\right) \cdot F_{3}\left(x_{3}\right) \cdot \ldots F_{N}\left(x_{N}\right)
$$

where $\mathrm{N}$ is now the number of uncertain parameters and the $F_{n}(0), n=1,2,3, \ldots, N$, are respectively the CDFs of the individual parameters, $x_{n}, n=1,2,3, \ldots, N$. The form expressed in Equation 3-5 is computationally convenient when certain efficient sampling methods (e.g., the Latin Hypercube Sampling method) are used to implement a stochastic simulation. Techniques for constructing CDFs of individual members of the parameter set are next briefly discussed.

Techniques for constructing CDFs for uncertain model parameters fall roughly into three classes: (1) direct use of data to construct an empirical CDF; (2) finding the analytic distribution that best fits available data (e.g., through use of the Kolmogorov-Smirnov test or the chi-squared test), and (3) Bayesian techniques that combine available data and expert judgment to form prior and posterior CDFs (Martz and Waller, 1982). Class 3 includes purely subjective procedures in which no posterior CDF is formed and assignment of a prior CDF relies entirely upon expert judgement. The amount of subjective judgment required by these techniques increases from class 1 to 3 ; the "best" technique to be used in a particular situation depends strongly upon the kinds and amounts of empirical data and subjective information that are available concerning the parameter.

Construction of empirical CDFs using available empirical data is appropriate if the parameter is intrinsically discrete (e.g., Figure 3-2) or when there are many data points for an intrinsically continuous quantity and that quantity can be related to the parameter by a deterministic, continuous function. The advantage of this technique lies in the fact that the empirical CDF is an unbiased estimator of the population's true CDF (Blom, 1989, p. 121); its disadvantages are that the empirical CDF may not capture probabilities of extreme values at the tails of the distribution, andfor continuous parameters and few data points-the empirical CDF may be too coarse to permit a representative, uniform set of sample values of the parameter to be drawn (as is necessary in implementing certain sampling methods in a stochastic simulation; see Section 3.2.4). The latter disadvantage may in part be offset by joining the vertices of the piecewise constant empirical CDF with straight lines (e.g., Figure 3-3), a procedure that is computationally simple and is justified by the Maximum Entropy Principle (Tierney, 1990).

The two standard techniques for testing whether a data set is consistent with a proposed analytic distribution (i.e., normal, lognormal, beta distributions, etc.) are the chi-squared test and the Kolmogorov-Smirnov test. The 
4

5

6

8

9

11

12

13

14

16

18

19

20

21

22

23

24

25

26

27

28

29

30

31

32

33

34

35

36

37

38

39

40

41

42.

43

44

45

46

47

48

49

50

51

52

53

54

55

56

57

58

59

60

61

62

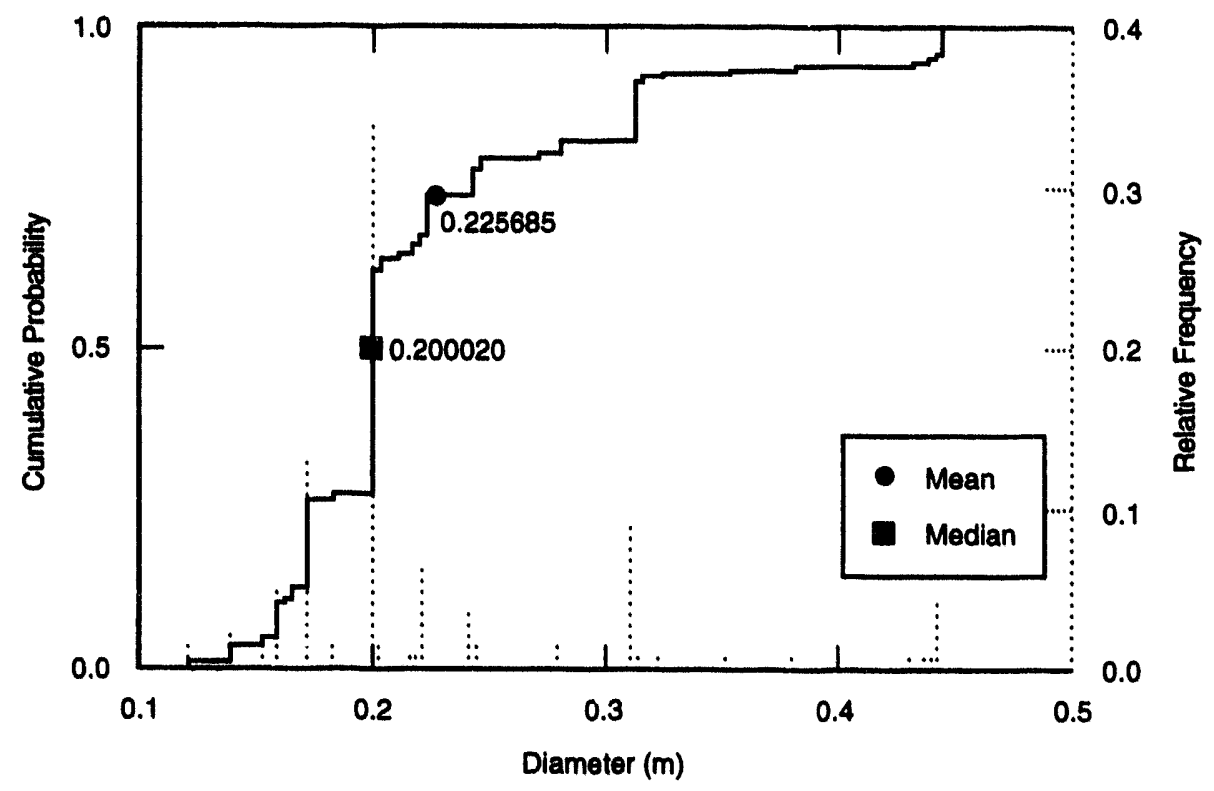

TA1-6342-1468-0

Figure 3-2. Distribution of historical drill bit diameter (Fig. 4.2-7, WIPP PA Division, 1991b). (Example of an intrinsically continuous parameter treated as a discrete quantity.)

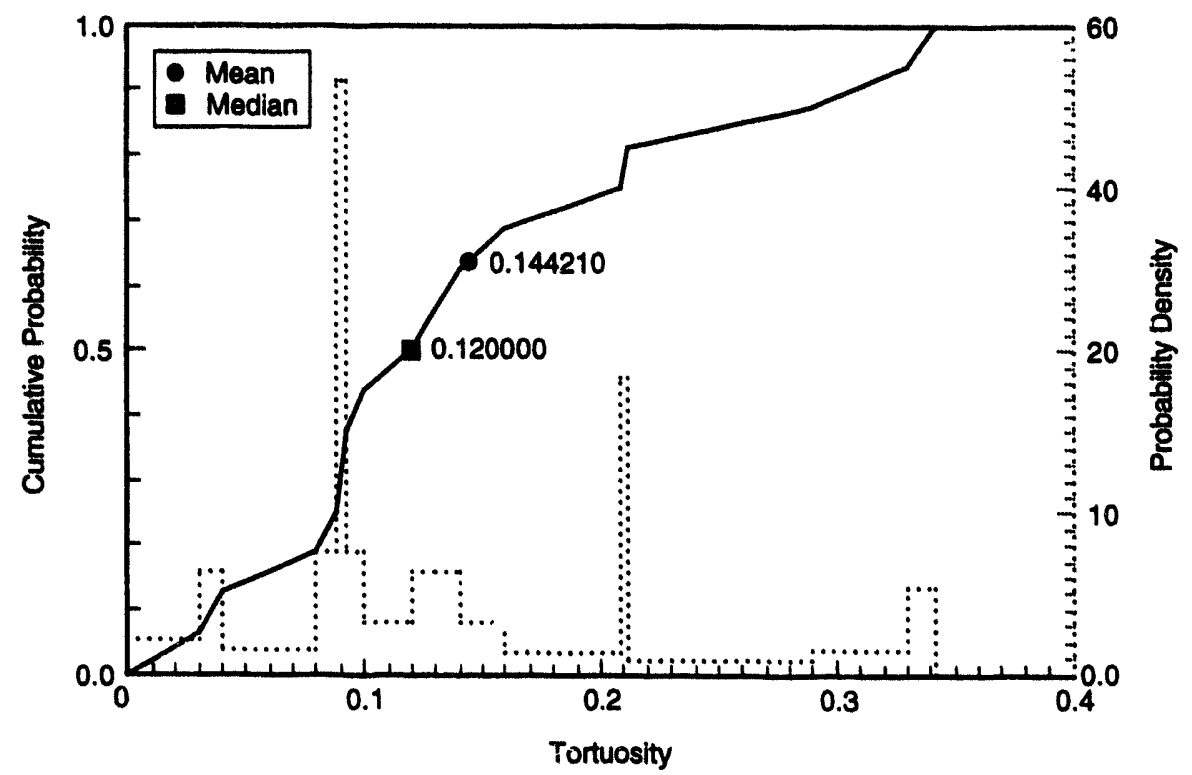

TRI-6342-1232-0

Figure 3-3. Measured distribution ( $p d f$ and cdf) for tortuosity of Culebra matrix (Fig. 2.6-i5, WIPP PA Division, 1991b). (Example of an intrinsically continuous parameter.) 
chi-squared test is best suited for intrinsically discrete quantities, whereas the Kolmogorov-Smirnov test is most appropriate for intrinsically continuous random variables (Morgan et al., 1990, p. 100). The use of either technique is seldom mentioned in published performance assessments, perhaps because of the sparse data sets so characteristic of measured quantities in the earth sciences or because these tests require lengthy calculations with the data before a distribution is chosen. In connection with PA for the WIPP Project, Pfeifle et al. (1992) have used the KolmogorovSmirnov test to find analytic distributions that are consistent with their measurements of certain constants (e.g., Figure 3-4) appearing in a mathematical model of salt creep used in the SANCHO code (see Table 3-1).

PA analysts in both WIPP and INEL programs have been compelled to adopt techniques from class 3 (Bayesian techniques; also see Section 3.2.2) to construct distributions for most of the uncertain parameters appearing in their consequence models (WIPP PA Division, 1991b). Because of the expense and time required to make and interpret measurements of earth-science quantities or poorly characterized waste-form quantities, the process of constructing CDFs for consequence model parameters has largely bypassed the classical techniques of Bayesian estimation (Martz and Waller, 1982) and his instead relied heavily on subjective judgments of scientific specialists. The elicitation of the judgment of specialists can be either an informal process involving only a few responsible investigators (Figure 3 5), or a formal process involving many experts and rigid controls (Bonano et al., 1990). Examples of distributions obtained by informal and formal elicitations are shown respectively in Figures 3-6 and 3-7.

\subsubsection{Stochastic Simulation}

Stochastic simulation is a class of computational methods that have been used to explore quantitative uncertainty in a model system's output variables, given measures of uncertainty in the model's input variables; in simple terms, stochastic simulation is a tool for the propagation of parametric uncertainty through complex mathematical models. The distinguishing characteristic of stochastic simulation is the opportunistic exploitation of the mathematical equivalence of taking the mean value of a set of numbers independently sampled from some distribution and calculating the expected value of a function of a random variable $(R V)$ by direct integration with respect to the $R V$ 's underlying probability density function. Those computational methods that emphasize drawing independent samples of the RV are called Monte Carlo methods (Hammersley and Handscomb, 1964; Ripley, 1987); the methods emphasizing direct integration have no special name but are centered around techniques of analytical or numerical evaluation of integrals. Most successful applications of stochastic simulation involve a mixture of the two points of view.

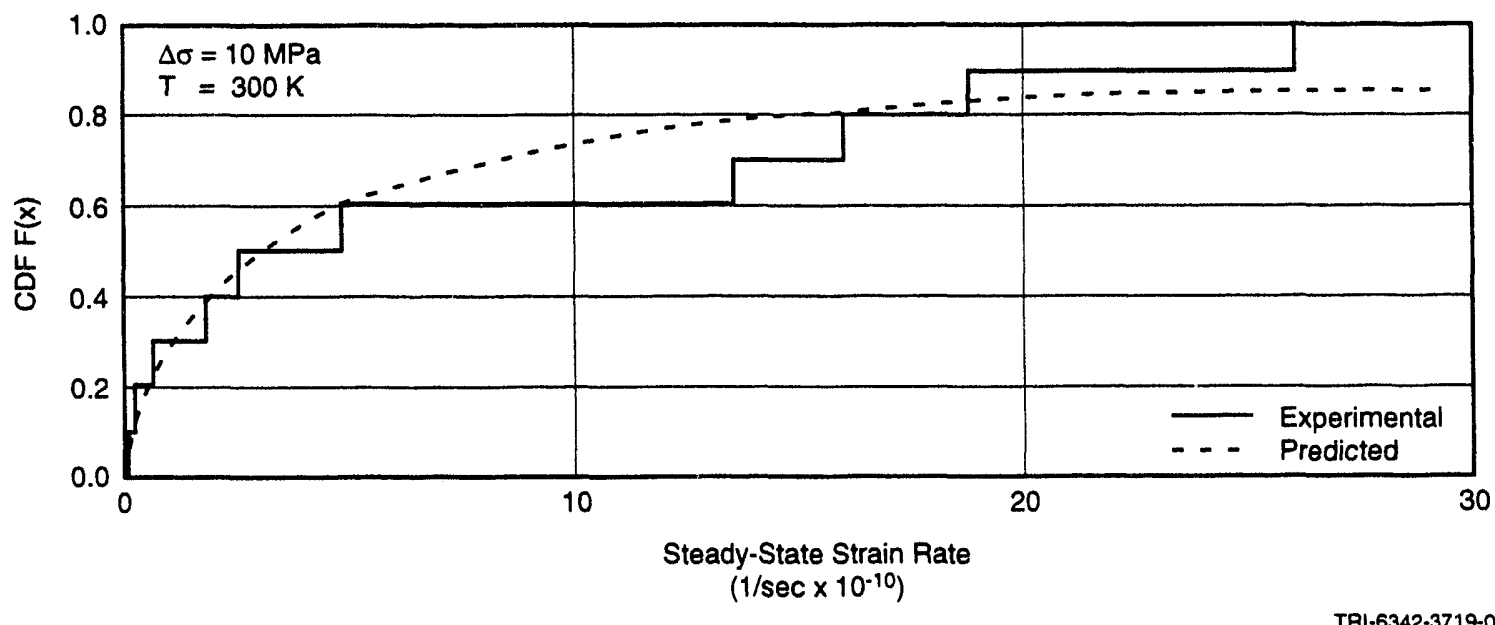

Figure 3-4. Typical comparison between predicted and experimental $C D F$ for the parameter, $\varepsilon_{s}$, at $T=300 \mathrm{~K}$ and $\Delta \sigma=10 \mathrm{MPa}$ (from Pfeifle et al., 1992). (Example of use of Kolmogorov-Smirnov Test to fit a distribution.) 
1

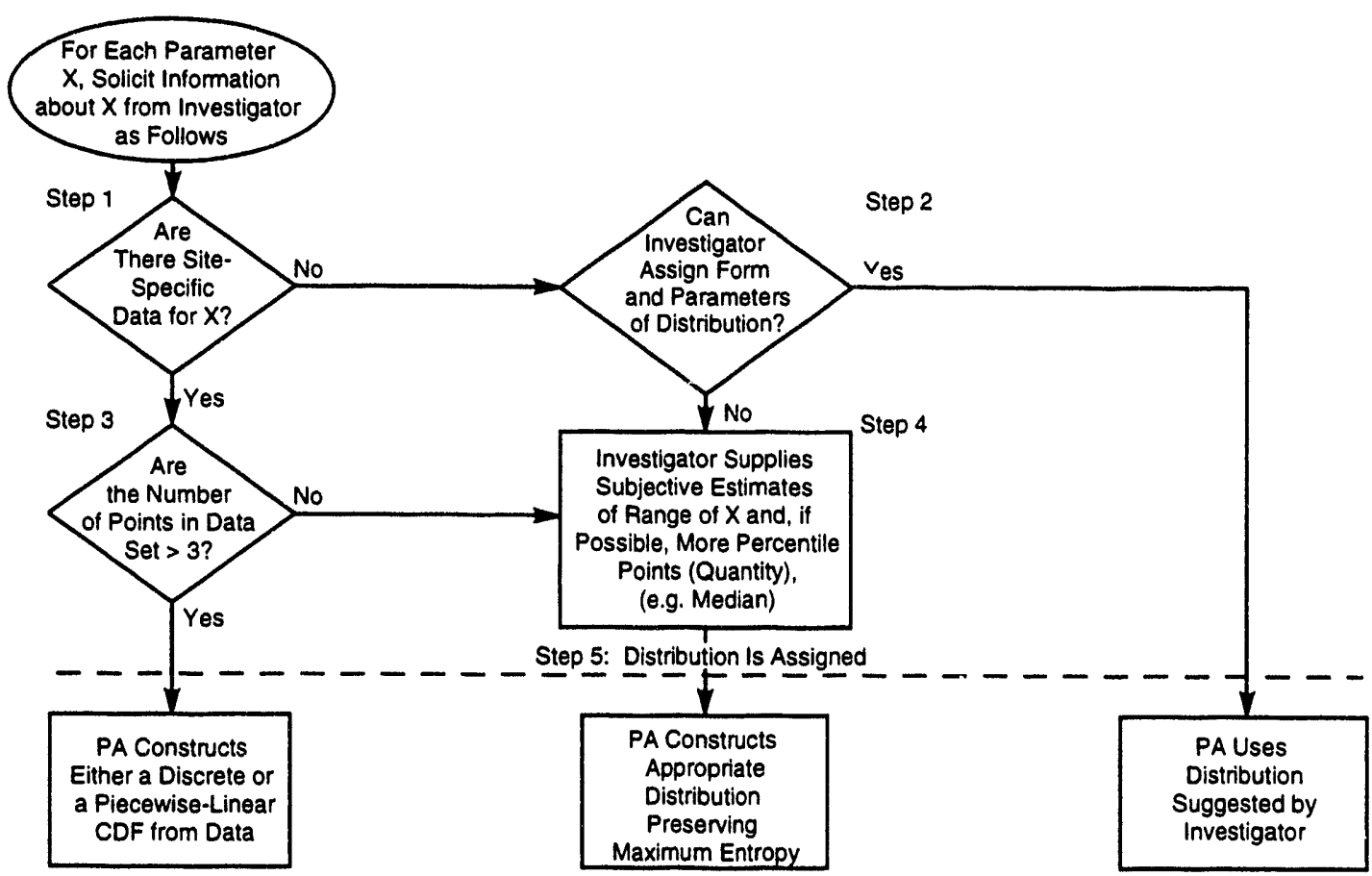

TRI-6342-634-1

Figure 3-5. An informal five-step procedure used to construct cumulative distribution functions (CDFs) for the 1990 WIPP performance simulations (Fig. E-1, Tierney, 1990). (See also Sandia WIPP Project, 1992, for example of formal procedure adopted for WIPP Project.)

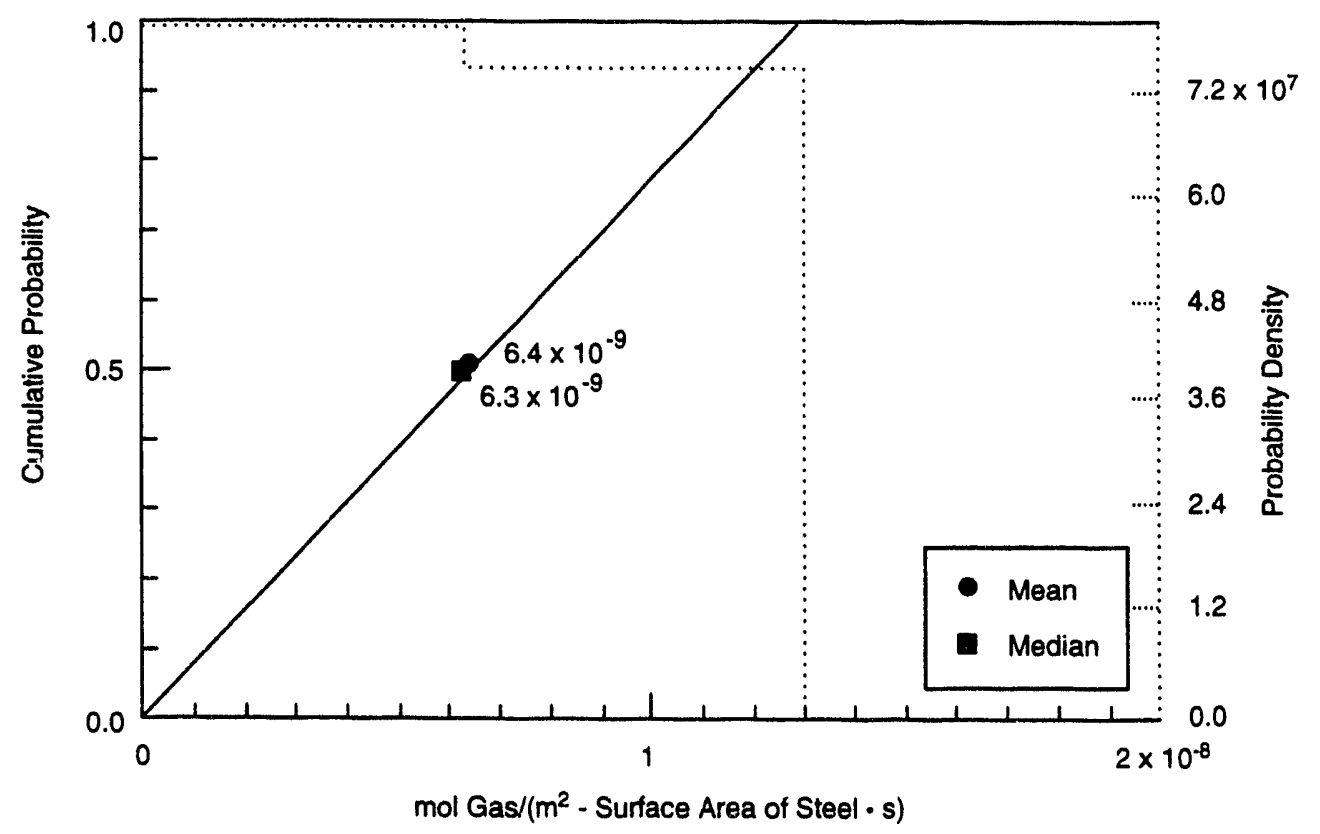

TRI-6342-1267-0

Figure 3-6. Constructed distribution for gas production rates from corrosion under inundated conditions (Fig. 3.311, WIPP PA Division, 1991b). (Distribution obtained from informal elicitation.) 


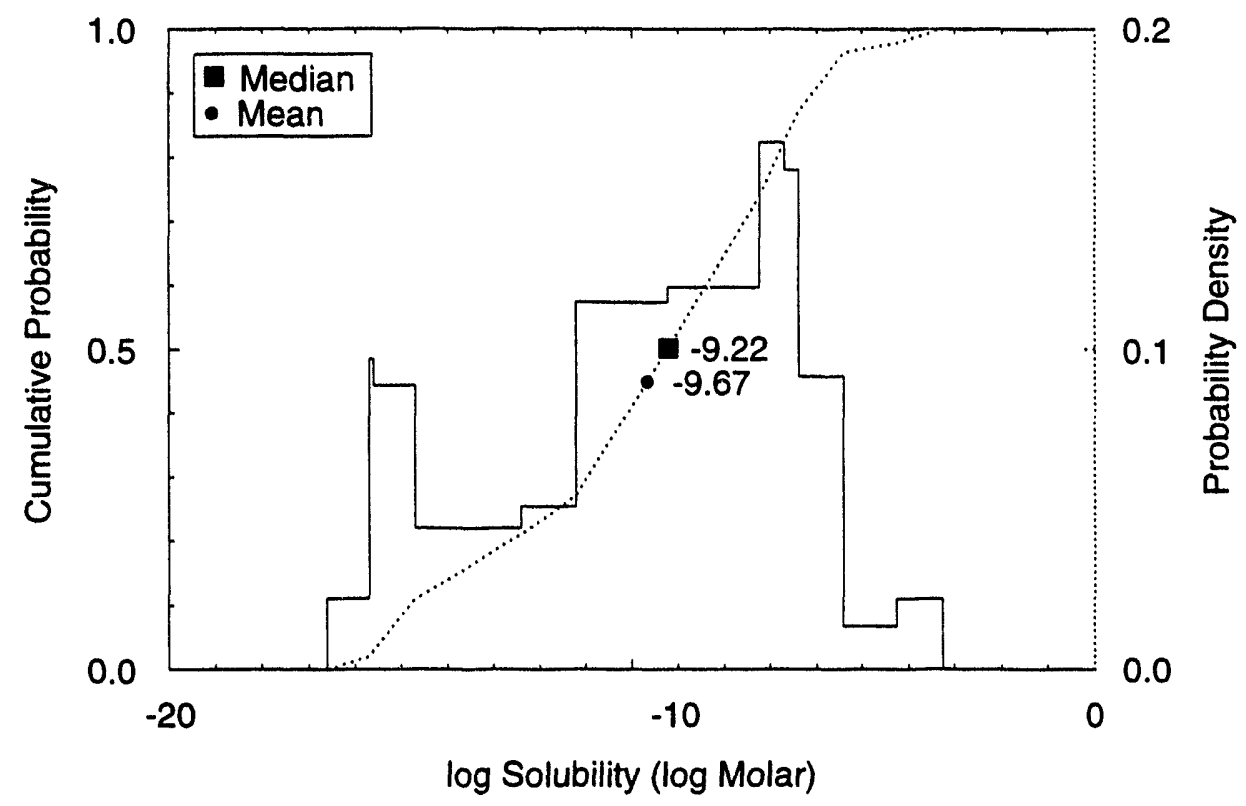

Figure 3-7. Constructed distribution for solubility of plutonium (Fig. 3.3-3(e), Sandia WIPP Project, 1992; see also Figure 9-1, this report). (Distribution obtained from formal elicitation.)

A stochastic simulation requires knowledge of the three objects described in the foregoing three sections of this overview: If uncertain system parameters are regarded as a vector-valued random variable $\mathbf{X}$ defined on the parameter space $D_{\mathrm{N}}$ (Section 3.2.2) and are distributed according to a total-system probability model $F(x)$ (Section 3.2.3), then stochastic simulation with the total-system consequence model $\mathbf{f}\left({ }^{\circ}\right)$ (Section 3.2.1) can give useful estimates of the statistical properties of model consequences, $\mathbf{c}=\mathbf{f}(\mathbf{x})$, or any system performance measure expressible as a function of model consequences, say $\mathbf{g}(\mathbf{c})$.

Note on System Performance Measures: Since model "consequences" have so far been given no special meaning in terms of particular types of quantities, there is no loss in generality in assuming that $\mathbf{c}$ [instead of $\mathbf{g}(\mathbf{c})$ ] represents whatever numerical measures of system performance are to be explored by the user of the model. Thus, in the remainder of this overview, $\mathbf{c}$ [or $\mathbf{f}(\mathbf{x})$ ] will mean either a vector of consequences or a vector whose elements are system performance measures. If there is only one performance measure, then that measure will be denoted by a scalar (not bold) " $c$ ". For example, the performance measure that is to be tested against EPA's standard (see Section 2.3.2) is of the form

$$
c=\sum_{i=1}^{l} \frac{M_{i}}{L_{i}}
$$

where the sequence of numbers $\left\{\mathrm{M}_{\mathrm{i}}\right\}$ are model consequences (in this example, the cumulative radioactivity released to the accessible environment in the form of the ith radionuclide during a 10,000-yr period); the numbers $\left\{\mathrm{L}_{\mathrm{i}}\right\}$ are normalization factors defined by the EPA; and $I$ is the number of radionuclides considered in forming releases to the environment.

Uncertainty in a performance measure $\mathrm{c}$ can be quantified in several ways: At the very least, estimates of the mean value and variance of the distribution of $\mathbf{C}$ are needed to roughly center the distribution and estimate its width. In many applications (e.g., compliance with EPA's standard; see Section 3.1), an estimate of the entire CDF for the performance measure is required. According to the mathematical theory of probability (see Feller, 1966), all moments 


\section{PA Methodology Overview}

and distributions of the function of a random variable, $\mathbf{C}=\mathbf{f}(\mathbf{X})$, may in principle be calculated by taking the expected value of certain functions of $\mathbf{C}$, i.e., by evaluating integrals of the form

$$
\mathcal{E}[h]=\int_{D_{N}} h[\mathbf{f}(\mathbf{x})] d F(\mathbf{x})
$$

where the integral is taken over the entire parameter space, $D_{N}$, and $d F(x)$ can be interpreted as a generalized derivative of the total-system probability model $\mathbf{F}(\mathbf{x})$. (Note: integrals of the kind shown in Expression 3-6 are called Lebesgue-Stieltjes integrals [Feller, 1966]; this generalized theory of integration becomes necessary when $F(x)$ is not differentiable in all of its arguments.) Possible choices for the functions $h($.$) will be described below along with prac-$ tical methods of numerically evaluating Lebesgue-Stieltjes integrals over multidimensional spaces.

Expression 3-6 defines the expectation operator, $\mathcal{E}[0]$, whose operational meaning should be obvious: take an arbitrary but well-behaved function $h(\mathbf{x})$, weight it with the infinitesimal probability $\mathrm{dF}(\mathbf{x})$, and integrate the product over all values of $x \in D_{N}$. The expected (or mean) value of the components of performance measure $\mathbf{C}$ can be calculated by taking

$$
h(\mathbf{x})=f_{i}(\mathbf{x}) \text {, for each } i=1,2,3, \ldots, N \text {. }
$$

The covariance matrix of the performance measure $\mathbf{C}$ is found by taking

$$
\begin{gathered}
h(\mathbf{x})=\left[f_{i}(\mathbf{x})-\mathcal{E}_{i}(h)\right]\left[f_{j}(\mathbf{x})-\mathcal{E}_{j}(h)\right], \\
\text { for all } i, j=1,2,3, \ldots, N,
\end{gathered}
$$

where the expressions $\mathcal{F}_{i}(h)$ and $\mathcal{F}_{j}(h)$ stand for the expected (or mean) values of the ith or jth component of $f$.

The full joint $\mathrm{CDF}$ for the performance measure $\mathbf{C}$ may be calculated by taking

$$
h(\mathbf{x})=\prod_{i=1}^{N} u\left[f_{i}(\mathbf{x})-c_{i}\right]
$$

where $u(0)$ stands for the unit step function, a function having the properties $u(z)=1$ if $z \geq 0$ and $u(z)=0$ if $z \prec 0$. In this case, application of the expectation operator to $h(\mathbf{x})$ gives $G(\mathbf{c})$, the probability that

$$
\mathrm{C}_{1} \leq \mathrm{c}_{1}, \mathrm{C}_{2} \leq \mathrm{c}_{2}, \mathrm{C}_{3} \leq \mathrm{c}_{3}, \ldots, \mathrm{C}_{\mathrm{N}} \leq \mathrm{c}_{\mathrm{N}},
$$

where $c_{1}, c_{2}, c_{3}, \ldots, c_{N}$ are now independent variables; i.e., they can take arbitrary values within their natural ranges. As a last example of the expectation-operator formalism, the full expression for the CDF of a scalar performance measure $\mathrm{C}$ is displayed below.

$$
G(c)=\int_{D_{N}} u[c-f(\mathbf{x})] d F(\mathbf{x})
$$

In the context of the EPA standard, if $f(x)$ is the normalized cumulative release of radioactivity in a $10,000-y r$ period following closure of a waste-disposal site that is predicted by the total-consequence model when given a parameter set $\mathbf{x}$, then the "overall probability distribution" called for by the Standard is precisely the complementary cumulative distribution function (CCDF), $1-\mathrm{G}(\mathrm{c})$, with $0 \leq \mathrm{c} \prec \infty$.

Practical Stochastic Simulation. In stochastic simulation, the problem of computing the multi-dimensional integrals of Expression 3-6 is second in importance only to the problems of creating realistic and computationally efficient consequence and probability models. It is seldom possible to evaluate all integrals appearing in Expression 3-6 by analytical means or by direct numerical integration: the dimension of integration is usually large, possibly involving tens or hundreds of parameters, and one or both of the integrands, $h[f(x)]$ and dF(x), may be communicable only in terms of complex numerical algorithms or computer code. Monte Carlo methods are perhaps the only universally applicable techniques for evaluating these multi-dimensional integrals. The following is a brief outline of the simplest of the Monte Carlo methods, the "random-sampling" method. 
To calculate an estimate of $\mathcal{E}[\mathrm{h}]$ (Expression 3-6) with the random-sampling method, one begins by drawing $M \gg 1$ sample values of the parameter-set vector, say $\mathbf{X}_{1}, \mathbf{X}_{2}, \mathbf{X}_{3}, \ldots, \mathbf{X}_{\mathrm{M}}$, from the total-system probability model $F(\mathbf{x})$. (Chapter 11 of Ross [1985] is a good introduction to techniques for "drawing" independent samples of a random variable from its associated CDF.) Next, one computes the quantities $h\left[f\left(\mathbf{X}_{m}\right)\right], 1 \leq m \leq M$, and forms their arithmetic mean:

$$
<h>=\frac{1}{M} \sum_{m=1}^{M} h\left[\mathbf{f}\left(\mathbf{X}_{m}\right)\right]
$$

The quantity $\langle\mathrm{h}\rangle$ is called an estimator of the expected value of $\mathrm{h}[\mathbf{f}(\mathbf{x})]$; by the equivalence mentioned earlier, it is also an estimate of the integral shown in Expression 3-6. Depending upon the size of the sample, $M$, there will be an error (i.e., the difference between the exact value of Expression 3-6 and the estimated value given by Expression 3-8) associated with this procedure. Brief discussions of the error term can be found in Ross (1985) and in Tierney (1991, p. 3-11); in general, the error associated with the random-sampling method is inversely proportional to the square root of the sample size $M$.

Note again that by an appropriate choice of the function h[.] in Expression 3-9, one may calculate estimators of mean value, variance, and of the $C D F$ of the performance measure $C$. In particular, the estimator of the CCDF of the EPA's performance measure (expression 3-8) takes the form

$$
<1-G(c)>=\frac{1}{M} \sum_{m=1}^{M} u\left[f\left(\mathbf{X}_{m}\right)-c\right]
$$

This estimator takes the form of an empirical CCDF (see Section 3.2.3), i.e., the sum of $M$ unit step functions, and has the appearance of a series of descending stair steps (Figure 3-8). As the sample size $M$ becomes large, the empirical CCDF will approach the smooth curve representing the true CCDF for the population of performance measures that is implicit in the total-system model under consideration.

The major drawback associated with the random-sampling method is that a large number of samples of the parameter-set vector must be taken to keep the sampling error within acceptable bounds. Random sampling may be impracticable when used with consequence models implemented by large and complex computer codes that require substantial time and storage to complete a calculation of a single sample consequence. In these situations, computertime requirements may be reduced by employing other variants of Monte Carlo methods such as stratified sampling (Kalos and Whitlock, 1986) or the related Latin hypercube sampling (LHS) (Iman and Conover, 1982); use of these variant methods will, for the same sample size $M$, often reduce variance of the estimates of the performance measures below the variance dictated by the random-sampling method. Unfortunately, making a priori estimates of the error associated with use of the LHS estimator in a non-linear model is difficult, and one usually has to estimate the sampling error after the fact of the calculation. Iman and Helton (1985, p. 2-5) have conducted numerical experiments with LHS on a variety of models; their experience shows that tolerable levels of sampling error are achieved when $M$ is chosen to be greater than $(4 / 3) \mathrm{N}$, where $\mathrm{N}$ is the number of uncertain parameters to be sampled.

An Example from the WIPP PA. The WIPP PA Department at Sandia National Laboratories has employed a combination of LHS and direct integration in their preliminary calculations of conditional CCDFs for the normalized cumulative release of radioactivity from the WIPP (this is the performance measure expressed above in Equation 3-8). A full description of the theoretical approach to these calculations is given in Helton (1993) and the particular computational approximations used in these calculations are described in Chapter 3 of WIPP PA Division (1991a); typical results from these simulation exercises are shown on Figure 3-9. A technical but brief discussion of the WIPP PA Department's approach to stochastic simulation is also supplied in the last section (3.3) of this overview.

The reader will notice that Figure 3-9 displays a family of CCDFs. Each curve in this family is the result of a conditional simulation in which integration is performed (approximately and numerically in this example) over a small subset of the uncertain parameters, while the remainder of elements of the parameter-set vector are held fixed at values chosen by the LHS sampling scheme. Thus there is one conditional CCDF for each of the LHS sample vectors, 


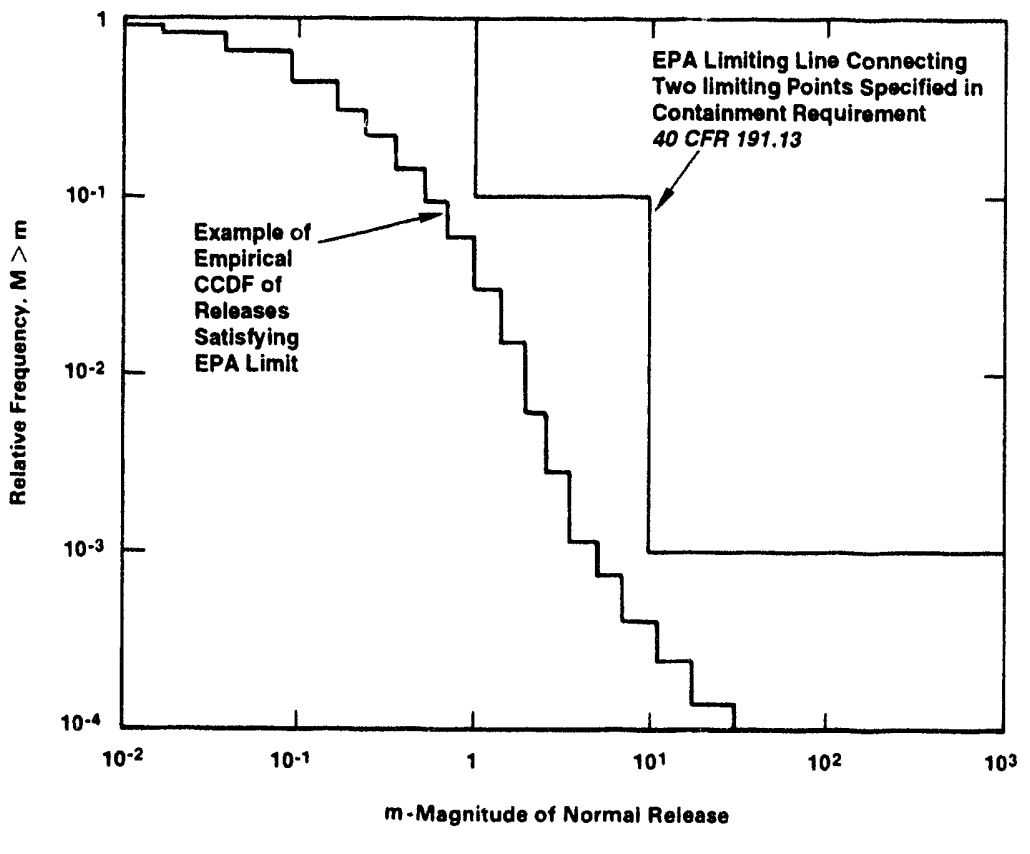

Figure 3-8. An example of an empirical CCDF (modified from Fig. 3.6 in Rechard, 1989).

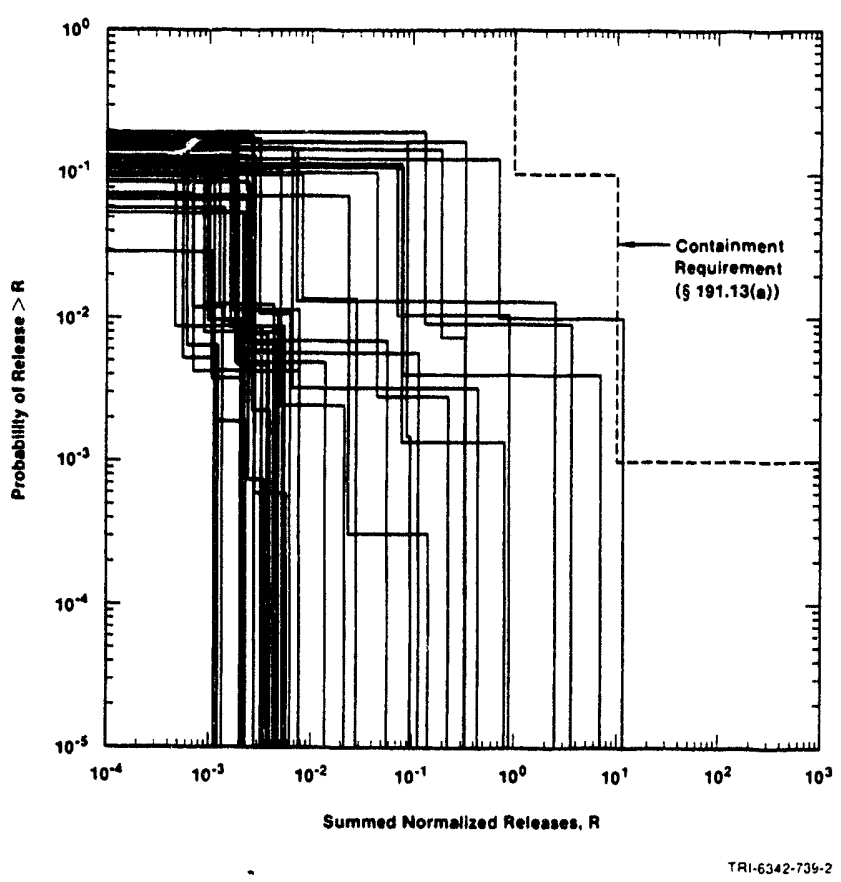

Figure 3-9. Example family of CCDFs generated by Latin hypercube sampling for comparison with the EPA release limits (Fig. 13 in Helton, 1993). 
say $\mathbf{X}_{m}, m=1,2,3, \ldots, M$. These $M$ curves may be combined and analyzed with the methods of ordinary statistics: the arithmetic average of the $\mathrm{M}$ curves is an estimator of the true CCDF for the population of performance measures implicit in the PA Department's consequence and probability models of the WIPP; and various "percentile" CCDFs may be formed by simple numerical interpolation of the $M$ curves (Figure 3-10, taken from Helton, 1993).

Caveats for Interpretations of Figures 3-9 and 3-10. The reader may also have noticed that one member of the family of CCDFs displayed in Figure 3-9 exceeds the limits of the Containment Requirements. This observation may lead to the conclusion that the WIPP system, at least as it is presently being modeled, is not likely to comply with the Containment Requirements of 40 CFR 191 (see Chapter 2). In reply to such a conclusion, we can only remark that one should be cautious about making regulatory interpretations of conditional CCDFs such as those displayed in Figure 3-9. There are at least two good reasons for exercising caution: First, conditional distributions (CDFs or $\mathrm{CCDFs}$ ) are themselves chance quantities (or random variables) to which probabilities should be attached before any interpretations of their significance are made. Realizations of conditional distributions, such as those displayed in Figure 3-9, can be viewed as "samples" independently drawn from an unknown "distribution of distributions" whose structure can be investigated using standard techniques of statistics; one instance of the use of standard techniques is the plotting of various percentiles and the calculation of a mean curve as shown in Figure 3-10. Another way of analyzing the significance of the set of curves in Figure 3-9 would use repeated sampling to estimate the frequency that the sample curves exceed the limits of the Containment Requirements; for the sample shown in Figure 3-9, the estimated frequency of exceeding the limits is obviously $1 / 40(0.025)$.

Second, and most important, a conditional distribution of the kind whose samples or realizations are exemplified in Figure 3-9 cannot be a unique and statistically stable measure of performance for the total-system model. To see why this is so, recall that conditional distributions are obtained by holding fixed certain members of the set of uncertain parameters while computing the expectation of the performance measure (Eq. 3-6) with respect to the remaining members of the uncertain-parameters set. The choice of the parameters to be held fixed in computing conditional distributions is arbitrary and usually depends upon the purposes of the analysis. In the example of Figure 3-9, the expectation integral was analytically taken over the small set of parameters that specified the number of inadvertent

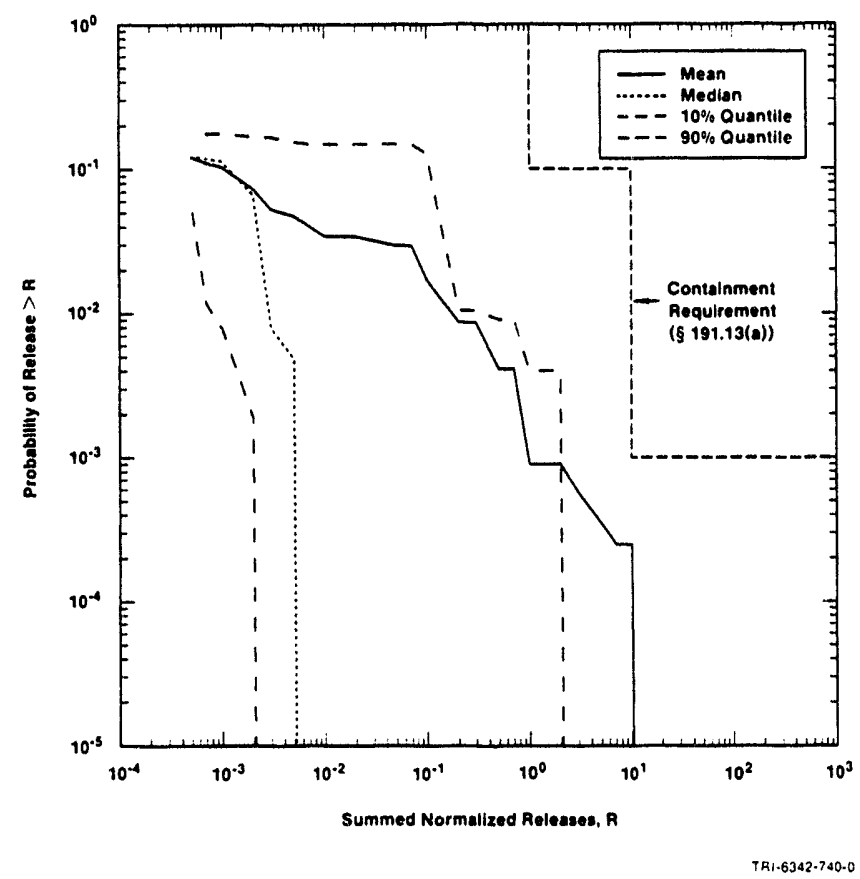

Figure 3-10. Mean and percentile curves for the family of CCDFs shown in Figure 3-9 (Fig. 14 in Helton, 1993). 
intrusions into the WIPP site during five 2000-yr time intervals; the remaining uncertain parameters, called "subjectively uncertain" parameters by Helton (1993, p. 37), were consecutively assigned the 40 vector values sampled by the LHS scheme. This choice of the set of fixed parameters and the set of parameters over which the expectation integral was taken was dictated only by considerations of computational economy and convenience; other considerations might have dictated a different choice that would lead to a family of realizations that differ considerably from those shown in Figure 3-9.

The cautions offered above may suggest an answer to a question that frequently arises in connection with families of empirical CCDFs such as those exemplified in Figure 3-9 and statistically ordered or averaged in Figure 3-10: Which curve should be used to assess compliance with the Containment Requirements? The mean curve, the median curve, or the 90th percentile? We believe the answer is the mean curve, for the mean curve is the result of taking the expectation of the performance measure with respect to all uncertain parameters and is therefore independent of arbitrary choices of the conditioning parameters (it is also an unbiased estimator of the unknown, underlying CCDF for the model system). Of course, uncertainty in the estimated mean curve must be quantified, but this can be done using standard statistical techniques as indicated above.

\subsection{Scenarios and Conditional Simulation}

This section is a treatment of several topics of special interest to performance assessment of geologic waste-disposal systems. The many definitions of "scenario" are sampled and briefly discussed in Sections 3.3.1 and 3.3.2 and a practically usable definition of "scenario" is proposed in the latter section. Section 3.3 .3 is a development of the idea of expanding a performance assessment in a series of "scenarios", an idea which is shown to be equivalent to expansion in a series of conditional simulations. Section 3.3.4 is a reconciliation of the formalism of stochastic simulation presented in Section 3.2 with the Kaplan-Garrick representation of risk (Kaplan and Garrick, 1981) that forms the basis of performance assessments of the WIPP system.

\subsubsection{Definitions of 'Scenario'}

The wide range of meaning attached by PA workers to the noun "scenario" has rendered the word nearly useless for the purpose of technical communication. Six different definitions of "scenario" are mentioned below; even among these few definitions there are instances in which the authors' intentions are not entirely clear.

1. Scenario selection and scenario screening name two steps in the mainly heuristic act of identifying important events, processes and features that can cause or influence significant releases of radioactivity to the accessible environment from a waste-disposal system (Cranwell et al., 1990; Hunter, 1989; Guzowski, 1990).

2. A summary scenario (WIPP PA Division, 1991a, p. 1-3) is a member of the class of all subsets (including the "empty" set) of the set of all distinct disruptive events, uncertain features, and alternative conceptual models that were identified by scenario selection and screening (Tierney, 1991, pp. 3-22 to 3-26). The empty set is often called the base-case scenario in PA work.

3. A scenario is "a set of similar occurrences" (Helton, 1993, p. 26), i.e., a set of things that can happen and, if they happen, have in some sense similar outcomes or consequences.

4. A computational scenario (WIPP PA Division, 1991a, p. 2-7) is a member of a class of subsets of parameter space obtained by dividing the parameter space of a summary scenario into units that are computationally convenient for partial evaluation of expectation integrals of the kind typified in Equation 3-6.

1

5. A scenario is a point in parameter space; $x \in D_{N}$ (Morgan et al., 1990, p. 174; Tierney, 1991).

6. A scenario class is a subset of parameter space (Tierney, 1991, Appendix A). 
Regarding definition 1: In Chapter 7, Scenario Development, the terms scenario selection and scenario screening are used as synonyms for two necessary steps in the construction of any model $\mathrm{f}$ a natural or engineered system: (1) deciding upon the physical, chemical and anthropogenic phenomena that might play a significant role in determining the performance of the system; and (2) deciding upon which of the phenomena identified in Step 1 must be incorporated in a mathematical model of the system if that model is to predict all consequences of interest to investigators. It appears that Steps 1 and 2 correspond to the steps of "translation from real-world data" and "induction from experience" that are at the heart of the cyclical process of mathematical modeling (Figure 3-11). The scenario-selection methodology proposed by Cranwell et al. (1990) is no more than a set of guidelines that a PA analyst may profitably follow in making the necessary inductions and translations from real-world data and experiences.

Regarding definition 3: Helton's intentions in posing this definition of scenario (Helton, 1993, p. 26) are not entirely clear, but from the context in which the word is used in his paper, one may infer that he is trying to connect certain computational scenarios (definition 4) with the first member of Kaplan and Garrick's representation of risk as a triplet: $\left\langle s_{i}, p_{i}, x_{i}\right\rangle$, where "s $s_{i}$ is a scenario identification or description; $p_{i}$ is the probability of that scenario; and $x_{i}$ is the consequence or evaluation measure of that scenario, i.e., the measure of damage" (Kaplan and Garrick, 1981, p. 13). An interpretation of the Kaplan-Garrick triplet in terms of subsets of, and probability measures on, the totalsystem parameter space is supplied in Section 3.3.4.

The reader may convince herself that the remaining definitions of "scenario"- $-2,4,5$, and 6 -have a practical meaning only after a mathematical model has been constructed and the analyst is ready to make numerical predictions with the model (the "prediction-deduction" step in Figure 3-11). At this point in the modeling cycle, a (perhaps preliminary) total-system consequence model (Section 3.2.1) and its associated parameter space (Section 3.2.2) exist and their concrete features can be displayed, examined and analyzed. The need for precise communication of model features dictates that, if used at all, the word "scenario" should at this point denote some common property of those features that is also useful as an analytical or computational tool. Since definitions 5 and 6 are already clear definitions in the sense of denoting concrete model features, only definitions 2 and 4 require further discussion and analysis. In the next section, it is proposed that the terms summary scenario and computational scenario be used to denote certain subsets of the model's parameter space.

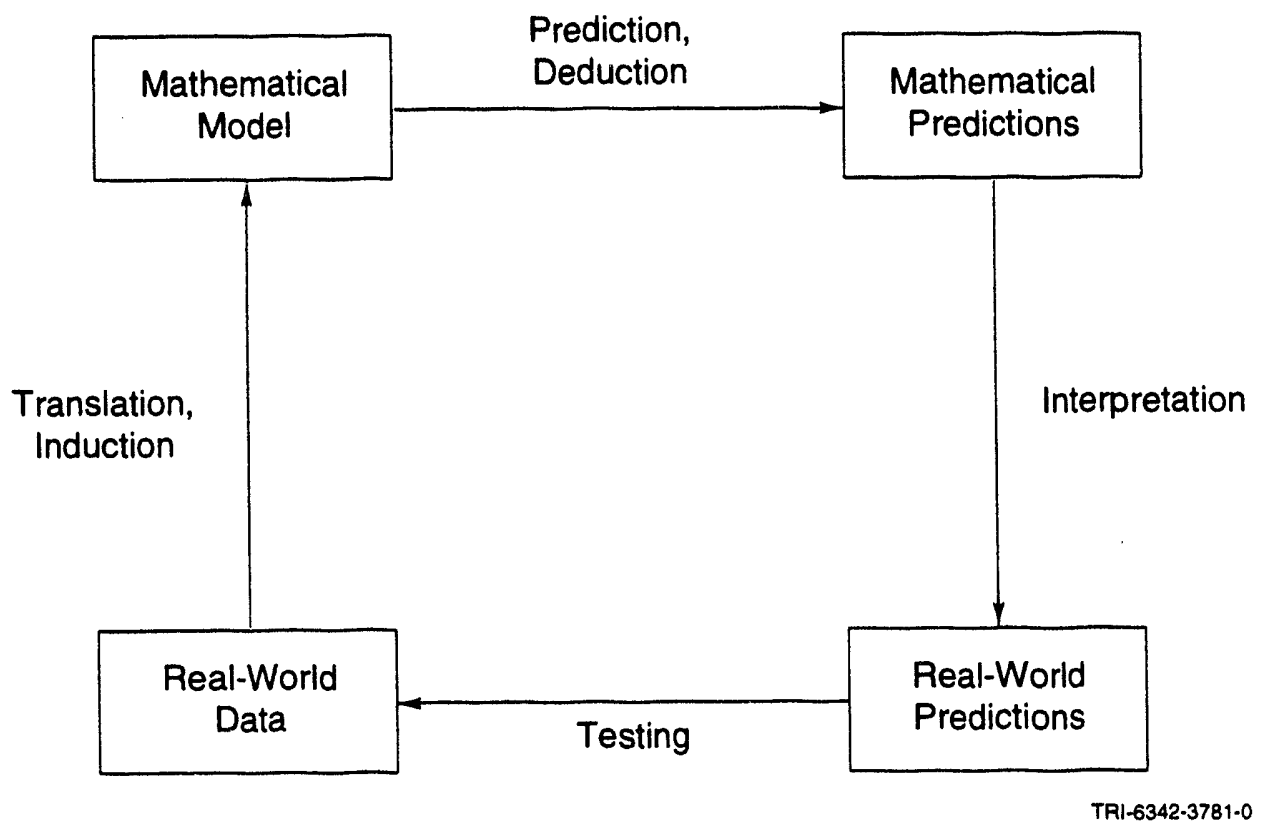

Figure 3-11. The cyclical nature of mathematical modeling (Roberts, 1976; Figure 1-1). 


\subsubsection{Scenarios as Subsets of Parameter Space}

The dictionary definition of "scenario" judged to be nearest to PA analysts' definitions is "an outline of a hypothetical chain of events" (American Heritage, 1975). Note that such an outline of events could take precise and economic narrative form as a series of simple statements-e.g., "Xavier is blue., Mary is less than three., John is ill."linked by the conjunction "and". In each simple statement, a subject (e.g., Xavier, Mary, John) is assigned a quality or property (e.g., respectively, blue, less than three, ill). Note also that a family of outlines of events can be generated by allowing one or more of the qualities/properties appearing in the simple statements to be free variables, e.g.,

$$
\begin{aligned}
& \text { "Xavier is } \mathrm{m} \text { ", where } \mathrm{m} \text { is degree of pleasure; } \\
& \text { "Mary is less than a", where a is age in years; } \\
& \text { "John is h", where } \mathrm{h} \text { is degree of health. }
\end{aligned}
$$

Simplistic reflections of this kind concerning the commonly accepted definition of scenario lead to the idea of defining the "scenarios" of performance assessment in a similar but slightly stronger way:

In PA work, a scenario is a compound statement, $\mathrm{A}(\mathbf{x})$, that is built by linking with the conjunction "and" any series of simple statements about values taken by elements of the parameter vector, $\mathbf{x}$, and/or the vector of consequences, $\mathbf{f}(\mathbf{x})$.

It is easily seen that this definition establishes a correspondence between properly phrased, qualitative statements about the behavior of the model system and certain sets of points in the parameter space $\mathrm{D}_{\mathrm{N}}$. For any given scenario $A(\mathbf{x})$, there is a (possibly empty) subset of $D_{N}$ defined by

$$
S=\left\{\mathbf{x}: \mathbf{x} \in D_{N} \text { and } A(x) \text { is true }\right\} \text {. }
$$

The expression above reads as follows: " $S$ is the set of all $\mathbf{x}$ contained in the parameter space for which the statement $\mathrm{A}(\mathbf{x})$ is true". The complement of $\mathrm{S}$,

$$
S^{c}=\left\{x: x \in D_{N} \text { and } A(x) \text { is false }\right\},
$$

is also a subset of $D_{N}$; it corresponds to the statement,

$$
A^{\prime}(\mathbf{x})=\text { the negation of } A(x) \text {. }
$$

Thus, subsets of parameter space defined in this way, i.e., by the truth value of a statement, may also be called "scenarios" without confusion.

In addition to establishing an operationally useful association between most qualitative assertions about the behavior of the system (i.e., "scenarios" in the sense of Hunter, 1989 and Guzowski, 1990) and subsets of the parameter space, this definition of scenario solves the long-standing problem of assigning probabilities to scenarios: The probability of a scenario, $A(\mathbf{x})$, is simply the probability of its associated subset $S$, a quantity that can be calculated by means of the total system's probability model (Section 3.2.3). A discussion of methods for calculating scenario probabilities is deferred to Section 3.3.3. At this point, it is appropriate to give some examples of different kinds of scenarios and their associated subsets. The first example is a simple but general one that demonstrates some of the advantages to be gained by defining scenarios in terms of subsets of the parameter space.

Example 1. Envelope of acceptable system performance. Suppose that $f(x)$ is a scalar performance measure for a total system and that the performance of the system is judged to be acceptable if that performance measure falls within a prescribed interval $(a, b)$ of the real line. The scenario of acceptable system performance, expressed as a subset of parameter space, is obviously 


$$
S(\text { acceptable })=\left\{\mathbf{x}: \mathbf{x} \in \mathrm{D}_{\mathrm{N}}, \mathrm{a}<\mathrm{f}(\mathbf{x})<\mathrm{b}\right\} .
$$

Normally, the system's analysts are not interested in the scenario of acceptable performance per se, but in the likelihood that the particular system (here specified by the function $\mathrm{f}[\cdot]$ ) will perform acceptably. As shown in the following section, the likelihood (probability) that the system performs acceptably is identical to the probability measure attached to the set $\mathrm{S}$ (acceptable); the latter can be estimated (perhaps at considerable computational expense) by a Monte Carlo simulation with the total system model that calculates $f(x)$ and the total-system probability model (Section 3.2.3) that calculates the joint $C D F, F(x)$, for the vector of uncertain parameters $\mathbf{x}$.

Example 2. Summary Scenarios as subsets of parameter space can be exemplified using a hypothetical situation in which R1 and R2 designate two different kinds of chance events that could disturb the operation of the waste-disposal system (e.g., exploratory drilling, earthquakes), and T1, T2, T3 designate three different kinds of uncertain features of the waste-disposal system (e.g., an undetected fault zone, an undetected brine pocket, and an undetected universal flaw in waste containers). Suppose that these five agents are the only significant chance events and uncertain features that were identified during the qualitative scenario screening process and that mathematical submodels of the effects of each agent upon system performance measures have been constructed and included in the total-system consequence model. It is also assumed that certain processes (e.g., groundwater flow) are operating everywhere and at all times, though at imprecisely known levels.

The five agents may be listed as elements of a set, viz., $\{R 1, R 2, T 1, T 2, T 3\}$, whose subsets, e.g., $\{R 2, T 3\},\{R 1\}$, etc., have the following meaning: the elements that appear in a subset occur at least once (in the case of an event) or are present (in the case of a feature) during the period of performance of the waste-disposal system. Since none of the five disturbing agents necessarily occurs or is necessarily present during the period of performance, an "empty" subset-here denoted simply by \{\} -must be introduced to allow for the base case of "undisturbed" or "nominal" performance. The set $\{\mathrm{R} 1, \mathrm{R} 2, \mathrm{~T} 1, \mathrm{~T} 2, \mathrm{~T} 3\}$ has exactly 32 subsets including the empty subset (i.e., $2^{\mathrm{N}}$ subsets, where $\mathrm{N}=5$ in this example); these subsets can be enumerated and classified by means of a logic diagram (Figure 3-12). The 31 subsets plus the base case are called "summary scenarios" in WIPP PA practice (WIPP PA Division, 1991a).

A practical meaning may be associated with each summary scenario by a statement expressing the presence or absence of each event or feature in the subset: for example, the subset $\{\mathrm{R} 1, \mathrm{~T} 2, \mathrm{~T} 3\}$ can be interpreted by the statement

"R1 occurs at least once during the period of performance and

$\mathrm{T} 2$ is present and

$\mathrm{T} 3$ is present and $\mathrm{R} 2$ does not occur and $\mathrm{T} 1$ is not present."

It is next shown that such compound statements place unique numerical constraints on uncertain parameters of the total system: If $\mathrm{N} 1, \mathrm{~N} 2$ are parameters representing respectively the number of times events $\mathrm{R} 1, \mathrm{R} 2$ occur in the period of performance and I1, I2, I3 are binary parameters representing respectively the presence $(I=1)$ or absence (I $=0$ ) of features $\mathrm{T} 1, \mathrm{~T} 2, \mathrm{~T} 3$, then the statement above can be recast in the symbolic form

$$
\mathrm{B}(\mathbf{x})=(\mathrm{N} 1>0, \mathrm{~N} 2=0, \mathrm{I} 1=0, \mathrm{I} 2=1, \mathrm{I} 3=1)
$$

where it is understood that all elements of the vector $x$ but elements $(\mathrm{N} 1, \mathrm{~N} 2, \mathrm{I1}, \mathrm{I2}, \mathrm{I3})$ can take arbitrary values within their allowable ranges. The set defined by

$$
S=\left\{\mathbf{x}: \mathbf{x} \in \mathrm{D}_{\mathrm{N}} \text { and } \mathrm{B}(\mathbf{x}) \text { is true }\right\}
$$

is a unique subset of the parameter space $D_{N}$. Furthermore, this set is but one of 32 subsets of $D_{N}$ that can be built from the 32 summary scenarios (Figure 3-12) using the procedure described above (i.e., translation of presence or absence of a feature or event into a statement about problem parameters). The reader may convince himself that these 
3. PA Methodology Overview

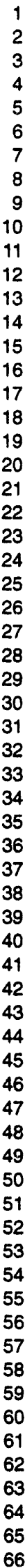

"Special Subset" of Features, Events, and Processes that the Analysts Wish to Focus Attention

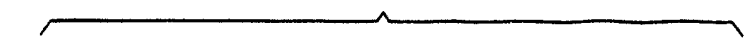

Two Hypothetical

Release Events

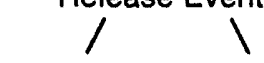

R2
Distinct "Future" (for 10,000 yr)

Examined by Consequence Modeling
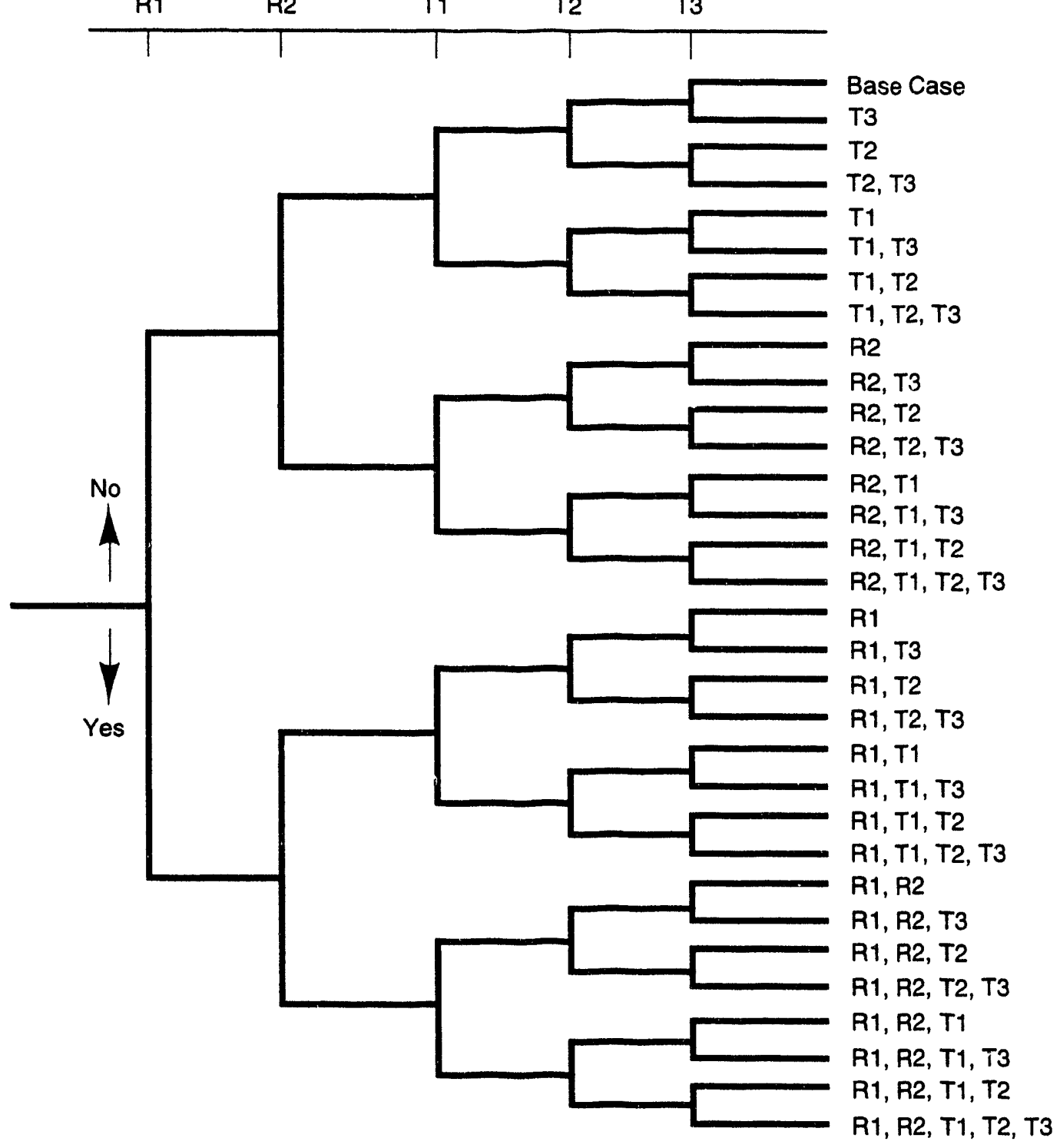

TRI-6342-222-6

Figure 3-12. Logic diagram for the construction of summary scenarios (adapted from Guzowski, 1990). 
32 subsets exhaust the parameter space in the sense that their union equals $D_{N}$; furth?rmore, the 32 subsets are mutually disjoint in the sense that no two of them share a common element $\mathbf{x}$. As will be seen in the next section (3.3.3), the fact that metrors of the class of subsets representing the class of summary scenarios are both exhaustive and mutually disjoint permits a convenient expansion of a PA calculation for a CCDF into a series of conditional simulations each involving a summary scenario.

Example 3. A computational scenario is a member of a class of subsets of parameter space that is obtained by subdividing the subspace S spanned by parameters of a summary scenario (Figure 3-13). As an object on which to focus this example, consider the summary scenario $\{R 1, T 2, T 3\}$ discussed in Example 2: the statement defining this summary scenario was expressed in symbolic form as

$$
\mathrm{B}(\mathrm{x})=(\mathrm{N} 1>0, \mathrm{~N} 2=0, \mathrm{I} 1=0, \mathrm{I} 2=1, \mathrm{I} 3=1),
$$

where $\mathrm{N} 1$ counts the number of times the event $\mathrm{R} 1$ occurs during the period of performance (see Example 2 for definitions of the oti.er parameters). As argued in Example 2, the summary scenario $B(\mathbf{x})$ corresponds to a unique set of points in parameter space.

$$
S=\left\{x: x \in D_{N} \text { and } B(x) \text { is true }\right\} .
$$

Notice that $\mathbf{B}(\mathbf{x})$ is true for all $\mathbf{x}$ in which the parameter $\mathbf{N} 1$ takes integer values greater than zero.

Now suppose that, for converiance in numerical calculations, an investigator needs to distinguish the occurrences of the chance event $\mathrm{R} 1$ in each of five, disjoint time intervals dividing the period of performance: fo make this distinction, he might begin by creating a vector of random variables, $N=\left(N_{1}, N_{2}, N_{3}, N_{4}, N_{5}\right)$, in which the $N_{i}, 1 \leq i \leq$ 5 , stand for the number of times event $\mathrm{R} 1$ occurs during the ith interval of time; the constraints imposed by the choice of summary scenario on $\mathrm{N}$ are only that

$$
\mathrm{N} 1=\mathrm{N}_{1}+\mathrm{N}_{2}+\mathrm{N}_{3}+\mathrm{N}_{4}+\mathrm{N}_{5}
$$

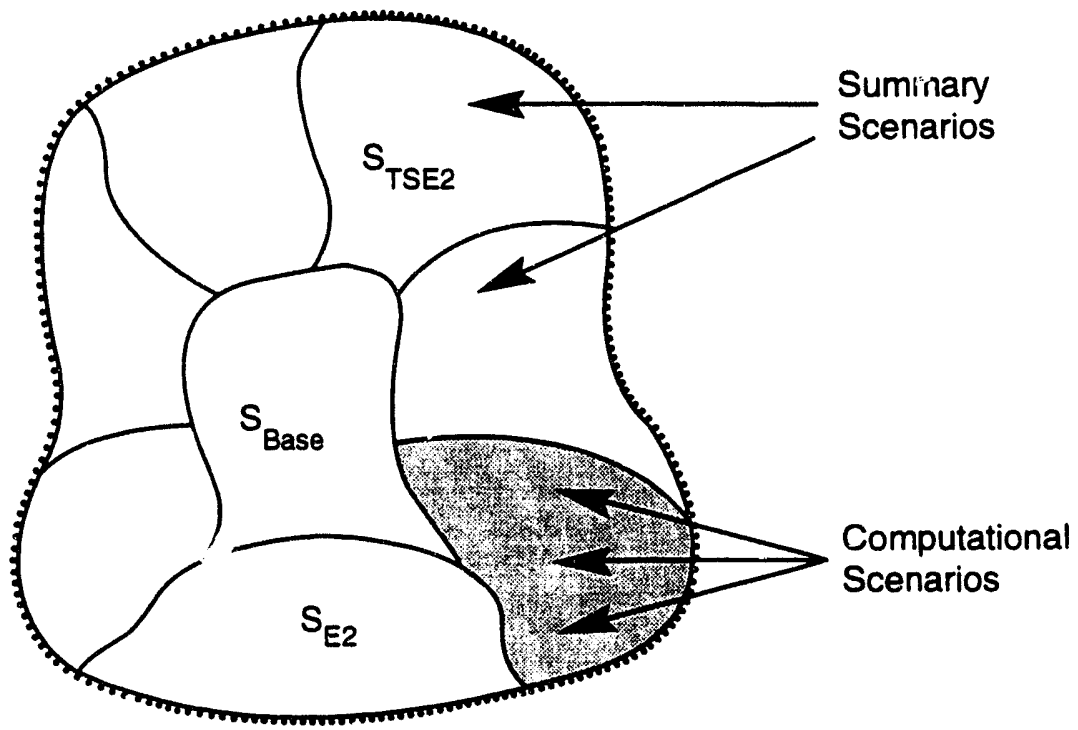

TR1-6342-3782-0

Figure 3-13. Schematic illustrating partitioning of parameter space into summary scenarios and computational scenarios. 


\section{PA Methodology Overview}

be an integer greater than zero. The hypothetical investigator might next define a family of scenarios by the statement (given here in symbolic form),

$$
C(\mathbf{x}, \mathbf{n})=\left(N_{1}=n_{1}, N_{2}=n_{2}, N_{3}=n_{3}, N_{4}=n_{4}, N_{5}=n_{5}\right),
$$

where $\mathbf{n}=\left(\mathrm{n}_{1}, \mathrm{n}_{2}, \mathrm{n}_{3}, \mathrm{n}_{4}, \mathrm{n}_{5}\right)$ is vector of non-negative integers not all of which are zero. The family of scenarios induced by this statement corresponds to a family of sets,

$$
S(n)=\left\{\mathbf{x}: \quad x \in D_{N} \text { and }[B(x) \text { and } C(x, n)] \text { is true }\right\},
$$

that composes an exhaustive, mutually exclusive partition of the summary scenario's parameter space. Members of a family of sets constructed in this way during preliminary PAs for the WIPP have been given the name computational scenarios (WIPP PA Division, 1991a)

\subsubsection{Probability of a Scenario, Conditional Simulation}

The idea of an indicator function of a set $S$ will be helpful in explaining scenario probabilities and conditional simulation. If $S$ is a subset of $D_{N}$, then the indicator function of $S$ is the function defined on $D_{N}$ with the properties,

$$
\begin{aligned}
& I(S, x)=1 \text { if } x \in S, \\
& I(S, x)=0 \text { if } x \notin S .
\end{aligned}
$$

If the set $S$ is a scenario in the sense of Section 3.3.2 (i.e., a subset of $D_{N}$ defined by a statement $A(x)$ ) then, since $x \in$ $S$ if and only if $A(x)$ is true, one can also define its indicator function by

$$
\begin{aligned}
& I(S, x)=1 \text { if } A(x) \text { is true, } \\
& I(S, x)=0 \text { if } A(x) \text { is false. }
\end{aligned}
$$

The latter definition of indicator function is useful for numerical calculations: Given a sample parameter vector $\mathbf{X}$, the value for $I(S, X)$ can be calculated simply by testing whether $A(X)$ is true or false.

Probability of a Scenario. A meaning can now be given to probability of a scenario. The probability of a scenario, $P(S)$, is the expectation of the scenario's indicator function, i.e.,

$$
P(S)=\int_{D_{N}} I(S, \mathbf{x}) d F(\mathbf{x})
$$

A Monte Carlo estimator for $\mathrm{P}(\mathrm{S})$ will therefore look like

$$
<P(S)>=\frac{1}{M} \sum_{m=1}^{M} I\left(S, \mathbf{X}_{\mathbf{m}}\right)
$$

(see Eq. 3-9), where the $\mathbf{X}_{m}, m=1,2,3, \ldots, M$, are independent samples of the parameter vector obtained by random sampling or LHS.

The Monte Carlo estimator for $P(S)$ clearly expresses the frequentist's definition of probability: Draw $M$ independent sample variates and count the number of samples that satisfy some criteria (i.e., are "successful" trials); the raio of the number of successes to the total number of trials gives an estimate of the probability that the criteria are 
satisfied. In spite of its simplicity, Monte Carlo estimation of scenario probabilities is rare in PA practice, perhaps because of the expense involved in evaluating the truth of $\mathrm{A}(\mathbf{x})$ whenever that statement places conditions on an expensive-to-calculate performance measure $f(\mathbf{x})$; in fact, the only instance of a Monte Carlo estimation of scenario probability of which we are aware is the calculation of the CCDF for the EPA performance measure (see Eq. 3-10). If the statement defining the scenario places no conditions on performance measures, it should be quite easy to estimate scenario probabilities by Monte Carlo simulation; admittedly, in such a case it may be even more efficient to calculate those probabilities using logic diagrams (Figure 3-12) and probability models of the agents (events, features, process representations) that are included in the underlying summary scenario.

Conditional Simulations. It has proven useful and revealing in PA work to study system performance by restricting the model system's configurations to those allowed by a particular summary scenario; in other words, the total-system model's "universe" is narrowed to the sum of all things that could happen in the more restricted universe of a summary scenario. Some (Helton, 1993) call this technique "importance sampling," because the summary scenario chosen for special attention is usually the one investigators believe will contain the most unfavorable, hence important, consequences. In the context of stochastic simulation, importance sampling is a subcase of a technique usually called conditional simulation.

All of the formulae of Section 3.2.4 that applied to stochastic simulation of the total system will, with some simple modifications, also apply to a conditional simulation with respect to a scenario $S$. Assuming that $P(S)>0$, the conditional expectation of a performance measure, $h[\mathbf{f}(\mathbf{x})]$, with respect to a scenario $S$ can be defined:

$$
\mathbb{E}[h / S]=\frac{1}{P(S)} \int_{D_{N}} I(S, \mathbf{x}) h[f(\mathbf{x})] d F(\mathbf{x})
$$

The left side of the above expression is to be read as "the conditional expectation of $h[f(x)]$ given the scenario S". Note the similarities and differences between this expression and Equation 3-6. As an exercise, the reader should try to form the expression for the Monte Carlo estimator of the conditional expectation of a performance measure $h[\mathbf{f}(\mathbf{x})]$ (see Eq. 3-9).

A technique frequently used in PA work is the expansion of a total-system performance measure in a series of performance measures of "scenarios", i.e., a series of conditional simulations, each of which is constrained to a given member of a set of summary scenarios. Let the parameter space $D_{N}$ be partitioned into $L$ summary scenarios, $S_{1}, S_{2}, S_{3}, \ldots, S_{L}$, which obey the following conditions:

$$
S_{l} \cap S_{m}=\phi, l \neq m ; \bigcup_{l=1}^{L} s_{l}=D_{N}
$$

and suppose that all the $P\left(S_{1}\right)$ are $>0$. Then, because of the second of the two conditions above,

$$
I\left(D_{N}, \mathbf{x}\right)=\sum_{l=1}^{L} I\left(S_{l}, \mathbf{x}\right)=1 \quad \text { for all } \mathbf{x} \in \mathbf{D}_{\mathbf{N}}
$$

and it follows that

$$
\mathbb{E}[h]=\sum_{l=1}^{L} \int_{D_{N}} I\left(S_{1}, \mathbf{x}\right) h[f(\mathbf{x})] d F(\mathbf{x})=\sum_{l=1}^{L} P\left(S_{1}\right) \mathbb{\{}\left[h \mid S_{1}\right] .
$$


For example: If $f(x)$ is the performance measure for the EPA standard and $h[f(x)]=u[f(x)-c]$, (see Eq. 3-8 and following text), then $\mathcal{E}$ h] is the CCDF for the normalized cumulative release of radioactivity in a 10,000 -yr period following closure and the $\mathcal{E}\left[\mathrm{h} \mid \mathrm{S}_{1}\right]$ are $\mathrm{CCDFs}$ for normalized cumulative release that are conditioned upon the realization of only those events and features included in the lth summary scenario; note that in forming the sum over all summary scenarios, each conditional CCDF is weighted by the probability of its summary scenario.

\subsubsection{Construction of CCDFs in the WIPP PA}

A formalism developed by Kaplan and Garrick (1981) has been extensively used in WIPP PA work to justify certain approximate methods for the calculation of conditional CCDFs (WIPP PA Division, 1991a). This section reconciles the Kaplan-Garrick formalism with the theory of stochastic simulation presented in Sections 3.2.4 and 3.3.3. In Helton's notation (Helton, 1993), the Kaplan-Garrick risk triplet is defined as follows:

$$
\mathrm{R}=\left\{\left(\mathrm{E}_{\mathrm{i}}, \mathrm{pE}_{\mathrm{i}}, \mathrm{cE}_{\mathrm{i}}\right), \mathrm{i}=1,2,3, \ldots \mathrm{nE}\right\}
$$

where

$$
\begin{aligned}
& \mathrm{E}_{\mathrm{i}}=\text { a set of similar occurrences } \\
& \mathrm{pE}_{\mathrm{i}}=\text { probability that an occurrence in set } \mathrm{E}_{\mathrm{i}} \text { will take place } \\
& \mathbf{c E}_{\mathrm{i}}=\text { a vector of consequences associated with } \mathrm{E}_{\mathrm{i}} \\
& \mathrm{nE}=\text { number of sets selected for consideration. }
\end{aligned}
$$

The correct correspondence between the formulae of this overview (e.g., see Eq. 3-10) and the risk-triplet formalism is made by letting $S_{1} \leftrightarrow E_{i}, P\left(S_{1}\right) \leftrightarrow p E_{i}, L \leftrightarrow n E$ and, for a vector performance measure of the form $h[f(x)]=f(x)$ (i.e., where the performance measure is consequences),

$$
\mathcal{E}\left[\mathbf{h} \mid \mathrm{S}_{\mathbf{l}}\right] \leftrightarrow \mathbf{c E}_{\mathrm{i}}
$$

Helton (1993, p. 19) shows how to construct an empirical CCDF for a scalar consequence value $c E$ using the KaplanGarrick formalism; this is done by plotting the points, .

$$
\left(c E_{i}, \sum_{j=i}^{n E} p E_{j}\right) \quad i=1,2,3, \ldots, n E
$$

to form the usual "descending stair steps" histogram. 


\title{
4. Description of Waste and Waste Parcels
}

\author{
J. W. Berglund and J. S. Rath
}

Characterizing the waste as to its inventory of radionuclides and other hazardous constituents is fundamental to evaluating the performance of the disposal system. For the purposes of this performance assessment, waste refers to the contents of a nuclear waste canister and comprises components such as fuel rods, brackets, hardware, and modified high-level waste, except any internal backfill that may be packed inside the canister. The canister is one component of the waste parcel, which includes the waste form, canister, internal backfill, and overpack (optional). For this performance assessment, 26 different waste parcels are considered.

\subsection{Volumes and Masses of Current Waste}

The waste types being studied for this performance assessment originate with spent fuel that has been accepted for interim storage and reprocessing since 1953 by DOE at the Idaho Chemical Processing Plant (referred to herein as the Chem Plant) at the INEL reservation. The fuels were reprocessed to recover uranium and other resources. As a result, $3800 \mathrm{~m}^{3}$ calcine (a granular solid) has been produced, and an inventory of $1100 \mathrm{~m}^{3}$ of high-level waste converted to $120 \mathrm{~m}^{3}$ calcine and $5700 \mathrm{~m}^{3}$ of greater than Class $C$ low-level radioactive, sodium-bearing liquid waste (Berreth, 1988; Ermold, 1992; Donovan, 1989). The inventory at INEL also contains 343 MT graphite spent fuels, 474 MT special fuels, and 240 MT spent fuel from the U.S. Navy (U.S. DOE, 1992; Public Service of Colorado, 1991; Fillmore, 1993).

The total amount of spent fuels and high-level waste located at INEL (666 metric tons of heavy metal [MTHM] or equivalent waste units) is very small* and therefore when evaluating the proposed waste forms as a group the decision was made to represent a repository of reasonable size (about 10,000 MTHM) by including immobilized defense high-level waste from three other DOE complexes: Defense Waste Processing Facility at the Savannah River Plant, Aiken, GA; Hanford Waste Vitrification Plant, Hanford, WA; and the West Valley Demonstration Project, West Valley, NY. The volume and MTHM of the Idaho fuel and waste are $49 \%$ and $6.7 \%$, respectively, of the total for the hypothetical repository.

The five main types of waste materials this performance assessment considers are:

Spent Nuclear Fuel:

1. Graphite spent fuel from

- Fort St. Vrain reactor in Colorado,

- Peach Bottom reactor 1 Cores I and II in Pennsylvania.

2. Special spent fuel (over 90 types of "experimental" spent fuel rods) categorized as

- Highly enriched (HEU), i.e., spent fuel with over $20 \%$ fissile $\left({ }^{235} \mathrm{U}\right)$

- Low enriched (LEU), i.e., spent fuel with less than $20 \%$ fissile $\left({ }^{235} \mathrm{U}\right)$

High-Level Waste:

3. Calcine high-level waste, from oxidation of liquid high-level waste created when uranium and plutonium are extracted during the reprocessing of some spent fuel.

4. Immobilized high-level waste from Savannah River, Hanford, and West Valley.

5. Sodium-bearing liquid high-level radioactive waste (greater than Class $\mathrm{C}$ low-level radioactive)

As mentioned in the introduction, the fifth waste type, sodium-bearing liquid high-level waste, is not considered directly in this performance assessment because it is assumed to have been conditioned through calcination. At present, liquid wastes stored at the Chem Plant are one of two basic types. The first type comes from fuel dissolution

- The potential Yucca Mountain Project repository, for example, has a design capacity of 70,000 MTHM. 


\section{Description of Waste and Waste Parcels}

during the first cycle of uranium recovery operations. First-cycle wastes, although varied in composition based on the type of fuel dissolved, have always been converted into dry granular solids using a fluidized-bed process.

At present, the second type of liquid waste is also assumed to be converted into calcine or vitrified in glass. This liquid waste originates primarily from chemical decontamination, but also from the second and third cycles of uranium recovery operations, and evaporator residue. The nature of this waste is particularly dependent upon the past use of decontamination chemicals at the Chem Plant. Although the emphasis over the past $15 \mathrm{yr}$ has been to adopt more remote technology to avoid extensive chemical decontamination, the current backlog of approximately $5600 \mathrm{~m}^{3}$ waste contains significant quantities of waste with sodium and potassium. This waste does not lend itself to fluid-bed calcination in the concentrated form and so blending with the first-cycle wastes has been the only option, which is viable until the first-cycle wastes are depleted. Another possible process option is the separation of the sodium from the strontium/cesium. In this option, the strontium/cesium would be calcined with aluminum nitrate, and the sodium waste would be solidified and disposed of as a low-level waste, perhaps in grout form.

\subsection{Description of Waste}

The sections below describe the graphite spent fuel, special spent fuel, calcined wastes, and borosilicate glass (from Savannah River, Hanford, and West Valley) as it is expected to be configured prior to its disposition into five groups of waste treatment options. The five groups will be discussed in Section 4.6. The radionuclide inventories for these wastes are given in Appendix $A^{\dagger}$ For this performance assessment, the inventories corresponding to "anticipated wastes" (year 2030) were used in analyzing the graphite spent fuels, special spent fuels, and calcines (Tables A.2.1.8, A.2.1.9, A.2.1.10). For Savannah, Hanford, and West Valley, the current radionuclide inventory was used (Tables A.2.1.5, A.2.1.6, A.2.1.7) but was modified to account for radioactive decay to the year 2030. In presenting the thermal output and activity of each waste type (see data summary tables in this section), all 177 radionuclides (Appendix A) were considered. However, many of these radionuclides have very short half-lives and their contribution to the development of CCDFs is insignificant. Consequently, only 49 radionuclides were included for CCDF development. However, for waste parcel heat production used to establish waste parcel spacing within the repository, the thermal output of all 177 radionuclides contained in the waste types was utilized.

\subsubsection{Description of Graphite Spent Fuel}

Two kinds of graphite spent fuel are included in the inventory: fuel from Fort St. Vrain (Figure 4-1) and Peach Bottom Cores I and II (Figure 4-2) (Table 4-1). Both fuels contain a homggeneous mixture of two types of particles, fissile and fertile. As manufactured and prior to irradiation, fissile particles contain thorium and $93.5 \%$ enriched uranium; fertile particles contain only thorium. The fuel kernels are coated with three fission-product-retaining layers by means of a fluidized-bed vapor-phase deposition process. The inner and outer layers are of isotropic carbon, and the middle layer is of silicon carbide (also referred to as TRISO-coated). A fourth layer of porous carbon, called the "buffer," is located next to the kernel of fissile or fertile material and provides a volume for accumulation of fissionproduct gases without excessive pressure buildup. The $\mathrm{SiC}$ layer is highly resistant to both oxidation and moisture, even at extremely high temperatures.

The fuel-rod design differs for Fort St. Vrain and Peach Bottom Cores I and II fuel. In the Fort St. Vrain design, the fissile and fertile particles are blended and then molded into $1.27-\mathrm{cm}$-diameter $\times 5.08-\mathrm{cm}$-long fuel rods (compacts) (Figure 4-1). In the Peach Bottom design, the compacts are 6.86-cm-outside-diameter, 4.25-cm-inside-diameter $\times 7.57-\mathrm{cm}$-long hollow cylinders (Figure 4-2). The total anticipated radionuclide inventory is given in Appendix A (Table A.2.1.8).

\footnotetext{
† The inventories provided in Appendix A reflect the current best available measured values. They differ from the quantities and characteristics presented in IDB (1992) and U.S. DOE (1992), which contain projected values based on continued processing of spent fuel. Reprocessing is currently being phased out, based on April, 1992, DOE guidelines that spent fuel no longer be reprocessed to recover enriched uranium. Thus, future evaluations will continue to refine the data and include new calcine production information. Note that other performance assessment programs (e.g., WIPP PA Department, 1992a; TSPA, in preparation) use the inventories from IDB (1992) and U.S. DOE (1992) in conformance with their current program guidance.
} 


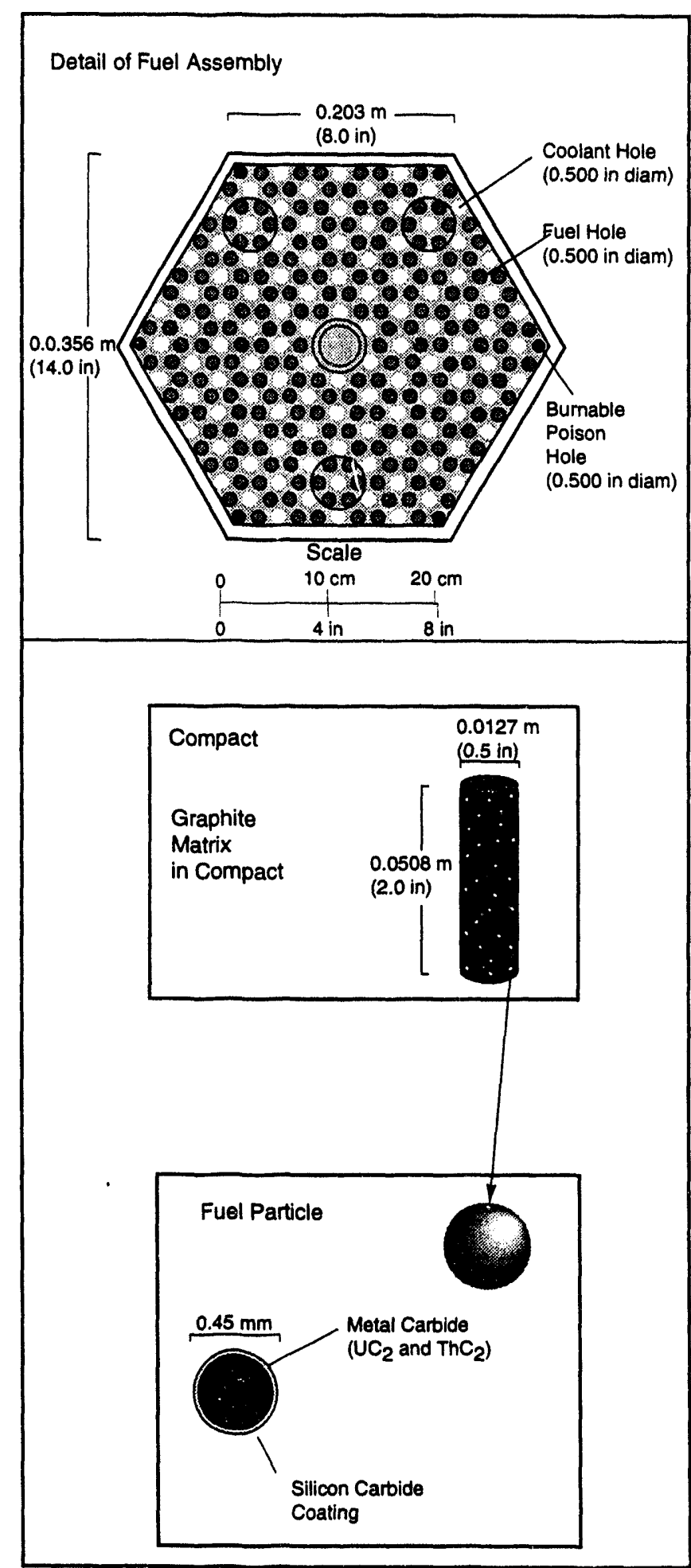

TR1-6342-3751-0 
1

5

6

7

8

10

11

12

13

14

15

16

17

18

19

20

21

22

23

24

25

26

27

28

29

30

31

32

33

34

35

36

37

38

39

40

41

42

43

44

45

46

47

48

49

50

51

52

53

54

55

56

57

58

59

60

61

62

63

64

65

66
Detail of Fuel Assembly

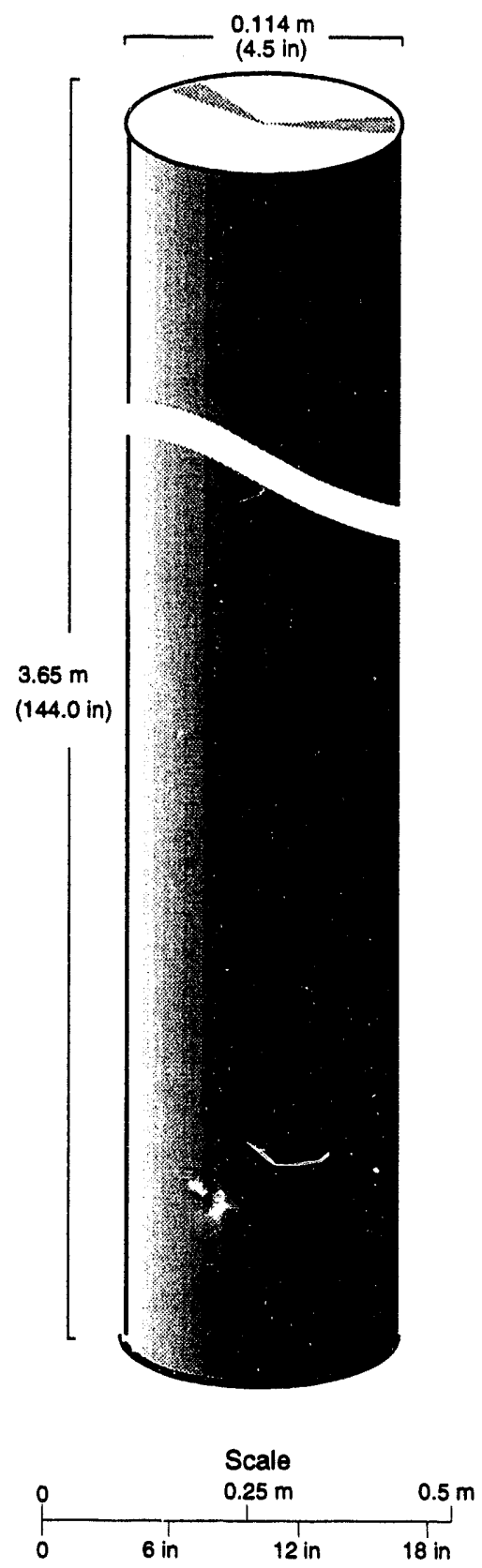

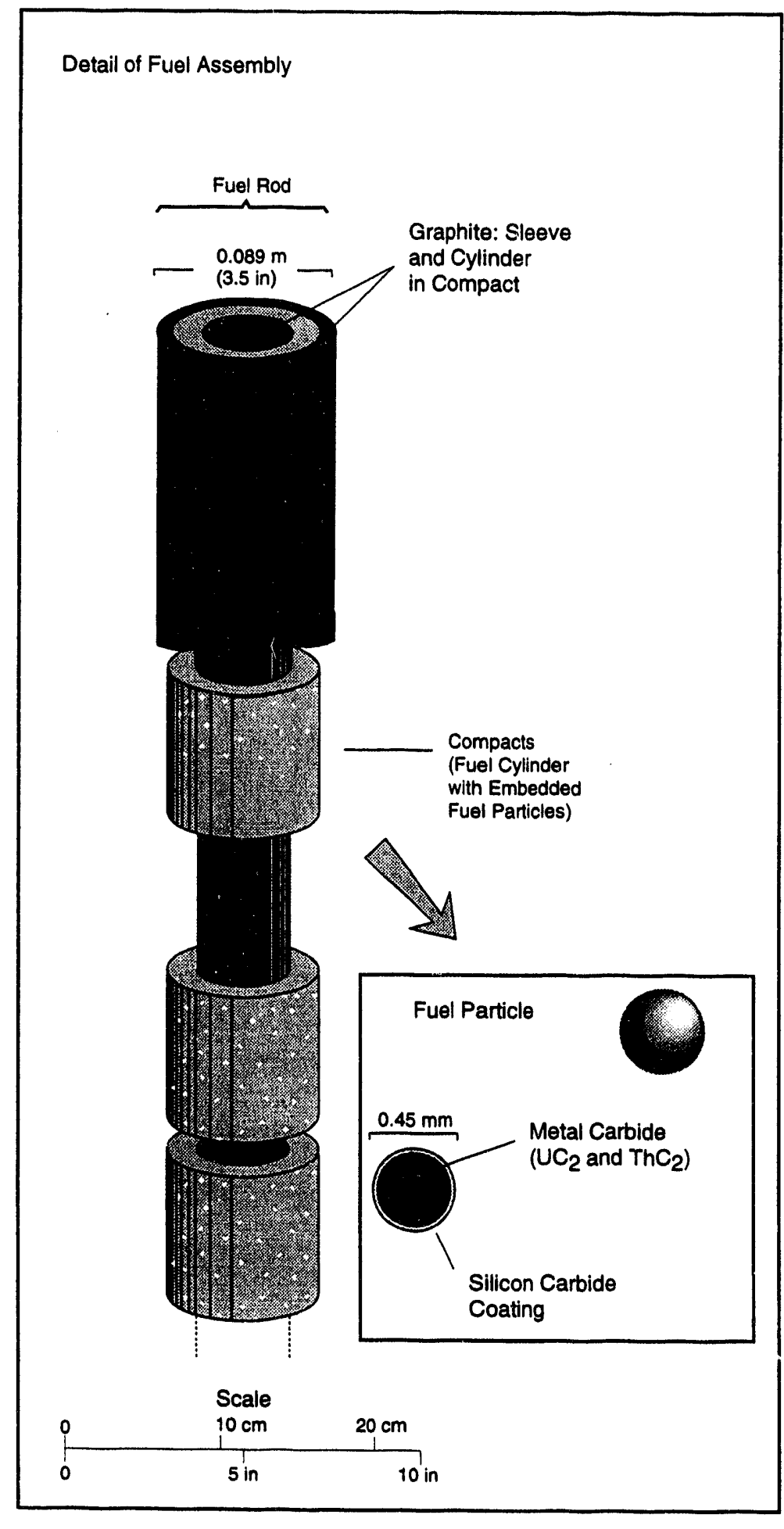

TRI-6342-3754-0

Figure 4-2. Peach Bottom fuel element. 
Table 4-1. Data Summary for Graphite Spent Fuel *

\begin{tabular}{lc}
\hline \multicolumn{1}{c}{ Description } & Units \\
\hline Volume (as is) & $259.8 \mathrm{~m}^{3}$ \\
Mass & $0.342 \mathrm{Gg}$ \\
Thermal Output (year 2030)** & $25.6 \mathrm{~W} / \mathrm{m}^{3}$ \\
Radionuclide Inventory** & $4.31 \times 10^{6} \mathrm{Ci}$ \\
& \\
\hline * See also Appendix A, Attachment 4. & \\
"* The activity and thermal output values were recalculated as additional infor- \\
mation was gathered to develop data to represent the w'aste parcels, and so \\
they differ from those values shown in Appendix A, Attachment 4.
\end{tabular}

\subsubsection{Description of Special Spent Fuel}

The special spent fuel is grouped into two categories: low and high ${ }^{235} \mathrm{U}$ enrichment (LEU and HEU) (Table 4-2) (Figures 4-3 and 4-4). The total anticipated radionuclide inventory is given in Appendix A, Table A.2.1.9.

Most of the special spent fuel consists of small, short fuel assemblies (typically $10^{-8}$ by $168.3 \mathrm{~cm}$ ) (HEU). However, some of the fuel is full-sized commercial spent fuel assemblies (LEU), which are about $28 \times 410 \mathrm{~cm}$ and consist of bundles of zircaloy-clad uranium oxide fuel rods.

Table 4-2. Data Summary for Special Spent Fuel (Low and High Enriched)*

\begin{tabular}{lc}
\hline \multicolumn{1}{c}{ Description } & Units \\
\hline Volume (as is) & $89 \mathrm{~m}^{3}$ \\
Mass & $0.474 \mathrm{Gg}$ \\
Thermal Output (year 2030)** & LEU $429.6 \mathrm{~W} / \mathrm{m}^{3}$ \\
& HEU $196.6 \mathrm{~W} / \mathrm{m}^{3}$ \\
Radionuclide Inventory** & LEU $4.19 \times 10^{7} \mathrm{Ci}$ \\
& HEU $1.20 \times 10^{7} \mathrm{Ci}$ \\
\hline - See also Appendix A, Attachment 4. & \\
".* The activity and thermal output values were recalculated as additional \\
information was gathered to develop data to represent the waste parcels, \\
and so they differ from those values shown in Appendix A, Attachment 4.
\end{tabular}

\subsubsection{Description of Calcine Waste}

Two types of calcines are stored at the Chem Plant: aluminum and zirconium calcines.

The aluminum wastes were the first high-level wastes generated (from the dissolution of Materials Test Reactor elements) at the Chem Plant. Use of a fluidized-bed to convert these liquids into a solid form was first demonstrated in 1963. The process used indirect heating to denitrate the acidic, aqueous waste and convert it into a dry, granular 

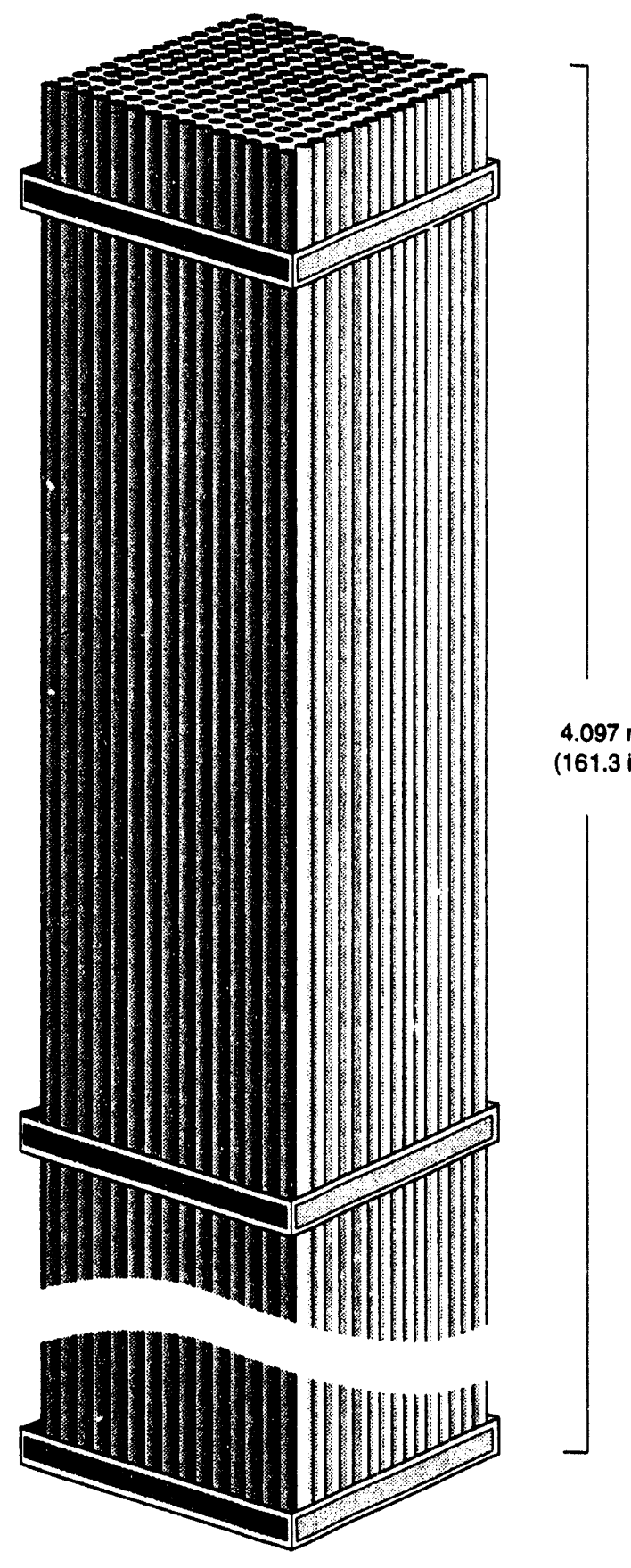

Detail of Fuel Assembly

$4.097 \mathrm{~m}$ (161.3 in)
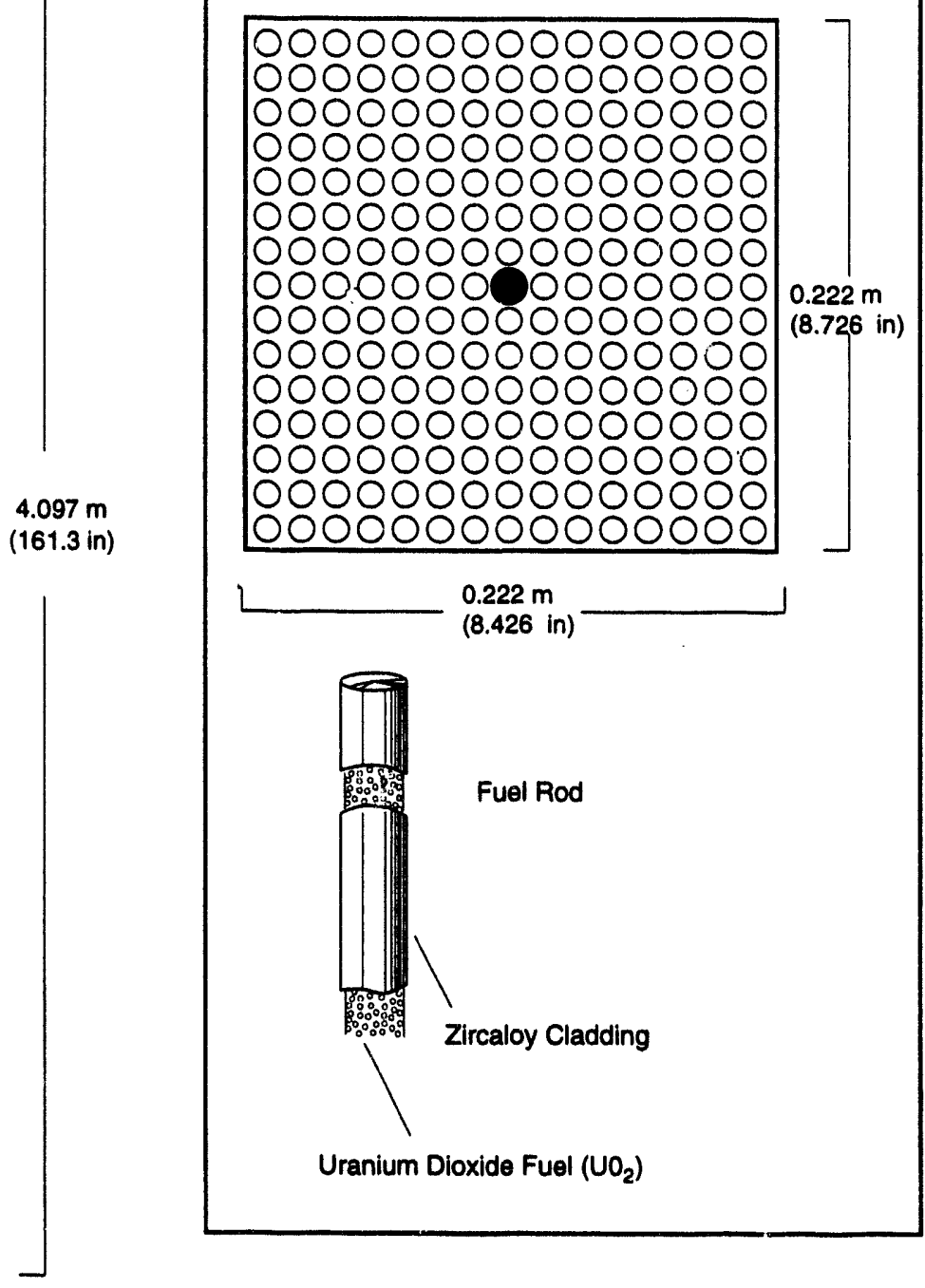

Uranium Dioxide Fuel $\left(\mathrm{UO}_{2}\right)$

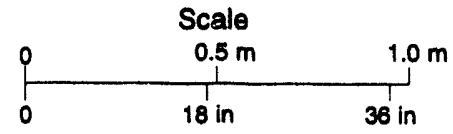

TRI-6342-3753-0

Figure 4-3. Representative low enriched uranium (LEU) fuel assembly. 
1
$0.108 \mathrm{~m}$ (4.25 in)

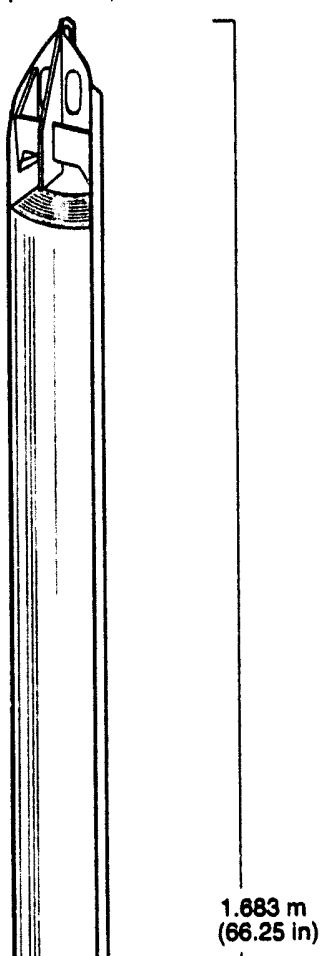
(66.25 in)

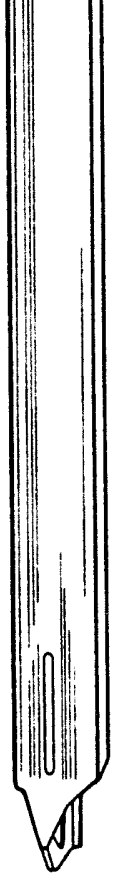

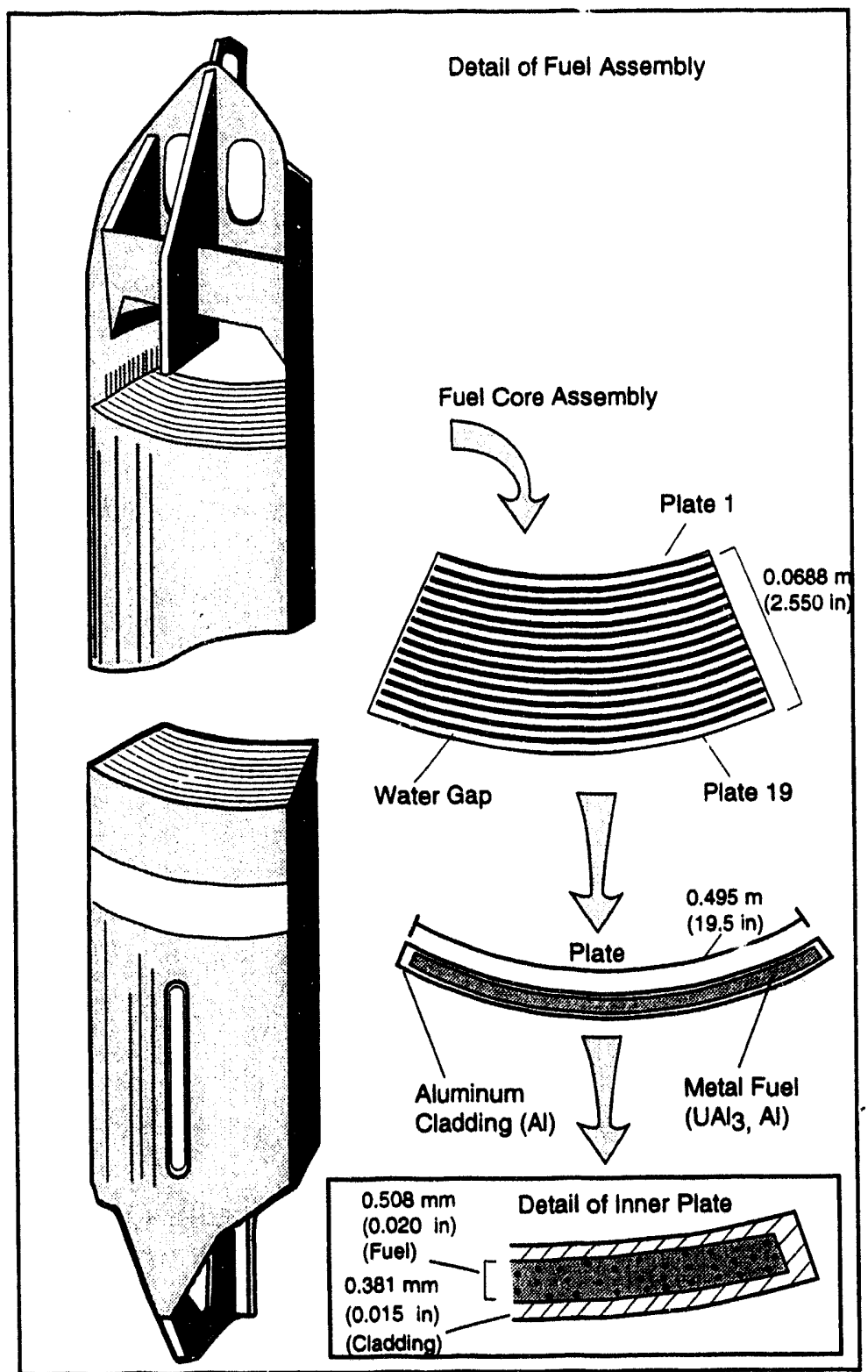

Note: Plates 1 and 19 are $2.03 \mathrm{~mm}(0.080 \mathrm{in})$ thick instead of $1.27 \mathrm{~mm}(0.050 \mathrm{in})$ as shown.

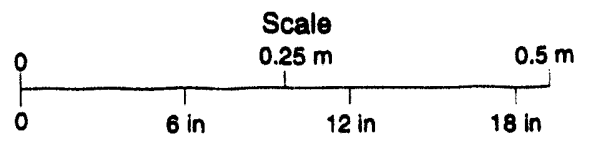

TR1-6342-3752-0

Figure 4-4. Representative highly enriched uranium (HEU) fuel assembly. (Aluminum clad fuel is shown as an example.) 


\section{Description of Waste and Waste Parcels}

solid that was stored in underground bins. In the calcination process, the volume reduction of the wastes was about 8-10:1. The dry solids consist of both a granular material and a powder (about 3 to $18 \%$ of the granular in wt percent) (Staples et al., 1979); both the granules and powder are pneumatically retrievable and transportable. In terms of short-lived radionuclide concentrations, the aluminum fuels and the resulting wastes provide the highest watt densities $\left(\mathrm{kW} / \mathrm{m}^{3}\right)$ of any of the wastes processed in the waste calcination facilities at the Chem Plant.

The zirconium waste originated from the processing of U.S. Navy nuclear fuels with their zirconium cladding. The Navy fuel dissolution requires a more complex process, using aqueous hydrofluoric acid to chemically dissolve the fuel assemblies. Aluminum nitrate was blended-first as a cold feed stock and then later as a coprocessing effort-with the zirconium dissolver product to complex the free fluorides. The calcination of zirconium wastes requires the addition of calcium nitrate to form calcium fluoride; all the other components of the waste are converted to their oxide form. Because of the cold chemical addition to the fluoride wastes, liquid to solid volume reduction factors are closer to 6-7:1. The watt densities are moderate compared with the aluminum calcines.

The grarular material ranges in size from 0.2 to $0.5 \mathrm{~mm}$. The grain size distribution is commonly described by the mass median particle diameter (mmipd) where $1 / 2$ the mass of particles in a given sarnple pass through a hypothetical screen having openings of that particular size. So for any given mmpd, there is actually a distributed range of diameters. The granular calcine has a grain density of 2.4 to $2.7 \mathrm{~g} / \mathrm{cm}^{3}$; however, when combined with the fine powder, the bulk density ranges between 1.0 and $1.7 \mathrm{~g} / \mathrm{cm}^{3}$, with $1.4 \mathrm{~g} / \mathrm{cm}^{3}$ the most representative value.

The total anticipated radionuclide inventory for calcines is given in Appendix A, Table A.2.1.10. A summary of calcine waste data is given in Table 4-3.

Table 4-3. Data Summary for Calcine Waste*

\begin{tabular}{lc}
\multicolumn{1}{c}{ Description } & Units \\
\hline Volume (as is) & $4250 \mathrm{~m}^{3}$ \\
Mass & $0.595 \mathrm{Gg}$ \\
Thermal Output (year 2030) & $9.74 \mathrm{~W} / \mathrm{m}^{3}$ \\
Radionuclide Inventory** & $2.02 \times 10^{7} \mathrm{Ci}$ \\
& \\
\hline - See also Appendix A, Attachment 4. & \\
The activity and thermal output values were recalculated as additional \\
information was gathered to develop data to represent the waste par- \\
cels, and so they differ from those values shown in Appendix A, \\
Attachment 4.
\end{tabular}

\subsubsection{Description of Borosilicate Glass from Other DOE Sites}

The borosilicate glass from Savannah River, Hanford, and West Valley is being produced from neutralized highlevel waste. The borosilicate glass is similar to the glass produced at the Chem Plant. The average radionuclide contents per canister for Savannah River, Hanford, and West Valley are 234,000 Ci, 298,000 Ci, and 109,500 Ci, respectively. The respective containers contain $1682 \mathrm{~kg}, 1650 \mathrm{~kg}$, and $1900 \mathrm{~kg}$ of glass. Because the influence of the mass of glass is very minor, $1900 \mathrm{~kg}$ was used for all three containers in the PA calculations. The DOE wastes will be accepted as received with one disposal option used in all five disposal groups (Section 4.6). A summary of the waste data for the immobilized high-level waste from Savannah River, Hanford, and West Valley is given in Table 4-4. 
Table 4-4. Data Summary for Waste from Savannah River, Hanford, and West Valley*

\begin{tabular}{lccc}
\hline \multicolumn{1}{c}{ Description } & $\begin{array}{c}\text { Savannah } \\
\text { River }\end{array}$ & Hanford & $\begin{array}{c}\text { West } \\
\text { Valley }\end{array}$ \\
\hline Volume (as is) & $3307 \mathrm{~m}^{3}$ & $1227 \mathrm{~m}^{3}$ & $193 \mathrm{~m}^{3}$ \\
Mass & $8.88 \mathrm{Gg}$ & $3.23 \mathrm{Gg}$ & $0.523 \mathrm{Gg}$ \\
Thermal Output (year 2030)** & $375 \mathrm{~W} / \mathrm{m}^{3}$ & $447 \mathrm{~W} / \mathrm{m}^{3}$ & $168 \mathrm{~W} / \mathrm{m}^{3}$ \\
Radionuclide Inventory** & $2.08 \times 10^{8} \mathrm{Ci}$ & $7.84 \times 10^{7} \mathrm{Ci}$ & $6.44 \times 10^{6} \mathrm{Ci}$ \\
& & & \\
\hline * See also Appendix A, Attachment 4. & & \\
"- The activity and thermal output values were recalculated as additional information was gathered to develop data to repre- \\
sent the waste parcels, and so they differ from those values shown in Appendix A, Attachment 4.
\end{tabular}

\subsection{Distribution of Radionuclides in Waste}

The inventory of the radionuclides in the waste and the decay chains are presented below.

\subsubsection{Radionuclide Inventory}

The anticipated radionuclide inventory for the graphite spent fuel, special spent fuel, and calcine high-level waste is provided in Appendix A, Tables A.2.1.8, A.2.1.9, and A.2.1.10. The anticipated radionuclide inventory is the projected radionuclide inventory of the spent fuel or high-level waste that needs to be disposed of in a repository by the year 2030. Note that for the anticipated high-level waste, all the liquid wastes are assumed to be converted into calcine. Thus, no liquid waste is included in the anticipated or design radionuclide inventory.

The current radionuclide inventories for the immobilized high-level waste from the three DOE complexes are given in Appendix A, Tables A.2.1.5 through A.2.1.7.

Of the 177 radionuclides shown in Appendix A, only 49 radionuclides are considered in the performance assessment (Table 4-5). ${ }^{* *}$ Radionuclides that appear on the anticipated inventory tables (Tables A.2.1.5 through A.2.1.10) but are not shown on Table 4-5 are not being considered either because they had a short half-life or made an insignificant contribution to the inventory when normalized by the EPA release limits. ${ }^{\dagger \dagger}$ All 49 radionuclides are accounted for in all waste parcels, although for some waste parcels the contribution of a particular radionuclide may be zero.

The initial activities (in curies) of the 49 radionuclides considered for the INEL and DOE wastes are shown in Figure 4-5. Eleven radionuclides (indicated by asterisks in Figure 4-5) were considered in the transport calculations for the Complex PA (see Chapter 7 for a discussion of the two approaches). A total of 22 radionuclides were considered in the Simple PA. All 49 radionuclides were used for the calculations for direct release due to drilling. ${ }^{137} \mathrm{Cs}$, ${ }^{238} \mathrm{Pu}$, ${ }^{241} \mathrm{Pu}$, and ${ }^{90} \mathrm{Sr}$ dominate the initial activity for most waste types.

\# Appendix A also contains current and design inventories. The current inventory includes curies in 1992 . The design inventory considers uncertainty in the projection and includes a margin for the uncertainty of about $10 \%$. Neither of these inventories was used in this performance assessment.

- All 177 radionuclides were considered for waste parcel heat production, which was used to determine waste parcel spacing within the repository.

${ }^{\text {t+ }}$ Other radionuclides could emerge as key radionuclides for other performance measures such as individual doses evaluated over very lcing time frames (e.g., one million years). 
4. Description of Waste and Waste Parcels

1

3

4

A 西 10

Carbon

Cadmium

Chlorine

Curium

Cesium

Europium

lodine

Neodymium

Nickel

Neptunium

Protactinium

Lead

Palladium

Promethium

Plutonium

Radium

Rubidium

Selenium

Samarium

Table 4-5. Radionuclides Considered in Performance Assessment

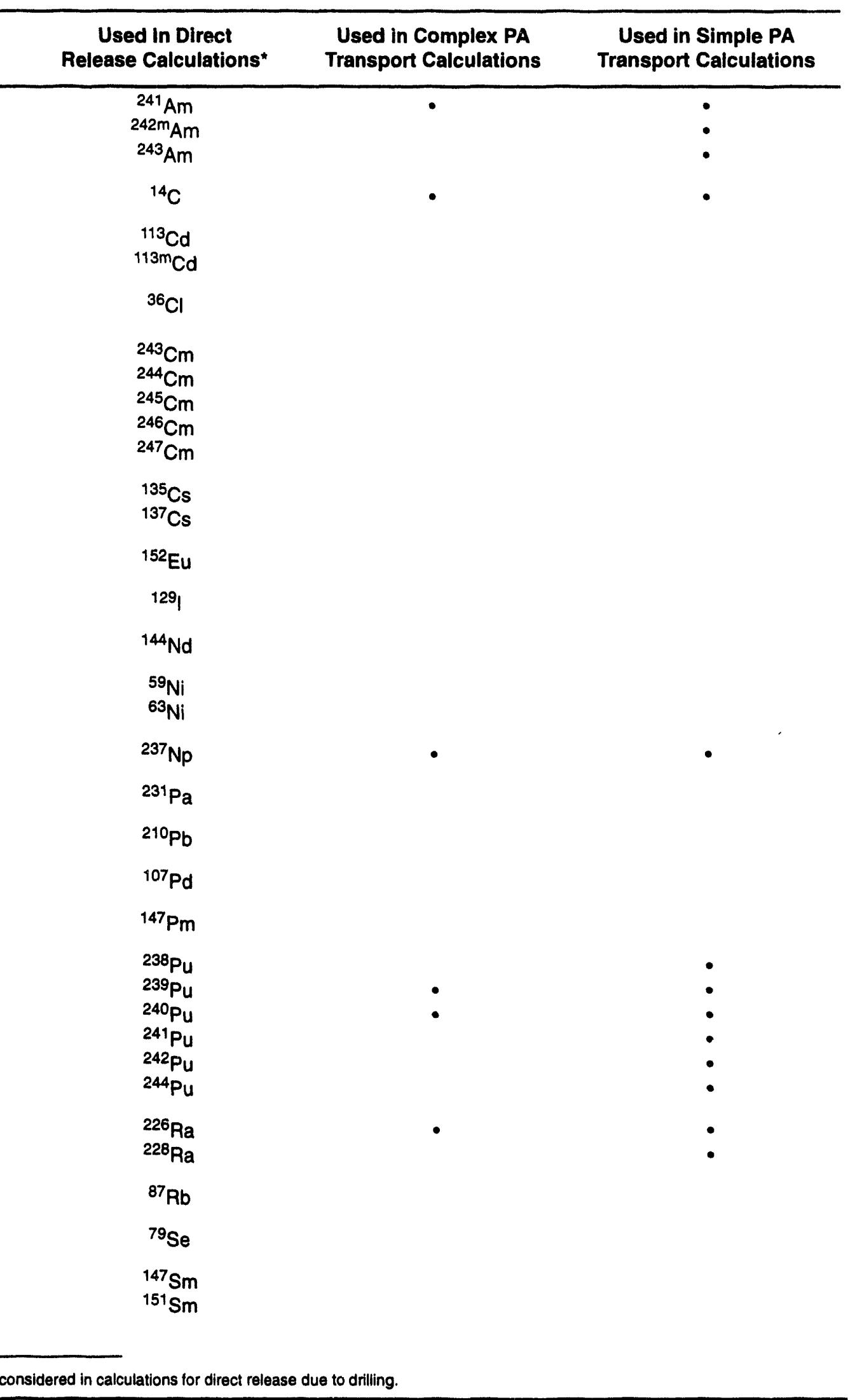

- All 49 radionuclides were considered in calculations for direct release due to drilling. 
2 3$$
\text { stro }
$$$$
\text { Strontium }
$$

Technetium

$\begin{array}{ll}\text { Thorium } & 229 \mathrm{Th} \\ & 230 \mathrm{Th} \\ & 232 \mathrm{Th}\end{array}$

Uranium

(1)

Zirconium

Total Radionuclides

Table 4-5. Radionuclides Considered in Performance Assessment (Continued)

\begin{tabular}{|c|c|c|c|}
\hline Element Name & $\begin{array}{l}\text { Used In Direct } \\
\text { Release Calculations* }\end{array}$ & $\begin{array}{l}\text { Used in Complex PA } \\
\text { Transport Calculations }\end{array}$ & $\begin{array}{l}\text { Used in Simple PA } \\
\text { Transport Calculations }\end{array}$ \\
\hline Tin & ${ }^{126} \mathrm{Sn}$ & & \\
\hline Strontium & ${ }^{90} \mathrm{Sr}$ & & \\
\hline Technetium & ${ }^{99} \mathrm{TC}$ & & \\
\hline Thorium & $\begin{array}{l}{ }^{229} \mathrm{Th} \\
230 \mathrm{Th} \\
232 \mathrm{Th}\end{array}$ & - & : \\
\hline Uranium & $\begin{array}{l}232 \mathrm{U} \\
233 \mathrm{U} \\
234 \mathrm{U} \\
235 \mathrm{U} \\
236 \mathrm{U} \\
238 \mathrm{U}\end{array}$ & • & $\begin{array}{l}\dot{\bullet} \\
\dot{\bullet} \\
\dot{\bullet}\end{array}$ \\
\hline Zirconium & ${ }^{93} \mathrm{Zr}$ & & \\
\hline Total Radionuclides & 49 & 11 & 22 \\
\hline
\end{tabular}

The decay of selected radionuclides in the waste inventory normalized to the EPA release limits (see Section 2.3.1) for the 10,000-yr regulatory period is shown in Figure 4-6. These include a total of 21 radionuclides that exceed a normalized release of 0.1 during the period of 1000 to $10,000 \mathrm{yr}$ and 28 radionuclides that exceed a normalized release of 0.01 during the period of 1000 to $10,000 \mathrm{yr}$. The activities of the remaining radionuclides in the inventory do not exceed the normalized value of $10^{-4}$ during the period from 1000 to $10,000 \mathrm{yr}$.

The radionuclides selected for transport for this performance assessment (11 for the Complex PA and 22 for the Simple PA) were chosen early in the performance assessment and were limited to data available on the decay of longlived radionuclides contained only in the graphite spent fuel. These radionuclides included all the radionuclides considered in receding WIPP performance assessments (WIPP PA Division, 1991c) and contained isotopes of carbon, plutonium, thorium, americium, neptunium, radium, and uranium. A comparison of the radionuclides selected for transport in this performance assessment and a list of those exceeding the normalized limit of 0.1 and 0.01 during the period of 1000 to $10,000 \mathrm{yr}$ are shown in Table 4-6. A consistent assessment of performance for transport based on nornalized inventory $>0.1$ would consider at least six additional radionuclides not considered in this performance assessment $\left({ }^{135} \mathrm{Cs},{ }^{59} \mathrm{Ni},{ }^{151} \mathrm{Sm},{ }^{126} \mathrm{Sn},{ }^{99} \mathrm{Tc}\right.$, and ${ }^{93} \mathrm{Zr}$. ${ }^{\ddagger \ddagger}$

\subsubsection{Radionuclide Chains and Half-Lives}

The decay chains for the 49 initial radionuclides in the INEL and high-level waste inventory are shown in Figure 4-7. The half-life for each radionuclide listed in the literature by ICRP Publication 38 (ICRP, 1983) is also on Figure 4-7. Because many of the daughter radionuclides have extremely short half-lives and make a small contribution to the curie inventory, these have been eliminated from the decay chains shown.

\# Funding and time limitations prevented these additional radionuclides from being considered for transport in every performance assessment calculation. However, all of these radionuclides were included in cuttings releases and one calculation did consider these additional radionuclides (see Section 16.8.1). 


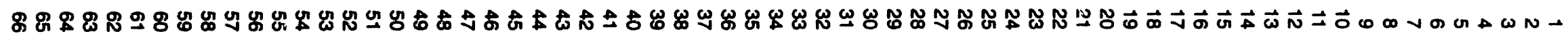

$\frac{t}{N}$

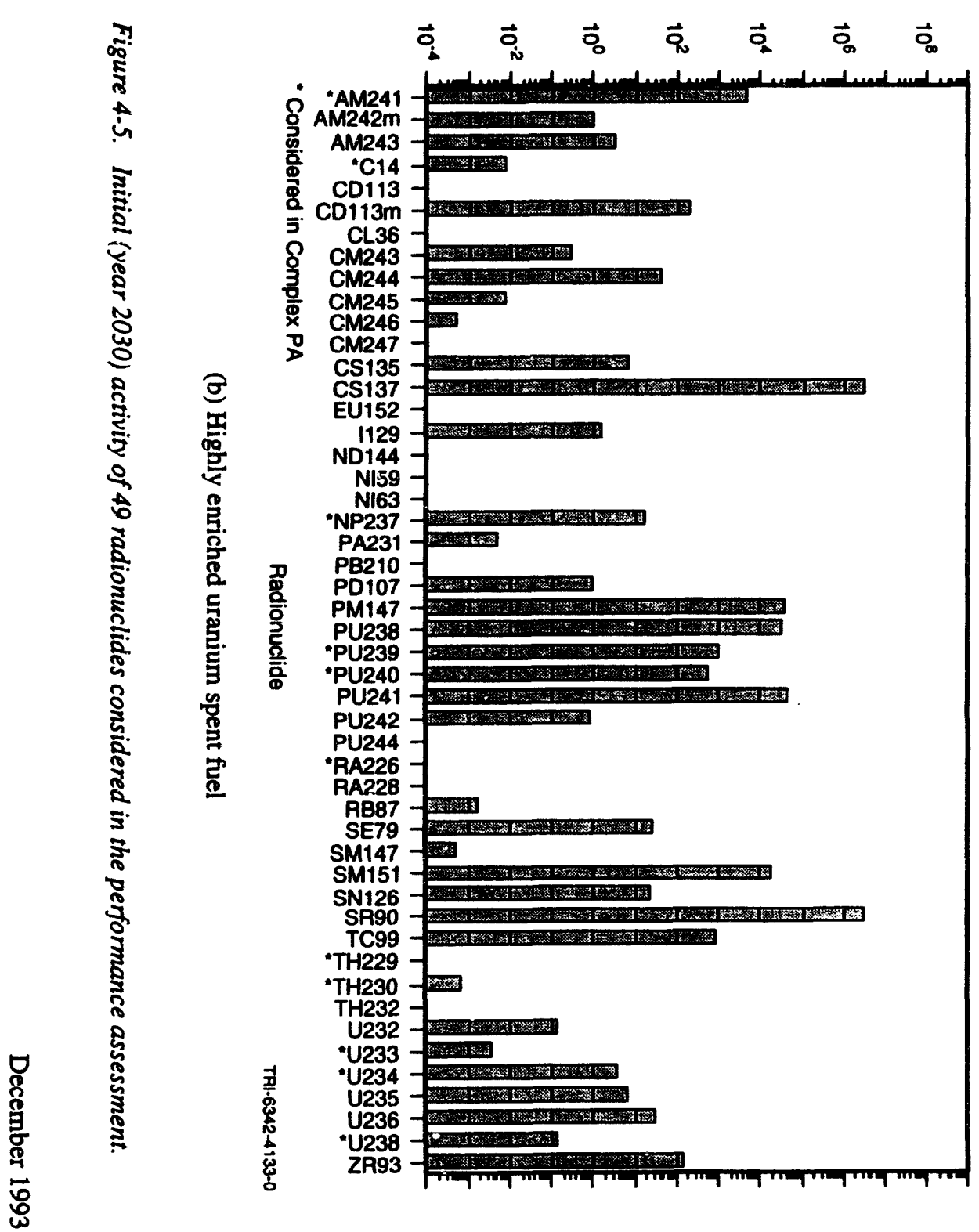




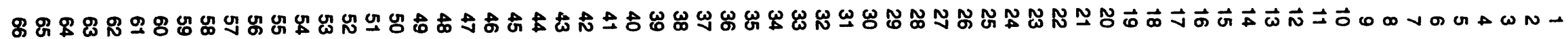
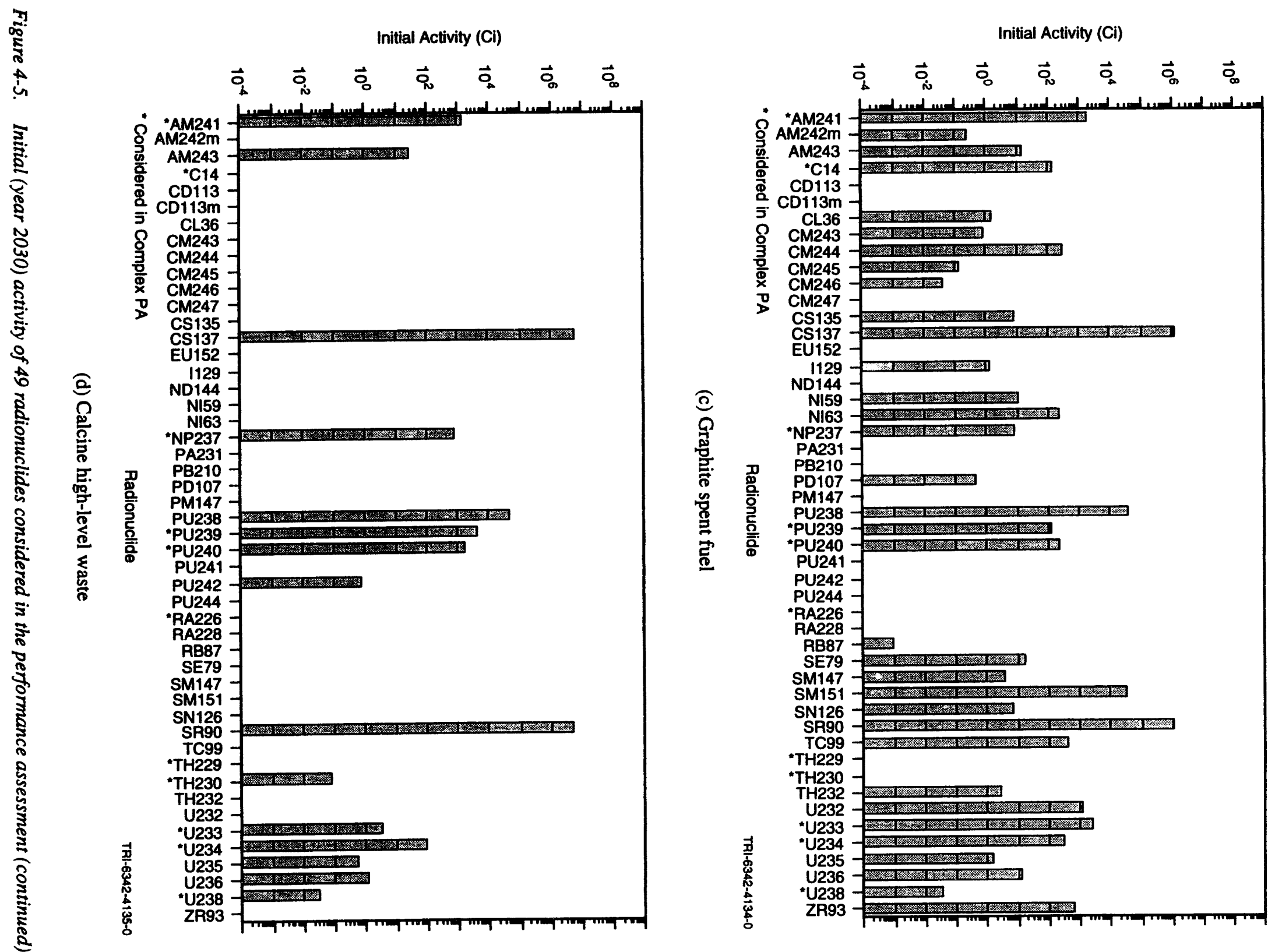


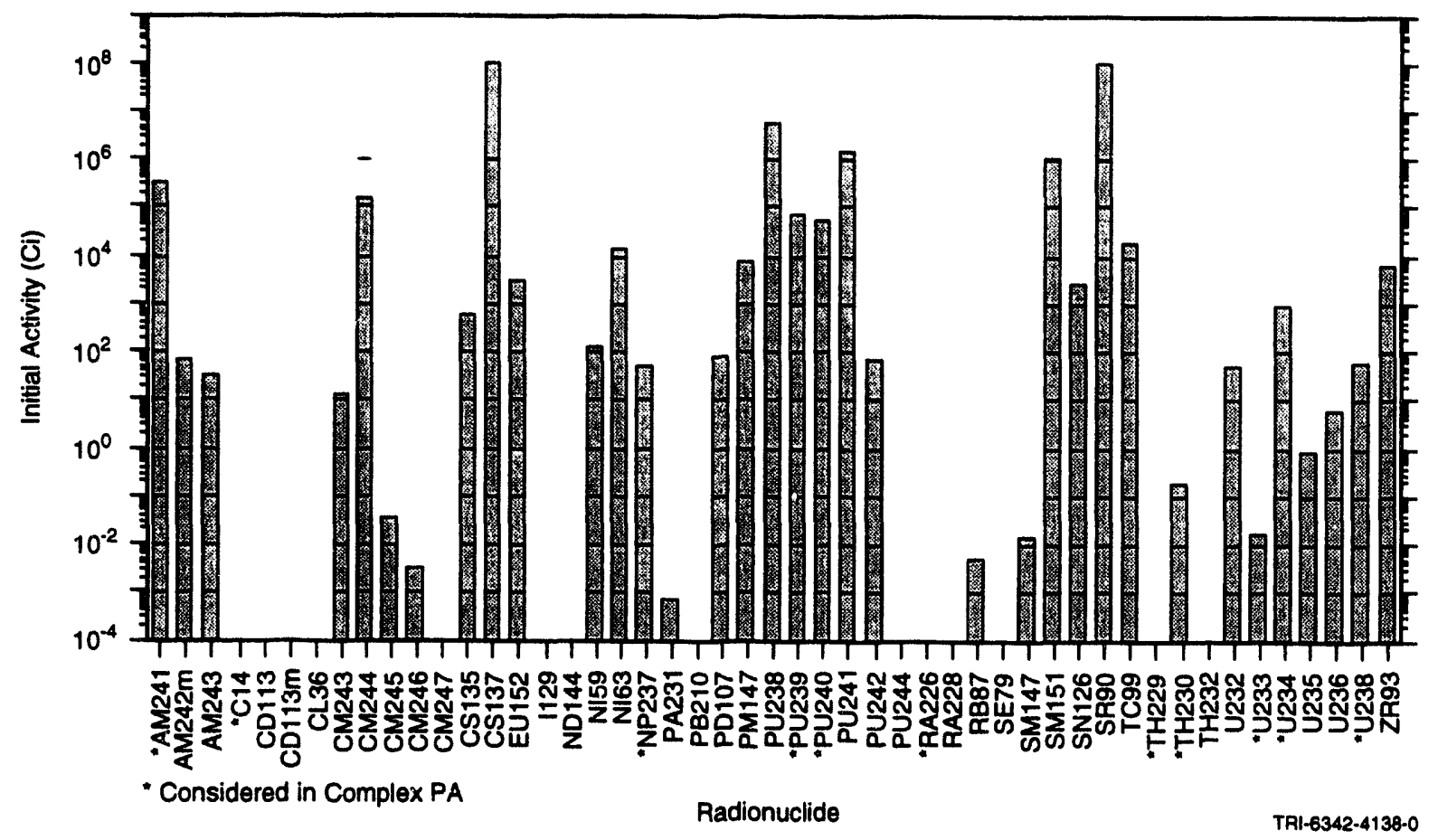

(e) Savannah River high-level waste

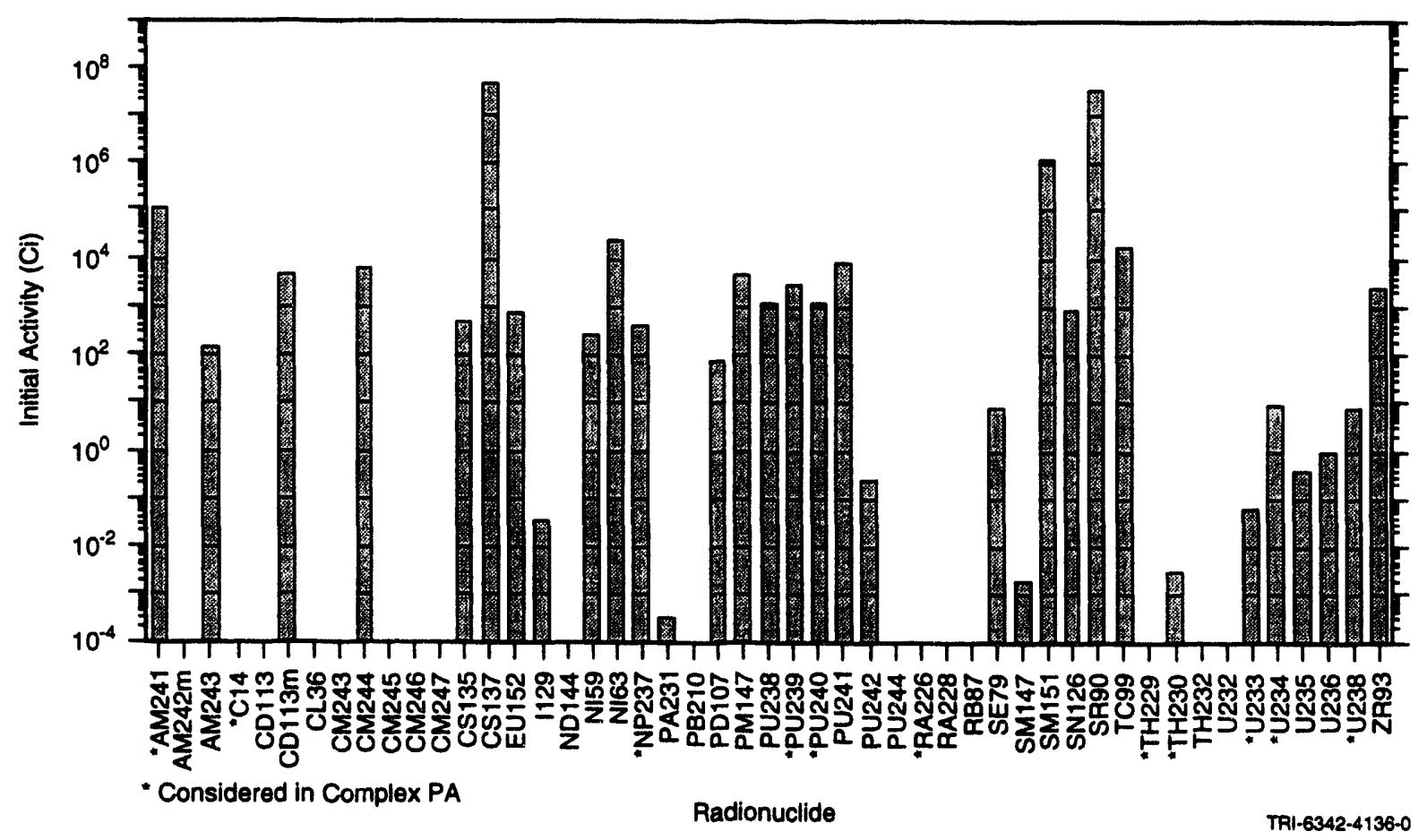

(f) Hanford high-level waste

Figure 4-5. Initial (year 2030) activity of 49 radionuclides considered in the performance assessment (continued). 


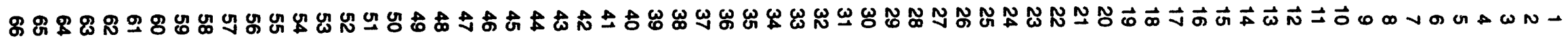

Initial Activity (Ci)

$\begin{array}{lllllll}\vec{o}_{i} & \overrightarrow{0} & \overrightarrow{0} & \vec{i} & \overrightarrow{0} & \vec{o}_{0} & \vec{o}_{\mathbf{O}}\end{array}$ \& AM241 AM243

-C14

C14

CD113m

$5.0136-$

CM243-

CM244-

CM245 -

CM246 -

CM247

CS135 -

EU152 -

$1129-$

ND144

N159-

NP237

PA231

产

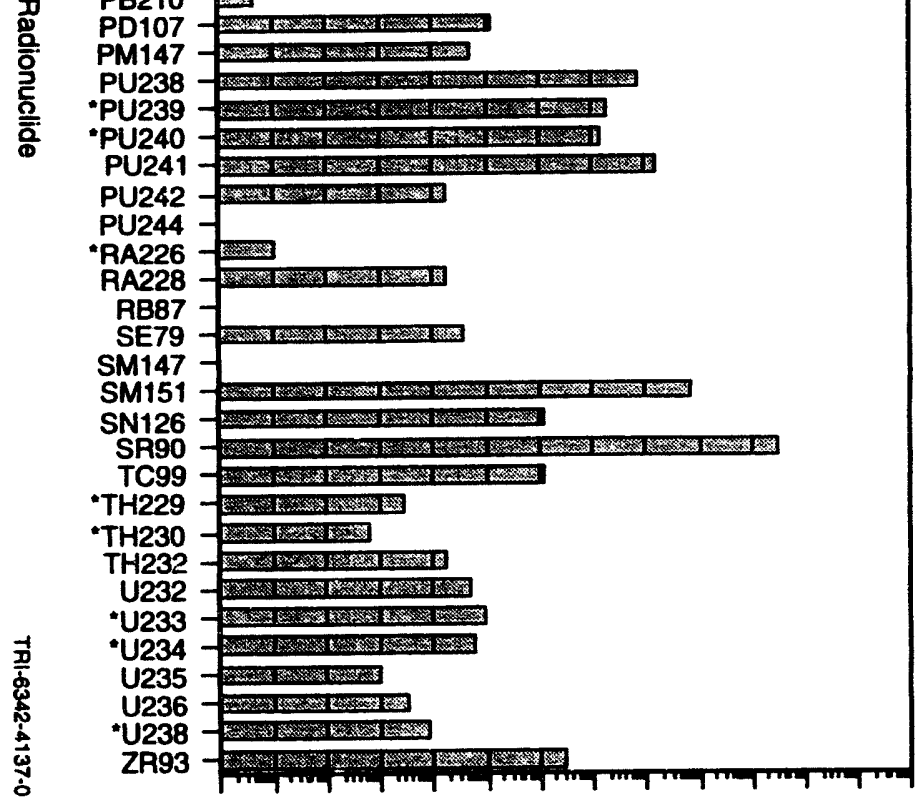



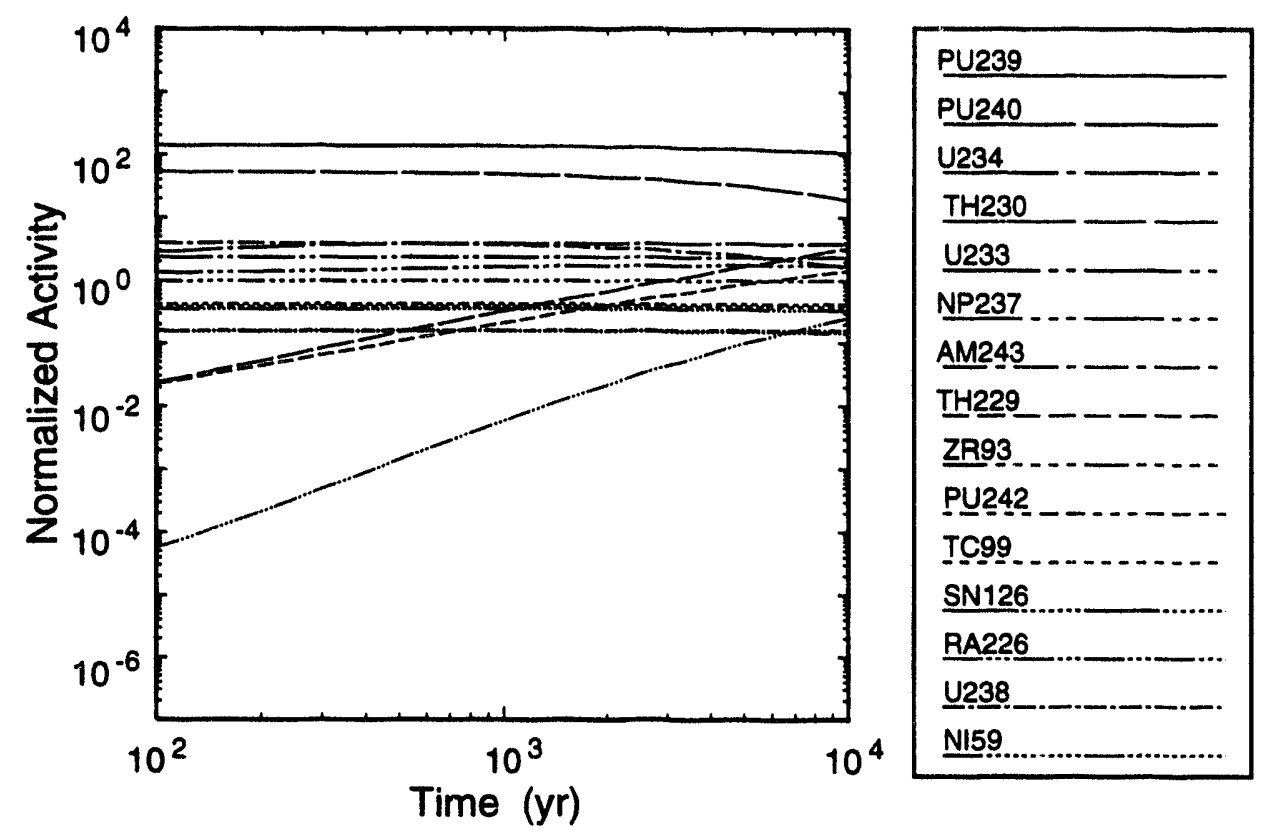

TR1-6342-4001-0
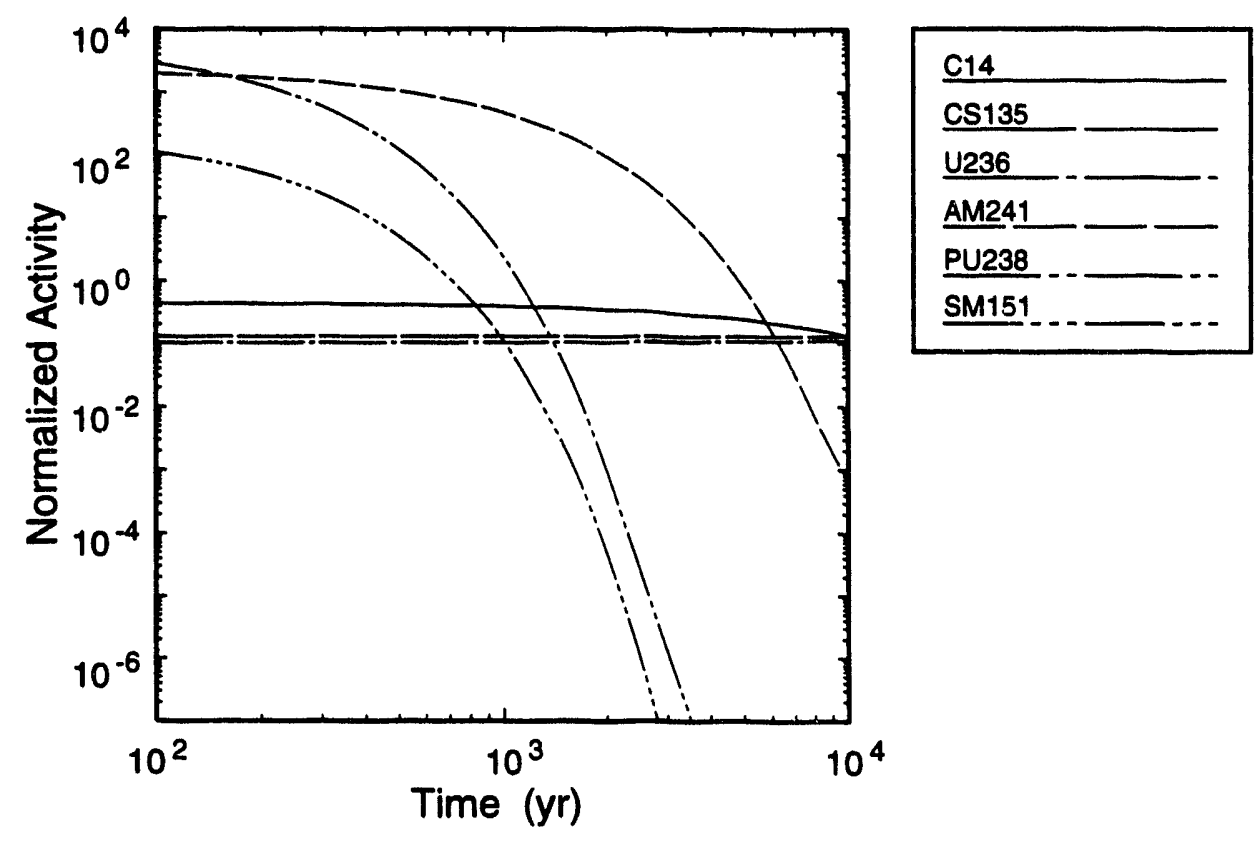

TR1-6342-4002-0

(a)

Figure 4-6. Normalized activity decay history of individual radionuclides that exceed a normalized release of (a) 0.1 and (b) 0.01 , based on 40 CFR 191 criterion. (Two plots each are shown to accommodate the individual radionuclides since they could not be graphically represented on one plot.) (Plots similar to these, see page 4-16.) 

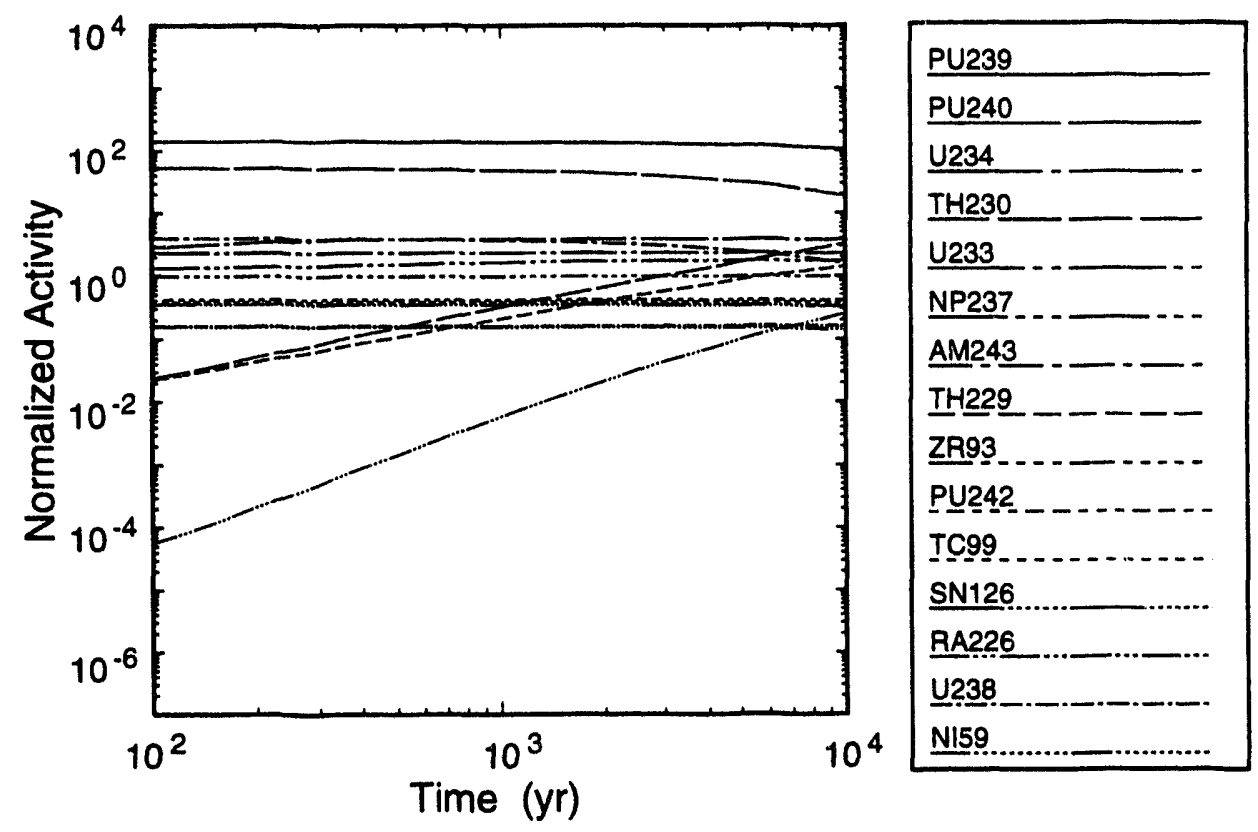

TR1-6342-4003-0
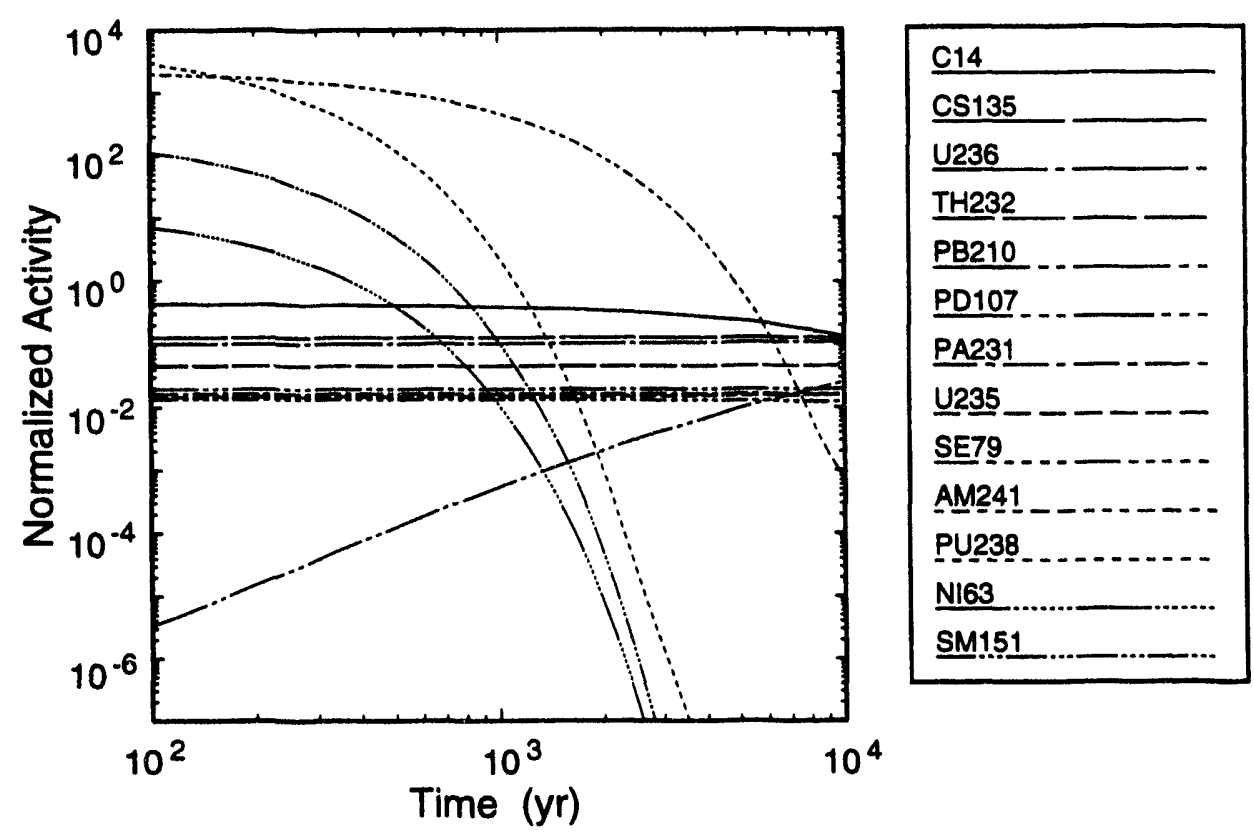


\section{Table 4-6. Comparison of Radionuclides Selected for Transport (Simple and Complex PA) with Inventory exceeding a Normalized Value of 0.1 and 0.01 during the Period of $1000 \mathrm{yr}$ in the $10,000 \mathrm{yr}$ Regulatory Period}

\begin{tabular}{|c|c|c|c|c|c|}
\hline \multicolumn{4}{|c|}{$\begin{array}{l}\text { Radionuclide with Normalized Inventory } \\
\left(Q, f_{w} L_{1}\right) \text { during the Period } 1000-10,000 \mathrm{yr}\end{array}$} & \multicolumn{2}{|c|}{ Radionuclides Used } \\
\hline$>10$ & $>1$ & $>0.1$ & $>0.01$ & Simple PA & Complex PA \\
\hline \multirow[t]{9}{*}{${ }^{241} \mathrm{Am}$} & ${ }^{241} \mathrm{Am}$ & ${ }^{241} \mathrm{Am}$ & ${ }^{241} \mathrm{Am}$ & $\begin{array}{l}241 \mathrm{Am} \\
242 \mathrm{~m}_{\mathrm{Am}}\end{array}$ & ${ }^{241} \mathrm{Am}$ \\
\hline & ${ }^{243} \mathrm{Am}$ & ${ }^{243} \mathrm{Am}$ & ${ }^{243} \mathrm{Am}$ & ${ }^{243} \mathrm{Am}$ & \\
\hline & & ${ }^{14} \mathrm{C}$ & ${ }^{14} \mathrm{C}$ & ${ }^{14} \mathrm{C}$ & ${ }^{14} \mathrm{C}$ \\
\hline & & ${ }^{135} \mathrm{Cs}$ & ${ }^{135} \mathrm{Cs}$ & & \\
\hline & & ${ }^{59} \mathrm{Ni}$ & $\begin{array}{l}{ }^{59} \mathrm{Ni} \\
{ }^{63} \mathrm{Ni}\end{array}$ & & \\
\hline & ${ }^{237} \mathrm{~Np}$ & ${ }^{237} \mathrm{~Np}$ & $\begin{array}{l}{ }^{237} \mathrm{~Np} \\
{ }^{231} \mathrm{~Pa}\end{array}$ & ${ }^{237} \mathrm{~Np}$ & ${ }^{237} \mathrm{~Np}$ \\
\hline & & & ${ }^{210} \mathrm{pb}$ & & \\
\hline & & & ${ }^{107} \mathrm{Pd}$ & & \\
\hline & ${ }^{238} \mathrm{Pu}$ & ${ }^{238} \mathrm{Pu}$ & ${ }^{238} \mathrm{Pu}$ & ${ }^{238} \mathrm{Pu}$ & ${ }^{238} \mathrm{Pu}$ \\
\hline${ }^{239} \mathrm{p}$ & ${ }^{239} \mathrm{P}$ & ${ }^{239} \mathrm{Pu}$ & ${ }^{239} \mathrm{Pu}$ & ${ }^{239} \mathrm{Pu}$ & ${ }^{239} \mathrm{Pu}$ \\
\hline \multirow[t]{14}{*}{${ }^{240} \mathrm{Pu}$} & ${ }^{240} \mathrm{Pu}$ & ${ }^{240} \mathrm{Pu}$ & ${ }^{240} \mathrm{Pu}$ & $\begin{array}{l}{ }^{240} \mathrm{Pu} \\
{ }^{241} \mathrm{Pu}\end{array}$ & \\
\hline & & ${ }^{242} \mathrm{Pu}$ & ${ }^{242} \mathrm{Pu}$ & $\begin{array}{l}{ }^{242} \mathrm{Pu} \\
{ }^{244} \mathrm{Pu}\end{array}$ & \\
\hline & & ${ }^{226} \mathrm{Ra}$ & $\begin{array}{l}{ }^{226} \mathrm{Ra} \\
{ }^{79} \mathrm{Se}\end{array}$ & $\begin{array}{l}{ }^{226} \mathrm{Ra} \\
{ }^{228} \mathrm{Ra}\end{array}$ & ${ }^{226} \mathrm{Ra}$ \\
\hline & & ${ }^{151} \mathrm{Sm}$ & ${ }^{151} \mathrm{Sm}$ & & \\
\hline & & ${ }^{126} \mathrm{Sn}$ & ${ }^{126} \mathrm{Sn}$ & & \\
\hline & & ${ }^{99} \mathrm{TC}$ & ${ }^{99} \mathrm{TC}$ & & \\
\hline & ${ }^{229} \mathrm{Th}$ & ${ }^{229} \mathrm{Th}$ & ${ }^{229} \mathrm{Th}$ & ${ }^{229} \mathrm{Th}$ & ${ }^{229} \mathrm{Th}$ \\
\hline & ${ }^{230} \mathrm{Th}$ & ${ }^{230} \mathrm{Th}$ & ${ }^{230} \mathrm{Th}$ & ${ }^{230} \mathrm{Th}$ & ${ }^{230} \mathrm{Th}$ \\
\hline & & & ${ }^{232} \mathrm{Th}$ & $\begin{array}{c}{ }^{232} \mathrm{Th} \\
232 \mathrm{U}\end{array}$ & \\
\hline & $233 u$ & ${ }^{233} U$ & ${ }^{233} \mathrm{U}$ & $233 \mathrm{U}$ & ${ }^{233} \mathrm{U}$ \\
\hline & ${ }^{234} \mathrm{U}$ & ${ }^{234} U$ & $\begin{array}{l}234 U \\
235 U\end{array}$ & ${ }^{234} \mathrm{U} U$ & ${ }^{234} \mathrm{U}$ \\
\hline & & $236 \mathrm{U}$ & $236 U$ & ${ }^{236} \mathrm{U}$ & \\
\hline & & $238 \mathrm{U}$ & $238 \mathrm{U}$ & ${ }^{238} \mathrm{U}$ & ${ }^{238} \mathrm{U}$ \\
\hline & & ${ }^{93} \mathrm{Zr}$ & ${ }^{93} \mathrm{Zr}$ & & \\
\hline 3 & 10 & 21 & 28 & 22 & 11 \\
\hline
\end{tabular}




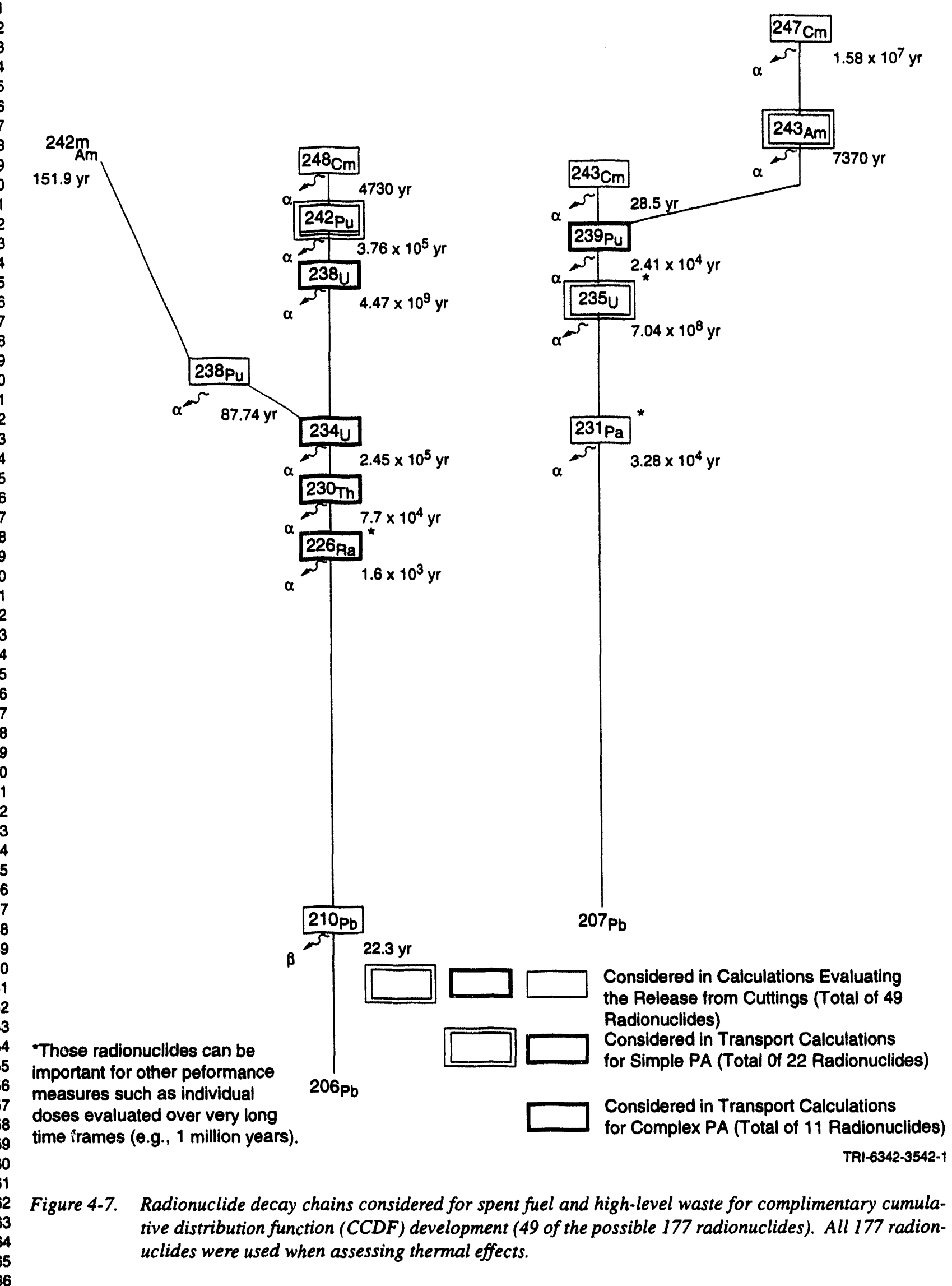



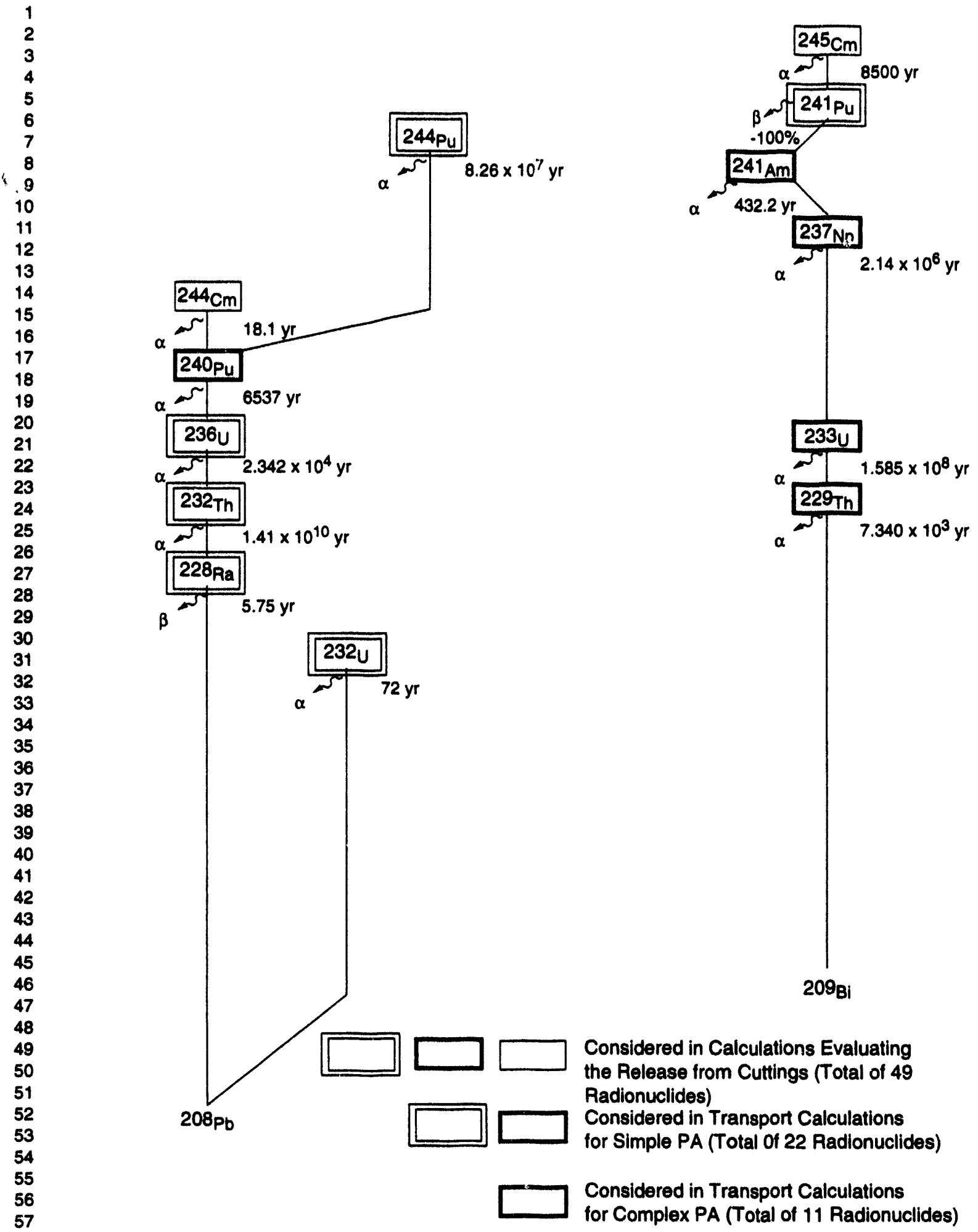

TRI-6342-3541-1

Figure 4-7. Radionuclide decay chains considered for spent fuel and high-level waste for complementary cumulative distribution function (CCDF) development (49 of the possible 177 radionuclides). All 177 radionuclides were used when assessing thermal effects (continued). 

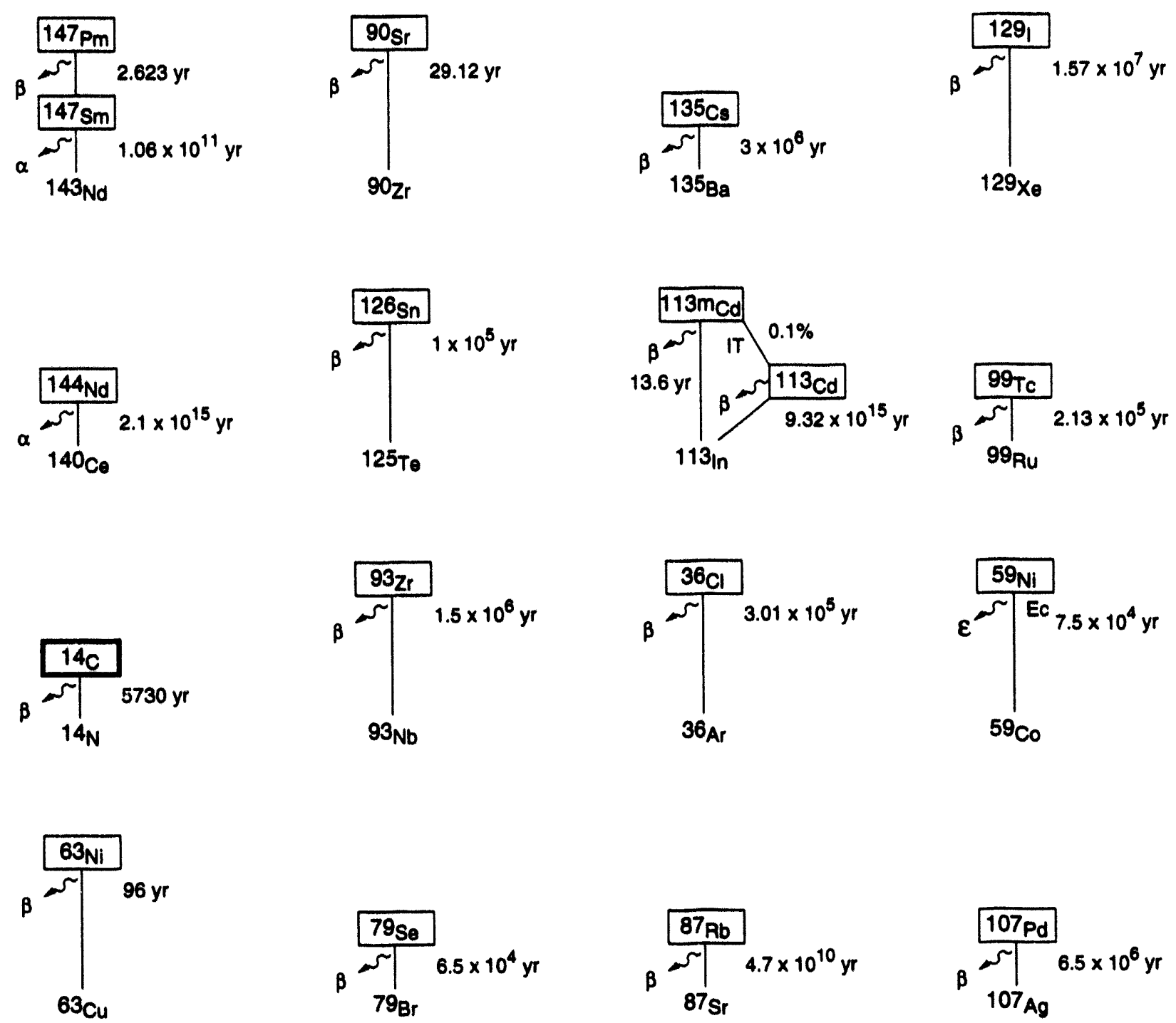

$$
\begin{array}{|l|l|}
137 \mathrm{Cs} \\
\hline \Gamma & \mathrm{yr}
\end{array}
$$
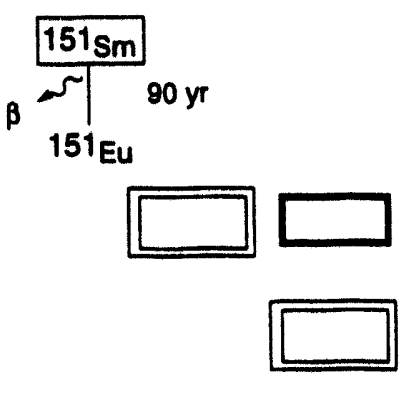

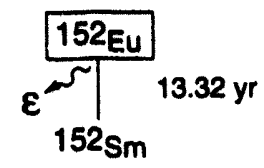

Considered in Calculations Evaluating the Release from Cuttings (Total of 49 Radionuclides)

Considered in Transport Calculations for Simple PA (Total of 22 Radionuclides)

Considered in Transport Calculations for Complex PA (Total of 11 Radionuclides)

Figure 4-7. Radionuclide decay chains considered for spent fuel and high-level waste for complementary cumulative distribution function (CCDF) development (49 of the possible 177 radionuclides). All 177 radionuclides were used when assessing thermal effects (concluded). 


\section{Description of Waste and Waste Parcels}

\subsubsection{Waste Unit Factor}

For the Containment Requirements of 40 CFR 191, the amount of radionuclides that can be released from the repository is proportional to the amount of the radionuclides emplaced. The proportional constant is called a waste unit factor $\left(f_{w}\right)$. The waste unit factor, $f_{w}$, that was used in the performance assessment calculations when disposal of waste forms in a group was evaluated (including borosilicate glass from Savannah River, Hanford, and West Valley) is the sum of the following:

1. The amount of the INEL spent fuels (in metric tons of heavy metal [MTHM]), as defined in 40 CFR 191 , Appendix A. Note 1(a), divided by 1000

2. The curies of radionuclides of gamma or beta emitters in the HLW with half-lives greater than 100 years, or any alpha emitters with half-lives greater than 20 years, as defined by 40 CFR 191, Appendix A, Note 1(d), divided by $1,000,000$.

The waste unit factor determined for the INEL PA is 9.934 (Table 4-7).

Table 4-7. Units of Waste for the INEL PA, Based on 40 CFR 191, Appendix A

\begin{tabular}{lccc}
\hline $\begin{array}{c}\text { Contributing DOE } \\
\text { Complexes }\end{array}$ & $\begin{array}{c}\text { Alpha Nuclides }>20 \mathrm{yr} \text {; } \\
\text { Beta and Gamma } \\
\text { Nuclides }>100 \mathrm{yr} \\
\text { (curies) }\end{array}$ & $\begin{array}{c}\text { Units of Waste } \\
\text { Based on Note 1(a) }\end{array}$ & $\begin{array}{c}\text { Units of Waste } \\
\text { Based on Note 1(d) }\end{array}$ \\
\hline $\begin{array}{l}\text { INEL } \\
\text { Graphite Fuels }\end{array}$ & N/A \\
$\begin{array}{l}\text { Special Fuels } \\
\text { Calcine }\end{array}$ & $3.39 \times 10^{5}$ & 0.027 & N/A \\
West Valley & $7.15 \times 10^{4}$ & $0.3^{*}$ & N/A \\
Savannah River & $8.04 \times 10^{6}$ & N/A & 0.339 \\
Hanford & $1.16 \times 10^{6}$ & N/A & 0.0715 \\
Total & & N/A & 8.036 \\
& Waste Unit Factor & 0.327 & 1.160 \\
\hline No correction was made for low bumup of some of the highly enriched fuels. & 9.607 \\
\hline
\end{tabular}

Waste unit factors were not used in this performance assessment when single waste parcels were compared (Simple PA). In addition, the depleted uranium added to Waste Group 4 was considered for this performance assessment to be a low-level waste and was not included in calculations of the waste unit factors. Including the depleted uranium in the waste unit factor calculation for waste group 4 would result in lower normalized releases for that group than reported here. The depleted uranium was, however, included in the inventory of the appropriate waste parcel in all the calculations and had a significant effect on the calculated releases.

\subsection{Treatment Options for the Waste}

Before the waste is placed into a container for disposal, the waste is treated with the intent of making the waste form more resistant to transport in repository groundwater and eventual release. The treatments being considered in this performance assessment differ for each type of spent fuel and high-level waste because characteristics vary among types (e.g., how susceptible the material is to becoming critical in certain hypothetical situations). Based on scoping calculations using conservative assumptions (Appendix $\mathrm{C}$ ), no more than $0.7 \mathrm{~kg}$ of ${ }^{235} \mathrm{U}$ is placed in a waste 
parcel for disposal in a granite repository because of criticality concerns. This requirement means that some spent fuel must be physically cut. Because of salt's low final permeability and porosity (which means that only a small amount of water is available to moderate a nuclear reaction) and the presence of chlorine that absorbs neutrons, the salt repository is not restricted to the $0.7 \mathrm{~kg}{ }^{235} \mathrm{U}$ limit, based on the conservative criticality assumptions (Appendix C). Two waste treatment options for the salt repository (Groups 1 and 3 ) were chosen to take advantage of this relaxation in criticality concerns and permit up to $10 \mathrm{~kg}$ of ${ }^{235} \mathrm{U}$. The heat generated by radioactive decay is also important as this can affect canister spacing and, thus, repository size.

In this performance assessment, five treatment options are considered for the spent fuel and high-level waste stored at INEL (Figures 4-8 through 4-10). Although many combinations of treatments could be constructed, the treatment options selected range from placing currently existing spent fuel and high-level waste directly into waste parcels to encapsulating or dissolving spent fuel and high-level waste into a glass-ceramic matrix. These options roughly correspond to one minimum, one moderate, and three high levels of stabilization or reprocessing of the spent fuel and high-level waste. In some options, waste types may be commingled.

Options 1 through 5-discussed separately in Sections 4.4.1 through 4.4.3 for graphite spent fuel, special spent fuel, and calcine waste-are the basis for the waste disposal groupings, which are discussed later in this chapter. For example, Waste Disposal Group 1 includes waste parcels that are designed to hold either graphite spent fuel, special spent fuel, or calcine waste that has been treated according to Option 1 for salt and/or granite repositories. Also note that the liquid, slurries, and solid high-level waste stored at Savannah River, Hanford, and West Valley are assumed to be immobilized in a borosilicate glass.

\subsubsection{Processing Options for Graphite Spent Fuel}

For graphite spent fuel (Figure 4-8), the five options include (1) disposal of whole or cut graphite blocks with the fuel plugs in canisters at a repository (whole block disposal), (2) separation of the fuel and graphite block, disposal of the graphite block at a low-level waste site (Lotts et al., 1992), clean glass encapsulation of the spent fuel, and spent fuel disposal in canisters at a repository (rod/block separation), (3) graphite burning, with or without shredding, after which the residue (i.e., the ash) is directly immobilized by melting with additives and calcine to form a glass (graphite burning), (4) burning the graphite and separating plutonium and enriched uranium for future use (national resource), calcining the raffinate and immobilizing the calcine using the preferred treatment for high-level waste calcine (e.g., glass or glass-ceramic) (spent fuel recycling), and (5) burning up the graphite, oxidizing the fuel and treating it with nitric acid to form uranyl nitrate, and adding depleted uranium at a ratio of about $200: 1$ to give a ${ }^{235} \mathrm{U}$ content of the waste equivalent to naturally occurring uranium before placing in canisters for repository disposal (oxidation then dilution with depleted uranium).

\subsubsection{Processing Options for Special Spent Fuel}

The five options for special spent fuel include (Figure 4-9) (1) placing spent fuel in canisters and filling the void space with lead (spent fuel/lead disposal), (2) placing spent fuel in canisters and filling the void space with the glassceramic product of calcine immobilization (spent fuelglass-ceramic), (3) placing spent fuel in canisters and surrounding the spent fuel with the borosilicate glass product of calcine immobilization (spent fuel/borosilicate glass), (4) separating plutonium and enriched uranium for future use (national resource), calcining the raffinate, and then immobilizing the calcine using the preferred treatment for high-level waste calcine (e.g., glass or glass-ceramic) (spent fuel recycling), and (5) oxidizing the fuel, mixing with depleted uranium oxide to reduce the enrichment, and then immobilizing the resulting uranium oxide mixture by melting with additives and calcine to form a glass (oxidation then dilution with depleted uranium). 


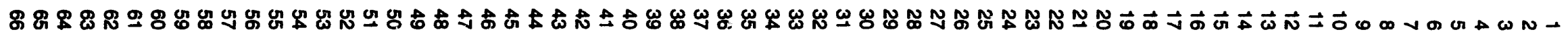
$\stackrel{+}{+}$
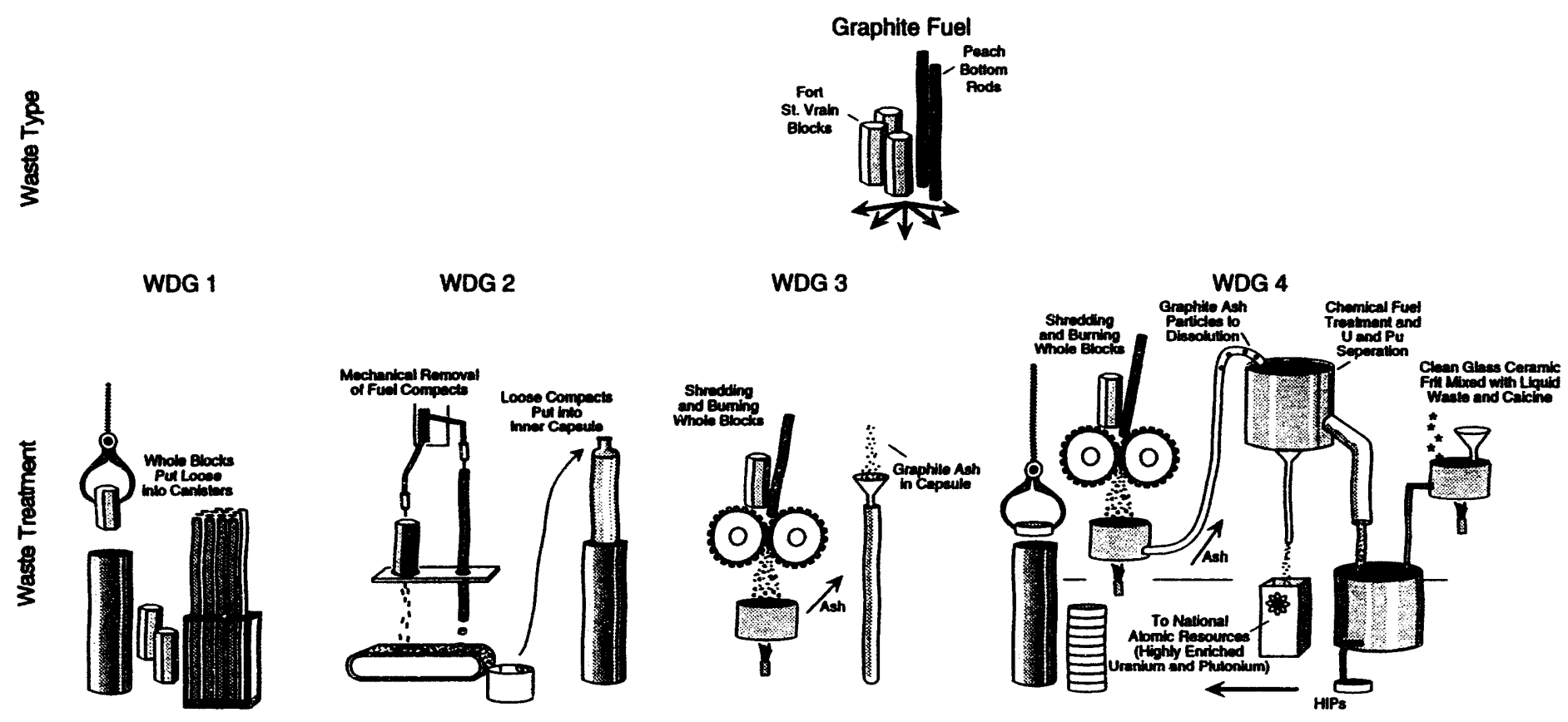

WDG 5
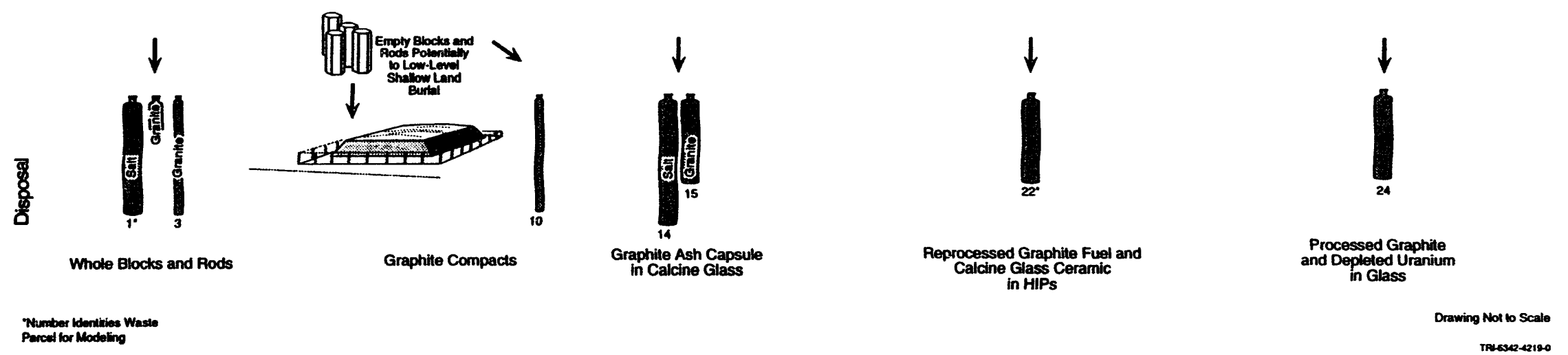

Figure 4-8. Treatment options used for graphite spent fuel. 
8Я

8
8
8
8
$\frac{8}{8}$
巡
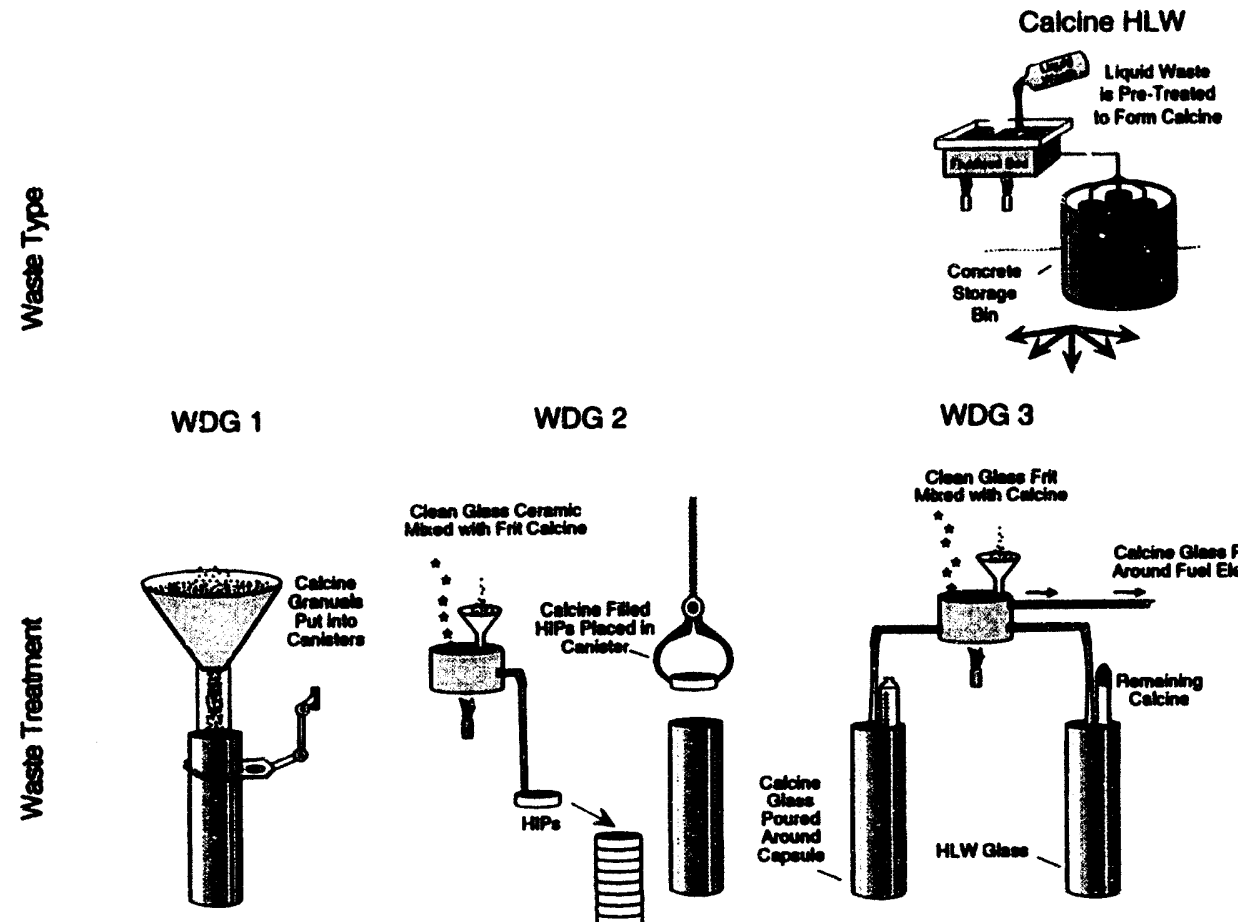

WDG 2

WDG 3

WDG 4
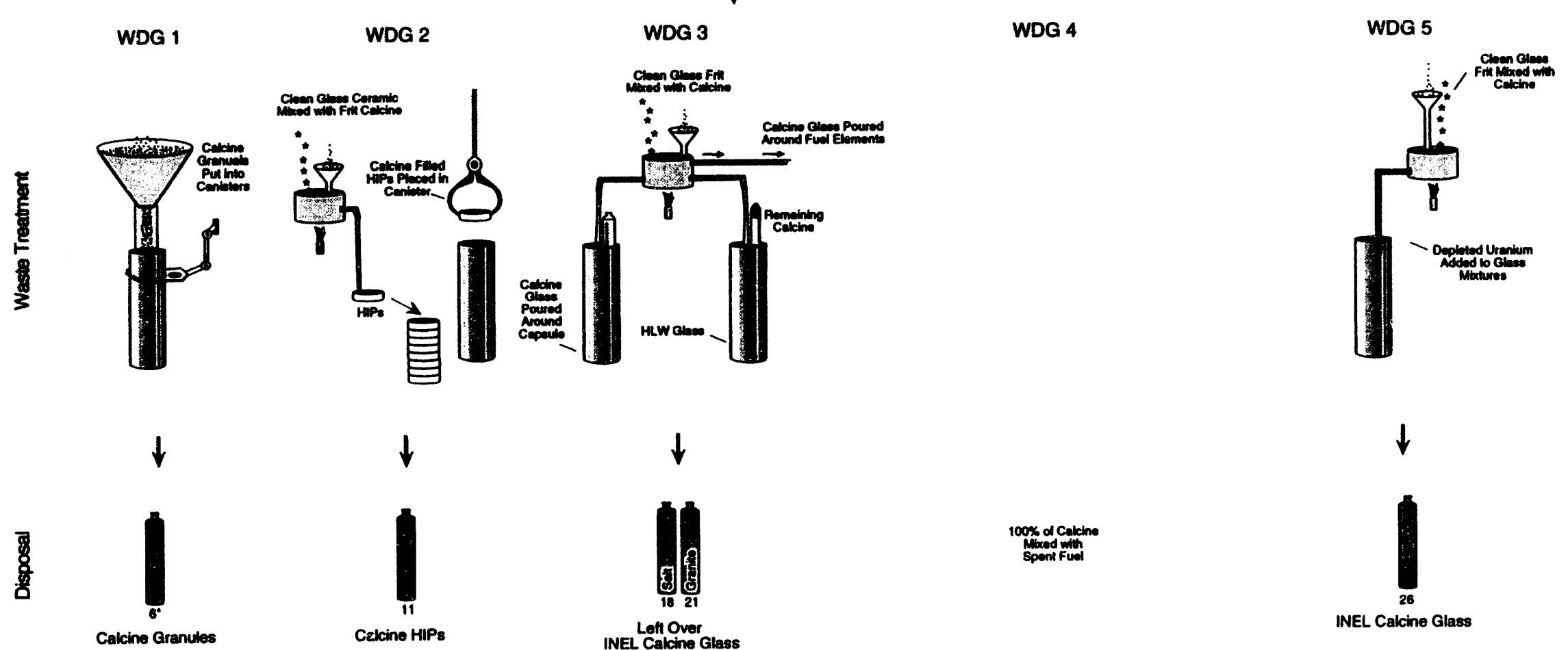

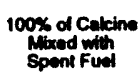

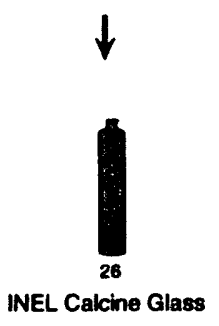

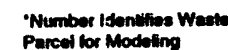
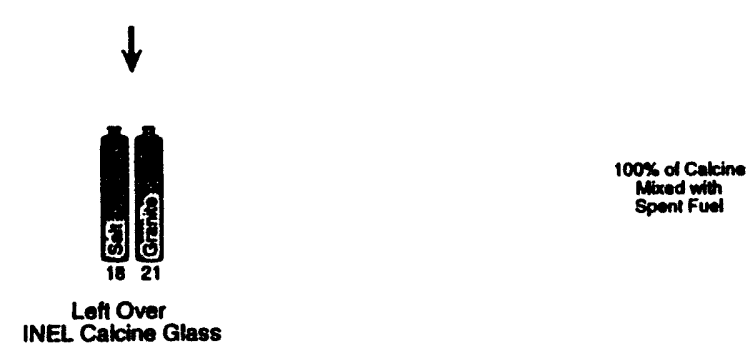

INEL Calche Glass

Draming Not to Scelb 


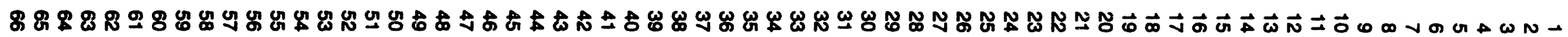
స̃
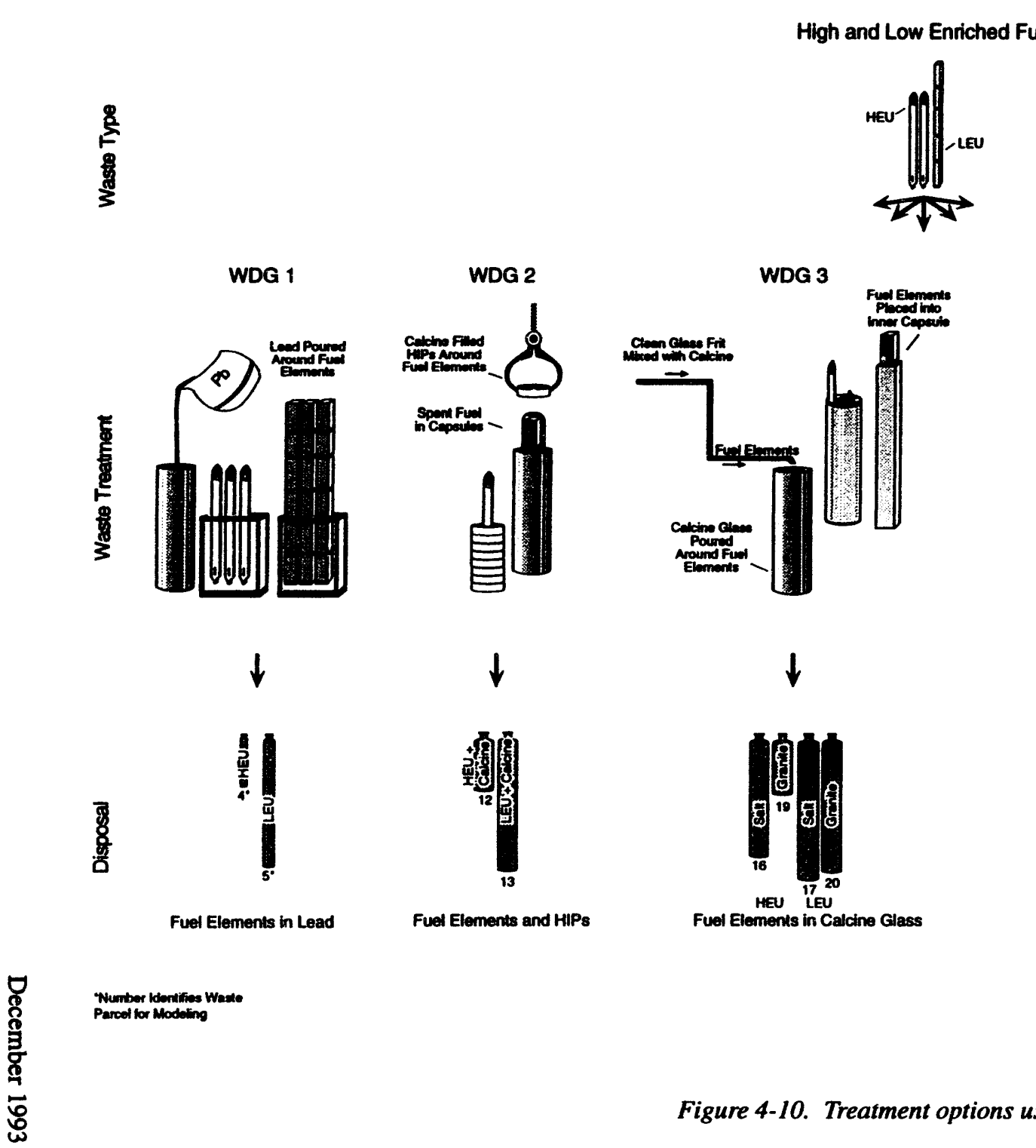
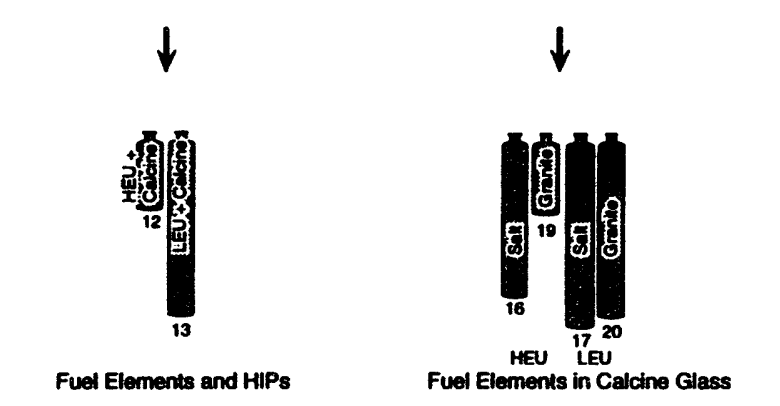

Fuel Elements in Calcine Glass

Fuel Elements and HIPs
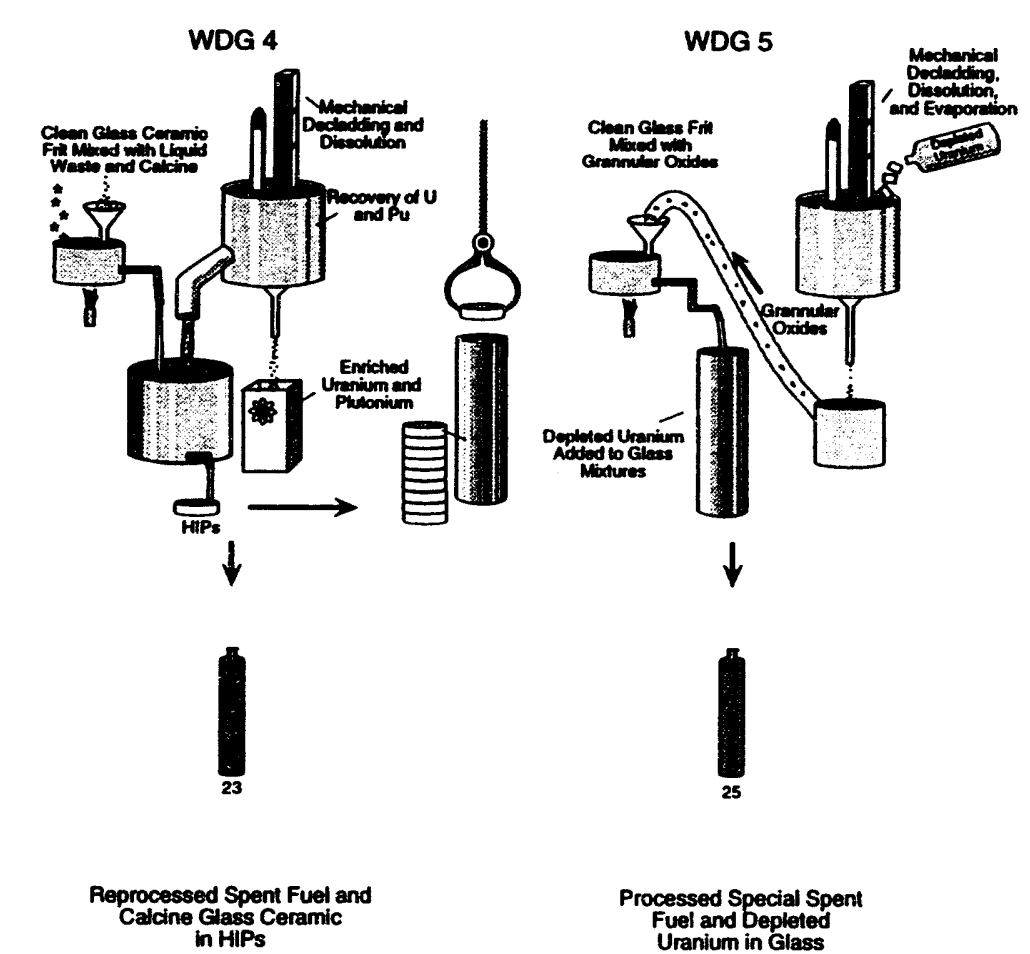

Fuel and Depleted

Drawing Not 10 Scalb 


\subsubsection{Processing Options for Calcine Waste}

For the calcine high-level waste (Figure 4-10), the five options include (1) calcining all liquid high-level waste and placing all the granular calcine in canisters (granular calcine), (2) calcining all liquid high-level waste and immobilizing the calcine by mixing with additives, then using the Hot Isostatic Press (HIP) process to form a glassceramic, and packaging all the glass-ceramic either separately or with the special spent fuel (spent fuel/glassceramic), (3) immobilizing the liquid high-level waste and calcine by mixing with additives and melting to form a glass, and either packaging separately or filling the void space around the special spent fuel (spent fuel/borosilicate glass), (4) mixing the calcine with the reprocessed spent fuels, immobilizing the calcine by mixing with additives, and then using the HIP process to form a glass-ceramic (spent fuel recycling/glass-ceramic), and (5) immobilizing the calcine by mixing with additives, and then melting to form glass (spent fuel recycling/borosilicate glass).

\subsection{Description of Waste Parcels}

The waste parcel (WP) is defined as the waste form, waste canister, internal backfill (or redundant barrier), and overpack for structural reinforcement or corrosion-resistant protection (Figure 4-11). Two types of waste containers are included in the waste parcel: a canister and an overpack. The waste canister encloses the waste form; for this performance assessment, the canister material is either stainless steel $304 \mathrm{~L}(70.89 \%-\mathrm{Fe}, 18 \%-\mathrm{Cr}, 8 \%-\mathrm{Ni}, 2 \%-\mathrm{Mn}, 1 \%$ $\mathrm{Si}, 0.05 \%-\mathrm{P}, 0.03 \%-\mathrm{C}, 0.03 \%-\mathrm{S})$ or Inconel $625(69 \%-\mathrm{Ni}, 22 \%-\mathrm{Cr}, 9 \%-\mathrm{Mo})$. The overpack provides structural reinforcement and corrosion resistance; the overpack material for this study is either Inconel 625 or Titanium ASTM Grade $12(98.9 \%-\mathrm{Ti}, 0.8 \%-\mathrm{Mo}, 0.3 \%-\mathrm{Ni})$, denoted here as Ti-Grade 12.

For this performance assessment, neither the overpack nor the internal backfill was modeled in the majority of the calculations. The overpack was omitted in response to preliminary calculations, which showed that the over-

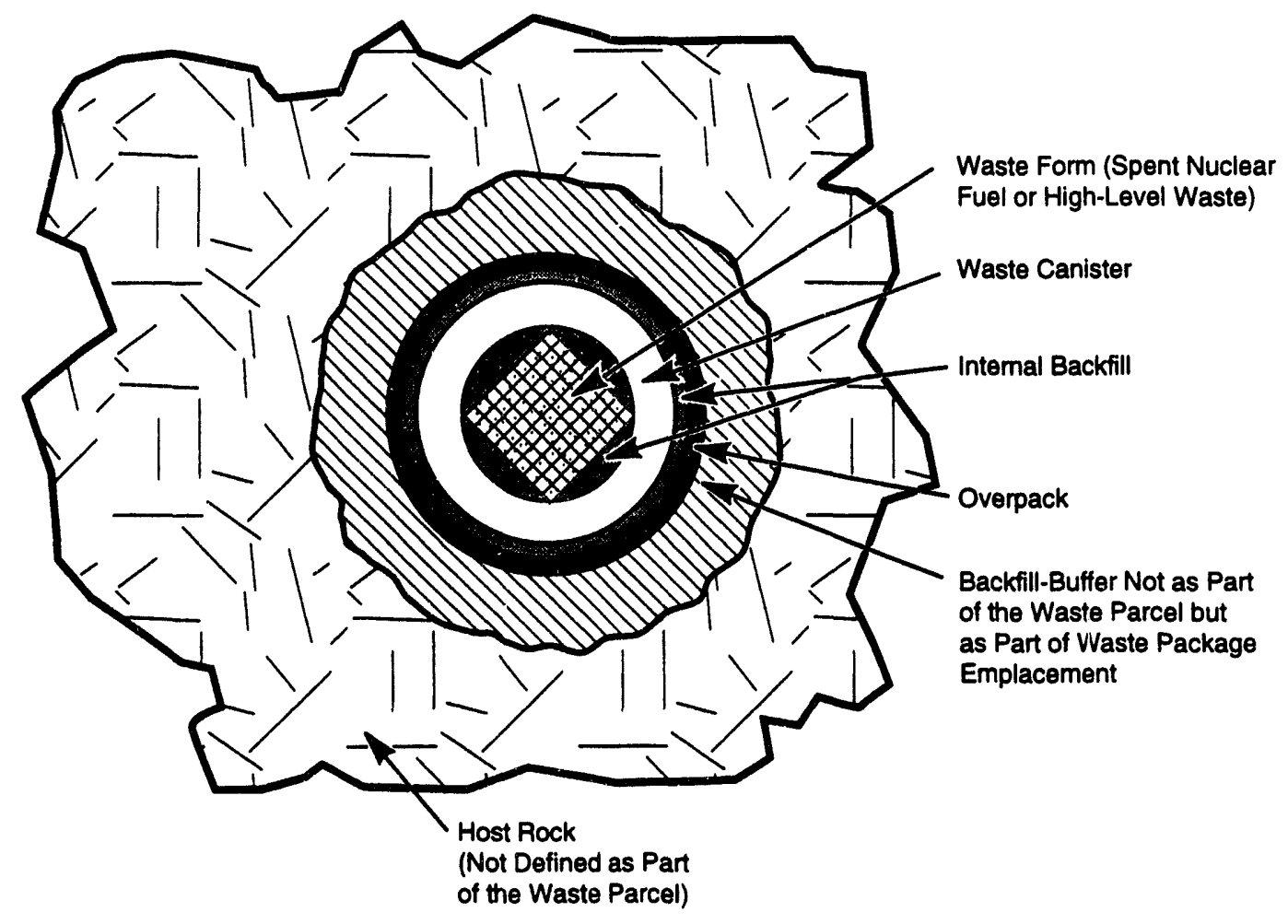

TRI-6342-2075-0

Figure 4-11. Schematic of cross-section of waste parcel, consisting of waste form, waste containers (canister and overpack), and internal backfill. 


\section{Description of Waste and Waste Parcels}

pack's role as a barrier tended to overwhelm the effects of the waste treatments. Therefore to maintain the focus of the calculations on the waste-treatment options, the waste parcel for the calculations primarily consists of the waste form and waste canister. The canister material is stainless steel 304L. To study the effect of the overpack, one set of calculations was run with an overpack (see Chapter 16). For the salt repository, the waste canister was Inconel 625 with a Ti-Grade 12 overpack. For the granite repository, both waste canister and overpack were Inconel 625 . A lead internal backfill was used for both the salt and granite repositories. Ti-Grade 12 is much more suitable for the corrosive environment in the salt repository and thus it was chosen as the overpack material for the salt site. Inconel 625 was chosen for the granite site based on its lower cost and adequate corrosion resistance.

\subsubsection{Waste Canister}

The waste canister is a physical barrier for containment. It must provide sufficient mechanical strength for waste transportation and handling operations. It must also be able to withstand the high temperature of the waste processing (e.g., glassification inside the canister or ceramic heater processes) (Molecke et al., 1981, p. 8). Canister designs for the salt and granite repositories were selected based on several canister concepts, including corrosion designs, corrosion-resistant designs, and self-shielding designs. For both the salt and granite repositories, the standard waste canister is assumed to be a stainless steel $304 \mathrm{~L}$ canister, with a wall thickness of $9.5 \mathrm{~mm}$.

Diameters and lengths of the canisters considered in this performance assessment vary greatly depending on waste type and form (see Figure 4-12). The standard canisters are thin-walled cylindrical canisters with domed tops. The domed or curved head is a more efficient canister design and allows less material to be subjected to high stress levels (Trabia and Kiley, 1992, p. 1252).

\subsubsection{Internal Backfill}

Usually a gap exists between the waste canister and the overpack, which is filled with molten metal. This gap between the inner and outer containers is to be filled with lead to transfer structural and heat loads between the two containers (Molecke et al., 1981, p. 30; Goodwin and Westerman, 1992). Such transfer of loads allows the overpack thickness to be designed on a corrosion-resistance criterion rather than structural considerations. Lead is being considered as a filler material in several waste management programs, e.g., in Sweden, Canada, Germany, Switzerland, and in the U.K (U.S. DOE, 1989). It should be noted that RCRA regulations may affect the decision to use lead in this application.

\subsubsection{Optional Canister Overpack}

The overpack is a container that acts as a primary containment barrier. Its function is to provide containment during the operating period (transfer, placement, and potential retrieval) and for thousands of years after repository closure (decommissioning).

As a primary containment barrier, the overpack should be designed for at least a 100 -yr corrosion lifetime (Tang and Saling, 1990, p. 125). In addition, the overpack should also be designed (with sufficient thickness) to resist crushing from external, lithostatic loads with sufficient allowance to prevent failure from any corrosion processes (Kircher and Bradley, 1983, p. 384).

Steel, copper, nickel-base alloys (such as Hastelloy $\mathrm{C} 4$ [66.9\%-Ni, 19.4\%-Cr, 13.8\%-Mo]), and titanium alloys (such as Ti-Grade 12 and Titanium ASTM Grade 2 [100\%-Ti]) are common overpack materials for waste parcels in various geologic-type repositories. The overpack material is an important (critical) compatibility selection when combined with the geological environment of the repository (i.e., granite, volcanic tuff, basalt, salt). Perhaps the most important material consideration for overpack longevity is resistance to corrosion (assuming the designed canister dimensions prevent structural failure from lithostatic loads and/or thermal-induced stress fields). 
2

3

4

6

7

8

9
10

11

12

13

14

15

16

17

18

19

20

21

22

23

24

25

26

27

28

29

30

31

32

33

34

35

36

37

38

39

40

41

42

43

44

45

46

47

48

49

50

51

52

53

54

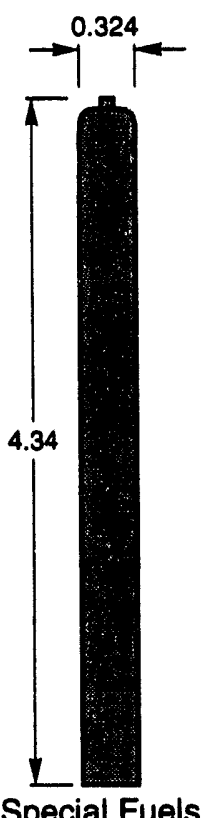

Group 1

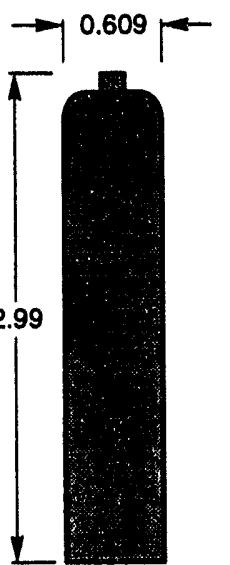

Special Fuels Group 4 Group 5

Graphite

Group 4

Group 5

Calcines

Group 1

Group 2

Group 3

Group 4

Group 5

DOE

All

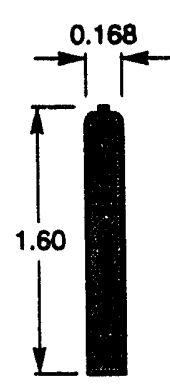

Special Fuels

Group 1
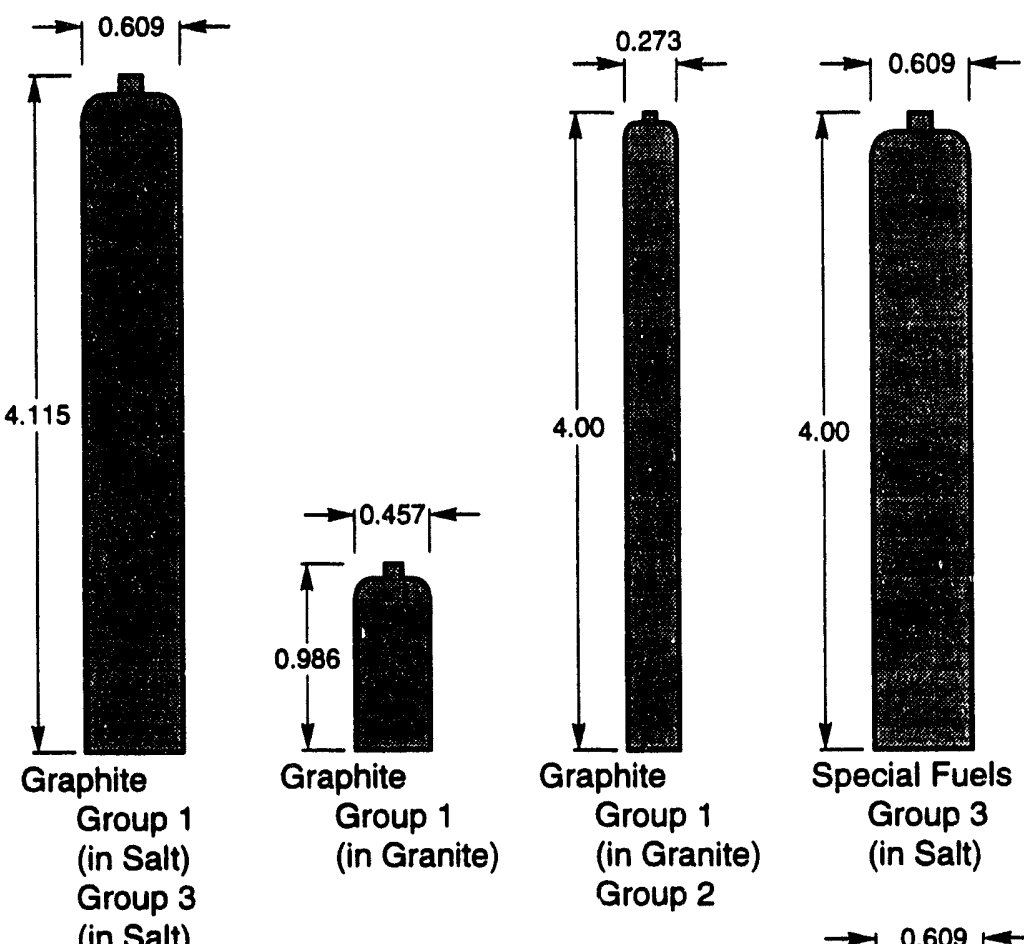

Graphite

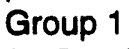

(in Granite)

Group 2

Special Fuels

Group 3

(in Salt)

(in Salt)
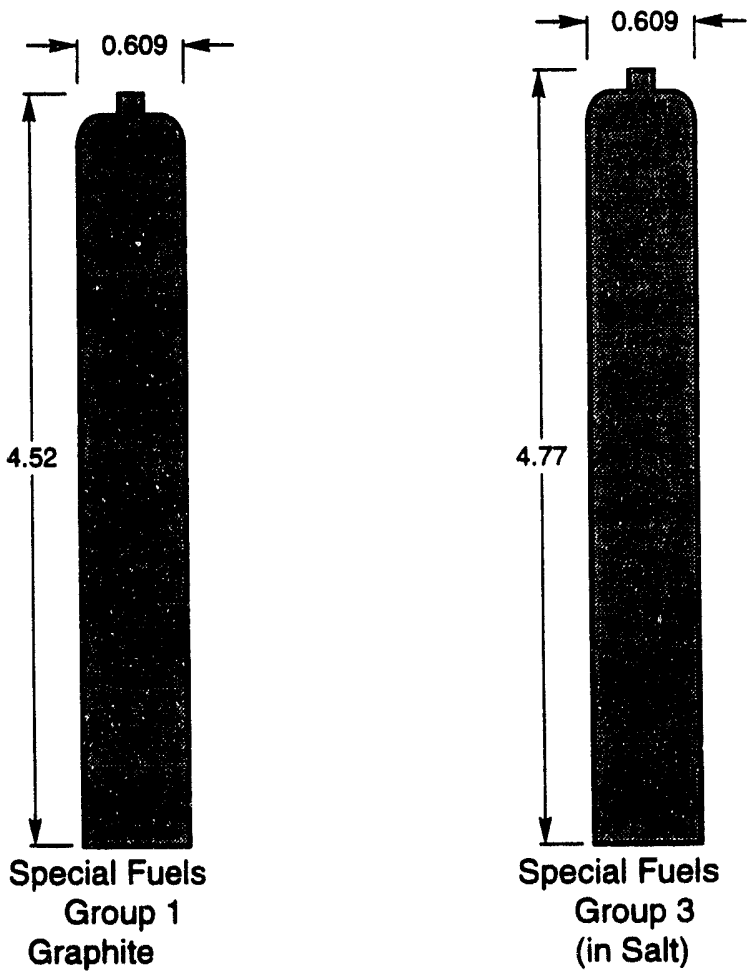

Calcines

Group 2

TRI-6342-3514-1

Figure 4-12. Relative sizes of 10 different canisters considered in this performance assessment (dimensions in meters). 


\section{Description of Waste and Waste Parcels}

As noted above, the overpack was not modeled in the majority of the calculations; only one set of calculations was run with the overpack and internal backfill (see Chapter 16). In this calculation, it was assumed that an Inconel 625 canister (not stainless steel 304L) is emplaced in an overpaci sometim's before being emplaced in the repository. The overpack is a $6.4 \mathrm{~mm}$ (1/4-in.) thick container (Molecke et ai., 1982; WEC, 1981) and is expected to provide enhanced corrosion resistance and capability, thus providing barrier protection for a long period of time.

\subsection{Waste Disposal Groups}

The five waste treatment options (Section 4.4) are the basis for the waste disposal groupings, which were created to study the effects of specific treatment options. For example, Waste Disposal Group 1 includes waste parcels that are designed to hold either graphite spent fuel, special spent fuel, or calcine waste that has been treated according to Option 1 for salt and/or granite repositories. Note that for the majority of the calculations, the waste parcel consists of the waste form and waste canister only, i.e., the overpack and internal backfill are not modeled (see Section 4.5 ).

This section describes the waste disposal groups, including treatment options per waste type and corresponding waste parcels. Table 4-8 provides a listing, by waste parcel number, of the waste parcels found in each waste disposal group. For all disposal groups, the liquid, slurries, and solid high-level waste stored at Savannah River, Hanford, and West Valley are assumed to be immobilized in a borosilicate glass.

Table 4-8. Waste Parcels (WPs) Found in Waste Disposal Groups 1 through 5

\begin{tabular}{|c|c|c|c|c|c|}
\hline & \multicolumn{5}{|c|}{ Waste Disposal Groups } \\
\hline & 1 & 2 & 3 & 4 & 5 \\
\hline Waste Parcels from INEL & $1-6$ & $10-13$ & $14-21$ & $22-23$ & $24-25$ \\
\hline \multicolumn{6}{|l|}{ Waste Parcels from DOE } \\
\hline $\begin{array}{l}\text { Waste Parcels } 7 \text { through } 9 \\
\text { River, Hanford, and West }\end{array}$ & $\begin{array}{l}\text { d to } e \\
\text { ctivel }\end{array}$ & Dorosilicat & ste fror & DOE cor & Savannah \\
\hline
\end{tabular}

\subsubsection{Waste Disposal Group 1}

Waste Disposal Group 1 includes six waste parcels (WPs) that correspond to treatment option 1 for graphite spent fuel, special spent fuel, and calcine waste (Table 4-9). Waste Disposal Group 1 also includes Waste Parcels 7 through 9, which were designed to enclose the borosilicate glass waste form from the three DOE sites, i.e., Savannah River, Hanford, and West Valley, respectively. (Note that Waste Parcels 7 through 9 are found in all five waste disposal groups.)

Of the five waste disposal groups, Waste Disposal Group 1 is subjected to the least degree of stabilization or reprocessing prior to disposal. As a consequence, the total volume of waste in Group 1 requires the largest number of waste parcels (up to 23,584$)^{* * *}$ of which 7519 are from the three DOE sites (Savannah River, Hanford, and West Valley). Waste Disposal Group 1 was also chosen to take advantage of relaxed ${ }^{235} \mathrm{U}$ criticality limits for the salt repository, and thus the waste parcel designs for disposal in salt are different from those for disposal in the granite repository.

*** 20,858 for salt repository and 23,584 for granite (see Table 6-1 in Chapter 6). The total number of waste parcels was recalculated based on waste volumes and waste parcel dimensions and does not necessarily agree with the numbers indicated in Appendix $A$. 
Selected Treatment Options. The treatment options for the waste stored at Idaho considered in Waste Disposal Group 1 include the following:

- Graphite spent fuel: Disposal of whole or cut graphite blocks with the fuel plugs in canisters at a repository (whole block disposal).

- Special spent fuel: Placing spent fuel in canisters and filling the void space with lead (spent fuel/lead disposal).

- Calcine Waste: Calcining all liquid high-level waste and placing all the granular calcine in canisters (granular calcine)

Corresponding Waste Parcels. A description of the waste parcels for Waste Disposal Group 1 is provided in Table 4-9 and shown in Figures 4-13 through 4-21.

Table 4-9. Waste Parcels (WPs) for Waste Disposal Group 1

\begin{tabular}{|c|c|c|c|c|c|}
\hline $\begin{array}{c}\text { Waste } \\
\text { Parcel } \\
\text { ID }\end{array}$ & & $\begin{array}{l}\text { sitory } \\
\text { pe }\end{array}$ & $\begin{array}{l}\text { Waste } \\
\text { Type }\end{array}$ & Source & $\begin{array}{l}\text { Treatment } \\
\text { Option }\end{array}$ \\
\hline WP 1 & Salt & & Spent fuel, graphite & $\begin{array}{l}\text { Fort St. Vrain } \\
\text { Peach Bottom }\end{array}$ & $\begin{array}{l}\text { Whole block } \\
\text { fuel assemblies }\end{array}$ \\
\hline WP 2 & & Granite & Spent fuel, graphite & Fort St. Vrain & Cut or whole block \\
\hline WP 3 & & Granite & Spent fuel, graphite & Peach Bottom & Fuel assembly in lead \\
\hline WP 4 & Salt & Granite & Spent fuel, special, HEU & Special & Fuel assembly in lead \\
\hline WP 5 & Salt & Granite & Spent fuel, special, LEU & Special & Fuel assembly in lead \\
\hline WP 6 & Salt & Granite & High-level waste & Chem Plant & Calcine, loose powder \\
\hline WP 7 & Salt & Granite & High-level waste & Savannah River & Borosilicate glass \\
\hline WP 8 & Salt & Granite & High-level waste & Hanford & Borosilicate glass \\
\hline WP 9 & Salt & Granite & High-level waste & West Valley & Borosilicate glass \\
\hline
\end{tabular}




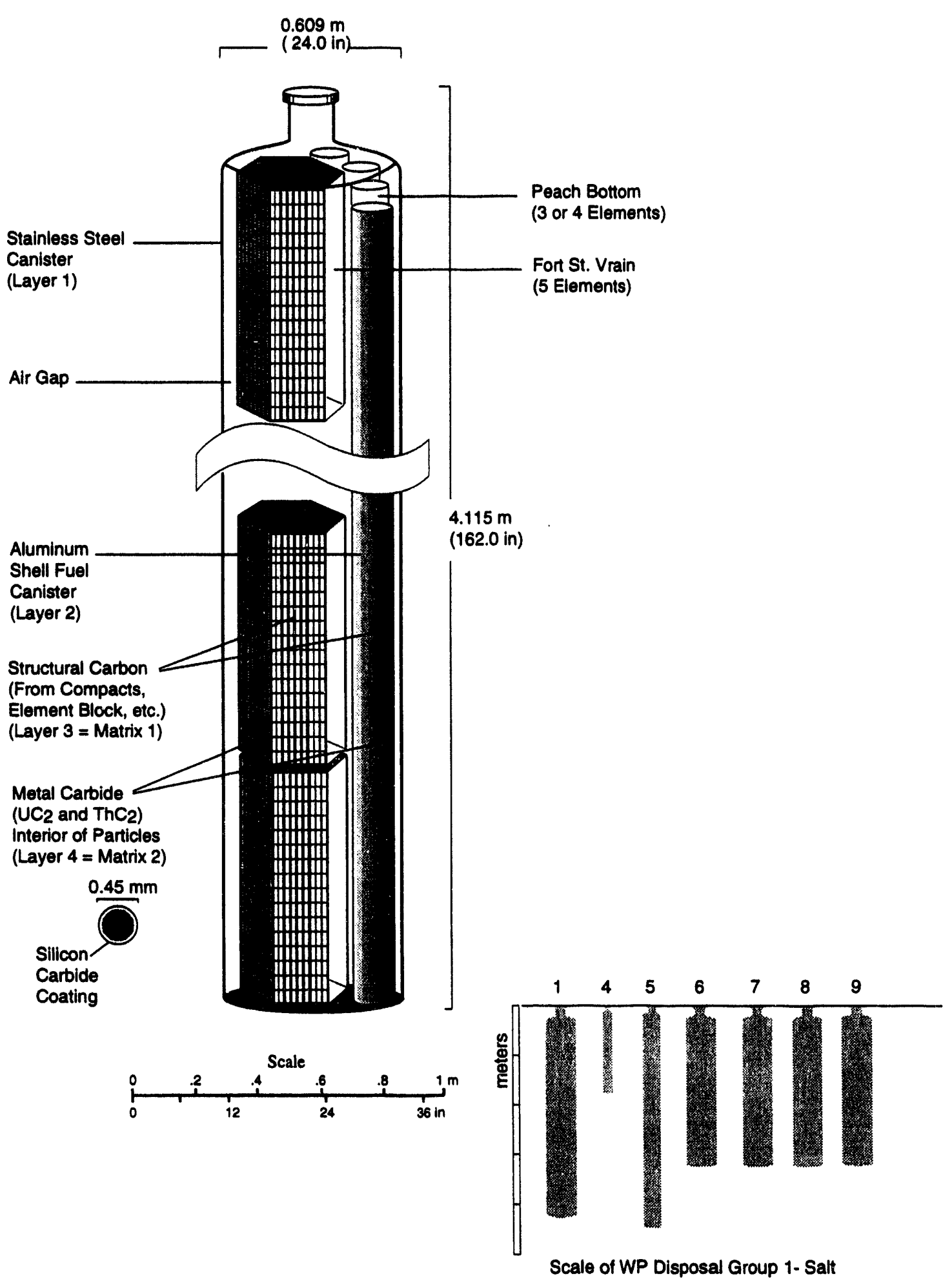

TRI-6342-3755-0

Figure 4-13. Waste Parcel 1 in Waste Disposal Group 1. This waste parcel contains whole block graphite spent fuel from Fort St. Vrain and Peach Bottom and is intended for disposal in a salt repository. (The layer and matrix designations are for the gas generation and source term submodels described in Chapter 11. The pertinent data for these layers are tabulated in Appendix D.) 

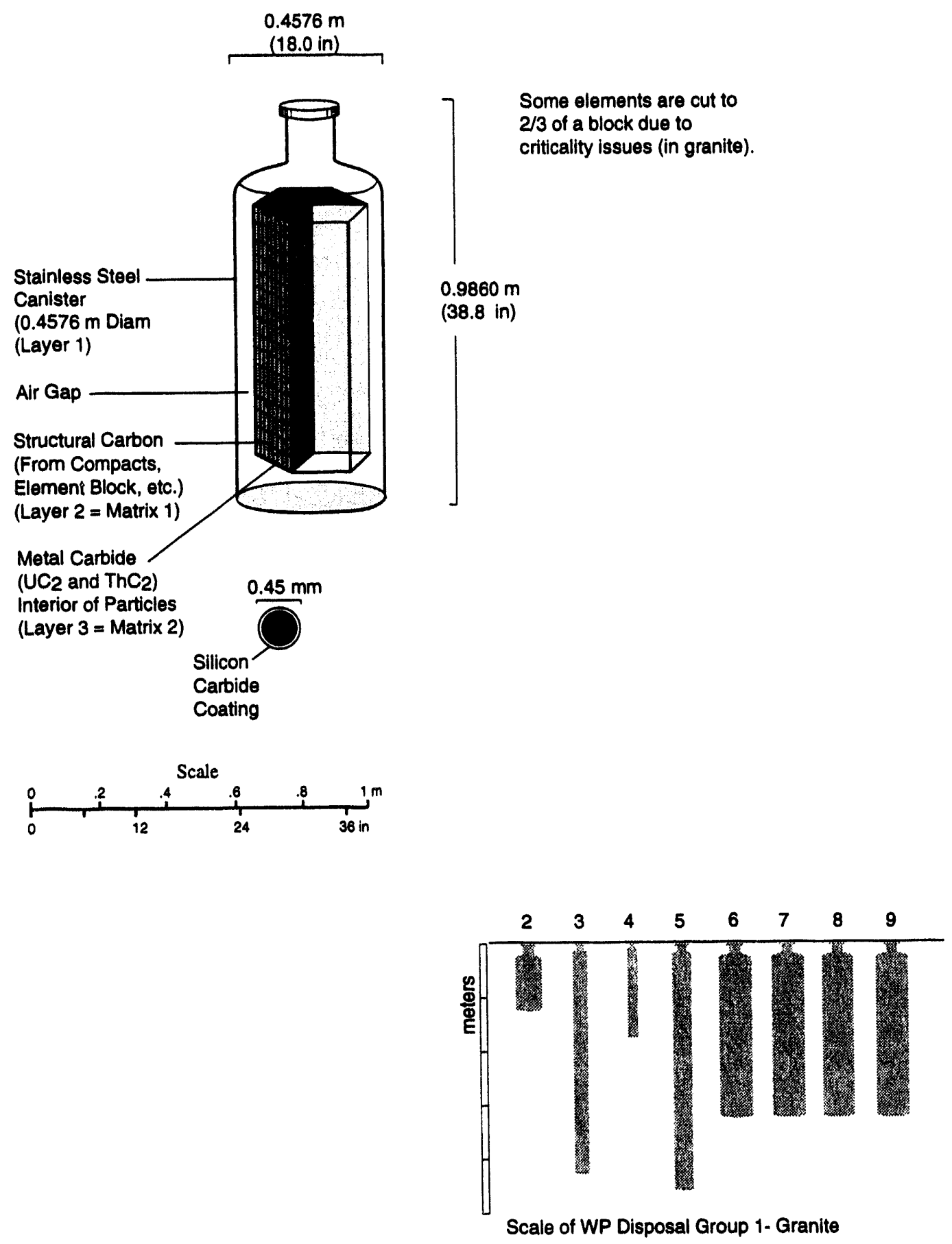

TRI-6342-3758-0

Figure 4-14. Waste Parcel 2 in Waste Disposal Group 1. This waste parcel contains cut or whole block graphite spent fuel from Fort St. Vrain and Peach Bottom and is intended for disposal in a granite repository. 


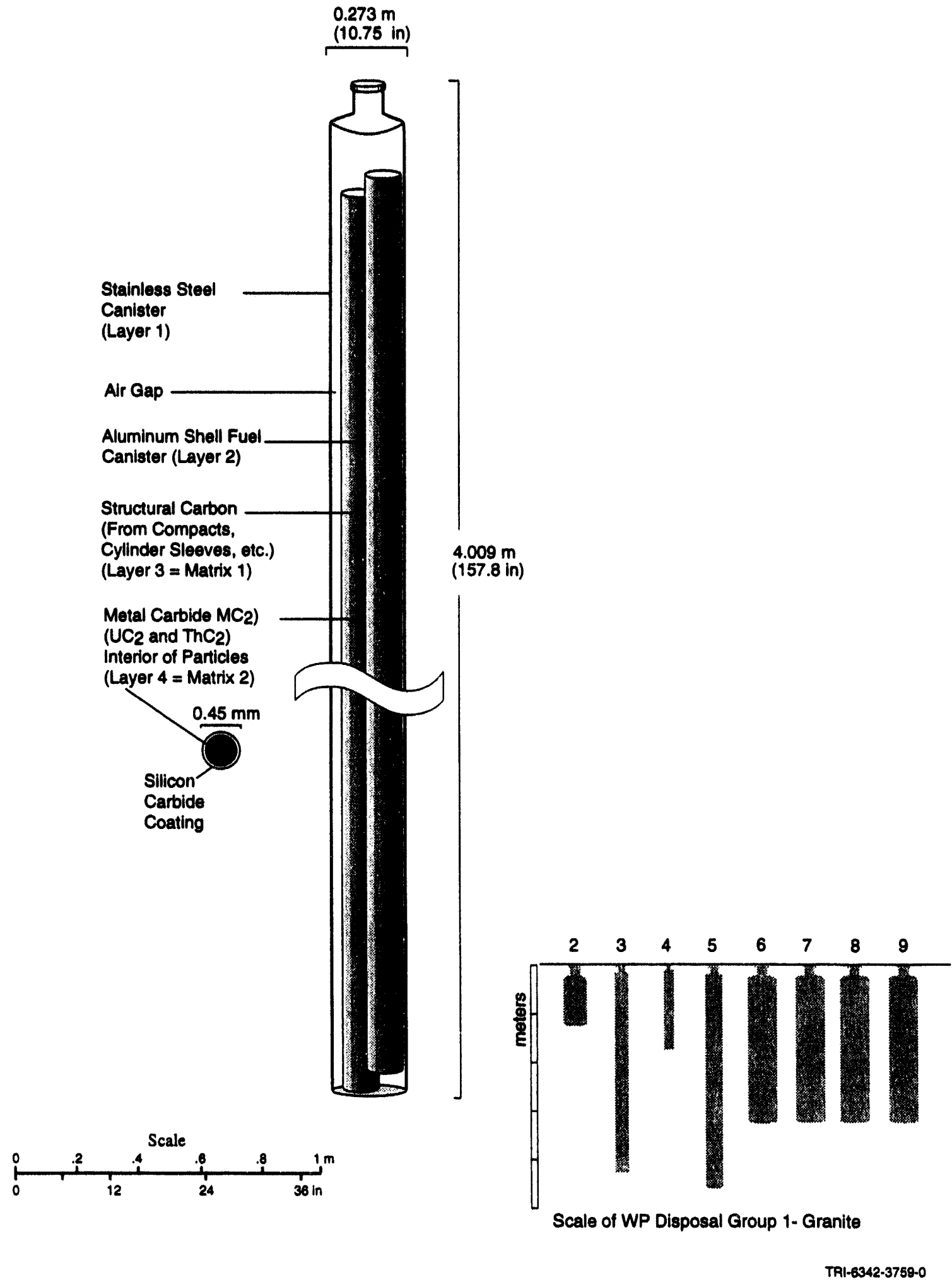

Figure 4-15. Waste Parcel 3 in Waste Disposal Group 1. This waste parcel contains fuel assemblies from Peach Bottom and is intended for disposal in a granite repository. 
1

3
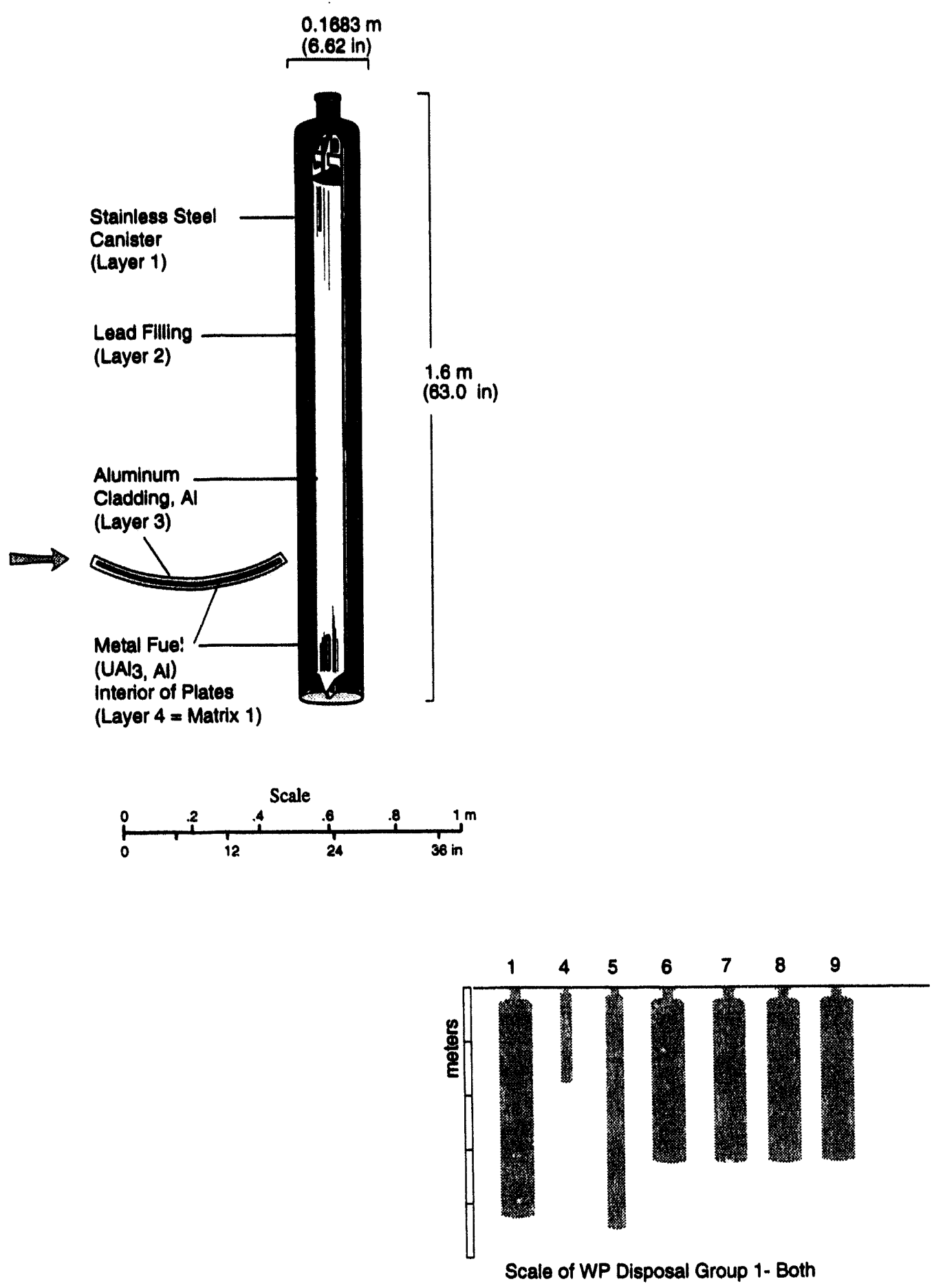

Figure 4-16. Waste Parcel 4 in Waste Disposal Group 1. This waste parcel contains fuel assemblies from special highly enriched uranium spent fuel and is intended for disposal in both salt and granite repositories. 
1

3

5

6

7

8

10

11

12

13

14

15

16

17

18

19

20

21

22

23

24

25

26

27

28

29

30

31

32

33

34

35

36

37

38

39

40

41

42

43

44

45

46

47

48

49

50

51

52

53

54

55

56

57

58

59

60

61

62

63

64

65

66

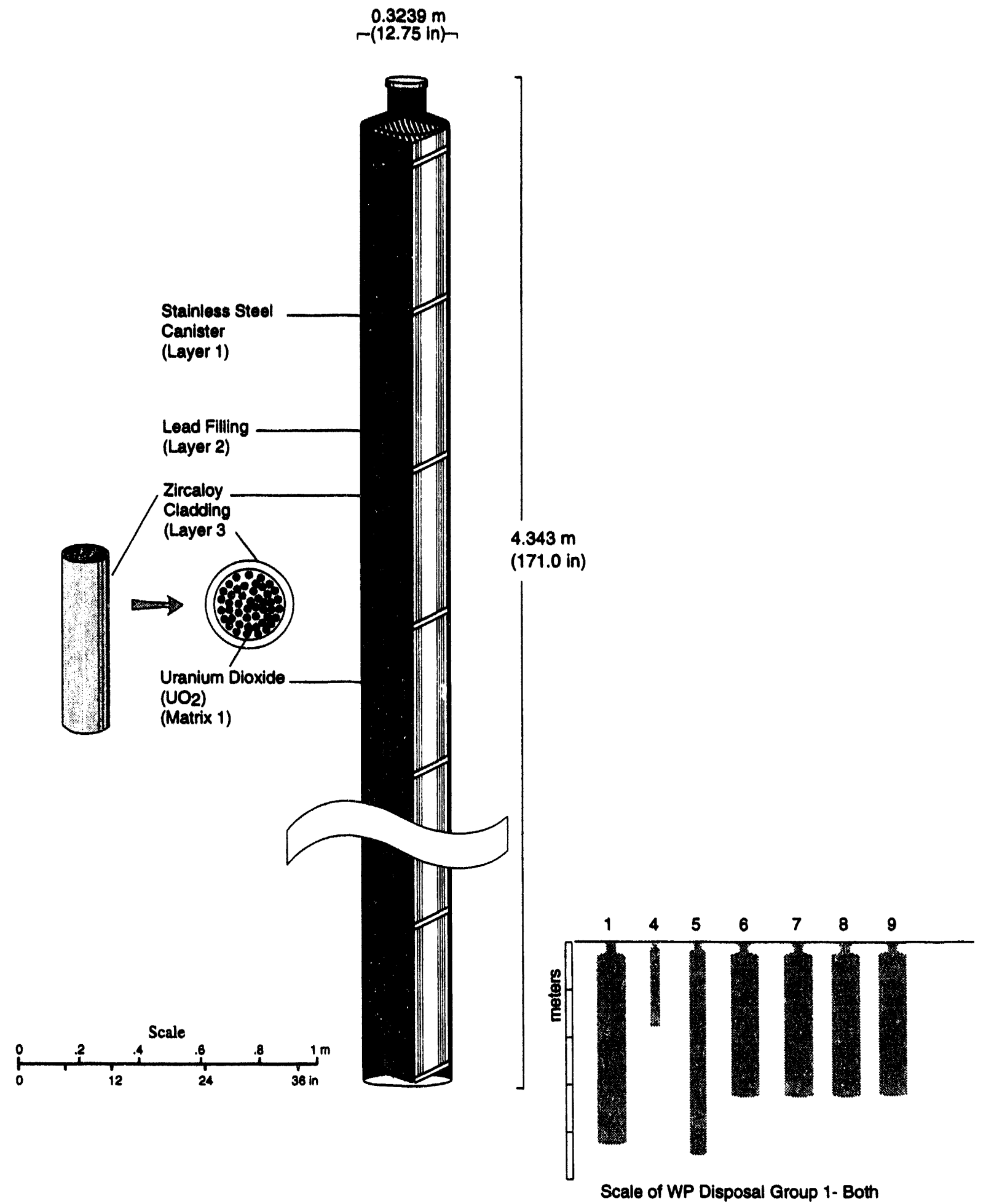

TR1-6342-3761.0

Figure 4-17. Waste Parcel 5 in Waste Disposal Group 1. This waste parcel contains fuel assemblies from special low enriched uranium spent fuel and is intended for disposal in both salt and granite repositories. 


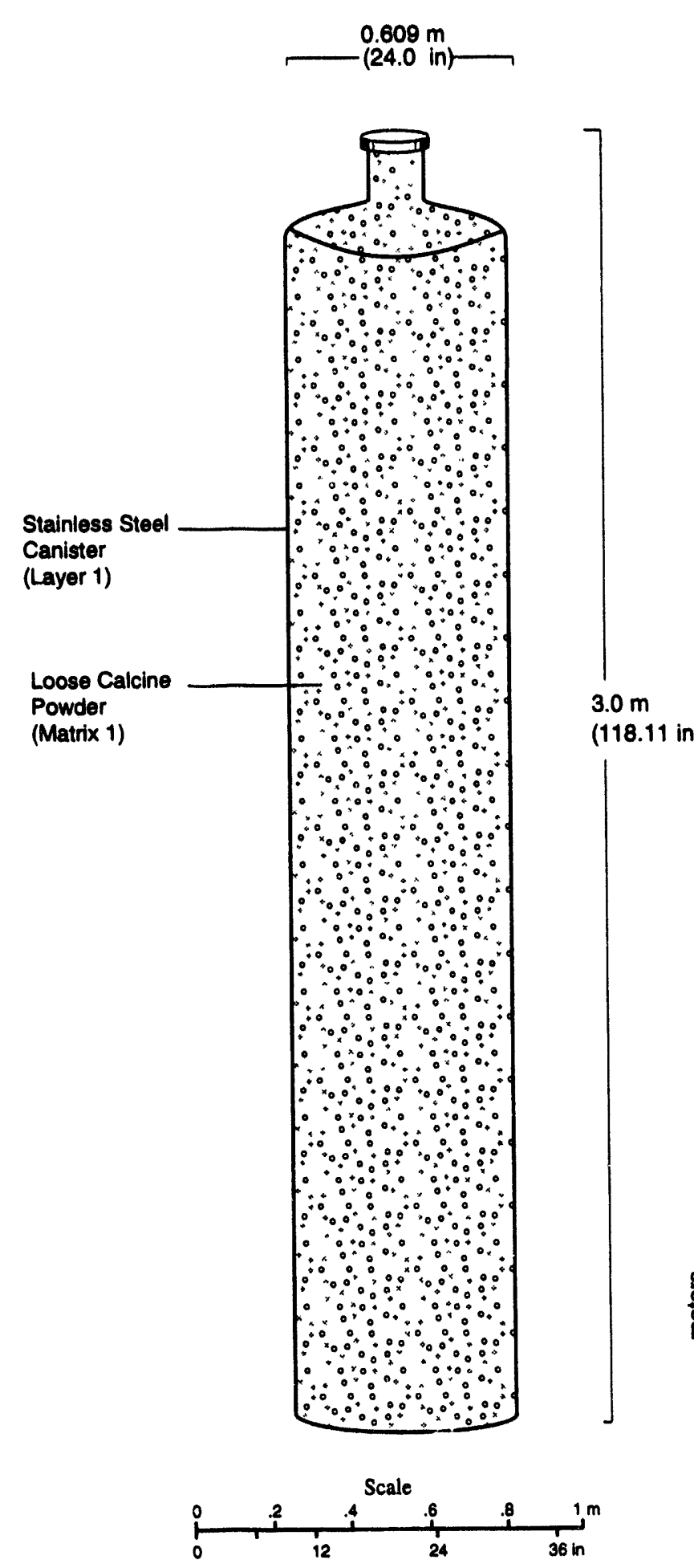

TRI-6342-3762-0

Figure 4-18. Waste Parcel 6 in Waste Disposal Group 1. This waste parcel contains calcine high-level waste and is intended for disposal in both salt and granite repositories. 

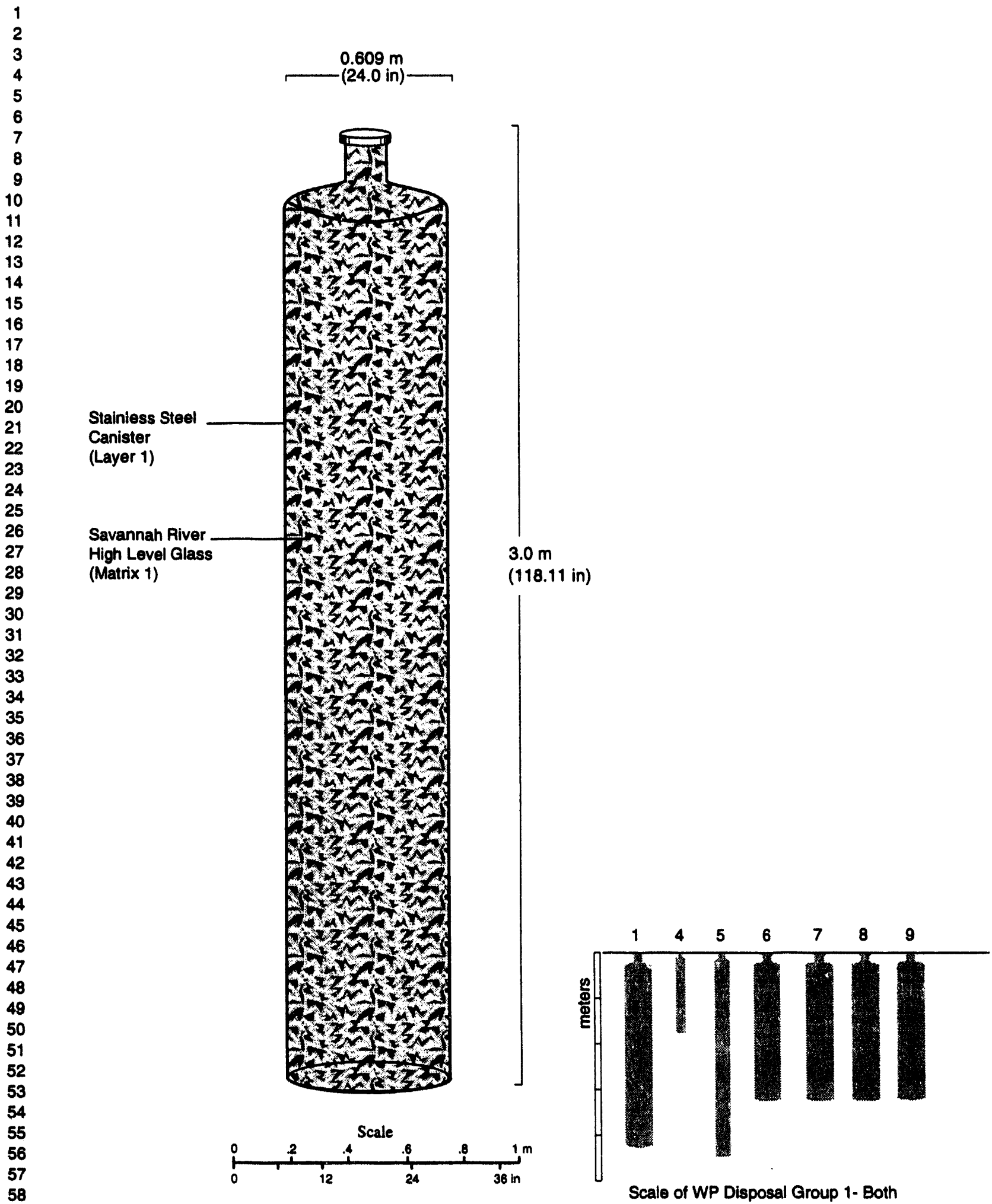

TRI-6342-3763-0

Figure 4-19. Waste Parcel 7 in all waste disposal groups. This waste parcel contains high-level waste in borosilicate glass from Savannah River and is intended for disposal in both salt and granite repositories. 
1

3

6

8

9
10

11

12

13

14

15

16

17

18

19

20

21

22

23

24

25

26

27

28

29

30

31

32

33

34

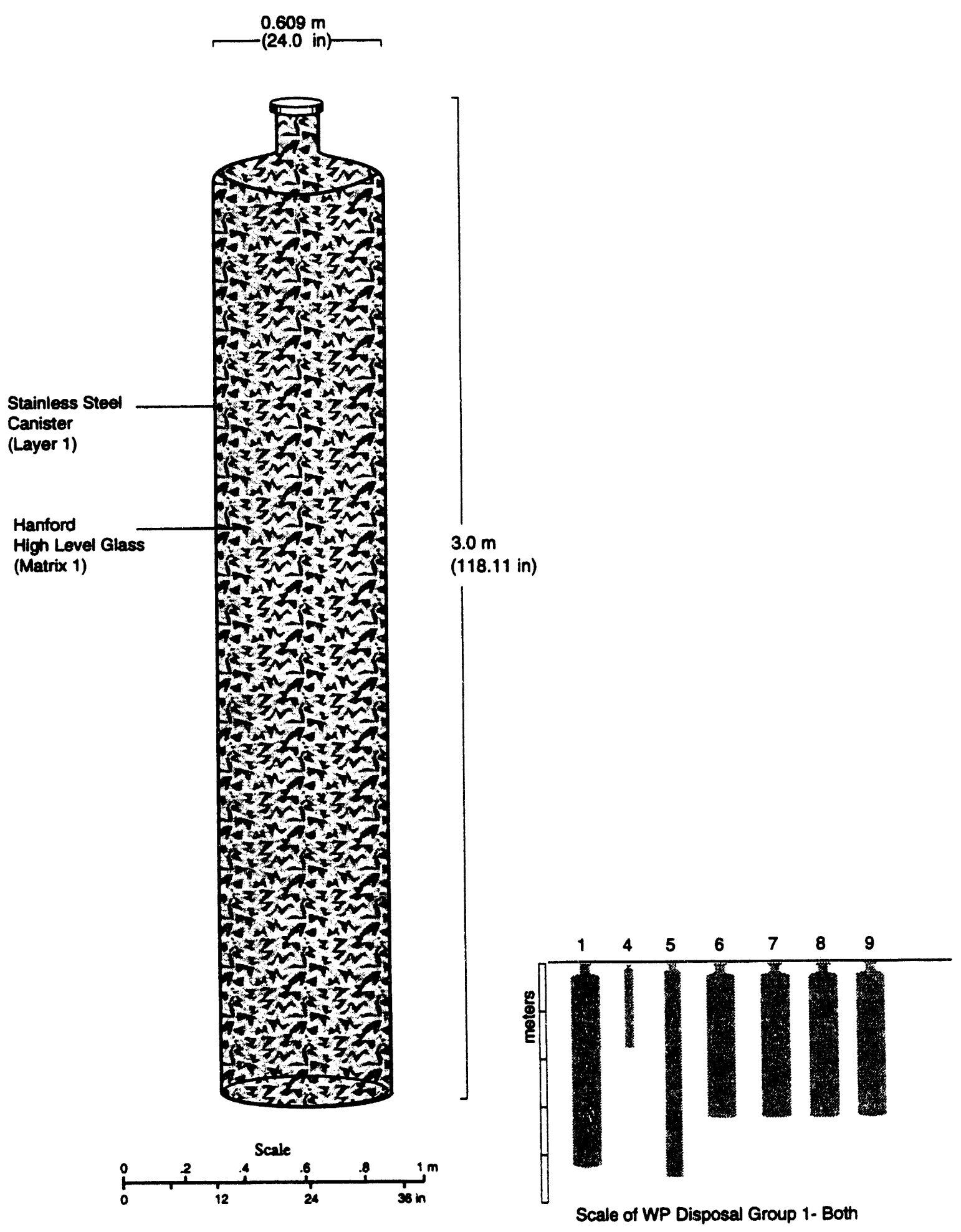

TR1-6342-3764-0

Figure 4-20. Waste Parcel 8 in all waste disposal groups. This waste parcel contains high-level waste in borosilicate glass from Hanford and is intended for disposal in both salt and granite repositories. 


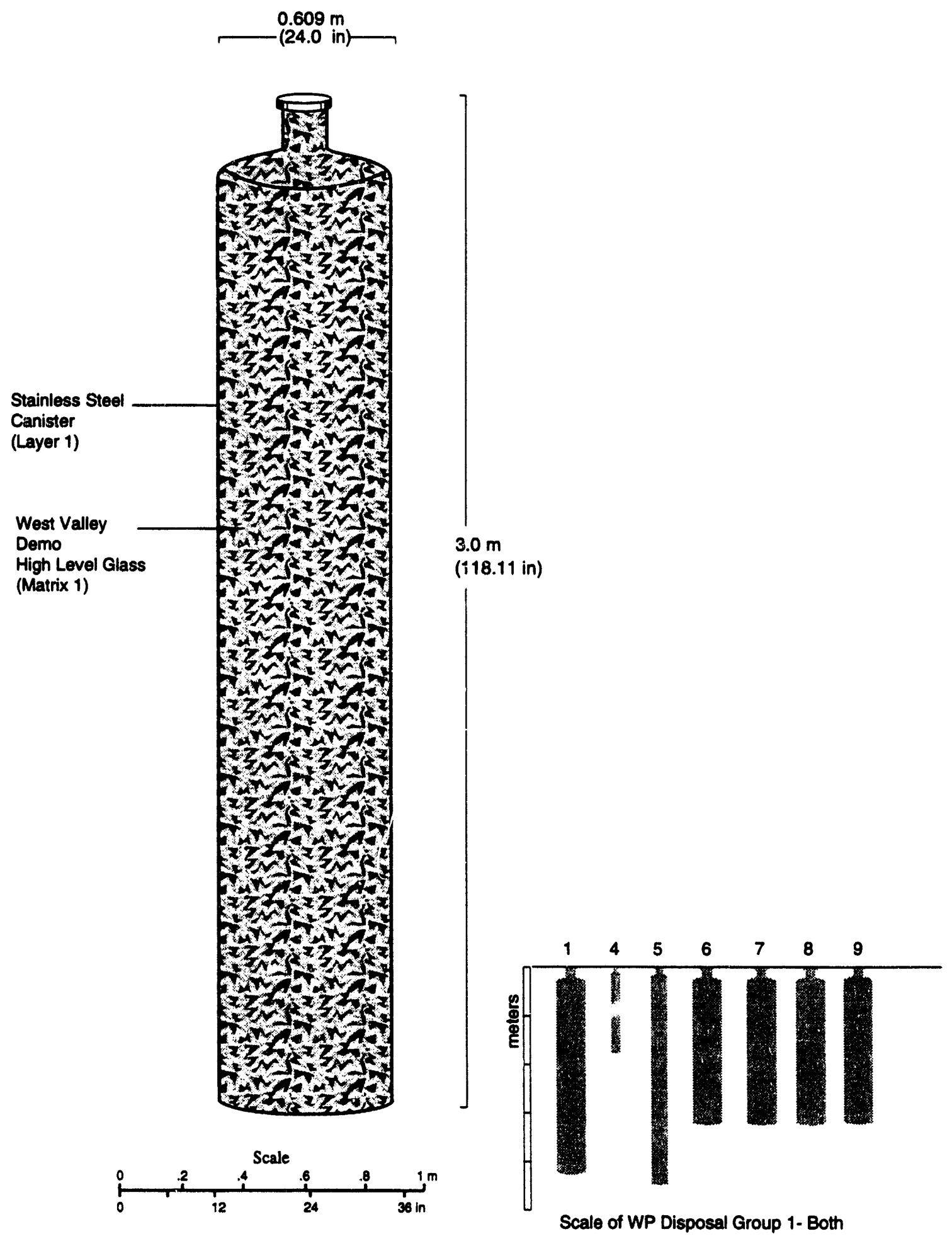

TR1-6342-3765-0

Figure 4-21. Waste Parcel 9 in all waste disposal groups. This waste parcel contains high-level waste in borosilicate glass from West Valley and is intended frr disposal in both salt and granite repositories. 


\subsubsection{Waste Disposal Group 2}

Waste Disposal Group 2 includes four waste parcels that correspond to treatment option 2 for graphite spent fuel, special spent fuel, and calcine waste (Table 4-10). Waste Disposal Group 2 also includes Waste Parcels 7 through 9 , which were designed to enclose the borosilicate glass waste form from the three DOE sites, i.e., Savannah River, Hanford, and West Valley, respectively.

This "moderate" reprocessing option requires 16,262 waste parcels, ${ }^{\dagger \dagger \dagger}$ of which 7519 are from the three DOE sites. The number of waste parcels required for the salt and granite repositories is the same.

Selected Treatment Options. The treatment options considered in Waste Disposal Group 2 include the following:

- Graphite spent fuel: Separation of the fuel and graphite block, disposal of the graphite block at a low-level waste site, clean glass encapsulation of the spent fuel, and spent fuel disposal in canisters at a repository (rod/ block separation)

- Special spent fuel: Placing spent fuel in canisters and filling the outer void space with calcine in compressed glass ceramic HIPs (spent fuel/glass-ceramic calcine)

- Calcine Waste: Calcining all the liquid high-level waste, adding glass-ceramic-forming additives to the calcine, and heating up to $1050^{\circ} \mathrm{C}$ and $140 \mathrm{MPa}$ in a Hot Isostatic Press (HIP) process and packaging all the calcine separately or with the special spent fuel (spent fuel/glass-ceramic calcine)

Corresponding Waste Emplacement Packages. A description of the waste parcels for Waste Disposal Group 2 is provided in Table 4-10; WPs 10 through 13 are shown in Figures 4-22 through 4-25 (waste parcels 7 through 9 were shown in Figures 4-19 through 4-21).

Table 4-10. Waste Parcels for Waste Disposal Group 2

\begin{tabular}{|c|c|c|c|c|c|}
\hline \multirow{2}{*}{$\begin{array}{c}\text { Waste } \\
\text { Parcel } \\
\text { ID }\end{array}$} & \multicolumn{2}{|c|}{$\begin{array}{c}\text { Repository } \\
\text { Type }\end{array}$} & \multirow{2}{*}{$\begin{array}{r}\begin{array}{c}\text { Waste } \\
\text { Type }\end{array} \\
\text { Spent fuel, graphite }\end{array}$} & \multirow{2}{*}{$\begin{array}{l}\text { Source } \\
\text { Fort St. Vrain } \\
\text { Peach Bottom }\end{array}$} & \multirow{2}{*}{ 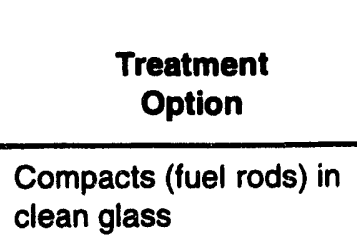 } \\
\hline & Salt & Granite & & & \\
\hline WP 11 & Salt & Granite & High-level waste & Chem Plant & $\begin{array}{l}\text { Calcine in HIPs" of glass } \\
\text { ceramic }\end{array}$ \\
\hline WP 12 & Salt & Granite & Spent fuel, special, HEU & Special & HIPS of glass ceramic \\
\hline WP 13 & Salt & Granite & Spent fuel, special, LEU & Special & HIPS of glass ceramic \\
\hline WP 7 & Salt & Granite & High-level waste & Savannah River & Borosilicate glass \\
\hline WP 8 & Salt & Granite & High-level waste & Hanford & Borosilicate glass \\
\hline WP 9 & Salt & Granite & High-level waste & West Valley & Borosilicate glass \\
\hline
\end{tabular}

${ }^{+\dagger \dagger} 16,262$ for both salt and granite repositories (see Table 6-1 in Chapter 6). The total number of waste parcels was recalculated based on waste volumes and waste parcel dimensions and does not necessarily agree with the numbers indicated in Appendix $A$. 


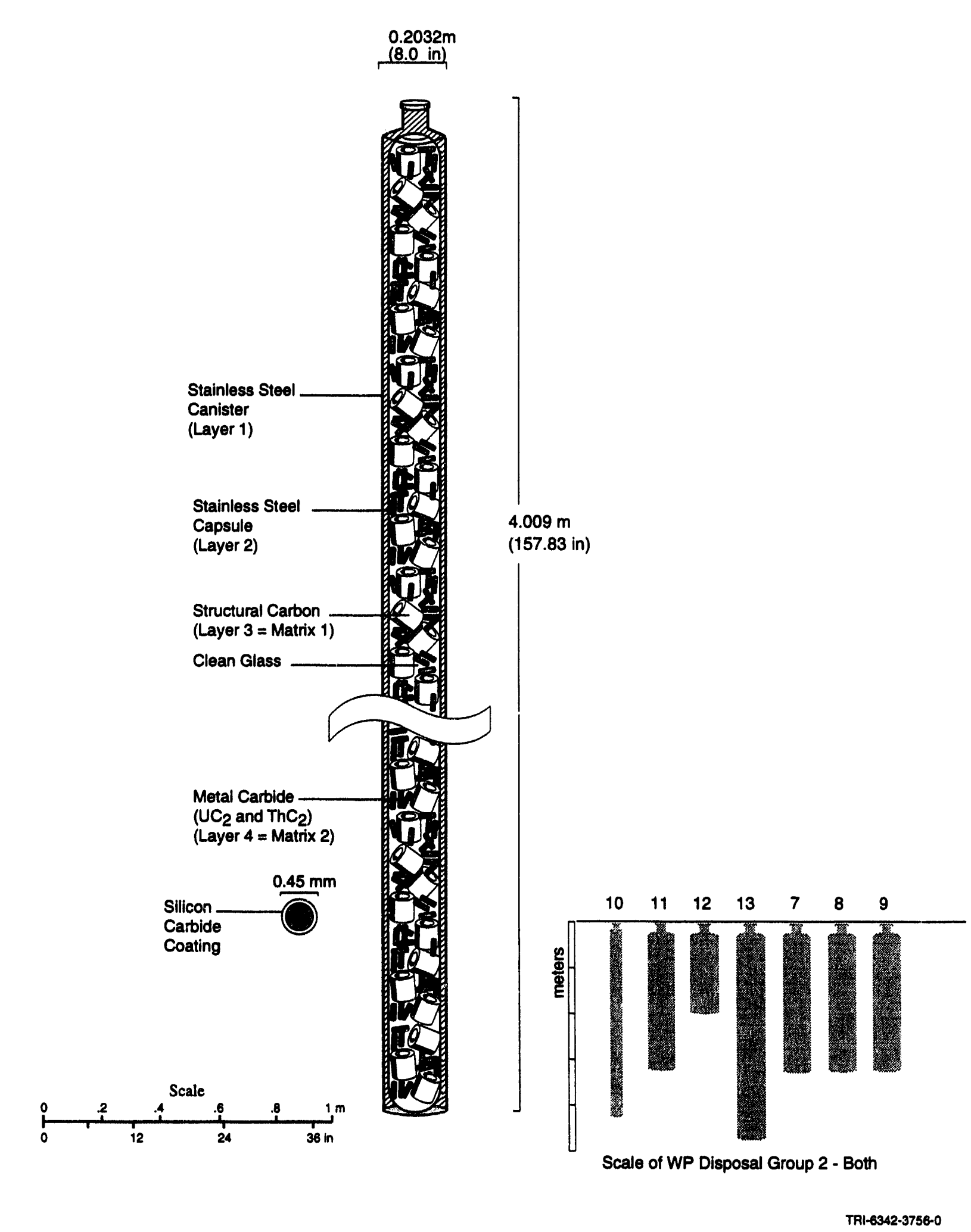

Figure 4-22. Waste Parcel 10 in Waste Disposal Group 2. This waste parcel contains compacts (fuel rods) in clean glass from Fort St. Vrain and Peach Bottom reactors and is intended for disposal in both salt and granite repositories. 


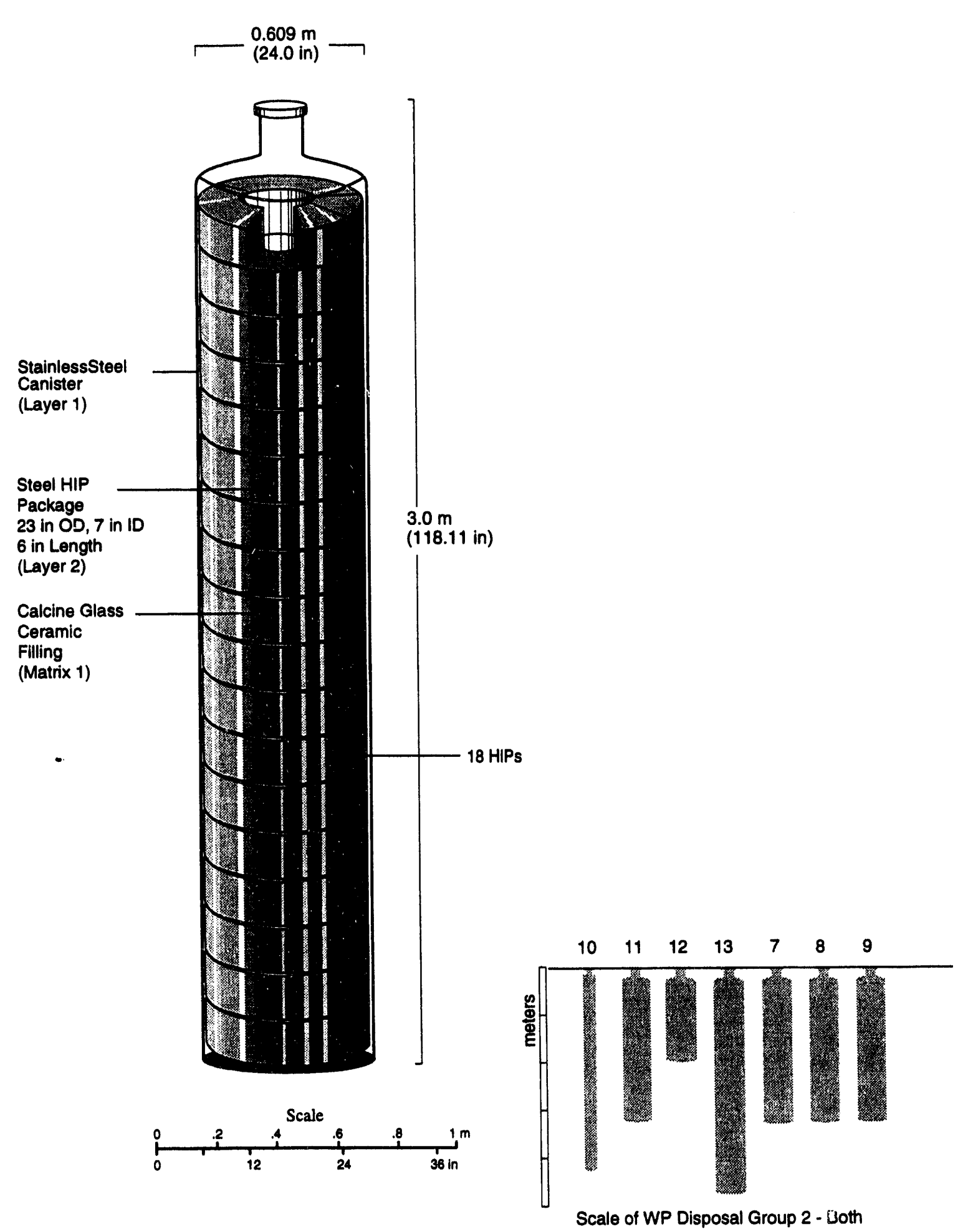

TRI-6342-3757-0

Figure 4-23. Waste Parcel 11 in Waste Disposal Group 2. This waste parcel contains calcine high-level waste in glass ceramic within 18 Hot Isostatic Press packages and is intended for disposal in both salt and granite repositories. 
2

3

4

6

7

8

9
10

11

12

13

14

15

16

17

18

19

20

21

22

23

24

25

26

27

28

29

30

31

32

33

34

35

36

37

38

39

40

41

42

43

44

45

46

47

48

49

50

51

52

53

54

55

56

57

58

59

60

61

62

63

64

65

66
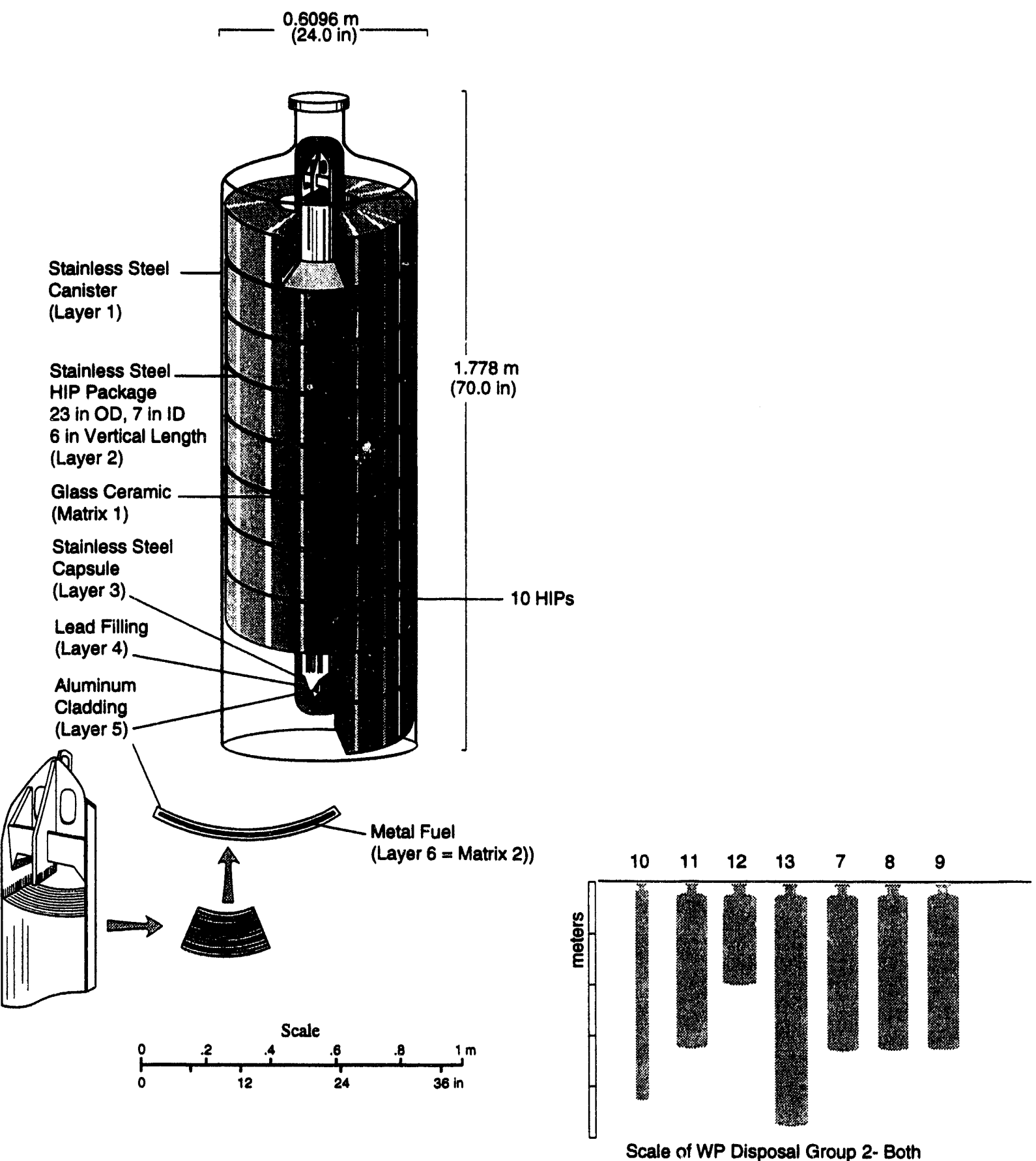

TRI-63\$2-3766-0

Figure 4-24. Waste Parcel 12 in Waste Disposal Group 2. This waste parcel contains special highly enriched uranium spent fuel in glass ceramic within 10 stainless-steel Hot Isostatic Press packages and is intended for disposal in both salt and granite repositories. 
2

3

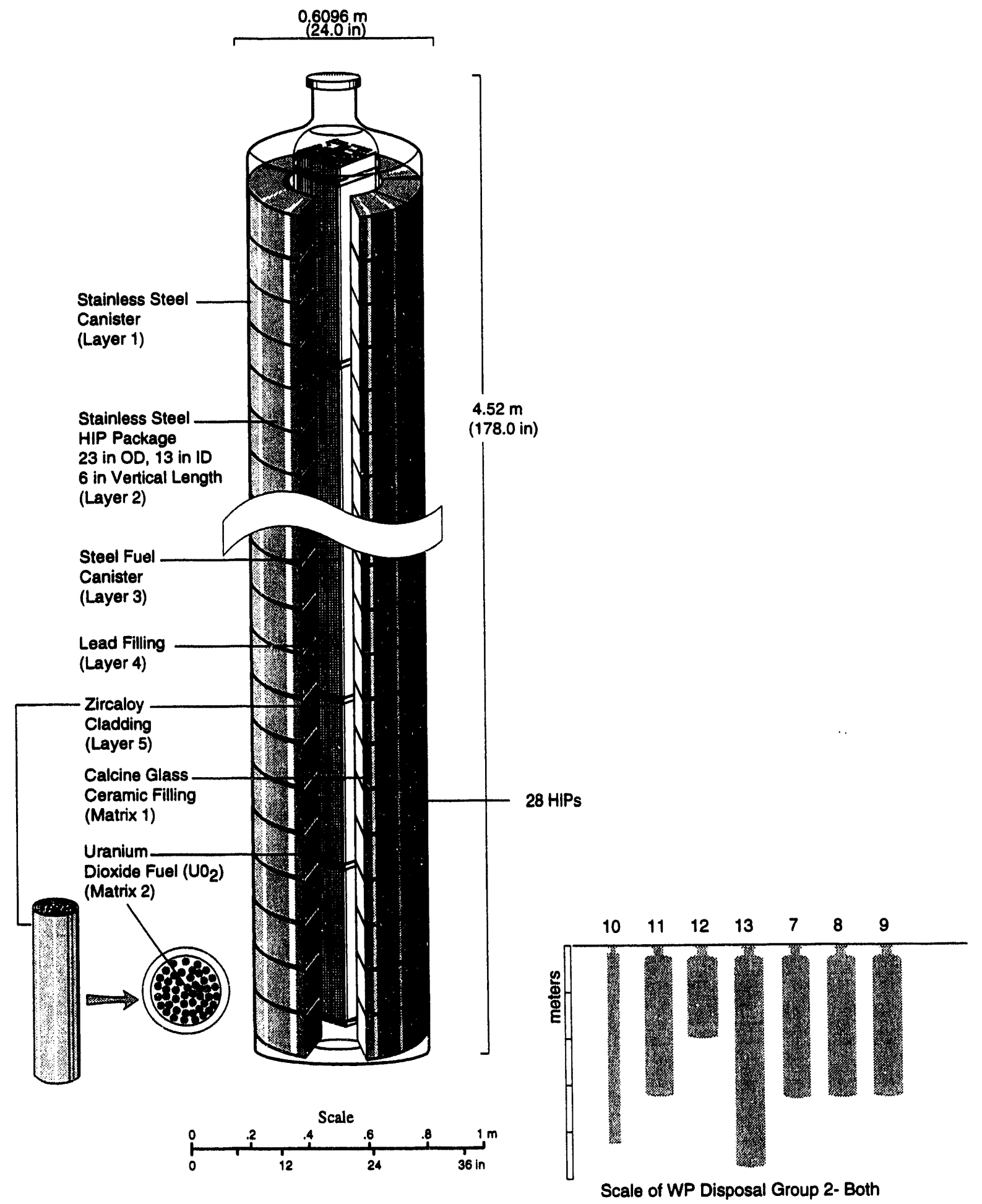

TRI-6342-3767-0

Figure 4-25. Waste Parcel 13 in Waste Disposal Group 2. This waste parcel contains special low enriched uranium spent fuel in glass ceramic within 28 stainless steel Hot Isostatic Press packages and is intended for disposal in both salt and granite repositories. 


\section{Description of Waste and Waste Parcels}

\subsubsection{Waste Disposal Group 3}

Waste Disposal Group 3 includes eight WPs that correspond to treatment option 3 for graphite spent fuel, special spent fuel, and calcine waste (Table 4-11). Waste Disposal Group 3 also includes Waste Parcels 7 through 9, which were designed to enclose the borosilicate glass waste form from the three DOE complexes, i.e., Savannah River, Hanford, and West Valley, respectively.

Up to 17,408 waste parcels ${ }^{\ddagger \ddagger \ddagger}$ are required for disposal of Group 3 waste, of which 7519 are from the three DOE sites. Like Waste Disposal Group 1, Group 3 was chosen to take advantage of relaxed ${ }^{235} \mathrm{U}$ criticality limits for disposal in a salt repository. Thus, the waste parcel designs for the salt and granite repositories are different.

Selected Treatment Options. The treatment options considered in Waste Disposal Group 3 include the following:

- Graphite spent fuel: Burning up the graphite and glass-ceramic encapsulation of the fuel particles (possibly after grinding up the fissile particles and additional graphite burning) (graphite burning)

- Special spent fuel: Placing spent fuel in canisters and filling the void space with lead and surrounding spent fuel with a borosilicate glass matrix (spent fuel/glass-ceramic calcine)

- Calcine Waste: Placing the liquid high-level waste and calcine in a borosilicate glass matrix and packaging separately or filling the void space around the special spent fuel (spent fuel/borosilicate calcine)

Corresponding Waste Emplacement Packages. A description of the waste parcels for Waste Disposal Group 3 is provided in Table 4-11; WPs 14 through 21 are shown in Figures 4-26 through 4-33 (waste parcels 7 through 9 shown in Figures 4-19 through 4-21).

Table 4-11. Waste Parcels for Waste Disposal Group 3

\begin{tabular}{|c|c|c|c|c|c|}
\hline $\begin{array}{l}\text { Waste } \\
\text { Parcel } \\
\text { ID }\end{array}$ & Rep & sitory & $\begin{array}{l}\text { Waste } \\
\text { Type }\end{array}$ & Source & $\begin{array}{l}\text { Treatment } \\
\text { Option }\end{array}$ \\
\hline WP 14 & Salt & & Spent fuel, graphite & $\begin{array}{l}\text { Fort St Vrain } \\
\text { Peach Bottom }\end{array}$ & $\begin{array}{l}\text { Compacts, pulverized } \\
\text { graphite burned off; result- } \\
\text { ant powder }\end{array}$ \\
\hline WP 15 & & Granite & Spent fuel, graphite & $\begin{array}{l}\text { Fort St Vrain } \\
\text { Peach Bottom }\end{array}$ & $\begin{array}{l}\text { Compacts, pulverized } \\
\text { graphite burned off; result- } \\
\text { ant powder }\end{array}$ \\
\hline WP 16 & Salt & & Spent fuel, special, HEU & Special & 16 fuel assemblies in glass \\
\hline WP 17 & Salt & & Spent fuel, special LEU & Special & 3 fuel assemblies in glass \\
\hline WP 18 & Salt & & High-level waste & Chem Plant & Calcine in INEL glass \\
\hline WP 19 & & Granite & Spent fuel, special HEU & Special & $\begin{array}{l}\text { Single element in glass } \\
\text { ceramic }\end{array}$ \\
\hline WP 20 & & Granite & Spent fuel, special, LEU & Special & Element in glass ceramic \\
\hline WP 21 & & Granite & High-level waste & Chem Plant & Calcine in INEL glass \\
\hline WP 7 & Salt & Granite & High-level waste & Savannah River & Borosilicate glass \\
\hline WP 8 & Salt & Granite & High-level waste & Hanford & Borosilicate glass \\
\hline WP 9 & Salt & Granite & High-level waste & West Valley & Borosilicate glass \\
\hline
\end{tabular}

$\$ \neq 13,858$ for salt repository and 17,408 for granite (see Table 6-1 in Chapter 6). The total number of waste parcels was recalculated based on waste volumes and waste parcel dimensions and does not necessarily agree with the numbers indicated in Appendix $A$.

$-.6$ 


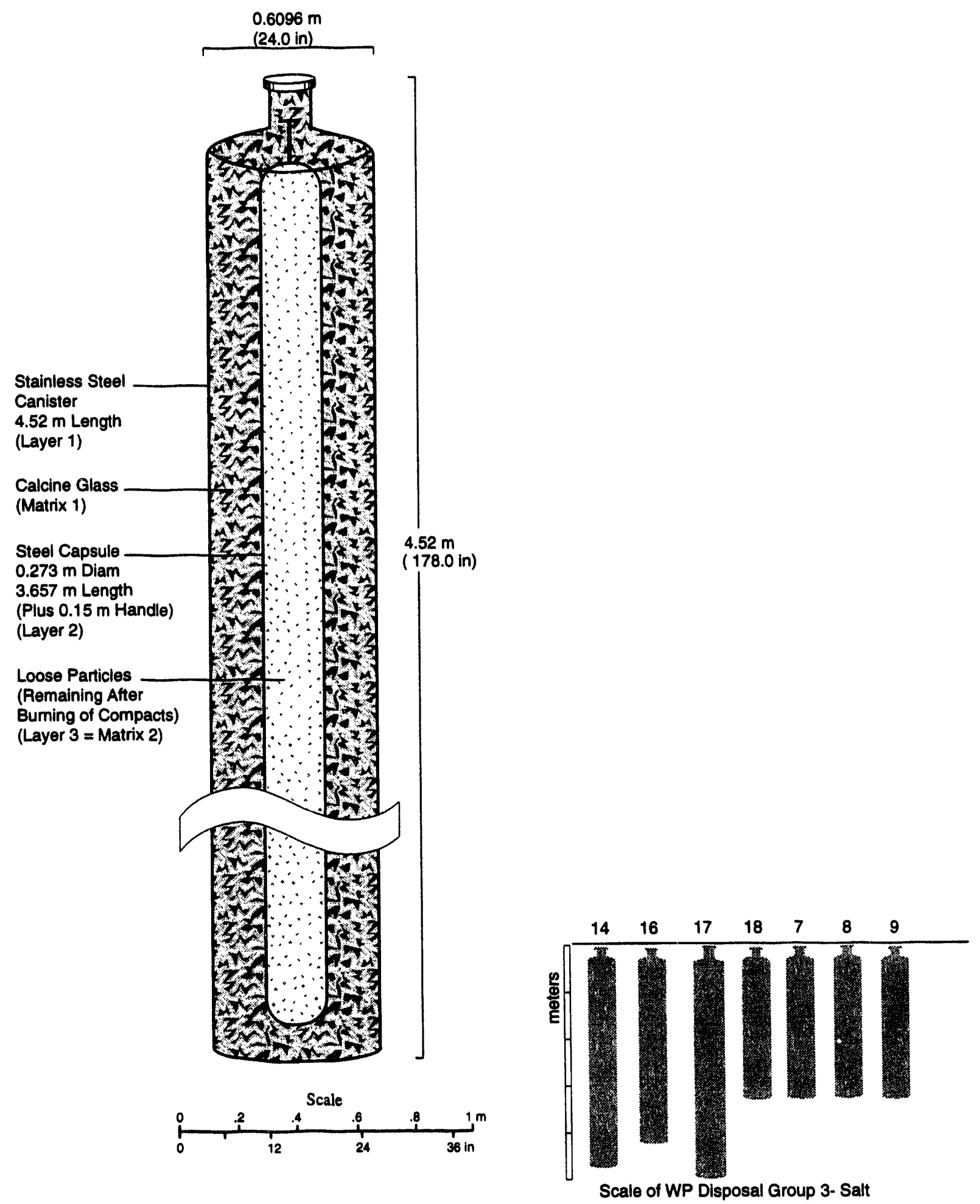

TA1-6342-3768-0

Figure 4-26. Waste Parcel 14 in Waste Disposal Group 3. This waste parcel contains compacts of pulverized graphite from Fort St. Vrain and Peach Bottom that has been burned off and is intended for disposal in the salt repository. 

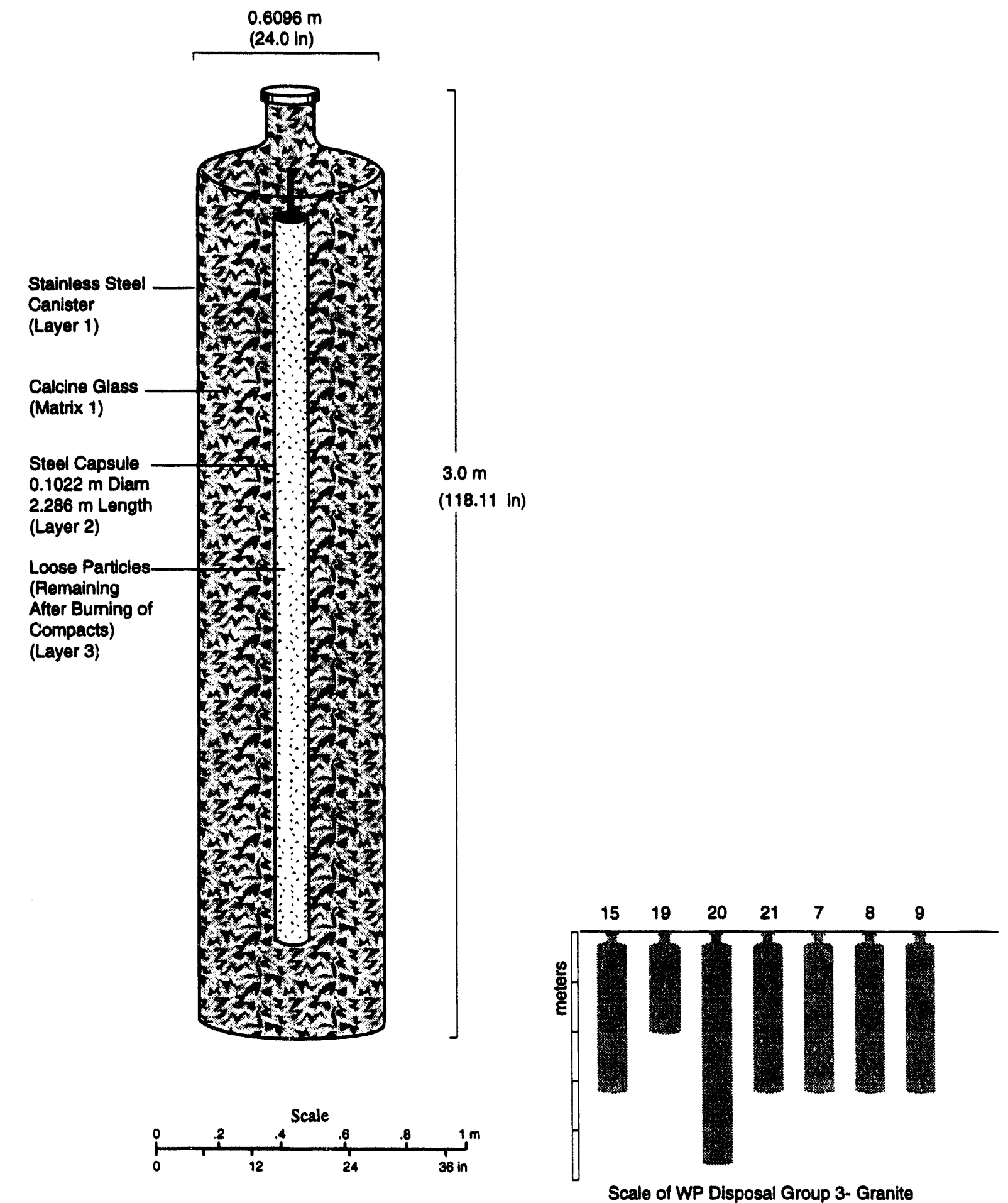

TRI-6342-3769-0

Figure 4-27. Waste Parcel 15 in Waste Disposal Group 3. This waste parcel contains compacts of pulverized graphite from Fort St. Vrain and Peach Bottom that has been burned off and is intended for disposal in the granite repository. 
2 3

32

33

34

35

36

37

38

39
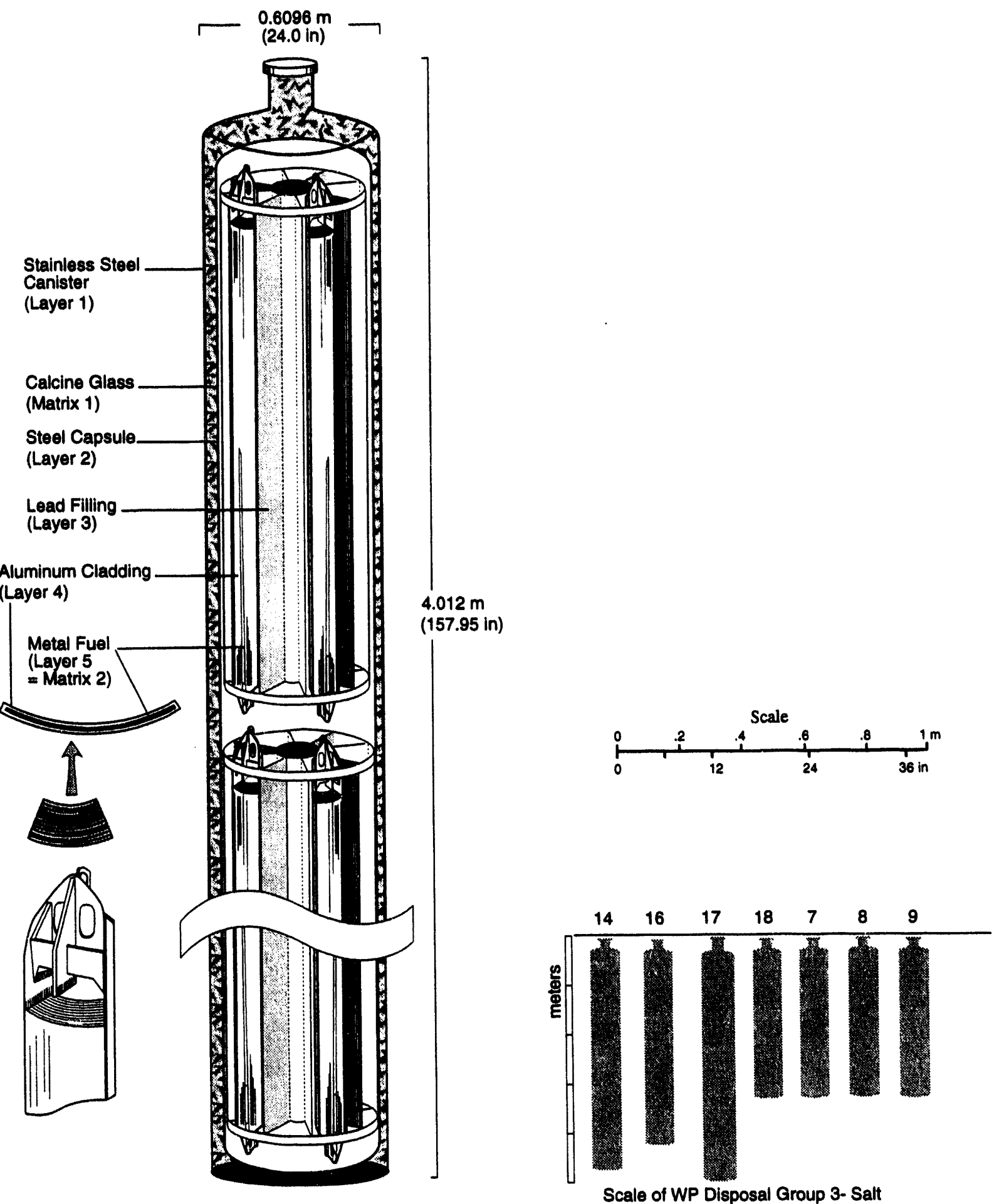

TRI-6342-3770-0

Figure 4-28. Waste Parcel 16 in Waste Disposal Group 3. This waste parcel contains 16 fuel assemblies in glass of special highly enriched uranium spent fuel and is intended for disposal in the salt repository. 

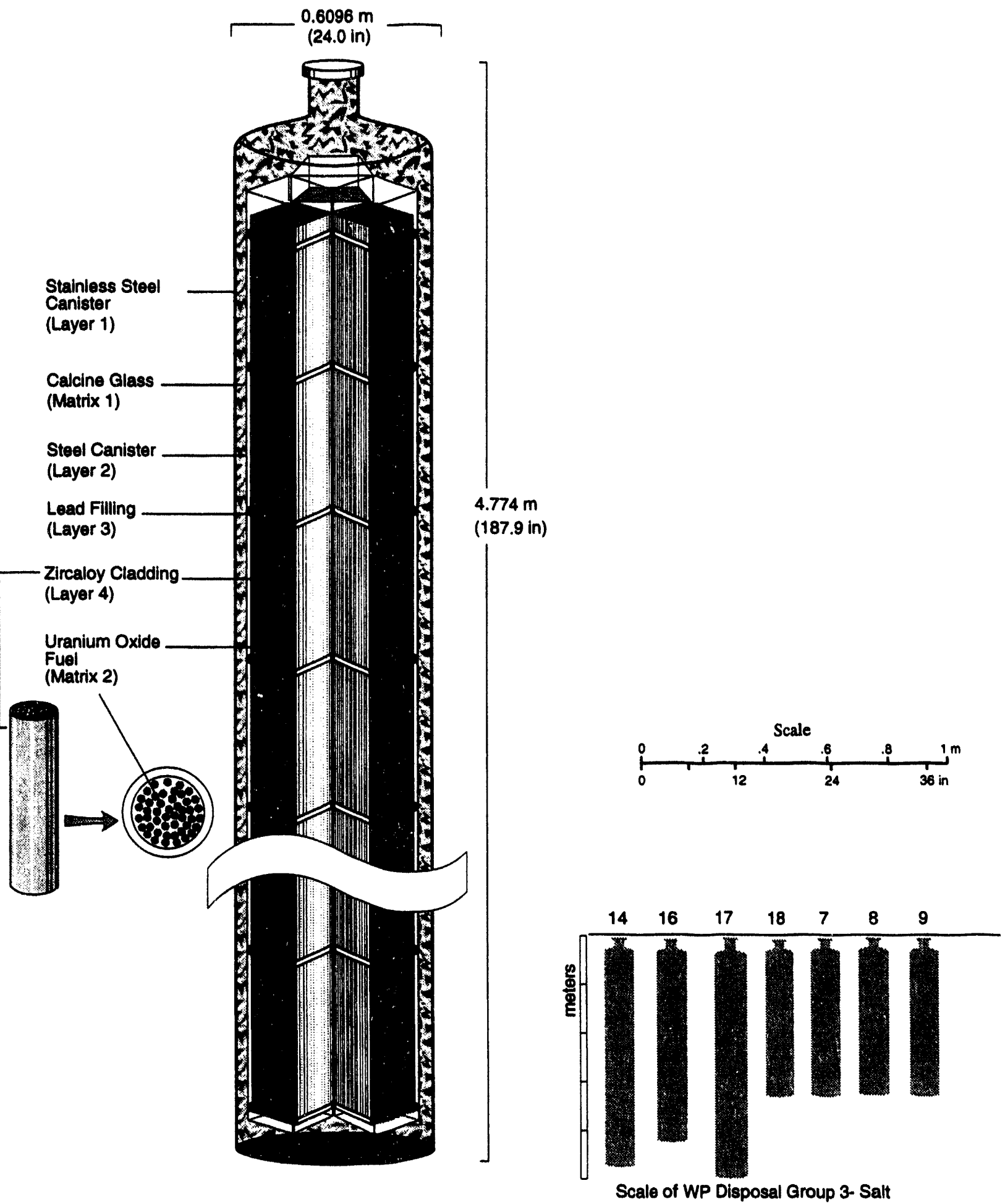

TR1-6342-3771-0

Figure 4-29. Waste Parcel 17 in Waste Disposal Group 3. This waste parcel contains three special low enriched uranium spent fuel in glass and is intended for disposal in the salt repository. 

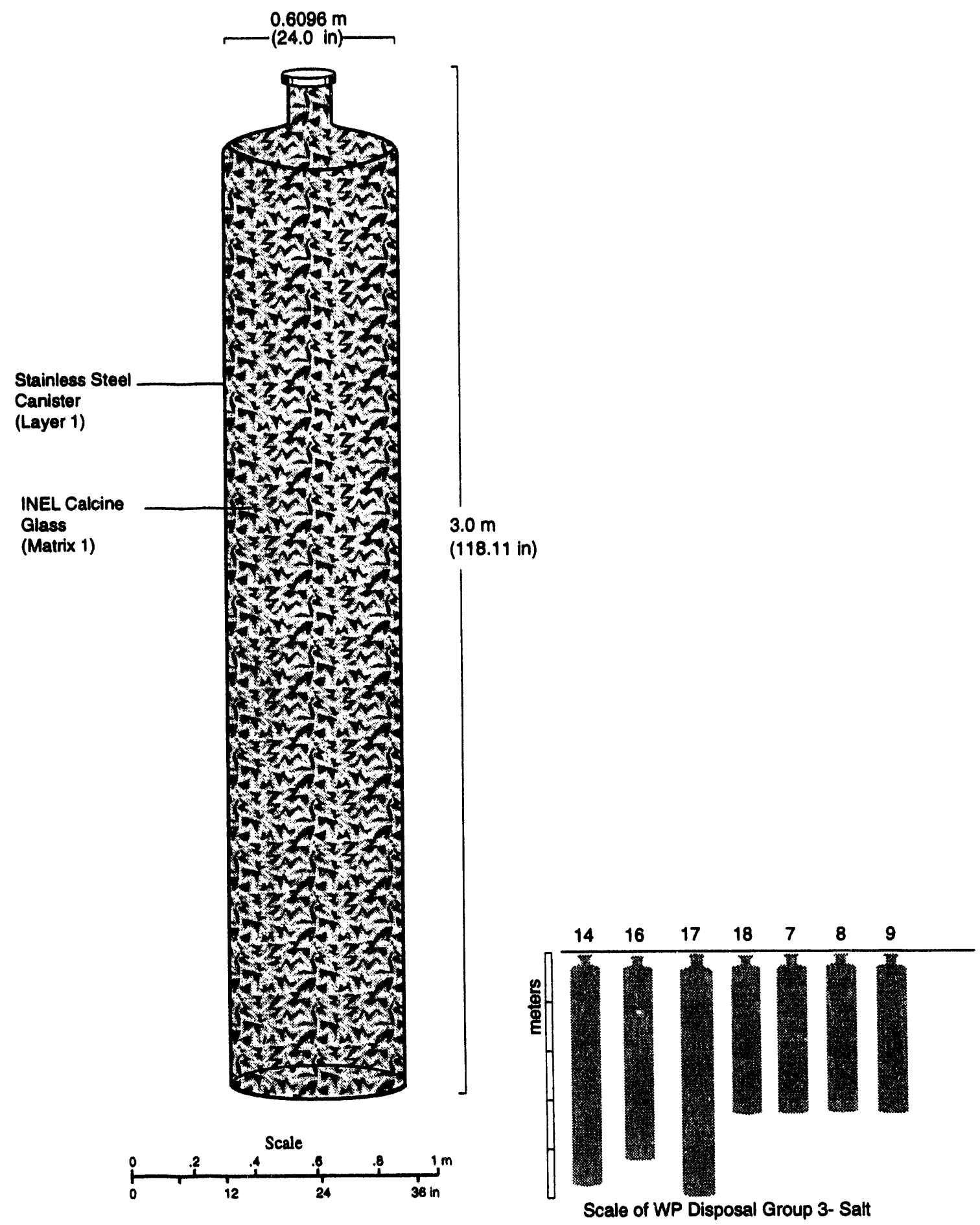

TRI-6342-3775-0

Figure 4-30. Waste Parcel 18 in Waste Disposal Group 3. This waste parcel contains high-level waste in glass and is intended for disposal in the salt repository. 
4. Description of Waste and Waste Parcels

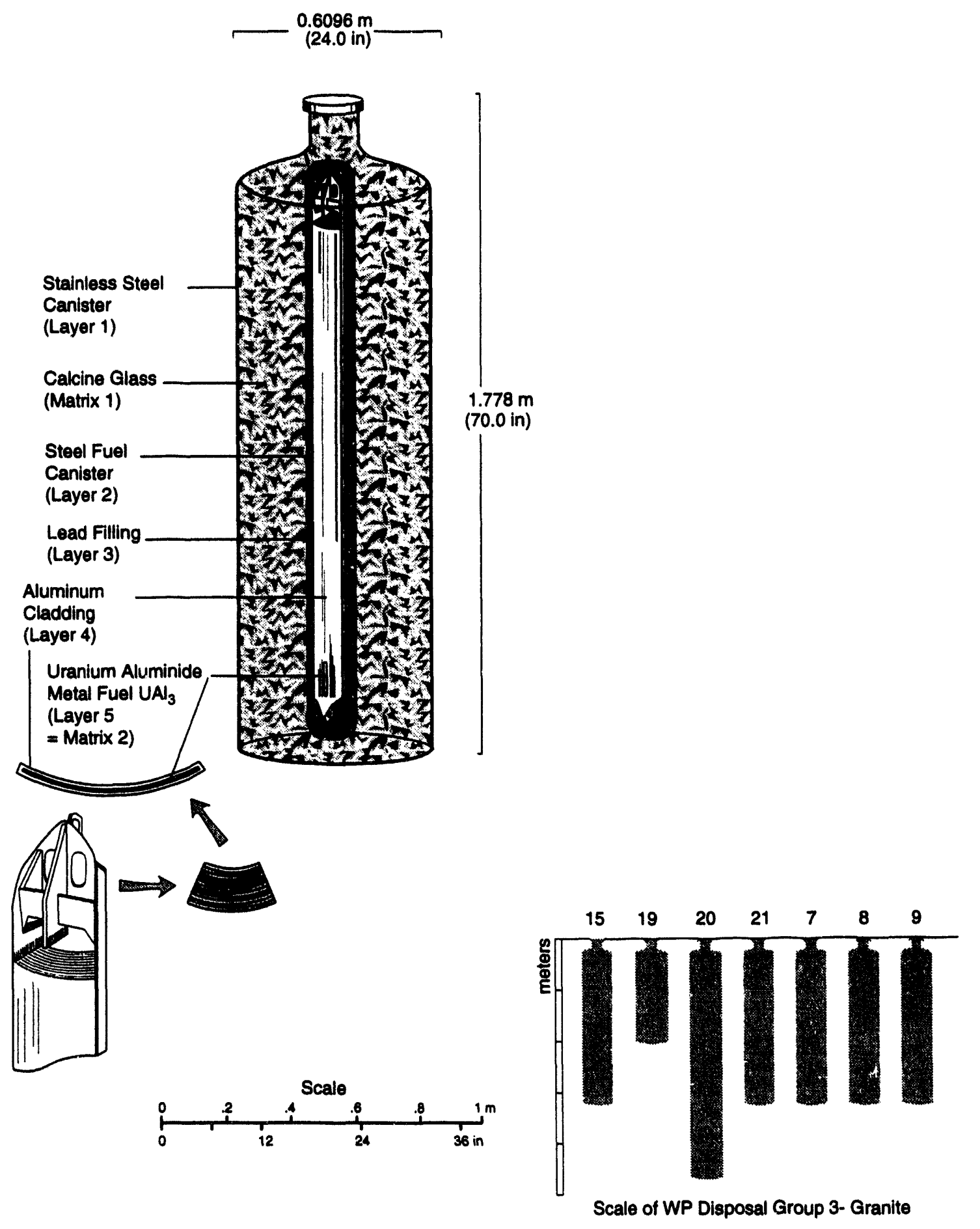

TRI-6342-3776-0

Figure 4-31. Waste Parcel 19 in Waste Disposal Group 3. This waste parcel contains one fuel assembly in glass of special highly enriched uranium spent fuel and is intended for disposal in the granite repository. 


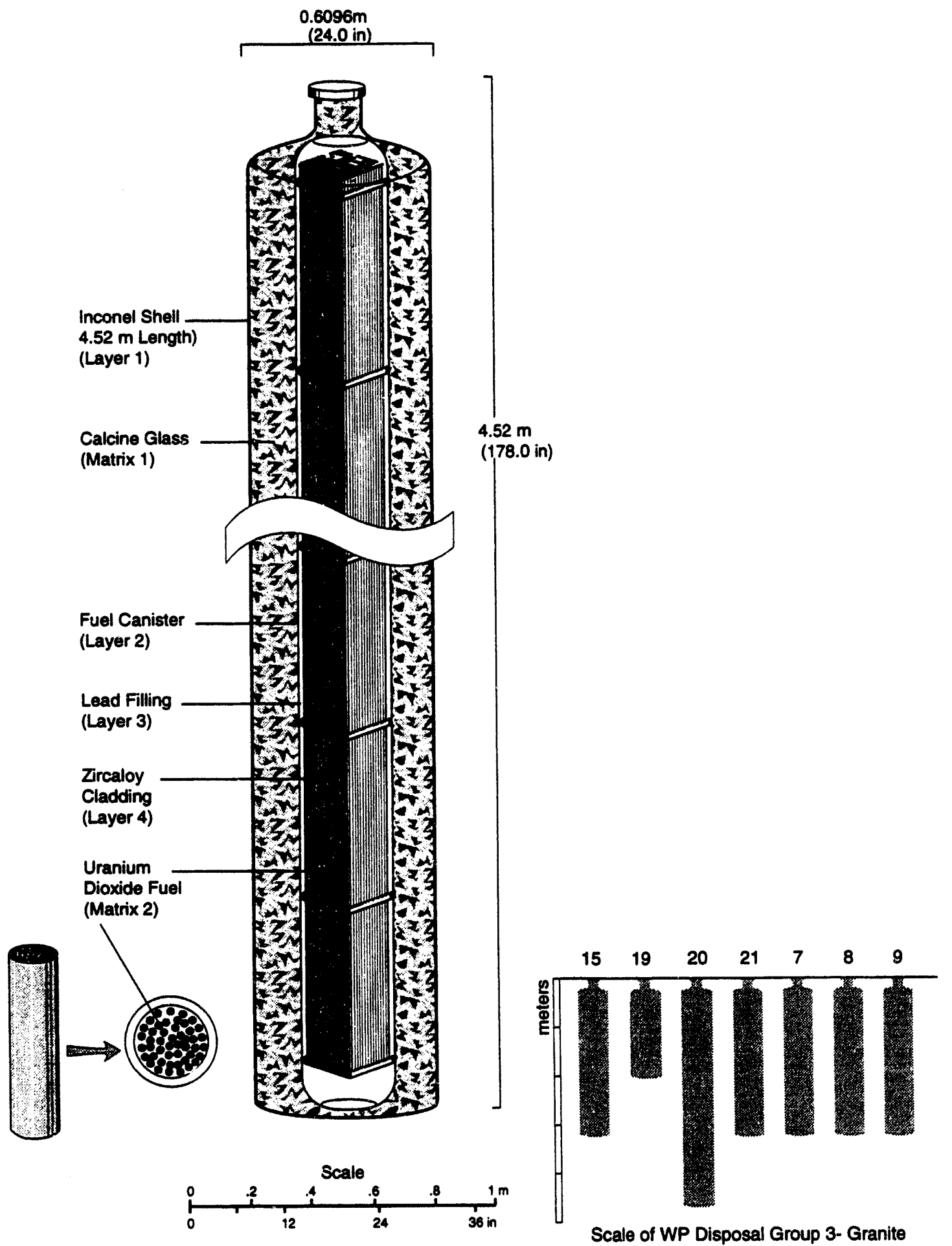

TRI-6342-3777-0

Figure 4-32. Waste Parcel 20 in Waste Disposal Group 3. This waste parcel contains special low enriched uranium spent fuel in glass and is intended for disposal in the granite repository. 

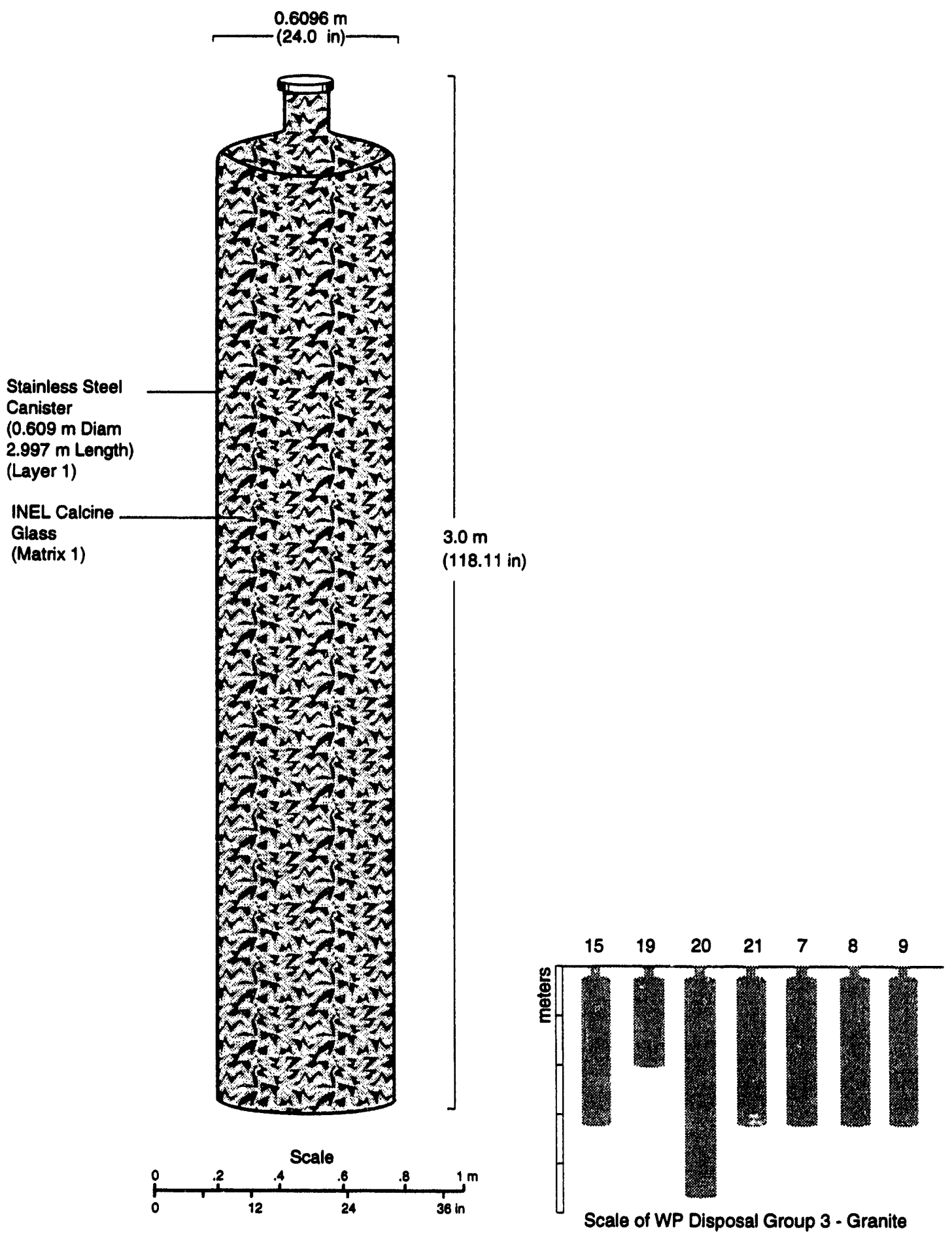

TR1-6342-3778-0

Figure 4-33. Waste Parcel 21 in Waste Disposal Group 3. This waste parcel contains high-level waste in INEL glass and is intended for disposal in the granite repository. 


\subsubsection{Waste Disposal Group 4}

Waste Disposal Group 4 includes two waste parcels that correspond to treatment option 4 for graphite spent fuel, special spent fuel, and calcine waste (Table 4-12). Waste Disposal Group 4 also includes Waste Parcels 7 through 9 , which were designed to enclose the borosilicate glass waste form from the three DOE complexes, i.e., Savannah River, Hanford, and West Valley, respectively.

A total of 12,618 waste parcels ${ }^{* * * *}$ are required to dispose of the waste in Group 4, of which 7519 are from the three DOE sites. The same number and design of waste parcels are used for the salt and granite repositories.

Selected Treatment Options. The treatment options considered in Waste Disposal Group 4 include the following:

- Graphite spent fuel: Burning up the graphite and separation of thorium and enriched uranium for future use (national resource) and mixing the remaining waste with borosilicate glass or glass-ceramic (spent fuel recycling)

- Special spent fuel: Separating the enriched uranium and thorium for future use (national resource) and mixing the remaining waste with borosilicate glass or glass-ceramic (spent fuel recycling)

- Calcine Waste: Calcining all the liquid high-level waste, adding glass-ceramic-forming additives to the calcine, mixing with reprocessed special spent fuel and graphite fuel, and heating up to $1050^{\circ} \mathrm{C}$ and $140 \mathrm{MPa}$ in a Hot Isostatic Press (HIP) process (glass-ceramic calcine)

Corresponding Waste Parcels. A description of the waste parcels for Waste Disposal Group 4 is provided in Table 4-12; WPs 22 and 23 are shown in Figures 4-34 and 4-35 (waste parcels 7 through 9 shown in Figures 4-19 through 4-21).

Table 4-12. Waste Parcels for Waste Disposal Group 4

\begin{tabular}{llllll}
$\begin{array}{c}\text { Waste } \\
\text { Parcel } \\
\text { ID }\end{array}$ & $\begin{array}{c}\text { Repository } \\
\text { Type }\end{array}$ & \multicolumn{1}{c}{$\begin{array}{c}\text { Waste } \\
\text { Type }\end{array}$} & \multicolumn{1}{c}{ Source } & \multicolumn{1}{c}{$\begin{array}{c}\text { Treatment } \\
\text { Option }\end{array}$} \\
\hline WP 22 & Salt & Granite & $\begin{array}{l}\text { Graphite spent fuel } \\
\text { High-level waste }\end{array}$ & $\begin{array}{l}\text { Fort St. Vrain } \\
\text { Peach Bottom } \\
\text { Chem Plant }\end{array}$ & $\begin{array}{l}\text { Glass ceramic within } \\
\text { HIPs* }\end{array}$ \\
WP 23 & Salt & Granite & $\begin{array}{l}\text { Special spent fuel } \\
\text { High-level waste }\end{array}$ & $\begin{array}{l}\text { Special } \\
\text { Chem Plant }\end{array}$ & $\begin{array}{l}\text { Glass ceramic within } \\
\text { HIPs }\end{array}$ \\
WP 7 & Salt & Granite & $\begin{array}{l}\text { High-level waste } \\
\text { High-level waste }\end{array}$ & Savannah River & Borosilicate glass \\
WP 8 & Salt & Granite & Hanford & Borosilicate glass \\
WP 9 & Salt & Granite & High-level waste & West Valley & Borosilicate glass \\
\hline -HIP = Hot lsostatic Press & & & & \\
\hline
\end{tabular}

**** 12,618 for both salt and granite repositories (see Table 6-1 in Chapter 6). The total number of waste parcels was recalculated based on waste volumes and waste parcel dimensions and does not necessarily agree with the numbers indicated in Appendix A. 
4. Description of Waste and Waste Parcels

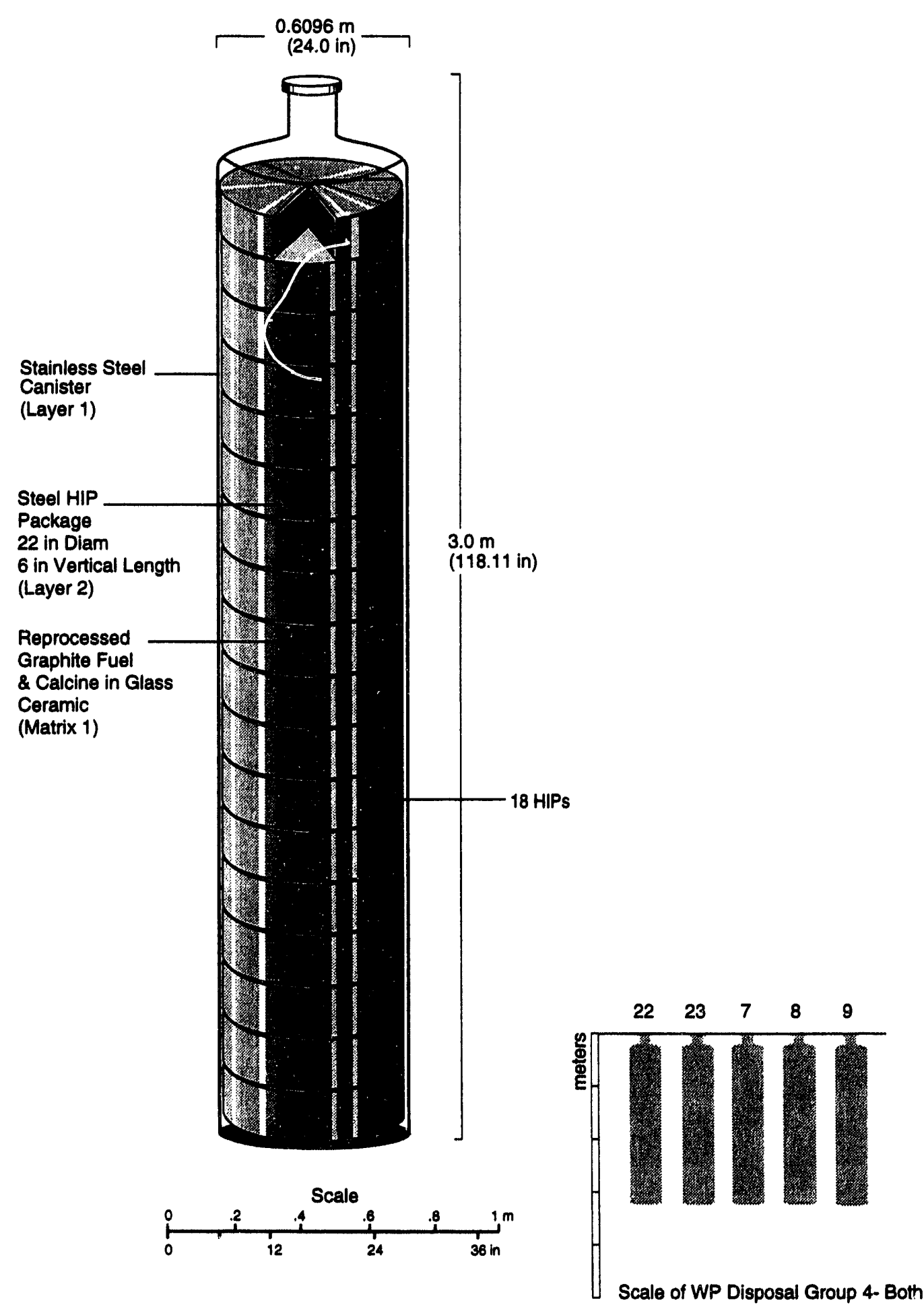

TR1-6342-3779-0

Figure 4-34. Waste Parcel 22 in Waste Disposal Group 4. This waste parcel contains graphite spent fuel from Fort St. Vrain and Peach Bottom reactors and calcine in 18 steel-clad Hot Isostatic Press packages and is intended for disposal in both salt and granite repositories. 
2 3 4

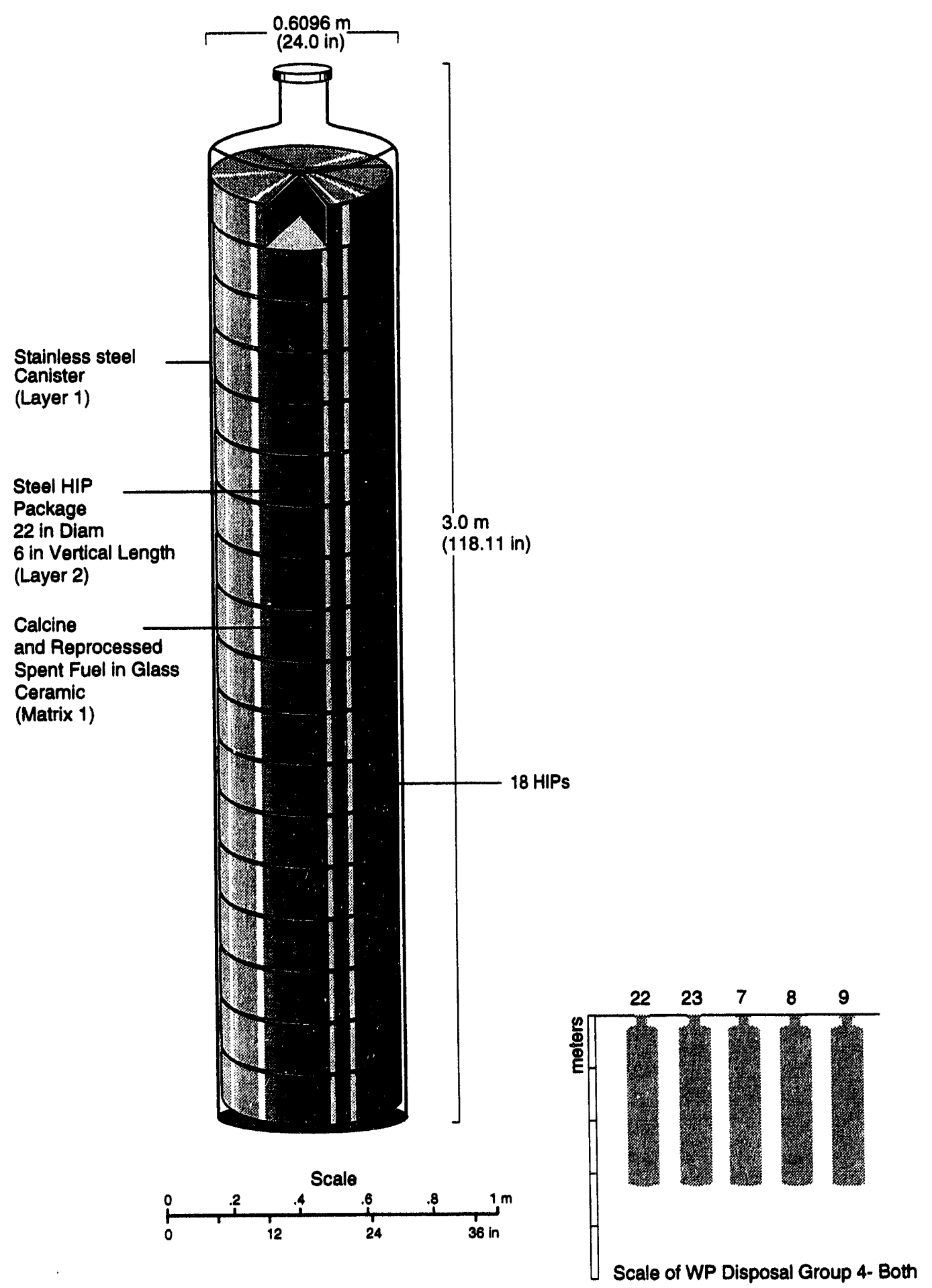

TRI-6342-3780-0

Figure 4-35. Waste Parcel 23 in Waste Disposal Group 4. This waste parcel contains special spent fuel from low and highly enriched uranium spent fuel and calcine in 18 steel-clad Hot Isostatic Press packages and is intended for disposal in both salt and granite repositories. 


\subsubsection{Waste Disposal Group 5}

Waste Disposal Group 5 includes three waste parcels that correspond to treatment option 5 for graphite spent fuel, special spent fuel, and calcine waste (Table 4-13). Waste Disposal Group 5 also includes Waste Parcels 7 through 9, which were designed to enclose the borosilicate glass waste form from the three DOE complexes, i.e., Savannah River, Hanford, and West Valley, respectively.

A total of 16,430 waste parcels ${ }^{\dagger \dagger \dagger \dagger}$ are required to dispose of the waste in Waste Disposal Group 5, of which 7519 are from the three DOE sites. The same number and design of waste parcels are used for the salt and granite repositories.

Selected Treatment Options. The treatment options considered in Waste Disposal Group 5 include the following:

- Graphite spent fuel: Burning up the graphite, oxidizing the fuel and treating with nitric acid to form uranyl nitrate, and adding depleted uranium at a ratio of about 200:1 to give a ${ }^{235} U$ content of the waste equivalent to naturally occurring uranium before placing in canisters for repository disposal (oxidation then dilution with depleted uranium)

- Special spent fuel: Oxidizing the fuel and adding depleted uranium at a ratio of about 100:1 (oxidation then dilution with depleted uranium)

- Calcine Waste: Placing the liquid high-level waste and calcine in a borosilicate glass matrix and filling the void space (borosilicate calcine)

Corresponding Waste Parcels. A description of the waste parcels for Waste Disposal Group 5 is provided in Table 4-13; WPs 24 through 26 are shown in Figures 4-36 through 4-38 (waste parcels 7 through 9 shown in Figures 4-19 through 4-21).

Table 4-13. Waste Parcels for Waste Disposal Group 5

\begin{tabular}{llllll}
\hline $\begin{array}{c}\text { Waste } \\
\text { Parcel } \\
\text { ID }\end{array}$ & $\begin{array}{c}\text { Repcsitory } \\
\text { Type }\end{array}$ & \multicolumn{1}{c}{$\begin{array}{c}\text { Waste } \\
\text { Type }\end{array}$} & \multicolumn{1}{c}{ Source } & \multicolumn{1}{c}{$\begin{array}{c}\text { Treatment } \\
\text { Option }\end{array}$} \\
\hline WP 24 & Salt & Granite & Spent fuel, graphite & $\begin{array}{l}\text { Fort St. Vrain } \\
\text { Peach Bottom }\end{array}$ & $\begin{array}{l}\text { Processed and mixed } \\
\text { with depleted uranium in } \\
\text { glass }\end{array}$ \\
WP 25 & Salt & Granite & $\begin{array}{l}\text { Spent fuel, special, HEU } \\
\text { and LEU }\end{array}$ & Special & $\begin{array}{l}\text { Mixed with depleted ura- } \\
\text { nium (in glass) }\end{array}$ \\
WP 26 & Salt & Granite & High-level waste & Chem Plant & Calcine in glass \\
WP 7 & Salt & Granite & High-level waste & Savannah River & Borosilicate glass \\
WP 8 & Salt & Granite & High-level waste & Hanford & Borosilicate glass \\
WP 9 & Salt & Granite & High-level waste & West Valley & Borosilicate glass \\
\hline
\end{tabular}

${ }^{+t+t} 16,430$ for both salt and granite repositories (see Table 6-1 in Chapter 6). The total number of waste parcels was recalculated based on waste volumes and waste parcel dimensions and does not necessarily agree with the numbers indicated in Appendix A. 
$0.6096 \mathrm{~m}$

(24.0 in)

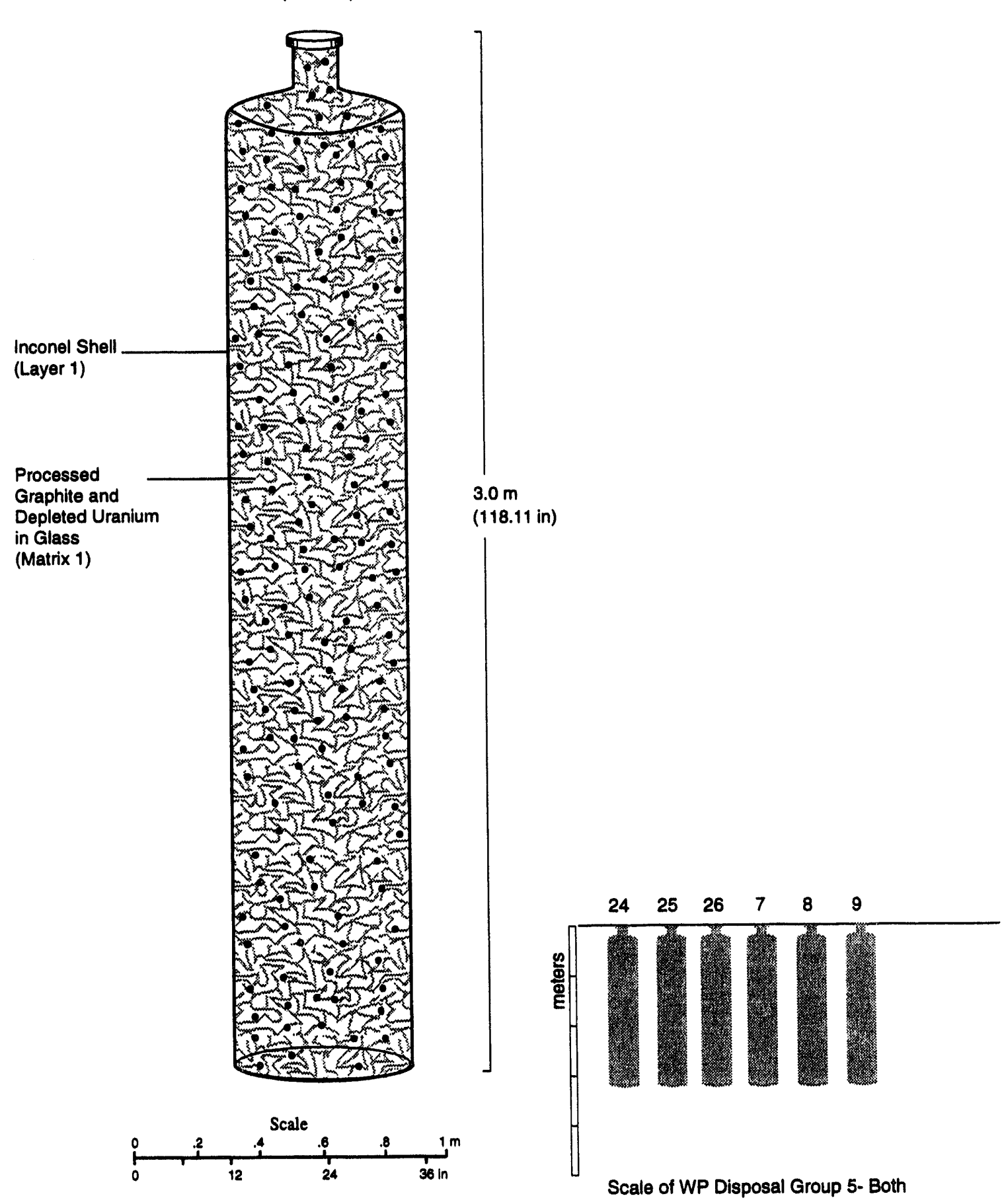

TR1-6342-3774-0

Figure 4-36. Waste Parcel 24 in Waste Disposal Group 5. This waste parcel contains graphite spent fuel from Fort St. Vrain and Peach Bottom reactors processed and mixed with depleted uranium in glass and is intended for disposal in both salt and granite repositories. 


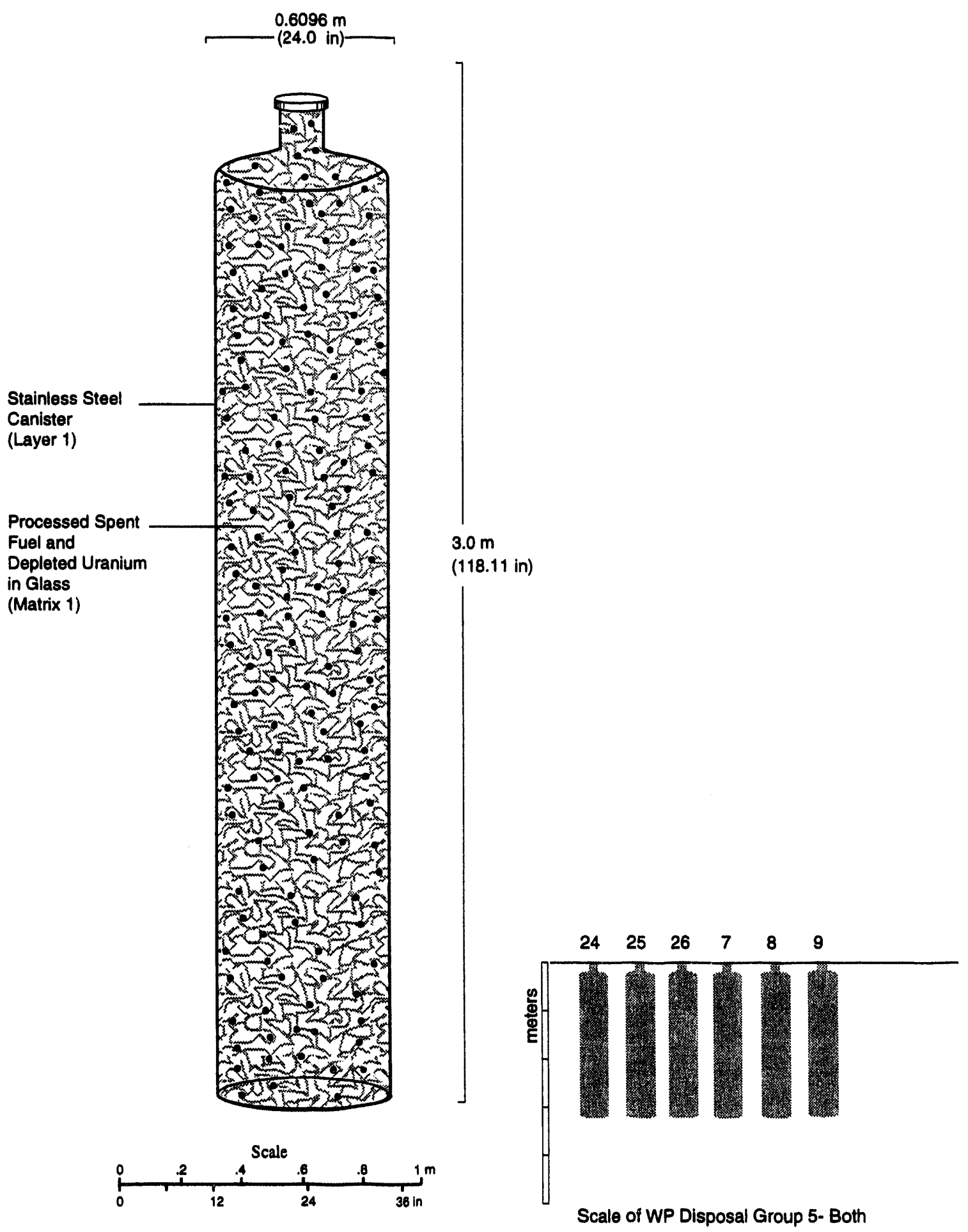

TRI-6342-3773-0

Figure 4-37. Waste Parcel 25 in Waste Disposal Group 5. This waste parcel contains special highly enriched and low enriched uranium spent fuel processed and mixed with depleted uranium in glass and is intended for disposal in both salt and granite repositories. 
2

3

4

38

39

40

41

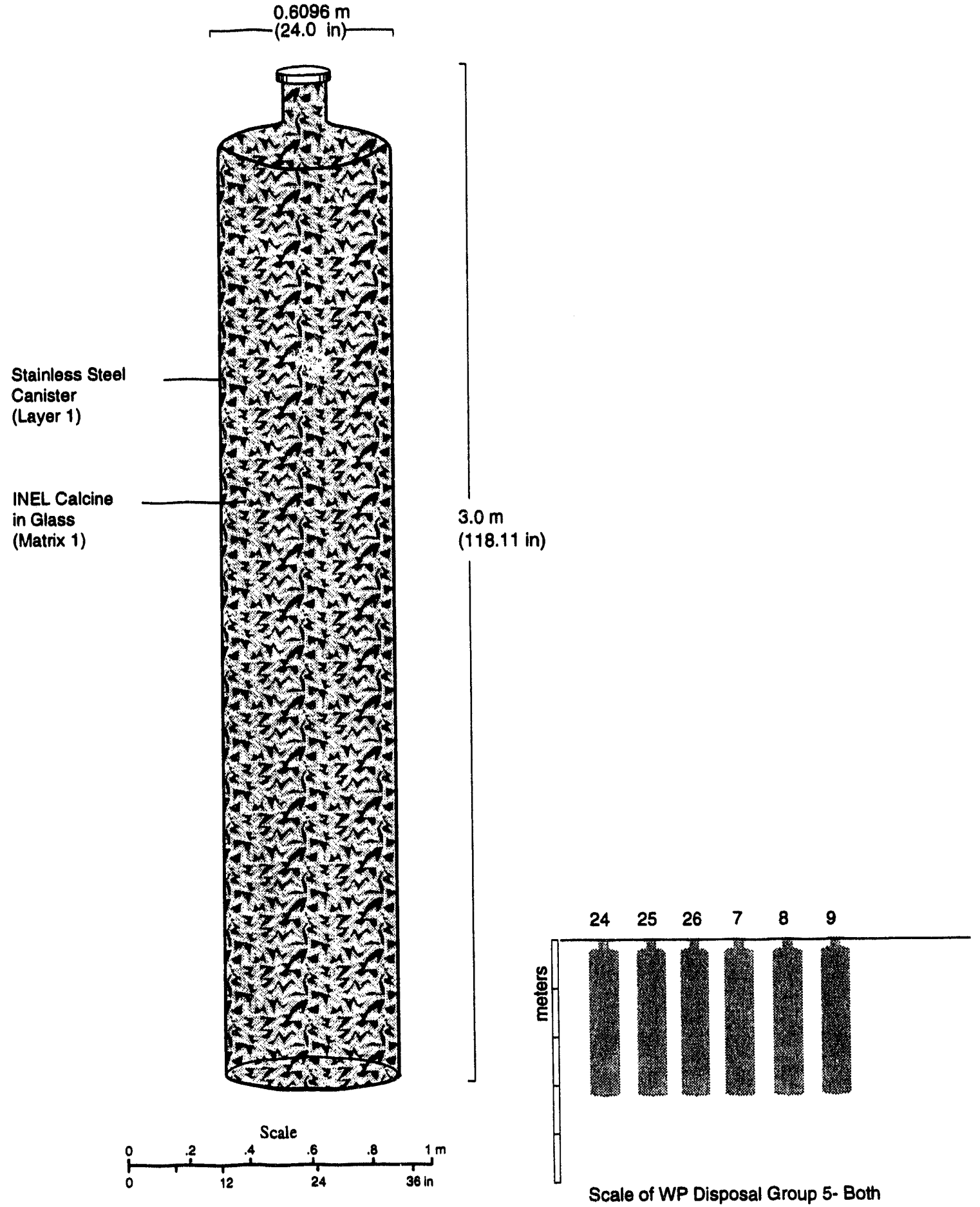

TRI-6342-3772-0

Figure 4-38. Waste Parcel 26 in Waste Disposal Group 5. This waste parcel contains calcine high-level waste in glass and is intended for disposal in both salt and granite repositories. 


\subsection{Waste Parcel Inventory}

The curie content of individual waste parcels for the full inventory of 177 radionuclides and for the 49 selected radionuclides used in the performance assessment is shown in Figure 4-39 for emplacement in the year 2030. If all the radionuclides were included (Appendix A), the curie amounts would be as much as twice as high. The difference arises from radionuclides with very short half-lives that do not contribute significantly to waste parcel activity at time scales of hundreds or thousands of years in the future. However, the high initial activity from all the radionuclides is important when waste parcel shielding and head output are considered.

Heat rates for the 26 different waste parcels in the year 2030 are shown in Figure 4-40. All 177 radionuclides were used to determine waste parcel heat production.

\subsection{Waste Parcel Shielding}

For this performance assessment, neither the overpack nor the internal backfill was modeled in the majority of the calculations. These components normally provide a significant contribution to the radiation shielding of a waste package. For the canister concept used in this performance assessment, additional shielding would have to be provided during handling operations and during emplacement in the vertical boreholes. A shield plug and cover plate might also be necessary to provide radiation shielding after emplacement (Stahl and Harrison, 1993).

\subsection{Summary of Modeling Assumptions for Waste Forms and Waste Parcels}

The following assumptions were made for the waste forms and waste parcels:

- Disposal of the waste inventory into a salt and saturated granite geologic repository is assumed to occur in the year 2030.

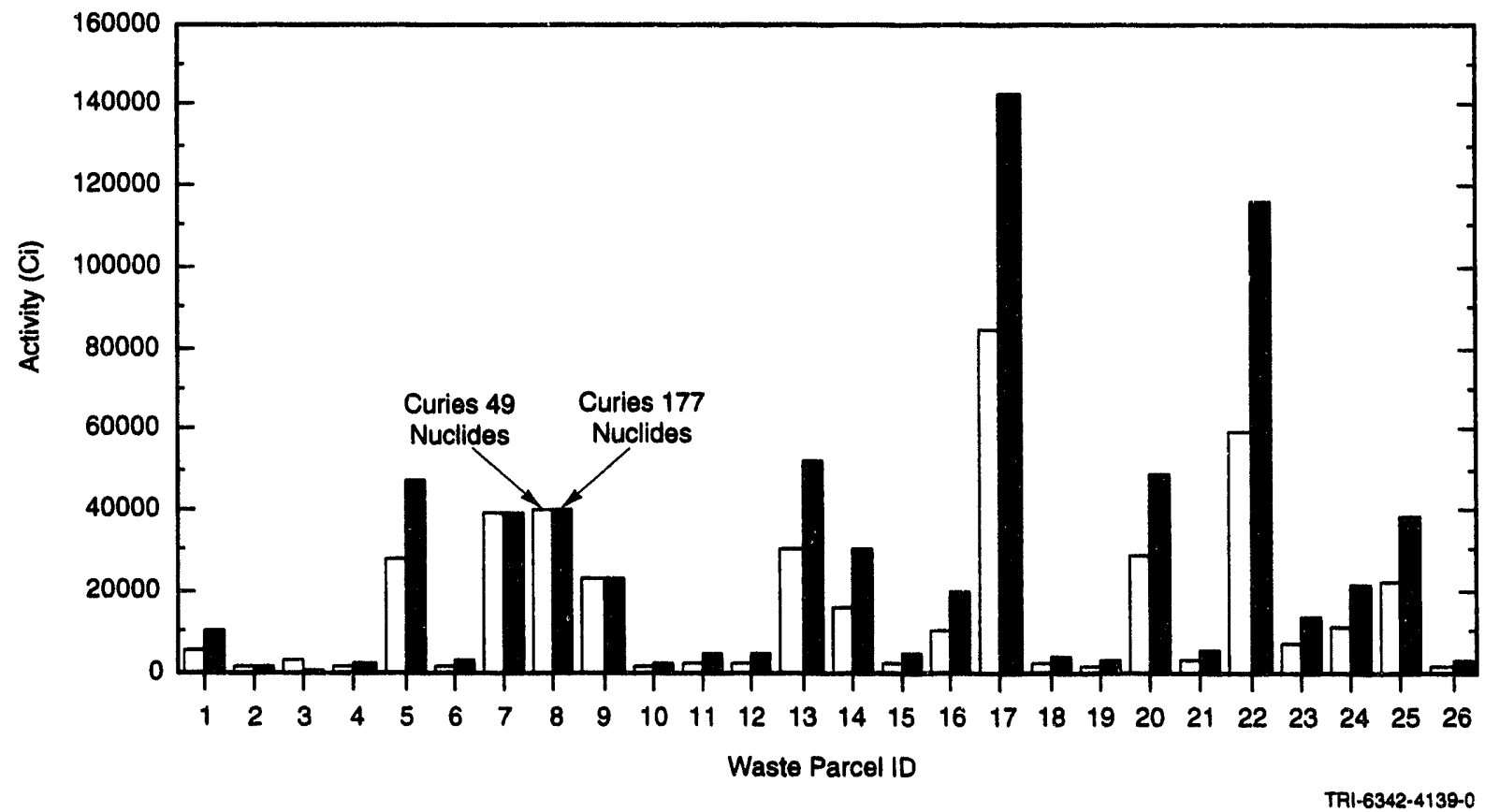

Figure 4-39. Curie content of waste parcels based on full inventory of 177 radionuclides and on reduced inventon of 49 radionuclides at time of emplacement (year 2030). 


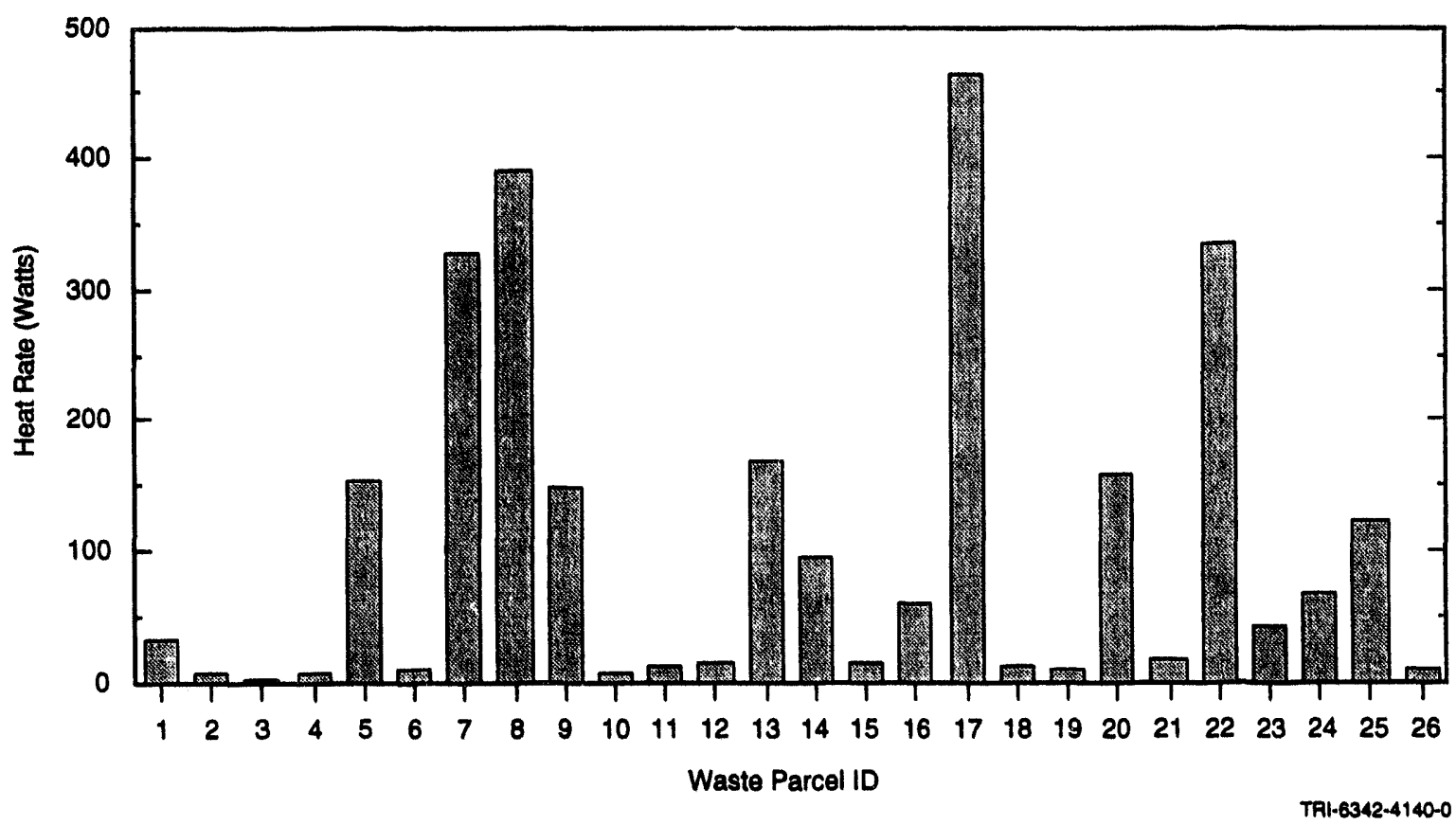

Figure 4-40. Heat rate of waste parcels at time of emplacement (year 2030) accounting for all 177 radionuclides.

- The number of radionuclides considered is limited to 49 for cuttings removal, 11 for transport in the groundwater (Complex PA), and 22 for transport in the groundwater (Simple PA).

- Five different processing options (waste groups) are considered for disposal of the waste inventory.

- Waste from each group is placed in waste parcels of several types. Depending on the waste disposal group, up to 10 different types of waste parcels are used. The total number of waste parcel types for all waste groups is 26.

- Except for a few test cases, the performance assessment assumes the waste parcels consist of the waste form, internal backfill, and canister. The waste parcels do not have overpacks.

- The material of the outer canister is assumed to be $304 \mathrm{~L}$ stainless steel.

- A 0.3-m external backfill-buffer of bentonite clay is assumed to surround canisters in a granite repository. Crushed salt backfill is used around the canisters in the salt repository (also $0.3 \mathrm{~m}$ thick).

- Canisters are buried vertically $1 \mathrm{~m}$ below the disposal room floor. The $1-\mathrm{m}$ space is backfilled with bentonite in the granite repository and crushed salt in the salt repository.

- A shield plug and cover plate will be added to the disposal borehole for radiation shielding, if necessary.

- Canisters sit on the bottom of borehole.

- Stainless steel HIP packages and zircaloy cladding are assumed to have been breached at time zero. 


\title{
5. Hypothetical Geologic Barrier
}

\author{
K. F. Brinster and R. P. Rechard
}

Characteristics of two hypothetical geologic barriers-salt" and granite ${ }^{\dagger}$-are summarized in this chapter. The description focuses on the major concepts and features of the barriers as they relate to the conceptual model for the performance assessment. General criteria for site selection are presented first, and then specific characteristics of the geologic barriers for salt and granite are provided.

\subsection{General Site Selection Criteria}

Several rock types have been considered as regional barriers for the disposal of spent nuclear fuel and high-level radioactive waste. These lithologies-granite, salt, basalt, and tuff-all have different geologic, geomechanical, and geochemical characteristics that can influence the overall design of a repository system. Some of the most important geologic variables to be considered in locating a waste disposal facility within a deep geologic formation are the flow-related properties such as permeability, porosity, and storativity of the geologic members. In addition, knowledge of the local and regional groundwater fluxes is necessary to assess the long-term flow and transport of waste from a breached canister system. During the first decades after emplacement, the decaying radioactive wastes generate heat that can drive fluid flow, create thermal stresses, and possibly accelerate temperature-dependent creep. Thus, thermal, transport, and mechanical properties of the site geology are also important.

In this performance assessment, two generic sites were selected: one in bedded salt and the other in fractured granite. Internationally, both lithologies are under active consideration as potential sites for deep disposal of radioactive waste.

Three main criteria are considered during selection of a site for radioactive waste disposal. The first criterion evaluates land-use conflicts (both human and natural), the second studies the technical suitability of the site, and the third examines how well the waste can be isolated from the human population (Carter, 1987). In this performance assessment only the latter two were considered since the sites were hypothetical and thus assumed to have met the first criterion.

\subsubsection{Technical Suitability}

In this performance assessment, the one basic criterion used to determine technical suitability of the geologic barrier was: its ability to provide physical and chemical stability. Technical suitability also depends on various other criteria, including how easily the site can be mined, how resistant it is to water, and its mechanical and chemical conditions (Carter, 1987). According to Paktunc (1993), relevant factors ${ }^{\ddagger}$ include

1. Candidate site should possess simple hydrological and geologic elements.

2. Site should have geologic media at the elevation of the proposed repository that is massive, competent, uniform, largely unfractured, and of low porosity and permeability.

3. Site should possess geologic media with a low frequency of faults.

4. Site should be at a location of low seismicity, where natural processes such as groundwater flow, erosion, and land movements are occurring at consistent and predictable rates.

\footnotetext{
"Throughout this report, the term "salt" refers to evaporite deposits that are predominantly sodium chloride ( $\mathrm{NaCl})$. Mineralogic names such as halite $(\mathrm{NaCl})$, sylvite $(\mathrm{KCL})$, gypsum $(\mathrm{CaSO} \cdot \mathrm{H} 2 \mathrm{O})$, and anhydrite (CaSO4) are used when referring to specific evaporite minerals. The term evaporite includes all of the above minerals; evaporites are formed by the evaporation of a saline solution such as sea water.

+ Throughout this report, the term "granite" refers to coarse-grained rock formed at great depth (plutonic) such as occurs at the Canadian site (Lac du Bonnet, Manitoba) (Everitt and Read, 1989). When modified by a descriptive term, such as "massive grey," we are referring to a specific granite, essentially of alkali feldspar and quartz.

\# Note that some of these factors imply that a fully saturated site would be used; features such as fractures or high porosity could be beneficial at an unsaturated site.
} 
5. Site should be large enough to allow predictive modeling to be undertaken with confidence.

The ease with which waste can be retrieved may also be a criterion. For waste retrieval, an example of a suitable site would be underground openings that are stable enough to make backfilling with crushed rock unnecessary.

\subsubsection{Reasonable Isolation}

The site must also meet the criterion of providing reasonable isolation of the waste from the biosphere, especially the human population. To satisfy this criterion, site characteristics in terms of hydrology, tectonics, chemistry, and geology must be considered with regard to whether they are conducive to isolation. Another approach would be to ensure that the canisters or casks are long-lived, thus making siting possible in a variety of geologic and geographic environments (Carter, 1987). The primary consideration of this criterion in the current performance assessment was to specify burial at sufficient thickness and depth in the geologic media.

\subsubsection{Salt Deposits as Geologic Barrier}

This section lists current investigations of salt, the advantages and disadvantages of salt as a geologic barrier, and site-selection criteria specific to salt.

Current Investigations of Salt. In the United States, a salt repository under active consideration is the Waste Isolation Pilot Plant (WIPP), located near Carlsbad, NM. Countries other than the United States that are actively investigating the suitability of bedded salt or domal salt formations for deep disposal of radioactive wastes are France, Germany, the Netherlands, and Spain. Salt repositories are being examined as part of the Commission of the European Communities (CEC) PAGIS project (Performance Assessment of Geological Isolation Systems) (Storck et al., 1988). The objective of PAGIS is to evaluate the ability of various geological formations to confine vitrified highlevel waste. Other CEC studies have considered disposal of alpha-contaminated wastes and intermediate-level wastes in German salt domes (Hirsekorn et al., 1991) and disposal of low- and intermediate-level wastes in various types of salt deposits in the Netherlands.

Advantages and Disadvantages of Salt Deposits. Certain characteristics of salt beds provide particular advantages and disadvantages for a disposal system for radioactive waste. The advantages are:

- Salt beds entomb the waste. (Note that although this is an advantage in that it provides isolation, it is also a disadvantage because it inhibits future retrieval.)

- Salt beds can be found in regions that are stable tectonically.

- Fairly large salt basins can be found relatively near the surface throughout the continental U.S. (see Section 5.2.1, "Origin and Occurrence of Salt Deposits").

- Salt beds are easy to mine.

- Salt beds have extremely small groundwater fluxes.

- Salt beds are relatively homogeneous, i.e., salt beds of a thickness and purity sufficient to contain a repository can be found.

- Salt beds provide good heat conduction, thus decreasing the temperature of the waste and so preventing critical temperatures at the waste parcel/salt interface.

- At elevated temperatures, the rate of creep increases around waste parcel boreholes, thus reducing the time required for reconsolidation of the salt around boreholes. (Salt reconsolidation is an advantage because reconsolidated crushed salt regains properties close to those of the original host rock properties, e.g., permeability, density, porosity.)

- Saturated site can entomb wastes containing radionuclides in gases $\left(\right.$ e.g., $\left.{ }^{14} \mathrm{C}\right)$ better than an unsaturated site.

The disadvantages are as follows:

- Waste is difficult to retrieve after disposal. 
- Keeping the repository open is difficult and requires extensive maintenance over long periods of time.

- There is a record of drilling in salt bed areas (see Appendix B of 40 CFR 191).

- Potential co-location with economically valuable minerals exists in salt bed (there is a record of drilling in salt bed areas [see Appendix B of 40 CFR 191]).

Site-Selection Guidelines Specific to Salt. Siting requirements specific to a salt repository were developed for the Waste Isolation Pilot Plant (WIPP) Project in southeastern New Mexico (Powers et al., 1978). These requirements are

- Avoidance of boreholes (through the evaporites) within $1.6 \mathrm{~km}$ of site ${ }^{* *}$,

- Salt of high purity,

- Depth of 300 to $900 \mathrm{~m}$,

- Avoidance of dissolution,

- Avoidance of deformation,

- Extensive horizontal bedding,

- Reasonable lack of valuable resources (i.e., avoidance of oil or gas resources),

- Existing resources not unique to site (i.e., resources can be found relatively easily elsewhere).

\subsubsection{Granite Batholiths as Geologic Barrier}

This section lists current investigations of granite, the advantages and disadvantages of granite as a geologic barrier, and site-selection criteria specific to granite.

Current Investigations of Granite. Several countries are investigating the suitability of crystalline rock for deep disposal of radioactive waste in specially engineered repositories. Among these countries are Canada, Finland, France, Japan, Spain, Sweden, Switzerland, and the United Kingdom. The granite units now being studied are quite deep $(<250 \mathrm{~m})$ and sparsely fractured.

No operating repositories yet exist in crystalline rock for disposal of high-level waste. However, Sweden and Finland have deep repositories in operation for the disposal of low- and intermediate-level wastes. The Swedish Radioactive Waste Management Company (SKB) has been evaluating the performance of repositories for both high, low, and intermediate-level wastes since 1977 (Eng, 1989). The Swedish Final Repository for low- and intermediatelevel waste has been in operation since 1988. This repository is located at a 60 -m depth in granite bedrock below the Baltic Sea. It is accessed from a tunnel extending from the Forsmark nuclear site. Finland was the second country to have an operating deep repository in crystalline rock for low- and intermediate-level wastes. The repository was constructed at a depth of 50 to $125 \mathrm{~m}$ by the Finnish Industrial Power Company at the Olkiluoto nuclear site.

The remaining countries have research in progress for radioactive waste disposal. Work being performed by Atomic Energy of Canada Limited for high-level waste disposal is focused on repository development at 500 to 1000 $m$ in igneous rocks of the Canadian Shield. In France, a reference granite site (Auriat) was chosen for the PAGIS project sponsored by the Commission of European Communities. The repository design consists of waste canisters stacked in vertical boreholes drilled from horizontal galleries excavated at depths of 500 to $1000 \mathrm{~m}$. In Switzerland, the Gewahr Project, undertaken by the Swiss Co-Operative for the Disposal of Radioactive Wastes (NAGRA), includes a performance assessment for a generic high-level waste repository located at a depth of 500 to $1000 \mathrm{~m}$ in basement rocks of northern Switzerland (van Dorp and Vigfusson, 1989). In the United Kingdom, current work by the implementing agency, UK NIREX Limited, is focused on investigating the suitability of the somewhat similar deep metamorphic rocks below the Sellafield site in Cumbria for the disposal of low- and intermediate-level waste.

Advantages and Disadvantages of Granite. Certain characteristics of granite provide particular advantages for use as a disposal system for radioactive waste. The advantages are as follows:

\footnotetext{
"* Although a scarcity of boreholes was considered an important criterion in the early 1970s, the EPA standard promulgated in 1985 has since made the point less critical. The EPA regulation specifies that human intrusion by means of an exploratory borehole must be examined.
} 
- Batholiths (huge rock bodies of intrusive rock over $1 \times 10^{8} \mathrm{~m}^{2}\left[40 \mathrm{mi}^{2}\right]$ ) are widespread throughout the United States (see Section 5.3.1, "Origin and Occurrence of Granite Batholiths").

- Batholiths may be very stable tectonically (particularly those that are very old).

- Batholiths are geochemically stable.

- Fairly large (tens of square kilometers) and thick (several kilometers) plutons can be found.

- Intact batholiths have low porosity and permeability.

- Many batholiths do not have minerals with high economic value.

The disadvantages are as follows:

- Granite is often highly fractured and the potential for short groundwater travel times exists.

- Excavation is difficult and thus expensive.

- Excavation creates a disturbed rock zone with higher permeability than host rock.

Site-Selection Guidelines Specific to Granite. Generic siting requirements developed for 10 CFR 60 for disposai of high-level radioactive waste can be applied to granite. These requirements include

- Favorable geologic, geochemical, and hydrological conditions (e.g., low permeability, inhibition of radionuclide transport, widely spaced, easily discernible fracture zones),

- Low population density and remote location,

- Emplacement of waste at a minimum depth of $300 \mathrm{~m}$,

- Lengthy groundwater travel time from the disturbed zone to the accessible environment,

- Reasonable lack of valuable resources,

- Existing resources not unique to site (i.e., resources can be found relatively easily elsewhere),

- Avoidance of potential for flooding.

Fracture density is also an important consideration for the granite repository. Typically, intact granite is not considered a groundwater source, although data to that end are scarce. However, fractures may allow for the flow of water. Fractures occur when compressional, tension $\quad$ ar stresses act on a rock mass.

Fracture density is determined by the depth, thic d composition of the unit and the stresses to which the unit has been subjected. Fracture sets at different depths may or may not be connected.

\subsection{Salt Site Description}

The geologic barrier consists of geologic components such as the stratigraphy. This section discusses the occurrences of salt deposits, stratigraphy for the hypothetical salt repository, important parameters, and specific parameter values for the geological formations modeled.

\subsubsection{Origin and Occurrence of Salt Deposits}

Salt deposits can originate in a variety of settings, and under proper conditions thick sequences of gypsum and halite can accumulate.

For an evaporite deposit to be preserved, it must be protected from dissolution by undersaturated water. Evaporitic sequences protected by sufficient thickness of overuurden that inhibits dissolution of the soluble evaporites exist all around the world. In the United States, salt deposits are located in about half the states and cover a wide span of geologic time ranging in age from the Silurian to the Pliocene. Figure 5-1 and Table 5-1 summarize salt deposits of sufficient thickness for a waste repository in the United States, and were compiled from the reports of Pierce and Rich (1962) and Isherwood (1981). All the beds occur in association with anhydrite, gypsum, shales, dolomites, and other minor lithologies. 


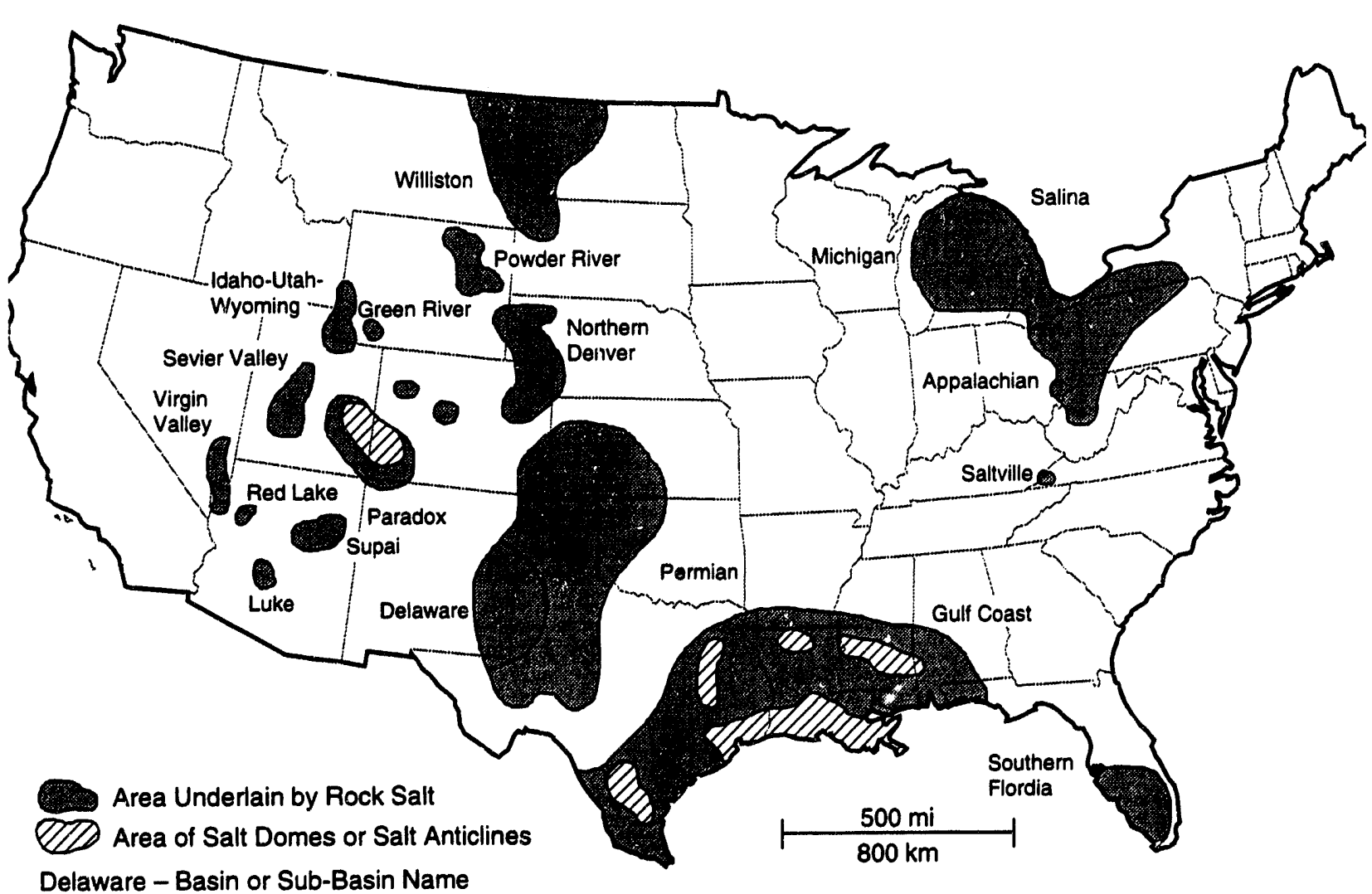

TRI-6342-3523-0

Figure 5-1. Location of salt basins in United States (after Pierce and Rich, 1962).

\subsubsection{Hypothetical Salt Stratigraphy}

Examination of salt beds as a potential nuclear waste repository site began in the early 1960s. Laboratory and field experiments indicated that no phenomena existed that were serious enough to eliminate salt beds as an acceptable geologic medium for waste disposal (Powers et al., 1978, p. 2-26). Potential repository sites were considersand a site with sufficient purity, depth, and thickness in the Permian basin in so. thwestern New Mexico was prnposed for a full-scale Waste Isolation Pilot Plant (WIPP). Because the sequence of marine evaporites of the WIPP is typical, and because extensive data are already availabie, data from this site will be used in the following discussion of a generic salt repository (Figures 5-2 and 5-3). Geologic names used here are fictitious, although properties of the units are based on WIPP-specific data.

Tower Formation. The oldest and lowest formation considered in the conceptual model is a thick (500-m) formation that consists of seven lithologic units: four anhydrite members intercalated with three halite members. The formation is of interest because it contains a pressurized brine reservoir. Pressurized brine reservoirs have been intersected in wells around the site and cannot be conclusively ruled out beneath the site by geophysi al methods. If brine is present below the repository, it may affect releases if a future drill hole connects the brine reservoir to the repository and material is removed from the repository as the brine moves upward into other units or to the land surface. Hydrologic and geochemical data indicate that the brine reservoirs are hydraulically isolated and contain stagnant fluid. The origin of the fluids is probably interstitial entrapment of connate water (fossil water) at time of deposition, or water of dehydration of original gypsum to anhydrite, or meteoric water moving from the adjacent more permeable limestone into the fractured anhydrites of the Tower Formation. Brine at pressures greater than hydrostatic occurs west of the site in a fractured part of the Tower Formation. The pressurized brine reservoir is assumed to exist for the next 10,000 yr with a totally impermeable pocket of material around it. 
Table 5-1. Summary of Salt Deposits in Coterminous United States (from Pierce and Rich, 1962)

ŭ

\begin{tabular}{|c|c|c|c|c|c|}
\hline Location & Type & $\begin{array}{c}\text { Aggregate } \\
\text { Thickness* } \\
\text { (m) }\end{array}$ & $\begin{array}{c}\text { Maximum } \\
\text { Single Bed* } \\
(\mathrm{m})\end{array}$ & $\begin{array}{l}\text { Depth to Top of } \\
\text { Salt* } \\
\text { (m) } \\
\end{array}$ & Remarks \\
\hline \multicolumn{6}{|l|}{ Northeastem States } \\
\hline Appalachian Basin & Bedded & 213-244 & 46 & -305 & $\begin{array}{l}\text { Locally } 300-\mathrm{ft} \text { unit } \\
\text { usually around } 30 \mathrm{ft}\end{array}$ \\
\hline Michigan Basin & Bedded & $152-549$ & & $152-1829$ & \\
\hline Pennsylvania & Bedded & $30-61$ & & & \\
\hline $\begin{array}{l}\text { Ohio } \\
\text { Gulf Coast }\end{array}$ & $\begin{array}{l}\text { Bedded } \\
\text { Domes }\end{array}$ & $\begin{array}{l}30-91 \\
\text { Vertical } \\
\text { very thick }\end{array}$ & & $\begin{array}{l}-305 \\
152-305\end{array}$ & $\begin{array}{l}\text { Thin beds at shallow depth } \\
\text { Impurities do not } \\
\text { occur as beds or pockets }\end{array}$ \\
\hline
\end{tabular}

Permian Basin

NM

TX, OK, KY

\section{Bedded}

Bedded

853

122

Bedded

\section{3-792}

Varies greatly

See notes

Paradox Basin

Bedded

19
9
See notes

Williston Basin

Devonian Age

Mississippian Age

Permian

Triassic-Jurassic

Utah

Nevada-Arizona

Wyoming-Nebraska

Nebraska

Playa deposits in

SW U.S.

$\begin{array}{ll}\text { Bedded } & >122 \\ \text { Bedded } & 91 \\ \text { Bedded } & >46 \\ \text { Bedded } & 122 \\ & \\ \text { Complex } & >61\end{array}$

Com

$>61$

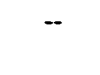

Bedded

Bedded

Bedded

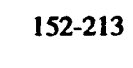

$\sim 29$

$>30$

$\begin{array}{ll}>122 & 122 \\ 91 & 46 \\ >46 & 30 \\ 122 & 91 \\ >61 & 61 \\ 152-213 & 15 \\ >29 & 9 \\ & 12 \\ >30 & >6\end{array}$

122
46
30
91
61
15
9
12
$>6$

407

183

122-1658

\section{"Pure" salt rare \\ Large lateral \\ variation}

\begin{tabular}{ll}
$\begin{array}{l}\text { Similar to Gulf Coast } \\
\text { bedded salt containing }\end{array}$ & $\begin{array}{l}\text { Some very thick } \\
\text { beds on crusts of } \\
\text { many shale } \& \text { anhydrite } \\
\text { beds in anticlines }\end{array}$ \\
\hline
\end{tabular}

beds in anticlines

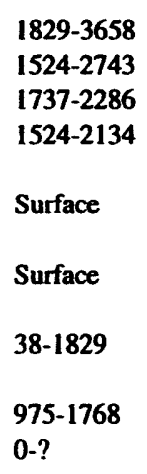

At $3600 \mathrm{ft}$, only 20 -ft bed

Much too deep for mining

(a)

Cover only a small area

About 1600-ft long $x$

1000-ft wide

Cover a small area

Not very much data

May be thick beds

$\sim 0.3-12$
Range of
Thickness*

(m)

6-24

$6-24$

6

$\sim 3$

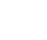

verage*

(m)

Age

*Units are as reported in sources as feet, Pierce and Rich (1962) and Isherwood (1981). To obtain feet, multiply values by 3.281. 


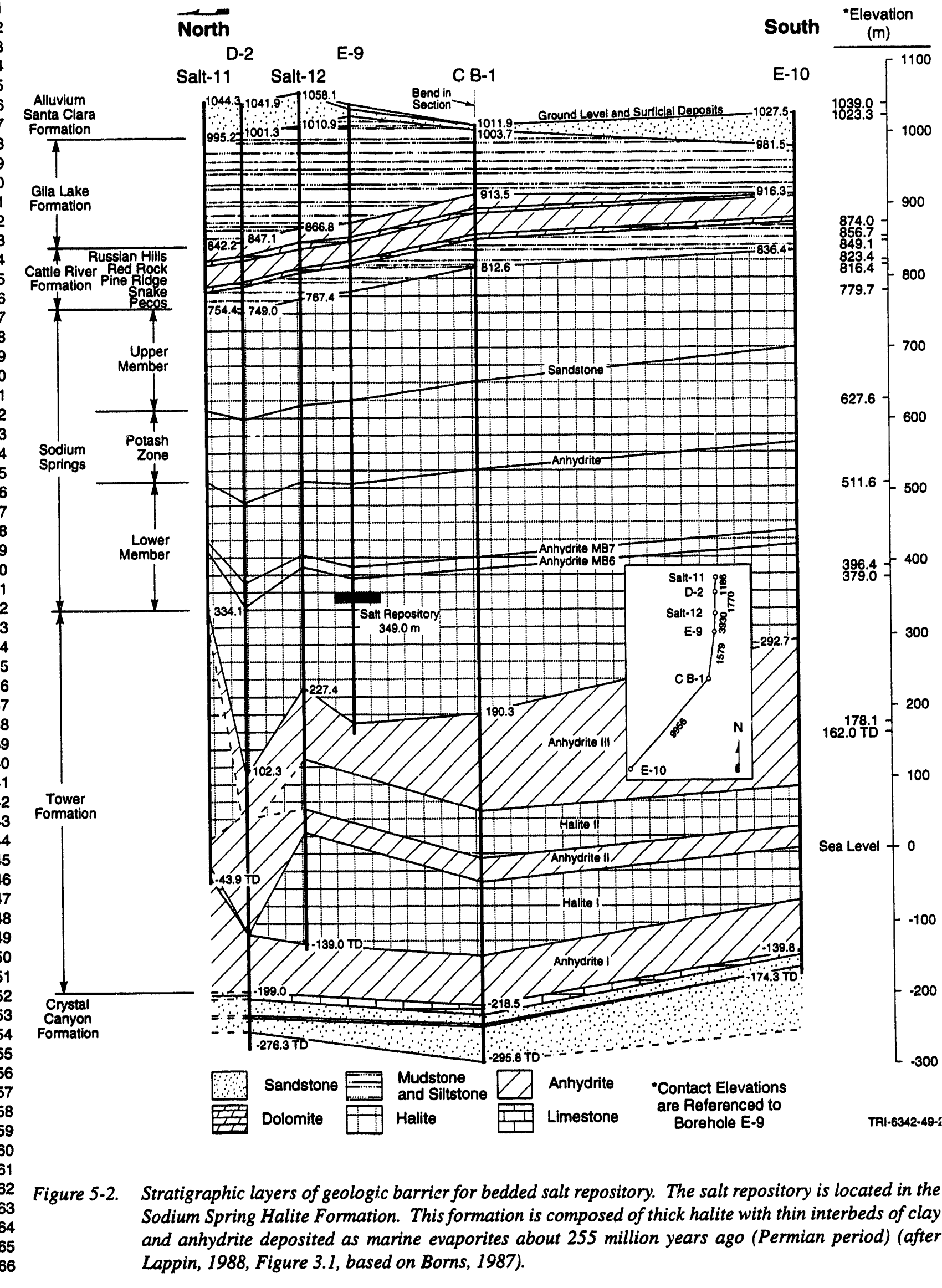

December 1993 


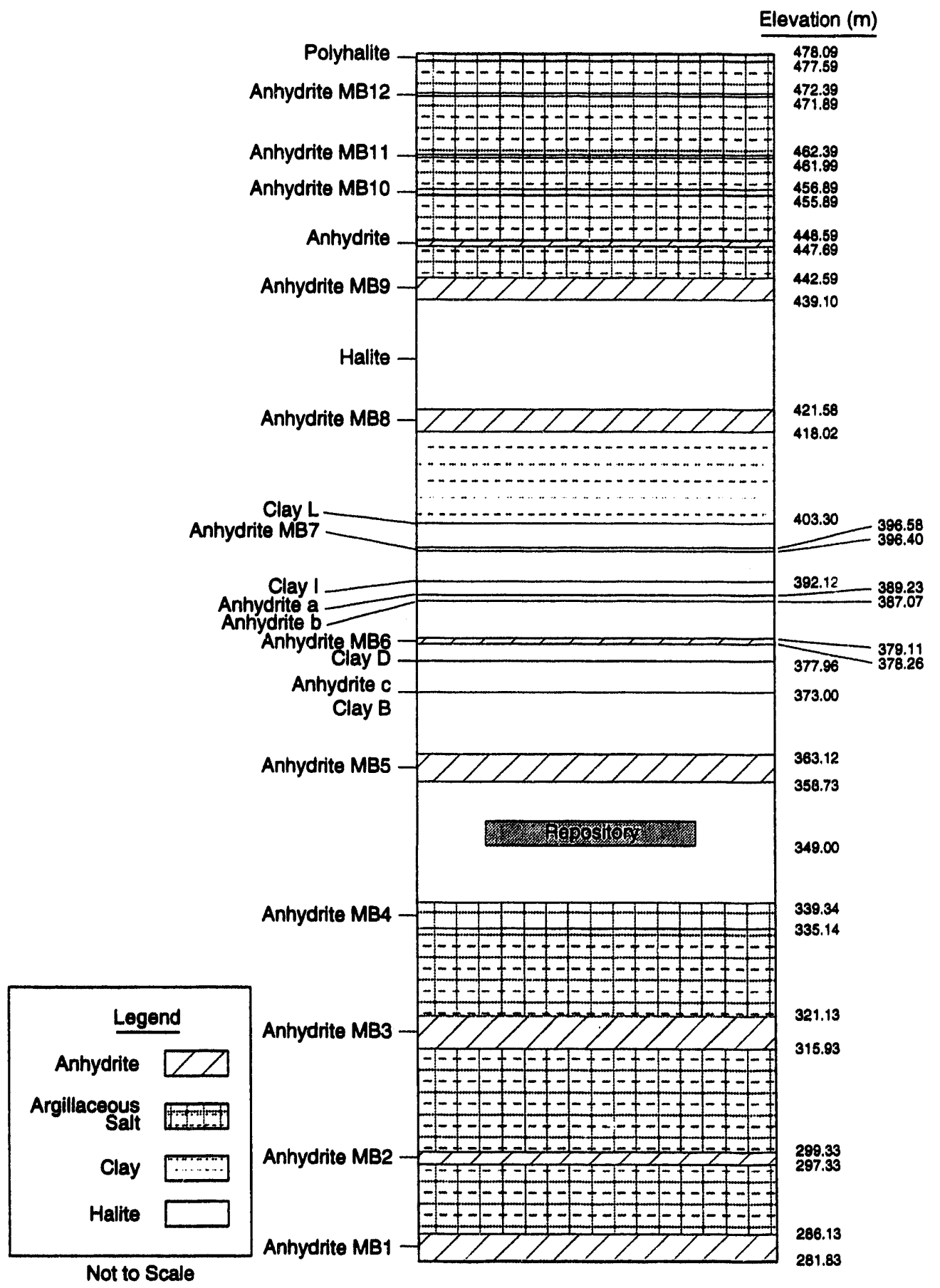

TRI-6342-1070-1

Figure 5-3. Stratigraphy in Sodium Springs Formation near repository (after Munson et al., 1989, Figure 3-3). 
Sodium Springs Formation. The second formation, the halite unit, is the host rock for the repository. The halite unit is about $600 \mathrm{~m}$ thick and consists of three informal members. The lower member is about $340 \mathrm{~m}$ thick and is mostly halite with lesser amounts of anhydrite, polyhalite, and glauberite. It ranges in color from light gray at the bottom to red. The repository is located in this unit about $180 \mathrm{~m}$ above the lower contact with the Tower Formation. The middle member is about $110 \mathrm{~m}$ thick and consists of halite interbedded with sylvite and langbeinite from which potassium salts (potash) are mined nearby. It is separated from the lower member by a thin silty sandstone and from the upper unit by a thin anhydrite. The upper unit is $150 \mathrm{~m}$ of reddish-orange-to-brown halite interbedded with polyhalite, anhydrite, and sandstone.

Cattle River Formation. The Cattle River Formation conformably overlies the Sodium Springs Formation and is the youngest unit of the evaporite series. The following description of the Cattle River Formation is general and may vary locally. The formation is a cyclical series of deposits consisting of $10 \%$ carbonates (dolomite), $30 \%$ sulfates (gypsum and anhydrite), $40 \%$ salts (halite and some polyhalite), and $20 \%$ clastic rocks (mudstone and shale). In the surrounding region where the Cattle River Formation is close to the surface, the anhidrites have been hydrated and converted to gypsum.

The Cattle River Formation has been divided into five members (Figure 5-4). The structure of the tops of each of the five members is very similar to the structure of Sodium Springs Formation; anticlines and synclines are present throughout the section. The five members have an average thickness (from the basal member up) of $40 \mathrm{~m}, 8 \mathrm{~m}, 40 \mathrm{~m}$, $7.5 \mathrm{~m}$ (Snake Dolomite), and $20 \mathrm{~m}$. The Cattle River Formation has a total average thickness of $110 \mathrm{~m}$ but ranges from 8 to $216 \mathrm{~m}$.

The Snake Dolomite Member of the Cattle River Formation (lower dolomite) is a hydrostratigraphic unit that may provide a potential pathway for radionuclides to the accessible environment. This member is a microcrystalline grayish dolomite or dolomitic limestone with solution cavities containing some gypsum and anhydrite filling. The member ranges in thickness from 3 to $14 \mathrm{~m}$. The dolomite has a nearly uniform thickness of $7.5 \mathrm{~m}$ close to the repository site.

\subsubsection{Modeling Assumptions for Geologic Barrier in Salt}

\section{General}

- Fluid (gas and liquid) flow obeys Darcy's law.

- Dissolution of gas into liquid phase is negligible.

- Nuclear criticality does not occur along the flow path (porosities within the geologic barrier are sufficiently low).

- Host salt formation is overlain by one major horizontal aquifer (Snake Dolomite Member of Cattle River Formation).

- Host salt formation is potentially underlain by brine reservoir in Tower Formation.

- Other strata are assumed not to affect the repository.

- Bedding planes are horizontal.

\section{Sodium Springs Formation}

- Repository horizon is in a thick, relative pure halite sequence (Sodium Springs Formation).

- Formation is of low porosity and permeability.

- Formation is initially brine saturated.

\section{Snake Dolomite Member of Cattle River Formation}

- Thickness of brine aquifer in Snake Dolomite is constant.

- Aquifer in Snake Dolomite is hydrologically confined with no vertical leakage.

- Aquifer flow is unaffected by potential subsidence from any nearby potash mining or the repository.

- Fluctuations in aquifer flow caused by climate changes are minor and thus inconsequential. 


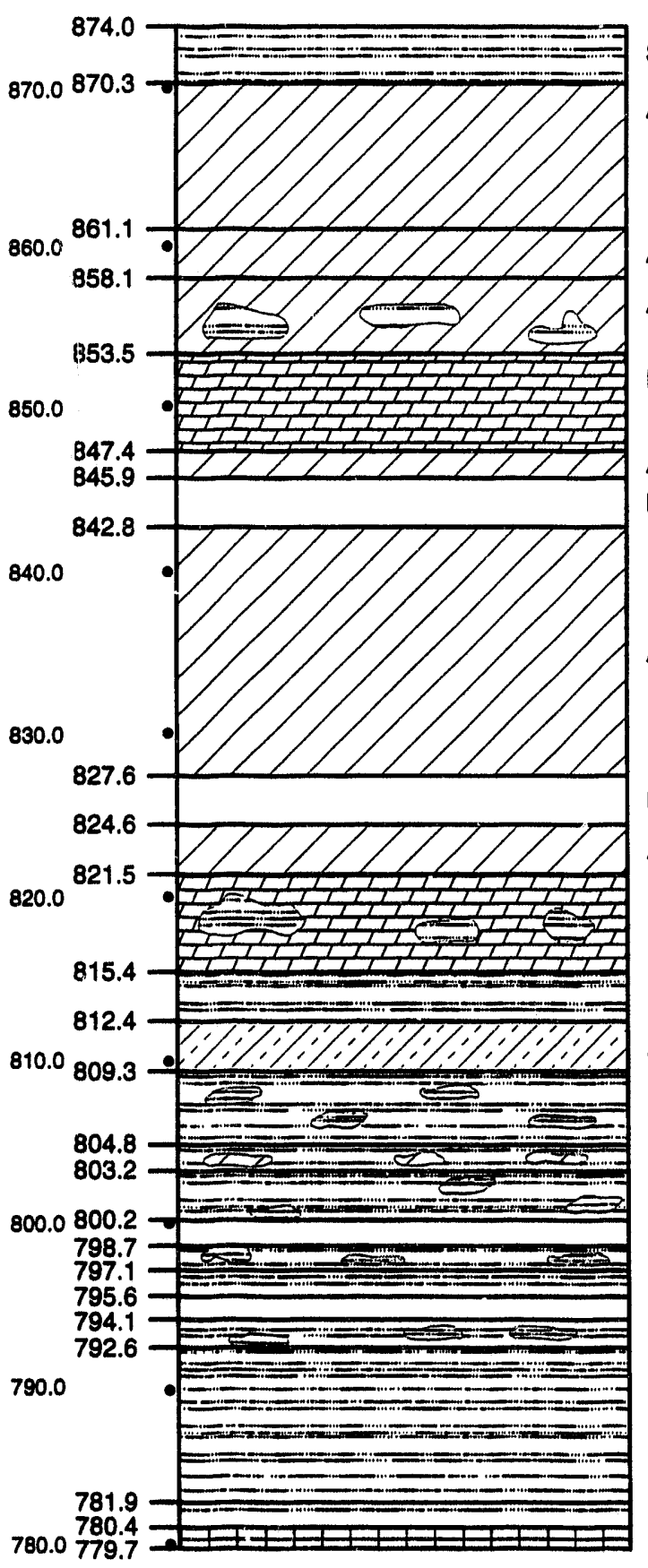

No Sample

Anhydrite

Dolomite(Mudstone)

Mudstone

Anhydrite(Gypsum)

Siltstone(Argillaceous)

Siltstone(Anhydrite)

Siltstone(Argillaceous)

No Sample

Siltstone(Argillaceous)

Siltstone

Siltstone(Argillaceous)

Siltstone

Siltstone

Halite
Russian Hills

Member

Red Rock

Dolomite

Member

$\zeta$

Fine Ridge

Member

Snake

Dolomite

Member

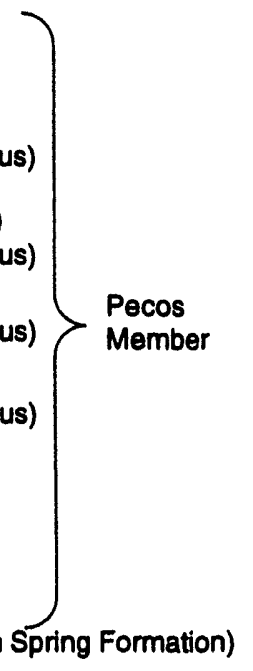

TRI-6342-527-2

Figure 5-4. Detailed lithology of hypothetical Cattle River Formation (after SNL and U.S. Geological Survey, 1983). 
- Dual porosity model without chemical retardation (see Chapter 12 ) adequately describes radionuclide transport in the Snake Dolomite aquifer.

- Brine flow from intrusion borehole does not alter flow in Snake Dolomite (Reeves et al., 1991).

The groundwater-flow model for the Snake Dolomite includes hydrologic characteristics based on extensive data available for the Culebra at the WIPP site, including the following:

- Flow occurs through a densely interconnected network of fractures and allows the Snake Dolomite to be modeled as a porous medium at the scale of interest $(5 \mathrm{~km})$.

- Fluid may be exchanged between fractures and the matrix pore space, in response to pressure differences between the fracture and matrix. The fluid exchange with the dolomite matrix is sufficiently rapid to permit approximation as a single porosity medium for the purpose of modeling flow (Reeves et al., 1991).

- Variations in fracture density, degree of fracture filling, and other factors produce large variability in bulk permeability of the fracture network over the site and, consequently, create tortuous advective pathways between potential intrusion borehole locations and the accessible environment boundary; thus, specific groundwater discharge (Darcy velocity) can vary greatly along the path.

Uncertainty in transmissivity is represented using the geostatistics approach described for the WIPP (LaVenue and RamaRao, 1992) (see Chapter 12).

\subsubsection{Model Parameters for Salt}

This section lists the parameters used in the computational models that are discussed in Chapters 12 and 14 . Although it is convenient in terms of organization to include the model parameters for the geologic barrier here, the reader may wish to defer examining them until he or she has read the discussion on the conceptual and mathematical models (Chapters 12 and 14).

Formation properties used in the Complex $\mathrm{PA}^{\dagger \dagger}$ of the salt repository are tabulated in Tables 5-2 through 5-6 for the stratigraphic units described in the Section 5.2.2. The Simple PA used a subset of these properties. The range of the parameters, the median value, and the source of the data are provided in these tables. ${ }^{\ddagger \ddagger}$ The parameters shown in bold were sampled. Because the emphasis of the performance assessment was on the waste treatment options, only a few geologic barrier parameters were sampled. Several importanit parameters are described below.

Some of the parameters in Tables 5-2 through 5-5 were selected based on the judgment of the analyst because of a lack of data on the parameter, simplifying assumptions, or a specific modeling objective. When this is the case, the term "analyst decision" appears as the source.

Three parameters appear repeatedly; they are the initial brine saturation (Saturatn), the thermal expansion reference temperature (DnsTmpRf), and the reference pressure used in the compressibility calculations (PorPrsRf). All materials, except the crushed salt backfill ${ }^{* * *}$ (see Chapter 6), were assumed to be brine saturated initially. The reference temperature and pressure for all materials were chosen as $298 \mathrm{~K}\left(25^{\circ} \mathrm{C}\right)$ and $1.013 \times 10^{5} \mathrm{MPa}(1 \mathrm{~atm})$, respectively, except in the Jower Formation and the brine reservoir within the Tower Formation.

Parameters for Halite and Polyhalite within Sodium Springs Formation. At the repository site, groundwater flow is minimal because of the plastic nature of halite within salt deposits, which results in low porosity and few open fractures. Permeability of the Sodium Springs Formation is low but measurable. Formation pressure varies from hydrostatic to lithostatic. Although the formation is believed to be brine saturated, the low permeability allows for very little groundwater movement.

\footnotetext{
†† Refer to Section 7.5, "Conceptual Model," for a description of the Simr'e and Complex PA formulations.

\# Refer to Section 9.1, "Model Parameters," for a general description of how a distribution is assigned.

The initial brine saturation in the crushed-salt backfill was assumed equal to the residual brine saturation, 0.20 , simulating dry emplacement.
} 


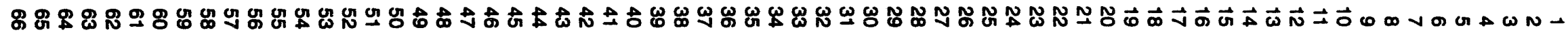

Table 5-2. Parameter Values for Halite and Polyhalite within Sodium Springs Formation near Repository

\begin{tabular}{|c|c|c|c|c|c|c|c|c|}
\hline \multirow{2}{*}{$\begin{array}{c}\begin{array}{c}\text { Record } \\
\text { No. }\end{array} \\
834\end{array}$} & \multirow{2}{*}{$\frac{\text { Parameter }}{\text { BrkCorEx }}$} & \multirow{2}{*}{$\begin{array}{l}\text { Parameter Description } \\
\text { Brooks-Corey Exponent for capillary } \\
\text { pressure }\end{array}$} & \multirow{2}{*}{$\frac{\text { Median }}{7.00 e-01}$} & \multicolumn{2}{|c|}{ Range } & \multirow{2}{*}{$\frac{\text { Units }}{\text { none }}$} & \multirow{2}{*}{$\begin{array}{c}\text { Distribution } \\
\text { Type }\end{array}$} & \multirow{2}{*}{$\begin{array}{l}\text { Sources In } \\
\text { Sandia WIPP } \\
\text { Project, } 1992\end{array}$} \\
\hline & & & & $2.00 e-01$ & $1.00 e+01$ & & & \\
\hline 2279 & Compres & Compressibility & $4.80 e-11$ & & & $\mathrm{~Pa}^{-1}$ & Constant & Krieg, 1984 \\
\hline 847 & LogPrmU & Log permeability undisturbed & -21.20 & -24.00 & -19.00 & $\log \left(m^{2}\right)$ & Constructed & $\begin{array}{l}\text { Sandia WIPP } \\
\text { Project, } 1992\end{array}$ \\
\hline 2273 & DnsGm & Material grain density & $2.16 e+03$ & & & $\mathrm{~kg} / \mathrm{m}^{3}$ & Constant & $\begin{array}{l}\text { Sandia WIPP } \\
\text { Project, } 1992\end{array}$ \\
\hline 849 & Pore_U & Porosity, undisturbed & $1.00 \mathrm{e}-02$ & $1.00 e-03$ & $3.00 e-02$ & none & Constructed & $\begin{array}{l}\text { Sandia WIPP } \\
\text { Project, } 1992\end{array}$ \\
\hline 2162 & PorPrsRf & Reference pressure for porosity & $1.01 e+05$ & & & $\mathbf{P a}$ & Constant & $\begin{array}{l}\text { Ramsey, analyst } \\
\text { decision }\end{array}$ \\
\hline 2161 & DnsTmpHf & Reference temperature for density & $2.98 e+02$ & & & $\mathbf{K}$ & Constant & $\begin{array}{l}\text { Ramsey, analyst } \\
\text { decision }\end{array}$ \\
\hline 2276 & SatRGP & Residual gas saturation & $2.00 e-01$ & 0 & $4.00 e-01$ & none & Unitorm & $\begin{array}{l}\text { Sandia WIPP } \\
\text { Project, } 1992\end{array}$ \\
\hline 2275 & SatRWP & Residual wetting phase (liquid) saturation & $2.009-01$ & 0 & $4.000-01$ & none & Uniform & $\begin{array}{l}\text { Sandia WIPP } \\
\text { Project, } 1992\end{array}$ \\
\hline 2163 & Saturatn & Saturation, initial & $1.00 e+00$ & & & none & Constant & $\begin{array}{l}\text { Ramsey, analyst } \\
\text { decision }\end{array}$ \\
\hline 2156 & SpHeat & Specific heat & $8.60 e+02$ & & & $\mathrm{~J} / \mathrm{kg} \cdot \mathrm{K}$ & Constant & Krieg, 1984 \\
\hline 2188 & ThExp & Thermal expansion coefficient & $4.500-05$ & & & $1 / K$ & Constant & Krieg, 1984 \\
\hline 2280 & ThCnd & Thermal conductivity & $5.00 e+00$ & & & $\mathrm{~W} / \mathrm{m} \bullet \mathrm{K}$ & Constant & Krieg, 1984 \\
\hline
\end{tabular}




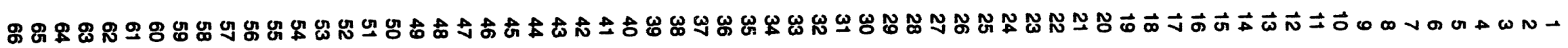

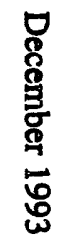

Table 5-3. Parameter Values for Anhydrite Marker Bed within Sodium Spring Formation near Repository

\begin{tabular}{|c|c|c|c|c|c|c|c|c|}
\hline \multirow{2}{*}{$\begin{array}{c}\text { Record } \\
\text { No. }\end{array}$} & \multirow{2}{*}{$\bar{B} \cdot \frac{\text { Prameter }}{\text { SorEx }}$} & \multirow{2}{*}{$\begin{array}{l}\text { Parameter Description } \\
\text { Brooks-Corey Exponent for capillary } \\
\text { pressure }\end{array}$} & \multirow{2}{*}{$\frac{\text { Median }}{7.00 \mathrm{e}-01}$} & \multicolumn{2}{|c|}{ Range } & \multirow{2}{*}{$\frac{\text { Units }}{\text { none }}$} & \multirow{2}{*}{$\begin{array}{c}\text { Distribution } \\
\text { Type }\end{array}$} & \multirow{2}{*}{$\begin{array}{l}\text { Sources In: } \\
\text { Sandia WIPP Project, } \\
1992\end{array}$} \\
\hline & & & & $2.000-01$ & $1.00 e+01$ & & & \\
\hline 2197 & Compres & Compressibility & $1.20 e-11$ & & & $\mathrm{~Pa}^{-1}$ & Constant & Krieg, 1984 \\
\hline 2167 & Elevat & Elevation above mean sea level & $3.79 e+02$ & & & $\mathbf{m}$ & Constant & SNL and USGS, 1983 \\
\hline 626 & LogPrmU & Log permeability undisturbed & -19.30 & -21.00 & -16.00 & $\log \left(m^{2}\right)$ & Constructed & $\begin{array}{l}\text { Sandia WIPP Project, } \\
1992\end{array}$ \\
\hline 2198 & DnsGm & Material grain density & $2.96 e+03$ & & & $\mathrm{~kg} / \mathrm{m}^{3}$ & Constant & $\begin{array}{l}\text { Sandia WIPP Project, } \\
1992\end{array}$ \\
\hline 629 & Pore_U & Porosity, undisturbed & $1.00 e-02$ & $1.000-03$ & $3.00 e-02$ & none & Constructed & $\begin{array}{l}\text { Sandia WIPP Project, } \\
1992\end{array}$ \\
\hline 2166 & Pressure & Pressure & $1.25 e+07$ & $1.20 e+07$ & $1.30 \mathrm{e}-07$ & $\mathbf{P a}$ & Uniform & Davies et al., 1992 \\
\hline 2196 & PorPrsRf & Reference pressure for porosity & $1.01 e+05$ & & & $\mathrm{~Pa}$ & Constant & $\begin{array}{l}\text { Ramsey, analyst } \\
\text { decision }\end{array}$ \\
\hline 2195 & DnsTmpRf & Reference temperature for density & $2.98 e+02$ & & & K & Constant & $\begin{array}{l}\text { Ramsey, analyst } \\
\text { decision }\end{array}$ \\
\hline 2201 & SatRGP & Residual gas saturation & $2.00 e-01$ & 0 & $4.00 e-01$ & none & Uniform & $\begin{array}{l}\text { Sandia WIPP Project, } \\
1992\end{array}$ \\
\hline 2202 & SatRWP & Residual wetting phase (liquid) saturation & $2.00 e-01$ & 0 & $4.00 \mathrm{e}-01$ & none & Uniform & $\begin{array}{l}\text { Sandia WIPP Project, } \\
1992\end{array}$ \\
\hline 2200 & Saturatn & Saturation, initial & $1.00 e+00$ & & & none & Constant & $\begin{array}{l}\text { Ramsey, analyst } \\
\text { decision }\end{array}$ \\
\hline 2194 & SpHeat & Specific heat & $8.60 e+02$ & & & $\mathrm{~J} / \mathrm{kg} \cdot \mathrm{K}$ & Constant & Krieg, 1984 \\
\hline 2193 & ThExp & Thermal expansion coefficient & $2.00 e-05$ & & & $1 / K$ & Constant & Krieg, 1984 \\
\hline 2192 & ThCnd & Thermal conductivity & $4.70 e+00$ & & & $\mathrm{~W} / \mathrm{m} \cdot \mathrm{K}$ & Constant & Krieg, 1984 \\
\hline
\end{tabular}




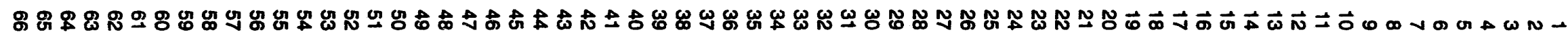

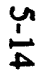

Tabłe 5-4. Parameter Values for Snake Dolomite Member near Repository

\begin{tabular}{|c|c|c|c|c|c|c|c|c|}
\hline \multirow{2}{*}{$\begin{array}{l}\text { Record } \\
\text { No. } \\
2309\end{array}$} & \multirow{2}{*}{$\frac{\text { Parameter }}{\text { BrkCorEx }}$} & \multirow{2}{*}{\begin{tabular}{l}
\multicolumn{1}{c}{ Parameter Description } \\
$\begin{array}{l}\text { Brooks-Corey Exponent for capillary } \\
\text { pressure }\end{array}$
\end{tabular}} & \multirow{2}{*}{$\frac{\text { Median }}{7.00 e-01}$} & \multicolumn{2}{|c|}{ Range } & \multirow{2}{*}{$\frac{\text { Units }}{\text { none }}$} & \multirow{2}{*}{$\begin{array}{c}\text { Distribution } \\
\text { Type }\end{array}$} & \multirow{2}{*}{$\begin{array}{l}\text { Sources In: } \\
\text { Sandia WIPP Project, } \\
1992\end{array}$} \\
\hline & & & & $2.00 e-01$ & $1.00 e+01$ & & & \\
\hline 2217 & Compres & Compressibility & $1.000-09$ & $1.00 \mathrm{e}-08$ & $1.000-10$ & $\mathrm{~Pa}^{-1}$ & Uniform & $\begin{array}{l}\text { Freeze and Cherry, } \\
1979\end{array}$ \\
\hline 2210 & DnsGm & Material grain density & $2.82 \theta+03$ & $2.78 e+03$ & $2.86 e+03$ & $\mathrm{~kg} / \mathrm{m}^{3}$ & Normal & $\begin{array}{l}\text { Kelley and Saulnier, } \\
\text { 1990; WIPP PA Div., } \\
\text { 1991c }\end{array}$ \\
\hline 2205 & Porosity & Matrix porosity & $1.45 \theta-01$ & $9.500-02$ & $2.52 e-01$ & none & Data & $\begin{array}{l}\text { Lappin et al., 1989; } \\
\text { Kelley and Saulnier, } \\
1990\end{array}$ \\
\hline 2209 & Prm & Permeability & $1.00 e-14$ & $1.00 e-17$ & $1.00 e-11$ & $m^{2}$ & Lognormal & Brinster, 1991 \\
\hline 2211 & Pressure & Pressure & $1.05 e+06$ & & & $\mathrm{~Pa}$ & Constant & $\begin{array}{l}\text { Ramsey, analyst } \\
\text { decision }\end{array}$ \\
\hline 2215 & PorPrsAf & Reference pressure for porosity & $1.01 e+05$ & & & $\mathbf{P a}$ & Constant & $\begin{array}{l}\text { Ramsey, analyst } \\
\text { decision }\end{array}$ \\
\hline 2214 & DnsTmpAf & Reference temperature for density & $2.98 e+02$ & & & K & Constant & $\begin{array}{l}\text { Ramsey, analyst } \\
\text { decision }\end{array}$ \\
\hline 2208 & SatRGP & Residual gas saturation & $2.00 e-01$ & 0 & $4.00 e-i 1$ & none & Uniform & $\begin{array}{l}\text { Sandia WIPP Project, } \\
1992\end{array}$ \\
\hline 2207 & SatRWP & Residual wetting phase (liquid) saturation & $2.00 e-01$ & 0 & $4.00 e-01$ & none & Uniform & $\begin{array}{l}\text { Sandia WIPP Project, } \\
1992\end{array}$ \\
\hline 2206 & Saturatn & Saturation, initial & $1.00 e+00$ & & & none & Constant & $\begin{array}{l}\text { Ramsey, analyst } \\
\text { decision }\end{array}$ \\
\hline 2216 & SpHeat & Specific heat & $8.92 e+02$ & $8.54 e+02$ & $9.30 e+02$ & $\mathrm{~J} / \mathrm{kg}$ & Uniform & $\begin{array}{l}\text { Weast, 1976: Lide, } \\
1991\end{array}$ \\
\hline 2213 & ThExp & Thermal expansion coefficient & $6.00 e-06$ & $2.00 e-06$ & $1.00 e-05$ & $1 / K$ & Uniform & Durham et al., 1987 \\
\hline 2212 & ThCnd & Thermal conductivity & $3.00 \theta+00$ & $2.60 e+00$ & $3.40 e+00$ & W/moK & Uniform & Durham et al., 1987 \\
\hline
\end{tabular}




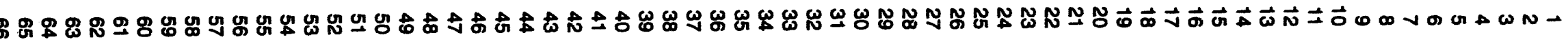

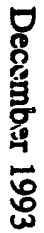

Table 5-5. Parameter Values for Tower Formation beneath Repository

\begin{tabular}{|c|c|c|c|c|c|c|c|c|}
\hline $\begin{array}{l}\text { Record } \\
\text { No. }\end{array}$ & Parameter & Parameter Description & Median & & & Units & $\begin{array}{l}\text { Dlstribution } \\
\text { Type }\end{array}$ & Sources in: \\
\hline 2301 & BrkCorEx & $\begin{array}{l}\text { Brooks-Corey Exponent for capillary } \\
\text { pressure }\end{array}$ & $7.00 e-01$ & $2.00 e-01$ & $1.00 e+01$ & none & Constructed & $\begin{array}{l}\text { Sandia WIPP } \\
\text { Project, } 1992\end{array}$ \\
\hline 2305 & Compres & Compressibility & $8.50 \theta-11$ & $3.00 e-11$ & $1.40 e-10$ & $\mathbf{P a}^{-1}$ & Uniform & Popielak et al., 1983 \\
\hline 2307 & DnsGrn & Material grain density & $2.96 e+03$ & & & $\mathrm{~kg} / \mathrm{m}^{3}$ & Constant & $\begin{array}{l}\text { Sandia WIPP } \\
\text { Project, } 1992\end{array}$ \\
\hline 2306 & Porosity & Matrix porosity & $5.00 \theta-03$ & $1.00 e-03$ & $1.000-02$ & none & Constructed & $\begin{array}{l}\text { Sandia WIPP } \\
\text { Project, } 1992\end{array}$ \\
\hline 2295 & Prm_X & Permeability in $\mathrm{X}$ direction & $1.000-19$ & $1.00 \theta-20$ & $1.00 e-18$ & $\mathrm{~m}^{2}$ & Constructed & $\begin{array}{l}\text { Sandia WIPP } \\
\text { Project, } 1992\end{array}$ \\
\hline 2296 & Prm_Y & Permeability in $Y$ direction & $0.00 e+00$ & & & none & Constant & $\begin{array}{l}\text { Ramsey, analyst } \\
\text { decision }\end{array}$ \\
\hline 2308 & PorPrsAf & Reference pressure for porosity & $1.48 e+07$ & $1.01 e+07$ & $1.74 e+07$ & $\mathrm{~Pa}$ & Data & $\begin{array}{l}\text { Sandia WIPP } \\
\text { Project, } 1992\end{array}$ \\
\hline 2323 & ModRîPrs & Reference pressure, model number & $1.70 e+07$ & & & $\mathrm{~Pa}$ & Constant & $\begin{array}{l}\text { Ramsey, analyst } \\
\text { decision }\end{array}$ \\
\hline 2297 & DnsTmpRf & Reference temperature for density & $2.98 \theta+02$ & & & K & Data & $\begin{array}{l}\text { Sandia WIPP } \\
\text { Project, } 1992\end{array}$ \\
\hline 2302 & SatRGP & Residual gas saturation & $2.00 e-01$ & 0 & $4.00 e-01$ & none & Uniform & $\begin{array}{l}\text { Sandia WIPP } \\
\text { Project, } 1992\end{array}$ \\
\hline 2303 & SatPWP & Residual wetting phase (liquid) saturation & $2.00 \theta-01$ & 0 & $4.00 e-01$ & none & Uniform & $\begin{array}{l}\text { Sandia WIPP } \\
\text { Project, } 1992\end{array}$ \\
\hline 2304 & Saturatn & Saturation, initial & $1.00 e+00$ & & & none & Constant & $\begin{array}{l}\text { Ramsey, analyst deci- } \\
\text { sion }\end{array}$ \\
\hline 2300 & SpHeat & Specific heat & $8.60 e+02$ & & & J/kg-K & Constant & Krieg, 1984 \\
\hline 2298 & ThExp & Thermal expansion coefficient & $2.00 e-05$ & & & $1 / K$ & Constant & Krieg, 1984 \\
\hline 2299 & ThCnd & Thermal conductivity & $4.70 e+00$ & & & W/moK & Constant & Krieg, 1984 \\
\hline
\end{tabular}




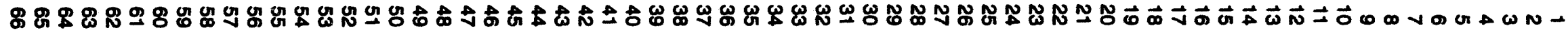

Table 5-6. Parameter Values for Global Materials and Agents Acting on the Salt Disposal System

\begin{tabular}{|c|c|c|c|c|c|c|c|c|}
\hline \multirow{2}{*}{$\begin{array}{l}\begin{array}{c}\text { Record } \\
\text { No. }\end{array} \\
30\end{array}$} & \multirow{2}{*}{$\frac{\text { Parameter }}{\text { Diameter }}$} & \multirow{2}{*}{$\begin{array}{l}\text { Parameter Description } \\
\text { Borehole diameter }\end{array}$} & \multirow{2}{*}{$-\frac{\text { Median }}{2.000 \theta-01}$} & \multicolumn{2}{|c|}{ Range } & \multirow{2}{*}{$\frac{\text { Units }}{m}$} & \multirow{2}{*}{$\begin{array}{l}\begin{array}{c}\text { Distribution } \\
\text { Type }\end{array} \\
\text { Delta }\end{array}$} & \multirow{2}{*}{\begin{tabular}{l}
\multicolumn{1}{c}{ Sources In: } \\
Sandia WIPP \\
Project, 1992
\end{tabular}} \\
\hline & & & & $1.207 \theta-01$ & $4.445 e-01$ & & & \\
\hline 2218 & SatRWP & Residual liquid saturation & $2.000 e-01$ & 0 & $4.000 \theta-01$ & none & Uniform & $\begin{array}{l}\text { Sandia WIPP } \\
\text { Project, } 1992\end{array}$ \\
\hline 2219 & BrkCorEx & Brooks-Corey exponent & $7.000 e-01$ & $2.000 e-01$ & $1.0000+01$ & none & Cumulative & $\begin{array}{l}\text { Sandia WIPP } \\
\text { Project, } 1992\end{array}$ \\
\hline 2220 & Saturatn & Saturation & $2.000 e-01$ & & & none & Constant & $\begin{array}{l}\text { Ramsey, analyst } \\
\text { decision }\end{array}$ \\
\hline 2222 & DnsBlk & Density, bulk & $2.030 e+03$ & & & $\mathrm{~kg} / \mathrm{m}^{2}$ & Constant & Rechard, Ed., 1992 \\
\hline 2223 & SpHeat & Specific Heat & $8.600+02$ & & & J/kgeK & Constant & Krieg, 1984 \\
\hline 2224 & ThCond & Thermal Conductivity & $5.000+00$ & & & W/moK & Constant & Krieg, 1984 \\
\hline 2225 & ThExp & Thermal expansion coefficient & $4.500 \theta-05$ & & & $1 / K$ & Constant & Krieg, 1984 \\
\hline 2226 & DnsTmpRf & Reference temperature, density & $2.980 e+02$ & & & K & Constant & $\begin{array}{l}\text { Ramsey, anatyst } \\
\text { decision }\end{array}$ \\
\hline 2227 & PorPrsRf & Reference pressure, porosity & $1.013 e+05$ & & & $\mathrm{~Pa}$ & Constant & $\begin{array}{l}\text { Ramsey, analyst } \\
\text { decision }\end{array}$ \\
\hline 2228 & Compres & Compressibility & 0 & & & 1/Pa & Constant & $\begin{array}{l}\text { Ramsey, analyst } \\
\text { decision }\end{array}$ \\
\hline 2229 & Porosity & Porosity & $3.750 \theta-01$ & $2.500 e-01$ & $5.000 e-01$ & none & Normal & $\begin{array}{l}\text { Sandia WIPP } \\
\text { Project, } 1992\end{array}$ \\
\hline 2231 & Tintr & Time of Intrusion & $3.156 \theta+10$ & 0 & $3.154 e+11$ & s & Uniform & $\begin{array}{l}\text { Ramsey, analyst } \\
\text { decision }\end{array}$ \\
\hline 2232 & SatRGP & Residual gas saturation & 0 & & & none & Constant & $\begin{array}{l}\text { Ria.nsey, analyst } \\
\text { decision }\end{array}$ \\
\hline 2233 & Prm & Permeability & $3.1600-13$ & $1.000 e-14$ & $1.000 e-11$ & $\mathrm{~m}^{2}$ & Lognormal & $\begin{array}{l}\text { Sandia WIPP } \\
\text { Project, } 1992\end{array}$ \\
\hline
\end{tabular}


A summary of the parameters for the halite and polyhalite within the Sodium Springs Formation near the repository is given in Table 5-2.

Permeability. Permeability of the Sodium Springs Formation is, like the Tower Formation, very low. Undisturbed permeability of the halites range from $10^{-19}$ to $10^{-24} \mathrm{~m}^{2}$ with a median of $6 \times 10^{-22} \mathrm{~m}^{2}$ (Sandia WIPP Project, 1992, p. 2-29). Disturbed permeability ranges from $10^{-22}$ to $10^{-15} \mathrm{~m}^{2}$ with a median of $2 \times 10^{-21} \mathrm{~m}^{2}$.

Porosity. Undisturbed porosity of the Sodium Springs Formation ranges from 0.001 to 0.03 with a median of 0.01. Porosity of disturbed halite near excavations is estimated at 0.06 (Sandia WIPP Project, 1992, p. 2-11).

Elevations. The elevations of all modeled units are fixed relative to the elevation of the anhydrite marker bed, set at $379.11 \mathrm{~m}$ above sea level.

Threshold Pressure. Threshold pressure plays an important role in controlling which formation lithologies are accessible to gas and at what pressure gas will flow. In this performance assessment, threshold pressure is equal to the capillary pressure at a saturation equal to the residual gas saturation (Sandia WIPP Project, 1992). This definition was adopted because flev $v$ of waste-generated gas outward from the repository requires that outward flowing gas penetrate and establish a gas-filled network of flow paths in the surrounding bedded salt.

Threshold pressure is also a key parameter in the Brooks and Corey (1964) model used to characterize the 2phase properties of analogue materials for preliminary gas-flow calculations (Davies and LaVenue, 1990).

Parameter values related to residual saturation are the Brooks and Corey exponent, residual wetting phase, residual gas saturation, and initial saturation.

Thermomechanical Parameters. During the first decades after emplacement, the decaying radioactive wastes generate heat that can drive fluid flow, create thermal stresses, and possibly exacerbate temperature-dependent creep. The thermomechanical parameters are compressibility, specific heat, thermal conductivity, and thermal expansion coefficient.

Compressibility - Compressibility $(\beta)$ of each material is the relative change in density $(\rho)$ with a change in pressure (P). It can also be expressed as the reciprocal of the bulk modulus (K). Krieg $(1984, \mathrm{p}$. 16) lists values of the bulk moduli of halite and anhydrite as $20.7 \mathrm{GPa}$ and $83.4 \mathrm{GPa}$ respectively, which yield compressibilities of $4.83 \times$ $10^{-11} \mathrm{~Pa}^{-1}$ and $1.2 \times 10^{-11} \mathrm{~Pa}^{-1}$. A second method of determining compressibility is presented by Munson (1992, $\mathrm{p}$. A-109). Values are reduced by a factor of 12.5 to conform to a "reduced modulus" model. This yielded a bulk modulus of $1.66 \mathrm{GPa}$ (or a compressibility of $6.04 \times 10^{-10} \mathrm{~Pa}^{-1}$ ). A third method of calculating the compressibility is a method using the elastic properties of the rock (from Green and Wang, 1990, p. 1632, in a memo dated 04/07/93):

$$
\beta=\frac{1}{K+4 G / 3}
$$

$$
\text { where } \quad \begin{aligned}
& \beta=\text { Rock compressibility }\left(\mathrm{Pa}^{-1}\right) \\
& \mathrm{K}=\text { Bulk modulus }(\mathrm{Pa})=2.07 \mathrm{GPa} \\
& \mathrm{G}=\text { Shear modulus }(\mathrm{Pa})=12.4 \mathrm{GPa}(\text { from Krieg, 1984) }
\end{aligned}
$$

This method yields a bulk compressibility of $2.7 \times 10^{-11} \mathrm{~Pa}^{-1}$ for halite and $8.3 \times 10^{-12} \mathrm{~Pa}^{-1}$ for anhydrite. Pore volume compressibility is calculated as:

$$
\beta_{p}=\frac{\beta}{\phi}
$$

where $\phi=$ porosity $=0.01$ (assumed for halite and anhydrite). This yields pore volume compressibilities of $2.7 \times 10^{-9}$ $\mathrm{Pa}^{-1}$ and $8.3 \times 10^{-10} \mathrm{~Pa}^{-1}$ for halite and anhydrite, respectively. 
Specific Heat-The specific heat, $C_{p}$, of evaporite minerals is used to calculate the thermal response of a geologic repository to heat generation of waste using the expression

$$
\mathrm{C}_{\mathrm{p}}=\mathrm{K}_{\mathrm{T}} / \rho \mathrm{d}^{*}
$$

where $K_{T}$ is the thermal conductivity, $d^{*}$ is thermal diffusivity, and $\rho$ is grain density. Tien et al. (1983) report that results from measurements and theoretical calculations produce approximately the same results. The specific heat of crystals of halite ranges from 0.20 to $0.22 \mathrm{cal} / \mathrm{g}^{\circ} \mathrm{C}$. The specific heat of rock salt varies due to the present of impurities (Tien et al., 1983).

The specific heat for evaporites can be calculated if the composition is known and can also be adjusted for the presence of fluid. The expression for calculating specific heat is

$$
C_{p}(\text { rock })=\sum_{i=1}^{n} x_{i} C_{p}(i)
$$

where $C_{p}(i)$ is the specific heat of the ith mineral, and $x_{i}$ is the weight fraction of the mineral. If fluids are present, the expression is

$$
\mathrm{C}_{\mathrm{p}} \text { (rock) }=(1-\phi) \mathrm{C}_{\mathrm{p}} \text { (rock) }+\mathrm{C}_{\mathrm{p}} \text { (fluid) }
$$

where $\phi$ is the total porosity and $C_{p}$ (rock) is the adjusted specific heat (Tien et al., 1983). Porosity is very low for halite and Tien et al. (1983) considered the affects of brine to be negligible. Tien et al. (1983) give a range of 0.19 to $0.23 \mathrm{cal} / \mathrm{g}^{\circ} \mathrm{C}$ for specific heat of bedded salt that consists of halite and anhydrite, which should be representative of most bedded salt.

Thermal Conductivity - Thermal conductivity generally decreases as temperature of the material increases, and increases as pressure increases at any given temperature. Thermal conductivity of $11.9 \times 10^{-3} \mathrm{cal} / \mathrm{cm}-\mathrm{s}^{\circ} \mathrm{C}(5.0 \mathrm{~W} /$ $\mathrm{mK}$ ) for halite was compared to data from other studies for salt by Krieg (1984). It was found that the values varied

\begin{tabular}{|c|c|}
\hline $\mathrm{T}\left({ }^{\circ} \mathrm{C}\right)$ & $\alpha_{L}\left(10^{-6} /{ }^{\circ} \mathrm{C}\right)$ \\
\hline 25 & $36-42$ \\
\hline 50 & $40-44$ \\
\hline 100 & $41-51$ \\
\hline 150 & $43-53$ \\
\hline 200 & $44-55$ \\
\hline
\end{tabular}
only $\pm 3 \%$ from room temperature to $200^{\circ} \mathrm{C}$.

Thermal Expansion Coefficient-Tien et al. (1983) suggests the following ranges for halite:

Parameters for Anhydrite Marker Beds within Sodium Springs Formation. An anhydrite marker bed located about $4 \mathrm{~m}$ above the repository is a potential transport pathway and so of interest in this performance assessment. A summary of the parameters for the anhydrite layers near the repository within the Sodium Springs Formation is given in Table 5-3.

Parameters for Snake Dolomite Member of Cattle River Formation. A summary of the parameters for the Snake Dolomite member is provided in Table 5-4. Groundwater flow in the Cattle River Formation is restricted mostly to the two dolomite members and the contact zone at the top of the Sodium Springs Formation. The intervening units, considered as aquitards, are composed of thin clay and mudstones, halite, and anhydrite. Estimated permeability of the aquitard units ranges from $10^{-19}$ to $10^{-16} \mathrm{~m}^{2}$. 
The Snake Dolomite Member has been studied extensively in the vicinity of the repository because it is the most transmissive water-saturated unit above the repository. It is therefore the most likely unit to provide a subsurface transport pathway away from the repository to the accessible environment.

Permeability. Log of the permeability $\left(\mathrm{m}^{2}\right)$ of the Snake Dolomite ranges from -17.0 to -11.0 with a median value of -14.0. The range of six orders of magnitude is due to fracturing of the dolomite above areas where halite has been dissolved in the upper Sodium Springs Formation. Differential removal of rock mass has increased permeability west and south of the repository as well as increasing secondary porosity. Porosity of the matrix ranges from 0.095 to 0.252 and has a median value of 0.145 . Fracture porosity ranges from $10^{-4}$ to $10^{-2}$ with a median of $10^{-3}$.

Parameters for Brine Reservoir in Tower Formation. A summary of the parameters for the Tower Formation is provided in Table 5-5.

Porosity and Permeability. Primary effective porosity in the unfractured matrix (porosity of interconnected voids through which fluid can flow) was measured in cores taken from wells near the repository and ranges from 0.002 to 0.016 . Model porosity ranges from 0.001 to 0.01 with a median of 0.005 (Sandia WIPP Project, 1992, p. 4-10). Permeability ranges from $10^{-18}$ to $10^{-20} \mathrm{~m}^{2}$ for intact anhydrite and $10^{-10}$ to $10^{-16} \mathrm{~m}^{2}$ for fractured anhydrite. To prevent depressurization of the brine reservoir due to vertical flow through the Tower Formation, the permeability of the Tower Formation in the vertical direction (Prm_Y) was set equal to zero.

Brine Pressure. Pressure at the brine reservoir ranges from hydrostatic to lithostatic (11 to $21 \mathrm{MPa}$, with a median value of $12.6 \mathrm{MPa}$ (Sandia WIPP Project, 1992, p. 4-10).

Grain Density. Grain density of the anhydrite is $2.96 \times 10^{3} \mathrm{~kg} / \mathrm{m}^{3}$.

Bulk Storativity. Bulk storativity of the Tower Formation (defined as the total volume of fluid discharged from the reservoir per unit decrease in reservoir pressure $(\Delta V / \Delta P))$ is estimated from well head pressure changes and discharge volumes or from the compressibility of the reservoir matrix and fluid and total volume and porosity of the reservoir (see Sandia WIPP Project, 1992, p. 4-16 for a complete discussion). Based on these calculations, Sandia WIPP Project (1992) estimated the reservoir bulk storativity to be between $2 \times 10^{-2}$ and $2 \mathrm{~m}^{3} / \mathrm{Pa}$ with a median of the log distribution of $0.2 \mathrm{~m}^{3} / \mathrm{Pa}$.

In the Tower Formation, the initial pressure of the brine reservoir, $17.0 \mathrm{MPa}$, was used as the reference pressure rather than $1.013 \times 10^{5} \mathrm{MPa}$. The initial pressure was used because the coefficient of compressibility can be assumed to be constant only over relatively small pressure ranges. Therefore, to minimize the pressure range to which the coefficient must be applied, the reference pressure was set equal to a value much closer to the pressure expected at these depths. Another analyst decision concerned the behavior of the overpressurized brine reservoir.

Parameters of Global Materials and Agents Acting on Disposal System. Table 5-6 tabulates parameter values for global parameters such as brine densities and parameters of the intrusion borehole. The borehole was assumed to be drilled $1000 \mathrm{yr}$ after filling the disposal site (Tintr), was incompressible, and had no residual gas phase (SatRGP). Incompressibility was assumed so that the porosity did not increase beyond the median value of 0.375 . The borehole residual gas saturation was set to zero because BRAGFLO (the parent code of BRAGFLO_T) performed significantly faster with this value in a similar modeling effort. Also, there are no data to support any value for this parameter, and a value of zero is conservative with respect to the migration of gas. The borehole permeability distribution was taken from the 1991 WIPP performance assessment where the boreholes were assumed plugged with concrete that eventually degrades to a silty sand (Sandia WIPP Project, 1992). The borehole permeability distribution is representative of silty sand.

Fluid density of the saturated brine is $1.2 \times 10^{3} \mathrm{~kg} / \mathrm{m}^{3}$. 


\subsection{Granite Site Description}

This section discusses the occurrences of granite batholiths, stratigraphy for the hypothetical granite repository, important parameters, and specific parameter values for the lithologies modeled.

\subsubsection{Origin and Occurrence of Granite Batholiths}

Igneous rocks are classified broadly into two types: (1) Intrusive or plutonic and (2) extrusive or volcanic. The igneous rock under discussion, granite, falls into the first category. These rocks intrude into other layers of rocks. The source is called magma and may form tabular structures that travel either across bedding, forming dikes, or parallel to bedding, forming sills. Very large units that are often very deep and intrude across bedding are called batholiths. Large structures that intrude between layers are called laccoliths.

Numerous granite batholiths are located in the United States (Figure 5-5). (Although metamorphic rock bodies are also quite extensive, they are excluded from consideration here.) A summary of geologic information about the batholiths in the United States is provided in Table 5-7. The site characterization data for the hypothetical granite repository, however, come primarily from the Lac Du Bonnet site in Canada.

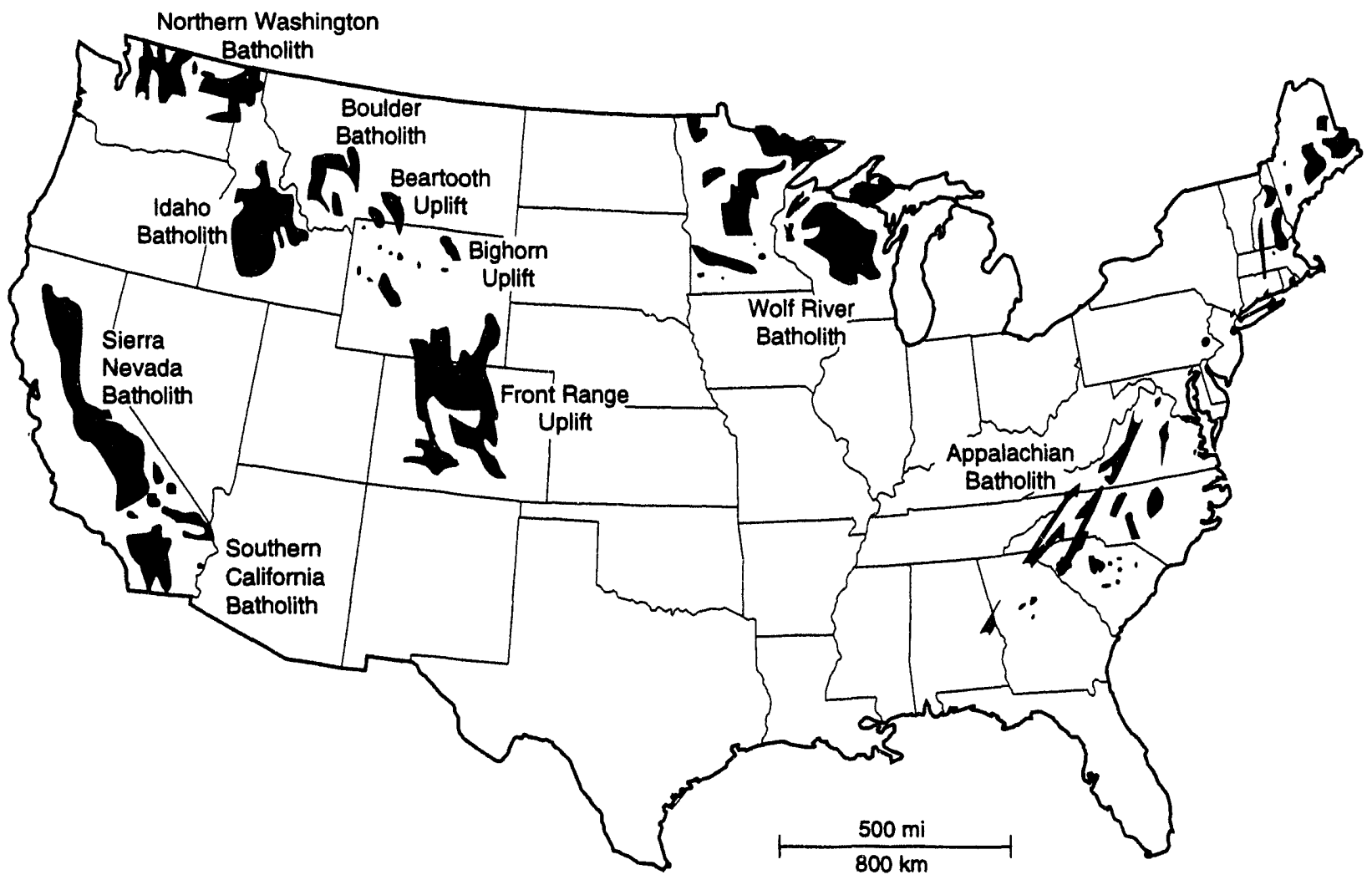

TRI-6342-3520-0

Figure 5-5. Generalized locations of surface and subsurface stocks and batholiths in the United States (after King, 1969). 


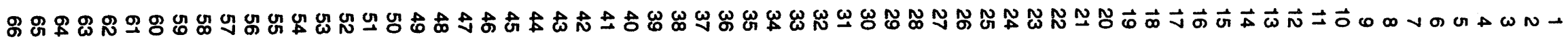

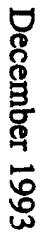

Table 5-7. Summary of Information about Batholiths

\begin{tabular}{|c|c|c|c|c|c|}
\hline Name & Location & $\begin{array}{r}\text { Size } \\
\left(\mathrm{km}^{2}\right)\end{array}$ & Age & Petrology & Structure \\
\hline $\begin{array}{l}\text { Front Range } \\
\text { Uplift }\end{array}$ & $\begin{array}{l}\text { Southern Colorado } \\
\text { to } \\
\text { southern Wyoming }\end{array}$ & many(?) $10^{6}$ & $\begin{array}{l}\text { Began } 1.7 \text { BYA } \\
\text { periodically to late Tertiary }\end{array}$ & $\begin{array}{l}\text { Boulder Creek Granodiorite } \\
\text { Pike's Peak Granite } \\
\text { Silver Plume Monzonite }\end{array}$ & $\begin{array}{l}\text { Precambrian deformation } \\
\text { and uplift. Active tronds } \\
\text { NW, NE, E }\end{array}$ \\
\hline $\begin{array}{l}\text { Bighorn } \\
\text { Uplift }\end{array}$ & $\begin{array}{l}\text { North central } \\
\text { Wyoming }\end{array}$ & $\sim 3100$ & Precambrian & Fine-grained gray granite & $\begin{array}{l}\text { Jointed, with dikes } \\
\text { Thrust, normal, and reverse } \\
\text { faults, folds }\end{array}$ \\
\hline $\begin{array}{l}\text { Beartooth } \\
\text { Uplift }\end{array}$ & $\begin{array}{l}\text { South central } \\
\text { Montana }\end{array}$ & $\sim 4800$ & Precambrian 2.7 BYA & $\begin{array}{l}\text { Metamorphosed granite } \\
\text { and granodiorite }\end{array}$ & $\begin{array}{l}\text { Precambrian dikes, } \mathrm{K}-\mathrm{T} \\
\text { folding and uplift }\end{array}$ \\
\hline $\begin{array}{l}\text { Wolf River } \\
\text { Batholith }\end{array}$ & $\begin{array}{l}\text { Northeast } \\
\text { Wisconsin }\end{array}$ & $\sim 9300$ & Precambrian 1.5 BYA & $\begin{array}{l}\text { Quartz monzonite w/granite } \\
\text { syenite and porphyry }\end{array}$ & $\begin{array}{l}\text { Jointing \& fracturing } \\
\text { at surface disappearing } \\
\text { at denth }\end{array}$ \\
\hline $\begin{array}{l}\text { Sierra Nevada } \\
\text { Batholith }\end{array}$ & $\begin{array}{l}\text { East central } \\
\text { California }\end{array}$ & $\sim 7800$ & Mesozoic & $\begin{array}{l}\text { E.-quartz monzonite \& diorite } \\
\text { W.-quartz diorite \& grandionite }\end{array}$ & $\begin{array}{l}\text { Trough of large syncline } \\
\text { Trend } \mathrm{N} 40^{\circ} \mathrm{W} w / \text { strike slip } \\
\& \text { normal faults }\end{array}$ \\
\hline $\begin{array}{l}\text { Southern California } \\
\text { Batholith }\end{array}$ & $\begin{array}{l}\text { Southern California, } \\
\text { Mexico }\end{array}$ & 207,000 & Mid Cretaceous & $\begin{array}{l}\text { Series of plutons ranging from } \\
\text { Gabbro (Intrusive Basalt) to } \\
\text { Granite-most common is } \\
\text { granodiorite }\end{array}$ & $\begin{array}{l}\text { Structures trend NW; faults } \\
\text { trend NW also }\end{array}$ \\
\hline $\begin{array}{l}\text { Northern Washington } \\
\text { Batholith }\end{array}$ & Northern Washington & 181 & Cretaceous & $\begin{array}{l}\text { Center granite grading outward } \\
\text { to granodiorite \& quartz diorite } \\
\text { at edges }\end{array}$ & $\begin{array}{l}\text { NW trending faults; jointing \& } \\
\text { fracturing; } 3 \text { sets of joints- } \\
2 \text { horizontal \& } 1 \text { vertical }\end{array}$ \\
\hline
\end{tabular}


용요

Table 5-7. Summary of Information about Batholiths (Concluded)

\begin{tabular}{llcclc}
\hline \multicolumn{1}{c}{ Name } & \multicolumn{1}{c}{ Location } & $\begin{array}{c}\text { Size } \\
\left(\mathbf{k m}^{2}\right)\end{array}$ & \multicolumn{1}{c}{ Age } & \multicolumn{1}{c}{ Petrology } & Structure \\
\hline $\begin{array}{l}\text { Idaho } \\
\text { Batholith }\end{array}$ & $\begin{array}{l}\text { Central \& western } \\
\text { Idaho }\end{array}$ & 41,000 & Mid Cretaceous & $\begin{array}{l}\text { Range from quartz gabbro to } \\
\text { granite average in granodiorite }\end{array}$ & $\begin{array}{l}\text { Complex because of size, NW } \\
\text { trending thrust, NE } \\
\text { shear zones }\end{array}$ \\
$\begin{array}{l}\text { Boulder } \\
\text { Batholith }\end{array}$ & $\begin{array}{l}\text { Southwest } \\
\text { Montana }\end{array}$ & 5000 & Late Cretaceous & $75 \%$ quartz monzonite & $\begin{array}{l}\text { Thrust faulting w/strike slip } \\
\text { shear zones }\end{array}$ \\
$\begin{array}{l}\text { Canadian Shield } \\
\text { Central to eastern Canada } \\
\text { Northern Minnesota, } \\
\text { Wisconsin }\end{array}$ & $>200,000$ & Precambrian & Mostly granite & \\
\hline
\end{tabular}




\subsubsection{Hypothetical Granite Lithologies}

The stratigraphy of the generic granite repository is based on a study by the Atomic Energy of Canada Limited (AECL) Research Company of a batholith at the Underground Research Laboratory (URL) near Lac du Bonnet, Manitoba, Canada (Everitt and Read, 1989). An objective of the AECL Research Company is to develop a methodology for site selection and characterization by examining drill cores and conducting geological, geophysical, and hydrological studies at the Underground Research Laboratory (Everitt and Read, 1989).

The repository is located at a depth of $300 \mathrm{~m}$ in a granite batholith (Figure 5-6). In plan view, the batholith covers several thousand square kilometers and reaches a depth of about 10 kilometers. It is almost completely covered with a thin $(6 \mathrm{~m})$ veneer of glacial till that forms low rolling hills with valleys where there are shallow lakes and swamps. Below the till, the batholith consists of several granite phases. The upper phase is a pink, moderately fractured granite that grades downward into a massive unfractured grey granite.

There are two extensive fracture zones; one separates the pink granite from the upper part of the grey granite and another, thinner, zone is below the repository level. The fracture zones are highly conductive and shallow-dipping. The remainder of batholith is a competent body of igneous rock of good quality and has very few fractures. Existing fractures are near vertical and become less frequent with depth. These fractures may be open and water-saturated, and contribute to vertical hydraulic permeability.

Gray Granite. Massive gray quartz monzonite granite occurs in two layers: a 75-m layer at the base of the model below the lower fracture zone, and a $175-\mathrm{m}$ layer between the fracture zones. The repository is located in the $175-\mathrm{m}$ unit at an elevation of $-25 \mathrm{~m}$, or $300 \mathrm{~m}$ from the surface; the location was selected to match work being performed by Atomic Energy of Canada Limited for high-level waste disposal (Everitt and Read, 1989). The massive gray granite has a much lower frequency of fractures than the other units.

Fractures Zones. The lower fracture zone, Fracture Zone 1, is a 10-m fracture zone below the massive gray granite layer in which the repository is located. The upper fracture zone, Fracture Zone 2, is a 75-m fracture zone above the repository.

Pink Granite. The upper granite unit is a $125-\mathrm{m}$ pink, porphyritic, biotite-rich granite with a xenolith-rich zone that grades into gray granite.

Glacial Till. The topmost unit is a 6-m veneer of glacial till (silty clay).

\subsubsection{Modeling Assumptions for Geologic Barrier in Granite}

The concept of storing radioactive waste in saturated granite rock has spawned considerable effort in modeling fluid in low permeability rocks. The studies at the Stripa mine in Sweden are a notable example. In low-permeability, water-saturated rock, water flows preferentially through fractures. Thus, the ability to predict water flow through such formations hinges on the ability to predict fluid flow through fractures.

One approach in developing fracture flow models has been to construct fracture networks from statistical orientation data and hydraulic aperature data on the fractures. Several computer codes have been developed for this approach. Of these codes, FracMan (Dershowitz et al., 1991) is widely known. Although we did investigate using FracMan to generate continuum fluid flow properties for fracture networks for a hypothetical granite site, we had little basis for choosing the necessary statistical orientation data and hydraulic aperature data. Furthermore, Long et al. (1991) have reported two findings that present major problems for this general approach.

The two findings are as follows: First, although fractures are ubiquitous in igneous and metamorphic rock formations, only an extremely small percentage of the often thousands of visible fractures are hydrologically connected and conductive, and thus active (termed "irregular" or "heterogeneous" connectivity). Second, Long et al. (1991) have inferred from observations reported in the literature at the Fanay Augeres mine in France (Billaux et al., 1989), 
5. Hypothetical Geologic Barrier

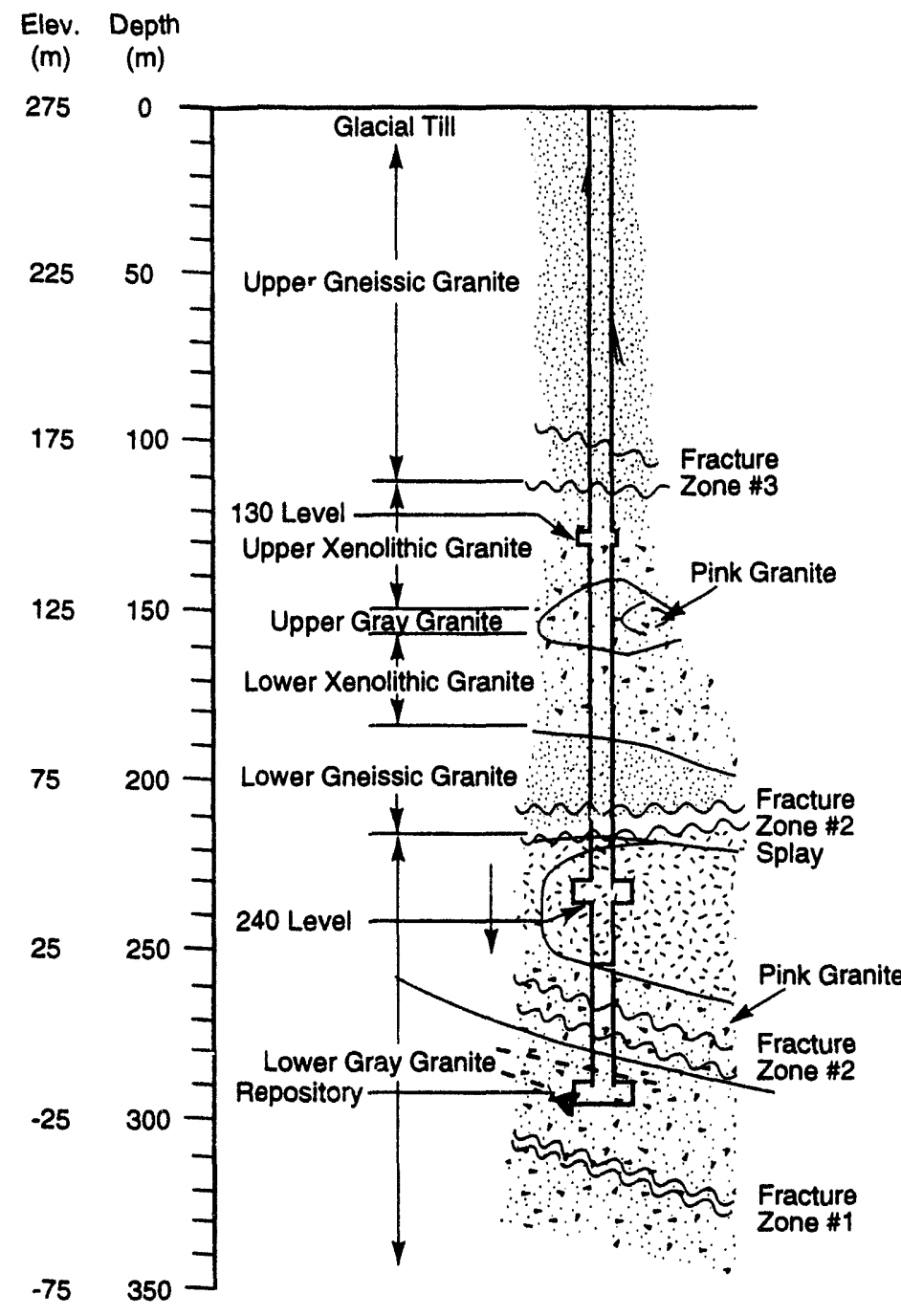

TRI-6? 42-3598-1

Figure 5-6. Geologic barrier for granite repository. The repository is located in the massive grey granite, with fracture zones above and below. 
the Stripa mine in Sweden (Olsson et al., 1989; Carlsten et al., 1988), and a ubiquitously fractured granite pluton (Hsieh and Neuman, 1985), that on any scale of interest the hydrology is controlled by the major unique features present at that scale, not the general ubiquitous aspects. Long et al. (1991) go on to argue that conceptual models for predicting fluid flow should instead acknowledge these findings and demonstrate the approach of the Stripa mine in which several fracture zones are assumed to be the dominant hydrologic features (Figure 5-7).

\section{General}

- Fluid flow obeys Darcy's law.

- Dissolution of gas into liquid phase is negligibls.

- Nuclear critical conditions do not occur along the flow path (porosities within geologic barrier are sufficiently low).

- Host gray granite lithology is over and underlain by a horizontal fracture zones.

- Other granite lithologies do not affect the repository.

\section{Gray Granite}

- Lithology of zero permeability except at fracture zones and connecting secondary fractures.

- Lithology has small nonzero porosity.

\section{Fracture Zones}

- Thickness of fracture zones is constant.

- Fluctuations in aquifer flow caused by climate changes are inconsequential.

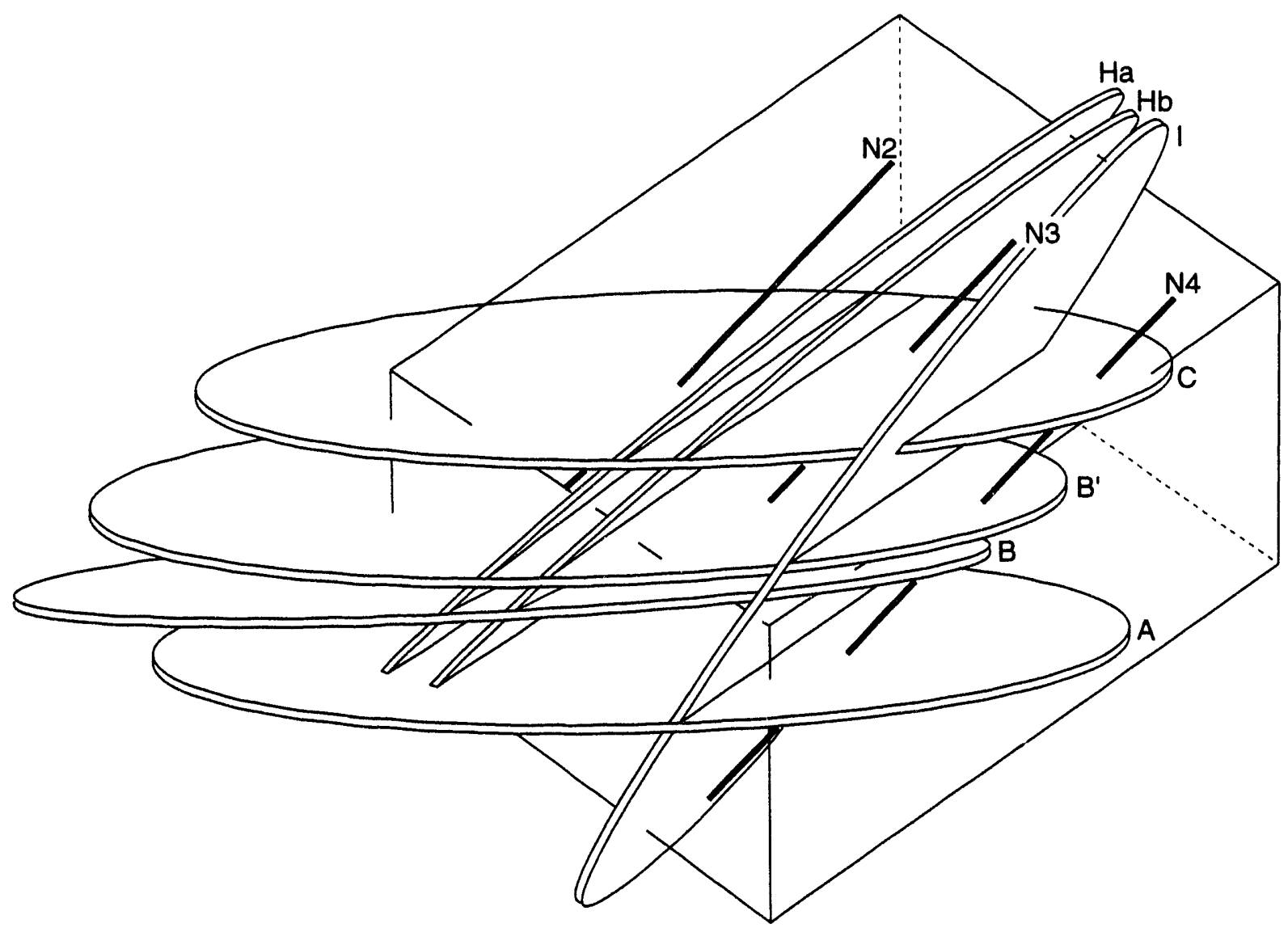

TRI-6342-3732-0

Figure 5-7. Seven dominant fracture zones used in conceptual model at Stripa mine in Sweden. The hydrologic zone model shown in perspective from the northwest looking down. A similar type of hydrologic zone model was used for modeling the hypothetical granite repository (Long, 1991, Figure 12-30). 
- Flow is through a densely interconnected network of fractures that allows fracture zones to be modeled as a single porous media.

- Flow in fracture zones is hydrologically confined with no vertical leakage.

- Singe porosity model without retardation adequately describes radionuclide transport.

\subsubsection{Model Parameters for Granite}

This section lists the parameters used in the computational models that are discussed in Chapters 13 and 15 . Although it is convenient in terms of organization to include the model parameters for the geologic barrier here, the reader may wish to defer examining them until he or she has read the discussion on the conceptual and mathematical models (Chapters 13 and 15).

Formation properties used in the Complex $\mathrm{PA}^{\dagger \dagger}$ of the granite repository are tabulated in Tables 5-8 through 5-14. The Simple PA used a subset of these properties. The range of the parameters, the median value, and the source of the data are provided in these tables. ${ }^{\ddagger} \neq$ The parameter shown in bold, fracture influx rate, was sampled.

Weitzman (1985) estimated the hydraulic conductivity of granite from single and double packer tests in several boreholes at the underground research laboratory (URL) in Canada. Permeabilities were computed from these measurements and included in Tables 5-9 and 5-12. We assumed that flow predominately occurred through the fractures and that no fluid passed through the matrix itself. Therefore, the permeability values presented in Tables 5-9 and 5-12 were treated as indications of flow through the fracture network father than the permeability of intact granite.

Some of the parameters in Tables 5-8 through 5-14 were selected based on the judgment of the analyst because of a lack of data on the parameter, simplifying assumptions, or a specific modeling objective. When this is the case, the term "analyst decision" appears in the source column.

Two parameters appear repeatedly in these tables; they are initial water saturation (Saturatn), and the exponent used in the capillary pressure/relative permeability equations (BrkCorEx) (Brooks and Corey, 1964). All material except the backfill regions (see Chapter 6) were assumed to be water saturated initially. The exponent was set to 0.7 for all materials.

Parameters Common to All Lithologies. Three parameters selected by analyst decision are included in Table 5-8. These parameters were applied to all or some of the material regions. As in salt, the thermal expansion reference temperature (DnsTmpRf) was $298 \mathrm{~K}\left(25^{\circ} \mathrm{C}\right)$ and used in all the materials.

Compressibility Reference Pressure. On the other hand, the compressibility reference pressure (PorPrsRf) was used in the excavated regions (shaft backfill, drift backfill, annular backfill, waste parcel) and set cqual to $1.01 \times 10^{5}$ $\mathrm{Pa}(1 \mathrm{~atm})$. In all other material regions, the compressibility reference pressure was set in the preprocessor MATSET to a pressure repi ssentative of the initial water pressure (Table 5-15).

Threshold Pressure. The Brooks and Corey model (1964) characterizes the 2-phase properties of analogue materials for preliminary gas calculations (Davies and LaVenue, 1990). Threshold pressure is a key parameter in this model and, in this performance assessment, is equal to the capillary pressure at a saturation equal to the residual saturation (Sandia WIPP Project, 1992). This definition was adopted because flow of waste-generated gas outward from the repository requires that outward flowing gas penetrate and establish a gas-filled network of flow paths in the surrounding host rock.

Parameter values related to threshold pressure are the Brooks and Corey exponent, residual wetting phase, residual gas saturation, and initial saturation. Parameter values for these four are common to all zones.

\footnotetext{
${ }^{\dagger+t}$ Refer to Section 7.5, "Conceptual Model," for a description of the Simple and Complex PA formulations.

${ }_{\ddagger \ddagger}$ Refer to Section 9.1, "Model Parameters," for a general description of how a distribution is assigned.
} 


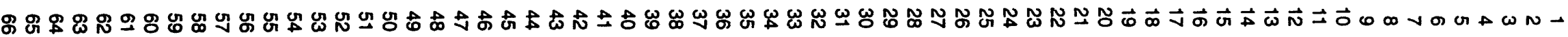

Table 5-8. Parameter Values for All Lithologies

\begin{tabular}{|c|c|c|c|c|c|c|c|c|}
\hline $\begin{array}{l}\text { Record } \\
\text { No. }\end{array}$ & Parameter & Parameter Description & Median & $\mathbf{R a}$ & & Units & $\begin{array}{l}\text { Distribution } \\
\text { Type }\end{array}$ & Sources In: \\
\hline 4203 & BrkCorEx & $\begin{array}{l}\text { Brooks-Corey Exponent for capillary } \\
\text { pressure }\end{array}$ & $7.00 e-01$ & $2.00 e-01$ & $1.00 e+01$ & none & Constructed & $\begin{array}{l}\text { Ramsey, analyst } \\
\text { decision }\end{array}$ \\
\hline 4147 & CoThExp & Coefficient of Linear thermal expansion & $2.66 \mathrm{e}+03$ & $2.59 e+03$ & $2.74 e+03$ & $\mathrm{~kg} / \mathrm{m}^{3}$ & Uniform & Percival et al., 1984 \\
\hline 4009 & FPore & Effective fracture porosity of crystalline & N/A & $1.00 e-06$ & $1.00 e-03$ & none & Uniform & Davison, 1980 \\
\hline 4022 & FPoreEff & Effective porosity of unfractured crystalline & $1.70 e-03$ & $3.20 e-04$ & $3.10 e-03$ & none & Uniform & Katsube et al., 1986 \\
\hline 4002 & FracDisL & Fracture dispersivity (Longitudinal & $3.50 e+02$ & $1.00 e+02$ & $6.00 e+02$ & m & Data & Heinrich, 1984 \\
\hline 4003 & Tortusty & Matrix tortuosity $(r)$ & $1.82 e+00$ & $1.00 e+00$ & $3.00 e+00$ & none & Data & LeNeveu, 1986 \\
\hline 4262 & PorPrsRf & Reference pressure for porosity & $1.01 e+05$ & & & $\mathrm{~Pa}$ & Constant & $\begin{array}{l}\text { Ramsey, analyst } \\
\text { decision }\end{array}$ \\
\hline 4263 & DnsTmpRf & Reference temperature for density & $2.98 e+02$ & & & K & Constant & $\begin{array}{l}\text { Ramsey, analyst } \\
\text { decision }\end{array}$ \\
\hline 4012 & RepDep & Repository depth & $3.00 e+02$ & & & m & Data & $\begin{array}{l}\text { Ramsey, analyst } \\
\text { decision }\end{array}$ \\
\hline 4265 & SatRGP & Residual gas saturation & $2.00 e-01$ & 0 & $4.00 e-01$ & none & Uniform & $\begin{array}{l}\text { Sandia WIPP Project, } \\
1992\end{array}$ \\
\hline 4264 & SatRWP & Residual wetting phase (liquid) saturation & $2.00 e-01$ & 0 & $4.00 e-01$ & none & Uniform & $\begin{array}{l}\text { Sandia WIPP Project, } \\
1992\end{array}$ \\
\hline 4025 & SurfEl & Surface elevation above sea level & $2.75 e+02$ & & & m & Constant & $\begin{array}{l}\text { Intera Technologies } \\
\text { Ltd., } 1985\end{array}$ \\
\hline 4152 & TStrngth & Tensile strength & $7.60 e+00$ & & & $\mathrm{MPa}$ & Data & Wai and Tsai, 1985 \\
\hline 4023 & ThGrad & Thermal gradient between $100-400$ meters & $1.30 \mathrm{e}-02$ & & & $\mathrm{~K} / \mathrm{m}$ & Data & Wai and Tsai, 1985 \\
\hline 4151 & CStrngth & Uniaxial Compressive strength & $1.94 e+02$ & & & $\mathrm{MPa}$ & Data & Wai and Tsai, 1985 \\
\hline
\end{tabular}




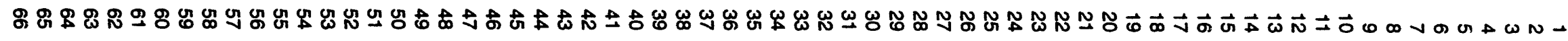
密

Table 5-9. Parameter Values for Massive Gray Granite

\begin{tabular}{|c|c|c|c|c|c|c|c|c|}
\hline \multirow{2}{*}{$\begin{array}{c}\begin{array}{c}\text { Record } \\
\text { No. }\end{array} \\
4200\end{array}$} & \multirow{2}{*}{$\begin{array}{l}\text { Parameter } \\
\text { DnsBlk }\end{array}$} & \multirow{2}{*}{$\frac{\text { Parameter Description }}{\text { Bulk density }}$} & \multirow{2}{*}{$\frac{\text { Median }}{2.65 e+03}$} & \multicolumn{2}{|c|}{ Range } & \multirow{2}{*}{$\frac{\text { Units }}{\mathrm{kg} / \mathrm{m}^{3}}$} & \multirow{2}{*}{$\begin{array}{c}\begin{array}{c}\text { Distribution } \\
\text { Type }\end{array} \\
\text { Uniform }\end{array}$} & \multirow{2}{*}{$\begin{array}{l}\text { Sources In: } \\
\begin{array}{l}\text { Percival et al., } \\
1984\end{array}\end{array}$} \\
\hline & & & & $2.62 e+03$ & $2.68 e+03$ & & & \\
\hline 4199 & Compres & Compressibility & $3.30 \mathrm{e}-13$ & & & $1 / \mathrm{Pa}$ & Constant & Weitzman, 1985 \\
\hline 4045 & HyCnd & Hydraulic Conductivity & $1.00 e-12$ & & & $\mathrm{~m} / \mathrm{s}$ & Data & Weitzman, 1985 \\
\hline 4039 & Porosity & Matrix porosity & $1.00 e-05$ & & & none & Data & Weitzman, 1985 \\
\hline 4204 & Prm & Permeability & $1.00 e-19$ & & & $\mathrm{~m}^{2}$ & Lognormal & Weitzman, 1985 \\
\hline 4201 & Saturatn & Saturation, initial & $1.00 e+00$ & & & none & Uniform & $\begin{array}{l}\text { Ramsey, analyst } \\
\text { decision }\end{array}$ \\
\hline 4196 & SpHeat & Specific heat & $8.45 e+02$ & & & $\mathrm{~J} / \mathrm{kg} \cdot \mathrm{K}$ & Constant & Wai and Tsai, 1985 \\
\hline 4033 & StorageC & Storage Coefficient (S) & $5.00 e-11$ & & & $1 / m$ & Data & Wai and Tsai, 1985 \\
\hline 4198 & ThExp & Thermal Expansion Coefficient & $8.00 e-06$ & & & $1 / K$ & Constant & Wai and Tsai, 1985 \\
\hline 4197 & ThCnd & Thermal conductivity & $3.60 e+00$ & & & $\mathrm{~W} / \mathrm{m} \cdot \mathrm{K}$ & Constant & Wai and Tsai, 1985 \\
\hline
\end{tabular}




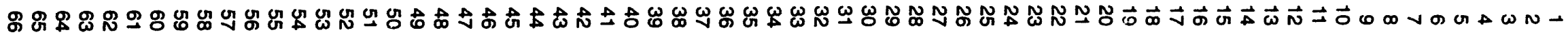

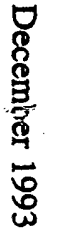

Table 5-10. Parameter Values for Fracture Zone 1

\begin{tabular}{|c|c|c|c|c|c|c|c|}
\hline $\begin{array}{l}\text { Record } \\
\text { No. }\end{array}$ & Parameter & Parameter Description & Median & Range & Units & $\begin{array}{c}\text { Distribution } \\
\text { Type }\end{array}$ & Sources In: \\
\hline 4192 & DnsBlk & Bulk density & $2.50 e+03$ & & $\mathrm{~kg} / \mathrm{m}^{3}$ & Constant & Tsang et al., 1983 \\
\hline 4191 & Compres & Compressibility & $1.00 e-09$ & & $1 / \mathrm{Pa}$ & Constant & Weitzman, 1985 \\
\hline 4040 & Porosity & Matrix porosity & $1.00 \mathrm{e}-03$ & & none & Data & Weitzman, 1985 \\
\hline 4195 & Prm & Permeability & $5.00 \mathrm{e}-13$ & & $\mathrm{~m}^{2}$ & Lognormal & Weitzman, 1985 \\
\hline 4193 & Saturatn & Saturation, initial & $1.00 e+00$ & & none & Uniform & $\begin{array}{l}\text { Ramsey, analyst } \\
\text { decision }\end{array}$ \\
\hline 4188 & SpHeat & Specific heat & $8.79 e+02$ & & $\mathrm{~J} / \mathrm{kg} \cdot \mathrm{K}$ & Constant & Tsang et al., 1983 \\
\hline 4034 & StorageC & Storage Coefficient (S) & $1.00 \mathrm{e}-05$ & & $1 / \mathrm{m}$ & Data & Weitzman, 1985 \\
\hline 4190 & ThExp & Thermal Expansion Coefficient & $1.11 \mathrm{e}-05$ & & $1 / K$ & Constant & Tsang et al., 1983 \\
\hline 4189 & ThCnd & Thermal conductivity & $3.20 e+00$ & & $\mathrm{~W} / \mathrm{m} \cdot \mathrm{K}$ & Constant & Tsang et al., 1983 \\
\hline 4028 & LyrThick & Thickness of the geologic layer & $1.00 e+01$ & & m & Data & Weitzman, 1985 \\
\hline
\end{tabular}




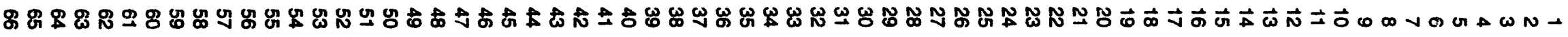

Table 5-11. Parameter Values for Fracture Zone 2

\begin{tabular}{|c|c|c|c|c|c|c|c|}
\hline $\begin{array}{l}\text { Record } \\
\text { No. }\end{array}$ & Parameter & Parameter Description & Median & Range & Units & $\begin{array}{c}\text { Distribution } \\
\text { Type }\end{array}$ & Sources in: \\
\hline 4176 & DnsBlk & Bulk density & $2.69 e+03$ & & $\mathrm{~kg} / \mathrm{m}^{3}$ & Constant & $\begin{array}{l}\text { Percival et al., } \\
1984\end{array}$ \\
\hline 4175 & Compres & Compressibility & $6.80 e-08$ & & $1 / \mathrm{Pa}$ & Constant & Weitzman, 1985 \\
\hline 4042 & Porosity & Matrix porosity & $1.00 \mathrm{e}-02$ & & none & Data & Weitzman, 1985 \\
\hline 4179 & Prm & Permeability & $1.00 e-13$ & & $\mathrm{~m}^{2}$ & Lognormal & Weitzman, 1985 \\
\hline 4177 & Saturatn & Saturation, initial & $1.00 e+00$ & & none & Uniform & $\begin{array}{l}\text { Ramsey, analyst } \\
\text { decision }\end{array}$ \\
\hline 4172 & SpHeat & Specific heat & $8.79 e+02$ & & $\mathrm{~J} / \mathrm{kg} \cdot \mathrm{K}$ & Constant & Tsang et al., 1983 \\
\hline 4036 & StorageC & Storage Coefficient (S) & $7.00 e-05$ & & $1 / \mathrm{m}$ & Data & Weitzman, 1985 \\
\hline 4174 & ThExp & Thermal Expansion Coefficient & $1.11 e-05$ & & $1 / K$ & Constant & Tsang et al., 1983 \\
\hline 4173 & ThCnd & Thermal conductivity & $3.20 \mathrm{e}+0 \mathrm{n}$ & & $\mathrm{W} / \mathrm{m} \cdot \mathrm{K}$ & Constant & Tsang et al., 1983 \\
\hline 4030 & LyrThick & Thickness of the geologic layer & $7.50 e+01$ & & $\mathbf{m}$ & Data & Weitzman, 1985 \\
\hline
\end{tabular}




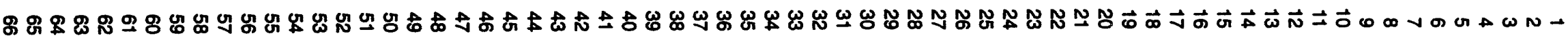

Table 5-12. Parameter Values for Pink Granite

\begin{tabular}{|c|c|c|c|c|c|c|c|c|}
\hline $\begin{array}{l}\text { Record } \\
\text { No. }\end{array}$ & Parameter & Parameter Description & Median & \multicolumn{2}{|c|}{ Range } & Units & $\begin{array}{l}\text { Distribution } \\
\text { Type }\end{array}$ & Sources In: \\
\hline 4168 & DnsBlk & Bulk density & $2.64 e+03$ & & & $\mathrm{~kg} / \mathrm{m}^{3}$ & Constant & $\begin{array}{l}\text { Percival et al., } \\
1984\end{array}$ \\
\hline 4167 & Compres & Compressibility & $3.30 e-10$ & & & $1 / \mathrm{Pa}$ & Constant & Weitzman, 1985 \\
\hline 4049 & Porosity & Matrix porosity & $1.00 e-03$ & & & none & Data & Weitzman, 1985 \\
\hline 4171 & Prm & Permeability & $1.00 \mathrm{e}-19$ & & & $m^{2}$ & Lognormal & Weitzman, 1985 \\
\hline 4169 & Saturatn & Saturation, initial & $1.00 e+00$ & & & none & Uniform & $\begin{array}{l}\text { Ramsey, analyst } \\
\text { decision }\end{array}$ \\
\hline 4164 & SpHeat & Specific heat & $8.45 e+02$ & & & $\mathrm{~J} / \mathrm{kg} \bullet \mathrm{K}$ & Constant & Wai and Tsai, 1985 \\
\hline 4037 & StorageC & Storage Coefficient (S) & $5.000-04$ & & & $1 / m$ & Date & Weitzman, 1985 \\
\hline 4166 & ThExp & Thermal Expansion Coefficient & $8.00 e-06$ & & & $1 / K$ & Constant & Wai and Tsai, 1985 \\
\hline 4165 & ThCnd & Thermal conductivity & $3.60 e+00$ & & & $\mathrm{~W} / \mathrm{m} \bullet \mathrm{K}$ & Constant & Wai and Tsai, 1985 \\
\hline 4031 & LyrThick & Thickness of the geologic layer & $1.25 e+02$ & $5.00 e+01$ & $1.50 e+02$ & m & Data & Weitzman, 1985 \\
\hline
\end{tabular}




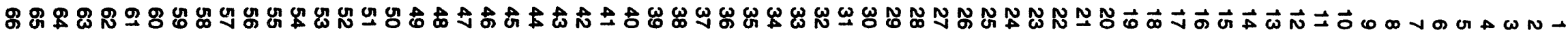

Table 5-13. Parameter Values for Glacial Till

\begin{tabular}{|c|c|c|c|c|c|c|c|}
\hline $\begin{array}{l}\text { Record } \\
\text { No. }\end{array}$ & Parameter & Parameter Desc "iption & Median & Range & Units & $\begin{array}{c}\text { Distribution } \\
\text { Type }\end{array}$ & Sources In: \\
\hline 4160 & DnsBlk & Bulk density & $1.40 e+03$ & & $\mathrm{~kg} / \mathrm{m}^{3}$ & Constant & $\begin{array}{l}\text { Radhakrishna and } \\
\text { Chan, } 1985\end{array}$ \\
\hline 4159 & Compres & Compressibility & $1.00 \mathrm{e}-07$ & & $1 / \mathrm{Pa}$ & Constant & $\begin{array}{l}\text { Freeze and Cherry, } \\
1979\end{array}$ \\
\hline 4044 & Porosity & Matrix porosity & $4.00 e-01$ & & none & Data & $\begin{array}{l}\text { Freeze and Cherry, } \\
1979\end{array}$ \\
\hline 4163 & Prm & Permeability & $3.20 e-18$ & & $m^{2}$ & Lognormal & $\begin{array}{l}\text { Freeze and Cherry, } \\
1979\end{array}$ \\
\hline 4161 & Saturatn & Saturation, initial & $1.00 e+00$ & & none & Uniform & $\begin{array}{l}\text { Ramsey, analyst } \\
\text { decision }\end{array}$ \\
\hline 4156 & SpHeat & Specific heat & $9.21 e+02$ & & $\mathrm{~J} / \mathrm{kg} \bullet \mathrm{K}$ & Constant & $\begin{array}{l}\text { Domenico and } \\
\text { Schwartz, } 1990\end{array}$ \\
\hline 4038 & StorageC & Storage Coefficient (S) & $1.00 e-02$ & & $1 / \mathrm{m}$ & Data & Betcher, 1983 \\
\hline 4158 & ThExp & Thermal Expansion Coefficient & $3.40 e-05$ & & $1 / K$ & Constant & Wai and Tsai, 1985 \\
\hline 4157 & ThCnd & Thermal conductivity & $1.05 e+00$ & & $W / m \cdot K$ & Constant & $\begin{array}{l}\text { Domenico and } \\
\text { Schwartz, } 1990\end{array}$ \\
\hline 4032 & LyrThick & Thickness of the geologic layer & $6.00 e+00$ & & m & Data & Betcher, 1983 \\
\hline
\end{tabular}




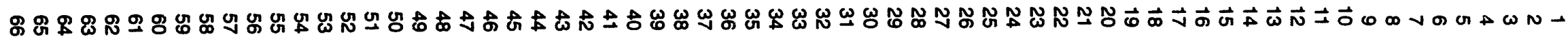

Table 5-14. Parameter Values for Global Materials and Agents Acting on the Granite Disposal System

\begin{tabular}{|c|c|c|c|c|c|c|c|c|}
\hline $\begin{array}{l}\text { Record } \\
\text { No. }\end{array}$ & Parameter & Parameter Description & Median & Rai & & Units & $\begin{array}{c}\text { Distribution } \\
\text { Type }\end{array}$ & Sources In: \\
\hline 4026 & DnsFluid & Density of Fluid & $1.01 \theta+03$ & $1.00 e+03$ & $1.01 \mathrm{e}+03$ & $\mathrm{~kg} / \mathrm{m}^{3}$ & Uniform & Gascoyne, 1988 \\
\hline 4250 & ComprsFI & Fluid compressibility & $5.20 e-10$ & & & $1 / \mathrm{Pa}$ & Constant & Weitzman, 1985 \\
\hline 4013 & HydGradV & Vertical hydraulic gradient & 0.02 & 0 & 0.4 & $\mathrm{~m} / \mathrm{m}$ & Data & Heinrich, 1984 \\
\hline 4014 & HydGradH & Horizontal hydraulic gradient & 0.005 & 0.001 & 0.02 & $\mathrm{~m} / \mathrm{m}$ & Data & Wuschke et al., 1985 \\
\hline 4026 & Dnsfluid & Fluid density & $1.007 e+03$ & & & $\mathrm{~kg} / \mathrm{m}^{3}$ & Constant & Gascoyne, 1988 \\
\hline 4253 & Porosity & Fracture porosity & 0.5 & & & none & Data & Weitzman, 1985 \\
\hline 4154 & FFlux & Fracture flux & $6.900 \mathrm{e}-06$ & $2.000 e-06$ & $2.000 e-05$ & $\mathrm{~m}^{3} / \mathrm{s}$ & Loguniform & Neretnieks, 1986 \\
\hline 4262 & PorPrsRf & Reference pressure, porosity & $1.013 e+05$ & & & $\mathrm{~Pa}$ & Constant & $\begin{array}{l}\text { Ramsey, analyst } \\
\text { decision }\end{array}$ \\
\hline 4263 & DnsTmpRf & Reference temperature, density & $2.980 e+02$ & & & $\mathrm{~K}$ & Constant & $\begin{array}{l}\text { Ramsey, analyst } \\
\text { decision }\end{array}$ \\
\hline 30 & Diameter & Borehole diameter & 0.355 & 0.1207 & 0.4445 & m & Delta & $\begin{array}{l}\text { Sandia WIPP } \\
\text { Project, } 1992\end{array}$ \\
\hline 4277 & SatRWP & Residual liquid saturation & $2.000 e-01$ & 0 & $4.000 \mathrm{e}-01$ & none & Uniform & $\begin{array}{l}\text { Sandia WIPP } \\
\text { Project, } 1992\end{array}$ \\
\hline 4278 & DnsBlk & Bulk density & $2.030 e+03$ & & & $\mathrm{~kg} / \mathrm{m}^{3}$ & Constant & Rechard, Ed., 1992 \\
\hline 4279 & SpHeat & Specific heat & $8.600 e+02$ & & & $\mathrm{~J} / \mathrm{kg} \cdot \mathrm{k}$ & Constant & Krieg, 1984 \\
\hline 4280 & ThCnd & Thermal conductivity & $5.000 e+00$ & & & $\mathrm{~W} / \mathrm{m} \cdot \mathrm{k}$ & Constant & Krieg, 1984 \\
\hline 4281 & ThExp & Thermal exponent & $4.500 e-05$ & & & $1 / K$ & Constant & Krieg, 1984 \\
\hline 4284 & Compress & Compressibility & 0 & & & $1 / \mathrm{Pa}$ & Constant & $\begin{array}{l}\text { Ramsey, analyst } \\
\text { decision }\end{array}$ \\
\hline 4285 & Porosity & Porosity & $3.750 e-01$ & $2.500 e-01$ & $5.000 \mathrm{e}-01$ & none & Normal & $\begin{array}{l}\text { Sandia WIPP } \\
\text { Project, } 1992\end{array}$ \\
\hline
\end{tabular}




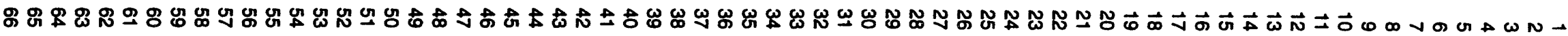

Table 5-14. Parameter Values for Global Materials and Agents Acting on the Granite Disposal System (continued)

\begin{tabular}{|c|c|c|c|c|c|c|c|c|}
\hline \multirow{2}{*}{$\begin{array}{c}\begin{array}{c}\text { Record } \\
\text { No. }\end{array} \\
4286\end{array}$} & \multirow{2}{*}{$\begin{array}{l}\text { Parameter } \\
\text { TIntr }\end{array}$} & \multirow{2}{*}{$\begin{array}{l}\text { Parameter Description } \\
\text { Intrusion }\end{array}$} & \multirow{2}{*}{$\frac{\text { Median }}{3.156 e+10}$} & \multicolumn{2}{|c|}{ Range } & \multirow{2}{*}{ Units } & \multirow{2}{*}{$\begin{array}{c}\text { Distribution } \\
\text { Type }\end{array}$} & \multirow{2}{*}{\begin{tabular}{l}
\multicolumn{1}{c}{ Sources In: } \\
$\begin{array}{l}\text { Ramsey, analyst } \\
\text { decision }\end{array}$
\end{tabular}} \\
\hline & & & & 0 & $3.154 e+11$ & & & \\
\hline 4287 & SatRGP & Residual gas saturation, borehole & 0 & & & none & Constant & $\begin{array}{l}\text { Ramsey, analyst } \\
\text { decision }\end{array}$ \\
\hline 4265 & SatRGP & $\begin{array}{l}\text { Residual gas saturation, unfractured } \\
\text { granite }\end{array}$ & 0.2 & 0 & 0.4 & none & Constant & $\begin{array}{l}\text { Sandia WIPP } \\
\text { Project, } 1992\end{array}$ \\
\hline 4288 & Prm & Permeability, borehole & $3.160 e-13$ & $1.000 e-14$ & $1.000 e-11$ & $m^{2}$ & Lognormal & $\begin{array}{l}\text { Sandia WIPP } \\
\text { Project, } 1992\end{array}$ \\
\hline 4266 & Prm & Permeability, unfractured granite & 0 & & & $\mathrm{~m}^{2}$ & Constant & $\begin{array}{l}\text { Ramsey, analyst } \\
\text { decision }\end{array}$ \\
\hline 4007 & TempSurf & Surface temperature & 281.2 & & & K & Constant & Davison, 1980 \\
\hline 4023 & ThGrad & Thermal gradient & 01013 & & & $\mathrm{C} / \mathrm{m}$ & Data & Davison, 1980 \\
\hline
\end{tabular}


Table 5-15. Compressibility Reference Pressures (Gronite Repository)

\begin{tabular}{lc}
\hline Material Name & $\begin{array}{c}\text { Compressibility Reference Pressure } \\
\text { (MPa) }\end{array}$ \\
\hline Gray Granite & 6.0 \\
Fracture Zone 1 & 4.0 \\
Secondary Fracture & 3.0 \\
Massive Gray Granite & 3.0 \\
Fracture Zone 2 & 2.0 \\
Pink Granite & 1.0 \\
Glacial Till & 0.2 \\
\hline
\end{tabular}

Hydraulic Gradient (HorizontalVertical). Horizontal hydraulic gradients at the granite repository were determined with the assumption that lakes in the area are hydraulically connected to the regional groundwater and that the flow paths are influenced by the water levels in the major lakes. Heinrich (1984) sampled flow paths between major lakes in the Atikokan region and recorded a trend with a range of $10^{-3}$ to $10^{-2}$; the mean horizontal gradient would be $5 \times 10^{-3}$. The horizontal hydraulic gradient in granite in the Atikokan area of Canada ranges from 0.002 to 0.016 . Table 5-16 shows the piecewise distribution of the hydraulic gradient.

For comparison, the horizontal gradient is 0.003 in the Finnsjon Area, 0.01 for Kamlunge, and 0.004 for Klyeperis (92-20). The high gradient at Kamlunge is indicative of a low hydraulic conductivity in that area.

Vertical hydraulic gradients in two wells in the Atikokan area showed that a gradient of 0.04 upward exists (Heinrich, 1984), but it should be stressed that this gradient is localized in nature.

Table 5-16. Distribution of Horizontal Hydraulic Gradient in Granite (Wuschke et al., 1985)

\begin{tabular}{cc} 
Range & Probability \\
\cline { 2 - 2 } $2 \times 10^{-3}$ to $4 \times 10^{-3}$ & 0.40 \\
$4 \times 10^{-3}$ to $6 \times 10^{-3}$ & 0.27 \\
$6 \times 10^{-3}$ to $1.2 \times 10^{-2}$ & 0.26 \\
$1.4 \times 10^{-2}$ to $1.6 \times 10^{-2}$ & 0.07
\end{tabular}

Gray Granite Parameters. The summary of parameter values for gray granite is provided in Table 5-10.

Porosity and Permeability. Total porosity of the rock mass is the volume of the voids (sum of the joint void space and intercrystalline void space divided by the total rock volume). At repository depth, the porosity of crystalline rocks is due primarily to fracturing and separation along faults, bedding planes, cracks, or fissures. The crystalline rock mass itself has a very low porosity because of the intergrown nature of the crystals. Fractures at depth may not be opened. Older joints may not be completely open because they may be partially or completely sealed with deposits such as clay, calcite, or hematite.

Porosity determines how much surface area of a rock mass will be exposed to fluids moving through it and how the retardation of radionuclides can be accomplished. Fractured crystalline rock can have a very low effective porosity. Domenico and Schwartz (1990) give an effective porosity range of $5 \times 10^{-7}$ to $1 \times 10^{-4}$ for fractured crystalline rock. Freeze and Cherry (1979) give a range of porosity for fractured crystalline rock of 0 to 0.10 and a range of 0 to 0.05 for dense crystalline rock. Porosity determinations of Canadian granites by Katsube et al. (1986) yielded an 
average value of $3.3 \times 10^{-3}$, with a range of $1.6 \times 10^{-3}$ to $4.9 \times 10^{-3}$, and an average effective porosity of $1.23 \times 10^{-3}$, with a range of $3.2 \times 10^{-4}$ to $3.1 \times 10^{-3}$ using water immersion and electrical resistivity methods. Diffusion experiments yielded an average value of porosity of $8.91 \times 10^{-3}$, with a range of $6.26 \times 10^{-4}$ to $4.13 \times 10^{-2}$, and an average effective porosity of $2.28 \times 10^{-3}$, with a range of $1.3 \times 10^{-4}$ to $6.43 \times 10^{-3}$. Tracer breakthrough studies of a site at Aspo, Sweden (Gustafsson et al., 1991, p. 55) yielded an effective porosity value for fracture zones of $2.0 \times 10^{-3}$ to $5.0 \times 10^{-2}$. Neretnieks (1986) reports a porosity for crystalline rocks of $1 \times 10^{-3}$ to $1 \times 10^{-2}$. Porosity of the unit is $1 \times 10^{-5}$, and the horizontal permeability is $1 \times 10^{-19} \mathrm{~m}^{2}$.

Fracture Zone Parameters. Summaries of parameter values for Fracture Zones 1 and 2 are found in Tables 5-11 and 5-12, respectively. Porosity of both units is $1 \times 10^{-2}$ and the horizontal permeability is $1 \times 10^{-13} \mathrm{~m}^{2}$.

Pink Granite Parameters. The summary of parameter values for pink granite is provided in Table 5-13. The porosity of the unit is $1 \times 10^{-3}$, and the horizontal permeability is $1 \times 10^{-12} \mathrm{~m}^{2}$.

Glacial Till Parameters. The summary of parameter values for the glacial till is provided in Table 5-14. The porosity of the unit is $4 \times 10^{-1}$, and the horizontal permeability is $1 \times 10^{-15} \mathrm{~m}^{2}$. The elevation of the ground surface at the site is $275 \mathrm{~m}$ above sea level.

Parameters of Global Material and Agents Acting on Disposal System. The summary of parameter values for global materials is provided in Table 5-15.

Fracture Influx Rate and Permeability. Although permeability of secondary connecting fractures could have been inferred from measurements of flow into the mine at the Underground Research Laboratory (URL), Canada, in this assessment, permeability is calculated for granite based on water inflow measurements for excavations at the Stripa research mine in Sweden as follows (Neretnicks, 1986; Abelin et al., 1985; Dverstorp and Andersson, 1989). Water flow into the Stripa mine was measured onto plastic sheets at a rate of $2 \times 10^{-7} \mathrm{~m}^{3} / \mathrm{s}(0.8 \mathrm{l} / \mathrm{hr})$ over a $700 \mathrm{~m}^{2}$ area. Because the granite repository is much larger than $700 \mathrm{~m}^{2}$, we assumed the secondary fracture connections would flow at between 10 and 100 times the observed value or an influx of between $2 \times 10^{-6}$ to $2 \times 10^{-5} \mathrm{~m}^{3} / \mathrm{s}$. We assumed a loguniform distribution over this range. The permeability of the fracture was then computed from a sampled influx assuming an average aperature of $0.01 \mathrm{~m}$, porosity of 0.5 , and mean horizontal and vertical gradients of 0.005 and 0.02 , respectively. The mean permeability was $2.6 \times 10^{-14} \mathrm{~m}^{2}$.

Dverstorp and Andersson (1989) used the same basic data and measured the lengths of the hydraulically active fractures in the $700 \mathrm{~m}^{2}$ area and assumed a unit gradient to estimate a mean transmissivity of $6.02 \times 10^{-10} \mathrm{~m}^{2} / \mathrm{s}$ per fracture and standard deviation of $4.05 \times 10^{-10} \mathrm{~m}^{2} / \mathrm{s}$ which, assuming an average fracture aperature of $0.01 \mathrm{~m}$, yields an equivalent mean permeability of about $6 \times 10^{-15} \mathrm{~m}^{2}$ per fracture. Hence, we have assumed around four hydraulically active fractures in the granite repository equivalent to those evaluated at the Stripa mine.

Fluid Density. The value selected for fluid density at the repository level is $1.01 \times 10^{3} \mathrm{~kg} / \mathrm{m}^{3}$ (Gascoyne, 1988). Saline groundwater from plutons and mines is predominantly rich in $\mathrm{Ca}, \mathrm{Na}$, and $\mathrm{Cl}$, and can be the result of intrusion of sea water or groundwater from adjacent sedimentary beds, dissolution of grain boundary salts, fluid inclusions in plutonic rocks, alteration of halogen-bearing minerals, or concentration of dissolved solids by in situ radiolysis (Gascoyne, 1986). Composition is controlled by rock type and solubility of the minerals. Gascoyne (1986) estimates the range of salinity for groundwater at about $1000 \mathrm{~m}$ in an undisturbed pluton to be 30 to $100 \mathrm{~g} /$ liter, which translates to a density range of about 1.023 to $1.068 \times 10^{3} \mathrm{~kg} / \mathrm{m}^{3}$ (CRC Handbook of Chemistry and Physics, Veazey and Hodgman, Eds., 2nd ed.). The most saline groundwater from a deep mine at about $1000 \mathrm{~m}$ can have a density of about $1.2 \times 10^{3} \mathrm{~kg} / \mathrm{m}^{3}$. 


\title{
6. Repository Design
}

\author{
J. S. Rath and J. W. Berglund
}

Although the geological environment surrounding the waste can be viewed as the ultimate barrier protecting the accessible environment, the engineered barrier can offer additional protection. Components of the engineered barrier may include (not all are necessary):

1. Durable waste form that is resistant to alteration (Chapter 4).

2. Containers that serve to protect the waste form from groundwater (Chapter 4).

3. Waste parcel placement that, for example, diminishes" temperatures of the host rock or diminishes groundwater movement, or diminishes neutron interaction between containers.

4. Subsurface facility layout that is constructed to accomplish particular objectives, for example, a layout that prevents undue host rock disturbances from waste decay heat, or diminishes groundwater movement by avoiding discovered fracture zones or orienting drifts perpendicular to regional groundwater gradients, yet also is able to be constructed.

5. Specially placed backfill in the shafts, drifts, rooms, and directly around the waste parcel, to limit groundwater movement and chemically retard radionuclide transport.

The latter three items, which are components of repository design (waste parcel placement, subsurface layout, and backfill), are discussed in this chapter. Because the design of the repository is governed by the total waste volume and the emplacement of the waste parcels, these subjects are discussed first, followed by repository dimensions (subsurface facility layout) and a description of the backfill.

Design considerations are discussed under emplacement method (waste decay heat, waste criticality, and, briefly, constructibility) and facility layout (location of fracture zones and direction of prevailing groundwater flow). Some design issues are not discussed in this report, e.g., operator safety, ventilation, stability of tunnels and shafts at the chosen repository depth, and in-depth study of constructibility.

\subsection{Volume of Waste}

Table 6-1 tabulates the number of waste parcels and rooms required per waste disposal group for the salt and granite repositories. These values are shown to indicate the differences per waste disposal group. Note that the expense of waste treatment can be partially offset by a decreased number of containers required, a decreased cost in transpe tation and handling of containers, and a reduction in repository size.

The number of waste parcels required for each waste disposal group assumes the amount of waste remains fixed. The number of required rooms is based on spacing of containers and a specific room size, which is discussed below.

For this performance assessment analysis, the dimensions of the repository were not changed per disposal group, but instead were assumed to be fixed (10 panels with 10 rooms each) at a convenient size required for the base case, Waste Disposal Group 1, which consists of $\sim 10,000 \mathrm{MTHM}^{\dagger}{ }^{\dagger}$ This initial heavy metal mass, equivalent waste activity, and volume was then proportionally increased for analysis of the other waste disposal groups and individual waste parcels. In the Containment Requirements of 40 CFR 191, acceptable releases are proportional to the activity of the repository and so whether the repository size is decreased for a fixed activity amount of waste or vice versa is generally not important. Although some subtle distinctions can occur (e.g., fluid flow through the repository may not scale linearly), fixing the repository size permitted the analysts to use identical applied models and assured that all differences in waste form behavior were based on waste form performance and not the reduced size of the repository (which, the reader should recall, is hypothetical).

* Some designs incorporate enhancement of temperatures to keep moisture from containers for a longer period of time.

+ MTHM indicates initial heavy metal mass. 
Table 6-1. Total Number of Waste Parcels and Rooms Required for Five Waste Disposal Groups in Salt and Granite Repositories

\begin{tabular}{|c|c|c|c|c|c|c|}
\hline \multirow[b]{2}{*}{$\begin{array}{l}\text { Parcel } \\
\text { ID }\end{array}$} & \multirow[b]{2}{*}{ Name } & \multicolumn{5}{|c|}{ Number of Parcels Required in Salt Repository } \\
\hline & & $\begin{array}{l}\text { Waste } \\
\text { Disposal } \\
\text { Group } 1 \\
\text { (10 kg } 1 \text { WP) }\end{array}$ & $\begin{array}{c}\text { Waste } \\
\text { Disposal } \\
\text { Group 2* } \\
(0.7 \mathrm{~kg} / \mathrm{P})\end{array}$ & $\begin{array}{l}\text { Waste } \\
\text { Disposal } \\
\text { Group } 3 \\
\text { (10 kg } / \text { WP) }\end{array}$ & $\begin{array}{c}\text { Waste } \\
\text { Disposal } \\
\text { Group } 4^{*} \\
(0.7 \mathrm{~kg} / \mathrm{P})\end{array}$ & $\begin{array}{l}\text { Waste } \\
\text { Disposal } \\
\text { Group 5* } \\
\text { (0.7 kg/WP) }\end{array}$ \\
\hline \multicolumn{7}{|c|}{ Salt Repository } \\
\hline WP01 & $\begin{array}{l}\text { Graphite spent fuel, Fort St. Vrain and } \\
\text { Peach Bottom }\end{array}$ & 442 & & & & \\
\hline WPO4 & $\begin{array}{l}\text { Special spent fuel, highly enriched ura- } \\
\text { nium (HEU) }\end{array}$ & 5064 & & & & \\
\hline WP05 & $\begin{array}{l}\text { Special spent fuel, low enriched ura- } \\
\text { nium (LEU) }\end{array}$ & 888 & & & & \\
\hline WP06 & Calcine $(\operatorname{Loos} \theta)$ & 6945 & & & & \\
\hline WP10 & $\begin{array}{l}\text { Graphite spent fuel, encapsulated in } \\
\text { glass }\end{array}$ & & 1801 & & & \\
\hline WP11 & $\begin{array}{l}\text { Calcine waste in glass ceramic, Hot } \\
\text { Isostatic Press (HIP) }\end{array}$ & & 990 & & & \\
\hline WP12 & Special spent fuel, HEU, HIP & & 5064 & & & \\
\hline WP13 & Special spent fuel, LEU, HIP & & 888 & & & \\
\hline WP14 & Graphite spent fuel in particles & & & 152 & & \\
\hline WP16 & Special spent fuel, HEU, in glass & & & 633 & & \\
\hline WP17 & Special spent fuel, LEU, in glass & & & 296 & & \\
\hline WP18 & Calcine in Chem Plant glass & & & 5238 & & \\
\hline WP22 & $\begin{array}{l}\text { Reprocessed graphite spent fuel, and } \\
\text { calcine }\end{array}$ & & & & 38 & \\
\hline WP23 & $\begin{array}{l}\text { Reprocessed special spent fuel, HEU } \\
\text { and LEU }\end{array}$ & & & & 5061 & \\
\hline WP24 & $\begin{array}{l}\text { Graphite spent fuel and depleted ura- } \\
\text { nium in glass }\end{array}$ & & & & & 202 \\
\hline WP25 & $\begin{array}{l}\text { Special spent fuel and depleted ura- } \\
\text { nium in glass }\end{array}$ & & & & & 1408 \\
\hline \multirow[t]{2}{*}{ WP26 } & Calcine, in glass & & & & & 7301 \\
\hline & $\begin{array}{l}\text { Subtotal of waste parcels } \\
\text { Subtotal of rooms required }\end{array}$ & $\begin{array}{r}13,339 \\
48.3\end{array}$ & $\begin{array}{r}8743 \\
31.7\end{array}$ & $\begin{array}{r}6319 \\
22.9\end{array}$ & $\begin{array}{r}5099 \\
18.5\end{array}$ & $\begin{array}{l}8911 \\
32.3\end{array}$ \\
\hline WP07 & Savannah River high-level waste & 5283 & 5283 & 5283 & 5283 & 5283 \\
\hline WP08 & Hanford high-level waste & 1960 & 1960 & 1960 & 1960 & 1960 \\
\hline \multirow[t]{3}{*}{ WP09 } & West Valley high-level waste & 276 & 276 & 276 & 276 & 276 \\
\hline & $\begin{array}{l}\text { Total Number of Waste Parcels for } \\
\text { Salt"t }\end{array}$ & 20858 & 16262 & 13838 & 12618 & 16430 \\
\hline & $\begin{array}{l}\text { Total Number of Rooms Required } \\
\text { for Salt** }\end{array}$ & 75.3 & 58.7 & 50 & 45.5 & 59 \\
\hline $\begin{array}{l}\text { We } \\
* \text { Th } \\
\text { wa }\end{array}$ & $\begin{array}{l}\text { Disposal Groups 2, } 4 \text {, and } 5 \text { are the sam } \\
\text { falues are shown to illustrate difference } \\
\text { arcels were modeled for each disposal }\end{array}$ & $\begin{array}{l}\text { for the salt a } \\
\text { in number } 0 \\
\text { oup (i.e., } 100\end{array}$ & $\begin{array}{l}\text { granite repos } \\
\text { aste parcels }\end{array}$ & $\begin{array}{l}\text { required } \\
\text { is each.) }\end{array}$ & moun in the & odel, 27,600 \\
\hline
\end{tabular}


Table 6-1. Total Number of Waste Parcels and Rooms Required for Five Waste Disposal Groups in Salt and Granite Repositories (Continued)

\begin{tabular}{|c|c|c|c|c|c|c|}
\hline \multirow[b]{2}{*}{$\begin{array}{l}\text { Parcel } \\
\text { ID }\end{array}$} & \multirow[b]{2}{*}{ Name } & \multicolumn{5}{|c|}{ Number of Parcels Required in Granite Repository } \\
\hline & & $\begin{array}{l}\text { Waste } \\
\text { Disposal } \\
\text { Group } 1 \\
(0.7 \text { kgWP) }\end{array}$ & $\begin{array}{l}\text { (Same as } \\
\text { Salt*) }\end{array}$ & $\begin{array}{c}\text { Waste } \\
\text { Disposal } \\
\text { Group } 3 \\
\text { (0.7 kg/WP) }\end{array}$ & $\begin{array}{l}\text { (Same as } \\
\text { Sallt") }\end{array}$ & $\begin{array}{l}\text { (Same as } \\
\text { Salt") }\end{array}$ \\
\hline \multicolumn{7}{|c|}{ Granite Repository } \\
\hline WP02 & Graphite spent fuel, Fort St. Vrain & 2368 & & & & \\
\hline WP03 & Graphite spent fuel, Peach Bottom & 800 & & & & \\
\hline WP04 & $\begin{array}{l}\text { Special spent fuel, highly enriched ura- } \\
\text { nium (HEU) }\end{array}$ & 5064 & & & & \\
\hline WP05 & $\begin{array}{l}\text { Special spent fuel, low enriched ura- } \\
\text { nium (LEU) }\end{array}$ & 888 & & & & \\
\hline WP06 & Calcine (Loose) & 6945 & & & & \\
\hline WP15 & $\begin{array}{l}\text { Graphite spent fuel, encapsulated in } \\
\text { glass }\end{array}$ & & & 1801 & & \\
\hline WP19 & Special spent fuel, HEU, in glass & & & 5064 & & \\
\hline WP20 & Special spent fuel, LEU, in glass & & & 888 & & \\
\hline \multirow[t]{2}{*}{ WP21 } & Calcine in Chem Pant glass & & & 2136 & & \\
\hline & $\begin{array}{l}\text { Subtotal of waste parcels } \\
\text { Subtotal of rooms required }\end{array}$ & $\begin{array}{c}16065 \\
58.2\end{array}$ & & $\begin{array}{l}9889 \\
35.8\end{array}$ & & \\
\hline WP07 & Savannah River high-level waste & 5283 & 5283 & 5283 & 5283 & 5283 \\
\hline WP08 & Hanford high-level waste & 1960 & 1960 & 1960 & 1960 & 1960 \\
\hline \multirow[t]{3}{*}{ WP09 } & West Valley high-level waste & 276 & 276 & 276 & 276 & 276 \\
\hline & $\begin{array}{l}\text { Total Number if Waste Parcels for } \\
\text { Granite** }\end{array}$ & 23584 & 16262 & 17408 & 12618 & 16430 \\
\hline & $\begin{array}{l}\text { Total Number of Rooms Required } \\
\text { for Granite** }\end{array}$ & 85 & 58.7 & 62.8 & 45.5 & 59 \\
\hline $\begin{array}{l}\text { "Wa } \\
\text { " The } \\
\text { was }\end{array}$ & $\begin{array}{l}\text { isposal Groups } 2,4 \text {, and } 5 \text { are the san } \\
\text { ralues are shown to illustrate differenc } \\
\text { arcels were modeled for each disposal }\end{array}$ & $\begin{array}{l}\text { or the salt ar } \\
\text { in number o } \\
\text { up (i.e., } 10 \mathrm{~F}\end{array}$ & $\begin{array}{l}\text { ranite repc } \\
\text { ste parcel } \\
\text { is of } 10 \text { ro }\end{array}$ & $\begin{array}{l}\text { es. } \\
\text { ns required } p \\
\text { with } 276 \text { parc }\end{array}$ & $\begin{array}{l}\text { roup; in th } \\
\text { per room.) }\end{array}$ & odel, 27,600 \\
\hline
\end{tabular}




\subsection{Placement of Waste Parcel}

This performance assessment assumes that waste parcels are (1) placed in vertical holes in the floor of the disposal rooms, (2) uniformly spaced throughout the disposal region, and (3) uniformly mixed in the disposal rooms.

\subsubsection{Orientation of Waste Parcels}

Both vertical and horizontal borehole emplacement designs were considered for the hypothetical repositories, as was tunnel or drift disposal. Although tunnel disposal has some advantages over borehole disposal (mechanical stability of engineered barrier systems, stability of tunnels, ease of excavation, and spacing flexibility [Hara et al., 1991]), the borehole design also has benefits: ease of providing backfill, emplacing the waste, and maintaining a ventilation path. An additional advantage is that, with the borehole design, radionuclides usually must diffuse into the drift before they can exit from the repository and, after emplacement, the waste parcel can be shielded sufficiently so that access to the area for continued repository operations is unlimited. Because borehole waste emplacement simplifies mining procedures and can require a smaller repository volume than tunnel disposal, borehole emplacement was selected for this study. The vertical orientation of the borehole was selected so that gravity could assist in the emplacement of the waste parcels.

\subsubsection{Spacing of Waste Parcels}

The spacing of the waste parcels depends primarily upon three factors: (1) thermal limits of the waste form, backfill-buffer, and adjacent geology, (2) considerations of waste criticality, and (3) limits on constructibility that include waste parcel diameter, backfill thickness, and boring equipment access. ${ }^{\ddagger}$

Based on these three factors, the disposal pattern selected within rooms is a $2.6-\mathrm{m}$ hexagonal pattern (Figure 6-1). The minimum spacing pattern of 2.6-m hexagonal was chosen for ease of borehole construction; also, the low thermal output from the waste parcels permits spacing at these distances (see Appendix $C$ for a discussion of scoping calculations regarding spacing of waste parcels, including criticality considerations). The influence of the three factors is discussed below.

Temperature Limitations. Generally, the centerline temperatures of certain waste forms (borosilicate glass, in particular) should not exceed $500^{\circ} \mathrm{C}$ for any length of time to avoid crystalline growth in the glass (Tang and Saling, 1990).

Temperature Limitations in Salt Repository. Because salt undergoes temperature-dependent creep when subjected to deviatoric stress, the mined shafts, drifts, and disposal rooms in a salt repository will eventually close due to creep after a period of time. The creep closure of the mined and backfilled disposal rooms and drifts eventually encapsulates the waste and returns the disturbed salt to a state of low permeability. The low permeability of the fully compacted salt provides the principal geologic barrier to the transport of the stored radionuclides. Because of salt creep, the repository design must account for a limited access time before the excavated rooms become unstable and cannot easily be accessed. Therefore, design dimensions of disposal rooms in the salt reference design will accommodate a period of up to $3 \mathrm{yr}$ of repository access prior to the onset of instability. This time period is based on a maximum areal thermal loading for the waste of $25 \mathrm{~W} / \mathrm{m}^{2}$, which indicates a $10 \%$ volumetric closure in $3 \mathrm{yr}$ (U.S. DOE, 1986a).

* Generally, the waste parcels in this performance assessment do not include overpacks or internal backfill materials and thus self shielding within each waste parcel is minimal. During handling operations, temporary shielding will be necessary in the design of the transport and emplacement equipment. Shielding may also be necessary during the placement of backfill-buffer materials around each waste parcel. After emplacement, a shield plug and cover plate will, if necessary, be placed above each waste parcel (Stahl and Harrison, 1993). 


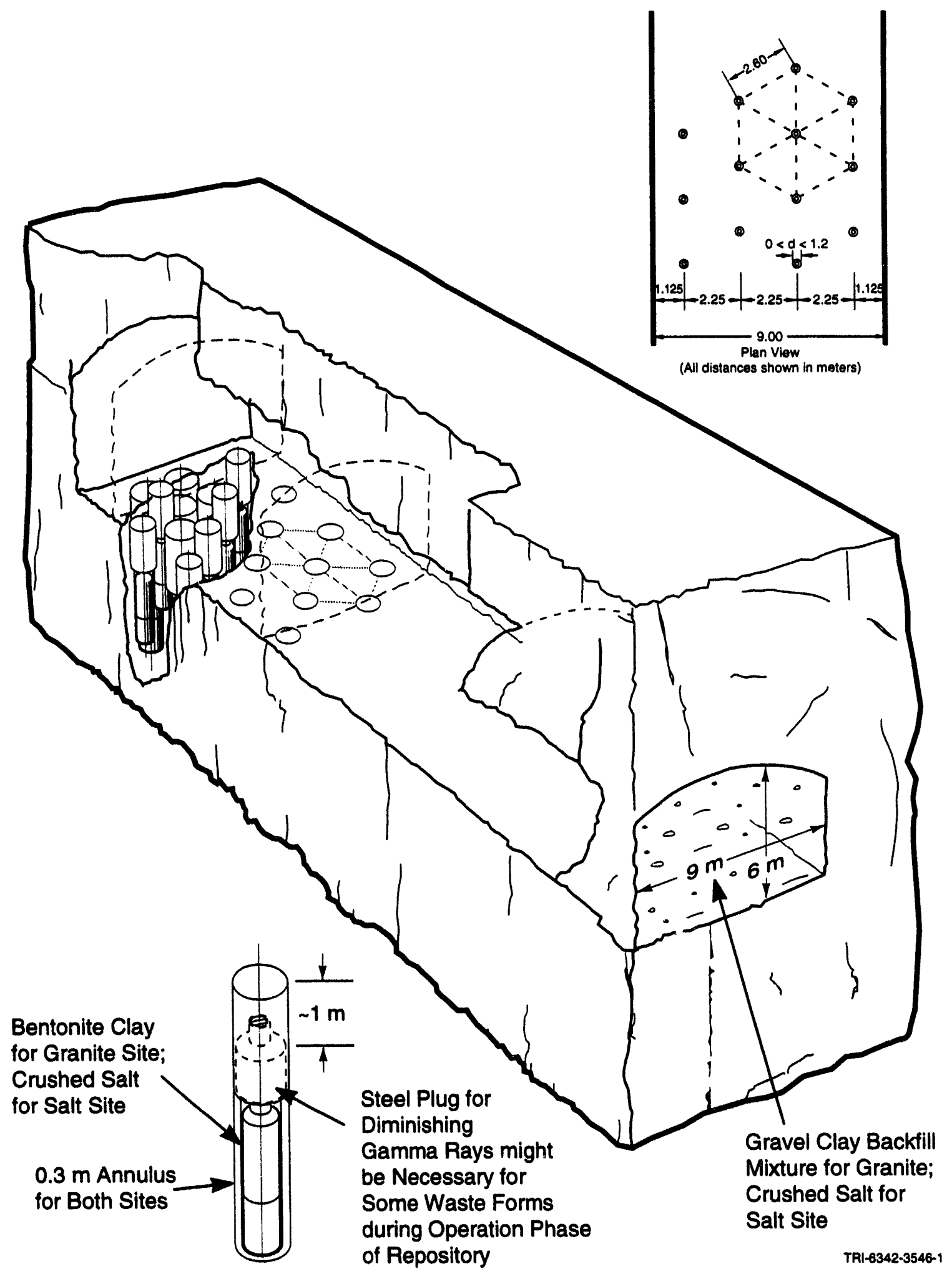

Figure 6-1. Disposal pattern within rooms for waste parcels. 


\section{Repository Design}

In addition, a governing factor for container spacing in the salt repository is the maximum temperature allowed by the backfill-buffer material(s). Typically, when a backfill/salt interface temperature exceeds $250^{\circ} \mathrm{C}$, salt decrepitation can occur (Moss and Molecke, 1983, p. 724). A final consideration is the vaporization of brine in the salt pores adjacent to the canisters. Because the consequences of vaporization are not well understood or easily simulated with the current set of computational models, ${ }^{* *}$ the waste package/host-salt boundary was conservatively kept below $100^{\circ} \mathrm{C}$ (water boiling point).

Several simplified design calculations were performed using the code, SUMQ_3D, for the five disposal groups. In addition, other scoping calculations were performed using the DOE wastes generated by the West Valley Demonstration Project, Defense Waste Processing Facility (Savannah River), and the Hanford Waste Vitrification Plant, assuming a total mass of $12,640 \mathrm{MT}$ and a total volume of $4706 \mathrm{~m}^{3}$. These calculations are reported in Appendix C.

Possibly because of the anticipated long interim storage time before emplacing the waste, none of the five waste forms to be emplaced in the salt repository generated heat at rates that required more than the minimum spacing (Table 6-2). Thus the minimum spacing for the waste parcels (2.6-m center-to-center, hexagonal) was used within the rooms for the mixed waste. In this configuration, a room ( $180 \mathrm{~m}$ length) has a capacity of $\sim 276$ vertically emplaced waste parcels. The number of rooms required for the five waste disposal groups is shown in Table 6-1. Room totals vary from 45.5 to 75.3 .

Temperature Limitations in Granite. Clay backfill barrier materials (e.g., bentonite) between the host rock and overpack must be maintained below a temperature of $130^{\circ} \mathrm{C}$ to remain stable (SKB, 1992b). Also, to avoid two-phase flow from vaporization of groundwater in the saturated granite repository, the backfill-buffer/host-rock interface temperature adjacent to the waste parcel was conservatively kept below $100^{\circ} \mathrm{C}$. Details on the calculations to assess the importance of the temperatures experienced in a bentonite backfill-buffer material are provided in Appendix C.

Like the salt repository, none of the five waste forms to be emplaced in the saturated granite repository generated heat at rates that required more than the minimum spacing (Table 6-2). ${ }^{\dagger \dagger}$ Thus the minimum spacing (2.6-m hexagonal pattern) was used within the rooms as described above. The number of rooms required for the five waste disposal groups is shown in Table 6-1. Room totals vary from 45.5 to 85 .

Criticality Considerations. A number of factors that are associated with criticality safety must be considered in repository design. Among these are (1) the composition and physical characteristics of the fuel, (2) the material and the distance between neighboring fissile regions, (3) the canister material and dimensions, (4) the repository type (e.g., salt or granite), and (5) the likelihood that the fuel remains intact and confined to a very specific configuration. Criticality safety applies to spent fuels only and is an issue principally within individual waste parcels but can also affect spacing between waste parcels within the disposal rooms of the repository.

Constructibility. A description of the borehole drilling and the mining of the waste disposal rooms is provided in this section (Sandia WIPP Project, 1992).

Drilling of Waste Parcel Boreholes. Boreholes for waste parcel emplacement are to be drilled vertically into the floors of the waste disposal rooms. This excavation method decreases the potential of collateral damage (MacDougall et al., 1987a, p. 6-36). The vertical drilling operation is envisioned to consist of two stages: pilot drilling and reamer drilling (MacDougall et al., 1987b, p. D-28). Such a two-stage drilling operation may require two different drilling machines. Both the pilot-hole drilling system and the following larger hole reaming system are to be mounted on a crawl-mounted machine that will move readily from hole to hole. Both pilot drilling and reamer drilling should be possible by modifying commercially available equipment (Robbins, 1984).

\footnotetext{
** Consideration of a very hot repository (from closely spaced containers) in initially saturated geologic media was not part of the scope of work, but could be explored in future performance assessments.

Rock temperatures for Waste Parcel 8 (Hanford high-level waste) actually exceeded the $100^{\circ} \mathrm{C}$ limit by about $3^{\circ} \mathrm{C}$ in a $2.6-\mathrm{m}$ hexagonal array. However, these computations assumed all waste parcels in the array generated heat at this rate. Waste parcels with different thermal outputs (and in most cases, very low thermal outputs) will be mixed within the rooms, and so the actual rock temperature will be lower than that calculated. Moreover, SUMQ_3D ignores drift ventilation cooling, which can amount to a significant fraction of heat produced (Montan, 1981).
} 
Table 6-2. Salt Repository Waste Parcel (WP) Heat Rates

\begin{tabular}{|c|c|c|c|c|}
\hline ID & Name & $\begin{array}{c}\text { Heat } \\
\text { Rate } \\
(W)\end{array}$ & Container Dimensions & $\begin{array}{c}\text { Heat Rate } \\
\text { Flux } \\
\left(\mathrm{W} / \mathrm{m}^{3}\right)\end{array}$ \\
\hline \multicolumn{5}{|c|}{ Waste Disposal Group 1} \\
\hline \multicolumn{5}{|c|}{ Salt Repository } \\
\hline WP01 & SNF/Graphite/FSV+PB & 30.79 & 0.6096 dia. $\times 4.1150$ & 25.64 \\
\hline WP04 & SNF/Special/HEU & 6.998 & 0.1683 dia. $\times 1.6000$ & 196.6 \\
\hline WP05 & SNF/Special/LEU & 153.7 & 0.3239 dia. $\times 4.3430$ & 429.6 \\
\hline WP06 & Calcine (Loose) & 8.52 & 0.6096 dia. $\times 2.9970$ & 9.74 \\
\hline WPO7* & DOE/HLW/DWPP(Savannah) & 328.0 & 0.6096 dia. $\times 2.9970$ & 375.0 \\
\hline WP08 & DOE/HLW/HWVP(Hanford) & 391.0 & 0.6096 dia. $\times 2.9970$ & 447.0 \\
\hline WP09 & DOE/HLWMVDP(West Valley) & 147.0 & 0.6096 dia. $\times 2.9970$ & 168.1 \\
\hline \multicolumn{5}{|c|}{ Granite Repository } \\
\hline WPO2 & SNF/Graphite/FSV & 5.06 & 0.4576 dia. $\times 0.9860$ & 31.2 \\
\hline WP03 & SNF/Graphite/PB & 2.04 & 0.2731 dia. $\times 4.0090$ & 8.7 \\
\hline WP04 & SNF/Special/HEU & 6.998 & 0.1683 dia. $\times 1.6000$ & 196.6 \\
\hline WP05 & SNF/Special/LEU & 153.7 & 0.3239 dia. $\times 4.3430$ & 429.6 \\
\hline WPO6 & Calcine (Loose) & 8.52 & 0.6096 dia. $\times 2.9970$ & 9.74 \\
\hline \multicolumn{5}{|c|}{ Waste Disposal Group 2} \\
\hline \multicolumn{5}{|c|}{ Salt and Granite Repositories } \\
\hline WP10 & SNF/Graphite/Capsule in Glass & 7.56 & 0.2032 dia. $\times 4.0090$ & 58.1 \\
\hline WP11 & Calcine/Glass Ceramic HIP & 12.25 & 0.6096 dia. $\times 2.9970$ & 14.01 \\
\hline WP12 & SNF/Special/HEU/HIP & 13.79 & 0.6096 dia. $\times 1.7780$ & 26.57 \\
\hline WP13 & SNF/Special/LEU/HIP & 168.0 & 0.6096 dia. $\times 4.5210$ & 127.3 \\
\hline \multicolumn{5}{|c|}{ Waste Disposal Group 3} \\
\hline \multicolumn{5}{|c|}{ Salt Repository } \\
\hline WP14 & SNF/Graphite/Particles & 94.21 & $0.6096 \mathrm{dia} . \times 4.5200$ & 71.41 \\
\hline WP16 & SNF/Special/HEU/Glass & 59.3 & 0.6096 dia. $\times 4.0120$ & 50.6 \\
\hline WP17 & SNF/Special/LEU/Glass & 463.8 & 0.6096 dia. $\times 4.7740$ & 332.9 \\
\hline WP18 & Calcine/Leftover ICPP Glass & 10.62 & 0.6096 dia. $\times 2.9970$ & 12.14 \\
\hline \multicolumn{5}{|c|}{ Granite Repository } \\
\hline WP15 & SNF/Graphite/Capsule in Glass & 13.6 & 0.6096 dia. $\times 4.5200$ & 10.31 \\
\hline WP19 & SNF/Special/HEU/Glass & 9.25 & 0.6096 dia. $\times 1.7780$ & 17.8 \\
\hline WP20 & SNF/Special/LEU/Glass & 158.2 & 0.6096 dia. $\times 4.5210$ & 120.0 \\
\hline WP21 & Calcine/Leftover ICPP Glass & 15.4 & 0.6096 dia. $\times 2.9970$ & 17.6 \\
\hline
\end{tabular}


Table 6-2. Salt Repository Waste Parcel (WP) Heat Rates (continued)

\begin{tabular}{|c|c|c|c|c|}
\hline ID & Name & $\begin{array}{l}\text { Heat } \\
\text { Rate } \\
\text { (W) }\end{array}$ & Container Dimensions & $\begin{array}{c}\text { Heat Rate } \\
\text { Flux } \\
\left(\mathrm{W} / \mathrm{m}^{3}\right)\end{array}$ \\
\hline \multicolumn{5}{|c|}{ Waste Disposal Group 4} \\
\hline \multicolumn{5}{|c|}{ Sait and Granite Repositories } \\
\hline $\begin{array}{l}\text { WP22 } \\
\text { WP23 }\end{array}$ & $\begin{array}{l}\text { SNF/Reproc. Graphite \& Calcine } \\
\text { SNF/Special/Reoroc. HEU \& LEU }\end{array}$ & $\begin{array}{r}335.1 \\
425\end{array}$ & 0.6096 dia. $\times 2.9970$ & $\begin{array}{r}383.1 \\
48.6\end{array}$ \\
\hline \multicolumn{5}{|c|}{ Waste Disposal Group 5} \\
\hline \multicolumn{5}{|c|}{ Salt and Granite Repositories } \\
\hline $\begin{array}{l}\text { WP24 } \\
\text { WP25 } \\
\text { WP26 }\end{array}$ & $\begin{array}{l}\text { SNF/Graphite/+DU in Glass } \\
\text { SNF/Special/Reproc. DU \& Glass } \\
\text { Calcine/NEL in Glass }\end{array}$ & $\begin{array}{c}67.39 \\
122.1 \\
8.1\end{array}$ & $\begin{array}{l}0.6096 \text { dia. } \times 2.9970 \\
0.6096 \text { dia. } \times 2.9970 \\
0.6096 \text { dia. } \times 2.9970\end{array}$ & $\begin{array}{c}77.04 \\
139.6 \\
9.26\end{array}$ \\
\hline
\end{tabular}

The hypothetical saturated granite repository design uses a backfill-buffer material different from the host rock (i.e., bentonite). Since the thermal conductivity of bentonite backfill-buffer materials is typically lower than the host rock (granite or other crystalline rocks), waste parcel heat dissipation is controlled by the backfill-buffer thickness. The backfill materials behave as insulators around the waste parcels. For this reason, borehole drilling diameters for waste parcels must be tailored to the specific waste parcel, according to its final diameter. Doing so results in several drill-bit sizes, because a unique diameter-sized drill-bit is used for each waste parcel.

The hypothetical salt repository design incorporates a crushed-salt backfill material between the waste parcel and the host rock. The crushed salt is expected to compact due to creep from lithostatic pressure and reach nearly the density of intact halite in a short time $(-100 \mathrm{yr})$. However, when first emplaced, the crushed-salt backfill-buffer has a lower density and thermal conductivity than intact salt. In addition, waste parcel heat generation is greatest when the parcel is first placed in the borehole. Thus, like granite emplacement, it is important to tailor drilling diameters in salt to specific waste parcels so that peak backfill-buffer temperatures can be controlled.

Mining of the Disposal Rooms. Repository designs for both geologic media specify that the waste disposal room be $9.0 \mathrm{~m}$ wide by $6.3 \mathrm{~m}$ high (as shown in Figure 6-1). For the salt repository, it is assumed that current technology exists to excavate such tunnels, e.g., the mined WIPP repository panels and drifts are $10 \mathrm{~m}$ wide by $4 \mathrm{~m}$ wide. For the saturated granite repository, it is envisioned that the conventional drill-and-blast mining techniques will be used to excavate the waste disposal rooms (as well as additional shafts, passageways, and ventilation paths) in the proposed granite repository (MacDougall et al., 1987a, p. 6-36). Drill-and-blast techniques inherently introduce a small amount of damage to the host rock surrounding the drift. This damage region can be controlled to some degree through the use of smooth-wall blasting techniques. Typical drill-and-blast equipment and full-face mechanized boring machines used for excavation include: a drill jumbo, a load/haul/dump machine, a rock bolting jumbo, a scaling machine, and a tunnel-boring machine (MacDougall et al., 1987b, Vol. 3, p. D-27).

\subsection{Dimensions of the Repository}

Three components of the repository are assumed to affect the behavior of the disposal system: waste disposal region, access drifts, and shafts (Figure 6-2). Tible 6-3 tabulates area measurements for these components. 
4

7

9

10

11

13

14

15

16

18

19

20

21

22

23
24

25

26

27

28

29

30

31

32

33

34

35

36

37

38

39

40

41

42

43

44

45
46

47

48

49

50

51

52

53

54

55

56

57

58

59

60

61

62

63

64

65

66
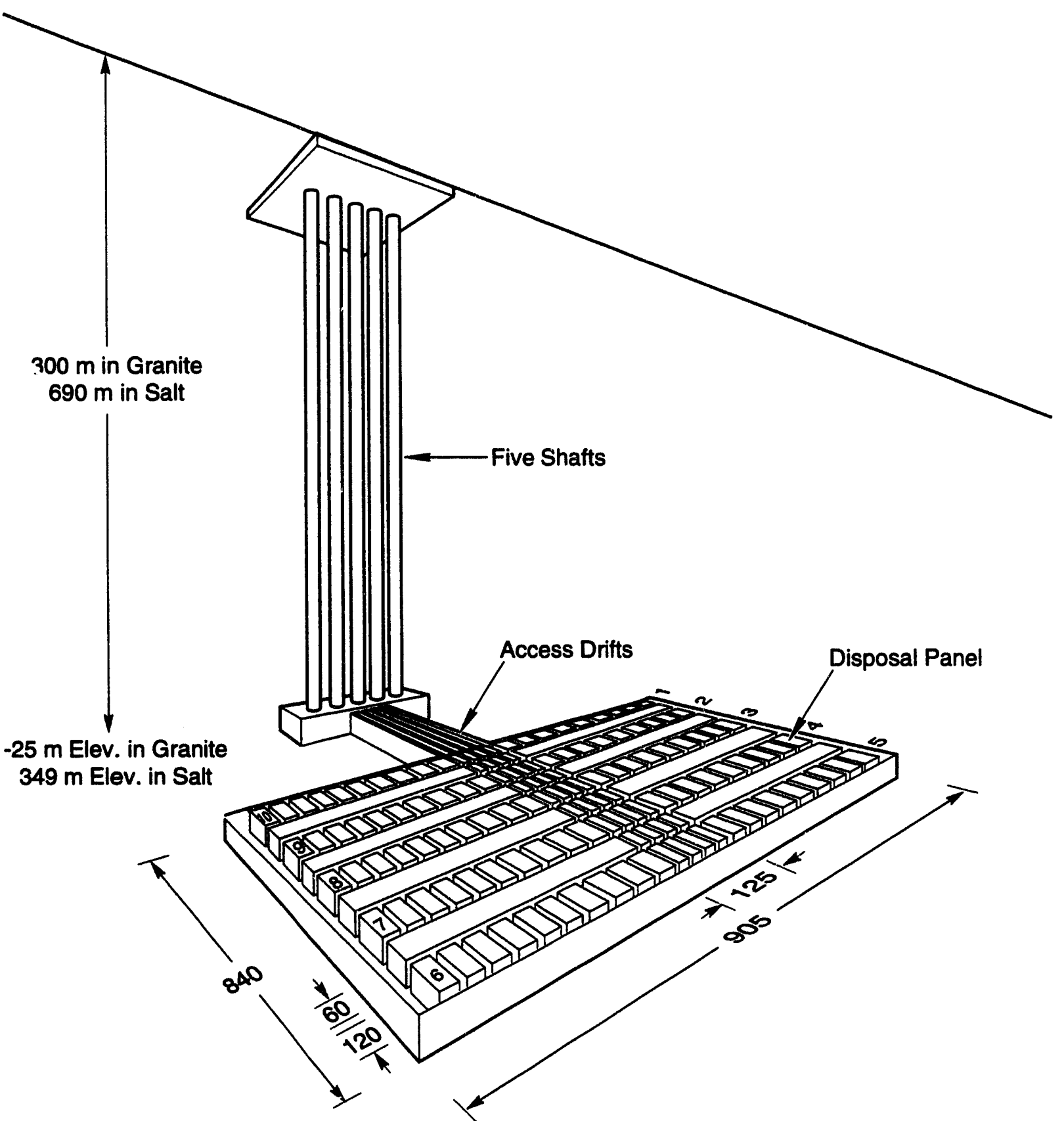

TRI-6342-2090-1

Figure 6-2. Schematic of hypothetical repository. 


\section{Repository Design}

Table 6-3. Areas in Subsurface Facilities

\begin{tabular}{lcc}
\hline & \multicolumn{2}{c}{ Area $\left(\mathrm{m}^{2}\right)$} \\
\cline { 2 - 3 } \multicolumn{1}{c}{ Region } & Excavated & Enclosed \\
\hline Main leg & 1,080 & $\ldots-$ \\
Side leg (2 per waste room) & 270 & $\cdots$ \\
Waste room (10 per panel) & 1620 & $\cdots$ \\
Panel (10 per repository) & 16,200 & 46,800 \\
Disposal region & 162,000 & 655,200 \\
Access dritts & 55,350 & 153,750 \\
Shatts (6 m dia) (5 per repository) & 28 & 28 \\
Repository & 217,400 & 809,000 \\
\hline
\end{tabular}

\subsubsection{Waste Disposal Region}

The waste disposal region for the hypothetical salt repository resides at an elevation of $349 \mathrm{~m}(690 \mathrm{~m}$ below the surface) in a unit of relatively pure halite $340 \mathrm{~m}$ thick. The hypothetical granite repository resides at an elevation of $-25 \mathrm{~m}$ ( $300 \mathrm{~m}$ below the surface); no dip is assumed in either repository.

The waste disposal region consists of excavated rooms and drifts for the spent fuel and high-level waste, and the main and branch access drifts for the transport of material, canisters, and ventilation (Figure 6-3). Generally, all drifts, and disposal rooms are rectangular in cross-section, $6 \mathrm{~m}$ high and $9 \mathrm{~m}$ wide.

The waste disposal rooms are $9 \mathrm{~m}$ wide, $6 \mathrm{~m}$ high, and $180 \mathrm{~m}$ long (Figure 6-3). A grouping of rooms is called a panel. The repository has 10 panels, each with 10 rooms (Figure 6-2).

Each panel within the repository consists of a sequence of parallel disposal rooms separated by salt or granite pillars (as shown in Figure 6-1). Access legs on both sides of the main leg permit access for excavation equipment and drilling machinery, and provide ventilation. These access legs have cross-sectional dimensions identical to the connecting main legs. The panel access legs will be excavated first, and then the connecting room segment at the end of each panel (Room A in Figure 6-3). Once the room is filled with waste, it will be backfilled with crushed salt (salt repository) or crushed rock mixed with local clay (granite repository); the crushed salt or rock will come from the excavation of the adjacent disposal room.

The number of rooms and waste parcels required for the salt and granite repositories is shown in Table 6-1. The waste disposal groups include both the waste stored at Idaho and the waste from the DOE sites (Savannah River, Hanford, and West Valley). Because of the lower thermal conductivity of granite as compared to salt, generally more rooms are required to dispose of the waste in a granite repository compared to a repository in salt (see totals for Waste Disposal Groups 1 and 3 in Table 6-1). Ten panels are still sufficient, however, to accommodate the increased number of disposal rooms. The values are based upon the calculations described in Appendix C, "Scoping Calculations." 


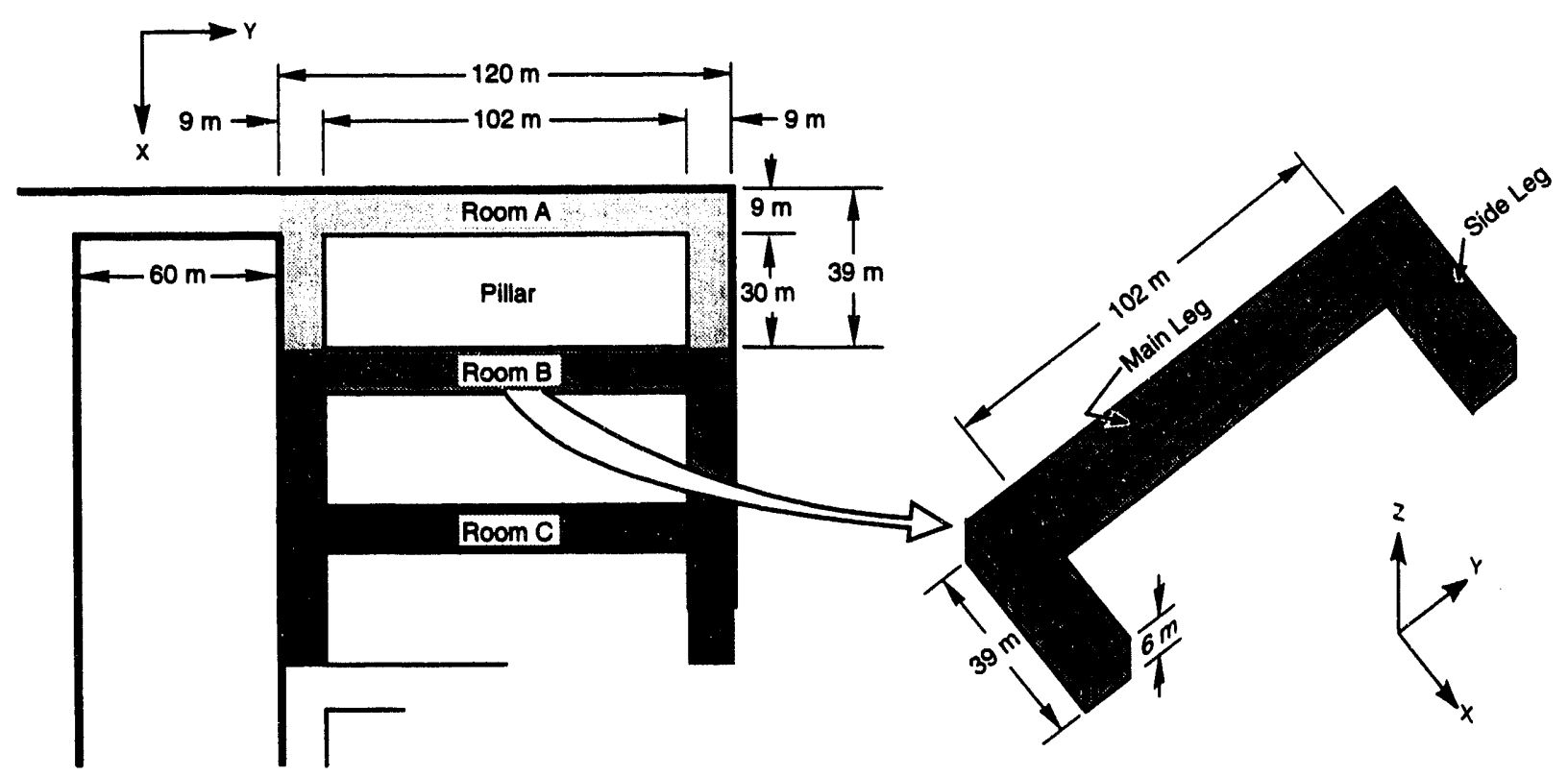

TRI-6342-3513-0

Figure 6-3. Schematic of panel in disposal region of repository.

\subsubsection{Access Drifts and Shafts}

Five horizontal main passageways traverse the center of the repository disposal area. They provide (1) a pathway for fresh air to the development and emplacement areas, (2) an exhaust air path from the development areas, (3) a passageway for mined salt transport, (4) a passageway for radioactive waste transport, and (5) access for personnel and equipment to the development and emplacement areas. Accessways are located at the perimeter of the disposal area.

All shafts are circular in cross-section, with either a 9-m-finished diameter (salt repository) or a 6-m-finished diameter (granite repository). It may be necessary to use an annular ring of grouting material (such as concrete) to prevent the disturbed rock zone (salt or granite) from falling into the shafts. In general, the shafts are located upstream of the disposal drifts and rooms when orienting the repository and accounting for the prevailing groundwater flow path. Shafts terminate at the repository level.

\subsection{Backfill}

As noted in the Introduction, backfill is the material used to fill the excavated openings of the repository. Generally, the backfill performs several functions, the importance of which varies according to the location of the backfill in the repository. For example, the backfill may prevent undue disturbance from room, drift, and shaft collapse or diminish groundwater movement around the container and thus limit advective transport. Specific primary functions are noted below. Special categories of backfill include (1) backfill-buffer (backfill in the annulus around the waste parcel) and (2) seals (e.g., concrete) for providing immediate sealing (albeit possibly short-term sealing) of the shafts and access drifts. Only the backfill-buffer and general backfill in the rooms, drifts, and shafts are discussed here. Seals provide operator safety functions or special functions that depend on unique circumstances at each specific site, circumstances that were not hypothesized for this performance assessment. 


\subsubsection{Backfill-Buffer around Waste Parcel}

The backfill-buffer is an important component of a waste-emplacement package (which includes the waste form, canister, internal backfill, overpack, and backfill-buffer). (The source term submodel [Chapter 11] groups the waste parcel and backfill-buffer as a modeling unit, the waste emplacement package.) The backfill-buffer material may provide up to five primary physical and chemical functions (not all are applicable to all sites) (Molecke et al., 1982, p. 4; Börgesson, 1993):

1. May serve as a hydrological barrier that significantly limits groundwater movement near the waste parcel.

2. May provide a supplemental, long-term radionuclide sorptive barrier retarding the release of radionuclides leached from the waste form.

3. May act as a near-field groundwater chemical conditioner, buffering $\mathrm{pH}$, and ionic composition.

4. May serve as an adequate heat-conduction material.

5. May reduce the severity of rock shear effects on the waste parrel when a shear plane intersects the waste parcel.

It is the general moderation of the release of contaminants to the host rock that is implied by the word "buffer" in backfill-buffer but, as noted above, the backfill may function as a buffer in the chemistry sense by containing both a weak acid and conjugate base to resist changes in $\mathrm{pH}$.

Although potentially assisting in containment, the backfill-buffer may also have detrimental side effects. For example, it may increase the possibility of diffusion or reduce heat conduction away from the waste parcel. Because transport from the waste parcel is primarily by diffusion, a backfill-buffer with a high porosity (e.g., clay) may aid diffusion. Furthermore, it is desirable for the backfill-buffer material to have a high thermal conductivity that permits adequate heat transfer away from the waste parcels to keep temperatures below specific limits. Low backfill-buffer thermal conductivities allow unacceptable temperatures to be reached in the waste parcel, backfill-buffer, and/or the geological host rock. Both waste parcel/backfill-buffer and backfill-buffer/host rock interface temperatures are important factors in material selection and design thickness of the backfill. In addition, backfill-buffer materials with low thermal conductivities invoke an economic penalty because fewer waste parcels can be distributed in a waste disposal room, which increases the spacing of waste parcels per disposal room, and thus requires a larger repository (Moss and Molecke, 1983, p. 724).

Salt Repository. Crushed salt was selected as the backfill-buffer material to be used with waste parcels stored in a salt repository. Crushed salt provides good heat conduction away from the waste parcel and also has the ability to creep and heal any existing cracks. This creeping phenomenon, from the surrounding lithostatic pressure, will decrease the backfill-buffer permeability, nearly matching the host rock (intact salt) within $100 \mathrm{yr}$ (based on predicted WIPP disposal room closure, Lappin et al., 1989, p. 4-85; WIPP PA Division, 1991a, p. 3-17). A major conclusion reached in other salt repository waste parcel designs was that "engineered" backfill-buffer materials were not necessary (Tang and Saling, 1990, p. 129). For a salt repository, the waste container is expected to perform adequately even with an unlimited amount of anoxic brine. Furthermore, because of the lack of a significant quantity of brine and the low permeability of the salt, any radionuclides released from the waste form in the vicinity of the waste parcel would be retained (Tang and Saling, 1990, p. 129). Thus, crushed salt is a suitable backfill material and generally will provide adequate heat transfer away from the waste form and container. ${ }^{* * *}$ For ease in computations, a backfillbuffer thickness of $0.3 \mathrm{~m}$ (of crushed salt) was chosen for all waste parcels.

Table 6-4 provides parameter values for the (creep compacted) backfill-buffer material in the salt repository.

\# The latter detrimental effects of the backfill-buffer suggest than an optimal size for the backfill-buffer annulus around the waste parcel exists. This optimization was not performed in this performance assessment except to check (as described later) that the selected size of the annulus did not raise temperatures greatly at the waste parcel/backfill-buffer interface or the backfill-buffer/host rock interface.

-.. Crushed-salt backfill-buffer has a lower thermal conductivity and density than intact salt (Molecke and Beraun, 1986). Since waste parcel heat rates are greatest immediately after emplacement and prior to creep consolidation, these lower conductivities are likely to have an effect on the local temperature field. The temperature rise in the backfill is not, however, expected to rise to salt-decrepitation temperatures $\left(-250^{\circ} \mathrm{C}\right)$. (See discussion of granite repository backfill-buffer, Appendix C.) 


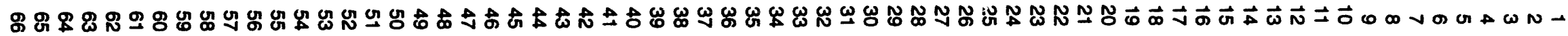

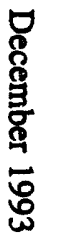

Table 6-4. Backfill-Buffer Between Waste Parcel and Host Rock (Salt)

\begin{tabular}{|c|c|c|c|c|c|c|c|c|}
\hline \multirow{2}{*}{$\begin{array}{l}\text { Recond } \\
\text { No. } \\
2258\end{array}$} & \multirow{2}{*}{$\begin{array}{l}\text { Parameter } \\
\text { BrkCorEx }\end{array}$} & \multirow{2}{*}{\begin{tabular}{l}
\multicolumn{1}{c}{ Parameter Description } \\
$\begin{array}{l}\text { Brooks-Corey Exponent for capillary } \\
\text { pressure }\end{array}$
\end{tabular}} & \multirow{2}{*}{$\frac{\text { Median }}{7.00 e-01}$} & \multicolumn{2}{|c|}{ Range } & \multirow{2}{*}{ Units } & \multirow{2}{*}{$\frac{\text { Type }}{\text { Cumulative }}$} & \multirow{2}{*}{$\begin{array}{l}\text { Sources In: } \\
\text { Sandia WIPP } \\
\text { Project, } 1992\end{array}$} \\
\hline & & & & $2.00 e-01$ & $1.00 e+01$ & & & \\
\hline 2250 & DnsBlk & Bulk density & $2.03 e+03$ & & & $\mathrm{~kg} / \mathrm{m}^{3}$ & Constant & $\begin{array}{l}\text { Sandia WIPP } \\
\text { Project, } 1992\end{array}$ \\
\hline 2247 & Compres & Compressibility & $3.70 e-10$ & & & $\mathrm{~Pa}^{-1}$ & Constant & $\begin{array}{l}\text { Sjaardema and } \\
\text { Krieg, } 1987\end{array}$ \\
\hline 2248 & Porosity & Matrix porosity & $1.20 e-01$ & $5.00 e-02$ & $1.90 e-01$ & none & Uniform & $\begin{array}{l}\text { IT Corporation, } \\
1987\end{array}$ \\
\hline 2249 & Prm & Permeability & $1.00 e-20$ & $3.30 e-21$ & $3.30 e-20$ & $m^{2}$ & Lognormal & $\begin{array}{l}\text { Sandia WIPP } \\
\text { Project, } 1992\end{array}$ \\
\hline 2255 & PorPrsRf & Reference pressure for porosity & $1.01 e+05$ & & & $\mathrm{~Pa}$ & Constant & $\begin{array}{l}\text { Ramsey, analyst } \\
\text { decision }\end{array}$ \\
\hline 2254 & DnsTmpAf & Reference temperature for density & $2.98 e+02$ & & & $\mathrm{~K}$ & Constant & $\begin{array}{l}\text { Ramsey, analyst } \\
\text { decision }\end{array}$ \\
\hline 2259 & SatRGP & Residual gas saturation & $2.00 e-01$ & 0 & $4.00 e-01$ & none & Uniform & $\begin{array}{l}\text { Sandia WIPP } \\
\text { Project, } 1992\end{array}$ \\
\hline 2257 & SatRWP & $\begin{array}{l}\text { Residual wetting phase (liquid) satura- } \\
\text { tion }\end{array}$ & $2.00 e-01$ & 0 & $4.00 e-01$ & none & Uniform & $\begin{array}{l}\text { Sandia WIPP } \\
\text { Project, } 1992\end{array}$ \\
\hline 2256 & Saturatn & Saturation, initial & $2.00 e-01$ & & & none & Constant & $\begin{array}{l}\text { Ramsey, analyst } \\
\text { decision }\end{array}$ \\
\hline 2251 & SpHeat & Specific heat & $8.60 e+02$ & & & $\mathrm{~J} / \mathrm{kg} \cdot \mathrm{K}$ & Constant & Krieg, 1984 \\
\hline 2253 & ThExp & Thermal expansion coefficient & $4.50 \theta-05$ & & & $1 / K$ & Constant & Krieg, 1984 \\
\hline 2252 & ThCnd & Thermal conductivity & $5.00 e+00$ & & & $W /(m \cdot K)$ & Constant & Krieg, 1984 \\
\hline
\end{tabular}


Granite Repository. Bentonite clay and sand mixtures have received great attention over the years as a potential backfill-buffer material for a waste parcel in a saturated granite repository. This mixture has been suggested for use to enhance radionuclide-sorption and hydrological-barrier functions of a high-density backfill-buffer material (Nowak, 1989). In addition, the mixture has the ability to swell upon contact with an aqueous solution, and if confined, reduce groundwater flow (Kircher and Bradley, 1983, p. 383). However, because bentonite clay and sand mixtures have a lower thermal conductivity than the host rock, the waste parcel backfill-buffer thickness must be carefully determined to avoid high temperatures at the backfill-buffer/host-rock interface, within the backfill-buffer, and at the waste centerline.

Another consideration is that bentonite clays have been shown to change crystalline structure from montmorillonite to illite at elevated temperatures. The change in crystalline structure of the bentonite clays causes a significant loss of swelling capability and a dramatic increase in hydraulic conductivity. This is illustrated in SKB (1992b) where French FO-CA clay was found to be altered to claystone with a total loss in swelling capacity and a significant increased in hydraulic conductivity at $170^{\circ} \mathrm{C}$. This temperature limit was reduced further $\left(130^{\circ} \mathrm{C}\right)$ in SKB, $1992 \mathrm{a}$. Thus if bentonite is used, $130^{\circ} \mathrm{C}$ can be considered as a design criterion limiting the maximum temperature allowed at the container/backfill-buffer interface. Scoping calculations reported in Appendix C for a bentonite backfill-buffer thickness of $0.3 \mathrm{~m}$ surrounding Waste Parcel 8 indicate that the $130^{\circ} \mathrm{C}$ limit is not exceeded in the backfill-buffer. Thus $0.3 \mathrm{~m}$ was chosen as the thickness for the bentonite backfill-buffer for the granite repository. Because of varying canister sizes, Waste Disposal Group i for granite contains one waste parcel with a 0.37 -m-thick backfill-buffer, and another with a 0.35 -m-thick backfill-buffer. For simplicity, $0.3 \mathrm{~m}$ was also chosen for the thickness of the crushed-salt backfill-buffer used in the bedded salt repository.

Table 6-5 provides parameter values for the backfill-buffer material in the granite repository.

\subsubsection{General Backfill in Rooms, Access Drifts, and Shaft}

After the waste parcels are emplaced in each room, the rooms and drifts will be filled with a backfill material: crushed salt (at a density of $14.9 \mathrm{kN} / \mathrm{m}^{3}$ [95 lb/ft ${ }^{3}$ ], occupying $97 \%$ of the excavated volume) for the salt repository and a crushed granite mixed with a local clay for the granite repository.

Salt Repository. Under the stress and heat conditions of the repository, the backfill in the salt repository will eventually (tens to hundreds of years) reform into a solid mass of salt similar to the original undisturbed salt. The low permeability of this recompacted salt and the surrounding undisturbed salt acts as a barrier to radionuclide migration from a breached canister, hence no additional material was thought necessary to add to the crushed salt. Individual panels will be isolated with blocks of precompressed salt stacked as masonry walls to provide isolation while the crushed salt is recompacting due to creep. Shafts will also be backfilled with crushed salt. ${ }^{\dagger \dagger}$ Table 6-6 summarizes model parameters for the backfill at the salt repository.

Granite Repository. After the waste is emplaced and backfilled, the drifts and disposal rooms will be filled with a mixture of crushed rock from the mining operation and a local clay to limit water movement in the mined drifts. Table 6-7 summarizes model parameters for the backfill at the granite repository.

In a saturated granite repository, two areas were assumed to include local fractures that were unacceptable for waste placement. Although special seals might be devised for these areas, the analysis assumed that they had degraded such that only the general backfill provided resistance to fluid flow.

\footnotetext{
${ }^{t+t}$ Special seals may be necessary to restrict the flow of water from a water-bearing layer at a higher elevation into the shafts, but were not modeled.
} 


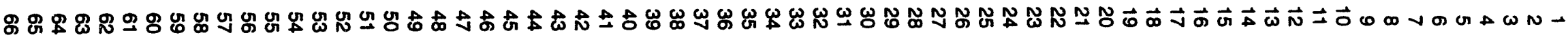

Table 6-5. Backfill-Buffer Between Waste Parcel and Host Rock (Granite)

\begin{tabular}{|c|c|c|c|c|c|c|c|c|}
\hline $\begin{array}{l}\text { Record } \\
\text { No. }\end{array}$ & Parameter & Parameter Description & Median & \multicolumn{2}{|c|}{ Range } & Units & Type & Sources In: \\
\hline 4231 & BrkCorEx & $\begin{array}{l}\text { Brooks-Corey Exponent for capillary } \\
\text { pressure }\end{array}$ & $7.00 e-01$ & $2.00 e-01$ & $1.00 e+01$ & none & Constructed & $\begin{array}{l}\text { Ramsey, analyst } \\
\text { decision }\end{array}$ \\
\hline 4223 & DnsBlk & Bulk density & $1.38 \theta+00$ & & & $\mathrm{~kg} / \mathrm{m}^{3}$ & Constant & $\begin{array}{l}\text { Radhakrishna and } \\
\text { Chan, } 1985\end{array}$ \\
\hline 4224 & Compres & Compressibility & $3.00 e-08$ & & & $1 / \mathrm{Pa}$ & Constant & $\begin{array}{l}\text { Radhakrishna and } \\
\text { Chan, } 1985\end{array}$ \\
\hline 4234 & HyCnd & Hydraulic Conductivity & $1.00 e-10$ & & & none & Constant & $\begin{array}{l}\text { LeNeveu, 1986; } \\
\text { Garisto and } \\
\text { LeNeveu, } 1989\end{array}$ \\
\hline 4222 & Porosity & Matrix porosity & $5.00 e-01$ & $4.00 e-01$ & $6.00 \mathrm{e}-01$ & none & Uniform & $\begin{array}{l}\text { Garisto and } \\
\text { LeNeveu, 1989; } \\
\text { Radhakrishna and } \\
\text { Chan, } 1985\end{array}$ \\
\hline 4233 & Prm & Permeability & $1.00 e-17$ & & & $\mathrm{~m}^{2}$ & Constant & $\begin{array}{l}\text { LeNeveu, 1986; } \\
\text { Garisto and } \\
\text { LeNeveu, } 1989\end{array}$ \\
\hline 4225 & PorPrsRf & Reference pressure for porosity & $1.01 e+05$ & & & $\mathrm{~Pa}$ & Constant & $\begin{array}{l}\text { Ramsey, analyst } \\
\text { decision }\end{array}$ \\
\hline 4226 & DnsTmpRf & Reference temperature for density & $2.98 e+02$ & & & K & Constant & $\begin{array}{l}\text { Ramsey, analyst } \\
\text { decision }\end{array}$ \\
\hline 4230 & SatRGP & Residual gas saturation & $2.00 e-01$ & & & none & Constant & $\begin{array}{l}\text { Ramsey, analyst } \\
\text { decision }\end{array}$ \\
\hline 4229 & SatRWP & $\begin{array}{l}\text { Residual wetting phase (liquid) satura- } \\
\text { tion }\end{array}$ & $4.00 e-01$ & & & none & Constant & $\begin{array}{l}\text { Ramsey, analyst } \\
\text { decision }\end{array}$ \\
\hline 4228 & Saturatn & Saturation, initial & $4.00 e-01$ & & & none & Constant & $\begin{array}{l}\text { Ramsey, analyst } \\
\text { decision }\end{array}$ \\
\hline 4235 & SpHeat & Specific heat & $9.21 e+02$ & & & $\mathrm{~J} / \mathbf{k g} \odot \mathrm{K}$ & Constant & $\begin{array}{l}\text { Domenico and } \\
\text { Schwartz, } 1990\end{array}$ \\
\hline 4227 & ThExp & Thermal expansion coefficient & $3.40 e-05$ & & & $1 / \mathrm{K}$ & Constant & Wai and Tsai, 1985 \\
\hline 4232 & ThCnd & Thermal conductivity & $1.05 e+00$ & & & $W /(m \cdot k)$ & Constant & $\begin{array}{l}\text { Domenico and } \\
\text { Schwartz, } 1990\end{array}$ \\
\hline
\end{tabular}




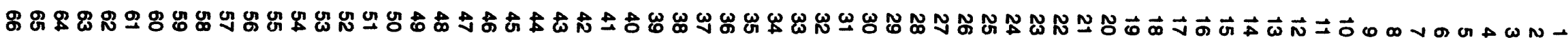
$\frac{9}{a}$

Table 6-6. Backfill in Drift and Panels (Salt)

\begin{tabular}{|c|c|c|c|c|c|c|c|c|}
\hline $\begin{array}{l}\text { Recond } \\
\text { No. }\end{array}$ & Parameter & Parameter Description & Median & & & Units & Type & Sources in: \\
\hline 2271 & BrkCorEx & $\begin{array}{l}\text { Brooks-Corey Exponent for capillary } \\
\text { pressure }\end{array}$ & $7.00 e-01$ & $2.00 \mathrm{e}-01$ & $1.00 e+01$ & none & Constructed & $\begin{array}{l}\text { Sandia WIPP } \\
\text { Project, } 1992\end{array}$ \\
\hline 2268 & DnsBlk & Bulk density & $2.03 e+03$ & & & $\mathrm{~kg} / \mathrm{m}^{3}$ & Constant & $\begin{array}{l}\text { WIPP PA Division, } \\
\text { 1991c }\end{array}$ \\
\hline 2260 & Compres & Compressibility & $3.70 e-10$ & & & $1 / \mathrm{Pa}$ & Constant & $\begin{array}{l}\text { Sjaardema and } \\
\text { Krieg, } 1987\end{array}$ \\
\hline 2267 & Porosity & Matrix porosity & $1.20 e-01$ & $5.00 \mathrm{e}-02$ & $1.90 \mathrm{e}-01$ & none & Uniform & IT Corporation, 1987 \\
\hline 2261 & Prm & Permeability & $1.00 \Theta-20$ & $3.30 e-21$ & $3.300-20$ & $m^{2}$ & Lognormal & $\begin{array}{l}\text { Sandia WIPP } \\
\text { Project, } 1992\end{array}$ \\
\hline 2133 & Prm_U & Permeability, undisturbed & $1.00 e-19$ & & & $\mathrm{~m}^{2}$ & Constant & $\begin{array}{l}\text { Vaughn, analyst } \\
\text { decision }\end{array}$ \\
\hline 2262 & PorPrsRf & Reference pressure for porosity & $1.01 e+05$ & & & $\mathbf{P a}$ & Constant & $\begin{array}{l}\text { Ramsey, analyst } \\
\text { decision }\end{array}$ \\
\hline 2263 & DnsTmpRf & Reference temperature for density & $2.98 e+02$ & & & K & Constant & $\begin{array}{l}\text { Ramsey, analyst } \\
\text { decision }\end{array}$ \\
\hline 2269 & SatRGP & Residual gas saturation & $2.00 e-01$ & 0 & $4.00 e-01$ & none & Uniform & $\begin{array}{l}\text { Sandia WIPP } \\
\text { Project, } 1992\end{array}$ \\
\hline 2272 & SatRWP & $\begin{array}{l}\text { Residual wetting phase (liquid) satura- } \\
\text { tion }\end{array}$ & $2.00 e-01$ & 0 & $4.00 e-01$ & none & Uniform & $\begin{array}{l}\text { Sandia WIPP } \\
\text { Project, } 1992\end{array}$ \\
\hline 2270 & Saturatn & Saturation, initial & $2.00 e-01$ & & & none & Constant & $\begin{array}{l}\text { Ramsey, analyst } \\
\text { decision }\end{array}$ \\
\hline 2266 & SpHeat & Specific heat & $8.60 e+02$ & & & $\mathrm{~J} / \mathrm{kg} \cdot \mathrm{K}$ & Constant & Krieg, 1984 \\
\hline 4227 & ThExp & Thermal expansion coefficient & $3.40 e-05$ & & & $1 / K$ & Constant & Krieg, 1984 \\
\hline 4232 & ThCnd & Thermal conductivity & $1.05 \theta+00$ & & & $W /(m \circ K)$ & Constant & Krieg, 1984 \\
\hline 2136 & Pt & Threshold Pressure, undisturbed & $2.10 e+06$ & & & $\mathrm{~Pa}$ & Constant & $\begin{array}{l}\text { Sandia WIPP } \\
\text { Project, } 1992\end{array}$ \\
\hline
\end{tabular}




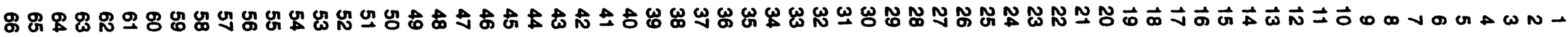

Table 6-7. Backfill in Drift and Panels (Granite)

\begin{tabular}{|c|c|c|c|c|c|c|c|c|}
\hline \multirow{2}{*}{$\begin{array}{l}\begin{array}{c}\text { Record } \\
\text { No. }\end{array} \\
4237 \\
\end{array}$} & \multirow{2}{*}{$\begin{array}{l}\text { Parameter } \\
\text { BrkCorEx }\end{array}$} & \multirow{2}{*}{$\begin{array}{l}\text { Parameter Description } \\
\text { Brooks-Corey Exponent for capillary } \\
\text { pressure }\end{array}$} & \multirow{2}{*}{$\frac{\text { Median }}{7.000-01}$} & \multicolumn{2}{|c|}{ Range } & \multirow{2}{*}{$\frac{\text { Units }}{\text { none }}$} & \multirow{2}{*}{$\frac{\text { Type }}{\text { Constructed }}$} & \multirow{2}{*}{\begin{tabular}{l}
\multicolumn{1}{c}{ Sources in: } \\
$\begin{array}{l}\text { Ramsey, analyst } \\
\text { decision }\end{array}$
\end{tabular}} \\
\hline & & & & $2.000-01$ & $1.000+01$ & & & \\
\hline 4241 & DnsBlk & Bulk density & $1.52 \theta+03$ & & & $\mathrm{~kg} / \mathrm{m}^{3}$ & Constant & $\begin{array}{l}\text { Garisto and } \\
\text { LeNeveu, 1989; } \\
\text { Radhakrishna, and } \\
\text { Chan, } 1985\end{array}$ \\
\hline 4243 & Compres & Compressibility & $3.00 e-08$ & & & $1 / \mathrm{Pa}$ & Constant & $\begin{array}{l}\text { Radhakrishna, and } \\
\text { Chan, } 1985\end{array}$ \\
\hline 4247 & HyCnd & Hydraulic conductivity & $1.00 \mathrm{e}-08$ & & & none & Constant & $\begin{array}{l}\text { LeNeveu, 1986; } \\
\text { Garisto and } \\
\text { LeNeveu, } 1989\end{array}$ \\
\hline 4242 & Porosity & Matrix porosity & $4.30 e-01$ & $2.000-01$ & $6.500-01$ & none & Uniform & $\begin{array}{l}\text { LeNeveu, 1986; } \\
\text { Garisto and } \\
\text { LeNeveu, } 1989\end{array}$ \\
\hline 4236 & Prm & Permeability & $1.00 \theta-14$ & & & $m^{2}$ & Constant & $\begin{array}{l}\text { LeNeveu, 1986; } \\
\text { Garisto and } \\
\text { LeNeveu, } 1989\end{array}$ \\
\hline 4238 & SatRGP & Residual gas saturation & $2.00 e-01$ & & & none & Constant & $\begin{array}{l}\text { Ramsey, analyst } \\
\text { decision }\end{array}$ \\
\hline 4239 & SatRWP & $\begin{array}{l}\text { Residual wetting phase (liquid) satura- } \\
\text { tion }\end{array}$ & $4.00 \mathrm{e}-01$ & & & none & Constant & $\begin{array}{l}\text { Ramsey, analyst } \\
\text { decision }\end{array}$ \\
\hline 4240 & Saturatn & Saturation, initial & $4.00 \mathrm{e}-01$ & & & none & Constant & $\begin{array}{l}\text { Ramsey, analyst } \\
\text { decision }\end{array}$ \\
\hline 4246 & SpHeat & Specific heat & $8.00 e+02$ & & & $\mathrm{~J} / \mathrm{kg} \cdot \mathrm{K}$ & Constant & Wai and Tsai, 1985 \\
\hline 4244 & ThExp & Thermal expansion coefficient & $3.40 e-05$ & & & $1 / K$ & Constant & Wai and Tsai, 1985 \\
\hline 4245 & ThCnd & Thermal conductivity & $2.00 \theta+00$ & & & $W /(m \circ K)$ & Constant & Wai and Tsai, 1985 \\
\hline
\end{tabular}


6. Repository Design

\subsection{Modeling Assumptions for Repository}

The following assumptions were made for the repository component of the engineered barrier:

- Individual waste parcels are emplaced vertically in bored holes in the repository floor. A distance of $1 \mathrm{~m}$ separates the top of the waste parcels from the disposal room floor.

- Each repository includes 10 panels of 10 rooms each, each room holding about 276 waste parcels.

- Waste parcels from all waste groups are distributed randomly throughout the repository disposal rooms in a hexagonal pattern on $2.6-\mathrm{m}$ centers.

- Waste parcels in the salt repository are backfilled with crushed salt, with annular thickness of $0.3 \mathrm{~m}$; in the granite repository, the backfill-buffer is a bentonite clay, $0.3 \mathrm{~m}$ thick (except for two cases in Waste Group 1).

- A shield plug and cover plate will be used above each waste parcel, if necessary.

- Drifts and accessways in the salt repository are backfilled with crushed salt that recompacts in time to conditions close to those of intact salt due to salt creep.

- Drifts and accessways in the granite repository are backfilled with a mixture of crushed rock and local clay. 


\title{
7. Scenario* Development
}

\author{
M. S. Tierney, R. V. Guzowski, and R. P. Rechard
}

\subsection{Purpose and Components of Scenario Development}

Evaluating the performance of a disposal system requires not only a scientific understanding of the current system but also a prediction of its behavior for $10,000 \mathrm{yr}$. Consequently, a model must be developed to simulate plausible futures of the disposal system. Scenario development is the second phase of conceptual model development (the first being disposal system characterization, Chapters 4 through 6 ). In conjunction with system characterization, the scenario development process establishes how the real world will be represented in the conceptual model(s) (Figure $7-1)$.

Generally speaking, scenario development is the process of deciding what may happen to the disposal system in the future and how to model it. The process entails selecting features (e.g., a brine reservoir under the repository), events (e.g., humans drilling into the repository), and processes (e.g., generation of gas in the repository after disposal of the waste). Scenario development consists of several steps: ${ }^{\dagger}$

1. Identifying the full scope (i.e., "universe") of features, events, and processes of the disposal system. (Events are also referred to as short-term phenomena; processes, as long-term phenomena.)

2. Selecting for the model those features, events, and processes that might contribute to contaminants being released to the accessible environment. (Regulations and standard modeling practice guide the selection process. For example, regulatory guidance indicates that some events may be discarded, and standard modeling practice is to assume that most aspects of the biosphere remain as currently exists for $10,000 \mathrm{yr}$.)

3. Creating conceptual models based on (a) the disposal system characterization and (b) the selected features, events, and processes.

4. Incorporating those conceptual models into the performance assessment calculations.

During the scenario development phase, many decisions are made about what to include in the model. However, the decisions themselves are more conveniently discussed in the chapters that describe either system characterization or consequence models. For example, features are discussed in the chapters on geologic and engineered barriers (Chapters 4, 5, and 6); processes and events are discussed with the consequence models (Chapters 11 through 15).

\subsection{Identification and Selection of Features, Events, and Processes}

Generating a comprehensive set of features, events, and processes (Step 1 in Section 7.1) is important so that significant contributions to contaminant release to the accessible environment are not overlooked. To ensure traceability and repeatability of the results, Sandia has developed a formal procedure for identifying and selecting features, events, and processes.

The procedure was developed by Sandia (Campbell et al., 1978) to specifically address the performance assessment requirements of and regulatory compliance with EPA's standard. Development of this procedure was funded by

\footnotetext{
In this chapter, the noun "scenario" is a synonym for the term "the set of all features, events, and processes considered for incorporation in the conceptual model of the disposal system." Other meanings of "scenario" are described in Sections 3.3.1 and 3.3.2; instances of the use of one of these other meanings in this chapter are noted by citation to Sections 3.3.1 or 3.3.2.

+ Other authors have used the term scenario development to refer to steps 1 and 2 only, because these two steps initially exact greater attention than steps 3 and 4 . In this report, however, scenario development includes not only the selection of the features, events, and processes but also the incorporation of those components into the computational model.
} 
7. Scenario Development

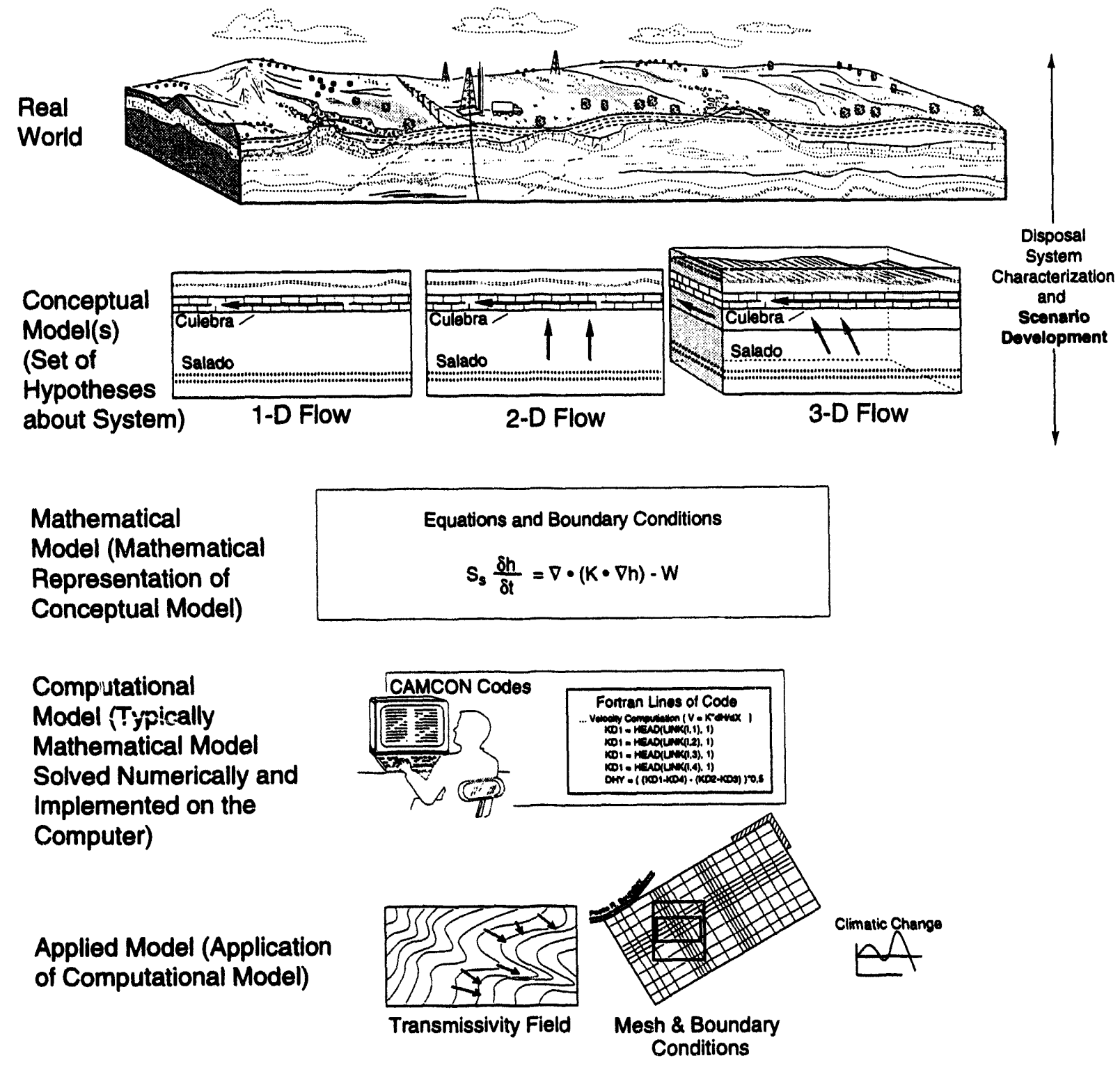

TR1-6342-3908-0

Figure 7-1. Scenario development, in conjunction with system characterization, establishes how the real world will be represented in the applied model. 
the U.S. Nuclear Regulatory Commission. This procedure, or a modification of this procedure, has been used in a joint exercise in scenario development by the Swedish Nuclear Power Inspectorate and the Swedish Nuclear Fuel and Waste Management Company (Andersson et al., 1989), the Canadian Nuclear Fuel Waste Management Program (Stephens and Goodwin, 1989), and Sandia National Laboratories for the Waste Isolation Pilot Plant (Guzowski, 1990; Guzowski and Helton, 1991).

The identification and selection procedure consists of four steps (Figure 7-2):

1. Compiling or adopting a comprehensive list of features, events, and processes that could affect the performance of a disposal system.

2. Classifying the features, events, and processes as an organizational tool and as an aid in addressing completeness arguments.

3. Providing rough estimates of probabilities and consequences of the features, events, and processes.

4. Screening the features, events, and processes to identify those that can be eliminated from consideration in the performance assessment without affecting regulatory compliance.

\subsubsection{Step 1: Identifying the 'Universe' of Features, Events, and Processes}

An initial list of features, events, and processes can be either developed as an original list or adopted (or adapted) from one or more existing sources. The question of whether the list is comprehensive must be addressed regardless of which approach is selected. A major concern to compliance assessment is the question of whether the list is complete.

Cranwell et al. (1990) provides a list of events and processes that was derived from events and processes identified by a panel of experts with diverse professional backgrounds. This panel met in 1976 and again in 1977 under the auspices of the U.S. Nuclear Regulatory Commission (Campbell et al., 1978). The task of the panel was to identify those events and processes that could influence the escape of radionuclides from a generic engineered disposal facility or affect the transport of radionuclides beyond the confines of the engineered disposal facility. The list was intended to be modified on a site-specific or performance-measure basis. Table 7-1 shows an example of a modification of the Cranwell et al. (1990) list.

\subsubsection{Step 2: Classifying Features, Events, and Processes}

Classifying the events and processes by any of a variety of classification schemes can serve as an organizational tool and can aid in determining the completeness of the list. Two classification schemes were used in Cranwell et al. (1990). One scheme was based on the origin of the events and processes (i.e., naturally occurring, human induced, and waste/repository induced), and a second scheme classified each event and process as to the primary effect on the disposal system (i.e., primarily affecting release of radionuclides from the engineered facility, and primarily affecting the transport of radionuclides from the facility toward the accessible environment). Note that other schemes are also possible. The first scheme was used for this performance assessment.

\subsubsection{Step 3: Providing Rough Estimates of Probabilities and Consequences of Features, Events, and Processes}

To identify scenarios that are possible and of interest, it is necessary to estimate the probabilities and consequences of the features, events, and processes. The PA analyst uses his or her own judgment, existing data, and simple modeling to develop the rough estimates. The estimates are then used to screen the features, events, and processes. 


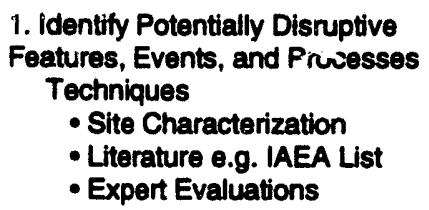

See Table 7-1

2. Estimate Probabilities and

Consequences

Techniques

- Subjective Estimate

- Use Existing Data

- Simple Modeling

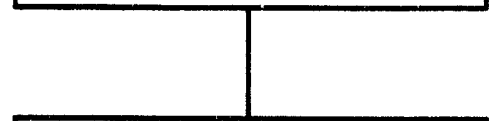

3. Screen Features, Events, and Processes Criteria

- Regulatory Guidance,

(e.g. Only Consider

Exploratory Drilling)

- Low Probability

- Low Consequence

(A)

See Figure 7-3

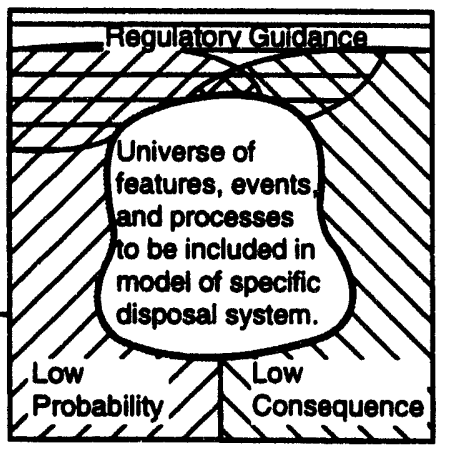

TR1-6342-3553-0

Figure 7-2. Identification and selection of features, events, and processes to model. 
Table 7-1. Potentially Disruptive Events and Processes for a Generic Disposal System (after Guzowski, 1990; and Cranwell et al., 1990)

\author{
Natural Features, Events, and Processes \\ Celestial Bodies Event \\ Meteorite Impact \\ Surficial Events and Processes

\begin{tabular}{|c|c|}
\hline Events & Processes \\
\hline Hurricar & Erosion/Sedimentation \\
\hline Seiches & Glaciation \\
\hline Tsunamis & Pluvial Periods \\
\hline Mass Wasting & Sea-Level Variations \\
\hline Flooding & Regional Subsidence or Uplift \\
\hline
\end{tabular}

Subsurface Events, Processes, and Features

\begin{tabular}{l}
\multicolumn{1}{c}{ Events } \\
\hline Earthquakes \\
Volcanic Activity \\
Magmatic Activity \\
Faulting
\end{tabular}

\begin{tabular}{l} 
Processes \\
\hline Diapirism \\
Formation of Dissolution Cavities \\
Formation of Interconnected Fracture \\
Systems
\end{tabular}

Features

Undetected:

- Fluid Reservoirs

- Fracture Zones
Human-Induced Events and Processes
Inadvertent Intrusion Subsurface Events
Mining
Drilling
Injection Wells
Withdrawal Wells
Explosions

Human-Induced Hydrologic Stresses

Withdrawal Well

Irrigation

Damming of Streams or Rivers

Waste- and Repository-Induced Processes

Subsidence and Caving

Shaft and Borehole Seal Degradation

Thermally Induced Stresses/Fracturing in Host Rock

Excavation-Induced Stress/Fracturing in Host Rock 


\subsubsection{Step 4: Screening Features, Events, and Processes to Establish History Space}

The third step in this procedure screens the events and processes to eliminate those from scenario development that are not applicable to a particular disposal system or do not have the potential of significantly contributing to the integrated radionuclide releases to the accessible environment. Specific screening criteria are to some extent dependent on the original list of events and processes. Three screening criteria were used: regulatory guidance, probability of occurrence, and consequence.

Regulatory Guidance. Guidance in 40 CFR 191 restricts the events and processes that must be included in performance assessments. This guidance limits the assessments to the time period after closure of the disposal facility, limits consideration of human intrusion to inadvertent intrusion, limits the severity of human intrusion at the disposalfacility location, and eliminates the need to consider surface transport of radionuclides and biological pathways to humans.

Probability of Occurrence. Screening by probability of occurrence is based on guidance in Appendix B of 40 CFR 191, which states that “...performance assessments need not consider categories of events or processes that are estimated to have less than one chance in 10,000 of occurring in 10,000 years" (EPA, 1985, p. 38088). The basic philosophy behind this screening criterion is that extremely rare events and processes should not influence the performance measure for a disposal system no matter how severe the specific consequence associated with such events and processes. For addressing 40 CFR 191, events and processes with less than one chance in 10,000 of occurring within the next $10,000 \mathrm{yr}$ are too rare to be of concern.

Physical reasonableness is considered with probability of occurrence. It is based on subjective judgment, which is founded on logical arguments, as to whether conditions can exist within the time period of regulatory concern that will result in the occurrence of a particular event or process of sufficient magnitude to affect disposal system performance. The logical arguments are founded in turn upon available data and information possibly supplemented by calculations.

Consequence. The criterion "consequence" refers to whether an event or process alone or in combination with other events and processes has the potential to affect the performance of the disposal system through the alteration of transport pathways or the creation of new pathways. Simplified conceptual and mathematical models can be used to estimate consequence. Considerable caution must be used to screen based on this criterion. A single event or process may have no consequence by itself on the disposal system but could have a major consequence when combined with one or more other events and processes.

Results of Screening Events and Processes. Appendix F contains information on the specific screening criterion used for each event and process, the disposition of each event and process, and a discussion of the rationale for each disposition based on the screening criterion as applied to the bedded-salt and saturated granite disposal systems. A summary of the screening results for these two locations is provided in Tables 7-2 and 7-3.

For the bedded-salt location, seven features, events, and processes were retained for consideration in scenario development after the screening process (those in bold were evaluated for this performance assessment; see Section 7.5):

1. Exploratory drilling for resources,

2. Mining of resources outside the boundaries of the disposal facility,

3. Drilling of injection wells,

4. Subsidence over the engineered facility,

5. Nuclear criticality,

6. Undetected fluid reservoir,

7. Colloid transport. 
Table 7-2. Summary of Screened Events and Processes-Bedded-Salt Location

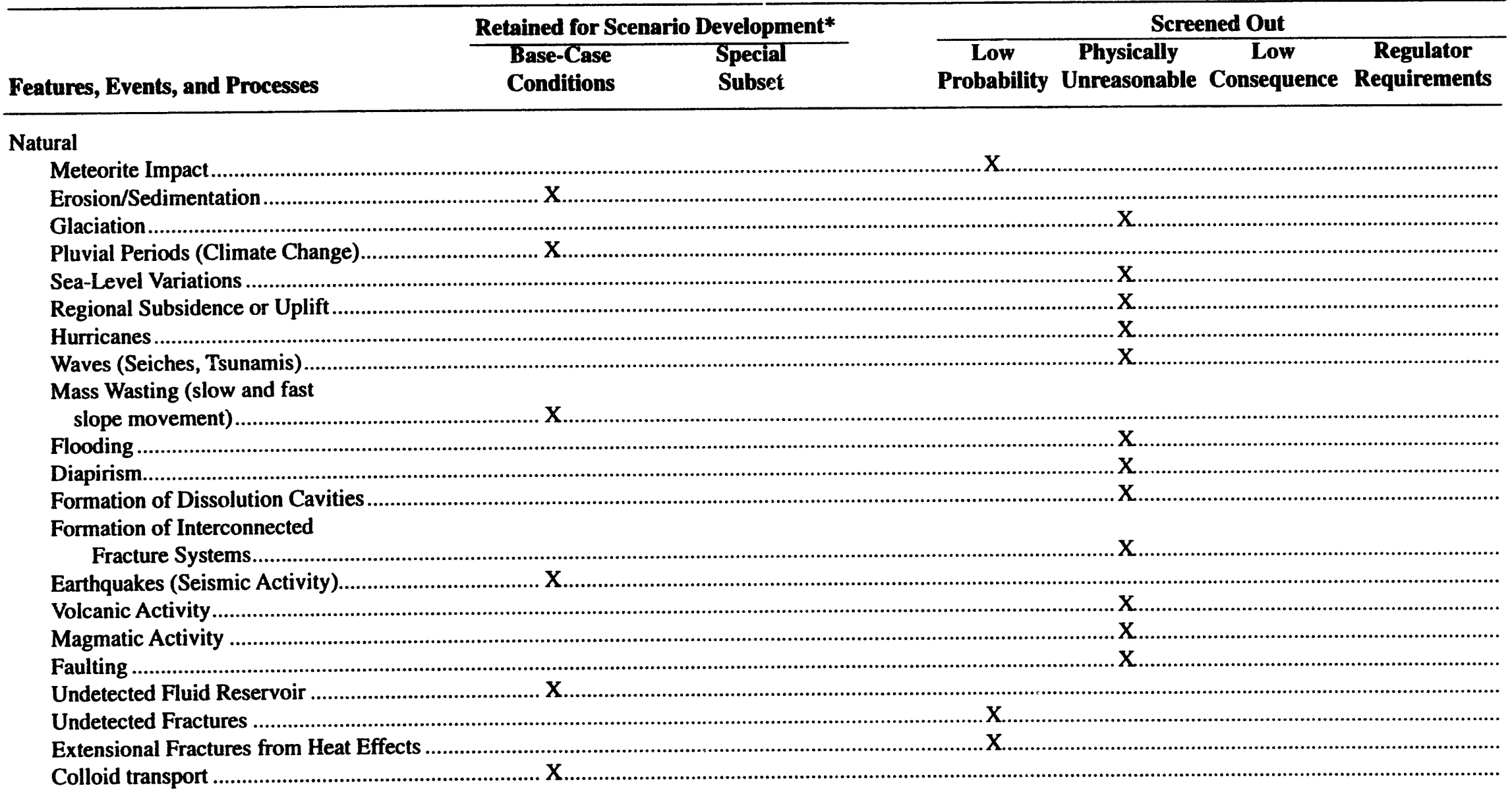

- The retained features, events, and processes have been categorized as "base case conditions" or "special subset" in preparation for combining them into summary scenarios; see Section 7.3. 
Table 7-2. Summary of Screened Events and Processes-Bedded-Salt Location (Concluded)

\begin{tabular}{|c|c|c|c|}
\hline \multirow[b]{2}{*}{ Features, Events, and Processes } & Retained for Scenario Development* & \multicolumn{2}{|l|}{ Screened Out } \\
\hline & $\begin{array}{c}\text { Base-Case } \\
\text { Conditions }\end{array}$ & $\begin{array}{ccc}\text { Low } & \text { Physically } & \text { Low } \\
\text { Probability } & \text { Unreasonable Consequence }\end{array}$ & $\begin{array}{c}\text { Regulator } \\
\text { Requirements }\end{array}$ \\
\hline \multicolumn{4}{|l|}{ Human-Induced } \\
\hline \multicolumn{4}{|l|}{ Explosions } \\
\hline \multicolumn{4}{|l|}{ At Waste-Panels Location.. } \\
\hline \multicolumn{4}{|l|}{ Near Waste-Panels Location } \\
\hline \multicolumn{4}{|c|}{ At Surface/Warfare..................... } \\
\hline \multirow{2}{*}{\multicolumn{4}{|c|}{ 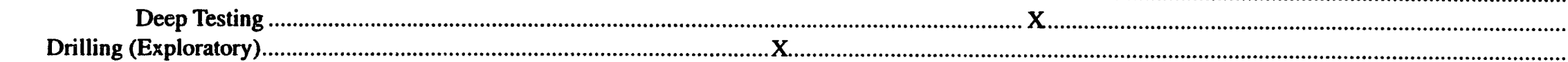 }} \\
\hline & & & \\
\hline \multicolumn{4}{|l|}{ Mining } \\
\hline \multicolumn{4}{|l|}{ At Waste-Panels Location.. } \\
\hline \multirow{2}{*}{\multicolumn{4}{|c|}{ 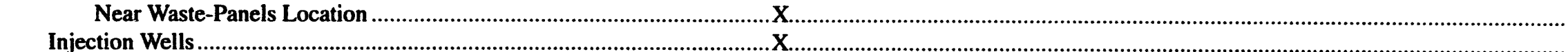 }} \\
\hline & & & \\
\hline \multicolumn{4}{|c|}{$\begin{array}{l}\text { Injection Wells } \\
\text { Withdrawal Wells ... }\end{array}$} \\
\hline \multicolumn{4}{|c|}{ Withdrawal Wells s. } \\
\hline \multicolumn{4}{|c|}{ 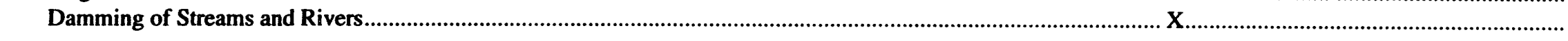 } \\
\hline \multicolumn{4}{|l|}{ Repository- and Waste-Induced } \\
\hline \multicolumn{4}{|c|}{ Subsidence and Caving......................... } \\
\hline \multicolumn{4}{|c|}{ Shaft \& Borehole Seal Degradation .................................. } \\
\hline \multicolumn{4}{|c|}{ 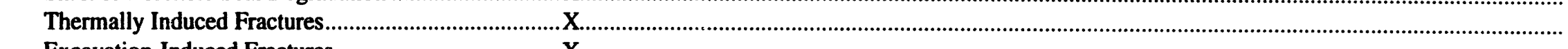 } \\
\hline xcavation-Induced Fracture & . X & & \\
\hline Nuclear Criticality ................. & $\mathrm{X}$ & & \\
\hline
\end{tabular}

- The retained features, events, and processes have been categorized as "base case conditions" or "special subset" in preparation for combining them into summary scenarios; see Section 7.3. 


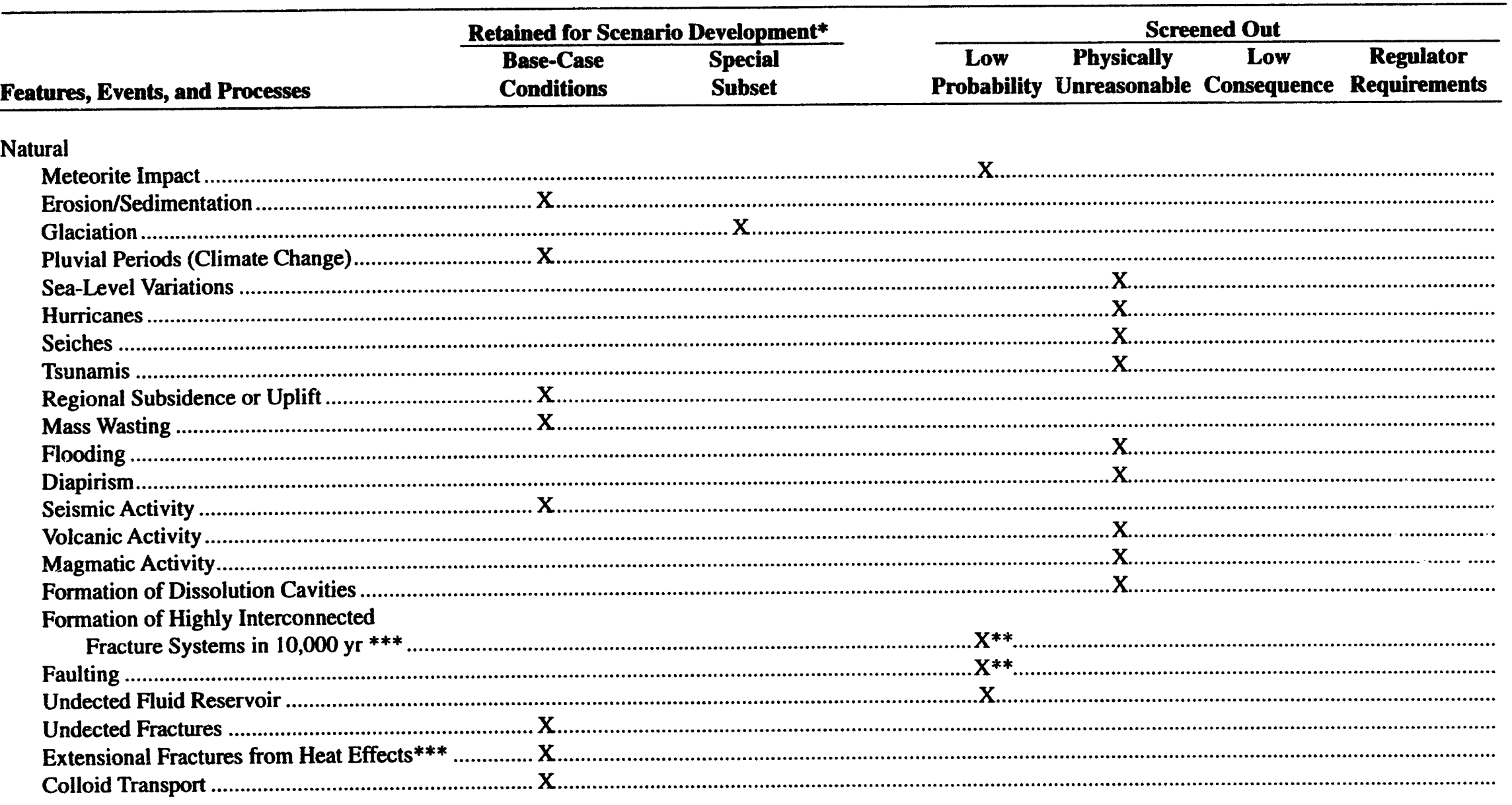

- The retained features, events, and processes have been categorized as "base case conditions" or "special subset" in preparation for combining them into summary scenarios: see Section 7.3.

* We have assumed that the region for the repository is tectonically stable. Because the consequences of this event are similar to the "undetected fractures" and "formation of extensional fractures from heat effects," another option would be to retain this event and then combine it with the other two events into a summary scenario.

... In the Complex PA, this feature was modeled by placing waste parcels in the fractures; in the Simple PA, the fracture was assumed to have been detected since the repository was assumed to end at this point but that sealing of fractures eventually failed. 
Table 7-3. Summary of Screened Events and Processes-Saturated Granite Location (Concluded)

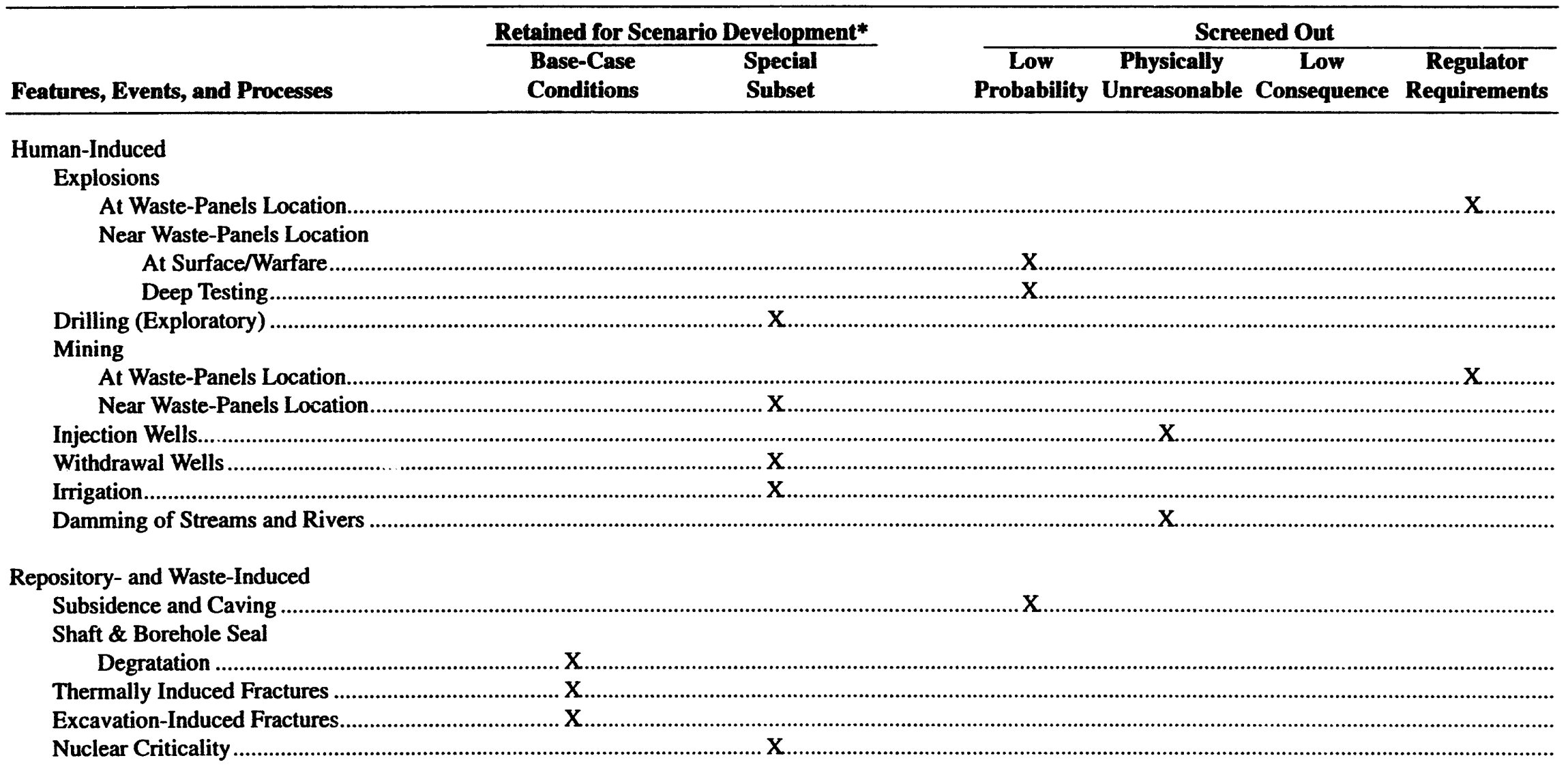

- The retained features, events, and processes have been categorized as "base case conditions" or "special subser" in preparation for combining them into summary scenarios; see Section 7.3 . 
For the saturated granite location, nine events and processes were retained:

1. Glaciation,

2. Exploratory drilling for resources,

3. Mining of resources outside the boundaries of the disposal system,

4. Drilling of withdrawal wells,

5. Irrigation,

6. Nuclear criticality,

7. Detected fractures,

8. Extensional fractures from effects of repository,

9. Colloid transport.

Additional screening based on consequence and modeling directed at determining physical reasonableness may reduce the number of features, events, and processes for each location. However, additional site characteristics can increase their number.

\subsection{Optionally Grouping Features, Events, and Processes}

When the features, events, and processes to be modeled are identified, the parameter space can be grouped into scenarios. The scenarios can be classified in various ways, e.g., by type of disruptive force (natural, human, or repository). The advantage of scenario groupings-which are called summary scenarios (see Section 3.3)-is that attention can be focused in the analysis on certain events and processes. This grouping is feasible provided the probabilities of residing in certain regions of the sample space are easily calculated. At this stage of scenario development, it is assumed that the behavior within the summary scenarios is similar enough that a representative (or a key subset) single history can be used to characterize the whole subset.

The process of grouping scenarios consists of two steps (Figure 7-3):

1. Combining features, events, and processes in summary scenarios (Section 3.3.2).

2. Grouping summary scenarios having identical consequences.

\subsubsection{Step 1: Combining Features, Events, and Processes into Summary Scenarios}

A major assumption of this step of combining features, events, and processes into summary scenarios is that the combinations of the remaining events and processes define all possible future states of the disposal system that are of regulatory concern. (In some cases, regulatory guidance in Appendix B of 40 CFR 191 (see Chapter 2 and Appendix B of this report) was used to eliminate some events and processes from consideration, e.g., mining the buried spent fuel so that it could be reprocessed.) Thus, Step 1 could also be titled "Dividing the History Space into Subsets."

Logic diagrams assist in developing the set of summary scenarios (Section 3.3.2), i.e., the set of all possible combinations of events and processes. Figure 7-4 is an example of a logic diagram for events and processes for hypothetical transport and release. At each junction within the diagram, a yes/no decision is made as to whether the next event or process across the top of the diagram occurs in combination with the previous event(s) and/or process(es) along the pathway through the logic diagram. Each pathway results in a summary scenario that contains events and processes that occur and those that do not occur, although the non-occurring features, events, and processes generally are not listed to simplify notation. ${ }^{\ddagger}$ The complete diagram develops all possible col binations of the events and processes located across the top of the diagram with the total number of scenarios equal to $2^{n}$, where $n$ equals the number of events and processes.

\# The summary scenario, in which no specific features, events, or processes occur, is often called the base case, especially when the probability of occurrence is significant. 


\section{Scenario Development}

2

3

4

6

8

9

10

11

12

13

14

17

18

19

20

21

22

23

24

25

26

27

28

29

30

31

32

33

34

35

36

37

38

39

40

41

42

43

44

45

46

47

48

49

50

51

52

53

54

55

56

57

58

59

60

61

62

63

64

65

66

See Figure 7-2

(A)

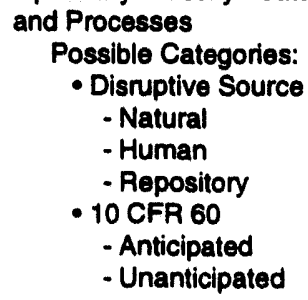
and Processes

Possible Categories:

- Disruptive Source

- Natural

- Human

- Repository

- 10 CFR 60

- Anticipated

- Unanticipated

Optionally Classify Features, Events,

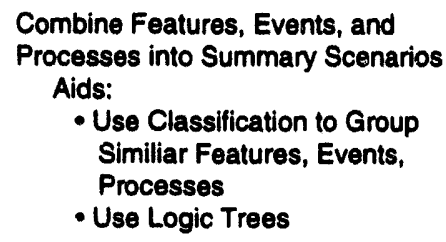

Combine Features, Events, and

Processes into Summary Scenarios Aids:

- Use Classification to Group Similiar Features, Events,

Processes

- Use Logic Trees

When possible, combine those summary scenarios that logical analyses show as having identical consequences.

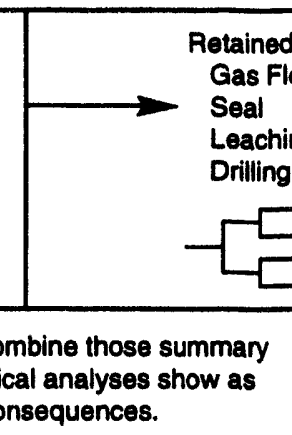
(

Base Case
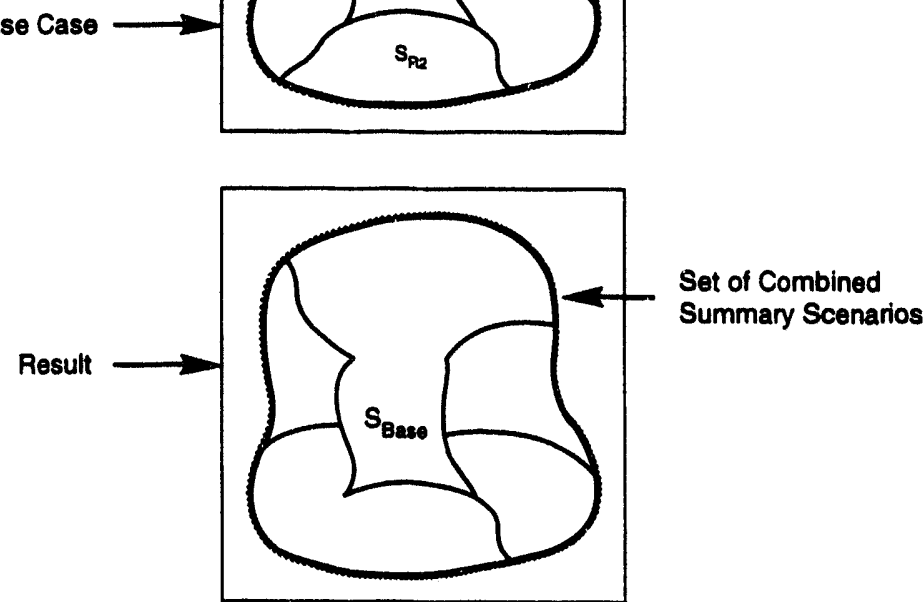

TR1-6342-3552-0

Figure 7-3. Grouping of features, events, and processes that survive initial screening into summary scenarios. 


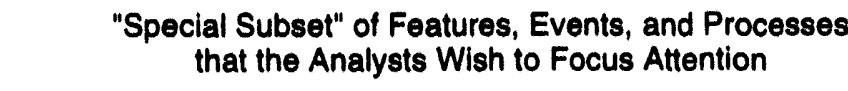
that the Analysts Wish to Focus Attention
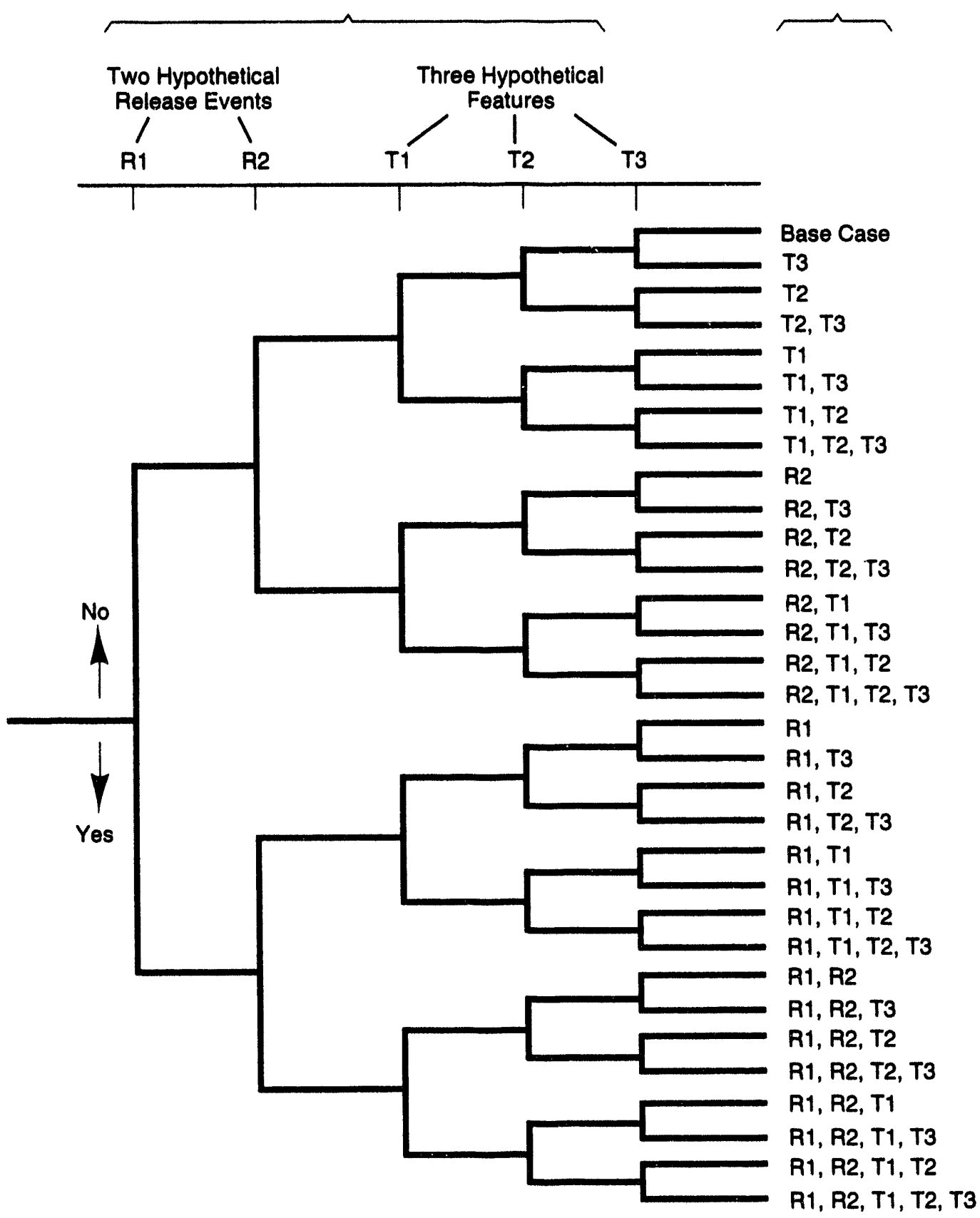

TRI-6342-222-6

Distinct "Future" (for 10,000 yr) Examined by Consequence Modeling
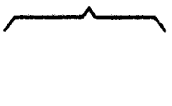
Because of the assumption stated above that these combinations define all possible future states of the disposal system that are of regulatory concern, the sum of the probabilities of the summary scenarios developed in Figure 7-2 must be 1 for this conditional probability. Scenario probabilities must include both the probabilities of occurrence and nonoccurrence for the appropriate events and processes (Figure 7-3). The inclusion of non-occurring events and processes in each scenario assures that the sum of the scenario probabilities is 1.

\subsubsection{Step 2: Grouping Summary Scenarios Having Similar Consequences}

In general, separate calculations of performance measures (CCDFs, etc.) must be made for each summary scenario (this is always the case if the method of conditional simulation described in Section 3.3.3 is adopted). To minimize the number of calculations, it is therefore desirable to reduce the number of distinct summary scenarios to the absolute minimum (Tierney, 1991, p. 3-30). A reduction of this kind may be possible by means of a careful, logical examination of the nature of the agents (features, events, and processes) that occur in each summary scenario and the way those agents can interact to produce consequences. In many cases, a logical, non-numerical, analysis will show that one or more of the summary scenarios will have consequences no different from the base-case summary scenario, or that two or more summary scenarios will have identical consequences; such an analysis is particularly easy if the base case has zero consequences. Two summary scenarios having identical consequences may be combined in a single scenario; the probability of this single scenario (see Section 3.3.3) is the sum of the probabilities attached to each of the two scenarios in the combination.

\subsection{Construction of CCDF}

The ultimate goal of the PA is to produce a performance measure for the real system using a model of that system and then compare the measure against a standard. Equally important, the statistical properties of the performance measure, which are captured in the complementary cumulative distribution function (CCDF), are evaluated. In the following sections, the merits of the Monte Carlo method are described and a general overview of constructing a CCDF is presented. The construction depends on whether the sample space of screened futures of the disposal system has been divided into scenarios. In order to complete the discussion about identification and selection of features, events, and processes (Section 7.2), the subtle differences between these alternatives are described.

\subsubsection{Advantages of Monte Carlo Method}

As discussed in Chapter 3 (Performance Assessment Methodology Overview), the Monte Carlo method is a universally applicable method for evaluating the statistical properties of a performance measure. The Monte Carlo method was selected for six reasons (Helton et al., 1991): (1) it can be used when several linked codes are needed to model the system by propagating the same parameter values and preceding code results through the next linked code and so on; (2) it produces a mapping of input to output that can be studied by a variety of standard statistical techniques (e.g., scatterplots or regression analysis); (3) it does not require an intermediate model (e.g., regression model), which may obscure discontinuities and transitions between regimes of behavior; (4) it does not require modifying deterministic codes or developing a code with a statistical formulation of the conceptual model; (5) it can include parameters with empirical or subjective distributions having wide ranges and discontinuities; and (6) it allows model parameters to be correlated.

To reduce the number of samples required from the sample space and still adequately cover the sample space, this performance assessment uses Latin hypercube sampling (LHS) (McKay et al., 1979). Normally, the rule of thumb is that the number of samples required, $n K$, is $4 / 3 n V$, where $n V$ is the number of uncertain parameters that are to be sampled. (As discussed in Chapter 9, "Probability Modeling," only a small number of parameters were varied.) Most model parameters were held constant. With LHS, it is even possible to determine the effects of different distributions for the parameters on the estimated distribution of the results without rerunning the consequence models (Iman and Conover, 1980). 


\subsubsection{Construction of CCDF without Scenarios (without Importance Sampling)}

If the sample space of possible futures of the disposal system was not divided into summary scenarios or computational scenarios, the CCDF is calculated as follows (Figure 7-5):

1. $n K$ Latin Hypercube samples are drawn from the $n P$-dimensional parameter space, where $n P$ is the total number of model parameters in the model of the disposal system.

2. Radionuclide releases over time, $q_{i}$, are evaluated using each of the $n K$ sample sets of uncertain parameters; all other parameters are held constant in the modeling system. Results of each of the $\mathrm{nK}$ evaluations are recorded.

3. The performance measure, $R_{k}$ (e.g., the EPA summed normalized release), is evaluated for each of the $\mathrm{k}=1$, . $\ldots n K$ releases

$$
R_{k}=\sum_{i=1}^{n R} \frac{Q_{i}}{f_{w} L_{i}}, \quad k=1,2, \ldots, n K
$$

where

$$
\begin{aligned}
f_{w} & =\text { the waste unit factor for scaling EPA release limits } \\
L_{i} & =\text { the EPA release limit for } i \text { th radionuclide } \\
n R & =\text { number of radionuclides monitored along the release pathway by } 40 \text { CFR } 191 \\
n K & =\text { number of samples } \\
Q_{i} & =\text { cumulative release for } i \text { th radionuclide } \\
& =\int q_{i} d t \\
q_{i} & =\text { release rate at time } t \text { for radionuclide } i \text { calculated for the consequence models. }
\end{aligned}
$$

4. The performance measure, $R_{k}$, are ranked.

5. The probability of the performance measure exceeding a specified value, $P\left(R_{k}>r\right)$ is estimated from the frequency:

$$
P\left(R_{k}>r\right)=n G / n K
$$

where

$n G=$ number of simulations with $R_{k}$ exceeding $r$

$n K=$ number of samples

$r \quad=$ specified value (on graph abscissa).

6. The value of the performance measure, $R_{k}$ is then paired with the probability $P\left(R_{k}>r\right)$ to produce a mean CCDF.

\subsubsection{Construction of CCDF with Scenarios}

When the sample space is discretized, results may be presented in several ways: (1) a CCDF can be calculated for each scenario, conditional on the scenario occurring; (2) a CCDF can be calculated for all the scenarios using the sample space set for each scenario; or (3) the mean CCDF can be calculated from either 1 or 2. This mean CCDF is approximately equal to the mean CCDF calculated without discretizing the sample space into scenarios (provided the scenario discretization is not so coarse that it ignores significant variations, and the approximations in calculating the 


\section{Calculate Mean CCDF}

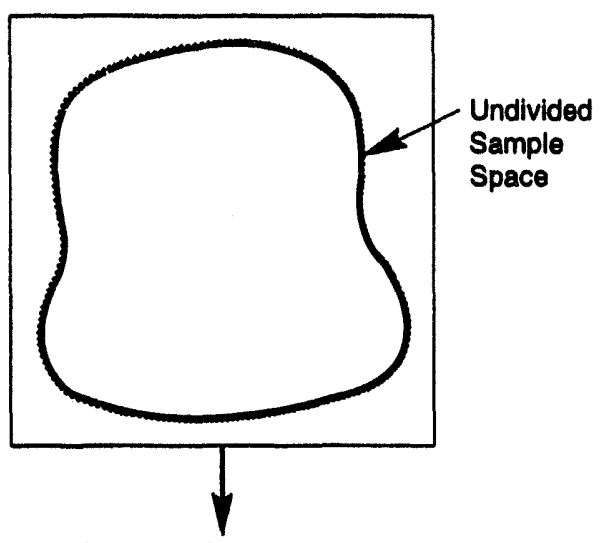

Sample All Parameters

(e.g. Time of Intrusion Drilling Position and Number)

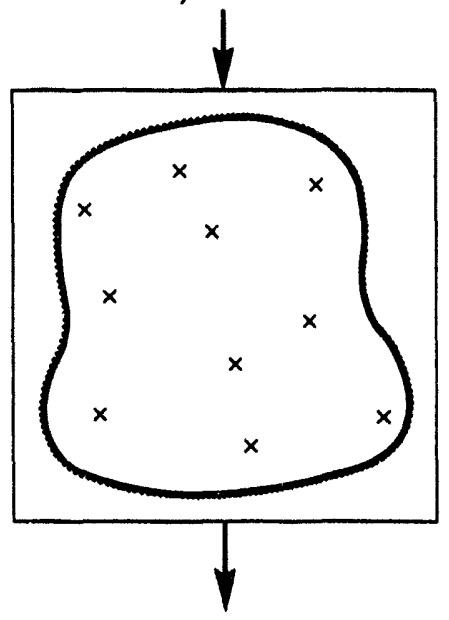

Order All Performance Metric Values, R, Evaluated from the

Consequences, C

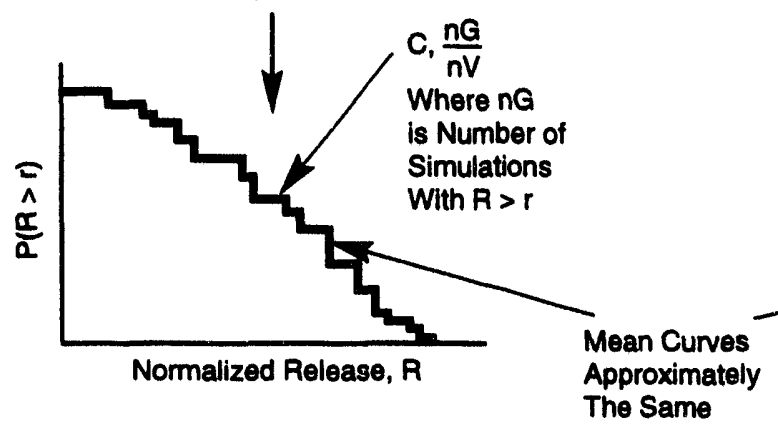

2. Calculate Family of CCDFs

(Since Several Parameter Held Constant to Define Scenarios

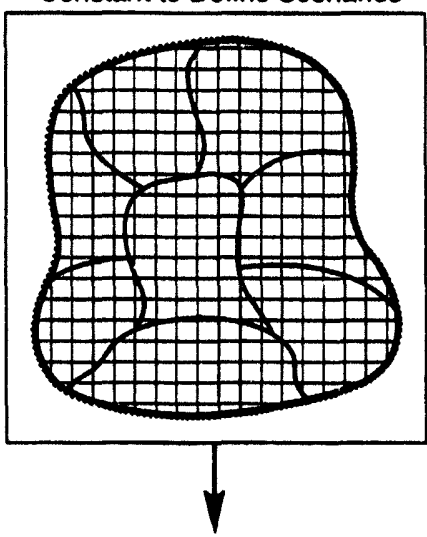

Sample Parameters that were Not Used to Define Scenarios

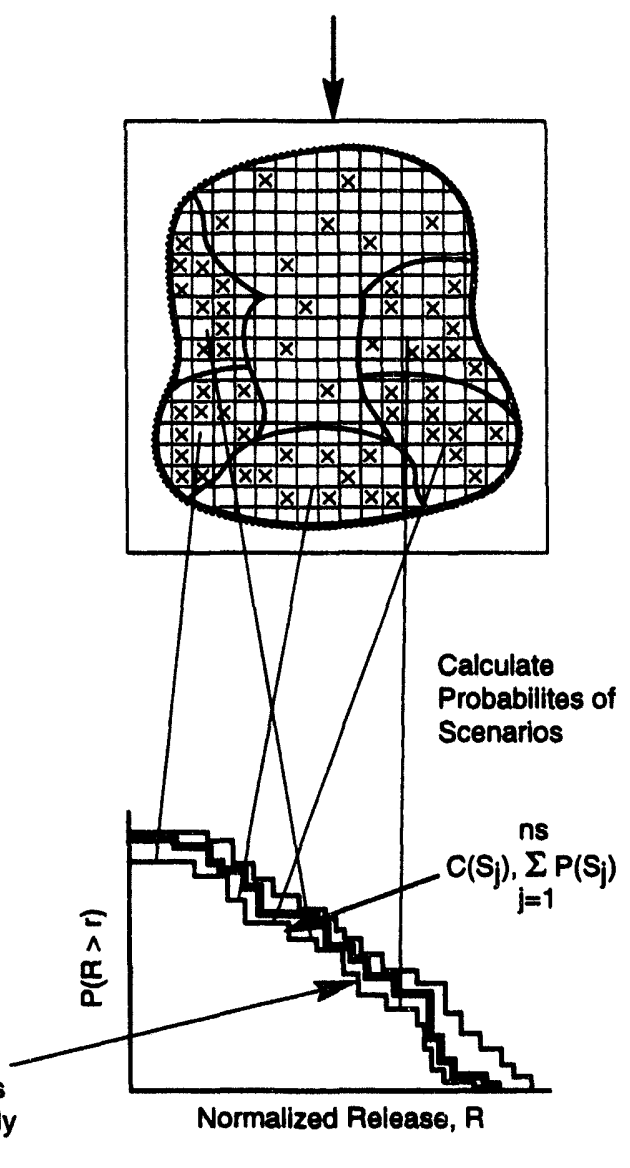

TRI-6342-3554-0

Figure 7-5. Method of calculating CCDF depends on whether sample space is discretized. 
individual scenario probabilities $P\left(S_{j}\right)$, are acceptable). The following sections describe the steps necessary for presenting the results by the three methods.

$C C D F$ Conditional on Scenario $S_{j}$ Occurring. The method of calculating a CCDF conditional on the scenario occurring is identical to that described for CCDF construction without scenarios except that only those performance metric values, $R\left(S_{j}\right)$, from the scenario of interest, $S_{j}$, are used.

CCDF of All Scenarios Using One Sample Set. To calculate one CCDF for a sample set over all scenarios, only subtle differences exist in a few of the steps:

1. $n K$ Latin hypercube sample sets are drawn from the $n P$-dimensional parameter space, where $n P$ is the total number of model parameters in the model of the disposal system.

2. Radionuclide releases over time, $q_{i}$, are evaluated using one sample set of varied parameters with all other parameters held constant in the modeling system. The sample sets of varied parameters are slightly different from those without scenario discretization because those parameters that define the scenario are now held constant. This step is repeated for each sample set.

3. The performance measure, $R$, (i.e., the EPA summed normalized release) is evaluated:

$$
R_{k}=\sum_{i=1}^{n R} \frac{Q_{i}}{f_{w} L_{i}}, \quad k=1,2, \ldots, n K
$$

where

$f_{w}=$ the waste unit factor for scaling EPA release limits

$L_{i}=$ the EPA release limit for $i$ th radionuclide

$n R=$ number of radionuclides monitored along the release pathway by 40 CFR 191

$n K=$ number of samples for set of scenarios, $S_{\mathrm{j}}$

$Q_{i}=$ cumulative release for $i$ th radionuclide

$=\int q_{i} d t$

$q_{i}=$ release rate at time $t$ for radionuclide $i$ calculated for the consequence models.

4. The performance measures are ranked such that $R\left(S_{j}\right) \leq R\left(S_{j+1}\right)$ for $j=1, \ldots, n S-1$.

5. The probability of a scenario, $P\left(S_{j}\right)$, is evaluated analytically or numerically from the probability model of the scenario.

6. The value of the performance measure, $R$, for one sample set is then paired with the cumulative probability

$$
\sum_{m=j+1}^{n S} P(S m)
$$

This is repeated for each scenario $S_{j}, j=1, \ldots, n S$, to produce a CCDF.

7. Step 6 is repeated for each sample set to produce a family of $n K$ CCDFs. The position of the outliers of the family (e.g., those CCDFs beyond the 20th and 80th quantiles) can vary substantially with the type of discretization of the sample space. The position of the mean CCDF as described below, however, is likely quite stable with appropriate changes in scenario discretization.

Mean CCDF. The mean CCDF can be estimated from either the family of CCDFs produced above or the CCDFs conditional on the scenario. The mean CCDF from the family of CCDFs is the arithmetic average of the probabilities of the family of CCDFs at specified values of $R$. 
The mean CCDF from the CCDFs conditional on the scenario is calculated as follows:

1. Produce a family of $n S$ CCDFs by evaluating the scaled CCDF, i.e., $P(R>r)=P\left(R>r \mid S_{j}\right) \bullet P\left(S_{j}\right)$.

2. Provided parameters in the probability model used to evaluate $P\left(S_{j}\right)$ were not varied, the mean CCDF is the arithmetic average of this family of CCDFs.

3. If parameters in the probability model were varied, then $P\left(S_{j} x\right)$ varies with each sample, $x_{k}$, so that each conditional CCDF results in $n K$ scaled CCDFs, i.e., $P_{k}(R>r)=P\left(R>n S_{j}\right) \bullet P_{k}\left(S_{j}, x_{k}\right)$. This family of $n K \bullet n S$ CCDFs can now be averaged as before to calculate the mean CCDF.

\subsection{Conceptual Model}

The conceptual model of the repository and the emplaced waste parcels is created from information gathered during (1) disposal system characterization, and (2) selection of features, events and processes. For this performance assessment, one conceptual model for each repository was constructed.

\subsubsection{Levels of Process Simplification}

In every analysis, physical phenomena (e.g., processes in the repository and the host rock) are simplified. Although the level of simplification that is appropriate cannot always be know a priori, evaluating the correct level is an important task. In this analysis, two levels of simplification were used so that results could be compared. The first level is referred to as the "Simple PA" and the second as the "Complex PA." However, the terms simple and complex are relative only to each other; compared to many risk assessments, the Simple PA is quite detailed."

In the "Simple PA," phenomena were described using ordinary differential equations (lumped parameter model). In the "Complex PA," phenomena were described using partial differential equations, which were then evaluated numerically on a coarse grid. Several codes were used to simulate the physical components of the disposal system in both levels of simplification.

\subsubsection{Scenario Selected for Study in Calculations}

As a result of the scenario selection procedure, one basic scenario was selected for investigation in the PA calculations. The criterion used to select the basic scenario was the potential for large consequences; use of this criterion ensures that differences between the waste forms will be accentuated and thus provides some basis for the comparison of waste-treatment options. However, any comparisons with the EPA probabilistic release limits must be qualified by the fact that only one subset of the sample space of all possible "futures" of the model disposal system (see Section 3.3.2) is being explored; the CCDFs are primarily intended to provide a relative ranking of the several wasteform options. The CCDFs developed on this part of the sample space are not suitable for comparison with the EPA standard unless the additional assumption is made that the consequences from other scenarios are similar or at least bound by the consequences of this one scenario. Based on the scoping calculations (Appendix C), this latter assumption was made. ${ }^{\dagger \dagger}$

- In this report, a "simple" level of modeling means a consequence model that can be quickly set up and is geared toward fast run times.

\# Before the effect of the low permeability of the crushed-salt backfill was known, the scoping calculations for the salt disposal system (Appendix C) evaluated the consequences of two nearby intrusions into the same panel within the same 1000-yr time interval, where at least one borehole intersected the brine reservoir. The purpose was to examine a scenario that allowed for increased circulation of brine to carry away radionuclides. As suggested in the scoping calculations (see Appendix C) and confirmed in the Complex PA (see Chapter 12 and Section 16.9), the low permeability of the rooms and drifts prevented increased circulation. Thus the consequences calculated in the Complex PA were assumed similar to those for two singie intrusions. For different reasons, two intrusion boreholes were also not evaluated for the CCDF of the granite disposal system. In the granite disposal system, extensive circulation usually occurred with one intrusion borehole because of the shaft and secondary fractures. Thus it was assumed that an additional intrusion borehole would only increase direct releases through cuttings. 
Summary Scenario for Salt Repository. For the salt repository, the summary scenario can be characterized as follows: One borehole is drilled into the repository after 1000 years; this event is followed by faulty borehole plugging or plug degradation, which leads to flow from a hypothetical brine reservoir into the waste repository. A similar summary scenario has been found to produce the largest consequences for the WIPP (Helton et al., 1992; WIPP PA Department, 1993).

Summary Scenario for Granite Repository. For the granite repository, the summary scenario can be characterized as follows: One borehole is drilled into the repository after 1000 years; this event is followed by faulty borehole plugging or plug degradation, which leads to flow from a hypothetical fracture channel (located above or below the repository) into the waste drifts.

Discretization of Summary Scenarios. The summary scenario for each repository was further discretized into computational scenarios using the parameter, time of intrusion. Six intervals were defined, with the time of intrusion assumed to occur in the middle of the interval:

$$
\begin{array}{cccccc}
125 & 175 & 350 & 1000 & 3000 & 7250 \\
{[100-150]} & {[150-200]} & {[200-500]} & {[500-1500]} & {[1500-4500]} & {[4500-10,000]}
\end{array}
$$

The probability of having an intrusion at one of these time intervals is evaluated by the probability model based on the Poisson distribution (Chapter 9). Except for the cuttings/cavings calculations (Chapter 10), most comparisons of the consequence results are made at $1000 \mathrm{yr}$. At this time, domination of total activity by short-lived radionuclides is waning, while the activity of long-lived daughter radionuclides is beginning to increase.

\subsubsection{Conceptual Model for Disposal System in Salt}

The significant event was an exploratory drill hole through the repository that was subsequently filled with a sand-like material (Figure 7-6 and Section 7.5.2). Also shown on Figure 7-6 are the computational models for the salt repository (described in more detail in Chapters 12 and 14).

The processes are (1) drill cuttings, and (2) gas generation and contaminant source term. The conceptual submodels and corresponding computational submodels are described in Chapters 10 and 11, respectively.

The essential geologic features retained for modeling a human intrusion event into a salt repository are (1) thick formation of sait, (2) brine aquifer above the salt formation, (3) possibility of brine reservoir below the salt formation, and (4) anhydrite marker bed directly above the repository (Chapter 5). Details within the repository (especially those in the horizontal plane) are not included here since different conceptualizations within the repository are required to accommodate the further simplification necessary to move to two dimensions for the Complex PA and one dimension for the Simple PA. The conceptual differences can be important when comparing results between the two efforts (see Table 7-4 in Section 7.6.2).

\subsubsection{Conceptual Model for Disposal System in Granite}

The essential geologic features retained for modeling a human intrusion event into a granite repository are (1) thick, intact pluton of granite, (2) fracture zone above the intact granite, (3) fracture zone below the intact granite, and (4) secondary fracture channel connecting the two fracture zones (from the Canadian Underground Research Laboratory and described in Chapter 5). The permeability of the connecting secondary fractures was estimated from the Swedish Stripa mine (Neretnieks, 1986). Again, details within the repository (especially those in the horizontal plane) are not included here since different conceptualizations within the repository are required to accommodate the further simplification necessary to move to two dimensions for the Complex PA and one dimension for the Simple PA. 


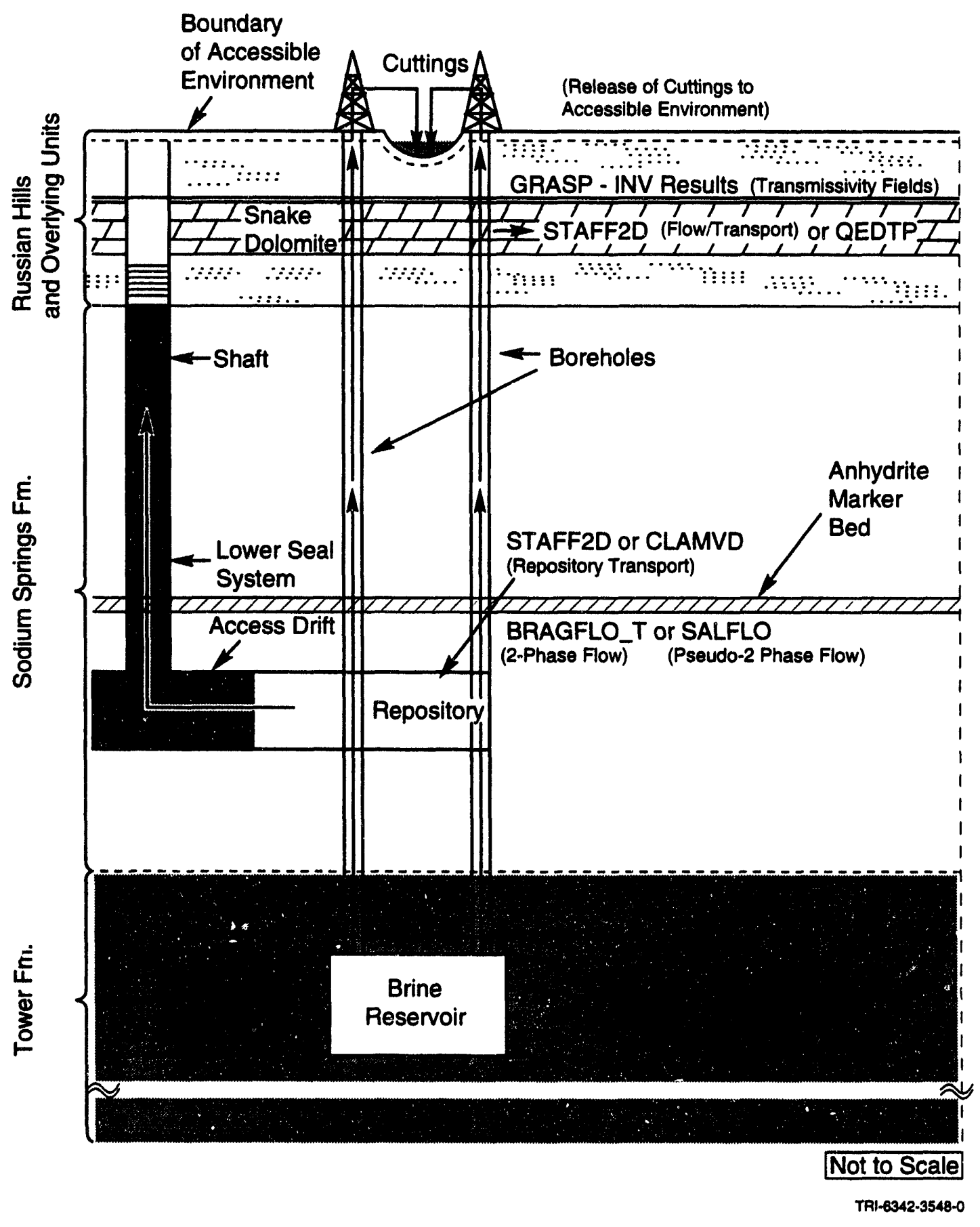

Figure 7-6. General conceptual model for salt disposal system. Computational models used in the Complex and Simple PA calculations are listed. 
The significant event was an exploratory drill hole through the repository that was subsequently filled with a sand-like material (Figure 7-7 and Section 7.5.2). Also shown on Figure 7-7 are the computational models for the granite repository (described in more detail in Chapters 13 and 15).

Like the conceptual model for the salt repository, the processes are gas generation, contaminant source term, and drill cuttings. The conceptual submodels and corresponding computational submodels are described in Chapters 10 and 11.

\subsection{Computational Submodels and Calculation Design}

Following site characterization and the choice of features, events, and processes, ${ }^{\ddagger \ddagger}$ design of a PA calculation can begin. The part of calculational design discussed in this section is the modeling system that was selected and the number of cases that were run. Other design choices are discussed elsewhere: the number of parameters to be varied is discussed in Chapter 9, "Probability Modeling"; simplifying assumptions made for some features are discussed along with geologic and engineered barriers in Chapters 4 through 6; and simplifications of processes are discussed along with the consequence models (Chapters 10 through 15).

\subsubsection{Modeling System Assembled}

Numerous categories of software (e.g., consequence models) are necessary for a performance assessment. These categories basically mirror the fundamental steps of a performance. The generic categories are modeling setup, consequence modeling, probability modeling, compliance assessment (CCDF construction), and sensitivity analysis. For this PA, the consequence modeling category is divided into repository modeling and groundwater flow and transport modeling, with probability modeling and compliance assessment grouped together. Each of these categories usually has a few main modeling codes. For this performance assessment, with two levels of simplification, two sets of main modeling codes are used in some of the software categories. Figure 7-8 depicts assembly of the main modeling codes of the Complex PA modeling system; Figure 7-9 depicts the main modeling codes of the Simple PA modeling system. These figures show the linkage of codes, while the previous figures of the conceptual model showed the disposal system component that each code was modeling. The linkage of these software components into a modeling system is through CAMCON (Rechard, Ed., 1992; Rechard et al., 1993). Not shown in Figures 7-6 and 7-7 are several codes that support the use of the main modeling codes, but they are included in the detailed figures of the modeling system in Appendix G. Appendix $\mathrm{G}$ also gives the version numbers used and a description of the function of each code. Following is a brief description of the software categories of the modeling system shown in Figures 7-6 and 7-7.

Modeling Setup. The main function of the modeling setup is parameter selection. The processes for parameter selections are the following:

1. Select computational model parameters $\left(x=x_{1}, \ldots, x_{n V}\right)$ consistent with manner of use in models.

2. Request that the data base manager place the parameters in secondary data base file (parameter values extracted with MATSET or PRELHS.

3. Select subset of parameters $\left(x=x_{1}, \ldots, x_{n V}\right)$ to vary (parameter uncertainty).

4. Sample parameters with Latin hypercube sampling (LHS).

Repository Consequence Modeling. The repository consequence modeling consists of three main groups of software: drill cuttings, two-phase flow, and source contamination. The repository model develops a source term for transport calculations by incorporating the complex processes in the waste parcel, disposal room, drifts, shafts, and backfill. Specifically, the repository module calculates

\$ Recall that the PA process is iterative; at any point in the PA process, the site characterization may be preliminary and incomplete and the choices of features, events, and processes may accordingly be tentative. 


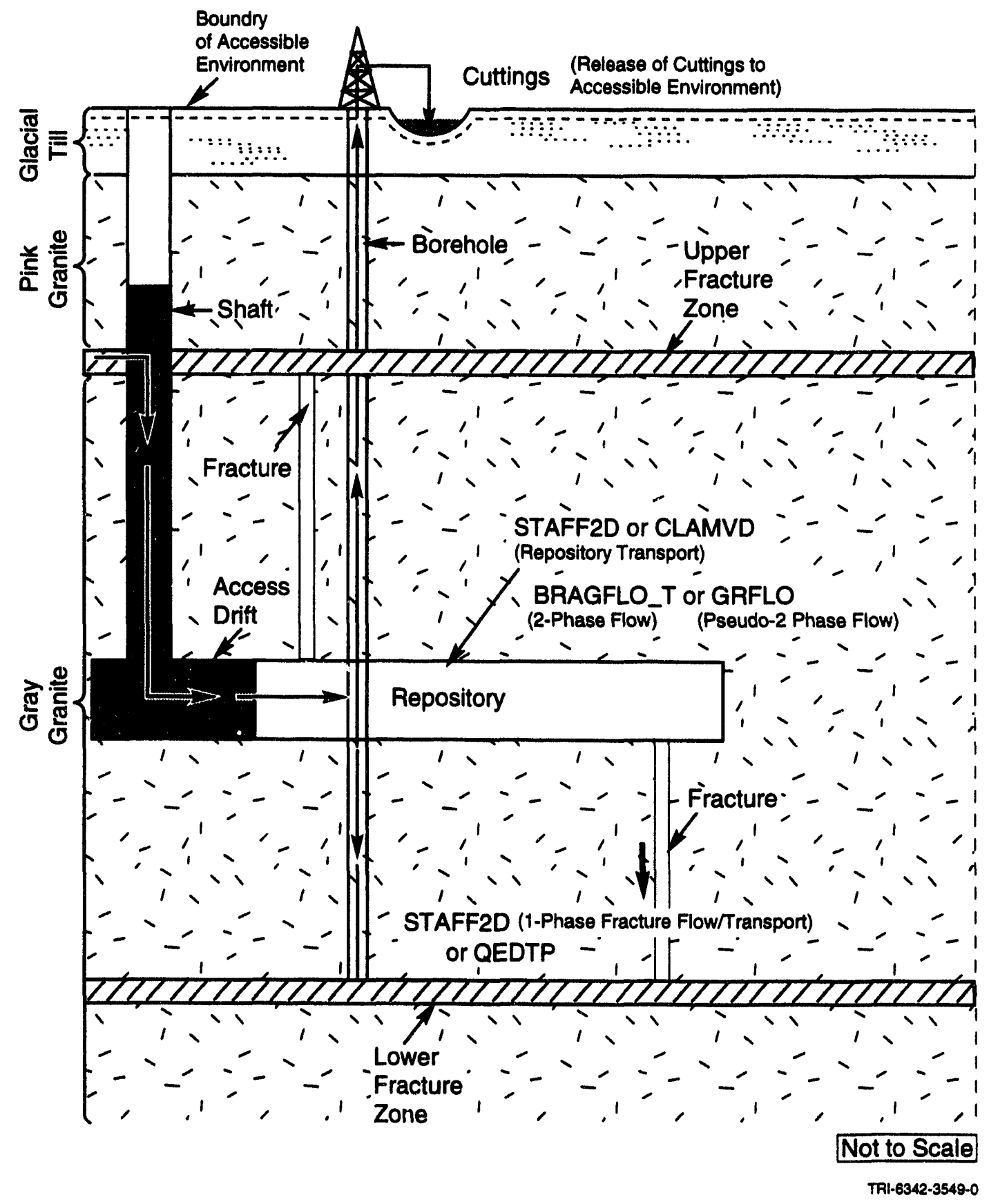

Figure 7-7. General conceptual model for granite disposal system. Computational models used in the Complex and Simple PA calculations are listed. 


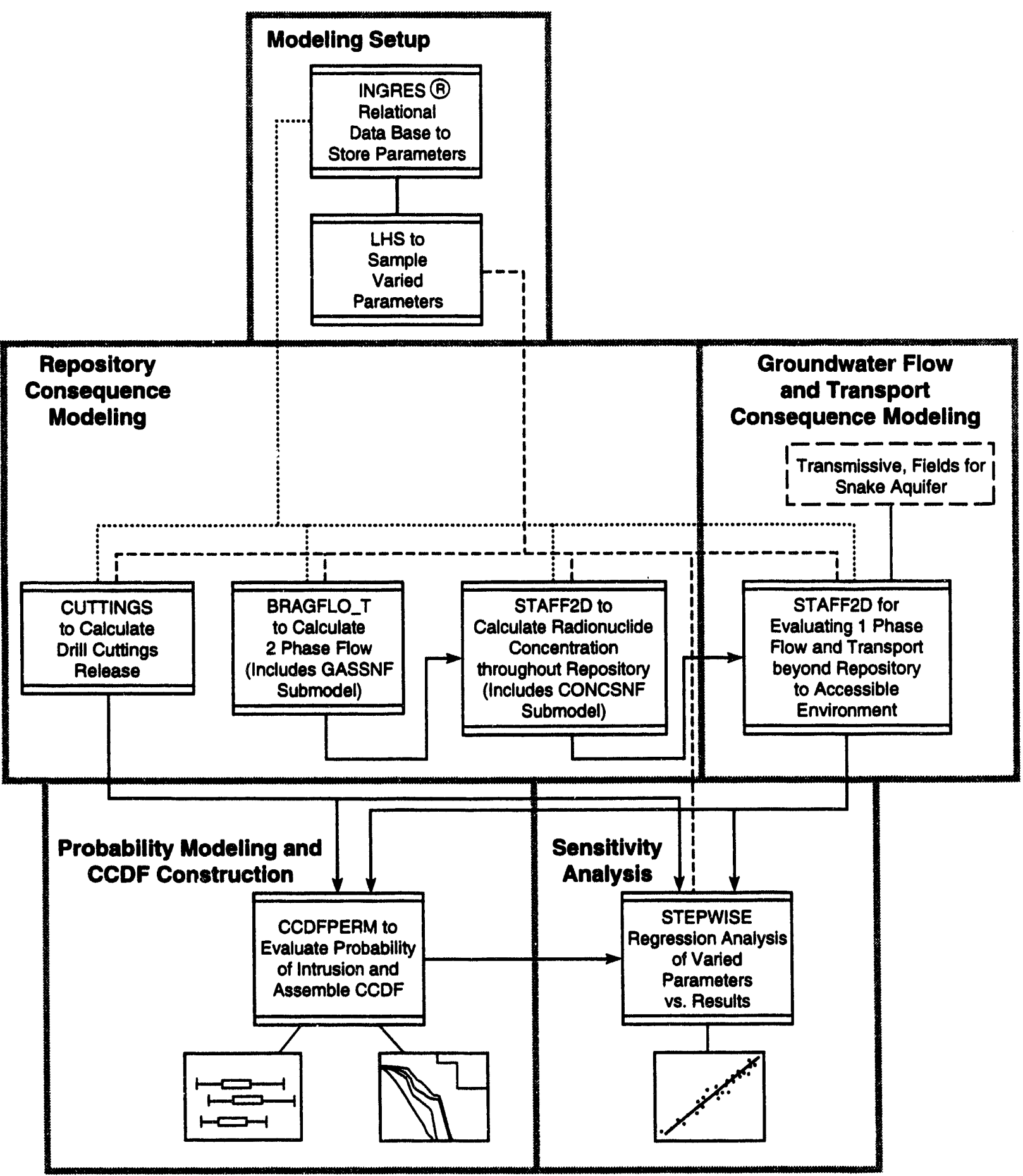

TRI-6342-3577-0

Figure 7-8. The modeling system assembled for the Complex PA consists of a suite of support codes plus the computational modules of BRAGFLO_T, STAFF2D, CUTTINGS, and CCDFPERM. 
7. Scenario Development

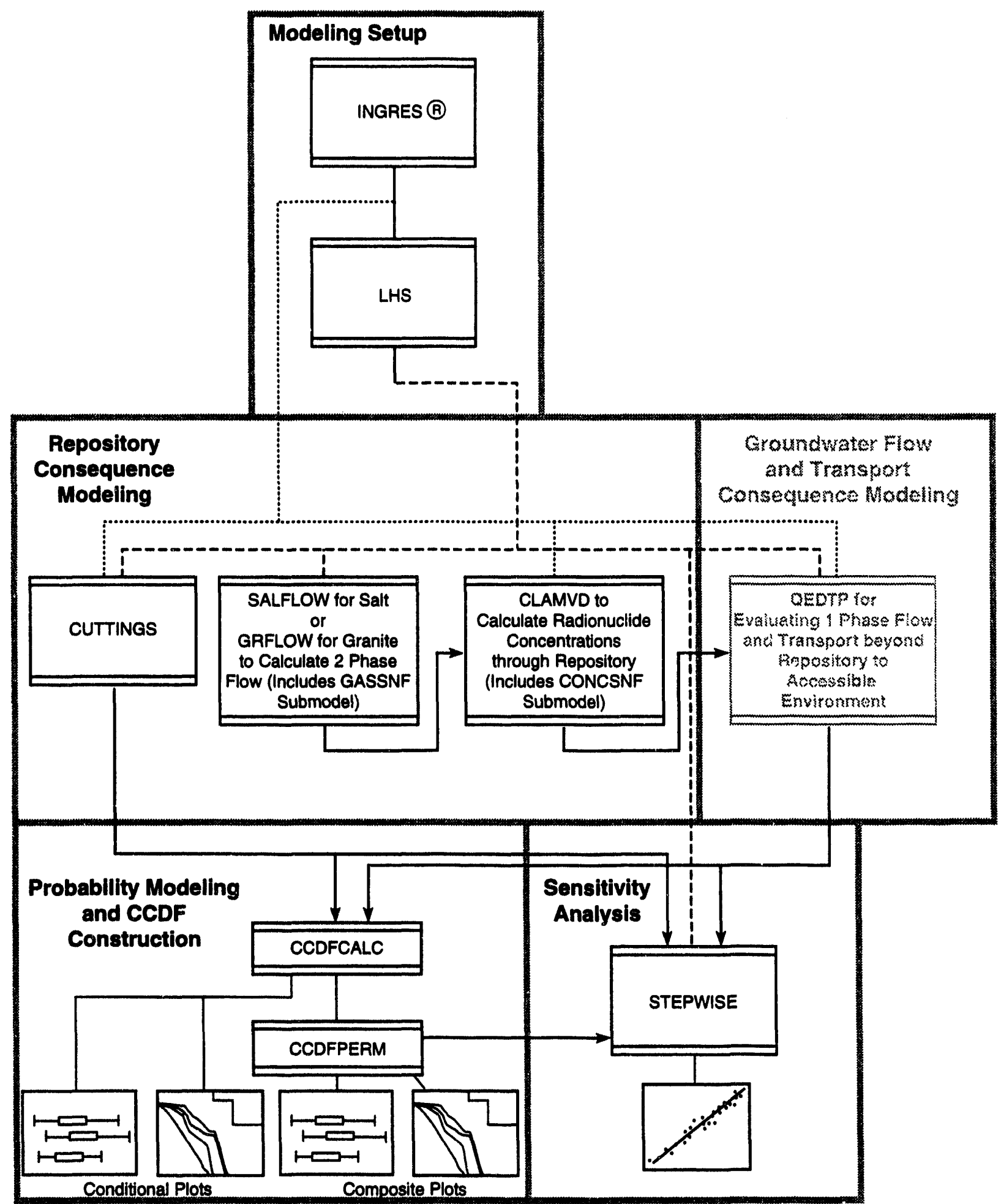

TRI-6342-3731-0

Figure 7-9. The modeling system assembled for the Simple PA consists of a suite of support codes plus the computational modules of SALFLOW, GRFLOW, CLAMVD, CUTTINGS, and CCDFPERM. Although QEDTP was used in the scoping calculations (Appendix $C$ ), it was not used in the Simple PA. 
- fluid flux through the disposal area.

- contaminant concentrations in any fluid (gas or liquid) flowing through the disposal area of the repository.

- contaminant releases to the accessible environment from drilling into the repository.

Groundwater Flow and Transport Modeling. The groundwater flow and transport modeling consists of three main groups of software: transmissivity fields, groundwater flow, and groundwater transport. The transmissivity fields module generates flow field around the salt disposal system (not necessary for granite disposal system). The groundwater flow module establishes regional and local fluid flow conditions within and directly surrounding the disposal system. The groundwater transport module predicts radionuclide migration from the repository source to the disposal system boundary.

Probability Modeling, CCDF Construction, and Results Analyses. The probability modeling, CCDF construction, and results analyses consist of two main groups of software: (1) probability modeling and CCDF construction, and (2) sensitivity analyses. Using results from the groundwater contaminant transport module, two different approaches (containment calculations or groundwater concentration and dose calculations) are taken depending on the EPA environmental standard for which measures are being evaluated. Sensitivity analyses evaluate variable importance by reporting the partial correlation coefficients and standardized regression coefficients on either the rank or raw data. Provided useful regression models can be developed, the absolute values of the standardized regression coefficients (or mathematically related partial correlation coefficients) can be used to rank variable importance.

\subsubsection{Cases Simulated}

The acceptability of the various waste forms was studied with a parametric sensitivity analysis (ceteris paribus) in two ways ${ }^{* * *}$. The Simple PA described above places a single waste parcel in a repository. Twenty-five cases were run for waste parcels in the salt repository and 26 cases for the granite repository (Table 7-4). The advantages of modeling a single waste parcel per repository are that

- Results (e.g., CCDFs) are easily compared; thus, waste forms are easy to rank.

- The acceptability of placing individual waste types in a repository is easily evaluated.

The Complex PA (and some of the Simple PA cases) uses a uniform distribution of the waste disposal groups in each room. Five cases each were run for waste disposal groups in the salt and granite repositories (Table 7-4). The advantages of modeling a uniform distribution of the waste disposal groups are that

- Heat and gas generation are more representative of an actual repository.

- Synergism between waste forms is possible.

- Results indicate whether robust waste forms can moderate the impact of nonrobust waste forms such that a waste group in the repository stays below the release limits.

- Fewer calculations are required (because only a few combinations of the waste parcels are of primary interest). 
2

3

4

7

Waste
Parcel ID

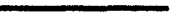

Repository Type

1

Salt

2

3

4

5

6

7

8

9

10

11

12

13

Salt

Salt Granite

Salt Granite

Salt

Granite

Granite

Granite

Granite

Granite

Granite

Granite

Granite

Granite

Waste Disposal Group 2

Spent fuel, graphite, compacts in glass

High-level waste, calcine in Hot Isostatic Press (HIP)

Spent fuel, HEU, and high-level waste, calcine in HIP, spent fuel in lead

Granite

Spent fuel, LEU, and high-level waste, calcine in HIP, spent fuel in lead

7,8,9 Salt Granite High-level waste, glass

\section{Waste Disposal Group 3}

$14 \quad$ Salt

15

16

Salt

Spent fuel, graphite, whole block/
Spent fuel, graphite, particles in glass

Granite Spent fuel, graphite, particles in glass

Spent fuel, HEU, and high-level waste; 8 elements in lead/calcine glass

\section{Simple PA}
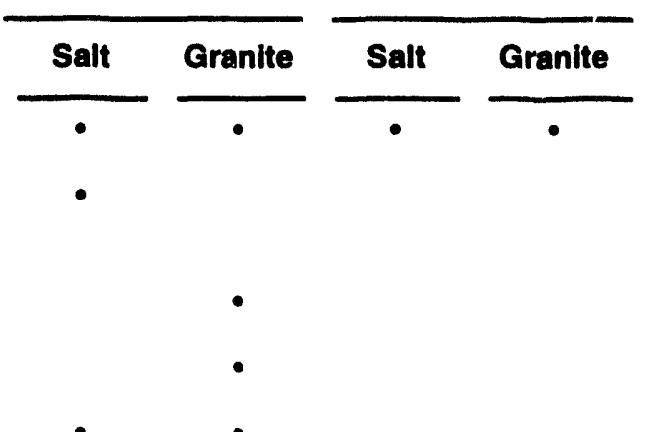

\section{Complex PA}

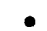

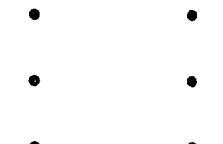
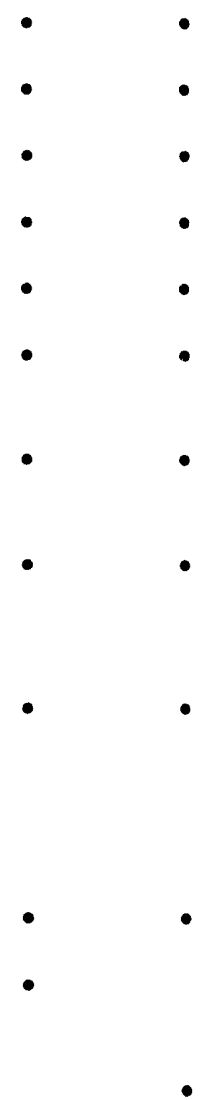

Spent fuel, HEU, and high-level
waste; 8 elements in lead/calcine
glass

- Comparisons are possible between the results for the waste disposal groups from the Complex and Simple PAs. However, recall that the details of the conceptual modeis differ because the Complex PA uses a two-dimensional representation and the Simple PA uses a one-dimensional representation. Also, because the modeling efforts were concurrent rather than sequential, it was not possible for the analysts performing the Simple PA to use the information about important parameters that was gathered during the Complex PA; thus a few features that were shown to be important in the Complex PA were ignored in the Simple PA.

* HEU = highly enriched uranium; LEU = low enriched uranium. 
Table 7-4. Cases Run to Evaluate Waste Forms (Continued)

\begin{tabular}{|c|c|c|c|c|c|c|c|}
\hline \multirow{2}{*}{$\begin{array}{l}\text { Waste } \\
\text { Parcel ID }\end{array}$} & \multirow{2}{*}{\multicolumn{2}{|c|}{ Repository Type }} & \multirow[b]{2}{*}{ Description } & \multicolumn{2}{|c|}{ Simple PA } & \multicolumn{2}{|c|}{ Complex PA } \\
\hline & & & & Salt & Granite & Salt & Granite \\
\hline \multirow[t]{2}{*}{17} & Salt & & $\begin{array}{l}\text { Spent fuel, LEU, and high-level } \\
\text { waste; } 3 \text { elements in lead/calcine } \\
\text { glass }\end{array}$ & - & & & \\
\hline & & & $\begin{array}{l}\text { (Waste Disposal Group } 3 \\
\text { Continued) }\end{array}$ & & & & \\
\hline 18 & Salt & & $\begin{array}{l}\text { High-level waste; remaining cal- } \\
\text { cine and liquild in glass }\end{array}$ & $\bullet$ & & & \\
\hline 19 & & Granite & $\begin{array}{l}\text { Spent fuel, HEU, and high-level } \\
\text { waste; } 1 \text { element in lead/calcine } \\
\text { glass }\end{array}$ & & $\bullet$ & & \\
\hline 20 & & Granite & $\begin{array}{l}\text { Spent fuel, LEU, and high-level } \\
\text { waste; } 1 \text { element in lead/calcine } \\
\text { glass }\end{array}$ & & $\bullet$ & & \\
\hline 21 & & Granite & $\begin{array}{l}\text { High-level waste; remaining cal- } \\
\text { cine and liquid in glass }\end{array}$ & & $\bullet$ & & \\
\hline \multirow[t]{2}{*}{$7,8,9$} & Salt & Granite & High-level waste, glass & & & & \\
\hline & & & Waste Disposal Group 4 & $\bullet$ & - & - & - \\
\hline 22 & Salt & Granite & $\begin{array}{l}\text { High-level waste, reprocessed } \\
\text { graphite spent fuel and calcine in } \\
\text { HIP }\end{array}$ & $\bullet$ & $\bullet$ & & \\
\hline \multirow[t]{2}{*}{23} & Salt & Granite & $\begin{array}{l}\text { High-level waste, reprocessed } \\
\text { special spent fuel and calcine in } \\
\text { HIP }\end{array}$ & - & $\bullet$ & & \\
\hline & & & Waste Disposal Group 5 & $\bullet$ & $\bullet$ & $\bullet$ & - \\
\hline 24 & Salt & Granite & $\begin{array}{l}\text { Spent fuel, graphite, blended } \\
\text { with depleted uranium }\end{array}$ & $\bullet$ & - & & \\
\hline 25 & Salt & Granite & $\begin{array}{l}\text { Spent fuel, HEU and LEU, } \\
\text { blended with depleted uranium }\end{array}$ & - & $\bullet$ & & \\
\hline \multirow[t]{2}{*}{26} & Salt & Granite & High-level waste, calcine in glass & - & - & & \\
\hline & TOTAL & & & 25 & 26 & 5 & 5 \\
\hline $\begin{array}{l}\text { Comparis } \\
\text { recall that } \\
\text { Simple PA } \\
\text { it was not } \\
\text { gathered } \\
\text { Simple PA } \\
\text { - HEU = his }\end{array}$ & $\begin{array}{l}\text { are pos } \\
\text { details } \\
\text { s a one } \\
\text { sibls ic } \\
g \text { the C }\end{array}$ & $\begin{array}{l}\text { lo between } \\
\text { he concept } \\
\text { nensional i } \\
\text { ne analysts } \\
\text { plex PA; th }\end{array}$ & $\begin{array}{l}\text { the results for the waste disposal grou } \\
\text { ual models differ because the Complex } \\
\text { epresentation. Also, because the mode } \\
\text { performing the Simple PA to use the } \\
\text { us a few features that were shown to b } \\
J=\text { low enriched uranium. }\end{array}$ & $\begin{array}{l}\text { rom t } \\
\text { uses a } \\
\text { effort: } \\
\text { imatic } \\
\text { portar }\end{array}$ & $\begin{array}{l}\text { Oomplex anc } \\
\text {-dimension } \\
\text { re concurre } \\
\text { bout import } \\
\text { the Comple }\end{array}$ & $\begin{array}{l}\text { imple } P \\
\text { represen } \\
\text { rather th } \\
\text { t parame } \\
\text { PA were }\end{array}$ & $\begin{array}{l}\text { However, } \\
\text { tion and the } \\
\text { sequential, } \\
\text { is that was } \\
\text { nored in the }\end{array}$ \\
\hline
\end{tabular}




\title{
8. Modeling Conclusions Drawn from Scoping Calculations
}

\author{
R. P. Rechard
}

To provide information about how to model the disposal system (scenario development), scoping or preliminary calculations are run on specific aspects of the system. This chapter lists the information that was gathered from the scoping calculations and used in the calculational design. Details about the scoping calculations for the preliminary performance assessment of both the salt and granite repositories, and the single canister calculations for both the salt and granite repositories are presented in Appendix C.

Although many tentative conclusions can be drawn from the scoping calculations, only those conclusions on which decisions were made about how to model or construct the calculations for the formal performance calculations are tabulated here (Table 8-1). (Those conclusions that were confirmed by the formal PA are described in Chapter 16, "Results and Conclusions.") Besides these conclusions, the scoping calculations did provide some intangible benefits: (1) discovering that resolution was needed on some waste characterization data prior to the formal calculations, and (2) providing a measure of insight about the disposal system and what to expect in the final results (e.g., the current waste forms provided sufficient durability without extensive treatment). 
1. Using very conservative assumptions, the maximum ${ }^{235} \mathrm{U}$ content of the container should not exceed $10 \mathrm{~kg}$ for a salt repository and $0.7 \mathrm{~kg}$ for a granite repository to prevent critical conditions within a corroded container.

2. The differences in consequences between the two scenarios considered at the salt site were minor (two versus one borehole into an underlying brine reservoir), thus

- the probability of hitting a brine reservoir could be assigned a value of one with little additional consequence

- a reasonable approximation to the CCDF could be constructed from the consequence results from only one basic scenario which includes a brine reservoir.

3. Many of the possible combinations of connections to the fracture zones at the granite site resulted in zero consequences, thus

- a reasonable approximation to the CCDF could be constructed using (1) consequences from a repository with all connections present, and (2) a probability model only for an intrusion into a granite repository ${ }^{1}$ while setting the probability of a secondary fracture connection to one.

4. When modeling with BRAGFLO_T, a smooth transition between the fully saturated corrosion rate to a zero corrosion rate at residual saturation was necessary to avoid numerical oscillations.

5. Corrosion rates and breach times show significant vertical variability. This variation is, to some extent, a consequence of the adopted relative permeability curve, which goes to 0 at residual saturation: corrosion will proceed until all liquid in contact with the container is consumed; at residual saturation, fluid is prevented from moving to the container/backfill boundary because the liquid phase is not connected.

6. The backfill-buffer surrounding the canister serves as an important conduit in the transporting of fluid to and from the room above.

7. A waste parcel spacing of $2.6 \mathrm{~m}$ center-to-center is sufficient to keep the temperature of the host rock (salt or granite) below $373 \mathrm{~K}\left(100^{\circ} \mathrm{C}\right)$.

8. A backfill-buffer thickness of $0.3 \mathrm{~m}$ is small enough to prevent the temperature of the bentonite backfill-buffer from exceeding $403 \mathrm{~K}\left(130^{\circ} \mathrm{C}\right)$.

\section{Secondary Conclusions}

1. Because few releases reached a $5-\mathrm{km}$ boundary for the disposal system, a smaller disposal system with a boundary of $2.4 \mathrm{~km}$ from the edge of the waste was used for calculating total-system CCDFs.

2. Minimum spacing of waste parcels should be $2 \mathrm{~m}$ (surface-to-surface) to prevent critical conditions.

3. The hydraulic conductivity values for the secondary fractures of the granite site should be reexamined for rellability.

4. The host rock usually remains saturated adjacent to the waste package, implying that the water saturation in the backfill around the waste parcel could be calculated from the "bulk water saturation" of an element containing many waste parcels, the backfill, and host rock.

5. The salt site transports more heat away from the waste parcel because of the higher conductivity and because assuming permeabilities (albeit low) for the salt allows heat to be advected away.

6. The relative difference in threshold pressure of the room backfill and canister backfill-buffer influences the brine flux rate to the container and, consequently, the life of the container.

1 In the formal PA, the CCDFs presented are conditional on the particular combinations of events occurring; however, the EPA release limits are shown on the graphs since the CCDFs can be considered rough approximations to the complete CCDF. 


\title{
9. Probability Modeling
}

\author{
M. S. Tierney
}

A probability model estimates the likelihoods of uncertain parameter values and also the likelihood of the events and processes of a scenario (Section 3.2.3). Although a probability model can be a computer algorithm, in this report it is either (1) a subjective distribution estimated by experts or (2) an analytic function. The general background necessary for describing the distributions and their limitations is presented in this chapter. In many cases, subjective estimates were used for uncertain model parameters, and the basis for these estimates is found in the chapters in which the specific parameters are discussed (e.g., Chapter 5, Geologic Barrier). The Poisson analytic function provides the estimation of the probability of the event of human intrusion into either the salt or granite repository-essentially the same as reported for the WIPP (Helton, 1991); however, because the parameters have changed somewhat, a short discussion of this topic is included here.

\subsection{Model Parameters}

Model parameters $\left(x=x_{1}, \ldots, x_{n}, \ldots, x_{n V}\right)$ are the underlying fundamental entities of computational probability and consequence models (see Sections 3.2.1 and 3.2.2). For this performance assessment, they are all scalar quantities and are all coefficients in the mathematical models underlying the computational models.

\subsubsection{Characterizing Parameter Uncertainty}

Characterizing the uncertainty in $x$ requires developing a joint probability distribution, $F(x)$; usually, the joint distribution is approximated by the product of distributions of the individual parameters $\left(F\left(x_{1}\right) \cdot\left(F\left(x_{2}\right) \cdot \ldots \cdot F\left(x_{n V}\right)\right.\right.$ (i.e., the parameters are assumed to be independent of each other). These individual distributions may be defined by probability density or cumulative distribution functions (PDFs or CDFs; see below). The distribution (either CDF or PDF) of a parameter, $x_{n}$, ideally represents both what we know and what we do not know about that parameter and should reflect the best, current knowledge of the range and likelihoods of the appropriate parameter value when used in a consequence or probability model. It is possible that the $F\left(x_{n}\right)$ may be obtained by classical statistical techniques for some parameters. However, in many cases, each $F\left(x_{n}\right)$ will be a subjective distribution based on the degree of belief that is developed from available information through a suitable review process.

For a continuous parameter, say $\mathrm{X}$, the probability density function (PDF) is a function $\mathrm{f}(\mathrm{x})>0$ with the properties

$$
\begin{aligned}
& \int_{a}^{b} f(x) d x=\text { probability that uncertain parameter } X \text { lies in interval }(a, b) \text { : } \\
& \qquad \int_{-\infty}^{+\infty} f(x) d x=1 .
\end{aligned}
$$

The cumulative distribution function (CDF) associated with $\mathrm{f}(\mathrm{x})$ is defined by

$$
F(x)=\int_{-\infty}^{x} f(s) d s=\text { probability that uncertain parameter } X \text { is less than or equal to } x \text {. }
$$


Probability density functions (PDFs) and CDFs can be similarly defined for uncertain parameters that take on a denumerable number of values, $x_{i}, i=1,2, \ldots$. The sequence $\left\{f_{i}\right\}, i=1,2, \ldots$, such that $f_{i}>0$ and

$$
\sum_{i} f_{i}=1
$$

is the discrete analogue of the continuous PDF, and

$$
F(x)=\sum_{\text {all } x_{i}<x} f_{i}
$$

is the discrete analogue of the continuous CDF.

Range. The range of a distribution is denoted by $(a, b)$, the pair of numbers in which $a$ and $b$ are respectively the minimum and maximum values that can reasonably be taken by the uncertain parameter $\mathbf{X}$.

Mean and Sample Mean. The mean value (or, simply, mean) of a distribution is one measure of the central tendency of a distribution; it is analogous to the arithmetic average of a series of numbers. The population mean, $\mu$, is defined by

$$
\begin{aligned}
\mu & =\int_{-\infty}^{\infty} x f(x) d x \text { for continuous distributions } \\
& =\sum_{\text {all } x_{i}} x_{i} f_{i} \text { for discrete distributions. }
\end{aligned}
$$

The sample mean, denoted by $\bar{x}$, is the arithmetic average of values in an empirical data set. In general, a sample mean may not be assigned to CDFs derived solely from subjective estimates of experts.

Median and Sample Median. The median value of a CDF is denoted by $\mathrm{x}_{50}$ and is that value in the range at which $50 \%$ of all values lie above and below (i.e., the 0.5 quantile). Sample medians, here denoted by $\bar{x}_{50}$, can be obtained directly from empirical CDFs in the obvious way.

Variance and Coefficient of Variation. The variance of a distribution, $\sigma^{2}$, is the second moment of the distribution about its mean, i.e.,

$$
\begin{aligned}
\sigma^{2} & =\int_{-\infty}^{\infty}(x-\mu)^{2} f(x) d x \text { for continuous distributions } \\
& =\sum_{\text {all } x_{i}}\left(x_{i}-\mu\right)^{2} f_{i} \text { for discrete distributions. }
\end{aligned}
$$

The standard deviation, $\sigma$, is the positive square root of the variance. The coefficient of variation, the ratio of standard deviation to mean, $\sigma / \mu$, is a convenient measure of the relative width of a distribution.

The sample variances of a set of measurements of parameter $x_{n}$, say $x_{1}, x_{2}, x_{3}, \ldots x_{n V}$ is the sum

$$
s=\frac{1}{(n V-1)} \sum_{n=1}^{n V}\left(x_{n}-\bar{x}^{2}\right) \text {. }
$$


The sample variances of independent measurements of some quantity is an unbiased estimator of the population variance of that quantity (Blom, 1989, p. 197). (A variance can also be formally calculated for empirical CDFs derived from subjective estimates of experts; this is not a sample variance, however.)

Categories of Distributions. Distributions used in this report are grouped into four categories:

1. Continuous analytical distributions: normal, lognormal, uniform or loguniform (Table 9-1),

2. Discrete analytical distributions: Poisson (Table 9-1),

3. Constructed distributions based on judgment ("Subjective Estimates"),

4. Miscellaneous categories (null distributions): constant, and table.

Continuous Distributions. Four continuous, analytical distributions used in this report are described below.

Normal. Normal designates the normal PDF, a good approximation to the distribution of many physical parameters. The normal distribution arises naturally from the central limit theorem (Johnson and Kotz, 1970, p. 40; Miller and Freund, 1977, p. 104). For purposes of performance assessment, the distribution is arbitrarily truncated at the 0.01 and 0.99 quantities (i.e., the probability that the parameter will be smaller or larger is $1 \%$ ), which corresponds to $\bar{x} \pm 2.33 \mathrm{~s}$, where $s$ is the sample standard deviation.

Lognormal. Lognormal designates a lognormal PDF, a distribution of a variable whose logarithm follows a normal distribution. Like a normal distribution, the lognormal distribution is arbitrarily truncated at the 0.01 and 0.99 quantiles.

Uniform. Uniform designates a PDF that is constant in the interval $(a, b)$ and zero outside of that interval.

Loguniform. Loguniform designates a loguniform PDF, a distribution of a variable whose logarithm follows a uniform distribution.

Discrete Distributions. The discrete distribution used in this report is the Poisson distribution. The Poisson PDF is often used to model the number of events taking place over intervals of time, such as the arrival of telephone calls at a switch station (queuing problem) or the number of imperfections per unit length produced in a bolt of cloth.

Constructed Distributions (Subjective). Constructed distributions of Subjective type are histograms based on subjective estimates of range (the 0 and 100 percentile) and at least one interior percentile point (usually the 50 percentile or median). The subjective estimates of percentile points are usually obtained directly from experts in the subject matter of the parameter of concern. Histogram PDFs for intrinsically continuous parameters are always converted to piecewise linear CDFs by joining the subjective percentile points with straight lines.

Miscellaneous Categories. Other "null" categories of distributions are described below:

Constant. When a distribution type is listed as constant, a distribution has not been assigned and a constant value is used in all PA calculations.

Table. The table category of data indicates that the parameter varies with another property and the result is a tabulated value. For example, relative permeability varies with saturation; its distribution type is listed as table (the median value is not meaningful and is therefore omitted in the table).

\subsubsection{Some Limitations on Distributions}

A major limitation of ensuring valid probability distributions is the lack of an actual disposal site for the INEL spent nuclear fuel and waste. However, other limitations on ensuring the validity of the probability distributions assigned to parameters in the performance assessment are thought to be a consequence of two assumptions that are frequently made in analyses of geologic disposal systems: 
Table 9-1. Description of Several Probability Distributions

$\stackrel{\wp}{\not}$

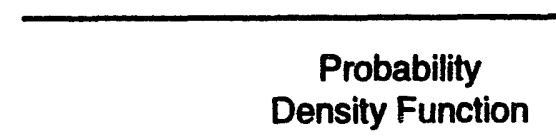

$f(x)$

1. Normal $\mathrm{N}\left(\mu, \sigma^{2}\right)$

$$
\frac{1}{\sqrt[\sigma]{2 \pi}} \exp \left[-\frac{(x-\mu)^{2}}{2 \sigma^{2}}\right]
$$

$-\infty \leq x \leq \infty$

but for PA $a \leq x \leq b$ where $P(x>a)=0.99$ and

$P(x>b)=0.01$ $\int_{-\infty}^{x} f(x) d x$
Variance

$\sigma^{2}$
2. Lognormal

$$
\frac{1}{\sigma x \sqrt{2 \pi}} \exp \left[-\frac{1}{2 \sigma^{2}}(\ln x-\mu)^{2}\right]
$$

$x \geq 0$

$$
x=e^{y} \text { where } y=N\left(\mu, \sigma^{2}\right)
$$

but for PA

$P(y>a)=0.99$ and

$P(y>b)=0.01$

$\mu(y)=\frac{a+b}{2}$

$\sigma^{2}(y)=\left(\frac{b-a}{4.66}\right)^{2}$ 
Table 9-1. Description of Several Probability Distributions (Concluded)

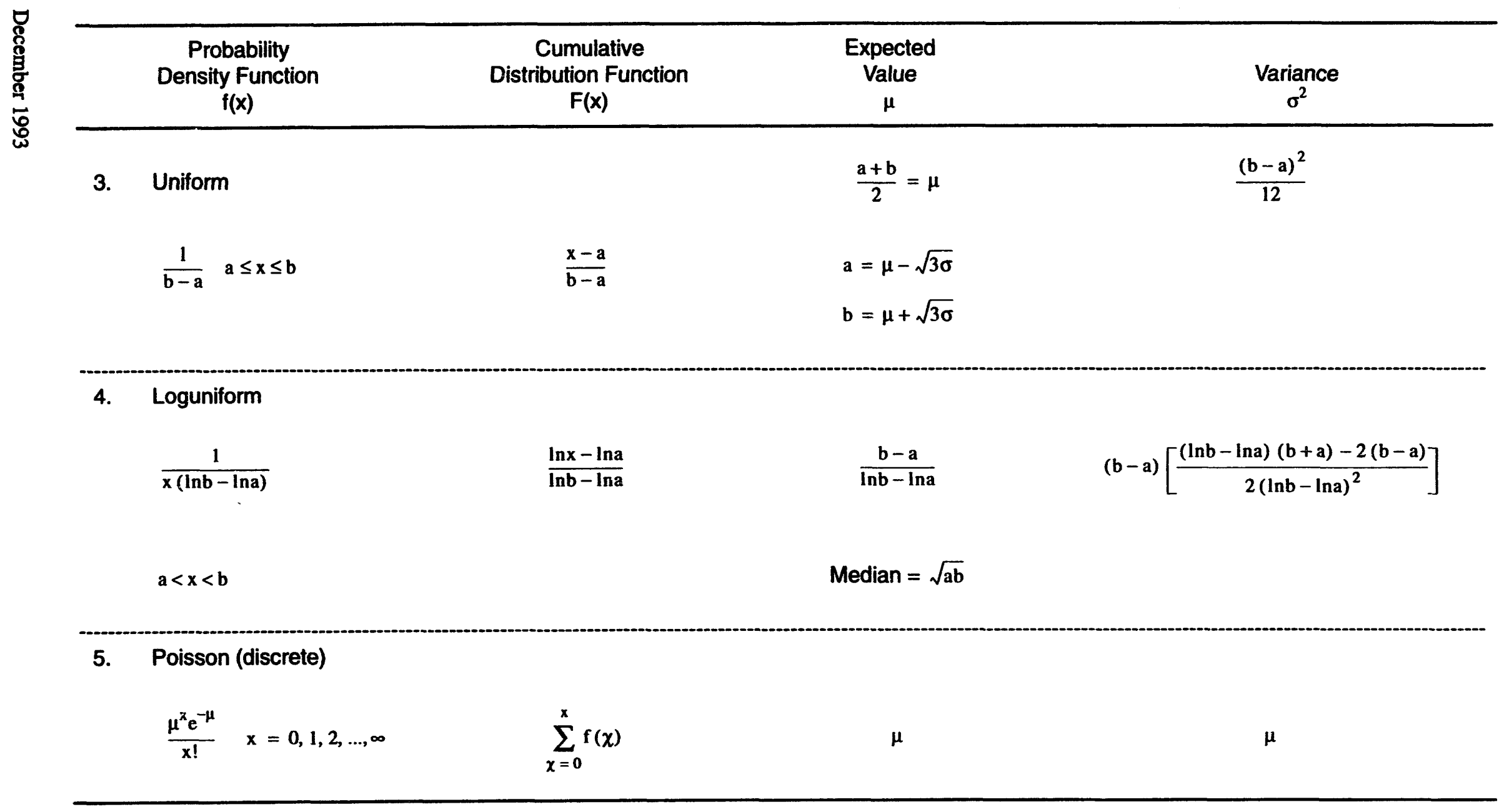


- The equating of the variability of a parameter in space (e.g., spatial variation of porosity) with parameter uncertainty, in a model (where the model uses a constant value), particularly for that class of parameters called material-property parameters.

- The neglect of obvious correlations between model parameters (e.g., permeability and porosity).

Current methods of assigning uncertainty to some of the material-property parameters (e.g., including smallscale spatial variability as a source of uncertainty) may distort results of sensitivity analyses, but will probably not affect the validity of results of the uncertainty analyses that are used to make preliminary comparisons with EPA standards. The following argument attempts to explain the two limitations described above. The following text is taken from Sandia WIPP Project, 1992, Volume 3, Chapter 1.

No Scaling of Variability for Material-Property Parameters. The performance models described in Chapters 11 through 15 of this report are based on the numerical solution of one or more of three types of equations:

(a) Partial differential equations - which are reduced to a set of algebraic equations or ordinary differential equations in order to effect a solution by finite-difference or finite-element methods. Examples: the equations of groundwater and brine flow, solute transport, gas flow, and salt creep (see Chapters 12 and 13).

(b) Ordinary differential equations - which may be the result of a reduction of a partial differential equation or may directly model the dynamics of a lumped-parameter system, e.g., punctured brine reservoirs, leaching and decay of radioactive waste stored in a panel (see Chapter 14, Simplified Consequence Modeling for Salt).

(c) Algebraic equations of the form

$$
F\left(x_{1}, x_{2}, x_{3}, \ldots, x_{n} ; y\right)=0
$$

which may arise indirectly from equilibrium solutions of ordinary differential equations (i.e., solutions for time $\rightarrow \infty$ ) or may directly express a model of some physical relationship between performance-model variables $\left(x_{1}, x_{2}, x_{3}, \ldots, x_{n}\right)$ and $y$, an intermediate dependent variable.

In addition to dependent variables and independent variables of position and time, certain constant quantities appear in each of the three types of equations. In most cases, these parameters are intended to represent physical and chemical properties of real materials of the disposal system: e.g., the hydraulic conductivity, porosity, and specific storage in models of fluid flow. This kind of parameter is called a material-property purameter in the remainder of this section.

Several of the material-property parameters of performance models have been included in the set of uncertain variables sampled in this study.

The distribution of a material-property parameter needs to reflect spatial variability of the material property and also the scale of the model. The zones or cells of numerical models (finite-element, finite-difference, or lumpedparameter models) must be few in number to minimize computational time and expense; in a typical problem involving geologic media, these cells will have dimensions of tens of meters or more and volumes of thousands of cubic meters. Material-property parameters must therefore represent the effects of a physical or chemical property of matter in these relatively large, arbitrarily defined volumes of space. It follows that material-property parameters are model dependent and usually not observable quantities, i.e., quantities that can be measured in the field or in the laboratory. On the other hand, with few exceptions most physical and chemical properties of geologic materials are actually measured on spatial scales typical of the laboratory or an exploratory borehole, a matter of at most a few tens of centimeters. In addition, natural materials and many man-made materials (e.g., defense waste) tend to be inhomogeneous on spatial scales that are smaller than the scales that characterize model cell sizes; accordingly, a set of measurements of a material property taken randomly from large volumes of real material may show wide variability. The question is: How to assign a distribution to material-property parameters in a way that correctly reflects both cell size and the small-scale variability that may appear in measurements of the corresponding material property? 
One way of approaching the problem of scaling is as follows. Assume that the material property can be represented as a scalar field in space, say $\phi(x)$, where $x=(x, y, z)$ denotes position in space. (The assumptions of a scalar quantity in three dimensions are for the sake of simplicity of argument and involve no loss of generality; the property could be a vector or tensor.) It is argued in some modern textbooks (e.g., de Marsily, 1986, Chapter 13 and Section 4.4) that the material-property parameter, say $\Phi$, to be used in the numerical solution of the partial differential equation should be taken as a spatial average of $\phi$ over the cell or zone; for instance, in a cell or zone of volume V,

$$
\Phi(\mathrm{V})=\frac{1}{\mathrm{~V}} \int_{\mathrm{V}} \phi(\mathbf{x}) \mathrm{dx}
$$

where $\mathrm{dx}$ is the volume element dxdydz. (Again, no loss of generality is involved; if appropriate, a line or surface average could replace the volume average.)

To account for spatial variability of $\phi(\mathbf{x})$, it can be assumed that $\phi$ is a stationary, random scalar field within a cell volume $V$, with realizations $\phi(\mathbf{x}, \mu)$ and the following statistical properties:

$$
\text { Expectation of } \phi(\mathbf{x}, \mu)=E[\phi(\mathbf{x})]=\bar{\phi}, \text { a constant, }
$$

and

$$
\text { Covariance of } \begin{aligned}
\phi(\mathbf{x}, \mu) & =E\{[\phi(\mathbf{x})-\bar{\phi}][\phi(\mathbf{y})-\bar{\phi}]\} \\
& =\sigma^{2} \rho(|\mathbf{x}-\mathbf{y}|),
\end{aligned}
$$

where $\sigma^{2}$ is a variance of $\phi$, and $\rho(\cdot)$ is a function of $r=|x-y|$ with the properties,

$$
\begin{aligned}
& \rho(r) \geq 0 \text { for } r \in 0, \infty \\
& \rho(r) \rightarrow 1 \text { as } r \rightarrow 0, \\
& \rho(r) \rightarrow 0 \text { as } r \rightarrow \infty .
\end{aligned}
$$

The function $\rho(\bullet)$ is called the autocorrelation function (Yaglom, 1962); it is a measure of the statistical dependence of the values of $\phi$ measured at two different points $\mathbf{x}$ and $\mathbf{y}$. The stationarity assumptions of constant mean value $\bar{\phi}$ and variance $\sigma^{2}$ can be slightly weakened by allowing these quantities to depend on the coordinates of the center of the volume $V$, i.e., $\bar{\phi}$ and $\sigma^{2}$ may vary from cell to cell.

Treating $\phi(x)$ as a stationary random field with statistical properties 9-2 through 9-4 allows estimates of the mean value and variance of the volume average of $\phi, \Phi(V)$, to be made. It is shown in textbooks (see for instance Yaglom, 1962, pgs. 23-24) that

$$
\text { Expectation of } \Phi(\mathrm{V})=\mathrm{E}[\Phi(\mathrm{V})]=\bar{\phi} \text {, }
$$

and

$$
\text { Variance of } \Phi(V)=\frac{\sigma^{2}}{V^{2}} \iint_{V} \rho(|x-y|) d x d y
$$

If $\bar{\phi}, \sigma^{2}$ and $\rho(r)$ were kn $: * n$, the problem would be essentially solved in that the distribution of the materialproperty parameter, $\Phi(\mathrm{V})$, could be approximated by a normal distribution with mean and variance given respectively by Eqs. 9-5 and 9-6. In general, $\bar{\phi}, \sigma^{2}$ and the function $\rho(r)$ must be estimated using sets of spatially coordinated measurements of the material property $\phi$, say $\left(\phi_{1}, \phi_{2}, \ldots, \phi_{N}\right)$. The estimators of $\bar{\phi}$ and $\sigma^{2}$ are the usual unbiased estimators of mean and variance (see Tierney, 1990, pp. II-4,5) and, given a sufficiently large set of spatially coordinated measurements of $\phi$, approximations to the autocorrelation function could be constructed and used in numerical evaluations of the volume integrals in Eq. 9-6. This ideal solution to the problem cannot be implemented, 


\section{Probability Modeling}

however, since there are few measurements of the material properties for the disposal system models and most are not spatially indexed. Thus, one must try to use available measurements and insight to infer the statistical properties, given by Eqs. 9-5 and 9-6, of material-property parameters, $\Phi(V)$. The following two general observations are useful in inferring statistical properties of material-property parameters.

First, variance of a material-property parameter is less than or equal to the apparent variance of the material property. Note that because of the properties of $\rho(r)$ (Eq. 9-4), the integrand in the double volume integral of Eq. 9-5 is always less than one so that

$$
\text { Variance of } \Phi(V) \leq \sigma^{2}
$$

In particular, if the special form of autocorrelation function is taken ("cookie cutter"),

$$
\begin{aligned}
\rho(|x-y|) & =1 \text { if }|x-y| \leq a \\
& =0 \text { otherwise, }
\end{aligned}
$$

then

$$
\text { Variance of } \Phi(V) \approx \frac{v}{v} \sigma^{2}
$$

where $v=\frac{4 \pi}{3} \mathrm{a}^{3}$ can be called the volume of correlation. Equation 9-8 suggests that if the volume of correlation is $<<$, then the distribution of $\Phi(V)$ is peaked about the mean value of the material property, $\bar{\phi}$. If the coefficient of variation of the material property, $\sigma / \bar{\phi}$, is not large (say, of the order of one), the distribution of $\Phi(V)$ is more sharply peaked about the mean value, $\bar{\phi}$, than is the distribution of the material property, $\phi(x)$. If this tendency is strong enough, then $\Phi(V)$ can simply be assigned the mean value,

$$
\Phi(\mathrm{V}) \approx \bar{\phi}
$$

This is what is usually done in studies with numerical models that are not probabilistic; that is, not directed explicitly towards sensitivity and uncertainty analyses.

Second, if as suggested above, $\Phi(\mathrm{V}) \approx \bar{\phi}$, then one must consider the uncertainty inherent in estimating the mean value $\bar{\phi}$, that arises from two sources: (1) a limited number of measurements of the material property, and (2) relationships between $\bar{\phi}$ and other uncertain problem parameters. Uncertainty of the first type can be handled by fitting available data to a "t-distribution" (Blom, 1989) which, in a Bayesian approach, gives the distribution of the true mean of the material property about the sample mean of measurements. However, this was not done in assigning ranges to parameters here. Also, the standard techniques cannot be used when the distribution of the material property, $\phi(x)$, must be gained by subjective means, i.e., the elicitation of expert judgment. In such cases, it is necessary to make the assumption that the distribution of the material property, $\phi(x)$, is also the distribution of the materialproperty parameter, $\Phi(V)$. Instances where this assumption was made are found in the estimation of waste-form solubility. Uncertainty of the second type is usually model dependent and must be handled on a case-by-case basis (see remarks on correlations below).

General Absence of Correlation Among Parameters. Most of the 23 parameters varied during the current performance assessment exercise were assumed to be independent random variables even though it was known that some were dependent upon others, i.e., correlated in some way. Correlations of the model variables may arise from the fact that there are natural correlations between the local quantities used to determine the form of the model variable (e.g., local porosity could be strongly correlated with local permeability); or correlations of model variables may be implicit in the form of the mathematical model in which they are used. The effects of neglecting correlations on the sensitivity/uncertainty analyses are generally unknown. 


\subsubsection{Parameters Selected for Sampling}

A parameter was initially selected as imprecisely known in this preliminary PA evaluation if (1) the parameter had proved to be highly sensitive in WIPP PA calculations (Helton et al., 1992), or (2) the parameter was thought to be at least moderately sensitive in a new consequence model for this PA. This initial list was then reduced to those parameters primarily used in gas generation and source term submodels. (For the performance assessment, 18 model parameters were varied [13 of which are identical] for the salt and granite sites.) The modeling processes that these 23 parameters $(13+5+5)$ influence are shown in Tables 9-2 and 9-3. The assumed distributions for these 23 parameters are shown in Figures 9-1 through 9-8.

Parameters sampled in the salt repository were the permeability of the Sodium Springs Formation and the anhydrite marker bed, the permeability in the upper segment of the deep borehole, and the permeability and porosity of the crushed salt backfill (see Chapter 6). With the exception of the borehole permeability, all the sampled parameter distributions were obtained from the 1992 WIPP performance assessment (Sandia WIPP Project, 1992). To simulate both the E1 and E1E2 human intrusion scenarios, the permeability distribution in the upper segment of the intrusion borehole (LogPrmU) was set such that half the sampled values fell in the range of $10^{-21}$ to $3.16 \times 10^{-13} \mathrm{~m}^{2}$ and the other half ranged from $3.16 \times 10^{-13}$ to $10^{-12} \mathrm{~m}^{2}$ (see Figure 9-1). The permeability of the shallow borehole and the lower segment of the deep borehole was set to the median value of $3.16 \times 10^{-13} \mathrm{~m}^{2}$. The median borehole permeability is identical to the median value used in the 1992 WIPP performance assessment; however, the permeability distribution is quite different. A high upper segment borehole permeability resulted in a scenario similar to the E1, while a low permeability led to an E1E2 scenario.

\subsubsection{Parameters Held Constant}

A total of about 650 and 700 parameters were required by the consequence and probability models for the salt and granite sites, respectively. The dimensions of important engineered and geologic features of the site are not considered as part of the total number of parameters. Of the total parameters, about 200 are the same for both the salt and granite sites.

Because only 18 parameters were varied per site, the majority of modeling parameters were held constant, usually at the median value. Yet ranges and distributions were estimated for many of these parameters to examine their potential validity. The tables of the model parameters are presented in Chapters 4,5 , and 6 for the waste parcel, geologic barrier, and repository, respectively. Model parameters for the gas generation and source term submodels are provided in Chapter 11.

\subsection{Poisson Model for Probability of Human Intrusion}

\subsubsection{Probability Model}

The probability model of human intrusion by exploratory drilling is based upon the assumption that the number of inadvertent drilling events in a time interval [a,b] follows a homogeneous Poisson process, i.e., the probability $P(n)$ that $n=0,1,2, \ldots$ events occur in $[a, b]$ is given by

$$
P(n)=\frac{[\lambda(b-a)]^{n}}{n !} \exp [-\lambda(b-a)]
$$

where $\lambda$ is a constant called the drilling intensity (see Tierney, 1991, Appendix C) (Figure 9-9). In practice,

$$
\lambda=A_{p} \lambda^{*}
$$

December 1993 
9. Probability Modeling

Table 9-2. Parameters Varied at Salt Repository for Formal Performance Assessment Calculations

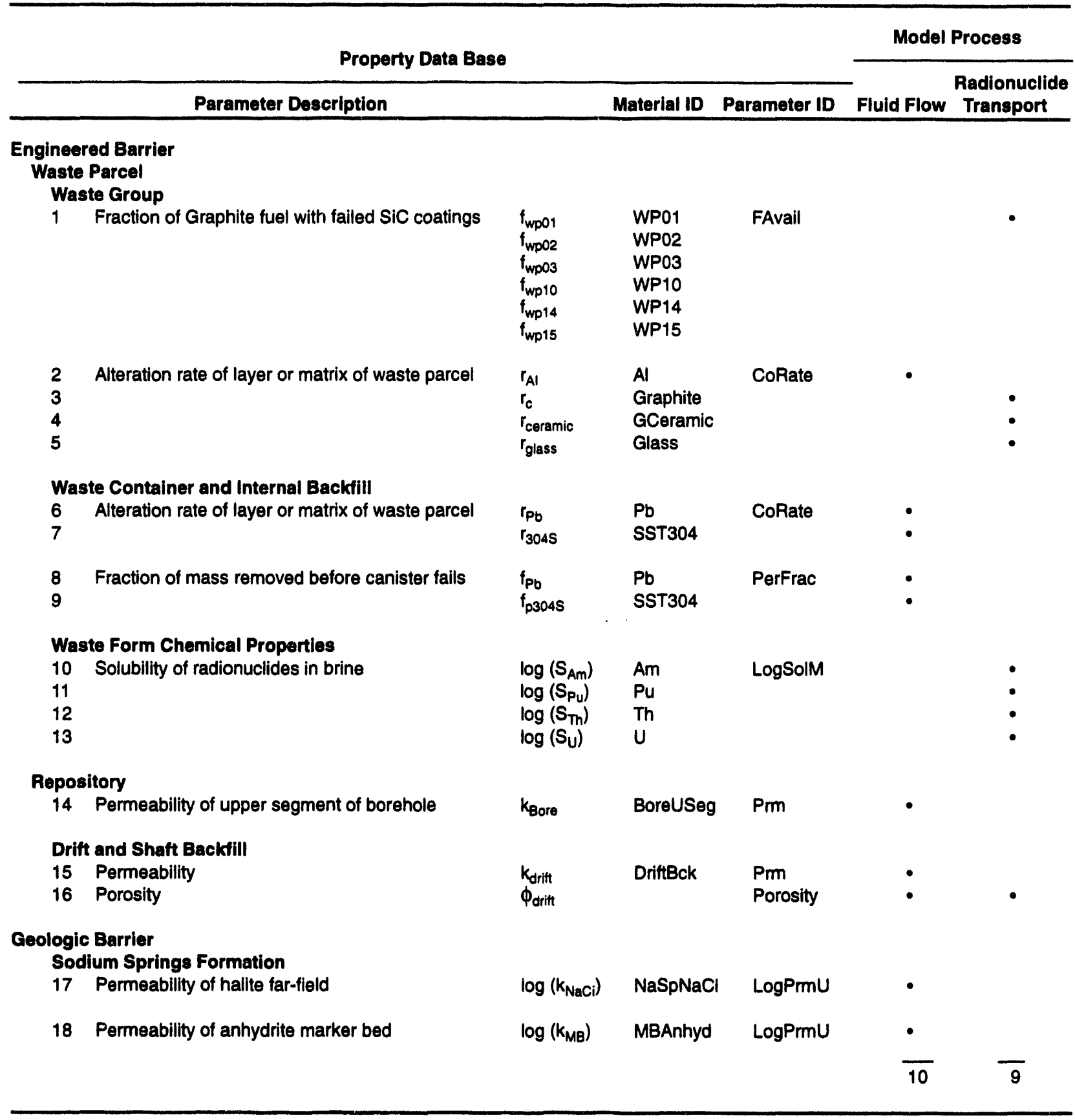


Table 9-3. Parameters Varied at Granite Repository for Formal Performance Assessment Calculations

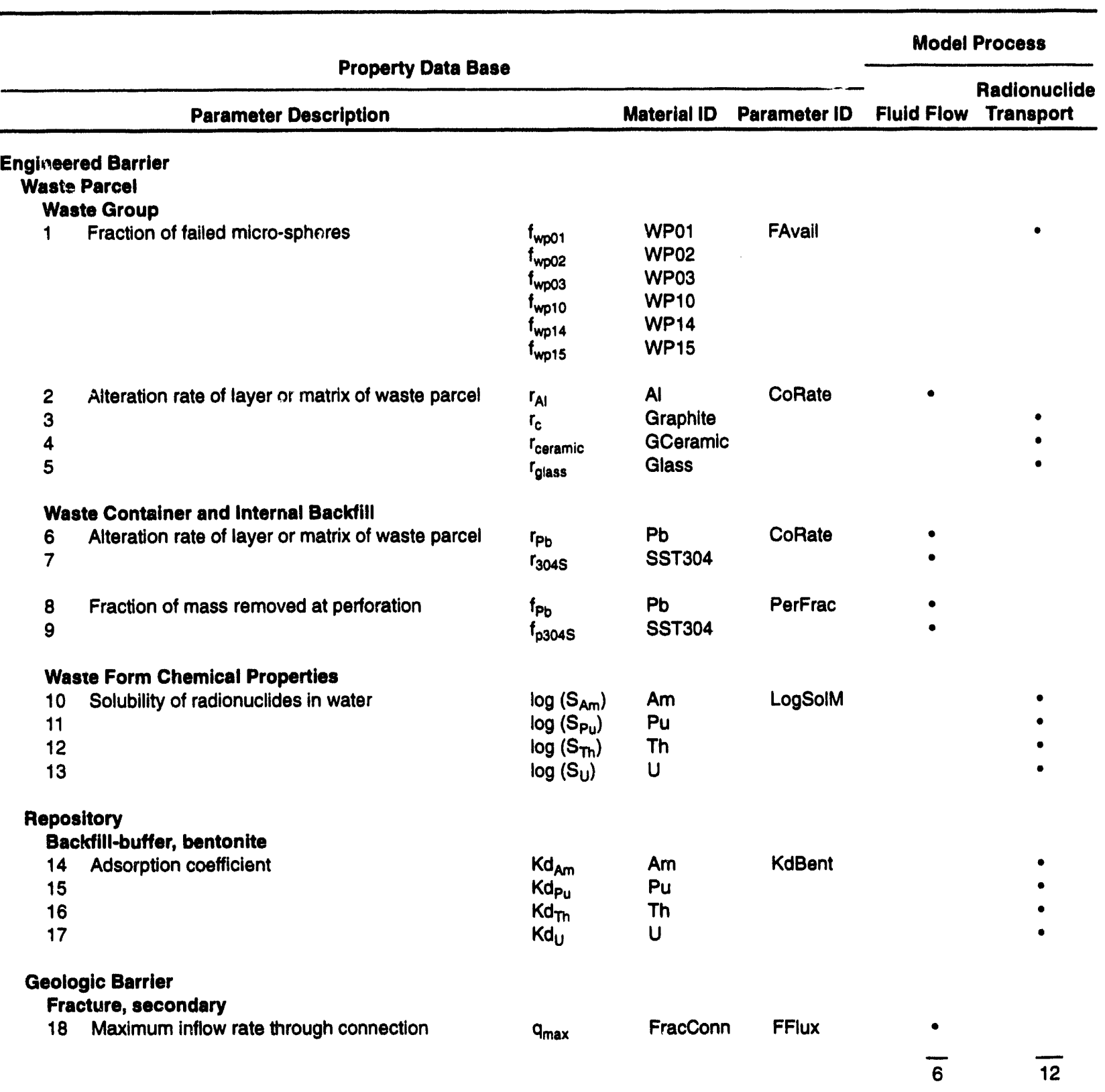



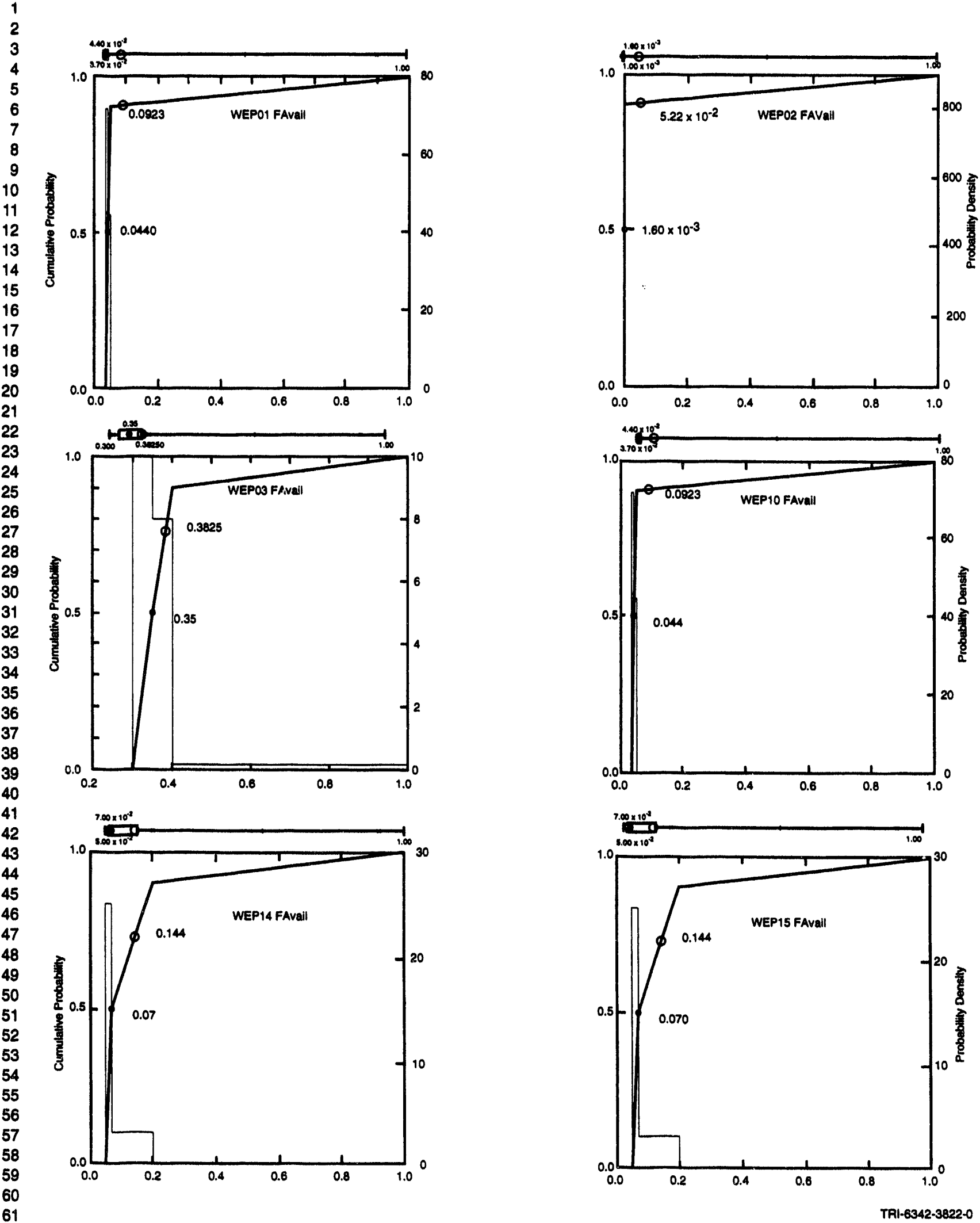

Figure 9-1. Probability distributions of fraction of silicon-carbide coatings that have failed in the waste parcels containing graphite spent nuclear fuel in both salt and granite repositories. 

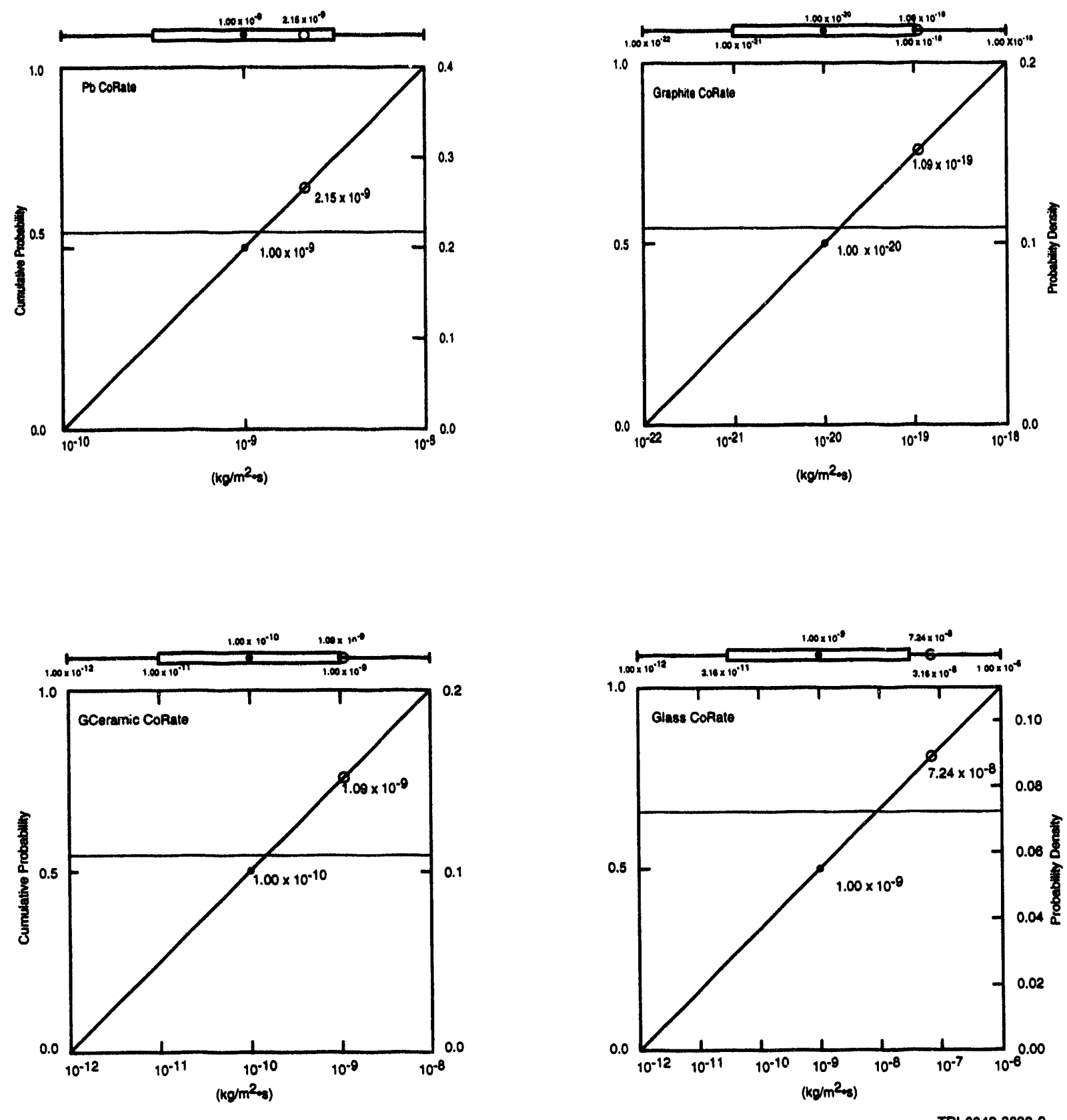

Figure 9-2. Uniform probability distributions of alteration rates for layers and matrices of the waste parcel in both salt and granite. 

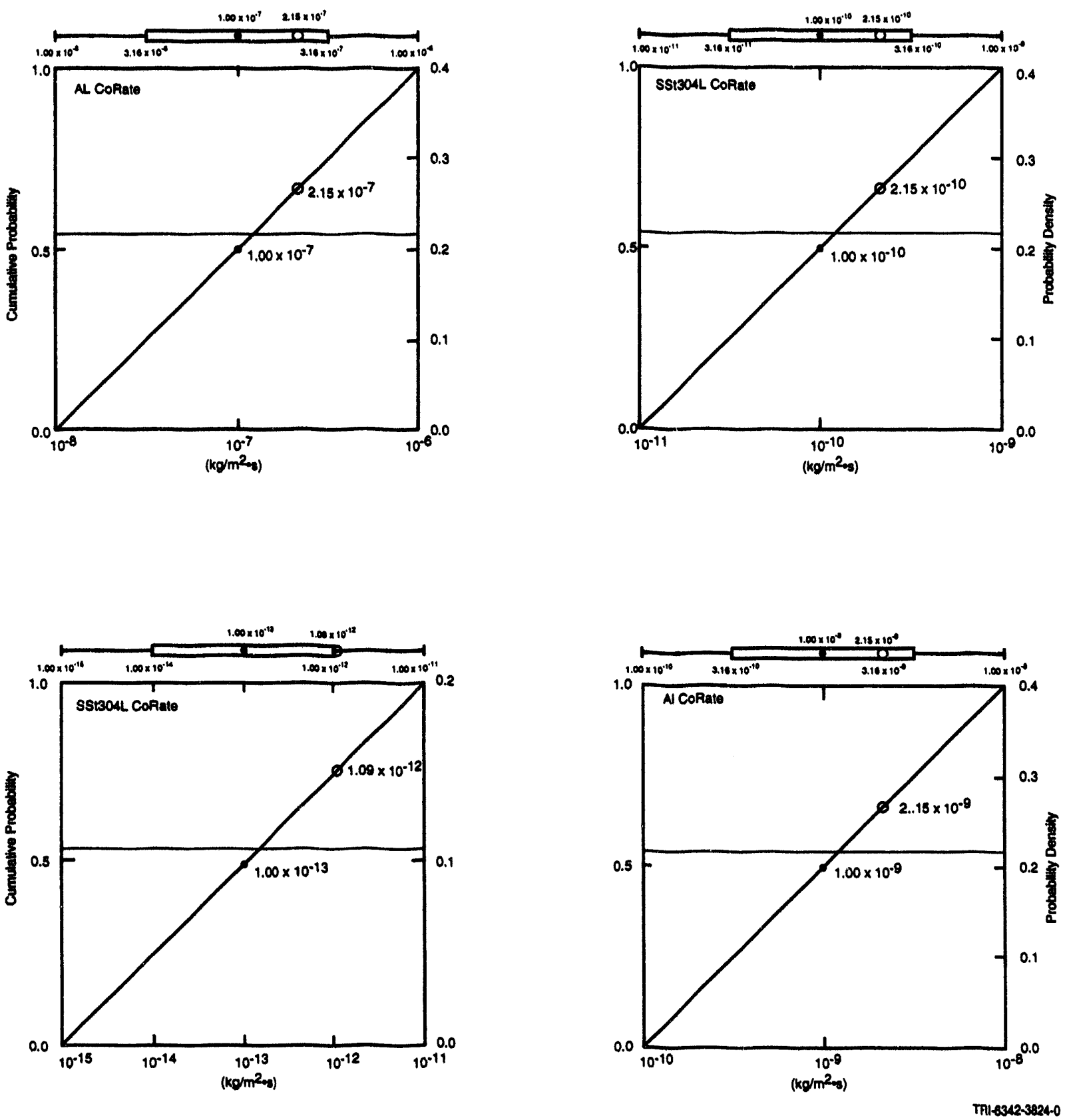

Figure 9-3. Uniform probability distributions of alteration rates for aluminum and stainless steel in salt and granite. 

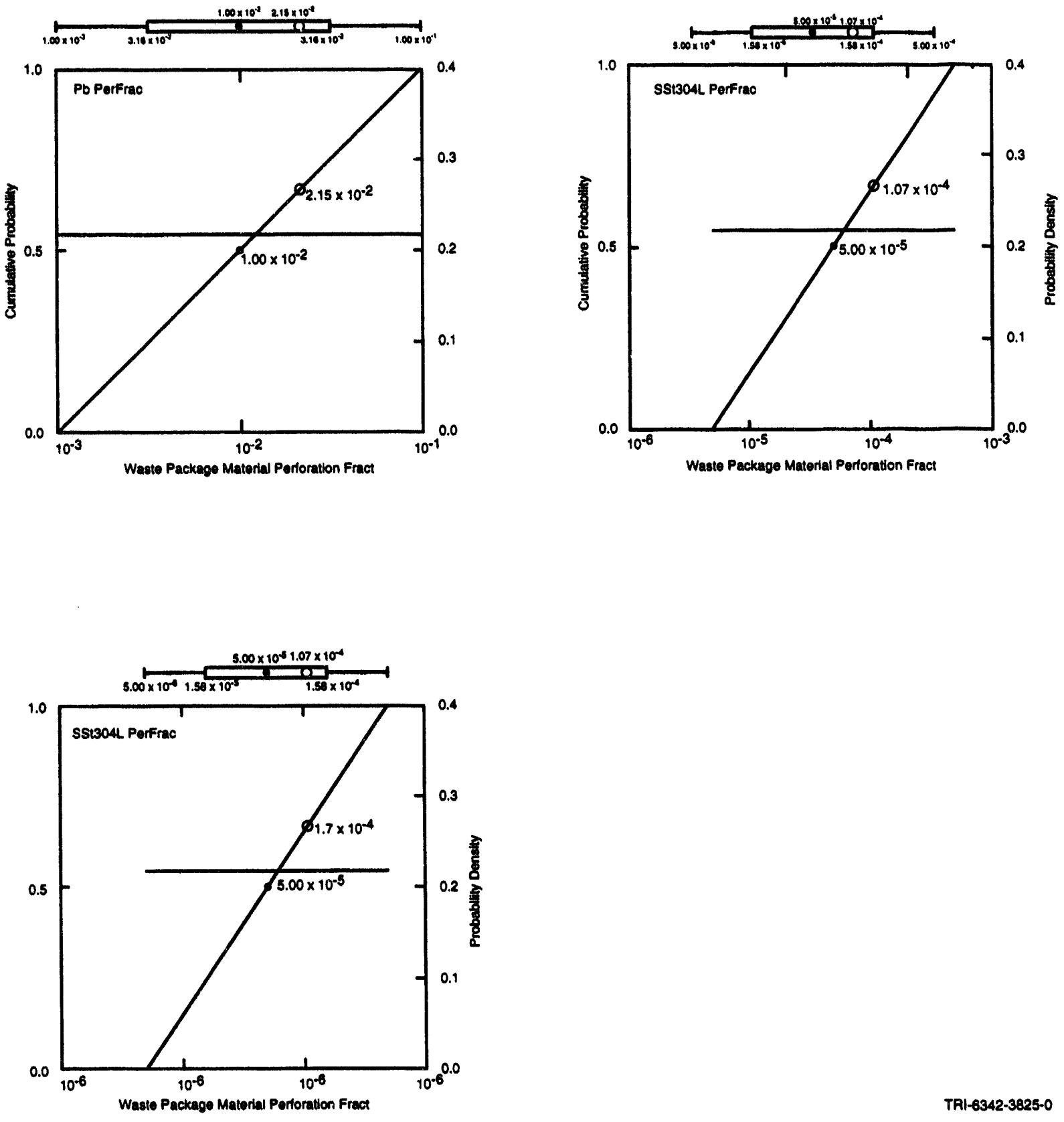

TRI-6342-3825-0

Figure 9-4. Probability distributions of fraction of mass removed at perforation of lead and stainless steel in salt and granite repositories. 

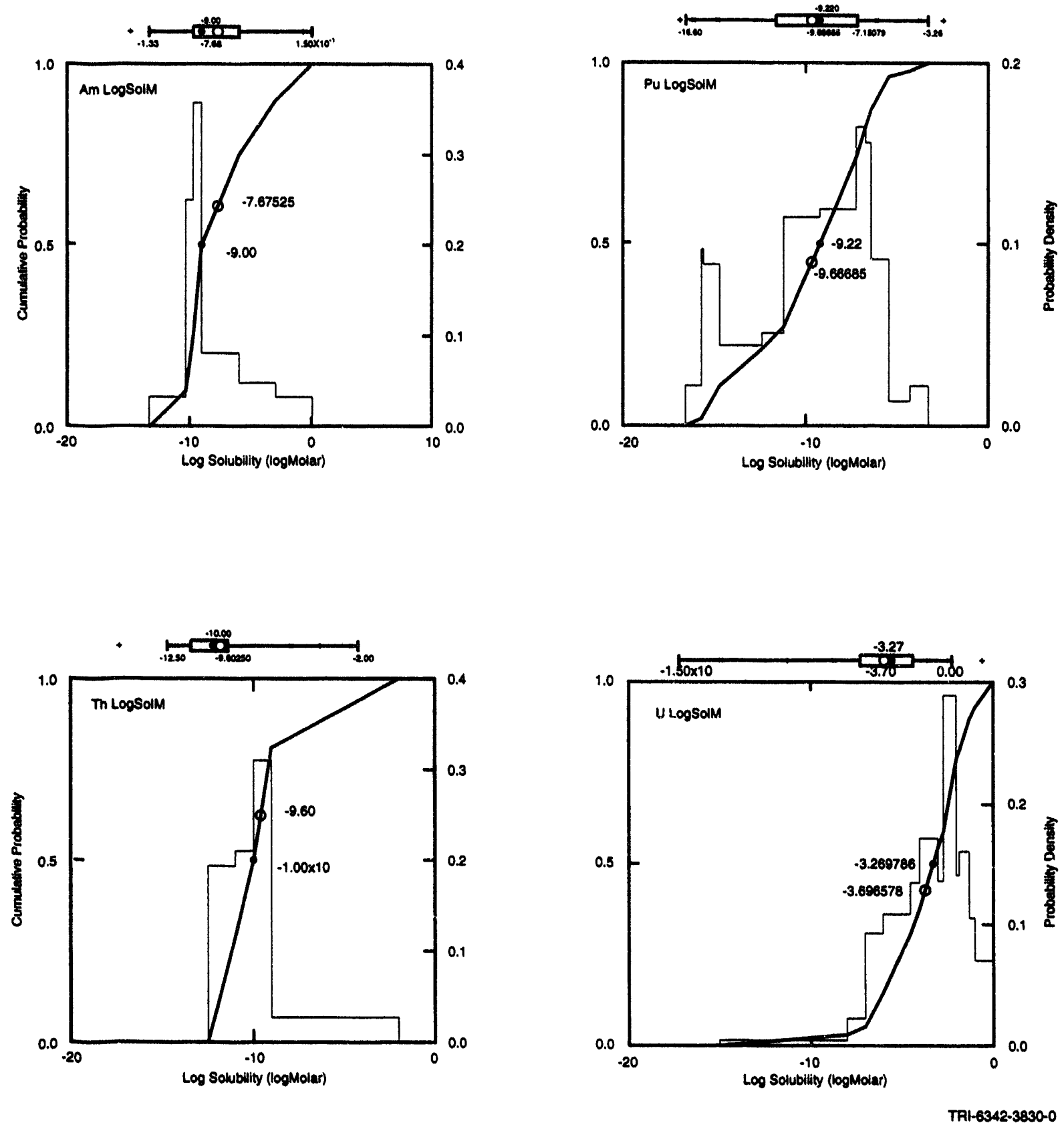

Figure 9-5. Probability distributions of solubility of americium, plutonium, thorium, and uranium radionuclides in brine in salt repository. 

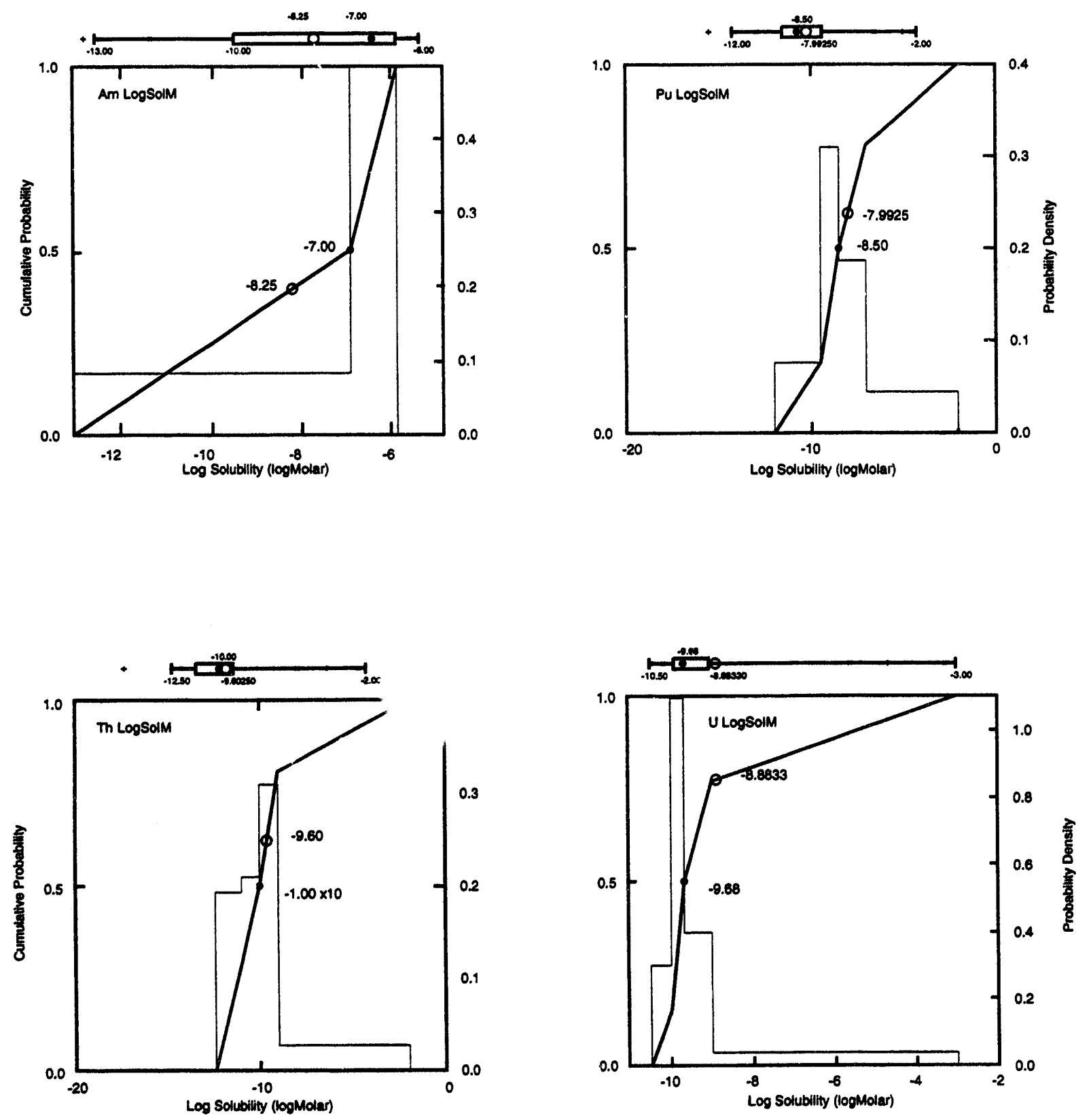

TR1-6342-3831-0

Figure 9-6. Probability distributions of solubility of americium, plutonium, thorium, and uranium radionuclides in fresh water in granite repository. 

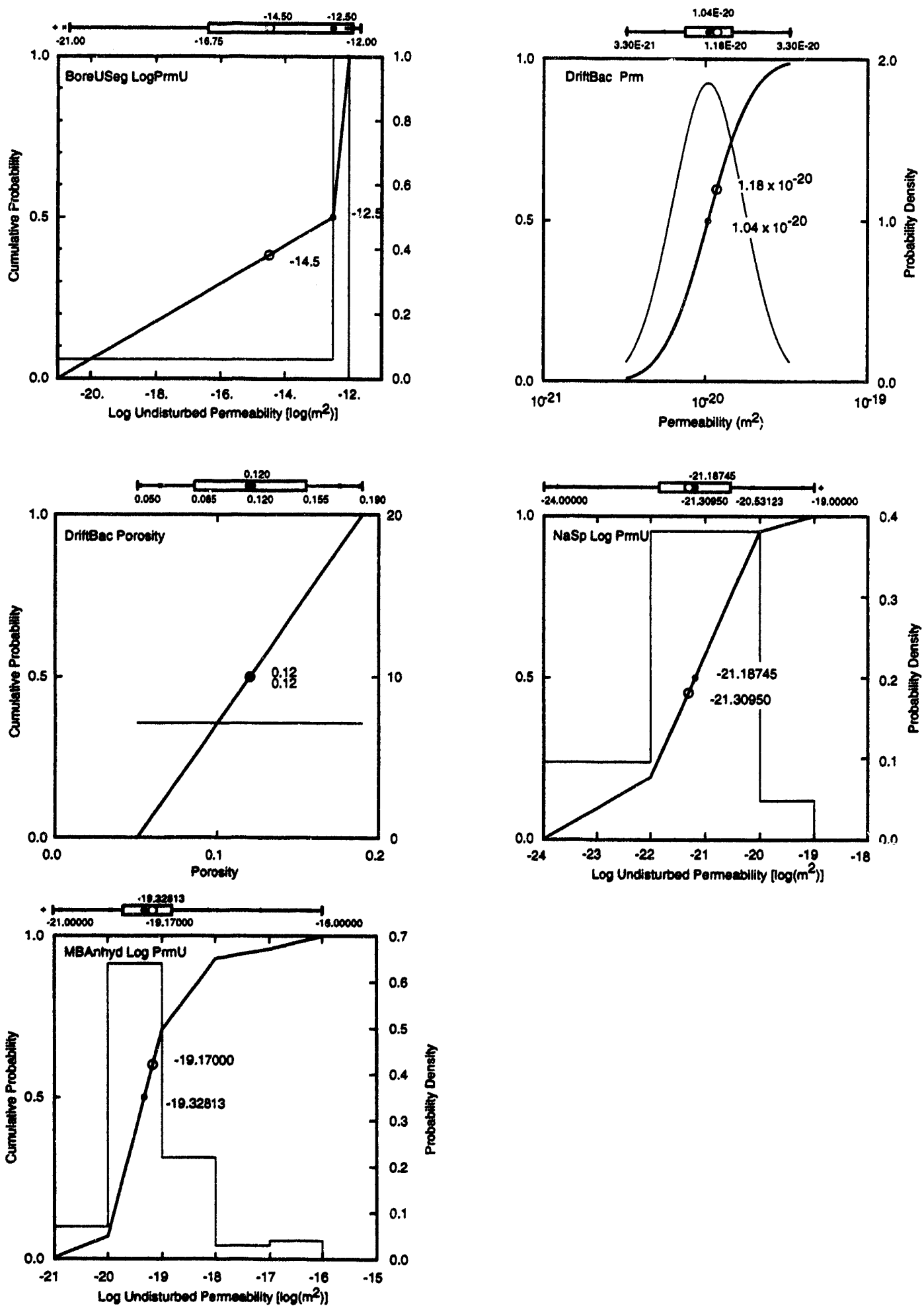

TRI-6342-3827-0

Figure 9-7. Probability distributions for repository and geologic barrier model parameters in salt. 
2

5

6

8

9

11

12

13
14

14
15

16

18

19

20

21

22

23

24

25

26

27

28

29

30

32

33

34

35

36

37

38

39

40

42

43

44

45

46

47

48

49

50

51

52

53

54

55

56

57

58

59

60

61

62

63

64

66
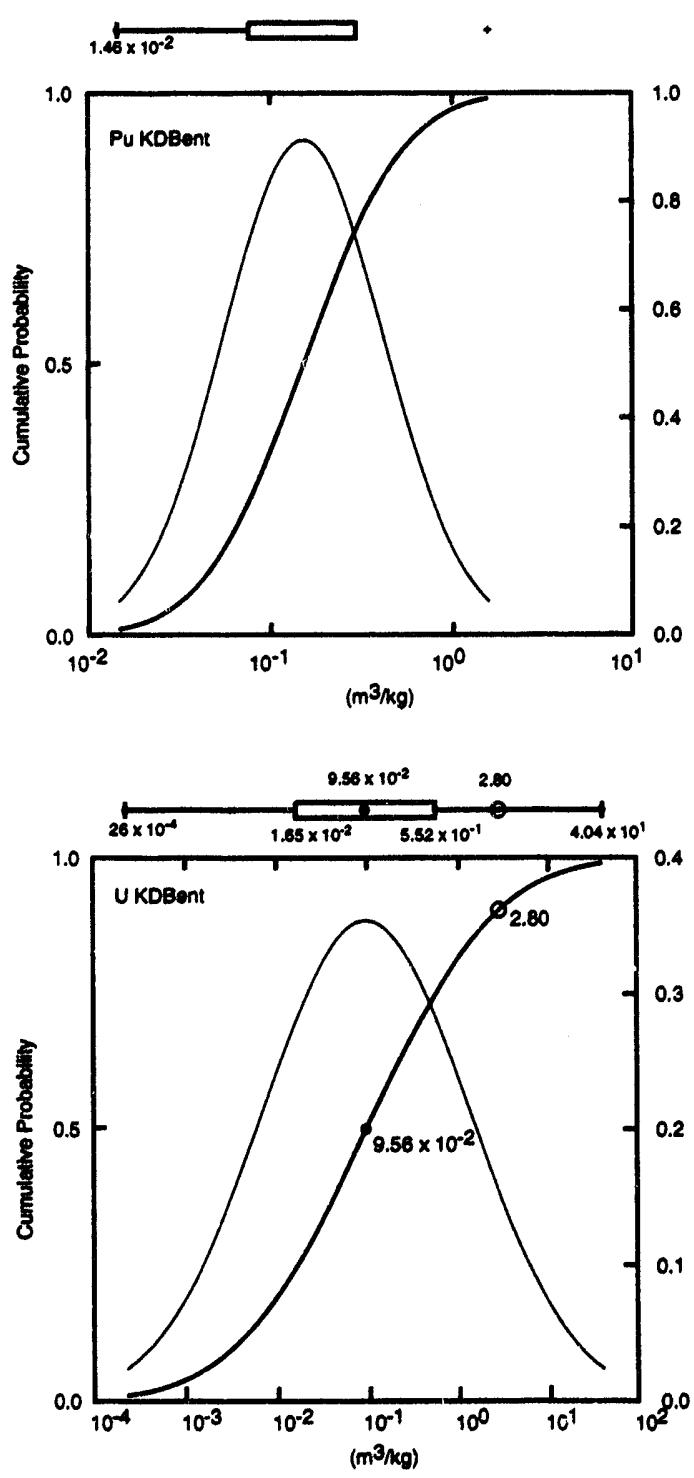
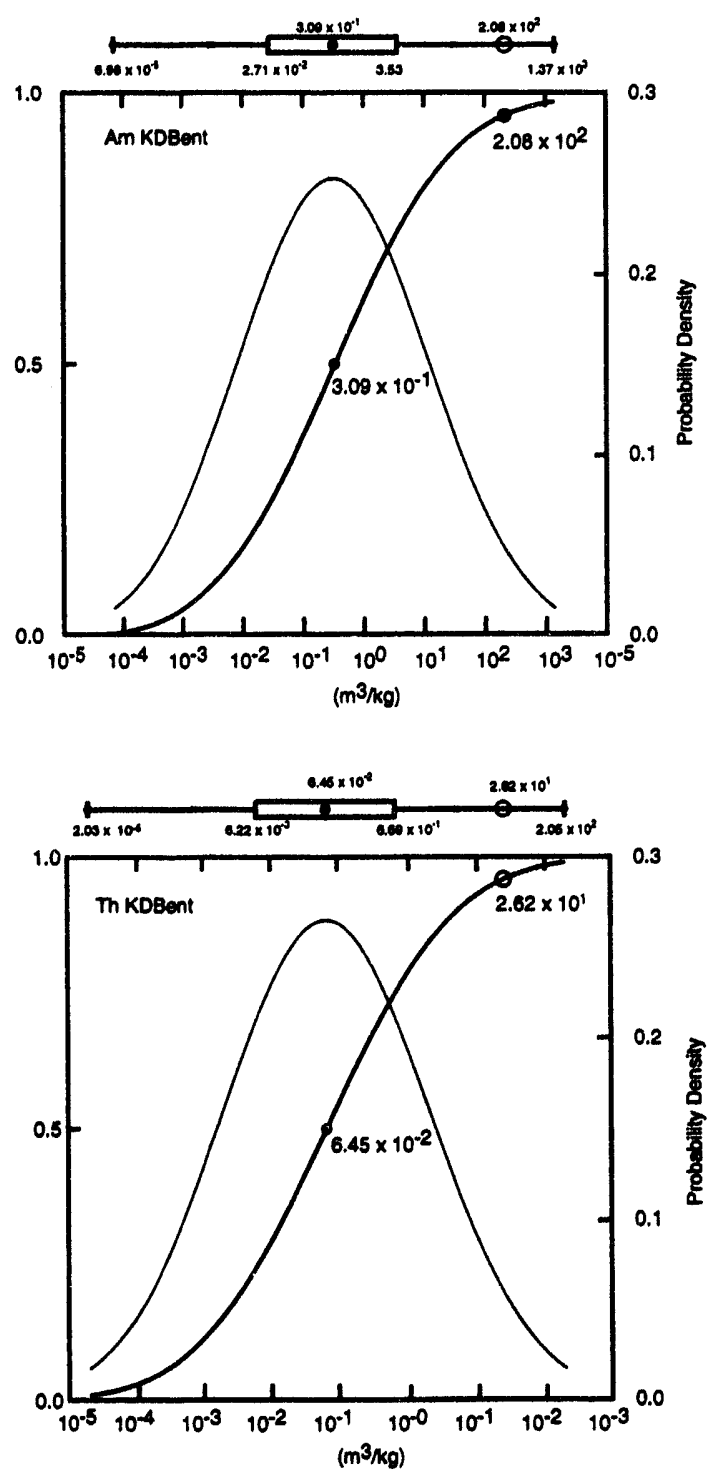

TR1-6342-3828-0

Figure 9-8. Probability distributions for repository and geologic barrier model parameters in granite. 
9. Probability Modeling

a)

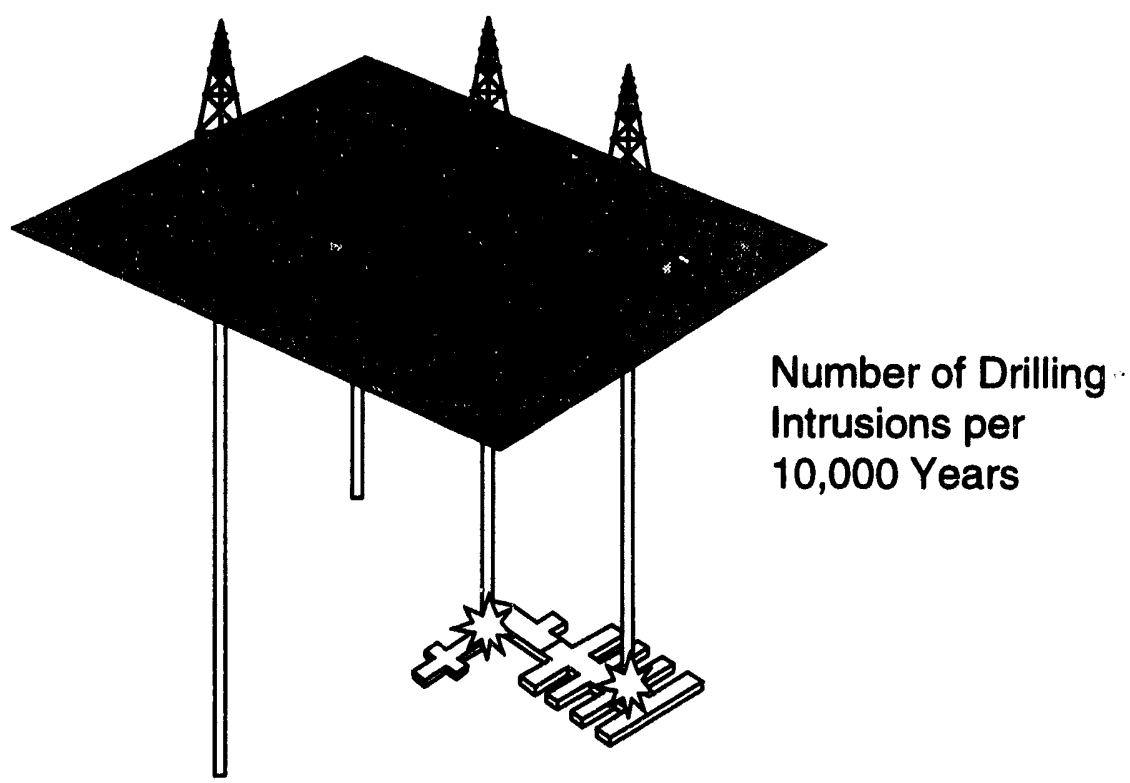

b)

$$
P\{N=n\}=\frac{(\lambda t)^{n}}{n !} e^{-\lambda t}
$$

Poisson Analytic

Probability Model

c)

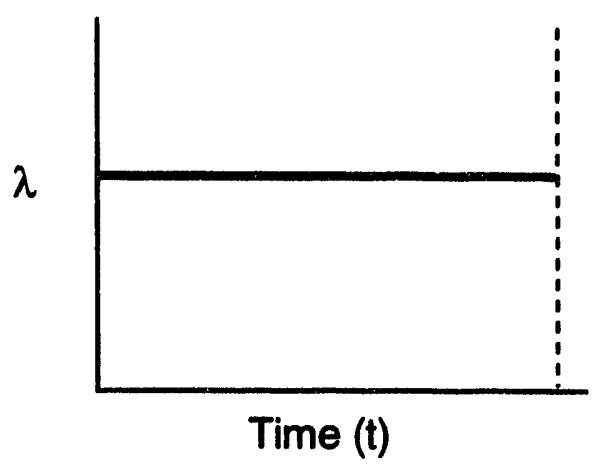

TRI-6342-3555-0

Figure 9-9. Probability of a human-intrusion estimated by Poisson analytic probability model with constant $\lambda$ 
where $A_{p}$ is the total of the projected areas of all containers $\left(A_{p}=\mathrm{Na}, N\right.$ the total number of canisters and $a$ the projected area of a canister on the ground's surface above the repository). The quantity $\lambda^{*}$ is a mean drilling rate for which the EPA (1985, Appendix B) has set an upper limit of

$$
\lambda_{\max , \text { salt }}^{*}=\frac{30 \text { boreholes }}{10^{6} \mathrm{~m}^{2} \cdot 10^{4} \mathrm{yr}}=3 \times 10^{-9} \mathrm{~m}^{-2} \cdot \mathrm{yr}^{-1} \text {, }
$$

for sites involving sedimentary rocks and

$$
\lambda_{\text {max, granite }}^{*}=\frac{3 \text { boreholes }}{10^{6} \mathrm{~m}^{2} \cdot 10^{4} \mathrm{yr}}=3 \times 10^{-10} \mathrm{~m}^{-2} \bullet \mathrm{yr}^{-1}
$$

Although $\lambda^{*}$ is uniformily varied between 0 and $\lambda^{*} \max _{\text {ax }}$ in the scoping calculations for the salt repository (Appen$\operatorname{dix}(\mathrm{C})$, in the formal calculations, $\lambda^{*}$ is treated as a constant parameter that is set at the maximum, $\lambda_{\max }^{*}\left[0, \lambda_{\max }^{*}\right]$, calculated above.

\subsubsection{General Implications}

Some numbers will help show how rare are the events that are predicted by this model using current design parameters for the salt repository and Waste Disposal Group 1. If there are $N=4406$ containers in a panel, 10 panels in a salt repository, and each container has a projected area of $0.2 \mathrm{~m}^{2}$, then $A p=8.8 \times 10^{3} \mathrm{~m}^{2}$. Taking $\lambda^{*}$ max $=$ $3 \times 10^{-9} / \mathrm{m}^{-2} \cdot \mathrm{yr}^{-1}$, it is found that

1. The mean time between inadvertent drilling events is about $38,000 \mathrm{yr}(1 / \lambda)$,

2. The expected number of events in a 10,000 -yr period following closure is about $0.26(\lambda \cdot 10,000 \mathrm{yr})$,

3. There is a $77 \%$ chance of not directly hitting a container in the 10,000 -yr period following closure $(n=0)$.

\subsubsection{Area Ratios for Probability of Hitting Different Waste Parcels}

To evaluate the complementary cumulative distribution function of releases from a repository filled with different waste parcels, the probability of a drillbit hitting a particular type of waste parcel must be known since the radionuclide content of each waste parcel type varies. In evaluating the probability of a drillbit hitting a specific type of waste parcel, the location of the drilling rig is assumed to be random such that the probability of hitting a waste parcel type, given that a hit has occurred, is the ratio of the cross-sectional area of the waste parcel type to the total crosssectional area of all waste types. The ratios for all five waste disposal groups for both the salt and granite repositories are presented in Table 9-4.

\subsection{Probability of Exploratory Drillhole Intersecting a Brine Reservoir}

The current conceptual model of the stratigraphy for the salt repository assumes that a brine reservoir exists under the repository (probability equals one). This value differs from that used in the WIPP performance assessment, for which the largest probability used has been 0.55 .

\subsection{Summary of Modeling Assumptions for Human Intrusion}

- Human intrusion from drilling follows a homogeneous Poisson process with constant expectation for each simulation.

- Low probability of borehole plug configuration for E1E2 scenario at salt repository (maximizing flow through panel) is ignored in calculating scenario probability (some plugs deteriorate, others remain intact). 
Granite (Inventory = $16.860 \mathrm{M} \mathrm{Cl}$ )

$7 \quad 0.0214 \quad 2.943 \mathrm{E}+00$

$8 \quad 0.0079 \quad 2.663 E+00$

$\begin{array}{lll}8 & 0.0011 & 1.509 \mathrm{E}+00\end{array}$

$10 \quad 0.0023 \quad 8.595 \mathrm{E}-02$ $11 \quad 0.0040 \quad 1.314 E-01$

$12 \quad 0.0205$

$13 \quad 0.0036$

No Hit: $\quad 0.9391$ 1.477E-01 $2.066 E+00$

\subsection{E-02} 2.807E-01 $2.775 \mathrm{E}+00$ 1.325E-01 $4.268 E+00$

$2.188 E+00$
1.146E-02

3.392E-02

8.591E-03

9.730E-01

9.582E-03

5.974E-01

5.856E-01

2.792E-01 EPA-Normalized Contents at Time of Intrusion (yr)

5

175

350

1000

3000

7250

$\begin{array}{lllll}6.724 E-03 & 2.536 E-03 & 1.375 E-03 & 1.356 E-03 & 1.543 E-03 \\ 1.990 E-02 & 7.505 E-03 & 4.070 E-03 & 4.015 E-03 & 4.567 E-03 \\ 4.108 E-03 & 1.240 E-03 & 4.704 E-04 & 2.387 E-04 & 1.903 E-04 \\ 8.118 E-01 & 5.594 E-01 & 2.288 E-01 & 6.748 E-02 & 5.356 E-02 \\ 4.268 E-03 & 1.184 E-03 & 7.384 E-04 & 6.497 E-04 & 5.580 E-04 \\ 3.574 E-01 & 1.126 E-01 & 2.992 E-02 & 1.705 E-02 & 1.359 E-02 \\ 4.259 E-01 & 2.741 E-01 & 9.645 E-02 & 5.881 E-03 & 1.809 E-03 \\ 1.903 E-01 & 1.166 E-01 & 4.650 E-02 & 1.092 E-02 & 7.614 E-03\end{array}$

$\begin{array}{lllll}2.464 \mathrm{E}-01 & 7.767 \mathrm{E}-02 & 2.063 \mathrm{E}-02 & 1.176 \mathrm{E}-02 & 9.368 \mathrm{E}-03 \\ 2.937 \mathrm{E}-01 & 1.890 \mathrm{E}-01 & 6.651 \mathrm{E}-02 & 4.055 \mathrm{E}-03 & 1.248 \mathrm{E}-03 \\ 1.312 \mathrm{E}-01 & 8.042 \mathrm{E}-02 & 3.206 \mathrm{E}-02 & 7.529 \mathrm{E}-03 & 5.250 \mathrm{E}-03 \\ 6.066 \mathrm{E}-03 & 2.269 \mathrm{E}-03 & 1.219 \mathrm{E}-03 & 1.208 \mathrm{E}-03 & 1.386 \mathrm{E}-03 \\ 4.233 \mathrm{E}-03 & 1.174 \mathrm{E}-03 & 7.322 \mathrm{E}-04 & 6.442 \mathrm{E}-04 & 5.533 \mathrm{E}-04 \\ 5.178 \mathrm{E}-03 & 1.506 \mathrm{E}-03 & 7.301 \mathrm{E}-04 & 5.216 \mathrm{E}-04 & 4.378 \mathrm{E}-04 \\ 5.647 \mathrm{E}-01 & 3.871 \mathrm{E}-01 & 1.586 \mathrm{E}-01 & 4.728 \mathrm{E}-02 & 3.758 \mathrm{E}-02\end{array}$

$\begin{array}{llllll}4.120 E-01 & 2.464 E-01 & 7.767 E-02 & 2.063 E-02 & 1.176 E-02 & 9.368 E-03 \\ 4.038 E-01 & 2.937 E-01 & 1.890 E-01 & 6.651 E-02 & 4.055 E-03 & 1.248 E-03 \\ 1.925 E-01 & 1.312 E-01 & 8.042 E-02 & 3.206 E-02 & 7.529 E-03 & 5.250 E-03 \\ 1.036 E-02 & 6.066 E-03 & 2.269 E-03 & 1.219 E-03 & 1.208 E-03 & 1.386 E-03 \\ 9.501 E-03 & 4.233 E-03 & 1.174 E-03 & 7.322 E-04 & 6.442 E-04 & 5.533 E-04 \\ 1.119 E-02 & 5.178 E-03 & 1.506 E-03 & 7.301 E-04 & 5.216 E-04 & 4.378 E-04 \\ 6.820 E-01 & 5.647 E-01 & 3.871 E-01 & 1.586 E-01 & 4.728 E-02 & 3.758 E-02\end{array}$

4.120E-01 4.038E-01 1.925E-01 1.036E-02 9.501E-03 1.119E-02 6.820E-01
1.201E-02

1.097E-03

$1.047 \mathrm{E}-03$

9.963E-02

2.424E-01

1.031E-01
6.515E-03

4.160E-04

2.023E-01

6.530E-04

2.646E-02

8.530E-02

4.112E-02
6.426E-03

2.112E-04

5.968E-02.

5.746E-04

$1.508 \mathrm{E}-02$

5.201E-03

9.657E-03
7.310E-03

.683E-04

$4.935 E-04$

1.202E-02

$1.600 \mathrm{E}-03$

6.734E-03

\footnotetext{
- Although the use of more than two significant digits may indicate a certainty beyond what is considered reasonable, we have maintained the four digits so that data output in subsequent calculations could be traced back to its original form in tables such as this.

** The probabilities are based on a borehole diameter of $0.30480 \mathrm{~m}$. If the borehole diameter is sampled, each vector will have its own table of hit probabilities. Repository Area $=162000 . \mathrm{m}^{2}$
} 


\title{
10. Modeling Drilling into Repository (CUTTINGS)
}

\author{
J. W. Berglund
}

Of the possible events that can influence the quantity of radionuclides reaching the accessible environment, one of the most important for a repository in bedded salt or granite is that caused by the inadvertent penetration of the disposal region by exploratory drilling. Such drilling can create a pathway that over time allows radionuclides to be released through groundwater flow; in fact, for example, the consequence models for the salt repository are solely concerned with this pathway. However, exploratory drilling can also cause an immediate release of radionuclides through the cuttings, cavings, or spallings brought to the surface in the drilling fluid (mud). Although the development of EPA release limits in 40 CFR 191 as promulgated in 1985 neglected direct release as a pathway and instead considered only the pathway to humans from drinking surface water, as noted in Chapter 2 and Appendix B, recent calculations for the WIPP Project have included the cuttings and cavings release (e.g., Bertram-Howery et al., 1990; WIPP Performance Assessment Division, 1991a; WIPP Performance Assessment Department, 1992a).

For the sake of completeness in this performance assessment, the direct release of radionuclides to the surface from cuttings and cavings was modeled. The discussion in Chapter 16 indicates those results for which these releases were included. A description of the cuttings model and intermediate results is provided below.

\subsection{Description of Drilling Mechanisms}

Three mechanisms have been identified that can influence the quantity of radionuclides brought to the ground surface when a WIPP-type waste disposal room is inadvertently penetrated by an exploratory borehole (Berglund, 1992). The first mechanism is due to the cutting action of the drill bit, which removes a volume of waste (cuttings) equal to the product of the drill bit area times the repository thickness. The second mechanism is due to the erosion of the borehole wall (cavings) adjacent to the waste caused by the flowing drilling fluid. The third mechanism concerns the borehole srall caused by the flow of waste-generated gas to the borehole. A model for the latter mechanism is still under development but this mechanism is not likely to affect releases from a repository based on disposal of containers in the floor of the disposal room.

For cuttings and cavings releases, the computer code, CUTTINGS, was developed (Rechard, Ed., 1992). The CUTTINGS code, which is currently being used to assess performance of the WIPP repository, has been modified to accommodate canister disposal of spent fuel and high-level waste. The modifications are required because direct removal of wastes to the accessible environment must be treated somewhat differently for the disposal technique of placing containers in the floor compared to the drum-in-drift disposal technique proposed for the WIPP.

\subsection{Description of Model}

For this performance assessment, any hit on a canister is assumed to remove the original but decayed contents of a container to the drilling mud pit and the surface; in essence, no material is assumed to diffuse out of the waste parcel in 10,000 yr. For consistency with this first assumption, no radionuclides are removed when the canister is missed. In actuality, after corrosion of the waste parcel, a plume of dissolved radionuclides would be transported from the waste parcel by advection and diffusion. Although this phenomenon is modeled in the groundwater flow situation, it was thought that here that the minor increase in accuracy would not warrant the difficulty of coupling the evaluation of the plume with the cuttings calculations. 


\subsection{Pertinent Model Information}

Forty-nine of the radionuclides in the inventory listed in Appendix A were accounted for using the CUTTINGS code. The remaining radionuclides were not considered because of their short half-life or small contribution to the overall inventory. Abbreviated chains for radioactive decay were also used in the CUTTINGS computations (see Figure 4-8 in Chapter 4).

The radionuclide inventory used is based on the "anticipated" wastes to be emplaced in the year 2030. This inventory is assumed to be divided among the waste parcels in each of the five disposal groups (Chapter 4). The actual release to the ground surface is governed by the probability of a drill bit intercepting a waste parcel during an intrusion, and thus is dependent upon the spacing of the waste parcels in the repository, the cross-sectional area of the waste parcels, drillbit diameter," and the type of waste parcel (Chapter 4).

\subsection{Intermediate Results}

As noted in Chapter 7 (Scenario Development), six time intervals (100-150; $150-200 ; 200-500 ; 500-1500 ; 1500$ 4500; and 4500-10,000 yr) were used to discretize the 10,000-yr regulatory period for calculating releases through drill cuttings. Drilling intrusions were assumed to occur at the midpoints of each of the six time intervals, i.e., 125, $175,350,1000,3000$, and $7250 \mathrm{yr}$. Releases through groundwater, however, were calculated using only a single intrusion at 1000 yr. For comparison, Table 10-1 shows the initial and potential activity release in curies from cuttings for each of the 26 waste parcels at the 1000 -yr intrusion. The potential activity releases for all six time intervals are tabulated in Appendix D.

A comparison of the radioactive decay within each of the 26 waste parcels is shown in Figures 10-1a through 10-1c. Generally, activity in a waste parcel decreases by factors of 100 to 1000 during the first $1000 \mathrm{yr}$. The most important radionuclides to the total activity at $1000 \mathrm{yr}$ can be evaluated from Table 10-1 (and, for other times, from the tables in Appendix D).

\subsection{Summary of Modeling Assumptions for Cuttings Removal}

The following assumptions were made for cuttings removal:

- Future drilling techniques during 10,000 -yr regulatory period are the same as today.

- Drilling that hits a canister removes entire original but decayed contents of a canister to the surface.

- Drilling that misses a canister removes no radionuclides.

- Boreholes are vertical.

- Effects of spalling from high gas pressure within the spent nuclear fuel and repository can be neglected.

- Activity of abbreviated decay chains for 49 inventory radionuclides are accurate enough to evaluate release.

- Intrusion drillbit diameter is $0.3048 \mathrm{~m}$.

Intrusion drillbit diameter is assumed to be $0.3048 \mathrm{~m}$. 
Table 10-1. Contents (in Curies) of 49 Radionuclides of Waste Parcels at Time of Emplacement (Year 2030) and after $1000 \mathrm{Yr}$

\begin{tabular}{|c|c|c|c|c|c|c|}
\hline \multirow[b]{2}{*}{ Nuclide } & \multicolumn{2}{|c|}{ Waste Parsel 1} & \multicolumn{2}{|c|}{ Waste Parcel 2} & \multicolumn{2}{|c|}{ Waste Parcel 3} \\
\hline & $\begin{array}{l}\text { Time of } \\
\text { Emplacement } \\
\text { (year 2030) }\end{array}$ & $\begin{array}{l}\text { Time of } \\
\text { Intrusion } \\
\text { (year 3030) }\end{array}$ & $\begin{array}{l}\text { Time of } \\
\text { Emplacement } \\
\text { (year 2030) }\end{array}$ & $\begin{array}{l}\text { Time of } \\
\text { Intrusion } \\
\text { (year 3030) }\end{array}$ & $\begin{array}{l}\text { Time of } \\
\text { Emplacement } \\
\text { (year 2030) }\end{array}$ & $\begin{array}{c}\text { Time of } \\
\text { Intrusion } \\
\text { (year 3030) }\end{array}$ \\
\hline${ }^{14} \mathrm{C}$ & $2.928 E-01$ & 2.594E-01 & 5.465E-02 & 4.842E-02 & 1.617E-01 & 1.433E-01 \\
\hline${ }^{36} \mathrm{Cl}$ & $3.287 E-03$ & $3.279 E-03$ & 5.400E-04 & 5.388E-04 & $2.179 E-04$ & 2.174E-04 \\
\hline${ }^{59} \mathrm{Ni}$ & 2.294E-02 & 2.273E-02 & 3.768E-03 & 3.733E-03 & $1.521 \mathrm{E}-03$ & 1.507E-03 \\
\hline${ }^{63} \mathrm{Ni}$ & 4.955E-01 & 3.604E-04 & 8.139E-02 & 5.919E-05 & $3.285 E-02$ & 2.389E-05 \\
\hline${ }^{79} \mathrm{Se}$ & 3.563E-02 & 3.525E-02 & 5.853E-03 & 5.791E-03 & 2.362E-03 & 2.337E-03 \\
\hline${ }^{87} \mathrm{Rb}$ & 2.122E-06 & 2.122E-06 & 3.486E-07 & 3.486E-07 & $1.407 \mathrm{E}-07$ & 1.407E-07 \\
\hline${ }^{90} \mathrm{Sr}$ & $2.319 E+03$ & 1.065E-07 & $3.809 E+02$ & 1.749E-08 & $1.538 \mathrm{E}+02$ & 7.062E-09 \\
\hline${ }^{93} \mathrm{Zr}$ & $1.411 E+00$ & $1.410 E+00$ & 2.318E-01 & 2.317E-01 & 9.357E-02 & 9.353E-02 \\
\hline${ }^{99} \mathrm{TC}$ & 8.921E-01 & 8.892E-01 & $1.465 E-01$ & $1.460 \mathrm{E}-01$ & 5.914E-02 & 5.895E-02 \\
\hline${ }^{107} \mathrm{Pd}$ & $1.047 E-03$ & 1.047E-03 & 1.720E-04 & $1.720 \mathrm{E}-04$ & 6.944E-05 & $6.943 \mathrm{E}-05$ \\
\hline${ }^{113} \mathrm{Cd}$ & $4.842 E-15$ & $4.842 E-15$ & 7.953E-16 & 7.953E-16 & $3.210 E-16$ & $3.210 E-16$ \\
\hline${ }^{113 m} \mathrm{Cd}$ & $0.000 E+00$ & $0.000 E+00$ & $0.000 E+00$ & $0.000 E+00$ & $0.000 E+00$ & $0.000 E+00$ \\
\hline${ }^{126} \mathrm{Sn}$ & 1.553E-02 & $1.542 E-02$ & 2.551E-03 & $2.533 E-03$ & $1.030 E-03$ & 1.023E-03 \\
\hline $129 \mid$ & 2.670E-03 & 2.670E-03 & 4.385E-04 & 4.385E-04 & $1.770 \mathrm{E}-04$ & $1.770 E-04$ \\
\hline${ }^{135} \mathrm{Crs}$ & 1.697E-02 & $1.696 \mathrm{E}-02$ & 2.788E-03 & 2.787E-03 & $1.125 \mathrm{E}-03$ & $1.125 \mathrm{E}-03$ \\
\hline${ }^{137} \mathrm{Cs}$ & $2.541 E+03$ & 2.347E-07 & 4.173E+02 & 3.855E-08 & $1.684 E+02$ & $1.556 \mathrm{E}-08$ \\
\hline${ }^{144} \mathrm{Nd}$ & 2.385E-06 & 2.385E-06 & 3.917E-07 & 3.917E-07 & $1.581 \mathrm{E}-07$ & $1.581 E-07$ \\
\hline${ }^{147} \mathrm{Pm}$ & $0.000 E+00$ & $0.000 E+00$ & $0.000 E+00$ & $0.000 E+00$ & $0.000 E+00$ & $0.000 E+00$ \\
\hline${ }^{147} \mathrm{Sm}$ & 7.683E-03 & 7.683E-03 & $1.262 E-03$ & $1.262 E-03$ & $5.094 E-04$ & 5.094E-04 \\
\hline${ }^{151} \mathrm{Sm}$ & $6.851 E+01$ & 3.079E-02 & $1.125 E+01$ & 5.057E-03 & $4.542 E+00$ & 2.042E-03 \\
\hline${ }^{152} \mathrm{Eu}$ & $0.000 E+00$ & $0.000 E+00$ & $0.000 E+00$ & $0.000 E+00$ & $0.000 E+00$ & $0.000 E+00$ \\
\hline${ }^{210} \mathrm{~Pb}$ & $0.000 E+00$ & $0.000 E+00$ & $0.000 E+00$ & $0.000 E+00$ & $0.000 E+00$ & $0.000 E+00$ \\
\hline${ }^{226} \mathrm{Ra}$ & $0.000 E+00$ & 1.127E-03 & $0.000 E+00$ & $1.852 E-04$ & $0.000 E+00$ & 7.471E-05 \\
\hline${ }^{228} \mathrm{Ra}$ & $0.000 E+00$ & $0.000 E+00$ & $0.000 E+00$ & $0.000 E+00$ & $0.000 E+00$ & $0.000 E+00$ \\
\hline${ }^{229} \mathrm{Th}$ & $0.000 E+00$ & 4.699E-01 & $0.000 E+00$ & 7.719E-02 & $0.000 E+00$ & 3.116E-02 \\
\hline${ }^{230} \mathrm{Th}$ & $0.000 E+00$ & $6.203 E-03$ & $0.000 E+00$ & $1.019 \mathrm{E}-03$ & $0.000 E+00$ & 4.113E-04 \\
\hline${ }^{232} \mathrm{Th}$ & $6.165 \mathrm{E}-03$ & 6.165E-03 & 1.013E-03 & $1.013 E-03$ & 4.087E-04 & 4.087E-04 \\
\hline
\end{tabular}


10. Modeling Drilling into Repository (CUTTINGS)

Table 10-1. Contents (in Curies) of 49 Radionuclides of Waste Parcels at Time of Emplacement (Year 2030) and after $1000 \mathrm{Yr}$ (continued)

\begin{tabular}{|c|c|c|c|c|c|c|}
\hline \multirow[b]{2}{*}{ Nuclide } & \multicolumn{2}{|c|}{ Waste Parcel 1} & \multicolumn{2}{|c|}{ Waste Parcel 2} & \multicolumn{2}{|c|}{ Waste Parcel 3} \\
\hline & $\begin{array}{l}\text { Time of } \\
\text { Emplacement } \\
\text { (year 2030) }\end{array}$ & $\begin{array}{c}\text { Time of } \\
\text { Intrusion } \\
\text { (year 3030) }\end{array}$ & $\begin{array}{l}\text { Time of } \\
\text { Emplacement } \\
\text { (year 2030) }\end{array}$ & $\begin{array}{l}\text { Time of } \\
\text { Intrusion } \\
\text { (year 3030) }\end{array}$ & $\begin{array}{l}\text { Time of } \\
\text { Emplacement } \\
\text { (year 2030) }\end{array}$ & $\begin{array}{c}\text { Time of } \\
\text { Intrusion } \\
\text { (year 3030) }\end{array}$ \\
\hline${ }^{231} \mathrm{~Pa}$ & $0.000 E+00$ & $6.503 E-05$ & $0.000 E+00$ & $1.068 \mathrm{E}-05$ & $0.000 E+00$ & 4.311E-06 \\
\hline $232 U$ & $2.600 E+00$ & $1.706 \mathrm{E}-04$ & 4.270E-01 & 2.802E-05 & 1.723E-01 & 1.131E-05 \\
\hline${ }^{233} \mathrm{U}$ & $5.226 E+00$ & $5.203 E+00$ & 8.584E-01 & 8.547E-01 & 3.465E-01 & $3.450 E-01$ \\
\hline $234 \mathrm{U}$ & 6.670E-01 & 6.951E-01 & $1.096 \mathrm{E}-01$ & 1.142E-01 & 4.422E-02 & 4.608E-02 \\
\hline${ }^{235} U$ & 3.106E-03 & $3.106 \mathrm{E}-03$ & 5.102E-04 & 5.102E-04 & 2.059E-04 & 2.059E-04 \\
\hline${ }^{236} \mathrm{U}$ & 2.572E-02 & 2.573E-02 & 4.225E-03 & 4.227E-03 & $1.705 E-03$ & $1.706 \mathrm{E}-03$ \\
\hline${ }^{238} \mathrm{U}$ & $7.156 E-05$ & $7.156 E-05$ & 1.175E-05 & $1.175 \mathrm{E}-05$ & 4.744E-06 & 4.744E-06 \\
\hline${ }^{237} \mathrm{~Np}$ & 1.852E-02 & $1.920 \mathrm{E}-02$ & $3.042 E-03$ & 3.154E-03 & $1.228 \mathrm{E}-03$ & $1.273 E-03$ \\
\hline${ }^{238} \mathrm{Pu}$ & $8.376 E+01$ & $3.108 \mathrm{E}-02$ & $1.376 \mathrm{E}+01$ & 5.106E-03 & $5.553 E+00$ & 2.061E-03 \\
\hline${ }^{239} \mathrm{Pu}$ & 2.595E-01 & 2.530E-01 & 4.262E-02 & 4.156E-02 & $1.720 \mathrm{E}-02$ & 1.677E-02 \\
\hline${ }^{240} \mathrm{Pu}$ & 4.670E-01 & 4.216E-01 & 7.670E-02 & $6.924 \mathrm{E}-02$ & $3.096 \mathrm{E}-02$ & 2.795E-02 \\
\hline${ }^{241} \mathrm{Pu}$ & $0.000 E+00$ & $0.000 E+00$ & $0.000 E+00$ & $0.000 E+00$ & $0.000 E+00$ & $0.000 E+00$ \\
\hline${ }^{242} \mathrm{Pu}$ & $0.000 E+00$ & $1.450 E-07$ & $0.000 E+00$ & 2.381E-08 & $0.000 E+00$ & 9.611E-09 \\
\hline${ }^{244} \mathrm{Pu}$ & $0.000 E+00$ & $0.000 E+00$ & $0.000 E+00$ & $0.000 \mathrm{E}+00$ & $0.000 E+00$ & $0.000 E+00$ \\
\hline${ }^{241} \mathrm{Am}$ & $4.247 E+00$ & $8.546 \mathrm{E}-01$ & $6.975 E-01$ & 1.403E-01 & 2.815E-01 & 5.664E-02 \\
\hline${ }^{242 m} \mathrm{Am}$ & 5.344E-04 & $5.570 E-06$ & 8.778E-05 & $9.150 \mathrm{E}-07$ & 3.543E-05 & 3.693E-07 \\
\hline${ }^{243} \mathrm{Am}$ & 3.285E-02 & $2.990 E-02$ & $5.396 \mathrm{E}-03$ & 4.911E-03 & 2.178E-03 & $1.982 E-03$ \\
\hline${ }^{243} \mathrm{Cm}$ & 1.873E-03 & $5.051 E-14$ & $3.076 \mathrm{E}-04$ & 8.294E-15 & $1.242 E-04$ & $3.349 E-15$ \\
\hline${ }^{244} \mathrm{Cm}$ & 6.271E-01 & $0.000 E+00$ & 1.030E-01 & $0.000 E+00$ & 4.158E-02 & $0.000 E+00$ \\
\hline${ }^{245} \mathrm{Cm}$ & $2.928 E-04$ & 2.699E-04 & 4.809E-05 & 4.432E-05 & $1.941 E-05$ & $1.789 \mathrm{E}-05$ \\
\hline${ }^{246} \mathrm{Cm}$ & 8.471E-05 & 7.316E-05 & 1.391E-05 & $1.201 E-05$ & $5.616 \mathrm{E}-06$ & $4.850 \mathrm{E}-06$ \\
\hline${ }^{247} \mathrm{Cm}$ & $2.511 E-10$ & $2.511 E-10$ & 4.125E-11 & 4.125E-11 & $1.665 \mathrm{E}-11$ & $1.665 \mathrm{E}-11$ \\
\hline Total & $4.030 E+03$ & $1.074 E+01$ & $8.261 E+02$ & $1.766 E+00$ & $3.336 E+02$ & 8.365E-01 \\
\hline
\end{tabular}

Note: Boldface values represent the most dominant radiolsotope (largest activity) in a waste parcel. 
Table 10-1. Contents (in Curies) of 49 Radionuclides of Waste Parcels at Time of Emplacement (Year 2030) and after $1000 \mathrm{Yr}$ (continued)

\begin{tabular}{|c|c|c|c|c|c|c|}
\hline \multirow[b]{2}{*}{ Nuclide } & \multicolumn{2}{|c|}{ Waste Parcel 4} & \multicolumn{2}{|c|}{ Waste Parcel 5} & \multicolumn{2}{|c|}{ Waste Parcel 6} \\
\hline & $\begin{array}{l}\text { Time of } \\
\text { Emplacement } \\
\text { (year 2030) }\end{array}$ & $\begin{array}{c}\text { Time of } \\
\text { Intrusion } \\
\text { (year 3030) }\end{array}$ & $\begin{array}{c}\text { Time of } \\
\text { Emplacement } \\
\text { (year 2030) }\end{array}$ & $\begin{array}{c}\text { Time of } \\
\text { Intrusion } \\
\text { (year 3030) }\end{array}$ & $\begin{array}{l}\text { Time of } \\
\text { Emplacement } \\
\text { (year 2030) }\end{array}$ & $\begin{array}{c}\text { Time of } \\
\text { Intrusion } \\
\text { (year 3030) }\end{array}$ \\
\hline${ }^{14} \mathrm{C}$ & $1.408 E-06$ & 1.247E-06 & $3.468 \mathrm{E}-01$ & 3.073E-01 & $0.000 E+00$ & $0.000 \mathrm{E}+00$ \\
\hline${ }^{36} \mathrm{Cl}$ & $0.000 E+00$ & $0.000 E+00$ & 2.568E-03 & 2.562E-03 & $0.000 E+00$ & $0.000 E+00$ \\
\hline${ }^{59} \mathrm{NI}$ & $0.000 E+00$ & $0.000 E+00$ & $1.171 E+00$ & $1.160 E+00$ & $0.000 E+00$ & $0.000 E+00$ \\
\hline${ }^{63} \mathrm{Ni}$ & $0.000 E+00$ & $0.000 E+00$ & $1.194 \mathrm{E}+02$ & 8.684E-02 & $0.000 E+00$ & $0.000 E+00$ \\
\hline${ }^{79} \mathrm{Se}$ & 5.075E-03 & $5.021 \mathrm{E}-03$ & $9.212 E-02$ & $9.114 \mathrm{E}-02$ & $0.000 E+00$ & $0.000 E+00$ \\
\hline${ }^{87} \mathrm{Rb}$ & 3.416E-07 & $3.416 E-07$ & $0.000 E+00$ & $0.000 E+00$ & $0.000 E+00$ & $0.000 E+00$ \\
\hline${ }^{90} \mathrm{Sr}$ & $5.766 E+02$ & $2.648 E-08$ & $8.063 E+03$ & 3.702E-07 & $6.782 E+02$ & $3.114 E-08$ \\
\hline${ }^{93} \mathrm{Zr}$ & 2.607E-02 & $2.606 \mathrm{E}-02$ & 4.065E-01 & 4.063E-01 & $0.000 E+00$ & $0.000 E+00$ \\
\hline${ }^{99} \mathrm{TC}$ & 1.710E-01 & $1.704 E-01$ & $2.939 E+00$ & $2.929 E+00$ & $0.000 E+00$ & $0.000 E+00$ \\
\hline${ }^{107} \mathrm{Pd}$ & 1.971E-04 & 1.971E-04 & 2.523E-02 & 2.523E-02 & $0.000 E+00$ & $0.000 E+00$ \\
\hline${ }^{113} \mathrm{Cd}$ & $0.000 E+00$ & $0.000 E+00$ & $0.000 E+00$ & $0.000 E+00$ & $0.000 E+00$ & $0.000 E+00$ \\
\hline${ }^{113 m} \mathrm{Cd}$ & $3.930 \mathrm{E}-02$ & $0.000 E+00$ & $0.000 E+00$ & $0.000 E+00$ & $0.000 E+00$ & $0.00 \cup E+00$ \\
\hline${ }^{126} \mathrm{Sn}$ & $4.542 E-03$ & 4.511E-03 & $1.745 \mathrm{E}-01$ & $1.733 E-01$ & $0.000 E+00$ & $0.000 E+00$ \\
\hline $129 j$ & 2.804E-04 & $2.804 E-04$ & 7.117E-03 & 7.117E-03 & $0.000 E+00$ & $0.000 E+00$ \\
\hline${ }^{135} \mathrm{Cs}$ & 1.325E-03 & 1.325E-03 & 7.770E-02 & 7.768E-02 & $0.000 E+00$ & $0.000 E+00$ \\
\hline${ }^{137} \mathrm{Cs}$ & $6.122 E+02$ & 5.655E-08 & $1.171 E+04$ & $1.082 E-06$ & $7.905 E+02$ & 7.302E-08 \\
\hline${ }^{144} \mathrm{Nd}$ & $0.000 E+00$ & $9.085 E-20$ & $0.000 E+00$ & $2.620 \mathrm{E}-22$ & $0.000 E+00$ & 1.133E-37 \\
\hline${ }^{147} \mathrm{Pm}$ & $7.109 E+00$ & $0.000 E+00$ & $1.124 E+01$ & $0.000 E+00$ & $1.055 E-08$ & $0.000 E+00$ \\
\hline${ }^{147} \mathrm{Sm}$ & 9.597E-08 & $9.615 E-08$ & $0.000 E+00$ & $2.783 E-10$ & $0.000 E+00$ & 2.612E-19 \\
\hline${ }^{151} \mathrm{Sm}$ & $3.594 E+00$ & $1.615 E-03$ & $6.453 E+01$ & 2.901E-02 & $0.000 E+00$ & $0.000 E+00$ \\
\hline${ }^{152} \mathrm{Eu}$ & $0.000 E+00$ & $0.000 E+00$ & $0.000 E+00$ & $0.000 E+00$ & $0.000 E+00$ & $0.000 E+00$ \\
\hline${ }^{210} \mathrm{pb}$ & $2.192 E-10$ & $4.745 E-08$ & $1.408 E-07$ & 2.734E-05 & $0.000 E+00$ & $3.394 \mathrm{E}-06$ \\
\hline${ }^{226} \mathrm{Ra}$ & 8.393E-10 & $1.283 E-06$ & 5.372E-07 & $5.246 E-04$ & $0.000 E+00$ & 2.339E-05 \\
\hline${ }^{228} \mathrm{Ra}$ & $0.000 E+00$ & $0.000 E+00$ & $0.000 E+00$ & $0.000 E+00$ & $0.000 E+00$ & $0.000 E+00$ \\
\hline${ }^{229} \mathrm{Th}$ & $1.286 \mathrm{E}-09$ & 5.903E-08 & 3.356E-08 & 1.168E-06 & $0.000 E+00$ & 4.312E-05 \\
\hline${ }^{230} \mathrm{Th}$ & 1.376E-07 & 2.302E-05 & 7.917E-05 & $3.909 E-03$ & 9.957E-06 & $1.324 E-04$ \\
\hline${ }^{232} \mathrm{Th}$ & $0.000 E+00$ & 2.857E-10 & $0.000 E+00$ & 2.853E-09 & 2.986E-11 & 3.797E-11 \\
\hline
\end{tabular}

Note: Boldface values represent the most dominant radioisotope (largest activity) in a waste parcel. 


\section{1} 3 3 5 6 8
9 9
10 11 12

Table 10-1. Contents (in Curies) of 49 Radionuclides of Waste Parcels at Time of Emplacement (Year 2030) and after $1000 \mathrm{Yr}$ (continued)

\begin{tabular}{|c|c|c|c|c|c|c|}
\hline \multirow[b]{2}{*}{ Nuclide } & \multicolumn{2}{|c|}{ Waste Parcel 4} & \multicolumn{2}{|c|}{ Waste Parcel 5} & \multicolumn{2}{|c|}{ Waste Parcel 6} \\
\hline & $\begin{array}{l}\text { Time of } \\
\text { Emplacement } \\
\text { (year 2030) }\end{array}$ & $\begin{array}{l}\text { Time of } \\
\text { Intrusion } \\
\text { (year 3030) }\end{array}$ & $\begin{array}{l}\text { Time of } \\
\text { Emplacement } \\
\text { (year 2030) }\end{array}$ & $\begin{array}{c}\text { Time of } \\
\text { Intrusion } \\
\text { (year 3030) }\end{array}$ & $\begin{array}{l}\text { Time of } \\
\text { Emplacement } \\
\text { (year 2030) }\end{array}$ & $\begin{array}{l}\text { Time of } \\
\text { Intrusion } \\
\text { (year 3030) }\end{array}$ \\
\hline${ }^{231} \mathrm{~Pa}$ & 9.795E-07 & 3.044E-05 & 5.236E-06 & 8.623E-05 & $0.000 E+00$ & 1.510E-06 \\
\hline${ }^{232} \mathrm{U}$ & $2.409 E-05$ & 1.581E-09 & $0.000 E+00$ & $0.000 E+00$ & $0.000 E+00$ & $0.000 E+00$ \\
\hline${ }^{233} \mathrm{U}$ & 6.418E-07 & 1.581E-05 & 1.261E-05 & $3.388 E-04$ & 4.795E-04 & 9.118 E-04 \\
\hline${ }^{234} \mathrm{U}$ & 7.306E-04 & 2.812E-03 & 2.939E-01 & 4.462E-01 & 1.178E-02 & 1.393E-02 \\
\hline${ }^{235} U$ & 1.408E-03 & 1.408E-03 & 3.874E-03 & 3.942E-03 & 7.214E-05 & 7.264E-05 \\
\hline${ }^{236} \mathrm{U}$ & 5.786E-03 & 5.789E-03 & 5.777E-02 & 5.781E-02 & 1.613E-04 & $1.674 E-04$ \\
\hline${ }^{238} \mathrm{U}$ & 2.587E-05 & 2.587E-05 & 7.151E-02 & 7.151E-02 & 4.046E-06 & 4.046E-06 \\
\hline${ }^{237} \mathrm{~Np}$ & 3.476E-03 & 3.675E-03 & 7.477E-02 & 2.276E-01 & 9.957E-02 & 9.957E-02 \\
\hline${ }^{238} \mathrm{Pu}$ & 5.825E+00 & 2.165E-03 & 4.279E+02 & 1.587E-01 & $6.091 E+00$ & 2.259E-03 \\
\hline${ }^{239} \mathrm{Pu}$ & 1.850E-01 & 1.798E-01 & 7.050E+01 & $6.860 E+01$ & 5.140E-01 & 4.995E-01 \\
\hline${ }^{240} \mathrm{Pu}$ & $1.055 E-01$ & 9.491E-02 & 1.182E+00 & 1.335E+00 & 2.189E-01 & 1.969E-01 \\
\hline${ }^{241} \mathrm{Pu}$ & $8.886 E+00$ & $0.000 E+00$ & $6.700 E+03$ & $0.000 E+00$ & $0.000 E+00$ & $0.000 E+00$ \\
\hline${ }^{242} \mathrm{Pu}$ & 1.584E-04 & 1.581E-04 & 3.964E-01 & 3.957E-01 & 1.073E-04 & 1.071E-04 \\
\hline${ }^{244} \mathrm{Pu}$ & 2.883E-11 & 2.883E-11 & $0.000 E+00$ & $0.000 E+00$ & $0.000 E+00$ & $0.000 E+00$ \\
\hline${ }^{241} \mathrm{Am}$ & 9.439E-01 & $2.4945-01$ & $7.241 E+02$ & $1.906 \Xi+02$ & 1.991E-01 & 4.005E-02 \\
\hline $242 m A m$ & 1.744E-04 & $1.818 E-06$ & $0.000 E+00$ & $0.000 E+00$ & $0.000 E+00$ & $0.000 E+00$ \\
\hline${ }^{243} \mathrm{Am}$ & 6.536E-04 & 5.949E-04 & $3.829 E+00$ & $3.485 E+00$ & 3.893E-03 & 3.543E-03 \\
\hline${ }^{243} \mathrm{Cm}$ & 5.450E-05 & $1.470 E-15$ & $0.000 E+00$ & $0.000 E+00$ & $0.000 E+00$ & $0.000 E+00$ \\
\hline${ }^{244} \mathrm{Cm}$ & 8.689E-03 & $0.000 E+00$ & $1.090 E+02$ & $0.000 E+00$ & $0.000 E+00$ & $0.000 E+00$ \\
\hline${ }^{245} \mathrm{Cm}$ & 1.374E-06 & 1.266E-06 & $0.000 E+00$ & $0.000 E+00$ & $0.000 E+\cap 0$ & $0.000 E+00$ \\
\hline${ }^{246} \mathrm{Cm}$ & $9.459 E-08$ & 8.169E-08 & $0.000 E+00$ & $0.000 E+00$ & $0.000 E+00$ & $0.000 E+00$ \\
\hline${ }^{247} \mathrm{Cm}$ & $0.000 E+00$ & $0.000 E+00$ & $0.000 E+00$ & $0.000 E+00$ & $0.000 E+00$ & $0.000 E+00$ \\
\hline Total & $1.216 E+03$ & 7.502E-01 & 2.801E+04 & $2.706 E+02$ & $1.476 E+03$ & 8.572E-01 \\
\hline
\end{tabular}

Note: Boldface values represent the most dominant radioisotope (largest activity) in a waste parcel. 
Table 10-1. Contents (in Curies) of 49 Radionuclides of Waste Parcels at Time of Emplacement (Year 2030) and after $1000 \mathrm{Yr}$ (continued)

\begin{tabular}{|c|c|c|c|c|c|c|}
\hline \multirow[b]{2}{*}{ Nuclitle } & \multicolumn{2}{|c|}{ Waste Parcel 7} & \multicolumn{2}{|c|}{ Waste Parcel 8} & \multicolumn{2}{|c|}{ Waste Parcel 9} \\
\hline & $\begin{array}{l}\text { Time of } \\
\text { Emplacement } \\
\text { (year 2030) }\end{array}$ & $\begin{array}{c}\text { Time of } \\
\text { Intrusion } \\
\text { (year 3030) }\end{array}$ & $\begin{array}{c}\text { Time of } \\
\text { Emplacement } \\
\text { (year 2030) }\end{array}$ & $\begin{array}{l}\text { Time of } \\
\text { Intrusion } \\
\text { (year 3030) }\end{array}$ & $\begin{array}{l}\text { Time of } \\
\text { Emplacement } \\
\text { (year 2030) }\end{array}$ & $\begin{array}{c}\text { Time of } \\
\text { Intrusion } \\
\text { (year 3030) }\end{array}$ \\
\hline${ }^{14} \mathrm{C}$ & $0.000 E+00$ & $0.000 \mathrm{E}+00$ & $0.000 E+00$ & $0.000 E+00$ & $0.000 E+00$ & $0.000 \mathrm{E}+00$ \\
\hline${ }^{36} \mathrm{Cl}$ & $0.000 E+00$ & $0.000 E+00$ & $0.000 E+00$ & $0.000 E+00$ & $0.000 E+00$ & $0.000 E+00$ \\
\hline${ }^{59} \mathrm{Ni}$ & 2.396E-02 & 2.374E-02 & $1.360 \mathrm{E}-01$ & 1.347E-01 & 4.145E-01 & 4.107E-01 \\
\hline${ }^{63} \mathrm{Ni}$ & $2.271 E+00$ & $1.652 \mathrm{E}-03$ & $1.199 E+01$ & $8.720 \mathrm{E}-03$ & $2.299 E+01$ & 1.672E-02 \\
\hline${ }^{79} \mathrm{Se}$ & $0.000 E+00$ & $0.000 E+00$ & $3.898 \mathrm{E}-03$ & 3.857E-03 & $1.374 \mathrm{E}-02$ & 1.359E-02 \\
\hline${ }^{87} \mathrm{Rb}$ & 8.717E-07 & 8.717E-07 & $0.000 \mathrm{E}+00$ & $0.000 E+00$ & $0.000 E+00$ & $0.000 E+00$ \\
\hline${ }^{90} \mathrm{Sr}$ & $1.925 E+04$ & $8.839 \mathrm{E}-07$ & $1.722 E+04$ & 7.907E-07 & $1.080 E+04$ & 4.959E-07 \\
\hline${ }^{93} \mathrm{Zr}$ & $1.117 E+00$ & $1.117 E+00$ & $1.290 E+00$ & $1.289 E+00$ & $1.066 \mathrm{E}+00$ & $1.066 \mathrm{E}+00$ \\
\hline${ }^{99} \mathrm{TC}$ & $3.078 E+00$ & $3.068 E+00$ & $9.352 E+00$ & $9.322 E+00$ & 4.264E-01 & 4.250E-01 \\
\hline${ }^{107} \mathrm{Pd}$ & 1.473E-02 & $1.473 E-02$ & $4.070 \mathrm{E}-02$ & $4.070 \mathrm{E}-02$ & $4.315 \mathrm{E}-02$ & 4.315E-02 \\
\hline${ }^{113} \mathrm{Cd}$ & 5.009E-14 & $5.009 \mathrm{E}-14$ & $0.000 E+00$ & $0.000 E+00$ & $0.000 E+00$ & $0.000 E+00$ \\
\hline${ }^{113 m} \mathrm{Cd}$ & $0.000 E+00$ & $0.000 E+00$ & $2.184 E+00$ & $0.000 E+00$ & $1.243 E+00$ & $0.000 E+00$ \\
\hline${ }^{126} \mathrm{Sn}$ & 4.412E-01 & 4.381E-01 & 4.599E-01 & 4.567E-01 & 4.076E-01 & 4.048E-01 \\
\hline 129 & 2.786E-20 & $2.786 E-20$ & $1.630 \mathrm{E}-05$ & $1.630 \mathrm{E}-05$ & $0.000 E+00$ & $0.000 E+00$ \\
\hline${ }^{135} \mathrm{Cs}$ & $9.941 \mathrm{E}-02$ & $9.938 \mathrm{E}-02$ & $2.510 \mathrm{E}-01$ & 2.509E-01 & 6.319E-01 & 6.317E-01 \\
\hline${ }^{137} \mathrm{Cs}$ & $1.835 E+04$ & 1.695E-06 & 2.157E+04 & 1.993E-06 & $1.192 E+04$ & 1.101E-06 \\
\hline${ }^{144} \mathrm{Nd}$ & 4.863E-09 & 4.863E-09 & $1.105 E-11$ & $1.105 \mathrm{E}-11$ & $9.460 \mathrm{E}-19$ & $9.460 \mathrm{E}-19$ \\
\hline${ }^{147} \mathrm{Pm}$ & $1.286 E+00$ & $0.000 E+00$ & $2.111 E+00$ & $0.000 E+00$ & $1.828 \mathrm{E}-02$ & $0.000 E+00$ \\
\hline${ }^{147} \mathrm{Sm}$ & 2.597E-06 & 2.597E-06 & $9.832 E-07$ & 9.832E-07 & 8.514E-09 & 8.515E-09 \\
\hline${ }^{151} \mathrm{Sm}$ & $1.859 E+02$ & 8.356E-02 & $6.276 E+02$ & 2.821E-01 & $2.475 E+02$ & 1.112E-01 \\
\hline${ }^{152} \mathrm{Eu}$ & 5.308E-01 & $0.000 E+00$ & 3.944E-01 & $0.000 E+00$ & 2.051E-01 & $0.000 E+00$ \\
\hline${ }^{210} \mathrm{~Pb}$ & $0.000 E+00$ & $1.207 E-05$ & $0.000 E+00$ & $5.549 \mathrm{E}-07$ & $1.538 \mathrm{E}-06$ & 8.466E-05 \\
\hline${ }^{226} \mathrm{Ra}$ & $0.000 E+00$ & 2.996E-04 & $0.000 E+00$ & 8.831E-06 & $3.764 \mathrm{E}-06$ & 1.196E-04 \\
\hline${ }^{228} \mathrm{Ra}$ & $0.000 E+00$ & $0.000 E+00$ & $0.000 E+00$ & $0.000 E+00$ & $6.424 E-03$ & $0.000 E+00$ \\
\hline${ }^{229} \mathrm{Th}$ & 5.561E-09 & 2.779E-07 & $0.000 E+00$ & 2.915E-06 & $9.435 \mathrm{E}-04$ & $4.040 \mathrm{E}-03$ \\
\hline${ }^{230} \mathrm{Th}$ & 3.542E-05 & 4.657E-03 & $1.628 \mathrm{E}-06$ & $4.698 \mathrm{E}-05$ & $2.411 E-04$ & 4.813E-04 \\
\hline${ }^{232} \mathrm{Th}$ & $2.131 E-12$ & $6.421 \mathrm{E}-11$ & $8.980 E-13$ & $2.476 \mathrm{E}-11$ & $6.428 \mathrm{E}-03$ & $6.428 E-03$ \\
\hline
\end{tabular}

Note: Boldface values represent the most dominant radioisotope (largest activity) in a waste parcel. 
Table 10-1. Contents (in Curies) of 49 Radionuclides of Waste Parcels at Time of Emplacement (Year 2030) and after 1000 Yr (continued)

\begin{tabular}{|c|c|c|c|c|c|c|}
\hline \multirow[b]{2}{*}{ Nuclide } & \multicolumn{2}{|c|}{ Waste Parcel 7} & \multicolumn{2}{|c|}{ Waste Parcel 8} & \multicolumn{2}{|c|}{ Waste Parcel 9} \\
\hline & $\begin{array}{c}\text { Time of } \\
\text { Emplacement } \\
\text { (year 2030) }\end{array}$ & $\begin{array}{l}\text { Time of } \\
\text { Intrusion } \\
\text { (year 3030) }\end{array}$ & $\begin{array}{c}\text { Time of } \\
\text { Ernplacement } \\
\text { (year 2030) }\end{array}$ & $\begin{array}{c}\text { Time of } \\
\text { Intrusion } \\
\text { (year 3030) }\end{array}$ & $\begin{array}{c}\text { Time of } \\
\text { Emplacement } \\
\text { (year 2030) }\end{array}$ & $\begin{array}{c}\text { Time of } \\
\text { Intrusion } \\
\text { (year 3030) }\end{array}$ \\
\hline${ }^{231} \mathrm{~Pa}$ & $1.239 \mathrm{E}-07$ & 3.425E-06 & $1.552 E-07$ & $4.276 E-06$ & $5.946 \mathrm{E}-02$ & $5.822 E-02$ \\
\hline $232 U$ & $1.287 \mathrm{E}-02$ & 8.445E-07 & $0.000 E+00$ & $0.000 E+00$ & 1.903E-02 & 1.249E-06 \\
\hline${ }^{233} \mathrm{U}$ & $3.034 E-06$ & 4.396E-05 & 3.242E-05 & $9.302 E-04$ & 3.538E-02 & 3.564E-02 \\
\hline${ }^{234} U$ & $1.700 \mathrm{E}-01$ & 5.648E-01 & $4.890 E-03$ & $5.081 E-03$ & $1.941 \mathrm{E}-02$ & 2.811E-02 \\
\hline $235 \mathrm{U}$ & $1.578 \mathrm{E}-04$ & $1.703 \mathrm{E}-04$ & $1.970 E-04$ & $1.984 \mathrm{E}-04$ & $3.529 \mathrm{E}-04$ & $3.591 E-04$ \\
\hline${ }^{236} \mathrm{U}$ & $1.137 \mathrm{E}-03$ & $1.388 E-03$ & 4.756E-04 & 4.917E-04 & $1.101 E-03$ & $1.234 \mathrm{E}-03$ \\
\hline${ }^{238} \mathrm{U}$ & $1.050 \mathrm{E}-02$ & $1.050 \mathrm{E}-02$ & $3.720 E-03$ & 3.720E-03 & 3.129E-03 & $3.129 E-03$ \\
\hline${ }^{237} \mathrm{~Np}$ & $9.383 E-03$ & $1.975 \mathrm{E}-02$ & 2.058E-01 & 2.936E-01 & $9.399 \mathrm{E}-02$ & 1.274E-01 \\
\hline${ }^{238} \mathrm{Pu}$ & $1.105 E+03$ & 4.102E-01 & $5.740 E-01$ & 2.129E-04 & $2.448 E+01$ & $3.250 \mathrm{E}-02$ \\
\hline${ }^{239} \mathrm{Pu}$ & $1.289 E+01$ & $1.252 E+01$ & $1.409 E+00$ & $1.371 E+00$ & $6.362 E+00$ & $6.218 E+00$ \\
\hline${ }^{240} \mathrm{Pu}$ & $8.870 E+00$ & $8.042 E+00$ & $5.658 \mathrm{E}-01$ & 5.164E-01 & 4.707E+00 & $4.251 E+00$ \\
\hline${ }^{241} \mathrm{Pu}$ & $2.779 E+02$ & $0.000 E+00$ & $4.294 \mathrm{E}+00$ & $0.000 E+00$ & $5.257 E+01$ & $0.000 E+00$ \\
\hline${ }^{242} \mathrm{Pu}$ & $1.224 E-02$ & $1.222 \mathrm{E}-02$ & 1.310E-04 & $1.308 E-04$ & $6.348 \mathrm{E}-03$ & 6.337E-03 \\
\hline${ }^{244} \mathrm{Pu}$ & 2.147E-19 & $5.967 E-18$ & $0.000 E+00$ & $0.000 E+00$ & $0.000 E+00$ & $0.000 E+00$ \\
\hline${ }^{241} \mathrm{Am}$ & $5.501 E+01$ & $1.293 E+01$ & $5.444 E+02$ & $1.095 E+02$ & $2.055 E+02$ & 4.169E+01 \\
\hline${ }^{242 m} \mathrm{Am}$ & 1.221E-02 & $1.273 E-04$ & $0.000 E+00$ & $0.000 E+00$ & 9.837E-01 & 1.025E-02 \\
\hline${ }^{243} \mathrm{Am}$ & 5.766E-03 & $5.248 \mathrm{E}-03$ & $6.735 E-02$ & $6.130 \mathrm{E}-02$ & $1.350 E+00$ & $1.229 E+00$ \\
\hline${ }^{243} \mathrm{Cm}$ & 2.247E-03 & $6.059 \mathrm{E}-14$ & $0.000 E+00$ & $0.000 E+00$ & 2.121E-01 & $5.719 \mathrm{E}-12$ \\
\hline${ }^{244} \mathrm{Cm}$ & $2.586 E+01$ & $0.000 E+00$ & $3.004 \mathrm{E}+00$ & $0.000 E+00$ & $7.185 E+00$ & $0.000 E+00$ \\
\hline${ }^{245} \mathrm{Cm}$ & 6.693E-06 & $6.169 \mathrm{E}-06$ & $0.000 E+00$ & $0.000 E+00$ & $3.437 E-03$ & 3.168E-03 \\
\hline${ }^{246} \mathrm{Cm}$ & 5.313E-07 & 4.588E-07 & $0.000 E+00$ & $0.000 E+00$ & 3.895E-04 & 3.364E-04 \\
\hline${ }^{247} \mathrm{Cm}$ & $6.602 E-13$ & $6.602 E-13$ & $0.000 E+00$ & $0.000 E+00$ & $0.000 E+00$ & $0.000 E+00$ \\
\hline Total & $3.928 E+04$ & $3.937 E+01$ & $4.000 E+04$ & $1.236 E+02$ & $2.330 E+04$ & $5.683 E+01$ \\
\hline
\end{tabular}


Table 10-1. Contents (in Curies) of 49 Radionuclides of Waste Parcels at Time of Emplacement (Year 2030) and after $1000 \mathrm{Yr}$ (continued)

\begin{tabular}{|c|c|c|c|c|c|c|}
\hline \multirow[b]{2}{*}{ Nuclide } & \multicolumn{2}{|c|}{ Waste Parcel 10} & \multicolumn{2}{|c|}{ Waste Parcel 11} & \multicolumn{2}{|c|}{ Waste Parcel 12} \\
\hline & $\begin{array}{c}\text { Time of } \\
\text { Emplacement } \\
\text { (year 2030) }\end{array}$ & $\begin{array}{c}\text { Time of } \\
\text { Intrusion } \\
\text { (year 3030) }\end{array}$ & $\begin{array}{c}\text { Time of } \\
\text { Emplacement } \\
\text { (year 2030) }\end{array}$ & $\begin{array}{l}\text { Time of } \\
\text { Intrusion } \\
\text { (year 3030) }\end{array}$ & $\begin{array}{c}\text { Time of } \\
\text { Emplacement } \\
\text { (year 2030) }\end{array}$ & $\begin{array}{l}\text { Time of } \\
\text { Intrusion } \\
\text { (year 3030) }\end{array}$ \\
\hline${ }^{14} \mathrm{C}$ & $1.940 \mathrm{E}-02$ & $1.719 \mathrm{E}-02$ & $0.000 E+00$ & $0.000 E+00$ & 1.408E-06 & $1.247 \mathrm{E}-06$ \\
\hline${ }^{36} \mathrm{Cl}$ & 8.068E-04 & 8.049E-04 & $0.000 E+00$ & $0.000 \mathrm{E}+00$ & $0.000 E+00$ & $0.000 E+00$ \\
\hline${ }^{59} \mathrm{Ni}$ & $5.630 \mathrm{E}-03$ & $5.578 E-03$ & $0.000 E+00$ & $0.000 \mathrm{E}+00$ & $0.000 E+00$ & $0.000 E+00$ \\
\hline${ }^{63} \mathrm{Ni}$ & $1.216 \mathrm{E}-01$ & 8.844E-05 & $0.000 E+00$ & $0.000 E+00$ & $0.000 E+00$ & $0.000 E+00$ \\
\hline${ }^{79} \mathrm{Se}$ & 8.745E-03 & 8.652E-03 & $0.000 E+00$ & $0.000 E+00$ & $5.075 E-03$ & $5.021 E-03$ \\
\hline${ }^{87} \mathrm{Rb}$ & $5.208 E-07$ & $5.208 E-07$ & $0.000 E+00$ & $0.000 E+00$ & $3.416 \mathrm{E}-07$ & $3.416 \mathrm{E}-07$ \\
\hline${ }^{90} \mathrm{Sr}$ & $5.691 E+02$ & 2.613E-08 & $9.753 E+02$ & 4.478E-08 & 1.117E+03 & $5.129 \mathrm{E}-08$ \\
\hline${ }^{93} \mathrm{Zr}$ & $3.464 \mathrm{E}-01$ & 3.462E-01 & $0.000 E+00$ & $0.000 \mathrm{E}+00$ & 2.607E-02 & $2.606 \mathrm{E}-02$ \\
\hline${ }^{99} \mathrm{TC}$ & 2.189E-01 & 2.182E-01 & $0.000 E+00$ & $0.000 E+00$ & $1.710 \mathrm{E}-01$ & $1.704 E-01$ \\
\hline${ }^{107} \mathrm{Pd}$ & $2.570 E-04$ & $2.570 \mathrm{E}-04$ & $0.000 E+00$ & $0.000 E+00$ & 1.971E-04 & $1.971 E-04$ \\
\hline${ }^{113} \mathrm{Cd}$ & $1.188 \mathrm{E}-15$ & $1.188 \mathrm{E}-15$ & $0.000 E+00$ & $0.000 E+00$ & $0.000 E+00$ & $0.000 E+00$ \\
\hline${ }^{113 m} \mathrm{Cd}$ & $0.000 E+00$ & $0.000 E+00$ & $0.000 E+00$ & $0.000 E+00$ & $3.930 \mathrm{E}-02$ & $0.000 E+00$ \\
\hline${ }^{126} \mathrm{Sn}$ & $3.812 \mathrm{E}-03$ & $3.786 \mathrm{E}-03$ & $0.000 E+00$ & $0.000 E+00$ & $4.542 E-03$ & 4.511E $\cdots 03$ \\
\hline 129 & $6.552 E-04$ & $6.552 E-04$ & $0.000 E+00$ & $0.000 E+00$ & 2.804E-04 & $2.804 \mathrm{E}-04$ \\
\hline${ }^{135} \mathrm{Cs}$ & 4.165E-03 & 4.164E-03 & $0.000 \mathrm{E}+00$ & $0.000 E+00$ & 1.325E-03 & $1.325 \mathrm{E}-03$ \\
\hline${ }^{137} \mathrm{Cs}$ & $6.235 E+02$ & 5.760 E-08 & $1.137 E+03$ & $1.050 \mathrm{E}-07$ & $1.242 E+03$ & 1.147E-07 \\
\hline${ }^{144} \mathrm{Nd}$ & 5.852E-07 & 5.852E-07 & $0.000 E+00$ & $1.628 \mathrm{E}-37$ & $0.000 E+00$ & $9.085 E-20$ \\
\hline${ }^{147} \mathrm{Pm}$ & $0.000 E+00$ & $0.000 E+00$ & $1.518 E-08$ & $0.000 E+00$ & $7.109 E+00$ & $0.000 E+00$ \\
\hline${ }^{147} \mathrm{Sm}$ & $1.886 \mathrm{E}-03$ & 1.886E-03 & $0.000 E+00$ & 3.759E-19 & 9.597E-08 & 9.615E-08 \\
\hline${ }^{151} \mathrm{Sm}$ & $1.681 E+01$ & 7.556E-03 & $0.000 E+00$ & $0.000 E+00$ & $3.594 E+00$ & $1.615 \mathrm{E}-03$ \\
\hline${ }^{152} \mathrm{Eu}$ & $0.000 E+00$ & $0.000 E+00$ & $0.000 E+00$ & $0.000 E+00$ & $0.000 E+00$ & $0.000 E+00$ \\
\hline${ }^{210} \mathrm{~Pb}$ & $0.000 E+00$ & $0.000 E+00$ & $0.000 E+00$ & $4.881 E-06$ & 2.192E-10 & 2.752E-06 \\
\hline${ }^{226} \mathrm{Ra}$ & $0.000 E+00$ & 2.766E-04 & $0.000 E+00$ & 3.363E-05 & 8.393E-10 & 1.992E-05 \\
\hline${ }^{228} \mathrm{Ra}$ & $0.000 E+00$ & $0.000 E+00$ & $0.000 E+00$ & $0.000 E+00$ & $0.000 E+00$ & $0.000 E+00$ \\
\hline${ }^{229} \mathrm{Th}$ & $0.000 E+00$ & 1.154E-01 & $0.000 E+00$ & $6.200 E-05$ & $1.286 E-09$ & 3.443E-05 \\
\hline${ }^{230} \mathrm{Th}$ & $0.000 E+00$ & 1.522E-03 & $1.432 E-05$ & $1.903 \mathrm{E}-04$ & 8.073E-06 & $1.285 E-04$ \\
\hline${ }^{232} \mathrm{Th}$ & 1.513E-03 & 1.513E-03 & 4.295E-11 & $5.461 \mathrm{E}-11$ & $2.380 \mathrm{E}-11$ & $3.159 \mathrm{E}-10$ \\
\hline
\end{tabular}

Note: Boldface values represent the most dominant radioisotope (largest activity) in a waste parcel. 
Table 10-1. Contents (in Curies) of 49 Radionuclides of Waste Parcels at Time of Emplacement (Year 2030) and after $1000 \mathrm{Yr}$ (continued)

\begin{tabular}{|c|c|c|c|c|c|c|}
\hline \multirow[b]{2}{*}{ Nuclide } & \multicolumn{2}{|c|}{ Waste Parcel 10} & \multicolumn{2}{|c|}{ Waste Parcel 11} & \multicolumn{2}{|c|}{ Waste Parcel 12} \\
\hline & $\begin{array}{c}\text { Time of } \\
\text { Emplacement } \\
\text { (year 2030) }\end{array}$ & $\begin{array}{c}\text { Time of } \\
\text { Intrusion } \\
\text { (year 3030) }\end{array}$ & $\begin{array}{c}\text { Time of } \\
\text { Emplacement } \\
\text { (year 2030) }\end{array}$ & $\begin{array}{c}\text { Time of } \\
\text { Intrusion } \\
\text { (year 3030) }\end{array}$ & $\begin{array}{c}\text { Time of } \\
\text { Emplacement } \\
\text { (year 2030) }\end{array}$ & $\begin{array}{c}\text { Time of } \\
\text { Intrusion } \\
\text { (year 3030) }\end{array}$ \\
\hline${ }^{231} \mathrm{~Pa}$ & $0.000 E+00$ & $1.596 \mathrm{E}-05$ & $0.000 E+00$ & 2.171E-06 & 9.795E-07 & 3.163E-05 \\
\hline${ }^{232} U$ & $6.380 E-01$ & 4.186E-05 & $0.000 E+00$ & $0.000 E+00$ & 2.409E-05 & $1.581 \mathrm{E}-09$ \\
\hline${ }^{233} \mathrm{U}$ & $1.283 E+00$ & $1.277 E+00$ & $6.895 \mathrm{E}-04$ & $1.311 E-03$ & $3.828 E-04$ & 7.425E-04 \\
\hline${ }^{234} U$ & 1.637E-01 & $1.706 \mathrm{E}-01$ & $1.694 \mathrm{E}-02$ & 2.003E-02 & $1.012 E-02$ & 1.391E-02 \\
\hline $235 \mathrm{U}$ & 7.624E-04 & 7.625E-04 & $1.037 E-04$ & $1.044 E-04$ & $1.465 \mathrm{E}-03$ & $1.466 \mathrm{E}-03$ \\
\hline${ }^{236} \mathrm{U}$ & 6.313E-03 & $6.316 \mathrm{E}-03$ & 2.319E-04 & 2.407E-04 & $5.914 \mathrm{E}-03$ & $5.922 \mathrm{E}-03$ \\
\hline${ }^{238} \mathrm{U}$ & $1.756 \mathrm{E}-05$ & $1.756 \mathrm{E}-05$ & 5.819E-06 & 5.819E-06 & $2.909 \mathrm{E}-05$ & 2.909E-05 \\
\hline${ }^{237} \mathrm{~Np}$ & $4.546 \mathrm{E}-03$ & 4.713E-03 & $1.432 E-01$ & $1.432 E-01$ & 8.283E-02 & 8.303E-02 \\
\hline${ }^{238} \mathrm{Pu}$ & $2.056 E+01$ & $7.630 \mathrm{E}-03$ & $8.759 E+00$ & 3.249E-03 & $1.068 E+01$ & 3.966E-03 \\
\hline${ }^{239} \mathrm{Pu}$ & 6.369E-02 & $6.210 \mathrm{E}-02$ & 7.392E-01 & 7.184E-01 & 5.947E-01 & $5.779 E-01$ \\
\hline${ }^{240} \mathrm{Pu}$ & $1.146 \mathrm{E}-01$ & $1.035 \mathrm{E}-01$ & 3.147E-01 & $2.830 \mathrm{E}-01$ & 2.799E-01 & 2.518E-01 \\
\hline${ }^{241} \mathrm{Pu}$ & $0.000 E+00$ & $0.000 E+00$ & $0.000 E+00$ & $0.000 E+00$ & $8.886 E+00$ & $0.000 E+00$ \\
\hline${ }^{242} \mathrm{Pu}$ & $0.000 E+00$ & 3.558E-08 & $1.543 E-04$ & $1.540 \mathrm{E}-04$ & $2.439 E-04$ & 2.435E-04 \\
\hline${ }^{244} \mathrm{Pu}$ & $0.000 E+00$ & $0.000 E+00$ & $0.000 E+00$ & $0.000 E+00$ & $2.883 E-11$ & 2.883E-11 \\
\hline${ }^{241} \mathrm{Am}$ & $1.042 E+00$ & 2.097E-01 & 2.864E-01 & $5.761 \mathrm{E}-02$ & $1.103 E+00$ & 2.814E-01 \\
\hline${ }^{242 m}$ Am & $1.311 E-04$ & 1.367E-06 & $0.000 E+00$ & $0.000 E+00$ & $1.744 \mathrm{E}-04$ & 1.818E-06 \\
\hline${ }^{243} \mathrm{Am}$ & 8.062E-03 & 7.338E-03 & 5.599E-03 & $5.096 \mathrm{E}-03$ & 3.757E-03 & $3.420 E-03$ \\
\hline${ }^{243} \mathrm{Cm}$ & 4.596E-04 & $1.239 \mathrm{E}-14$ & $0.000 E+00$ & $0.000 E+00$ & $5.450 E-05$ & $1.470 E-15$ \\
\hline${ }^{244} \mathrm{Cm}$ & $1.539 \mathrm{E}-01$ & $0.000 E+00$ & $0.000 E+00$ & $0.000 E+00$ & 8.689E-03 & $0.000 E+00$ \\
\hline${ }^{245} \mathrm{Cm}$ & 7.185E-05 & $6.622 E-05$ & $0.000 E+00$ & $0.000 E+00$ & $1.374 \mathrm{E}-06$ & $1.266 \mathrm{E}-06$ \\
\hline${ }^{246} \mathrm{Cm}$ & $2.079 E-05$ & $1.796 \mathrm{E}-05$ & $0.000 E+00$ & $0.000 E+00$ & $9.459 \mathrm{E}-08$ & $8.169 E-08$ \\
\hline${ }^{247} \mathrm{Cm}$ & 6.163E-11 & $6.163 E-11$ & $0.000 E+00$ & $0.000 E+00$ & $0.000 E+00$ & $0.000 E+00$ \\
\hline Total & $1.234 E+03$ & $2.584 E+00$ & $2.123 E+03$ & $1.233 E+00$ & $2.392 E+03$ & $1.434 E+00$ \\
\hline
\end{tabular}


Table 10-1. Contents (in Curies) of 49 Radionuclides of Waste Parcels at Time of Emplacement (Year 2030) and after $1000 \mathrm{Yr}$ (continued)

\begin{tabular}{|c|c|c|c|c|c|c|}
\hline \multirow[b]{2}{*}{ Nuclide } & \multicolumn{2}{|c|}{ Waste Parcel 13} & \multicolumn{2}{|c|}{ Waste Parcel 14} & \multicolumn{2}{|c|}{ Waste Parcel 15} \\
\hline & $\begin{array}{c}\text { Time of } \\
\text { Emplacement } \\
\text { (year 2030) }\end{array}$ & $\begin{array}{c}\text { Time of } \\
\text { Intrusion } \\
\text { (year 3030) }\end{array}$ & $\begin{array}{c}\text { Time of } \\
\text { Emplacement } \\
\text { (year 2030) }\end{array}$ & $\begin{array}{c}\text { Time of } \\
\text { Intrusion } \\
\text { (year 3030) }\end{array}$ & $\begin{array}{l}\text { Time of } \\
\text { Emplacement } \\
\text { (year 2030) }\end{array}$ & $\begin{array}{c}\text { Time of } \\
\text { Intrusion } \\
\text { (year 3030) }\end{array}$ \\
\hline${ }^{14} \mathrm{C}$ & 3.468E-01 & 3.073E-01 & $5.959 \mathrm{E}-02$ & $5.280 \mathrm{E}-02$ & 5.029E-03 & 4.456E-03 \\
\hline${ }^{36} \mathrm{Cl}$ & $2.568 E-03$ & $2.562 E-03$ & $9.559 \mathrm{E}-03$ & $9.537 E-03$ & 8.068E-04 & 8.049E-04 \\
\hline${ }^{59} \mathrm{Ni}$ & $1.171 E+00$ & $1.160 E+00$ & $6.671 \mathrm{E}-02$ & $6.610 \mathrm{E}-02$ & $5.630 \mathrm{E}-03$ & $5.578 \mathrm{E}-03$ \\
\hline${ }^{63} \mathrm{Ni}$ & $1.194 E+02$ & 8.684E-02 & $1.441 E+00$ & $1.048 E-03$ & $1.216 \mathrm{E}-01$ & 8.844E-05 \\
\hline${ }^{79} \mathrm{Se}$ & $9.212 E-02$ & $9.114 \mathrm{E}-02$ & $1.036 \mathrm{E}-01$ & $1.025 E-01$ & 8.745E-03 & 8.652E-03 \\
\hline${ }^{87} \mathrm{Rb}$ & $0.000 E+00$ & $0.000 E+00$ & 6.171E-06 & $6.171 E-06$ & 5.208E-07 & 5.208E-07 \\
\hline${ }^{90} \mathrm{Sr}$ & $9.200 E+03$ & 4.225E-07 & $7.115 E+03$ & 3.267E-07 & $1.050 E+03$ & $4.821 E-08$ \\
\hline${ }^{93} \mathrm{Zr}$ & 4.065E-01 & 4.063E-01 & $4.104 E+00$ & $4.102 E+00$ & 3.464E-01 & 3.462E-01 \\
\hline${ }^{99} \mathrm{TC}$ & $2.939 E+00$ & $2.929 E+00$ & $2.594 E+00$ & $2.586 \mathrm{E}+00$ & 2.189E-01 & 2.182E-01 \\
\hline${ }^{107} \mathrm{Pd}$ & 2.523E-02 & 2.523E-02 & 3.045E-03 & $3.045 E-03$ & $2.570 E-04$ & $2.570 E-04$ \\
\hline${ }^{113} \mathrm{Cd}$ & $0.000 E+00$ & $0.000 E+00$ & $1.408 E-14$ & $1.408 \mathrm{E}-14$ & $1.188 \mathrm{E}-15$ & $1.188 \mathrm{E}-15$ \\
\hline${ }^{113 m} \mathrm{Cd}$ & $0.000 E+00$ & $0.000 \Xi+00$ & $0.000 E+00$ & $0.000 E+00$ & $0.000 E+00$ & $0.000 E+00$ \\
\hline${ }^{126} \mathrm{Sn}$ & $1.745 \mathrm{E}-01$ & $1.733 E-01$ & 4.516E-02 & 4.485E-02 & $3.812 E-03$ & 3.786E-03 \\
\hline 129 & 7.117E-03 & 7.117E-03 & 7.763E-03 & 7.763E-03 & 6.552E-04 & 6.552E-04 \\
\hline${ }^{135} \mathrm{Cs}$ & 7.770E-02 & 7.768E-02 & 4.936E-02 & 4.935E-02 & 4.165E-03 & 4.164E-03 \\
\hline${ }^{137} \mathrm{Cs}$ & $1.304 E+04$ & $1.205 E-06$ & $7.822 E+03$ & $7.226 \mathrm{E}-07$ & $1.184 E+03$ & 1.094E-07 \\
\hline${ }^{144} \mathrm{Nd}$ & $0.000 E+00$ & $2.620 E-22$ & $6.934 \mathrm{E}-06$ & $6.934 \mathrm{E}-06$ & 5.852E-07 & 5.852E-07 \\
\hline${ }^{147} \mathrm{Pm}$ & $1.124 E+01$ & $0.000 E+00$ & 5.787E-09 & $0.000 E+00$ & 7.489E-09 & $0.000 E+00$ \\
\hline${ }^{147} \mathrm{Sm}$ & $0.000 E+00$ & 2.783E-10 & 2.234E-02 & 2.234E-02 & $1.886 \mathrm{E}-03$ & $1.886 \mathrm{E}-03$ \\
\hline${ }^{151} \mathrm{Sm}$ & $6.453 E+01$ & $2.901 E-02$ & $1.992 E+02$ & 8.954E-02 & $1.681 E+01$ & $7.556 \mathrm{E}-03$ \\
\hline${ }^{152} \mathrm{Eu}$ & $0.000 E+00$ & $0.000 E+00$ & $0.000 E+00$ & $0.000 E+00$ & $0.000 E+00$ & $0.000 E+00$ \\
\hline${ }^{210} \mathrm{~Pb}$ & $1.408 \mathrm{E}-07$ & 3.303E-05 & $0.000 \mathrm{E}+00$ & $1.861 \mathrm{E}-06$ & $0.000 E+00$ & $2.408 E-06$ \\
\hline${ }^{226} \mathrm{Ra}$ & $5.372 E-07$ & $5.639 \mathrm{E}-04$ & $0.000 E+00$ & 3.290E-03 & $0.000 E+00$ & $2.930 \mathrm{E}-04$ \\
\hline${ }^{228} \mathrm{Ra}$ & $0.000 E+00$ & $0.000 E+00$ & $0.000 E+00$ & $0.000 E+00$ & $0.000 E+00$ & $0.000 E+00$ \\
\hline${ }^{229} \mathrm{Th}$ & $3.356 \mathrm{E}-08$ & 7.343E-05 & $0.000 E+00$ & $1.367 E+00$ & $0.000 E+00$ & $1.154 \mathrm{E}-01$ \\
\hline${ }^{230} \mathrm{Th}$ & $9.585 \mathrm{E}-05$ & 4.131E-03 & 5.459E-06 & 1.811E-02 & 7.065E-06 & $1.616 \mathrm{E}-03$ \\
\hline${ }^{232} \mathrm{Th}$ & $5.005 E-11$ & 2.916E-09 & 1.793E-02 & 1.793E-02 & $1.513 E-03$ & $1.513 E-03$ \\
\hline
\end{tabular}

Note: Boldface values represent the most dominant radioisotope (largest activity) in a waste parcel. 
10. Modeling Drilling into Repository (CUTTINGS)

Table 10-1. Contents (in Curies) of 49 Radionuclides of Waste Parcels at Time of Emplacement (Year 2030) and after $10 M 0$ Yr (continued)

\begin{tabular}{|c|c|c|c|c|c|c|}
\hline \multirow[b]{2}{*}{ Nuclide } & \multicolumn{2}{|c|}{ Waste Parcel 13} & \multicolumn{2}{|c|}{ Waste Parcel 14} & \multicolumn{2}{|c|}{ Waste Parcel 15} \\
\hline & $\begin{array}{c}\text { Time of } \\
\text { Emplacement } \\
\text { (year 2030) }\end{array}$ & $\begin{array}{c}\text { Time of } \\
\text { Intrusion } \\
\text { (year 3030) }\end{array}$ & $\begin{array}{l}\text { Time of } \\
\text { Emplacement } \\
\text { (year 2030) }\end{array}$ & $\begin{array}{l}\text { Time of } \\
\text { Intrusion } \\
\text { (year 3030) }\end{array}$ & $\begin{array}{c}\text { Time of } \\
\text { Emplacement } \\
\text { (year 2030) }\end{array}$ & $\begin{array}{c}\text { Time of } \\
\text { Intrusion } \\
\text { (year 3030) }\end{array}$ \\
\hline${ }^{231} \mathrm{~Pa}$ & $5.236 \mathrm{E}-06$ & 8.877E-05 & $0.000 E+00$ & $1.899 \mathrm{E}-04$ & $0.000 E+00$ & 1.703E-05 \\
\hline $232 U$ & $0.000 E+00$ & $0.000 E+00$ & $7.559 E+00$ & $4.960 \mathrm{E}-04$ & $6.380 \mathrm{E}-01$ & 4.186E-05 \\
\hline${ }^{233} U$ & $8.162 E-04$ & 1.867E-03 & $1.520 E+01$ & $1.513 E+01$ & $1.283 E+00$ & $1.278 E+00$ \\
\hline${ }^{234} U$ & 3.137E-01 & 4.695E-01 & $1.946 E+00$ & $2.029 \mathrm{E}+00$ & $1.720 \mathrm{E}-01$ & 1.804E-01 \\
\hline${ }^{235} U$ & 3.995E-03 & 4.064E-03 & $9.072 E-03$ & $9.073 E-03$ & 8.135E-04 & 8.139E-04 \\
\hline${ }^{236} \mathrm{U}$ & $5.804 \mathrm{E}-02$ & 5.809E-02 & 7.489E-02 & 7.493E-02 & $6.428 \mathrm{E}-03$ & $6.435 \mathrm{E}-03$ \\
\hline $238 \mathrm{U}$ & 7.152E-02 & 7.152E-02 & 2.103E-04 & 2.103E-04 & 2.043E-05 & 2.043E-05 \\
\hline${ }^{237} \mathrm{~Np}$ & 2.417E-01 & $3.945 \mathrm{E}-01$ & 1.085E-01 & $1.105 E-01$ & 7.519E-02 & 7.536E-02 \\
\hline${ }^{238} \mathrm{Pu}$ & $4.381 E+02$ & $1.625 \mathrm{E}-01$ & $2.469 E+02$ & $9.162 E-02$ & $2.488 E+01$ & $9.232 E-03$ \\
\hline${ }^{239} \mathrm{Pu}$ & $7.136 E+01$ & $6.944 E+01$ & $1.036 E+00$ & $1.009 E+00$ & $4.284 \mathrm{E}-01$ & 4.165E-01 \\
\hline${ }^{240} \mathrm{Pu}$ & $1.549 E+00$ & $1.665 \mathrm{E}+00$ & $1.478 E+00$ & $1.334 E+00$ & $2.699 \mathrm{E}-01$ & $2.431 E-01$ \\
\hline${ }^{241} \mathrm{Pu}$ & $6.700 E+03$ & $0.000 E+00$ & $0.000 E+00$ & $0.000 E+00$ & $0.000 E+00$ & $0.000 E+00$ \\
\hline${ }^{242} \mathrm{Pu}$ & 3.966E-01 & 3.959E-01 & 5.882E-05 & 5.913E-05 & 7.611E-05 & 7.601E-05 \\
\hline${ }^{244} \mathrm{Pu}$ & $0.000 E+00$ & $0.000 E+00$ & $0.000 E+00$ & $0.000 E+00$ & $0.000 E+00$ & $0.000 E+00$ \\
\hline${ }^{241} \mathrm{Am}$ & $7.244 E+02$ & $1.906 E+02$ & $1.246 E+01$ & $2.507 E+00$ & $1.183 E+00$ & 2.380E-01 \\
\hline${ }^{242 m} \mathrm{Am}$ & $0.000 E+00$ & $0.000 E+00$ & $1.554 E-03$ & $1.620 \mathrm{E}-05$ & $1.311 \mathrm{E}-04$ & 1.367E-06 \\
\hline${ }^{243} \mathrm{Am}$ & $3.835 \mathrm{E}+00$ & $3.490 E+00$ & $9.766 \mathrm{E}-02$ & 8.889E-02 & $1.082 E-02$ & $9.848 E-03$ \\
\hline${ }^{243} \mathrm{Cm}$ & $0.000 E+00$ & $0.000 E+00$ & $5.445 E-03$ & $1.468 \mathrm{E}-13$ & $4.596 \mathrm{E}-04$ & $1.239 E-14$ \\
\hline${ }^{244} \mathrm{Cm}$ & $1.090 E+02$ & $0.000 E+00$ & $1.824 E+00$ & $0.000 E+00$ & $1.539 \mathrm{E}-01$ & $0.000 E+00$ \\
\hline${ }^{245} \mathrm{Cm}$ & $0.000 \mathrm{E}+00$ & $0.000 E+00$ & 8.513E-04 & $7.846 \mathrm{E}-04$ & $7.185 E-05$ & 6.622E-05 \\
\hline${ }^{246} \mathrm{Cm}$ & $0.000 E+00$ & $0.000 E+00$ & 2.463E-04 & 2.127E-04 & 2.079E-05 & 1.796E-05 \\
\hline${ }^{247} \mathrm{Cm}$ & $0.000 E+00$ & $0.000 E+00$ & 7.303E-10 & 7.303E-10 & $6.163 E-11$ & 6.163E-11 \\
\hline Total & $3.049 E+04$ & $2.721 E+02$ & $1.543 E+04$ & $3.093 E+01$ & $2.281 E+03$ & $3.179 E+00$ \\
\hline
\end{tabular}


Table 10-1. Contents (in Curies) of 49 Radionuclides of Waste Parcels at Time of Emplacement (Year 2030) and after $1000 \mathrm{Yr}$ (continued)

\begin{tabular}{|c|c|c|c|c|c|c|}
\hline \multirow[b]{2}{*}{ Nuclide } & \multicolumn{2}{|c|}{ Waste Parcel 16} & \multicolumn{2}{|c|}{ Waste Parcel 17} & \multicolumn{2}{|c|}{ Waste Parcel 18} \\
\hline & $\begin{array}{l}\text { Time of } \\
\text { Emplacement } \\
\text { (year 2030) }\end{array}$ & $\begin{array}{c}\text { Time of } \\
\text { Intrusion } \\
\text { (year 3030) }\end{array}$ & $\begin{array}{c}\text { Time of } \\
\text { Emplacement } \\
\text { (year 2030) }\end{array}$ & $\begin{array}{c}\text { Time of } \\
\text { Intrusion } \\
\text { (year 3030) }\end{array}$ & $\begin{array}{l}\text { Time of } \\
\text { Emplacement } \\
\text { (year 2030) }\end{array}$ & $\begin{array}{c}\text { Time of } \\
\text { Intrusion } \\
\text { (year 3030) }\end{array}$ \\
\hline${ }^{14} \mathrm{C}$ & 1.126E-05 & $9.976 \mathrm{E}-06$ & $1.041 E+00$ & $9.223 \mathrm{E}-01$ & $0.000 E+00$ & $0.000 E+00$ \\
\hline${ }^{36} \mathrm{Cl}$ & $0.000 E+00$ & $0.000 E+00$ & 7.703E-03 & $7.685 \mathrm{E}-03$ & $0.000 E+00$ & $0.000 E+00$ \\
\hline${ }^{59} \mathrm{Ni}$ & $0.000 E+00$ & $0.000 E+00$ & $3.514 E+00$ & $3.482 E+00$ & $0.000 E+00$ & $0.000 E+00$ \\
\hline${ }^{63} \mathrm{Ni}$ & $0.000 E+00$ & $0.000 E+00$ & $3.581 E+02$ & $2.604 \mathrm{E}-01$ & $0.000 E+00$ & $0.000 E+00$ \\
\hline${ }^{79} \mathrm{Se}$ & $4.060 E-02$ & 4.017E-02 & 2.764E-01 & 2.735E-01 & $0.000 E+00$ & $0.000 E+00$ \\
\hline${ }^{87} \mathrm{Rb}$ & 2.733E-06 & $2.733 E-06$ & $0.000 E+00$ & $0.000 E+00$ & $0.000 E+00$ & $0.000 E+00$ \\
\hline${ }^{90} \mathrm{Sr}$ & $4.873 E+03$ & $2.238 \mathrm{E}-07$ & $2.440 E+04$ & $1.120 \mathrm{E}-06$ & $8.452 E+02$ & $3.881 E-08$ \\
\hline${ }^{93} \mathrm{Zr}$ & 2.085E-01 & $2.084 \mathrm{E}-01$ & 1. $J E+00$ & $1.219 E+00$ & $0.000 E+00$ & $0.000 E+00$ \\
\hline${ }^{99} \mathrm{TC}$ & $1.368 E+00$ & $1.364 E+00$ & $8.818 E+00$ & $8.789 E+00$ & $0.000 E+00$ & $0.000 E+00$ \\
\hline${ }^{107} \mathrm{Pd}$ & $1.577 \mathrm{E}-03$ & $1.577 \mathrm{E}-03$ & $7.568 \mathrm{E}-02$ & 7.567E-02 & $0.000 E+00$ & $0.000 E+00$ \\
\hline${ }^{113} \mathrm{Cd}$ & $0.000 E+00$ & $0.000 E+00$ & $0.000 E+00$ & $0.000 E+00$ & $0.000 E+00$ & $0.000 E+00$ \\
\hline${ }^{113 m} \mathrm{Cd}$ & 3.144E-01 & $0.000 E+00$ & $0.000 E+00$ & $0.000 E+00$ & $0.000 E+00$ & $0.000 E+00$ \\
\hline${ }^{126} \mathrm{Sn}$ & 3.633E-02 & 3.608E-02 & $5.236 \mathrm{E}-01$ & $5.200 E-01$ & $0.000 E+00$ & $0.000 E+00$ \\
\hline $129 \mid$ & $2.243 E-03$ & $2.243 E-03$ & 2.135E-02 & 2.135E-02 & $0.000 E+00$ & $0.000 E+00$ \\
\hline${ }^{135} \mathrm{Cs}$ & $1.060 \mathrm{E}-02$ & $1.060 \mathrm{E}-02$ & $2.331 E-01$ & 2.330E-01 & $0.000 E+00$ & $0.000 E+00$ \\
\hline${ }^{137} \mathrm{Cs}$ & $5.201 E+03$ & 4.805E-07 & $3.538 \mathrm{E}+04$ & $3.268 \mathrm{E}-06$ & $9.852 E+02$ & $9.101 E-08$ \\
\hline${ }^{144} \mathrm{Nd}$ & $0.000 E+00$ & 7.267E-19 & $0.000 E+00$ & 7.857E-22 & $0.000 E+00$ & $1.412 E-37$ \\
\hline${ }^{147} \mathrm{Pm}$ & $5.687 E+01$ & $0.000 E+00$ & $3.372 E+01$ & $0.000 E+00$ & $1.315 \mathrm{E}-08$ & $0.000 E+00$ \\
\hline${ }^{147} \mathrm{Sm}$ & 7.678E-07 & 7.692E-07 & $0.000 E+00$ & $8.349 \mathrm{E}-10$ & $0.000 E+00$ & 3.256E-19 \\
\hline${ }^{151} \mathrm{Sm}$ & $2.875 E+01$ & $1.292 E-02$ & $1.936 E+02$ & 8.702E-02 & $0.000 E+00$ & $0.000 E+00$ \\
\hline${ }^{152} \mathrm{Eu}$ & $0.000 E+00$ & $0.000 E+00$ & $0.000 E+00$ & $0.000 E+00$ & $0.000 E+00$ & $0.000 E+00$ \\
\hline${ }^{210} \mathrm{~Pb}$ & $1.754 \mathrm{E}-09$ & $1.683 E-06$ & 4.223E-07 & 8.304E-05 & $0.000 E+00$ & 4.230E-06 \\
\hline${ }^{226} \mathrm{Ra}$ & $6.714 E-09$ & $1.925 \mathrm{E}-05$ & $1.611 \mathrm{E}-06$ & $1.581 \mathrm{E}-03$ & $0.000 E+00$ & 2.914E-05 \\
\hline${ }^{228} \mathrm{Ra}$ & $0.000 E+00$ & $0.000 E+00$ & $0.000 E+00$ & $0.000 E+00$ & $0.000 E+00$ & $0.000 E+00$ \\
\hline${ }^{229} \mathrm{Th}$ & $1.028 \mathrm{E}-08$ & 1.703E-05 & 1.007E-07 & $1.666 \mathrm{E}-05$ & $0.000 E+00$ & 5.374E-05 \\
\hline${ }^{230} \mathrm{Th}$ & 4.925E-06 & $2.350 E-04$ & $2.405 E-04$ & $1.177 \mathrm{E}-02$ & $1.241 \mathrm{E}-05$ & 1.650E-04 \\
\hline${ }^{232} \mathrm{Th}$ & $1.147 \mathrm{E}-11$ & 2.300E-09 & $9.109 \mathrm{E}-12$ & $8.572 E-09^{\circ}$ & $3.722 \mathrm{E}-11$ & 4.733E- 11 \\
\hline
\end{tabular}

Note: Boldface values represent the most dominant radioisotope (largest activity) in a waste parcel. 
Table 10-1. Contents (in Curies) of 49 Radionuclides of Waste Parcels at Time of Emplacement (Year 2030) and after $1000 \mathrm{Yr}$ (continued)

\begin{tabular}{|c|c|c|c|c|c|c|}
\hline \multirow[b]{2}{*}{ Nuclide } & \multicolumn{2}{|c|}{ Waste Parcel 16} & \multicolumn{2}{|c|}{ Waste Parcel 17} & \multicolumn{2}{|c|}{ Waste Parcel 18} \\
\hline & $\begin{array}{l}\text { Time of } \\
\text { Emplacement } \\
\text { (year 2030) }\end{array}$ & $\begin{array}{l}\text { Time of } \\
\text { Intrusion } \\
\text { (year 3030) }\end{array}$ & $\begin{array}{l}\text { Time of } \\
\text { Emplacement } \\
\text { (year 2030) }\end{array}$ & $\begin{array}{c}\text { Time of } \\
\text { Intrusion } \\
\text { (year 3030) }\end{array}$ & $\begin{array}{l}\text { Time of } \\
\text { Emplacement } \\
\text { (year 2030) }\end{array}$ & $\begin{array}{c}\text { Time of } \\
\text { Intrusion } \\
\text { (year 3030) }\end{array}$ \\
\hline${ }^{231} \mathrm{~Pa}$ & 7.836E-06 & $2.440 \mathrm{E}-04$ & $1.571 E-05$ & $2.591 E-04$ & $0.000 E+00$ & $1.882 E-06$ \\
\hline${ }^{232} U$ & 1.927E-04 & $1.264 \mathrm{E}-08$ & $0.000 E+00$ & $0.000 \mathrm{E}+00$ & $0.000 E+00$ & $0.000 E+00$ \\
\hline $233 \mathrm{U}$ & $1.893 E-04$ & 4.766E-04 & $1.841 \mathrm{E}-04$ & $1.295 \mathrm{E}-03$ & 5.976E-04 & $1.136 \mathrm{E}-03$ \\
\hline${ }^{234} \mathrm{U}$ & $1.037 E-02$ & 2.785E-02 & 8.853E-01 & $1.343 E+00$ & $1.468 \mathrm{E}-02$ & $1.735 \mathrm{E}-02$ \\
\hline $235 \mathrm{U}$ & $1.129 \mathrm{E}-02$ & 1.129E-02 & $1.164 \mathrm{E}-02$ & $1.185 \mathrm{E}-02$ & 8.991E-05 & 9.053E-05 \\
\hline${ }^{236} \mathrm{U}$ & 4.635E-02 & 4.637E-02 & $1.734 \mathrm{E}-01$ & $1.735 \mathrm{E}-01$ & $2.010 E-04$ & 2.087E-04 \\
\hline${ }^{238} \mathrm{U}$ & 2.085E-04 & 2.085E-04 & $2.145 E-01$ & 2.145E-01 & 5.043E-06 & 5.043E-06 \\
\hline${ }^{237} \mathrm{~Np}$ & $6.604 E-02$ & 6.763E-02 & 2.547E-01 & $7.131 E-01$ & $1.241 \mathrm{E}-01$ & $1.241 \mathrm{E}-01$ \\
\hline${ }^{238} \mathrm{Pu}$ & $4.894 E+01$ & $1.819 \mathrm{E}-02$ & $1.286 E+03$ & $4.770 E-01$ & $7.591 E+00$ & $2.816 \mathrm{E}-03$ \\
\hline${ }^{239} \mathrm{Pu}$ & $1.678 E+00$ & $1.630 E+00$ & $2.116 E+02$ & $2.059 E+02$ & 6.407E-01 & $6.226 E-01$ \\
\hline${ }^{240} \mathrm{Pu}$ & $9.276 E-01$ & 8.345E-01 & $3.614 E+00$ & $4.065 E+00$ & $2.728 \mathrm{E}-01$ & 2.454E-01 \\
\hline${ }^{241} \mathrm{Pu}$ & $7.109 E+01$ & $0.000 E+00$ & $2.010 E+04$ & $0.000 E+00$ & $0.000 E+00$ & $0.000 E+00$ \\
\hline${ }^{242} \mathrm{Pu}$ & $1.308 E-03$ & $1.306 \mathrm{E}-03$ & $1.189 E+00$ & $1.187 E+00$ & $1.337 E-04$ & $1.334 \mathrm{E}-04$ \\
\hline${ }^{244} \mathrm{Pu}$ & $2.306 \mathrm{E}-10$ & $2.306 \mathrm{E}-10$ & $0.000 E+00$ & $0.000 E+00$ & $0.000 E+00$ & $0.000 E+00$ \\
\hline${ }^{241} \mathrm{Am}$ & $7.628 E+00$ & $2.011 E+00$ & $2.172 E+03$ & $5.716 E+02$ & $2.482 E-01$ & 4.993E-02 \\
\hline${ }^{242 m} \mathrm{Am}$ & $1.395 \mathrm{E}-03$ & $1.454 \mathrm{E}-05$ & $0.000 E+00$ & $0.000 E+00$ & $0.000 E+00$ & $0.000 E+00$ \\
\hline${ }^{243} \mathrm{Am}$ & $6.724 \mathrm{E}-03$ & $6.120 \mathrm{E}-03$ & $1.149 E+01$ & $1.046 \mathrm{E}+01$ & 4.853E-03 & 4.417E-03 \\
\hline${ }^{243} \mathrm{Cm}$ & 4.360E-04 & $1.176 E-14$ & $0.000 E+00$ & $0.000 E+00$ & $0.000 E+00$ & $0.000 E+00$ \\
\hline${ }^{244} \mathrm{Cm}$ & 6.951E-02 & $0.000 E+00$ & $3.270 E+02$ & $0.000 E+00$ & $0.000 E+00$ & $0.000 E+00$ \\
\hline${ }^{245} \mathrm{Cm}$ & 1.100E-05 & $1.014 \mathrm{E}-05$ & $0.000 E+00$ & $0.000 E+00$ & $0.000 E+00$ & $0.000 E+00$ \\
\hline${ }^{246} \mathrm{Cm}$ & 7.567E-07 & $6.535 \mathrm{E}-07$ & $0.000 E+00$ & $0.000 E+00$ & $0.000 E+00$ & $0.000 E+00$ \\
\hline${ }^{247} \mathrm{Cm}$ & $0.000 E+00$ & $0.000 E+00$ & $0.000 E+00$ & $0.000 E+00$ & $0.000 E+00$ & $0.000 E+00$ \\
\hline Total & $1.029 E+04$ & $6.331 E+00$ & $8.450 E+04$ & $8.121 E+02$ & $1.839 E+03$ & $1.068 E+00$ \\
\hline
\end{tabular}


Table 10-1. Contents (in Curies) of 49 Radionuclides of Waste Parcels at Time of F mblacement (Year 2030) and after 1000 Yr (continued)

\begin{tabular}{|c|c|c|c|c|c|c|}
\hline \multirow[b]{2}{*}{ Nuclide } & \multicolumn{2}{|c|}{ Waste Parcel 19} & \multicolumn{2}{|c|}{ Waste Parcel 20} & \multicolumn{2}{|c|}{ Waste Parcel 21} \\
\hline & $\begin{array}{c}\text { Time of } \\
\text { Emplacement } \\
\text { (year 2030) }\end{array}$ & $\begin{array}{l}\text { Time of } \\
\text { Intrusion } \\
\text { (year 3030) }\end{array}$ & $\begin{array}{c}\text { Time of } \\
\text { Emplacement } \\
\text { (year 2030) }\end{array}$ & $\begin{array}{c}\text { Time of } \\
\text { Intrusion } \\
\text { (year 3030) }\end{array}$ & $\begin{array}{l}\text { Time of } \\
\text { Emplacement } \\
\text { (year 2030) }\end{array}$ & $\begin{array}{c}\text { Time of } \\
\text { Intrusion } \\
\text { (year 3030) }\end{array}$ \\
\hline${ }^{14} \mathrm{C}$ & $1.408 \mathrm{E}-06$ & 1.247E-06 & $3.468 E-01$ & 3.073E-01 & $0.000 E+00$ & $0.000 E+00$ \\
\hline${ }^{36} \mathrm{Cl}$ & $0.000 E+00$ & $0.000 E+00$ & $2.568 \mathrm{E}-03$ & $2.562 E-03$ & $0.000 E+00$ & $0.000 \mathrm{E}+00$ \\
\hline${ }^{59} \mathrm{Ni}$ & $0.000 E+00$ & $0.000 E+00$ & $1.171 E+00$ & $1.160 E+00$ & $0.000 E+00$ & $0.000 E+00$ \\
\hline${ }^{63} \mathrm{Ni}$ & $0.000 E+00$ & $0.000 E+00$ & $1.194 E+02$ & 8.684E-02 & $0.000 E+00$ & $0.000 E+00$ \\
\hline${ }^{79} \mathrm{Se}$ & 5.075E-03 & $5.021 E-03$ & $9.212 E-02$ & $9.114 \mathrm{E}-02$ & $0.000 E+00$ & $0.000 E+00$ \\
\hline${ }^{87} \mathrm{Rb}$ & 3.416E-07 & 3.416E-07 & $0.000 E+00$ & $0.000 E+00$ & $0.000 E+00$ & $0.000 \mathrm{E}+00$ \\
\hline${ }^{90} \mathrm{Sr}$ & $7.561 E+02$ & $3.472 E-08$ & $8.418 E+03$ & 3.865E-07 & $1.226 \mathrm{E}+03$ & $5.630 \mathrm{E}-08$ \\
\hline${ }^{93} \mathrm{Zr}$ & 2.607E-02 & $2.606 \mathrm{E}-02$ & 4.065E-01 & 4.063E-01 & $0.000 E+00$ & $0.000 E+00$ \\
\hline${ }^{99} \mathrm{TC}$ & $1.710 \mathrm{E}-01$ & $1.704 \mathrm{E}-01$ & $2.939 E+00$ & $2.929 E+00$ & $0.000 E+00$ & $0.000 E+00$ \\
\hline${ }^{107} \mathrm{Pd}$ & $1.971 \mathrm{E}-04$ & $1.971 E-04$ & 2.523E-02 & 2.523E-02 & $0.000 E+00$ & $0.000 E+00$ \\
\hline${ }^{113} \mathrm{Cd}$ & $0.000 E+00$ & $0.000 E+00$ & $0.000 E+00$ & $0.000 E+00$ & $0.000 E+00$ & $0.000 E+00$ \\
\hline${ }^{113 m} \mathrm{Cd}$ & $3.930 \mathrm{E}-02$ & $0.000 \mathrm{E}+00$ & $0.000 E+00$ & $0.000 E+00$ & $0.000 E+00$ & $0.000 \mathrm{E}+00$ \\
\hline${ }^{126} \mathrm{Sn}$ & $4.542 \mathrm{E}-03$ & $4.511 \mathrm{E}-03$ & $1.745 \mathrm{E}-01$ & $1.733 E-01$ & $0.000 E+00$ & $0.000 E+00$ \\
\hline $129_{1}$ & 2.804E-04 & 2.804E-04 & 7.117E-03 & 7.117E-03 & $0.000 E+00$ & $0.000 E+00$ \\
\hline${ }^{135} \mathrm{Cs}$ & $1.325 \mathrm{E}-03$ & 1.325E-03 & $7.770 E-02$ & $7.768 \mathrm{E}-02$ & $0.000 E+00$ & $0.000 E+00$ \\
\hline${ }^{137} \mathrm{Cs}$ & 8.214E+02 & 7.588E-08 & $1.213 E+04$ & $1.120 \mathrm{E}-06$ & $1.429 E+03$ & 1.320E-07 \\
\hline${ }^{144} \mathrm{Nd}$ & $0.000 E+00$ & $9.085 E-20$ & $0.000 E+00$ & $2.620 E-22$ & $0.000 E+00$ & 2.047E-37 \\
\hline${ }^{147} \mathrm{Pm}$ & $7.109 E+00$ & $0.000 E+00$ & $1.124 E+01$ & $0.000 E+00$ & $1.908 \mathrm{E}-08$ & $0.000 E+00$ \\
\hline${ }^{147} \mathrm{Sm}$ & 9.597E-08 & $9.615 \mathrm{E}-08$ & $0.000 E+00$ & 2.783E-10 & $0.000 E+00$ & 4.724E-19 \\
\hline${ }^{151} \mathrm{Sm}$ & $3.594 E+00$ & 1.615E-03 & $6.453 E+01$ & 2.901E-02 & $0.000 E+00$ & $0.000 E+00$ \\
\hline${ }^{152} \mathrm{Eu}$ & $0.000 E+00$ & $0.000 E+00$ & $0.000 E+00$ & $0.000 E+00$ & $0.000 E+00$ & $0.000 E+00$ \\
\hline${ }^{210} \mathrm{~Pb}$ & 2.192E-10 & $9.458 \mathrm{E}-07$ & $1.408 \mathrm{E}-07$ & 2.912E-05 & $0.000 E+00$ & $6.136 \mathrm{E}-06$ \\
\hline${ }^{226} \mathrm{Ra}$ & 8.393E-10 & 7.472E-06 & 5.372E-07 & 5.369E-04 & $0.000 E+00$ & 4.227E-05 \\
\hline${ }^{228} \mathrm{Ra}$ & $0.000 E+00$ & $0.000 E+00$ & $0.000 E+00$ & $0.000 E+00$ & $0.000 E+00$ & $0.000 \mathrm{E}+00$ \\
\hline${ }^{229} \mathrm{Th}$ & $1.286 \mathrm{E}-09$ & $1.148 E-05$ & $3.356 \mathrm{E}-08$ & 2.377E-05 & $0.000 E+00$ & 7.795E-05 \\
\hline${ }^{230} \mathrm{Th}$ & 2.773E-06 & 5.805E-05 & 8.438E-05 & 3.978E-03 & $1.800 \mathrm{E}-05$ & 2.392E-04 \\
\hline${ }^{232} \mathrm{Th}$ & $7.904 \mathrm{E}-12$ & $2.958 \mathrm{E}-10$ & $1.565 \mathrm{E}-11$ & 2.872E-09 & 5.399E-11 & $6.865 \mathrm{E}-11$ \\
\hline
\end{tabular}


Table 10-1. Contents (in Curies) of 49 Radionuclides of Waste Parcels at Time of Emplacement (Year 2030) and after 1000 Yr (continued)

\begin{tabular}{|c|c|c|c|c|c|c|}
\hline \multirow[b]{2}{*}{ Nuclide } & \multicolumn{2}{|c|}{ Waste Parcel 19} & \multicolumn{2}{|c|}{ Waste Parcel 20} & \multicolumn{2}{|c|}{ Waste Parcel 21} \\
\hline & $\begin{array}{l}\text { Time of } \\
\text { Emplacement } \\
\text { (year 2030) }\end{array}$ & $\begin{array}{l}\text { Time of } \\
\text { Intrusion } \\
\text { (year 3030) }\end{array}$ & $\begin{array}{l}\text { Time of } \\
\text { Emplacement } \\
\text { (year 2030) }\end{array}$ & $\begin{array}{c}\text { Time of } \\
\text { Intrusion } \\
\text { (year 3030) }\end{array}$ & $\begin{array}{l}\text { Time of } \\
\text { Emplacement } \\
\text { (year 2030) }\end{array}$ & $\begin{array}{c}\text { Time of } \\
\text { Intrusion } \\
\text { (year 3030) }\end{array}$ \\
\hline${ }^{231} \mathrm{~Pa}$ & $9.795 E-07$ & $3.083 E-05$ & $5.236 \mathrm{E}-06$ & 8.703E-05 & $0.000 E+00$ & $2.730 \mathrm{E}-06$ \\
\hline${ }^{232} \mathrm{U}$ & $2.409 \mathrm{E}-05$ & $1.581 E-09$ & $0.000 E+00$ & $0.000 E+00$ & $0.000 E+00$ & $0.000 E+00$ \\
\hline${ }^{233} \mathrm{U}$ & $1.276 \mathrm{E}-04$ & $2.572 E-04$ & $2.639 E-04$ & 8.164E-04 & 8.668E-04 & $1.648 \mathrm{E}-03$ \\
\hline${ }^{234} \mathrm{U}$ & $3.848 E-03$ & 6.498E-03 & $3.001 E-01$ & 4.535E-01 & 2.129E-02 & 2.517E-02 \\
\hline $235 \mathrm{U}$ & $1.427 \mathrm{E}-03$ & 1.427E-03 & $3.912 E-03$ & $3.981 E-03$ & $1.304 \mathrm{E}-04$ & 1.313E-04 \\
\hline${ }^{236} \mathrm{U}$ & $5.829 \mathrm{E}-03$ & $5.833 E-03$ & 5.785E-02 & 5.789E-02 & 2.915E-04 & 3.026E-04 \\
\hline${ }^{238} \mathrm{U}$ & $2.694 \mathrm{E}-05$ & 2.694E-05 & 7.151E-02 & 7.151E-02 & 7.314E-06 & 7.314E-06 \\
\hline${ }^{237} \mathrm{~Np}$ & 2.983E-02 & $3.003 E-02$ & $1.269 \mathrm{E}-01$ & 2.797E-01 & $1.800 \mathrm{E}-01$ & $1.800 \mathrm{E}-01$ \\
\hline${ }^{238} \mathrm{Pu}$ & $7.438 E+00$ & 2.763E-03 & 4.311E+02 & $1.599 \mathrm{E}-01$ & $1.101 E+01$ & 4.084E-03 \\
\hline${ }^{239} \mathrm{Pu}$ & $3.211 \mathrm{E}-01$ & 3.120E-01 & $7.076 E+01$ & $6.886 E+01$ & 9.293E-01 & $9.031 E-0 i$ \\
\hline${ }^{240} \mathrm{Pu}$ & $1.634 \mathrm{E}-01$ & $1.470 \mathrm{E}-01$ & $1.297 E+00$ & $1.438 E+00$ & 3.957E-01 & $3.559 \mathrm{E}-01$ \\
\hline${ }^{241} \mathrm{Pu}$ & $8.886 E+00$ & $0.000 E+00$ & $6.700 E+03$ & $0.000 E+00$ & $0.000 E+00$ & $0.000 E+00$ \\
\hline${ }^{242} \mathrm{Pu}$ & $1.868 \mathrm{E}-04$ & $1.865 E-04$ & 3.965E-01 & $3.958 \mathrm{E}-01$ & $1.939 \mathrm{E}-04$ & $1.935 \mathrm{E}-04$ \\
\hline${ }^{244} \mathrm{Pu}$ & 2.883E-11 & $2.883 E-11$ & $0.000 E+00$ & $0.000 E+00$ & $0.000 E+00$ & $0.000 E+00$ \\
\hline${ }^{241} \mathrm{Am}$ & $9.966 \mathrm{E}-01$ & 2.600E-01 & $7.242 E+02$ & $1.906 E+02$ & $3.600 \mathrm{E}-01$ & 7.242E-02 \\
\hline${ }^{242 m} \mathrm{Am}$ & $1.744 \mathrm{E}-04$ & $1.818 \mathrm{E}-06$ & $0.000 E+00$ & $0.000 E+00$ & $0.000 E+00$ & $0.000 E+00$ \\
\hline${ }^{243} \mathrm{Am}$ & $1.684 \mathrm{E}-03$ & $1.532 E-03$ & $3.831 E+00$ & $3.487 E+00$ & $7.039 E-03$ & 6.407E-03 \\
\hline${ }^{243} \mathrm{Cm}$ & 5.450E-05 & $1.470 \mathrm{E}-15$ & $0.000 E+00$ & $0.000 E+00$ & $0.000 E+00$ & $0.000 E+00$ \\
\hline${ }^{244} \mathrm{Cm}$ & 8.689E-03 & $0.000 E+00$ & $1.090 E+02$ & $0.000 E+00$ & $0.000 E+00$ & $0.000 E+00$ \\
\hline${ }^{245} \mathrm{Cm}$ & 1.374E-06 & $1.266 \mathrm{E}-06$ & $0.000 E+00$ & $0.000 E+00$ & $0.000 E+00$ & $0.000 E+00$ \\
\hline${ }^{246} \mathrm{Cm}$ & $9.459 \mathrm{E}-08$ & $8.169 E-08$ & $0.000 E+00$ & $0.000 E+00$ & $0.000 E+00$ & $0.000 E+00$ \\
\hline${ }^{247} \mathrm{Cm}$ & $0.000 E+00$ & $0.000 E+00$ & $0.000 E+00$ & $0.000 E+00$ & $0.000 E+00$ & $0.000 E+00$ \\
\hline Total & $1.606 E+03$ & $9.772 E-01$ & $2.879 E+04$ & $2.711 E+02$ & $2.668 E+03$ & $1.550 E+00$ \\
\hline
\end{tabular}


Table 10-1. Contents (in Curies) of 49 Radionuclides of Waste Parcels at Time of Emplacement (Year 2030) and after $1000 \mathrm{Yr}$ (continued)

\begin{tabular}{|c|c|c|c|c|c|c|}
\hline \multirow[b]{2}{*}{ Nuclide } & \multicolumn{2}{|c|}{ Waste Parcel 22} & \multicolumn{2}{|c|}{ Waste Parcel 23} & \multicolumn{2}{|c|}{ Waste Parcel 24} \\
\hline & $\begin{array}{l}\text { Time of } \\
\text { Emplacement } \\
\text { (year 2030) }\end{array}$ & $\begin{array}{l}\text { Time of } \\
\text { Intrusion } \\
\text { (year 3030) }\end{array}$ & $\begin{array}{c}\text { Time of } \\
\text { Emplacement } \\
\text { (year 2030) }\end{array}$ & $\begin{array}{c}\text { Time of } \\
\text { Intrusion } \\
\text { (year 3030) }\end{array}$ & $\begin{array}{l}\text { Time of } \\
\text { Emplacement } \\
\text { (year 2030) }\end{array}$ & $\begin{array}{c}\text { Time of } \\
\text { Intrusion } \\
\text { (year 3030) }\end{array}$ \\
\hline${ }^{14} \mathrm{C}$ & $0.000 E+00$ & $0.000 E+00$ & $0.000 E+00$ & $0.000 E+00$ & $0.000 E+00$ & $0.000 E+00$ \\
\hline${ }^{36} \mathrm{Cl}$ & 3.824E-02 & 3.815E-02 & 4.505E-04 & 4.495E-04 & 7.193E-03 & 7.176E-03 \\
\hline${ }^{59} \mathrm{Ni}$ & $2.668 E-01$ & 2.643E-01 & $2.055 E-01$ & $2.036 \mathrm{E}-01$ & 5.020E-02 & 4.974E-02 \\
\hline${ }^{63} \mathrm{Ni}$ & $5.763 E+00$ & 4.191E-03 & $2.094 E+01$ & $1.523 E-02$ & $1.084 E+00$ & 7.884E-04 \\
\hline${ }^{79} \mathrm{Se}$ & 4.145E-01 & 4.101E-01 & 2.124E-02 & $2.101 E-02$ & 7.797E-02 & 7.714E-02 \\
\hline${ }^{87} \mathrm{Rb}$ & 2.468E-05 & $2.468 E-05$ & 3.418E-07 & $3.418 E-07$ & 4.644E-06 & 4.644E-06 \\
\hline${ }^{90} \mathrm{Sr}$ & $2.790 E+04$ & $1.281 E-06$ & $2.915 E+03$ & $1.338 E-07$ & $5.074 E+03$ & 2.330E-07 \\
\hline${ }^{93} \mathrm{Zr}$ & $1.642 E+01$ & $1.641 E+01$ & $9.741 E-02$ & 9.737E-02 & $3.088 E+00$ & $3.087 E+00$ \\
\hline${ }^{99} \mathrm{TC}$ & $1.038 E+01$ & $1.035 E+01$ & $6.868 E-01$ & $6.846 \mathrm{E}-01$ & $1.952 E+00$ & $1.946 \mathrm{E}+00$ \\
\hline${ }^{107} \mathrm{Pd}$ & $1.218 \mathrm{E}-02$ & $1.218 E-02$ & 4.623E-03 & $4.622 \mathrm{E}-03$ & 2.292E-03 & 2.292E-03 \\
\hline${ }^{113} \mathrm{Cd}$ & 5.632E-14 & $5.632 E-14$ & $0.000 E+00$ & $0.000 E+00$ & $1.059 E-14$ & $1.059 E-14$ \\
\hline${ }^{113 m} \mathrm{Cd}$ & $0.000 E+00$ & $0.000 E+00$ & $3.932 E-02$ & $0.000 E+00$ & $0.000 E+00$ & $0.000 E+00$ \\
\hline${ }^{126} \mathrm{Sn}$ & $1.807 E-01$ & 1.795E-01 & 3.517E-02 & 3.493E-02 & 3.399E-02 & 3.376E-02 \\
\hline 129 & 3.105E-02 & 3.105E-02 & $1.529 \mathrm{E}-03$ & $1.529 \mathrm{E}-03$ & $5.942 E-03$ & $5.842 \mathrm{E}-03$ \\
\hline${ }^{135} \mathrm{Cs}$ & $1.974 \mathrm{E}-01$ & 1.973E-01 & 1.496E-02 & $1.496 \mathrm{E}-02$ & 3.714E-02 & 3.713E-02 \\
\hline${ }^{137} \mathrm{Cs}$ & $3.064 E+04$ & $2.830 \mathrm{E}-06$ & $3.744 E+03$ & 3.459E.-07 & $5.559 E+03$ & 5.135E-07 \\
\hline${ }^{144} \mathrm{Nd}$ & $2.774 E-05$ & 2.774E-05 & $0.000 E+00$ & $9.093 \mathrm{E}-20$ & 5.218E-06 & $5.218 \mathrm{E}-06$ \\
\hline${ }^{147} \mathrm{Pm}$ & $1.447 E-08$ & $0.000 E+00$ & $9.085 E+00$ & $0.000 E+00$ & $0.000 E+00$ & $0.000 E+00$ \\
\hline${ }^{147} \mathrm{Sm}$ & 8.937E-02 & 8.937E-02 & $9.603 E-08$ & $9.626 \mathrm{E}-08$ & 1.681E-02 & $1.681 E-02$ \\
\hline${ }^{151} \mathrm{Sm}$ & $7.968 E+02$ & 3.582E-01 & $1.492 E+01$ & $6.706 \mathrm{E}-03$ & $1.499 E+02$ & $6.738 \mathrm{E}-02$ \\
\hline${ }^{152} \mathrm{Eu}$ & $0.000 E+00$ & $0.000 E+00$ & $0.000 E+00$ & $0.000 E+00$ & $0.000 E+00$ & $0.000 E+00$ \\
\hline${ }^{210} \mathrm{~Pb}$ & $0.000 E+00$ & 4.653E-06 & 2.492E-08 & $9.467 \mathrm{E}-06$ & $0.000 E+00$ & $0.000 E+00$ \\
\hline${ }^{226} \mathrm{Ra}$ & $0.000 E+00$ & $9.758 \mathrm{E}-05$ & $9.509 E-08$ & 3.725E-05 & $0.000 \mathrm{E}+00$ & 2.646E-03 \\
\hline${ }^{228} \mathrm{Ra}$ & $0.000 E+00$ & $0.000 E+00$ & $0.000 E+00$ & $0.000 E+00$ & $0.000 E+00$ & $0.000 E+00$ \\
\hline${ }^{229} \mathrm{Th}$ & $0.000 E+00$ & 2.739E-02 & 7.174E-09 & $5.874 E-05$ & $0.000 E+00$ & $1.029 E+00$ \\
\hline${ }^{230} \mathrm{Th}$ & 1.365E-05 & $5.421 E-04$ & 2.759E-05 & $1.976 \mathrm{E}-04$ & $0.000 E+00$ & 1.453E-02 \\
\hline${ }^{232} \mathrm{Th}$ & 7.171E-02 & 7.171E-02 & 4.067E-11 & $5.565 E-11$ & 1.349E-02 & 1.349E-02 \\
\hline
\end{tabular}

Note: Boldface values represent the most dominant radioisotope (largest activity) in a waste parcel. 
Table 10-1. Contents (in Curies) of 49 Radionuclides of Waste Parcels at Time of Emplacement (Year 2030) and after $1000 \mathrm{Yr}$ (continued)

\begin{tabular}{|c|c|c|c|c|c|c|}
\hline \multirow[b]{2}{*}{ Nuclide } & \multicolumn{2}{|c|}{ Waste Parcel 22} & \multicolumn{2}{|c|}{ Waste Parcel 23} & \multicolumn{2}{|c|}{ Waste Parcel 24} \\
\hline & $\begin{array}{c}\text { Time of } \\
\text { Emplacement } \\
\text { (year 2030) }\end{array}$ & $\begin{array}{c}\text { Time of } \\
\text { Intrusion } \\
\text { (year 3030) }\end{array}$ & $\begin{array}{c}\text { Time of } \\
\text { Emplacement } \\
\text { (year 2030) }\end{array}$ & $\begin{array}{c}\text { Time of } \\
\text { Intrusion } \\
\text { (year 3030) }\end{array}$ & $\begin{array}{c}\text { Time of } \\
\text { Emplacement } \\
\text { (year 2030) }\end{array}$ & $\begin{array}{c}\text { Time of } \\
\text { Intrusion } \\
\text { (year 3030) }\end{array}$ \\
\hline${ }^{231} \mathrm{~Pa}$ & $0.000 E+00$ & 5.852E-06 & 1.899E-06 & 4.135E-06 & $0.000 E+00$ & $2.822 E-04$ \\
\hline${ }^{232} \mathrm{U}$ & $1.512 \mathrm{E}-01$ & $9.921 \mathrm{E}-06$ & $1.205 \mathrm{E}-07$ & 7.907E-12 & $5.688 E+00$ & $3.732 E-04$ \\
\hline${ }^{233} \mathrm{U}$ & $3.046 E-01$ & $3.048 \mathrm{E}-01$ & $6.531 E-04$ & $1.314 \mathrm{E}-03$ & $1.144 E+01$ & $1.139 E+01$ \\
\hline${ }^{234} \mathrm{U}$ & 5.493E-02 & $5.951 E-02$ & $1.630 \mathrm{E}-02$ & $1.937 \mathrm{E}-02$ & $1.566 \mathrm{E}+00$ & $1.627 \mathrm{E}+00$ \\
\hline $235 \mathrm{U}$ & 2.795E-04 & $2.802 E-04$ & $1.087 E-04$ & $1.094 \mathrm{E}-04$ & $1.348 \mathrm{E}-02$ & $1.348 \mathrm{E}-02$ \\
\hline${ }^{236} \mathrm{U}$ & $1.717 \mathrm{E}-03$ & 1.727E-03 & 2.993E-04 & $3.092 E-04$ & $5.629 \mathrm{E}-02$ & 5.632E-02 \\
\hline${ }^{238} \mathrm{U}$ & $9.708 E-06$ & 9.708E-06 & 6.837E-05 & $6.837 E-05$ & 2.871E-01 & 2.871E-01 \\
\hline${ }^{237} \mathrm{~Np}$ & $3.519 E-01$ & $3.598 \mathrm{E}-01$ & $1.522 \mathrm{E}-01$ & $1.729 \mathrm{E}-01$ & 4.053E-02 & 4.202E-02 \\
\hline${ }^{238} \mathrm{Pu}$ & $1.322 E+01$ & $5.054 \mathrm{E}-03$ & $8.700 E+00$ & $3.232 E-03$ & $1.833 E+02$ & $6.802 E-02$ \\
\hline${ }^{239} \mathrm{Pu}$ & 7.197E-01 & $7.098 \mathrm{E}-01$ & 7.629E-01 & $7.536 \mathrm{E}-01$ & $5.678 E-01$ & $5.536 \mathrm{E}-01$ \\
\hline${ }^{240} \mathrm{Pu}$ & 3.272E-01 & $3.125 \mathrm{E}-01$ & 2.996E-01 & $3.171 \mathrm{E}-01$ & $1.022 E+00$ & $9.226 \mathrm{E}-01$ \\
\hline${ }^{241} \mathrm{Pu}$ & $0.000 E+00$ & $0.000 E+00$ & $5.923 E+00$ & $0.000 \mathrm{E}+00$ & $0.000 E+00$ & $0.000 E+00$ \\
\hline${ }^{242} \mathrm{Pu}$ & $1.470 E-04$ & $1.484 E-04$ & $4.946 \mathrm{E}-04$ & 4.937E-04 & $0.000 E+00$ & 3.171E-07 \\
\hline${ }^{244} \mathrm{Pu}$ & $0.000 E+00$ & $0.000 E+00$ & $1.442 E-13$ & $1.442 E-13$ & $0.000 E+00$ & $0.000 E+00$ \\
\hline${ }^{241} \mathrm{Am}$ & 4.967E+01 & $9.994 E+00$ & $1.283 E+02$ & $2.585 E+01$ & $9.292 E+00$ & $1.870 E+00$ \\
\hline${ }^{242 m} \mathrm{Am}$ & $6.216 \mathrm{E}-03$ & $6.479 E-05$ & $1.745 \mathrm{E}-04$ & $1.819 \mathrm{E}-06$ & 1.169E-03 & $1.218 \mathrm{E}-05$ \\
\hline${ }^{243} \mathrm{Am}$ & 3.874E-01 & $3.526 \mathrm{E}-01$ & $6.778 E-01$ & $6.169 \mathrm{E}-01$ & 7.188E-02 & 6.542E-02 \\
\hline${ }^{243} \mathrm{Cm}$ & $2.178 E-02$ & $5.873 E-13$ & 5.453E-05 & $1.470 \mathrm{E}-15$ & 4.098E-03 & $1.105 \mathrm{E}-13$ \\
\hline${ }^{244} \mathrm{Cm}$ & $7.295 E+00$ & $0.000 E+00$ & $1.914 E+01$ & $0.000 E+00$ & $1.372 E+00$ & $0.000 E+00$ \\
\hline${ }^{245} \mathrm{Cm}$ & 3.405E-03 & $3.138 E-03$ & 1.375E-06 & 1.267E-06 & $6.406 \mathrm{E}-04$ & $5.904 \mathrm{E}-04$ \\
\hline${ }^{246} \mathrm{Cm}$ & $9.853 E-04$ & $8.509 \mathrm{E}-04$ & $9.465 \mathrm{E}-08$ & 8.174E-08 & 1.853E-04 & $1.600 \mathrm{E}-04$ \\
\hline${ }^{247} \mathrm{Cm}$ & 2.921E-09 & $2.921 E-09$ & $0.000 E+00$ & $0.000 E+00$ & 5.495E-10 & 5.495E-10 \\
\hline Total & $5.944 E+04$ & 4.055E+01 & $6.869 E+03$ & $2.883 E+01$ & $1.100 E+04$ & $2.329 E+01$ \\
\hline
\end{tabular}




\begin{tabular}{|c|c|c|c|c|}
\hline \multirow[b]{2}{*}{ Nuclide } & \multicolumn{2}{|c|}{ Waste Parcel 25} & \multicolumn{2}{|c|}{ Waste Parcel 26} \\
\hline & $\begin{array}{c}\text { Time of } \\
\text { Emplacement } \\
\text { (year 2030) }\end{array}$ & $\begin{array}{c}\text { Time of } \\
\text { Intrusion } \\
\text { (year 3030) }\end{array}$ & $\begin{array}{c}\text { Time of } \\
\text { Emplacement } \\
\text { (year 2030) }\end{array}$ & $\begin{array}{c}\text { Time of } \\
\text { Intrusion } \\
\text { (year 3030) }\end{array}$ \\
\hline${ }^{14} \mathrm{C}$ & $0.000 E+00$ & $0.000 E+00$ & $0.000 E+00$ & $0.000 E+00$ \\
\hline${ }^{36} \mathrm{Cl}$ & $1.619 \mathrm{E}-03$ & 1.615E-03 & $0.000 E+00$ & $0.000 E+00$ \\
\hline${ }^{59} \mathrm{Ni}$ & 7.386E-01 & 7.318E-01 & $0.000 E+00$ & $0.000 E+00$ \\
\hline${ }^{63} \mathrm{Ni}$ & $7.528 E+01$ & $5.475 \mathrm{E}-02$ & $0.000 \mathrm{E}+00$ & $0.000 E+00$ \\
\hline${ }^{79} \mathrm{Se}$ & 7.635E-02 & 7.554E-02 & $0.000 E+00$ & $0.000 E+00$ \\
\hline${ }^{87} \mathrm{Rb}$ & $1.229 E-06$ & $1.229 \mathrm{E}-06$ & $0.000 E+00$ & $0.000 E+00$ \\
\hline${ }^{90} \mathrm{Sr}$ & $7.159 E+03$ & $3.287 E-07$ & $6.451 E+02$ & 2.962E-08 \\
\hline${ }^{93} \mathrm{Zr}$ & $3.501 E-01$ & $3.499 E-01$ & $0.000 E+00$ & $0.000 E+00$ \\
\hline${ }^{99} \mathrm{TC}$ & $2.469 E_{+00}$ & $2.461 E+00$ & $0.000 E+00$ & $0.000 E+00$ \\
\hline${ }^{107} \mathrm{Pd}$ & $1.662 E-02$ & $1.662 E-02$ & $0.000 E+00$ & $0.000 E+00$ \\
\hline${ }^{113} \mathrm{Cd}$ & $0.000 E+00$ & $0.000 E+00$ & $0.000 E+00$ & $0.000 E+00$ \\
\hline${ }^{113 m} \mathrm{Cd}$ & $1.413 E-01$ & $0.000 E+00$ & $0.000 E+00$ & $0.000 E+00$ \\
\hline${ }^{126} \mathrm{Sn}$ & $1.264 E-01$ & $1.255 \mathrm{E}-01$ & $0.000 E+00$ & $0.000 E+00$ \\
\hline 129 & 5.497E-03 & 5.497E-03 & $0.000 E+00$ & $0.000 E+00$ \\
\hline${ }^{135} \mathrm{Cs}$ & 5.377E-02 & 5.375E-02 & $0.000 E+00$ & $0.000 E+00$ \\
\hline${ }^{137} \mathrm{Cs}$ & $9.588 E+03$ & 8.857E-07 & $7.520 E+02$ & $6.947 E-08$ \\
\hline${ }^{144} \mathrm{Nd}$ & $0.000 E+00$ & $3.269 E-19$ & $0.000 E+00$ & $1.077 \mathrm{E}-37$ \\
\hline${ }^{147} \mathrm{Pm}$ & $3.266 E+01$ & $0.000 E+00$ & $1.004 E-08$ & $0.000 E+00$ \\
\hline${ }^{147} \mathrm{Gm}$ & $3.452 E-07$ & $3.460 E-07$ & $0.000 E+00$ & 2.486E-19 \\
\hline${ }^{151} \mathrm{Sm}$ & $5.362 E+01$ & $2.410 E-02$ & $0.000 E+00$ & $0.000 E+00$ \\
\hline${ }^{152} \mathrm{Eu}$ & $0.000 E+00$ & $0 . C 00 E+00$ & $0.000 E+00$ & $0.000 E+00$ \\
\hline${ }^{210} \mathrm{~Pb}$ & 8.957E-08 & $1.741 E-05$ & $0.000 E+00$ & $3.228 E-06$ \\
\hline${ }^{226} \mathrm{Ra}$ & $3.41 E-07$ & 4.897E-04 & $0.000 E+00$ & 2.224E-05 \\
\hline${ }^{228} \mathrm{Ra}$ & $0.000 E+00$ & $0.000 E+00$ & $0.000 E+00$ & $0.000 E+00$ \\
\hline${ }^{229} \mathrm{Th}$ & $2.579 E-08$ & $9.486 \mathrm{E}-07$ & $0.000 E+00$ & $4.101 E-05$ \\
\hline${ }^{230} \mathrm{Th}$ & $5.042 E-05$ & 3.365E-03 & $9.471 E-06$ & $1.259 E-04$ \\
\hline${ }^{232} \mathrm{Th}$ & $0.000 E+00$ & 2.826E-09 & 2.841E-11 & $3.612 E-11$ \\
\hline
\end{tabular}


10. Modeling Drilling into Repository (CUTTINGS)

Table 10-1. Contents (in Curies) of 49 Radionuclides of Waste Parcels at Time of Emplacement (Year 2030) and after $1000 \mathrm{Yr}$ (continued)

\begin{tabular}{|c|c|c|c|c|}
\hline \multirow[b]{2}{*}{ Nuclide } & \multicolumn{2}{|c|}{ Waste Parcel 25} & \multicolumn{2}{|c|}{ Waste Parcel 26} \\
\hline & $\begin{array}{l}\text { Time of } \\
\text { Emplacement } \\
\text { (year 2030) }\end{array}$ & $\begin{array}{l}\text { Time of } \\
\text { Intrusion } \\
\text { (year 3030) }\end{array}$ & $\begin{array}{c}\text { Time of } \\
\text { Emplacement } \\
\text { (year 2030) }\end{array}$ & $\begin{array}{c}\text { Time of } \\
\text { Intrusion } \\
\text { (year 3030) }\end{array}$ \\
\hline${ }^{231} \mathrm{~Pa}$ & 6.825E-06 & $2.830 E-04$ & $0.000 E+00$ & $1.437 \mathrm{E}-06$ \\
\hline $232 U$ & 8.665E-05 & 5.685E-09 & $0.000 E+00$ & $0.000 E+00$ \\
\hline${ }^{233} U$ & $1.026 \mathrm{E}-05$ & 2.705E-04 & 4.561E-04 & 8.673E-04 \\
\hline${ }^{234} U$ & 2.793E-01 & 3.825E-01 & 1.120E-02 & $1.324 \mathrm{E}-02$ \\
\hline${ }^{235} U$ & $1.320 E-02$ & 1.324E-02 & 6.862E-05 & $6.909 \mathrm{E}-05$ \\
\hline${ }^{236} \mathrm{U}$ & 5.724E-02 & $5.728 \mathrm{E}-02$ & $1.534 E-04$ & $1.592 E-04$ \\
\hline${ }^{238} U$ & 2.898E-01 & 2.898E-01 & $3.849 \mathrm{E}-06$ & $3.849 \mathrm{E}-06$ \\
\hline${ }^{237} \mathrm{~Np}$ & 5.966E-02 & $1.568 \mathrm{E}-01$ & $9.471 E-02$ & $9.471 E-02$ \\
\hline${ }^{-236} \mathrm{Pu}$ & $2.908 E+02$ & 1.079E-01 & $5.794 E+00$ & $2.149 \mathrm{E}-03$ \\
\hline${ }^{239} \mathrm{Pu}$ & 4.513E+01 & $4.391 E+01$ & 4.890E-01 & $4.752 E-01$ \\
\hline${ }^{240} \mathrm{Pu}$ & $1.125 E+00$ & $1.183 E+00$ & 2.082E-01 & $1.873 E-01$ \\
\hline${ }^{241} \mathrm{Pu}$ & $4.258 E+03$ & $0.000 E+00$ & $0.000 E+00$ & $0.000 E+00$ \\
\hline${ }^{242} \mathrm{Pu}$ & 2.506E-01 & 2.501E-01 & $1.020 \mathrm{E}-04$ & $1.018 E-04$ \\
\hline${ }^{244} \mathrm{Pu}$ & 1.037E-10 & $1.037 \mathrm{E}-10$ & $0.000 E+00$ & $0.000 E+00$ \\
\hline${ }^{241} \mathrm{Am}$ & 4.601E+02 & $1.211 E+02$ & $1.894 \mathrm{E}-01$ & 3.810E-02 \\
\hline${ }^{242 m} \mathrm{Am}$ & $6.271 E-04$ & 6.537E-06 & $0.000 E+00$ & $0.000 E+00$ \\
\hline${ }^{243} \mathrm{Am}$ & $2.417 E+00$ & $2.200 E+00$ & $3.704 \mathrm{E}-03$ & 3.371E-03 \\
\hline${ }^{243} \mathrm{Cm}$ & $1.960 E-04$ & $5.285 \mathrm{E}-15$ & $0.000 E+00$ & $0.000 E+00$ \\
\hline${ }^{244} \mathrm{Cm}$ & $6.878 E+01$ & $0.000 E+00$ & $0.000 E+00$ & $0.000 E+00$ \\
\hline${ }^{245} \mathrm{Cm}$ & 4.943E-06 & 4.556E-06 & $0.000 E+00$ & $0.000 E+00$ \\
\hline${ }^{246} \mathrm{Cm}$ & $3.402 E-07$ & 2.938E-07 & $0.000 E+00$ & $0.000 E+00$ \\
\hline${ }^{247} \mathrm{Cm}$ & $0.000 E+00$ & $0.000 E+00$ & $0.000 E+00$ & $0.000 E+00$ \\
\hline Total & $2.204 E+04$ & $1.736 E+02$ & $1.404 E+03$ & 8.154E-01 \\
\hline
\end{tabular}

Note: Boldface values represent the most dominant radioisotope (largest activity) in a waste parcel. 

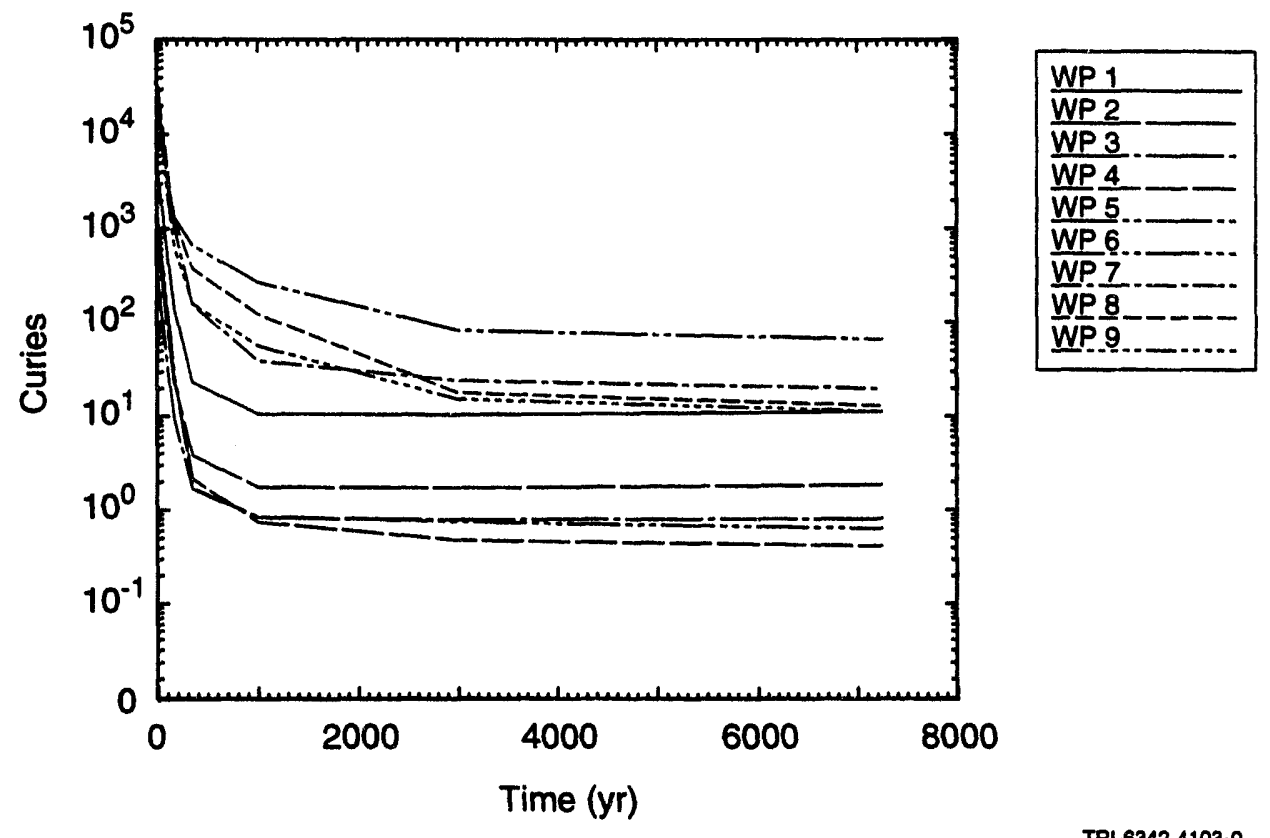

(a)
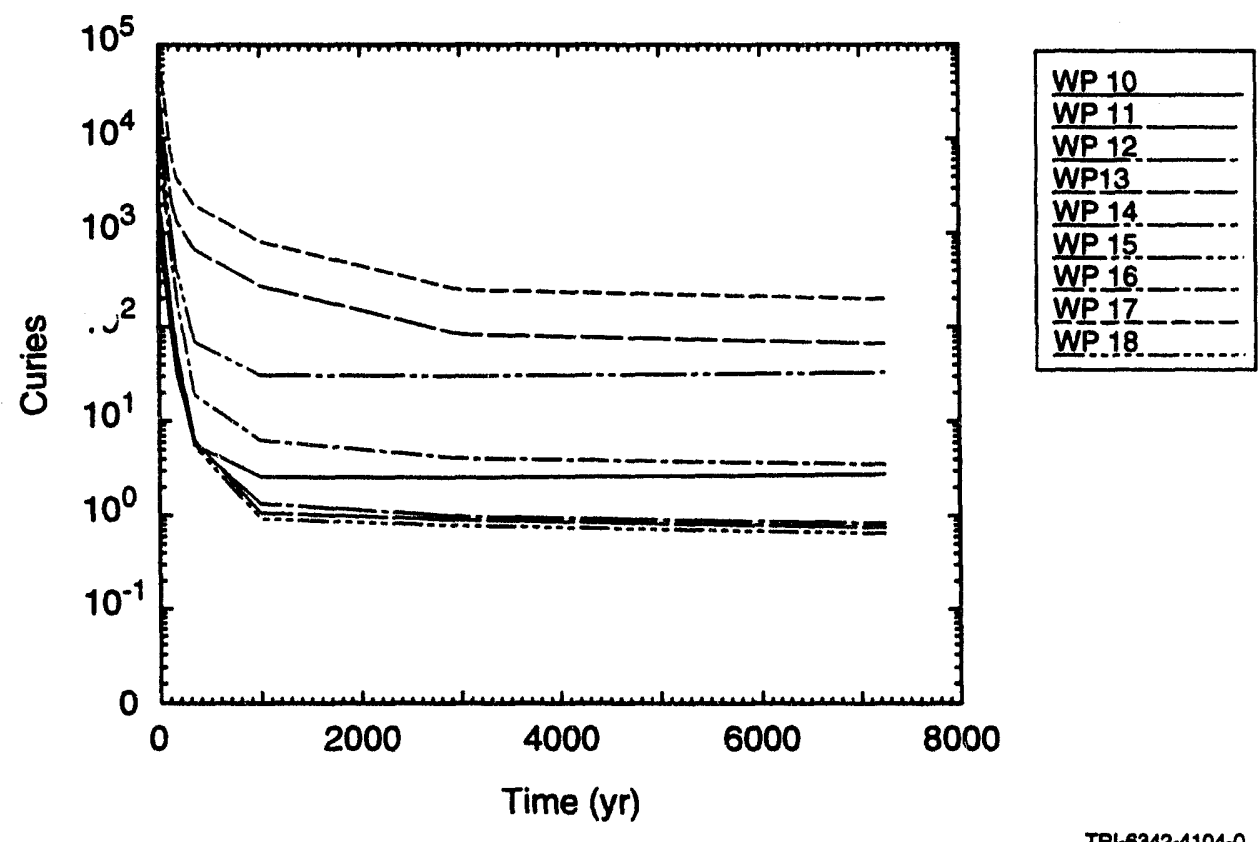

(b)

Figure 10-1. Total curie content at six intrusion times for (a) WP01 to WP09, (b) WP10 to WP18, and (c) WP19 to WP26. 
10. Modeling Drilling into Repository (CUTTINGS)
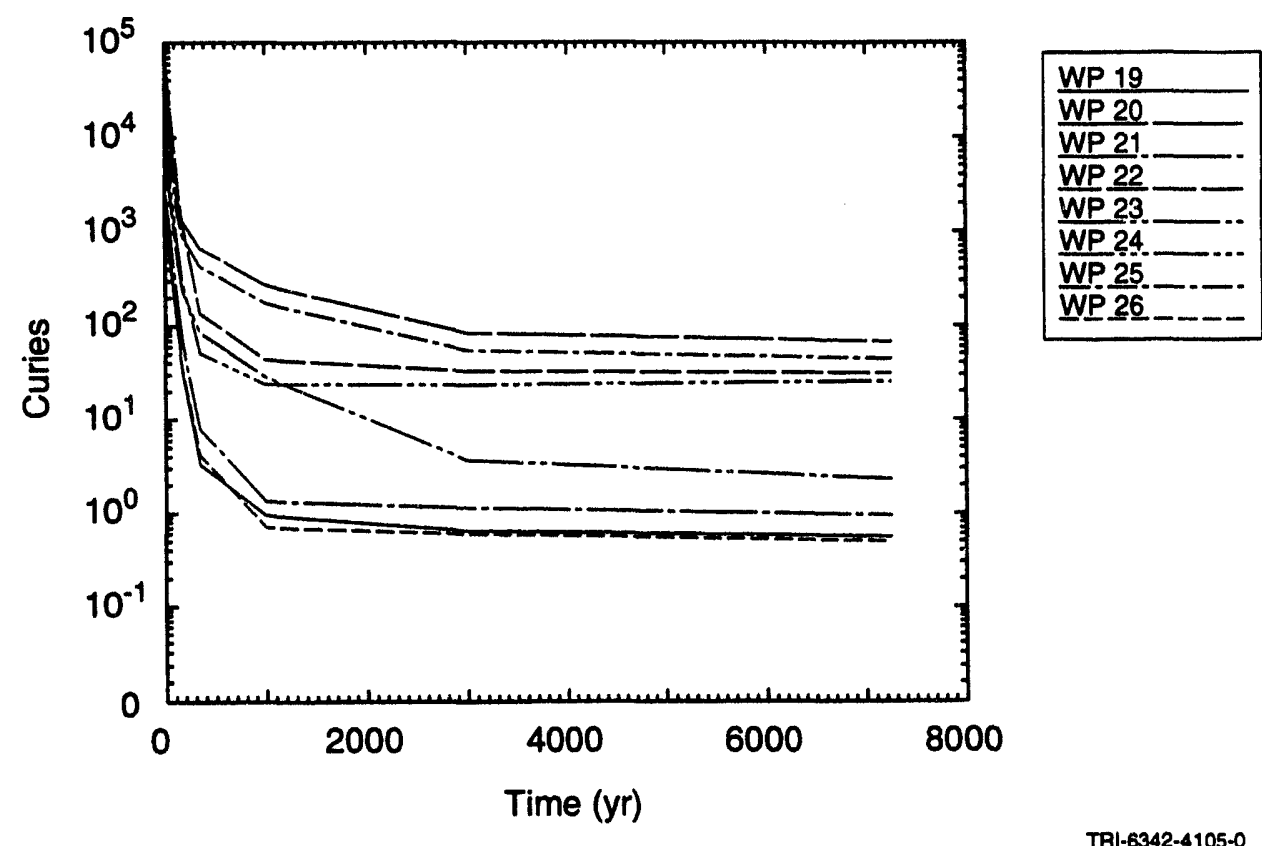

13

14

Figure 10-1. Total curie content at six intrusion times for (a) WP01 to WP09, (b) WP10 to WP18, and (c) WP19 to WP26 (continued). 


\title{
11. Gas Generation and Source Term Submodels
}

\author{
C. T. Stockman
}

In this performance assessment, an effort was made to model details of gas generation from the corrosion of the metal waste containers (gas generation submodel) and the resultant movement of contaminants out of the containers and into the repository (source term submodel). To some extent, the inclusion of these specifics ahout the waste form in a total system performance assessment distinguishes this effort from earlier assessments. A discussion of these submodels is presented in this chapter to provide information about how these processes were modeled. The gas generation and source term submodels were used in both the simple and complex assessments (Chapters 12 through 15). An understanding of the models will be beneficial in interpreting the results.

\subsection{Gas Generation Submodel}

This chapter describes the expected process by which the layers of the waste parcels corrode and breach and includes a description of the gas generation submodel, GASSNF, which simulates the process. The fluid flow model for the complex performance assessment is BRAGFLO_T; for the simple performance assessment, the model is SALFLOW (for salt repository) and GRFLOW (for granite repository). Modeling parameters for the gas generation submodel are discussed in Section 11.3.

\subsubsection{Types of Corrosion in a Waste Parcel}

To simulate the corrosion/breach process, the models had to incorporate an understanding of material corrosion, the important factors in the process, and the anticipated steps of the process. In general, corrosion is expected to occur when layers of the waste parcel react with oxidants. The oxidants expected in the hypothetical repositories are: oxygen, peroxide, water, sulfate and sulfides $\left(\mathrm{H}_{2} \mathrm{~S}\right.$ and $\left.\mathrm{HS}^{-}\right)$.

- Oxygen: Although there is little dissolved oxygen in deep groundwaters, because of the excavation there initially will be significant amounts of $\mathrm{O}_{2}$ in the air and dissolved in the groundwater in immediate contact with the air.

- Hydrogen Peroxide: Hydrogen peroxide is one of the expected products of the radiolysis of water. The amount produced around each waste parcel depends mainly on the amount and type of radiation entering the water surrounding the waste parcel. Calculations on the amount of radiation expected from two waste parcels were completed, but due to time constraints, further analysis has not been performed.

- Water: Water saturation is calculated by the flow model (see Section 13.1.4).

- Sulfate/Sulfides: Sulfate is present in both the salt and granite repositories, but in the absence of microbes to accelerate the reaction process, very little of it will react with container metals. We cannot totally dismiss the presence of microbes, because the waste and/or repository may contain organic contaminants. The overall level of nutrients is expected, however, to be quite low, so we expect that the amount of sulfate converted to sulfide by microbes will be small. Sulfides are present in low concentrations in granite groundwater and some bentonites. The total amount of sulfide available for reaction with container metals will be determined by the amount in the bentonite and flow rate of the groundwater past the containers.

The gas generation submodel was written to perform two major functions: calculate the gas generation and water consumption rates for use in the flow models, and calculate the breach times of the waste parcel layers. The breach times of the parcel layers have a direct effect on the calculated gas generation rate (by determining which metals are exposed and their total surface areas), but even more importantly, are used by the source term model to determine the time at which radionuclides come in contact with groundwater. Because of these functions, the submodel needed two pieces of information on each parcel material: the rate of bulk material reaction, and the rate of material penetration. This information was obtained or extrapolated from the literature reports of the various types of corrosion and failure modes for these materials. The types of corrosion include: generalized, pitting, crevice, 
intergranular, and stress-corrosion cracking, etc. In addition, some failure modes include: manufacturing defects, mechanical damage, hydrogen embrittlement, hydride realignment, aging, long-term creep, etc.

Generalized Corrosion. Generalized corrosion is characterized by a fairly uniform reaction across the entire metal surface. Note that material corrosion rates reported in the literature are often the bulk material reaction rates accompanied by a qualitative description of the uniformity of the attack. Presence of cracks, crevices, pits, etc. are usually noted but often not quantified.

Localized Corrosion. Localized corrosion includes corrosion within a pit or a crevice. It is caused by concentration cells in locations where oxygen or metal ions are depleted/enriched because of inadequate mixing. In the case of waste parcels, the concentration cells are expected to initiate at the site of manufacturing defects or mechanical damage in the containers.

Stress-Corrosion Cracking. Stress-corrosion cracking is a form of localized corrosion that occurs when the susceptible material is in a corrosive environment while under a tensile stress. The tensile stress can be active, such as those imposed by lithostatic loads, or residual, such as those caused by welding or other fabrication processes.

Corrosion-Resistant Materials. One important factor in selecting container materials is their corrosion resistance in the expected chemical environment of the repository. Materials resist corrosion because (a) they are "noble" or thermodynamically stable, (b) they are thermodynamically unstable, but develop a protective oxide coating that resists attack, or (c) they are unstable, but the reaction is slow because either the activation energy for reaction is very large or the rate at which reactants migrate to the canister is very slow. No container material is stable under all possible conditions.

Materials considered in gas generation layers of the waste parcels include Ti-Grade 12, lead, Inconel 625, stainless steel 304L, zircaloy, aluminum, and graphite. As seen in Table 11-1, most of these materials rely on a protective coating for their corrosion resistance. In these cases, generalized corrosion will be slow as long as the protective coating remains intact. When the coating is destroyed in a small area and not re-established, the material will undergo localized corrosion, which includes pitting, crevice corrosion, and stress-corrosion cracking. Conditions that contribute to the breakdown of the protective coating include low $\mathrm{pH}$, low Eh, corrosive anions such as chloride, high temperature, and mechanical stress.

Table 11-1. Corrosion Resistance in Waste Parcel Layer Materials

\begin{tabular}{|c|c|c|c|}
\hline & \multicolumn{3}{|c|}{ Corrosion Resistance } \\
\hline & $\begin{array}{c}\text { Protective } \\
\text { Coating }\end{array}$ & $\begin{array}{c}\text { Large Activation } \\
\text { Energy }\left(E_{\mathrm{a}}\right)\end{array}$ & $\begin{array}{c}\text { Limited } \\
\text { Reactants }\end{array}$ \\
\hline Ti-Grade 12 & $\bullet$ & & \\
\hline Lead & & & - \\
\hline Inconel 625 & $\bullet$ & & - \\
\hline Stainless steel 304L & - & & \\
\hline Zircaloy & $\bullet$ & & \\
\hline Aluminum & $\bullet$ & & \\
\hline Graphite & & - & \\
\hline
\end{tabular}




\subsubsection{Conceptual Model of Corrosion Process}

Because the gas generation model is called many times per time step for each grid block that contains waste, a major consideration in the design of the conceptual model was computational speed. Below we describe in detail what we believe may happen during the corrosion of a single waste parcel (Figure 11-1), and also how we have simplified the model to achieve computational speed. In the example shown in Figure 11-1, the waste form is special highly enriched uranium spent fuel and the treatment option is placing the fuel in canisters and filling the void space with lead (Waste Disposal Group 1). (Note, stainless steel is not a good corrosion-resistant material and was used in this performance assessment because significant container failure is necessary in order to distinguish different waste disposal options.)

In this example, the waste parcel includes four layers that may corrode to produce gas: the stainless steel canister (layer 1), the castable metal filler, e.g., lead (layer 2), the special fuel cladding, e.g., aluminum (layer 3), and the metallic fuel (layer 4).

Corrosion of Layer 1 - Stainless Steel. The breach of the stainless steel (layer 1) is assumed to be fairly fast due to stress-corrosion cracking, especially in brine. At early times, the primary oxidant in the reaction with the steel is expected to be residual oxygen dissolved into the groundwater during excavation and waste emplacement. Thus, during the breach of the steel, the corrosion process consumes gas. The steel continues to corrode after breach and eventually all the oxygen will be consumed (by all exposed metals). When the oxygen is depleted, the steel reacts with water and produces hydrogen gas. This process continues until all the steel is consumed. Reaction with other oxidants such as peroxide and sulfide, if present, will also occur. Sulfide may affect the passivating properties of the stainless steel.

To keep the model simple, a single reaction and reaction rate for stainless steel was chosen for the entire regulatory period. The reaction chosen was the reaction after oxygen was consumed, because oxygen is expected to be present for only a short time. (This assumption should be checked in future performance assessments.) The rate was sampled over a large range to compensate for the uncertainty in its value. To simulate breach from localized corrosion, a parameter was input to indicate the fraction of a layer that was removed at the time of breach (perforation fraction). Note that this method of determining breach time is different than using a penetration rate and material thickness, although the resultant breach times will be similar if the perforation fractions are based on perforation rate data. Since very little of the stainless steel total mass has reacted when stress corrosion cracking breaches the layer, the perforation fractions input for stainless steel are very small. A range of perforation fractions were used because of the inherent uncertainty in the relative speed of generalized corrosion versus penetration due to the many possible failure mechanisms. It is important to note that the perforation fraction is used only to determine the time of breach, it is not used to estimate the size of the breach. Instead, the model assumes that a breached layer offers no protection.

Corrosion of Layer 2 - Lead. Because the breach of the steel layer is expected to be caused by localized corrosion, the amount of dissolved oxygen will not have decreased significantly at the time of breach. Therefore, the initial reaction will be that of lead with dissolved oxygen. Lead is not expected to "wet" steel, and a small gap into which water can flow is assumed to exist between the lead and the steel. However, because of transport limitations most of the lead corrosion is expected to occur in the small area directly under the crack in the steel. The resultant lead oxide does not passivate the reaction, and the reaction continues at a rate determined by the transport of dissolved oxygen to the lead. When all the available oxygen is used up, peroxide or sulfides, if present, will react as fast as they are produced and/or transported toward the lead. This reaction will occur as long as the amount of sulfide is not great enough to form a passivating coat of lead sulfide. In the absence of these or other oxidants, the lead will start to react with the water to produce hydrogen gas. When the hydrogen fugacity approaches $1 \times 10^{-5} \mathrm{~atm}$, the reaction will slow and be limited by the rate at which the hydrogen can be transported away.

The available oxidants determine which reactions will occur, when they will occur, and how fast they will proceed. Once again we have condensed the many reactions and reaction rates down to a single reaction and a range of rates in our model. Breach of the lead occurs when any portion of the lead is perforated through to the aluminium clad of the fuel rod. Because the fuel rod is not cylindrical and because it may not be exactly in the center of the waste parcel, these is some uncertainty in the depth of lead that must be penetrated for breach to occur. Since we 


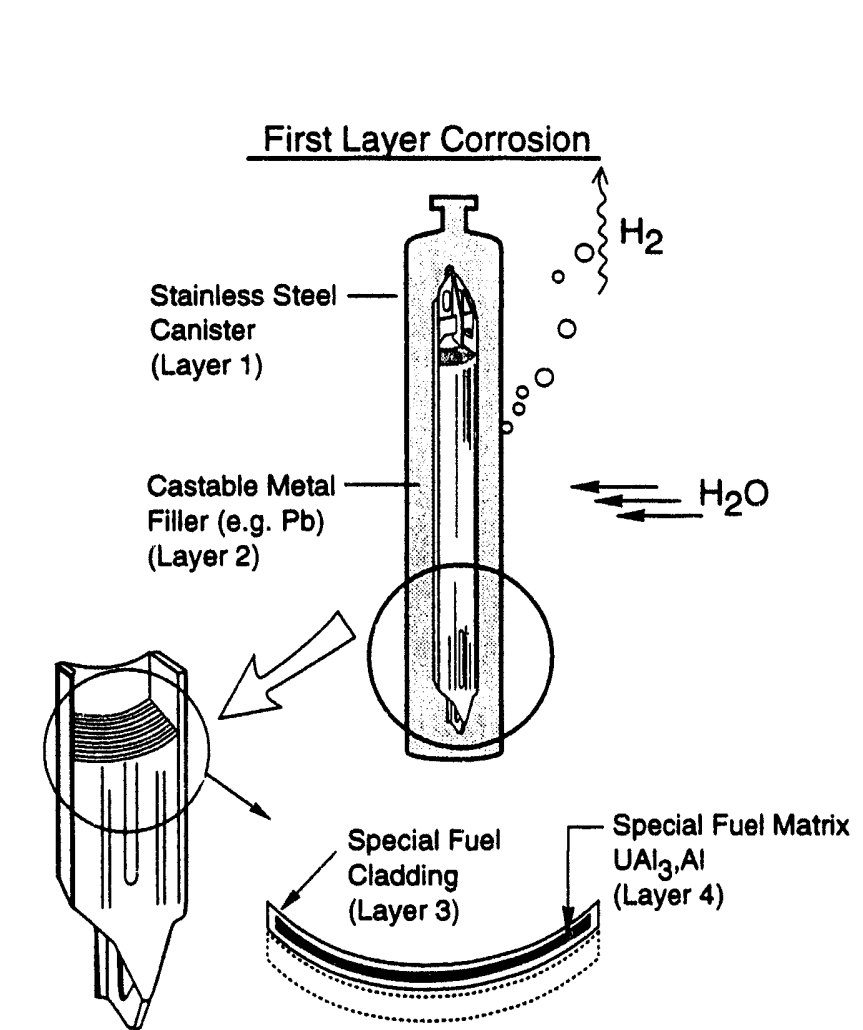

- Dissolved $\mathrm{O}_{2}$ (available at time of burial) is assumed to be quickly consumed.

- Stress corrosion cracking of stainless steel canister (Layer 1) occurs fairly fast.

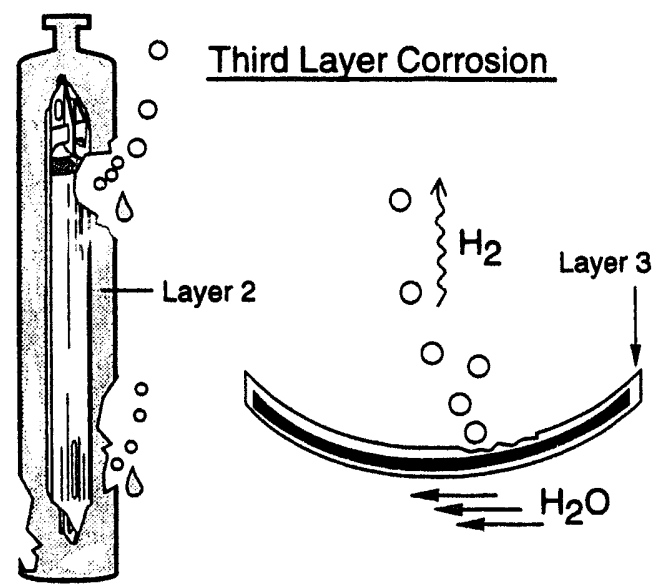

- Layer 3 corrosion begins after a specified mass fraction of Layer 2 has been corroded.

\section{Second Layer Corrosion}

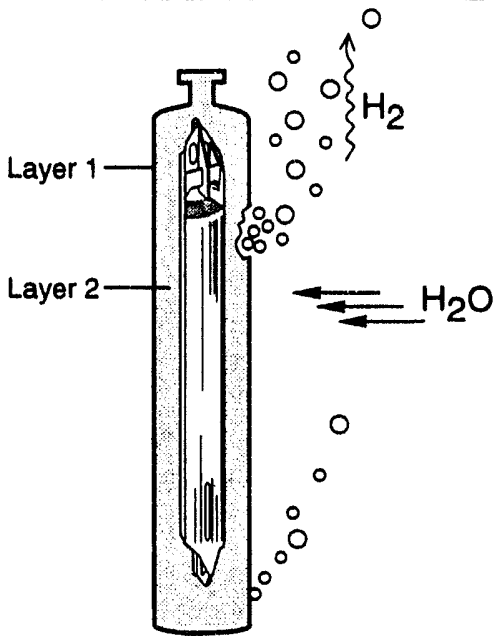

- Layer 2 corrosion begins after a specified mass fraction of Layer 1 has been corroded.

- In absence of dissolved $\mathrm{O}_{2}$ lead reacts with $\mathrm{H}_{2} \mathrm{O}$ and quickly creates equilibrium amounts of $\mathrm{H}_{2}$; after this time, corrosion rate is determined by $\mathrm{H}_{2}$ transport and radiolitic production of peroxide $\left(\mathrm{H}_{2} \mathrm{O}_{2}\right)$.

- Corrosion of each layer continues until entire layer is consumed.

\section{Fourth Laver Corrosion (Matrix)}

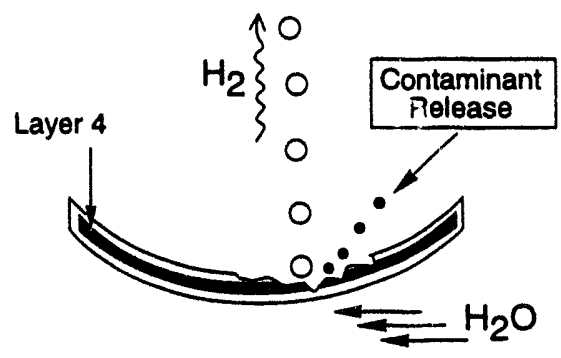

- When Layer 3 is breached, source term sub-model starts the release of radionuclides from Layer 4.

- Both the gas generation sub-model and the source term sub-model use the corrosion rate of Layer 4.

TA1-6342-3796.0

Figure 11-1. Corrosion and breach of waste container and waste form layers. In the example illustrated, the waste form is special highly enriched uranium spent fuel, and the waste treatment option is placing the spent fuel in canisters and filling the void space with lead (Waste Disposal Group 1). 
have used a perforation fraction to determine breach time and not a material thickness and penetration rate, we have added this uncertainty into our estimate of the uncertainty in the perforation fraction. A conservatively small perforation fraction was choser $(0.001$ to 0.1$)$ because, although the lead often undergoes fairly generalized corrosion, it is expected that the lead will preferentially corrode in the small area exposed by the breach in the steel canister. If the corrosion was assumed to not be localized, and a larger perforation fraction was included in the range, breach times for lead would be calculated to occur at later times.

Corrosion of Layer 3-Aluminum. When the lead layer is breached, the fuel cladding begins to corrode. In the presence of chloride, the passive coating of aluminum deteriorates and the aluminum corrodes at a relati:ely rapid rate.

Corrosion of Layer 4 - Uranium-Aluminum Alloy. The uranium-aluminum alloy fuel is the last layer to corrode. Little is known about the corrosion rate of this alloy in repository groundwaters. In the severe conditions of high acidity used in dissolving these fuels during reprocessing, the uranium-aluminum alloy fuel dissolves faster than the aluminum clad. Therefore, we have conservatively used a high corrosion rate. Because there are no layers inside the fuel, the perforation fraction is irrelevant.

The source term submodel uses the breach time of the layer protecting the radionuclide-containing matrix as the time to start its dissolution of the radionuclides (see Section 11.2). In this example, the radionuclides are part of Layer 4, which is also called matrix 1 for the source term submodel. The last protective layer is Layer 3; when Layer 3 is breached, the gas generation submodel corrodes Layer 4 for its gas-generating ability, and the source term model corrodes Layer 4 to release its radionuclides. The same corrosion rates are used by both submodels.

\subsubsection{Mathematical/Computational Model}

The gas generation submodel, GASSNF, is part of the fluid flow code, either BRAGFLO_T (Complex PA), SALFLOW (Simple PA for salt), or GRFLOW (Simple PA for granite). In the performance assessment, the following conditions were modeled for gas generation:

- Stoichiometry of the gas-producing corrosion reaction.

- Rate of the corrosion reaction for each material.

- Saturation and temperature.

Stoichiometry of Corrosion. The reaction of the container with water can be generalized as:

$$
\text { metal }+\mathrm{S}_{\mathrm{H}_{2} \mathrm{O}} \cdot \mathrm{H}_{2} \mathrm{O} \rightarrow \text { solid corrosion products }+\mathrm{s}_{\mathrm{gas}} \cdot \text { gas products }
$$

where

$\mathrm{s}_{\mathrm{H}_{2} \mathrm{O}}=$ stoichiometry of water, moles water consumed per mole of metal consumed

$\mathrm{s}_{\mathrm{gas}}=$ stoichiometry of gas, moles of gas produced per mole of metal consumed

A list of possible reactions in the repository environment was compiled from a cursory literature search and the use of the code REACT by Bethke (1992). REACT is a code similar to EQ3/6 using the same database provided by Wolery (1992). The database was derived from "data0.3245r46" using the 1 atmosphere steam saturation curve data and the debye-huckel equations. The stable corrosion products from many of the metals were dependant on the repository minerals. For example in the presence of sulfide, $\mathrm{PbS}$ and $\mathrm{Ni}_{3} \mathrm{~S}_{2}$ are important corrosion products, but in the presence of silicates, $\mathrm{Ni}_{2} \mathrm{SiO}_{4}$ becomes important. We expect calcium to be present in both repositories so we have included it in the reaction of zircaloy with water to produce $\mathrm{CaZrO}_{4}$. Table 11-2 shows some of the reactions that produced oxide corrosion products and their stoichiometries. Other corrosion products include carbonates, sulfates, sulfides, silicates, etc. The actual stoichiometries used in the calculations are averages of these and other reactions and are given in Section 11.3. When a dominant reaction for a metal could be identified, the stoichiometry of that reaction was used, otherwise a weighted average of the probable reactions was used. In this performance 
Table 11-2. Examples of Some Reactions of Waste/Container with Water*

\begin{tabular}{|c|c|c|}
\hline Reaction & $\begin{array}{l}\text { Liquid Stoichiometry } \\
\qquad\left(\mathrm{s}_{2} \mathrm{O}\right)\end{array}$ & $\begin{array}{c}\text { Gas Stoichiometry } \\
\left(\mathbf{s}_{\text {gas }}\right)\end{array}$ \\
\hline \multicolumn{3}{|l|}{ Reactions in absence of dissolved oxygen } \\
\hline $\mathrm{Al}+3 / 2 \mathrm{H}_{2} \mathrm{O}=1 / 2 \mathrm{Al}_{2} \mathrm{O}_{3}+3 / 2 \mathrm{H}_{2}$ & 1.5 & 1.5 \\
\hline $\mathrm{C}+2 \mathrm{H}_{2} \mathrm{O}=\mathrm{CO}_{2}+\mathrm{H}_{2}$ & 2 & 3 \\
\hline $\mathrm{C}+\mathrm{H}_{2} \mathrm{O}=\mathrm{CO}+\mathrm{H}_{2}$ & 1 & 1 \\
\hline Steel (average): & 1.7 & 1.17 \\
\hline $\mathrm{Fe}+2 \mathrm{H}_{2} \mathrm{O}=\mathrm{Fe}(\mathrm{OH})_{2}+\mathrm{H}_{2}$ & 2 & 1 \\
\hline $\mathrm{Fe}+4 / 3 \mathrm{H}_{2} \mathrm{O}=1 / 3 \mathrm{Fe}_{3} \mathrm{O}_{4}+4 / 3 \mathrm{H}_{2}$ & 1.3 & 1.3 \\
\hline Inconel (weighted average): & 1.29 & 1.29 \\
\hline $69 \% \mathrm{Ni}+\mathrm{H}_{2} \mathrm{O}+=\mathrm{H}_{2}+\mathrm{NiO}$ & 1 & 1 \\
\hline $22 \% \mathrm{Cr}+3 / 2 \mathrm{H}_{2} \mathrm{O}=1 / 2 \mathrm{Cr}_{2} \mathrm{O}_{3}+3 / 2 \mathrm{H}_{2}$ & 1.5 & 1.5 \\
\hline $9 \% \mathrm{Mo}+\mathrm{PbO}+3 \mathrm{H}_{2} \mathrm{O}=\mathrm{PbMoO}_{4}+3 \mathrm{H}_{2}$ & 3 & 3 \\
\hline $\mathrm{Pb}+\mathrm{H}_{2} \mathrm{O}=\mathrm{PbO}+\mathrm{H}_{2}$ & 1 & 1 \\
\hline $\mathrm{Ti}+2 \mathrm{H}_{2} \mathrm{O}=\mathrm{TiO}_{2}+2 \mathrm{H}_{2}$ & 2 & 2 \\
\hline \multicolumn{3}{|l|}{ Metal Carbide Fuel: } \\
\hline $\mathrm{ThC}_{2}+2 \mathrm{H}_{2} \mathrm{O}=\mathrm{ThO}_{2}+\mathrm{C}_{2} \mathrm{H}_{2}+\mathrm{H}_{2}$ & 2 & 2 \\
\hline $\mathrm{UC}_{2}+2 \mathrm{H}_{2} \mathrm{O}=\mathrm{UO}_{2}+\mathrm{C}_{2} \mathrm{H}_{2}+\mathrm{H}_{2}$ & 2 & 2 \\
\hline $\mathrm{U}_{.9} \mathrm{Al}_{4}+7.8 \mathrm{H}_{2} \mathrm{O}=.9 \mathrm{UO}_{2}+2 \mathrm{Al}_{2} \mathrm{O}_{3}+7.8 \mathrm{H}_{2}$ & 7.8 & 7.8 \\
\hline $\mathrm{Zr}+3 \mathrm{H}_{2} \mathrm{O}+\mathrm{CaH}_{3}=\mathrm{CaZrO}_{3}+2 \mathrm{H}_{2}+2 \mathrm{H}^{+}$ & 3 & 2 \\
\hline \multicolumn{3}{|l|}{ Reactions in presence of dissolved oxygen } \\
\hline $\mathrm{Al}+3 / 4 \mathrm{O}_{2}=1 / 2 \mathrm{Al}_{2} \mathrm{O}_{3}$ & 0 & -.75 \\
\hline $\mathrm{C}+\mathrm{O}_{2}=\mathrm{CO}_{2}$ & 0 & -1 \\
\hline $\mathrm{Fe}+1 / 3 \mathrm{O}_{2}=1 / 2 \mathrm{Fe}_{2} \mathrm{O}_{3}$ & 0 & -.33 \\
\hline $\mathrm{Ni}+1 / 2 \mathrm{O}_{2}+=\mathrm{NiO}$ & 0 & -.5 \\
\hline $\mathrm{Cr}+3 / 4 \mathrm{O}_{2}=1 / 2 \mathrm{Cr}_{2} \mathrm{O}_{3}$ & 0 & -.75 \\
\hline $\mathrm{Mo}+\mathrm{PbO}+3 / 2 \mathrm{O}_{2}=\mathrm{PbMoO}_{4}$ & 0 & -1.5 \\
\hline $\mathrm{Pb}+1 / 2 \mathrm{O}_{2}=\mathrm{PbO}$ & 1 & -.5 \\
\hline$\pi+O_{2}=\pi O_{2}$ & 0 & -1 \\
\hline $\mathrm{Zr}+\mathrm{O}_{2}+\mathrm{Ca}^{++}+\mathrm{H}_{2} \mathrm{O}=\mathrm{CaZrO}_{3}+2 \mathrm{H}^{+}$ & 1 & -1 \\
\hline
\end{tabular}

- Stoichiometries used in calculations can be found in Tables 11-3 and 11-4. Oxide corrosion products are shown here, although many other reactions will occur. 
assessment, the reactions given most weight were those that occur in the absence of dissolved oxygen. This decision was based on the expectation that the initial oxygen will be consumed rapidly compared to $10,000 \mathrm{yr}$ and that radiolysis will produce oxygen at a very slow rate. Since the time for initial oxygen consumption and the rate of radiolytic oxygen production have not been calculated, we cannot say how large an error is produced by neglecting the oxic reactions of container materials. Further calculations are recommended to confirm or refute that the error is small. The errors in the stoichiometries used in this performance assessment due to missing important anoxic reactions are expected to be no more than a factor of two. This error is small in comparison to the uncertainty in the corrosion reaction rates.

Rates of Reaction. The rate of reaction for corrosion was evaluated as follows:

$$
r_{\text {metal }}=r_{A} \cdot A \cdot\left[1-e^{-\alpha\left(S_{B}-S_{B r}\right)}\right] e^{-E a / R T}
$$

where
$r_{A}$
A
$\mathrm{E}_{\mathrm{a}}$
R
$\mathrm{T}$
$1-e^{-\alpha\left(S_{B}-S_{B r}\right)}$
$=$ corrosion rate per surface area (from literature)
$=$ surface area
$=$ activation energy
$=$ universal gas constant
$=$ temperature
ual saturation $\mathrm{S}_{\mathrm{Br}}$
$\alpha$
$=$ parameter controlling adjustment; large value approximates a step function (value of 20
$\mathrm{e}^{-\mathrm{E} a / \mathrm{RT}}$ selected based on scoping calculations reported in Appendix C).
$=$ Arhenius rate equation term

The reaction rates per surface area were taken from the literature, but often the rates were for conditions different from those being considered. In addition, while overall corrosion rates were reported, often only qualitative descriptions were given for the amount and type of localized corrosion. Thus, the analyst used professional judgment in estimating ranges for these rates (see Section 11-3).

Because most rates are specified as mass corroded per surface area and time, Equation 11-2 uses this method.

Also, the Arhenius equation for the rates has been incorporated into Equation 11-2 because rates can be highly temperature dependent. However, sufficient time was not available to search for temperature-dependent corrosion data. Because these data are not available for many of the reactions, a range of rates was used for these reactions with the activation energy set to zero.

Some simplifications have been used in evaluating the surface area of each layer for Equation 11-2. Although the simplifications cause errors, they are small compared to the uncertainty in the corrosion rates. The surface area is assigned a constant value that equals the initial outside surface area of the layer within the grid block. This constant value ignores the increase or decrease of the surface area as the layer corrodes and does not include the inside surface area after the layer is breached. Use of the outside surface area is also equivalent to assuming that any filler materials do not "wet" the other layers and thus a small gap exists into which water may migrate.

Production Rates. Both the gas production rate and water consumption rate used by the fluid rates are evaluated as follows (both depend upon the corrosion rate):

$$
\begin{gathered}
\text { gas production rate }=\mathrm{r}_{\text {metal }} \bullet \mathrm{s}_{\text {gas }} \bullet \frac{\mathrm{MW}_{\text {gas }}}{\mathrm{MW}_{\text {metal }}} \\
\text { water consumption rate }=\mathrm{r}_{\text {metal }} \bullet \mathrm{s}_{\mathrm{H} 2 \mathrm{O}} \cdot \frac{\mathrm{MW}_{\mathrm{H} 2 \mathrm{O}}}{\mathrm{MW}_{\text {metal }}}
\end{gathered}
$$

December 1993 
where

$$
\begin{array}{ll}
r_{\text {metal }} & =\text { metal corrosion rate, } \\
\mathrm{MW} & =\text { molecular weight } \\
\mathrm{S}_{\text {gas }} & =\text { stoichiometry of gas, } \\
\mathrm{S}_{\mathrm{H}_{2} \mathrm{O}} & =\text { stoichiometry of liquid. }
\end{array}
$$

Equations 11-3 and 11-4 must be evaluated for each gas-producing material that is exposed to brine. Gas-producing materials are modeled as nested layers, with a layer corroding only when all outer layers are breached.

\subsubsection{Interface of GASSNF with BRAGFLO_T}

GASSNF requires the liquid saturation of the element as input and calculates the rate of gas generation and rate of v:ater consumption. The fully flooded rate of corrosion for each reactive substance is assumed constant over the modeled time span. The model describes the corrosion and breach of nested layers of metal and can be used for any number of nested barriers. Gas generation from a layer occurs when (1) all overlying layers are breached, (2) metal remains in the current layer, and (3) there is sufficient water for reaction.

To calculate the saturation of the element, BRAGFLO_T requires the rates for total gas produced and brine consumed in each element. However, because production and consumption rates depend on saturation (Equation 11-2), GASSNF must be repeatedly called until BRAGFLO_T converges on a solution.

After convergence and at each timestep, GASSNF updates the mass of each layer in each type of waste parcel per element and checks to determine its status: protected, exposed, breached, or gone. A layer is breached when its mass is less than its initial mass multiplied by its "perforation fraction." The perforation fraction is a value that is input by the user and signifies the user's estimate of the fraction of material removed at time of breach:

where

$$
\text { perforation fraction }=\frac{\text { mass }_{\text {tot }}-\text { mass }_{\text {breach }}}{\text { mass }_{\text {tot }}}
$$

$$
\begin{array}{ll}
\text { mass }_{\mathrm{tot}} & =\text { original mass, } \\
\text { mass }_{\mathrm{t}} & =\text { current mass, } \\
\text { mass }_{\text {breach }} & =\text { mass remaining at breach. }
\end{array}
$$

Thus, breach occurs when

$$
\text { mass }_{\mathrm{t}}<\text { mass }_{\text {tot }} \cdot \text { perforation fraction. }
$$

For example, when the user believes that a material will fail by generalized corrosion, he/she will chose a perforation fraction close to 1 . When the material is expected to fail by localized corrosion, a value close to 0 will be chosen. When the material is not expected to provide any barrier to inflow of water, the value is set to 0 .

While BRAGFLO_T requires only the production and consumption rates for each element, the submodel must keep track of the mass of each layer corroded and not allow any masses to go negative. Accordingly, the submodel calculates the mass of each layer that would be consumed by Equation 11-2 in a time step. If Equation 11-2 indicates that more metal will be consumed than remains in the layer, only the amount in the layer is allowed to be corroded. Once the mass of metal consumed is calculated, the mass of gas produced and water consumed is easily calculated by equations similar to 11-3 and 11-4. The total mass of gas produced in the grid block is then given by:

$$
\Delta \text { Mass }_{\text {gas }}=\sum_{W P s} \sum_{\text {layers }} \Delta \text { Mass layer } \bullet s_{\text {gas }} \bullet \frac{M W_{\text {gas }}}{M W_{\text {layer }}},
$$


and the production rate is given by:

$$
\text { rate }=\Delta \text { Mass gas } / \Delta t .
$$

The important input, output, and model parameters are summarized below. The layer input parameters are constants.

Input from BRAGFLO_T:

Output to BRAGFLO_T:

Output to source term:

Model input:
Water saturation.

Mass gas generation rate each time step.

Mass water consumption rate each time step.

Time of each layer breach for each waste parcel and each element.

For each layer material:

$\mathrm{m}_{1}$, initial mass

$\mathrm{k}_{\mathrm{l}}$, fully flooded corrosion rate

$\mathrm{pr}_{1}$, perforation fraction

$\mathrm{SA}_{1}$, surface area

$\mathrm{s}_{\mathrm{w}, 1}$, moles of water consumed for each mole of metal corroded

$\mathrm{s}_{\mathrm{h}, \mathrm{l}}$, moles of gas $\left(\mathrm{H}_{2}\right)$ produced for each mole of metal corroded.

Model Geometry. The submodel was originally written for a grid system in which each waste parcel was totally enclosed within a single grid block. The grid selected for the Complex PA, however, cuts the waste parcel into up to five horizontal sections (Figure 11-2). Using multiple grid blocks raised the dilemma of how to model the breach of layers. For example, consider WP22, which spans three grid blocks. If a breach occurs near the top of the parcel, water will flow into the steel canister and flood all the Hot Isostatic Press (HIP) packages. Thus, Layer 1 is technically breached in all three grid blocks and corrosion of Layer 2 starts in all three grid blocks. In the hypothetical salt repositories, however, water flow is slow and will take some time to fill the entire containers. In addition, GASSNF does not make a distinction between a pinhole breach and a breach from generalized corrosion, which would remove the entire layer. In response to these difficulties and the additional bookkeeping required to track the breaching of layers in more than one grid block, we decided to model the waste parcel as three separate parcels. The mass and surface areas of the layers in the parcel were divided in proportion to the height of the grid blocks. Thus, when Layer 1 in the top grid block is breached, the other grid blocks are not affected; the surface area of Layer 2 that is used for corrosion is only the fraction of the HIP package surface area that lies in the top grid block. In cases where the breach times are very different in these horizontal sections, this simplification may lead to an artificially low overall gas generation rate. The maximum possible error that could occur is a gas production rate that is five times too low. The conditions that would produce this result are highly unlikely, and we decided that this error was acceptable in light of the large uncertainty in the corrosion rates.

A typical grid block containing waste is about one-half of a panel, or about five rooms. Because the waste region was divided into five horizontal layers, the salt repository contains about 88 grid blocks that contain waste, with 110 in the granite repository.

Simplifications for Submodel. In actual repositories, there is a distribution in the failure time of containers due to variations in local temperature, saturation, chemistry, and canister properties. In our performance assessment, variability in saturation and temperature was incorporated at the grid-block scale only; chemical variability was not incorporated. The only container property with variability is the potential for a manufacturing defect of the outermost metallic layer. For this property, a single parameter-the early breach fraction-indicates the fraction of all waste parcels in the entire repository with a manufacturing defect of the outer layer that would lead to early breach of the layer. The early breach time was assumed to be the start time of the run.

To properly treat variability within the repository, modeling temperature, saturation, chemistry, and canister properties should be varied at the waste package scale, not the grid-block scale. However, modeling at such a small 


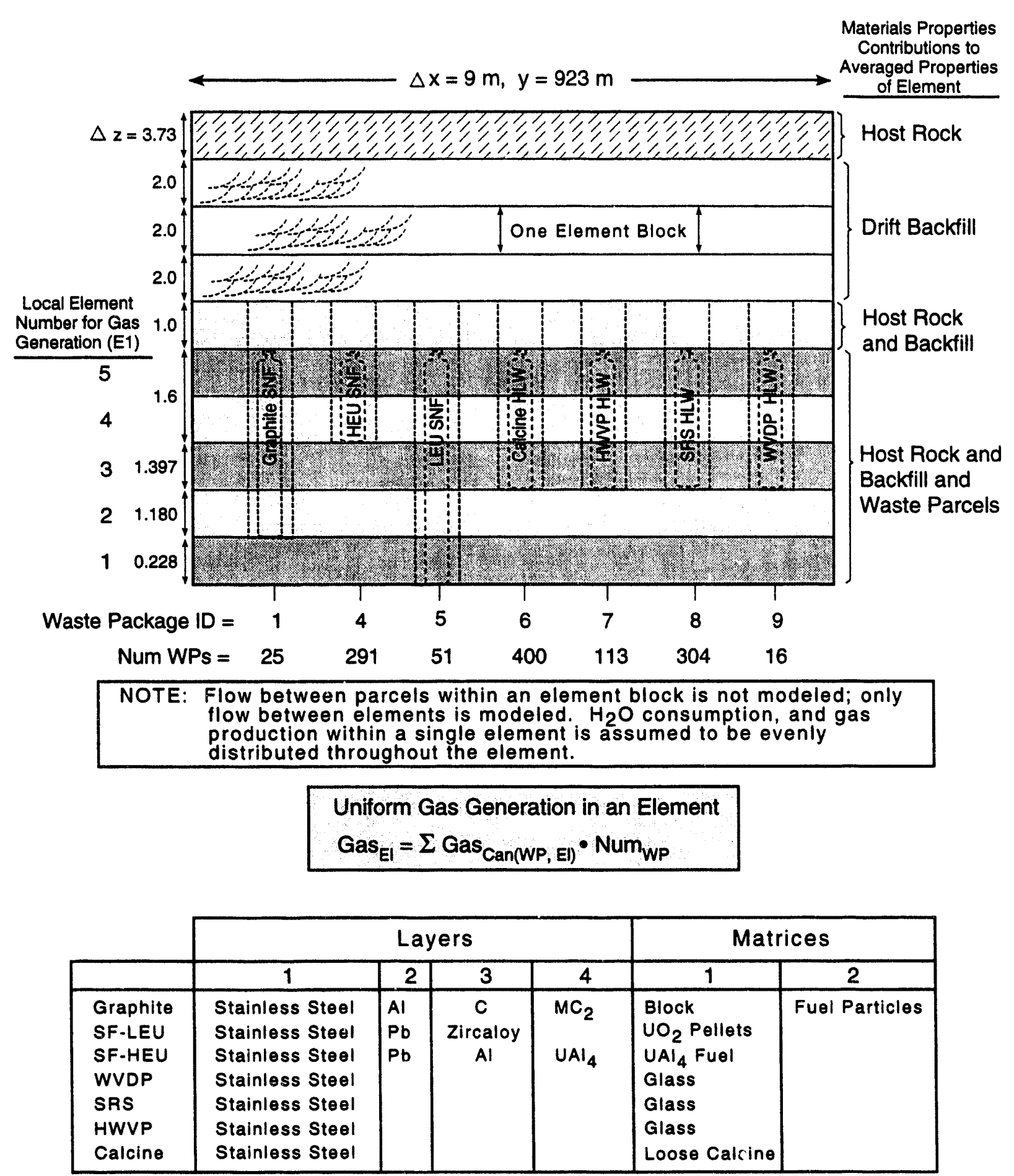

TR1-6342-3797-0

Figure 11-2. Interface of GASSNF with BRAGFLO_T. Waste Disposal Group 1 in salt is shown on side leg portion of the logical grid for BRAGFLO_T (see Figure 12-4, example $i=11$ ). In the complex performance assessment, where the gas-generation submodel, GASSNF, interfaces with the 2-phase flow model, BRAGFLO_T, the volume of gas generated in a local element is the sum calculated for each layer of each waste parcel in that element. 
scale creates computational problems. For example, if we took the present grid and divided each grid block into eight grid blocks (i.e., one for each waste parcel type in Waste Disposal Group 1 for granite), the run time would increase by a factor of between $8^{2}$ to $8^{3}$ ( 64 to 512). Thus, granite runs that now take about 2 hours would each take 5 to 43 days. Given 20 runs per waste disposal group, the runs would take between 3 months to 2.3 years. To become aware of potential problems, scoping calculations of a single canister were run (see Appendix C). Potential errors that can occur because of using a large-scale grid block and neglecting repository variability are described below.

Saturation. Saturation is dependent on water consumption and gas generation, which in turn are dependent on the corrosion rate. The corrosion rate, however, is also dependent on saturation because of a function that makes a smooth transition between the maximum corrosion rate (full saturation) and zero (residual saturation). Thus, a feedback loop exists, which is treated by successive iterations at each time step.

To illustrate the potential for error, consider an element that contains two waste parcel types: WP7 (Savannah River glass in a stainless steel 304L container) and WP12 (the highly enriched uranium spent fuel packed with $10 \mathrm{HIP}$ packages). While the single stainless steel layer of WP7 will breach quickly from stress corrosion cracking, the layer consumption will proceed at a steady slow rate until it is gone. WP12 will also undergo slow consumption of the stainless-steel layer, but at the time of breach due to stress corrosion cracking, the HIP packages and fuel canister will also be exposed, which greatly increases the total surface area for corrosion. At that time, the rate of water consumption and gas production will jump. Also, a jump in overall corrosion occurs when the spent fuel container and filler are breached and the highly reactive aluminum and metallic fuels are exposed. The higher corrosion rates in the waste package hole containing WP12 will consume the water within the borehole, thus cutting back the corrosion rate; therefore the corrosion rate for WP12 will be cut back more than the rate for WP7 because of decreased saturation.

Because BRAGFLO_T calculates saturation on an element level, however, the saturation passed to the gas model is an average saturation from both waste packages (WP"7 and WP12). Thus, the rates on both boreholes will be cut back equally, anc the corrosion of WP12 will be artificially high and, for WP7, artificially low. If fluid flows easily between waste package boreholes, then the error will be small, but if the room/drift is relatively impermeable, then the error can be large. The error is also more pronounced for the elements that contain bottoms of containers than those that contain tops of containers since the tops are hydraulically closer to one another. For this calculation, a large coefficient, $\alpha$, is used in the exponential smoothing function to approximate a step function. Thus, the corrosion rate remains at the fully saturated value until the element saturation is very close to residual saturation. (As described in Appendix $\mathrm{C}$, a continuous smoothing function was selected for estimating the corrosion rate rather than a step function to avoid numerical instability; full saturation $\left(S_{B}\right)$ equals 1 and residual saturation $\left(S_{B r}\right)$ equals 0 .

Temperature. Temperature-dependent corrosion rates were not used in this performance assessment, so there is h.i.ie effect from neglesting variability of temperature on the waste parcel scale within the repository. Including temperature dependence $\theta^{2}$, the corrosion rates is a suggested goal for future performance assessments if the data become available.

Chemistry. Factors that would cause chemistry to vary from one waste package borehole to another are as follows:

- Differing amounts of reactants such as $\mathrm{O}_{2}$ and products such as $\mathrm{H}_{2}$ occurring because of differing corrosion rates for the various materials.

- Differing amounts of reactants occurring because of differences in radiolysis.

- Differing chemistry as the result of different temperarares; varied amounts of evaporation and alteration of minerals affect the ionic strength, chloride content, and $\mathrm{pH}$ of pore fluids.

- Differing amounts and kinds of corrosion products forming from the different materials used in the waste parcel types.

These conditions not only are likely to vary fi om one waste package hole to another, but they are also guaranteed to change with time. 
However, modeling this complex behavior was not attempted; instead, corrosion rates for each material within the repository were used for the entire regulatory period. The uncertainty for all effects was taken into account by estimating ranges for fully inundated rates. Although tracking the $\mathrm{Eh}$ and $\mathrm{pH}$ of each waste parcel hole and using Ehand $\mathrm{pH}$-dependent corrosion rates may improve the predictive value of the model, such tracking is likely to be difficult to implement and the necessary data inay not be practical to obtain. Thus, further attempts to include more of these natural processes are probably not appropriate, although side calculations to support corrosion rate ranges may be enlightening.

Container Variability. Varying container properties is currently impractical, because using distributions for container properties, such as manufacturing defects and corrosion resistance, greatly increases the execution time of the submodel. The internal algorithm of GASSNF requires single values for the early breach fraction and perforation fraction of each type of waste parcel. Currently the early breached containers are modeled by creating a duplicate set of waste parcels with the same properties of the initially defined waste parcels, except that the outer layer is defined as breached at time 0 . Thus, the current algorithm would require that a new set of waste parcels be defined for each property that could vary. For example, if the early breach fraction was extended to $x$ relevant container materials instead of just the first layer, then the algorithm would be called $2 x$ times for each waste parcel. Currently, the algorithm is called 14 times for Waste Disposal Group 1 in salt, which has 7 waste parcel types. If early breach for the lead and aluminum layers were added, the algorithm would be called 24 times. Adding a zircaloy early breach fraction ould bring it to 28 .

\subsubsection{Interface of GASSNF with SALFLOW and GRFLOW}

GASSNF was designed as a submodel within BRAGFLO_T, which references its grid block in three dimensions by the letters, $\mathrm{i}, \mathrm{j}$, and $\mathrm{k}$. Since a large fraction of the repository does not contain waste, a separate grid-block number was assigned by GASSNF to the grid blocks that contain waste. A translation array between $i, j, k$ and the GASSNF grid number was necessary to pass grid information such as the grid volume and grid height. For the one-dimensional SALFLOW and GRFLOW, this step was not necessary, so the code was modified to eliminate this step. Also, the gas generation rate required by BRAGFLO_T in units of $\mathrm{kg} / \mathrm{m}^{3} \mathrm{sec}$ was replaced for the simple flow models with the total gas generation in units of $\mathrm{kg} / \mathrm{sec}$ for the time step.

Unlike BRAGFLO_T, which calculates the saturation within the waste package holes, SALFLOW and GRFLOW assume that if the fluid saturation within the drift is above $5 \%$, then the waste package holes are fully saturated. When the drift saturation falls below $5 \%$, the waste package hole saturation is assumed to follow a linear curve down to $0 \%$. This saturation behavior in the waste package hole is a conservative assumption, because the resultant corrosion rates are the maximum values, the fully flooded rates. Note that this simplifying assumption not only greatly reduces the execution time for the flow model, but also eliminates the problem of modeling different saturations for each waste package hole.

The simple flow models do not model the generation and transport of heat. Instead, temperature effects were handled by using a range of corrosion rates for the temperature range of $25^{\circ} \mathrm{C}$ to $100^{\circ} \mathrm{C}$, with the activation energy for corrosion set to zero.

The geometry used in the simple flow models is significantly different from the geometry used in the BRAGFLO_T calculations (Figure 11-3). Instead of five horizontal elements intersecting the waste parcels, the waste parcels are all assumed to be contained within one horizontal element. Thus, the coding that divided the waste parcels mass and surface area into the five elements has been removed from the version of GASSNF that interfaces with the simple flow models. 
One Leg of 100 in Panel (Salt) or Repository (Granite)

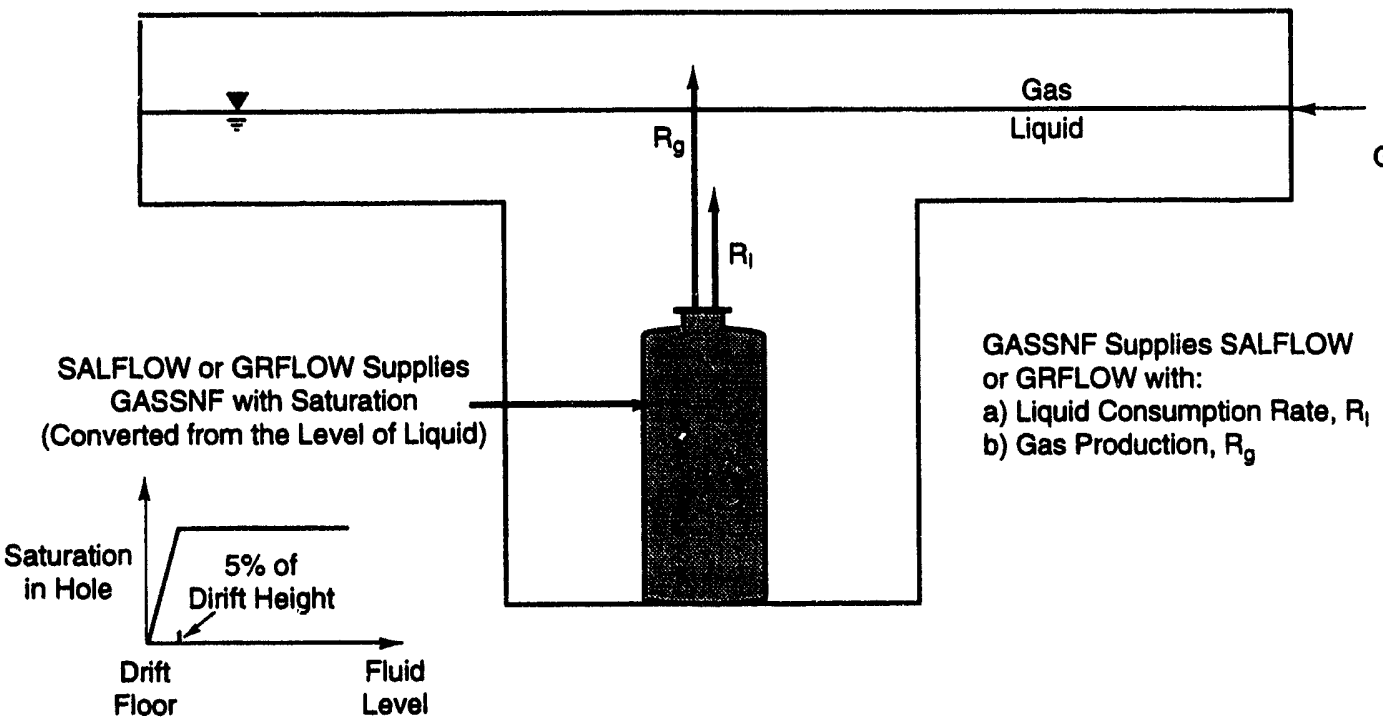

TR1-6342-3922-0

Figure 11-3. Interface of GASSNF with fluid flow models, SALFLOW and GRFLOW. For the simple performance assessment, the gas-generation rate from GASSNF is input at the repository node of the pseudo-2phase flow codes, SALFLOW and GRFLOW.

\subsubsection{Modeling Assumptions for Gas Generation Submodel (GASSNF)}

The following assumptions were made in the gas generation submodel:

- Constant rates for fully flooded conditions are used for each material.

- Calculated rates are dependent on saturation near the residual saturation $\left(s_{\mathrm{Br}}\right)$.

- Temperature-dependence of corrosion rates is included but not used.

- Breach of each layer is simulated.

- Surface area is a constant value that equals the initial outside surface area of the layer within the element. This includes layers such as the lead filler which are not expected to "wet" Ti-Grade 12 or stainless steel 304L, and thus a small gap will exist into which water may flow.

- $\mathrm{Gas}\left(\mathrm{H}_{2}, \mathrm{O}_{2}\right)$ from radiolysis of water is ignored, which is expected to result in a minor underestimation of the total gas production and water consumption.

\subsection{Source Term Submodel of Waste Emplacement Package}

The source term submodel, CONCSNF, is responsible for the dissolution, sorption, and radioactive buildup and decay of specified isotopes within the waste emplacement packages. (Note that the waste emplacement package includes both the waste parcel and the backfill in the waste borehole between the parcel and the host rock.) The transport code, STAFF2D in the Complex PA and CLAMVD in the Simple PA, is responsible for transporting the isotopes out of the pore fluid within the waste emplacement packages. Because these processes are inherently coupled, CONCSNF has been designed as a subroutine that is called by the transport computational model every time step.

For the decay calculations, a total of 49 isotopes was selected from the more than 160 isotopes listed in Appendix A of this report; the selection was based on the length of their half-lives and whether the isotopes were present in significant amounts (see Chapter 4). For transport modeling and to accommodate STAFF2D's limits in terms of 
computational speed, 11 isotopes were drawn from the 49 isotopes used in the decay calculations (see Chapter 4 ). The 11 isotopes selected were considered likely to exceed the regulatory limit after 1000 years, based on the original inventory and decay: ${ }^{14} \mathrm{C},{ }^{226} \mathrm{Ra},{ }^{229} \mathrm{Th},{ }^{230} \mathrm{Th},{ }^{233} \mathrm{U},{ }^{234} \mathrm{U},{ }^{238} \mathrm{U},{ }^{237} \mathrm{~Np},{ }^{239} \mathrm{Pu}$, ${ }^{240} \mathrm{Pu}$, and ${ }^{241} \mathrm{Am}$. Because solubility and sorption are properties of elements, not isotopes, the seven transport elements considered are Am, C, Np, Pu, $\mathrm{Ra}, \mathrm{Th}$, and $\mathrm{U}$.

The materials within the waste emplacement packages that contain radioactive isotopes are called matrices herein and are listed below:

- Glass, the borosilicate glass used to vitrify high-level waste.

- Glass-ceramic, one of the proposed materials used to immobilize INEL calcine.

- Calcine, INEL calcine that has not been immobilized.

- Metallic fuel, the uranium-aluminum alloy fuel, taken as representative of the highly enriched fuel stored at INEL.

- $\mathrm{UO}_{2}$ fuel, the fuel of a pressurized water reactor

- Metal carbide fuel, the fuel within the small particles used in graphite reactors.

- Structural carbon, the graphite and pyrolitic carbon used to hold the metal carbide fuel particles. This matrix is included because $93 \%$ of the ${ }^{14} \mathrm{C}$ found in the graphite fuels was formed from activation of ${ }^{13} \mathrm{C}$ and ${ }^{14} \mathrm{~N}$ within the structural carbon and not within the microspheres used to encapsulate the fuel.

- Other structural materials. Although other structural materials also contain activation products, for this performance assessment, the isotopes in these materials were treated as if they were in the fuel matrices. Including these materials as separate matrices in a future performance assessment can be easily accomplished.

- Gap inventory. Although gap inventory is not technically "in" the fuel matrix, it is treated as if it were until the time of cladding breach, when it is released instantly.

In CONCSNF, radionuclides are considered available for dissolution when all protective layers of the waste parcel are breached. The submodel conservatively assumes that the fluids within the boreholes are well mixed (at least within the control volume of the element) and that the breached containers do not inhibit this mixing.

Parameters that are input to the source term submodel from BRAGFLO_T, SALFLOW, or GRFLOW

- Breach time per layer for each type of waste parcel in each grid block.

- Fluid saturation of the backfill within the boreholes during that time step.

\subsubsection{Conceptual Model of Location of Radionuclides} 11-4):

CONCSNF defines five locations within the waste emplacement packages where the isotopes may reside (Figure

Location

1,2

3

4

5

\section{Description}

In up to two matrices.

As a precipitate within the canister.

Dissolved within the pore fluid in the borehole.

Absorbed onto the borehole backfill.

Isotopes are released at each location as follows (Figure 11-4):

1,2 Matrices. Initially, all radionuclide inventory resides in the matrix(es). Within spent fuel matrices, such as $\mathrm{UO}_{2}$, some separation of radionuclides from the bulk matrix into grain boundaries or the gap between the matrix and the cladding may have occurred. The fraction of each radionuclide that has migrated into these locations will be called the quick release fraction. Other than the quick release fraction, the remaining radionuclides are assumed to be homogenous within the matrix. At breach of the protective 


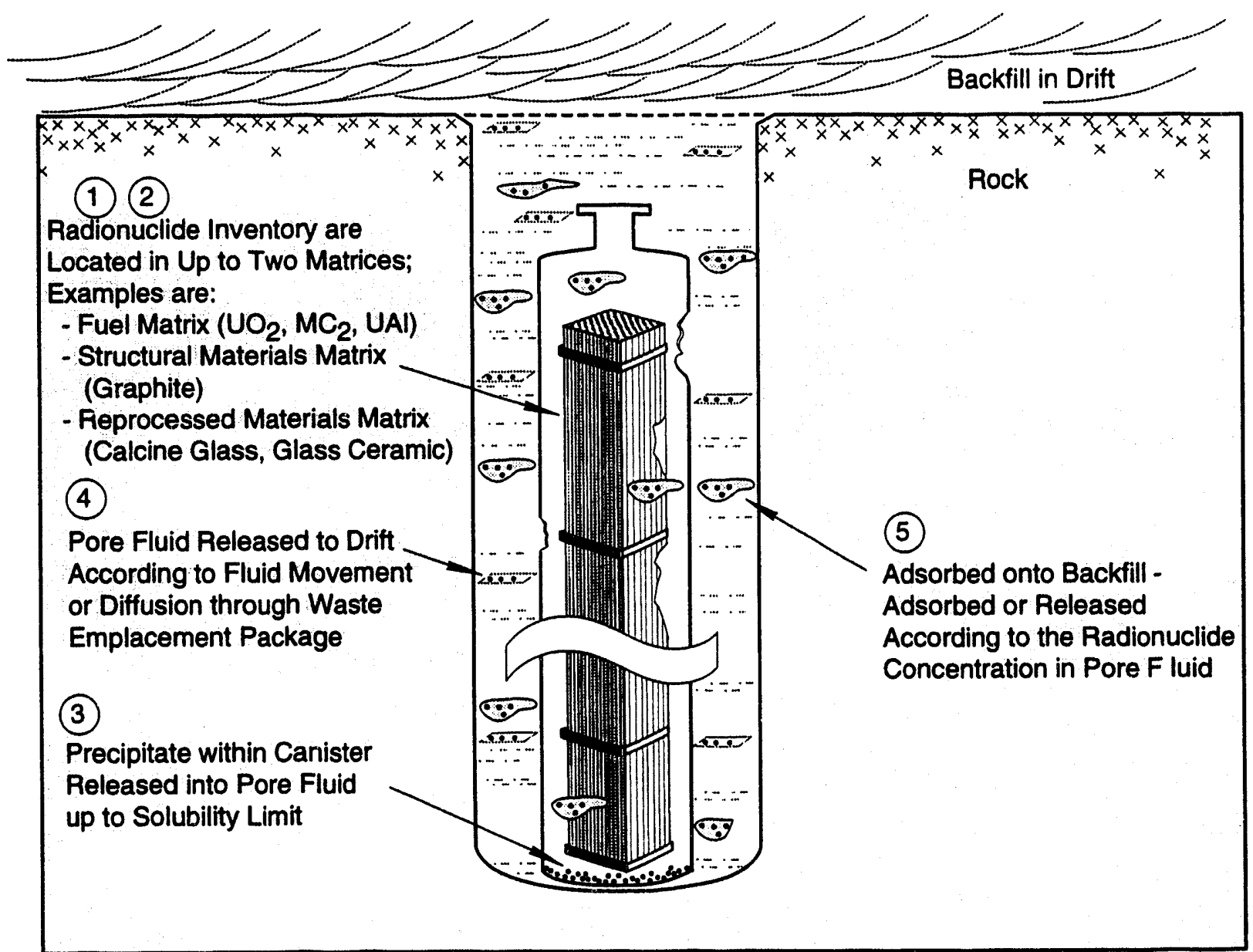

(3) Released into Pore Fluid up to Solubility Limit

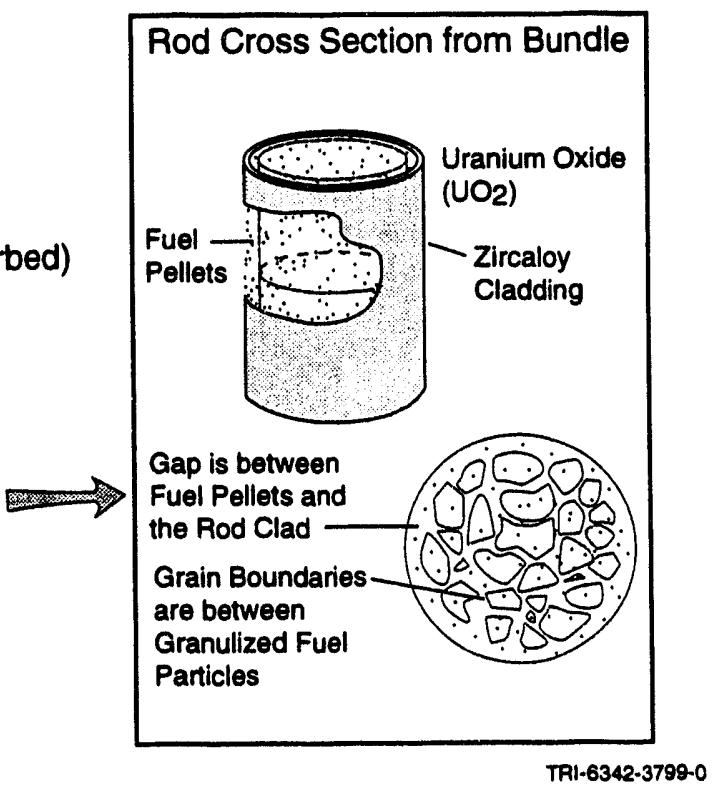

Figure 11-4. Locations of radionuclides that CONCSNF monitors within the waste emplacement package. 
layers, the quick release fraction of the radionuclides is immediately moved into the precipitate location, from which the radionuclides may dissolve up to their solubility limit. This movement of "released" radionuclides into the precipitate location prior to dissolution has been adopted for convenience only. It allows separation of the process that exposes the radionuclide from the actual dissolution which is solubility limited. For the bulk matrix, the process that exposes the radionuclides is dissolution or alteration of the matrix.

To keep the subroutine simple, we have chosen to model alteration of each matrix as occurring by one of two mechanisms: (1) constant-rate alteration for the thermodynamically unstable matrices or (2) solubility-limited dissolution for the stable matrices. Constant alteration rates are used for glass, glassceramic, carbon, calcine, metal carbide, and metal fuels. (While the rate of alteration is kinetically controlled for most of the matrices, all other processes are assumed to be controlled by chemical equilibrium.) We have used constant alteration rates in the model for computational speed only. Alteration reactions usually exhibit an initial rate followed by a long-term rate. The long-term rate may differ from the initial rate for many reasons including a change in the $\mathrm{pH}$, a buildup of dissolved alteration products, and a buildup of a semi-protective coating of alteration products. When possible, we have used the long-term rates when estimating the range of alteration rates.

The mineral portion of glass-ceramic was not modeled separately for this performance assessment, though it is stable and technically should be treated separately from the unstable glass portion. Currently, the radionuclide inventory cannot be listed separately for each phase because the partitioning of isotopes between phases is highly dependent on the batch composition, calcine particle size, and temperature and pressure history of the material.

The calcine has been modeled with a fast constant rate because of its small particle size and its observed fast leach rates.

The solubility limit for $\mathrm{UO}_{2}$ was used based on the assumption that by the time the $\mathrm{UO}_{2}$ matrix is exposed, the repository environment will have become reducing enough that $\mathrm{UO}_{2}$ is the stable phase.

3 Precipitate. Each element is released into the pore fluid up to its solubility limit. All pore fluid within the waste package borehole and within the local waste element is assumed to be available for dissolution. When more than one isotope exists for an element (e.g., uranium), each isotope is dissolved in proportion to its relative abundance. (This process is performed only for the transport elements: Am, C, $\mathrm{Np}, \mathrm{Pu}, \mathrm{Ra}, \mathrm{Th}$, and $\mathrm{U}$.)

Pore Fluid. The CONCSNF routines model the equilibration of radionuclides between precipitate, pore fluid, and backfill. Removal of the radionuclides from the waste emplacement package borehole by diffusion and/or advection is modeled by the transport codes, STAFF2D or CLAMVD. Note that the transport code does not necessarily remove all the isotopes of each of the seven transport elements. However, because dissolution and adsorption are elemental processes, the removal of sister isotopes not transported by STAFF2D or CLAMVD must be modeled by CONCSNF; failure to do so results in artificial suppression of the dissolution of the transported isotopes. Consequently, CONCSNF removes the "nontransported" isotopes in proportion to the amount of a specified transported sister isotope; the PA analyst assigns which transported isotope the nontransported isotope will mimic.

Borehole Backfill-Buffer. Elements are absorbed or released in proportion to the concentration within the pore fluid. Once again, the relative abundance of the isotopes of each element is used to scale the adsorption of individual isotopes. 


\subsubsection{Mathematical/Computational Model}

The main algorithm of CONCSNF calculates the radionuclide release for a single canister given a single set of saturations and containment failure time.

Release of radionuclides is described by two coupled processes: mobilization and transport. The mobilization step is limited for most nuclides by their solubilities and the volume of water available to the waste form in a time step. For very soluble nuclides, their mobilization is limited by the rate at which the matrix, in which they are contained, is dissolved or altered. A fraction of the soluble nuclides are available for immediate release on breach of containment.

Transport affects the mobilization stage in that it determines the volume of water available to the waste form in a time step. The transport of radionuclides may be divided into the two scales as modeled by the transport codes and by CONCSNF. CONCSNF is responsible for transport within the computational grid of the transport codes. The gridding is different for the two transport codes, (see Sections 11.1.4 and 11.1.5 for the model geometries), but in each case, transport up the waste emplacement package borehole is modeled by the transport code, and radial transport within the waste emplacement package borehole is modeled by CONCSNF. In most scenarios, radionuclides will dissolve into fluids filling a partially failed container and release from the container is determined by the rate of diffusion through the breach in the container. Depending on the mode of failure, the size of the container breach can range from a pinhole to the entire surface area of the container. Small holes have been calculated to reduce the release from the container by orders of magnitude (Romero et al., 1991; Nilsson et al., 1991; McKinley et al., 1992).

Given the uncertainty in the breach size, we decided to use the conservative approach adopted by several others (KBS, 1983; Nagra, 1985). Once breached, the container is assumed to offer no resistance to transport. Once at the container surface, radionuclides can then be transported through the $0.3-\mathrm{m}$ of backfill out to the waste emplacement package borehole boundary mainly by diffusion. We have assumed that the host rock in the immediate vicinity of the waste emplacement package borehole is not significantly disturbed by the drilling of the waste emplacement package borehole, and given the low permeability and low porosity of both salt and granite, the advective and diffusive transport into the host rock will be minor compared to transport up the borehole. Based in these assumptions, we have neglected any transport into the host rock. (This is a significant departure from other studies in granite which show diffusion into the granite [e.g., Skagius and Neretnieks, 1986, and Birgersson and Neretnieks, 1990]. However, Neretnieks, 1991, also reports that because diffusion through intact granite is very slow, one meter of intact granite is sufficient to effectively isolate two regions.) Finally, to make the code very fast, we decided not to model the diffusion from the waste form to the waste emplacement package borehole boundary, but instead assume instant diffusion within the backfill-buffer surrounding the waste parcel. This assumption is very conservative, and the result is an earlier time for the radionuclide plume to reach the drift. This assumption is especially conservative for estimating the releases of short-lived isotopes at early times. At later times, when a quasi-steady state is likely to be achieved, this assumption is likely to cause little error for long-lived isotopes.

Because the total inventory of each nuclide is not constant (due to the decay process), the total inventory is calculated from the inventory at an initial reference time, using input decay chains and half-lives.

The parameters for each of the $\mathbf{4 9}$ isotopes, $i$, are treated as constants:

$$
\begin{array}{ll}
\mathrm{t}_{1 / 2 \mathrm{i}} & =\text { half-life, } \\
\text { daughter }_{\mathrm{i}} & =\text { decay daughter, if daughter is not stable, } \\
\mathrm{t}_{0 \text { waste }} & =\text { reference time for the inventory data, } \\
\text { Inv }_{0 \mathrm{i}, \text { waste }} & =\text { inventory in curies in each waste type at } \mathrm{t}_{\text {Owaste }} .
\end{array}
$$

The parameters for each of the seven transport elements, $e$, are treated as constants:

$$
\begin{array}{lll}
\mathrm{S}_{e} & = & \text { Solubility in repository pore fluids, } \\
\mathrm{GF}_{\mathrm{e}, \text { matrix }} & =\text { Gap fraction in each matrix, }
\end{array}
$$




$\begin{array}{ll}\mathrm{Kd}_{\mathrm{e}}= & \begin{array}{l}\text { Adsorption coefficient in relevant materials. (Only salt and } \\ \text { bentonite were used in this stıdy; future work may consider } \\ \text { corrosion products.) }\end{array}\end{array}$

At each time step and for each type of waste parcel per grid block, CONCSNF performs the following steps (Figure 11-5; see also Figure 11-4):

1. Updates the liquid volume within the waste emplacement package based on the time step's borehole saturation.

2. Calculates the radioactive buildup and decay of all 49 isotopes in all locations and updates the isotope masses to the end of the time step.

3. Recalculates the mass fraction of each isotope within each matrix and the relative abundance of each isotope for each element released from the matrix into the precipitate, liquid, and backfill.

4. Updates the amount of each isotope for the seven transport elements and the $\mathrm{UO}_{2}$ matrix in the pore fluid based on the amount added or removed by the transport code during the last time step. Saves this amount of each isotope for use in Step 9.

5. Checks to see if any matrix within the parcel is exposed by the breach of the layers covering it. If no matrices are exposed, the model skips to the next parcel. If a matrix is exposed since the last time step, the quick release fraction of each isotope is moved from the matrix to the precipitate location.

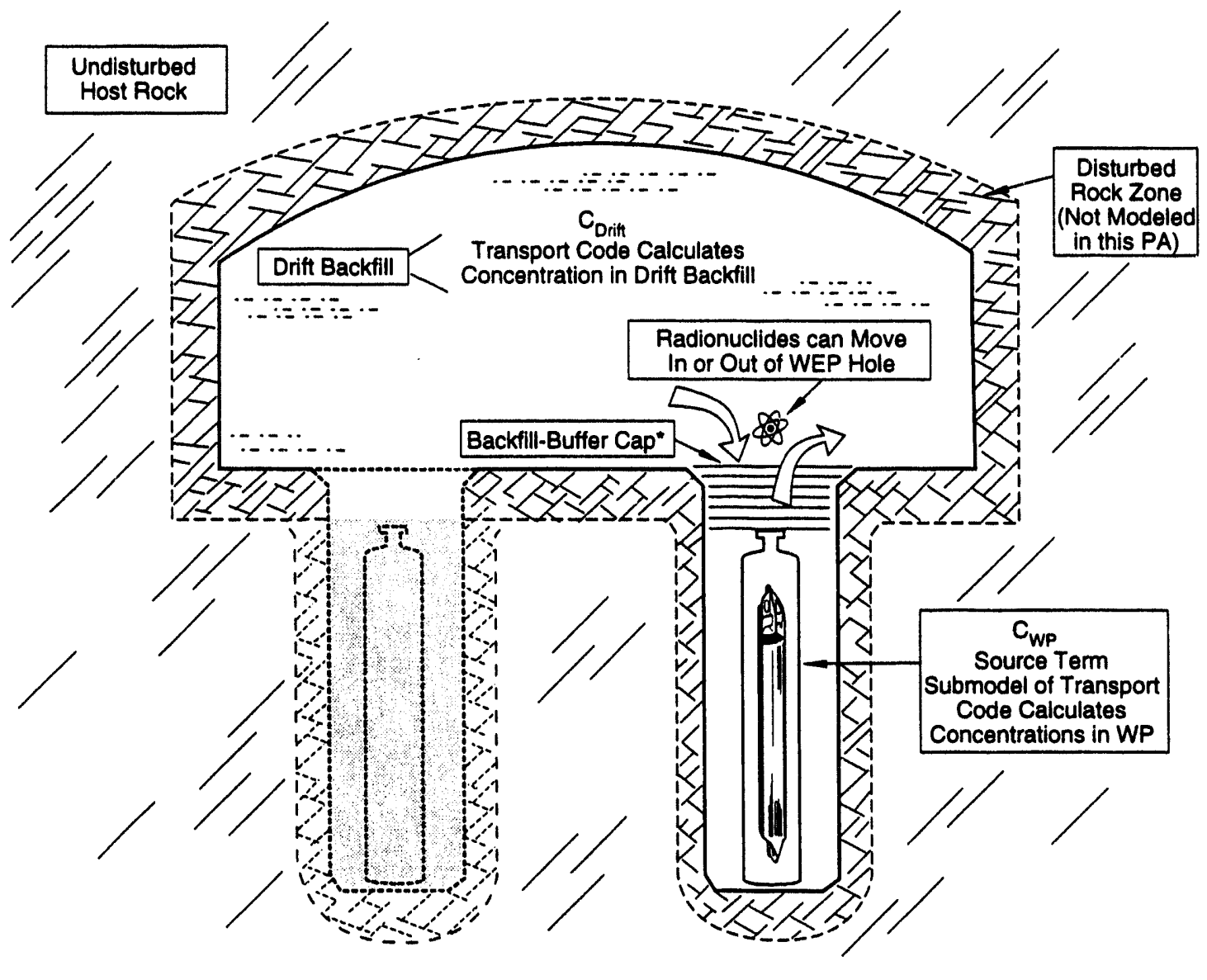

- Several Assumptions Necessary to Specify Movement through Backfill-Buffer Cap

TRI-6342-3800-0

Figure 11-5. General interface between source term submodel and transport model. The interface accounts for the movement of radionuclides out of and into the waste emplacement package. (Note that the disturbed rock zone was not modeled for this performance assessment.) 
6. Calculates the amount of matrix that either is dissolved or altered within the time step and updates the pore fluid concentration of $\mathrm{UO}_{2}$.

7. Based on the mass of matrix altered in Step 6 and the mass fraction of each isotope in the matrix calculated in Step 3, moves the appropriate amount of each isotope from the matrix to the precipitate.

8. Equilibrates all isotopes of the seven transport elements among the precipitate, pore fluids, and backfill using (a) the solubilities and Kd's of the elements and (b) the relative abundance of each isotope as calculated in Step 3.

9. Calculates the "amount dissolved during this time step" per isotope for each of the seven transport elements by subtracting the amount calculated in Step 8 from the amount calculated in Step 4 . The values calculated here are passed onto the transport code.

\subsubsection{Interface of CONCSNF with STAFF2D}

The interface between CONCSNF and STAFF2D involves injection of the isotopes into the borehole surrounding the waste parcel in each element. Like the BRAGFLO_T calculations (Section 11.1), this model treats each section of a waste emplacement package within an element as a separate package. In this interface, CONCSNF provides STAFF2D with a mass injection rate, and STAFF2D performs the transport out of the borehole (i.e., advection and diffusion) (Figure 11-6; see also Figure 11-5).

After STAFF2D calculates advection and diffusion, it returns the average concentration of each isotope for the entire element (Figure 11-6). CONFSNF, which needs the value for the mass per isotope removed from each borehole, stores this average grid-block concentration and the concentrations that CONCSNF calculated for each borehole, and then assumes that the fractional change in each borehole is equal to the fractional change in the average element concentration. The model also assumes that the concentration within the pore fluid of the intact rock is zero. CONCSNF then calculates new borehole concentrations and the mass change in the borehole liquid for each isotope.

The interface is performed using the following steps (Figure 11-6):

1. At the end of the call to CONCSNF during the last time step, CONCSNF saved both the concentrations within each waste package borehole and also the average concentrations within the element.

2. After the call to CONCSNF in the last time step, isotopes were transported by STAFF2D into or out of the element.

3. At the beginning of this time step, STAFF2D calls CONCSNF and passes it the new concentrations in the element.

4. Using the information saved in Step 1 and received in Step 3, CONCSNF calculates new waste package pore concentrations and saves them for use in Step 6.

5. CONCSNF dissolves matrices and re-equilibrates the precipitates, pore fluids, and backfill, producing new waste package pore concentrations.

6. CONCSNF subtracts the concentrations saved in Step 4 from the new concentrations calculated in Step 5 and reports the amount of each isotope that was added to the fluid during the time step to STAFF2D.

In CONCSNF, the volume of the liquid within the borehole is an important variable. The liquid volume is estimated as the product of the physical volume of the borehole times the porosity of the backfill times the saturation of the backfill. Because BRAGFLO_T calculates the bulk saturation for the entire element, a method was needed to estimate the saturation within the backfill-buffer. Because the permeability of the backfill was much larger than that of the intact host rock, it was decided to use the assumption that changes to the bulk saturation of the element are due entirely to changes in the saturation of the backfill-buffer, i.e., the saturation of the intact host rock does not vary. BRAGFLO_T uses this assumption to calculate the average saturation of all waste package boreholes in the element. Thus, although CONCSNF is designed to use the individual saturations in each waste package borehole, only the average saturations are available. 
"Volume averaged concentration in an element is calculated by CONCSNF. Change in this value due to dissolution is used to calculate the mass injection rate for passing back to STAFF2D.

* STAFF2D returns to CONCSNF new AveConc after transport. CONSNF calculates change in WEP hole concentrations, ConcBck, using the change in AveConc.

TRI-6342-3901-0

Figure 11-6. Interface of CONCSNF with STAFF2D. Waste Disposal Group 1 shown on a logical grid for one room in STAFF2D model. For the complex performance assessment, the interface between the source term submodel, CONCSNF, and the transport model, STAFF2D, is through the average concentration of radionuclides in an element. This value is the average of the concentrations calculated for the pore fluid in the backfill of each portion of the waste emplacement package and the host rock. 
Future studies may be required to assess the error that is introduced by averaging the saturation, since we expect that the saturation within the backfill-buffer will respond relatively quickly to brine consumption and gas generation from corrosion of the waste parcels, while saturation within the intact rock will change very slowly. Also, waste parcels with differing amounts of the more reactive materials (e.g., aluminum, steel, metal carbides) will consume brine and generate gas at significantly different rates. We expect, however, the error to be small in granite where the saturation is more uniform throughout the waste region.

\subsubsection{Interface of CONCSNF with CLAMVD}

CLAMVD is the transport model that is used for the Simple PA for both the salt and granite repositories. CLAMVD uses a single dimension array of drift legs (Figure 11-7) compared to the two-dimensional mesh of STAFF2D. In CLAMVD, the transport of isotopes out of the WEP holes is communicated to CONCSNF as the moles of the isotope removed from the WEP hole. This information is much easier for CONSNF to use than the concentration information passed from STAFF2D. The interface of CONCSNF with CLAMVD uses the following steps:

1. At the end of the call to CONCSNF during the last time step, CONCSNF saved the moles per isotope in each location for each waste package.

2. In the last time step, CLAMVD calculated the transport of isotopes between the waste package borehole and the room/drift.
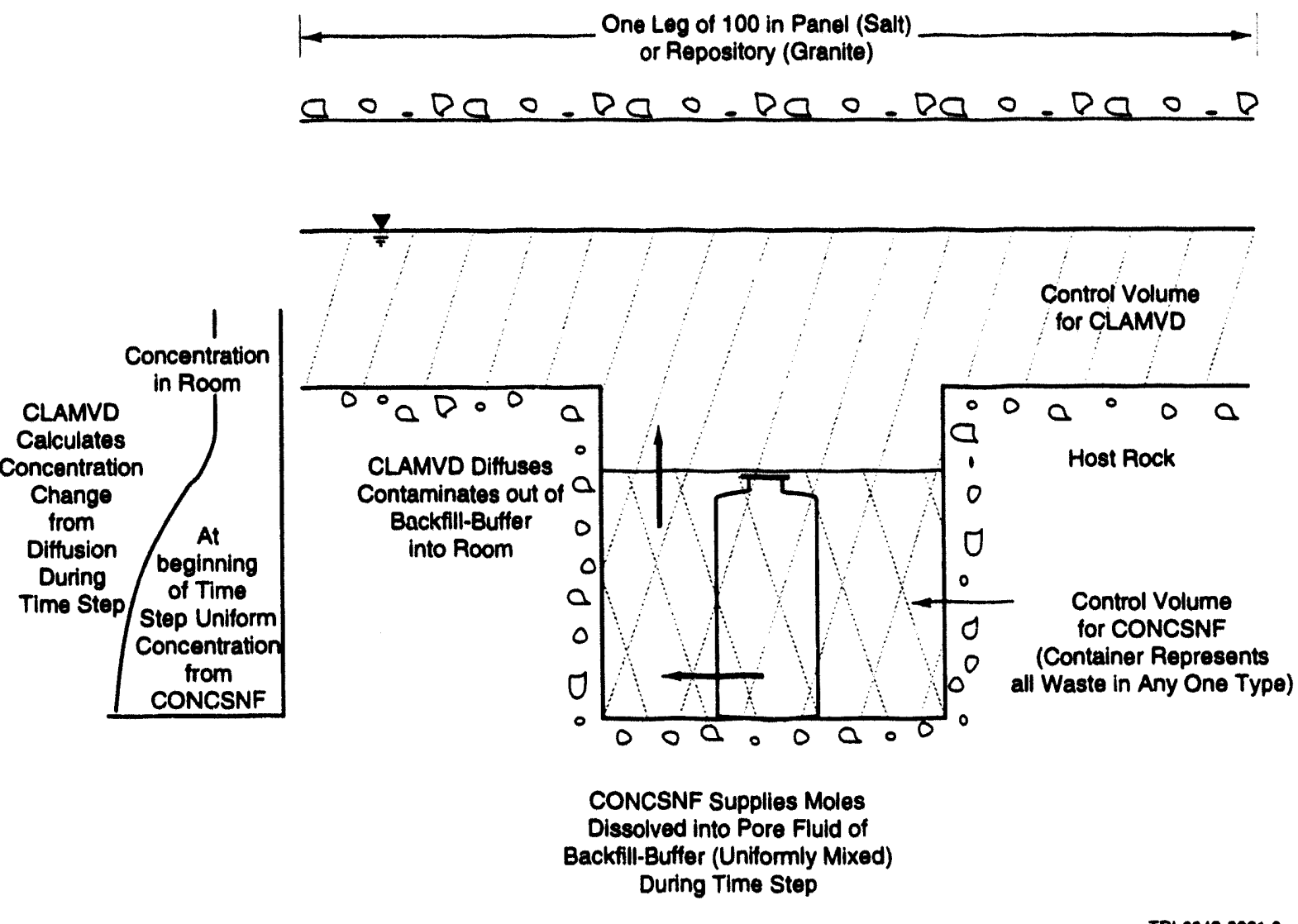

Figure 11-7. Interface of CONCSNF with CLAMVD. For the simple performance assessment, the interface between the source term submodel, CONCSNF, and the transport model, CLAMVD, is through the moles injected by dissolution during the timestep into the pore fluid of the backfill-buffer around each waste parcel and the moles removed by diffusion from the pore fluid of the waste package hole. 
3. At the beginning of this time step, CLAMVD calls CONCSNF and passes it the moles for each isotope that were removed per waste package during the last time step.

4. CONCSNF subtracts the moles of each isotope removed from the moles in the pore fluid and saves this new value.

5. CONCSNF dissolves matrices and equilibrates the precipitates, pore fluids, and backfill, computing moles in each location.

6. CONCSNF subtracts the moles in the fluid stored in Step 4 from the moles in the fluid calculated in Step 5 to obtain the moles that dissolved or desorbed into the fluid during the time step, which it then reports to CLAMVD.

\subsubsection{Summary of Modeling Assumptions}

- The submodel conservatively assumes that the fluids within the boreholes are well mixed (at least within the control volume of the grid block) and that the breached containers do not inhibit this mixing.

- The radionuclide inventory in the matrix(es) of the waste form is assumed to be released congruently as the matrix alters except for the quick release fraction of each isotope, which is released instantly at the time of matrix exposure. Released radionuclides are moved to the precipitate location from which they may dissolve up to their solubility limit.

- The concentration within the pore fluid of the intact rock is zero.

- Saturation of pore fluid is assumed to be the same within all waste package boreholes of an element because the fluid flow code, BRAGFLO_T, calculates saturations for an element and does not track individual waste package boreholes within the element.

- The temperature dependence of solubility is not directly modeled but is included in large solubility ranges.

- Pore space within the degraded container is not included in waste package hole pore volume.

- Matrix alteration occurs from one of two simplified mechanisms: solubility limited dissolution or constant rate alteration.

- The surface areas of each matrix used in alteration rate calculations are set to the original outside area and not changed with time. When the surface area is uncertain, for example with fractured glass, the simple outside area is used and the uncertainty in the surface area is added to the uncertainty in the alteration rate.

- Adsorption onto corrosion products is ignored.

- Mixing within the control volume used by CONCSNF is assumed to be instantaneous, i.e., degraded waste parcel layers and backfill-buffer offer no barrier to immediate transport out to the edge of the waste package hole.

\subsection{Modeling Parameters for Gas Generation and Source Term Submodels}

The time constraints imposed on this project meant that parameters for the gas generation and source term submodels for this performance assessment were chosen without the benefit of an exhaustive review of the literature. Instead, recently published literature, review articles, and personal communications with experts were used as guidance in making decisions about parameter values and their ranges. A discussion of the reasoning behind decisions regarding parameter values for these submodels is provided below.

Tables of these parameter values are provided as follows: GASSNF parameters for the salt repository (Table 11-3) and the granite repository (Table 11-4); CONCSNF parameters for the salt repository (Table 11-5) and the granite repository (Table 11-6).

\subsubsection{Corrosion Parameters}

The corrosion parameters were used by both the gas generation and source term submodels. Because information about corrosion rates and perforation fractions was not available for most parameters, loguniform distributions 


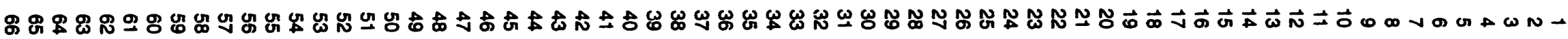

Table 11-3. GASSNF Model Parameters for Salt Repository

\begin{tabular}{|c|c|c|c|c|c|c|c|c|}
\hline $\begin{array}{l}\text { Record } \\
\text { No. }\end{array}$ & Parameter & Parameter Description & Median & \multicolumn{2}{|c|}{ Range } & Units & $\begin{array}{l}\text { Distribution } \\
\text { Type }\end{array}$ & Sources In:* \\
\hline \multicolumn{9}{|c|}{ Aluminum element } \\
\hline 2098 & CorEn & Activation energy for corrosion & $0.00 e+00$ & & & none & Constant & $\begin{array}{l}\text { Stockman, analyst } \\
\text { decision }\end{array}$ \\
\hline 2097 & CorrMode & $0=\mathrm{kg} / \mathrm{m}^{2} \mathrm{~s} \quad 1=1 / \mathrm{s} \quad 2=$ solubility control & $0.00 e+00$ & & & none & Constant & $\begin{array}{l}\text { Stockman, analyst } \\
\text { decision }\end{array}$ \\
\hline 2051 & CoRate & Corrosion rate of material & $1.00 e-07$ & $1.00 \mathrm{e}-08$ & $1.00 e-06$ & $\mathrm{~kg} / \mathrm{m}^{2} \mathrm{~s}$ & Loguniform & $\begin{array}{l}\text { Stockman, analyst } \\
\text { decision }\end{array}$ \\
\hline 2184 & PerFrac & $\begin{array}{l}\text { Fraction of material removed at perfora- } \\
\text { tion }\end{array}$ & $5.00 e-01$ & & & none & Constant & $\begin{array}{l}\text { Stockman, analyst } \\
\text { decision }\end{array}$ \\
\hline 2064 & MW & Molecular Weight & $2.70 e+01$ & & & $\mathrm{~g} / \mathrm{mole}$ & Constant & Lide, Ed., 1991 \\
\hline 2079 & StoicH2O & $\begin{array}{l}\text { Stoichiometry: moles of } \mathrm{H} 20 \text { per moles } \\
\text { of metal }\end{array}$ & $1.50 e+00$ & & & none & Constant & $\begin{array}{l}\text { Stockman, analyst } \\
\text { decision }\end{array}$ \\
\hline 2078 & StoicGas & $\begin{array}{l}\text { Stoichiometry: moles gas per moles of } \\
\text { metal }\end{array}$ & $1.50 e+00$ & & & none & Constant & $\begin{array}{l}\text { Stockman, analyst } \\
\text { decision }\end{array}$ \\
\hline \multicolumn{9}{|l|}{ Graphite } \\
\hline 2104 & CorEn & Activation energy for corrosion & $0.00 e+00$ & & & none & Constant & $\begin{array}{l}\text { Stockman, analyst } \\
\text { decision }\end{array}$ \\
\hline 2103 & CorrMode & $0=\mathrm{kg} / \mathrm{m}^{2} \mathrm{~s} \quad 1=1 / \mathrm{s} \quad 2=$ solubility control & $0.00 e+00$ & & & none & Constant & $\begin{array}{l}\text { Stockman, analyst } \\
\text { decision }\end{array}$ \\
\hline 2113 & CoRate & Corrosion rate of material & $1.00 e-20$ & $1.00 e-22$ & $1.00 e-18$ & $\mathrm{~kg} / \mathrm{m}^{2} \mathrm{~s}$ & Loguniform & $\begin{array}{l}\text { Stockman, analyst } \\
\text { decision }\end{array}$ \\
\hline 2186 & PerFrac & $\begin{array}{l}\text { Fraction of material removed at perfora- } \\
\text { tion }\end{array}$ & $0.00 \mathrm{e}-00$ & & & none & Constant & $\begin{array}{l}\text { Stockman, analyst } \\
\text { decision }\end{array}$ \\
\hline 2067 & MW & Molecular Weight & $1.20 e+01$ & & & $\mathrm{~g} /$ mole & Constant & Lide, Ed., 1991 \\
\hline 2085 & StoicH2O & $\begin{array}{l}\text { Stoichiometry: moles of } \mathrm{H} 20 \text { per moles } \\
\text { of meta }\end{array}$ & $2.00 e+00$ & & & none & Constant & $\begin{array}{l}\text { Stockman, analyst } \\
\text { decision }\end{array}$ \\
\hline 2084 & StoicGas & $\begin{array}{l}\text { Stoichiometry: moles gas per moles of } \\
\text { metal }\end{array}$ & $3.00 e+00$ & & & none & Constant & $\begin{array}{l}\text { Stockman, analyst } \\
\text { decision }\end{array}$ \\
\hline
\end{tabular}




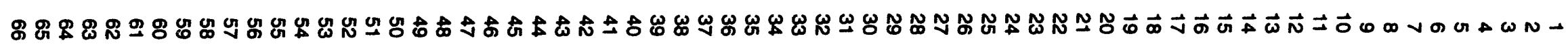

Table 11-3. GASSNF Model Parameters for Salt Repository (Continued)

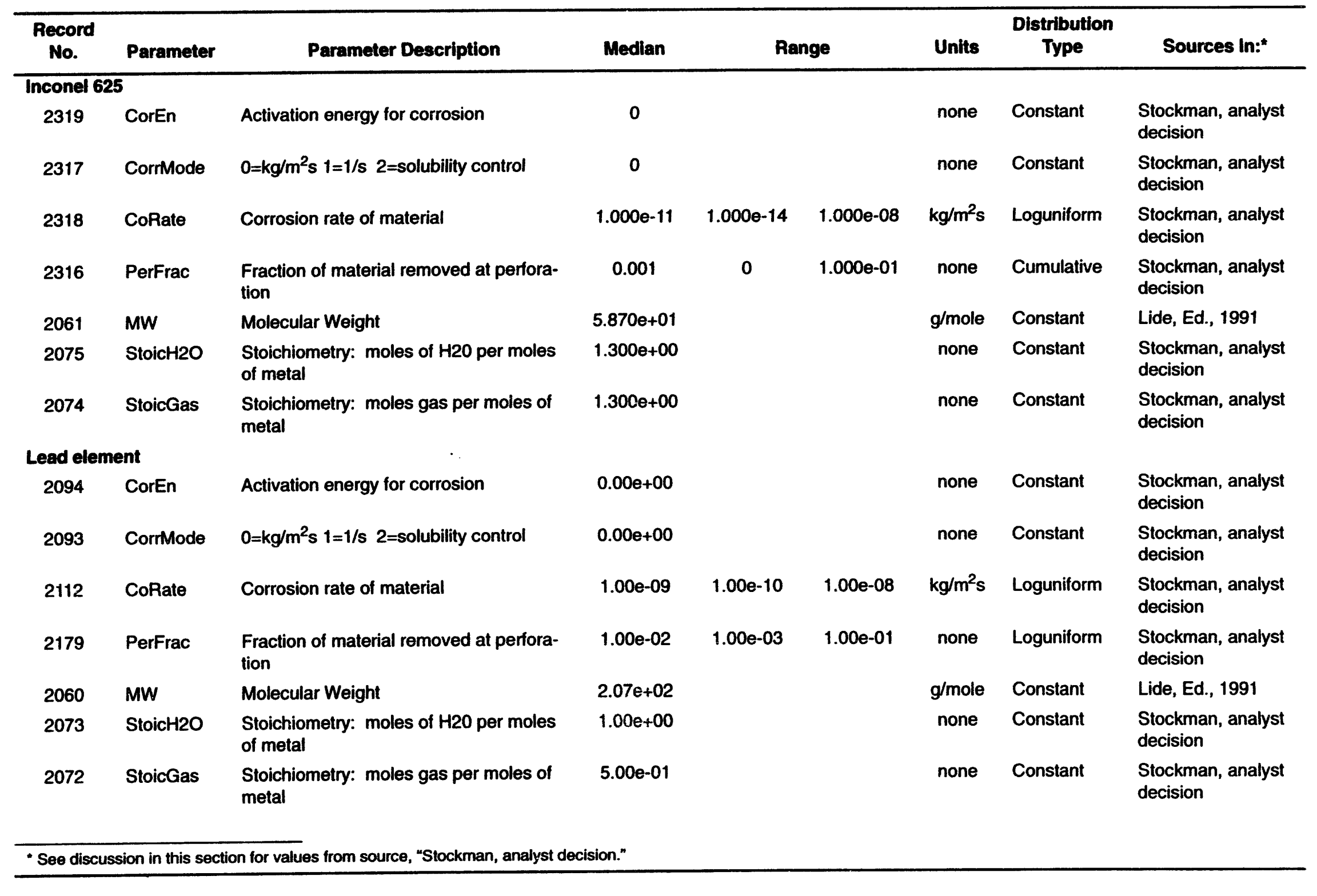




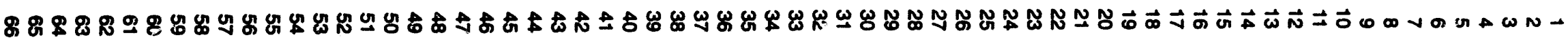

Table 11-3. GASSNF Model Parameters for Salt Repository (Continued)

\begin{tabular}{|c|c|c|c|c|c|c|c|c|}
\hline $\begin{array}{l}\text { Record } \\
\text { No. }\end{array}$ & Paramoter & Parameter Deecription & Median & \multicolumn{2}{|c|}{ Range } & Unitis & $\begin{array}{l}\text { Distribution } \\
\text { Type }\end{array}$ & Sources In:* \\
\hline \multicolumn{9}{|c|}{ Metal Carblde Fuel (Uranium and Thorlum) } \\
\hline 2105 & CorEn & Activation energy for corrosion & $0.00 \theta+00$ & & & none & Constant & $\begin{array}{l}\text { Stockman, analyst } \\
\text { decision }\end{array}$ \\
\hline 2088 & Corrmode & $0=\mathrm{kg} / \mathrm{m}^{2} \mathrm{~s} 1=1 / \mathrm{s} \quad 2=$ solubility control & $1.000+00$ & & & none & Constant & $\begin{array}{l}\text { Stockman, analyst } \\
\text { decision }\end{array}$ \\
\hline 2111 & CoRate & Corrosion rate of material & $1.00 \mathrm{e}-09$ & & & $1 / \mathrm{s}$ & Constant & $\begin{array}{l}\text { Stockman, analyst } \\
\text { decision }\end{array}$ \\
\hline 2187 & PerFrac & $\begin{array}{l}\text { Fraction of material removed at perfora- } \\
\text { tion }\end{array}$ & $0.000+00$ & & & none & Constant & $\begin{array}{l}\text { Stockman, analyst } \\
\text { decision }\end{array}$ \\
\hline 2068 & MW & Molecular Weight & $2.56 e+02$ & & & g/mole & Consiant & Lide, Ed., 1991 \\
\hline 2087 & StoicH2O & $\begin{array}{l}\text { Stoichiometry: moles of } \mathrm{H} 20 \text { per moles } \\
\text { of metal }\end{array}$ & $2.00 e+00$ & & & none & Constant & $\begin{array}{l}\text { Stockman, analyst } \\
\text { decision }\end{array}$ \\
\hline 2086 & StoicGas & $\begin{array}{l}\text { Stoichiometry: moles gas per moles of } \\
\text { metal }\end{array}$ & $2.00 e+00$ & & & none & Constant & $\begin{array}{l}\text { Stockman, analyst } \\
\text { decision }\end{array}$ \\
\hline \multicolumn{9}{|c|}{ Stainless Steel 3041} \\
\hline 2096 & CorEn & Activation energy for corrosion & $0.00 e+00$ & & & none & Constant & $\begin{array}{l}\text { Stockman, analyst } \\
\text { decision }\end{array}$ \\
\hline 2095 & CorrMode & $0=\mathrm{kg} / \mathrm{m}^{2} \mathrm{~s} \quad 1=1 / \mathrm{s} \quad 2=$ solubility control & $0.00 \theta+00$ & & & none & Constant & $\begin{array}{l}\text { Stockman, analyst } \\
\text { decision }\end{array}$ \\
\hline 2053 & CoRate & Corrosion rate of material & $1.00 e-10$ & $1.00 e-11$ & $1.00 \mathrm{e}-09$ & $\mathrm{~kg} / \mathrm{m}^{2} \mathrm{~s}$ & Loguniform & $\begin{array}{l}\text { Stockman, analyst } \\
\text { decision }\end{array}$ \\
\hline 2182 & PerFrac & $\begin{array}{l}\text { Fraction of material removed at perfora- } \\
\text { tion }\end{array}$ & $5.00 e-03$ & $5.00 e-04$ & $5.00 e-02$ & none & Loguniform & $\begin{array}{l}\text { Stockman, analyst } \\
\text { decision }\end{array}$ \\
\hline 2063 & MW & Molecular Weight & $5.580+01$ & & & g/mole & Constant & Lide, Ed., 1991 \\
\hline 2077 & StoicH2O & $\begin{array}{l}\text { Stoichiometry: moles of } \mathrm{H} 20 \text { per moles } \\
\text { of metal }\end{array}$ & $1.70 \theta+00$ & & & none & Constant & $\begin{array}{l}\text { Stockman, analyst } \\
\text { decision }\end{array}$ \\
\hline 2076 & StoicGas & $\begin{array}{l}\text { Stoichiometry: moles gas per moles of } \\
\text { metal }\end{array}$ & $1.17 \theta+00$ & & & none & Constant & $\begin{array}{l}\text { Stockman, analyst } \\
\text { decision }\end{array}$ \\
\hline
\end{tabular}

Е

See discussion in this section for values from source, "Stockman, analyst decision." 


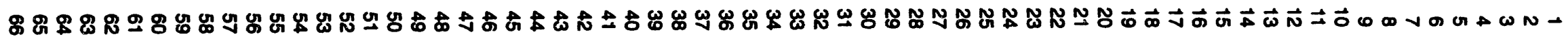
సั.

Table 11-3. GASSNF Model Parameters for Salt Repository (Continued)

\begin{tabular}{|c|c|c|c|c|c|c|c|c|}
\hline \multicolumn{2}{|c|}{$\begin{array}{l}\text { Record } \\
\text { No. Parameter } \\
\text { Stainieas Steel of HIP8 }\end{array}$} & Parameter Description & Median & \multicolumn{2}{|c|}{ Range } & Units & $\begin{array}{l}\text { Distribution } \\
\text { Type }\end{array}$ & Sources In:* \\
\hline 2173 & CorEn & Activation energy for corrosion & $0.00 e+00$ & & & none & Constant & $\begin{array}{l}\text { Stockman, analyst } \\
\text { decision }\end{array}$ \\
\hline 2074 & CorrMode & $0=\mathrm{kg} / \mathrm{m}^{2} \mathrm{~s} \quad 1=1 / \mathrm{s} \quad 2=$ solubility control & $0.00 e+00$ & & & none & Constant & $\begin{array}{l}\text { Stockman, analyst } \\
\text { decision }\end{array}$ \\
\hline 2172 & CoRate & Corrosion rate of material & $1.00 \theta-10$ & $1.00 e-11$ & $1.00 e-09$ & $\mathrm{~kg} / \mathrm{m}^{2} \mathrm{~s}$ & Loguniform & $\begin{array}{l}\text { Stockman, analyst } \\
\text { decision }\end{array}$ \\
\hline 2183 & Perfrac & $\begin{array}{l}\text { Fraction of material removed at perfora- } \\
\text { tion }\end{array}$ & $0.00 e+00$ & & & none & Constant & $\begin{array}{l}\text { Stockman, analyst } \\
\text { decision }\end{array}$ \\
\hline 2176 & MW & Molecular Weight & $5.58 e+01$ & & & g/mole & Constant & Lide, Ed., 1991 \\
\hline 2178 & StoicH2O & $\begin{array}{l}\text { Stoichiometry: moles of } \mathrm{H} 20 \text { per moles } \\
\text { of metal }\end{array}$ & $1.70 e+00$ & & & none & Constant & $\begin{array}{l}\text { Stockman, analyst } \\
\text { decision }\end{array}$ \\
\hline 2177 & StoicGas & $\begin{array}{l}\text { Stoichiometry: moles gas per moles of } \\
\text { metai }\end{array}$ & $1.17 e+00$ & & & none & Constant & $\begin{array}{l}\text { Stockman, analyst } \\
\text { decision }\end{array}$ \\
\hline \multicolumn{9}{|c|}{ The first container placed around the waste form } \\
\hline 2055 & EBFrac & Early breach fraction (Time Zero) & $5.00 e-03$ & $0.00+00$ & $1.00 e-02$ & none & Uniform & $\begin{array}{l}\text { Stockman, analyst } \\
\text { decision }\end{array}$ \\
\hline \multicolumn{9}{|c|}{ Titanium (TI-Grade 12) } \\
\hline 2312 & CorEn & Activation energy for corrosion & 0 & & & none & Constant & $\begin{array}{l}\text { Stockman, analyst } \\
\text { decision }\end{array}$ \\
\hline 2314 & CorrMode & $0=k g / \mathrm{m}^{2} \mathrm{~s} \quad 1=1 / \mathrm{s} \quad 2=$ solubility control & 0 & & & none & Constant & $\begin{array}{l}\text { Stockman, analyst } \\
\text { decision }\end{array}$ \\
\hline 2052 & CoRate & Corrosion rate of material & $5.00 \theta-12$ & $5.000 e-14$ & $5.000 e-10$ & $\mathrm{~kg} / \mathrm{m}^{2} \mathrm{~s}$ & Loguniform & $\begin{array}{l}\text { Stockman, analyst } \\
\text { decision }\end{array}$ \\
\hline 2313 & Perfrac & $\begin{array}{l}\text { Fraction of material removed at perfora- } \\
\text { tion }\end{array}$ & 0.05 & 0 & 1 & none & Cumulative & $\begin{array}{l}\text { Stockman, analyst } \\
\text { decision }\end{array}$ \\
\hline 2059 & MW & Molecular Weight & $4.788 e+01$ & & & $\mathrm{~g} /$ mole & Constant & Lide, Ed., 1991 \\
\hline 2071 & StoicH2O & $\begin{array}{l}\text { Stoichiometry: moles of } \mathrm{H} 20 \text { per moles } \\
\text { of metal }\end{array}$ & $2.000 e+00$ & & & none & Coristant & $\begin{array}{l}\text { Stockman, analyst } \\
\text { decision }\end{array}$ \\
\hline 2070 & StoicGas & $\begin{array}{l}\text { Stoichiometry: moles gas per moles of } \\
\text { metal }\end{array}$ & $2.000 e+00$ & & & none & Constant & $\begin{array}{l}\text { Stockman, analyst } \\
\text { decision }\end{array}$ \\
\hline
\end{tabular}




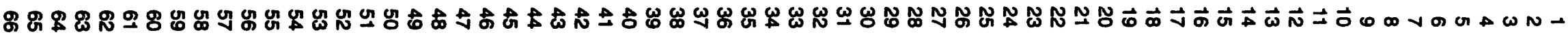

Table 11-3. GASSNF Model Parameters for Salt Repository (Continued)

\begin{tabular}{|c|c|c|c|c|c|c|c|}
\hline $\begin{array}{l}\text { Record } \\
\text { No. }\end{array}$ & Parameter & Parameter Description & Median & Range & Units & $\begin{array}{l}\text { Distribution } \\
\text { Type }\end{array}$ & Sources In:* \\
\hline \multicolumn{8}{|c|}{ Uranium Aluminide } \\
\hline 2100 & CorEn & Activation energy for corrosion & $0.00 e+00$ & & none & Constant & $\begin{array}{l}\text { Stockman, analyst } \\
\text { decision }\end{array}$ \\
\hline 2099 & CorrMode & $0=\mathrm{kg} / \mathrm{m}^{2} \mathrm{~s} \quad 1=1 / \mathrm{s} \quad 2=$ solubility control & $0.00 e+00$ & & none & Constant & $\begin{array}{l}\text { Stockman, analyst } \\
\text { decision }\end{array}$ \\
\hline 2180 & CoRate & Corrosion rate of material & $1.00 \mathrm{e}-06$ & & $\mathrm{~kg} / \mathrm{m}^{2} \mathrm{~s}$ & Constant & $\begin{array}{l}\text { Stockman, analyst } \\
\text { decision }\end{array}$ \\
\hline 2181 & PerFrac & $\begin{array}{l}\text { Fraction of material removed at perfora- } \\
\text { tion }\end{array}$ & $0.00 e+00$ & & none & Constant & $\begin{array}{l}\text { Stockman, analyst } \\
\text { decision }\end{array}$ \\
\hline 2065 & MW & Molecular Weight & $3.22 e+02$ & & $\mathrm{~g} / \mathrm{mole}$ & Constant & Lide, Ed., 1991 \\
\hline 2081 & StoicH2O & $\begin{array}{l}\text { Stoichiometry: moles of } \mathrm{H} 20 \text { per moles } \\
\text { of metal }\end{array}$ & $7.80 e+00$ & & none & Constant & $\begin{array}{l}\text { Stockman, analyst } \\
\text { decision }\end{array}$ \\
\hline 2080 & StoicGas & $\begin{array}{l}\text { Stoichiometry: moles gas per moles of } \\
\text { metal }\end{array}$ & $7.80 e+00$ & & none & Constant & $\begin{array}{l}\text { Stockman, analyst } \\
\text { decision }\end{array}$ \\
\hline \multicolumn{8}{|c|}{ Ziracloy cladding around nuclear fuel } \\
\hline 2102 & CorEn & Activation energy for corrosion & $0.00 e+00$ & & none & Constant & $\begin{array}{l}\text { Stockman, analyst } \\
\text { decision }\end{array}$ \\
\hline 2101 & CormMode & $0=k g / \mathrm{m}^{2} \mathrm{~s} \quad 1=1 / \mathrm{s} \quad 2=$ solubility control & $0.00 e+00$ & & none & Constant & $\begin{array}{l}\text { Stockman, arialyst } \\
\text { decision }\end{array}$ \\
\hline 2114 & CoRate & Corrosion rate of material & $1.00 \mathrm{e}-11$ & & $\mathrm{~kg} / \mathrm{m}^{2} \mathrm{~s}$ & Constant & $\begin{array}{l}\text { Stockman, analyst } \\
\text { decision }\end{array}$ \\
\hline 2185 & PerFrac & $\begin{array}{l}\text { Fraction of material removed at perfora- } \\
\text { tion }\end{array}$ & $0.00 e+00$ & & none & Constant & $\begin{array}{l}\text { Stockman, analyst } \\
\text { decision }\end{array}$ \\
\hline 2066 & MW & Molecular Weight & $9.12 e+01$ & & g/mole & Constant & Lide, Ed., 1991 \\
\hline 2083 & Stoich2O & $\begin{array}{l}\text { Stoichiometry: moles of } \mathrm{H} 20 \text { per moles } \\
\text { of metal }\end{array}$ & $2.00 e+00$ & & none & Constant & $\begin{array}{l}\text { Stockman, analyst } \\
\text { decision }\end{array}$ \\
\hline 2082 & StoicGas & $\begin{array}{l}\text { Stoichiometry: moles gas per moles of } \\
\text { metal }\end{array}$ & $3.00 e+00$ & & none & Constant & $\begin{array}{l}\text { Stockman, analyst } \\
\text { decision }\end{array}$ \\
\hline
\end{tabular}




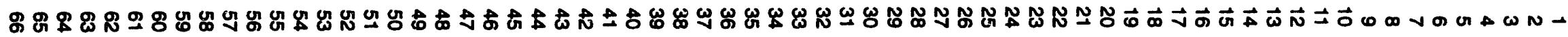

Table 11-4. GASSNF Model Parameters for Granite Repository

\begin{tabular}{|c|c|c|c|c|c|c|c|c|}
\hline $\begin{array}{l}\text { Record } \\
\text { No. }\end{array}$ & Parameter & Parameter Description & Median & \multicolumn{2}{|c|}{ Range } & Units & $\begin{array}{l}\text { Distribution } \\
\text { Type }\end{array}$ & Sources In:* \\
\hline \multicolumn{9}{|c|}{ Aluminum element } \\
\hline 2098 & CorEn & Activation energy for corrosion & $0.00 e+00$ & & & none & Constant & $\begin{array}{l}\text { Stockman, analyst } \\
\text { decision }\end{array}$ \\
\hline 2097 & CorrMode & $0=k g / \mathrm{m}^{2} s \quad 1=1 / \mathrm{s} \quad 2=$ solubility control & $0.00 e+00$ & & & none & Constant & $\begin{array}{l}\text { Stockman, analyst } \\
\text { decision }\end{array}$ \\
\hline 2051 & CoRate & Corrosion rate of material & $1.00 \theta-09$ & $1.00 e-10$ & $1.00 e-08$ & $\mathrm{~kg} / \mathrm{m}^{2} \mathrm{~s}$ & Loguniform & $\begin{array}{l}\text { Stockman, analyst } \\
\text { decision }\end{array}$ \\
\hline 2184 & PerFrac & $\begin{array}{l}\text { Fraction of material removed at perfora- } \\
\text { tion }\end{array}$ & $5.00 e-01$ & & & none & Constant & $\begin{array}{l}\text { Stockman, analyst } \\
\text { decision }\end{array}$ \\
\hline 2064 & MW & Molecular Weight & $2.70 e+01$ & & & $\mathrm{~g} /$ mole & Constant & Lide, Ed., 1991 \\
\hline 2079 & Stoich2O & $\begin{array}{l}\text { Stoichiometry: moles of } \mathrm{H} 20 \text { per moles } \\
\text { of metal }\end{array}$ & $1.50 \theta+00$ & & & none & Constant & $\begin{array}{l}\text { Stockman, analyst } \\
\text { decision }\end{array}$ \\
\hline 2078 & StoicGas & $\begin{array}{l}\text { Stoichiometry: moles gas per moles of } \\
\text { metal }\end{array}$ & $1.50 e+00$ & & & none & Constant & $\begin{array}{l}\text { Stockman, analyst } \\
\text { decision }\end{array}$ \\
\hline \multicolumn{9}{|l|}{ Graphite } \\
\hline 2104 & CorEn & Activation energy for corrosion & $0.00 e+00$ & & & none & Constant & $\begin{array}{l}\text { Stockman, analyst } \\
\text { decision }\end{array}$ \\
\hline 2103 & CorrMode & $0=\mathrm{kg} / \mathrm{m}^{2} \mathrm{~s} \quad 1=1 / \mathrm{s} 2=$ solubility control & $0.00 e+00$ & & & none & Constant & $\begin{array}{l}\text { Stockman, analyst } \\
\text { decision }\end{array}$ \\
\hline 2113 & CoRate & Corrosion rate of material & $1.00 \theta-20$ & $1.00 e-22$ & $1.00 e-18$ & $\mathrm{~kg} / \mathrm{m}^{2} \mathrm{~s}$ & Loguniform & $\begin{array}{l}\text { Stockman, analyst } \\
\text { decision }\end{array}$ \\
\hline 2186 & Perfrac & $\begin{array}{l}\text { Fraction of material removed at perfora- } \\
\text { tion }\end{array}$ & $0.00 e-00$ & & & none & Constant & $\begin{array}{l}\text { Stockman, analyst } \\
\text { decision }\end{array}$ \\
\hline 2067 & MW & Molecular Weight & $1.20 e+01$ & & & $g /$ mole & Constant & Lide, Ed., 1991 \\
\hline 2085 & StoicH2O & $\begin{array}{l}\text { Stoichiometry: moles of } \mathrm{H} 20 \text { per moles } \\
\text { of metal }\end{array}$ & $2.00 e+00$ & & & none & Constant & $\begin{array}{l}\text { Stockman, analyst } \\
\text { decision }\end{array}$ \\
\hline 2084 & StoicGas & $\begin{array}{l}\text { Stoichiometry: moles gas per moles of } \\
\text { metal }\end{array}$ & $3.00 e+00$ & & & none & Constant & $\begin{array}{l}\text { Stockman, analyst } \\
\text { decision }\end{array}$ \\
\hline
\end{tabular}




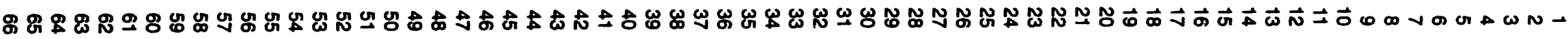

Table 11-4. GASSNF Model Parameters for Granite Repository (Continued)

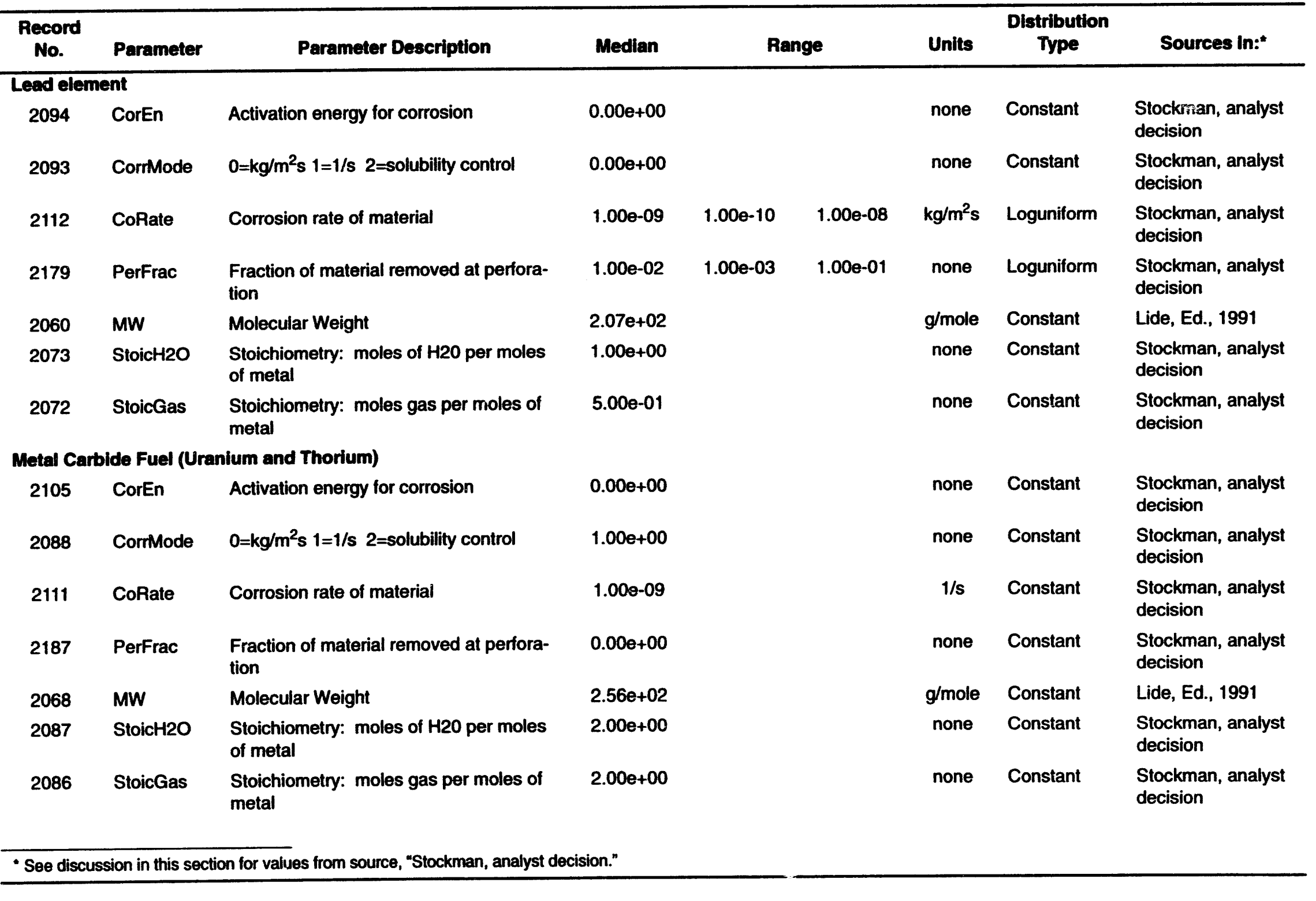




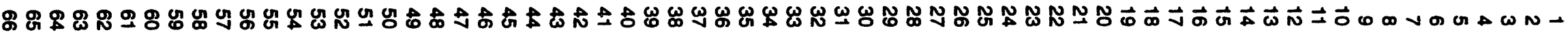

Table 11-4. GASSNF Model Parameters for Granite Repository (Continued)

\begin{tabular}{|c|c|c|c|c|c|c|c|c|}
\hline $\begin{array}{l}\text { Record } \\
\text { No. }\end{array}$ & Parameter & Parameter Description & Median & \multicolumn{2}{|c|}{ Range } & Units & $\begin{array}{l}\text { Distribution } \\
\text { Type }\end{array}$ & Sources In:* \\
\hline 2120 & CorEn & $0=\mathrm{kg} / \mathrm{m}^{2} \mathrm{~s} \quad 1=1 / \mathrm{s} \quad 2=$ solubility control & $1.00 e+00$ & & & none & Constant & $\begin{array}{l}\text { Stockman, analyst } \\
\text { decision }\end{array}$ \\
\hline 2122 & CoRate & Corrosion rate of material & $4.00 \mathrm{e}-07$ & & & $1 / s$ & Constant & $\begin{array}{l}\text { Stockman, analyst } \\
\text { decision }\end{array}$ \\
\hline \multicolumn{9}{|c|}{ Inconel 625} \\
\hline 2319 & CorEn & Activation energy for corrosion & 0 & & & none & Constant & $\begin{array}{l}\text { Stockman, analyst } \\
\text { decision }\end{array}$ \\
\hline 2317 & CorrMode & $0=\mathrm{kg} / \mathrm{m}^{2} \mathrm{~s} \quad 1=1 / \mathrm{s} \quad 2=$ solubility control & 0 & & & none & Constant & $\begin{array}{l}\text { Stockman, analyst } \\
\text { decision }\end{array}$ \\
\hline 2318 & CoRate & Corrosion rate of material & $3.162 e-13$ & $1.000 e-14$ & $1.000 e-11$ & $\mathrm{~kg} / \mathrm{m}^{2} \mathrm{~s}$ & Loguniform & $\begin{array}{l}\text { Stockman, analyst } \\
\text { decision }\end{array}$ \\
\hline 2316 & PerFrac & $\begin{array}{l}\text { Fraction of material removed at perfora- } \\
\text { tion }\end{array}$ & 0.001 & 0 & $1.000 e-01$ & none & Cumulative & $\begin{array}{l}\text { Stockman, analyst } \\
\text { decision }\end{array}$ \\
\hline 2061 & MW & Molecular Weight & $5.870 e+01$ & & & g/mole & Constant & Lide, Ed., 1591 \\
\hline 2075 & StoicH2O & $\begin{array}{l}\text { Stoichiometry: moles of } \mathrm{H} 20 \text { per moles } \\
\text { of metal }\end{array}$ & $1.300 e+00$ & & & none & Constant & $\begin{array}{l}\text { Stockman, analyst } \\
\text { decision }\end{array}$ \\
\hline 2074 & StoicGas & $\begin{array}{l}\text { Stoichiometry: moles gas per moles of } \\
\text { metal }\end{array}$ & $1.300 e+00$ & & & none & Constant & $\begin{array}{l}\text { Stockman, analysí } \\
\text { decision }\end{array}$ \\
\hline \multicolumn{9}{|c|}{ Stainless Steel 304L } \\
\hline 2096 & CorEn & Activation energy for corrosion & $0.00 e+00$ & & & none & Constant & $\begin{array}{l}\text { Stockman, analyst } \\
\text { decision }\end{array}$ \\
\hline 2095 & CorrMode & $0=\mathrm{kg} / \mathrm{m}^{2} \mathrm{~s} \quad 1=1 / \mathrm{s} \quad 2=$ solubility control & $0.00 e+00$ & & & none & Constant & $\begin{array}{l}\text { Stockman, analyst } \\
\text { decision }\end{array}$ \\
\hline 2053 & CoRate & Corrosion rate of material & $1.00 e-13$ & $1.00 e-15$ & $1.00 \mathrm{e}-11$ & $\mathrm{~kg} / \mathrm{m}^{2} \mathrm{~s}$ & Loguniform & $\begin{array}{l}\text { Stockman, analyst } \\
\text { decision }\end{array}$ \\
\hline 2182 & PerFrac & $\begin{array}{l}\text { Fraction of material removed at perfora- } \\
\text { tion }\end{array}$ & $5.00 \mathrm{e}-06$ & $5.00 e-08$ & $5.00 e-04$ & none & Loguniform & $\begin{array}{l}\text { Stockman, analyst } \\
\text { decision }\end{array}$ \\
\hline 2063 & MW & Molecular Weight & $5.58 e+01$ & & & g/mole & Constant & Lide, Ed., 1991 \\
\hline 2077 & Stoic $\mathrm{H} 2 \mathrm{O}$ & $\begin{array}{l}\text { Stoichiometry: moles of } \mathrm{H} 20 \text { per moles } \\
\text { of metal }\end{array}$ & $1.70 e+00$ & & & none & Constant & $\begin{array}{l}\text { Stockman, analyst } \\
\text { decision }\end{array}$ \\
\hline 2076 & StoicGas & $\begin{array}{l}\text { Stoichiometry: moles gas per moles of } \\
\text { metal }\end{array}$ & $1.17 e+00$ & & & none & Constant & $\begin{array}{l}\text { Stockman, analyst } \\
\text { decision }\end{array}$ \\
\hline
\end{tabular}




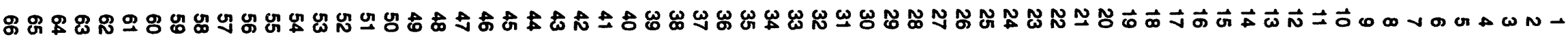

Table 11-4. GASSNF Model Parameters for Granite Repository (Continued)

\begin{tabular}{|c|c|c|c|c|c|c|c|c|}
\hline $\begin{array}{l}\text { Record } \\
\text { No. }\end{array}$ & Parameter & Parameter Description & Median & \multicolumn{2}{|c|}{ Range } & \multicolumn{2}{|r|}{$\begin{array}{c}\text { Distribution } \\
\text { Type }\end{array}$} & Sources In:* \\
\hline 2173 & CorEn & Activation energy for corrosion & $0.00 e+00$ & & & none & Constant & $\begin{array}{l}\text { Stockman, analyst } \\
\text { decision }\end{array}$ \\
\hline 2074 & CorrMode & $0=\mathrm{kg} / \mathrm{m}^{2} \mathrm{~s} \quad 1=1 / \mathrm{s} \quad 2=$ solubility control & $0.00 e+00$ & & & none & Constant & $\begin{array}{l}\text { Stockman, analyst } \\
\text { decision }\end{array}$ \\
\hline 2172 & CoRate & Corrosion rate of material & $1.00 e-13$ & $1.00 e-15$ & $1.00 e-11$ & $\mathrm{~kg} / \mathrm{m}^{2} \mathrm{~s}$ & Loguniform & $\begin{array}{l}\text { Stockman, analyst } \\
\text { decision }\end{array}$ \\
\hline 2183 & Perfrac & $\begin{array}{l}\text { Fraction of material removed at perfora- } \\
\text { tion }\end{array}$ & $0.00 e+00$ & & & none & Constant & $\begin{array}{l}\text { Stockman, analyst } \\
\text { decision }\end{array}$ \\
\hline 2176 & MW & Molecular Weight & $5.58 e+01$ & & & $\mathrm{~g} / \mathrm{mole}$ & Constant & Lide, Ed., 1991 \\
\hline 2178 & StoicH2O & $\begin{array}{l}\text { Stoichiometry: moles of } \mathrm{H} 20 \text { per moles } \\
\text { of metal }\end{array}$ & $1.70 e+00$ & & & none & Constant & $\begin{array}{l}\text { Stockman, analyst } \\
\text { decision }\end{array}$ \\
\hline 2177 & StoicGas & $\begin{array}{l}\text { Stoichiometry: moles gas per moles of } \\
\text { metal }\end{array}$ & $1.17 e+00$ & & & none & Constant & $\begin{array}{l}\text { Stockman, analyst } \\
\text { decision }\end{array}$ \\
\hline \multicolumn{9}{|c|}{ The first container placed around the waste form } \\
\hline 4267 & EBFrac & Early breach fraction (Time ،:ero) & $5.00 \mathrm{e}-03$ & $0.00+00$ & $1.00 e-02$ & none & Uniform & $\begin{array}{l}\text { Stockman, analyst } \\
\text { decision }\end{array}$ \\
\hline \multicolumn{9}{|c|}{ Uranium Aluminide } \\
\hline 2100 & CorEn & Activation energy for corrosion & $0.00 e+00$ & & & none & Constant & $\begin{array}{l}\text { Stockman, analyst } \\
\text { decision }\end{array}$ \\
\hline 2099 & CorrMode & $0=\mathrm{kg} / \mathrm{m}^{2} \mathrm{~s} \quad 1=1 / \mathrm{s} \quad 2-$ solubility control & $0.00 e+00$ & & & none & Constant & $\begin{array}{l}\text { Stockman, analyst } \\
\text { decision }\end{array}$ \\
\hline 2180 & CoRate & Corrosion rate of inaterial & $1.00 e-07$ & & & $\mathrm{~kg} / \mathrm{m}^{2} \mathrm{~s}$ & Constant & $\begin{array}{l}\text { Stockman, analyst } \\
\text { decision }\end{array}$ \\
\hline 2181 & PerFrac & $\begin{array}{l}\text { Fraction of material removed at perfora- } \\
\text { tion }\end{array}$ & $0.00 e+00$ & & & none & Constant & $\begin{array}{l}\text { Stockman, analyst } \\
\text { decision }\end{array}$ \\
\hline 2065 & MW & Molecular Weight & $3.22 e+02$ & & & $\mathrm{~g} /$ mole & Constant & Lide, Ed., 1991 \\
\hline 2081 & StoicH2O & $\begin{array}{l}\text { Stoichiometry: moles of } \mathrm{H} 20 \text { per moles } \\
\text { of metal }\end{array}$ & $7.80 e+00$ & & & none & Constant & $\begin{array}{l}\text { Stockman, analyst } \\
\text { decision }\end{array}$ \\
\hline 2080 & StoicGas & $\begin{array}{l}\text { Stoichiometry: moles gas per moles of } \\
\text { metal }\end{array}$ & $7.80 e+00$ & & & none & Constant & $\begin{array}{l}\text { Stockman, analyst } \\
\text { decision }\end{array}$ \\
\hline
\end{tabular}




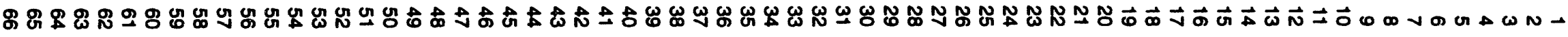
芯

Table 11-4. GASSNF Model Parameters for Granite Repository (Continued)

\begin{tabular}{|c|c|c|c|c|c|c|c|}
\hline $\begin{array}{l}\text { Record } \\
\text { No. }\end{array}$ & Parameter & Parameter Description & Median & Range & Units & $\begin{array}{l}\text { Distribution } \\
\text { Type }\end{array}$ & Sources in:* \\
\hline \multicolumn{8}{|c|}{ Ziracloy cladding around nuclear fuel } \\
\hline 2102 & CorEn & Activation energy for corrosion & $0.00 e+00$ & & none & Constant & $\begin{array}{l}\text { Stockman, analyst } \\
\text { decision }\end{array}$ \\
\hline 2101 & CorrMode & $0=k g / \mathrm{m}^{2} \mathrm{~s} \quad 1=1 / \mathrm{s} \quad 2=$ solubility control & $0.00 e+00$ & & none & Constant & $\begin{array}{l}\text { Stockman, analyst } \\
\text { decision }\end{array}$ \\
\hline 2114 & CoRate & Corrosion rate of material & $1.00 e-12$ & & $\mathrm{~kg} / \mathrm{m}^{2} \mathrm{~s}$ & Constant & $\begin{array}{l}\text { Stockman, analyst } \\
\text { decision }\end{array}$ \\
\hline 2185 & Perfrac & $\begin{array}{l}\text { Fraction of material removed at perfora- } \\
\text { tion }\end{array}$ & $0.00 e+00$ & & none & Constant & $\begin{array}{l}\text { Stockman, analyst } \\
\text { decision }\end{array}$ \\
\hline 2066 & MW & Molecular Weight & $9.12 e+01$ & & $g /$ mole & Constant & Lide, Ed., 1991 \\
\hline 2083 & StoicH2O & $\begin{array}{l}\text { Stoichiometry: moles of } \mathrm{H} 20 \text { per moles } \\
\text { of metal }\end{array}$ & $2.00 e+00$ & & none & Constant & $\begin{array}{l}\text { Stockman, analyst } \\
\text { decision }\end{array}$ \\
\hline 2082 & StoicGas & $\begin{array}{l}\text { Stoichiometry: moles gas per moles of } \\
\text { metal }\end{array}$ & $3.00 \theta+00$ & & none & Constant & $\begin{array}{l}\text { Stockman, analyst } \\
\text { decision }\end{array}$ \\
\hline
\end{tabular}

discussion in this section for values from source, "Stockman, analyst decision." 


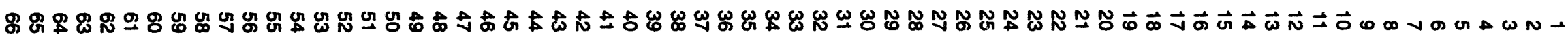

Table 11-5. CONCSNF Model Parameters for Salt Repository

\begin{tabular}{|c|c|c|c|c|c|c|c|c|}
\hline \multirow{2}{*}{\multicolumn{2}{|c|}{$\begin{array}{l}\begin{array}{c}\text { Record } \\
\text { No. Parameter }\end{array} \\
\text { Americium element }\end{array}$}} & \multirow{3}{*}{$\begin{array}{l}\text { Parameter Description } \\
\text { Log radionuclide solubility }\end{array}$} & \multirow{3}{*}{$\begin{array}{c}\text { Median } \\
-9.00 e+00\end{array}$} & \multicolumn{2}{|c|}{ Range } & \multirow{3}{*}{ Units } & \multirow{3}{*}{$\begin{array}{c}\begin{array}{c}\text { Distribution } \\
\text { Type }\end{array} \\
\text { Cumulative }\end{array}$} & \multirow{3}{*}{$\begin{array}{c}\text { Sources In:* } \\
\text { Trauth et al., } 1992\end{array}$} \\
\hline & & & & \multirow[b]{2}{*}{$1.33 e+01$} & \multirow[b]{2}{*}{$1.50 e-01$} & & & \\
\hline 4 & LogSolM & & & & & & & \\
\hline \multicolumn{9}{|c|}{ Borosilicate glass } \\
\hline 2119 & CorrMode & $0=\mathrm{kg} / \mathrm{m}^{2} \mathrm{~s} \quad 1=1 / \mathrm{s} \quad 2=$ solubility control & $0.00 e+00$ & & & none & Constant & $\begin{array}{l}\text { Stockman, analyst } \\
\text { decision }\end{array}$ \\
\hline 2054 & CoRate & Corrosion rate of material & $1.00 e-09$ & $1.00 e-12$ & $1.00 e-06$ & $\mathrm{~kg} / \mathrm{m}^{2} \mathrm{~s}$ & Loguniform & $\begin{array}{l}\text { Stockman, analyst } \\
\text { decision }\end{array}$ \\
\hline \multicolumn{9}{|c|}{ Carbon element } \\
\hline 4301 & LogSolM & Log radionuclide solubility & $1.00 e+00$ & & & logMolar & Constant & $\begin{array}{l}\text { Stockman, analyst } \\
\text { decision }\end{array}$ \\
\hline \multicolumn{9}{|c|}{ Cesium element } \\
\hline 2329 & LogSolM & Log radionuclide solubility & $7.900 e-01$ & & & logMolar & Constant & U.S. DOE, $1986 b$ \\
\hline \multicolumn{9}{|c|}{ Glass ceramic product of hot isostatic press of INEL high level waste } \\
\hline 2123 & CorrMode & $0=\mathrm{kg} / \mathrm{m}^{2} \mathrm{~s} \quad 1=1 / \mathrm{s} \quad 2=$ solubility control & $0.00 e+00$ & & & none & Constant & $\begin{array}{l}\text { Stockman, analyst } \\
\text { decision }\end{array}$ \\
\hline 2058 & CoRate & Corrosion rate of material & $1.00 \mathrm{e}-10$ & $1.00 e-12$ & $1.00 e-08$ & $\mathrm{~kg} / \mathrm{m}^{2} \mathrm{~s}$ & Loguniform & $\begin{array}{l}\text { Stockman, analyst } \\
\text { decision }\end{array}$ \\
\hline \multicolumn{9}{|l|}{ Graphite } \\
\hline 2103 & CorrMode & $0=\mathrm{kg} / \mathrm{m}^{2} \mathrm{~s} \quad 1=1 / \mathrm{s} \quad 2=$ solubility control & $0.00 e+00$ & & & none & Constant & $\begin{array}{l}\text { Stockman, analyst } \\
\text { decision }\end{array}$ \\
\hline 2113 & CoRate & Corrosion rate of material & $1.00 e-20$ & $1.00 e-22$ & $1.00 \mathrm{e}-18$ & $\mathrm{~kg} / \mathrm{m}^{2} \mathrm{~s}$ & Loguniform & $\begin{array}{l}\text { Stockman, analyst } \\
\text { decision }\end{array}$ \\
\hline \multicolumn{9}{|c|}{ Lead element } \\
\hline 2324 & LogSolM & Log radionuclide solubility & $-1.515 e+01$ & & & logMolar & Constant & $\begin{array}{l}\text { Bruno and Sellin, } \\
1992\end{array}$ \\
\hline
\end{tabular}

- See discussion in this section for values from source, "Stockman, analyst decision." 


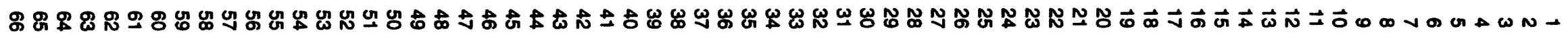

Table 11-5. CONCSNF Model Parameters for Salt Renository (Continued)

\begin{tabular}{|c|c|c|c|c|c|c|c|c|}
\hline $\begin{array}{l}\text { Record } \\
\text { No. }\end{array}$ & Parameter & Parameter Description & Median & \multicolumn{2}{|c|}{ Range } & Units & $\begin{array}{c}\text { Distribution } \\
\text { Type }\end{array}$ & Sources In:* \\
\hline \multicolumn{9}{|c|}{ Metal Carbide Fuel (Uranium and Thorium) } \\
\hline 2088 & CorrMode & $0=\mathrm{kg} / \mathrm{m}^{2} \mathrm{~s} \quad 1=1 / \mathrm{s} \quad 2=$ solubility control & $1.000+00$ & & & none & Constant & $\begin{array}{l}\text { Stockman, analyst } \\
\text { decision }\end{array}$ \\
\hline 2111 & CoRate & Corrosion rate of material & $1.00 \mathrm{e}-09$ & $1.00 \mathrm{e}-12$ & $1.00 e-08$ & $1 / \mathrm{s}$ & Constant & $\begin{array}{l}\text { Stockman, analyst } \\
\text { decision }\end{array}$ \\
\hline \multicolumn{9}{|c|}{ Neptunium element } \\
\hline 692 & LogSolM & Log radionuclide solubility & $-6.99 e+00$ & $-1.55 e+01$ & $-1.92 e+01$ & logMolar & Cumulative & Trauth et al., 1992 \\
\hline \multicolumn{9}{|c|}{ Nickel element } \\
\hline 2328 & LogSolM & Log radionuclide solubility & $-4.700 e+00$ & & & logMolar & Constant & $\begin{array}{l}\text { Bruno and Sellin, } \\
1992\end{array}$ \\
\hline \multicolumn{9}{|c|}{ Palladium element } \\
\hline 2330 & LogSolM & Log radionuclide solubility & $-5.820 e+00$ & & & logMolar & Constant & $\begin{array}{l}\text { Bruno and Sellin, } \\
1992\end{array}$ \\
\hline \multicolumn{9}{|c|}{ Piutonium element } \\
\hline 732 & LogSolM & Log radionuclide solubility & $-9.22 e+00$ & $-1.66 e+01$ & $-3.26 e+01$ & logMolar & Cumulative & Trauth et al., 1992 \\
\hline \multicolumn{9}{|c|}{ Radium element } \\
\hline 817 & LogSolM & Log radionuclide solubility & $1.04 e+00$ & $3.00 e-01$ & $1.26 e+00$ & logMolar & Cumulative & Trauth et al., 1992 \\
\hline \multicolumn{9}{|c|}{ Calcine: Solids from oxidation of INEL high level liquid wastes from reprocessing SNF } \\
\hline 2120 & CorrMode & $0=\mathrm{kg} / \mathrm{m}^{2} \mathrm{~s} \quad 1=1 / \mathrm{s} \quad 2=$ solubility control & $1.00 e+00$ & & & none & Constant & $\begin{array}{l}\text { Stockman, analyst } \\
\text { decision }\end{array}$ \\
\hline 2122 & CoRate & Corrosion rate of material & $4.00 e-07$ & & & $1 / \mathrm{s}$ & Constant & $\begin{array}{l}\text { Stockman, analyst } \\
\text { decision }\end{array}$ \\
\hline \multicolumn{9}{|c|}{ Samarium element } \\
\hline 2331 & LogSolM & Log radionuclide solubility & $-3.150 e+00$ & & & logMolar & Constant & $\begin{array}{l}\text { Bruno and Sellin, } \\
1992\end{array}$ \\
\hline
\end{tabular}




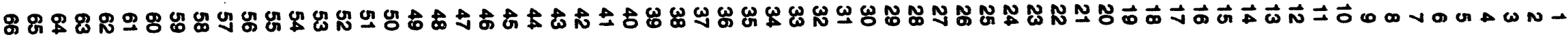

Table 11-5. CONCSNF Model Parameters for Salt Repository (Continued)

\begin{tabular}{|c|c|c|c|c|c|c|c|c|}
\hline $\begin{array}{l}\text { Record } \\
\text { No. }\end{array}$ & Parameter & Parameter Description & Median & \multicolumn{2}{|c|}{ Range } & Units & $\begin{array}{l}\text { Distribution } \\
\text { Type }\end{array}$ & Sources In:* \\
\hline \multicolumn{9}{|c|}{ Technetium element } \\
\hline 2326 & LogSolM & Log radionuclide solubility & $-7.700 e+00$ & & & logMolar & Constant & $\begin{array}{l}\text { Bruno and Sellin, } \\
1992\end{array}$ \\
\hline \multicolumn{9}{|c|}{ Thorium element } \\
\hline 868 & LogSolM & Log radionuclide solubility & $-1.00 e+01$ & $-1.530+01$ & $-5.66 \theta+00$ & logMolar & Cumulative & Trauth et al., 1992 \\
\hline \multicolumn{9}{|c|}{ Tin element } \\
\hline 2327 & LogSolM & Log radionuclide solubility & $-7.700 \theta+00$ & & & logMolar & Constant & $\begin{array}{l}\text { Bruno and Sellin, } \\
1992\end{array}$ \\
\hline \multicolumn{9}{|c|}{ Tracer element } \\
\hline 2332 & LogSolM & Log radionuclide solubility & $1.000 \theta+00$ & & & logMolar & Constant & $\begin{array}{l}\text { Stockman, analyst } \\
\text { decision }\end{array}$ \\
\hline \multicolumn{9}{|c|}{ Uranium Aluminide } \\
\hline 2099 & CorrMode & $0=k g / \mathrm{m}^{2} s \quad 1=1 / \mathrm{s} \quad 2=$ solubility control & $0.00 e+00$ & & & none & Constant & $\begin{array}{l}\text { Stockman, analyst } \\
\text { docision }\end{array}$ \\
\hline 2180 & CoRate & Corrosion rate of material & $1.000-06$ & & & $\mathrm{~kg} / \mathrm{m}^{2} \mathrm{~s}$ & Constant & $\begin{array}{l}\text { Stockman, analyst } \\
\text { docision }\end{array}$ \\
\hline \multicolumn{9}{|c|}{ Uranium dioxide } \\
\hline 2121 & CorrMode & $0=k g / \mathrm{m}^{2} s \quad 1=1 / \mathrm{s} \quad 2=$ solubility control & $1.00 e+00$ & & & none & Constant & $\begin{array}{l}\text { Stockman, analyst } \\
\text { decision }\end{array}$ \\
\hline 2168 & GF_C & $\begin{array}{l}\text { Quick release fraction of C-14 from UO2 } \\
\text { matrix }\end{array}$ & $1.25 e-01$ & $1.00 e-02$ & $2.50 e-01$ & none & Uniform & $\begin{array}{l}\text { Garisto and } \\
\text { Garisto, } 1991\end{array}$ \\
\hline \multicolumn{9}{|c|}{ Uranium element } \\
\hline 889 & LogSolM & Log radionuclide solubility & $-3.27 e+00$ & $-1.500+01$ & $0.00 e+00$ & logimolar & Cumulative & Trauth et al., 1992 \\
\hline \multicolumn{9}{|c|}{ Waste emplacement package } \\
\hline 2055 & EBFrac & Early breach fraction & $5.000-03$ & $0.00 e+00$ & $1.00 e-02$ & none & Uniform & $\begin{array}{l}\text { Stockman, analyst } \\
\text { decision }\end{array}$ \\
\hline
\end{tabular}




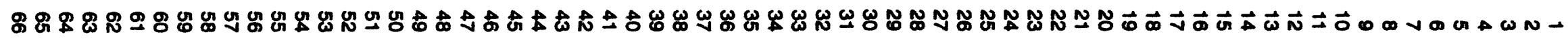

Table 11-5. CONCSNF Model Parameters for Salt Repository (Continued)

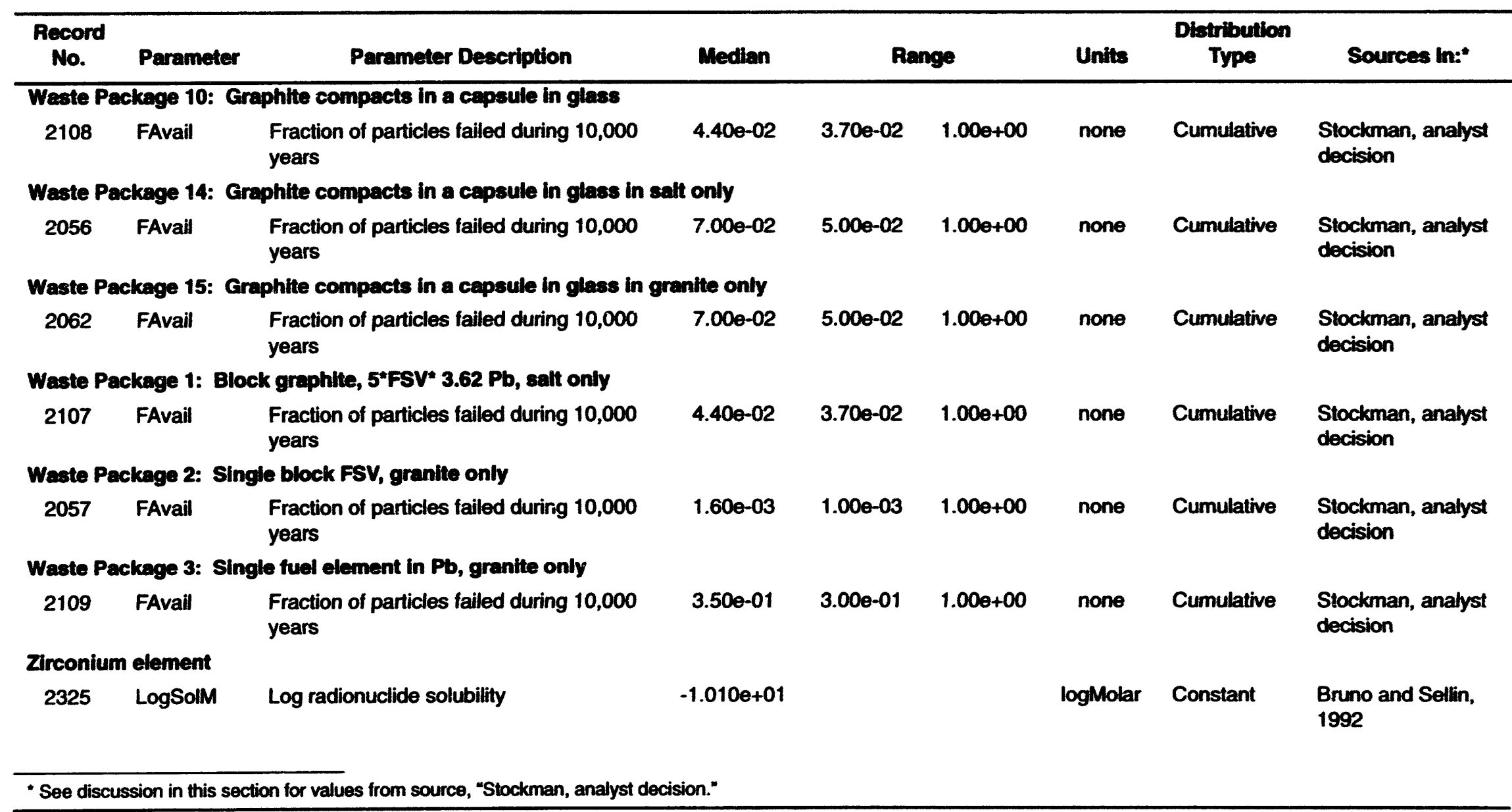




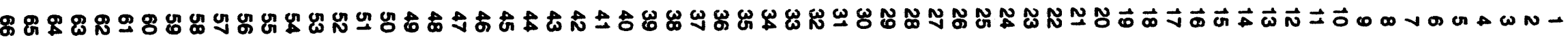

Table 11-6. CONCSNF Model Parameters for Granite Repository

\begin{tabular}{|c|c|c|c|c|c|c|c|c|}
\hline $\begin{array}{l}\text { Record } \\
\text { No. }\end{array}$ & Parameter & Parameter Description & Medlan & \multicolumn{2}{|c|}{ Range } & Units & $\begin{array}{l}\text { Distribution } \\
\text { Type }\end{array}$ & Sources In:* \\
\hline \multicolumn{9}{|c|}{ Americium element } \\
\hline 4929 & LogSolM & Log radionuclide solubility & $-7.000-00$ & $1.30 \theta+01$ & $-6.00 e+00$ & logMolar & Cumulative & EPRI, 1992 \\
\hline 4294 & KDBent & Partition coefficient for bentonite & $3.09 e-01$ & $6.98 e-05$ & $1.37 e+03$ & $\mathrm{~m}^{3} / \mathrm{kg}$ & Lognormal & LeNeveu, 1986 \\
\hline \multicolumn{9}{|c|}{ Borosilicate glass } \\
\hline 2119 & Cormode & $0=k g / \mathrm{m}^{2} \mathrm{~s} 1=1 / \mathrm{s} \quad 2=$ solubility control & $0.00 e+00$ & & & none & Constant & $\begin{array}{l}\text { Stockman, analyst } \\
\text { decision }\end{array}$ \\
\hline 2054 & CoRate & Corrosion rate of material & $1.000-09$ & $1.000-12$ & $1.00 e-06$ & $\mathrm{~kg} / \mathrm{m}^{2} \mathrm{~s}$ & Loguniform & $\begin{array}{l}\text { Stockman, analyst } \\
\text { decision }\end{array}$ \\
\hline \multicolumn{9}{|c|}{ Carbon element } \\
\hline 4293 & LogSolM & Log radionuclide solubility & $1.000+00$ & & & logMolar & Constant & $\begin{array}{l}\text { Stockman, analyst } \\
\text { decision }\end{array}$ \\
\hline 4300 & KDBent & Partition coefficient for bentonite & $0.00 e+00$ & & & $\mathrm{~m}^{3} / \mathrm{kg}$ & Constant & $\begin{array}{l}\text { Stockman, analyst } \\
\text { decision }\end{array}$ \\
\hline \multicolumn{9}{|c|}{ Cesium element } \\
\hline 4315 & LogSolM & Log radionuclide solubility & $7.900 e-01$ & & & logMolar & Constant & U.S. DOE, 1986b \\
\hline 4306 & KDBent & Partition coefficient for bentonite & $3.467 \theta-01$ & $2.116 e-03$ & $5.682 e+01$ & $\mathrm{~m}^{3} / \mathrm{kg}$ & Lognormal & LeNeveu, 1986 \\
\hline \multicolumn{9}{|c|}{ Glass ceramic product of hot isostatic press of INEL high level waste } \\
\hline 2123 & CorrMode & $0=k g / \mathrm{m}^{2} \mathrm{~s} \quad 1=1 / \mathrm{s} 2=$ solubility control & $0.000+00$ & & & none & Constant & $\begin{array}{l}\text { Stockman, analyst } \\
\text { decision }\end{array}$ \\
\hline 2058 & CoRate & Corrosion rate of material & $1.00 e-10$ & $1.00 \theta-12$ & $1.000-08$ & $\mathrm{~kg} / \mathrm{m}^{2} \mathrm{~s}$ & Loguniform & $\begin{array}{l}\text { Stockman, analyst } \\
\text { decision }\end{array}$ \\
\hline \multicolumn{9}{|l|}{ Graphite } \\
\hline 2103 & CorrMode & $0=k g / \mathrm{m}^{2} \mathrm{~s} \quad 1=1 / \mathrm{s} \quad 2=$ solubility control & $0.00 \theta+00$ & & & none & Constant & $\begin{array}{l}\text { Stockman, analyst } \\
\text { decision }\end{array}$ \\
\hline 2113 & CoRate & Corrosion rate of material & $1.00 e-20$ & $1.00 e-22$ & $1.00 e-18$ & $\mathrm{~kg} / \mathrm{m}^{2} \mathrm{~s}$ & Loguniform & $\begin{array}{l}\text { Stockman, analyst } \\
\text { decision }\end{array}$ \\
\hline
\end{tabular}




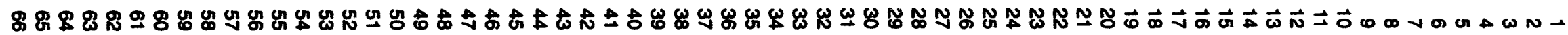

Table 11-6. CONCSNF Model Parameters for Granite Repository (Continued)

\begin{tabular}{|c|c|c|c|c|c|c|c|c|}
\hline \multirow{2}{*}{\multicolumn{2}{|c|}{$\begin{array}{l}\text { Record } \\
\text { No. Parameter } \\
\text { Lead element }\end{array}$}} & \multirow[t]{2}{*}{ Parameter Description } & \multirow[t]{2}{*}{ Median } & \multicolumn{2}{|c|}{ Range } & \multirow[t]{2}{*}{ Units } & \multirow[t]{2}{*}{$\begin{array}{c}\text { Distribution } \\
\text { Type }\end{array}$} & \multirow[t]{2}{*}{ Sources In:* } \\
\hline & & & & & & & & \\
\hline 4310 & LogSolM & Log radionuclide solubility & $-1.470 e+01$ & & & logMolar & Constant & $\begin{array}{l}\text { Bruno and Sellin, } \\
1992\end{array}$ \\
\hline 4301 & KDBent & Partition coefficient for bentonite & $1.698 \theta-02$ & $2.253 \theta-04$ & $1.280 e+00$ & $\mathrm{~m}^{3} / \mathrm{kg}$ & Lognormal & LeNeveu, 1986 \\
\hline \multicolumn{9}{|c|}{ Metal Carbide Fuel (Uranium and Thorium) } \\
\hline 2088 & CorrMode & $0=k g / \mathrm{m}^{2} \mathrm{~s} \quad 1=1 / \mathrm{s} \quad 2=$ solubility control & $1.00 e+00$ & & & none & Constant & $\begin{array}{l}\text { Stockman, analyst } \\
\text { decision }\end{array}$ \\
\hline 2111 & CoRate & Corrosion rate of material & $1.00 e-09$ & & & $1 / s$ & Constant & $\begin{array}{l}\text { Stockman, analyst } \\
\text { decision }\end{array}$ \\
\hline \multicolumn{9}{|c|}{ Neptunium element } \\
\hline 4292 & LogSolM & Log radionuclide solubility & $-7.50 \theta+00$ & $-9.50 e+00$ & $-2.00 e+00$ & logMolar & Cumulative & $\begin{array}{l}\text { Lemire and } \\
\text { Garisto, } 1989\end{array}$ \\
\hline 4294 & KDBent & Partition coefficient for bentonite & $3.39 e-02$ & $3.60 e-03$ & $3.19 e-01$ & $\mathrm{~m}^{3} / \mathrm{kg}$ & Lognormal & LeNeveu, 1986 \\
\hline \multicolumn{9}{|c|}{ Nickel element } \\
\hline 4314 & LogSolM & Log radionuclide solubility & $-4.0000+00$ & & & logMolar & Constant & $\begin{array}{l}\text { Bruno and Sellin, } \\
1992\end{array}$ \\
\hline 4305 & KDBent & Partition coefficient for bentonite & $1.230 e-02$ & $2.330 e-04$ & $6.496 e-01$ & $\mathrm{~m}^{3} / \mathrm{kg}$ & Lognormal & LeNeveu, 1986 \\
\hline \multicolumn{9}{|c|}{ Palladium element } \\
\hline 4316 & LogSolM & Log radionuclide solubility & $-5.820 e+00$ & & & logMolar & Constant & $\begin{array}{l}\text { Bruno and Sellin, } \\
1992\end{array}$ \\
\hline 4307 & KDBent & Partition coefficient for bentonite & $1.230 e-02$ & $2.330 \theta-04$ & $6.496 e-01$ & $\mathrm{~m}^{3} / \mathrm{kg}$ & Lognormal & LeNeveu, 1986 \\
\hline \multicolumn{9}{|c|}{ Plutonium element } \\
\hline 4291 & LogSolM & Log radionuclide solubility & $-8.50 \theta+00$ & $-1.20 e+01$ & $-2.00 e+00$ & logMolar & Cumulative & $\begin{array}{l}\text { Lemire and } \\
\text { Garisto, } 1989\end{array}$ \\
\hline 4296 & KDBent & Partition coefficient for bentonite & $1.51 \theta-01$ & $1.46 e-02$ & $1.57 \theta+00$ & $\mathrm{~m}^{3} / \mathrm{kg}$ & Lognormal & LeNeveu, 1986 \\
\hline
\end{tabular}




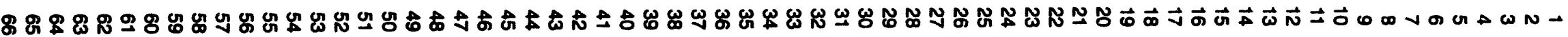

Table 11-6. CONCSNF Model Parameters for Granite Repository (Continued)

\begin{tabular}{|c|c|c|c|c|c|c|c|c|}
\hline $\begin{array}{l}\text { Record } \\
\text { No. }\end{array}$ & Parameter & Paremeter Description & Median & $\mathbf{R a}$ & 198 & Units & $\begin{array}{c}\text { Distribution } \\
\text { Type }\end{array}$ & Sources In:* \\
\hline \multicolumn{9}{|c|}{ Radium element } \\
\hline 817 & LogSolM & Log radionuclide solubility & $-9.00 e+00$ & $-1.10 e+01$ & $-7.00 \theta+00$ & logMolar & Cumulative & EPRI, 1992 \\
\hline 4297 & KDBent & Partition coefficient for bentonite & $5.62 \theta-01$ & $2.65 e-02$ & $1.19 e+00$ & $\mathrm{~m}^{3} / \mathrm{kg}$ & Lognormal & LeNeveu, 1986 \\
\hline \multicolumn{9}{|c|}{ Samarium element } \\
\hline 4317 & LogSolM & Log radionuclide solubility & $-4.000 e+00$ & & & logMolar & Constant & $\begin{array}{l}\text { Bruno and Sellin, } \\
1992\end{array}$ \\
\hline 4308 & KDBent & Partition coefficient for bentonite & $7.430 \mathrm{e}-03$ & $2.056 e-04$ & $2.685 e-01$ & $\mathrm{~m}^{3} / \mathrm{kg}$ & Lognormal & LeNeveu, 1986 \\
\hline \multicolumn{9}{|c|}{ Technetium element } \\
\hline 4312 & LogSolM & Log radionuclide solubility & $-7.700 e+00$ & & & logMolar & Constant & $\begin{array}{l}\text { Bruno and Sellin, } \\
1992\end{array}$ \\
\hline 4303 & KDBent & Partition coefficient for bentonite & $8.913 e-05$ & $8.655 e-08$ & $9.178 \mathrm{e}-02$ & $\mathrm{~m}^{3} / \mathrm{kg}$ & Lognormal & LeNeveu, 1986 \\
\hline \multicolumn{9}{|c|}{ The first container place around the waste form } \\
\hline 4267 & EBFrac & Early breach fraction (Time Zone) & $5.00 e-03$ & $0.00 e+00$ & $1.00 e-02$ & none & Uniform & $\begin{array}{l}\text { Stockman, analyst } \\
\text { decision }\end{array}$ \\
\hline \multicolumn{9}{|c|}{ Thorium element } \\
\hline 4290 & LogSolM & Log radionuclide solubility & $-1.00 e+01$ & $-1.25 e+01$ & $-2.00 e+00$ & logMolar & Cumulative & $\begin{array}{l}\text { Lemire and } \\
\text { Garisto, } 1989\end{array}$ \\
\hline 4298 & KDBent & Partition coefficient for bentonite & $6.46 e-02$ & $2.03 e-05$ & $2.05 e+02$ & $\mathrm{~m}^{3} / \mathrm{kg}$ & Lognormal & LeNeveu, 1986 \\
\hline \multicolumn{9}{|c|}{ Tin element } \\
\hline 4313 & LogSolM & Log radionuclide solubility & $-7.520 e+00$ & & & logMolar & Constant & $\begin{array}{l}\text { Bruno and Sellin, } \\
1992\end{array}$ \\
\hline 4304 & KDBent & Partition coefficient for bentonite & $1.698 e-02$ & $2.253 e-04$ & $1.280 e+00$ & $\mathrm{~m}^{3} / \mathrm{kg}$ & Lognormal & LeNeveu, 1986 \\
\hline
\end{tabular}




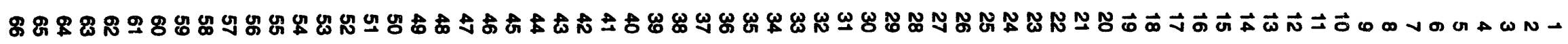

Table 11-6. CONCSNF Model Parameters for Granite Repository (Continued)

\begin{tabular}{|c|c|c|c|c|c|c|c|c|}
\hline $\begin{array}{l}\text { Record } \\
\text { No. }\end{array}$ & Paramater & Parameter Description & Median & \multicolumn{2}{|c|}{ Range } & Units & $\begin{array}{l}\text { Distribution } \\
\text { Type }\end{array}$ & Sources In:* \\
\hline \multicolumn{9}{|c|}{ Tracer element } \\
\hline 4318 & LogSolM & Log radionuclide solubility & $1.000 e+00$ & & & logMolar & Constant & $\begin{array}{l}\text { Stockman, analyst } \\
\text { decision }\end{array}$ \\
\hline 4309 & KDBent & Partition coefficient for bentonite & 0 & & & $\mathrm{~m}^{3} / \mathrm{kg}$ & Constant & $\begin{array}{l}\text { Stockman, analyst } \\
\text { decision }\end{array}$ \\
\hline \multicolumn{9}{|c|}{ Uranium Aluminide } \\
\hline 2099 & CorrMode & $0=\mathrm{kg} / \mathrm{m}^{2} \mathrm{~s} \quad 1=1 / \mathrm{s} \quad 2=$ solubility coritiol & $0.00 e+00$ & & & none & Constant & $\begin{array}{l}\text { Stockman, analyst } \\
\text { decision }\end{array}$ \\
\hline 2180 & CoRate & Corrosion rate of material & $1.00 e-07$ & & & $\mathrm{~kg} / \mathrm{m}^{2} \mathrm{~s}$ & Constant & $\begin{array}{l}\text { Stockman, analyst } \\
\text { decision }\end{array}$ \\
\hline \multicolumn{9}{|c|}{ Uranium dioxide } \\
\hline 2121 & CorrMode & $0=\mathrm{kg} / \mathrm{m}^{2} \mathrm{~s} \quad 1=1 / \mathrm{s} \quad 2=$ solubility control & $2.00 e+00$ & & & none & Constant & $\begin{array}{l}\text { Stockman, analyst } \\
\text { decision }\end{array}$ \\
\hline 2168 & GF_C & $\begin{array}{l}\text { Quick release fraction of C-14 from UO2 } \\
\text { matrix }\end{array}$ & $1.25 e-01$ & $1.000-02$ & $2.500-01$ & none & Uniform & $\begin{array}{l}\text { Garisto and } \\
\text { Garisto, } 1991\end{array}$ \\
\hline \multicolumn{9}{|c|}{ Uranium element } \\
\hline 4289 & LogSolM & Log radionuclide solubility & $-9.68 \theta+00$ & $-1.05 e+01$ & $-3.00 e+00$ & logMolar & Cumulative & $\begin{array}{l}\text { Lemire and } \\
\text { Garisto, } 1989\end{array}$ \\
\hline 4299 & KDBent & Partition coefficient for bentonite & $9.55 e-02$ & $2.26 e-04$ & $4.04 \theta+01$ & $\mathrm{~m}^{3} / \mathrm{kg}$ & Lognormal & LeNeveu, 1986 \\
\hline \multicolumn{9}{|c|}{ Waste Package 10: Graphite compacts in a capsule in glass } \\
\hline 2108 & FAvail & $\begin{array}{l}\text { Fraction of particles failed during } 10,000 \\
\text { years }\end{array}$ & $4.400-02$ & $3.700-02$ & $1.00 e+00$ & none & Cumulative & $\begin{array}{l}\text { Stockman, analyst } \\
\text { decision }\end{array}$ \\
\hline \multicolumn{9}{|c|}{ Waste Package 14: Graphite compacts in a capsule in glass in salt only } \\
\hline 2056 & FAvail & $\begin{array}{l}\text { Fraction of particles failed during } 10,000 \\
\text { years }\end{array}$ & $7.00 e-02$ & $5.000-02$ & $1.00 e+00$ & none & Cumulative & $\begin{array}{l}\text { Stockman, analyst } \\
\text { decision }\end{array}$ \\
\hline
\end{tabular}




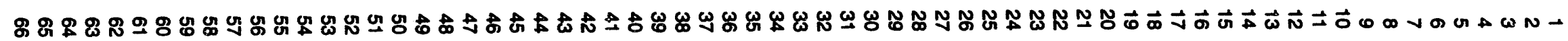

Table 11-6. CONCSNF Model Parameters for Granite Repository (Continued)

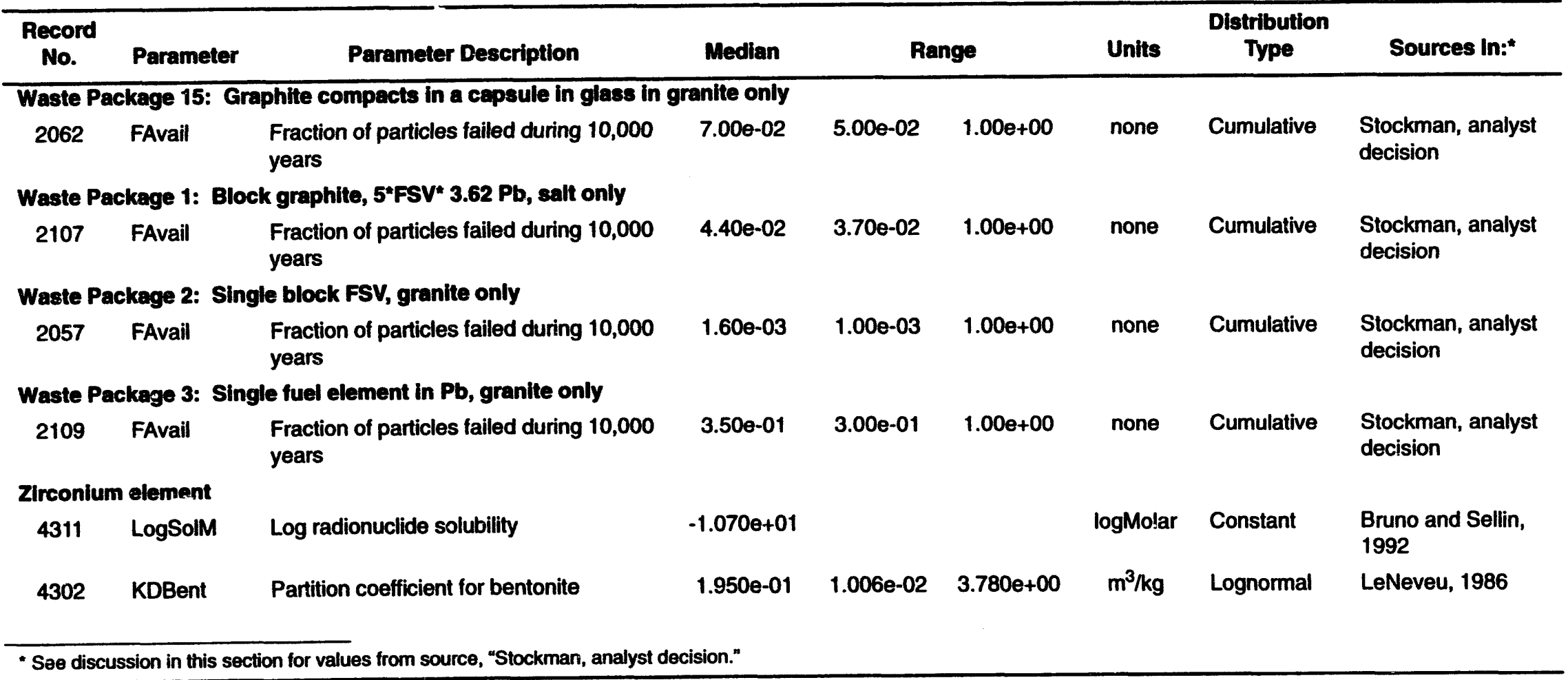


are used for these values unless noted otherwise. The discussion of these parameters is presented below in alphabetical order by material.

Aluminum. The corrosion rate and perforation fraction (fraction of material removed at breach) are used by GASSNF. The values for these parameters are provided below for both the salt and granite repositories.

\section{Salt Repository:}

\section{CoRate}

PerFrac
$1 \times 10^{-7}, 1 \times 10^{-8}$ to $1 \times 10^{-6} \mathrm{~kg} /\left(\mathrm{m}^{2} \cdot \mathrm{s}\right)$

0.5

These values were based on Marine Corrosion (LaQue, 1975), which quotes corrosion rates in seawater. According to LaQue $(1975$, p. 145), the perforation of an 0.0003-inch aluminum coating in quiet seawater in two

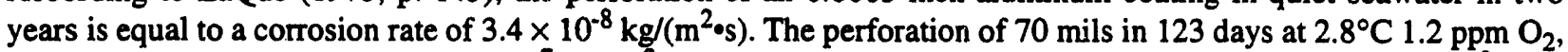
reduces to a corrosion rate of $4.5 \times 10^{-7} \mathrm{~kg} /\left(\mathrm{m}^{2} \cdot \mathrm{s}\right)$ (LaQue, 1975, p. 159). The corrosion rate of $25 \mathrm{mg} /\left(\mathrm{dm}^{2} \cdot\right.$ day) with an $\mathrm{Al}-\mathrm{Al}$ couple is $2.8 \times 10^{-8} \mathrm{~kg} /\left(\mathrm{m}^{2} \circ \mathrm{s}\right)(\mathrm{LaQue}, 1975, \mathrm{p}$. 197). Other couplings with stainless steel or titanium increase the corrosion rate. Because of the increased salinity in a salt repository, these values were used as indications of the low range for the corrosion rates. The upper range is simply conjecture. Studies currently underway by Westerman at Pacific Northwest Laboratory, Richland, WA, are expected to provide data to substantiate corrosion rates for aluminum (personal communication, 1993).

The perforation fraction of 0.5 was also an estimate; it is in the range of a material that undergoes mainly generalized corrosion. Subsequent comments by INEL personnel, however, suggest that it may have been more appropriate to use the more conservative value of zero. Because the generalized corrosion rate is high, however, the difference in breach times that results from using either 0 or 0.5 is about the same size as a time step.

\section{Granite Repository:}

$\begin{array}{ll}\text { CoRate } & 1 \times 10^{-9}, 1 \times 10^{-10} \text { to } 1 \times 10^{-8} \mathrm{~kg} /\left(\mathrm{m}^{2} \bullet \mathrm{s}\right) \\ \text { PerFrac } & 0.5\end{array}$

$$
0.5
$$

These values again are based on rates from LaQue (1975), but since groundwater in a granite site is less saline than seawater, the seawater value was set as the upper limit. The median and lower values are conjectures only.

A perforation fraction of 0.5 was used.

Graphite. The corrosion rate is used by GASSNF and CONCSNF; the perforation rate is used by GASSNF. The values for these parameters are provided below for both the salt and granite repositories.

\section{Salt and Granite Repositories:}

$$
\begin{array}{ll}
\text { CoRate } & 1 \times 10^{-20}, 1 \times 10^{-22} \text { to } 1 \times 10^{-18} \mathrm{~kg} /\left(\mathrm{m}^{2} \cdot \mathrm{s}\right) \\
\text { PerFrac } & 0
\end{array}
$$

Lotts et al. (1992) report an extrapolated oxidation rate of graphite at $100^{\circ} \mathrm{C}$ as $4.5 \times 10^{-20} \mathrm{~g} /\left(\mathrm{cm}^{2} \mathrm{~s}\right)$ for 0.1 atm $\mathrm{O}_{2}$ pressure, which is $4.5 \times 10^{-19} \mathrm{~kg} /\left(\mathrm{m}^{2} \cdot \mathrm{s}\right)$. Because these values not only were extrapolated to low temperatures but then applied to an aqueous repository instead of a steam environment; the values are uncertain but probably very conservative (because the rate in the absence of dissolved $\mathrm{O}_{2}$ would most likely be even lower). The possible effect of catalysis due to the presence of salt was neglected even though catalysis by calcium potassium oxides has been reported (Pereira et al., 1992) for the gasification of graphite at high temperatures. The results of the experiments indicated that the temperature necessary for commercially acceptable rates of gasification could be lowered from $1400 \mathrm{~K}$ to $800 \mathrm{~K}$. However, it is not clear whether the catalysis mechanism would be the same at $90^{\circ} \mathrm{C}$. Because of the expected error due to extrapolation, we chose a range of four orders of magnitude.

The perforation fraction was set to zero to echo our assumption that the time for water to soak through the graphite is very small compared to the time steps of the calculation. 


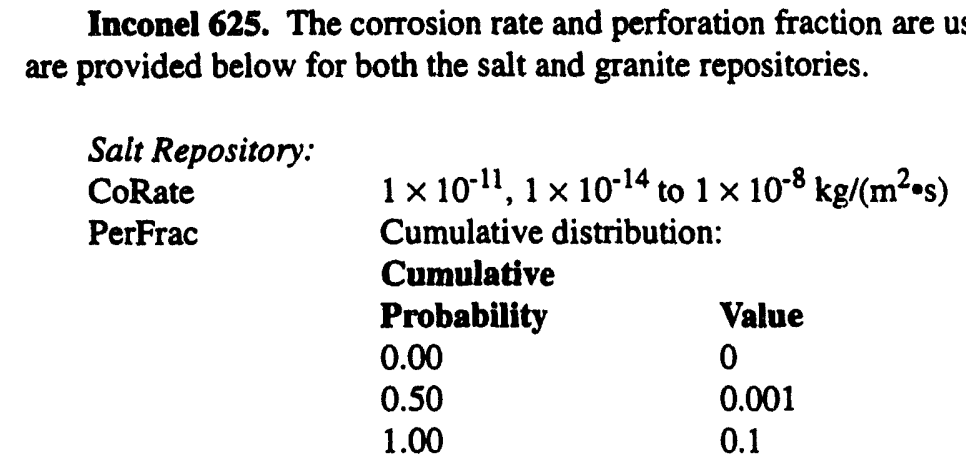

Sorensen and Molecke (1992) report: "Grade-12 Ti, grade-2 Ti and Inconel 625 exhibited excellent corrosion resistance." In earlier work by Molecke et al. (1981), the maximum rate quoted was $1.2 \times 10^{-5} \mathrm{~m} / \mathrm{yr}$, which equals $3 \times 10^{-9} \mathrm{~kg} /\left(\mathrm{m}^{2} \mathrm{~s}\right)$ at $250^{\circ} \mathrm{C}, 30 \mathrm{ppb} \mathrm{O}_{2}$ in seawater with pitting noted. With $1 \times 10^{7} \mathrm{rad} / \mathrm{hr}$ at $90^{\circ} \mathrm{C}$ in seawater, the rate ranged from $2 \times 10^{-6}$ to $9 \times 10^{-6} \mathrm{~m} / \mathrm{yr}$ depending on test time. When all dissolved oxygen is consumed, and in the absence of sufficient $\mathrm{H}_{2} \mathrm{~S}, \mathrm{Ni}$ becomes stable and the reaction rate goes to zero. Without further data, a range from $1 \times 10^{-8}$ to $1 \times 10^{-14}$ seemed prudent.

\section{Granite Repository:}

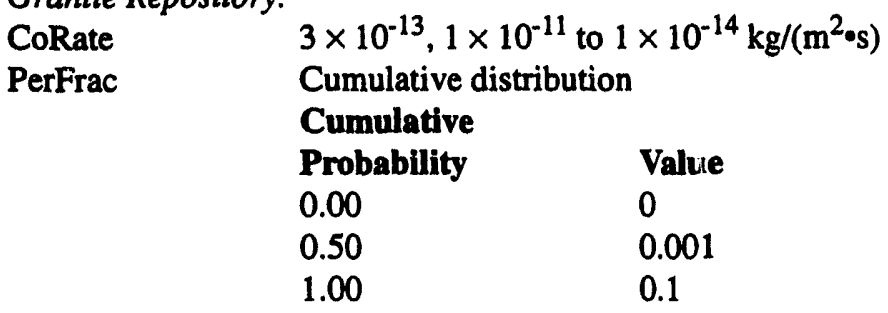

In a review of candidate canister materials for a potential Yucca Mountain repository (Van Konynenburg et al.,1993), Inconel 625 was reported to have good base-metal corrosion properties, similar to Incoloy 825; however, the addition of $\mathrm{Nb}$ makes it susceptible to ordering and precipitate formation. The reported generalized corrosion rate for Incoloy 825 was $3 \times 10^{-8} \mathrm{~m} / \mathrm{yr}$, and the reported localized corrosion rate was $1 \times 10^{-6} \mathrm{~m} / \mathrm{yr}$. These values correspond to $7 \times 10^{-12}$ and $2 \times 10^{-10} \mathrm{~kg} /\left(\mathrm{m}^{2} \bullet \mathrm{s}\right)$. We rounded the value $7 \times 10^{-12}$ up to $1 \times 10^{-11}$ and used it as the upper range. Because the Ni portion of the alloy will become stable as $\mathrm{O}_{2}$ is consumed, and reacts only as fast as $\mathrm{H}_{2} \mathrm{~S}$ can migrate in from the surroundings, $1 \times 10^{-14}$ seemed a reasonable lower limit.

Since there are few data for localized corrosion, our reasoning for selecting a value was similar to that used for Ti-Grade 12, but we gave an equal probability to localized-only corrosion and combined generalized and localized corrosion.

Lead. The corrosion rate and perforation fraction are used by GASSNF. The values for these parameters are provided below for both the salt and granite repositories.

$$
\begin{aligned}
& \text { Salt and Granite Repositories: } \\
& \begin{array}{ll}
\text { CoRate } & 1 \times 10^{-9}, 1 \times 10^{-10} \text { to } 1 \times 10^{-8} \mathrm{~kg} /\left(\mathrm{m}^{2} \cdot \mathrm{s}\right) \\
\text { PerFrac } & 1 \times 10^{-2}, 1 \times 10^{-3} \text { to } 1 \times 10^{-1}
\end{array}
\end{aligned}
$$

Goodwin and Westerman (1992) report corrosion of lead in simulated Yucca Mou:tain Repository conditions that range from $<5 \times 10^{-6}$ to $2 \times 10^{-4} \mathrm{~m} / \mathrm{yr}$. These values correspond to corrosion rates of $2 \times 10^{-9}$ to $7 \times 10^{-8} \mathrm{~kg} /$ $\left(\mathrm{m}^{2} \bullet \mathrm{s}\right)$. Sorensen and Molecke (1992, Figure 6) report 10 to $30 \mathrm{gms}$ lead lost in 1800 days in the MIIT tests in salt. A total of $10 \mathrm{gms}$ lost corresponds to $2 \times 10^{-8} \mathrm{~kg} /\left(\mathrm{m}^{2} \cdot \mathrm{s}\right)$, and $30 \mathrm{gms}$ lost equals $6 \times 10^{-8} \mathrm{~kg} /\left(\mathrm{m}^{2} \cdot \mathrm{s}\right)$. The data at 800 days gives a range of $4 \times 10^{-9}$ to $8 \times 10^{-8} \mathrm{~kg} /\left(\mathrm{m}^{2} \cdot \mathrm{s}\right)$. In these tests, access of oxygen from the air in the tunnels was not controlled by the experimentalists. These results are probably a good representation of the rates that would occur prior to and shortly after closure of the repository. As the dissolved oxygen is used up, however, the corrosion rate 
will fall dramatically and eventually reach a slow rate that is determined by the mass transport of $\mathrm{H}_{2}$ away, $\mathrm{H}_{2} \mathrm{~S}$ in, and/or the production of $\mathrm{O}_{2}$ or peroxide from radiolysis of water. Accordingly, we used the $1 \times 10^{-8}$ value as an upper limit and estimated the lower limit at $1 \times 10^{-10} \mathrm{~kg} /\left(\mathrm{m}^{2} \bullet \mathrm{s}\right)$. This lower limit is very conservative since it is possible in a very tight repository to get a corrosion rate of zero.

Sorensen and Molecke (1992) reported that lead underwent both local and generalized ccrrosion, but mainly generalized corrosion. Because the lead will always be surrounded by a layer of stainless steel in the packages modeled, it is likely that a small breach will occur in the overlying layer of steel, and it is possible that the lead will undergo generalized corrosion in a localized area under the steel breach. Accordingly, a perforation fraction range of $1 \times 10^{-1}$ to $1 \times 10^{-3}$ seemed reasonable.

Metal Carbide Fuel: ThC $_{2}$ and $\mathrm{UC}_{2}$. The corrosion rate is used by GASSNF and CONCSNF; the perforation fraction is used by GASSNF. The values for these parameters are provided below for both the salt and granite repositories.

$\begin{array}{ll}\text { Salt and Granite Repositories: } \\ \text { CorrMode } & 1 \text { corrosion is in units of } 1 / \mathrm{s} \\ \text { CorRate } & 1 \times 10^{-9} / \mathrm{s} \\ \text { PerFrac } & 0\end{array}$

The corrosion values are based on information in Section 2.6.1.1.1 in Appendix A of this document in which it was estimated that it will take $17 \mathrm{yr}$ to use up all the available carbides when a reasonable rate derived from a mechanism of a cyclic thermal suction of water into the cracked particles is used. Total consumption in $17 \mathrm{yr}$ is equivalent to $1.86 \times 10^{-9} \mathrm{~kg}$ removed per second. Since $17 \mathrm{yr}$ is about equal to the smallest times steps used in the simulation, this rate is effectively instantaneous, and we therefore decided that sampling over a range would not be necessary.

The perforation fraction is irrelevant because the carbide is the innermost layer.

Stainless Steel 304L. The corrosion rate and perforation fraction are used by GASSNF. The values for these parameters are provided below for both the salt and granite repositories.

Salt Repository:

CoRate

$1 \times 10^{-10}, 1 \times 10^{-11}$ to $1 \times 10^{-9} \mathrm{~kg} /\left(\mathrm{m}^{2} \cdot \mathrm{s}\right)$

PerFrac

$5 \times 10^{-3}, 5 \times 10^{-4}$ to $5 \times 10^{-2}$

Sorensen and Molecke (1992) report tests at $90^{\circ} \mathrm{C}$ at the WIPP site. The samples were immersed in a sample of brine (Brine A) in boreholes drilled into the floor of the repository. The boreholes quickly filled in with salt and no special steps were taken to control oxygen access to samples. The samples were disk shaped with surface areas of about $3049 \mathrm{~mm}^{2}$.

Results are summarized here (Sorensen and Molecke, 1992, Figure 1):

unwelded $304 \mathrm{~L}$ corrodes $0.02+/-0.001 \mathrm{~g}$ in 1800 days

welded, $0.04+/-0.02 \mathrm{~g}$ in 1800 days

outlier at $0.07 \mathrm{~g}$

$$
\begin{aligned}
& 4.22 \times 10^{-11}+/-2 \times 10^{-11} \mathrm{~kg} /\left(\mathrm{m}^{2} \bullet \mathrm{s}\right) \\
& 8.44 \times 10^{-11}+/-4 \times 10^{-11} \mathrm{~kg} /\left(\mathrm{m}^{2} \cdot \mathrm{s}\right) \\
& 1.48 \times 10^{-10} \mathrm{~kg} /\left(\mathrm{m}^{2} \bullet \mathrm{s}\right)
\end{aligned}
$$

For the corrosion rate, we used the welded data rounded to the nearest order of magnitude and used a range of one order of magnitude on either side. Other data, for example the study of stainless steel $304 \mathrm{~L}$ at $250^{\circ} \mathrm{C}$ with limited $\mathrm{O}_{2}$ access (Pitman et al., 1981), also fell in this range $\left(8 \times 10^{-11} \mathrm{~kg} /\left[\mathrm{m}^{2} \cdot \mathrm{s}\right]\right)$.

Sorensen and Molecke (1992) also noted a Cl-induced 4-mm crack at one year, which is equivalent to $4 \times 10^{-3} \mathrm{~m} /$ yr penetration rate. Compared to the penetration rate of $4 \times 10^{7} \mathrm{~m} / \mathrm{yr}$ from a $1 \times 10^{-10} \mathrm{~kg} /\left(\mathrm{m}^{2} \bullet \mathrm{s}\right)$ generalized corrosion rate, cracking is four orders of magnitude greater. Since all samples did not show this behavior, $5 \times 10^{-4}$ was selected as a lower bound for the perforation fraction; the upper bound of $5 \times 10^{-2}$ was arbitrarily chosen. 
Granite Repository:

$$
\begin{array}{ll}
\text { CoRate } & 1 \times 10^{-13}, 1 \times 10^{-15} \text { to } 1 \times 10^{-11} \mathrm{~kg} /\left(\mathrm{m}^{2} \cdot \mathrm{s}\right) \\
\text { PerFrac } & 5 \times 10^{-5}, 5 \times 10^{-6} \text { to } 5 \times 10^{-4}
\end{array}
$$

Van Konyenburg et al. (1993) considered the selection of canister material for a potential repository in Yucca Mountain. They concluded that in a Yucca Mountain environment (i.e., unsaturated tuff), stainless steel 304L would have a generalized corrosion rate of about $0.1 \mathrm{~m} / \mathrm{yr}$ or $2 \times 10^{-11} \mathrm{~kg} /\left(\mathrm{m}^{2} \cdot \mathrm{s}\right)$, a local corrosion rate of $100 \mu / \mathrm{yr}$ or $2 \times 10^{-7} \mathrm{~kg} /\left(\mathrm{m}^{2} \circ \mathrm{s}\right)$ and a high likelihood of stress corrosion cracking, $\mathrm{H}_{2}$ degradation, and microbe influence. Another study by Pitman et al. (1981) using Hanford water, $250^{\circ} \mathrm{C}$, and limited $\mathrm{O}_{2}$, showed a corrosion rate of 1.5 $\mathrm{mg} / \mathrm{dm}^{2}$ in 3 months or $2 \times 10^{-15} \mathrm{~kg} /\left(\mathrm{m}^{2} \cdot \mathrm{s}\right)$. We felt that the corrosion rates for Yucca Mountain were a conservative upper range, because less dissolved oxygen is expected in a granite repository than at Yucca Mountain, and the Hanford rates were a conservative lower range. Because the Hanford rates were measured at higher temperatures, this low range may not be a low enough. A further literature search is warranted to better identify the range of corrosion rates and the shape of the distribution.

The perforation fraction was estimated by comparing the localized penetration rate with the generalized penetration rate. The median rate of $1 \times 10^{-13} \mathrm{~kg} /\left(\mathrm{m}^{2} \cdot \mathrm{s}\right)$ corresponds to a penetration rate of $3.9 \times 10^{-10} \mathrm{~m} / \mathrm{yr}$. Farmer and McCright (1989) report a penetration rate of $2 \times 10^{-5} \mathrm{~m} / \mathrm{yr}$ for a U-bend specimen in $0.8 \% \mathrm{NaCl}$ and $0.5 \%$ $\mathrm{CH}^{3} \mathrm{COOH}$ at $141^{\circ} \mathrm{C}$, which is $5 \times 10^{+4}$ times faster, suggesting a perforation fraction of $2 \times 10^{-5}$. Using an equation for pitting rates reported by Bullen (1992), and parameters for poor aeration and $\mathrm{pH} 8$, yields a penetration rate of $1 \times 10^{-4} \mathrm{~m} / \mathrm{yr}$ suggesting a perforation fraction of $4 \times 10^{-6}$. Based on these limited data, we selected a range of $5 \times 10^{-4}$ to $5 \times 10^{-6}$ for the perforation fraction.

Stainless Steel 304L - HIP packages. The corrosion rate and perforation fraction are used by GASSNF. The values for these parameters are provided below for both the salt and granite repositories. Note that the corrosion rate is the same as for stainless steel $304 \mathrm{~L}$, but that the perforation fraction for both repositories is zero.

$$
\begin{array}{ll}
\begin{array}{l}
\text { Salt Repository. } \\
\text { CoRate } \\
\text { PerFrac }
\end{array} & 1 \times 10^{-10}, 1 \times 10-11 \text { to } 1 \times 10^{-9} \mathrm{~kg} /\left(\mathrm{m}^{2} \cdot \mathrm{s}\right) \\
\text { Granite Repository: } & 0 \\
\begin{array}{l}
\text { CoRate } \\
\text { PerFrac }
\end{array} & 1 \times 10^{-13}, 1 \times 10^{-15} \text { to } 1 \times 10^{-11} \mathrm{~kg} /\left(\mathrm{m}^{2} \cdot \mathrm{s}\right) \\
& 0
\end{array}
$$

The difference between stainless steel $304 \mathrm{~L}$ and $304 \mathrm{~L}$ in HIPs is the effect of heating at high pressures. We do not know what the effect will be, but expect that it will depend in part on how long the package is kept at high temperature and how fast it is cooled. For the perforation fraction, we have chosen to use the conservative assumption that the integrity of the package has been compromised; thus PerFrac is set to a constant 0 . While the generalized corrosion rate may also change in response to the heating cycle, we do not know how much; we have assumed that using the same rates as $304 \mathrm{~L}$ will not create a significant error in our calculations.

Titanium Grade 12. The corrosion rate and perforation fractions are used by GASSNF. The values for these parameters are provided below, for the salt repository only, because this material was not used for a container in the granite repository calculations. (Inconel-625 was used instead.)

Salt Repository:

CoRate

PerFrac

$5 \times 10^{-12}, 5 \times 10^{-14}$ to $5 \times 10^{-10} \mathrm{~kg} /\left(\mathrm{m}^{2} \cdot \mathrm{s}\right)$
Cumulative distribution:
$\begin{array}{ll}\text { Cumulative } \\ \text { Probability } & \text { Value } \\ 0.00 & 0 \\ 0.33 & 0.001 \\ 0.66 & 0.1 \\ 1.00 & 0.1\end{array}$


Garisto and LeNeveu (1989) report a uniform corrosion rate of less than $2 \times 10^{-6} \mathrm{~m} / \mathrm{yr}$ at $100^{\circ} \mathrm{C}\left(<3 \times 10^{-10} \mathrm{~kg} /\right.$ $\left[\mathrm{m}^{2} \bullet \mathrm{s}\right]$ ) for Ti-Grade 12, based on compiled data. In Molecke et al. (1981), the maximum rate reported was $3 \times 10^{-6}$ $\mathrm{m} / \mathrm{yr}$ at $250^{\circ} \mathrm{C}$ with $30 \mathrm{ppb} \mathrm{O}_{2}$ in Brine B (equivalent to $5 \times 10^{-10} \mathrm{~kg} /\left[\mathrm{m}^{2} \cdot \mathrm{s}\right]$ ). Molecke (personal communication, 1993) suggested that the observed oxidation in more realistic experiments occurs only during the first few days and the results from Molecke et al. (1981) should not be used as constant rates over long time periods. He was not comfortable in recommending any rate or range of rates but felt that the actual long-term rate would be close to 0 . We chose an upper limit of $5 \times 10^{-10} \mathrm{~kg} /\left(\mathrm{m}^{2} \cdot \mathrm{s}\right)$ and estimated a lower limit of $5 \times 10^{-14} \mathrm{~kg} /\left(\mathrm{m}^{2} \cdot \mathrm{s}\right.$ ) (penetration rate of $0.003 \mathrm{~mm}$ in $10,000 \mathrm{yr})$. With a loguniform distribution, the median is $5 \times 10^{-12} \mathrm{~kg} /\left(\mathrm{m}^{2} \bullet \mathrm{s}\right)$.

Asano et al. (1992) identify stress corrosion cracking due to $\mathrm{H}_{2}$ absorption and crevice corrosion as two mechanisms that must be studied further for unspecified titanium alloys. Magnani (1983) says the Swedish upper limit is $120^{\circ} \mathrm{C}$ for use of $\mathrm{Ti}$ in seawater, but their tests show crevice corrosion in $\mathrm{Ti}$ at temperatures of $150^{\circ} \mathrm{C}$ and up. They also state that Ti-Grade 12 is more resistant to crevice corrosion than other Ti alloys; no crevice corrosion has been observed in Ti-Grade 12; Ti-Grade 12 is not susceptible to $\mathrm{SCC}$ or embrittlement due to $\mathrm{H}_{2}$ in basaltic groundwater; and radiolytic production of $\mathrm{H}_{2}$ and $\mathrm{H}_{2} \mathrm{O}_{2}$ actually increases the healing rate of the oxide film. Ikeda et al. (1989) report crevice corrosion propagation but no pitting or hydride cracking under conditions expected in the Canadian granite repository. Preformed crevices were found to be active but oxygen starved after an initial period. Because the generalized corrosion rate is so slow (the upper limit of $5 \times 10^{-10} \mathrm{~kg} /\left(\mathrm{m}^{2} \bullet \mathrm{s}\right)$ would breach a 1-cm-thick container in about $3,000 \mathrm{yr}$; the median rate would take $300,000 \mathrm{yr}$ for breach), breach from other mechanisms will dominate, and the perforation fraction will be the crucial parameter. Unfortunately, it is difficult to assess the likelihood and rate of crevice corrosion in our hypothetical repository. Because of little information, the use of a range of 0 to 1 covers all possibilities. We decided to use a constructed distribution with uniform probabilities within three ranges: 0 to 0.001 for highly localized corrosion only; 0.001 to 0.1 for mixed localized and generalized corrosion; and 0.1 to 1 for generalized corrosion only. Since we do not know the likelihood of these regimes, we gave each regime an equal probability.

Uranium-Aluminum Alloy Fuel. The corrosion rate is used by GASSNF and CONCSNF; the perforation rate is used by GASSNF. The values for these parameters are provided below for both the salt and granite repositories.

$\begin{array}{ll}\begin{array}{l}\text { Salt Repository: } \\ \text { CoRate }\end{array} & 1 \times 10^{-6} \mathrm{~kg} /\left(\mathrm{m}^{2} \bullet \mathrm{s}\right) \\ \text { PerFrac } & 0 \\ \text { Granite Repository: } & \\ \text { CoRate } & 1 \times 10^{-7} \mathrm{~kg} /\left(\mathrm{m}^{2} \bullet \mathrm{s}\right) \\ \text { PerFrac } & 0\end{array}$

Although no information was available for this alloy regarding corrosion rates in either a salt or granite repository, INEL personnel indicated that during dissolution of the fuel in acid, the aluminum clad lasted longer than the fuel. Accordingly, we arbitrarily selected the upper limit of corrosion of aluminium in salt as the corrosion rate for the uranium-aluminum alloy, and used a rate in granite of one order of magnitude lower than the lower limit for aluminum. Because these rates result in total consumption of the layer in times that are relatively short compared with the time steps used in the calculations, we decided that sampling of these variable would not provide much additional information. The perforation fractions were set to zero because the fuel is the innermost layer and therefore the perforation fraction is irrelevant.

Zircaloy Cladding. The corrosion rate and perforation fraction are used by GASSNF. The values for these parameters are provided below for both the salt and granite repositories.

Salt Repository:

CoRate

PerFrac

$$
\int_{0}^{1 \times 10^{-11} \mathrm{~kg} /\left(\mathrm{m}^{2} \cdot \mathrm{s}\right)}
$$




\section{Salt and Granite Repositories}

EBFrac $\quad 0.005$ for both salt and granite

The early breach fraction is defined as the fraction of waste parcels within the repository that have a breached outer layer at time zero. This breach may be caused by manufacturing defects, damage that occurs during transportation and emplacement, or any other reason. SKB (1992a) used $1 \times 10^{-3}$. We decided to be more conservative and picked a range of 0 to $1 \%$. However, to reduce the number of sampled parameters so that run time could be improved, we chose the single value of $0.5 \%$.

\subsubsection{Matrix Alteration Parameters}

Borosilicate Glass. The corrosion rate is used by CONCSNF. The value for this parameter is provided below for both the salt and granite repositories.

\section{Salt and Granite Repositories:}

$$
\text { CoRate } 1 \times 10^{-9}, 1 \times 10^{-12} \text { to } 1 \times 10^{-6} \mathrm{~kg} /\left(\mathrm{m}^{2} \cdot \mathrm{s}\right)
$$

Strachan et al. (1990, Figure 3.1) report final dissolution rates for PNL 76-78, SRL-165, and EMS-11 glasses as $0.08,0.02$, and $0.002 \mathrm{~g} / \mathrm{m}^{2} \mathrm{~d}$, respectively. The final dissolution rate is defined as the rate when the solution is saturated with respect to SiO2. These rates correspond to $9 \times 10^{-10}, 2 \times 10^{-10}$, and $2 \times 10^{-11} \mathrm{~kg} /\left(\mathrm{m}^{2} \cdot \mathrm{s}\right)$, so we selected a range of $1 \times 10^{-8}$ to $1 \times 10^{-12}$ to cover the possible conditions. Uncertainty also exists in the surface area of the glass; in the calculation, the monolithic surface area was used. Because this is the low end of an actual range, we added two orders of magnitude to the upper limit of the alteration rate to account for glass fracturing that may increase the surface area.

Calcine - Loose. The corrosion mode and rates are used by CONCSNF. The values for these parameters are provided below for both the salt and granite repositories.

\section{Salt and Granite Repositories: \\ CorrMode $\quad 1$ units $=1 / \mathrm{s}$ \\ CoRate $\quad 4 \times 10^{-7} / \mathrm{s}$}


Leach experiments show nearly quantitative removal of non-solubility limited elements in 28 days (see McLain and Rhodes, 1958; Paige, 1966; and Wilding and Rhodes, 1969). Assuming a constant rate of dissolution/alteration in the 28 days gives a fractional release rate of $4 \times 10^{-7} / \mathrm{s}$.

Glass-Ceramic. The corrosion rate is used by CONCSNF. The value for this parameter is provided below for both the salt and granite repositories.

$$
\begin{aligned}
& \text { Salt and Granite Repositories: } \\
& \text { CoRate } 1 \times 10^{-10}, 1 \times 10^{-12} \text { to } 1 \times 10^{-8}
\end{aligned}
$$

Vinjamuri et al. (1992) report total mass removal rates of 0.17 to $1.5 \mathrm{~g} / \mathrm{m}^{2} \mathrm{~d}$ in MCC-1 static leach tests in deionized water at $90^{\circ} \mathrm{C}$ for 14 days. This rate is very similar to the forward reaction rates reported by Strachan et al. (1990) for glass $\left(0.08\right.$ to $\left.1.6 \mathrm{gm} / \mathrm{m}^{2} \mathrm{~d}\right)$. Because the leach behavior of glass-ceramic is highly dependant on the particle size of reactants and the exact recipe for the mix, and because little is known of the long-term leach rates, we elected to use the same range for glass-ceramic as for glass. Because we expect the glass-ceramic to have more strength than glass, we decided not to extend the glass-ceramic corrosion rates two order of magnitude as we did for glass to account for the uncertainty in surface area. We believe this treatment of glass-ceramic dissolution is crude because of the two phases in glass-ceramic: the glass phase and mineral grains. Although using an alteration rate is not an unreasonable approach for the glass, which is thermodynamically unstable, the mineral grains are stable and therefore the use of solubility limits would be more appropriate. Our model could more appropriately model the glass-ceramic waste form by treating it as more than one matrix but doing so would require information that is not available at this time, e.g., the radionuclide inventory within the glass and each separate mineral, the mass of the glass and each mineral, and the solubility of each mineral.

$\mathrm{UO}_{2}$ Fuel. The corrosion mode and quick release fraction of carbon-14 are used by CONCSNF. The values for these parameters are provided below for both the salt and granite repositories.

\section{Salt and Granite Repositories: \\ CorrMode 2 solubility limited \\ GF_C $\quad 0.125$}

Because the repository is expected to be reducing during all but the initial period, $\mathrm{UO}_{2}$ is the expected thermodynamically stable phase. Accordingly, the dissolution rate is determined by the solubility of $\mathrm{UO}_{2}$. Of the isotopes transported, ${ }^{14} \mathrm{C}$ is the only one likely to have a significant gap or grain boundary fraction. Garisto and Garisto (1991) report a uniform distribution from 0 to $25 \%$. We have used the median value for our calculation.

\subsubsection{Fraction of Metal Carbide Fuel Particles that Breach in $10,000 \mathrm{yr}$}

The values for the fraction of metal carbide fuel particles that breach in $10,000 \mathrm{yr}$ (FAvail) are shown below.

\begin{tabular}{clcccc} 
Waste Parcel & \multicolumn{1}{c}{ Fuel Type } & $0 \%$ & $50 \%$ & $90 \%$ & $100 \% *$ \\
\hline 1 & Fort St. Vrain and Peach Bottom & 0.037 & 0.044 & 0.053 & 1.0 \\
2 & Fort St. Vrain & 0.001 & 0.0016 & 0.005 & 1.0 \\
3 & Peach Bottom & 0.3 & 0.35 & 0.4 & 1.0 \\
10 & Fort St. Vrain and Peach Bottom & 0.037 & .044 & 0.053 & 1.0 \\
14 & Fort St. Vrain and Peach Bottom burned & 0.05 & 0.07 & 0.20 & 1.0 \\
15 & Fort St. Vrain and Peach Bottom burned & 0.05 & 0.07 & 0.20 & 1.0
\end{tabular}

- Probability that actual value is below given value. 
For our estimates we have divided the graphite fuel into three groups: Peach Bottom Core I, Peach Bottom Core II, and Fort St. Vrain. The inventory was estimated for these three groups using the following number of "Fort St. Vrain equivalents."

\begin{tabular}{lccc} 
& $\begin{array}{c}\text { Fort St. Vrain } \\
\text { Equivalent }\end{array}$ & $\begin{array}{c}\text { Percent of } \\
\text { Inventory }\end{array}$ & $\begin{array}{c}\text { Estimate of } \\
\text { Falled } \\
\text { Particles }\end{array}$ \\
\cline { 2 - 4 } Peach Bottom Core I & 165.5 & 6.6 & $64 \%$ \\
Peach Bottom Core II & 139.2 & 5.5 & $3 \%$ \\
Fort St. Vrain & 2208 & 87.9 & $0.16 \%$ \\
TOTAL & 2512 & &
\end{tabular}

Even though there was a difference in failure rates of the fissile vs. fertile fuel particles, and the final inventory is expected to be different in these two locations, good estimates of the proportion of each isotope in these two locations are not available so this effect has been neglected. Lotts et al. (1992) report expected failure rates of 0.3 to $0.5 \%$ in the first three reloads (726 elements) of the Fort St. Vrain reactor, and an order of magnitude lower failure rates in the later reloads (1482 elements). We estimated the lowest possible fraction (FAvail) for all of Fort St. Vrain waste (Waste Parcel 2 [WP2]) as 0.001 based on a weighted average of 0.003 for the early reloads and 0.00008 for the later ones. For the median probability value, we used a weighted average of 0.005 for the early reloads and 0.00008 for the later ones. For the $90 \%$ value, we used the highest guess for the early reloads and used 1.0 for the $100 \%$ value. This distribution assumes that the probability that unfailed particles will fail during the $10,000 \mathrm{yr}$ is about $10 \%$.

Lotts et al. (1992) report that only unknown mechanisms will breach unfailed particles within a repository. Further study is needed to provide a defensible estimate of the likelihood of failure within 10,000 yr. Examination of Peach Bottom Core I segments 10, 18, 25 and 27 showed a large amount of damaged and broken particles (Bob Kirkham, personal communication, 1993). Based on the percentage of damaged and broken particles in these four segments, we estimated the fraction of broken particles for the whole core as about $64 \%$. Core II was in much better shape, and our estimate has about twice the failed particles as the Fort St. Vrain fuel. The values of 0.3 and 0.4 for FAvail used for all of the Peach Bottom fuel (WP3) reflects weighted averages of FAvail values for Core I ( 0.55 and 0.73 ) and for Core $2(0.002$ and 0.01$)$. The FAvail values of WP1 are weighted averages from the FAvail values from Fort St. Vrain (WP 2) and Peach Bottom (WP3). For WP10, we used the same distribution as for WP1, assuming that mechanical removal of the compacts does not disturb any of the particles within the compacts. For WPs 14 and 15, the compacts have been burned, and based on this assumption, we estimated that up to $20 \%$ of the particles may be damaged (personal communication with Bob Kirkham and Larry Taylor, 1993). 


\title{
12. Complex Consequence Modeling for Salt Disposal System
}

\author{
J. L. Ramsey, M. E. Lord, D. K. Rudeen, D. M. Stoelzel
}

The complex performance assessment (Complex PA) used spatially distributed parameters and partial differential equations on a coarse numerical grid. For this approach, all four waste types (i.e., graphite spent fuel, special spent fuel, calcine high-level waste, and borosilicate high-level waste from the DOE sites) were place uniformly in the repository. Five full PA calculations were run per disposal site (salt and granite): one each per waste disposal group, which included all waste types. Several separate, complete codes were used to simulate the selected physical components of the disposal system (see Chapter 7, "Scenario Development," and Appendix G). The two categories of consequence modeling systems developed from these codes are (1) repository fluid flow and (2) radionuclide transport modeling, which are described below.

\subsection{Repository Fluid Flow Modeling}

\subsubsection{Mathematical Model Description}

BRAGFLO_T is the code (computational model that implements the numerical solution of the mathematical model on a computer) used to compute the flow of fluid and energy, fluid saturations, and pressure fields throughout the generic salt and granite repository for the Complex PA. BRAGFLO_T is an enhanced version of BRAGFLO (WIPP PA Division, 1991b), which has been used to evaluate the isothermal flow of brine and waste-generated gas throughout the Waste Isolation Pilot Plant (WIPP) repository in performance assessment calculations. The following discussion of the code emphasizes the mathematical model used rather than the various numerical approximations and implementation schemes available in the code.

Conservation of Mass and Energy. To account for the effects of heat generated by spent fuel and high-level waste and subsequent heat transfer on the flow of brine and gas, BRAGFLO_T solves simultaneously the three partial differential equations (PDEs) assuring mass conservation of gas, mass conservation of brine, and energy conservation. In addition to the mass and energy balance equations BRAGFLO_ $T$ imposes appropriate constraint equations, initial conditions, and boundary conditions. The fundamental equations can be found in Crookston et al. (1979). A total of five independent equations can be written to describe the two-phase flow phenomena that is assumed to occur in the vicinity of the repository.

Conservation of gas:

$$
\nabla \cdot\left[\frac{\alpha \rho_{g} k k_{r g}}{\mu_{g}}\left(\nabla P_{g}-\rho_{g} g \nabla D\right)\right]+\alpha q_{g}+\alpha q_{r g}=\alpha \frac{\partial\left(\phi \rho_{g} S_{g}\right)}{\partial t}
$$

Conservation of brine:

$$
\nabla \cdot\left[\frac{\alpha \rho_{B} k k_{r B}}{\mu_{B}}\left(\nabla P_{B}-\rho_{B} g \nabla D\right)\right]+\alpha q_{B}+\alpha q_{r B}=\alpha \frac{\partial\left(\phi \rho_{B} S_{B}\right)}{\partial t}
$$

Conservation of energy:

$$
\begin{aligned}
\nabla \cdot(\alpha \lambda \nabla T) & +\nabla \cdot\left[\frac{\alpha k_{r g} k \rho_{g} h_{g}}{\mu_{g}}\left(\nabla P_{g}-\rho_{g} g \nabla D\right)+\frac{\alpha k_{r B} k \rho_{B} h_{B}}{\mu_{B}}\left(\nabla P_{B}-\rho_{B} g \nabla D\right)\right] \\
& +\alpha\left[q_{g} h_{g}^{*}+q_{B} h_{B}^{*}\right]+\alpha Q_{S} \\
& =\alpha \frac{\partial}{\partial t}\left[(1-\phi) \rho_{f} U_{f}+\phi\left(s_{g} \rho_{g} U_{g}+S_{B} \rho_{B} U_{B}\right)\right]
\end{aligned}
$$


Saturation constraint:

$$
S_{B}+S_{g}=1
$$

Capillary pressure constraint:

$$
P_{g}-P_{B}=P_{C}
$$

where

$k=$ absolute permeability of the formation (assumed isotropic, but a function of the material) $\left[\mathrm{m}^{2}\right]$,

$k_{r g}, k_{r B}=$ relative permeability to gas and brine, respectively,

$\rho_{g}, \rho_{B}, \rho_{f}=$ density of gas, brine, and formation respectively $\left[\mathrm{kg} / \mathrm{m}^{3}\right]$,

$\mu_{g}, \mu_{B} \quad=$ viscosity of gas and brine, respectively [Pa-s],

$\mathrm{h}_{g}, \mathrm{~h}_{B} \quad=$ enthalpy of gas and brine, respectively [W-s/kg],

$U_{g}, U_{B}, U_{f}=$ internal energy of gas, brine, and formation respectively [W-s/kg],

$\mathrm{h}_{g}{ }^{*}, \mathrm{~h}_{B}{ }^{*} \quad=$ enthalpy of injected or produced gas and brine [W-s/kg],

$q_{g}, q_{B} \quad=$ rate of mass injected or produced for gas and brine $\left[\mathrm{kg} / \mathrm{s} / \mathrm{m}^{3}\right]$,

$q_{r g}, q_{r B} \quad=$ rate of mass produced from chemical reaction for gas and brine (evaluated from gas generation submodel) $\left[\mathrm{kg} / \mathrm{s} / \mathrm{m}^{3}\right]$,

Q $\quad=$ rate of heat generation (e.g., radiation decay) $\left[\mathrm{W} / \mathrm{m}^{3}\right]$,

$\lambda \quad=$ formation thermal conductivity $[\mathrm{W} / \mathrm{m} / \mathrm{k}]$,

$\phi \quad=$ formation porosity,

g $\quad=$ gravitational constant $\left[\mathrm{m} / \mathrm{s}^{2}\right]$,

D $\quad=$ elevation $[\mathrm{m}]$,

$\mathbf{P}_{g}, \mathbf{P}_{B} \quad=$ pressure of gas and brine $[\mathrm{Pa}]$ (unknown),

$S_{g}, S_{B} \quad=$ saturation of gas and brine (unknown),

$t \quad=$ time [s],

$\mathrm{T} \quad=$ temperature $[\mathrm{K}]$ (unknown),

$\alpha \quad=$ dimension dependent geometry factor (equal to width for two-dimensional formulation; spatial variation discussed under Section 12.1.3, "Applied Models").

Equation 12.1-1 states the rate at which gas is accumulated in a control volume is equal to the net rate at which gas is transported across the boundary of the control volume by convection plus the rate at which gas is added due to injection/production and the rate at which gas is added due to chemical reaction. A similar brine balance is done in Equation 12.1-2. It is assumed that there is no interchange between the gas component and the brine; that is to say, the gas does not go into solution with the brine. Similarly, brine is found only in the liquid phase (single wetting phase). This results from the assumption that the brine does not vaporize. Salt concentration was assumed to be constant and independent of temperature. (Brine migration by dissolution and precipitation within brine inclusions cannot occur under this assumption.)

Equation 12.1-3 states that the rate at which energy is accumulated within a control volume is equal to the rate at which energy is transferred through the formation by conduction and convection of the fluids, plus the rate at which energy is added to the formation due to production and radioactive heating. In formulating Equation 12.1-3, we have assumed that kinetic energy effects, viscous dissipation, and radiative heat transfer are negligible compared to conduction and convection. We further assume that heat from chemical reaction is small compared to heat generation due to the radioactive inventory.

The right-hand side of Equations 12.1-1 through 12.1-3 are expanded and a compressibility parameter is defined. For example, expanding the right-' and side of Equation 12.1-1 gives

$$
\alpha \frac{\partial}{\partial t}\left(\phi \rho_{g} S_{g}\right)=\alpha\left[\rho_{g} S_{g} \phi \beta_{s} \frac{\partial P_{B}}{\partial t}+\phi S_{g} \frac{\partial \rho_{g}}{\partial t}+\phi \rho_{g} \frac{\partial S_{g}}{\partial t}\right]
$$


where

$$
\beta_{s}=\frac{1}{\phi} \frac{\partial \phi}{\partial P_{B}}
$$

The formation compressibility of the pressurized brine reservoir was computed from the bulk storativity as follows (WIPP PA Division, 1991c, p. 4-57):

$$
\beta_{s}=\left(\frac{s_{b}}{v}-\phi \beta_{f}\right) / \phi
$$

where

$S_{b} \quad=$ bulk storativity of pressurized brine reservoir $\left(\mathrm{m}^{3} / \mathrm{Pa}\right)$

$v \quad=$ volume $\left(\mathrm{m}^{3}\right)$

$\phi \quad=$ porosity

$\beta_{\mathrm{f}}=$ brine compressibility.

These five equations are used to solve for the five unknowns: gas saturation, brine saturation, gas pressure, brine pressure, and temperature. The saturation constraint and capillary pressure constraint are used to eliminate the brine saturation and gas pressure from the three conservation equations. The gas saturation, brine pressure, and temperature are then solved from the three mass/energy balance conservation equations.

The three PDEs are strongly coupled and highly non-linear through the pressure, saturation, and temperature dependencies of the rock and fluid properties. These dependencies (constitutive equations) are described in the following subsection, followed by a discussion of the treatment of heat conduction from the repository site to the surrounding formation (boundary condition).

Constitutive Equations. The constitutive equations relate the model parameters of the mass/energy equations to the state variables or other model parameters. Unless described below, the model parameters were assigned constant values based on observed material response or analyst judgement; the parameters are described in Chapters 4,5 , and 6. For example, rock density and thermal conductivity were assigned a constant value dependent on material type (see Chapter 5, "Geologic Barrier").

Thermal Equation of State for Gas. The thermal (or kinetic) equation of state defines the natural relationship of pressure and density or specific volume. In BRAGFLO_T, the gas PVT properties are described by the RedlichKwong real gas equation of state (Reid et al., 1987, p. 43, Eqs. 3-6.1 to 3-6.4):

$$
\mathbf{P V}=\mathbf{Z R T} \text {, }
$$

where the real gas Z-factor is the largest real value solution of the cubic equation,

$$
\begin{aligned}
& A=a P /(R T)^{2}, \\
& B=b P /(R T),
\end{aligned}
$$

$$
Z^{3}-Z^{2}+\left(A-B-B^{2}\right) Z-A B=0,
$$

and

$\mathbf{R} \quad=$ universal gas constant,

T = temperature,

$\mathbf{P} \quad$ = pressure,

$\mathrm{V} \quad=$ specific volume,

a,b = pure component values for the gas (either $\mathrm{H}_{2}$ or $\mathrm{CO}_{2}$ ). 
Caloric Equation of State for Gas. The caloric equation of state relates the internal energy (or enthalpy) to the state variables, $\mathrm{T}$ and $\mathrm{V}$. The gas thermodynamic properties require evaluation of gas internal energy and enthalpy. The change in internal energy or enthalpy between two states $\left(P_{1}, T_{1}\right)$ and $\left(P_{2}, T_{2}\right)$ is evaluated by the change between a $(\mathrm{P}, \mathrm{T})$ state and the ideal gas state $(0, \mathrm{~T})$, which is called the departure function for internal energy or enthalpy, and the ideal gas change between $\left(0, \mathrm{~T}_{1}\right)$ and $\left(0, \mathrm{~T}_{2}\right)$.

The departure function for internal energy is computed from the real gas equation of state, in this case RedlichKwong, by an integration with respect to specific volume while temperature is held constant (Reid et al., 1987, p. 99, Example 5.1):

$$
U-U^{o}=-(3 a / 2 b \sqrt{T}) \ln ((V+b) / V),
$$

while departure enthalpy is related to the departure internal energy by

$$
H-H^{o}=U-U^{o}+R T(Z-1)
$$

where

$$
\begin{array}{ll}
\mathbf{H} & \text { = enthalpy, } \\
\mathrm{U} & \text { = internal energy, } \\
\mathbf{R} & \text { = universal gas constant, } \\
\mathbf{T} & \text { = temperature, } \\
\mathbf{Z} & \text { = real gas Z-factor, } \\
\mathbf{V} & \text { = specific volume, } \\
\text { a,b } & \text { = pure gas component constant. }
\end{array}
$$

The change in energy in the ideal gas state $(P=0)$ is evaluated by integration of the ideal gas specific heat

$$
C_{p}=A+B T+C T^{2}+D T^{3}
$$

where $A, B, C, D$ are gas component constants (Reid et al., 1987, Appendix A). The internal energy or enthalpy of gas at any $(P, T)$ is the change in property between $\left(P_{\text {ref }} T_{\text {ref }}\right)$ and $(P, T)$.

Gas Viscosity. Gas viscosity is modeled by the Chapman and Enskog theory, which results in the following estimate for viscosity at low pressures (Reid et al., 1987, p. 392, Eq. 9-3.9)

$$
\mu^{0}=26.69(\mathrm{MT})^{1 / 2} / \sigma^{2} \Omega_{v}
$$

where

$$
\begin{array}{ll}
\mu^{0} & =\text { low pressure gas viscosity } \\
\mathbf{M} & =\text { molecular weight } \\
\mathbf{T} & =\text { temperature, } \\
\sigma & =\text { hard sphere diameter of pure gas molecule, } \\
\Omega_{v} & =\text { collision integral. }
\end{array}
$$

The following empirical equation is given for the collision integral (Reid et al., 1987, .. 393, Eq. 9-4.3)

$$
\Omega_{v}=A T^{*-B}+C e^{-D T^{*}}+E e^{-F T}
$$

and

$$
\mathrm{T}^{*}=\frac{\mathbf{k T}}{\varepsilon},
$$


where

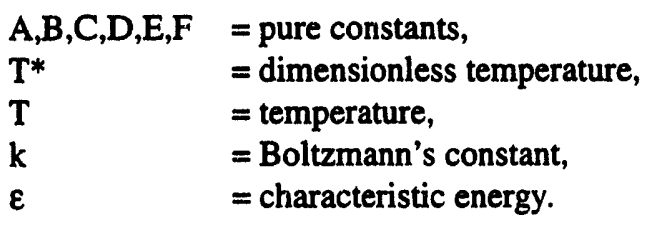

The viscosity correction for high pressure (dense gas) uses the method of Jossi, Stiel, and Thodos for nonpolar gases (Reid et al., 1987, p. 424, Eq. 9-6.11):

$$
\begin{aligned}
{\left[\left(\mu-\mu^{0}\right) \xi_{T}+1\right]^{1 / 4} } & =1.0230+0.23364 \rho_{r}+0.58533 \rho_{r}^{2} \\
& -0.40758 \rho_{r}^{3}+0.093324 \rho_{r}^{4}
\end{aligned}
$$

where

$$
\begin{array}{ll}
\mu & =\text { dense gas viscosity, } \\
\mu^{0} & =\text { low pressure gas viscosity }, \\
\xi_{T} & =\left(\mathrm{T}_{c}\left(\mathrm{M}^{3} \mathrm{P}_{c}\right)^{1 / 6},\right. \\
\mathrm{T}_{c} & =\text { critical temperature, } \\
\mathrm{P}_{c} & =\text { critical pressure, } \\
\mathrm{M} & =\text { molecular weight } \\
\rho_{r} & =\text { reduced gas density, } \rho / \rho_{c} .
\end{array}
$$

Thermal Equation of State for Liquid. Brine PVT properties are estimated from the linear approximation for density,

$$
\rho_{b}=\rho_{s c}\left(1+C_{w p}\left(P-P_{r e f}\right)+C_{w T}\left(T-T_{r e f}\right)\right) .
$$

The compressibility coefficient is approximated by the data from (McTigue et al., 1991, Table 3, p. 7),

$$
C_{w p}=(4.5-0.077 * \text { SALT }) * 10^{-10}\left[\mathrm{~Pa}^{-1}\right],
$$

where SALT $=\%$ salt by weight. The constant thermal expansion coefficient, $C_{w T}$, is taken from data in Brandshaug (1986, p. 5, Table 2-1).

$$
C_{w T}=0.000045[1 / \mathrm{K}] .
$$

Brine viscosity is computed from an industry standard software package, Hewlett-Packard, "Petroleum Fluids PAC"' (p. 102).

Caloric Equation of State for Liquid. Brine enthalpy is computed from specific heat values for water and salt. Specific heat for salt is found in Perry and Chilton (1973, Trible 3-174, p. 3-124) and was assigned the constant value $874 \mathrm{~J} / \mathrm{kg} / \mathrm{K}$. For water, a temperature-dependent specific heat was assigned (Crookston et al., 1979, p. 37-58):

$$
C_{p w}=9716.0-0.32748 * T+0.047276 * T^{2}[\mathrm{~J} / \mathrm{kg} / \mathrm{K}] .
$$

From the enthalpy of the salt and water, the brine enthalpy is computed as the average by weight fraction of the two enthalpies. Brine internal energy is set equal to the enthalpy.

Formation or material heat capacity is given by

$$
C_{p r}=C_{1}+C_{2} T+C_{3} T^{2}
$$


where $C_{1}, C_{2}$, and $C_{3}$ are material dependent coefficients.

Capillary Pressure. At the boundary between two phases, an imbalance in forces creates a component of force that acts tangential to the interface. This force component is commonly referred to as the interfacial tension. Interfacial tension acting on a curved surface between two phases is balanced, at equilibrium, by pressure differences in the two phases. The difference in pressure, called the capillary pressure, is defined as the capillary constraint

$$
P_{C}=P_{g}-P_{B}
$$

where $P_{B}$ and $P_{g}$ are the brine (wetting phase) and gas (non-wetting phase) pressures, respectively, and $P_{6}$ is the capillary pressure. Typically, capillary pressure is unbounded as the wetting phase saturation, $\mathrm{S}_{\mathrm{B}}$, approaches the residual (also referred to as the irreducible) saturation, $S_{\mathrm{Br}_{r}}$ from the right (Figure 12-1). Less than or equal to $\mathrm{S}_{\mathrm{Br}}$ the capillary pressure is undefined. The residual saturation is defined as the saturation at which the wetting phase no longer flows because of a pressure gradient."

The constitutive equation for capillary pressure is assumed as empirically derived by Brooks and Corey (1964): ${ }^{\dagger}$

$$
P_{c}=P_{t} S_{e}^{-1 / \eta}
$$

where $P_{t}$ is the threshold pressure, $P_{c}$ is the capillary pressure, $\eta$ is the pore size distribution exponent, and $S_{e}$ is the effective saturation defined for the brine (wetting phase) as

$$
S_{e B}=\left(S_{B}-S_{B r}\right) /\left(1-S_{B r}\right)
$$

where $\mathrm{S}_{\mathrm{B}}$ and $\mathrm{S}_{\mathrm{Br}}$ are the wetting phase and residual wetting phase saturations, respectively. Because of the lack of experimental data on the types of rocks used in this study, $\eta$ was assumed to be 0.7 for all rock types (see parameter tables in Chapter 5). The threshold pressure was correlated to the permeability of the rock (Davies, 1991) using the equation (Figure 12-2):

$$
P_{t}=5.6 \times 10^{-7} * k^{-0.346},
$$

where $P_{t}$ is the threshold pressure in MPa and $k$ is the permeability in $\mathrm{m}^{2}$.

Relative Permeabilities. Brooks and Corey (1964) also derived expressions for the wetting and non-wetting phase relative permeabilities, $\mathrm{k}_{\mathrm{rB}}$ and $\mathrm{k}_{\mathrm{rg}}$, using Equation 12.1-20 and the generalized form of the Kozeny-Carmen equations (see, for example, Corey, 1990). They are

$$
\begin{gathered}
k_{r B}=S_{e}^{\xi} \\
k_{r g}=\left(1-S_{e}\right)^{2}\left(1-S_{e}^{\gamma}\right)
\end{gathered}
$$

in which

$$
\xi=\frac{2+3 \eta}{\eta}
$$

\footnotetext{
"This does not mean, as the term "irreducible" would imply, that the wetting phase saturation cannot be reduced below $S_{\text {wr }}$ As discussed by Melrose (1991), the wetting phase is held in place at low saturations by a combination of adsorption to the solid surface and capillarity in the smallest pore spaces. Through desorption and vapor exchange, then, $S_{w}$ can be reduced to zero.

$\uparrow$ Another constitutive relationship proposed by Van Genuchten (1980), describing similar behavior, is available in BRAGFLO_T. Each relationship causes some numerical problems. We had more experience working with the numerical difficulties of the Brooks-Corey relationship, and so we used it instead of the Van Genuchten model.
} 

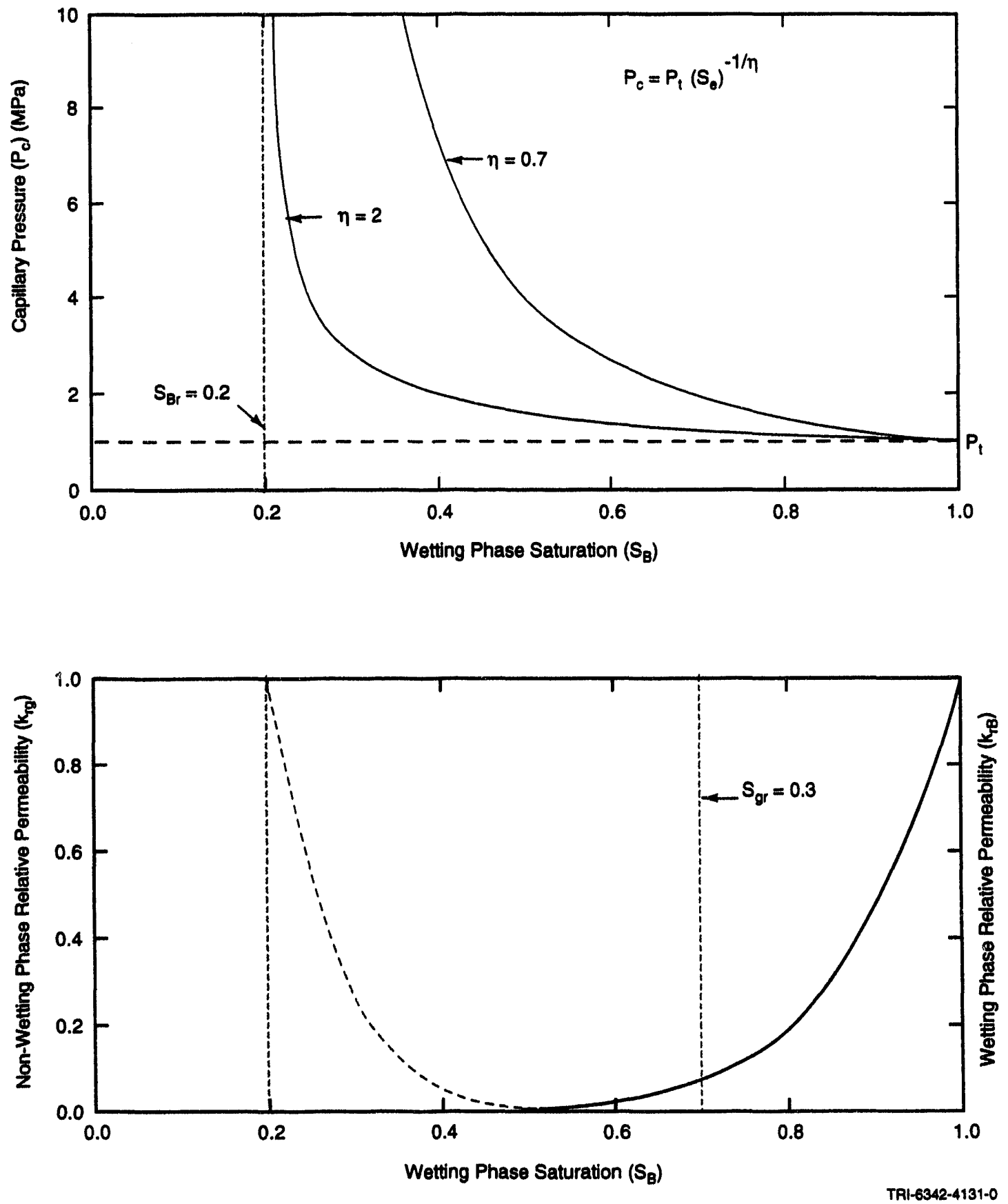

Figure 12-1. Sample capillary pressure and relative permeability diagrams. 


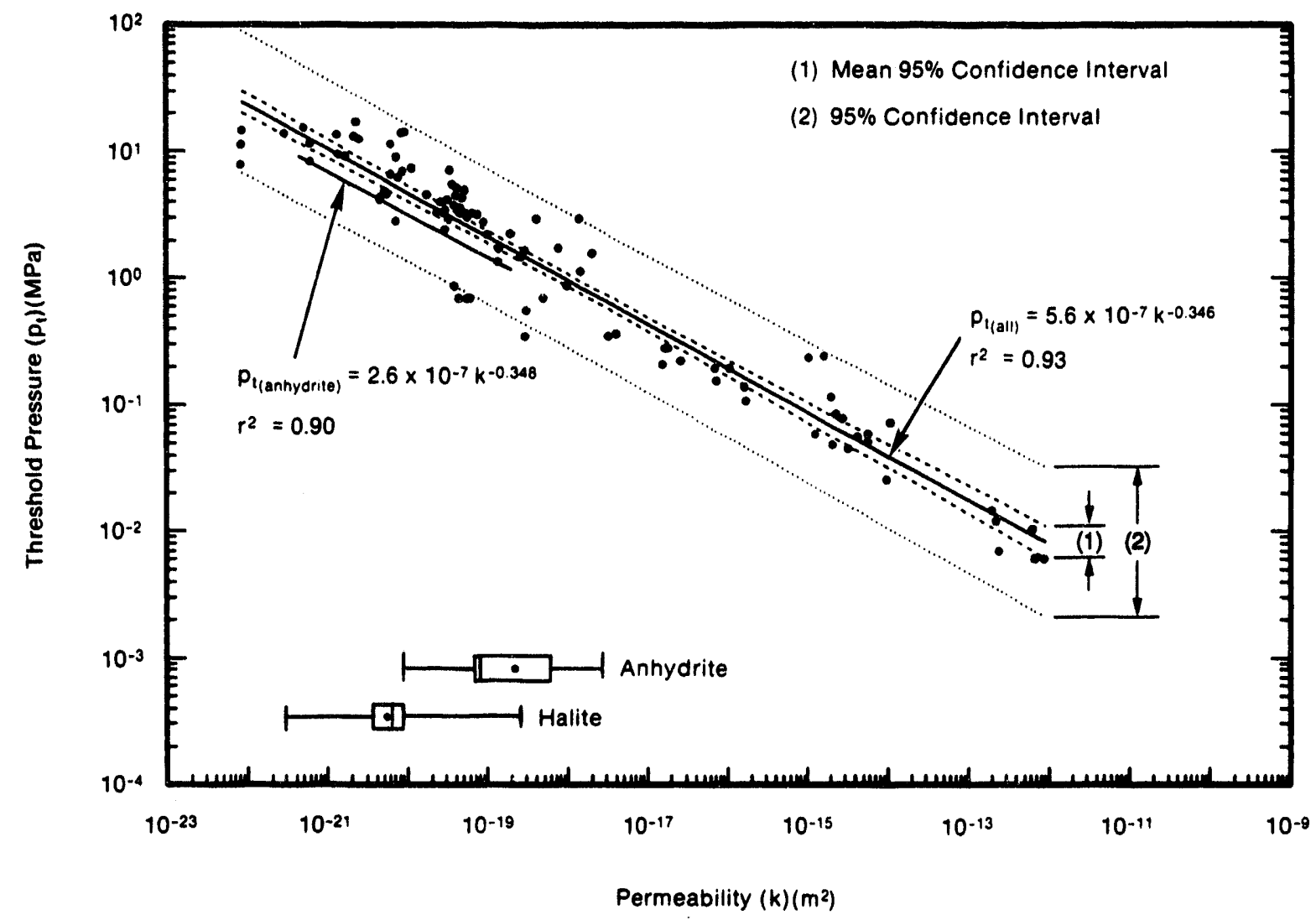

TA1-6344-730-1

Figure 12-2. Correlation of threshold pressure with permeability for a composite of data from all consolidated rock lithologies. Data from Ibrahim et al., 1970; Rose and Bruce, 1949; Thomas et al., 1968; and Wylie and Rose, 1950 (after Davies, 1991, Figures 5 and 8).

$$
\gamma=\frac{2+\eta}{\eta}
$$

To account for the entrapment and resulting immobility of the non-wetting phase as the non-wetting phase saturation approaches its residual saturation, $\mathrm{S}_{\mathrm{gr}}$ the original non-wetting phase relative permeability equation, Equation 12.1-24, was modified as follows:

$$
\begin{gathered}
S_{B} \leq S_{B r}, k_{r g}=1 \\
S_{B r}<S_{B}<1-S_{g r}, \quad k_{r g}=\left(1-S_{e}^{*}\right)^{2}\left(1-S_{e}^{* \xi}\right) \\
S_{B} \geq 1-S_{g r}, k_{r g}=0
\end{gathered}
$$

where $\mathrm{S}_{\mathrm{e}}^{*}$ is defined as

$$
S_{e}^{*}=\left(S_{B}-S_{B r}\right) /\left(1-S_{B r}-S_{g r}\right)
$$


Boundary Heat Loss. BRAGFLO_T normally models fluid flow with a no-flow boundary condition. (As described in Chapter 13, however, fluid flow across a boundary can be simulated with wells.) However, in the presence of a thermal source, heat at the flow boundary must be accounted for in the energy balance equation. Although this problem could be solved by extending the finite difference grid system to include the surrounding rock, a larger grid system will impose serious computer storage and CPU time demands.

The method adopted requires gridding only to the fluid flow boundary. We consider a one-dimensional analytical treatment of the heat loss from each boundary grid block to the surrounding rock. We conceptualize a semi-infinite rod on the positive $x$-axis with left boundary at $x=0$ and with uniform cross-section area $A$. This rod represents the surrounding rock with interface at the point $x=0$ with the repository site. The problem is to compute the heat flux across the left boundary of the semi-infinite rod when the temperature history is known at the left boundary. This temperature history at the left boundary represents the temperature history of a boundary grid block in BRAGFLO_T. The differential equation governing temperature within the surrounding rock is

where

$$
\frac{\partial T}{\partial t}=k \frac{\partial^{2} T}{\partial x^{2}}
$$

$$
\begin{aligned}
& \mathrm{k}=\text { thermal diffusivity }\left[\mathrm{m}^{2} / \mathrm{s}\right] \\
& \mathrm{T}=\text { temperature }[\mathrm{K}] \\
& \mathrm{t}=\text { time }[\mathrm{s}] \\
& \mathrm{x}=\operatorname{distance}[\mathrm{m}]
\end{aligned}
$$

The boundary condition is

$$
T(0, t)=F(t), t \geq 0 \text { (given temperature history), }
$$

and initial condition is

$T(x, 0)=T_{1}, x \geq 0$ (given initial temperature).

The diffusivity is expressed in terms of the formation properties as

$$
k=K / \rho c
$$

where

$\mathrm{K}=$ thermal conductivity $[\mathrm{J} / \mathrm{s} / \mathrm{m} / \mathrm{K}]$

$\rho=$ density $\left[\mathrm{kg} / \mathrm{m}^{3}\right]$

c $\quad=$ specific heat $[\mathrm{J} / \mathrm{kg} / \mathrm{K}]$

Let

$\mathrm{t}_{\mathrm{i}} \quad=$ ith time in simulation, $\mathrm{i}=1, \ldots, \mathrm{n}+1$

$T_{i} \quad=$ temperature at ith time

$\Delta T_{i}=T_{i+i}-T_{i}$ (temperature increment over ith time step).

Note $T_{1}$ is temperature at initial time $t_{1}$.

The solution of the differential equation with boundary and initial conditions is given as a generalization of a result in (Carslaw and Jaeger, 1959, p. 63, Eq. 2.5-3)

$$
T-T_{1}=\sum_{i=1}^{n} \Delta T_{i} e r f c\left[\frac{\mathrm{x}}{2 \sqrt{k\left(t-t_{i}\right)}}\right]
$$


where erfc is the complementary error function. The temperature gradient at the boundary $x=0$ is computed to be

$$
\frac{\partial T}{\partial \mathrm{x}}(0, t)=-\frac{1}{\sqrt{\pi k}} \sum_{i=1}^{n} \Delta T_{i} / \sqrt{t-t_{i}}, t_{n} \leq t \leq t_{n+1} .
$$

The cumulative heat flux over the time step is obtained from

$$
Q_{\text {boundary }}=-\int_{t_{n}}^{t_{n}+1} K A \frac{\partial T}{\partial x}(0, t) d t
$$

and is found to be

$$
Q_{\text {boundary }}=E(n)-E(n-1)
$$

where

$$
E(n)=\frac{2 K A}{\sqrt{\pi k}} \sum_{i=1}^{n} \Delta T_{i} \sqrt{t_{n+1}-t_{i}}
$$

In BRAGFLO_T the boundary heat loss term is treated implicitly in temperature. It was found that an explicit treatment was numerically unstable at large time steps.

\subsubsection{Computational Model}

Numerical Solution of PDEs. In BRAGFLO_T, three mathematical model equations are discretized using standard finite difference techniques. The finite difference formulation uses a fully implicit treatment of the primary variables. Integration across a time step of the resulting nonlinear algebraic equations is done using Newton-Raphson iteration with adaptive time-step control. The large system of algebraic equations, which is the result of discretization, is solved using a Banded LU decomposition algorithm. These numerical methods are chosen because of their quadratic convergence and robustness as well as their proven success in solving similar systems in the WIPP performance assessment (WIPP PA Division, 1991b; WIPP PA Department, 1992b).

Algorithm for Capillary Pressure Constitutive Equation. The computational implementation of the constitutive equations is straightforward except where it was necessary to implement the capillary constitutive equation to avoid unrealistically low brine (wetting phase) pressure gradients at low brine saturations.

In the backfill-buffer regions of the waste package, pressure of the non-wetting phase, gas, was set equal to 1 atm and the initial brine saturation was assumed equal to $S_{\mathrm{Br}}$. $S_{\mathrm{B}}$ was set equal to $\mathrm{S}_{\mathrm{Br}}$ under the assumption the backfillbuffer materials are kept as dry as possible during the operation phase to retard corrosion of the waste parcels. From the definition of capillary pressure, the initial wetting phase pressure is computed as

$$
\mathbf{P}_{\mathrm{B}, \mathrm{i}}=\mathrm{P}_{\mathrm{g}}-\mathbf{P}_{\mathrm{c}}
$$

Because of a low initial wetting phase saturation, $P_{c}$ can be much greater than the assumed $P_{g}$ of 1 atm in the PA calculation. Consequently, the initial wetting phase pressure $\left(\mathrm{P}_{\mathrm{B}, \mathrm{i}}\right)$ can be negative in the backfill-buffer regions.

Non-positive wetting phase pressures can also occur when the water consumption rate due to gas generation exceeds the surrounding water inflow rate. In time, $S_{B}$ can approach $S_{B r}$ when the capillary pressure increases dramatically. Increases in capillary pressure produced pressure changes in both phases. However, in a gas-liquid two phase system, pressure changes in the liquid phase are generally much larger than pressure changes in the gas phase. 
The large pressure change in the liquid is due to factors such as the compressibility of gas being much greater than the compressibility of liquid. Also, the viscosity of gas is much lower than the viscosity of liquid, and the relative permeability to the gas phase is much higher than the relative permeability to the liquid phase, at low liquid saturations. Therefore, the non-wetting phase is more mobile and compressible than the wetting phase. This mobility means the $P_{g}$ will tend to equilibrate much faster than $P_{B}$. Hence, an increase in capillary pressure results in a reduction of the wetting phase pressure of approximately the same magnitude, causing the wetting phase pressure to become negative.

Approach used in BRAGFLO_T to Avoid Negative Liquid Pressures. Melrose (1988) discussed the phenomena of negative wetting phase pressures and suggested that, for water, the pressure may be as low as $-210 \mathrm{MPa}$. Negative wetting phase pressures occur only when the capillary pressure is high. Consequently, a wetting phase with a negative pressure will generally have a saturation at or near $\mathrm{S}_{\mathrm{Br}}$, and in this report is assumed to be hydraulically discontinuous.

In BRAGFLO_T, we are concerned with the pressure distribution of interconnected mobile fluids. BRAGFLO_T currently does not model adsorption/desorption or vapor exchange of the wetting phase. So we are not concerned with the pressure of these immobile fluid pockets. Rather than impose what we thought were unrealistically large pressure gradients toward regions of disconnected, negative wetting phase pressures, ${ }^{\ddagger}$ a minimum wetting phase pressure was established in BRAGFLO_T, specifically, that the wetting phase pressure be greater than an arbitrarily small value, $\varepsilon$ or

$$
P_{B} \geq \varepsilon
$$

The actual value of $\varepsilon$ is not critical, but for numerical reasons $\varepsilon$ should be greater than the vapor pressure of the liquid at the prevailing temperature.

The specific procedure implemented in BRAGFLO_T is as follows. During a Newton Raphson time step iteration, if the inequality of Equation 12.1-38 is violated, the current capillary pressure, $P_{c}$, is set equal to the previous capillary pressure, $P_{c(\text { old })}$, less the amount that $\varepsilon$ exceds $P_{B}$, or,

$$
P_{c}=P_{c(\text { old })}-\left(\varepsilon-P_{B}\right)
$$

Once Equation 12.1-38 is violated, the capillary pressure continues to be evaluated using Equation 12.1-39 regardless of the status of Equation 12.1-38 until $P_{c}$ from Equation 12.1-39 is greater than $P_{c(\text { model }) \text {, }}$

$$
\mathbf{P}_{\mathrm{c}} \geq \mathrm{P}_{\mathrm{c}(\text { model })}
$$

where, $P_{c(\text { model })}$ is the capillary pressure computed with the selected capillary pressure model. Once 12.1 .40 is satisfied, the capillary pressure is set equal to $P_{c \text { (model) }}$, and the capillary pressure model is used to compute $P_{c}$ in subsequent iterations as long as the inequality in 12.1-38 is satisfied.

The primary solution variables solved for in BRAGFLO_T are the wetting phase pressure and the non-wetting phase saturation. The intent of the capillary pressure modifications was to conditionally specify the wetting phase pressure by adjusting $P_{c}$. If the $P_{c}$ computed from one of the available models forces the solution variable $P_{B}$ to fall below $\varepsilon, P_{c}$ is reduced so that $P_{B}$ approaches $\varepsilon$. Since $P_{B}$ remains a solution variable, it never equals $\varepsilon$ exactly but only converges toward $\varepsilon$. Also, since Equation 12.1-38 is used only in the computation of $P_{c}$ and not as a convergence criteria, Equation 12.1-38 is not necessarily satisfied in the final solution.

\footnotetext{
\$ We are not questioning whether the large negative pressure exists, but rather whether the negative pressures are "felt" throughout the large numerical mesh in our applied model through a connected liquid phase such that Darcy's law applies. The validity of large negative pressures and the applicability of Darcy's law will need to be explored in future work.
} 


\subsubsection{Applied Model}

Element Flaring Modeling Technique. The fluid- and heat-flow fields within and around the repository are three dimensional. Three-dimensional problems, however, are quite difficult technically and very expensive computationally. Because of the numerous computations required for the performance assessment, the repository was modeled in two rather than three dimensions.

The transformation from three dimensions to two dimensions was accomplished using a combination of element radial flaring and volumetrically averaged properties. Volume-averaged properties and their use in this study are discussed later in this section. Element flaring is the process of defining one or more subregions in a two-dimensional mesh where radially symmetric flow is assumed to occur, then evaluating the width ( $\alpha=$ width in Equations 12.1-1 through 12.1-3) of the element assuming radial coordinates. This process has been used quite successfully by the WIPP PA Department for over four years (Rechard et al., 1990, p. 37) to approximate flow as one moves away from the point of study of a system that is not fully axisymmetric. Specifically, a cylinder is defined in radial coordinates by the height, $\Delta z$, the internal radius, $r_{i}$, and the external radius, $r_{i}+\Delta r$, where $\Delta r$ is the width of the cylinder. If the element to model is not a complete cylinder, the angle $\theta$ represents the fraction of the cylinder, in radians. The distinction between element flaxing and fully axisymmetric modeling is that not all of the elements are automatically increased in thickness by radial distance times the full $2 \pi$ arc. Rather, several origins can be used and portions of the study domain with, for example, an approximately uniform flow can have constant widths. It is this latter part of the modeling technique (i.e., the use of several origins for flared elements mixed with constant width elements) that is difficult to convey when explaining the applied model, not the basic concept of translation to an equivalent cartesian coordinate system. The translation from radial to cartesian coordinates, shown in Figure 12-3, is made by setting

$$
\Delta \mathbf{x}=\Delta \mathbf{r}
$$

$$
\Delta y=\theta\left(r_{i}+\Delta r / 2\right)
$$

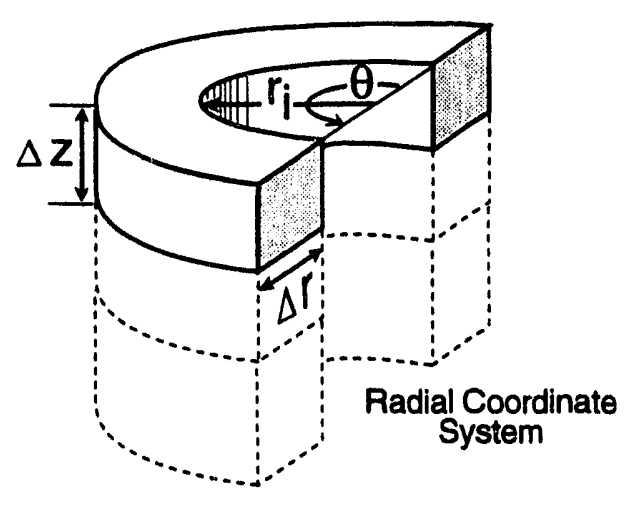

$$
\begin{aligned}
& \begin{array}{c}
\text { Translation to Cartesian } \\
\text { Coordinate System }
\end{array} \\
& \hline \begin{array}{c}
\Delta x=\Delta r \\
\Delta y=\theta\left(r_{i}+\frac{\Delta r}{2}\right)
\end{array}
\end{aligned}
$$

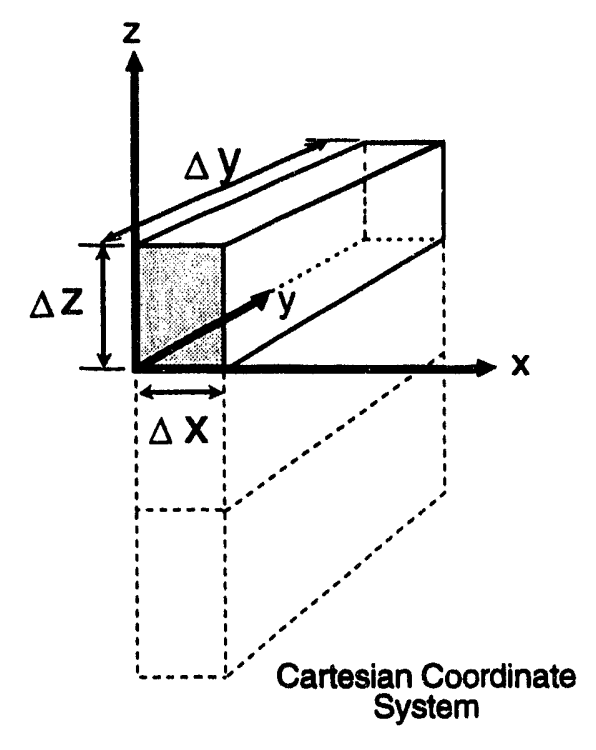

TRI-6342-3720-0

Figure 12-3. Element radial flaring. The distinction between element flaring and fully axisymmetric modeling is that not all of the elements are automatically increased in thickness by radial distance times the full $2 \pi$ arc. Rather, several axes can be used and portions of the study domain with, for example, an approximately uniform flow can have constant widths. 
Setting $\Delta x$ equal to $\Delta r$ maintains the distance parallel to the flow field; $\Delta y$ is simply the thickness in the direction normal to flow required to conserve the volume of the cylinder.

Model Geometry and Spatial Discretization. A three-dimensional conceptualization of the hypothetical salt repository is shown in Figure 12-4a and 12-4b. The coordinate system used in the simulation is included in this figure. The coordinate system was positioned such that the z-axis is oriented opposite gravity and parallel to the shaft, while the $x$-axis and $y$-axis form a plane parallel with the repository and geologic bedding planes. To introduce the flared elements used in this analysis, on artistic representation of an $x-y$ plane plan view of the repository is shown in Figure 12-4b.

Volume-averaged properties were used within the repository to combine heterogeneous materials into single homogeneous cells. Combined with element flaring and a few approximations of the flow field near the repository, spatial variations in the direction of the $y$-axis were removed. Hence, a cross-section in the $x-z$ plane with variable thickness in the y-direction completely describes the repository in two dimensions. (The Simple PA, discussed in Chapter 14, simplifies further to a one-dimensional mathematical model.)

Logical Mesh for Vertical Cross-Section. A logical mesh representative of the $\mathbf{x - z}$ plane is presented in Figure 12-5. (The $x-y$ plane is only one element wide.) Displaying the logical mesh emphasizes the mesh element discretization while still showing the features modeled. A logical mesh is not to scale; rather, each element or grid block is displayed with a unit dimension. The actual element dimensions are shown to the left, below, and above the drawing. The integers above and to the right of the drawing represent the $(i, j)$ element location.

The logical mesh shows the position of the major geologic units and the repository. Fluid and heat flow were modeled from the Snake Dolomite to the Tower Formation vertically, and $2.4 \mathrm{~km}$ away from the repository horizontally. Two intrusion boreholes penetrated the repository $1000 \mathrm{yr}$ after emplacement intc the side rooms of the center panel. One borehole was drilled into a brine reservoir within the Tower Formation, while the other stopped after completely penetrating the repository. Notice that the size of the elements increases dramatically near the boundaries of the mesh on both the left and right sides of Figure 12-5. The purpose of these elements was to reduce the influence of boundary conditions on flow around and within the repository.

Groupings of Rooms and Panels in Disposal Region. Within the repository, elements of constant thickness were used. These elements extended the width of the repository and were placed along the features within it. (Compare the $x-y$ cross-section [Figure 12-4b] with the detailed drawing of the repository [Figure 6-2].) For instance, the side legs (Figure 6-2) of each room within a panel were combined with the side legs of each room in the opposite panel along with a portion of the main passageway separating the panels to form a single composite grid block. In a similar fashion, composite grid blocks were created by combining the main legs, the pillars separating the rooms, and the passageway between opposite panels. For the most part, the constant thickness elements contained two or three different materials (backfill, host rock, canister) with independent fluid flow and thermodynamic properties. As a result, volume-averaged parameters were used to represent these elements.

Waste Parcel Placement. Regions containing waste emplacement packages were finely meshed to capture preferential corrosion of the waste parcels. Mesh lines were placed one meter below the repository floor and at the bottom of each canister. Since each waste disposal group contained canisters of different lengths, the mesh changed to accommodate each waste disposal group. However, to not disrupt the entire mesh, the sum of grid blocks $6,7,8,9$, and 10 in the $\mathrm{z}$ direction was always $10 \mathrm{~m}$.

Each panel contained a total of 2760 waste parcels: 1560 in the main leg and 600 in each side leg. Because the elements within the disposal region contained portions of two panels, 1200 waste packages were placed into each side panel element. The main panel group was split into two elements, so the number of the waste packages per element remained 1560. In the side legs containing the intrusion boreholes, the flared elements surrounding the borehole occupied a relatively small portion of the side leg (Figure 12-6). Rather than develop an elaborate scheme for placing waste parcels within these elements, the 1200 waste parcels were divided equally and placed into the two large elements on each end of the side leg (x-axis indices $21,29,32,40$ of Figure 12-5). 
12. Complex Consequence Modeling for Salt Disposal System

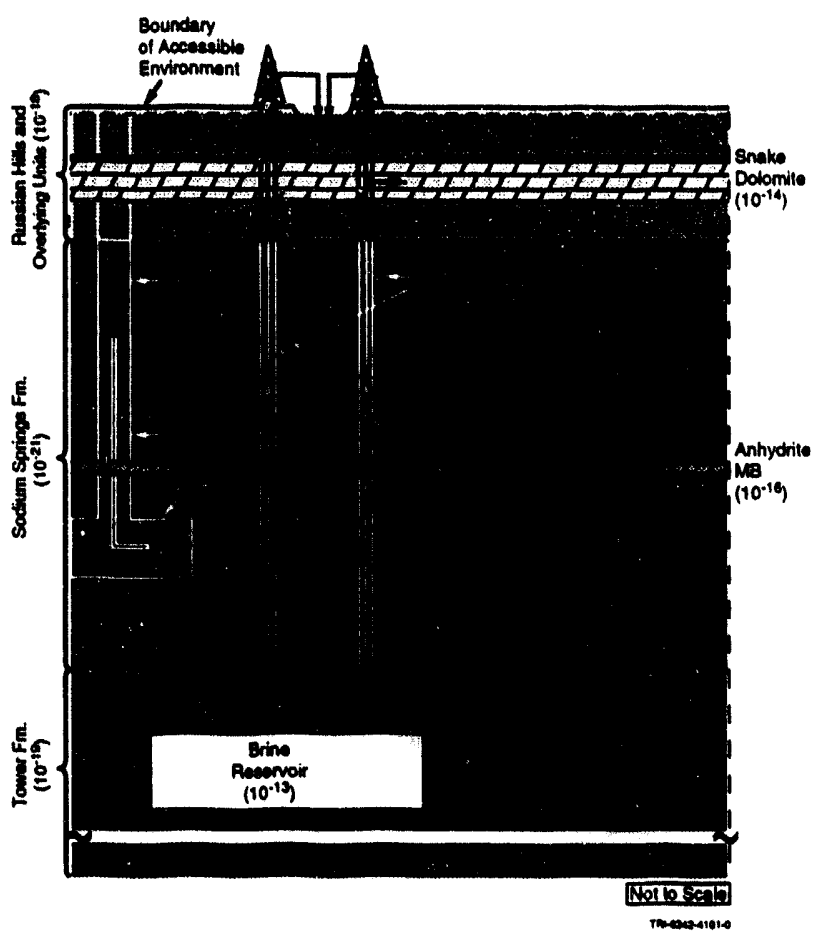

(a)

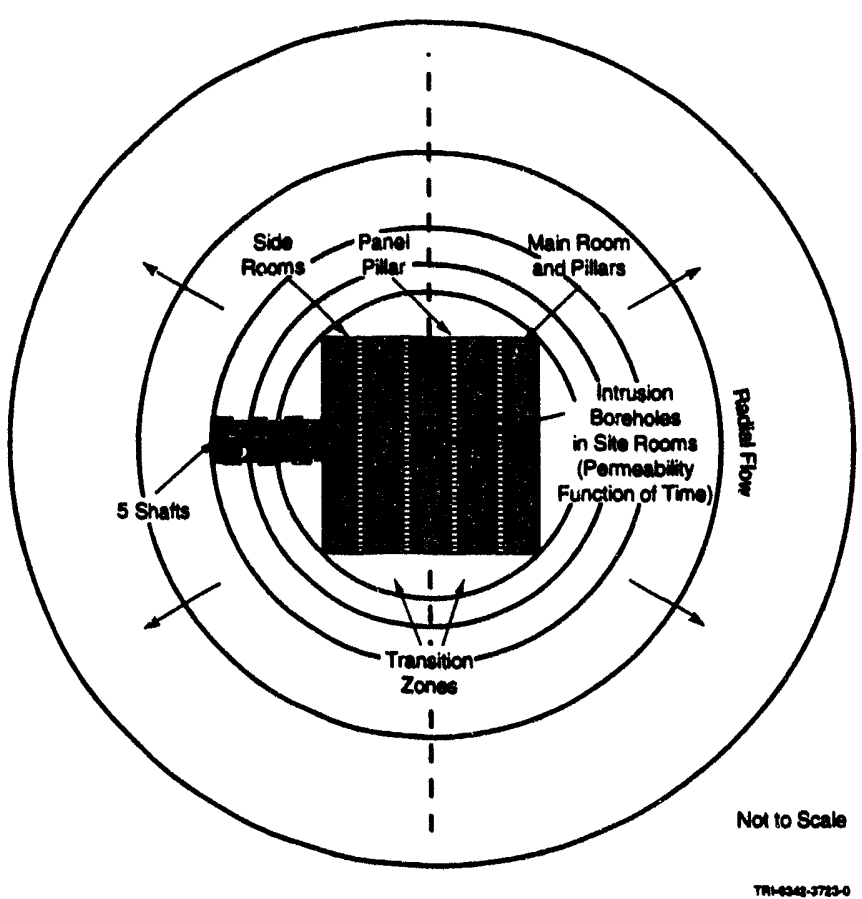

(b)

Figure 12-4. Conceptual model of salt repository and surrounding host rock for Complex PA. (a) $x-z$ vertical crosssection, and (b) $x-y$ plan view. 


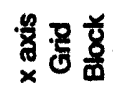

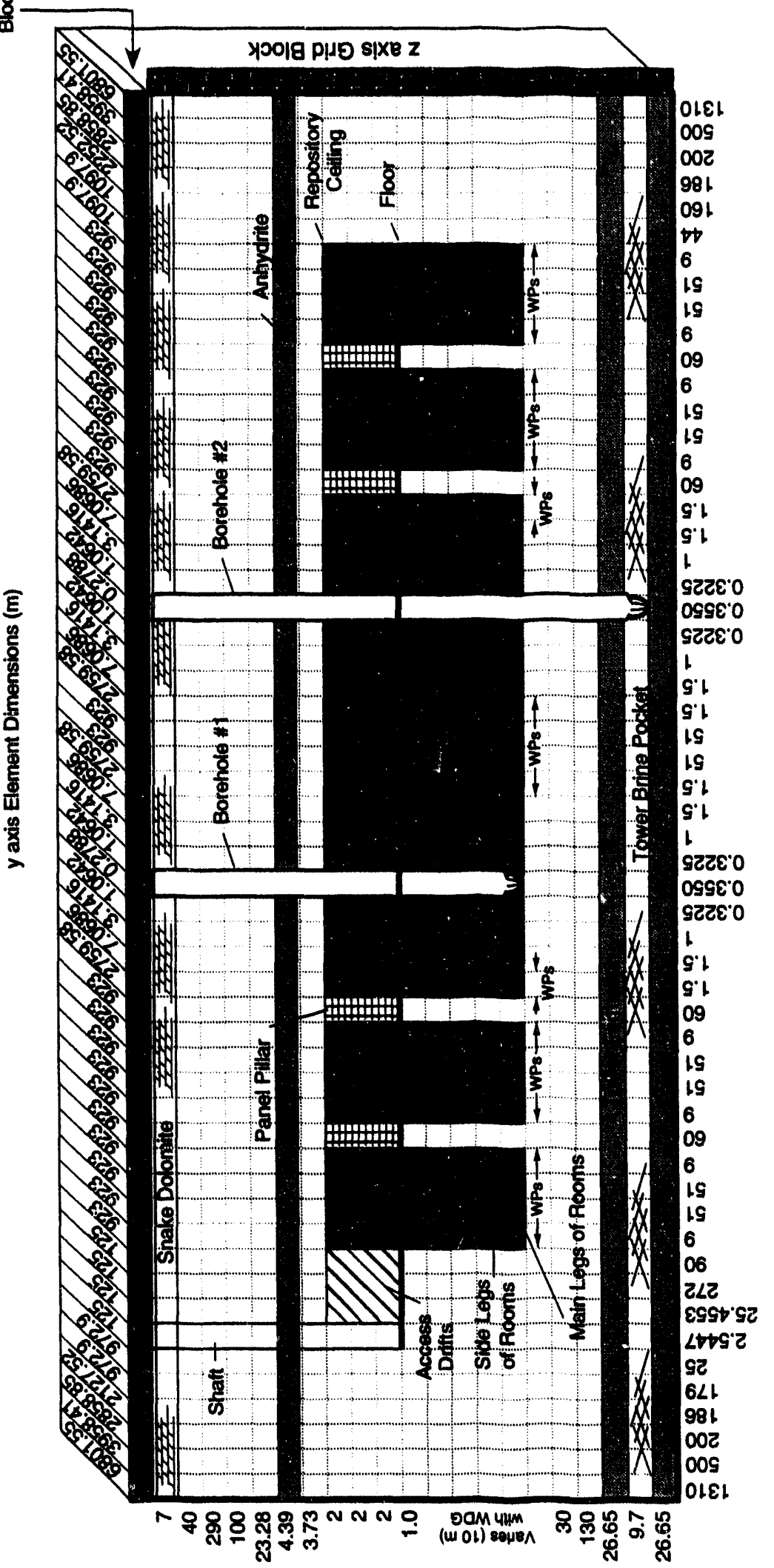

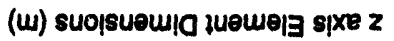

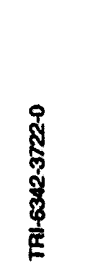

ะ

के

s

ปั

$8 \frac{8}{8}$

ริ

ธี่

28

รี

ษ

ล

6ิ

$y$

క

(2)

के

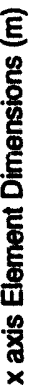

8

ชี้

.

$y$

3

క

ㅇํㅇ

ล

ช 0

जิ

ะี के

iे

ลे.

5

$\frac{8}{2}$

ป

ชิ

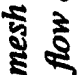

है

क्ष

ำ

5

ह

\&ิ

เ.

ปิ 


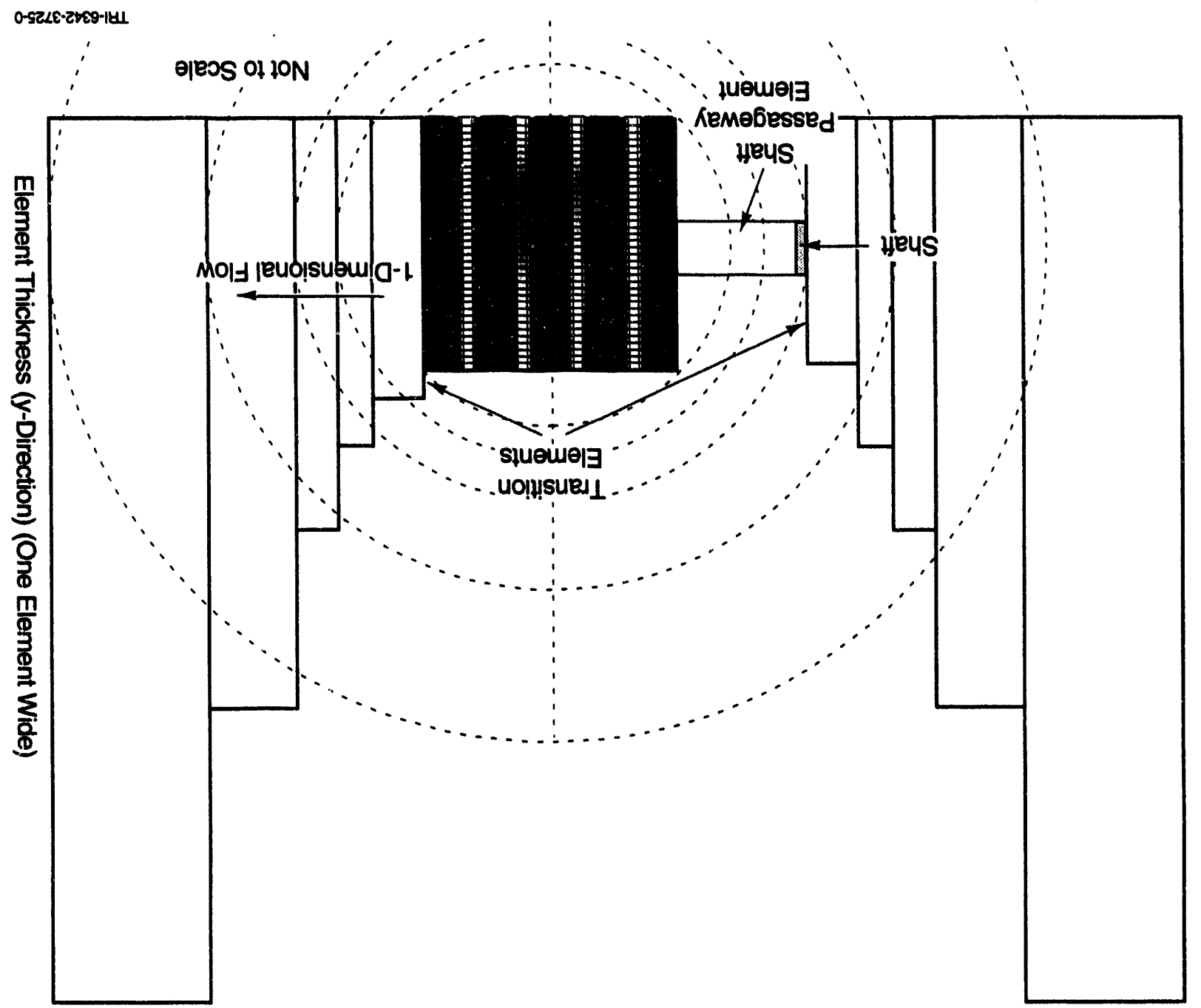

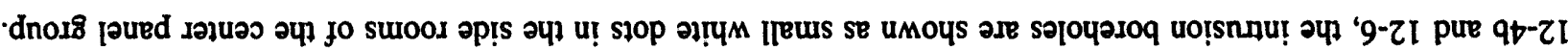

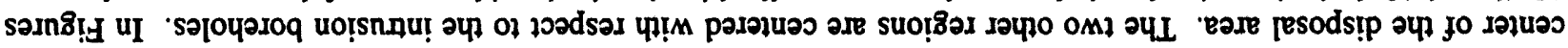

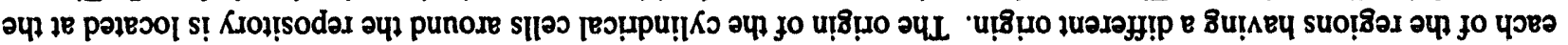

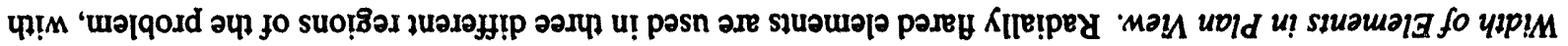

$$
6 \mathrm{IS} \sim \varepsilon t^{\circ} 6 \mathrm{IS}=\frac{8 S 8^{\circ} 0 Z}{S t 69} \times 09 S \mathrm{I}=
$$

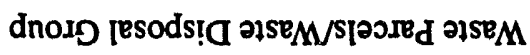

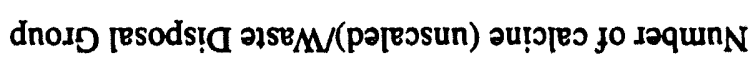

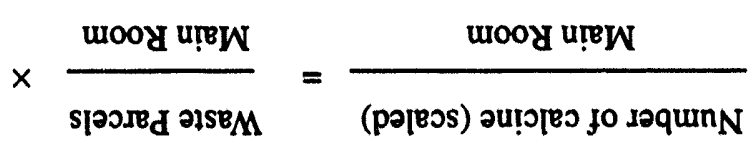

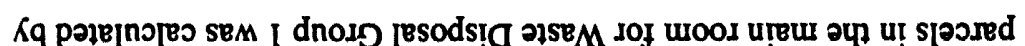
ә1seм әu!

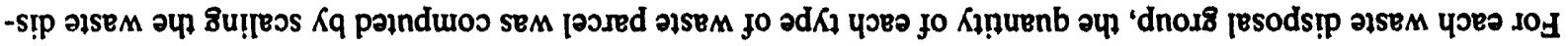


Flared elements were used around the boreholes to capture the geometry of the borehole as well as the locally radial fluid flow. A plan view of the region near the boreholes is shown in Figure 12-7.

In Figure 12-4b, four very large cylindrical grid blocks surround the repository and extend to the boundaries of the problem domain. The vertical line running through the center of the repository splits the domain into two parts: a left side and a right side. Consequently, the four large cylindrical grid blocks were split in eight radial grid blocks where $\theta$ was equal to $\pi$. The corresponding elements in the logical grid are located in the cells with $x$-axis indices of 1-4 and 53-56. The left side corresponds to the indices 1-4, and the right side corresponds to the indices 53-56. Working inward toward the repository, the next two indices (5-6 and 51-52) contain the transition zone. The transition zone is located between the cylindrical grid blocks around the repository and the rectangular grid blocks within the repository.

The three-dimensional conceptual model was converted into two dimensions by translating the radial elements into rectangular elements using Equations : 2.1-41 and 12.1.-42. The converted one-dimensional $x-y$ plane view is shown in Figure 12-6. The disposal region of the repository remained the same except for the radial elements around the intrusion boreholes. The borehole mesh is not shown here due to the size of the side rooms, but it can be inferred from Figure 12-7. The transitional blocks were placed at the end of the repository on the right side, and behind the shafts on the left side. Because fluid transport between the shaft and the disposal area was considered to be a potential source of brine to the repository as well as a release point for radionuclides, the shaft was modeled as a separate entity rather than using the volume averaging approach. Consequently, the distance of the transitional and radial blocks surrounding the repository to the center of the disposal region remained constant on the right side of the repository, but were shifted by the length of the access drift on the left side. Finally, the five shafts were combined into a single rectangular shaft. The thickness, or $\Delta y$, of the rectangular shaft was set equal to the thickness of the access drift, and the length, or $\Delta x$ was calculated to conserve the volume of the original five shafts.

Volume-Averaged Modeling Parameters. To evaluate the applied model in a reasonable amount of time, a coarse numerical mesh is required. In turn, this means that elements within the refository contain numerous heterogenous materials that must be averaged to obtain appropriate values of the required naterial property parameters for the element. This volume averaging (or areal averaging when the third dimension of all the materials in the element is equal) is straightforward for the most part; yet a few parameters, such as permeability and thermal conductivity, are different enough to warrant a discussion of the methodology adopted.

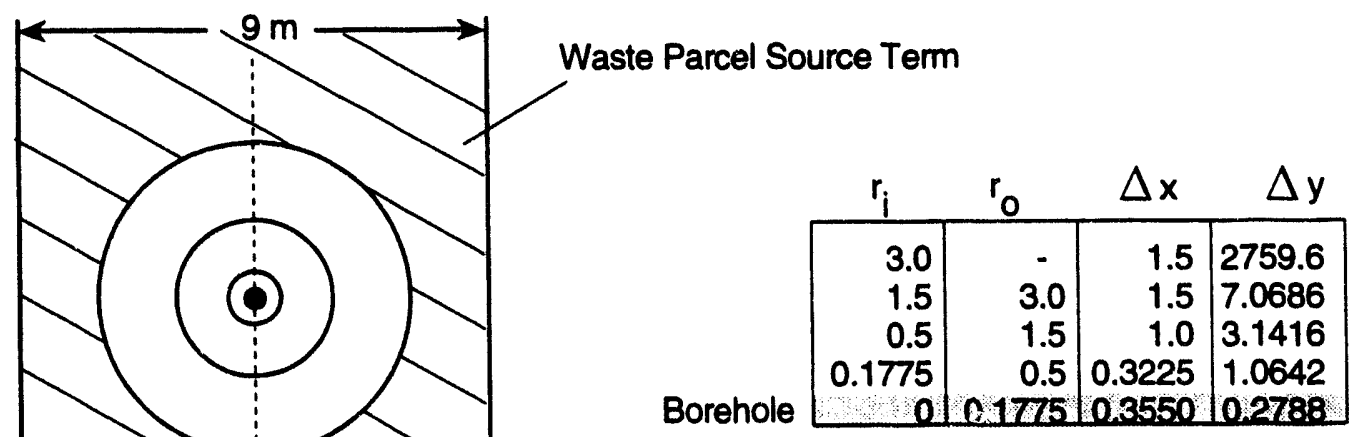

Figure 12-7. Plan view of conceptual model near borehole for salt repository centered in an element of side leg of rooms in panels 3 and 8 . Note that the waste parcel source is located in an element $\sim 3 \mathrm{~m}$ from the borehole. However, the fluid flow properties are the same throughout this region. 
Using the same approach, it can be shown that the grid-block relative permeabilities in a series flow configuration (the $\mathrm{x}$-direction) are computed by:

$$
\overline{k_{x B}}=d_{t} /\left[\Sigma d_{i} /\left(k_{r B V i} k_{x i}\right)\right]
$$

where $\bar{k}_{\mathrm{x}}$ and $\bar{k}_{\mathrm{z}}$ are the horizontal and vertical grid-block permeabilities, $\mathrm{k}_{\mathrm{xi}}$ and $\mathrm{k}_{\mathrm{zi}}$ are the horizontal and vertical component permeabilities, $d_{i}$ and $d_{t}$ are the component length and total length in the series formulation, and $A_{i}$ and $A_{t}$ are the component and total cross-sectional areas in the parallel formulation. In the grid blocks containing a mixture of host rock, waste parcel, and waste parcel 1..., fill, the host rock term in Equation 12.1.45 dominated over the waste

Two-Phase Flow and Relative Perm. „. For multiphase parallel flow, the effective permeability including

$$
\begin{aligned}
& \overline{k_{z B}}=\Sigma k_{r B i} k_{z i} A_{i} / A_{t}, \\
& \overline{k_{z g}}=\Sigma k_{r g i} k_{z i} A_{i} / A_{t},
\end{aligned}
$$

where $\overline{\mathrm{k}_{\mathrm{zB}}}$, and $\overline{\mathrm{k}_{\mathrm{zg}}}$ are the effective vertical permeability to the wetting and non-wetting phase, and $\mathrm{k}_{\mathrm{rBi}}$ and $\mathrm{k}_{\mathrm{rgi}}$ are the component wetting and non-wetting phase relative permeabilities. Once the effective permeability to each phase computed by:

$$
\begin{aligned}
& k_{r B z}=\overline{k_{z B}} / \overline{k_{z}}, \\
& k_{r g z}=\overline{k_{z g}} / \overline{k_{z}} .
\end{aligned}
$$




$$
\begin{gathered}
\overline{k_{x g}}=d_{t} /\left[\Sigma d_{i} /\left(k_{r g i} k_{x i}\right)\right], \\
k_{r B x}=\overline{k_{x B}} / \overline{k_{x}}, \\
k_{r g x}=\overline{k_{x g}} / \overline{k_{x}} .
\end{gathered}
$$

The difficulty with this approach is in the evaluation of the component relative permeabilities in Equations 12.1-47, 12.1-48, 12.1-51, and 12.1-52. Although it is possible to determine the functional relationship between the relative permeability and the wetting phase saturation for each component, there is no way to know the saturation of each component unless each component is modeled separately. Consequently, the component relative permeabilities cannot be evaluated without making some simplifying assumptions.

Because the threshold pressure of the host rock and the canister are very high, little or no gas is expected to penetrate these components. Hence, one can assume the host rock and the canister remain wetting phase saturated, $S_{B}=$ 1 , and that the non-wetting phase is found only in the backfill around the canister and the backfill in the drift. Using this assumption, $\mathrm{k}_{\mathrm{rB}}=1$, and $\mathrm{k}_{\mathrm{rg}}=0$, in the host rock and the canister. The relative permeability in the backfill is evaluated by first computing the non-wetting phase saturation of the backfill material, $S_{g b}$,

$$
S_{g b}=\overline{S_{g}}\left(\Sigma \phi_{i} V_{i}\right) /\left(\phi_{b} V_{b}\right)
$$

where, $\overline{\mathrm{S}_{\mathrm{g}}}$ is the grid-block non-wetting phase saturation, $\phi_{\mathrm{b}}$ is the backfill porosity, and $\mathrm{V}_{\mathrm{b}}$ is the backfill volume. Using $S_{g b}$, the relative permeability in the backfill to the wetting and non-wetting phases is calculated using the methods of Brooks and Corey (1964). $\mathrm{S}_{\mathrm{gb}}$ is also passed to the gas generation submodel in BRAGFLO_T, where it is used to compute the corrosion rate of the waste parcel overpack.

Initial Conditions. Initial conditions for the fluid state variables, saturation, pressures, and temperature, of the model are discussed here. The initial conditions for the gas generation submodel are discussed in Chapter 11.

Initial Saturation. All regions except the repository and the shaft were assumed to be fully saturated with liquid brine. The initial brine saturation in the shaft and the access drift to the storage area was set equal to the residual

\begin{tabular}{|c|c|c|}
\hline Component & Porosity & Saturation \\
\hline Waste Par & 0.00 & 1.00 \\
\hline Backfill & $0.05-0.19$ & 0.20 \\
\hline Halite & 0.01 & 1.00 \\
\hline
\end{tabular}
brine saturation of the salt backfill, 0.2 . Within the repository, elements contained a mixture of waste parcels, backfill, and intact halite. The effective initial grid block saturation was computed using Equation 12.1-34 (see Section 12.1.3), where the component initial porosity and brine saturation are as follows:

\begin{tabular}{|c|c|c|}
\hline Location & Elevation (m) & Pressure (MPa) \\
\hline Snak & 819.9 & 1.053 \\
\hline Anhydrite* & 379.0 & 12.5 \\
\hline Brine Reservoir & 146.5 & 17.0 \\
\hline
\end{tabular}

Initial Pressures. The initial brine and gas pressure of all cells in the repository and the shaft were assumed to be $1.01325 \times 10^{5} \mathrm{~Pa}(1 \mathrm{~atm})$. In all other regions, the initial brine pressure was interpolated from three measured values. The three measured values were

* Not the anhydrite layer modeled in the simulation 
Initial Temperature. The initial temperature of the entire mesh was computed from a measured value of $300 \mathrm{~K}$ in the anhydrite layer at an elevation of $379 \mathrm{~m}$ and a regional temperature gradient of $0.0078 \mathrm{~K} / \mathrm{m}$. Therefore, the initial temperature in the repository was $302 \mathrm{~K}$.

Boundary Conditions. The boundary conditions include fluid flow and heat flow boundaries.

Fluid Flow Boundary Conditions. BRAGFLO_T requires no-flow boundary conditions at all boundary locations. To minimize the impact of the boundary conditions on the solution, large grid blocks were placed at the left and right boundaries. The saturation, temperature, and pressure of these cells remained relatively constant over the simulation period. As a result, the regions of interest behaved as though the problem domain were infinite.

Heat Flow Boundary Conditions. Heat was transported across all boundaries using the boundary heat loss treatment discussed in Section 12.1.1.

\subsubsection{Intermediate Results}

Results are presented below from the fluid flow phase (or module) of the total system consequence model (or performance assessment process, refer to Figure 1-2 and Section 3.2). The results are intermediate in the process and become input to the transport model, described in Section 12.2. The 20 sets of different model parameters from Latin hypercube sampling were used for each of the five waste disposal groups; hence results from 100 simulations (or runs) are summarized here.

Monitoring Points. For each sample set, or "run," fluid flow to and from the disposal region of the repository through the shaft, the two boreholes, and the intact halite was evaluated. To analyze fluid transport between these features and the repository, five locations were selected as monitoring points (Table 12-1).

Table 12-1. Analysis Monitoring Points for Salt Disposal System

\begin{tabular}{clll}
\hline & \multicolumn{1}{c}{ Name } & Description & $(\mathbf{i}, \mathrm{j})$ index \\
\hline 1 & Shaft & Shaft at repository ceiling & $(7,15)$ \\
2 & Borehole 1 & Borehole at repository ceiling & $(25,15)$ \\
3 & Borehole 2 & Borehole at repository ceiling & $(36,15)$ \\
4 & Brine reservoir & Borehole 2 at repository floor & $(36,6)$ \\
5 & Halite & Repository/host rock boundary & - \\
\hline
\end{tabular}

In the discussion and figures that follow, the first monitoring point will be referred to as the shaft, the second as Borehole 1, the third as Borehole 2, the fourth as the brine reservoir, and the fifth as halite (surrounding the repository). The two boreholes penetrate the repository at $1000 \mathrm{yr}$, simulating the WIPP E1E2 (shallow well/deep well) human intrusion scenario. Monitoring point 4 is referred to as the brir: : reservoir because fluid flow through this locations consists primarily of brine released from the brine reservoir of the Tower Formation. The fifth point consists of the sum of the cells at the halite/repository interface.

Fluid Flow. At each monitoring location, the net flow of brine and gas was computed by integrating fluid flow across the interface of the monitoring location over the 10,000-yr regulation period. Net flow can be defined simply as the total fluid volume flowing out of the repository minus the total fluid volume flowing into the repository over 
10,000 yr. A negative value indicates the net fluid flow was into the repository. Because flow reverses direction at certain times during the simulation, it was necessary to split the net flow into two components: total inflow and total outflow. Total inflow was computed by integrating only the flow into the repository, while total outflow was computed by integrating only the flow out of the repository. The total inflow, total outflow, and net flow at each of the five monitoring locations was used as the basis to analyze fluid transport.

Net Brine and Gas Flow. Net brine and gas flow at all five monitoring points is presented in Figures 12-8 and 12-9. Net brine flow is almost identical for all waste disposal groups for a given model parameter sampling set, or "run," demonstrating that the waste disposal group had virtually no influence on the flow of brine between the repository and the surrounding environment. Therefore, the brine flow characteristics for any of the five waste disposal groups can be inferred from a detailed analysis of a single waste disposal group. On the other hand, there were some differences in net gas flow depending on the waste disposal group, particularly though the halite where the most significant gas flow occurred. Gas flow through the brine reservoir was purposely excluded from Figure 12-9 because there was no gas at this location in any of the simulations.

Total Brine Flow for Waste Disposal Group 1. In all 20 sample sets, the principal source of brine flow into the repository v'as through the : alite and the brine reservoir (Figure 12-10). However, as shown in Figure 12-11, most of the brine flowing from the brine reservoir continued to migrate up Borehole 2. Therefore, the primary source of brine to the waste parcels was through the intact halite, which as expected is highly dependent on the halite permeability (Figure 12-12).

By carefully looking at the direction of flow within the repository around Borehole 2, it was determined that brine never flowed from the repository into Borehole 2 during the entire 10,000-yr period. The direction of flow was always away from the borehole at all times for all 20 simulation of Waste Disposal Group 1. This result does not necessarily imply that contaminants are not discharged through Borehole 2, since radionuclides can move upstream via molecular diffusion, or possibly enter the borehole at locations outside the repository, such as the anhydrite marker bed. (Radionuclide transport is discussed in Section 12.2.)

The flow of brine away from the repository is very important to the transport of radionuclides and is presented in Figure 12-13. Brine moves away from the repository predominately through Borehole 2 and through the salt. Brine flow into the salt potentially will be highly contaminated; however, unless it can move into a more permeable material (anhydrite or the borehole), it is not likely to be a threat. On the other hand, brine flow up Borehole 2 could be quite influential. Even though the source of this fluid was found to be the brine reservoir, the potential for molecular diffusion into the borebole followed by advective transport to the Snake Dolomite is quite large.

Flow out Borehole 2 was strongly correlated to the permeability of the upper segment of the borehole (Figure 12-14). The upper segment is defined as the section of borehole 2 that extends from the repository ceiling to the Snake Dolomite. Because of the skewed permeability distribution used to simulate both the E1 and E1E2 scenarios (see Figure 9-7), half the sampled values fell between $10^{-12.5}$ and $10^{-12.0} \mathrm{~m}^{2}$. These values explain why 10 of the 20 sample sets had very large discharges out Borehole 2; each of these sample sets had an upper segment borehole permeability between $10^{-12.5}$ to $10^{-12.0} \mathrm{~m}^{2}$.

Surprisingly, in only one sample set, run 1, was there any brine released up Borehole 1 , and the quantity was rather small. This observation is significant because in the 1992 WIPP performance assessment, the largest contaminant releases occurred in the E1E2 scenario (WIPP PA Department, 1992a). The E1E2 scenario involves the flow of brine up the deep borehole, through the repository, then into the shallow borehole. This scenario never really developed in this study because brine flow through the repository was insufficient to force fluid up the shallow borehole. In the 1992 WIPP performance assessment, brine flow through the repository was for the most part neglected. Consequently, the validity of this scenario is questionable when flow through the repository is accounted for.

Total Gas Flowing into the Repository for Waste Disposal Group 1. Very small volumes of gas actually flow into the repository. The primary source of gas moving into the repository is what was initially contained in the shaft at the beginning of the simulation (Figure 12-15). In run 18, gas is shown flowing into the repository through the halite. Since the halite was initially saturated with brine, gas flow from the halite must consist of gas generated within the repository that flowed into the halite, then later flowed back into the repository. 
12. Complex Consequence Modeling for Salt Disposal System

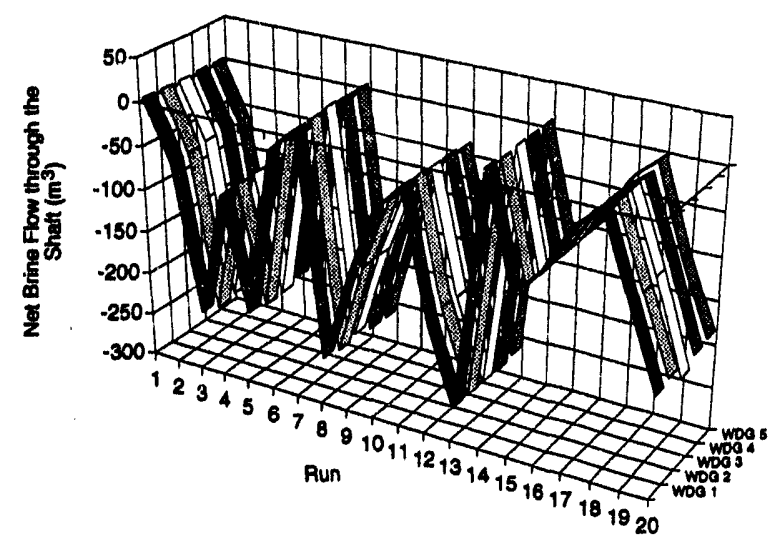

(a)

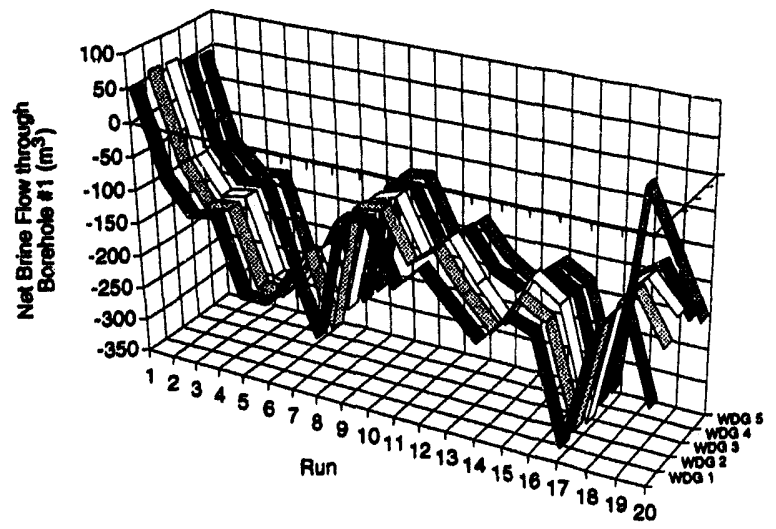

(b)

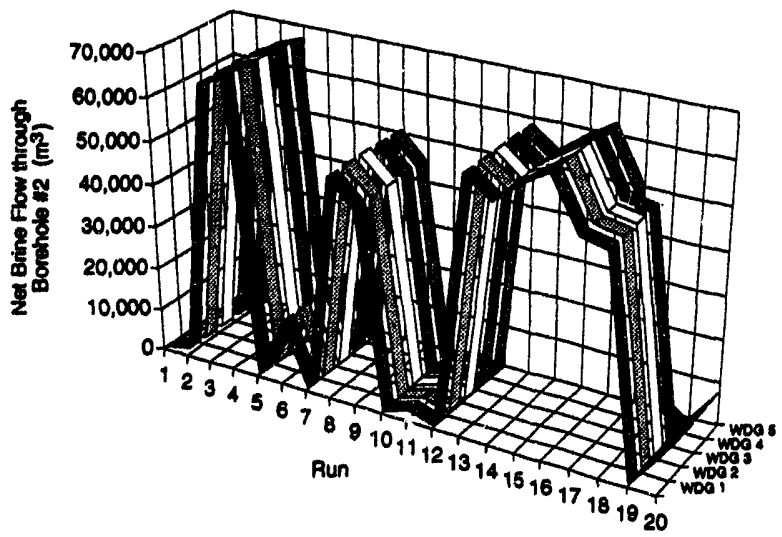

(c)

Figure 12-8. Integrated net brine flows at 10,000 yr for the five waste disposal groups at each of the monitoring points: (a) the shaft, (b) borehole 1, (c) borehole 2, (d) brine reservoir, and (e) surrounding halite. Although no functional relationship exists between the run number, the values of each run are connected to help the eye see the similar behavior among the waste disposal groups (WDGs). 


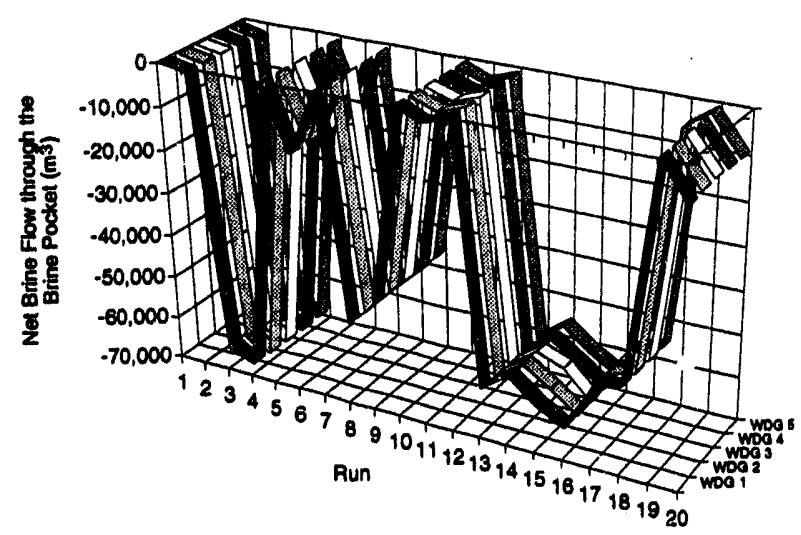

(d)

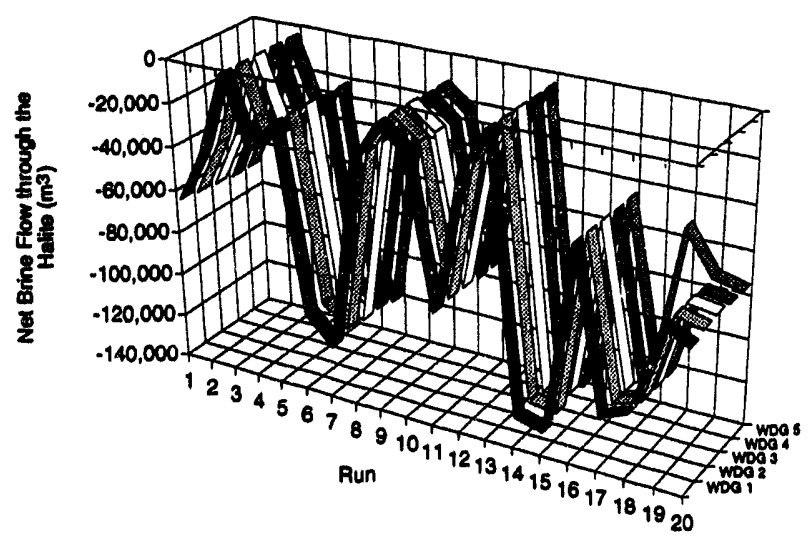

(e)

Figure 12-8. Integrated net brine flows at 10,000 yr for the five waste disposal groups at each of the monitoring points: (a) the shaft, (b) borehole 1, (c) borehole 2, (d) brine reservoir, and (e) surrounding halite. Although no functional relationship exists between the run number, the values of each run are connected to help the eye see the similar behavior among the waste disposal groups (WDGs) (continued). 


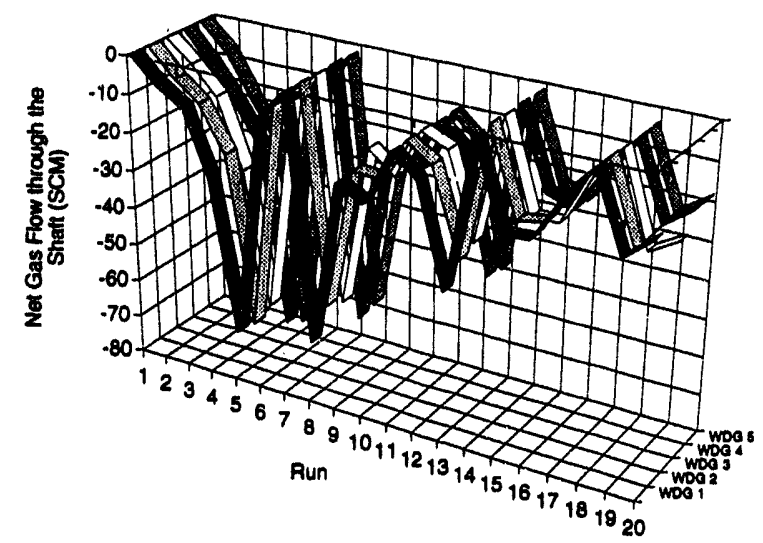

(a)

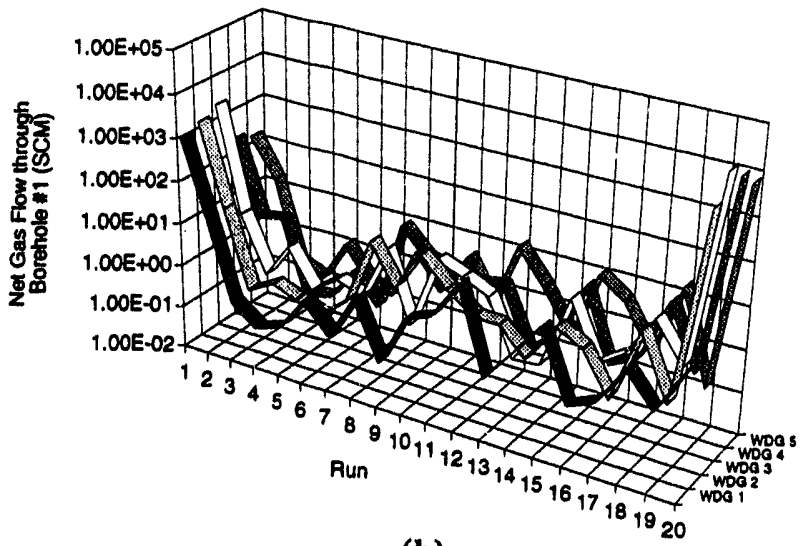

(b)

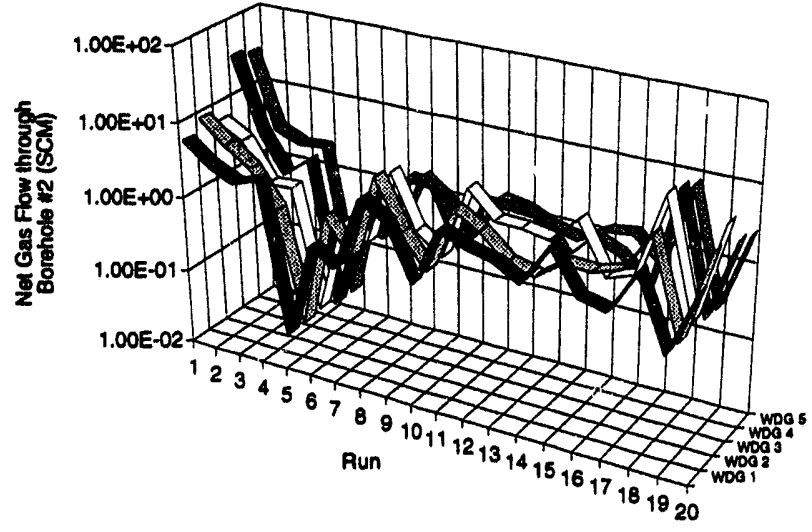

(c)

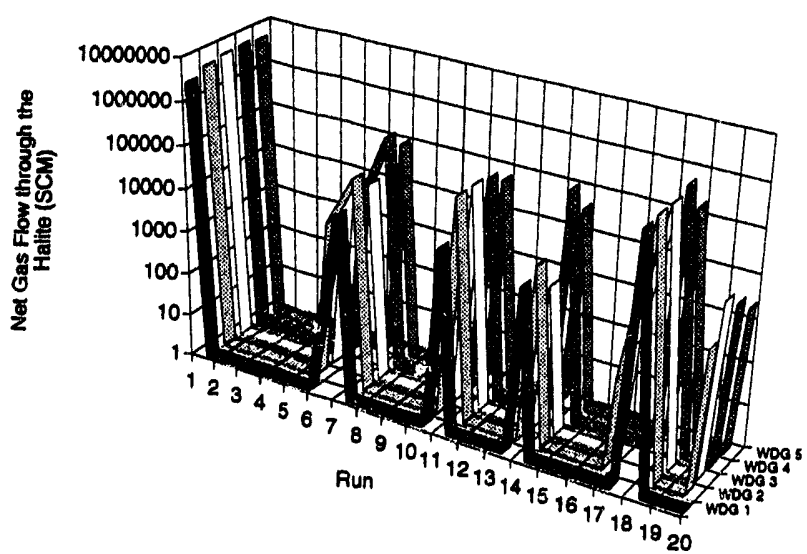

(d)

Figure 12-9. Integrated net gas flows at 10,000 yr for the five waste disposal groups at each of the monitoring points: (a) the shaft, (b) borehole 1, (c) borehole 2, (d) halite. 


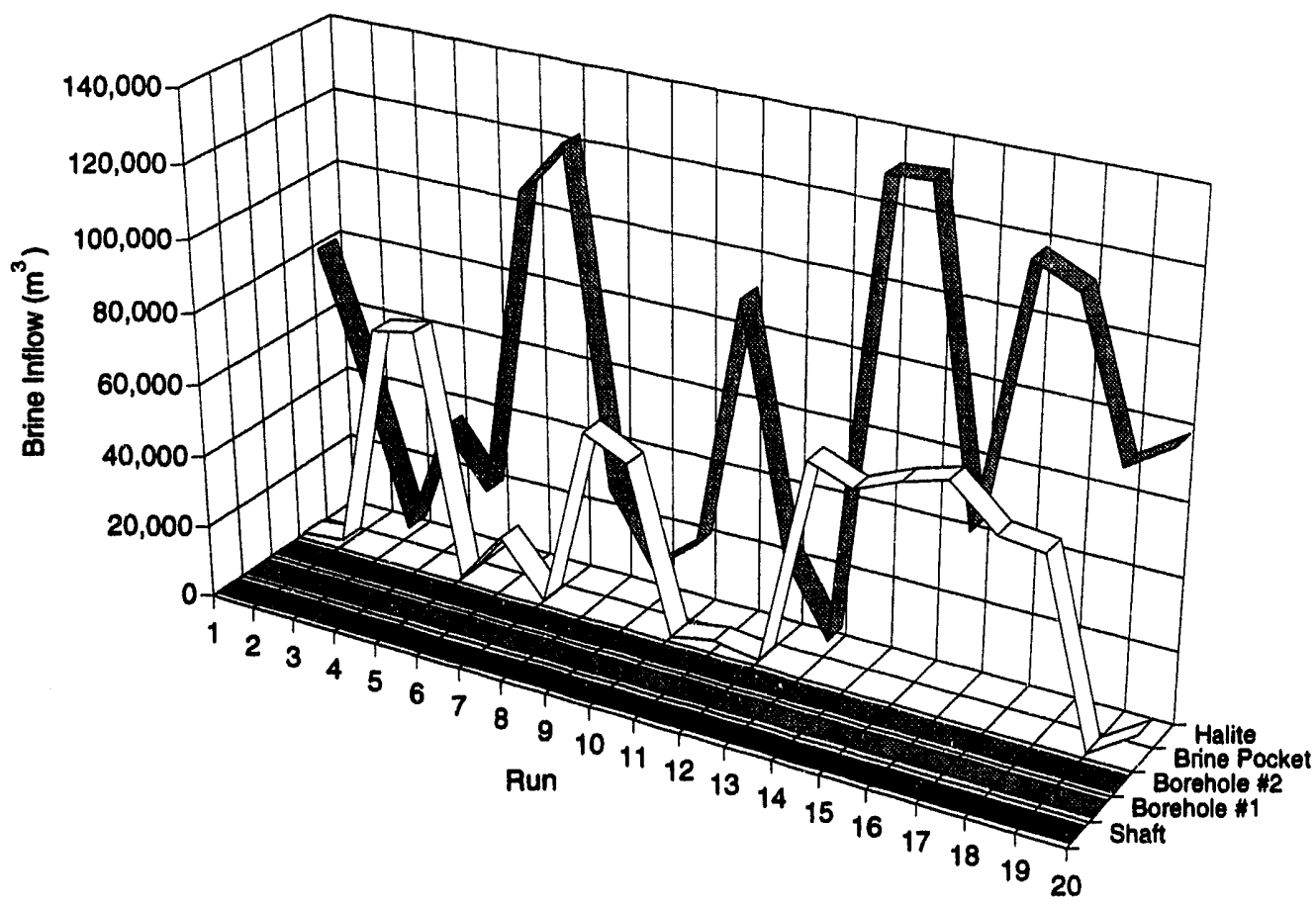

Figure 12-10. Total brine flow into salt repository by 10,000 yr at the five monitoring points for all 20 simulations in Waste Disposal Group 1.

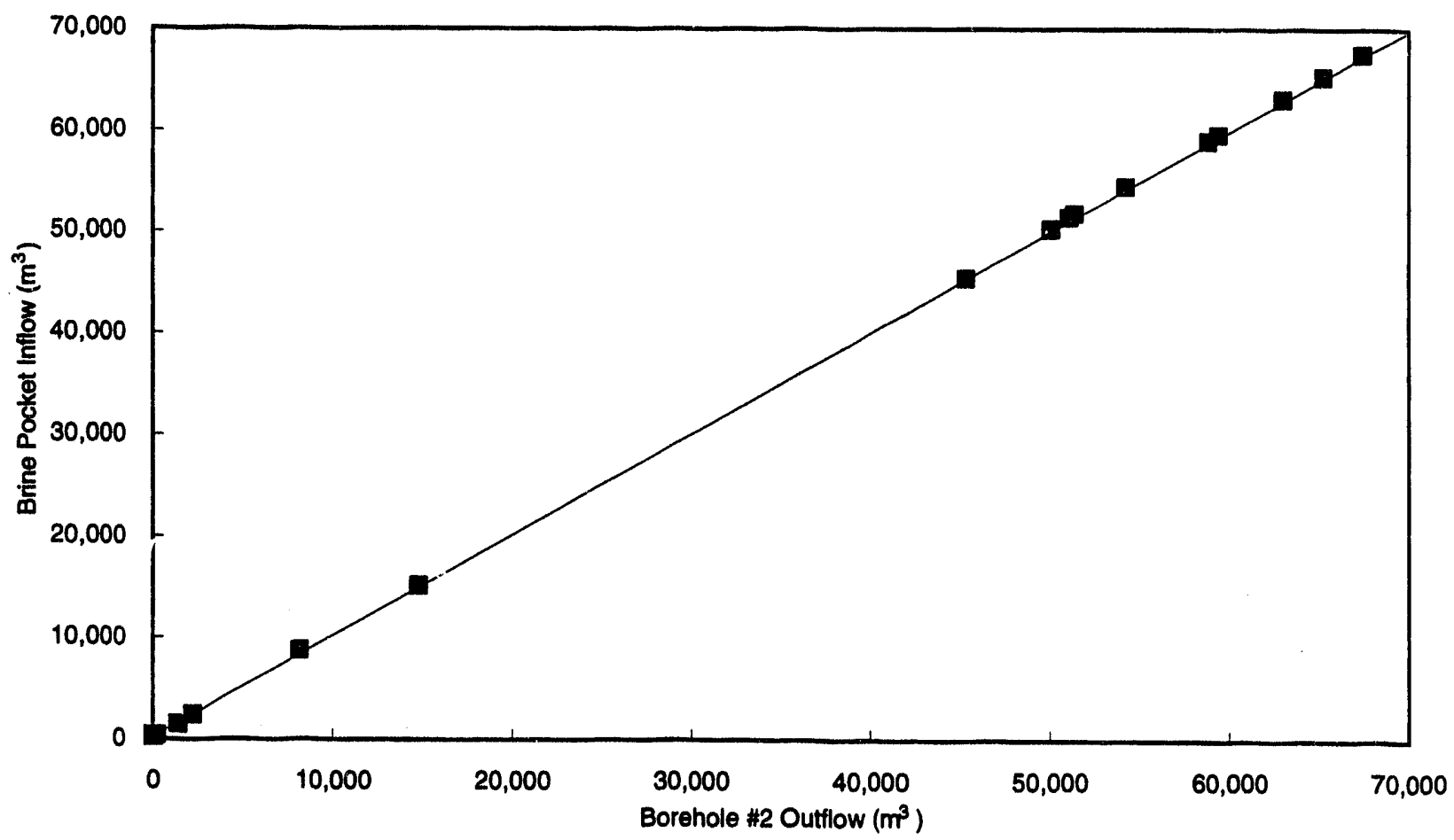

Figure 12-11. Brine inflow from the brine reservoir is only slightly greater than brine outflow through Borehole 2 indicating a slight loss of fluid to the repository in Waste Disposal Group 1. 
12. Complex Consequence Modeling for Salt Disposal System

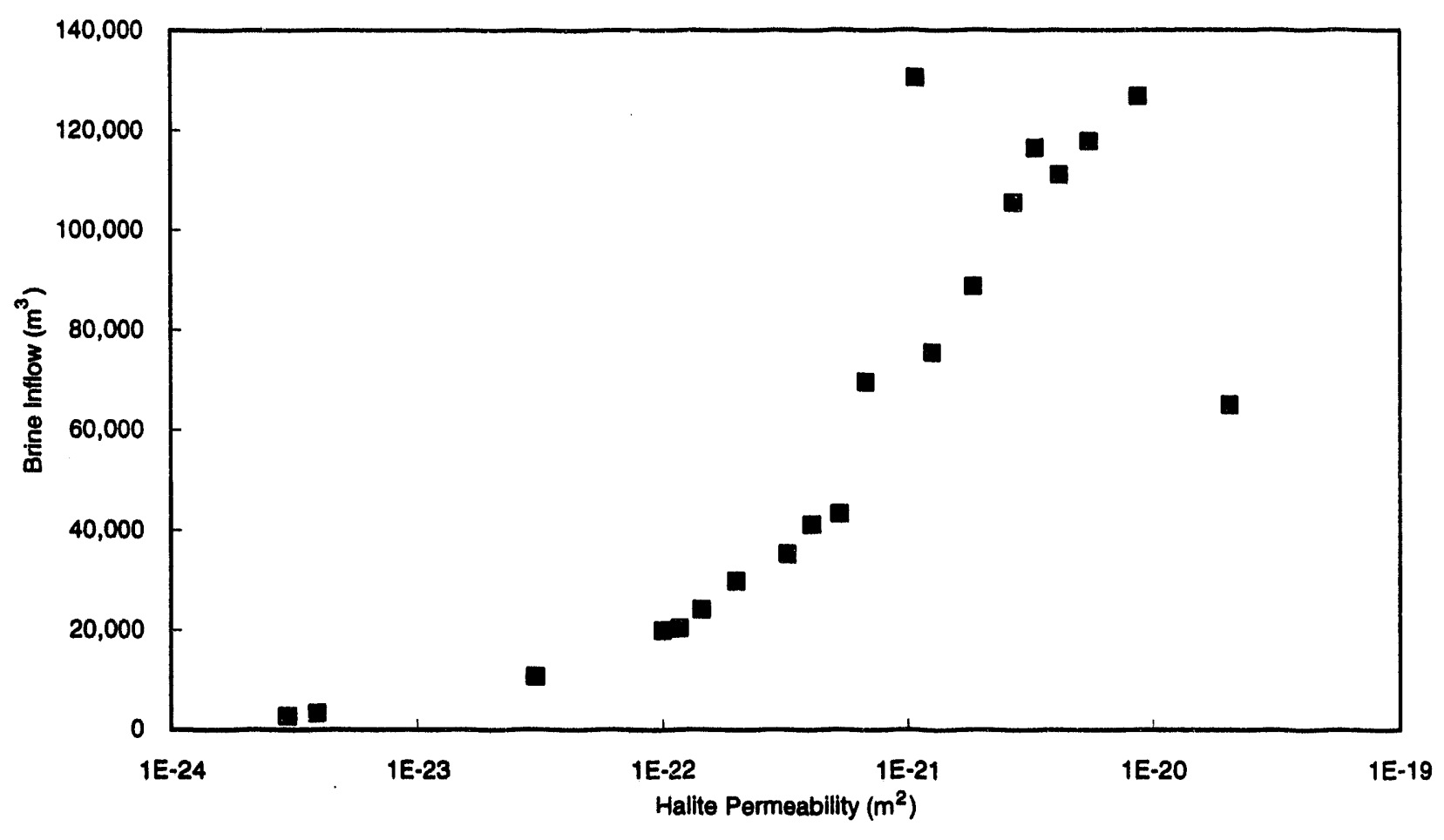

Figure 12-12. Brine inflow from the surrounding halite is highly dependent upon the sampled halite permeability.

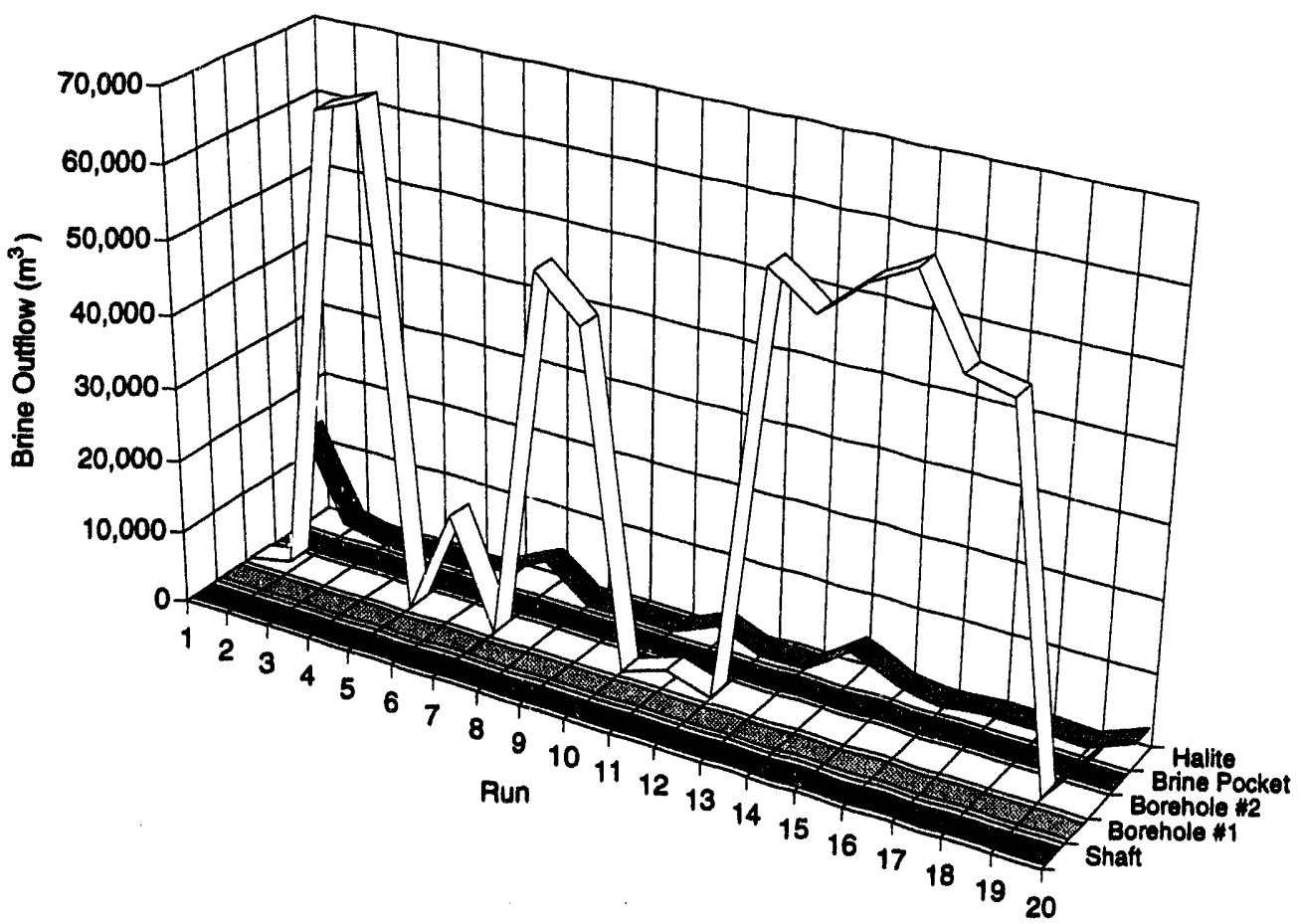

Figure 12-13. Total brine flow out the salt repository after 10,000 yr for all 20 simulations in Waste Disposal Group 1. 


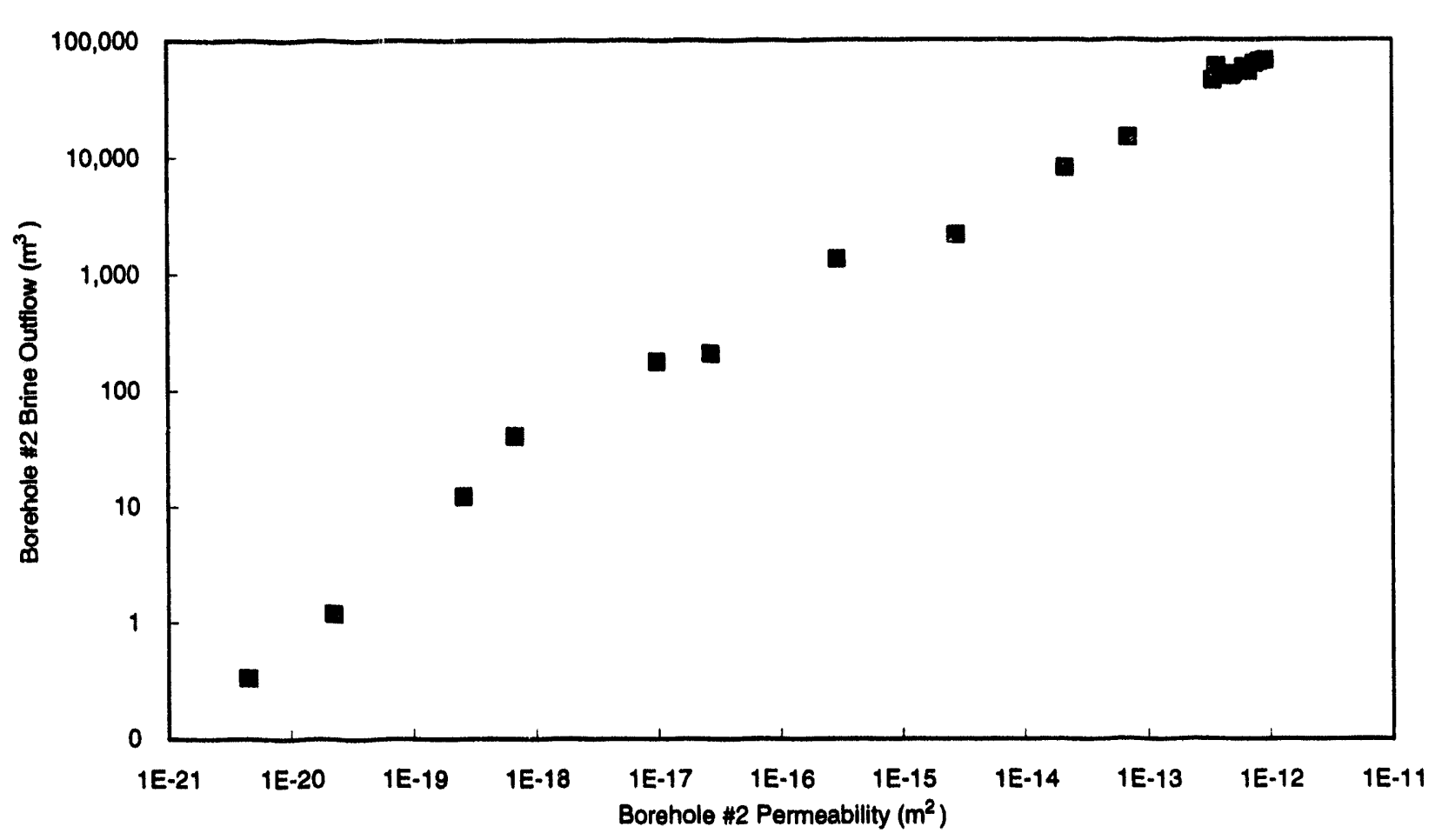

Figure 12-14. Total brine flow out Borehole 2 is highly dependent on the permeability of the upper segment of Borehole 2.

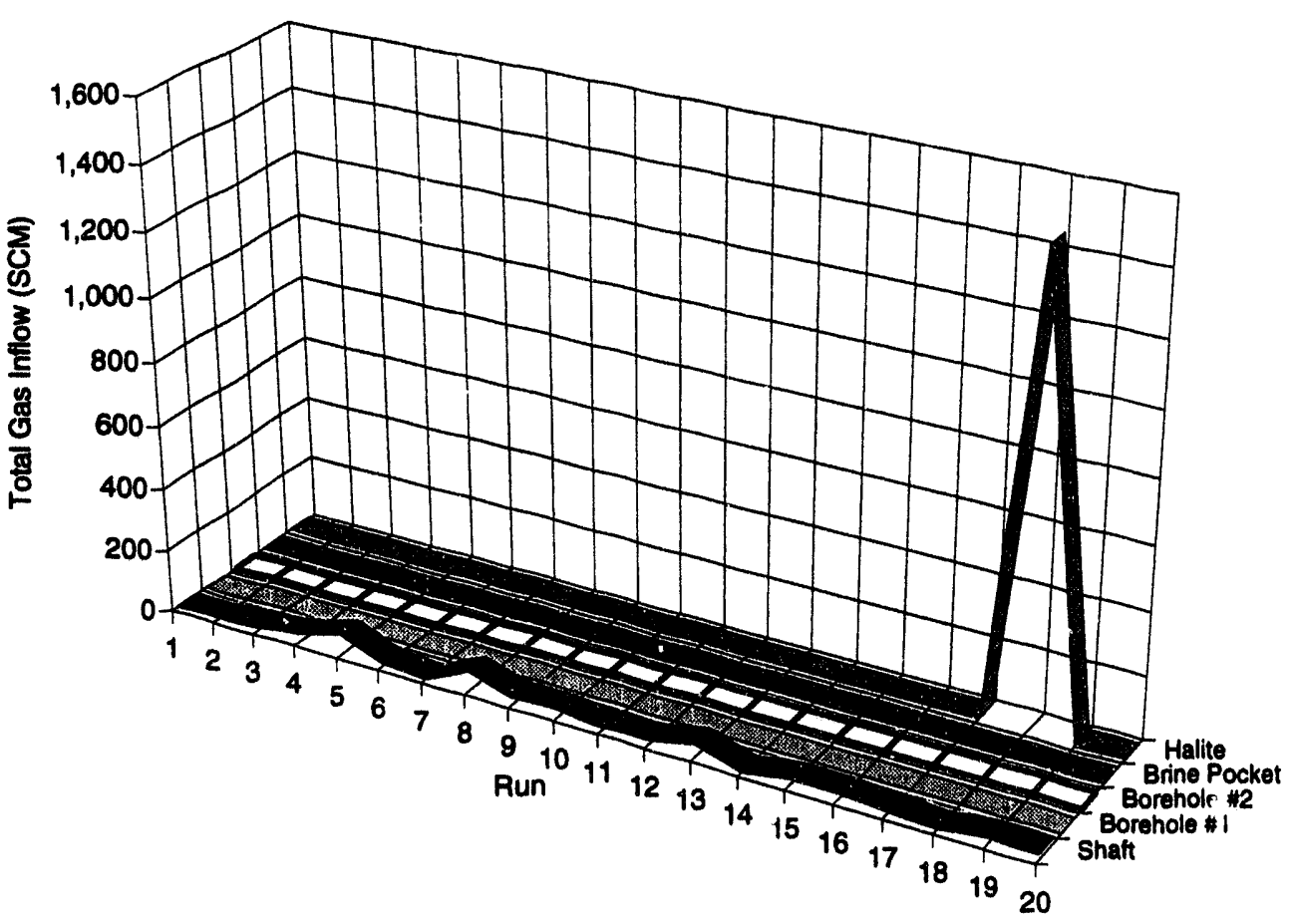

Figure 15-15. Total gas inflow for Waste Disposal Group 1 occurs primarily from the gas-saturated shaft, or from gas that initially moved into the salt but later flowed back into the repository. 
Total gas outflow is presented in Figure 12-16. The only appreciable gas flow out of the repository was into the adjacent halite. Sample sets 1 and 18 had the highest gas releases. These sample sets also had very high halite permeabilities, and therefore, large brine influx rates and low threshold pressures.

Gas Production. The dependence of gas flow on the waste disposal group is linked to the gas generation from the corrosion process. In Figure 12-17, the cumulative gas produced after 10,000 yr is plotted for all waste disposal groups and all sample sets. When gas production exceeded about 4 million standard cubic meters (SCM), Waste Disposal Group 3 produced the most gas. The largest volume of gas produced was slightly in excess of 8 million SCM by Waste Disposal Group 3 in sample set 20.

Cumulative gas production correlated quite well for all waste disposal groups with the corrosion rate of stainless steel (Figure 12-18). In general, the corrosion rate appears to control the cumulative volume of gas generated until the corrosion rate exceeds about $3.5 \times 10^{-10} \mathrm{~kg} /\left(\mathrm{m}^{2} \mathrm{~s}\right)$. At higher rates, the availability of the reactants (metal and brine) possibly control the process.

Repository Pressure and Saturation. Average repository brine saturation, brine pressure, and gas pressure are plotted as functions of time for all 20 sample sets of Waste Disposal Group 1 in Figure 12-19. In most simulations, brine flow into the repository is slow resulting in low brine saturations over most of the 10,000-yr period (Figure 12-19a). As a consequence, the repository brine pressure did not equilibrate with the pore pressure of the salt, and in many cases only slightly exceeder the initial repository brine $\mathrm{F}$ essure of $0.1 \mathrm{MPa}$ (Figure 12-19b). Gas pressures, however, were significantly higher due to the production of gas and consumption of brine as the waste parcels corrode (Figure 12-19c).

As further evidence that the waste disposal group has very little influence on fluid flow, brine and gas pressures at the end of the simulation period are presented in Figures 12-20a and 12-20b. Not surprisingly, the sample sets which generated the most gas also developed the highest gas pressures.

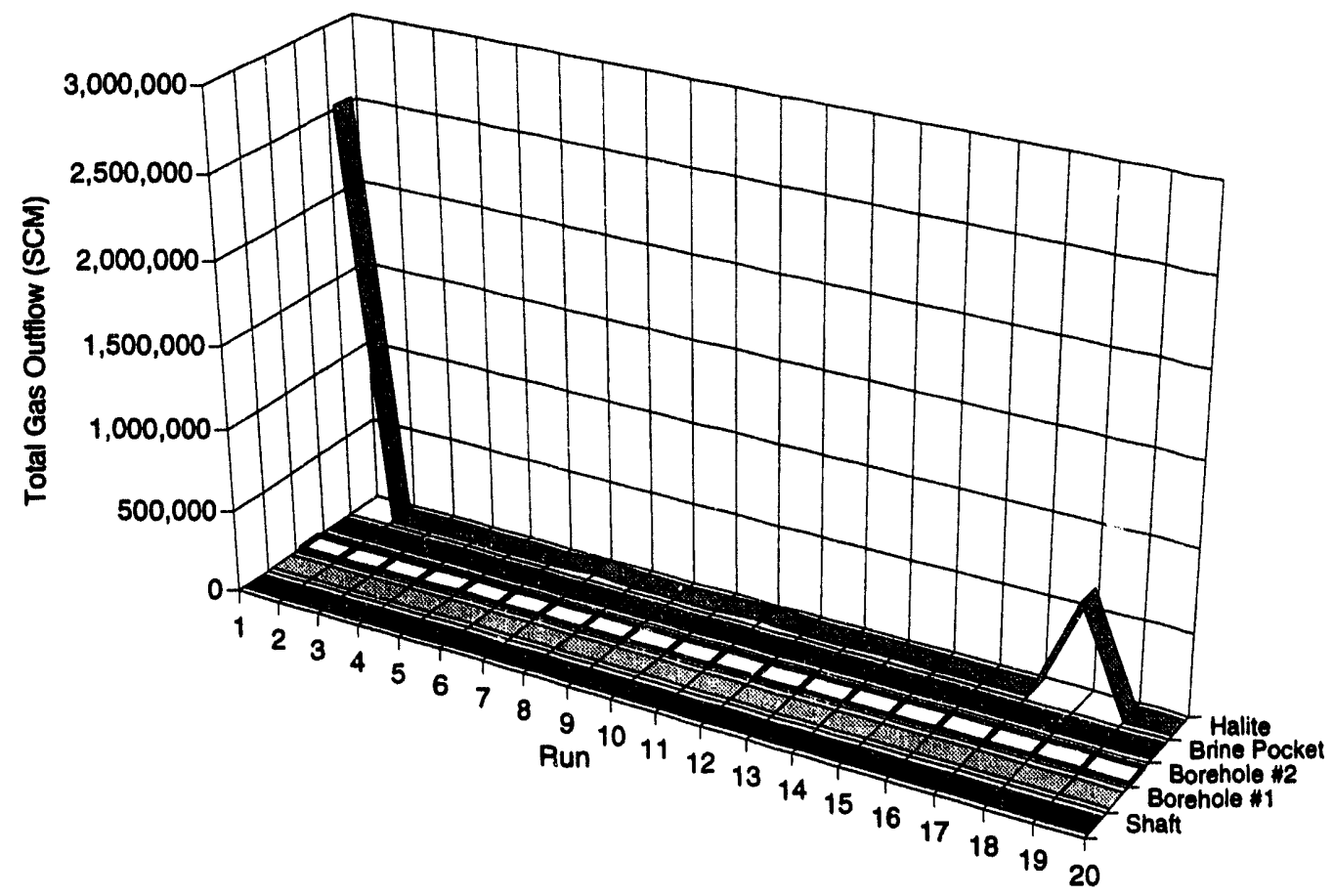

Figure 12-16. The only significant gas outflow occurs into the surrounding liquid-saturated salt. 


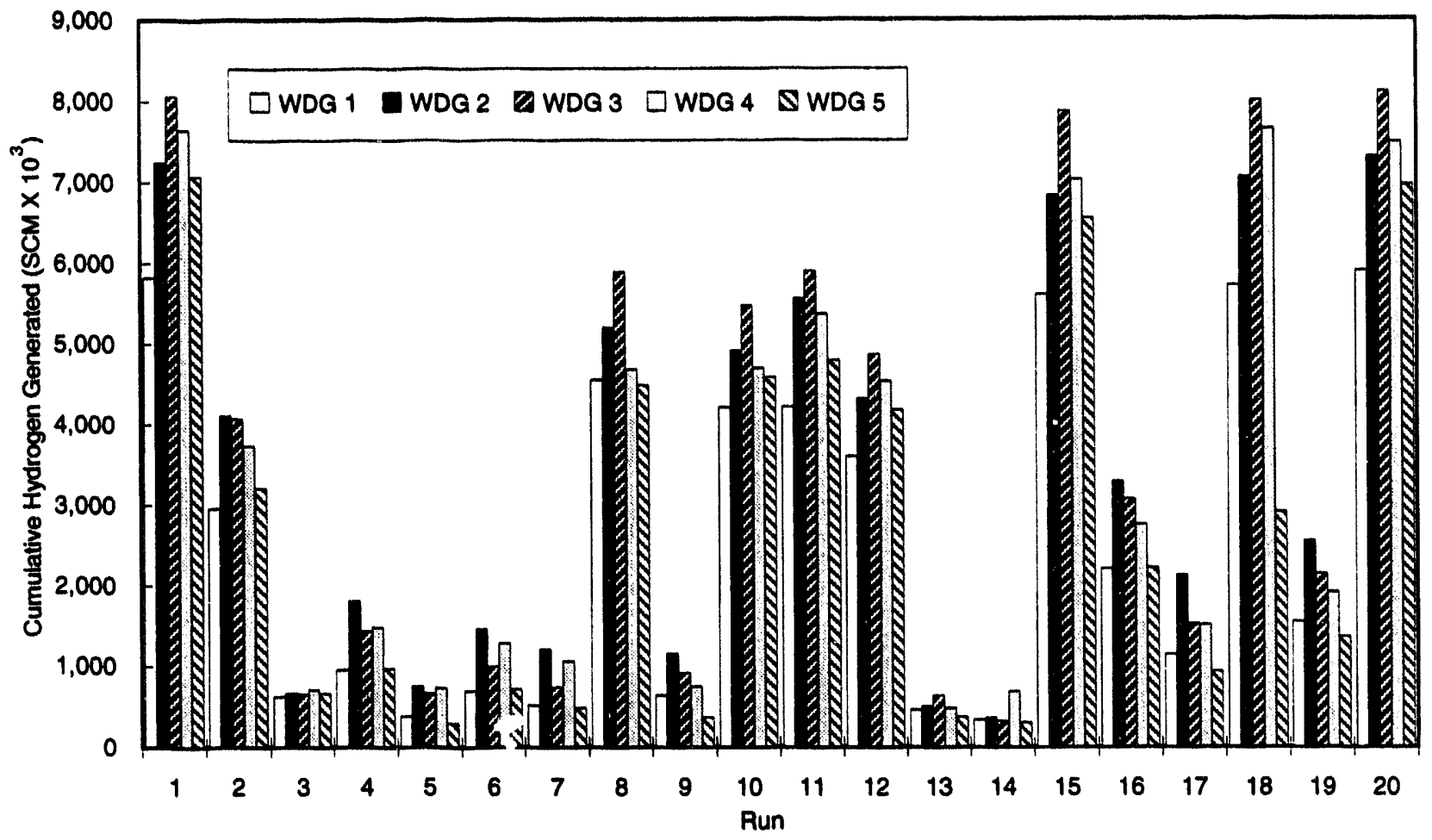

Figure 12-17. Cumulative hydrogen gas genercted by container corrosion at 10,000 yr in all 20 sample sets for all waste disposal groups (WDGs) in the salt repository.

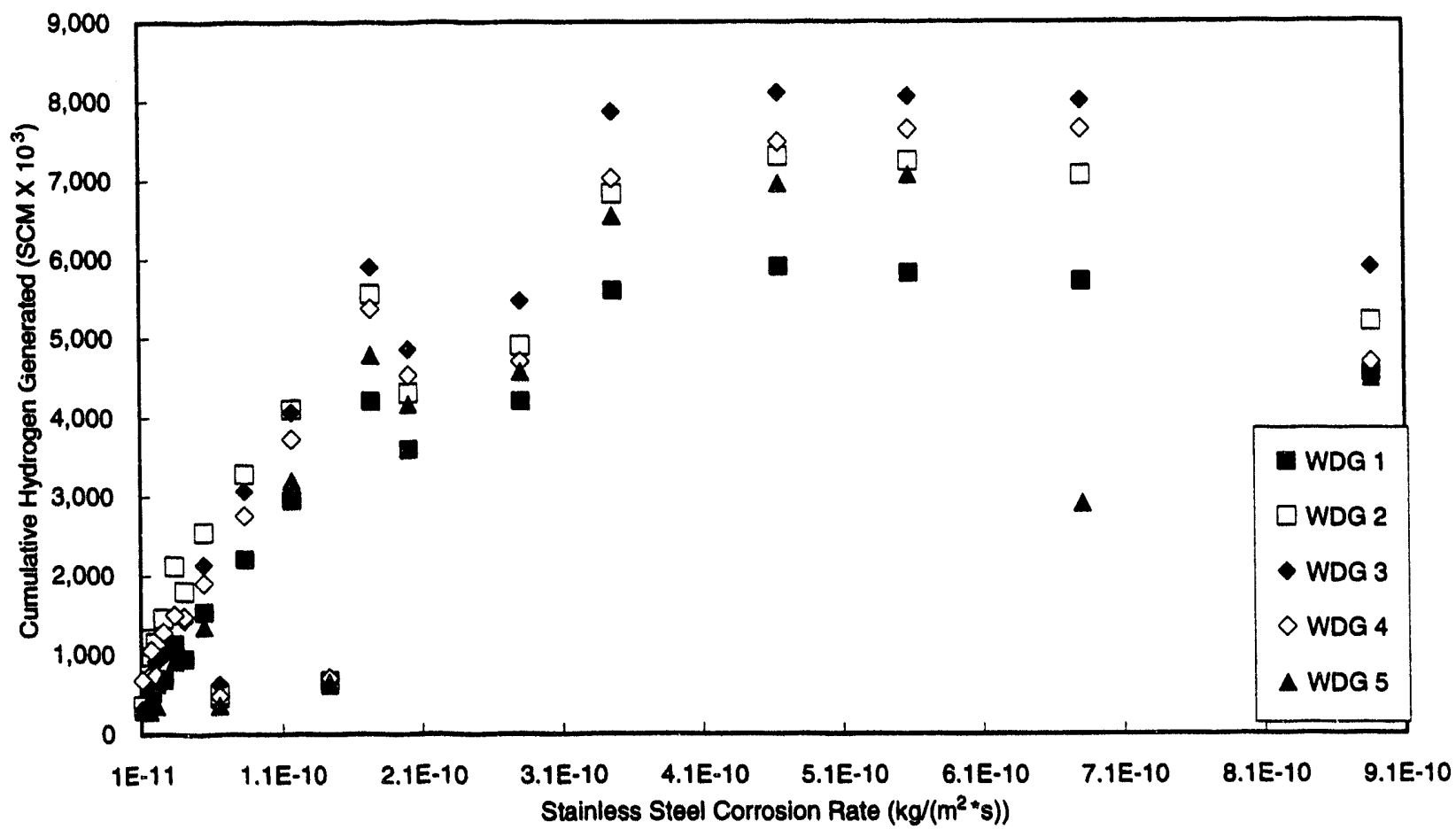

Figure 12-18. Cumulative hydrogen gas production correlates well with the stainless steel corrosion rate up to $3.5 \times 10^{-10} \mathrm{~kg} / \mathrm{m}^{2} \cdot \mathrm{s}$, at which point the availability of reactants possibly controls the process. 
12. Complex Consequence Modeling for Salt Disposal System

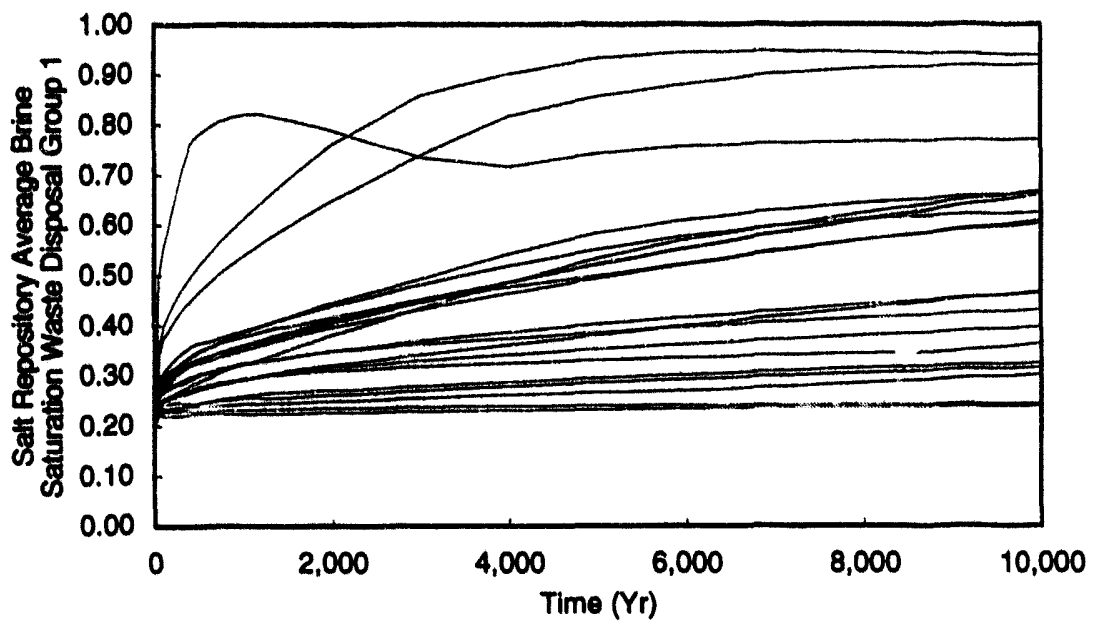

(a)

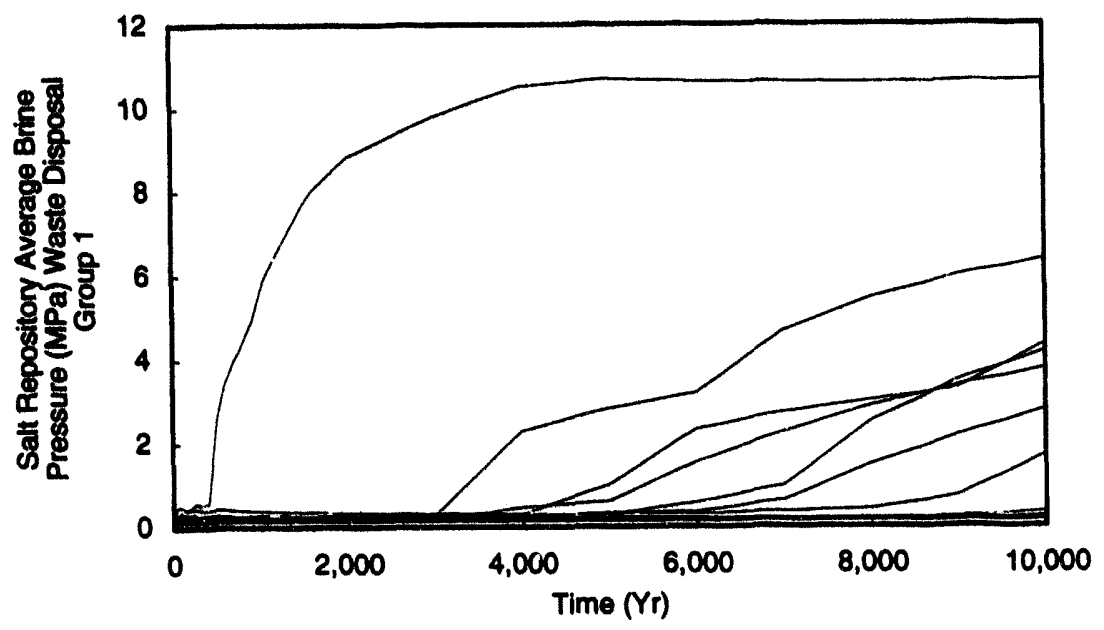

(b)

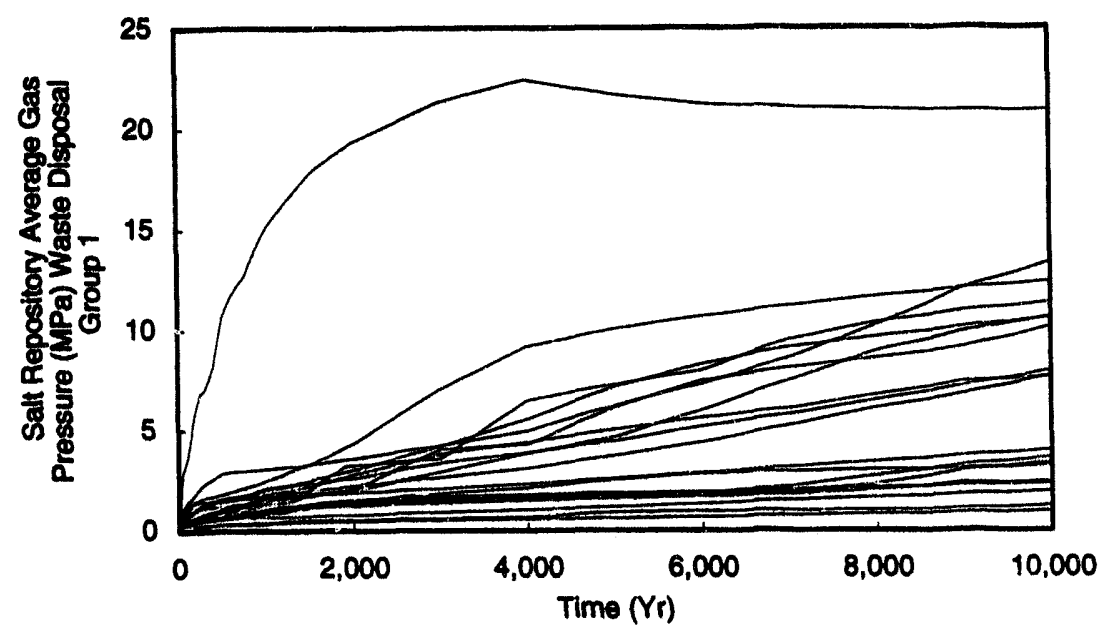

(c)

Figure 12-19. Salt repository average (a) brine saturation, (b) brine pressure, and (c) gas pressure for all 20 sample sets of Waste Disposal Group 1. 


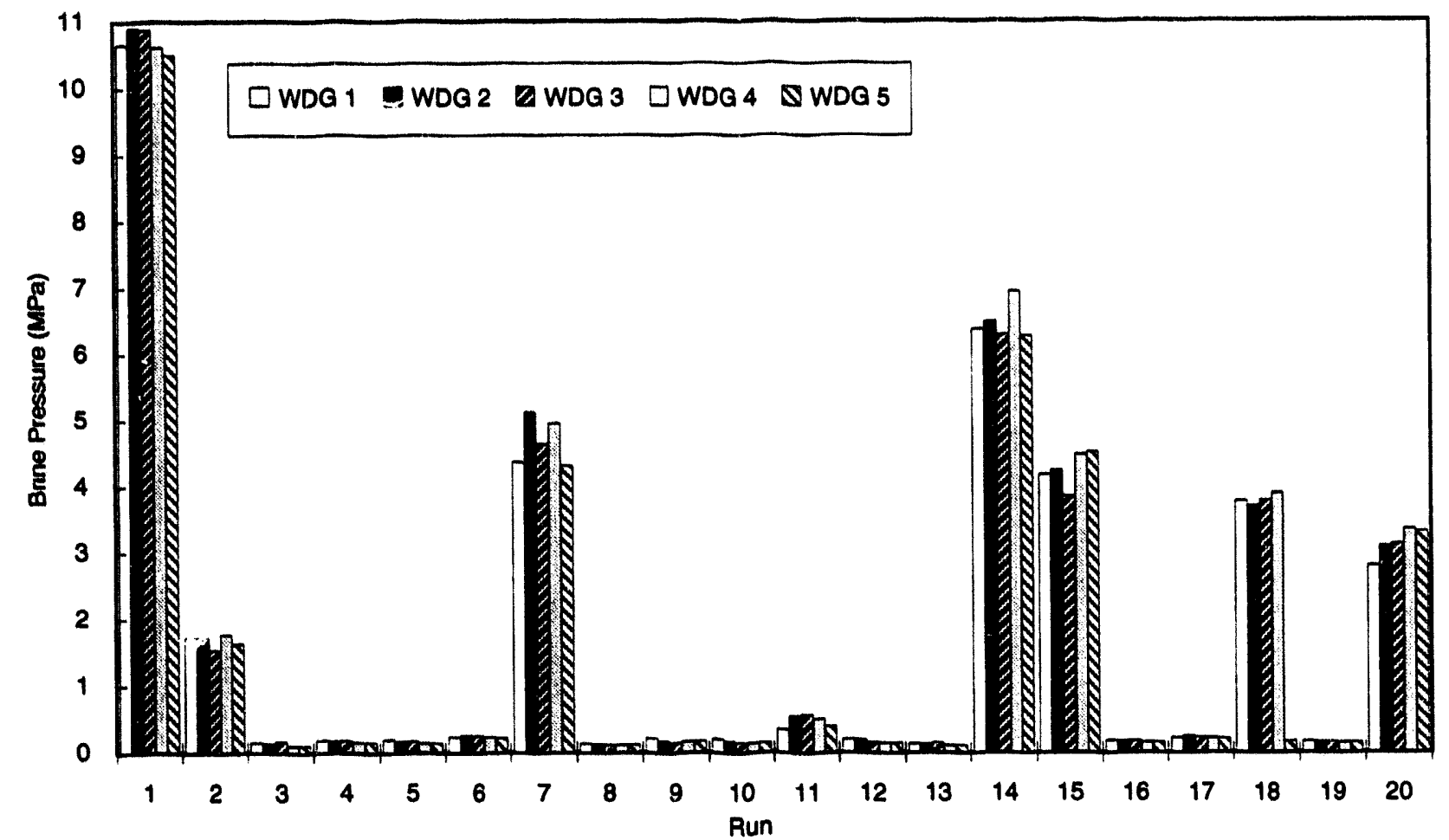

(a)

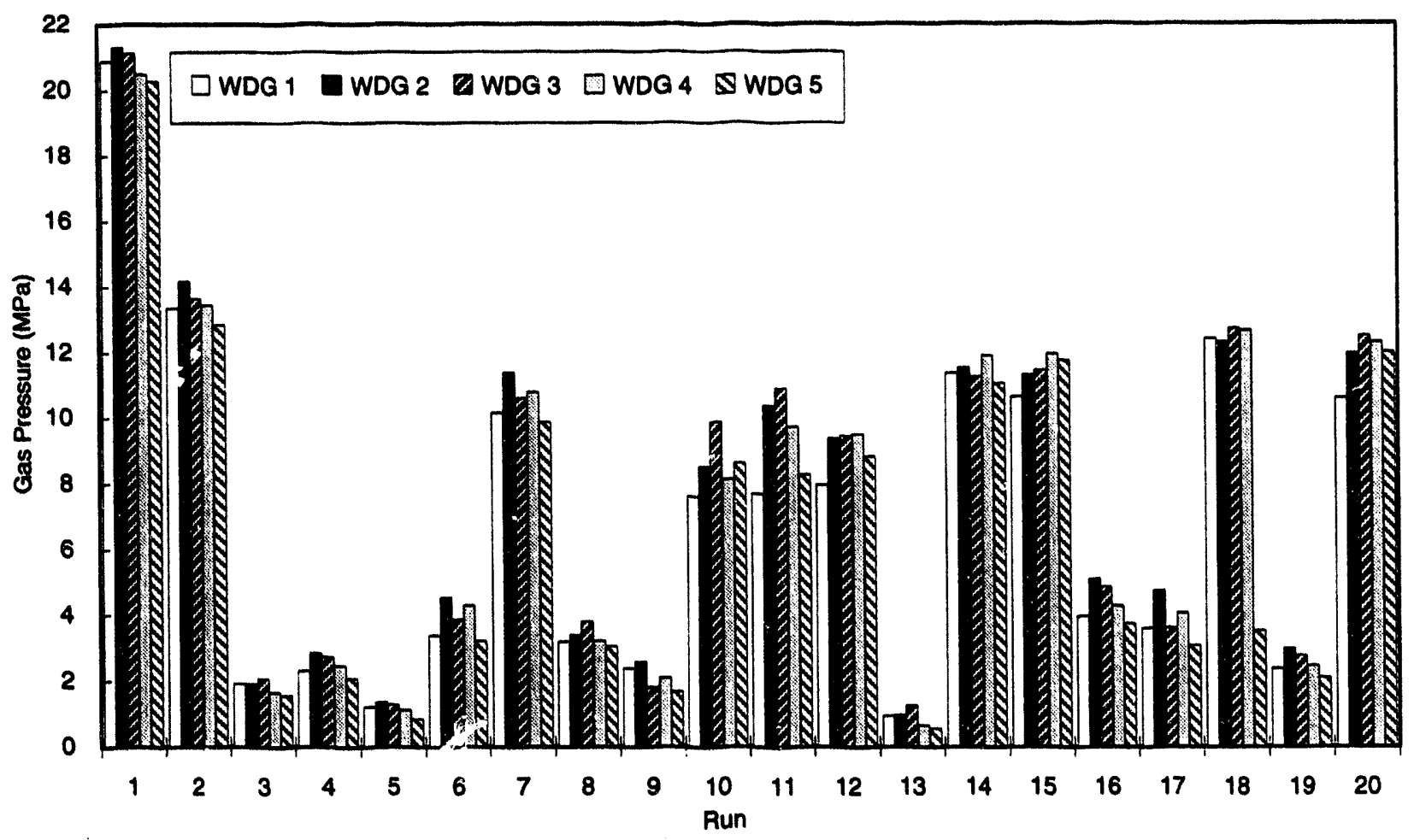

(b)

Figure 12-20. Final (a) brine pressure and (b) gas pressure attained in the salt repository for all five waste disposal groups. Brine pressure significantly increases above the $0.1 \mathrm{MPa}$ initial value in only seven of the twenty sample sets. 


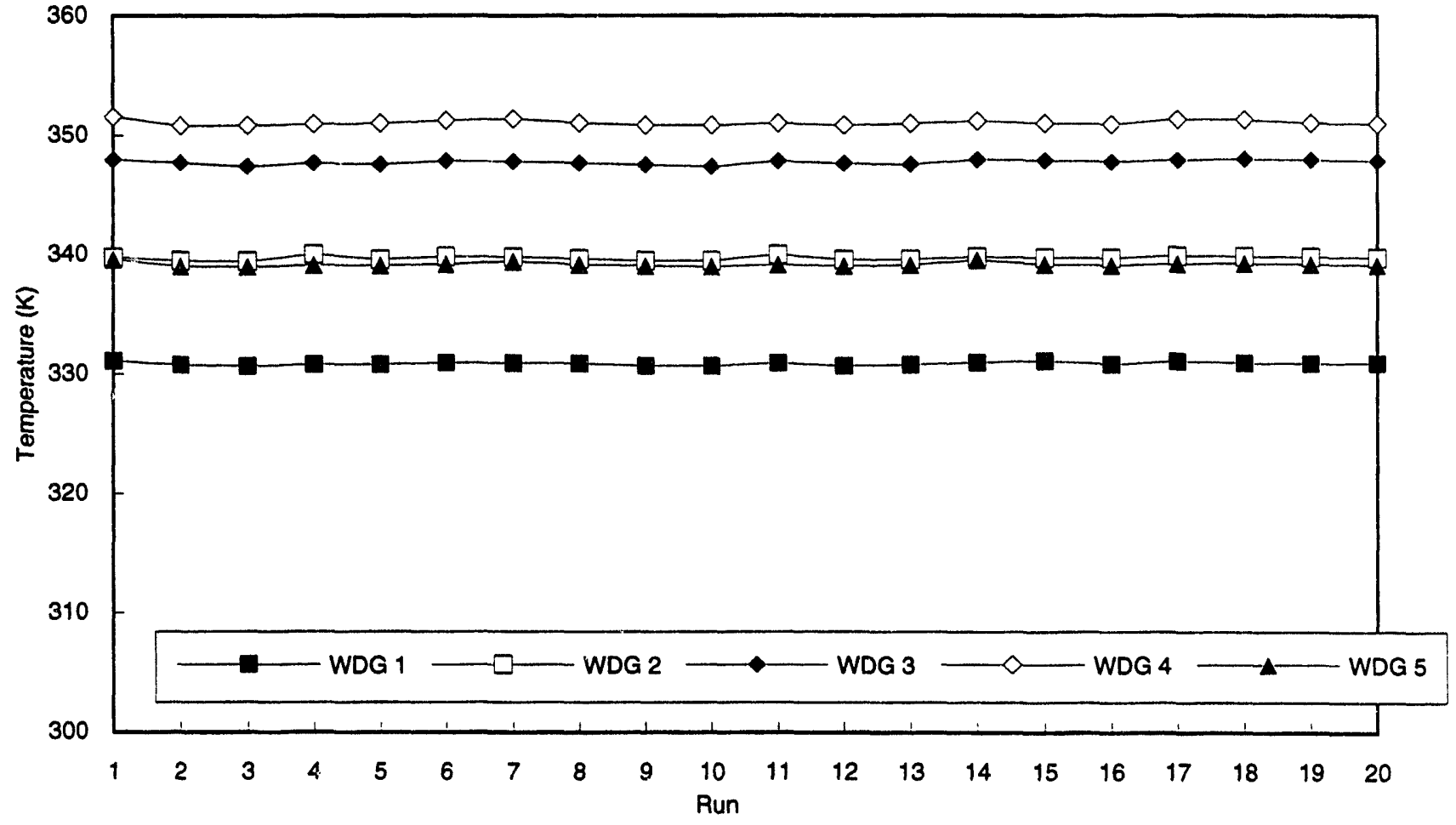

Figure 12-21. Maximum temperature observed throughout the salt repository for all five waste disposal groups. Temperature was a function of the waste disposal group and did not vary with sample sets.

Temperature. In contrast to the single canister scoping calculations (Appendix C), fluid transport had very little impact on the maximum temperature. For a given waste disposal group, each simulation attained essentially the same maximum temperature (Figure 12-21). On the other hand, the waste disposal group had a dramatic impact on the maximum temperature because of the different radionuclide contents. From an initial temperature slightly above 300 $\mathrm{K}$, Waste Disposal Group 1 increased by $30 \mathrm{~K}$, while Waste Disposal Group 4 increased by $50 \mathrm{~K}$.

Finally, it should be pointed out that the maximum temperature increases reported here are possibly low because of the coarse numerical mesh. However, the situation is compensated for somewhat as a result of the no-flow boundary condition in the third dimension. Also fluid transport, rather than heat transport, was emphasized in the passageway between the shaft and the disposal area (see Section 12.1.2 for further details). Consequently, heat flow from the disposal area out the left face first had to flow through the passageway before it could dissipate into the much larger transitional elements.

\subsection{Radionuclide Transport Modeling}

Radionuclide transport in and around the repository in the Complex PA was performed with the STAFF2D (Solute Transport and Fracture Flow in 2 Dimensions) finite-element program. STAFF2D can calculate either Darcy fluid flow or radionuclide transport. In this performance assessment, only the transport capabilities were used. The flow fields were calculated with the BRAGFLO_T code discussed in Section 12.1 and then passed to the STAFF2D transport model as input. The primary assumption allowing for decoupling of the flow and transport simulation is that solute concentrations are small and do not affect fluid density or thermal properties. The radionuclide source term (solute mass injection well) for STAFF2D was provided by CONCSNF, discussed in Chapter 11. CONCSNF, a subroutine of STAFF2D, provides mass injection rates per nuclide for each computational grid element that contains spent fuel or high-level waste. 
A transport simulation was run for each flow simulation; hence for the 20 sets of different model parameters from Latin hypercube sampling and five waste disposal groups, a total of 100 STAFF2D transport simulations were run for the salt disposal system.

\subsubsection{Mathematical Model Description}

STAFF2D is a two-dimensional finite-element code designed to simulate groundwater flow and solute transport in fractured or granular aquifers (Huyakorn et al., 1991). The original version was developed through a joint effort by HydroGeoLogic, Inc. and the International Ground Water Modelling Center of the Holcomb Research Institute. Improved versions of the code have since been commercially available through HydroGeoLogic, the latest being Version 3.2. CAMCON originally adapted Version 2.0 of the code and has since included upgrades from Version 3.2 . Changes to the code were made to accommodate CAMCON input/output requirements and to tailor the code inputs to the secondary database for the WIPP (Rechard et al., 1989); these changes also apply to this performance assessment. In addition, this study required that significant additional upgrades be made to accommodate variable fluid density, porosity, and saturation as well as the source term submodel.

Mass Balance Equations for Transport. The unsaturated transport mass balance equation, assuming variable fluid density, porosity, and saturation, linear equilibrium adsorption, and a fluid and solute source, is as follows:

$$
\begin{array}{r}
\nabla \cdot\left(\rho \theta D_{l J}\right) \cdot \nabla C_{l}-\nabla \cdot\left(\rho \theta V C_{l}\right)=\frac{\partial}{\partial t}\left(\rho \theta C_{l}\right)+\rho_{b} K d_{l} \rho \frac{\partial}{\partial t}\left(C_{l}\right) \\
-\lambda_{l} \rho\left(\theta+\rho_{b} K d_{l}\right) C_{l}+\lambda_{p} \rho\left(\theta+\rho_{b} K d_{p}\right) C_{p}+Q C_{l}^{*}
\end{array}
$$

where (SI units are shown),

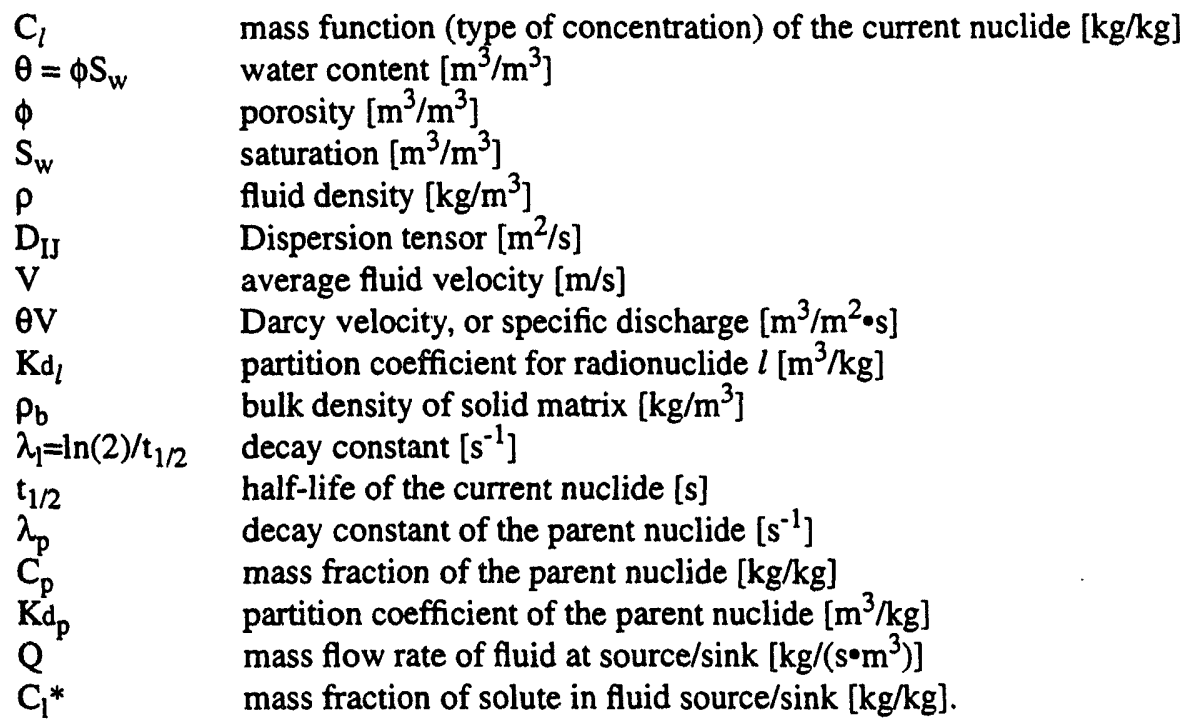

When the second term on the left-hand side and the first term on the right-hand side are expanded and rearranged, Equation 12.2-1 yields:

$$
\begin{aligned}
\nabla & \cdot\left(\rho \theta D_{l J}\right) \cdot \nabla C_{l}-\rho \theta V \cdot \nabla C_{l}-C_{l} \nabla \cdot(\rho \theta V) \\
& =C_{l} \frac{\partial}{\partial t}(\rho \theta)+\rho\left(\theta+\rho_{b} K d_{l}\right) \frac{\partial}{\partial t}\left(C_{l}\right)-\lambda_{l} \rho\left(\theta+\rho_{b} K d_{l}\right) C_{l}+\lambda_{p} \rho\left(\theta+\rho_{b} K d_{p}\right) C_{p}+Q C_{l}^{*} \\
& =C_{l} \frac{\partial}{\partial t}(\rho \theta)+\rho \theta R \frac{\partial}{\partial t}\left(C_{l}\right)-\lambda_{l} \rho \theta R C_{l}+\lambda_{p} \rho \theta R C_{p}+Q C_{l}^{*}
\end{aligned}
$$


where,

$$
\mathrm{R}=\left(1+\frac{\rho_{b}}{\theta} K d_{l}\right), \text { the retardation }
$$

$\mathrm{Kd}_{l}$ is the partition coefficient for linear equilibrium adsorption defined as follows:

$$
C_{\mathrm{s}}=K d \rho_{0} C_{l}
$$

where,

$\mathrm{C}_{\mathrm{s}}=$ adsorbate concentration $[\mathrm{kg} / \mathrm{kg}]$

$\rho_{0}=$ initial fluid density $\left[\mathrm{kg} / \mathrm{m}^{3}\right]$

The fluid mass balance equation can be expressed as follows:

$$
\nabla \cdot(\rho \theta V)+\frac{\partial}{\partial t}(\rho \theta)=Q
$$

Multiplying by solute concentration yields:

$$
C_{l} \nabla \cdot(\rho \theta V)+C_{l} \frac{\partial}{\partial t}(\rho \theta)=C_{l} Q
$$

Substituting Eq. 12.2-4 into 12.2-2 yields the final form of the advection/dispersion solute mass balance equations:

$$
\begin{aligned}
\nabla & \cdot\left(\rho \theta D_{I J}\right) \cdot \nabla C_{l}-\rho \theta V \cdot \nabla C_{l} \\
& =\rho\left(\theta+\rho_{b} K d_{l}\right) \frac{\partial}{\partial t}\left(C_{l}\right)-\lambda_{l} \rho\left(\theta+\rho_{b} K d_{l}\right) C_{l}+\lambda_{p} \rho\left(\theta+\rho_{b} K d_{p}\right) C_{p}+Q\left(C_{l}^{*}-C_{l}\right) \\
& =\rho \theta R \frac{\partial}{\partial t}\left(C_{l}\right)-\lambda_{l} \rho \theta R C_{l}+\lambda_{p} \rho \theta R C_{p}+Q\left(C_{l}^{*}-C_{l}\right)
\end{aligned}
$$

The elements of the dispersion tensor, $\mathrm{D}_{\mathrm{IJ}}$, are defined as (Scheidegger, 1960), ${ }^{* *}$

$$
\begin{aligned}
& D_{11}=\frac{\alpha_{L} V_{1}^{2}+\alpha_{T} V_{2}^{2}}{|V|}+D_{1}^{*},\left[\mathrm{~m}^{2} / \mathrm{s}\right] \\
& D_{12}=\left(\alpha_{L}-\alpha_{T}\right) \frac{V_{1} V_{2}}{|V|},\left[\mathrm{m}^{2} / \mathrm{s}\right] \\
& D_{22}=\frac{\alpha_{L} V_{2}^{2}+\alpha_{T} V_{1}^{2}}{|V|}+D_{2}^{*},\left[\mathrm{~m}^{2} / \mathrm{s}\right]
\end{aligned}
$$

where $\alpha_{\mathrm{L}}$ and $\alpha_{\mathrm{T}}[\mathrm{m}]$ are the longitudinal and transverse dispersitivites, and $D_{1}^{*}$ and $D_{2}^{*}\left[\mathrm{~m}^{2} / \mathrm{s}\right]$ are the effective coefficients of molecular diffusion, including tortuosity effects $\left(D_{l}^{o} \bullet \tau\right)$ where $D_{l}^{o}$ is the free water molecular

"During review of this report, it was noted that this form is not rigorously accurate for radial geometry. 
diffusion of species $l$, and $\tau$ [dimensionless] is the tortuosity defined as $\left(\mathrm{L} / \mathrm{L}_{\mathrm{e}}{ }^{2}\right.$ ), where $\mathrm{L}$ designates straight line length and $L_{e}$ designates actual path length for the fluid.

The decay constant is

$$
\lambda=\frac{\ln (2)}{t_{1 / 2}},\left[s^{-1}\right]
$$

where $t_{1 / 2}$ is the half-life of species 1 .

Assumptions and Limitations. The assumptions and limitations of the model are as follows

- Transport is governed by Fick's law.

- The implementation of the above mathematical model in STAFF2D model is limited to two dimensions.

- Solute effects on fluid density are assumed negligible so flow and transport can be decoupled.

- The dispersivity is assumed to correspond to an isotropic porous medium so that it can be represented by two constants in the principle directions of flow.

- Adsorption obeys a linear equilibrium isotherm.

- There is local chemical equilibrium between the liquid and the solid.

\subsubsection{Mathematical Model Implementation (Computational Model)}

Original STAFF2D. STAFF2D originally used an equation very similar to Equation 12.2-5. The equation can be derived by assuming that (1) the wetting phase saturation, $S_{w}$, is one and (2) the fluid density is constant. The constant fluid density can be combined with the mass fraction to get volumetric concentration, $c_{l}$. The resulting equation as originally solved by STAFF2D is as follows:

$$
\begin{aligned}
& \nabla \cdot\left(\phi D_{l J}\right) \cdot \nabla c_{l}-\phi V \cdot \nabla c_{l} \\
& \quad=\phi R \frac{\partial}{\partial t}\left(c_{l}\right)-\lambda_{l} \phi R c_{l}+\lambda_{p} \phi R c_{p}+q\left(c_{l}^{*}-c_{l}\right)
\end{aligned}
$$

Modified STAFF2D. STAFF2D was modified to solve the more general Equation 12.2-5 so that unsaturated transport could be modeled for this performance assessment. For STAFF2D to solve Equation 12.2-5, fluid density was added explicitly, concentration $c$ was changed to mass fraction $C, \phi$ was replaced with $\theta$ (which equals $S_{w} \phi$ ), and retardation was calculated from $\mathrm{Kd}$ and $\theta$.

These modifications to the transport equations required that the time-dependent saturation, porosity, and fluid density be imported from the finite-difference flow code (BRAGFLO_T) in addition to the velocities. Also, routines were developed that interpolated the velocities to insure that they were properly centered in space for consistency with the STAFF2D finite-element numerical algorithms. This modification was non-trivial because even small inconsistencies in velocities contribute significantly to fluid mass balance errors. These errors appear as sources or sinks in the transport solution.

Numerical Approach. The finite-element approximation technique applied to the convective-dispersive Equation 12.2-5 is an upstream-weighted technique (Huyakorn and Pinder, 1983) designed to overcome oscillations of the numerical solutions when the convective terms are dominant.

Verification. STAFF2D was verified with an analytical solution for saturated transport of a three-specie chain (Lester et al., 1975) and with density-dependent flow and solute transport (Henry, 1964). Results in both cases were very good. Because analytical solutions to unsaturated multiple radionuclide decay chain transport are difficult to 
find. SUTRA was also modified to solve Eq. 12.2-5 so that the two codes could be used to benchmark each other. The complementary capabilities of the original versions of the codes improved the reliability of the benchmarking. Given the two analytical solutions and the benchmarking of the two codes, a reliable, unsaturated multiple-specie transport capability was verified with a high degree of confidence. STAFF2D was chosen for this performance assessment because its numerics were more compatible with BRAGFLO_T.

\subsubsection{Applied Model}

Model Geometry and Spatial Discretization. The decoupled sequential nature of this performance assessment (a flow simulation, then a transport simulation) and the structure of CAMCON meant that the data output from the flow modeling becomes the input data to the transport modeling (see Chapter 7 and Appendix G). As a result, the geometry for the transport model is identical to the geometry for flow (see Section 12.1.2 for salt repository geometry). This situation facilitates the input or import of the flow-field data: velocities (fluxes), porosity, fluid density, and saturation from BRAGFLO_T to STAFF2D. The logical grid showing major features is shown in Figure 12-4.

Time Discretization. Time discretization for the transport simulations was controlled by the BRAGFLO_T flow simulations. The time steps used by BRAGFLO_T are controlled internally by the convergence criteria and the time scale of the physical phenomena taking place. The computational results were written to the CAMDAT (Compliance Assessment Methodology Data) data base at specified cycle intervals. These times on the data base were used directly in the transport simulations. Typically, time steps were small (initially on the order of fractions of years) growing to a maximum of 500 years; the smaller time steps occurred around the time of intrusion at $1000 \mathrm{yr}$.

\subsubsection{Initial and Boundary Conditions}

Flow field initial conditions are described in Section 12.1.3. Initial radionuclide concentrations throughout the model domain are assumed to be zero, $C_{l}(x, y, 0)=0$ for ail $x, y$. Initial conditions inside the backfill-buffer around the waste parcel are handled by the source term model, CONCSNF, as described in Chapter 11.

A zero-concentration-gradient boundary condition was set at the four external grid boundaries (model domain boundaries). This type of boundary condition allows for advection across the boundary, but not dispersion or diffusion. If there is flow at the boundaries, solute is allowed to flow out of but not into the mesh. However, as described earlier for the flow model, the domain of the flow simulations was assumed large enough to set no-flow boundary conditions. Therefore, regardless of what is set in STAFF2D, the boundaries are non-transporting. Consequently, no solute leaves the modeling domain.

The source term is implemented in STAFF2D as an internal mass flux boundary condition (injection well). Originally, the user supplied a rate history in tabular form. In performance assessments for the WIPP, this rate history table was generated by the PANEL code (parent of IPANEL discussed in Appendix C) and was uncoupled from the existing concentration fields. In this performance assessment, CONCSNF, the source-term submodel, is implemented in STAFF2D as a subroutine that is called for every element containing spent fuel or high-level radioactive waste. The model returns a mass flux to STAFF2D for each transported radionuclide, which is function of both the current state inside each waste emplacement package and the current state of the flow and transport fields: concentrations and saturations (refer to Figure 11-6 in Chapter 11). For the Complex PA for the salt repository, there were 88 elements with waste emplacement packages.

\subsubsection{Material Properties}

Two sets of material properties are required for the STAFF2D transport simulations: the porous media or host material properties and radionuclide properties. Property values were obtained from the property secondary data base (see Chapter 5, "Geologic Barrier," and Chapter 6, "Repository Design"). Porous media properties include porosity, tortuosity, longitudinal dispersivity, and transverse dispersivity. Nuclide properties include half-life, specific activity, 
chain descriptions, partition coefficients (retardation), and effective diffusion coefficients. Drift and backfill porosity was the only transport property sampled for this performance assessment (see Chapter 7, "Scenario Development"). However, several source term submodel properties were also sampled as discussed in Chapter 11.

Guidelines for applying the STAFF2D isotropic dispersion model suggest that the size of dispersitivities can be on the order either of the largest hydrogeologic or flow inhomogeneity along the transport reach or the distance between inhomogeneities, whichever is greater. Transverse dispersitivities are even less well defined and are typically chosen to be between one-tenth and one-third of the longitudinal value. Also, there is a numerical requirement that finite elements be no larger than roughly 4 to 20 times the longitudinal dispersivity to ensure stability and accuracy (note that elements vary between $0.3 \mathrm{~m}$ to $6800 \mathrm{~m}$ ). Because of the large geometric and material differences between the repository backfill and host material, these parameters are not constant and suggest a spatially varying dispersivity. However, because of these complexities and the lack of supporting data, a single minimum value for dispersivity that was numerically stable was chosen by trial and error to minimize upstream dispersion to the intrusion borehole. For the salt disposal system, both longitudinal and transverse dispersivity were set to $1.5 \mathrm{~m}$.

A total of 11 radionuclides were transported: two 4-isotope chains and 3 individual isotopes. The chains are as follows (see Figure 4-8 for complete chains): ${ }^{\dagger \dagger}$

(1) ${ }^{239} \mathrm{Pu}$

(2) ${ }^{240} \mathrm{Pu}$

(3) ${ }^{241} \mathrm{Am} \rightarrow{ }^{237} \mathrm{~Np} \rightarrow{ }^{233} \mathrm{U} \rightarrow{ }^{229} \mathrm{Th}$

(4) ${ }^{238} \mathrm{U} \rightarrow{ }^{234} \mathrm{U} \rightarrow{ }^{230} \mathrm{Th} \rightarrow{ }^{226} \mathrm{Ra}$

(5) ${ }^{14} \mathrm{C}$

\subsubsection{Intermediate Results}

Results from the radionuclide transport model (refer to Figure 1-2 and Section 3.2) of the total system consequence model are described below. These intermediate results become input to CCDF construction codes, CCDFCALC, and CCDFPERM (see Chapter 7 and Appendix G).

Typical solute contours in and around the repository are shown in Figures 12-22 and 12-23. The logical grid is used for ease of visualization. On the logical grid, all element dimensions are 1, which can be deceiving for this mesh since the outer elements are three orders of magnitude larger than the elements near the waste emplacement packages in the $\mathrm{x}$-direction and two orders of magnitude in the $\mathrm{z}$ direction. However, the entire mesh can be easily viewed in this manner. Shown on the figure are material interfaces, not individual elements.

Figure 12-22 shows contours of the solute concentrations in terms of mass fractions (mass solute/mass of groundwater, the calculated dependent variable in STAFF2D). The results shown are for ${ }^{234} U$, Waste Disposal Group 1 , and simulation 17, which had one of the larger summed normalized activity. The contours are representative of most simulations except in amplitude. Negative concentrations are due to oscillations in the numerical solution. Numerical parameters can control the negative concentration but only at the expense of increased numerical diffusion which spreads concentration gradients. The area of negative concentration is small and thus represents only a small negative mass and small mass balance error when compared to the total solute mass in the system. Because of unsaturation, these contours do not adequately display the distribution of the contaminant mass. The distribution is viewed better when expressed in terms of mass of contaminant/element volume (Figure 12-23). As demonstrated by these contours there is very little advective transport; the transport appears to be diffusion dominated. Furthermore, this transport is primarily from the waste packages into the repository with concentrations decreasing with time because of slow radial diffusion. Also apparent is the primary solute pathway up the intrusion borehole from the brine reservoir and slight buildup of solute at the intersection with the overlying aquifer. More significant pooling of solute

\footnotetext{
${ }^{+\dagger}$ As noted in Chapter 4, 49 radionuclides were included in the source term submodel, CONCSNF, but only a small subset was included in the transport calculations. The subset was selected based on the potential influence of the radionuclide on the CCDF of the EPA normalized releases up to $10,000 \mathrm{yr}$. Radionuclides not in the subset may become important after 10,000 yr; also some radionuclides may be important to consider for other performance measures (e.g., individual dose calculations) during the 10,000-yr regulatory period.
} 
12. Complex Consequence Modeling for Salt Disposal System

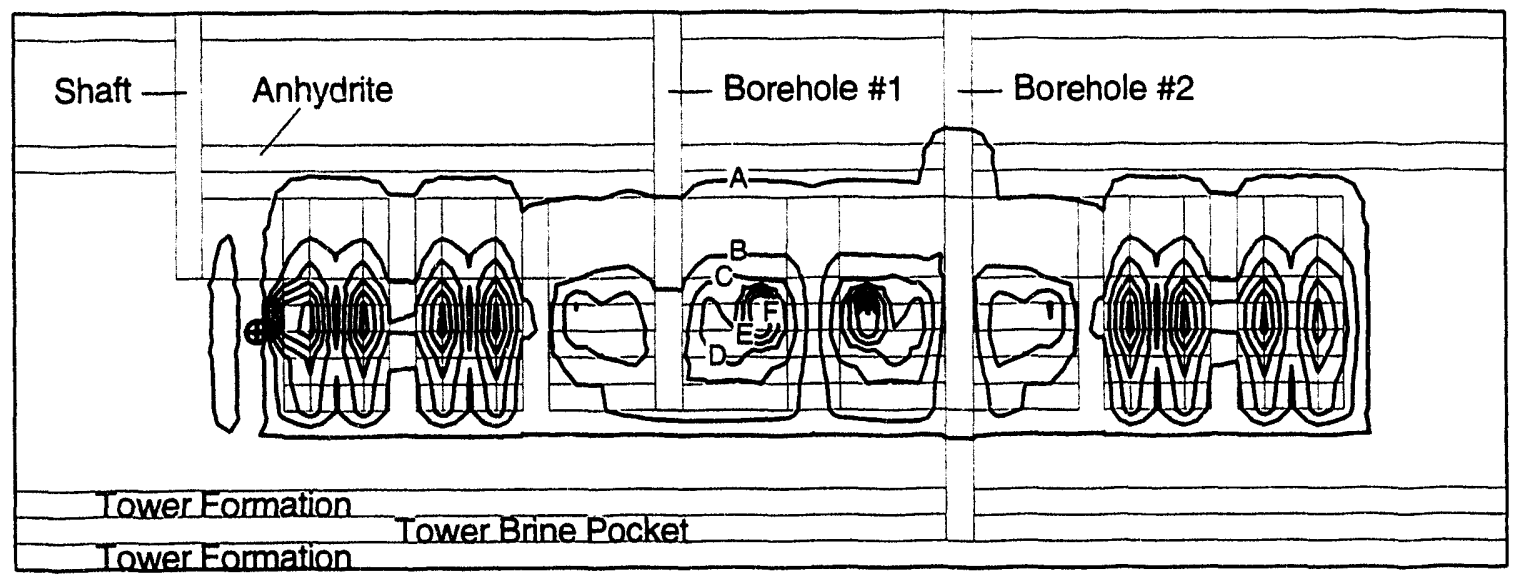

Concentration ${ }^{234} \mathrm{U}(\mathrm{kg} / \mathrm{kg}$ Fluid)

(A) $=0.0050 \times 10^{-6} \quad E=0.4050 \times 10^{-6}$

$B=0.1050 \times 10^{-6} \quad F=0.5050 \times 10^{-6}$

$C=0.2050 \times 10^{-6} \oplus=-0.1347 \times 10^{-6}$

$D=0.3050 \times 10^{-6} \quad$ * $=0.6833 \times 10^{-6}$

Note: $\bigcirc, \square$, Contour Levels Common to Figure $a$ and $b$.

TR1-6342-4114-0

(a) $1000 \mathrm{yr}$

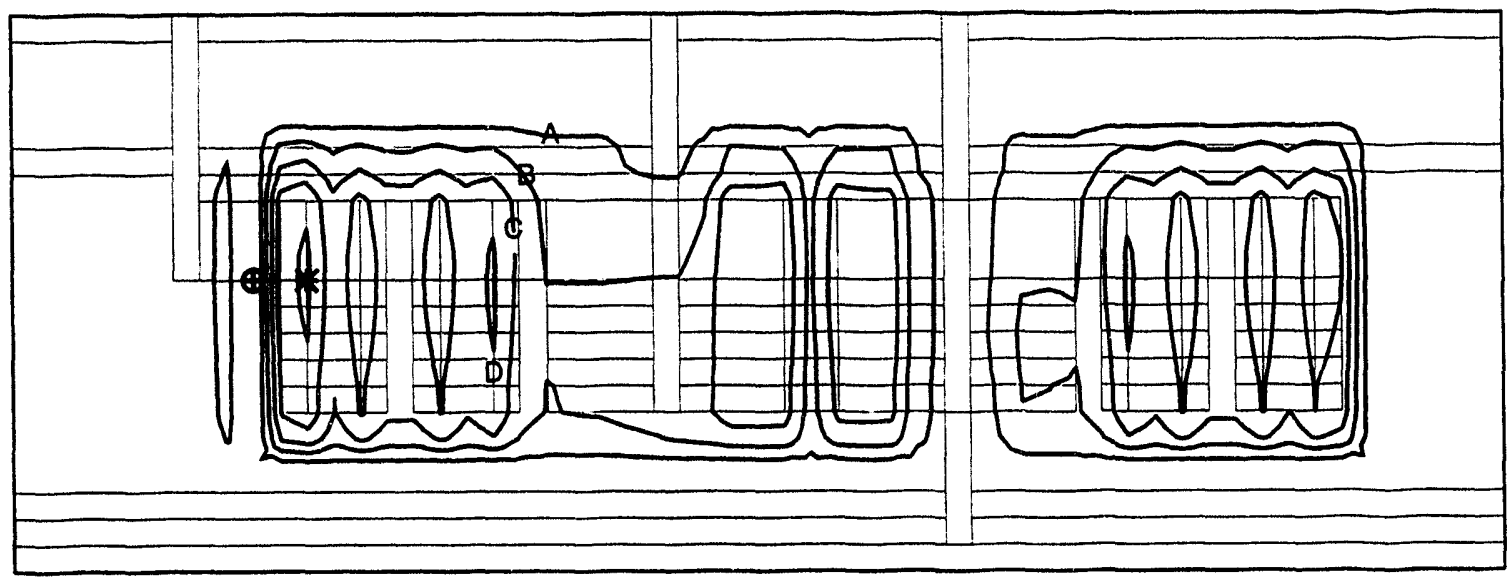

Concentration ${ }^{234} \mathrm{U}$ (kg/kg Fluid)

(A) $=0.0050 \times 10^{-6} \quad E=0.0850 \times 10^{-6}$

$B=0.0250 \times 10^{-6} \quad F=0.1050 \times 10^{-6}$

$C=0.0450 \times 10^{-6} \oplus=-22.350 \times 10^{-6}$

$D=0.0650 \times 10^{-6} \quad$ * $=88.760 \times 10^{-6}$

Note: $\bigcirc, \square$, Contour Levels Common to Figure $a$ and $b$.

TRI-6342-4115-0

(b) $10,000 \mathrm{yr}$

Figure 12-22. Contours of mass fraction of contaminant ( $\mathrm{kg} / \mathrm{kg}$ fluid) for salt repository, Waste Disposal Group 1, Run 17, at (a) $1000 \mathrm{yr}$ and (b) 10,000 yr. 

4

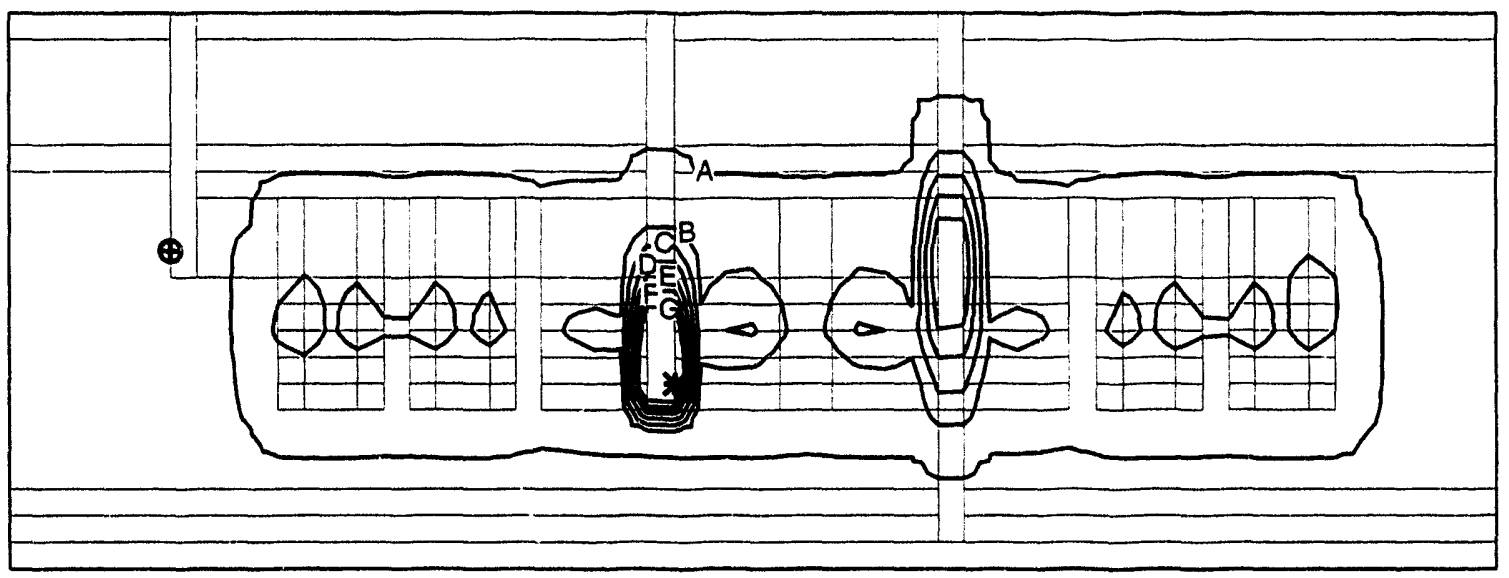

Concentration ${ }^{234} \cup(\mathrm{kg} / \mathrm{kg}$ Fluid)
(A) $=0.10 \times 10^{-6}$
B $=5.10 \times 10^{-6}$
(C) $=10.10 \times 10^{-6}$
$E=20.10 \times 10^{-6}$
$\bigoplus=-0.10 \times 10^{-6}$
$F=25.10 \times 10^{-3}$
* $=44.77 \times 10^{-6}$
$D=15.10 \times 10^{-6}$

Note: $\bigcirc, \square, \bigcirc$, Contour Levels Common to Figure a and $b$.

TRI-6342-4116-0

(a) $1000 \mathrm{yr}$

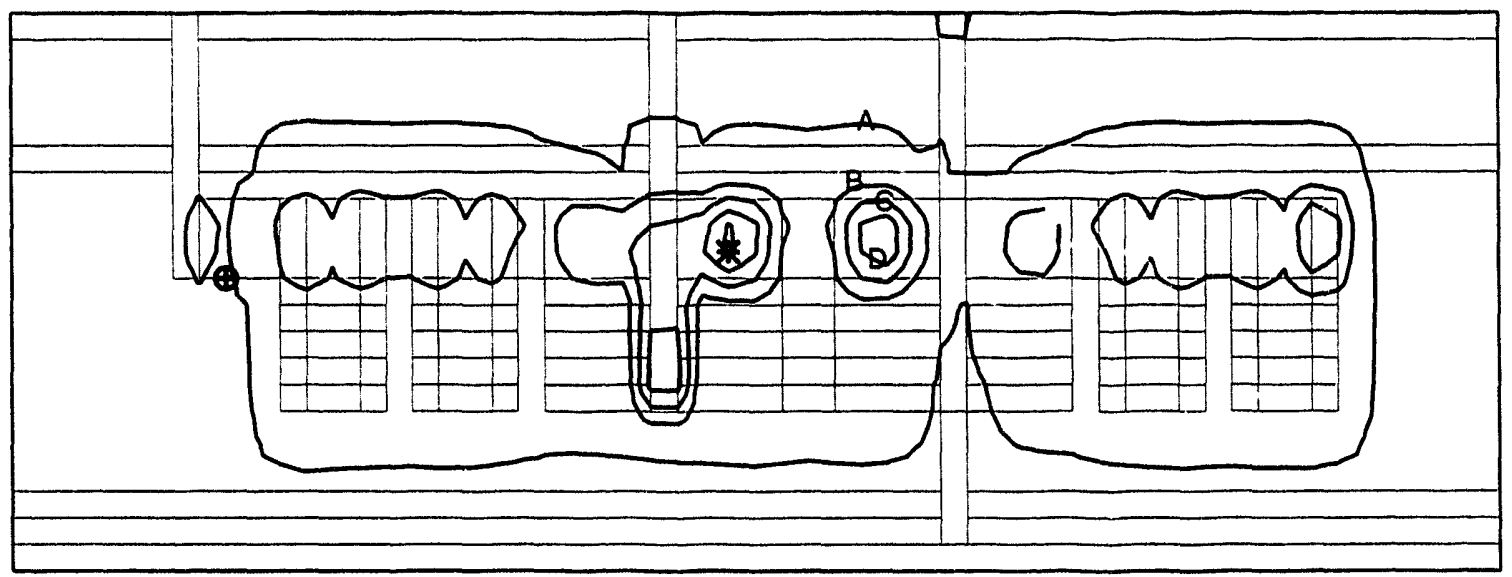

Concentration ${ }^{234} \mathrm{U}$ (kg/kg Fluid)

$$
\begin{array}{ll}
A=0.10 \times 10^{-6} & \mathbb{E}=10.10 \times 10^{-6} \\
B=2.60 \times 10^{-6} & \bigoplus=-0.02 \times 10^{-6} \\
C=5.10 \times 10^{-6} & \mathbb{*}=10.76 \times 10^{-6} \\
D=7.60 \times 10^{-6} & \\
\text { Note: } \bigcirc, \square, \bigcirc, \text { Contour Levels Common to Figure } a \text { and } b .
\end{array}
$$

\section{(b) $10,000 \mathrm{yr}$}

Figure 12-23. Contours of mass of contaminant per element volume ( $\mathrm{kg} / \mathrm{m} 3$ element) for salt repository, Waste Disposal Group 1, Run 17, at (a) 1000 yr and (b) 10,000 yr. 
occurs at the intersection of the left intrusion borehole and the repository. The described transport phenomena are directly attributable to the flow field shown in Figure 12-24. The figi:re shows a vect ${ }^{-n^{-}}$nlot of Darcy velocities or specific fluxes $\left[\mathrm{m}^{3} /\left(\mathrm{s}^{\bullet} \mathrm{m}^{2}\right)\right]$ that are representative of the flow field after intrusion and resaturation of the repository. Note that the vector scale is nonlinear in that a change in vector size by a factor of two represents a factor of ten change in amplitude. It is evident in the figure that groundwater flow is predominantly from the brine reservoir up the right intrusion borehole and out the overlying Dolomite aquifer with some flow from the Dolomite aquifer into the repository through the left intrusion borehole.

The primary results from the simulations used for this performance assessment are the positive discharge rates across a prescribed boundary. For the salt repository, the boundary was specified at two locations: one in each of the two intrusion boreholes just below the intersection with the overlying Snake aquifer. From Figure 12-5, the exact locations of the discharge boundaries correspond to logical z-axis gridblock index 19 . The integrated positive discharges are presented and discussed in Chapter 16 and represent the normalized activity discharge from the repository. Not all of this cumulative normalized activity is released to the accessible environment since the Snake aquifer acts as a significant barrier also.

\subsection{Transport Modeling in Overlying Snake Aquifer}

Radionuclide transport in the overlying Snake aquifer was also performed with the STAFF2D finite-element program. These simulations were performed to estimate groundwater releases to the accessible environment. Transport in the aquifer was modeled using a "dual porosity" conceptual model where flow and transport are assumed to take place in the fractures with a diffusion-controlled exchange of solute between the fractures and the pores of aquifer matrix. This process results in a mechanical retardation of the solute transport.

This performance assessment used 20 flow fields calculated for the Culebra Dolomite aquifer in the 1992 WIPP preliminary performance assessment (Sandia WIPP Project, 1992) as input. A brief discussion is provided below. (Details of the flow calculations can be found in the 1992 WIPP PA documentation). The radionuclide source term (solute mass injection well) was provided by the repository discharges up the two intrusion boreholes as discussed in Section 12.2. The discharges up the two intrusion boreholes were combined and applied as a single injection well

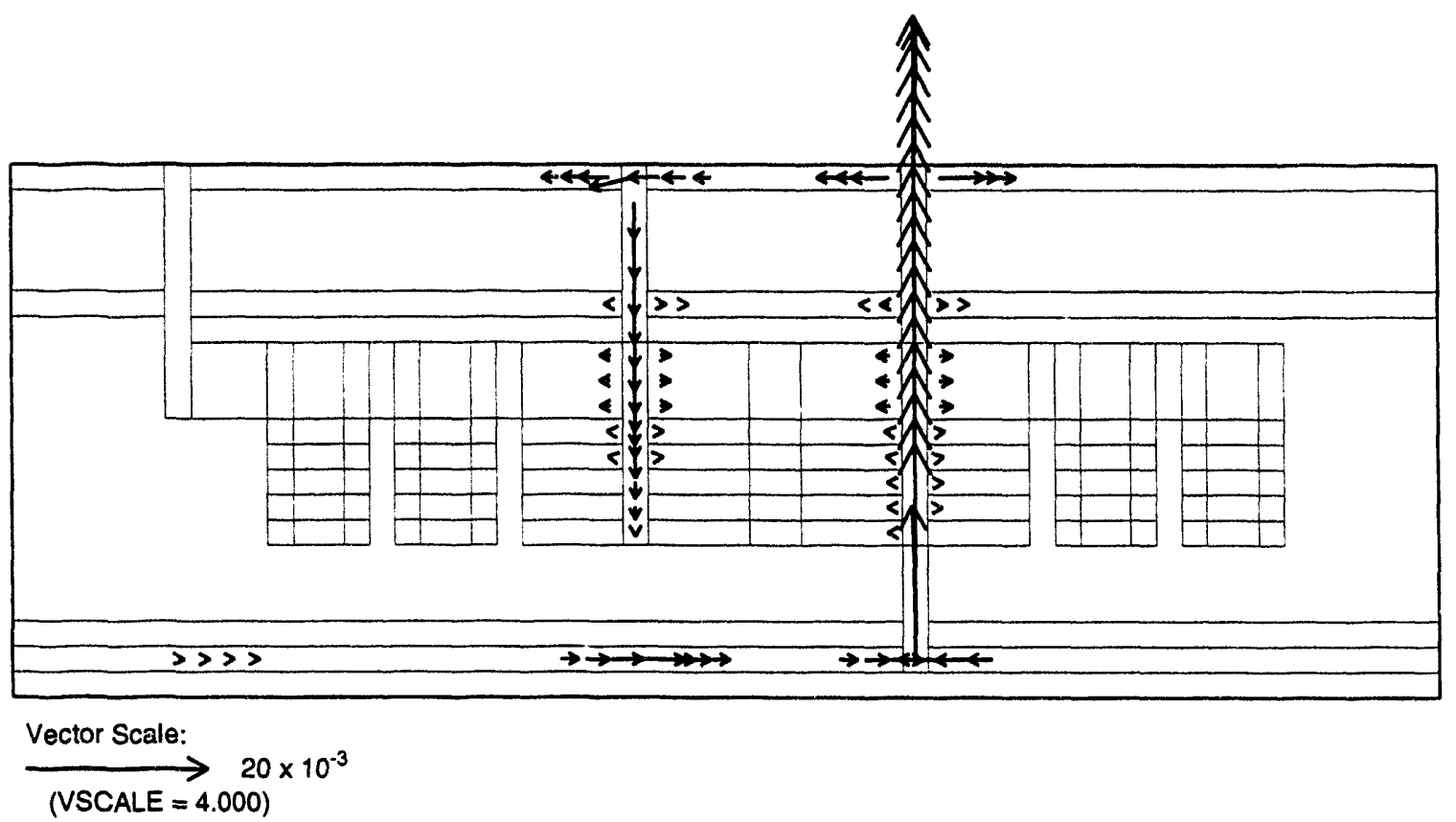

TRI.6342.4110-0

Figure 12-24. Darcy velocity vector plot representative of the flow field after intrusion and resaturation, salt repository, Waste Disposal Group 1, Run 17. 
intersecting the center of the repository. The same 11 radionuclides transported in the repository were also transported in the aquifer.

Snake aquifer transport simulations were run for the 20 repository simulations performed for Waste Disposal Group 1 (Section 4.6.1).

\subsubsection{Mathematical and Computational Model Description}

As described in Section 12.2.1, STAFF2D (Solute Transport and Fracture Flow in 2 Dimensions) is a two-dimensional finite-element code designed to simulate groundwater flow and solute transport in fractured or granular aquifers (Huyakorn et al., 1991). The dual porosity capability was used for the transport in the Snake aquifer as discussed below.

Mass Balance Equations for Dual Porosity Transport. The unsaturated transport mass balance equation assuming variable fluid density, porosity and saturation, linear equilibrium adsorption, and a fluid and solute source as shown in Eq. 12.2-1, was used to model dual-porosity transport using the following assumptions:

- Fluid density is constant so volumetric concentration can be calculated instead of mass fraction.

- The Snake Dolomite aquifer is fully saturated so the water content is equal to the fracture porosity, $\phi_{\mathrm{f}}=\mathrm{b} /$ $\left(b+b_{c}+B\right)$ (see Figure 12-25).

- The solute exchange $\left(\Gamma_{1},\left[\mathrm{~kg} /\left(\mathrm{m}^{3} \cdot \mathrm{s}\right)\right]\right)$ between the fractures and matrix is non-zero.

These assumptions result in the original STAFF2D mass balance equation for transport in the fractures as follows:

$$
\begin{aligned}
& \nabla \cdot\left(\phi_{f} D_{I J}\right) \cdot \nabla c_{l}-\phi_{f} V \cdot \nabla c_{l} \\
& =\phi_{f} R \frac{\partial}{\partial t}\left(c_{l}\right)-\lambda_{l} \phi_{f} R c_{l}+\lambda_{p} \phi_{f} R c_{p}+q\left(c_{l}^{*}-c_{l}\right)+\Gamma_{l}
\end{aligned}
$$

The one-dimensional transport equation for slab geometry and no flow conditions in the porous matrix is as follows:

$$
\frac{\partial}{\partial \chi}\left(D^{\prime} \frac{\partial c_{l}^{\prime}}{\partial \chi}\right)=\phi^{\prime} R_{l}^{\prime} \frac{\partial}{\partial t}\left(c_{l}^{\prime}\right)+\lambda_{l} \phi^{\prime} R_{l}^{\prime} c_{l}^{\prime}-\lambda_{p} \phi^{\prime} R_{p}^{\prime} c_{p}^{\prime}
$$

where

$$
\begin{aligned}
c_{l}^{\prime} & =\text { dissolved concentration of radionuclide } l \text { in matrix }\left[\mathrm{kg} / \mathrm{m}^{3}\right] \\
D^{\prime} & \left.=\text { effective molecular diffusion for radionuclide } l\left[\mathrm{~m}^{2} / \mathrm{s}\right]=\tau^{\prime} \mathrm{Dx} * \text { (see Equation } 12.2-6\right) \\
\phi^{\prime} & =\text { porosity of matrix } \\
R_{2}^{\prime} & =\text { retardation in matrix } \\
& =1+\frac{\rho_{s}\left(1-\phi^{\prime}\right) k_{d}^{\prime}}{\phi^{\prime}} \\
\rho_{s} & =\text { density of matrix solid }\left(\mathrm{kg} / \mathrm{m}^{3}\right) \\
K_{d}^{\prime} & =\text { partition coefficient for matrix }\left(\mathrm{m}^{3} / \mathrm{kg}\right) \\
\tau^{\prime} & =\text { tortuosity of matrix }(\mathrm{m} / \mathrm{m})
\end{aligned}
$$

For this performance assessment, no chemical retardation was used $\left(K_{d}^{\prime}=0 ; R^{\prime}=1.0\right)$. In addition, the solute flux at the center of the matrix block $(\chi=0)$ is zero, i.e., $\frac{\partial c^{\prime} l}{\partial \chi}(x, y, 0, t)=0$ 


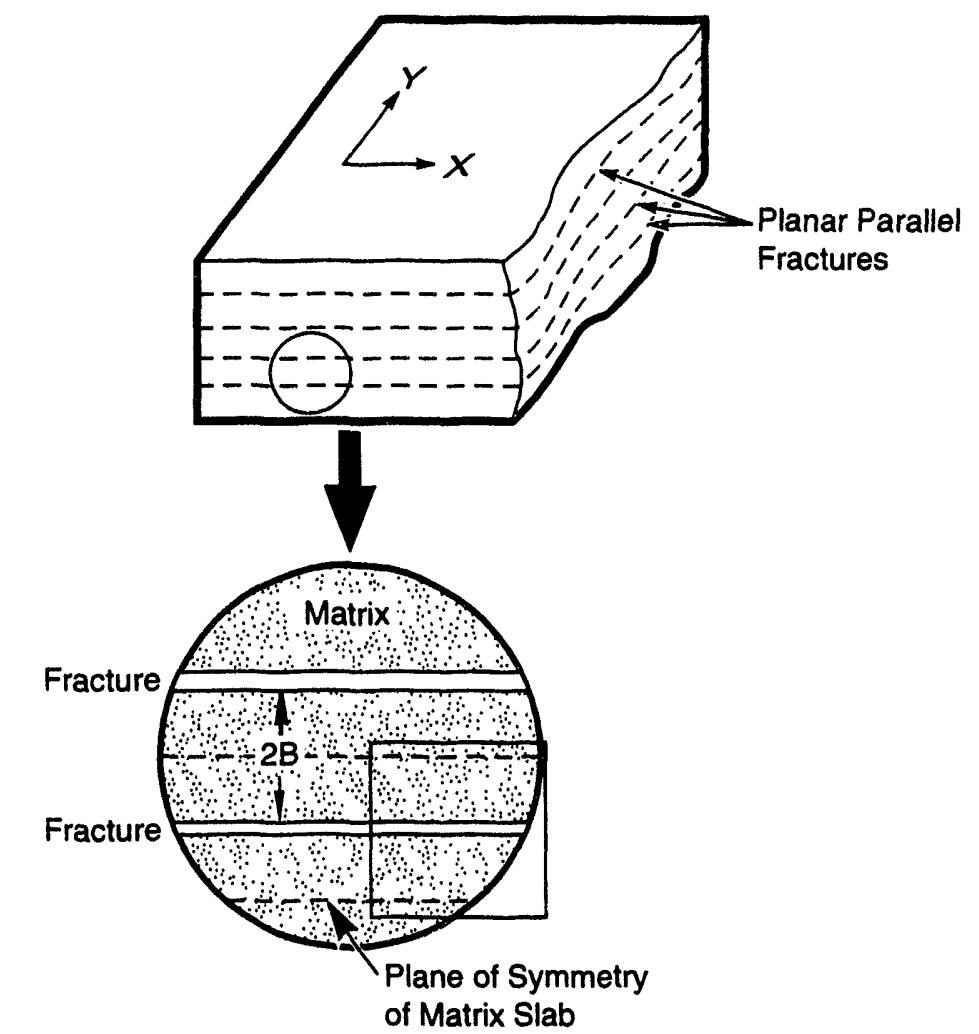

Detail for Fracture Spacing, 2B (Not To Scale)

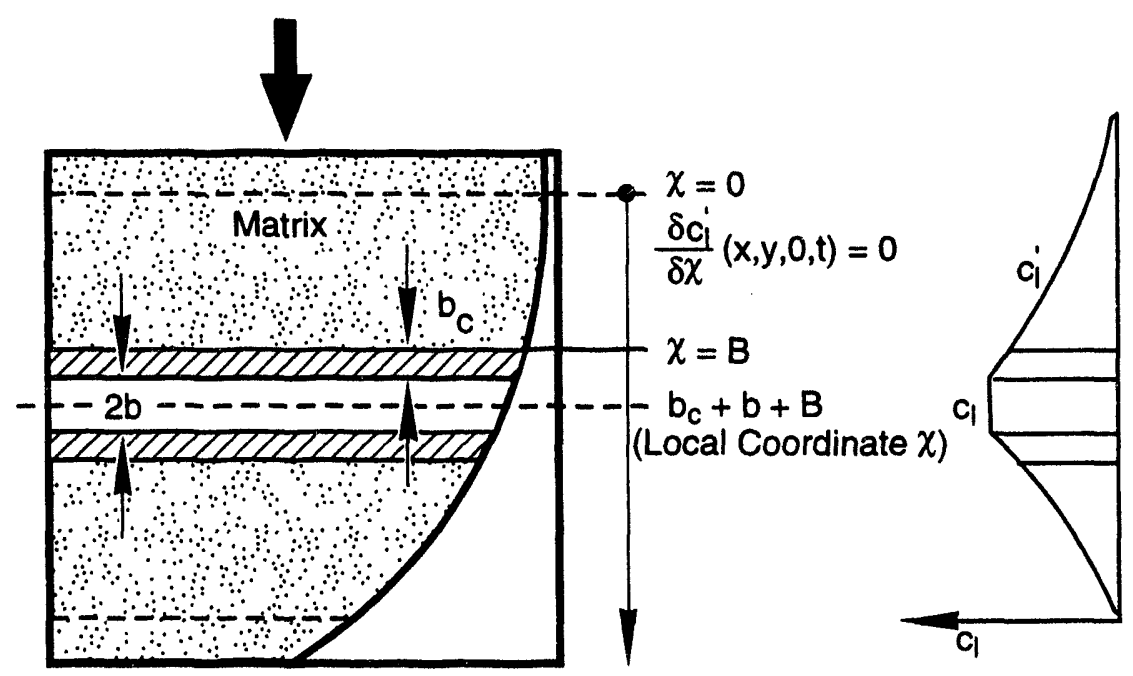

Detail for Fracture Aperture, 2b, Clay Coating

Thickness, $b_{c}=0$ for this PA, and Local Coordinate $\chi$. (Not To Scale)

TRI- 6342-1436-0

Figure 12-25. Conceptual hydrologic model of the Snake Dolomite Member. 
The equation for transport in the fractures (Equation 12.3-1) and transport in porous matrix (Equation 12.3-2) equates the flux at the surface of the matrix $D^{\prime} \partial c_{2}^{\prime} / \partial \chi$ to flux from the fluid in the fracture, $\Gamma_{l}$. The linkage must convert from the mass in the matrix control volume to the mass in the fracture/matrix control volume $\left(1-\phi_{f}\right)$ and the surface flux rate to a volume flux rate by multiplying by the ratio of surface area to volume $(\sigma)$. Hence,

$$
\begin{gathered}
\Gamma_{l}=-\left(\frac{D^{\prime} \partial c_{l}^{\prime}(x, y, B, t)}{\partial \chi}\right)\left(1-\phi_{f}\right) \sigma \\
\Gamma_{l}=\frac{1}{b+B}\left(\left.D^{\prime} \frac{\partial c_{l}^{\prime}}{\partial \chi}\right|_{\chi=B}\right)
\end{gathered}
$$

The corresponding boundary is that $c_{l}^{\prime}(\mathrm{x}, \mathrm{y}, \mathrm{B}, \mathrm{t})=c_{l}(\mathrm{x}, \mathrm{y}, \mathrm{t})$.

Assumptions and Limitations. The assumptions and limitations of the model are as follows:

- Transport is governed by Fick's law.

- Two dimensions adequately model phenomena.

- The dispersivity is assumed to correspond to an isotropic porous medium so that it can be represented by two constants in the principle directions of flow.

- Adsorption does not occur in matrix or fracture in Snake Dolomite, $\mathrm{Kd}=0$.

- No clay layer is in fractures.

- Solute effects on fluid density are assumed negligible so flow and transport can be decoupled.

- There is local chemical equilibrium between the liquid and the solid.

- For fractured systems, the fracture aperture is small compared to other dimensions in the fracture plane.

Numerical Approach. The finite-element approximation technique applied to the convective-dispersive equation (12.3-1 and 12.3-3) is an upstream-weighted technique (Huyakorn and Pinder, 1983) designed to overcome oscillations of the numerical solutions when the convective terms are dominant. The standard Galerkin approximation technique is used for the one-dimensional matrix transport.

A three step, direct sequential solution scheme is used to couple the fracture and matrix transport equations. The first step uses the Thomas algorithm to derive the flux term from the 1-D matrix equations. The second step involves incorporating the flux term into the fracture domain equations and performing a banded matrix solution to determine fracture concentrations. Finally, Step 3 involves determining the porous matrix concentrations by performing back substitution in the Thomas algorithm.

\subsubsection{Applied Model}

Model Geometry and Spatial Discretization. The Snake aquifer transport model geometry was identical to the local-scale flow geometry used in the 1992 WIPP preliminary performance assessment modeling of the Culebra Dolomite aquifer. In the WIPP PA, the Culebra flow simulations were run in two steps on two domains: a regional domain $(25 \times 30 \mathrm{~km})$ and an "embedded" local domain $(5.75 \times 6.625 \mathrm{~km})$ (see Figure 12-26). The regional grid domain was sized and oriented so its boundaries coincided with natural boundaries of the aquifer where climate effects could be incorporated. Grid-block dimensions varied from $50 \mathrm{~m}$ in the vicinity of the repository to $2800 \mathrm{~m}$ at the grid boundaries. The local grid domain was oriented north-south and sized to cover the repository, land withdrawal boundaries and the expected variability in flow paths and solute plumes originating at the intrusion borehole centered over the repository. All grid blocks in the local domain were $125 \times 125 \mathrm{~m}$. The thickness of the aquifer was $7.7 \mathrm{~m}$. The actual grids are shown in Figure 12-26.

Time discretization. Time discretization for the transport simulations was $500 \mathrm{yr}$, or 20 steps over the 10,000 -yr modeling period. 


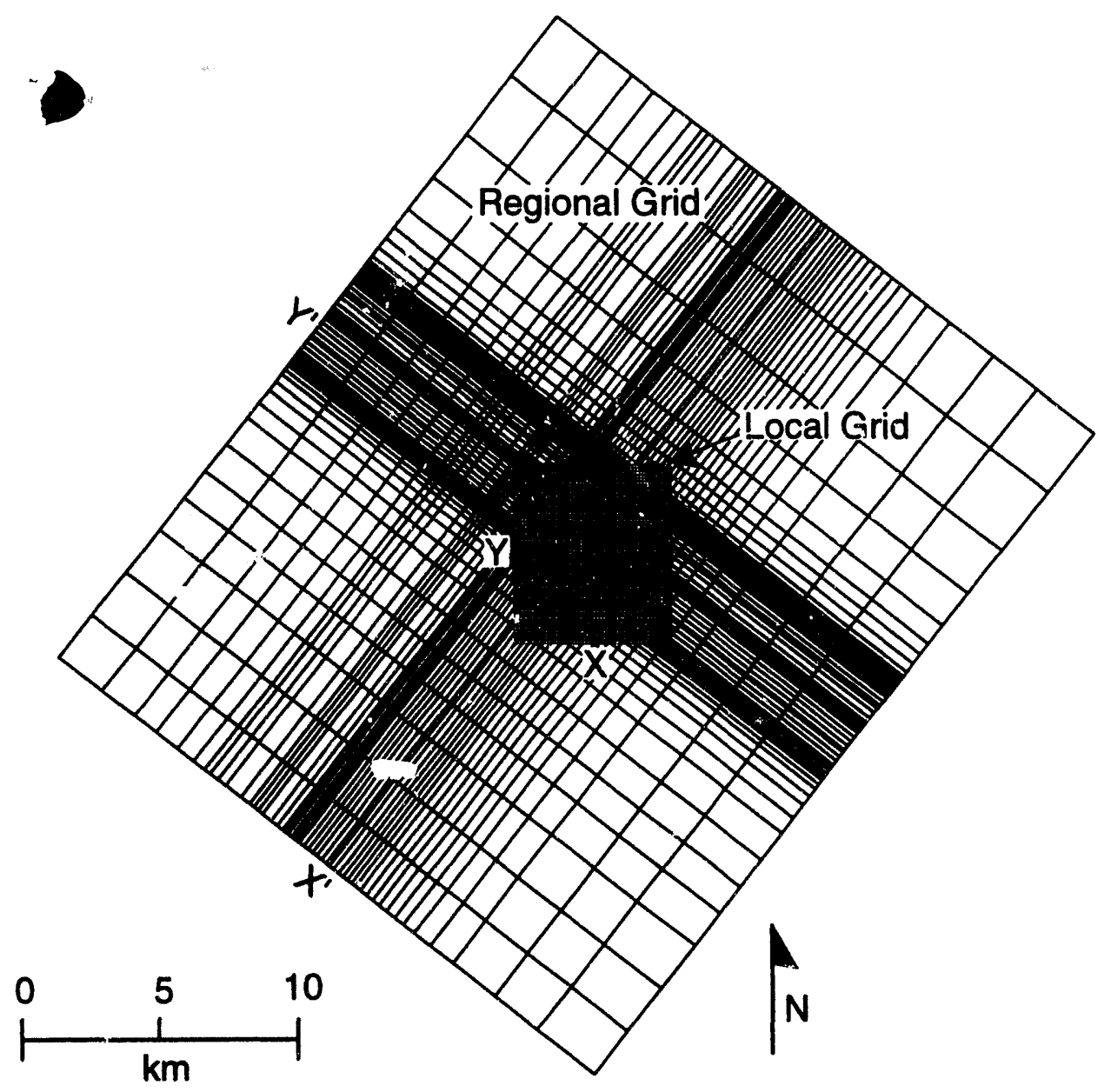

TRI-6342-2680-0

Figure 12-26. Example of regional and local grids used for disturbed fluid flow and transport calculations.

\subsubsection{Initial and Boundary Conditions}

A combination of fixed head, variable head, and no flow computational grid boundary conditions was used in the regional-scale flow calculations. The variable head was applied at the northwest corner of the regional grid to simulate climate effects. Time-dependent head boundary conditions were applied on the local-scale grid boundaries. These head values were interpolated from the calculated regional-scale head values. For the transport simulations, a zero concentration gradient boundary condition is assumed at all external, computational, grid boundaries, which allows for advection at the boundaries but ot dispersion. Solute is allowed to flow out of the grid but not into the grid.

Initial conditions for the flow calculations on both the regional and local scales were determined by a steady state solution assuming the boundary conditions at time zero. For transport, the initial concentrations were assumed to be zero: $c_{i}(x, y, \chi, 0)=0 ; c_{l}(x, y, 0)=0$.

The source term is implemented in STAFF2D as an interior grid point mass flux boundary condition (injection well). Originally, the user supplied a rate history in tabular form. For this performance assessment the rate histories were the repository discharges generated by the STAFF2D repository calculations discussed in Section 12.2. The positive (upward) discharges in the two intrusion boreholes were summed and injected into the aquifer flow at a 
single point centered over the repository. The fluid flow is assumed to have a negligible affect on the aquifer flow; only the solute mass is injected.

\subsubsection{Material Properties}

Flow Properties. The 20 flow fields, which originated with the WIPP performance assessment, include the effects of sampling several Culebra Dolomite parameters (which correspond to the Snake Dolomite). For this performance assessment, the sensitivity to these sampled parameters is ignored. The parameters are included to provide spatial and travel time variability to the Snake aquifer flow. For flow, the critical parameter is the transmissivity field. The WIPP performance assessment employs a multiple-realization technique to account for spatial variability of the transmissivity field within the corresponding Culebra Dolomite. The technique uses an automated inverse approach to calibrate a two-dimensional model to both steady-state and transient pressure data. The technique can be broken down into three steps:

1. Unconditional Simulation. An unconditional simulation of the WIPP transmissivity fields is generated. This is a random field that has the same spatial correlation structure as the transmissivity measurements, but does not necessarily match measured transmissivities at the location of their measurements.

2. Conditional Simulation. The random field produced in Step 1 is conditioned in this step so that it honors exactly the measured transmissivities at the locations of their measurements. The resulting field, called a "conditional simulation" of the transmissivity field, is used as the initial estimate of the Culebra transmissivity field.

3. Automated Calibration. The conditional simulation of the transmissivity field is then calibrated so that the pressures computed by the groundwater-flow model (both steady and transient state) agree closely (calibrated within the uncertainty in head measurements, i.e., between 1 and $2 \mathrm{~m}$ ) with the measured pressures in a leastsquare sense. Calibration is achieved by placing synthetic transmissivity values (pilot points) automatically where the sensitivity of the difference between observed and calculated pressure to changes in the transmissivity field is greatest. When calibration is completed, a conditionally simulated transmissivity field is obtained that conforms with all head and transmissivity data at the WIPP site and may be regarded therefore as a plausible version of the true distribution of transmissivity.

This process is repeated to produce the desired number of calibrated, conditionally simulated fields (LaVenue and RamaRao, 1992; Sandia WIPP Project, 1992). The travel times to the 3-km land-withdrawal boundary were ranked and the corresponding index was sampled.

Figure 12-27 shows the spatial variability of the flow fields as the paths followed by a neutrally buoyant particle released at the injection well for each of the 20 flow simulations. The time variability is shown in Figure 12-28 as a graph of travel times. Actual travel times are bounded by the fracture and matrix travel times shown in the figure due to the mechanical retardation (diffusion in and out of the matrix) of the dual porosity conceptual model.

Transport Properties. Three sets of material properties are required for the STAFF2D dual porosity transport simulations: fracture properties, porous media properties and radionuclide properties. Fracture and porous media properties include porosity, tortuosity, longitudinal dispersivity and transverse dispersivity and fracture spacing. The 1992 WIPP PA values were used (see Sandia WIPP Project, 1992, for details). Fracture porosity, matrix porosity, and block spacing were sampled. Dispersitivites were varied from element to element based on the element dimensions. Nuclide properties include half life, specific activity, chain descriptions, and effective diffusion coefficients. No chemical retardation was used $\left(R_{l}=1.0\right)$.

The same 11 radionuclides included in the repository transport were included in the aquifer transport (see Section 12.2.5). 
12. Complex Consequence Modeling for Salt Disposal System

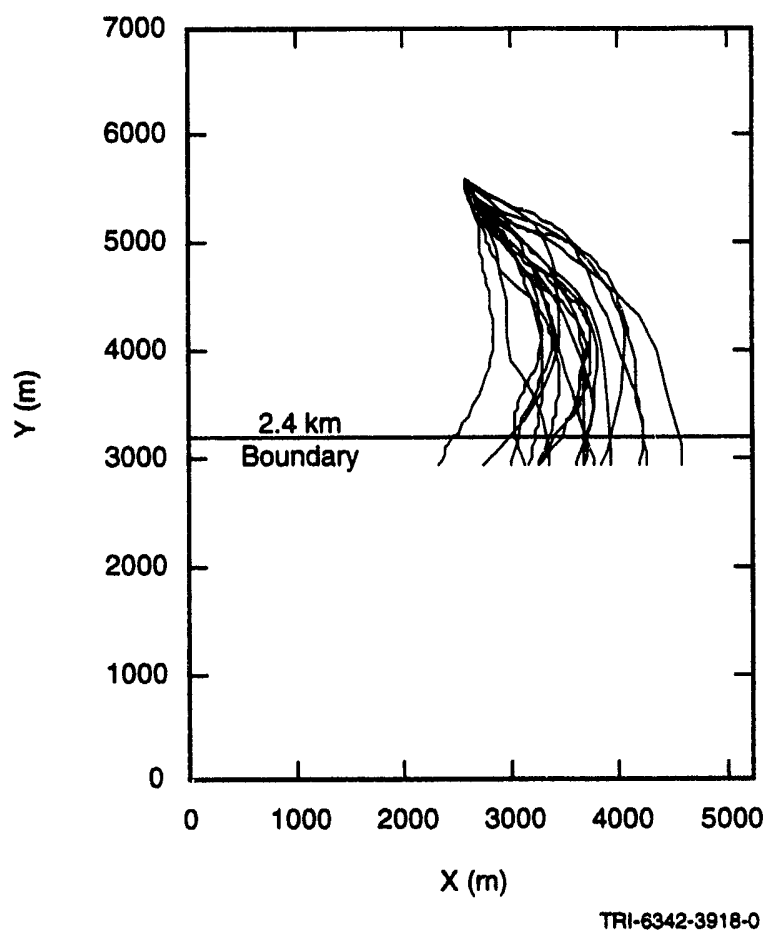

Figure 12-27. Particle paths for neutrally buoyant particle released at intrusion borehole centered in repository for 20 flow fields used in Snake Aquifer transport simulations.

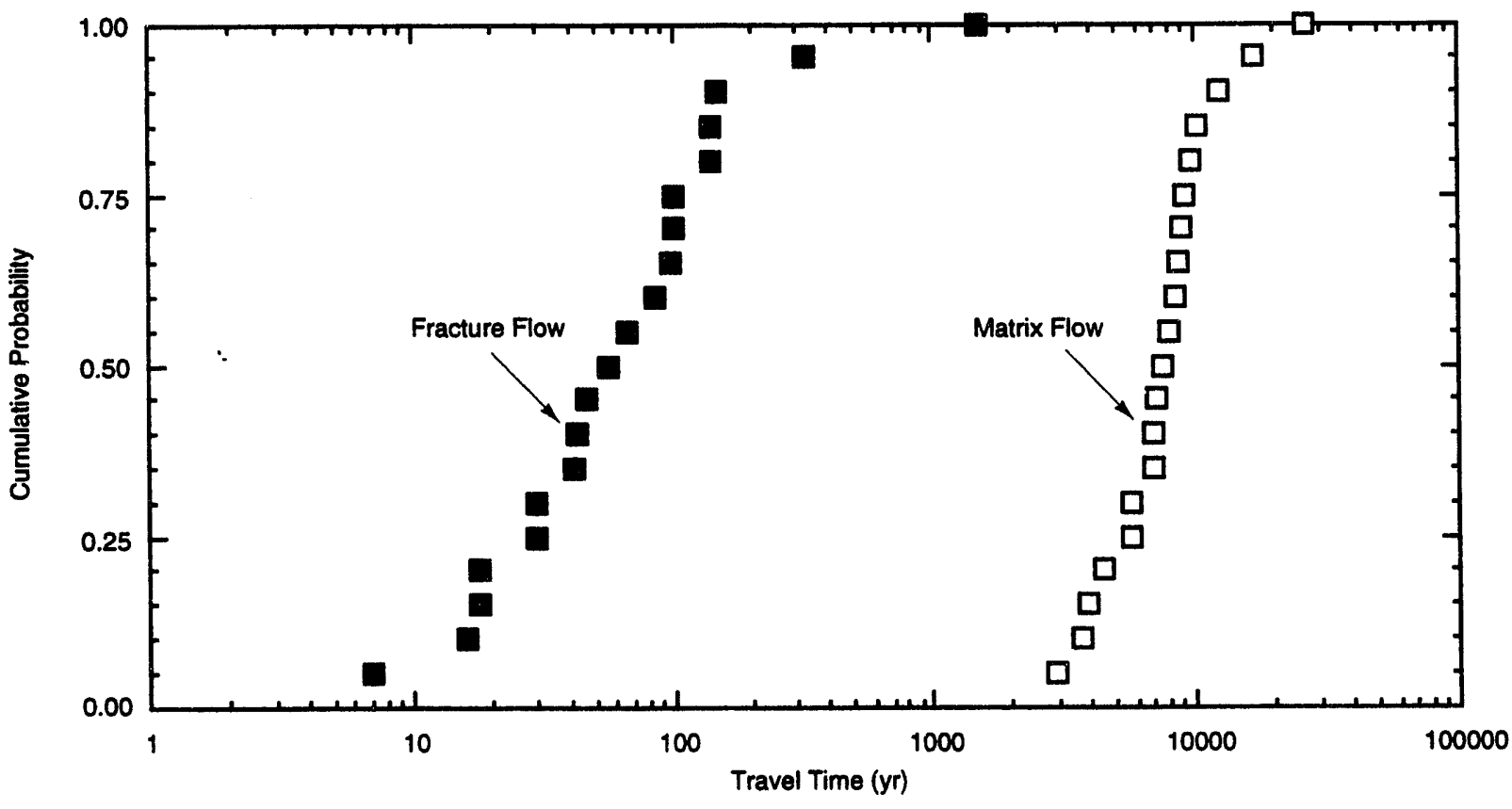

Figure 12-28. Travel times to 2.4-km boundary of Snake Aquifer (using first 20 realizations from WIPP 1992 performance assessment), assuming flow through fractures (as assumed in this performance assessment) versus flow through porous matrix (alternative conceptual model not used in this performance assessment). 


\subsubsection{Intermediate Results}

Typical solute contours showing a well developed plume are presented in Figure 12-29 at 1000 and 10,000 yr. 
12. Complex Consequence Modeling for Salt Disposal System

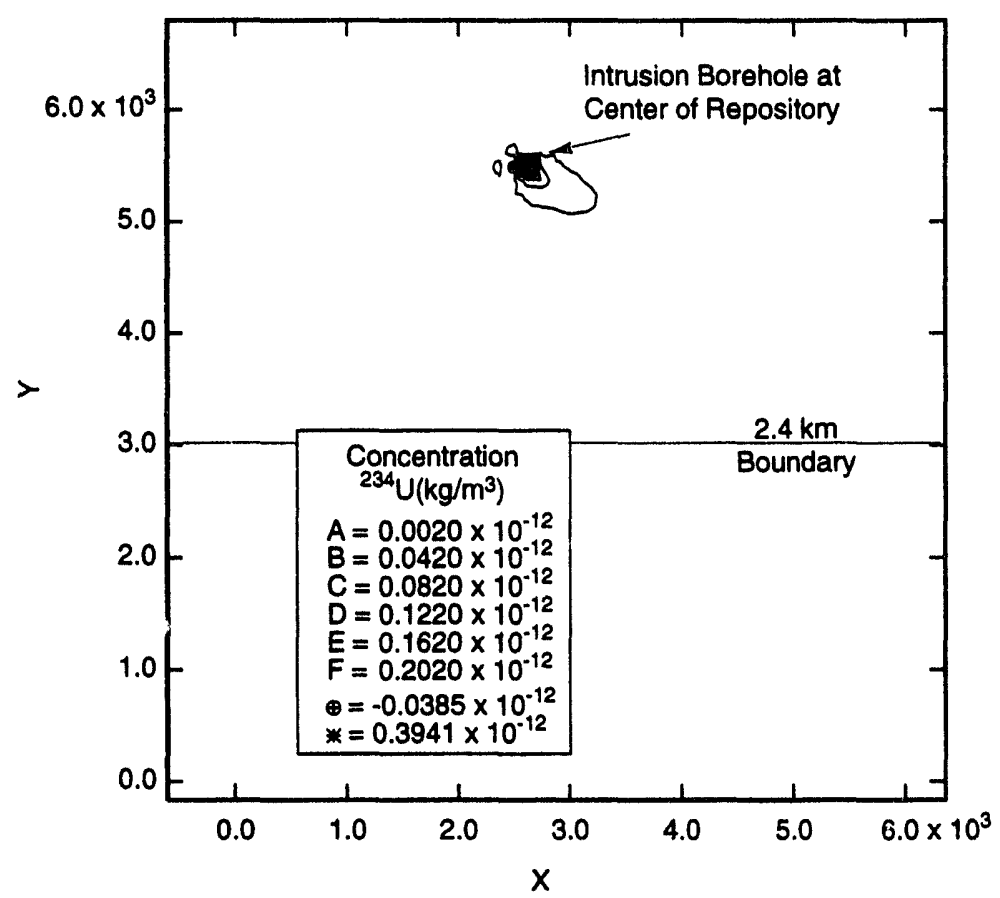

TRI-6342-4106-0

(a)

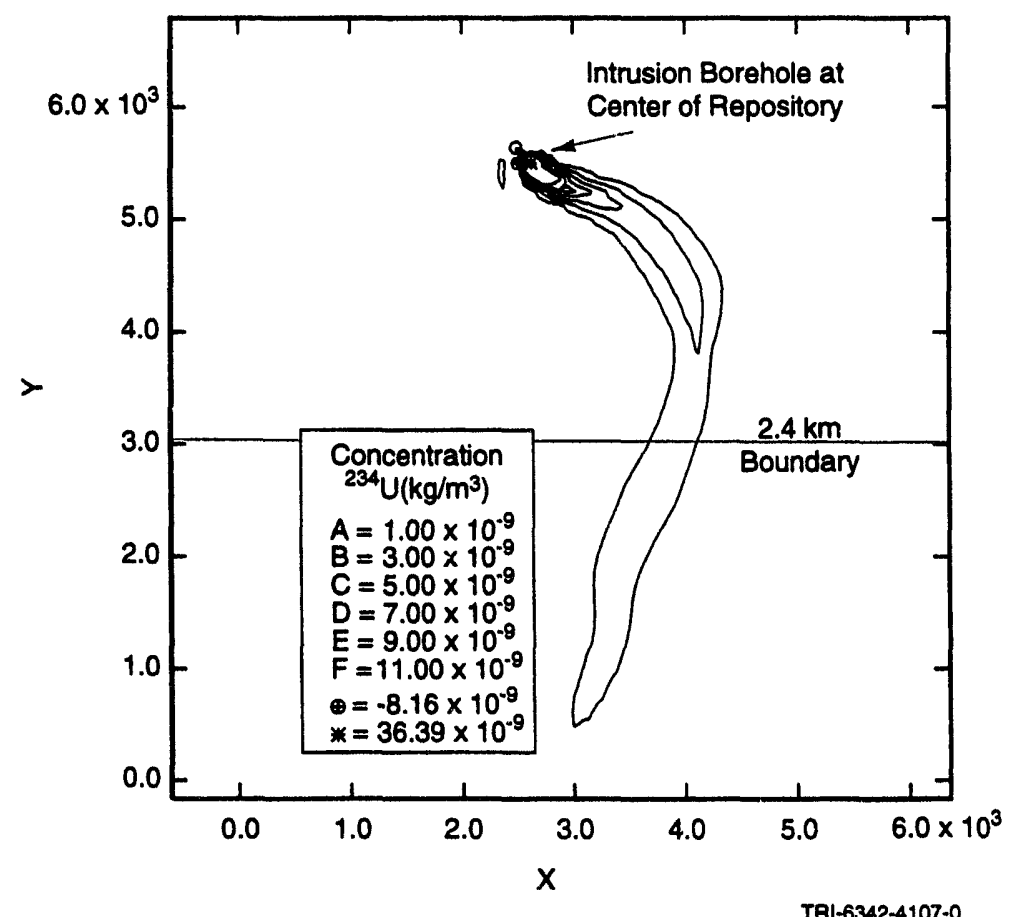

(b)

Figure 12-29. Concentration $\left(\mathrm{kg} / \mathrm{m}^{3}\right)$ contours for ${ }^{234} \mathrm{U}$ in Snake Aquifer at (a) $1000 \mathrm{yr}$ and (b) $10,000 \mathrm{yr}$ for Waste Disposal Group 1, salt disposal system. 


\title{
13. Complex Consequence Modeling for Granite Disposal System
}

\author{
J. L. Ramsey, D. K. Rudeen, and D. M. Stoelzel
}

The Complex performance assessment (PA) for the granite disposal system used the same methods as the salt disposal system (Chapter 12). The consequence models for the granite repository are similar to those used in the salt repository; exceptions are discussed below.

\subsection{Fluid Modeling in Disposal System}

\subsubsection{Mathematical and Computational Model Description}

The mathematical and computational model used to predict energy and two-phase flow in the vicinity of the granite repository is the same as the model of the repository described for disposal in salt (Section 12.1.1); however, the repository scale model was also used to simulate flow to the accessible environment without using an intervening larger scale model domain as was done for the salt disposal system.

\subsubsection{Applied Model}

Model Geometry and Spatial Discretization. A three-dimensional conceptualization of the proposed granite repository is shown in Figure 13-1. For reasons discussed in Chapter 12, it was necessary to model the granite repository in two rather than three dimensions. This was accomplished through the use of element flaring and volumetrically averaged material properties (see Sections 12.1 .2 and 12.1.3).

As with the salt repository, regions within the repository were finely meshed to model fluid transport and corrosion of the waste parcel. Near the boundaries of the mesh, the size of the elements increases dramatically. To emphasize the relatively large elements and discuss the flared elements used in this analysis, an artistic representation of a planar view at the level of the repository ceiling is shown in Figure 13-1b. Unlike the logical grid discussed later, in this plan view, the element sizes are meant to be somewhat representative of the actual size.

Although not a necessary requirement for modeling with BRAGFL,O_T, the radial geometry was maintained for the entire thickness ( $z$ direction) of the mesh. While appropriate in most of the mesh, it does imply radial flow in Fracture Zones 1 and 2. This radial flow assumption may be appropriate if, as assumed here, the density of the fractures is large, the distribution of the fractures is isotropic, and the fractures are highly connected hydrologically (Barker, 1988). The relatively high permeability values measured in the two fracture zones, $10^{-13}$ and $5 \times 10^{-13} \mathrm{~m}^{2}$, somewhat support this assumption. For evaluating total activity released, this assumption of radial flow is unimportant since activity released is integrated across the entire boundary; in contrast, this assumption could be indirectly important in that the travel times to the boundary are possibly not conservatively estimated; however, even in this case the effect is probably minor since in this PA analysis no retardation was assumed to occur in the fracture zones; thus, travel times were short relative to $10,000 \mathrm{yr}$.

Within the repository, elements extend the width of the repository and were placed along the features within it. (Compare the plan view [Figure 13-1b] with the detailed drawing of the repository [Figure 6-3].) For the most part, elements contained two or three different materials (backfill, host rock, canister) with independent fluid flow and thermodynamic properties. As a result, volume-averaged parameters were used to represent these elements. In Figure 13-1b, the intrusion borehole is shown as a small white dot in the center of the middle panel group. As in the salt repository, flared elements were used around the borehole to capture the geometry of the borehole and local radial fluid flow (Figure 13-2). 


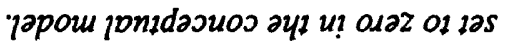

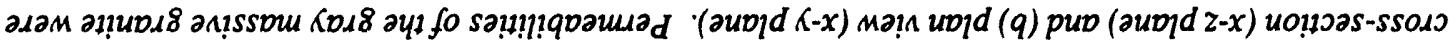

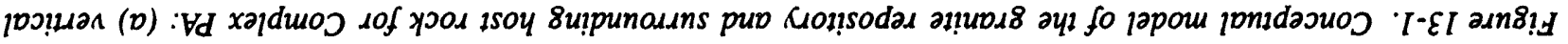

(q)

'I-EI aมn8! $]$

(q)

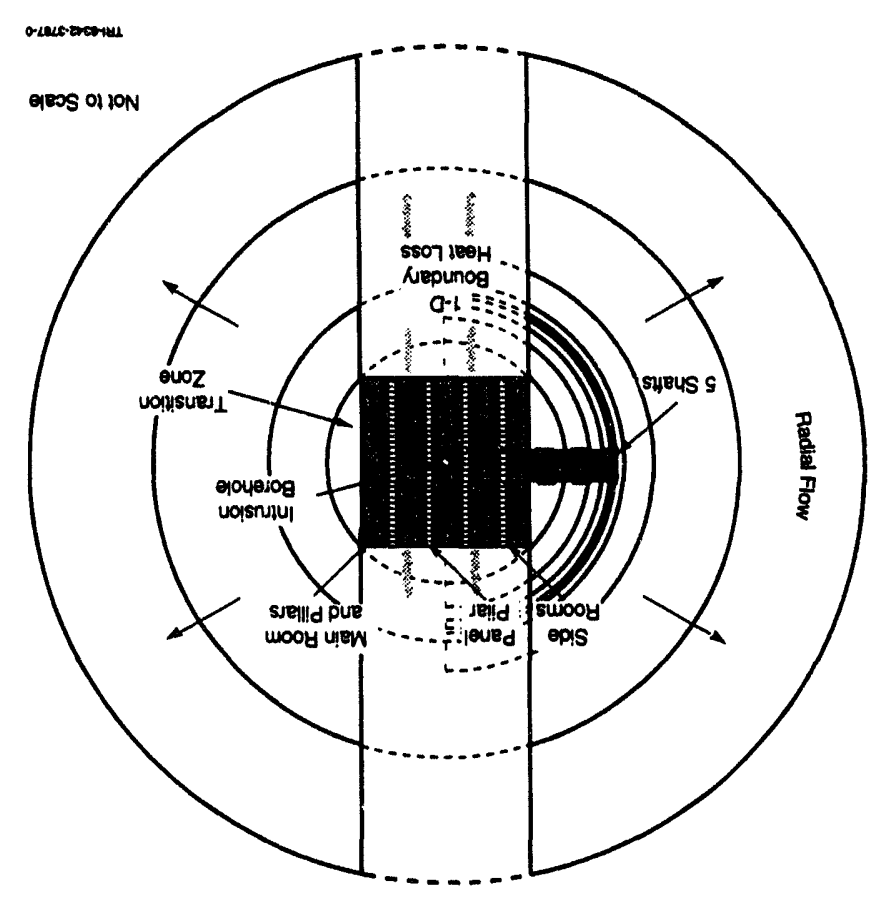

(e)

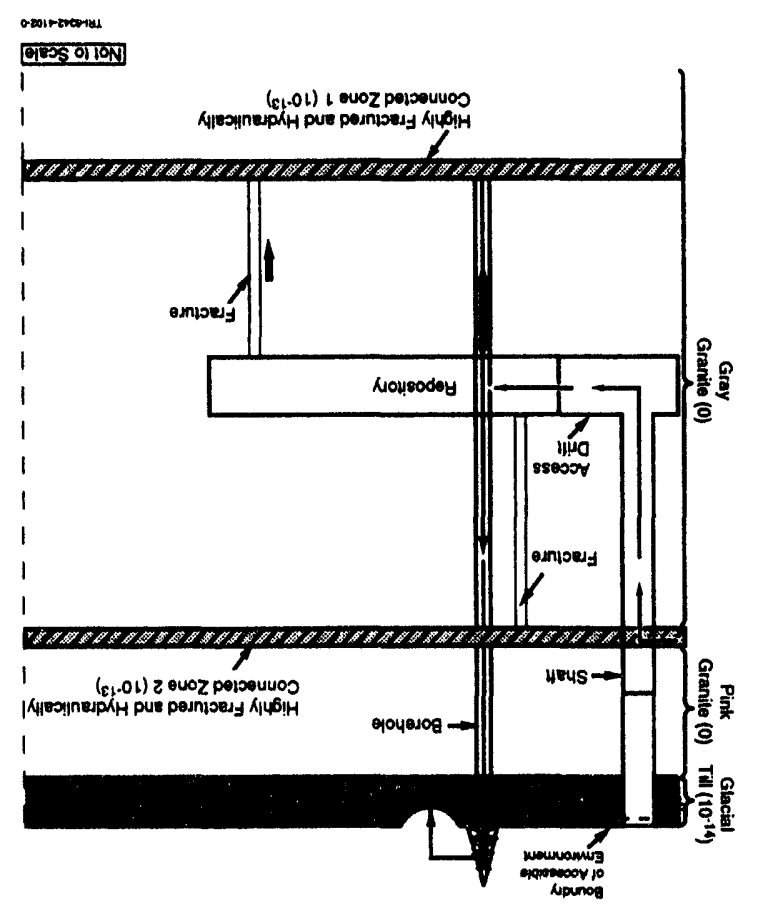




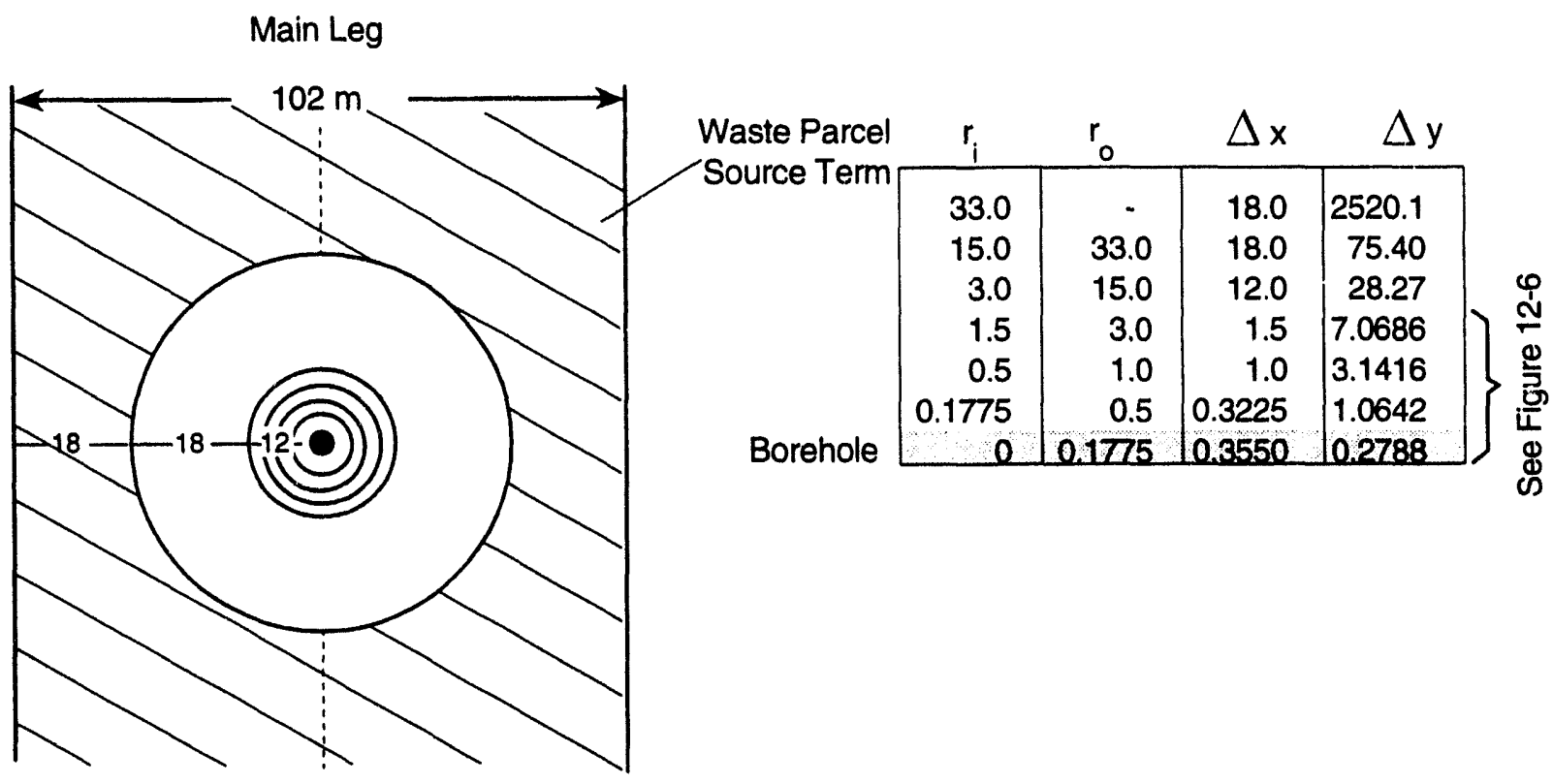

TRI-6342-3786-0

Figure 13-2. Plan view of borehole for granite repository centered in an element of the main legs of rooms in panels 3 and 8 . The fluid-flow properties of the mesh are the same throughout this portion.

Logical Mesh for Vertical Cross-Section. A vertical cross-section (x-z plane) through the conceptual model, showing the major features modeled and the computational grid, is presented using a logical grid (discussed in Section 12.1.2) in Figure 13-3. The granite repository contains more grid blocks along the vertical z-axis than does the salt repository, resulting in a finite-difference grid of 56 by 23 elements or 1288 elements total.

In a manner similar to the salt repository, regions containing waste parcels were finely meshed so that corrosion of the waste parcels could be investigated. Because of differences in the length of the waste parcels in each waste disposal group, the mesh was modified for each group. However, the sum of the dimensions in the z-direction of blocks 7 through 12 was always $10 \mathrm{~m}$, as occurred in the salt repository.

Fluid and heat inw were modeled from the surface to $275 \mathrm{~m}$ below Fracture Zone 1 and $2.4 \mathrm{~km}$ away from the repository horizontally. In addition to the geologic features and the repository, two undetected fractures were also included in the model; the undetected fractures connected the repository to Fracture Zones 1 and 2. Also, a single intrusion borehole was assumed to penetrate through the center of the repository, $1000 \mathrm{yr}$ after emplacement of the waste.

Grouping of Components in the Repository. There are two distinct differences between the discretization used in the salt disposal system and the discretization used in modeling the granite disposal system. First, volumetrically averaged properties were used to represent the shaft and the adjacent granite. Doing so eliminated the need to displace the transitional and flared elements on the shaft side of the repository. Second, the flared elements surrounding the repository do not wrap around the entire repository, $\Theta<\pi$ (Figure 13-1b). (Fluid flow through the host rock surrounding the repository could be neglected in the granite repository, but not in the salt repository.) This change allowed a one-dimensional boundary heat loss condition to be used on the sides of the repository. Both changes more realistically modeled heat transport by allowing heat to more easily dissipate from the repository.

Width of Elements in Plan View. The three-dimensional conceptual model was converted into two dimensions by translating the radial elements into rectangular elements using Equations 12.1-41 and 12.1-42. The converted onedimensional $x-y$ cross-section is shown in Figure 13-4. The disposal region of the repository remained exactly as 


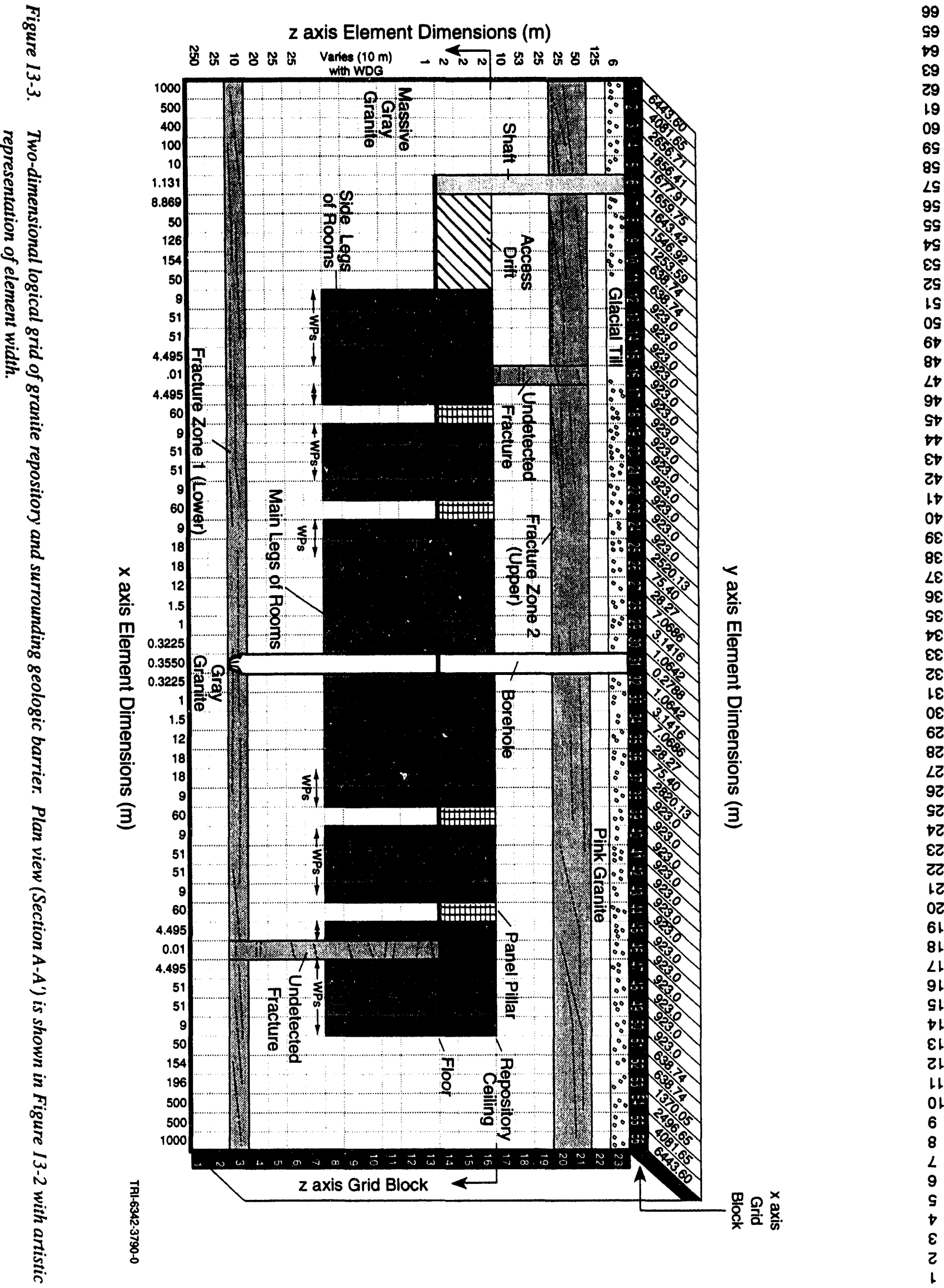




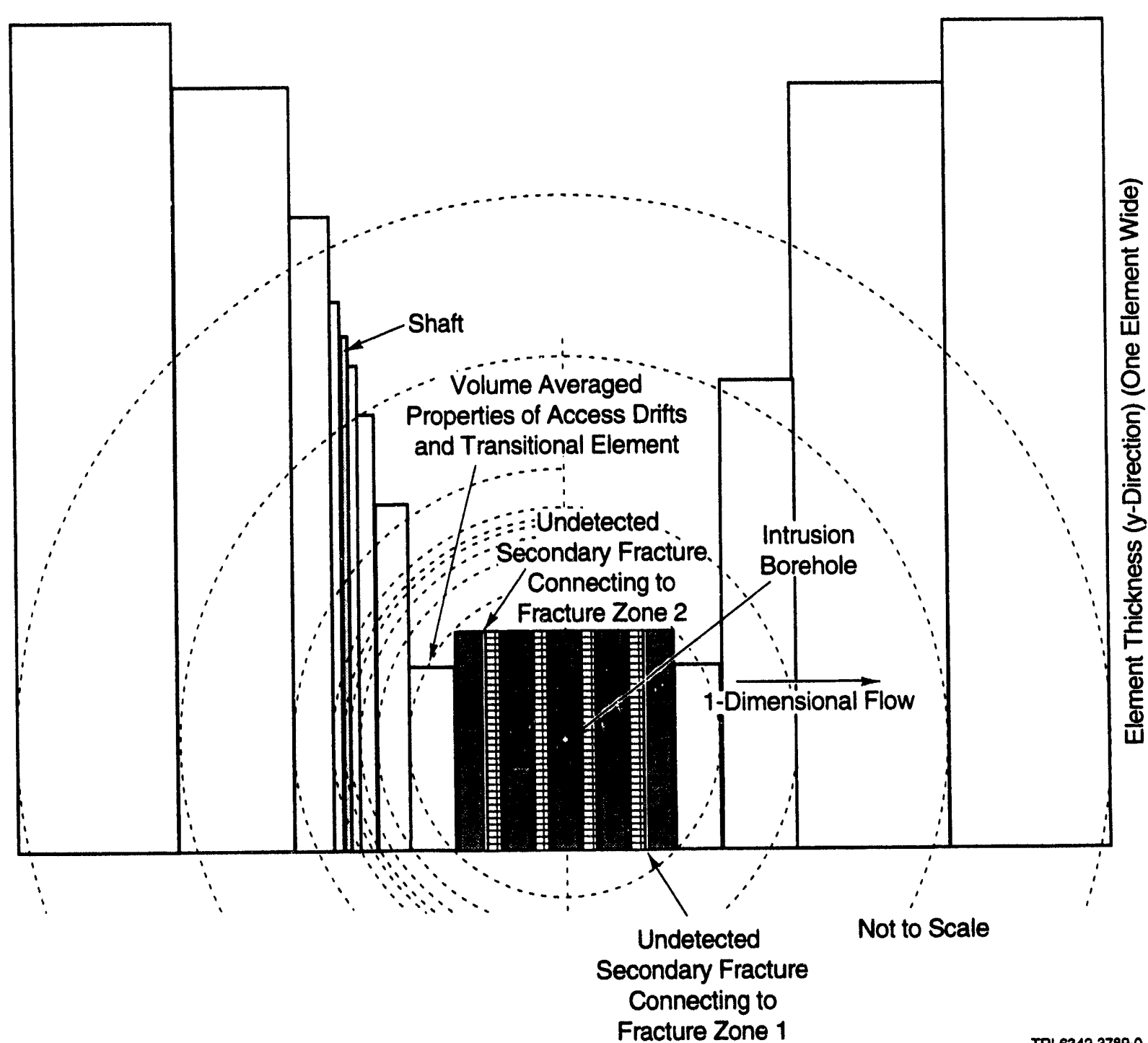

Figure 13-4. Schematic of granite disposal system depicting the area increase of elements to account for area increase in radial direction. (This schematic is similar to the salt repository [Figure 12-6] except for the portion modeling access drifts and shafts.) Flow is one-dimensional in $x-y$ plane.

shown in the $x-y$ cross-section except for the radial elements around the intrusion boreholes. The borehole mesh is not shown here due to the size of these elements, but it can be inferred from Figure 13-2.

Finally, the five shafts were combined into a single rectangular shaft. The thickness, or $\Delta y$, of the rectangular shaft was set equal to the thickness of the passageway, and the length, or $\Delta x$ was calculated to conserve the volume of the original five shafts.

Initial Conditions. Initial conditions for the fluid state variables (saturation, pressure, and temperature) are discussed here. The initial conditions for the gas generation submodel are discussed in Chapter 11.

Initial Saturation. As with the salt repository, all regions except for the repository and the shaft are assumed to be fully saturated with liquid water. The initial water saturation in the shaft and the access drifts to the disposal area was set equal to the residual brine saturation of the clay/granite backfill, 0.40 . Within the repository, most clements 
contained a mixture of waste parcels, backfill, and intact granite. The effective initial grid-block saturation was computed using Equation 12.1-34 where the component initial porosity and water saturation are as follows:

$\begin{array}{llc}\text { Component } & \text { Porosity } & \text { Saturatio } \\ \text { Waste parcel } & 0.00 & 1.00 \\ \text { Backfill } & 0.43 & 0.40 \\ \text { Granite } & 0.00001 & 1.00\end{array}$

Initial Pressures. The initial water and gas pressure of all cells in the repository and the shaft was assumed equal to $1.01325 \times 10^{5} \mathrm{~Pa}(1 \mathrm{~atm})$. The water pressure in all other regions was computed based on a vertical head gradient, 0.02 , and an assumed surface water pressure of $1.01325 \times 10^{5} \mathrm{~Pa}(1 \mathrm{~atm})$.

Initial Temperature. The initial temperature of the entire mesh was computed from the average surface temperature of $281.2 \mathrm{~K}$ and a regional temperature gradient of $0.013 \mathrm{~K} / \mathrm{m}$. Therefore, the initial temperature in the repository was $285 \mathrm{~K}$.

Boundary Conditions. The boundary conditions consist of fluid flow and heat flow boundaries.

Fluid Flow Boundary Conditions. In BRAGFLO_T, no flow (Neumann) boundary conditions are automatically estat.ished at all boundary locations. Constant head (Dirichet) boundary conditions can be approximated by establishing constant pressure wells in the grid blocks adjacent to the boundary for which this type of boundary condition is desired. Therefore, to create a horizontal head gradient across the problem domain, constant pressure wells were placed in the grid blocks along the left and right boundaries of the permeable layers (upper and lower fracture zones, glacial till).

The impact of these boundary conditions on the solution was minimized by using large elements along the left and right boundaries of the problem. Consequently, the saturation, temperature, and pressure of these cells remained relatively constant over the simulation period and the regions of interest behaved as though the problem domain were infinite.

Heat Flow Boundary Conditions. Heat was transported across all boundaries using the boundary heat loss treatment discussed in Section 12.1.1. At the surface, a relatively constant temperature was maintained by using an artificially large thermal conductivity of the atmosphere, $1000 \mathrm{~W} /(\mathrm{m} \cdot \mathrm{k})$.

Material Properties. The fluid flow properties of the materials in the granite disposal system are reported in Chapters 4, 5, and 6. The permeabilities of the intrusion borehole and the undetected fractures were the only fluid flow properties that were varied (see Chapter 7). All others remained constant. In addition, capillary pressure and relative permeability characteristic curves were derived using the same methodology used in the salt repository (see Section 12.1.4). Once again, the pore size distribution exponent, $\zeta$, was assumed to be 0.7 for all material types, and threshold pressure, $P_{t}$, was correlated to the material permeability (Davies, 1991) (see Chapter 5).

\subsubsection{Intermediate Fluid Flow Results}

Results from the fluid flow model (refer to Figure 1-2 and Section 3.2) of the total system consequence model are presented below. These intermediate results of the PA modeling process are input to the transport model described in Section 13.2.

Monitoring Points. Fluid flow to and from the disposal region of the repository was through the shaft, the borehole, and a single fracture. For these computations, the granite surrounding the repository was assumed to be impermeable. To analyze fluid transport between these features and the repository, five locations were selected as monitoring points (Table 13-1). 
13.1 Fluid Modeling in Disposal System

2

3

4

8

9

10

Table 13-1. Analysis Monitoring Points for Granite Disposal System

\begin{tabular}{|c|c|c|c|c|}
\hline & Name & Description & $(i, j)$ index & Static Head (m) \\
\hline 1 & Shaft & Shaft at repository ceiling & $(6,17)$ & 23.97 \\
\hline 2 & Borehole top & Borehole at repository ceiling & $(31,17)$ & 19.92 \\
\hline 3 & Borehole bottom & Borehole at repository floor & $(31,7)$ & 20.16 \\
\hline 4 & Top fracture & $\begin{array}{l}\text { Secondary connection (fracture at repository } \\
\text { ceiling) }\end{array}$ & $(16,17)$ & 21.44 \\
\hline 5 & Bottom fracture & Secondary connection (fracture at repository floor) & $(46,7)$ & 18.64 \\
\hline
\end{tabular}

In the discussion and figures that follow, the first monitoring point is referred to as the shaft, the second as the borehole top, the third as the borehole bottom, the fourth as the top fracture, and the fifth as the bottom fracture. The last column in Table 13-1 is the hydraulic head at each monitoring point under static conditions. These values are referred to later in the discussion concerning the impact of the regional hydraulic gradient on water flow.

Using the techniques discussed in Section 12.1.5, the total inflow, total outflow, and net flow at the end of the 10,000-yr regulatory period was computed and used to analyze fluid transport between the repository and the adjacent host rock. As in the salt repository simulations, the waste disposal group had little impact on water flow between the repository and the nearby environment (Figure 13-5). Consequently, the following discussion of water flow for Waste Disposal Group 1 can be applied to all five waste disposal groups.

Total Water Flowing into Repository for Waste Disposal Group 1. Water flows into the repository mainly through the shaft, the top fracture, and the bottom fracture (Figure 13-6). The combined inflow through these features was approximately equal to $65 \%$ of the repository pore volume, or roughly $5 \times 10^{5} \mathrm{~m}^{3}$. Inflow through the top and bottom of the borehole was always substantially lower than that of the shaft and the fractures. The reason for this is partly due to the fact that the borehole is not present until $1000 \mathrm{yr}$ into the simulation. Generally after $1000 \mathrm{yr}$, the more permeable regions of the repository, such as the drift, are already saturated with water.

Not surprisingly, the rate at which the repository filled with water was highly dependent on the permeability of the fractures connecting the repository to the two fracture zones. Plotting the flow rate in the vertical direction as a function of time at all five monitoring points for the vector with the lowest fracture permeability (run 12), and the vector with the highest fracture permeability (run 5), illustrates this dependency (Figures 13-7a and 13-7b). A positive flow rate indicates that the flow is upward, and negative flow rate indicates that flow is downward. Therefore, inflow includes positive flow from monitoring points below the repository bottom fracture, borehole bottom, and negative flow from monitoring points above the repository (shaft, top fracture, borehole top). When the connecting fracture permeability was low, all water flow was into the repository for about the first $2500 \mathrm{yr}$, and the siaft was by far the greatest contributor. However, when the connecting fracture permeability was high, the repository saturated with water much faster, and the major inflow features were the connecting fractures. Hence, the fracture permeability not only influences how rapidly the repository fills with water, but also affects where the water comes from.

Total Water Flowing out of the Repository for Waste Disposal Group 1. The behavior of the simulations once the repository is highly water saturated, can be attributed to the regional flow field imposed on the repository. Horizontal and vertical head gradients of 0.005 and 0.020 , respectively, were established in the permeable layers above and below the repository. Because the granite was assumed to be impermeable, the repository was isolated from the regional flow field except through the features monitored. Consequently, the direction of flow once the repository was saturated can be predicted from the static hydraulic head at each monitoring point. 


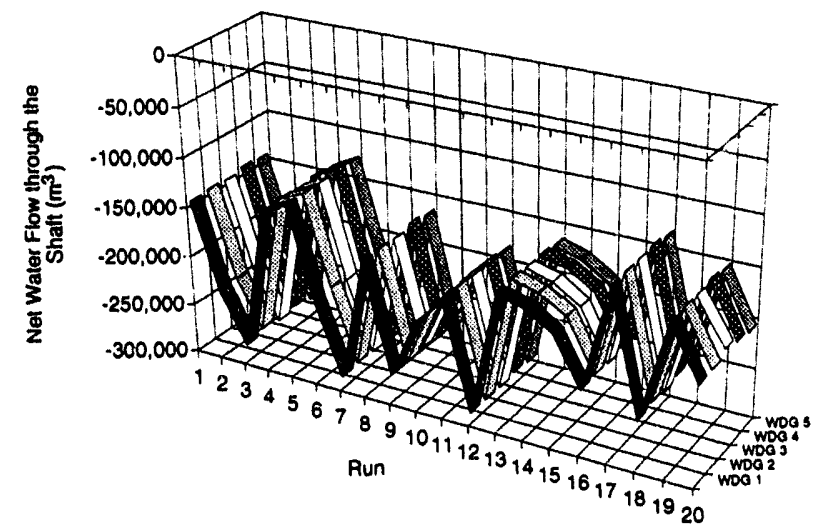

(a)

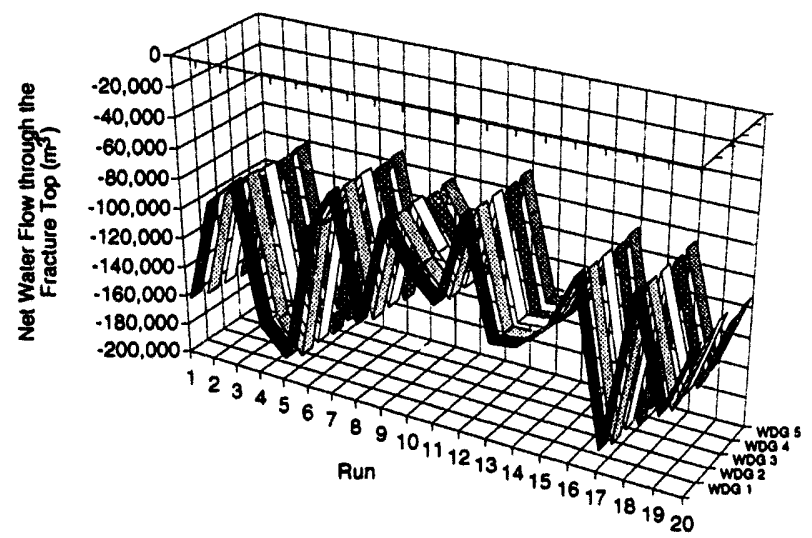

(b)

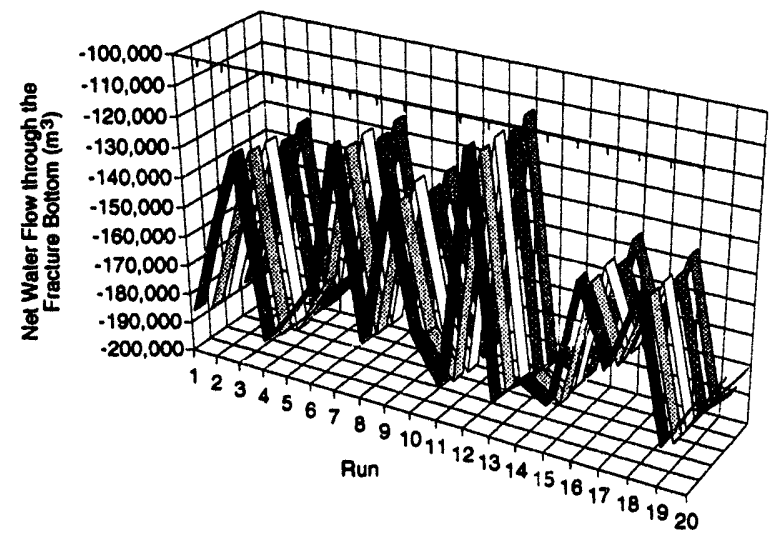

(c)

Figure 13-5. Net water flows through the granite repository are the same for each waste disposal group at each of the five monitoring points: (a) shaft, (b) top fracture, (c) bottom fracture, $(d)$ borehole top, and $(e)$ borehole bottom. 


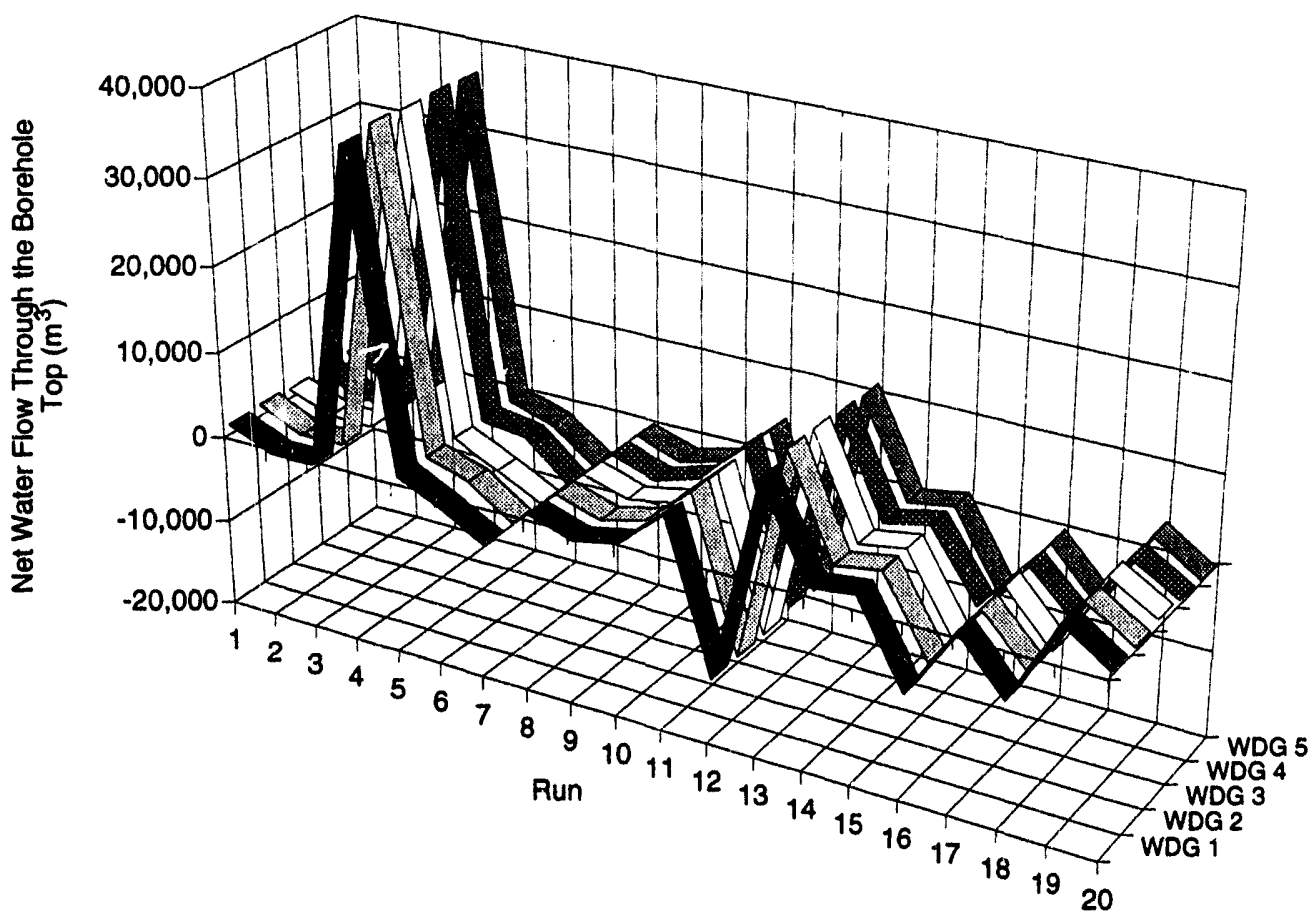

(d)

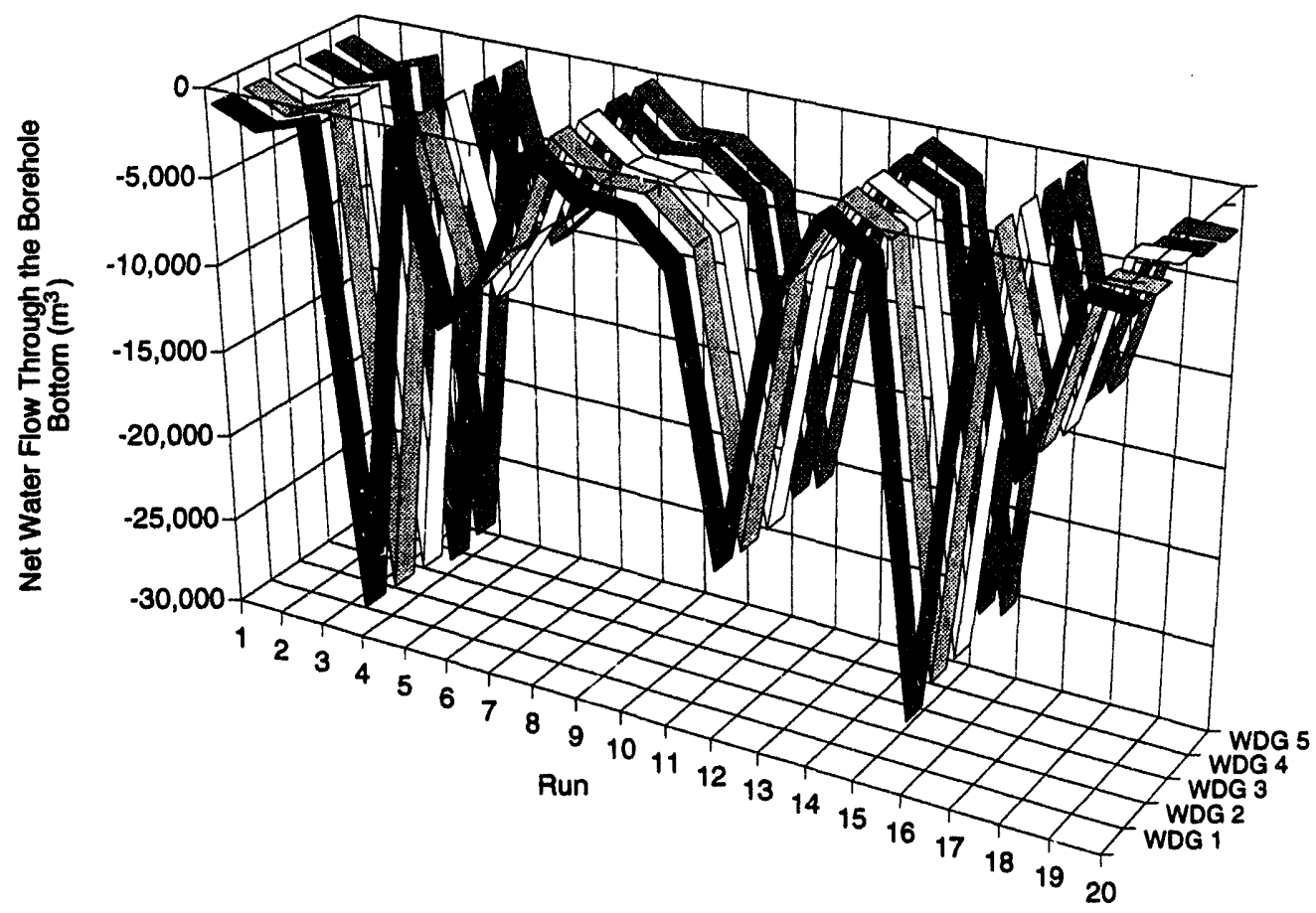

(e)

Figure 13-5. Net water flows through the granite repository are the same for each waste disposal group at each of the five monitoring points: (a) shaft, (b) top fracture, (c) bottom fracture, (d) borehole top, and (e) borehole bottom (continued). 


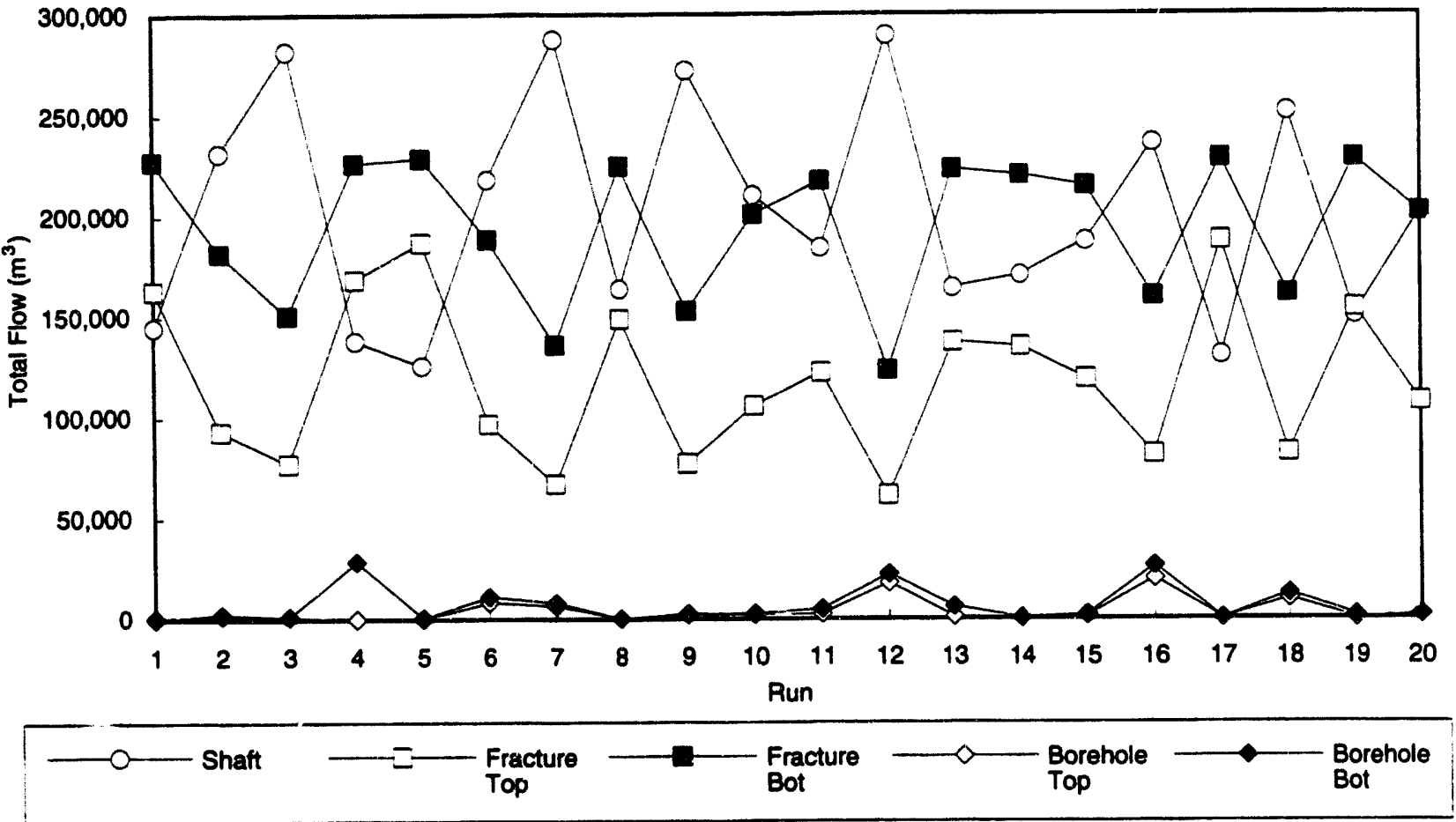

Figure 13-6. Total water flowing into granite repository by 10,000 yr through five monitoring points for all 20 simulations using W ste Disposal Group 1.

The static hydraulic head at each monitoring point was computed with respect to the center of the element indices $(56,21)$ (see Figure 13-3) and is presented in Table 13-1. The two highest potentials are at the shaft and the top fracture. The borehole top and bottom, along with the bottom fracture, have the lowest potential. Therefore, based on the regional flow field, one would expect water to enter the repository through the shaft and the top fracture, then flow out through the bottom fracture and possibly both sections of the borehole, but preferentially through the top section.

The total o . How of water for all 20 runs of Waste Disposal Group 1 is shown in Figure 13-8. As expected, most of the water that flows away from the repository through the bottom fracture. Smaller, but significant, quantities of water were also released out the top of the borchole. In half the simulations, some water escaped from the repository through the bottom of the borehole. Inspection of these sample sets revealed that flow out the borehole bottom occurred in only 10 sample sets, and these sets had the lowest secondary fracture permeability. Hence, the low permeability restricted flow through the secondary fracture, which diverted water down the intrusion borehole.

Total Gas Inflow and Outflow for Waste Disposal Group 1. Total gas inflow is presented in Figure 13-9a. Fairly small volumes of gas actually flow into the repository. The only gas that moves into the repository was initially contained in the shaft at the beginning of the simulation. Total gas outflow is presented in Figure 13-9b. As shown, gas escapes only out the top of the borehole and in relatively small quantities.

Net Gas Flow. Net gas flow through the borehole top is presented for all five waste disposal groups in Figure 13-10. As was the case for Waste Disposal Group 1, gas flowed from the repository only through the top of the borehole in the other waste disposal groups. As seen in Figure 13-10, there were noticeable differences in net gas flow. However, the volumes are very small.

Gas Production. In Figure 13-11, the cumulative hydrogen gas produced by 10,000 yr is plotted for all waste disposal groups and all runs. Waste Disposal Group 3 generally produced the most gas, usually followed by Waste 


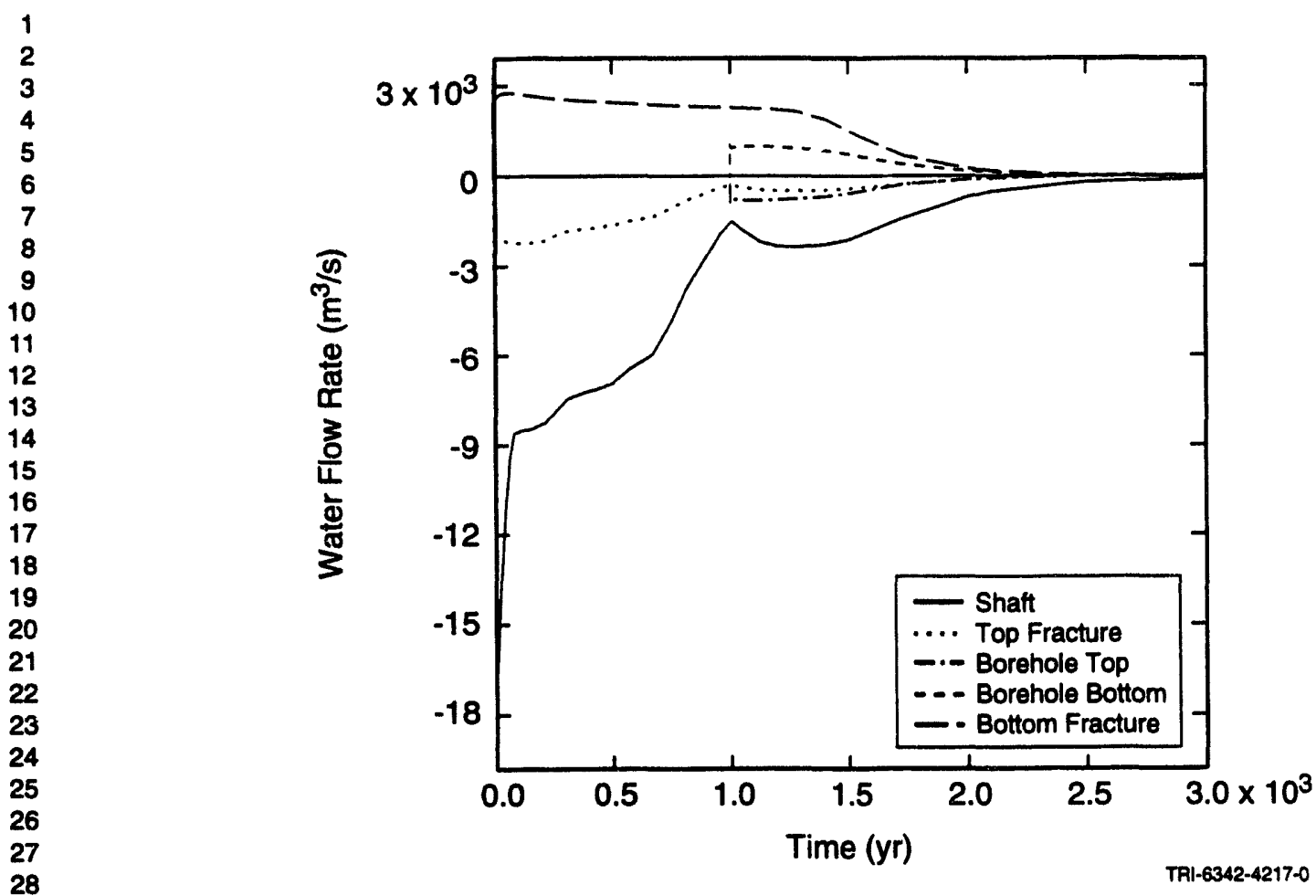

(a)

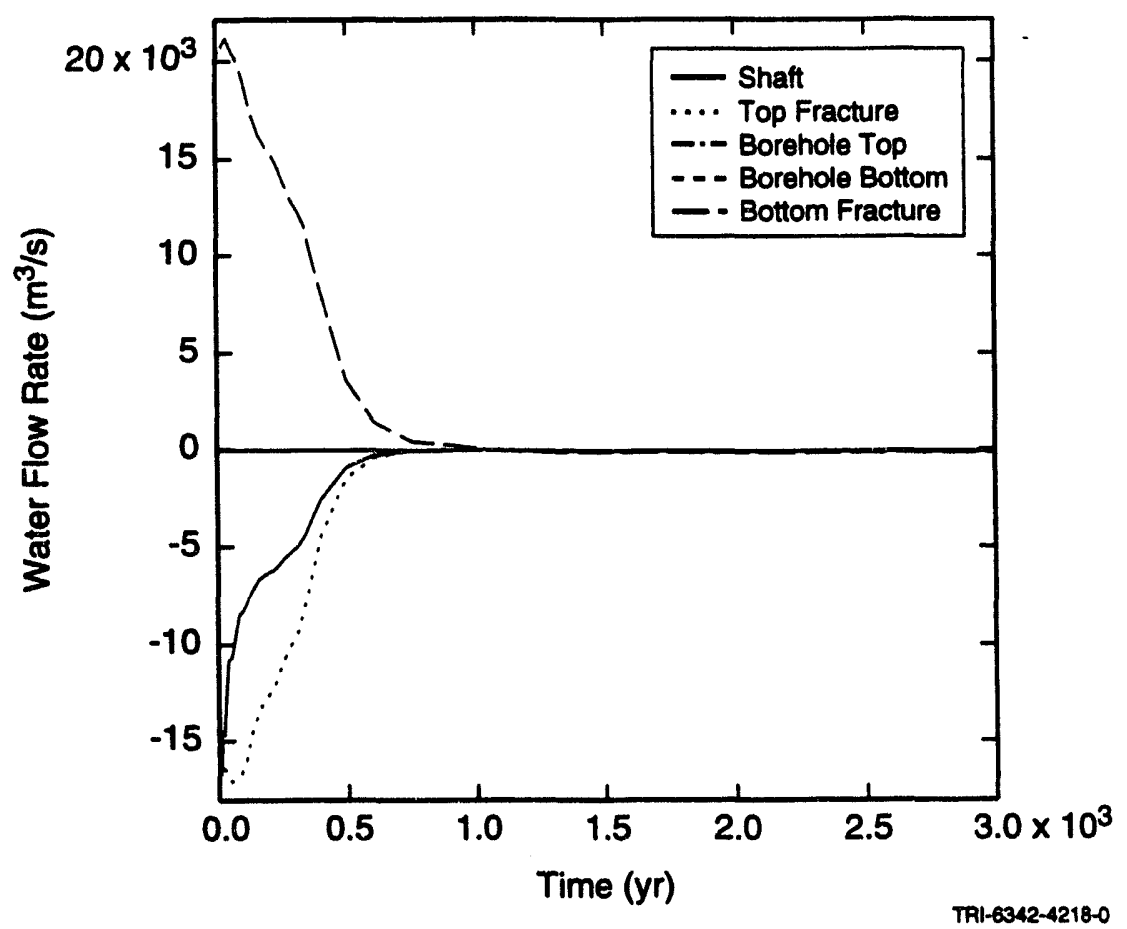

(b)

Figure 13-7. Water inflow into the granite repository when the connecting fracture permeability is $(a)$ low and (b) high. 


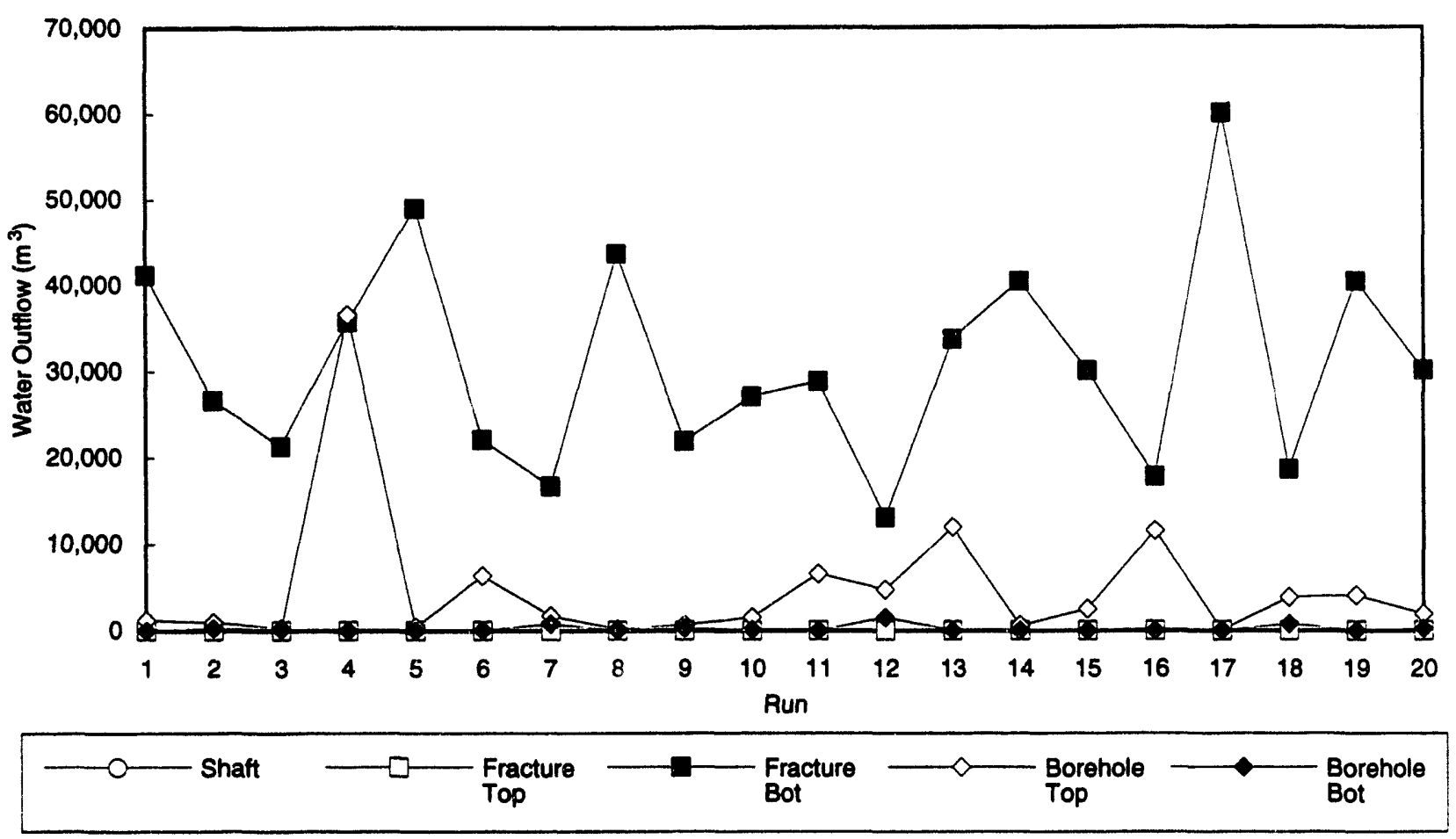

Figure 13-8. Total water outflow for all 20 runs, Waste Disposal Group 1, granite repository.

Disposal Group 1. The largest volume of gas produced was about 900,000 standard cubic meters (SCM) by Waste Disposal Group 3 in run 20 . Run 20 had the highest corrosion rates, but average fracture permeability and average borehole permeability.

Unlike the salt repository, cumulative gas production did not correlate well with any of the corrosion rates. There does appear to be some correlation between the gas generated by Waste Disposal Group 3 and the stainless steel corrosion rate (Figure 13-12a) or the lead corrosion rate (Figure 13-12b), but little else.

Repository Pressure and Saturation. Comparing the average water saturation in the salt and granite repositories (Figure 12-19a and 13-13a), it is clear the granite repository fills with water much faster and to a greater degree than the salt repository. The granite repository reaches an average water saturation close to 1.0 in the first 400 to 2000 $\mathrm{yr}$, whereas in the salt repository, much lower saturations prevailed over the entire 10,000 -yr period. Also, notice that after the initial fill-up period, flow through the granite repository was predominantly single phase. Because the repository fills up with water relatively quickly, the water pressure reaches hydrostatic long before the end of the simulation (Figure 13-13b). As a result, the final water pressure varied little from vector to vector, ranging from 3.21 to 3.25 $\mathrm{MPa}$. Gas pressures were only slightly higher than brine pressures in all simulations (Figure 13-13c). Gas pressures at the end of the simulation ranged from 3.26 to slightly more than $3.32 \mathrm{MPa}$.

Maximum Repository Temperature. The maximum temperature observed in the repository for all waste disposal groups and all runs is presented in Figure 13-14. As in the salt repository, each simulation attained essentially the same maximum temperature for a given waste disposal group, indicating fluid transport had little impact on the maximum repository temperature. Also like the salt repository, the maximum temperature varied substantially between waste disposal groups. The initial temperature of the repository was $285 \mathrm{~K}$. Waste Disposal Group 1 attained a maximum temperature of $314 \mathrm{~K}$, an increase of $29 \mathrm{~K}$. On the other hand, Waste Disposal Group 4 reached $340 \mathrm{~K}$, or a $55 \mathrm{~K}$ increase in temperature. 


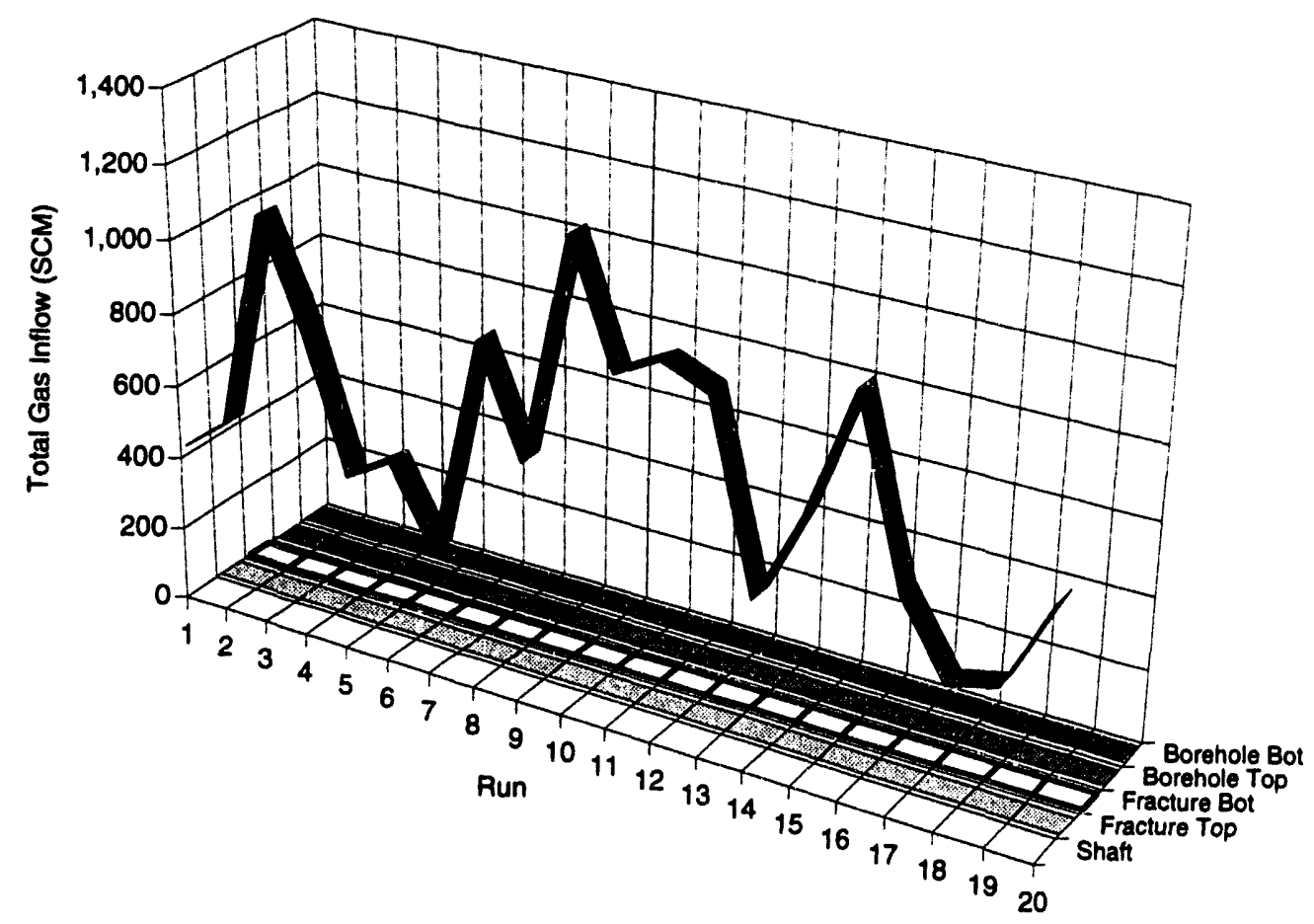

(a)

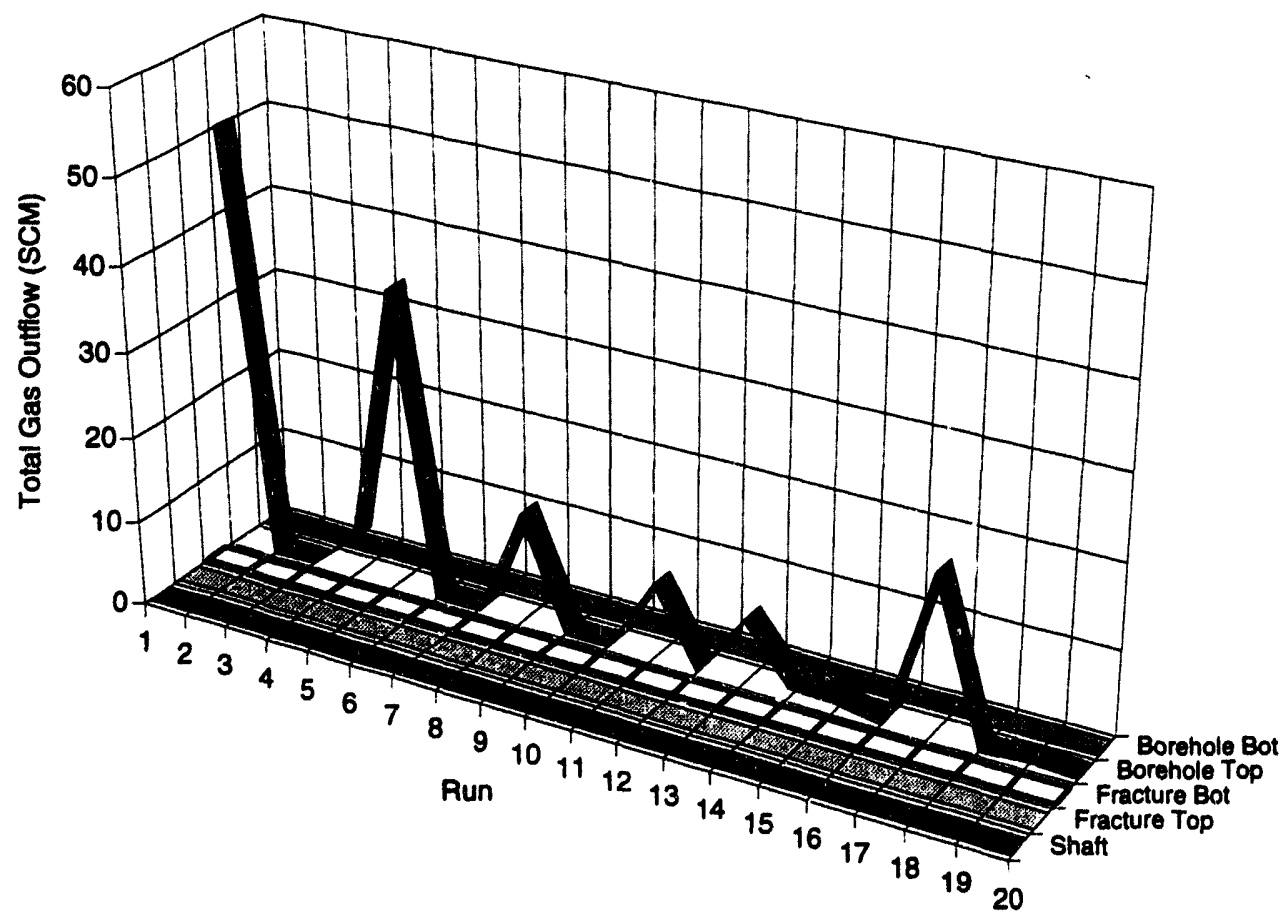

(b)

Figure 13-9. Total gas flow in the granite repository for all 20 simulations of Waste Disposal Group 1. (a) Gas inflow occurs only through the shaft, and (b) gas outflow occurs only through the borehole top. (Similar behavior was observed in the other waste disposal groups.) 
13. Complex Consequence Modeling for Granite Disposal System

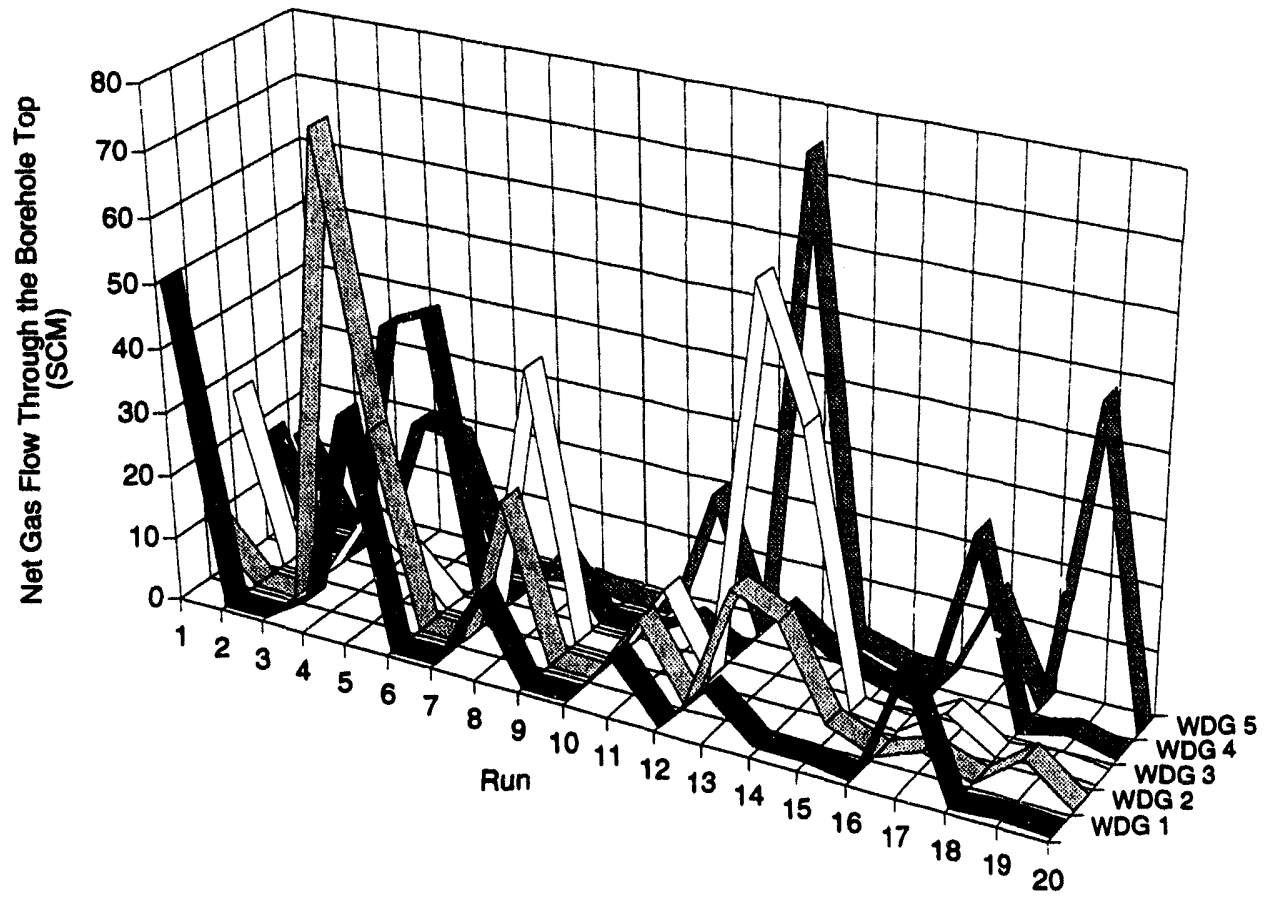

Figure 13-10. Net gas flow through the borehole top in the granite repository for all five waste disposal groups.

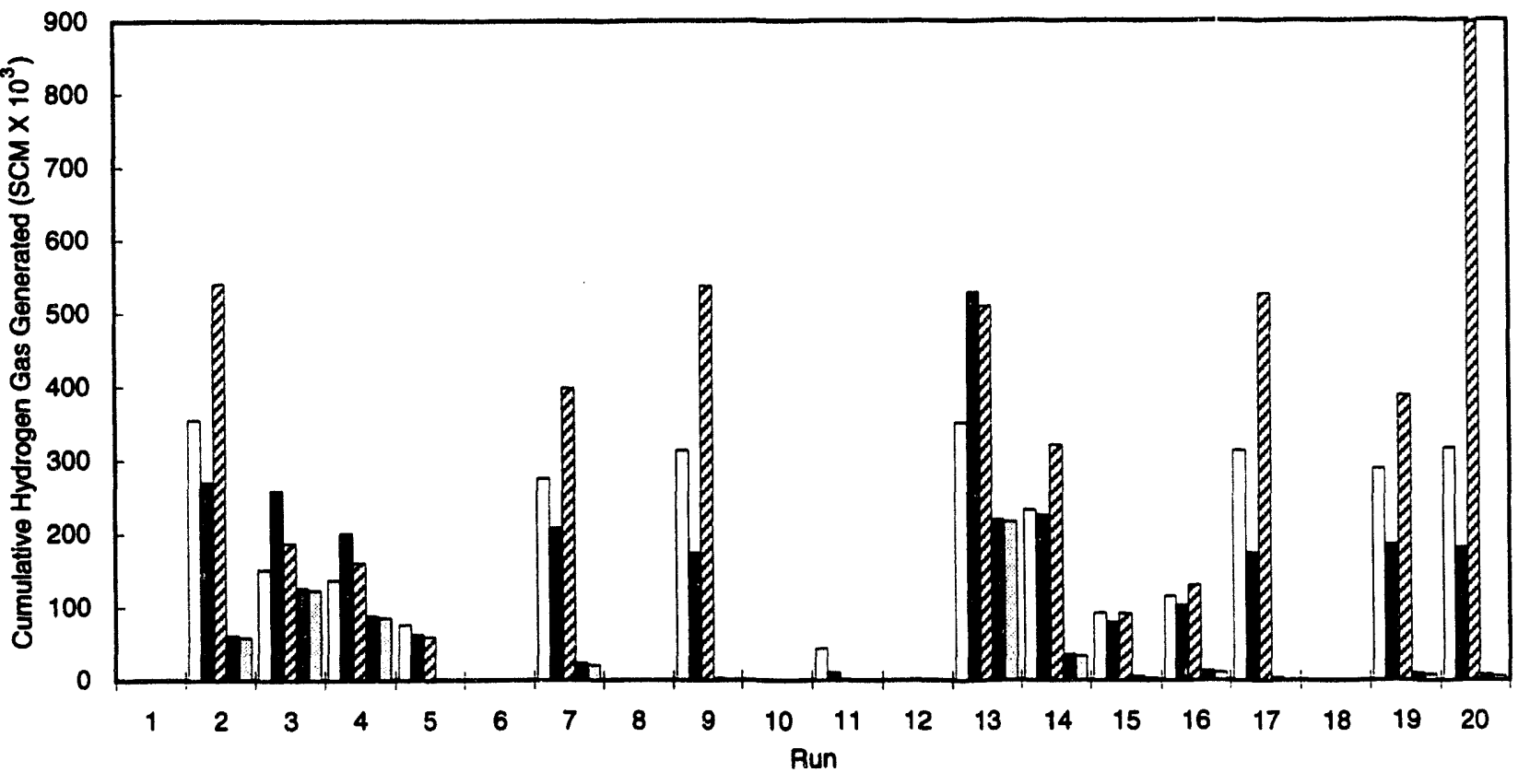

$\square$ WDG $1 \square$ WDG $2 \square$ WDG 3 WDG $4 \square$ WDG 5

Figure 13-11. Cumulative hydrogen gas generated by container corrosion by 10,000 yr in all 20 simulations for all waste disposal groups at the granite repository. 


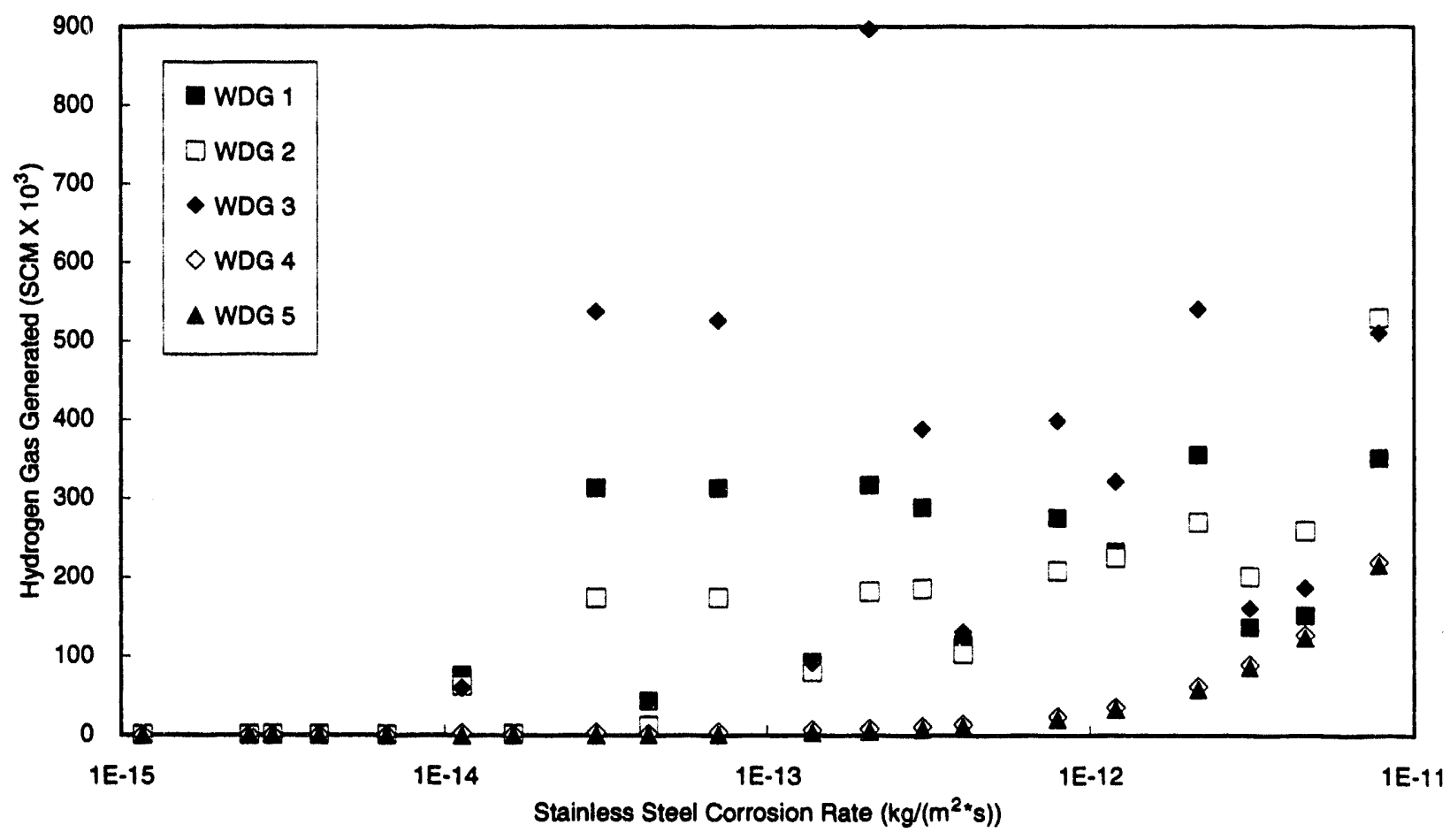

(a)

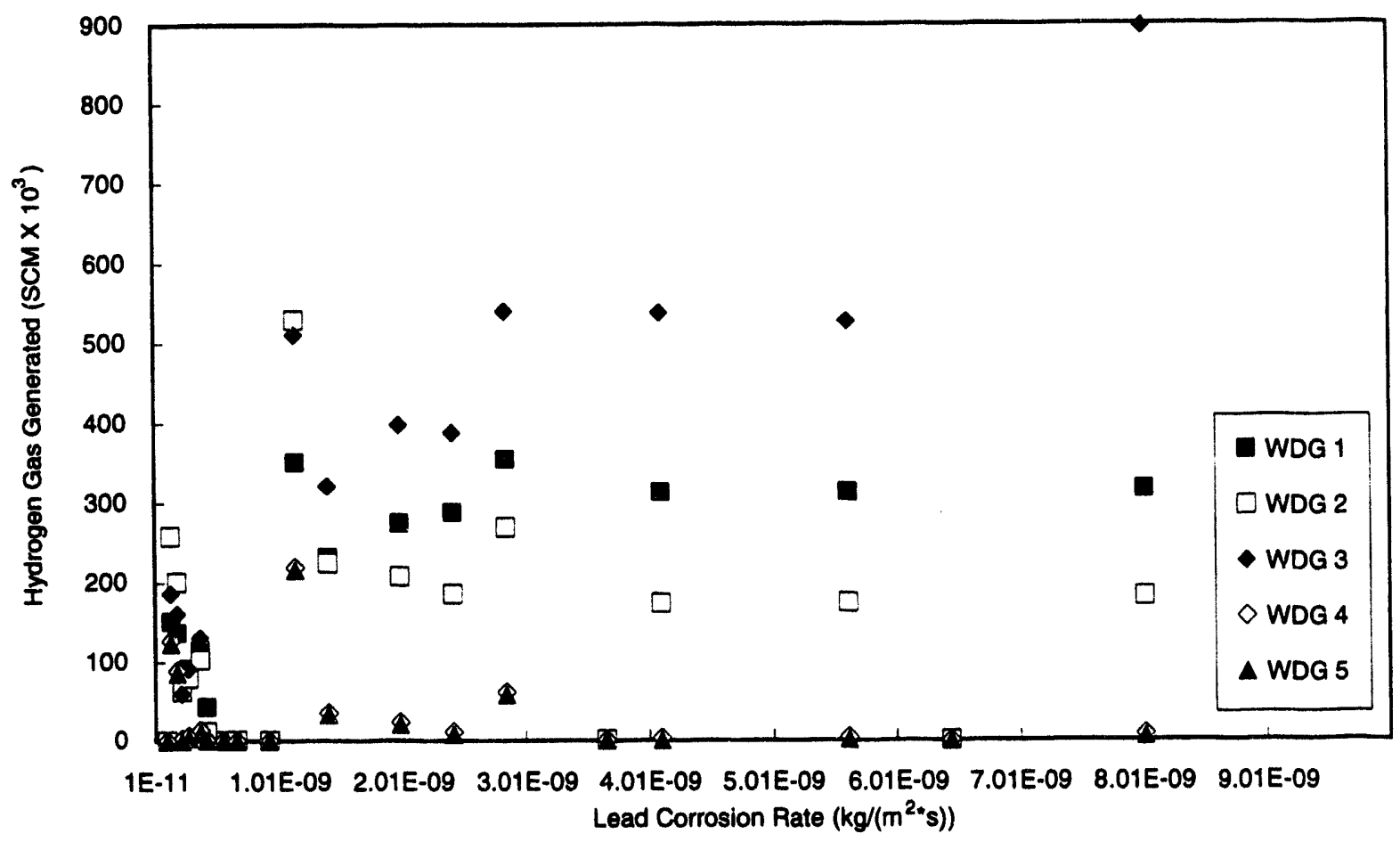

(b)

Figure 13-12. Scatterplot of volume of hydrogen gas generated vs. corrosion rate. (a) Volume of hydrogen gas generated vs. stainless steel corrosion rate shows weak correlation for Waste Disposal Groups 4 and 5 , (b) volume of hydrogen gas generated vs. lead corrosion rate shows a weak correlation for Waste Disposal Group 3.

December 1993 
13. Complex Consequence Modeling for Granite Disposal System

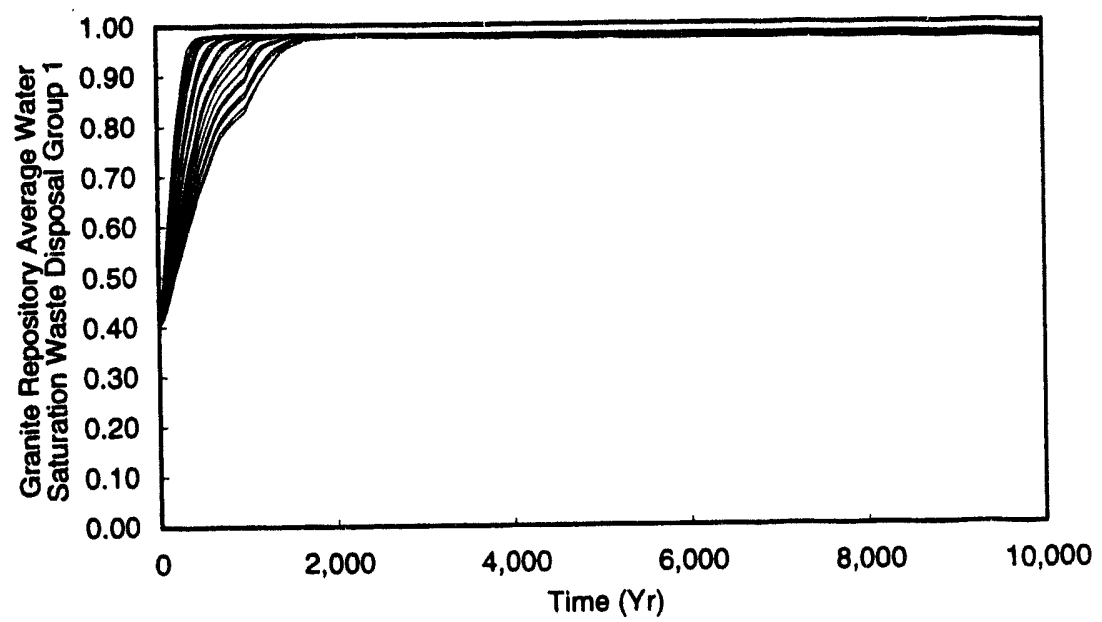

(a)

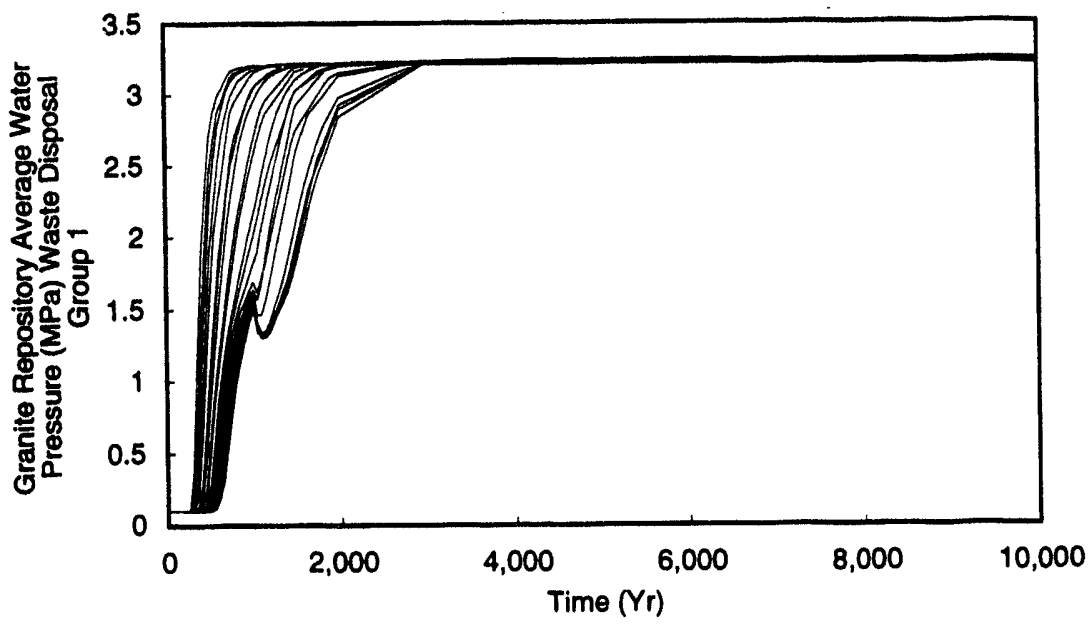

(b)

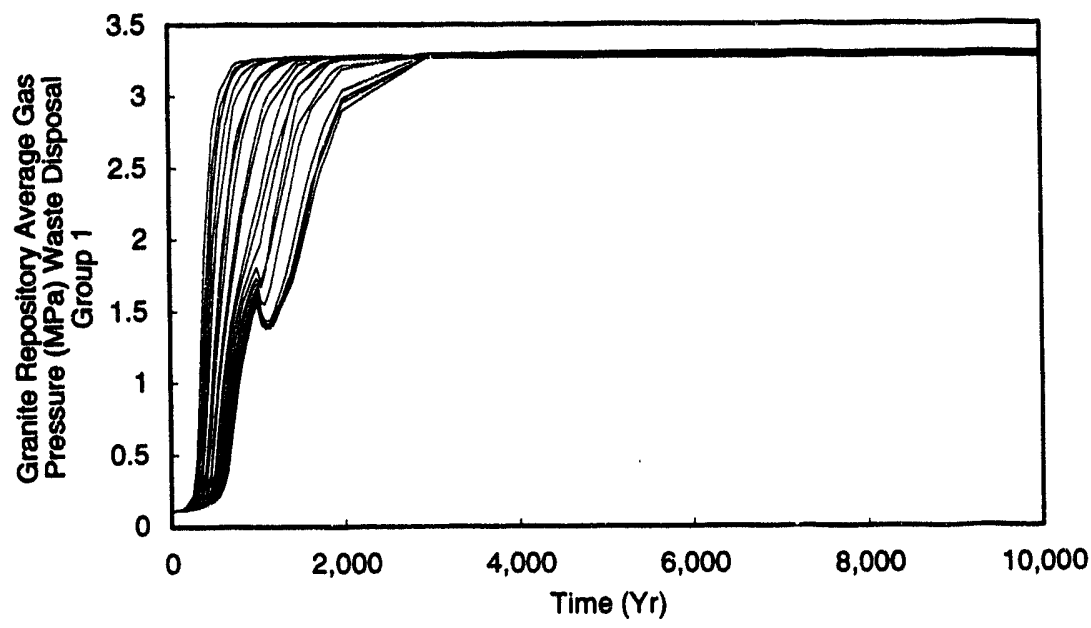

(c)

Figure 13-13. Granite repository average (a) water saturation, (b) water pressure, and (c) gas pressure for all 20 sample sets of Waste Disposal Group 1. 


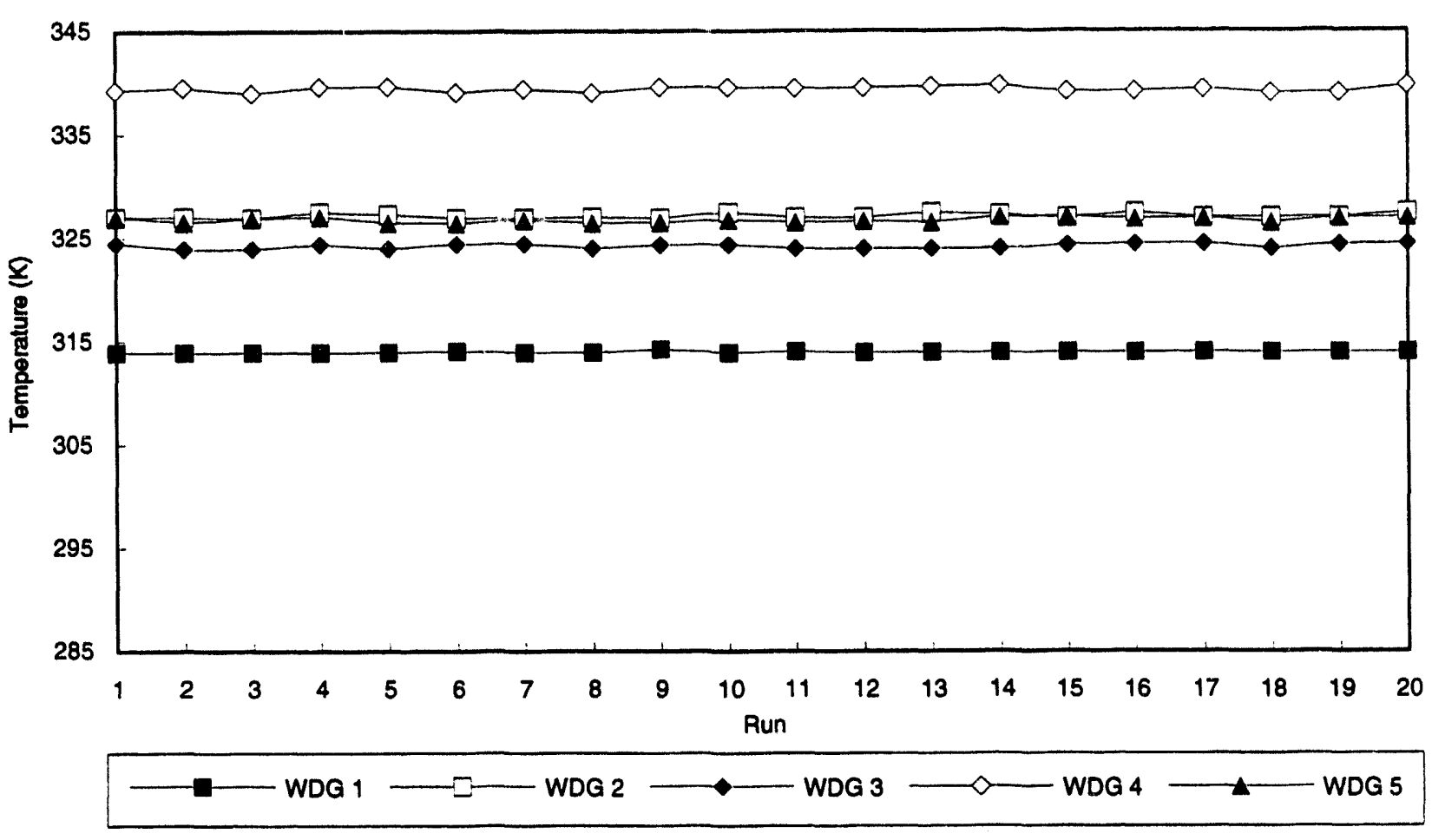

Figure 13-14. Maximum temperatures observed throughout granite repository for all five waste disposal groups.

The temperature increases observed in the granite repository are fairly close to those reported for the salt repository in Section 16.2. As discussed there, the temperature increases reported are possibly high because of the way the repository was discretized. However, in the granite disposal system, we were able to discretize the problem differently, enabling heat to dissipate from the repository more readily (see Section 13.2). Also, the one-dimensional boundary heat loss submodel allowed heat transport in the y-direction directly around the disposal region. Therefore, the temperatures reported here are likely to be more accurate than those reported for the salt repository (except for the influence of large mesh sizes).

\subsection{Radionuclide Transport Modeling}

The consequence model for radionuclide transport, STAFF2D, used the same approach for the granite disposal system as for the salt repository (Chapter 12), with the following exceptions in the applied model.

\subsubsection{Mathematical and Computational Model Description}

The mathematical and computational models are the same as described for the salt repository, Sections 12.2.1 and 12.2.2.

\subsubsection{Applied Model}

Model Geometry and Spatial Discretization. The geometry for the transport model was described in Section 13.1.2 and is identical to the flow model geometry used for the granite disposal system. 
Initial and Boundary Conditions. Initial radionuclide concentrations throughout the model domain are assumed to be zero $\left(C_{l}(x, y, 0)=0\right)$. Initial conditions inside the waste parcel boreholes are handled in the same fashion as for the salt disposal system computational model (using the source term submodel, CONCSNF, described in Chapter 11).

The boundary conditions for the granite disposal system are similar to those for the salt disposal system (Section 12.2.3), except for the constant pressure boundaries at the external grid domain boundaries of the horizontal fracture zones and the glacial till, where flow can either enter or exit the model domain, depending on the pressure gradients. (Because BRAGFLO_T must simulate a constant-pressure boundary with a well submodel, a similar type of technique was also used here.)

In the granite disposal system computational model, 110 elements had a source term (see Figure 13-3).

Material Properties. The material properties for porous media are taken from the secondary data base. Tables of these properties are provided in Chapters 4, 5, and 6. Partition coefficients are both material and element dependent and were the only transport parameters varied (see Chapters 7 and 9). Chemical retardation was modeled only in the waste parcel backfill-buffer and drift backfill. These values were scaled by the volumetric ratio of bentonite in the backfill.

As in the salt disposal system, a single value of longitudinal dispersivity that was numerically stable was chosen by trial and error for the granite repository. A value of $15 \mathrm{~m}$ was used for longitudinal dispersivity; a value of $1.5 \mathrm{~m}$ was used for transverse dispersivity. Also, as in the salt disposal system model, 11 radionuclide species in five decay chains were transported.

\subsubsection{Intermediate Results}

Results from the radionuclide transport model (refer to Figure 1-2 and Section 3.2) of the total system consequence model are described below. These intermediate results become input to CCDF construction codes CCDFCALC and CCDFPERM (see Chapter 7 and Appendix G).

Typical solute contours in and around the repository are shown in Figures 13-15 and 13-16 on the logical grid for ease of visualization. Figure 13-15 shows contours of the solute concentrations in terms of mass fractions (mass solute/mass of groundwater), the calculated, dependent variable in STAFF2D. The, results shown are for ${ }^{234} \mathrm{U}$, Waste Disposal Group 1, run 17, which had one of the larger summed normalized activities. This run was also selected for consistency with the salt repository discussions in Chapter 12. The contours are representative of most runs except in amplitude. Small negative concentrations are due to low-level oscillations in the numerical solution. Numerical input parameters can control the negative concentrations but only at the expense of increased numerical dispersion which spreads concentration gradients. Because of unsaturation, contours of mass fraction do not adequately display the distribution of the contaminant mass. The distribution is better viewed when expressed in terms of mass of contaminant/element volume (Figure 13-16).

As demonstrated by the contours, there is very little advective transport; the transport is diffusion dominated with little movement from the waste parcels into the repository due to the retardation of the backfill-buffer. Also visible is the primary pathway down the vertical fracture and out the Fracture Zone 1. The described transport phenomena are directly attributable to the flow field shown in Figure 13-17. The figure shows a vector plot of Darcy velocities or specific fluxes $\left[\mathrm{m}^{3} /\left(\mathrm{s}^{\bullet} \mathrm{m}^{2}\right)\right]$ that are representative of the flow field after intrusion and resaturation of the repository. Note that the vector scale is nonlinear in that a change in vector size by a factor of two represents a factor of ten change amplitude. It is evident in the figure that flow is predominantly in the horizontal fracture zones. Groundwater flows into the repository from the Fracture Zone 2 through the upper fracture and out the repository through the lower fracture to the Fracture Zone 1. The lower fracture actually intersects waste disposal rooms and therefore is the primary pathway to the accessible environment. The intrusion borehole provides a pathway from Fracture Zone 1 to the Fracture Zone 2 but is less significant for transport; because of radial flaring of the geometry, no waste was located adjacent to the borehole. 


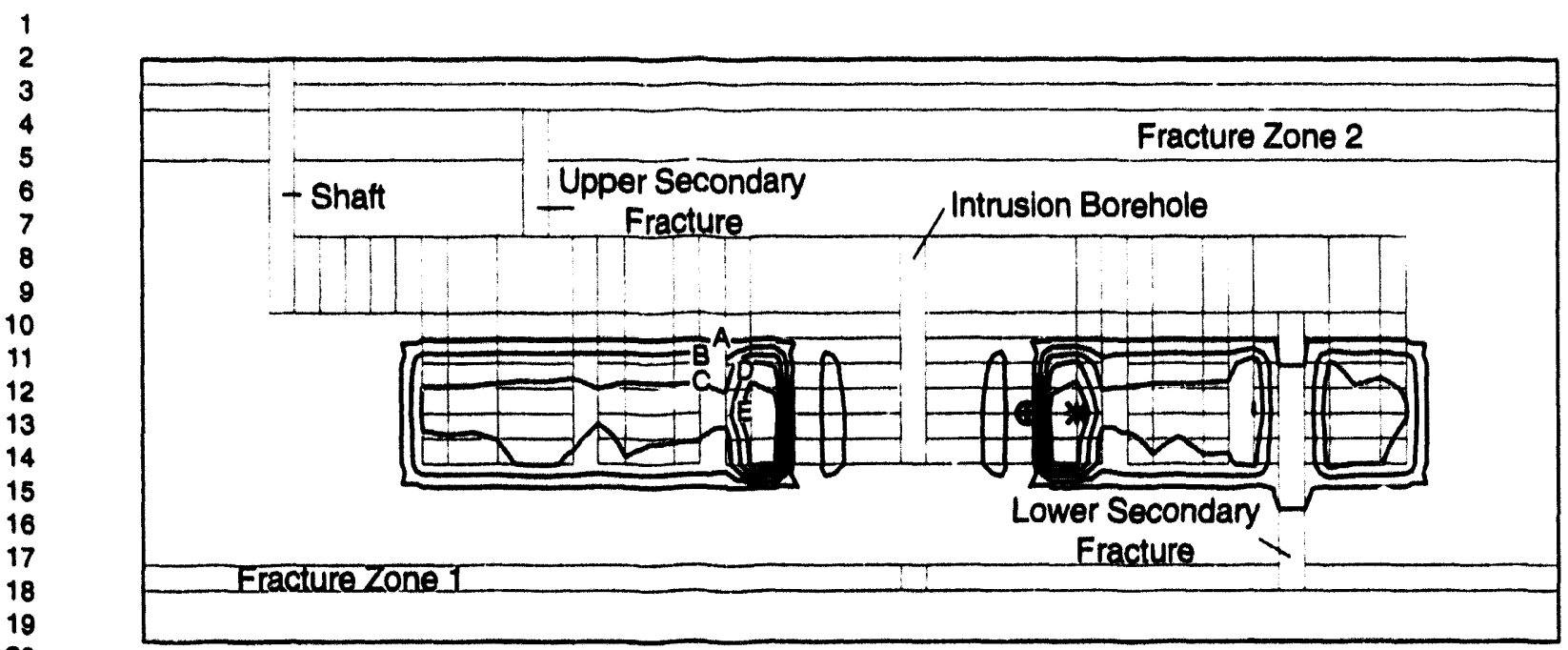

Concentration ${ }^{234} \mathrm{U}$ (kg/kg Fluid)
$A=1.00 \times 10^{-12}$
$B=6.00 \times 10^{-12}$
$C=11.00 \times 10^{-12} \oplus=-7.66 \times 10^{-12}$
$D=16.00 \times 10^{-12}$ 米 $=27.86 \times 10^{-12}$
Note: $\bigcirc$, Contour Level Common to Figure $a$ and $b$.

TA1-6342-4118-0
(a) $1000 \mathrm{yr}$

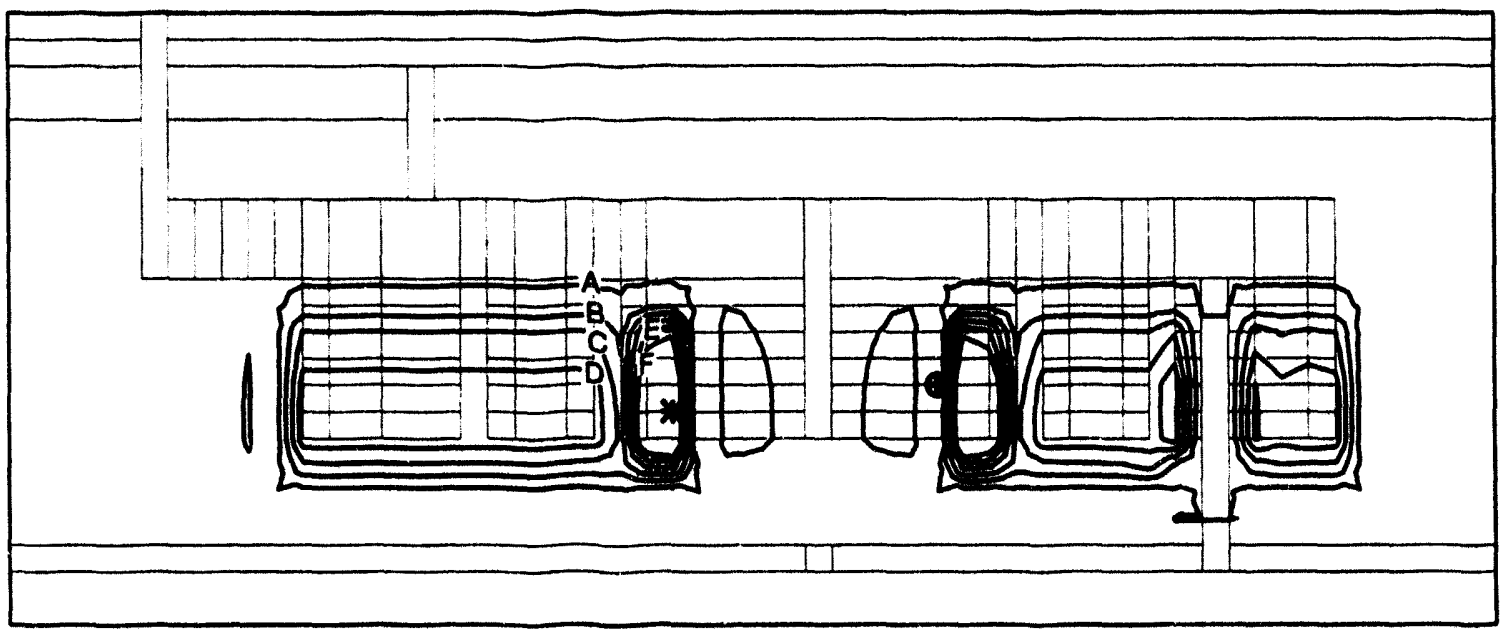

Concentration ${ }^{234} \mathrm{U}$ (kg/kg Fluid)
(A) $=0.021 \times 10^{-9} \quad E=0.821 \times 10^{-9}$
$B=0.221 \times 10^{-9} \quad F=1.021 \times 10^{-9}$
$C=0.421 \times 10^{-9} \quad \bigoplus=-0.471 \times 10^{-9}$
$D=0.621 \times 10^{-9} \quad$ 米 $=1.710 \times 10^{-9}$

Note: $\bigcirc$, Contour Level Common to Figure $a$ and $b$.

TR1-6342-4119.0
(b) $10,000 \mathrm{yr}$

Figure 13-15. Contours of mass fraction (kg/kg liquid) of contaminant for Waste Disposal Group 1, Run 17, in granite repository, at (a) $1000 \mathrm{yr}$ and (b) 10,000 yr. 
13. Complex Consequence Modeling for Granite Disposal System

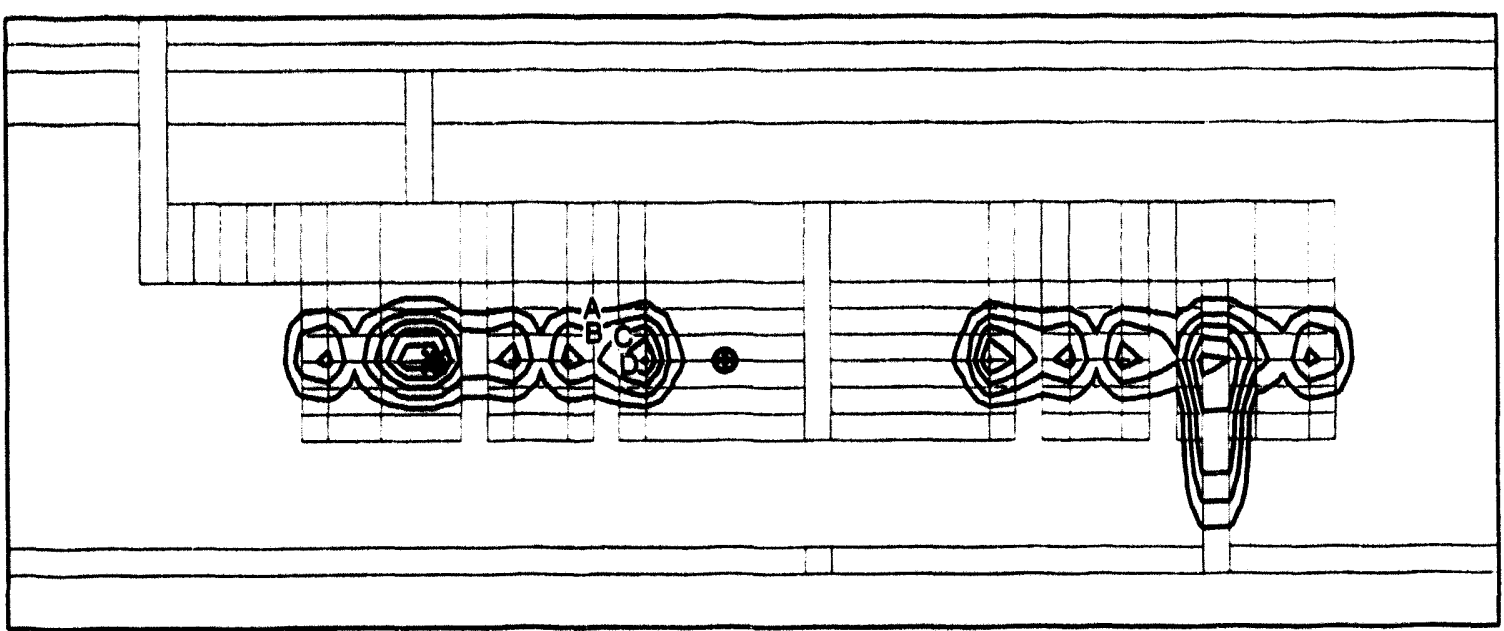

Concentration ${ }^{234} \mathrm{U}$ (kg/kg Fluid)
$A=0.100 \times 10^{-0}$
$B=0.200 \times 10^{-9}$
$C=0.300 \times 10^{-0}$
(E) $=0.500 \times 10^{-9}$
$D=0.400 \times 10^{-9}$
$\oplus=-0.0036 \times 10^{-9}$
米 $=0.5747 \times 10^{\circ}$

Note: $\bigcirc$. Contour Level Common to Figure $a$ and $b$.

TRI.6342-4120-0

(a) $1000 \mathrm{yr}$

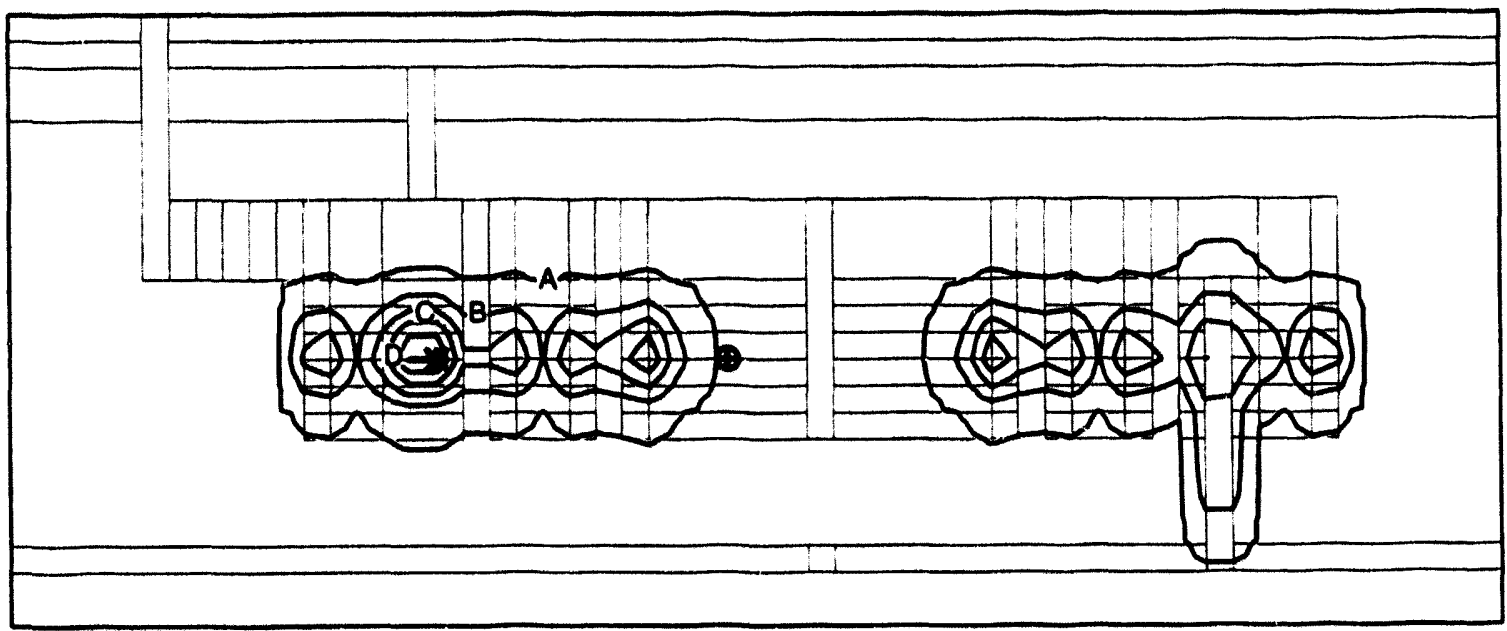

Concentration ${ }^{234} \mathrm{U}$ (kg/kg Fluild)

$(A)=0.50 \times 10^{-9} \quad E=20.50 \times 10^{-9}$

$B=5.50 \times 10^{-9} \quad F=25.50 \times 10^{-9}$

$C=10.50 \times 10^{-9} \oplus=-0.33 \times 10^{-9}$

$D=15.50 \times 10^{-9} \quad$ 米 $=25.76 \times 10^{-9}$

Note: $\bigcirc$, Contour Level Common to Figure a and $b$.

TRI.6342-4121-0

(b) $10,000 \mathrm{yr}$

Figure 13-16. Contours of mass contaminant per element volume $\left(\mathrm{kg} / \mathrm{m}^{3}\right.$ element) for Waste Disposal Group 1, Run 17, in granite repository, at (a) $1000 \mathrm{yr}$ and (b) 10,000 yr. 


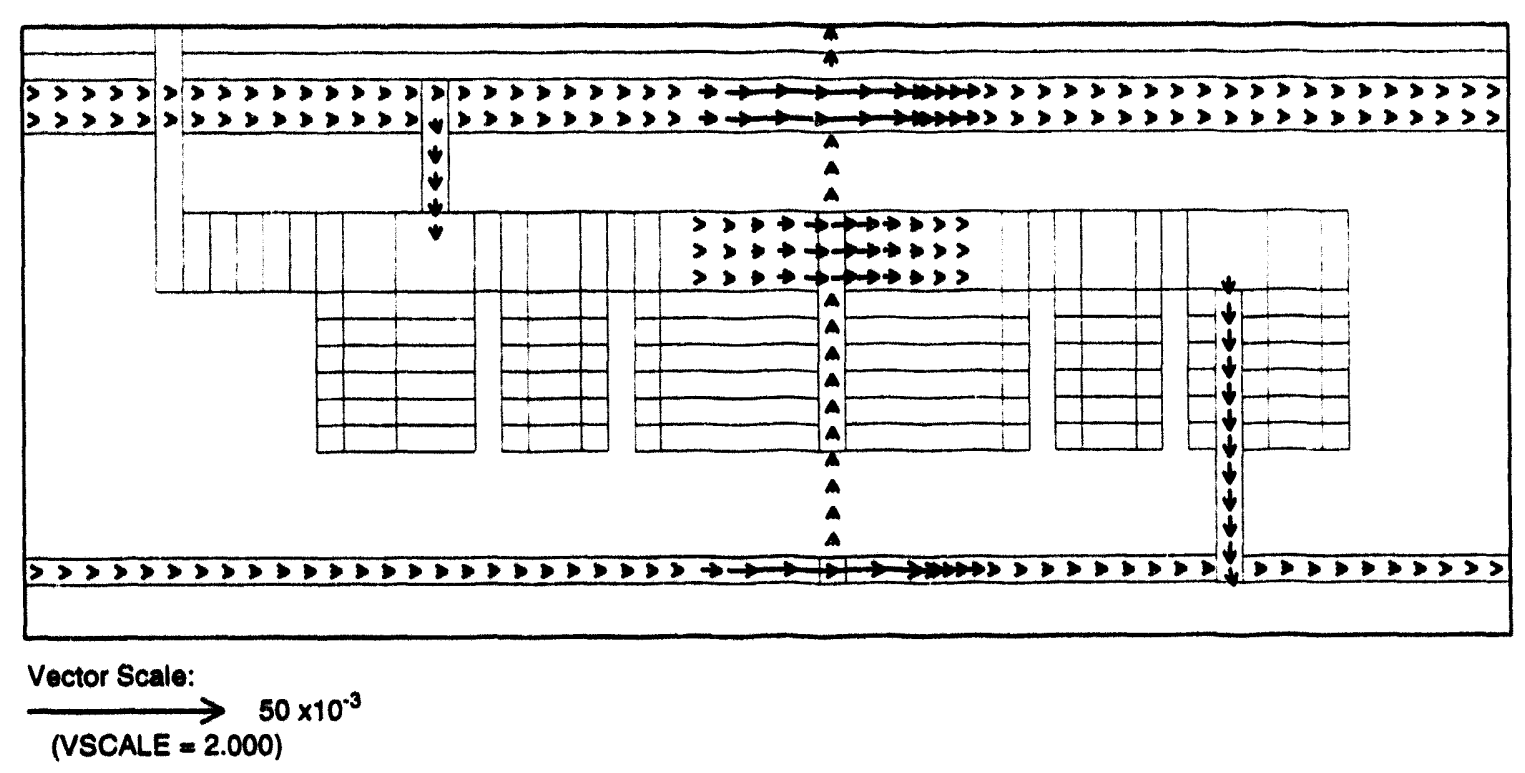

Figure 13-17. Darcy velocity vector plots representative of the flow field after intrusion and resaturation, granite repository, Waste Disposal Group 1, Run 17.

The primary results from the simulations for this performance assessment are the positive discharge rates across a prescribed boundary. For the granite repository, the boundary was specified at six locations: two in each of the two horizontal fractures above and below the repository and two in the glacial till. From Figure 13-3, the exact locations of the discharge boundaries correspond to logical $x$-axis grid-block index 4 (discharge to the left), and 52 (discharge to the right) for Fracture Zone 1, Fracture Zone 2, and the glacial till. These logical $\mathrm{x}$-axis grid-block numbers are positioned two zones beyond the repository shaft and two cells beyond the end of the repository along the horizontal ( $x$-direction). The integrated positive discharges are presented and discussed in Chapter 16 and represent the integrated discharge from the repository and the eventual release to the accessible environment. 


\title{
14. Simplified Consequence Modeling for Salt Repository
}

\author{
W. E. Beyeler
}

The consequence models for the "Simple PA" fall into two categories: Fluid flow and contaminant transport. A description of these models follow. The final release results, expressed as complementary cumulative distribution functions (CCDFs) are presented in Chapter 16, "Results and Conclusions."

\subsection{Repository Fluid Flow Modeling}

\subsubsection{Conceptual Model}

Figure 14-1 shows the general conceptual model of the repository and nearby geologic barrier in the simplified flow calculations for waste disposal in salt.

Estimates of flow rate from the salt repository were based on the following assumptions:

- The relatively low permeability of intact halite, compared to the anhydrite layer overlying the repository, suggests that the primary source for brine is the anhydrite layer. Both brine and gas flow in the halite are neglected in the simplified model, and the anhydrite is assumed to be in direct communication with the repository.

- Gas generated within the repository is assumed to be freely mobile within the repository, to remain within the repository, and to obey the ideal gas law.

- Liquid phase is assumed to be incompressible.

- Each panel is hydrologically isolated from the other panels.

- Liquid and gas phases are separate: a given repository pore volume is either saturated by liquid or saturated by gas.

- Gas phase and liquid phase pressures in the repository are identical.

- The pore volume in all components is constant over time.

- Heat generation and heat transport are neglected.

- Only liquid flow in the intrusion boreholes, brine pocket, and Snake Dolomite is considered.

- All flow is assumed to be described by Darcy's law.

Ranges of values of the hydrologic properties of the Cattle River and Snake Dolomite are found in Appendix B; values for the backfill-buffer material are found in Appendix D. Using the property values for the WIPP site, which is the basis of the parameter values for the hypothetical salt repository, an analysis of the hydrologic response of the repository following interconnection by a borehole of the repository, dolomite aquifer, and brine reservoir, suggests the following (Rechard et al., 1990; Table 4-4):

a. During discharge of the brine reservoir, pressure in the Snake Dolomite is not significantly elevated above its initial value.

b. Time constants for internal pressure transients in the Cattle River and borehole range from less than a year to tens of years.

c. The discharge time of the Cattle River brine reservoir ranges from thousands to hundreds of thousands of years. 
14. Simplified Consequence Modeling for Salt Repository

General Conceptual Model

Plan View of Panel

(10 Rooms in Sequence)

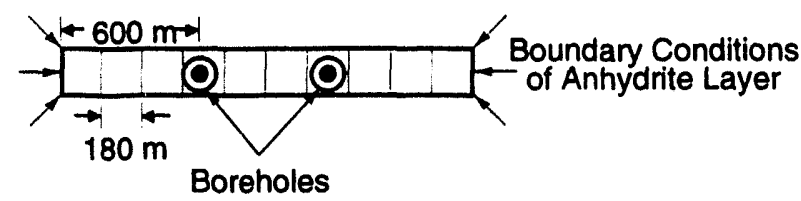

Cross-Section of

Domain Modeled

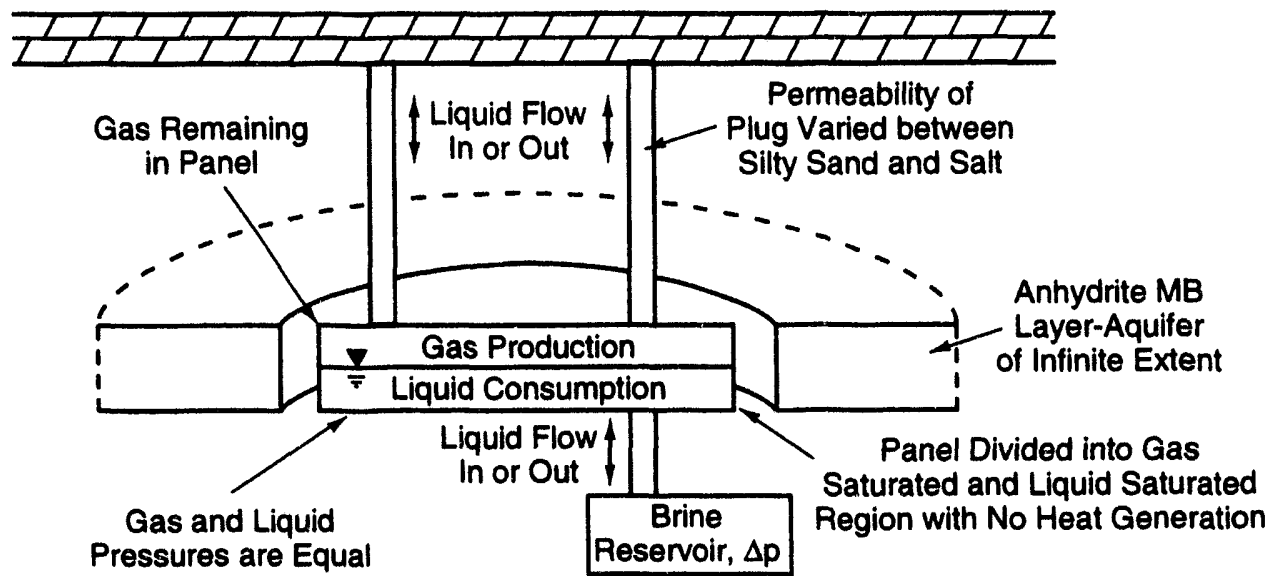

One Dimensional Mathematical Model

Anhydrite MB

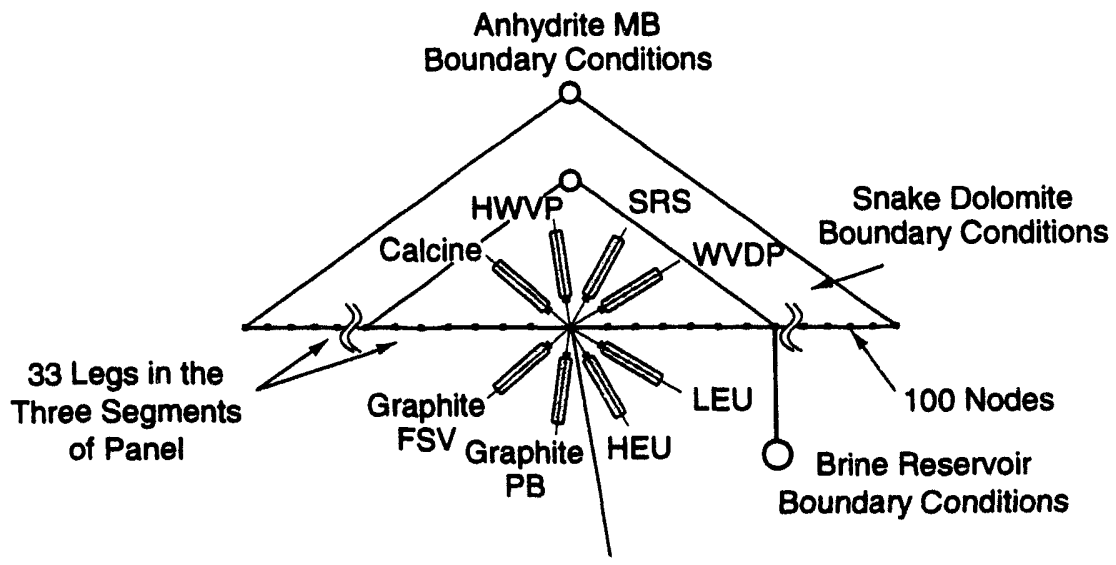

Gas and Source-Term

Submodel Input at Each Leg

TR1-6342-39190

Figure 14-1. General conceptual model and one-dimensional mathematical model of salt repository and nearby geologic barrier in Simple PA. 
On this basis, the following assumptions about the Cattle River, boreholes, drift, and Snake Dolomite have been made in the fluid flow model:

a. The Snake Dolomite acts as a fixed-pressure boundary for all boreholes.

b. The transient behavior of the system over the period of interest is governed by the depletion of the brine reservoir and the generation of gas within the repository, rather than by internal pressure transients within any component. Accordingly, all components are assumed to be at steady state with respect to boundary pressures at any given time. The assumption of quasi-equilibrium flow within the repository may not be justified for the drift backfill permeabilities in the salt repository, which are substantially less than corresponding permeabilities at the WIPP.

c. Pressure change in the brine reservoir is assumed to be a linear function of the change in reservoir brine volume (e.g., due to linear elastic expansion of reservoir fluid and anhydrite):

$$
\Delta \mathrm{V}_{\mathrm{p}}=\mathrm{S}_{\mathrm{v}} \Delta \mathrm{P}_{\mathrm{p}}
$$

Some of the assumptions made in the development of the flow model for the Simple PA for salt were not consistent with the results of the flow model, BRAGFLO_T, which was used in the Complex PA. In particular,

- Significant liquid flow from the halite into the repository was seen in the BRAGFLO_T results. This flow is precluded in the construction of the simple model.

- With higher values of halite permeability, gas moved from the repository into the surrounding halite in the BRAGFLO_T model. This behavior was not possible in the simple flow model. Gas flow into the halite tends to decrease the repository pressure, thereby increasing the tendency of liquid to move into the repository (or decreasing the tendency of liquid to move out of the repository).

The combined affect of these differences is to reduce the amount of brine available for canister dissolution and waste mobilization. This effect was mitigated by the differences in the control volumes used in the Simple and Complex PAs: the simple model was configured to represent a single panel, while the complex model was configured to represent the entire repository. The differences in control volume are consistent with assumptions made regarding permeability of intact halite, which is the design permeability of the panel seals: impermeable halite implies isolation of panels, while permeable halite implies communication between panels.

In application, sufficien* brine was not available to fill the larger pore volume of the repository in the complex model. In the simple model, the lower inflow rate vyas more than sufficient to fill the repository panel and to produce outflow from the panel through the intrusion boreholes in a majority of sample vectors. For the particular intrusion scenario considered in this analysis, flow rates calculated in the Simple PA result in larger radionuclide release because they result in flow upward from the repository to the Snake Dolomite.

\subsubsection{Mathematical Model}

The mathematical model is based on a mass balance equation for liquid and gas within the repository; mass balance equations for liquid in control volumes around borehole/repository intersections, and a mass balance equation for liquid in the brine reservoir. Because the liquid phase is assumed incompressible, volume balance equations are written for the liquid phase.

Mass balance equations are expressed in terms of fluid pressure using the assumption of darcian flow in all components. The repository state is characterized by a single average pressure for both the gas and liquid phase. Liquid flow to and from the anhydrite layer is assumed to be controlled by the average repository pressure. Liquid flow through the upper and lower borehole segments, and between the repository control volume and the borehole/repository intersection control volume, depend on pressure at the borehole/repository intersection, which may vary from 
borehole to borehole. Figure 14-2 is a schematic illustration of the relationship between the control volumes considered in the flow model.

As discussed above, the time constants for internal pressure transients in the boreholes and repository are assumed to be small, so that the flow rates through all fluid pathways are proportional to a hydraulic conductance, $\zeta$, which includes the permeability, length, and area normal to the flow direction. Conductance terms used to characterize the fluid pathways in Figure 14-2 are:

$\zeta_{U_{j}} \quad=$ Conductance of the upper segment of borehole $\mathrm{j}$

$\zeta_{L_{j}} \quad=$ Conductance of the lower segment of borehole $\mathrm{j}$

$\zeta_{P_{j}}=$ Conductance of drift backfill between borehole $\mathrm{j}$ and the waste panel

$\zeta_{X_{j, k}}=$ Conductance of drift backfill between boreholes $\mathrm{j}$ and $\mathrm{k}$.

The borehole segment conductances are a function of the borehole length, area, and the hydraulic conductivity of the borehole fill:

$$
\begin{gathered}
\zeta_{U_{j}}=\frac{K_{U_{j}} A_{j}}{\Delta Z_{2} \rho g} \\
\zeta_{L_{j}}=\frac{K_{L_{j}} A_{j}}{\Delta Z_{1} \rho g}
\end{gathered}
$$

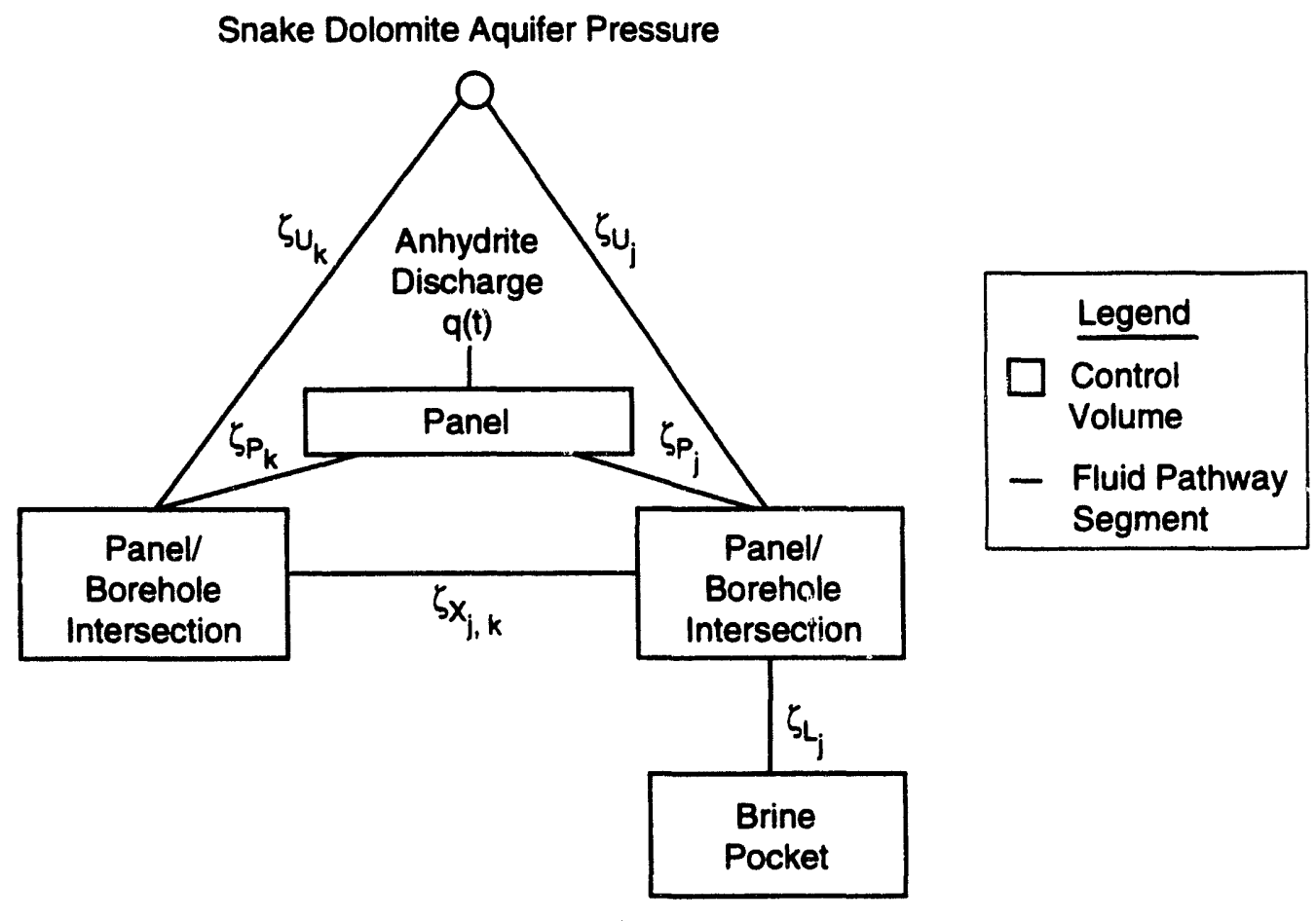

TRI-6342-3734-0

Figure 14-2. Relationship between control volumes in flow model of salt disposal system used in mathematical model. 


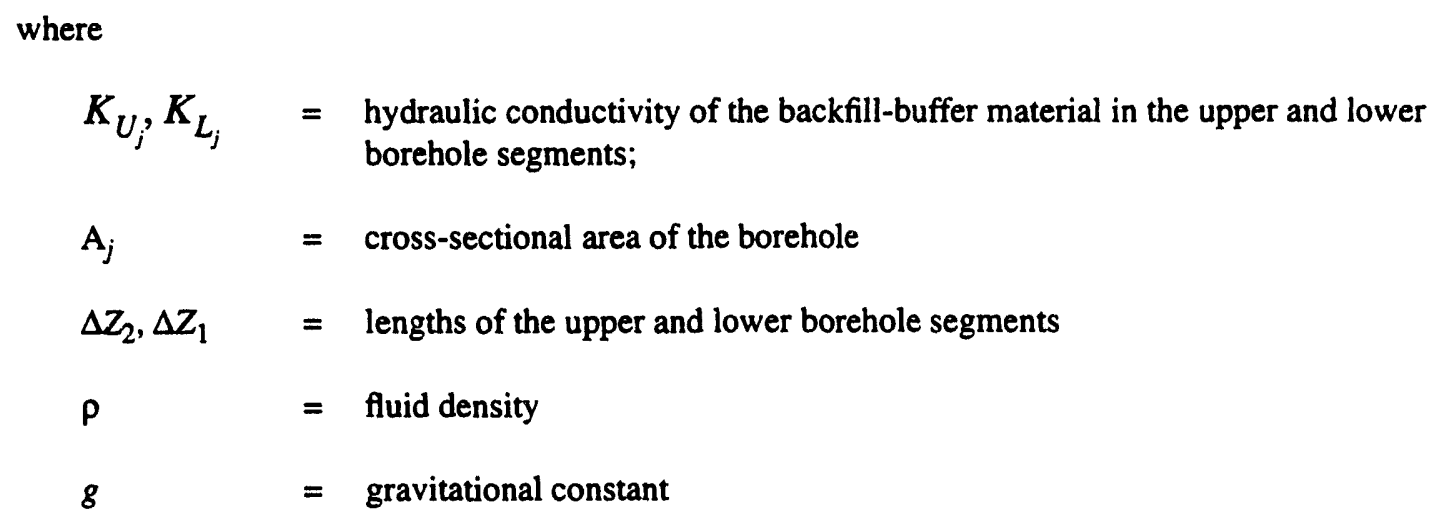

The borehole/repository conductance is estimated as the conductance of a homogeneous annulus with an inner radius equal to the borehole radius and an outer radius equal to the radius of a circle with an area equal to the excavated panel area:

$$
\zeta_{P_{j}}=\frac{2 \pi K_{D^{b}}}{\ln \left(\frac{r_{P}}{r_{w_{j}}}\right) \rho g}
$$

The inter-borehole conductance terms are composed of three parts, representing flow resistance near each borehole, where the convergent flow field is approximated as radially symmetric, and flow resistance through the rooms, where the flow field is approximated as parallel 1-D pathways (see Figure 14-3).

$$
\left(\zeta_{X_{j k}}\right)^{-1}=\left(\zeta_{w_{j}}\right)^{-1}+\left(\zeta_{w_{k}}\right)^{-1}+\left(\sum_{i} \zeta_{i D_{j k}}\right)^{-1}
$$

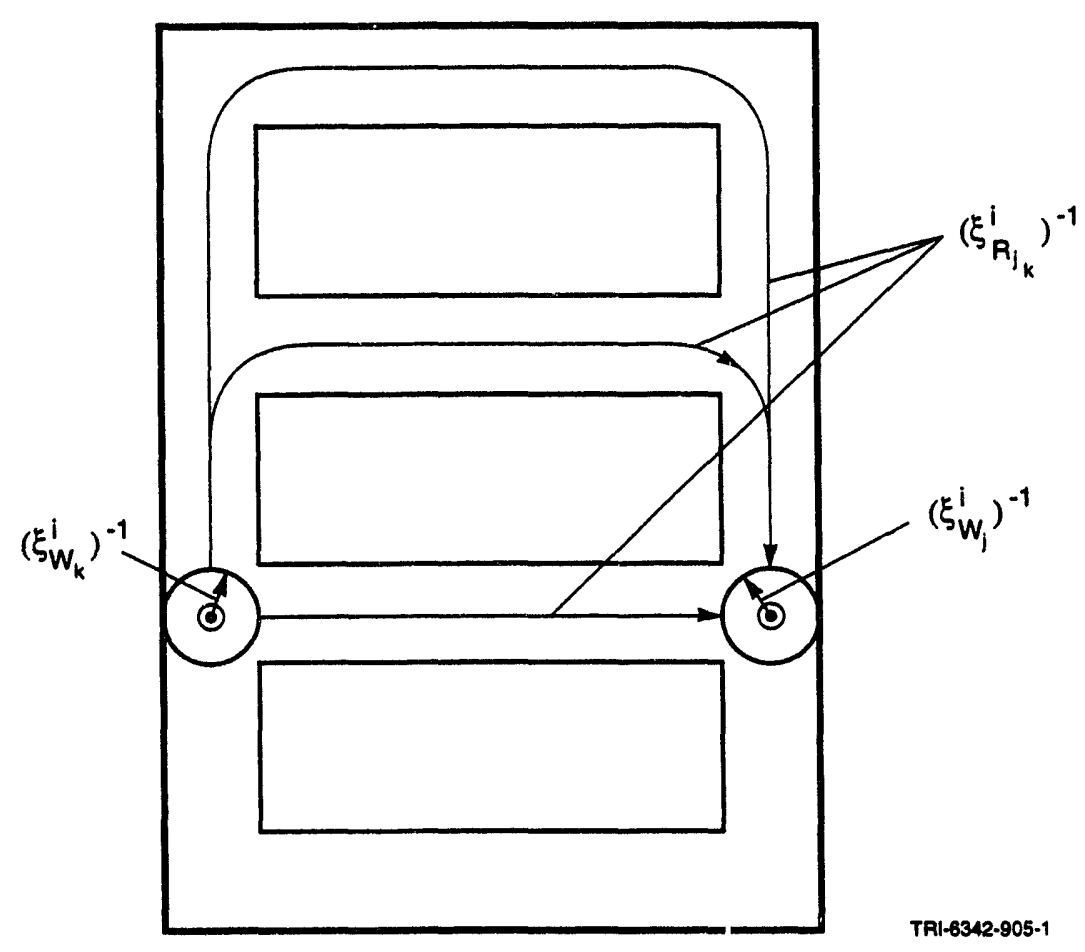

Figure 14-3. Convergent flow field is approximate' as radially symmetric. 


$$
\text { where, } \begin{aligned}
\zeta_{w_{j}} & =\frac{2 \pi K_{D} b}{\ln \left(\frac{r_{D}}{r_{w_{j}}}\right) \rho g} \\
\zeta_{i D_{j, k}} & =\frac{K_{D} 2 b r_{D}}{L_{i} \rho g},
\end{aligned}
$$

with $r_{D}$ being one-half the drift width, and $L_{i}$, the flow path length between boreholes.

Equations of motion in the several control volumes are next described.

Repository Control Volume. The mass balance and flow rates for the repository control volume are described below.

Mass Balance. For the repository control volume, the ideal gas law gives:

$$
P_{o} V_{g}=n_{g} R T
$$

where,

$$
\begin{array}{ll}
P_{o} & =\text { repository fluid (liquid and gas) pressure, } \\
V_{g} & =\text { volume of gas-saturated pore space, } \\
n_{g} & =\text { number of moles of gas, } \\
R & =\text { ideal gas constant, } \\
T & =\text { absolute temperature. }
\end{array}
$$

differentiation of Equation 14.1-3 gives:

$$
V_{g} \frac{d P_{o}}{d t}+P_{o} \frac{d V_{g}}{d t}=R T \frac{d n_{g}}{d t}
$$

The total repository pore volume $V_{P}$ is constant by assumption, so that

$$
V_{g}=V_{p}-V_{l},
$$

where $V_{l}$ is the volume of the liquid-saturated pore space. Substituting for $V_{g}$ in Equation 14.1-4 gives:

$$
\frac{n_{g} R T}{P_{o}} \frac{d P_{o}}{d t}-P_{o} \frac{d V_{1}}{d t}=R T \frac{d n_{g}}{d t}
$$

or

$$
\frac{d V_{l}}{d t}=\frac{n_{g} R T}{P_{o}^{2}} \frac{d P_{o}}{d t}-\frac{R T}{P_{o}} \frac{d n_{g}}{d t}=\sum Q_{\text {in }}-\sum Q_{o u t}+\frac{m_{l}}{\rho_{l}} \frac{d n_{l}}{d t}
$$

where

$\boldsymbol{Q}_{\text {in }}=$ volumetric repository inflow rate,

$Q_{\text {out }}=$ volumetric repository outflow rate,

$n_{l} \quad=$ number of moles of liquid produced in the repository,

$m_{l} \quad=$ liquid molecular weight,

$\rho_{l} \quad=$ liquid density. 
Finally,

$$
\frac{d P_{o}}{d t}=\frac{P_{o}^{2}}{n_{g} R T}\left[\sum Q_{\text {in }}-\sum Q_{\text {out }}\right]+\frac{P_{o}^{2}}{n_{g} R T} \frac{m_{l}}{\rho_{l}} \frac{d n_{l}}{d t}+\frac{P_{o}}{n_{g}} \frac{d n_{g}}{d t}
$$

Flow Rates. Net inflow to the repository control volume includes inflow from the anhydrite and outflow to each of the borehole/repository intersection control volumes. Flow in the anhydrite layer is approximated as darcian flow in a homogeneous annulus with impermeable upper and lower boundaries, and with the inner radius corresponding to the intersection of the anhydrite with the repository. Pressure at the inner radius is assumed to be equal to the average repository pressure, $P_{o}$. Outflow from the annulus is therefore a linear function of $P_{o}$, and may be calculated from:

$$
Q_{\text {in }}=\left(P_{F}-P_{o}\right) * h
$$

where:

* denotes convolution, and

$P_{F} \quad=\quad$ initial (pre-excavation) pressure in the anhydrite,

$h=$ inflow from the anhydrite resulting from impulse depressurization at the inner radius of the repository.

Outflow from the repository control volume to the intersection control volumes is simply,

$$
Q_{\text {out }}=\sum_{j} \zeta_{P_{j}}\left(P_{o}-P_{j}\right)
$$

where $P_{j}$ is the pressure at intersection $\mathrm{j}$.

Repository/Borehole Intersection Control Volumes. The mass balance and flow rates for the repository/borehole intersection control volumes are described.

Mass Balance. Volume balance expressions are written for each borehole $\mathrm{j}$ at the point of penetration of the waste panel (Figure 14-4).

Storage within the borehole control volume is assumed to be negligible, so that

$$
Q_{H_{j}}=Q_{R_{j}}+Q_{X_{j}}+Q_{P_{j}}
$$

where,

$Q_{H_{j}} \quad=\quad$ flow rate to the Snake Dolomite in borehole j,

$Q_{R_{j}} \quad=\quad$ flow rate from the repository to borehole $\mathrm{j}$,

$Q_{X_{j}} \quad=$ flow rate from all other boreholes to borehole j,

$Q_{P_{j}} \quad=\quad$ flow rate from the brine pocket in borehole $\mathrm{j}$.

Flow Rates. Flow rates in Equation 14.1-9 are

$$
Q_{H_{j}}=\zeta_{U_{j}}\left(P_{j}-P_{D}-\Delta P_{2}\right),
$$


14. Simplified Consequence Modeling for Salt Repository

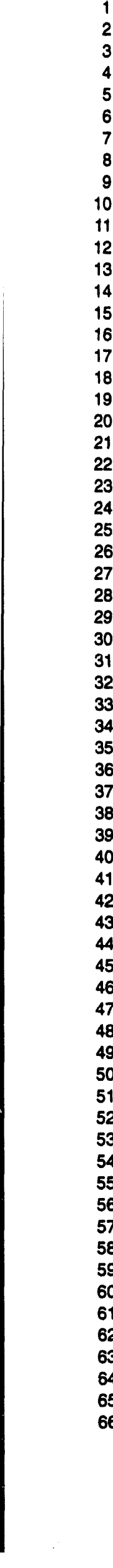

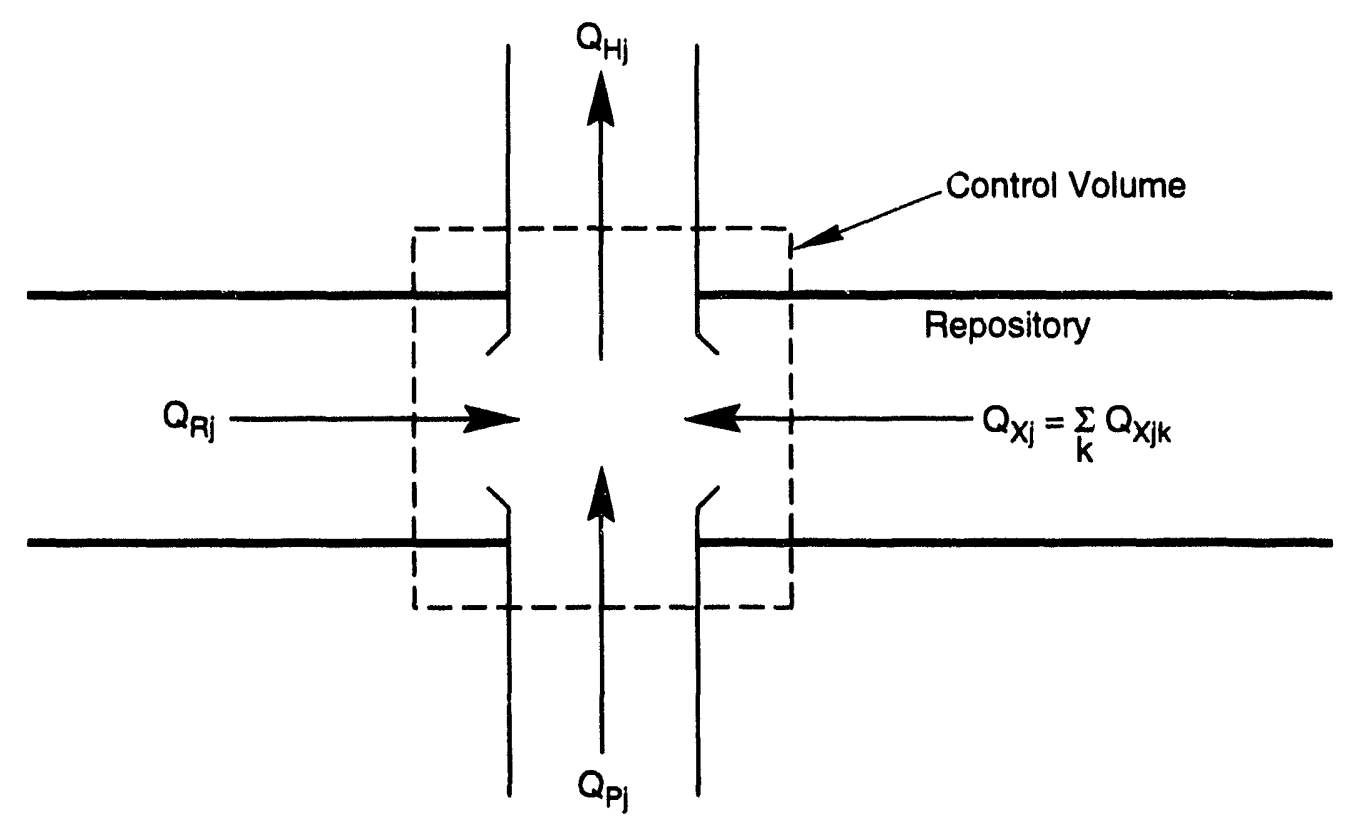

TRI-6342-3735-0

Figure 14-4. Schematic showing repository/borehole intersection control volume (see Figure 14-2).

$$
\begin{gathered}
Q_{R_{j}}=\zeta_{P_{j}}\left(P_{o}-P_{j}\right), \\
Q_{X_{j}}=\sum_{k \neq j} \zeta_{X_{j k}}\left(P_{k}-P_{j}\right), \\
Q_{P_{j}}=\zeta_{L_{j}}\left(P_{p}-P_{j}-\Delta P\right)
\end{gathered}
$$

where

$P_{D} \quad=$ constant pressure in the Snake Dolomite,

$P_{P} \quad=$ pressure in the brine reservoir,

$\Delta P_{1} \quad=\rho g \Delta Z_{1}$,

$\Delta P_{2} \quad=\rho g \Delta Z_{2}$.

Brine Reservoir Control Volume. The mass balance and flow rates for the brine reservoir control volume use the differential form of Equation 14.1-1:

$$
Q_{D}=\frac{d V_{p}}{d t}=S_{v} \frac{d P_{p}}{d t}
$$

where $Q_{D}$ is the total rate of recharge to the brine pocket, given by,

$$
Q_{D}=-\sum_{j} Q_{P_{j}}
$$




\subsubsection{Computational Model}

The numerical solution of the mathematical model is as follows. The mass balance expressions in terms of control volume pressures are from Equations 14.1-6, 14.1-7, and 14.1-8,

$$
\begin{aligned}
\frac{d P_{o}}{d t} & =\frac{P_{o}^{2}}{n_{g} R T}\left[\left(P_{F}-P_{o}\right) * h-\sum_{j} \zeta_{P_{j}}\left(P_{o}-P_{j}\right)\right] \\
& +\frac{P_{o}^{2}}{n_{g} R T} \frac{m_{l}}{P_{l}} \frac{d n_{l}}{d t}+\frac{P_{o}}{n_{g}} \frac{d n_{g}}{d t}
\end{aligned}
$$

from Equations 14.1-9 and 14.1-10,

$$
\begin{aligned}
\zeta_{U_{j}}\left(P_{j}-P_{D}-\Delta P_{2}\right) & =\zeta_{P_{j}}\left(P_{o}-P_{j}\right)+\sum_{k \neq j} \zeta_{X_{j k}}\left(P_{k}-P_{j}\right), \\
& +\zeta_{L_{j}}\left(P_{R}-P_{j}-\Delta P\right) \quad \forall j
\end{aligned},
$$

and from Equations 14.1-11, 14.1-12, and 14.1-10d,

$$
\frac{d P_{R}}{d t}=-\frac{1}{S_{V}} \sum_{j} \zeta_{L_{j}}\left(P_{R}-P_{j}-\Delta P\right)
$$

Equations 14.1-13 are solved using an explicit finite-difference approximation. Picard iteration is used to treat the non-linearity in Equation 14.1-13a. The gas generation and brine consumption terms in 14.1-13a are estimated explicitly using the corrosion model, GASSNF, while the convolution in 14.1-13a is approximated by a linear expansion around $P_{o}\left(t_{N}\right)$ using tabulated values of $h(t)$ and prior values of $P_{o}(t)$ :

$$
\left(P_{F}-P_{o}\right)^{*} h \approx Q_{o}+\alpha\left(P_{F}-P_{o}\left(t_{N}\right)\right)
$$

The finite-difference approximations of Equation 14.1-13 for the pressures in all control volumes at time $t_{2}$ given pressures at $t_{1}$ are then:

$$
\begin{gathered}
-\frac{\Delta t P_{o}^{2}\left(t_{*}\right)}{n_{8}\left(t_{2}\right) R T} \sum_{j} \zeta_{P_{j}} P_{j}\left(t_{2}\right) \\
+\left[1+\frac{\Delta t P_{o}^{2}\left(t_{*}\right)}{n_{g}\left(t_{2}\right) R T}\left(\alpha+\sum_{j} \zeta_{p_{j}}\right)-\frac{\Delta t}{n_{g}\left(t_{2}\right)} \frac{d n_{g}}{d t}\right] P_{o}\left(t_{2}\right) \\
=P_{o}\left(t_{1}\right)+\frac{\Delta t P_{o}^{2}\left(t_{*}\right)}{n_{g}\left(t_{2}\right) R T}\left(Q_{o}+\alpha P_{F}+\frac{m_{l}}{\rho_{l}} \frac{d n_{l}}{d t}\right) \\
\left(\begin{array}{c}
\left.\zeta_{U_{j}}+\zeta_{P_{j}}+\zeta_{L_{j}}+\sum_{k \neq j} \zeta_{X_{j k}}\right) P_{j}\left(t_{2}\right)-\zeta_{L_{j}} P_{P}\left(t_{2}\right)-\zeta_{P_{j}} P_{o}\left(t_{2}\right) \\
-\sum_{k \neq j} \zeta_{X_{j k}} P_{k}\left(t_{2}\right)=\left(P_{D}+\Delta P_{2}\right) \zeta_{U_{j}}-\Delta R \zeta_{L_{j}}
\end{array}\right.
\end{gathered}
$$




$$
\begin{gathered}
-\frac{\Delta t}{S_{V}} \sum_{j} \zeta_{L_{j}} P_{j}\left(t_{2}\right)+\left(1+\frac{\Delta t}{S_{V}} \sum_{j} \zeta_{L_{j}}\right) P_{P}\left(t_{2}\right) \\
=P_{P}\left(t_{1}\right)+\frac{\Delta t}{S_{V}} \sum_{j} \zeta_{L_{j}} \Delta P_{1}
\end{gathered}
$$

Equations 14.1-14 are solved iteratively, replacing $P_{o}\left(t_{*}\right)$ by the estimate of $P_{o}\left(t_{2}\right)$ from the previous iteration until the pressure change between iterations falls below a specified tolerance.

\subsection{Radionuclide Transport Modeling}

\subsubsection{Conceptual Model}

The same repository transport model was used in the simplified calculations for both the salt and granite repositories. In the repository transport model, discharge from the repository is assumed to result from interconnection of the repository and a stratigraphically isolated liquid source (brine reservoir) through a localized conduit (borehole). This interconnection induces flow through the repository drifts, which have a higher permeability than the surrounding rock. Radionuclides released from breached canisters move upward into the repository flow field, where they may be carried out of the repository through the localized conduits. Only transport in the liquid phase is considered in this analysis.

Placing waste parcels in the room/drift floor isolates them to some extent from the room/drift flow field (Figure 14-5). Transport from the canisters to the drift is therefore expected to proceed much more slowly than transport along the drift. To the extent waste emplacement package holes isolate individual waste packages from moving fluid, release from the waste packages can occur only by diffusion of dissolved nuclides, and may be limited by the rate at which diffusion can remove dissolved nuclides from pore fluid in contact with the waste emplacement packages.

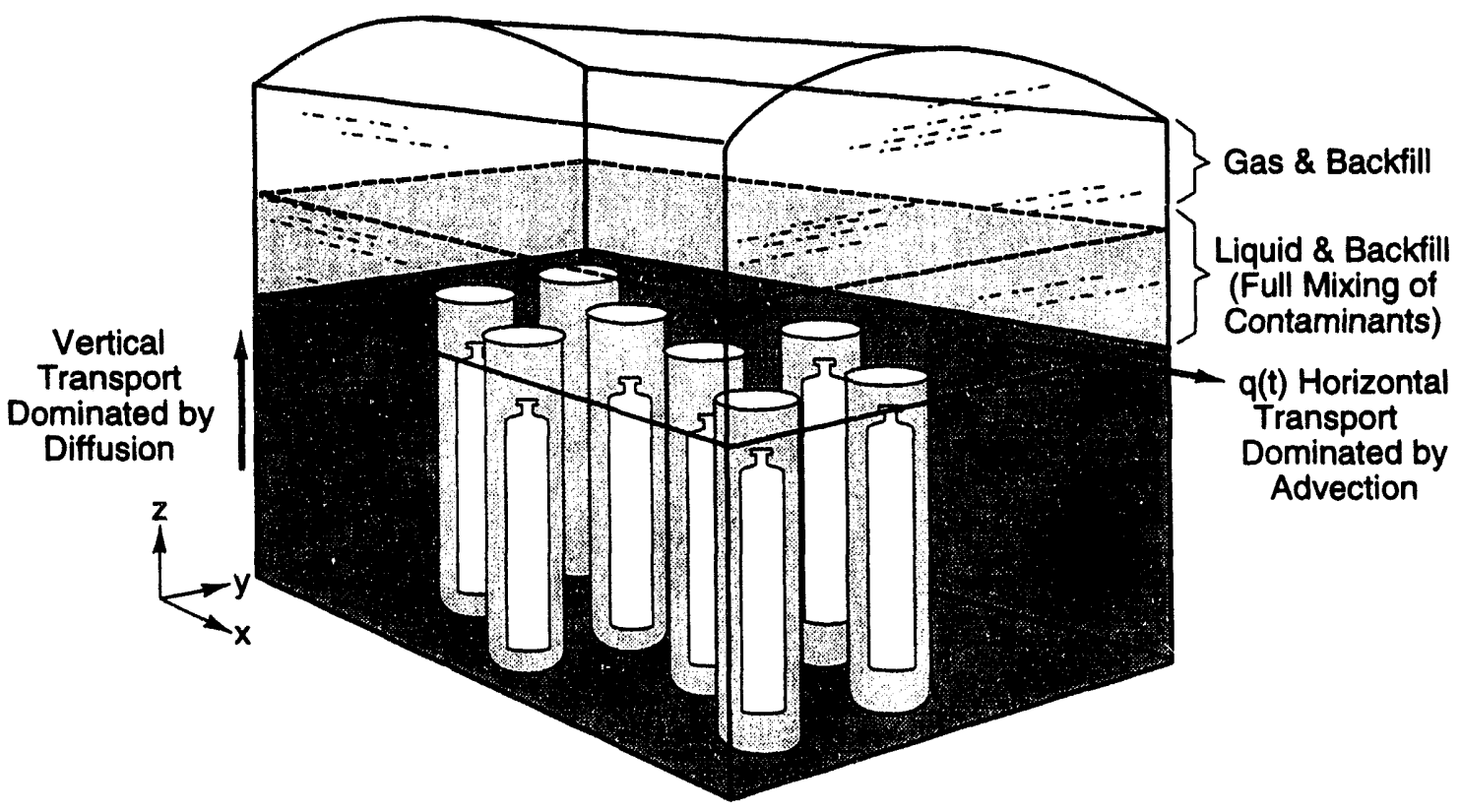

TRI-6342-3736-0

Figure 14-5. Canisters emplaced in floor are isolated to some extent from drift flow. 
Transport through the repository is divided into two domains: transport within the waste emplacement package holes and transport within the room/drifts. Fluid flow rates past waste emplacement packages will be slower than fluid flow rates through the drifts, and drift flow rates will be larger than flow rates through the host rock. The simplified transport model assumes a limiting case: within the waste emplacement holes, radionuclide migration is assumed to be caused by molecular diffusion, and diffusion is assumed to occur only vertically (i.e., diffusion radially outward into the host rock is neglected); within the drifts, transport occurs through advection of dissolved nuclides in the liquid phase. Bulk fluid motion is assumed to parallel the drift axes, and diffusion into the drift walls is neglected. Variations in concentration normal to flow direction ( $y$ and $z$ ) are assumed to be small compared to variations along the flow direction and are not resolved.

\subsubsection{Mathematical and Computational Model of Advection/Dispersion in Rooms/Drifts}

Define: $\bar{C}\left(x, t ; \Delta m, t_{s}, x_{s}\right)$ as the concentration [mole/L] (integrated over $\mathrm{y}$ and $\mathrm{z}$ ) at $\mathrm{x}$ due to introduction of mass $\Delta \mathrm{m}$ at time $\mathrm{t}_{\mathrm{s}}$ and position $\mathrm{x}_{\mathrm{s}}$. Assume $\bar{C}$ is approximately given by the solution of the advection-dispersion equation in 1 dimension with constant parameters:

$$
\bar{C}\left(x, t ; \Delta m, t_{s} x_{s}\right)=\frac{\Delta m}{2 \phi_{E}\left(\pi D\left(t-t_{s}\right)\right)^{1 / 2}} \exp \left\{-\frac{\left(x-x_{a}\right)^{2}}{4 D\left(t-t_{s}\right)}\right\} \exp \left(-\lambda\left(t-t_{s}\right)\right)
$$

where:

$$
\begin{array}{ll}
\phi_{E} & =\text { the drift backfill porosity-retardation product, } \\
x_{a}=x_{s}+\Delta x_{m} & =\text { the expected tracer position at time } t \\
D=\alpha_{L} \bar{V} & =\text { the dispersion coefficient, } \\
\alpha_{L} & =\text { the longitudinal dispersivity, } \\
\bar{V}=\bar{q} / \phi_{E} & =\text { the average particle velocity, } \\
\bar{q} & =\text { the average fluid specific discharge, } \\
\lambda & =\text { the radioactive decay constant, and } \\
\Delta x_{m} & =\text { the expected displacement during the interval }\left[t_{s} \rightarrow t\right], \text { given by } \\
\qquad \Delta x_{m}=\int_{t_{s}}^{t} \frac{q}{\phi_{E}} d t
\end{array}
$$

The disposal system is idealized as a number of connected segments (see Figure 14-2). The repository, in turn, is made up of a number of legs. Flow enters and leaves the repository at the leg junctions (Figure 14-6). The legs are assigned a length and width that characterize the available flow paths between junctions. The number of legs used to represent the salt repository is related to the number of discrete fluid sources and sinks (Figure 14-7). Junction flow rates for the salt repository are obtained from the salt flow models discussed in Section 14.1.

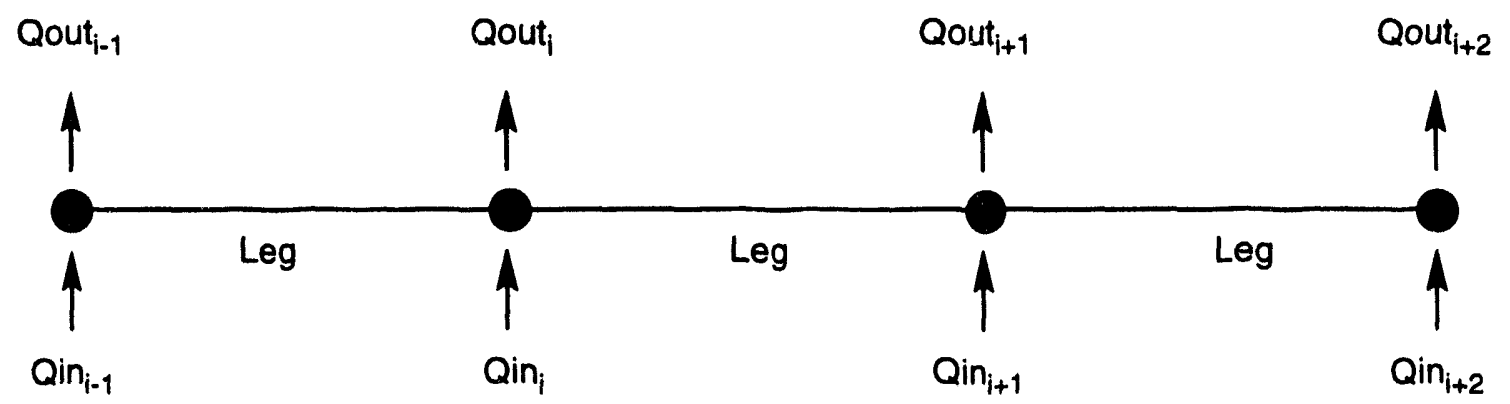

TRI-6342-3737-0

Figure 14-6. General repository flow network for repository control volume (see Figure 14-2). 
14. Simplified Consequence Modeling for Salt Repository

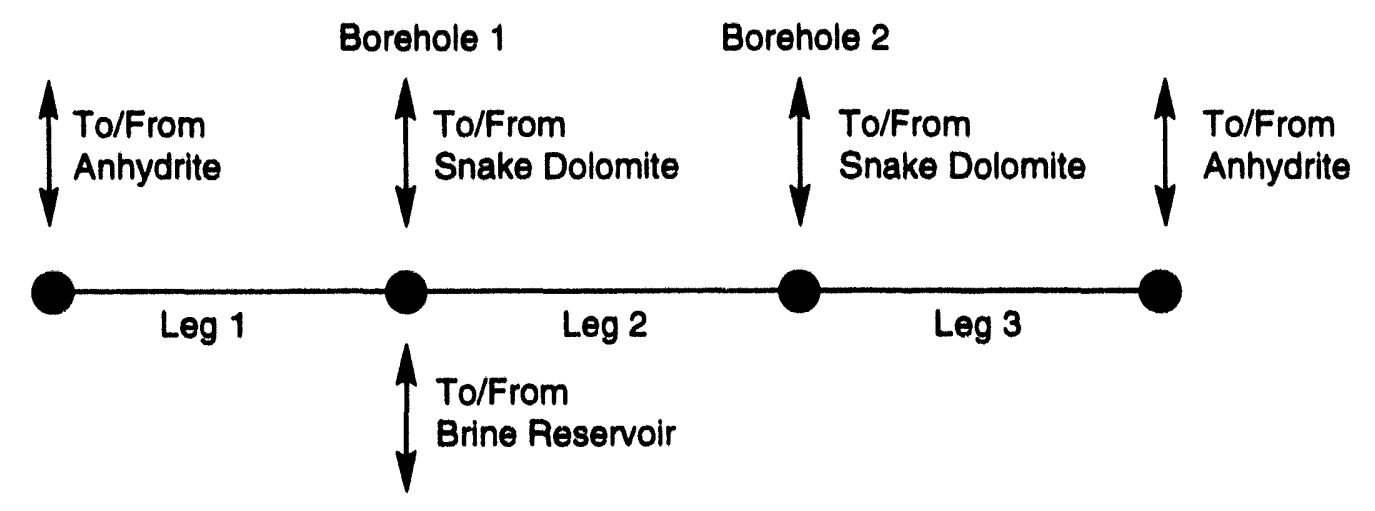

TRI-6342-3738-0

Figure 14-7. Specific flow network for control volume of salt repository.

Specific discharge within each leg is calculated from the net flow rates at each junction. Within each segment, specific discharge is assumed to vary linearly with distance (Figure 14-8).

$$
q\left(x_{l}\right)=q_{1}+\frac{x_{l}}{L}\left(q_{2}-q_{1}\right)
$$

The position of a particle at time $t_{2}, x_{a}$, introduced into the flow field at time $t_{1}$ can be found from Equation 14.2-3:

$$
\frac{d x_{l}}{d t}=V=\frac{q\left(x_{l}\right)}{\phi_{E}}=\frac{1}{\phi_{E}}\left[q_{1}+\frac{x_{l}}{L}\left(q_{2}-q_{1}\right)\right]
$$

Integration of this ODE gives

$$
\int_{t_{1}}^{t_{2}} d t=\int_{x_{s}}^{x_{a}} \frac{\phi_{E} d x_{l}}{q_{1}+\frac{x_{l}}{L}\left(q_{2}-q_{1}\right)}
$$

hence

$$
x_{a}=\left(x_{s}+\frac{q_{1}}{d}\right) e^{\frac{d}{\phi_{R}}\left(t_{2}-t_{1}\right)} \quad, d \neq 0
$$

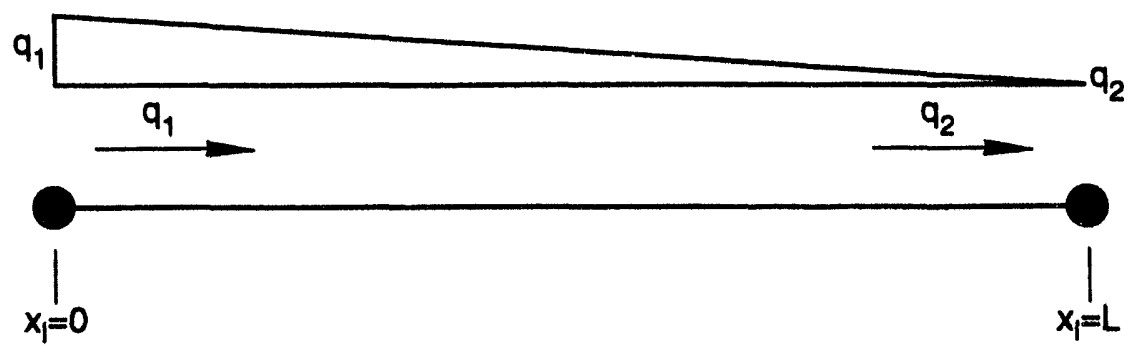

TA1-6342-3739-0

Figure 14-8. Storage can occur within the leg in the flow model. Specific discharge is assumed to vary linearly with distance for purposes of evaluating fluid flow between junctions. 
where

$$
x_{a}=x_{s}+\frac{q_{1}}{\phi_{E}}\left(t_{2}-t_{1}\right) \quad, d \neq 0
$$

$$
d=\frac{q_{2}-q_{1}}{L}
$$

Evaluating contaminant concentrations from advective transport typically requires more discretization. To resolve lateral concentration gradients within the repository flow network, each leg is divided into a number of cells of equal size (Figure 14-9).

Equation $14.2-1$ is then integrated to derive an expression for the average (over each cell) of $\bar{C}$ due to introduction uf a uniform distributed source in each cell at time $t_{s}$ as follows (Figure 14-10):

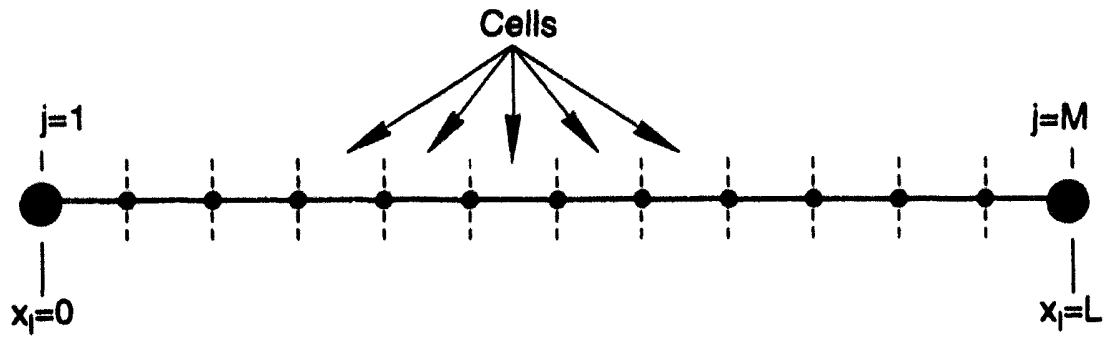

TA1-6342-3740-0

Figure 14-9. Each fluid flow leg in the repository is divided into a number of transport cells of equal size. Each cell has a source term.

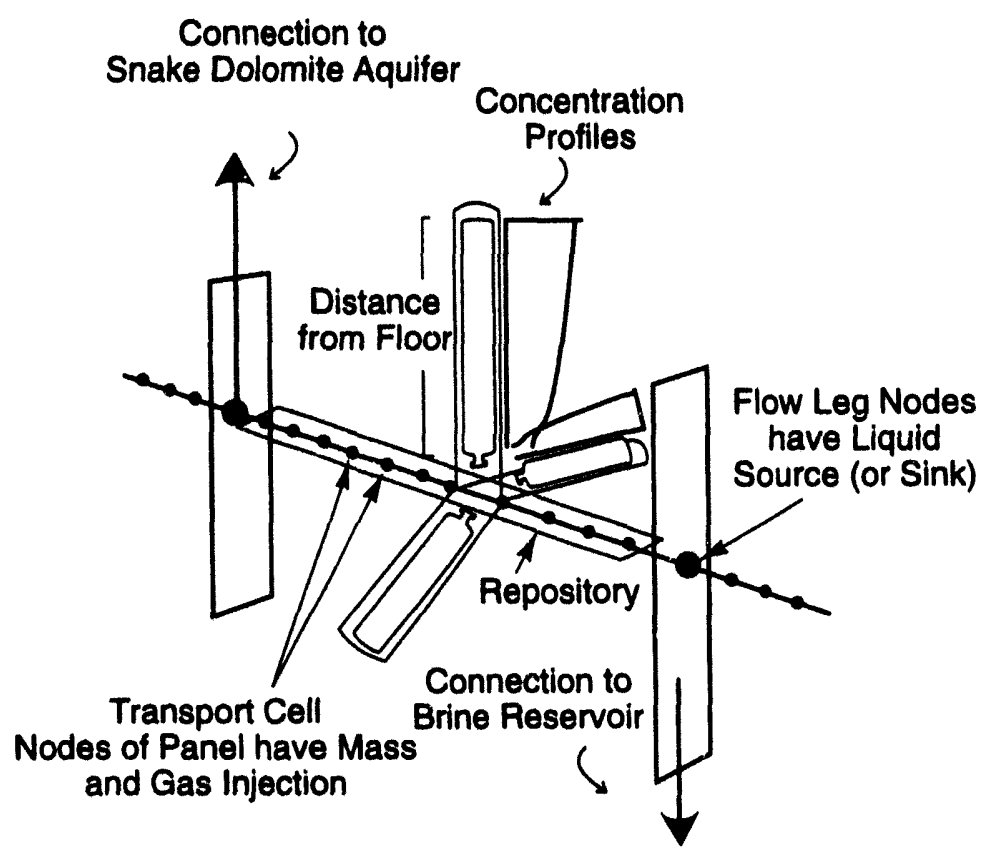

TP1-6342-3903-0

Figure 14-10. Mathematical one-dimensional transport model for repository. Note that rooms in panel are stacked end to end in the mathematical model (refer also to Figure 14-1). 
Define $\bar{C}_{P}\left(x_{1}, x_{w_{1}} t \Delta m, x_{s}, t_{s}\right)$ to be the average concentration over the cell with boundaries at $x_{1}$ and $x_{2}$ due to the introduction of mass $\Delta \mathrm{m}$ at position $x_{s}$. From Equation 14.2-1,

$$
\begin{aligned}
\bar{C}_{P}\left(x_{1}, x_{2}, t ; \Delta m, x_{s}, t_{s}\right) & =\frac{1}{x_{2}-x_{1}} \int_{x_{1}}^{x_{2}} \frac{\Delta m e^{-\lambda\left(t-t_{s}\right)}}{2 \phi_{E}\left[\pi D\left(t-t_{s}\right)\right]^{1 / 2}} \exp \left\{\frac{-\left(x-x_{a}\right)^{2}}{4 D\left(t-t_{s}\right)}\right\} d x \\
& =\frac{1}{x_{2}-x_{1}} \frac{\Delta m e^{-\lambda\left(t-t_{s}\right)}}{2 \phi_{E}}\left[\operatorname{erf}\left(\frac{x_{2}-x_{a}}{2 \sqrt{D\left(t-t_{s}\right)}}\right)-\operatorname{erf}\left(\frac{x_{1}-x_{a}}{2 \sqrt{D\left(t-t_{s}\right)}}\right)\right]
\end{aligned}
$$

Equation 14.2-5 is then used to derive an expression for the average concentration over the interval $\left[x_{1} \rightarrow x_{2}\right]$ due to a distributed source of strength $I_{m k}$ [moles/ength] on the interval $\left[x_{s 1} \rightarrow x_{s 2}\right]$ :

$$
\bar{C} D\left(x_{1}, x_{2}, t ; l_{m_{k}}, x_{s_{1}}, x_{s_{2}}, t_{s}\right)=\int_{x_{s_{1}}}^{x_{s_{2}}} \overline{C_{P}}\left(x_{1}, x_{2}, t ; I_{m_{k}} d x_{s}, x_{s}, t_{s}\right)
$$

The integral in Equation 14.2-6 is evaluated by noting that for any value of d, $x_{a}$ from Equation 14.2-4 is linear in $x_{s}$, and may be written:

$$
x_{a}=\sigma x_{s}+\delta
$$

The final expression for $\overline{\bar{C}}_{D}$ is then

$$
\begin{aligned}
& \bar{C}_{D}\left(j, t ; I_{m_{k}}, k . t_{s}\right)=\frac{I_{m_{k}} e^{-\lambda \Delta t}}{\sigma\left(x_{2}-x_{1}\right) \phi_{E}} \quad \frac{x_{1}-\delta}{2}\left(\operatorname{erf}\left(\frac{x_{1}-\sigma x_{s_{2}}-\delta}{\eta}\right)-\operatorname{erf}\left(\frac{x_{1}-\sigma x_{s_{1}}-\delta}{\eta}\right)\right) \\
& -\frac{x_{2}-\delta}{2}\left(\operatorname{erf}\left(\frac{x_{2}-\sigma x_{s_{2}}-\delta}{\eta}\right)-\operatorname{erf}\left(\frac{x_{2}-\sigma x_{s_{1}}-\delta}{\eta}\right)\right) \\
& +\frac{\sigma x_{s_{1}}}{2}\left(\operatorname{erf}\left(\frac{x_{1}-\sigma x_{s_{1}}-\delta}{\eta}\right)-\operatorname{erf}\left(\frac{x_{2}-\sigma x_{s_{1}}-\delta}{\eta}\right)\right) \\
& -\frac{\sigma x_{s_{2}}}{2}\left(\operatorname{erf}\left(\frac{x_{1}-\sigma x_{s_{2}}-\delta}{\eta}\right)-\operatorname{erf}\left(\frac{x_{2}-\sigma x_{2}-\delta}{\eta}\right)\right) \\
& +\frac{\eta}{2 \sqrt{\pi}}\left(\exp \left(-\frac{\left(x_{1}-\sigma x_{s_{2}}-\delta\right)^{2}}{\eta^{2}}\right)-\exp \left(\frac{\left(x_{1}-\sigma x_{s_{1}}-\delta\right)^{2}}{\eta^{2}}\right)-\exp \left(\frac{\left(x_{2}-\sigma x_{s_{2}}-\delta\right)^{2}}{\eta^{2}}\right)\right. \\
& +\exp \left(-\frac{\left(x_{2}-\sigma x_{s_{1}}-\delta\right)^{2}}{\eta^{2}}\right)
\end{aligned}
$$

where

$$
\begin{gathered}
\eta=2 \sqrt{D \Delta t} \\
\Delta t=t-t_{s}
\end{gathered}
$$

and $j$ and $k$ are the indices of the cells with boundary locations $\left(x_{1}, x_{2}\right)$ and $\left(x_{s 1}, x_{s 2}\right)$. The total concentration at time $t$ is the superposition of the contributions of all $M$ cells: 


$$
\bar{C}\left(j, t ; t_{s}\right)=\sum_{k=1}^{M} \overline{\bar{C}}_{D}\left(j, t ; I_{m_{k}}, k, t_{s}\right)
$$

The distributed sources $I_{m k}$ include all mass available for transport at time $t_{s}$, and consist of three parts: the initial mass present in the cell, mass introduced by decay, and the mass introduced by diffusion from the waste emplacement package holes in the cell. The first part is obtained from a prior solution for $\overline{\bar{C}}$, the second from the average activity of the parent in the cell, and the third from implicit coupling with the waste emplacement package hole diffusion model discussed in the next subsection.

In summary, concentrations in each cell in each leg are calculated at time $t_{2}$ given concentrations at time $t_{1}$ using the following procedure:

1. Equation $14.2-8$ is used to calculate the average concentration for each cell at time $t_{2}$ due to advection, dispersion, and decay of mass present at time $\mathrm{t}_{1}$;

2. For each cell, $\overline{\bar{C}}\left(\mathrm{t}_{2}\right)$ is modified to include diffusion from the waste emplacement package holes in the cell as discussed below. Continuous diffusion over $\left[t_{1} \rightarrow t_{2}\right]$ is approximated as instantaneous injection at $t_{2}$.

3. The activity in each cell, assuming a linear variation in $\overline{\bar{C}}$ between $t_{1}$ and $t_{2}$, is used to augment concentrations of transported daughter nuclides as follows:

a. decay between $t_{1}$ and $\left(t_{1}+t_{2}\right) / 2$ is approximated by an instantaneous daughter source at time $t_{1}$.

b. decay between $\left(t_{1}+t_{2}\right) / 2$ and $t_{2}$ is approximated by an instantaneous daughter source at time $t_{2}$.

\subsubsection{Mathematical and Computational Model of Diffusion in Waste Emplacement Package Holes}

All waste package hole boundaries are assumed to be impermeable: radial concentration variations are assumed to be small, so that each waste package hole concentration is only a function of time and elevation from the bottom of the waste package hole (Figure 14-11).

Nuclide transport is assumed to be due to diffusion alone. Radioactive decay is not included, so that concentrations below and above the canister $\left(c_{l}(z, t)\right.$ and $\left.c_{2}(z, t)\right)$ are solutions of the following PDE's:

$$
\begin{gathered}
D_{1} \frac{\partial^{2} c_{1}}{\partial z^{2}}+S_{1}=R \phi_{1} \frac{\partial c_{1}}{\partial t} \\
D_{2} \frac{\partial^{2} c_{2}}{\partial z^{2}}=R \phi_{2} \frac{\partial c_{2}}{\partial t}
\end{gathered}
$$

where:

$$
\begin{array}{ll}
\quad \phi_{1}\left(1-\left(\frac{r_{c}}{r_{h}}\right)^{2}\right) \phi_{f} & =\text { macroscopic porosity for } 0 \leq z \leq z_{c} \\
\phi_{2}=\phi_{f} & =\text { macroscopic porosity for } z_{c}<z \leq z_{h} \\
D_{1}=D_{m} \phi_{1} \tau & =\text { macroscopic diffusion coefficient for } 0 \leq z \leq z_{c} \\
D_{2}=D_{m} \phi_{2} \tau & =\text { macroscopic diffusion coefficient for } z_{c}<z \leq z_{h}
\end{array}
$$




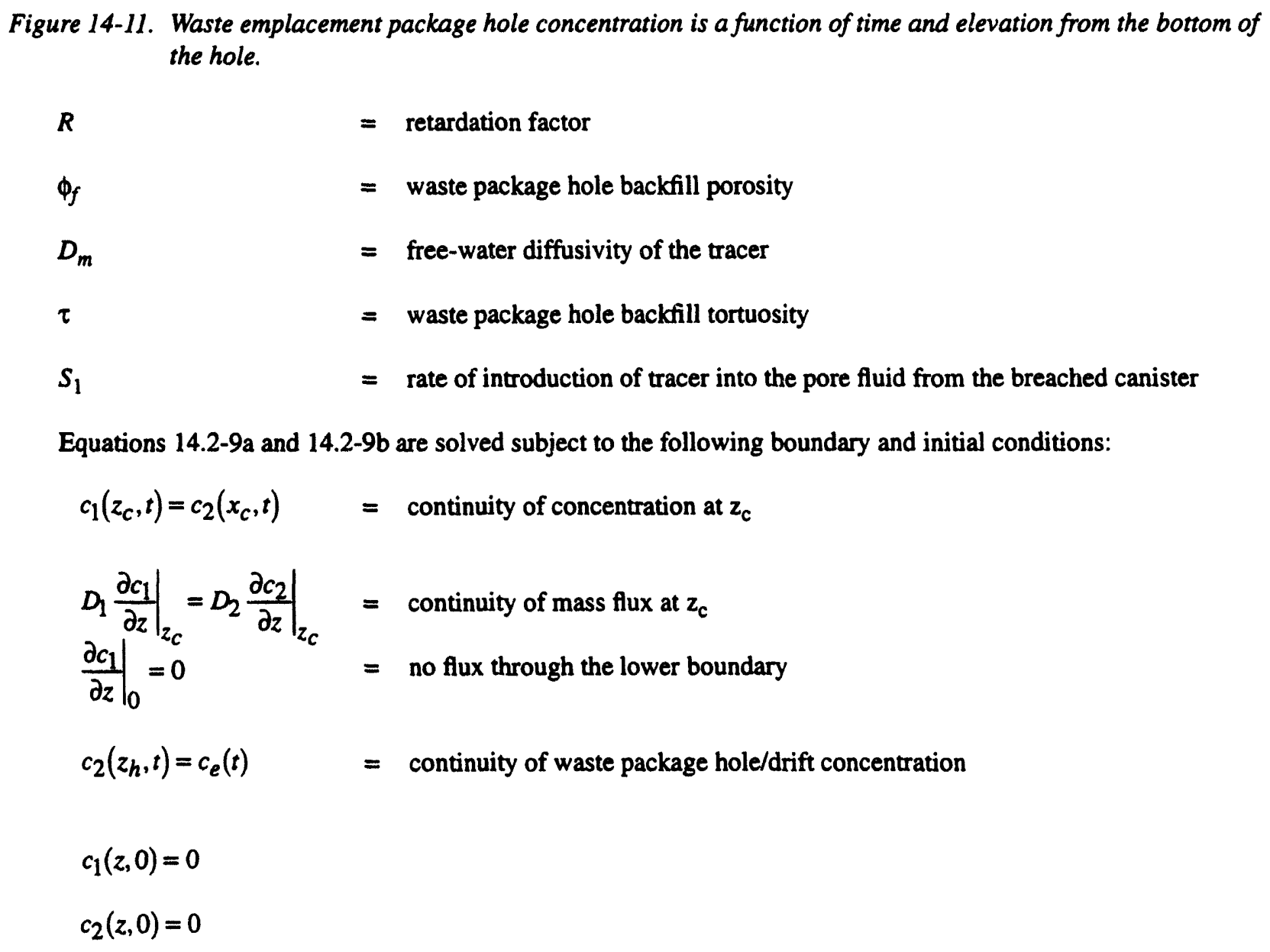

Figure 14-11. Waste emplacement package hole concentration is a function of time and elevation from the bottom of the hole.
$R$
$=$ retardation factor
$\phi_{f}$
= waste package hole backfill porosity
$D_{m}$
$=$ free-water diffusivity of the tracer
$\tau$
= waste package hole backfill tortuosity
$S_{1}$
$=$ rate of introduction of tracer into the pore fluid from the breached canister

Equations $14.2-9 \mathrm{a}$ and $14.2-9 \mathrm{~b}$ are solved subject to the following boundary and initial conditions:

$$
\begin{aligned}
& c_{1}\left(z_{c}, t\right)=c_{2}\left(x_{c}, t\right)=\text { continuity of concentration at } z_{c} \\
& \left.D_{1} \frac{\partial c_{1}}{\partial z}\right|_{z_{c}}=\left.D_{2} \frac{\partial c_{2}}{\partial z}\right|_{z_{c}}=\text { continuity of mass flux at } z_{c} \\
& \left.\frac{\partial c_{1}}{\partial z}\right|_{0} ^{\partial z_{c}}=0 \quad=\text { no flux through the lower boundary } \\
& c_{2}\left(z_{h}, t\right)=c_{e}(t) \quad=\text { continuity of waste package hole/drift concentration } \\
& c_{1}(z, 0)=0 \\
& c_{2}(z, 0)=0
\end{aligned}
$$
1 2 
The concentration at the top of the waste package hole is assumed equal to the average drift concentration in the cell containing the waste package. Note that Equations $14.2-9 \mathrm{a}$ and $14.2-9 \mathrm{~b}$ can be written for any number of waste parcel types in the same cell, which will have a common $c_{e}(t)$, but may have different sources and concentration profiles.

The diffusion model for the waste emplacement package borehole is used to couple the canister model with the drift transport model through the source rate, $S_{1}$, and the drift concentration, $c_{e}$. An implicit coupling is desirable for stability. A solution to the governing equations is therefore sought in terms of unknown source rates and boundary concentrations at the time of evaluation, $t$. We are not aware of a solution that satisfies this condition and includes decay in the governing equations. The method adopted here treats decay in the waste emplacement package holes only approximately: decay of nuclides in the pore space adjacent to the canister $\left(0 \leq z \leq z_{c}\right)$ is included in the source term, $S_{1}$, provided by the canister model. Decay in the pore space above the canister $\left(z_{c} \leq z \leq z_{h}\right)$ is ignored. Concentrations (and therefore fluxes) of nuclides whose halflife is not larger than the diffusion time through this region will be overestimated. Because decay is included in the drift transport model, the extent of overestimation will be small (even for short-lived nuclides) provided the residence time in the drift is large compared to the diffusion time through $\left(z_{c} \leq z \leq z_{h}\right)$

Following Özisik (1986), the solution is obtained in terms of an incremental concentration:

$$
\begin{aligned}
& \varphi_{1}(x, t)=c_{1}(z, t)-c_{e}(t) \\
& \varphi_{2}(x, t)=c_{2}(z, t)-c_{e}(t)
\end{aligned}
$$

as:

$$
\varphi_{i}(z, t)=\sum_{n=1}^{\infty} e^{-\beta_{n}^{2} t} X_{i_{n}}(z)\left[\int_{t^{\prime}=0}^{t}\left\{g_{n}^{*}\left(t^{\prime}\right)-I_{n}^{*} \frac{d c_{e}}{d t^{\prime}}\right\} e^{{ }^{2} t^{\prime}} d t^{\prime}\right] \quad i=1,2
$$

where:

$$
\begin{gathered}
g_{n}^{*}\left(t^{\prime}\right)=\frac{1}{N} S_{1}\left(t^{\prime}\right) \int_{0}^{z_{c}} X_{1_{n}}(z) d z \\
I_{n}^{*}=\frac{1}{N}\left[\frac{D_{1}}{\alpha_{1}} \int_{0}^{z_{c}} X_{1_{n}}(z) d z+\frac{D_{2}}{\alpha_{2}} \int_{0}^{z_{c}} X_{2_{n}}(z) d z\right] \\
N=\frac{D_{1}}{\alpha_{1}} \int_{0}^{z_{c}} X_{1_{n}}^{2}(z) d z+\frac{D_{2}}{\alpha_{2}} \int_{0}^{z_{c}} X_{2_{n}}^{2}(z) d z \\
\alpha_{1}=\frac{D_{1}}{R \phi_{1}}=\frac{D_{m} \tau}{R} \\
\alpha_{2}=\frac{D_{2}}{R \phi_{2}}=\frac{D_{m} \tau}{R}
\end{gathered}
$$


and $X_{1_{n}}$ and $X_{2 n}$ are solutions of the eigenvalue problem:

$$
\begin{array}{ll}
\alpha_{1} \frac{d^{2} X_{1_{n}}}{d z^{2}}+\beta_{n}^{2} X_{1_{n}}=0, & 0 \leq z \leq z_{c} \\
\alpha_{2} \frac{d^{2} X_{2_{n}}}{d z^{2}}+\beta_{n}^{2} X_{2_{n}}=0, & z_{c} \leq z \leq z_{h}
\end{array}
$$

subject to:

$$
\begin{gathered}
X_{1_{n}}\left(z_{c}\right)=X_{2_{n}}\left(z_{c}\right) \\
\left.D_{1} \frac{d X_{1_{n}}}{d z}\right|_{z_{c}}=\left.D_{2} \frac{d X_{2_{n}}}{d z}\right|_{z c} \\
\left.\frac{d X_{1_{n}}}{d z}\right|_{0}=0 \\
X_{2_{n}}\left(z_{h}\right)=0
\end{gathered}
$$

Noting that $\alpha=\alpha_{1}=\alpha_{2}$, and defining $\gamma_{n}=\beta_{n} / \sqrt{\alpha}$, the general solution of Equation 14.2-15 is

$$
\begin{gathered}
X_{1_{n}}=A_{1_{n}} \cos \left(\gamma_{n} z\right)+B_{1_{n}} \sin \left(\gamma_{n} z\right) \\
X_{2_{n}}=A_{2_{n}} \cos \left(\gamma_{n} z\right)+B_{2_{n}} \sin \left(\gamma_{n} z\right)
\end{gathered}
$$

Conditions (14.2-16) determine $A_{1}, B_{1_{n}}, A_{2}, B_{2}$ within a common multiplicative constant. Arbitrarily assigning $A_{1_{n}}=1$, Equations $14.2-16 \mathrm{a}, \mathrm{b}$, and $\mathrm{c}$ can be solved for the remaining coefficients:

$$
\begin{gathered}
B_{1_{n}}=0 \\
A_{2_{n}}=1-\left(\frac{r_{c}}{r_{h}}\right)^{2} \sin ^{2}\left(\gamma_{n} z_{c}\right) \\
B_{2_{n}}=\left(\frac{r_{c}}{r_{h}}\right)^{2} \cos \left(\gamma_{n} z_{c}\right) \sin \left(\gamma_{n} z_{c}\right)
\end{gathered}
$$

while Equation $14.2-16$ provides the transcendental equation satisfied by the normalized eigenvalues $\gamma_{n}$ :

$$
\frac{1}{2}\left(\frac{r_{c}}{r_{h}}\right)^{2} \cos \left(\gamma_{n}\left(2 z_{c}-z_{h}\right)\right)+\left(1-\frac{1}{2}\left(\frac{r_{c}}{r_{h}}\right)^{2}\right) \cos \left(\gamma_{n} z_{h}\right)=0
$$

The waste package hole diffusion model is coupled to the source term subrnodel, CONCSNF, and the drift advection/dispersion model through the nuclide source rate, $S_{1}$, and the drift concentration, $c_{e}$, in Equation 14.2-10. Mass transfer between the canister and waste package hole cont , volumes, and between the waste package hole and drift 
model control volumes, is based on discretization of the simulation period into $\mathrm{N}_{t}$ equal time intervals of size $\Delta_{\mathrm{t}}$. Over each interval, $S_{1}$ is assumed to be constant, while $c_{e}$ is assumed to vary linearly between values given by $\overline{\bar{C}}$ evaluated at the interval endpoints (Figure 14-12):

$$
\begin{aligned}
& \varphi_{i}\left(z, t_{N_{t}}\right) \text { can then be written in terms of } \mathrm{K} \text { values of } S_{1} \text { and } \frac{d c_{e}}{d t}: \\
& \varphi_{i}\left(z, t_{K}\right)=\sum_{n=1}^{\infty} \frac{X_{i_{n}}(z)}{\beta_{n}^{2}} \sum_{k=1}^{K}\left(g_{n}^{*} S_{1 k}-\left.I_{n}^{*} \frac{d c_{e}}{d t}\right|_{k}\right) e^{-\beta_{n}^{2}\left({ }^{\prime} K-t_{k}\right)}\left(1-e^{-\beta_{n}^{2}\left(t_{k}-t_{k-1}\right)}\right)
\end{aligned}
$$

where:

$$
\begin{aligned}
& g_{n}^{\prime *}=\frac{\sqrt{\alpha}}{N \beta_{n}} \sin \left(\frac{B_{n}}{\sqrt{\alpha}} z_{c}\right) \\
& S_{1_{k}}=\text { constant value of } S_{1} \text { between } t_{k-1} \text { and } t_{k} \\
& \left.\frac{d c_{e}}{d t}\right|_{k}=\text { constant value of } \frac{d c_{e}}{d t} \text { between } t_{k-1} \text { and } t_{k}
\end{aligned}
$$

The solution for incremental concentration $\varphi_{i}\left(z, t_{K}\right)$ is not explicitly required to couple waste package release to diffusive flux into the drift. All that is required is an expression for the total radionuclide mass exchanged between control volumes during the interval $\left[t_{K-1} \rightarrow t_{K}\right]$. The waste package model control volume includes the waste parcel and its backfill-buffer below $\mathrm{z}_{\mathrm{c}}$; the advection/dispersion model control volume is the drift. The total moles removed from the waste package model control volume during $\left[t_{K-1} \rightarrow t_{K}\right]$ is then

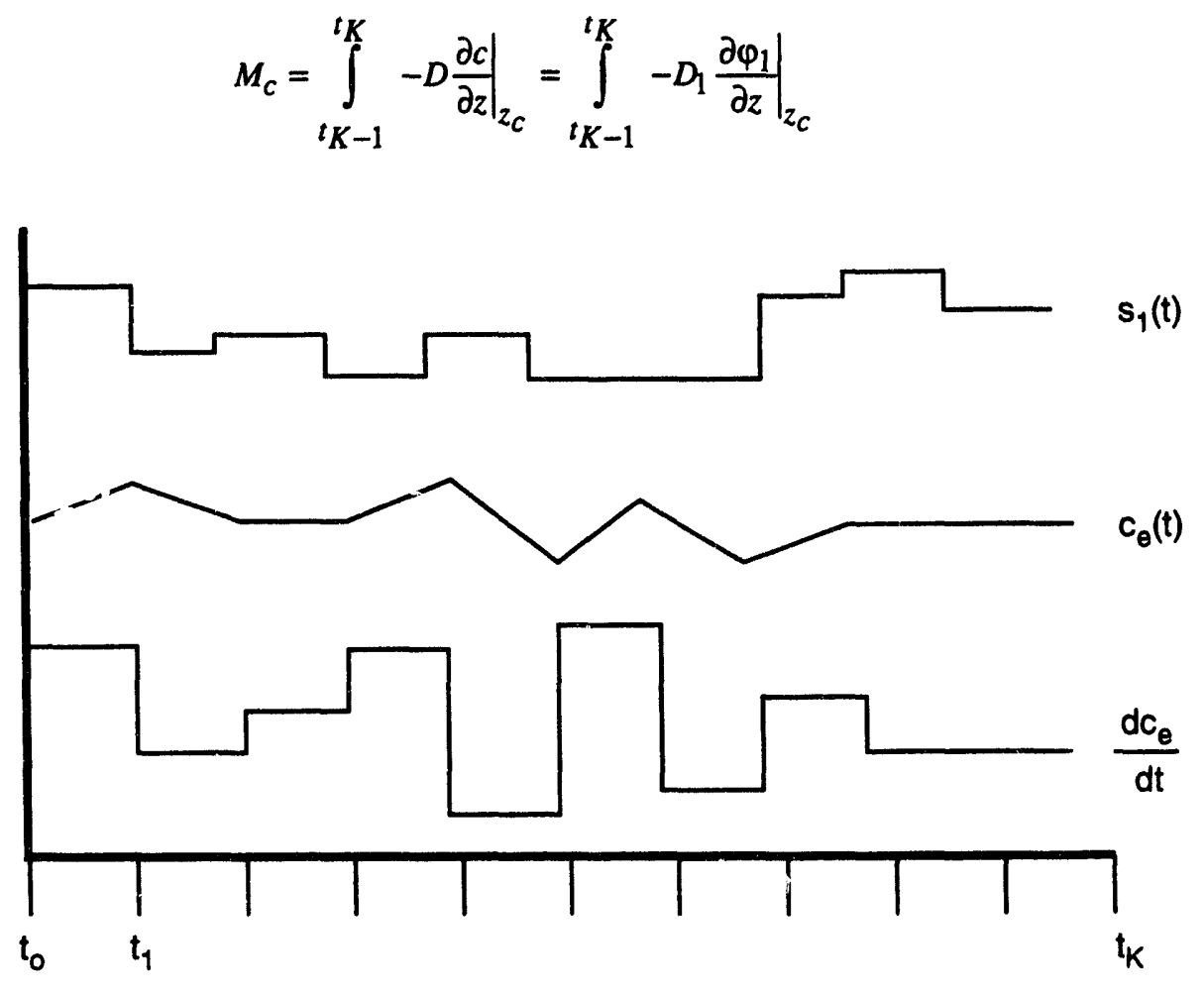

Figure 14-12. Approximation of source rate and drift concentration. 


\section{Simplified Consequence Modeling for Salt Repository}

while the total mass injected into the advection/dispersion model control volume during $\left[t_{\mathrm{K}-1} \rightarrow t_{\mathrm{K}}\right.$ ] is

$$
M_{D}=\int_{t_{K-1}}^{t_{K}}-\left.D \frac{\partial c}{\partial z}\right|_{z_{h}}=\int_{t_{K-1}}^{t_{K}}-\left.D_{2} \frac{\partial \varphi_{2}}{\partial z}\right|_{z_{h}}
$$

Both $M_{c}$ and $M_{p}$ can ultimately be expressed in a similar form, where the dependence of mass exchange on $S_{1}$ and $c_{e}\left(t_{K}\right)$ has been made explicit:

$$
\begin{aligned}
M_{X}= & P_{X}\left(\sum_{n=1}^{\infty} G_{X_{n}} H_{n, K}-\frac{c_{e_{K}-1}}{\Delta t} \sum_{n=1}^{\infty} G_{X_{n}} W_{n} I_{n}^{*}\right) \\
& -\left(P_{X} \sum_{n=1}^{\infty} G_{X_{n}} W_{n} g_{n}^{*}\right) S_{1_{K}}+\left(\frac{P_{X}}{\Delta t} \sum_{n=1}^{\infty} G_{X_{n}} W_{n} I_{n}^{*}\right) c_{e_{K}} \quad X=c, D
\end{aligned}
$$

where:

$$
\begin{aligned}
& P_{c}=\left(1-\left(\frac{r_{c}}{\eta_{h}}\right)^{2}\right) \frac{\phi_{f}}{D_{m} \tau} \\
& P_{D}=\frac{\phi_{f}}{D_{m} \tau} \\
& G_{c_{n}}=\left(\frac{\sqrt{\alpha}}{\beta_{n}}\right)^{3} \sin \left(\frac{\beta_{n} z_{c}}{\sqrt{\alpha}}\right) \\
& G_{D_{n}}=\left(\frac{\sqrt{\alpha}}{\beta_{n}}\right)^{3}\left\{A_{2} \sin \left(\frac{\beta_{n} z_{h}}{\sqrt{\alpha}}\right)-B_{2 n} \cos \left(\frac{\beta_{n} z_{h}}{\sqrt{\alpha}}\right)\right\} \\
& H_{n, K}=\left(1-e^{-\beta_{n}^{2} \Delta t}\right)^{2} \sum_{k=1}^{K-1}\left(g_{n}^{\prime *} S_{1_{k}}-\left.I_{n}^{*} \frac{d c_{e}}{d t}\right|_{k}\right)\left(e^{\left.-\beta_{n}^{2} \Delta t\right) K-1-k}\right. \\
& W_{n}=1-e^{-\beta_{n}^{2} \Delta t-\beta_{n}^{2} \Delta t}
\end{aligned}
$$

The source term, $S_{1_{K}}$, is treated explicitly. As discussed in the subsection above, drift cell concentrations, $C\left(t_{K}\right)$, are updated to reflect diffusion into the drift during the interval $\left[t_{K-1} \rightarrow t_{K}\right]$ using the linear relationship between $M_{D}$ and $c_{e}\left(t_{K}\right)$ given in Equation 14.2-22, which may be written:

$$
M_{D}^{l}=J_{D}^{l}+\gamma_{D}^{l} \frac{\overline{\bar{C}}}{\omega^{l}}
$$

where the superscript $l$ is an index of waste parcel type in the drift cell, and $\omega^{\prime}$ is the required conversion factor between $\bar{C}$ [mole/length of drift] and $c_{e}$ [moles/length of waste package hole].

If $\overline{\bar{C}}$ is the radionuclide concentration in the drift following redistribution by advection/dispersion, the augmented drift concentration, which includes diffusion from the waste package holes, is 


$$
\overline{\bar{C}}^{+}=\overline{\bar{C}}+\frac{\sum_{l=1}^{N_{w}} M_{D}^{l}}{\Delta x \phi_{E}}=\overline{\bar{C}}+\frac{\sum_{l=1}^{N_{w}}\left(J_{D}^{l}+\gamma_{D}^{l} \frac{\overline{\bar{C}}^{+}}{\omega^{l}}\right)}{\Delta x \phi_{E}}
$$

which can be solved for $\overline{\bar{C}}^{+}$:

$$
\overline{\bar{C}}^{+}=\frac{\Delta x \phi_{E} \overline{\bar{C}}+\sum_{l=1}^{N_{w}} J_{D}^{l}}{\Delta x \phi_{E}-\sum_{l=1}^{N_{w}} \frac{\gamma^{l} D}{\omega^{l}}}
$$

where $N_{w}$ is the number of waste package types in the cell.

In summary, the waste package hole diffusion model couples the source term submodel, CONCSNF, and drift transport model (Section 14.2.2) using the following procedure:

1. The drift transport model is used to calculate $\overline{\bar{C}}\left(t_{K}\right)$ given $\overline{\bar{C}}\left(t_{K-1}\right)$

2. Mass diffused into the drift from each waste emplacement package over $\left[t_{K-1} \rightarrow t_{K}\right]$ is used to calculate the augmented concentration $\overline{\bar{C}}^{+}\left(t_{K}\right)$ using Equation 14.2-23. This mass is available for transport in the drift during $\left[\mathrm{t}_{\mathrm{K}} \rightarrow \mathrm{t}_{\mathrm{K}+1}\right]$.

3. The mass removed from the waste package control volume, $M_{c}$, is calculated from Equation 14.2-22 using the updated concentration $\overline{\bar{C}}^{+}\left(t_{K}\right)$

4. $\mathrm{M}_{\mathrm{c}}$ is used by the canister model to calculate a new canister source term $\left(\mathrm{S}_{1}\right)$ for the waste package hole diffusion model. The source term provided by the canister model includes decay of dissolved and adsorbed nuclides in the pore space adjacent to the canister. This source is assumed to apply for the subsequent time interval $\left[t_{K} \rightarrow t_{K+1}\right]$. 


\title{
15. Simplified Consequence Modeling for Granite Repository
}

\author{
W. E. Beyeler
}

The consequence models for the "Simple PA" for the granite repository are similar to those described for the salt repository (Chapter 14). Hence, only the differences between the models are emphasized in the following discussion.

\subsection{Repository Fluid Flow Modeling}

\subsubsection{Conceptual Model}

The granite repository is assumed to be located entirely within an intact block of granite. Localized zones of high fracture density above and below the repository provide the only lateral pathways to the accessible environment. Repository access shaft(s) are assumed to pass through an overlying fracture zone. The arguments for modeling only large, prominent features are presented in Chapter 5 (see Figure 5-2). Fluid processes in the Simple PA model are shown in Figure 15-1.

Release from the repository can only occur following connection to one or more fracture zones at one or more locations. The most likely connection is through an access shaft following failure of a shaft seal. Other connections may be created through other shafts, by a borehole drilled through the repository, or through fracturing of the host granit around the repository induced by mechanical or thermal stresses (see Figures 7-6 and 8-8).

The simplest representation of flow through the system uses a single set of lumped parameters to describe the hydrologic properties of the primary features (Figure 15-2). Note that flow through the system is assumed to be limited by the permeabilities of the connections to the fracture zones. The permeabilities of the fracture zones are assumed to be effectively infinite in comparison. This assumption implies that pressures at the connection/fracture zone junctions are constants, independent of the flow rate through the upper and lower connections. The pressures are instead determined by external factors. The repository permeability is also assumed to be effectively infinite compared to connection permeabilities. The validity of this assumption depends on the permeabilities and areas of the connections. This assumption allows the repository state to be described by a single pressure in each phase. In the case of single-phase flow, fluid flow rates are conservatively overpredicted by neglecting resistance to flow through the backfill. In BRAGFLO_T, the flow model used in the Complex PA, the permeability of the repository backfill was a significant impediment to flow. Fluid flow rates through the repository are therefore overpredicted by the flow model used in the Simple PA.

The flow rate through any leg of the network system is a function of conductive properties of the legs. In the fracture zones, fracture frequency, extent, aperture, and connectivity, as well as the degree of flow channeling within individual fractures, combine to determine the hydraulic and transport properties of the fracture zone. The same types of parameters determine the bulk properties of any fracture connections to the repository. Although distributions for fracture parameters might be constructed from existing granite site data and expert opinion, and used to derive distributions of flow rates through the fracture zones, this process is difficult. Furthermore, the data can vary greatly from location to location within a given site. A similar uncertainty exists in characterizing the flow through failed shaft seals and boreholes because the mode and degree of failure are uncertain. Hence, the range in possible flow rates resulting from simulation using an actual fracture network is expected to be large because of the underlying uncertainty in fracture parameters.

All elements of the system were treated as porous media obeying Darcy's law, with uncertain lumped parameters describing flow and transport behavior (e.g., hydraulic conductivity and effective porosity). This method is not expected to mimic the response of a fractured system or failed seal for any particular value of the model parameters. 


\section{General Conceptual Model}

Plan View of Panel (100 Rooms in Sequence)
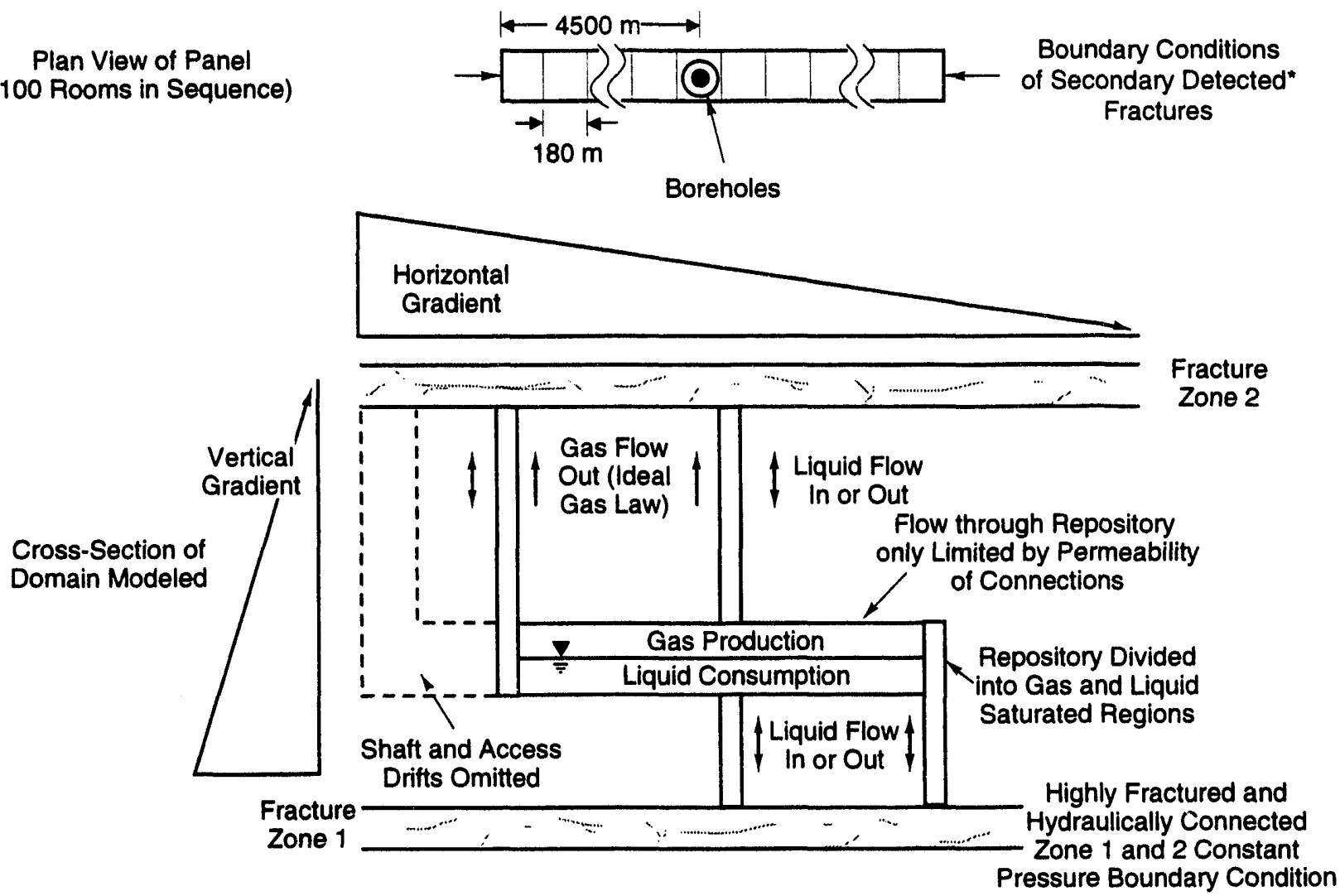

\section{One Dimensional Mathematical Model}

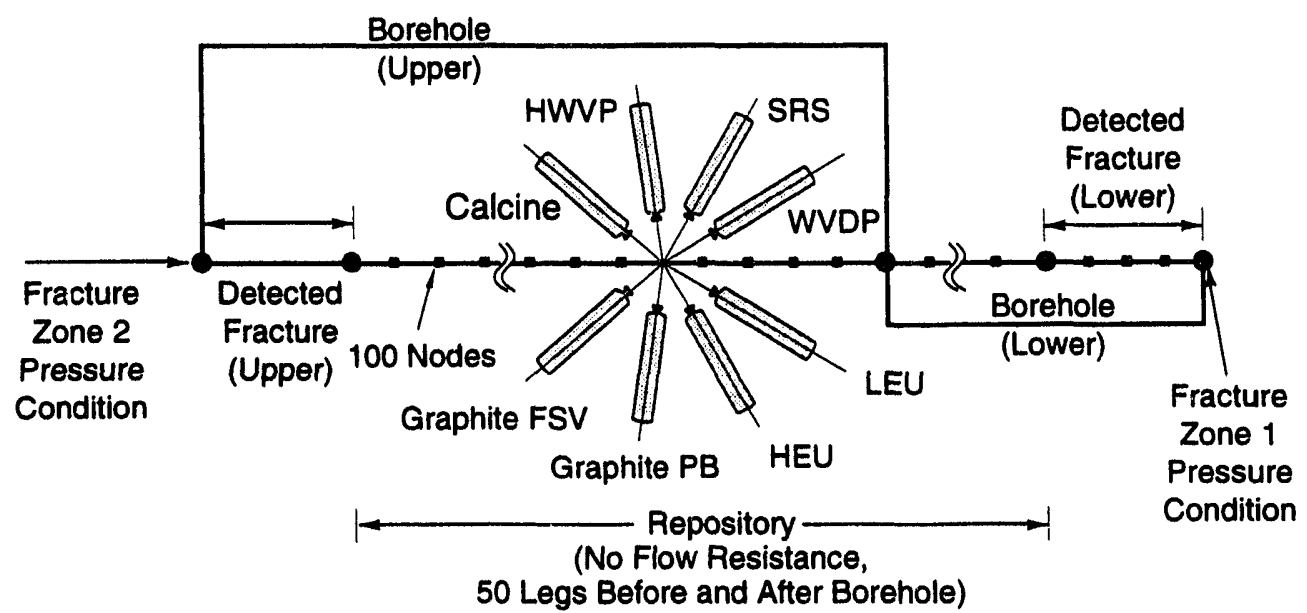

* Secondary Fractures Classified as Detected Since Repository

Assumed to Stop when Discovered

TRI-6342-3920-0

Figure 15-1. General conceptual model and one-dimensional mathematical model of granite repository and nearby geologic barrier in Simple PA. 


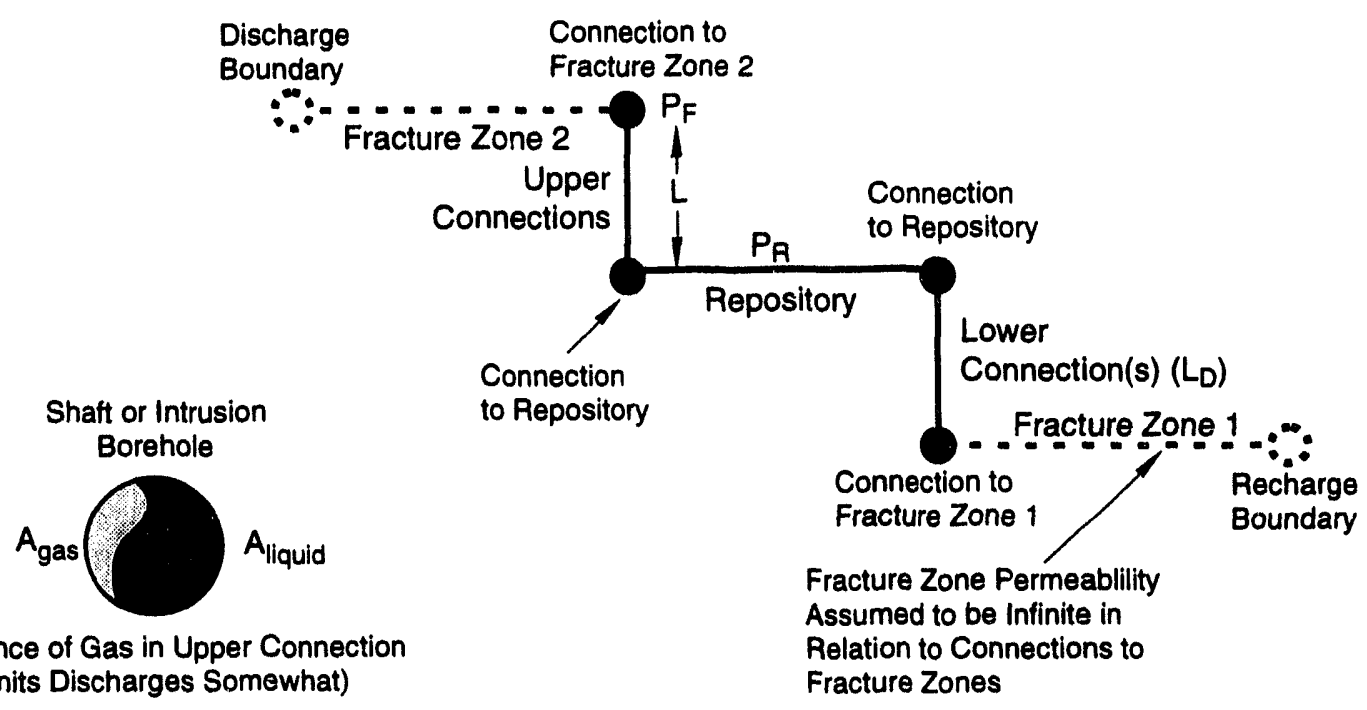

TR1-6342-3783-0

Figure 15-2. Representation of flow through granite disposal system (see also Figure 15-1).

Rather, the assumption is justified by the belief that using a more realistic model of fracture flow and transport would not improve our ability to predict cumulative discharge due to the uncertainty in the parameters of fracture models.

The upper connection of the flow network (Figures 5-1 and 5-2) is assumed to allow flow of either gas or liquid, or both simultaneously. The permeability of the connection to each phase depends on (1) the saturation, and (2) the type of pore space in the connection. The saturation of the connection pore space with respect to each phase is, in turn, a function of saturation and pressure in the fracture zone and in the repository near the connection. However, the Simple PA consequence model does not resolve spatial variations in the repository and does not include a model of capillary flow in the connection that would allow saturation to be calculated. Furthermore, the pore space might approximate a porous medium or might consist of discrete channels. Because of uncertainty in the saturation and geometry of the pore space, the functional connection between saturation and permeability (expressed as relative permeability) is unclear.

In this model, the relative permeability is assumed to be a function of the average relative cross-sectional area occupied by each phase. This assumption excludes the possibility of substantial phase interference in which connection pore space must be near saturation before a continuous connection is created. Flow rates in one or both phases may be overpredicted by this model. Three types of dependency of relative cross-sectional area on the saturation of the repository can be proposed (Figure 15-3):

1. The gas phase has preferential access to the connection. This situation might occur because the repository elevation is higher at the connection location, or because gas accumulates at the top of the repository, or because gas production occurs primarily near the connection. This condition might be represented as

$$
A_{g}=\left\{\begin{array}{cc}
0.99 A_{\text {total }} & M_{g} / \rho_{g}>V_{t h} \\
0 & \text { otherwise }
\end{array}\right.
$$

where $\mathrm{V}_{\mathrm{th}}$ is a small fraction of the repository volume.

2. The liquid phase has preferential access to the connection. This situation might occur for complementary conditions to the above (e.g., low repository elevation near the connection or production of gas primarily in 
15. Simplified Consequence Modeling for Granite Repository
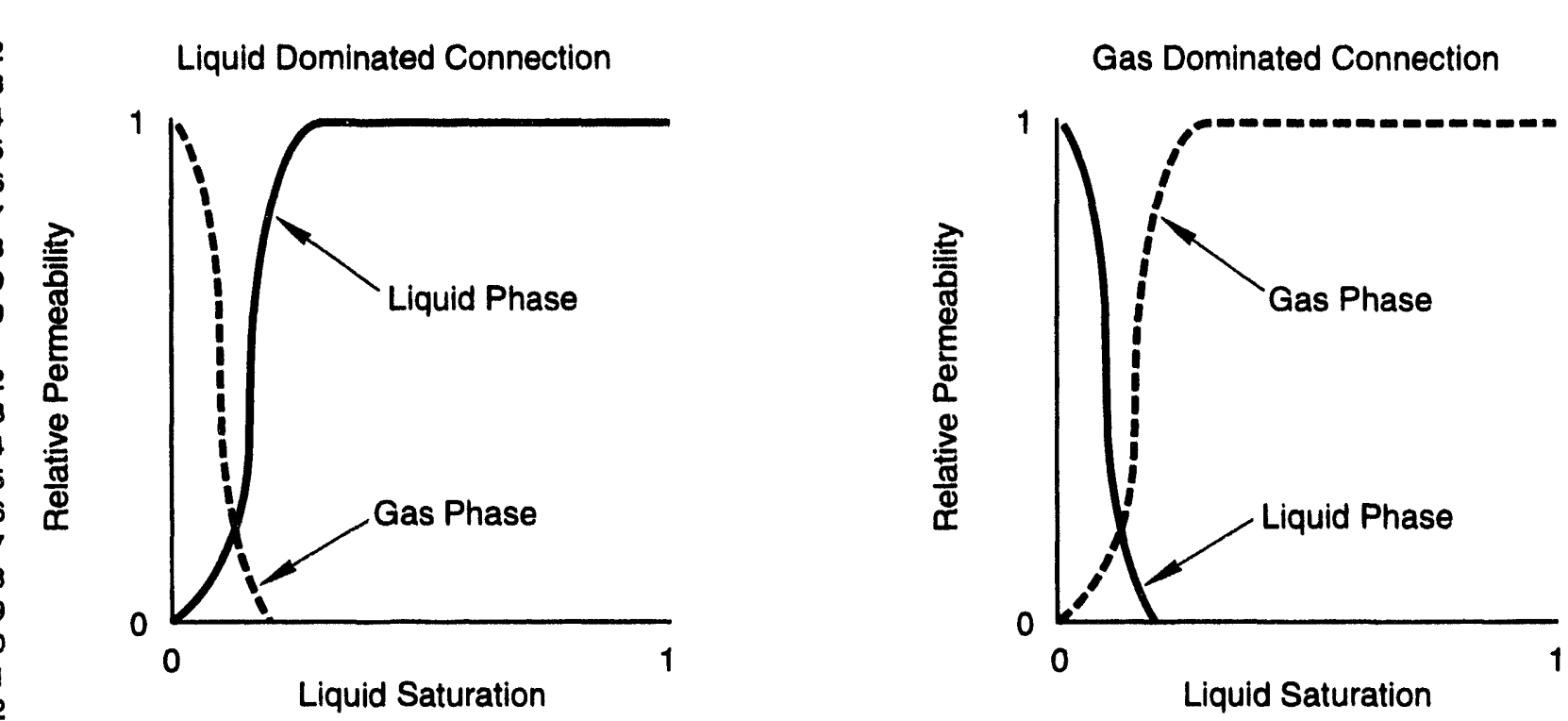

TRI-6342-3586-0

Figure 15-3. Relative permeability of a connection to the fracture zones in the granite repository is a function of sat. uration of the repository.

areas away from the connection. The area available to gas flow would be calculated as above, with $V_{\text {th }}$ replaced by some large fraction of the repository volume.

3. The area available to each phase is proportional to the saturation of the repository as a whole:

$$
A_{g}=\frac{M_{g}}{\rho_{g} V_{R}} .
$$

This condition represents an intermediate case between the preferential availability of the release pathway to gas or liquid. A corresponding physical situation might be failure of the seals in several access shafts (or intrusion boreholes) throughout the repository, or the development of many fracture connections throughout the repository.

The third type of dependency was selected for this analysis and expressed in terms of the cross-sectional area available for flow.

\subsubsection{Mathematical Model}

Following the above simplifications, the flow network is reduced to the following (Figure 15-2).

\section{Terminology}

$\mathrm{P}_{\mathrm{F}} \quad$ liquid and gas pressure in Fracture Zone 2

$L$ length of upper connection

$A_{1}$ average area available for flow of liquid

$\mathrm{A}_{\mathrm{g}}$ average area available for flow of gas

$k$ intrinsic permeability of the upper connection following seal failure

$\mu_{1} \quad$ liquid viscosity

$\mu_{\mathrm{g}}$ gas viscosity 


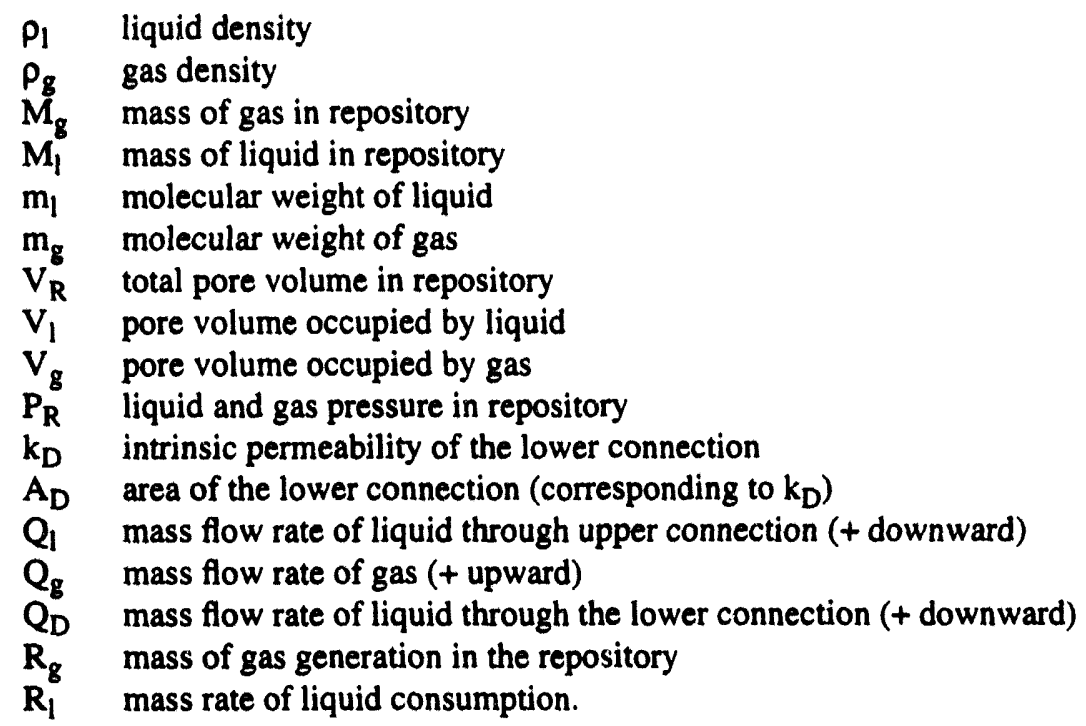

Relationships between State Variables. Gas Density is given by:

From the ideal gas law,

$$
\rho_{g}=\frac{m_{g} n}{V_{g}}
$$

$$
\begin{aligned}
& P V=P\left(\frac{m_{g} n}{\rho_{g}}\right)=n R T \\
& \Rightarrow \rho_{g}=\alpha P
\end{aligned}
$$

where

$$
\alpha=\frac{m_{g}}{R T}
$$

Pore volume in the repository is constant, and occupied by either gas or liquid:

$$
\begin{aligned}
& V_{R}=V_{l}+V_{g}=\frac{M_{l}}{\rho_{l}}+\frac{M_{g}}{\rho_{g}}=\frac{M_{l}}{\rho_{l}}+\frac{M_{g}}{\alpha P_{R}} \\
& \Rightarrow P_{R}=\frac{M_{g}}{a\left(V_{R}-\frac{M_{l}}{\rho_{l}}\right)}
\end{aligned}
$$

Mass Flux Terms. Darcy flow of liquid in the upper connection gives:

$$
Q_{l}=\frac{\rho_{l} g k}{\mu_{l}} A_{l} \rho_{l} \frac{h_{F}-h_{g}}{L}
$$


15. Simplified Consequence Modelling for Granite Repository

where

$$
h_{F}=\frac{P_{F}}{\rho_{18}}+L, \quad h_{R}=\frac{P_{R}}{\rho_{18}}
$$

so that

$$
Q_{l}=\frac{\rho_{l} g k}{\mu_{l}} A_{l} \rho_{l}\left[1+\frac{P_{F}-P_{R}}{\rho_{l} g L}\right]
$$

Darcy flow of liquid in the lower connection is similarly

$$
Q_{D}=\frac{\rho_{l} g k_{D}}{\mu_{l}} A_{D} \rho_{l}\left[1+\frac{P_{R}-P_{D}}{\rho_{l} g L_{D}}\right]
$$

Darcy flow of gas in the upper connection:

$$
Q_{g}=-\rho_{g} \frac{k}{\mu_{g}} A_{g}\left(\frac{d P}{d z}-\rho_{g} g\right)
$$

where $P$ is the gas pressure in the upper connection. Substituting of $\rho_{g}$ from (15-1), and defining

$$
\Gamma=A_{g}\left(\frac{k}{\mu_{g}}\right)
$$

gives

$$
P \frac{d P}{d z}=\alpha g P^{2}-\frac{Q_{g}}{\alpha \Gamma}
$$

Defining $X=P^{2}$, the solution is

$$
X=C_{l} e^{2 \alpha g z}+\frac{Q_{g}}{\alpha^{2} g \Gamma}
$$

where $C_{l}$ is an integration constant.

Applying boundary conditions

$$
\begin{aligned}
& P(0)=P_{R} \\
& P(L)=P_{F}
\end{aligned}
$$


gives the following expression for $\mathrm{Q}_{\mathrm{g}}$

$$
Q_{g}=\alpha^{2} g \Gamma\left[\frac{P_{R}^{2} e^{2 \alpha g L}-P_{F}^{2}}{e^{2 \alpha g L}-1}\right]
$$

Repository Mass Balance. The gas phase and liquid phase mass balance expressions are

$$
\begin{gathered}
\frac{d M_{g}}{d t}=-Q_{g}+R_{g} \\
\frac{d M_{l}}{d t}=Q_{l}-R_{l}-Q_{d}
\end{gathered}
$$

where

$\mathbf{R}_{\mathbf{g}} \quad$ = mass rate of gas generation in the repository

$\mathbf{R}_{1} \quad=$ mass rate of liquid consumption.

Gas production rates $\left(R_{g}\right)$ and liquid consumption rates $\left(R_{\mathcal{L}}\right)$ are calculated in the gas generation submodel GASSNF. (This was done in IPANEL in the scoping PA for the granite repository). Gas and liquid phases are assumed to be stratified in the repository, so that liquid saturation in the pore space surrounding the waste package hole backfill-buffer equals 1 , as long as the total volume of liquid in the repository is sufficient to saturate the backfillbuffer.

\subsubsection{Computational Model}

Flow rates from Equations 15-4, 15-5, and 15-6 can be expressed in terms of gas and liquid mass using Equation 15-3. The resulting coupled system can be solved numerically using trapezoidal integration based on Equations 15-7 and 15-8 with iterative updating of the rate terms. The gas generation and liquid consumption are evaluated explicitly given the repository state at the beginning of a timestep.

\subsubsection{Summary of Assumptions}

- Intact granite surrounding the repository and fracture zones is impermeable.

- Flow through the repository is limited by the permeability of failed shaft seals or other features connecting the repository with the fracture zones, not the repository itself (no flow resistance in repository). In the complex calculations, repository backfill conductivity did limit flow in most cases. The simplified model overestimates repository flow rates in these cases.

- Heat generation and transport are neglected.

- Gas and liquid phase pressures are equal (no capillary pressure).

- Ideal gas law applies.

- Liquid and solid phases are incompressible.

- Flow through all upper and lower connections is one dimensional.

- Flow through repository-fracture zone connections is quasi-steady state.

- Both gas and liquid can flow through the upper connection; only liquid can flow through the lower connection.

- Relative permeability of a repository connection (fracture, shaft, exploratory drillhole) is a function of repository saturation (relative amounts of gas and liquid in repository) and is expressed as cross-sectional area available for flow.

- The repository is segregated into gas-saturated and liquid-saturated regions.

- No horizontal gradient. 


\subsection{Radionuclide Transport Modeling}

\subsubsection{Conceptual Model}

Radionuclide release from the repository in this configuration is a function of the radionuclide release rate from the waste parcels in the repository and the transport characteristics of the upper connection and Zone 2. Canister release is, in general, a function of liquid flow rate past the canister (which removes dissolved species), and therefore of flow rate through the repository.

As in the salt repository, only transport of radionuclides dissolved in the liquid phase are considered. Concentrations are assumed to be sufficiently small to allow the transport problem to be decoupled from the flow problem. Once flow rates have been estimated, repository source-term concentrations and integrated release due to transport in Fracture Zone 2 are estimated using the same assumptions as in the scoping calculations for the salt repository (see Section 14.2.1).

\subsubsection{Mathematical and Computational Model}

The mathematical and computational models of advective and diffusion transport within and near a granite repository are the same as the models for the salt repository (described in Section 14.2). 


\section{Results and Conclusions}

This chapter presents results from both the complex performance assessment (Complex PA) and the simplified performance assessment (Simple PA) for both the salt and granite repositories. Results include a discussion of percentage of radionuclides leaving the waste parcel, normalized activity leaving the repository (for both the Simple and Complex PAs) and normalized release leaving the disposal system (Complex PA). Results for the breach time of containers and direct release to the ground surface during drilling are provided for both the salt and granite repositories. Also, the results from the Simple and Complex PAs are compared, after the conceptual model for the Complex PA was modified so that the two conceptual models would be similar. This chapter begins with a description of performance metrics and analysis techniques used in the assessment.

\subsection{Performance Metrics and Analysis Techniques (R. P. Rechard)}

\subsubsection{Performance Metrics}

The relative ranking of the waste form depends upon the performance metric. For this report, the overall performance metric is the cumulative release of radionuclides over $10,000 \mathrm{yr}$ to the accessible environment in the form of a complementary cumulative distribution function (CCDF) (40 CFR 191). When assessing the total disposal system, however, differences in waste form performance were not easy to discern with this metric. Although that fact in itself is important, a relative ranking of the waste is still necessary to understand the differences among the forms and possibly identify parameters that could be modified to enhance performance. Therefore, three other metrics are used in evaluating and comparing the results:

- EPA normalized discharge at intrusion boreholes or lateral pathway (e.g., fracture zone or marker bed) (presented as a CCDF).

- Percentage of radionuclide discharged to pore liquid in the backfill-buffer around containers (presented as a CCDF).

- Breach time of canisters.

A fourth metric, fluid flow out of the repository, was used also in the comparison and is discussed in the intermediate results of Chapters 12 and 13.

\subsubsection{Description of Terms and Graphical Presentation of Results}

This section discusses the terms that are used to describe the results and explains how the results are presented graphically. See also Chapter 3 in this report.

Distribution Functions. This chapter presents the complementary cumulative distribution function (CCDF) of the results. Because the cumulative distribution function (CDF) is the integral (or sum, as appropriate) of the probability density function (PDF), the CCDF is the "complement" of the CDF, or one minus the values of the CDF ( 1 CDF).

If the parameters (x) of the consequence or probability model vary, the variation (or uncertainty) is described mathematically as a CDF (or possibly, PDF). When this variation is propagated through the consequence model, then the results $\left(\mathbf{C}\left[\mathbf{x}, \mathrm{S}_{\mathrm{j}}(\mathbf{x})\right)\right.$ will vary and the variation can be described again as a CDF. To make comparisons with the EPA limiting points in the CCDF space specified in the Containment Requirements of 40 CFR 191, the CDF of the results is converted to a CCDF, which plots the probability of a consequence occurring versus the consequence, $C[x$, $\mathrm{S}_{\mathrm{j}}(\mathrm{x}) \mathrm{l}^{*}$

Typically, the model parameters are described with a CDF (instead of a CCDF), which prevents confusion. 
Conditional CCDFs. When "conditional" CCDFs are presented in this report, the term "conditional" means that the CCDF is contingent on certain events or processes occurring. Most frequently, the selected event is a single, or at most a double, intrusion into the repository (rather than many intrusions). The construction of the conditional CCDF is performed with CCDFCALC in the CAMCON modeling system (see Figures 7-8 and G-1). Although multiple intrusions are a distinct possibility, the probability of their occurrence rapidly decreases as the number of intrusions increases.

However, a clarification of the term "conditional" is necessary. Although CCDFs conditional on one selected event are presented, they actually are the mean of the CCDFs conditional on the one selected event and on the sampled parameters. (Recall that the mean of the CCDFs conditional on the sampled parameters was used to develop the EPA limiting line.) (Throughout the remainder of this chapter, we have shortened "EPA limiting points in the CCDF space specified in the Containment Requirements of 40 CFR 191" to "EPA limiting line.") The CCDFs that are conditional on both the selected event and sampled parameters are presented only once as a demonstration because a comparison with the EPA limiting line is inappropriate. (Note that the number of distributions is equal to the number of samples taken to propagate parameter uncertainty through the consequence or probability models; see Section 3.2.)

Composite CCDF. A composite, or complete, CCDF is rarely presented in this report. ${ }^{\dagger}$ The composite CCDF is the relevant measure of system performance for evaluating compliance with 40 CFR $191,{ }^{\ddagger}$ yet it subsumes so many events (such as multiple intrusions) that it is not very useful for understanding the behavior of a particular system component. For example, consider a composite CCDF such as that constructed using the summary scenarios in this report (refer also to Chapter 3, "Performance Assessment Overview," and Chapter 7, "Scenario Development"). Because it is very time consuming to evaluate the consequences of first one intrusion into one location, and then one intrusion into another location, we take one intrusion into a centrally located panel as indicative of all single intrusions. If the additional assumption is made that the effect of two or more intrusions is simply the sum of the consequences of single intrusions, then the single intrusion can be used to calculate multiple intrusions. "* The construction of the composite CCDF is performed with CCDFPERM in the CAMCON modeling system (see Figures 7-8 and G1).

Although the composite CCDF is the appropriate metric to compare with the EPA limiting line, no additional information from the consequence calculations is generated. Therefore, if an important goal of the analysis is understanding which parameters strongly influence the consequences, then using a CCDF conditional on one event, not a composite CCDF, is the more useful way to piesent these results.

Plotting CCDFs. For the most part, a linear-arc hyperbolic sine plot or semilog plot was used for displaying composite and conditional CCDFs, although a few CCDFs are shown on the commonly used log-log plot. To elaborate, because of the orders-of-magnitude change in consequences that make up the CCDF, the consequences are frequently displayed using a logarithmic scale on the abscissa. Here, however, the consequences (results from the consequence model) are sometimes shown on a similar arc hyperbolic sine scale $\left(\sinh ^{-1} x=\ln \left(x+\left[x^{2}+1\right]^{1 / 2}\right)\right.$ since zero consequences can be plotted. Although not always the case for other PAs, the probabilities for this PA did not vary over orders or magnitude and so a linear scale was usually used on the ordinate of the CCDF graph. (Note, however, that the probability will necessarily not be one since the ordinate is $P[\hat{R}>R]$ not $P[\hat{R} \geq R]$.) A logarithmic scale for probability tended to obscure differences in the CCDFs at high probabilities and accentuated the differences at low probabilities.

Presenting the Contribution of Individual Radionuclides. The performance metric $(R)$ for the Containment Requirements (Section 13) of 40 CFP 191 is the summed contribution from each radionuclide normalized by the corresponding EPA release limit $\left(L_{i}\right)$ and waste unit factor $\left(f_{w}\right)$ (see Chapters 2 and 3 ):

A CCDF is generally described without the modifiers, "composite" or "complete", i.e., in most literature, a CCDF is referred to either simply as a CCDF (meaning a complete CCDF) or a conditional CCDF. However, in this chapter, we have chosen to modify a CCDF as "composite" when it is important to emphasize that a particular CCDF is not a CCDF conditional cl one event.

* In actuality, the CCDF is still conditional because only exploratory drilling is assumed to occur and not, for example, purposeful intrusion. Nevertheless, the comparison with the EPA limiting line is appropriate because it also is conditional on only exploratory drilling occurring.

*- Other partitions of the sample space are also appropriate. For example, the scoping calculations evaluated the consequences of two nearby intrusions into the same panel within the same 1000-yr time interval. See Appendix C. 


$$
R=\frac{1}{f_{w}}\left(\frac{Q_{1}}{L_{1}}+\frac{Q_{2}}{L_{2}}+\ldots+\frac{Q_{i}}{L_{i}}+\ldots+\frac{Q_{n R}}{L_{n R}}\right)
$$

where

$$
\mathrm{Q}_{\mathrm{i}}=\text { activity released beyond a specified boundary }\left(\mathrm{q}_{\mathrm{i}}\right) \text { integrated over } 10,000 \mathrm{yr}=\int_{0}^{10,000 \mathrm{yr}} q_{i} d t
$$

Several times in this chapter, the distribution function of each normalized radionuclide $\left(Q_{i} / f_{w} L_{i}\right)$ is presented rather than the sum $(R)$. Rather than presenting numerous CCDFs of $\left(Q_{i} / f_{w} L_{i}\right)$, the PDFs are presented instead as "Tukey box plots," which are much easier to compare. ${ }^{\dagger \dagger}$

\subsubsection{Uncertainty Analysis Techniques}

The purpose of uncertainty analysis is to determine the contribution (importance) of the uncertainty in the model parameters used in an analysis and the associated uncertainty in the result (usually consequences, $C\left[x, S_{j}(x)\right]$, but sometimes probability, $\left.\mathrm{P}\left[\mathrm{x}, \mathrm{S}_{\mathrm{j}}(\mathrm{x})\right]\right)$. Hence, "uncertainty" primarily means measures of the uncertainty in estimates for results of interest, including means, variances, and distribution functions. This section describes briefly the uncertainty analysis techniques used, including scatterplots and stepwise regression analysis. A more detailed discussion of these techniques and their application can be found in Helton et al., 1992; 1991.

The generation of scatterplots is the simplest sensitivity analysis technique and is used here to support a stated finding. This approach consists of generating plots of the results (dependent variable value) vs. model parameters (independent variable value), probability and consequence models with one set of sampled model parameters). When there is no relationship between the independent and dependent variable, the individual points are incoherently scattered over the plot or spread along a horizontal line. In contrast, the existence of a well-defined relationship between the independent and dependent variable is often revealed by patterns in the distribution of the individual points such as a spread of points along a nonhorizontal locus.

In stepwise regression analysis, a sequence of regression models is constructed. The first regression model contains the single independent variable that has the largest impact on the dependent variable. The second regression model contains the two independent variables that have the largest impact on the dependent variable, the independent variable from the first step, plus whichever of the remaining variables has the largest impact on the variation not accounted for by the first step. Additional models are constructed in the same manner until a point is reached at which additional independent variables are unable to account meaningfully for the amount of variation in the dependent variable.

One way that the meaningful improvement is described mathematically is through the coefficient of determination $\mathrm{r}^{2}$. Further, the order in which the variables are selected in the stepwise procedure provides an indication of variable importance, with the most important variable usually being selected first, the next most important variable selected second, and so on. The $r^{2}$ values for each step in the regression analysis equal the fraction of the total variability in the dependent variable that can be accounted for by the individual independent variable added at that step. When the variation about the regression model locus is small, the corresponding $r^{2}$ value is close to 1 , which indicates that the regression model is accounting for most of the variability in the dependent variable. Conversely, an $\mathrm{r}^{2}$ value close to zero indicates that the regression model is not successful in accounting for the variability in the dependent variable. The absolute values of the standardized regression coefficients (SRCs) in the individual regression models

It A box plot succinctly displays a PDF, e.g., the PDF of the results. See Chapter 9, where the PDF, CDF, and the box plot of the PDF, are presented for the model parameters that were varied in this rejort.

\# The terms "dependent variable" and "independent variable" are used in the following paragraphs to emphasize that the result or model parameter may be modified to highl" ...t relationships (e.g., the rank of a result may become the dependent variable and the rank of two or more model parameters mathematically combined may become the independent variable). 
provide an indication of variable importance, and the sign of the SRC indicates whether the independent and depencient variables tend to increase and decrease together (a positive SRC) or tend to move in opposite directions (a negative SRC).

The statistical program, STEPWISE (Iman et al., 1980; Rechard, Ed., 1992, p. 12-13), was used to evaluate variable importance using the stepwise regression procedure on rank-transformed data. Regression analyses often perform poorly when the relationships between the independent and dependent variables are nonlinear. Poor linear fits to nonlinear data can often be avoided when the data are replaced with their corresponding ranks and the regression procedures are performed on these ranks (Iman and Conover, 1979).

It is necessary to have some criterion to stop the regression model construction process. In the analyses reported here, an alpha-value of 0.02 was used to add a variable to the regression model and a value of 0.05 to drop a variable from the model once added. (The alpha-valus is the probability of obtaining a stronger relationship than the one identified in the analysis as a result of chance variation.) In addition, the Predicted Error Sum of Squares (PRESS) criterion (Iman et al., 1980) was used to protect against oveifit.

As mentioned earlier in this report, sensitivity results are strongly dependent on the complexity of the models and the parameters that are $a$ priori assumed to vary substantially. If modified conceptual models were used and other parameters were varied, the results might differ from those presented here. 


\subsection{Complex PA Results for Disposal in Bedded Salt}

\subsubsection{Release from the Disposal System (R.P. Rechard and H.J. Iuzzolino)}

This section presents results in the form of composite CCDFs for radionuclide activity release from the salt disposal system. The influence of various phenomena and model parameters on these results is explored in Sections 16.2.2 through 16.2.4 by examining the conditional CCDFs that make up the composite CCDF.

The mean composite CCDF curve for Waste Disposal Group 1 lies to the left of the EPA limiting line, specified in the Containment Requirements of 40 CFR 191, at all levels of probability for the salt disposal system (Figure 16.2-1a and 16.2-1b). This composite CCDF of all releases from the disposal system to the accessible environment incorporates the likelihood of occurrence of all combinations of the basic summary scenario, using six intrusion times, and an upper bound on drilling frequency for a repository in sedimentary rock of 30 boreholes $/ \mathrm{km}^{2} / 10,000 \mathrm{yr}$.

The direct releases from drill cuttings/cavings dominate the total releases below a probability of about $25 \%$ (Figures 16.2-1a and 16.2-1b). Interestingly, the individual conditional CCDFs for each of the 20 samples from which the groundwater and direct releases are derived are grouped fairly tightly (recall, however, that only a few model parameters of the geologic barrier were sampled and so the complete variation of the geologic barrier is not included) (Figure 16.2-1c). Parameters affecting direct removal (e.g., drill bit size and drilling frequency) were not sampled: therefore there is no variability of release caused by direct removal across sample sets in one disposal group from minor changes in the inventory of each waste parcel. (A minor variability can occur, however, across waste disposal groups.)

The mean composite CCDFs for each waste disposal group are also grouped fairly tightly, indicating that the waste treatment options for the relatively small amount of INEL spent fuel and radioactive waste do not have much influence on the moderately sized salt repository (a large percentage of the inventory includes waste from other DOE sites) (Figure 16.2-2).

Accessibility of Radionuclides. As partially demonstrated by these results (e.g., Figure 16.2-2) (becoming more obvious as other results are presented), it is difficult to predict a priori the relative rank of waste disposal groups without detailed knowledge of the repository. Such things as the solubilities and Kds (granite site) are highly dependent on the geologic site conditions or the repository and will determine the relative importance of the radioactive elements for compliance. For example, in a hypothetical repository where uranium is disproportionately insoluble or highly sorbed, it could be irrelevant whether the uranium was removed by reprocessing. On the other hand, in any repository using containers with a high probability of complete containment during the regulatory period, the repository is irrelevant except in its ability to ensure the container integrity.

Clearly, if the radionuclides are packaged in such a way that they are effectively isolated from the environment, they may never be released during the 10,000 -yr regulatory period. There is evidence that certain package materials under some conditions can meet this goal, but the same is not true of the waste forms. One waste form that approaches this goal, however, is the Fort St. Vrain graphite fuel elements. The SiC coating (TRISO) on the (Th,U) $C_{2}$ fuel particles was designed to withstand high temperatures and pressures, and is expected to be highly resistant to moisture and oxidants. Lotts et al. (1992) believe that very few of these particles will breach during the regulatory period in an unsaturated-tuff type of repository. If the probability of breach of these particles during the regulatory period can be shown to be diminishingly small, then the most important parameter for this waste form is the fraction of these particles breached prior to disposal. Recall this parameter was estimated to be about $0.16 \%$ based on the study of several fuel elements (see Chapter 11).

\subsubsection{Percentage of Radionuclides Leaving the Waste Parcel (D. K. Rudeen)}

To evaluate the relative performance of the five different waste disposal groups in terms of repository-scale transport, the percentage of the initial radionuclide inventory that leaves the waste parcel was calculated for each waste 
16. Results and Conclusions

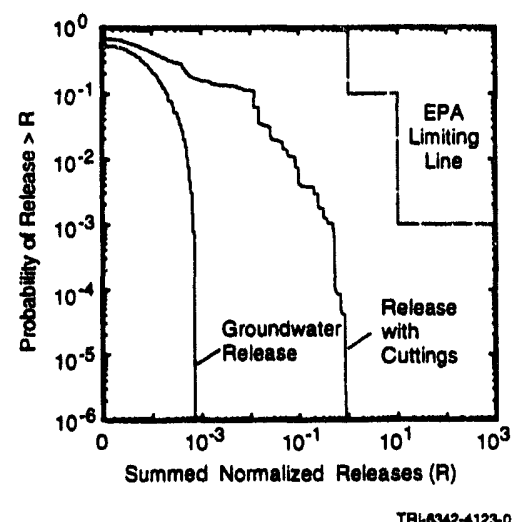

(a)

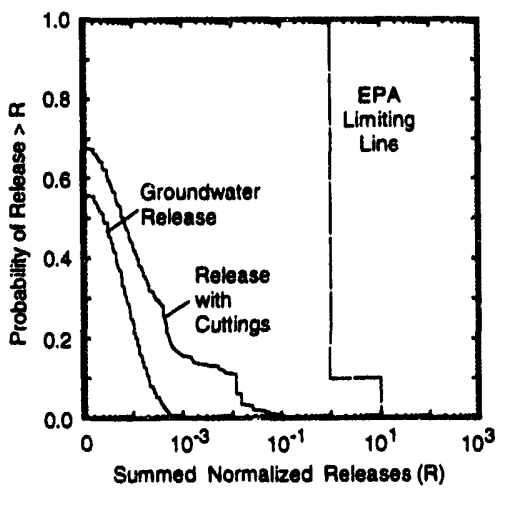

(b)

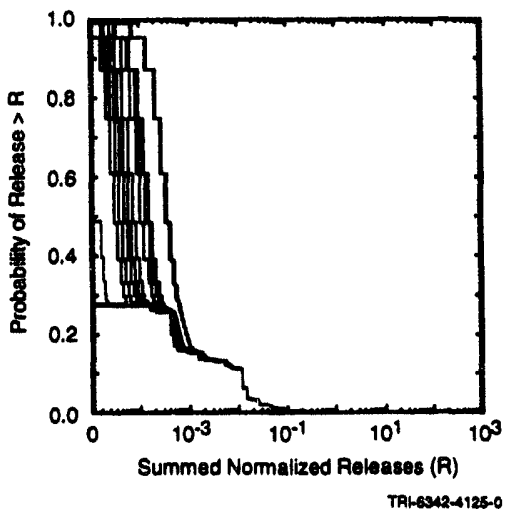

(c)

Figure 16.2-1. Mean composite compleme:ntary cumulative distribution functions (CCDFs) for Waste Disposal Group L salt evaluated at 2.4-km disposal sustem boundary in Snake Dolomite, assuming six intrusion times. (a) Mean composite CCDF of releases from groundwater advection and direct cuttings and mean composite CCDF of only groundwater releases on log arc hyperbolic sine axis, (b) the same data as above but plotted on linear-arc hyperbolic sine axis, and (c) conditional CCDFs of groundwater releases and direct cutting for 20 samples from which the mean composite CCDF of groundwater release with cuttings is derived. 


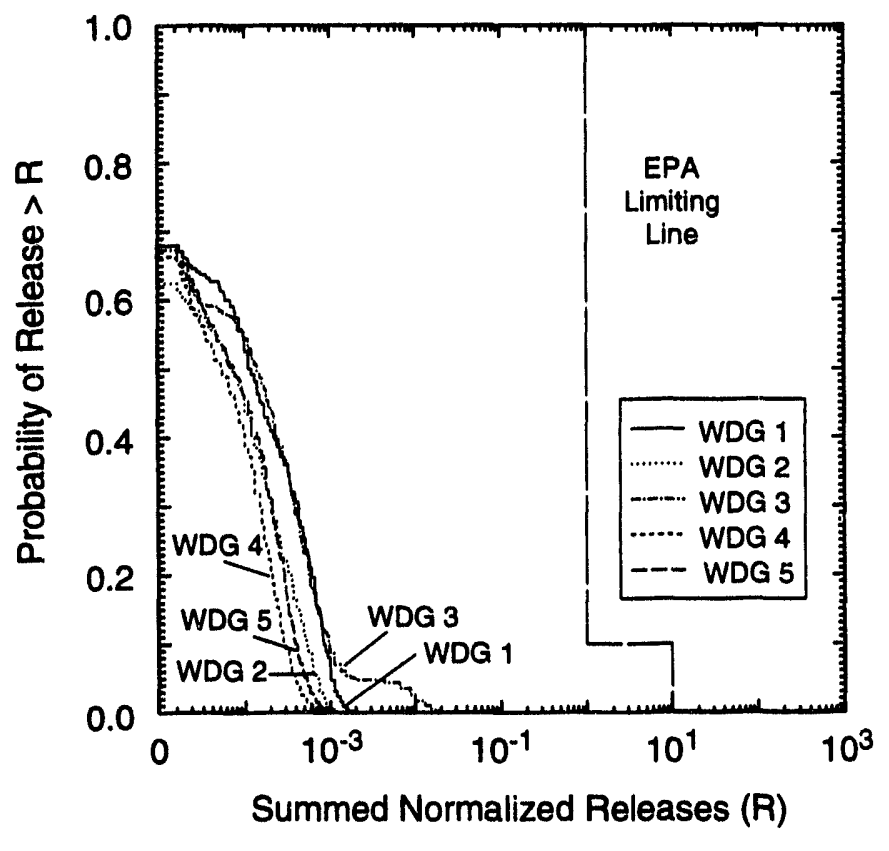

TR1-6342-4126-0

Figure 16.2-2. Mean composite CCDF of normalized integrated activity of only groundwater releases from the repository boundary of salt disposal system for all five waste disposal groups. (The waste unit factor, $f_{w}$ was not changed in evaluating $R$ for Waste Disposal Group 5 , even through depleted uranium was added to the inventory.)

disposal group. This calculation was accomplished in a manner similar to calculating the EPA summed normalized release. For each simulation, the mass injection rates of each of the 11 transported radionuclides calculated by the CONCSNF source term submodel in STAFF2D were converted to activity rates, integrated over time, normalized by the corresponding radionuclide EPA release linit, and summed. This sum was then scaled by the summed normalized initial inventory of the 11 transported radionuclides (Section 12.2 instead of the waste unit factor $\left(f_{w}\right)$, resulting in a waste parcel fraction $\left(F_{w p}\right)$. A value of 1 implies that the entire initial inventory is released from all waste parcels.

The results for the five waste disposal groups are shown as a CCDF conditional on two intrusions occurring in Figure 16.2-3 (for a description of a conditional CCDF, refer to Section 16.1.2). In Figure 16.2-3, each step represents the discharge fraction of a single simulation within a waste disposal group. Each simulation was assumed to have the same probability of occurrence ( 0.05 vertical step size). The largest waste parcel discharge fraction for a single simulation was $1.5 \%$, for Waste Disposal Group 3, parameter sample set 4 . Waste Disposal Group 4 generally had the lowest waste parcel discharge fraction, but the exact relative order for the remaining waste disposal groups is not as clear.

To quantify the relative performance of the five waste disposal groups, they were further evaluated by ranking for each sample set (or run) the waste parcel discharge fraction across the five disposal groups from least (rank 1) to most (rank 5). The frequency with which each waste disposal group received a particular ranking is plotted in Figure 16.2-4.*** Waste Disposal Group 4 has the lowest discharge fraction, followed by Waste Disposal Groups 5, 2, 3, and 1.

\footnotetext{
*** Although the frequency data could be plotted as bar graphs at each of the five rank points, we plotted the frequency without the bar and instead connected the values with lines to help the reader's eye note the changes. However, we do not mean to imply that a functional relationship exists between, for example, ranks 1 and 2 .
} 


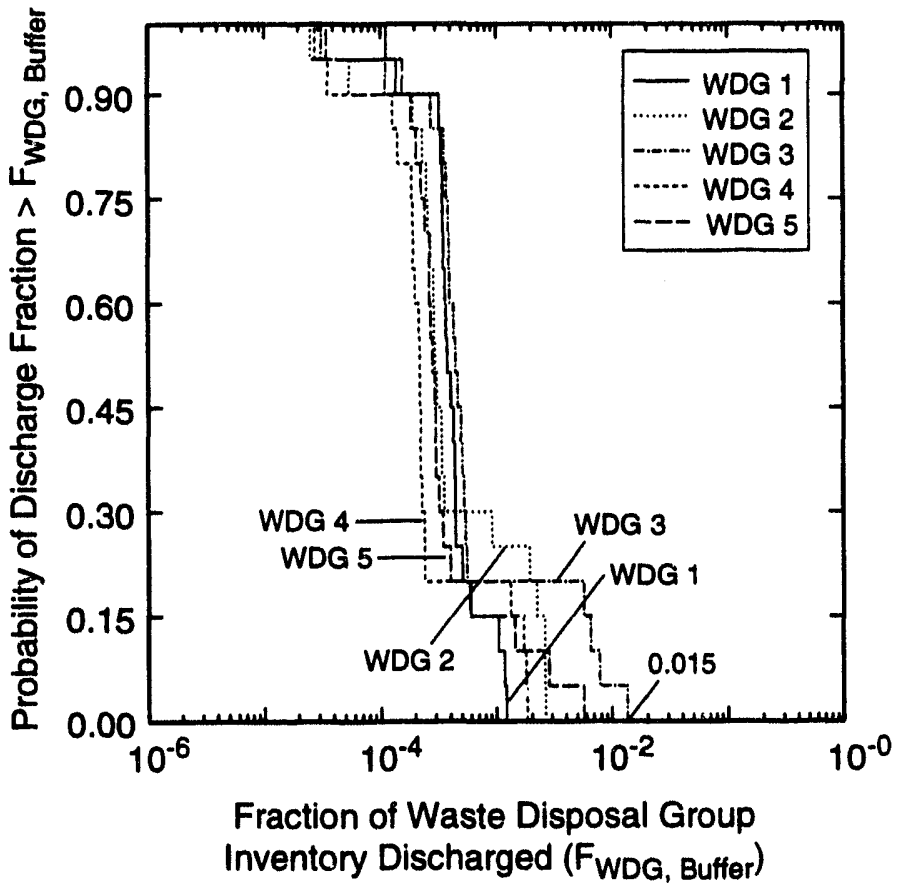

Figure 16.2-3. $\quad C C D F$, conditional on intrusion of fraction of initial, total waste disposal group inventory discharge into backfill-buffer for waste parcels in salt disposal system for all five waste disposal groups.

\subsubsection{Normalized Activity Leaving Repository (D. K. Rudeen)}

One of the primary results from the salt repository transport simulations for this performance assessment is the integrated positive discharge activities across prescribed boundaries or "monitoring points." For the salt repository, the discharge boundary was specified at two locations: one in each of the two intrusion boreholes just below their intersection with the overlying Snake Dolomite aquifer. (The exact locations of the discharge boundaries are found on Figure 12-5, corresponding to vertical element index 19, horizontal element indices 25 and 36 .) Total discharges are the sum of the upward discharges from the two intrusion boreholes.

The summed normalized discharges are presented as CCDF plots conditional on the occurrence of two exploratory boreholes for the five waste disposal groups (left set of CCDFs in Figure 16.2-5). These discharges are scaled by the waste unit factor $\left(f_{w}\right)$, not the initial total inventory as was presented in Figure 16.2-3. Each step in the conditional CCDF curve represents the summed normalized discharge leaving the repository for a single sample set. As before, each simulation of the sample set is assumed to have an equal likelihood of occurring or a probability of 0.05 . The summed normalized discharges are typically bounded by Waste Disposal Groups 1 (largest) and 4 (smallest).

The second set of five curves in Figure 16.2-5 (to the right) are summed normalized discharges from the waste parcels into the repository (scaled by the waste unit factor, not the initial normalized inventory). By comparing the two sets of summed normalized discharges, it can be seen that the repository itself provides a significant barrier to radionuclide transport. Discharges up the intrusion boreholes are four or more orders of magnitude smaller than discharges from the waste parcels. Again, to quantify the relative performance, the summed normalized repository discharges up the intrusion boreholes were ranked for each sample set (or run) across the five disposal groups from least (rank 1) to most (rank 5). The frequency with which each waste disposal group received a particular ranking is plotted in Figure 16.2-6. Waste Disposal Group 4 again had the lowest summed normalized discharge, followed by Disposal Groups 5, 2, 3 and 1. 


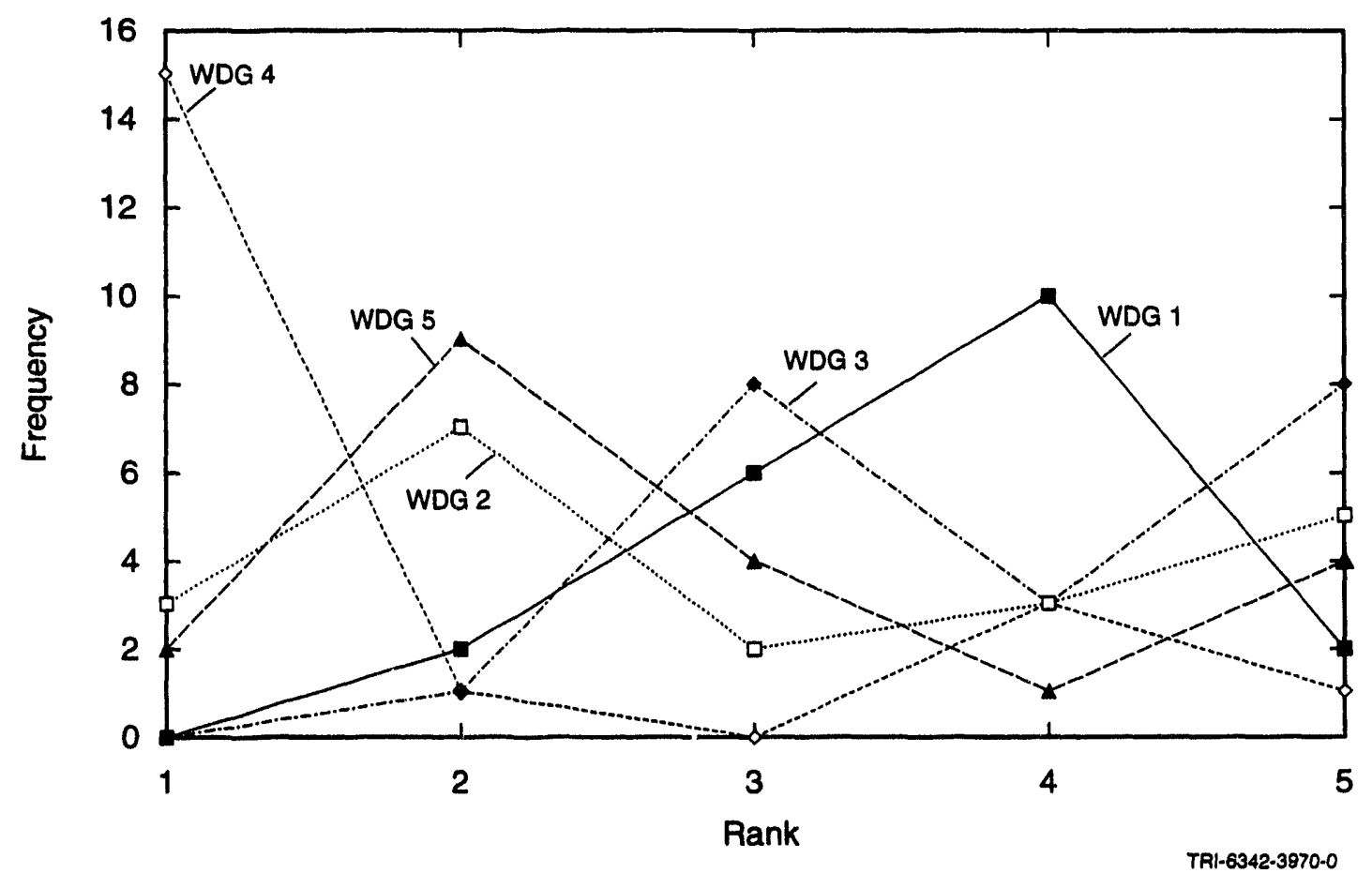

Figure 16.2-4. Frequency with which waste parcel activity fraction for each waste disposal group in the salt disposal system was ranked showing, in general, a ranking of 4, 5, 2, 3, and 1. (Rank 1 is the least discharge, and rank 5, the most.)

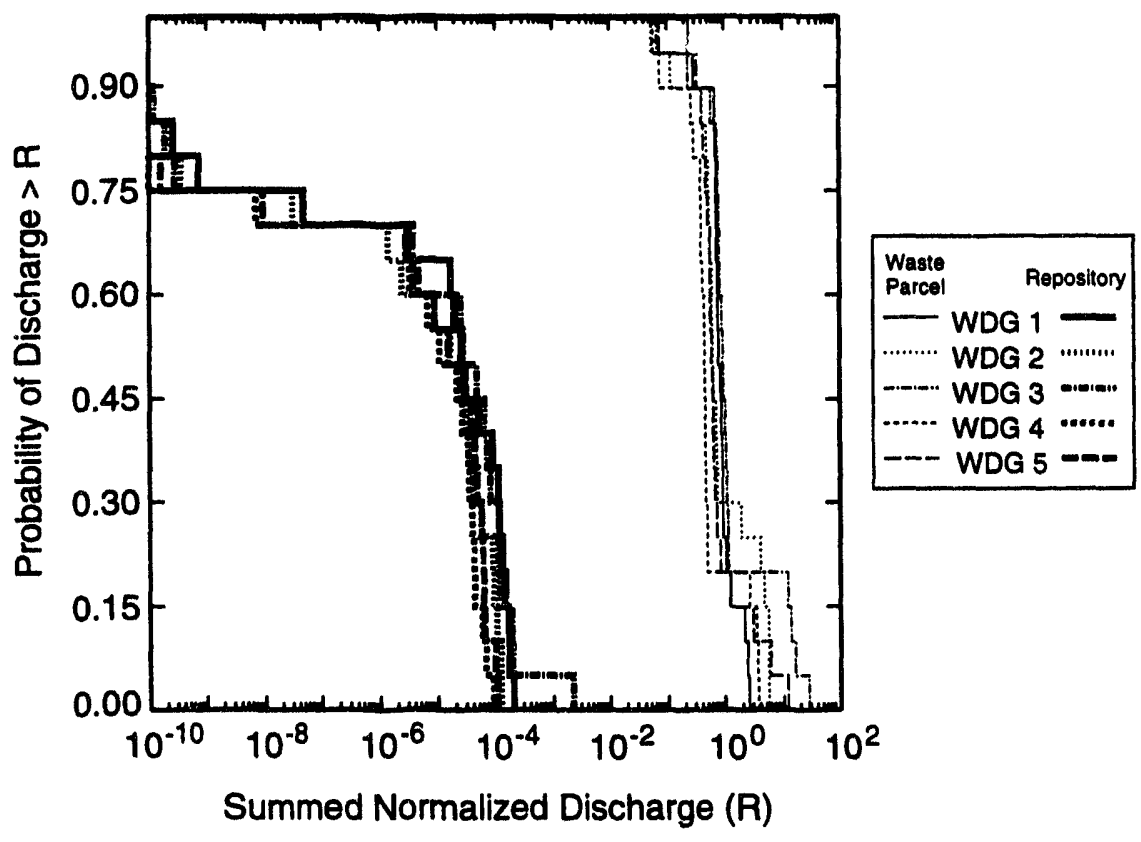

TA1-6342-4022-0

Figure 16.2-5. CCDF, conditional on intrusion, of summed normalized activity from the waste disposal groups into the pore space of the backfill-buffer surrounding the waste parcels (curves on right) and summed normalized discharges from repositorn (up the intrusion boreholes) (curves on lefi) for the salt disposal system. 
16. Results and Conclusions

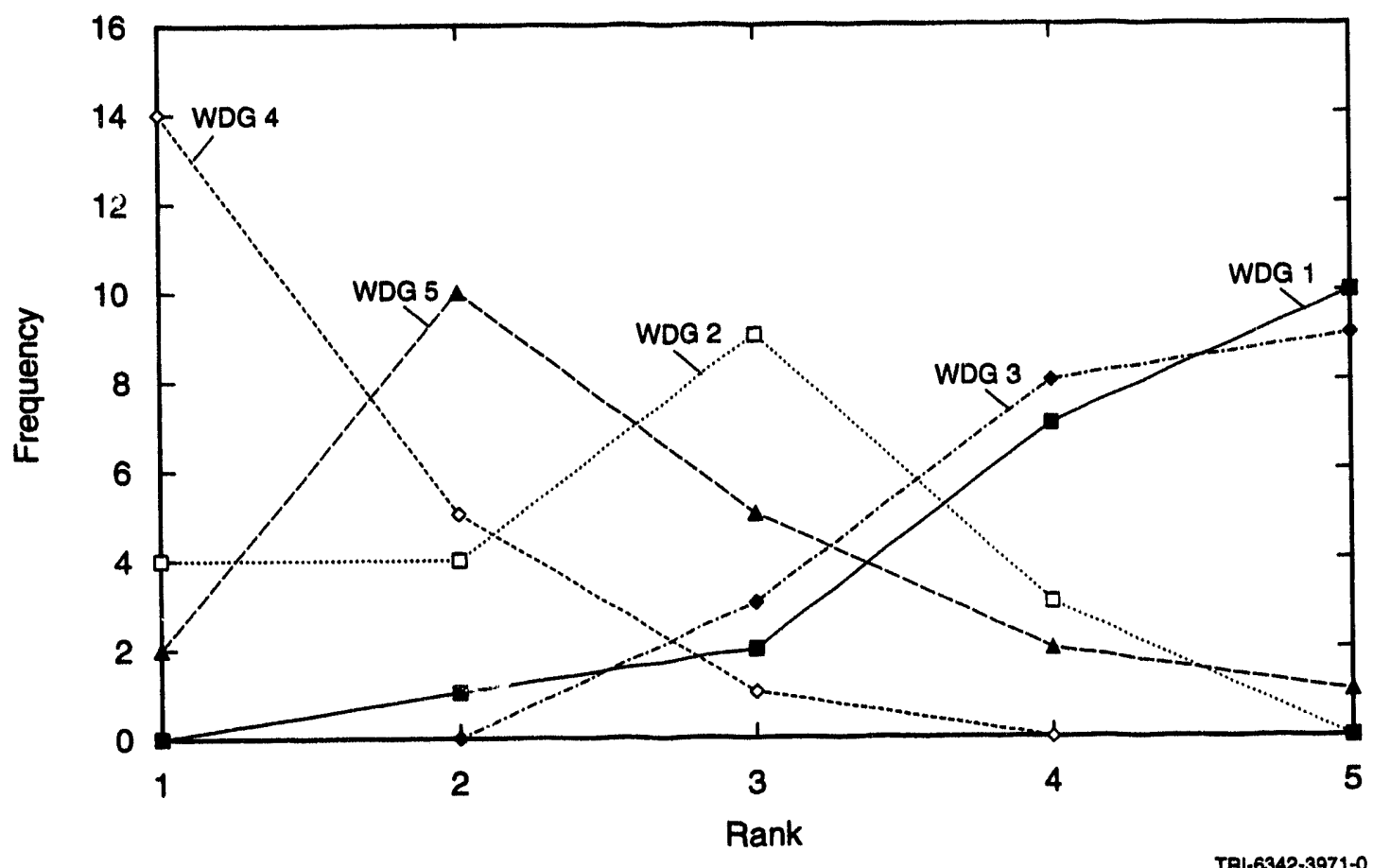

Figure 16.2-6. Frequency with which summed normalized activity from the repository for each disposal group in the salt disposal system was ranked showing, in general, a ranking of $4,5,2,3$ and 1. (Rank 1 is the least discharge, and rank 5, the most.)

Figures 16.2-7 through 16.2-11 summarize the contribution of individual isotopes $\left(Q_{i} / F_{w} L_{i}\right)$ to the total normalized discharge (R) for each of the disposal groups. Figures 16.2-7a through 16.2-11a show the distribution of the percent of total release for each isotope. Figures $16.2-7 \mathrm{~b}$ through $16.2-11 \mathrm{~b}$ show the distribution of normalized release for each isotope. The summed normalized discharges are dominated by ${ }^{233} \mathrm{U}$ and ${ }^{234} \mathrm{U}$ for Waste Disposal Groups 1 , 2 and 3. For Waste Disposal Groups 4 and $5,{ }^{226} \mathrm{Ra}$ also makes a significant contribution. The lower discharges for Waste Disposal Group 4 is due to the removal of ${ }^{14} \mathrm{C}$, uranium, and plutonium. For Waste Disposal Group 5, the lower discharges are due to the removal of ${ }^{14} \mathrm{C}$ and the addition of depleted uranium. Since solubility is by element, not isotope, the depleted uranium reduces the effective solubility of ${ }^{233} U$ and ${ }^{234} U$. Consequently, ${ }^{233} U$ and ${ }^{234} U$ make up a smaller fraction of the total dissolved uranium in Waste Disposal Group 5.

A sensitivity/uncertainty study using regression analysis was performed to see if summed normalized discharge consistently correlated with any of the sampled model parameters. The only significant correlation was with the log permeability of the upper borehole. The upper borehole permeability controls the amount of flow from the brine reservoir through the repository and up to the Snake aquifer and, consequently, the transport. A less significant correlation was seen with halite permeability (Figure 16.2-12b). This parameter controls resaturation of the repository or the amount of brine available for corrosion. Although scatterplots for only Waste Disposal Group 1 are shown, results are similar for the other waste disposal groups. 


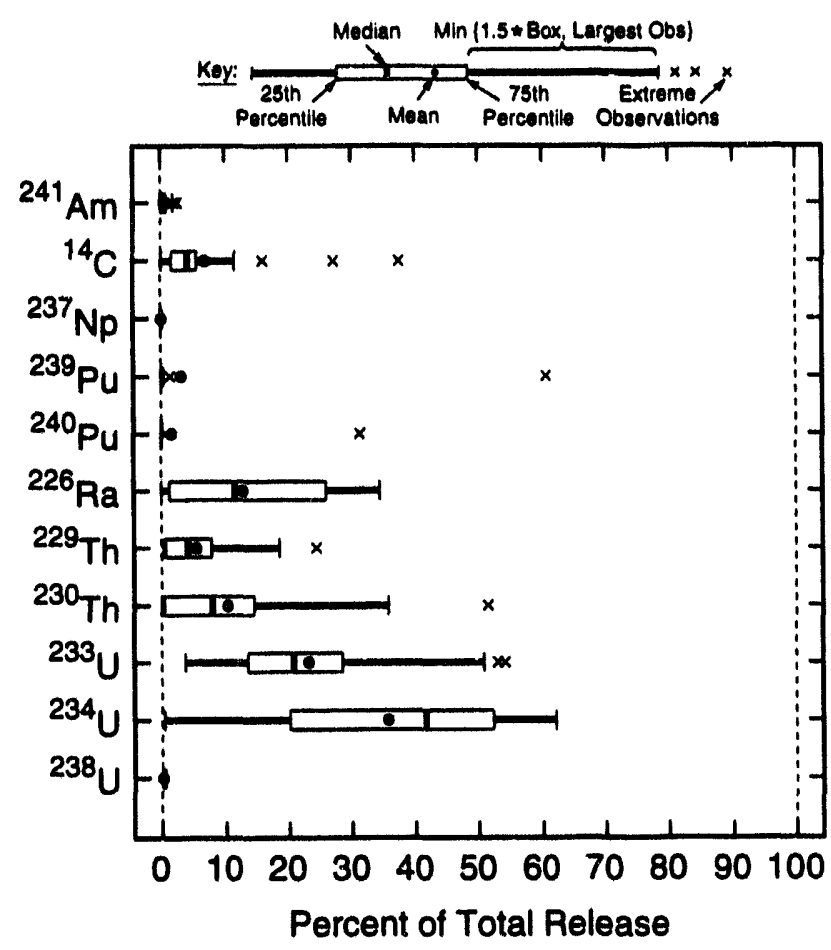

TR1-6342-4023-0

(a) Percent of total discharge

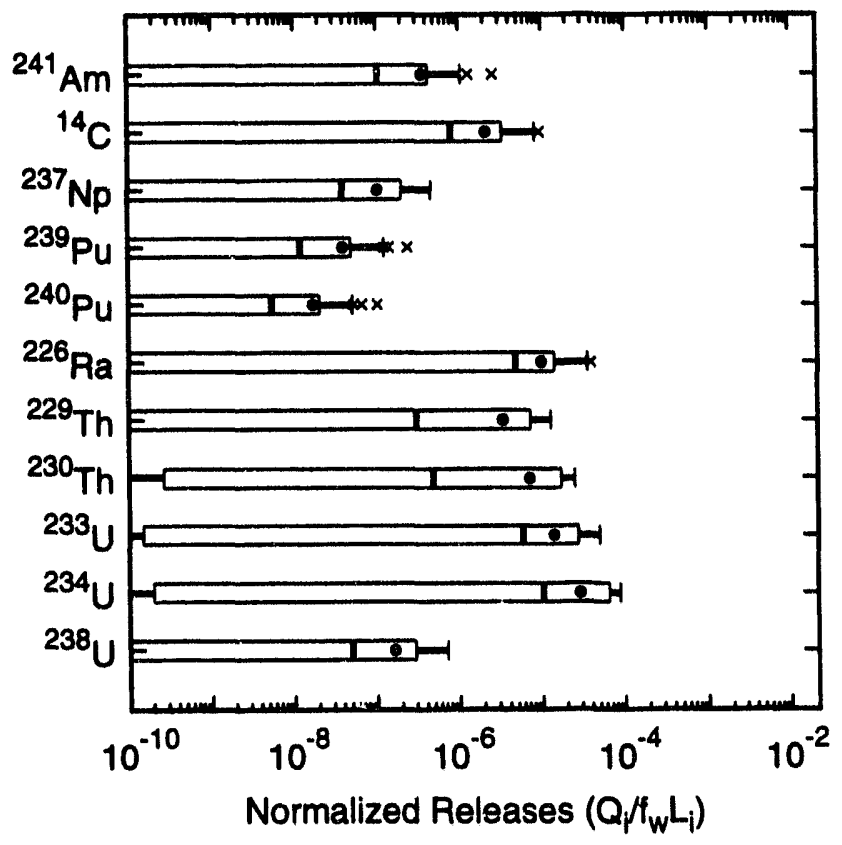

TRI-6342-4029-0

(b) Normalized discharge

Figure 16.2-7. Contributions of individual isotopes to summed normalized discharges from repository (up the intrusion borehole) for salt repository, Waste Disposal Group 1. 
16. Results and Conclusions

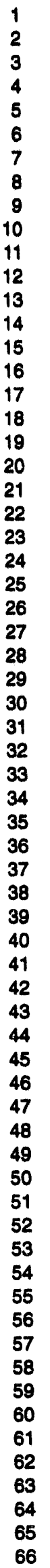

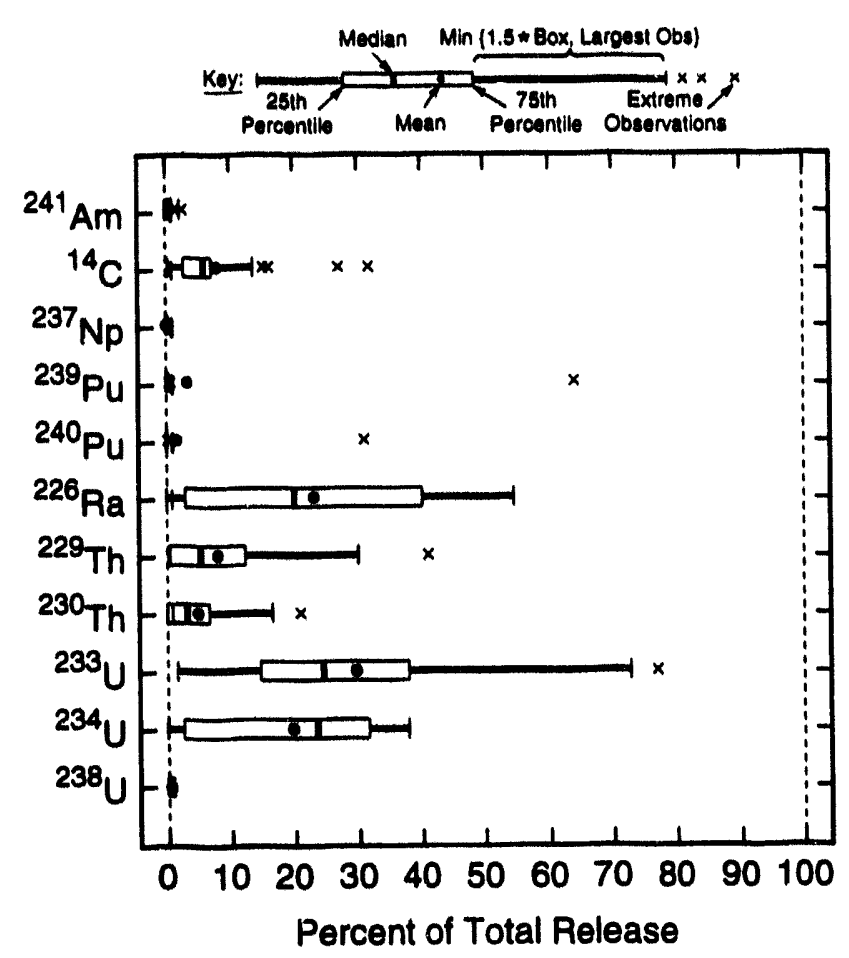

TRI-6342-4031-0

(a) Percent of total discharge

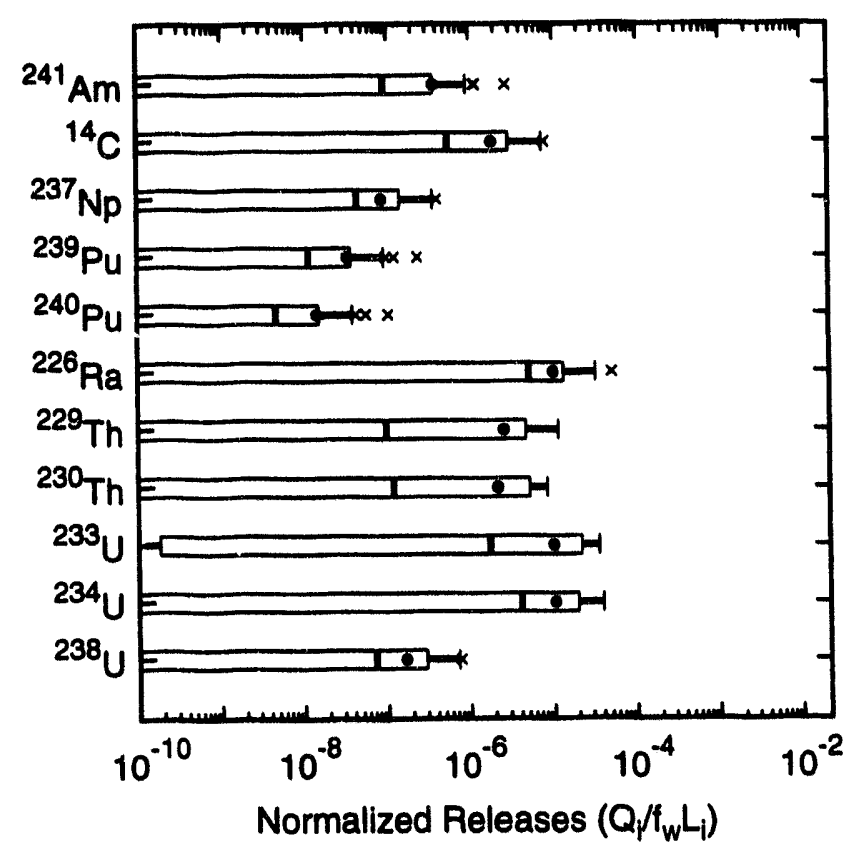

TA1-6342-4032.0

(b) Normalized discharge

Figure 16.2-8. Contributions of individual isotopes to summed normalized discharges from repository (up the intrusion borehole) for salt repository, Waste Disposal Group 2. 


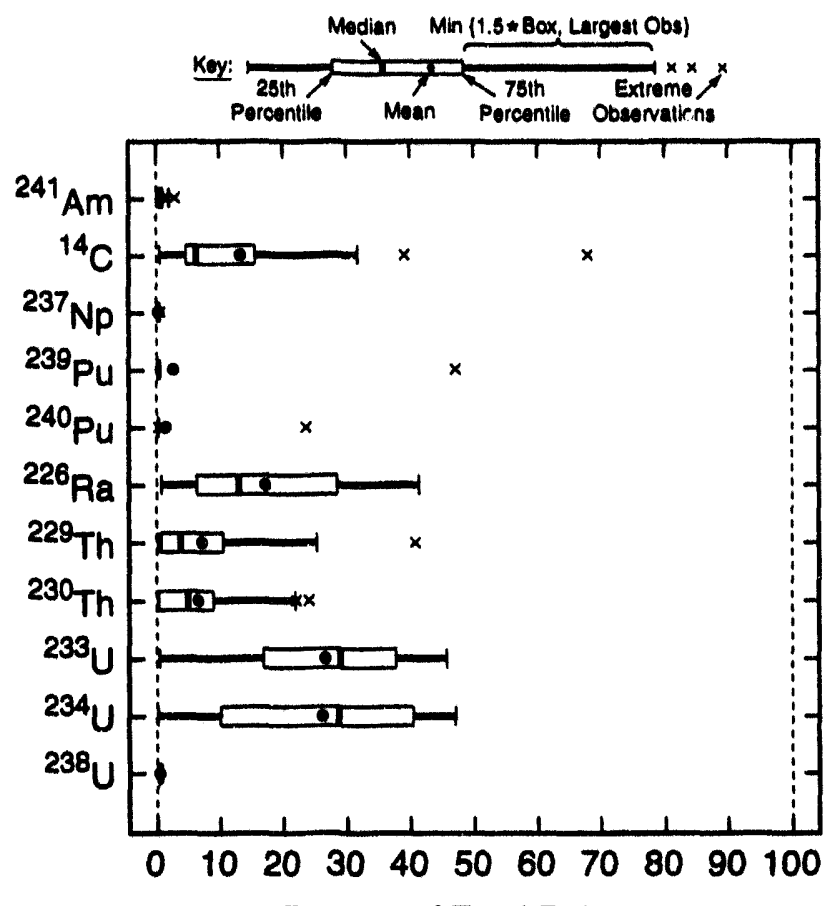

Percent of Total Release

TA1-6342-4033-0

(a) Percent of total discharge

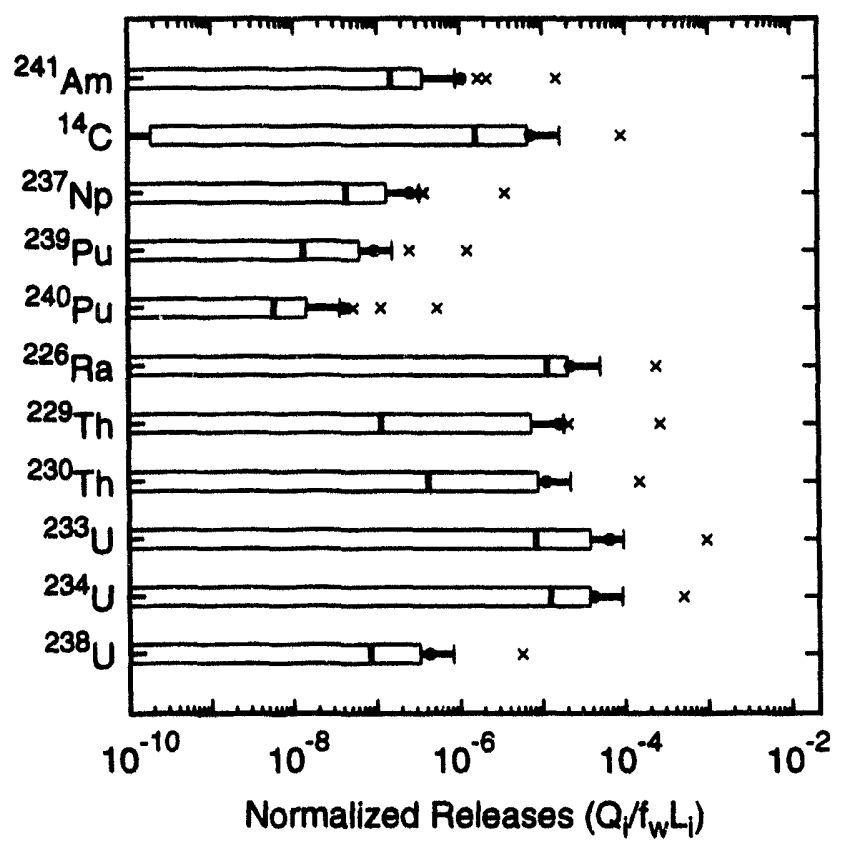

TR1.6342-4034-0

(b) Normalized discharge

Figure 16.2-9. Contributions of 11 individual isotopes in Complex PA to summed normalized discharges from repository (up the intrusion borehole) for salt repository, Waste Disposal Group 3. 
16. Results and Conclusions

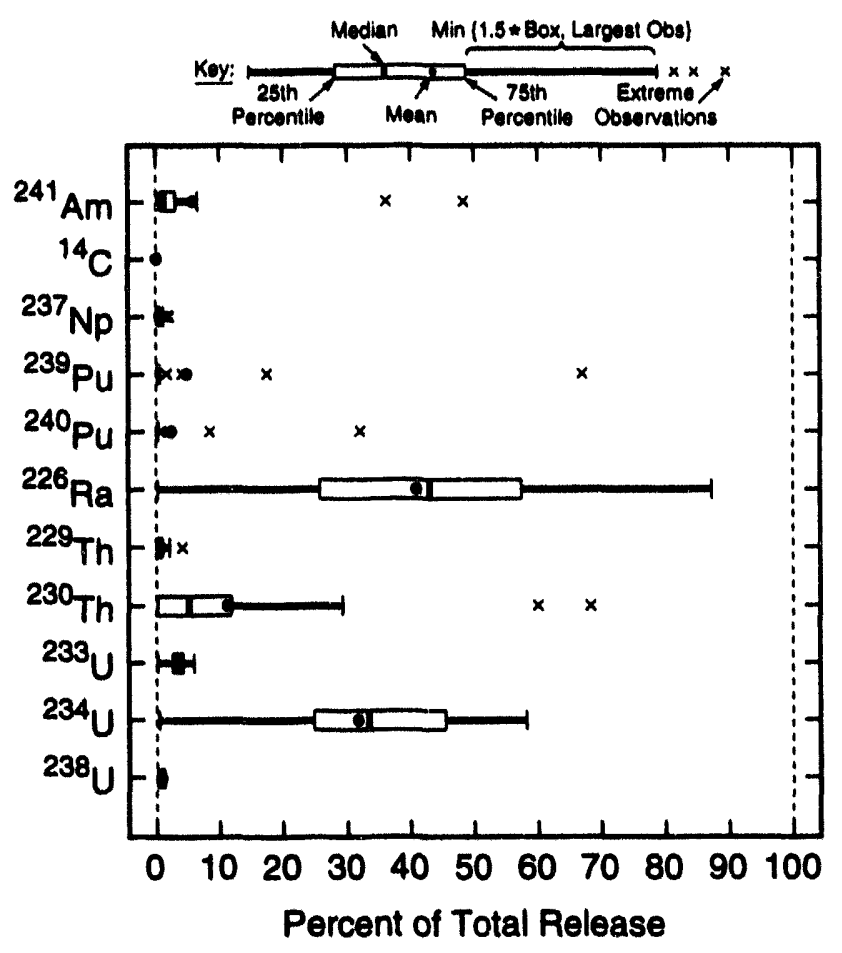

TRI-6342-4035-0

(a) Percent of total discharge

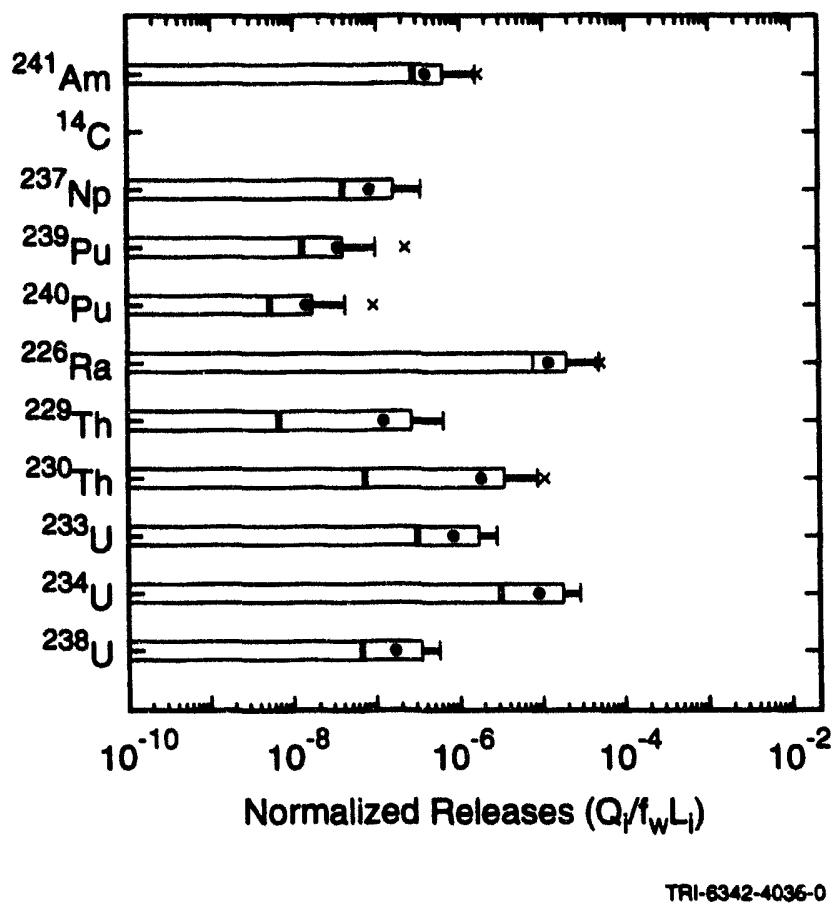

(b) Normalized discharge

Figure 16.2-10. Contributions of individual isotopes to summed normalized discharges from repository (up the intrusion borehole) for salt repository, Waste Disposal Group 4. 


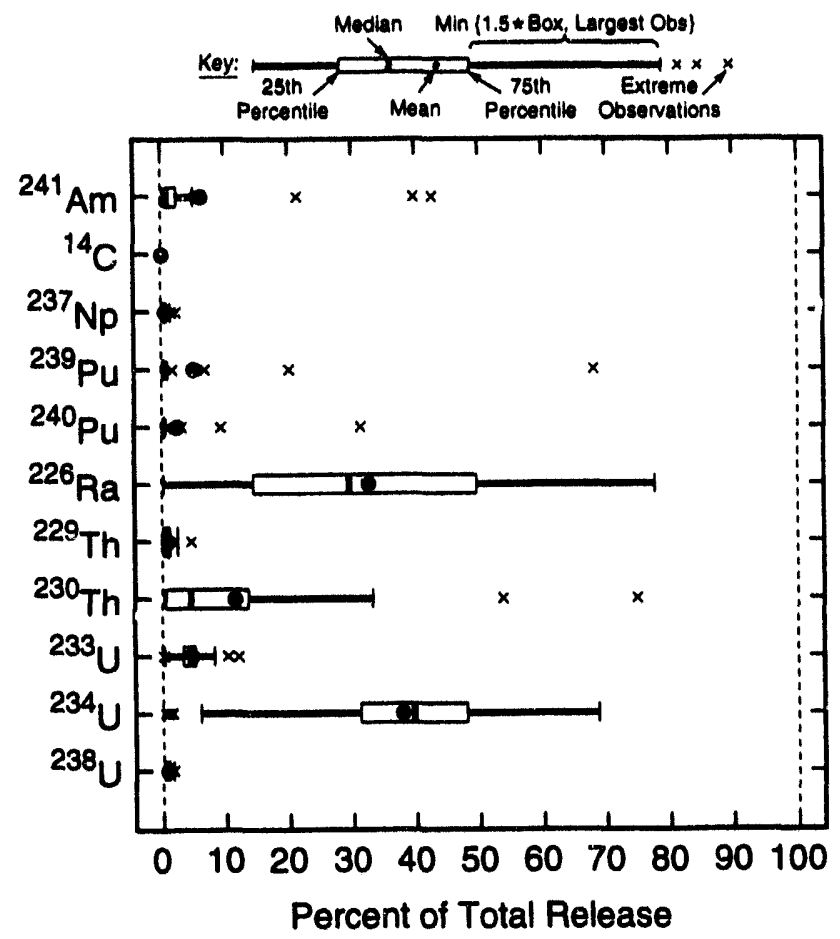

TR1-6342-4037-0

(a) Percent of total discharge

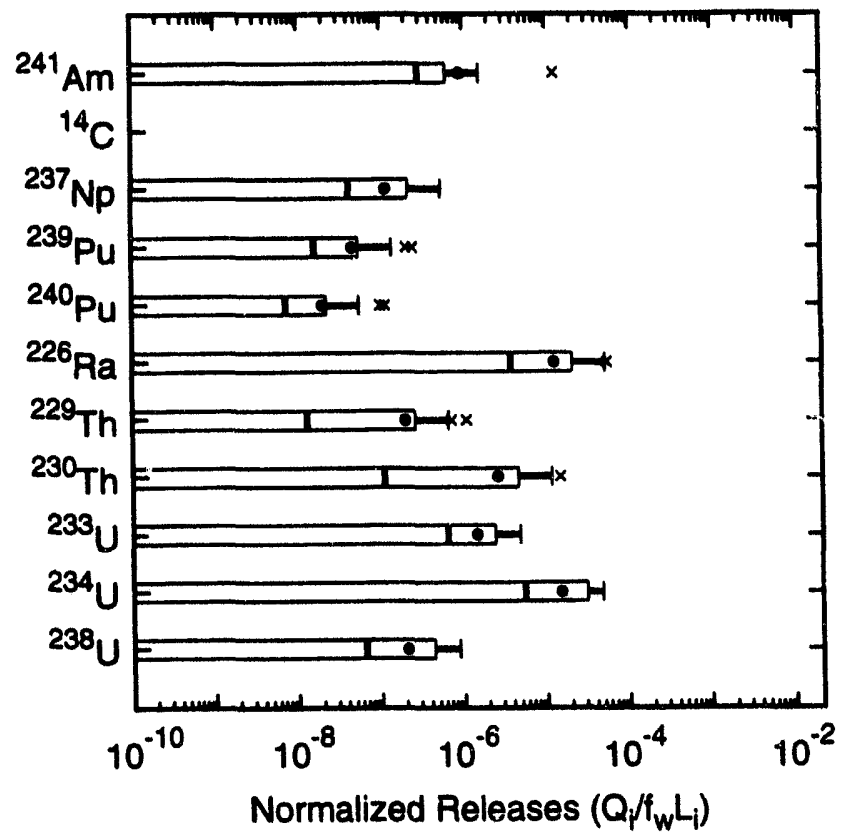

TRI-6342-4038-0

(b) Normalized discharge

Figure 16.2-11. Contributions of individual isotopes to summed normalized discharges from repository (up the intrusion borehole) for salt repository, Waste Disposal Group 5. 
16. Results and Conclusions

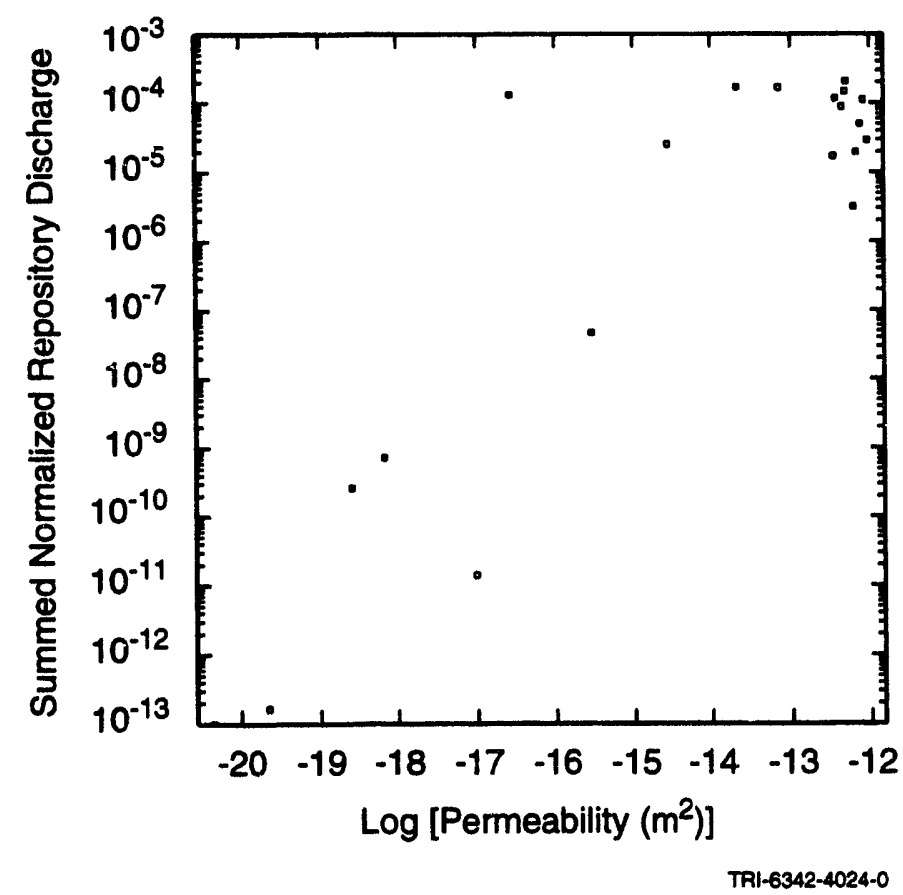

(a) Upper intrusion borehole

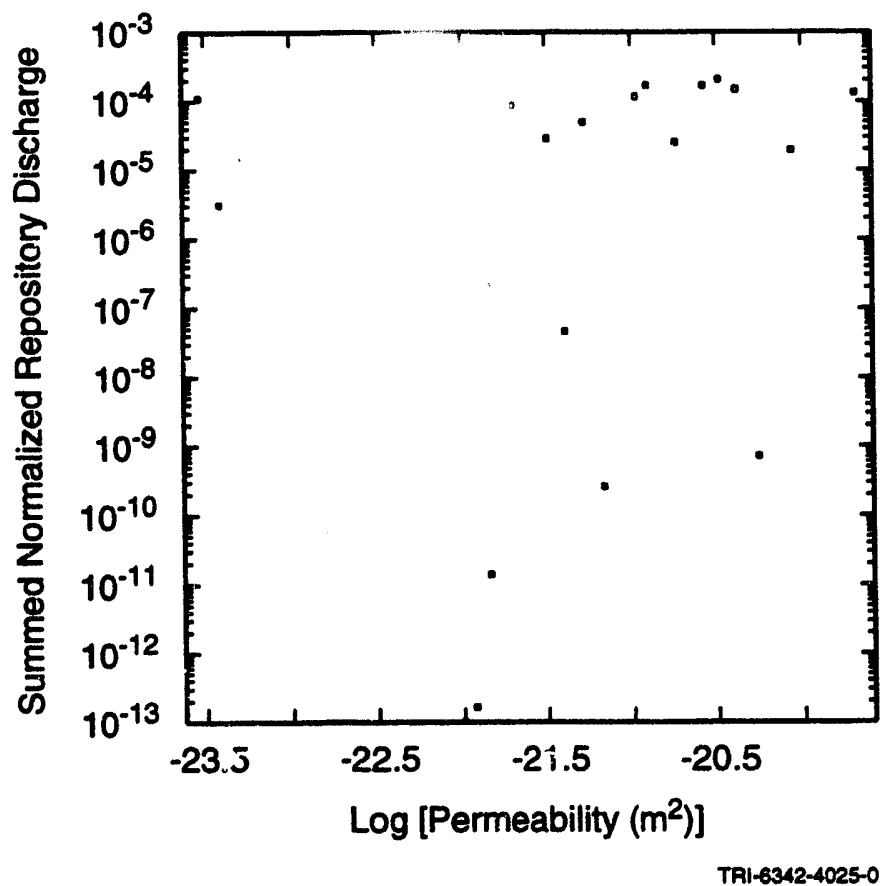

(b) Sodium Springs Formation

Figure 16.2-12. Scatterplot of activity passing repository boundary normalized by EPA release limits vs. (a) $\log$ permeability of the upper intrusion borehole, and (b) log permeability of surrounding Sodium Springs Formation for salt disposal system, Waste Disposal Groupl. 


\subsubsection{Normalized Activity Leaving the Disposal System in the Snake Aquifer (D. K. Rudeen)}

The releases from the disposal system to the accessible environment that occur in the overlying Snake Dolomite aquifer are significantly less than releases from the repository. The overlying Snake aquifer provides a natural barrier to radionuclide transport. The primary pathway to the accessible environment for the salt repository is through the intrusion boreholes, particularly Borehole 2, which intersects the brine reservoir in the Tower Formation, and into the overlying Snake aquifer. Once in the aquifer, the long travel times and retardation from mechanical (dual porosity) effects will significantly reduce the amount of radionuclides that get to the boundary of the disposal system (Figure 16.2-13). Releases are integrated at the $2.4-\mathrm{km}$ boundary of the salt disposal system. Note that for these simulations no chemical retardation (adsorption) was included in the Snake aquifer.

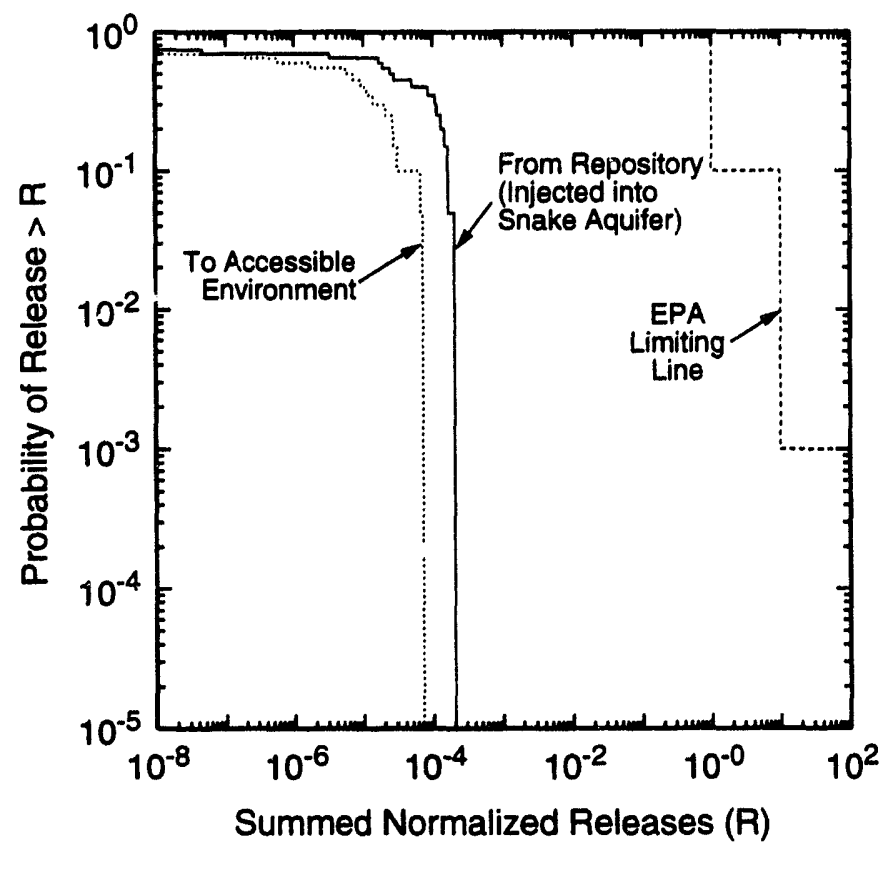

TRI-6342-4026-0

Figure 16.2-13. Comparison of CCDFs, conditional on intrusion of summed normalized release to the accessible environm nt in the Snake Dolomite aquifer of the salt disposal system and summed normalized activity discharge from the repository (injected into aquifer) (from Figure 16.2-5) Waste Disposal Group 1. (Note log-log scale.) 


\subsection{Complex PA Results for Disposal in Granite Batholith}

\subsubsection{Release from the Disposal System (R. P. Rechard and H. J. Iuzzolino)}

This section presents results in the form of composite CCDFs for release from the granite disposal system. The influence of various phenomena and model parameters on these results is explored in Sections 16.3.2 through 16.3.3 by examining the conditional CCDFs that make up this composite CCDF.

Similar to the results for the salt disposal system, the mean composite CCDF curve for all five waste disposal groups lies to the left of the EPA limiting line, specified in the Containment Requirements of 40 CFR 191, at all levels of probability, even at the boundary of the repository for the granite disposal system (Figure 16.3-1a). This composite CCDF incorporates the likelihood of occurrence of all combinations of the basic summary scenario, using six intrusion times, and an upper bound on drilling frequency for a repository in sedimentary rock of 3 boreholes $/ \mathrm{km}^{2} /$ $10,000 \mathrm{yr}$.

The direct releases from cuttings are often below the releases from groundwater advection (entirely below for Waste Disposal Group 1) (compare Figures 16.3.1-1 and Figure 16.8-2, found in Section 16.8).

The mean composite CCDFs for the first three waste disposal groups and the last two waste disposal groups are grouped fairly tightly, indicating that the minimal and moderate waste treatment options for the INEL spent fuel and radioactive waste do not have much influence on the moderately sized granite repository when a large percentage of the inventory includes waste from other DOE sites; however, extensive treatment does have some effect.

Because of the hypothetical nature of the two disposal systems, it is difficult to make absolute and conclusive comparisons. However, based on the assumed geologic barrier features and model parameters, the salt disposal

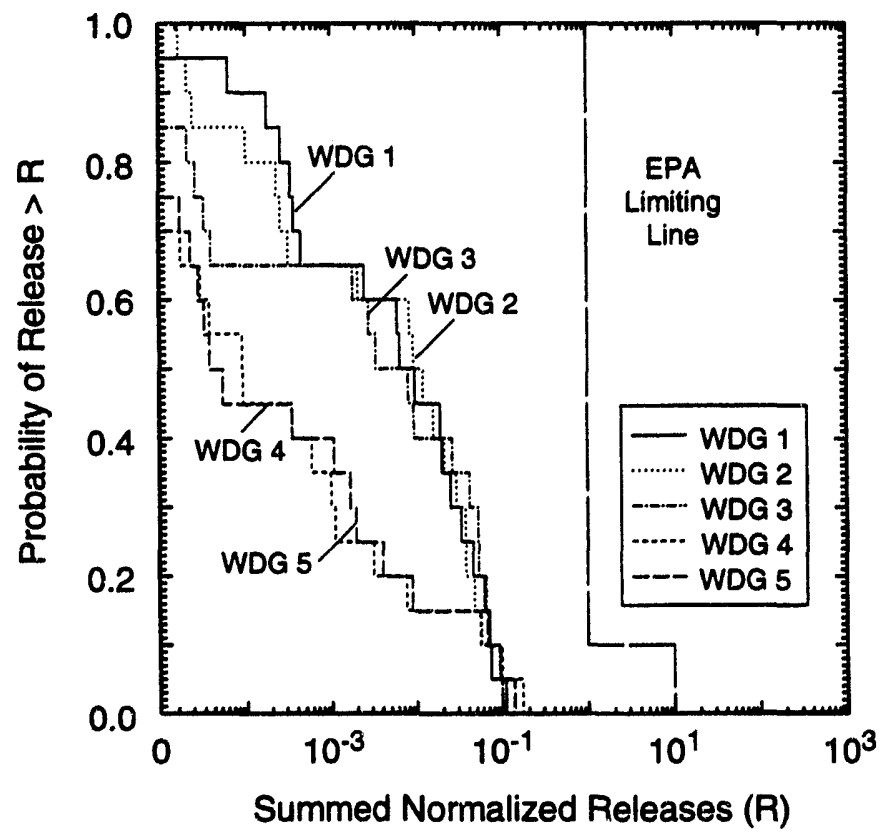

TRI-6342-1128-0

Figure 16.3-1. Mean composite CCDF of normalized integrated activity of only groundwater releases from the repository boundary of granite disposal system for all five waste disposai groups. (This figure is comparable to Figure 16.2-2 for the salt disposal system.) (Calculated direct releases from cuttings are usually below the groundwater releases and only tend to smooth the groundwater release curves shown; see Figures 16.10-2 in Section 16.10.) 
system is more robust than the granite disposal system (even at the boundary of the repository, which diminishes the influence of travel through the geologic system). Extensive treatment of the spent fuel and radioactive waste is required for the granite disposal system to reach a level of performance comparable to the salt disposal system (Figure 16.3-2). Also refer to Section 16.7, "Calculations Using Corrosion-Resistant Waste Parcel," where an alternative waste parcel with two corrosion-resistant layers is discussed. The corrosion-resistant waste parcel allowed the granite disposal system to reach a level of performance similar to the salt disposal system, even with Waste Disposal Group 1.

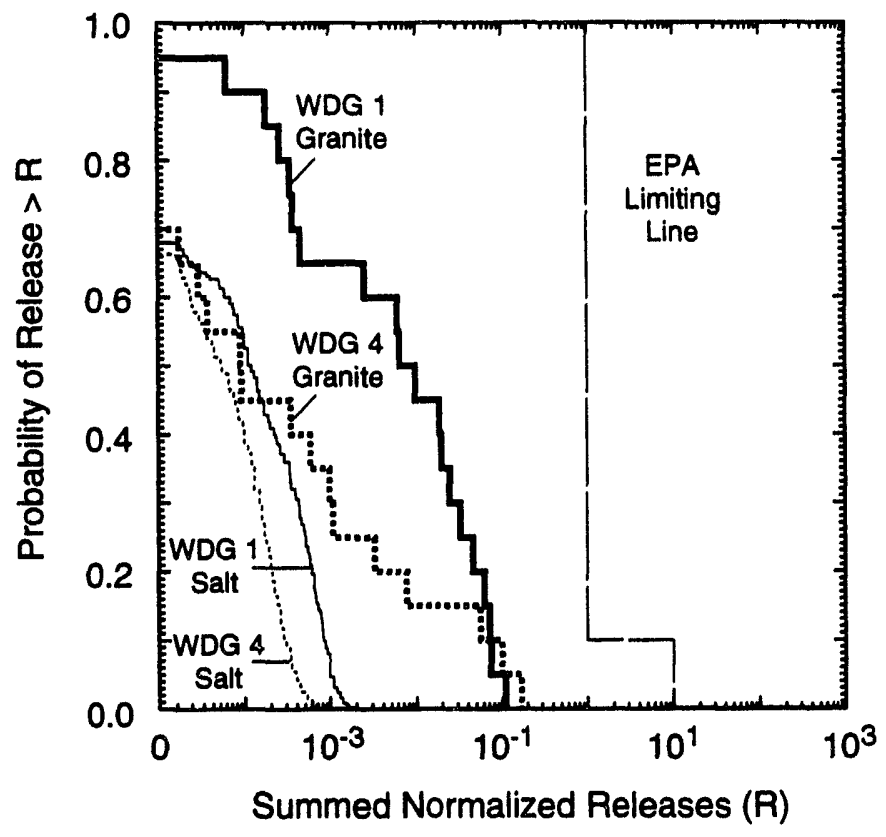

TRI-6342-4127-0

Figure 16.3-2. Comparison between salt and granite mean composite CCDFs of summed normalized activity of groundwater releases at the respective repositery boundaries for Waste Disposal Groups 1 and 4.

\subsubsection{Percentage of Radionuclides Leaving the Waste Parcel (D. K. Rudeen)}

As in the salt disposal system, the fraction of the inventory that leaves the waste parcel was evaluated for each of the five waste disposal groups for the granite disposal system. The results are shown as a conditional CCDF in Figure 16.3-3. The largest waste parcel discharge fraction $\left(F_{w p}\right)$ was $24 \%$ for Waste Disposal Group 3, Run 4 . As in the salt disposal system, Waste Disposal Group 4 generally had the lowest waste parcel activity fraction (Figure 16.3-3), but the relative order of the remaining waste disposal groups is not clear.

To quantify the relative performance, the five waste disposal groups were evaluated further by ranking the activity fraction for each run, or sample, across the five disposal groups from least (rank 1) to most (rank 5). The frequency with which a waste disposal group received a particular ranking is plotted in Figure 16.3-4. In the granite disposal system, Waste Disposal Group 4 has the lowest discharge fraction, followed by Waste Disposal Groups 5, 2, 3 , and 1 , which is the same order as for the salt disposal system.

\subsubsection{Normalized Activity Leaving Repository (D. K. Rudeen)}

One of the primary results from the transport simulations is the integrated positive discharge activities across a prescribed boundary or "monitoring points." For the granite repository, the boundary is specified at six locations: one in each of the two horizontal fractures above and below the repository and two in the glacial till. These logical 
16. Results and Conclusions

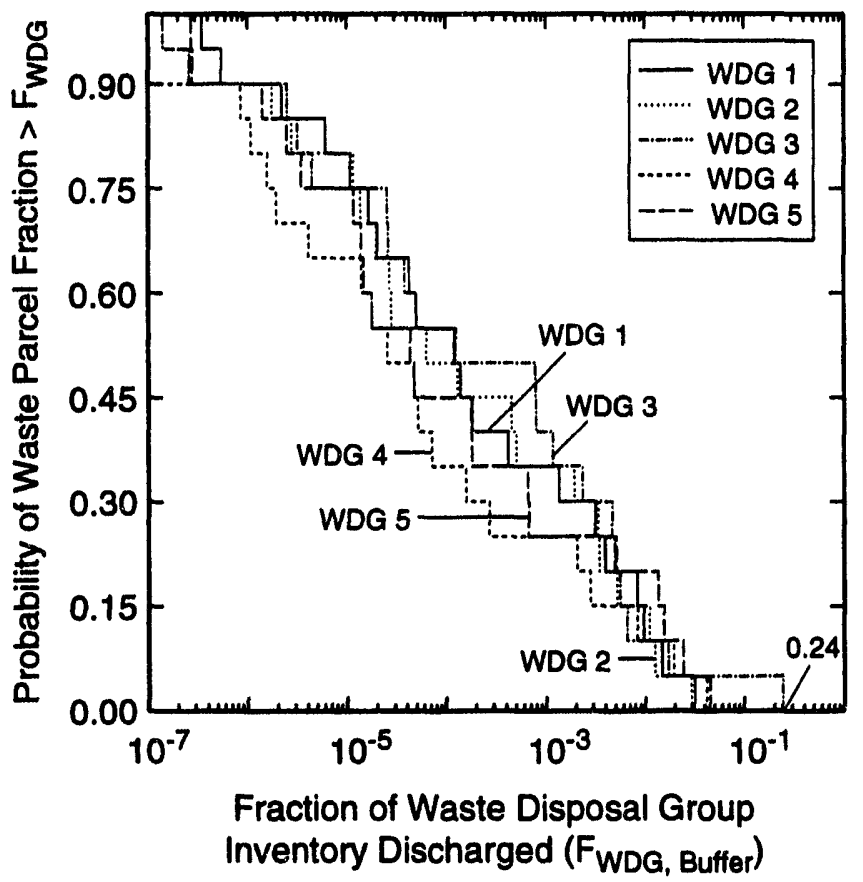

TR1-6342-4027-0

Figure 16.3-3. CCDF, conditional on intrusion, of fraction of initial waste disposal group inventory discharge inte backfill-buffer granite disposal system, for all five waste disposal groups. (This figure is comparable to Figure 16.2-3 for the salt.)

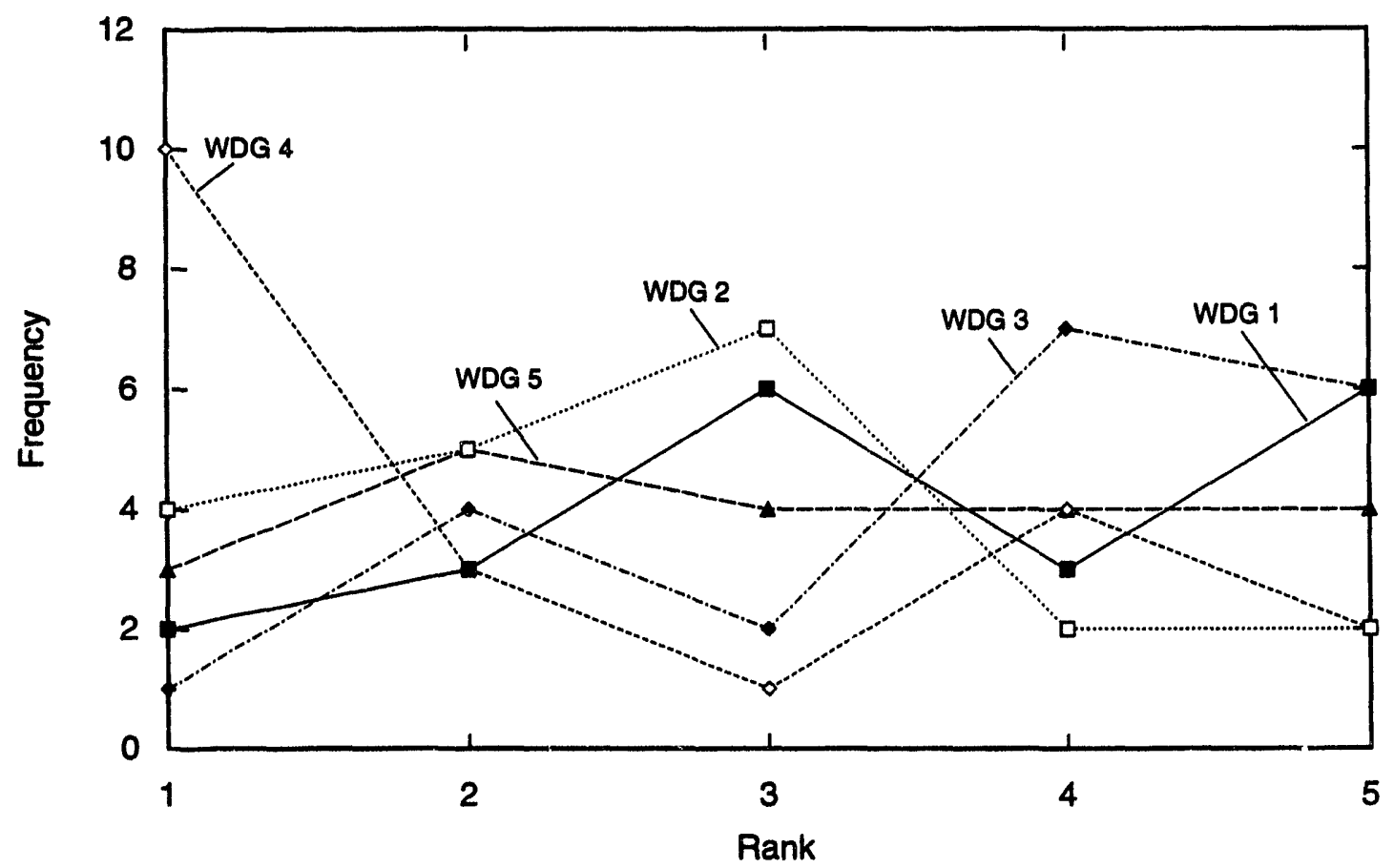

TRI-6342-3972-0

Figure 16.3-4. Frequency with which waste parcel activity fraction into backfill-buffer for each waste disposal group in granite repository was ranked showing, in general, a ranking of 4, 5, 2, 3, and 1. (Rank 1 is the least discharge, and rank 5 , is the most.) 
$\mathrm{x}$-axis element numbers are positioned two cells beyond the repository shaft and two cells beyond the end of the repository along the horizontal (x-direction). (The exact locations of the discharge boundaries are found on Figure 13-2, corresponding to logical $x$-axis element index 4 [discharge to the left], and 52 [discharge to the right], for Fracture Zone 1, Fracture Zone 2, and the glacial till.)

The summed normalized discharges are presented as CCDF plots conditional on the occurrence of an exploratory borehole in Figure 16.3-5 for the five waste disposal groups. The summed normalized activity discharges are not bound by specific disposal groups as was the case for the salt repository. Also seen in the CCDFs on the left of Figure 16.3-5 is a distinct separation in the curves for Waste Disposal Groups 1,2, and 3, and Waste Disposal Groups 4 and 5. This separation is due to the absence of ${ }^{14} \mathrm{C}$ in Waste Disposal Groups 4 and 5 . In the first three waste disposal groups, ${ }^{14} \mathrm{C}$ dominates the summed normalized discharge because it is not retarded or adsorbed during transport. In the absence of ${ }^{14} \mathrm{C}$, the elements that dominated the discharge are ${ }^{239} \mathrm{Pu},{ }^{240} \mathrm{Pu}$, and ${ }^{241} \mathrm{Am}$. Thus, the removal or dilution of the most important isotope, ${ }^{14} \mathrm{C}$, provided a larger margin of safety in comparison with the EPA limiting line.

The second set of five curves on the figure (to the right) are summed normalized discha ges from the waste parcels into the repository. As in the salt site, the repository itself provides a significant barrier to radionuclide transport. Discharges up the intrusion boreholes are 2 or 4 orders of magnitude smaller than discharges from the waste parcels. Again, to quantify the relative performance, the summed normalized repository discharges out the fracture zones were ranked for each sample set (or run) across the five disposal groups from least (rank 1) to most (rank 5). The rankings are plotted as a histogram in Figure 16.3-6. This time, Waste Disposal Group 5 had the lowest summed normalized discharge, followed by Disposal Groups 4, 3, 1 and 2.

Figures 16.3-7 through 16.3-11 summarize the contribution of individual isotopes to the total normalized discharge for each of the disposal groups. Figures 16.3-7a through 16.3-11a show the distribution of the percent of total release for each isotope. Figures $16.3-7 \mathrm{~b}$ through $16.3-11 \mathrm{~b}$ show the distribution of normalized release for each isotope. The summed normalized discharges are dominated by ${ }^{14} \mathrm{C}$ for Waste Disposal Groups 1,2 and 3. For Waste

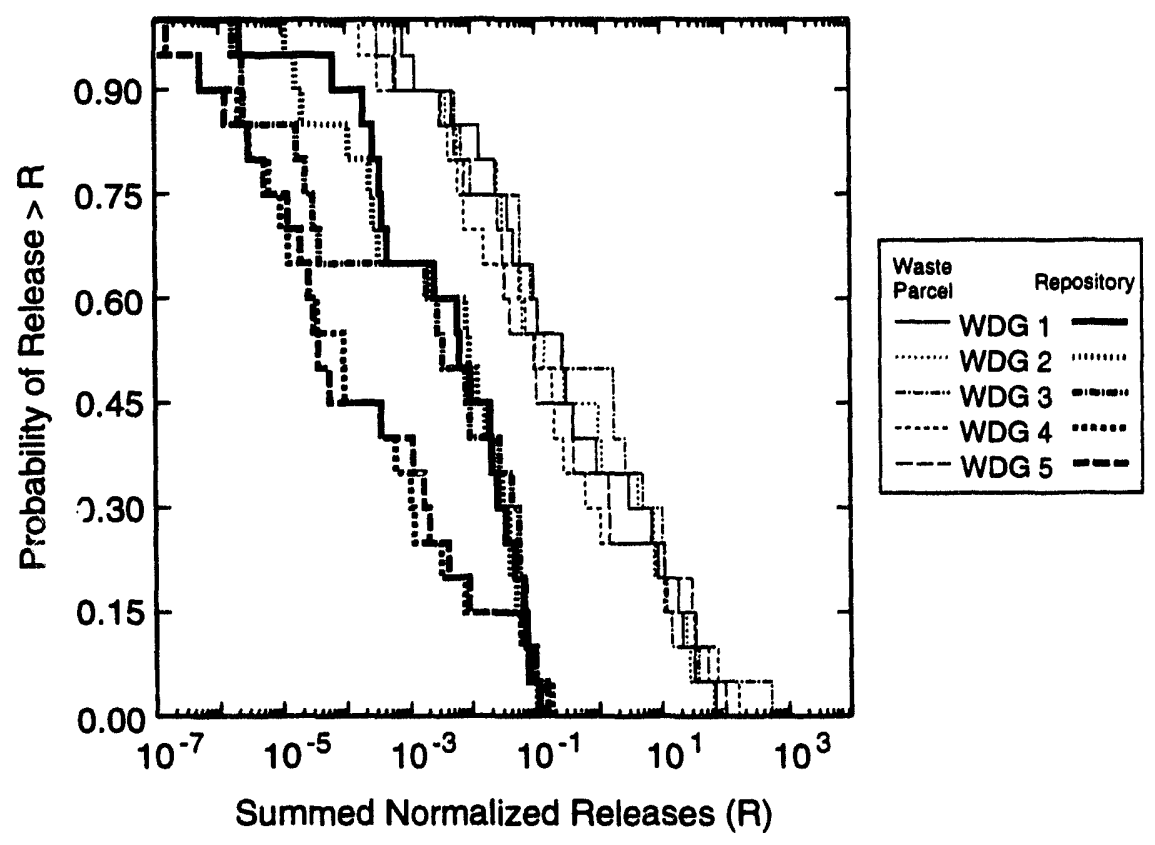

TAI-6342-4028-0

Figure 16.3-5. CCDF, conditional on intrusion, of summed normalized activity discharges from the waste parcels into the pore space of backfill-buffer surrounding the waste parcels (right curves) and from the repository at the horizontal fracture zones (left curves) from the granite disposal system. 


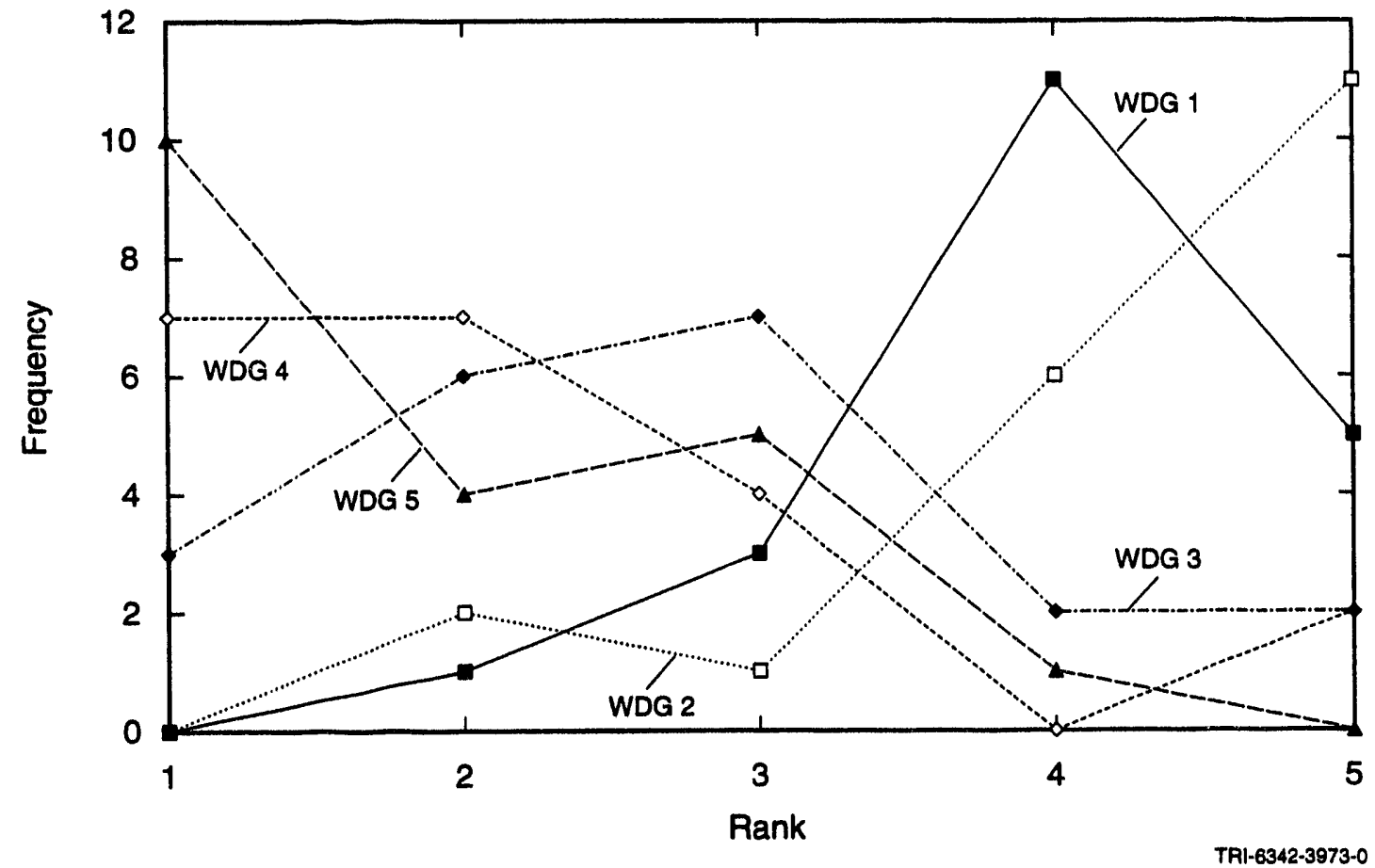

Figure 16.3-6. Frequency with which summed normalized activity discharge from the repository boundary for each disposal group in the granite repository was ranked showing, in general, a ranking of 5, 4, 3, 1 , and 2. (Rank 1 is the least discharge, and rank 5, the most.)

Disposal Groups 4 and $5,{ }^{241} \mathrm{Am},{ }^{239} \mathrm{Pu}$, and ${ }^{240} \mathrm{Pu}$ dominate. The lower discharges for Waste Disposal Group 4 are due to the removal of ${ }^{14} \mathrm{C}$, uranium, and plutonium. For Waste Disposal Group 5, the lower discharges are due to the removal of ${ }^{14} \mathrm{C}$ and the addition of depleted uranium. Because solubility is by element, not isotope, the depleted uranium reduces the effective solubility of ${ }^{233} \mathrm{U}$ and ${ }^{234} \mathrm{U}$. Consequently, ${ }^{233} \mathrm{U}$ and ${ }^{234} \mathrm{U}$ make up a smaller fraction of the total uranium dissolved in Waste Disposal Group 5.

A sensitivity/uncertainty study using regression analysis was performed to see if summed normalized discharge correlated with any of the sampled properties. The analysis showed a correlation with the stainless steel corrosion rate (Figure 16.3-12a). The corrosion rate controls the breach time and ronsequently the time of release of the radionuclides. The container corrosion rate in the granite disposal system was more important than in salt because the rate is much slower in the fresh water. There was also a slight correlation with the partition coefficients (Kds) for uranium and americium (not shown). This result was different than for the salt disposal system, probably because of the effect of retardation in the backfill-buffer surrounding the waste parcels and the backfill in the drifts of the granite disposal system (recall that all Kds in salt were zero). (To explore this issue further in future PAs, we should probability correlate the Kd with solubility; recall that Kd was not correlated with solubility when sampling in this performance assessment of the granite disposal system.) A second regression analysis was performed to check the sensitivity of the normalized discharge of a single isotope, ${ }^{234} U$, to the sampled parameters. As expected, discharge of ${ }^{234} U$ was correlated with solubility of uranium (Figure 16.3-12b) and also the partition coefficient (Kd) for uranium (not shown). Although Figure 16.3-12b presents only a scatterplot for Waste Disposal Group 1, results are simiiar for the other waste disposal groups.

Note that the release from the granite disposal system to the accessible environment is very similar to release from the repository. Because of the modeling assumptions, the zero permeability assumption of the host granite and the no-retardation assumption of the fracture zone, once radionuclides get into the horizontal fracture zones, thzy will stay in the fractures and travel with the liquid (these assumptions are discussed in Section 13.1). 


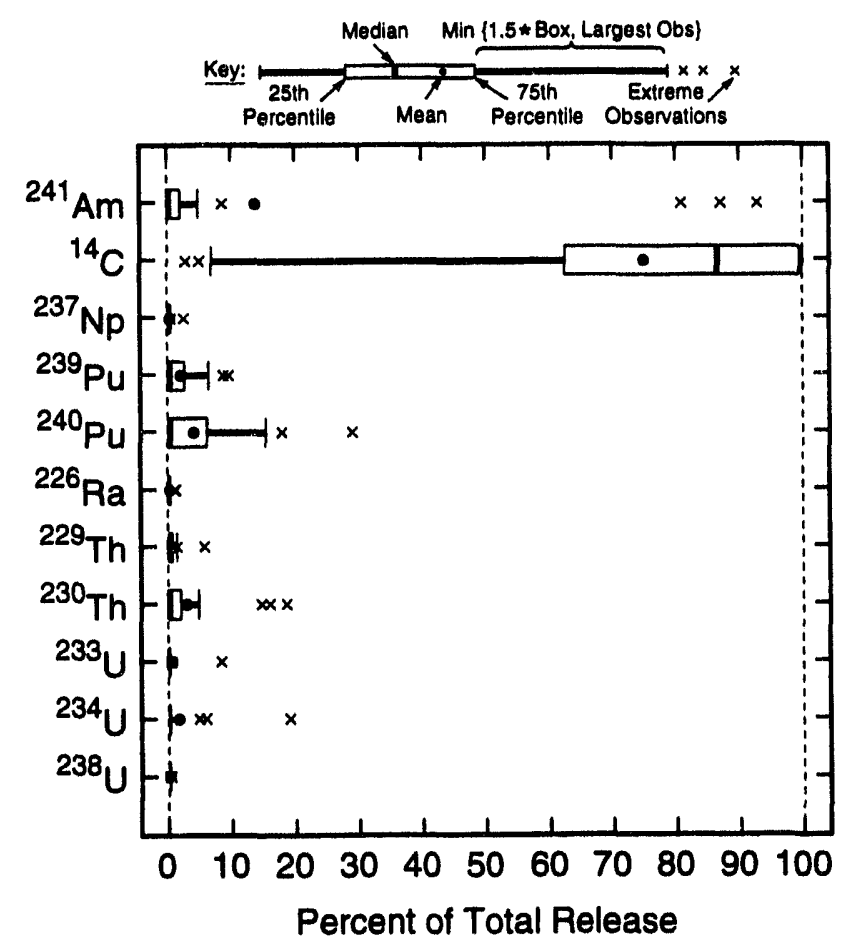

TRI-6342-4039-0

(a) Percent of total discharge

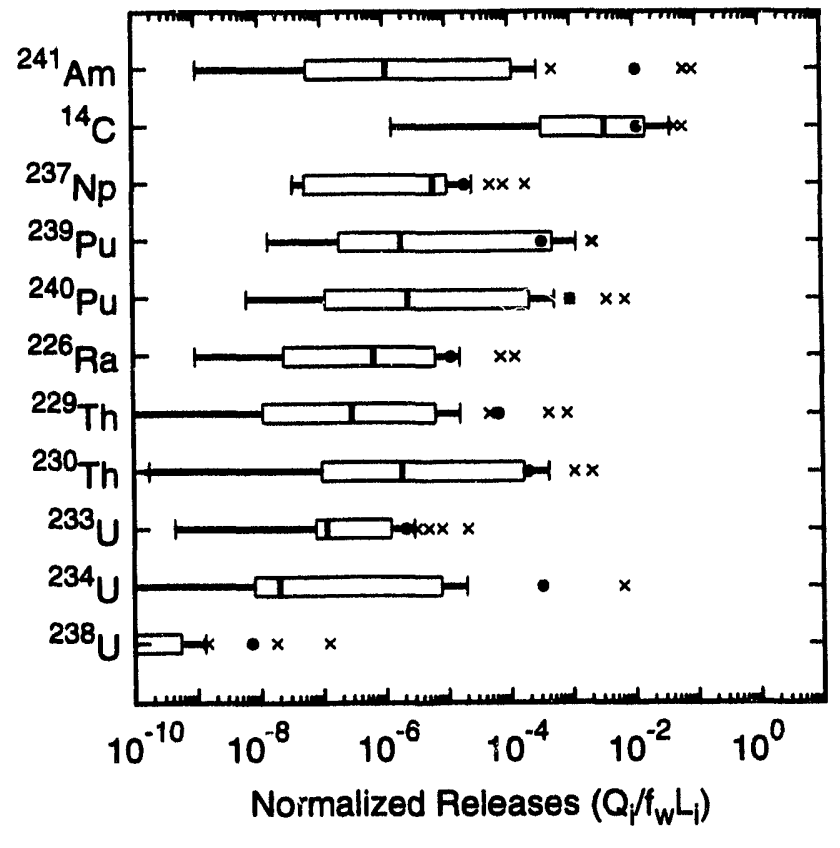

TRI-6342-4040-0

(b) Normalized discharge

Figure 16.3-7. Contributions of 11 individual isotopes in Complex PA to summed normalized discharges at hori zontal fracture zones for granite disposal system, Waste Disposal Groupl. 
16. Results and Conclusions

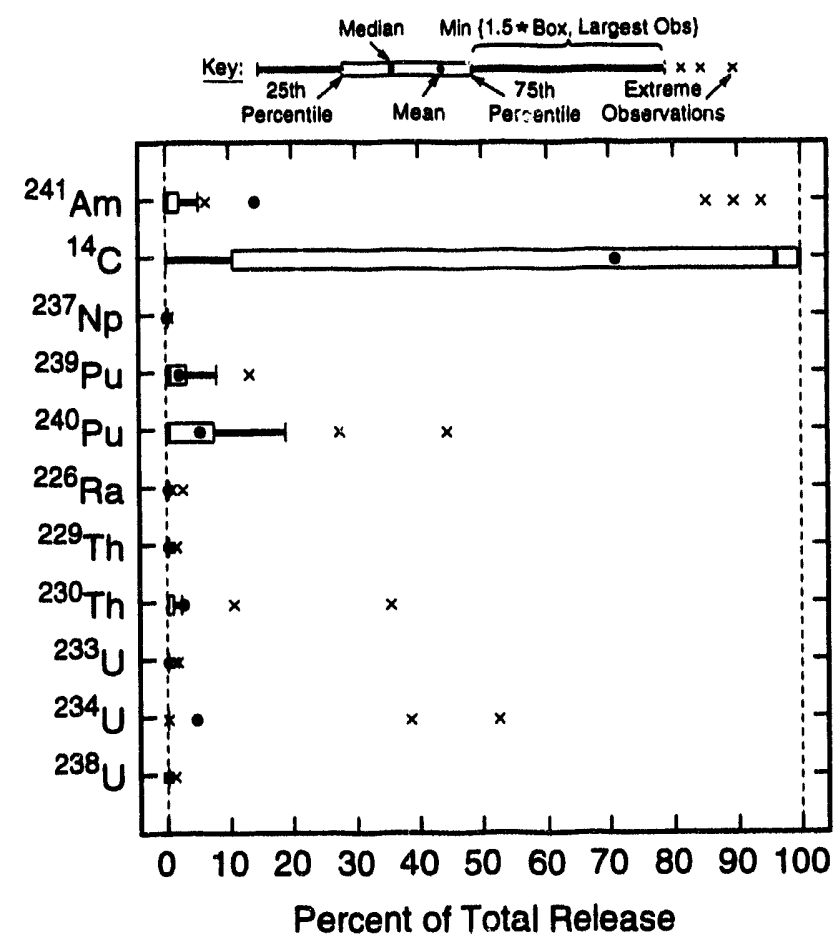

TRI-6342-4041-0

(a) Percent of total discharge

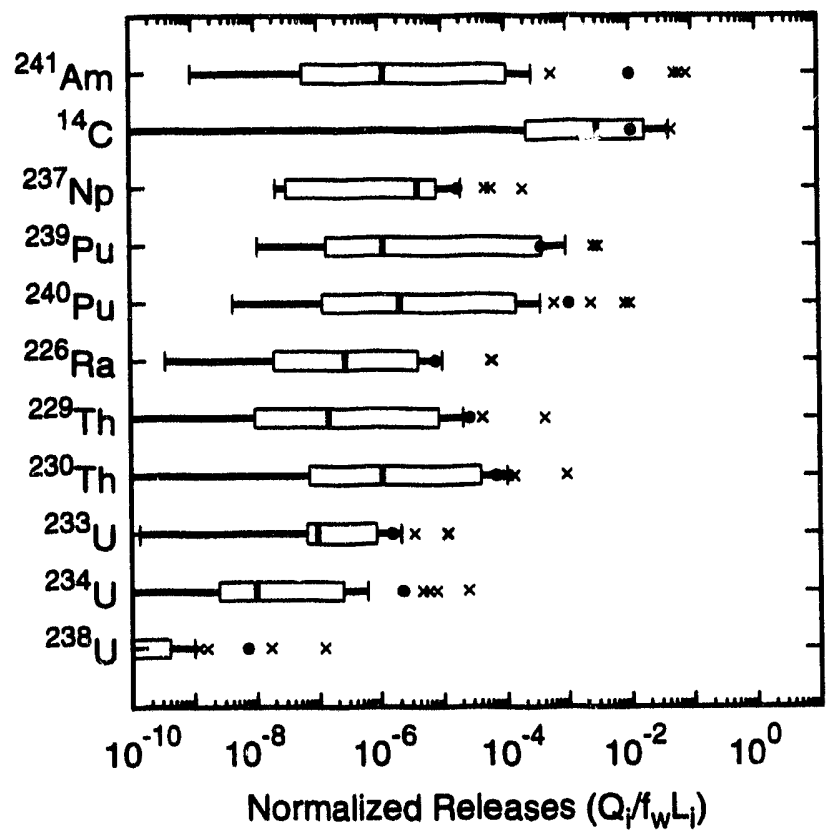

TRI-6342-4042-0

(b) Normalized discharge

Figure 16.3-8. Contributions of 11 individual isotopes in Complex PA to summed normalized discharges at horizontal fracture zones for granite disposal system. Waste Disposal Group2. 


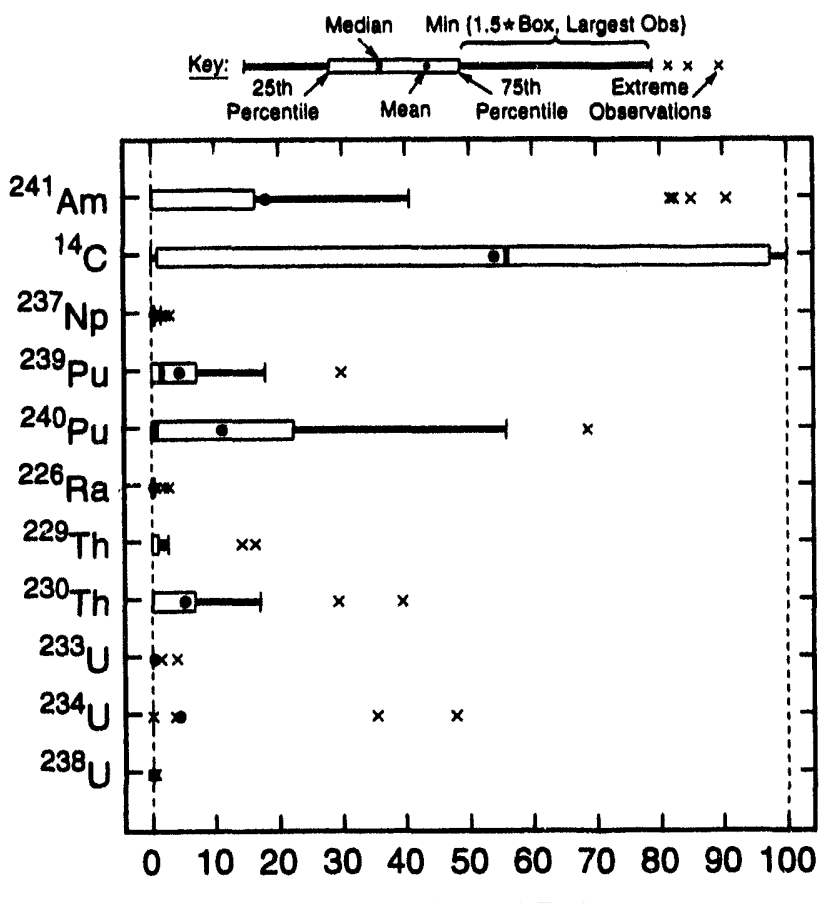

Percent of Total Release

TRI-6342-4043-0

(a) Percent of total discharge

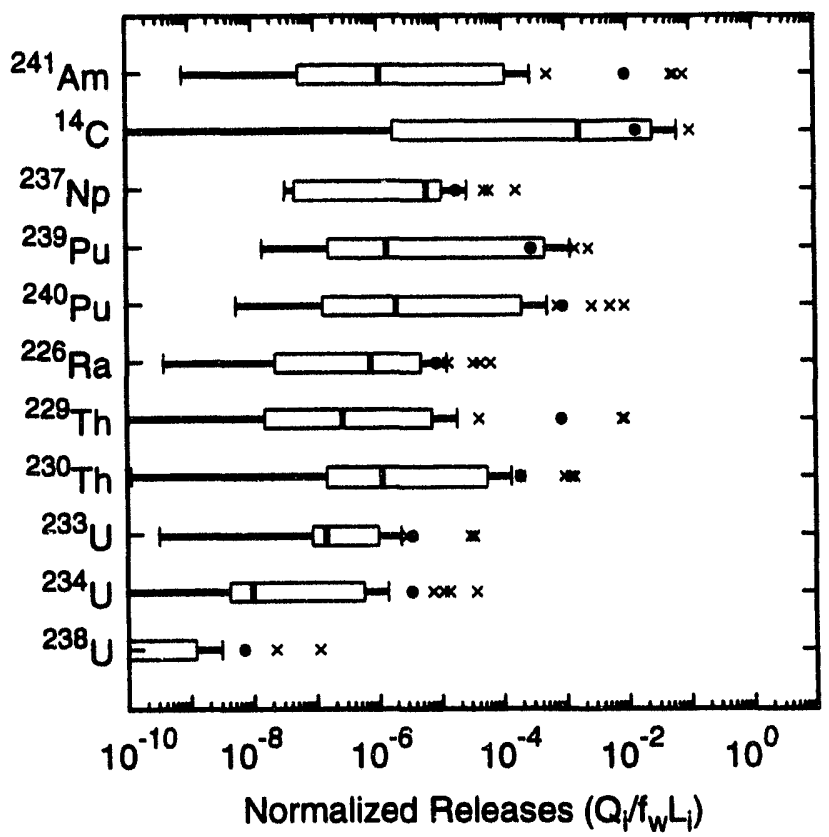

TRI-6342-4044-0

(b) Normalized discharge

Figure 16.3-9. $\quad C_{i}$...ributions of individual isotopes in Complex PA to summed normalized discharges at horizontal frecure zones for granite disposal system, Waste Disposal Group 3. 
16. Results and Conclusions

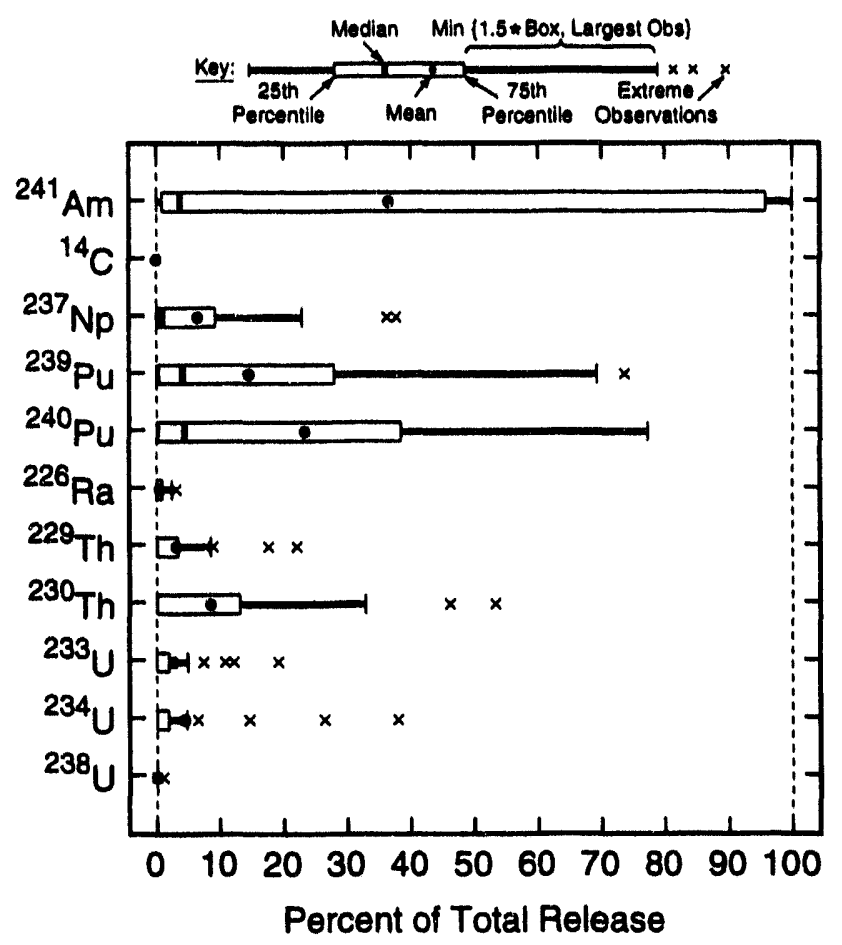

TR1-6342-4045-0

(a) Percent of total discharge

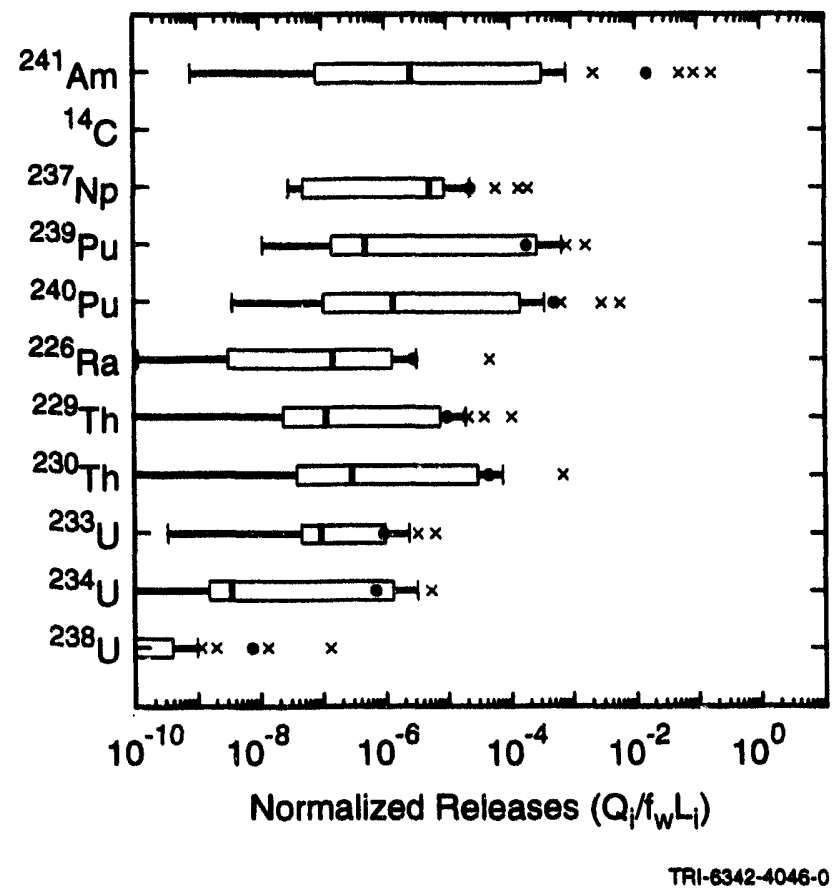

(b) Normalized discharge

Figure 16.3-10. Contributions of individual isotopes in Complex PA to summed normalized discharges at horizontal fracture zones for granite disposal system, Waste Disposal Group 4. 


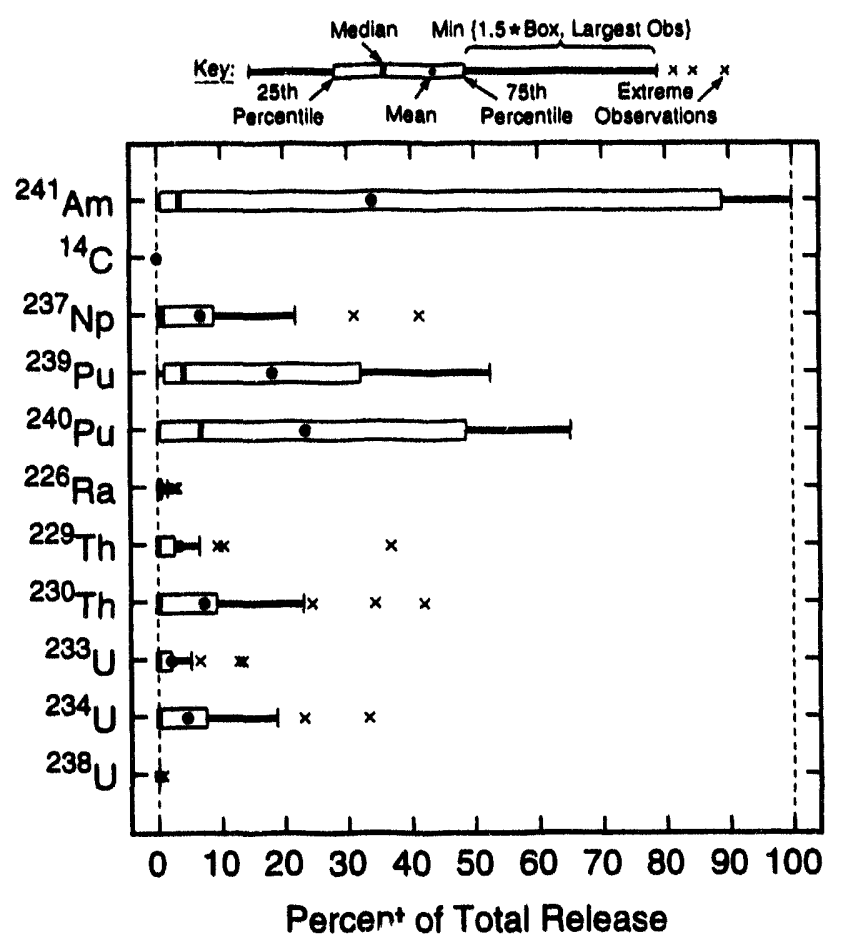

TR1-6342-4047-0

(a) Percent of total discharge

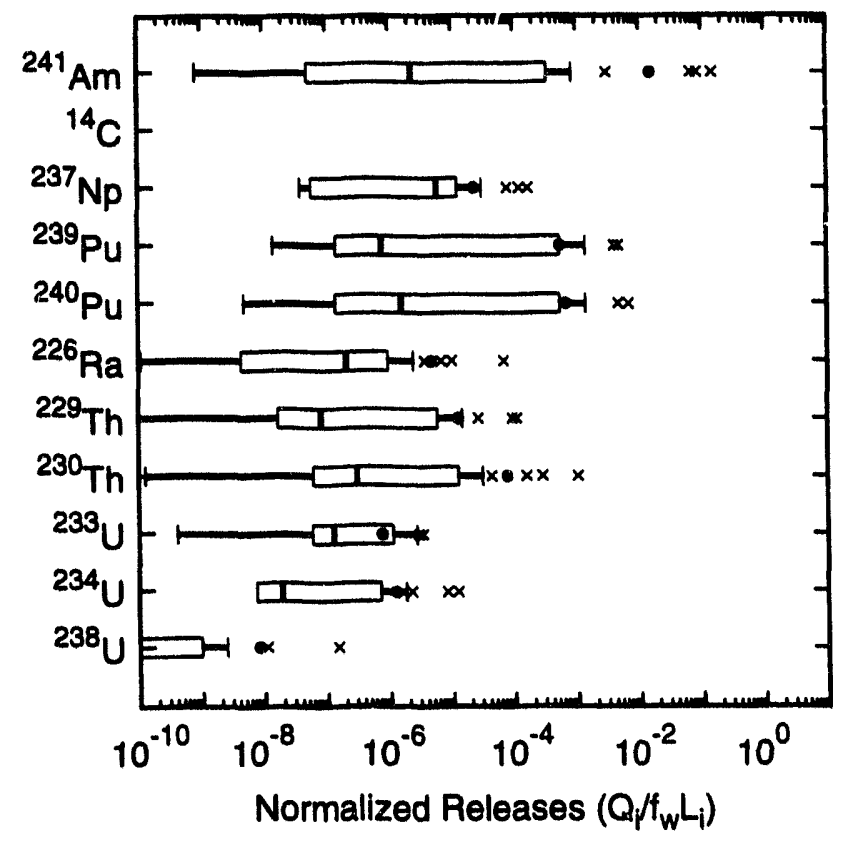

TR1-6342-4048-0

(b) Normalized discharge

Figure 16.3-11. Contributions of individual isotopes in Complex PA to summed normalized discharges at horizontal fracture zones for granite disposal system, Waste Disposal Group 5. 
16. Results and Conclusions

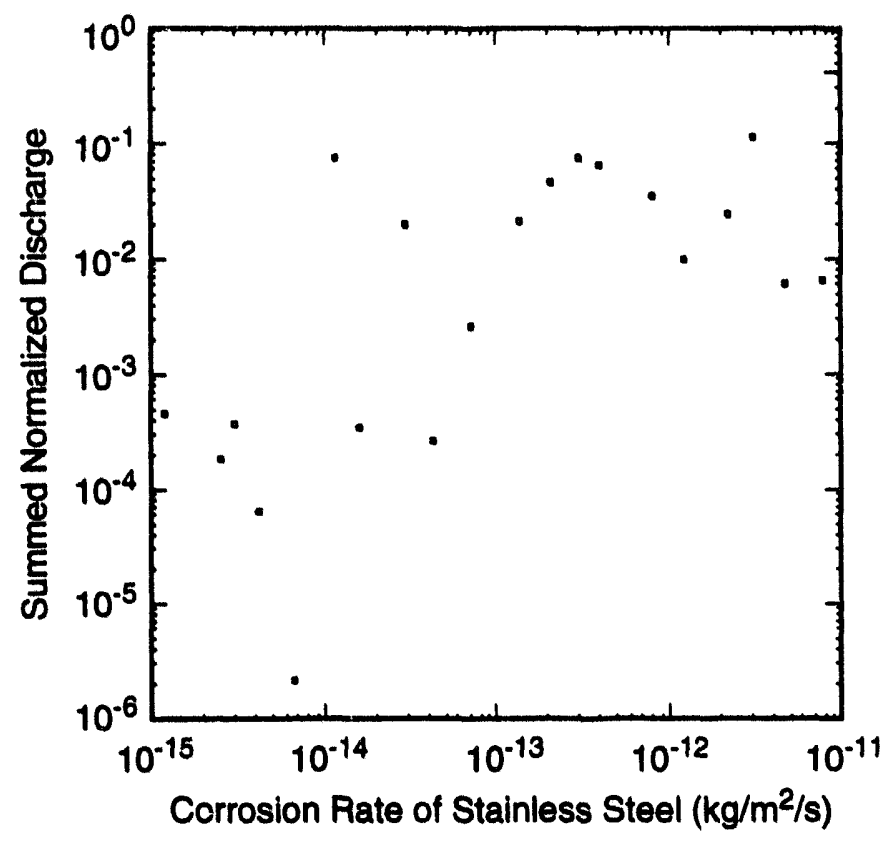

TRI-6342-3974-0

(a)

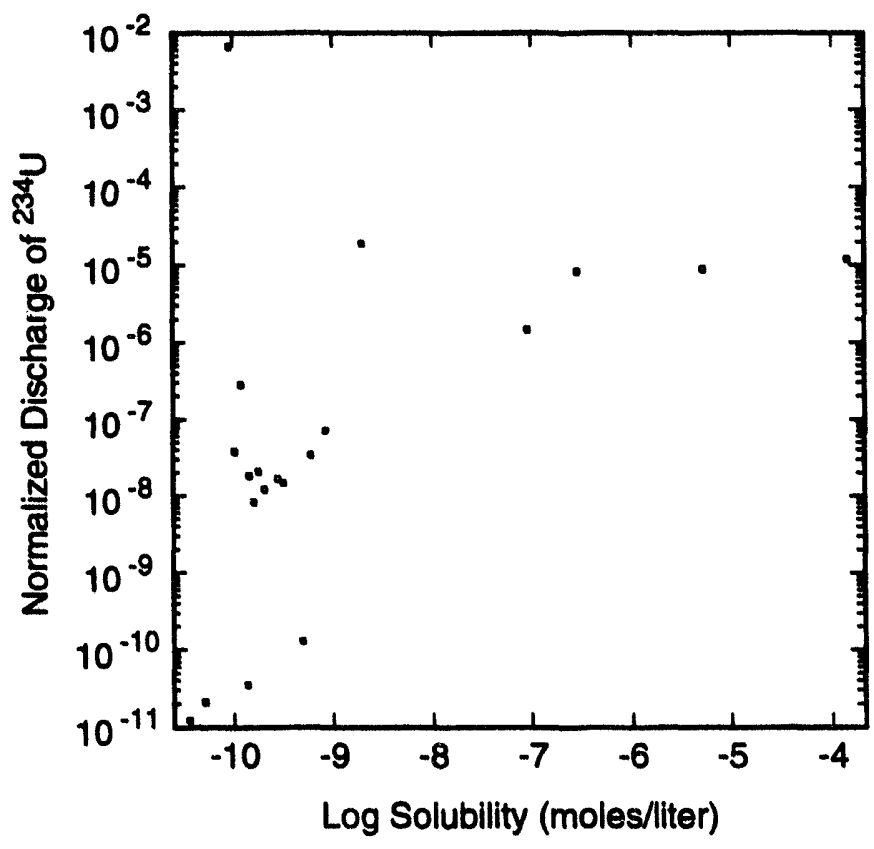

TR1-6342-4030-0

(b)

Figure 16.3-12. Scatterplot of (a) summed normalized discharge at horizontal fracture zones vs. stainless steel corrosion rate and (b) summed normalized discharge of ${ }^{234} U$ vs. its solubility, showing some correlation for granite disposal system, Waste Disposal Gmopl. 


\subsection{Simple PA Results for Disposal in Bedded Salt}

The simplified consequence model enabled us to assess the performance of individual waste parcels (WPs) (see Chapter 7 for a discussion of the Simple and Complex PAs. $)^{\dagger+\dagger}$ The samie set of sampled parameter values as in the waste disposal groups for the Complex PA were used in simulating each waste parcel type. Furthermore, the physical size of the repository (100 rooms of 27,600 waste parcels) was held constant. ${ }^{\ddagger \neq \ddagger}$ However, analysis of the Simple PA required that the performance metrics be modified slightly. These changes are discussed in the subsections below.

\subsubsection{Fluid Flow through Salt Repository (L. Smith)}

Using the techniques described in Section 16.1.3, sensitivity/uncertainty analyses were performed to determine factors that affect gas generation and the movement of gas and brine away from the repository for all 20 waste parcels considered in the Simple PA for the salt disposal system. These techniques are based on Latin hypercube sampling and include examination of scatterplots and stepwise regression analysis. Specific performance measures examined included cumulative gas and brine flow out of the repository. Also examined were cumulative measures of the mass of brine consumed and the mass of gas generated during chemical reactions.

Regression analyses were performed on results at the end of the 10,000-yr simulations. The importance of independent variables can change with time but this change was not examined in this report. Table 16.4-1 identifies the independent and dependent variables used in the stepwise regression analysis.

The scatterplots and stepwise regression analysis show that three parameters have a significant effect on cumulative brine flow out of the repository: undisturbed permeability of anhydrite marker bed, corrosion rate of stainless steel $304 \mathrm{~L}$, and room/drift backfill porosity. These independent parameters were deemed important to cumulative brine discharge in 18 of the 20 waste parcels considered, although the order of their importance varied among the different waste parcels. For the salt disposal system, cumulative brine discharge was negative in most outcomes for the waste parcels considered, indicating net inflow of brine into the repository occurred (as in the Complex PA) rather than net outflow. However, some simulations with individual waste parcels showed small net outflows from the repository. Figure 16.4-1 shows a representative scatterplot of cumulative mass of brine versus the undisturbed permeability of the anhydrite marker bed; the plot is for WP 6 (loose calcine in stainless steel container). A significant trend toward greater cumulative brine inflow into the repository with higher permeability values is apparent. For all 20 waste parcels, the value of the cumulative mass of gas discharged from the repository was zero for all sample sets, and no regression analyses were carried out for this dependent variable.

The remaining two performance measures, the cumulative mass of gas generated from chemical reactions, and the cumulative mass of brine consumed during chemical reactions, behaved virtually the same in the rank regression analysis because they are functionally related. Thus, only the cumulative mass of gas generated is discussed. Three of the seven independent variables selected for regression showed contributions to cumulative gas generation, but the corrosion rate of type $304 \mathrm{~L}$ stainless steel dominated the regression analyses, appearing as a significant variable for all 20 waste parcels. Scatterplots for the corrosion rate of type $304 \mathrm{~L}$ stainless steel showed a strong relationship to cumulative gas generation, with a plateau formed after the corrosion rate exceeded a certain value, likely indicating the minimum corrosion rate required to consume all of the stainless steel within the 10,000 -yr regulatory period under analysis. Figure 16.4-2 shows a scatterplot of the corrosion rate of type 304L stainless steel for WP 6 as an example of this behavior. Table 16.4-1 tabulates the number of waste parcels (out of a total of 20) that showed significant correlation between the independent flow parameter and dependent flow results.

${ }^{+++}$Examining the performance of the waste parcels individually allowed us to more easily gather information about the performance of the waste forms themselves and, therefore, ways in which they could be improved. Readers who are interested in specific aspects of the spent fuel and high-level waste should find this discussion of interest. However, because results indicated that all waste forms were acceptable in terms of compliance, the information presented here is nut emphasized in the last section of this chapter.

\$\$ A fixed repository size was used for modeling convenience for the Complex PA, because five disposal groups had to be un in a short time to complete the report and changes in repository size would have required new numerical meshes.

December 1993 


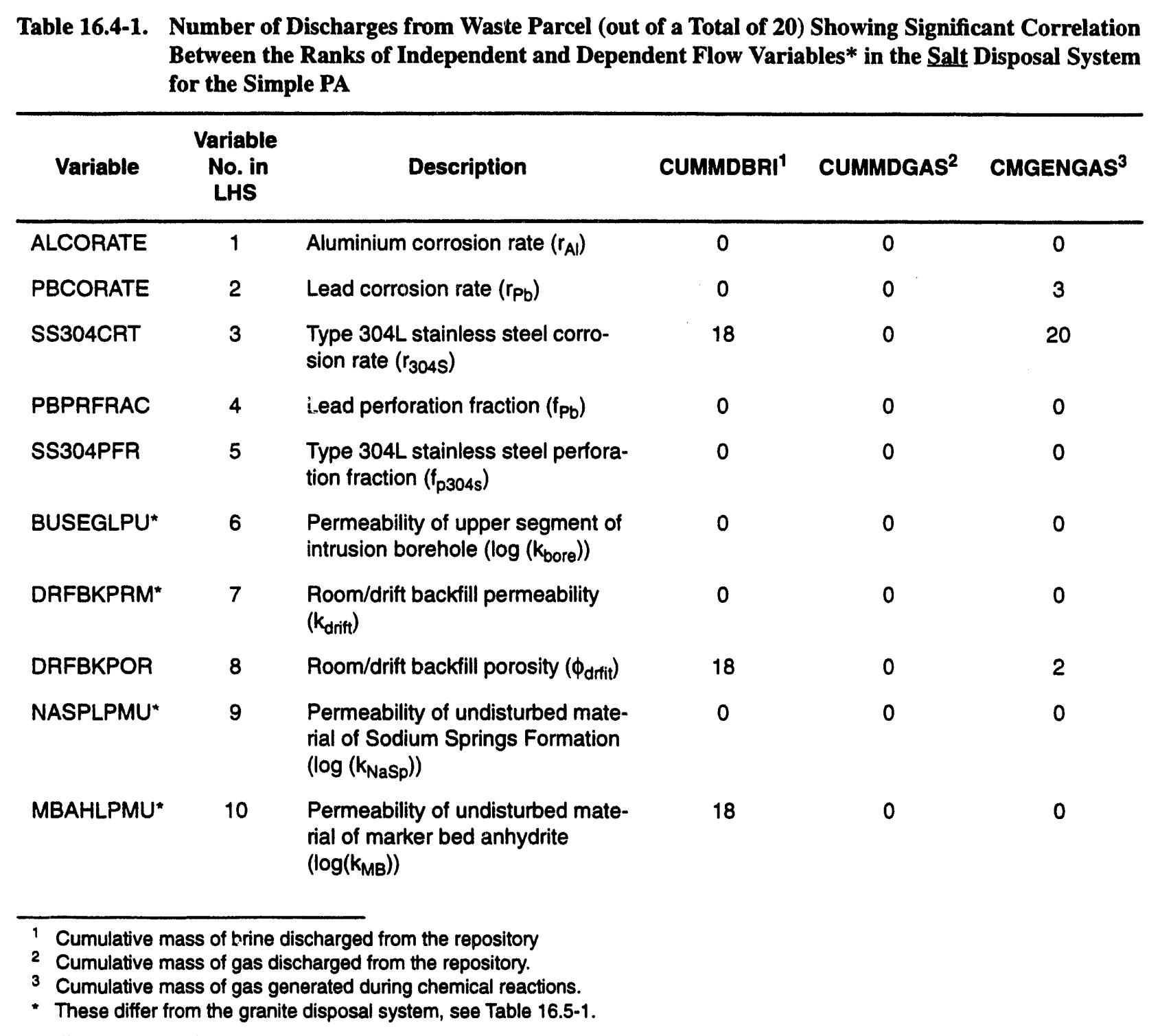

\subsubsection{Percentage of Radionuclides Leaving the Waste Parcel (W. E. Beyeler and C. T. Stockman)}

Discharges from the waste parcel and repository (not the disposal system boundary) were calculated separately for the 20 waste parcel designs for the salt disposal system. Waste parcel discharge was calculated as the total amount of each radionuclide dissolved into the pore fluid of the backfill-buffer around the waste parcel, similar to the Complex PA. (In contrast, repository discharge, discussed in the next section, consists of all radionuclide flux out of the repository control volume.)

As described earlier, we need to evaluate how well a given waste parcel design contains its material. Consequently, radionuclide discharges in the Complex and Simple PAs for the respective waste disposal groups and individual waste parcels are presented in terms of the fraction (or percentage) of the initial waste parcel inventory, calculated as the sum over all radionuclides of the initial (emplacement) activity in the waste parcel, normaiized by the EPA release limit for that radionuclide. However, a slight distinction exists. For the Complex PA, the fraction is of the entire inventory, while the fraction for the Simple PA is for one waste parcel. The numerical difference is 27,600 . Because the contents of more than one waste parcel is sometimes released, the fraction can be greater than 1. 


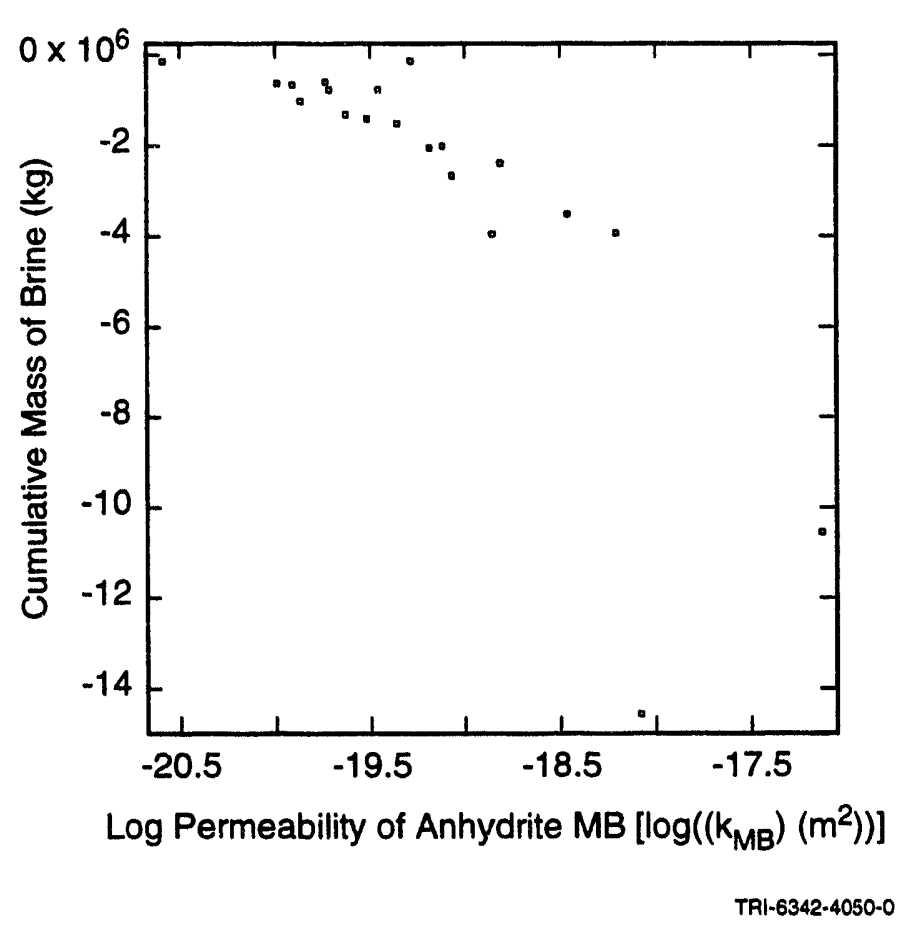

Figure 16.4-1. Representative scatterplot of cumulative mass of brine discharged from repository vs. logarithm of undisturbed permeability of the anhydrite marker bed for Waste Parcel 6 in salt disposal system. (Loose calcine in stainless steel container.)

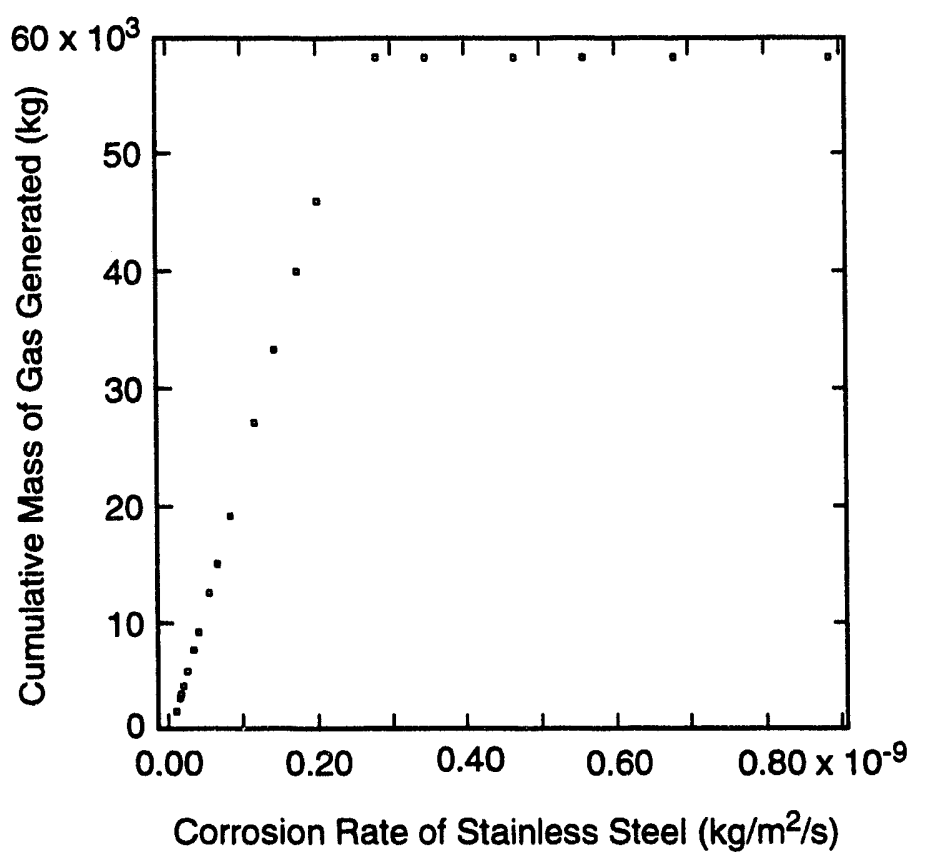

Figure 16.4-2. Scatterplot of cumulative mass of gas generated vs. the corrosion rate of type 304L stainless steel for Waste Parcel 6 in salt disposal system. (Loose calcine in stainless steel container.) 
We chose to maintain this distinction because, as discussed in Section 16.7, the conceptual models are different enough that direct comparisons were inappropriate.

Figures 16.4-3 through 16.4-7 show the CCDFs of discharge into the pore fluid of the backfill-buffer from each waste parcel in the salt disposal system, normalized as described above. The CCDFs are grouped to assist comparison of waste parceis containing similar material: high-level waste from three DOE facilities (WPs 7, 8, 9 in the first group); graphite (WPs 1, 10,14,22, and 24) in the second; highly enriched uranium [HEU] (WPs 4, 12, 16, 23, and 25 ) in the third; low enriched uranium [LEU] (WPs 5, 13, 17, 23, 25) in the fourth, and calcine (WPs 6, 11, 18, and 26) in the fifth.

For some waste parcel groups, a clear distinction can be made between discharges from different types of waste parcels: activity discharges from WP 14 are consistently larger than discharges from WP 10, while WP 10 discharges exceed WP 22 discharges (Figure 16.4-4). In other cases, the relative performance of waste parcel designs is harder to discern. For activity discharge of LEU from waste parcels, for example, WP 25 shows the largest maximum discharge (Figure 16.4-6), but also shows the lowest discharge for probabilities above 70\%. In general, waste parcel ranking changes with probability level. It is not clear from Figure 16.4-6, for instance, whether WP 17 is preferable to WP 5 .

As another way of assessing waste parcel performance (similar to the Complex PA results reported in Sections 16.2 and 16.3 for the waste disposal groups), rankings of waste parcels (within the groupings described above) were assigned for each of the 20 parameter sample sets. For example, for each sample set, the waste parcel with the smallest discharge was assigned a rank of 1 and the waste parcel with the largest discharge, a rank of 5. Figures 16.4-8 through 16.4-11 show the distributions of the ranks for waste parcel discharge in the salt repository.

For the graphite spent fuel (Figures 16.4-4 and 16.4-8), activity discharge (expressed as waste parcel equivalents) from a repository filled solely with WP 22 is usually smaller than activity discharge from the other waste parcels: the activity discharge from WP 22 in a lower bound at any level of probability in Figure 16.4-4 and is the minimum activity discharge of the five graphite waste parcels in 15 of the 20 sample sets (Figure 16.4-8). It might be expected from

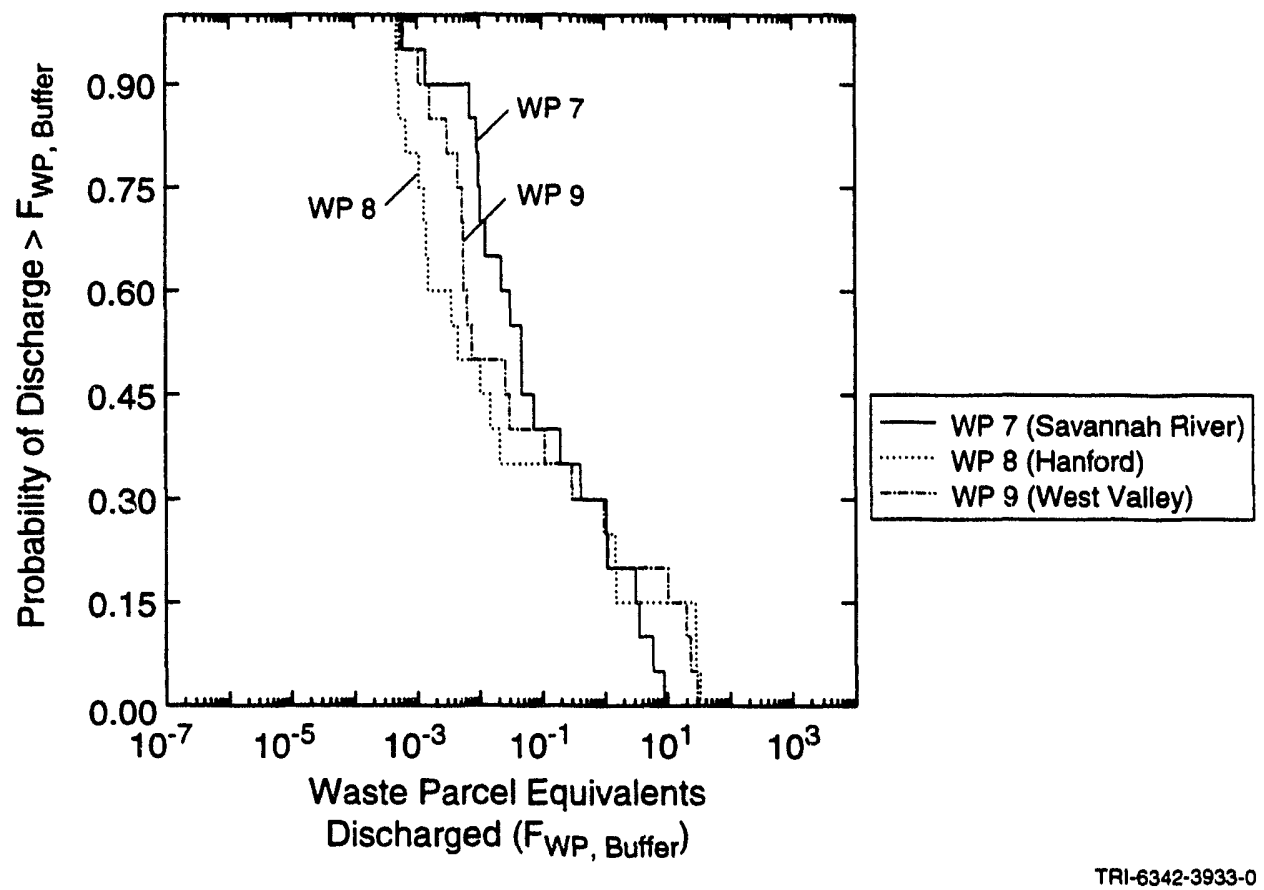

Figure 16.4-3. CCDF, conditional on two intrusions, of number of waste parcel inventory equivalents discharged into backfill-buffer for high-level waste in borosilicate glass from DOE sites (WPs 7, 8, and 9), salt disposal system. 


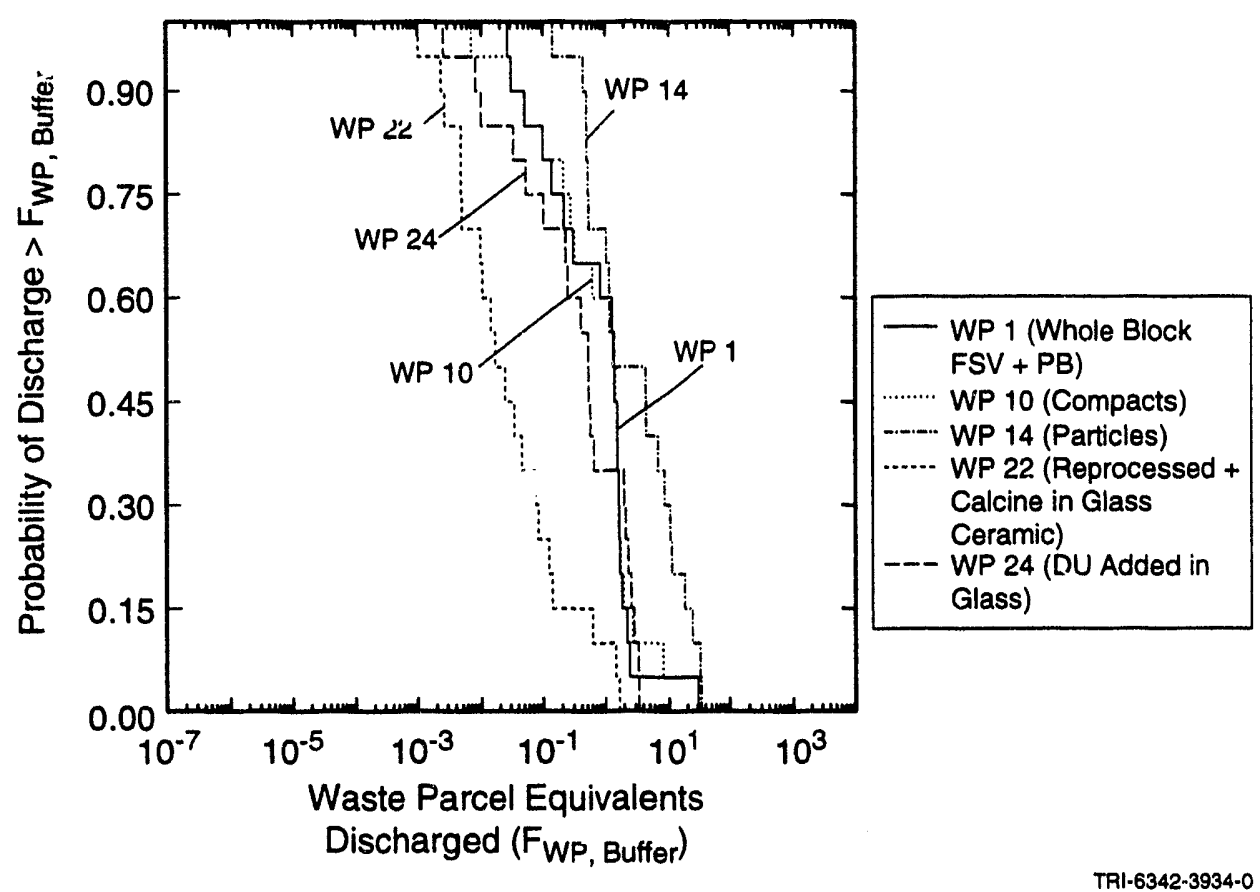

Figure 16.4-4. $\quad C C D F$, conditional on two intrusions, of number of waste parcel inventory equivalents discharged into backfill-buffer for graphite spent fuel (WPs 1, 10,14, 22, and 24), salt disposal system.

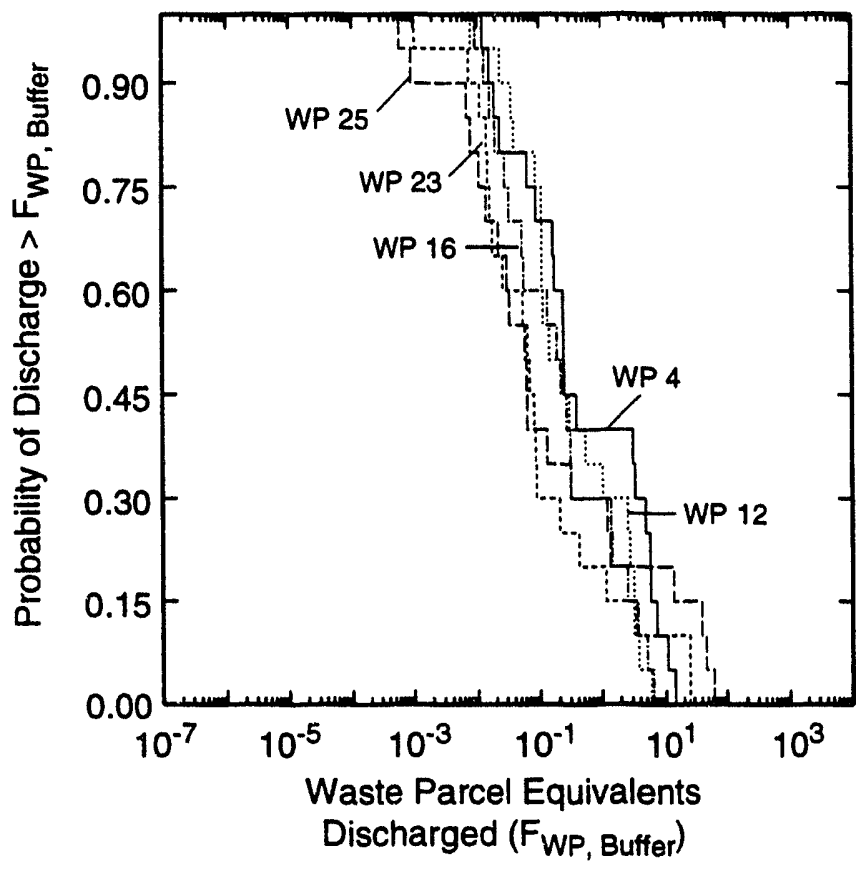
WP 4 (1 HEU) WP 12 ( 1 HEU + Ceramic)
W..... WP 16 (HEU + Glass)
..... WP 23 (Reprocessed HEU + LEU + Calcine in Glass Ceramic)
--- WP 25 (HEU + LEU + DU in Glass)

Figure 16.4-5. CCDF, conditional on two intrusions, of number of waste parcel inventory equivalents discharged into backfill-buffer for highly enriched uranium spent fuel (WPs 4,12,16,23, and 25), salt disposal system. 
16. Results and Conclusions

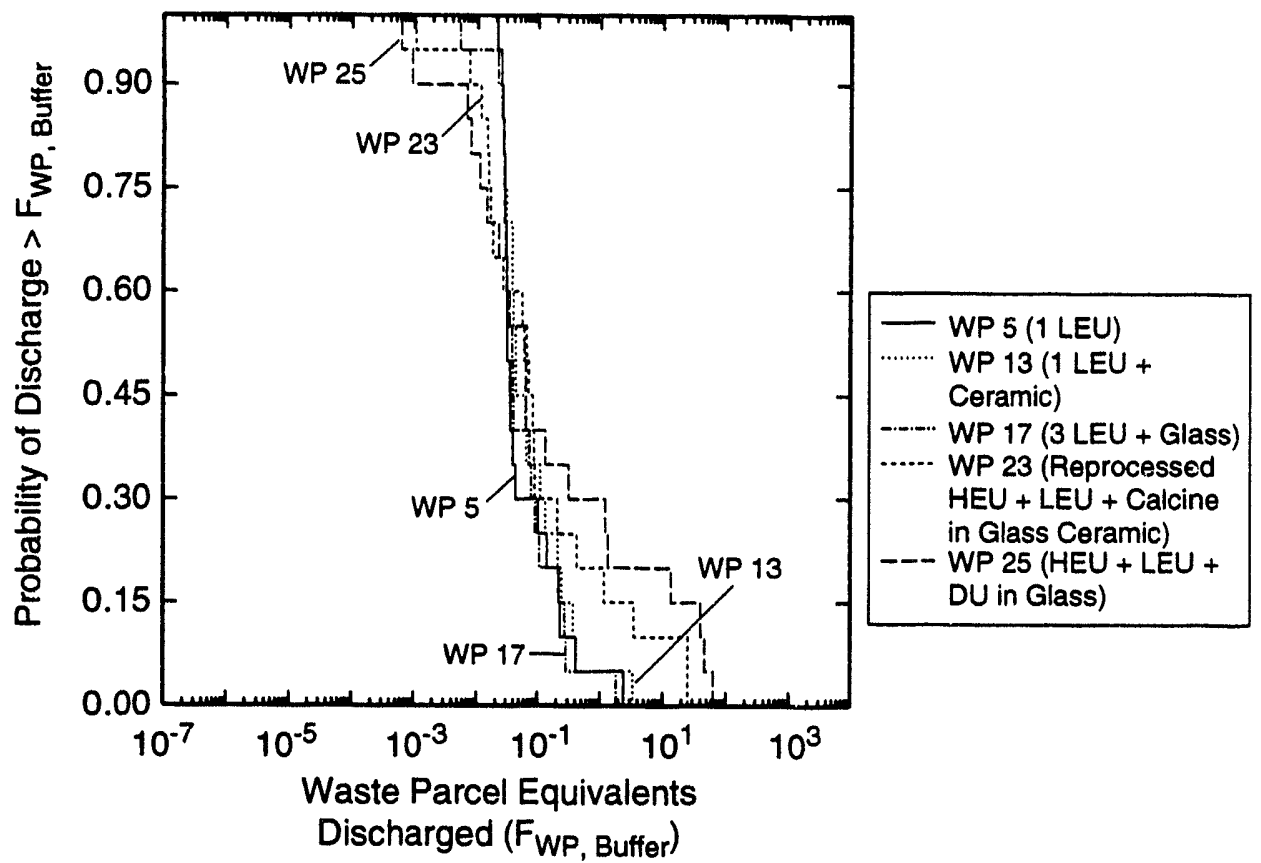

TRI-6342-3936-0

Figure 16.4-6. CCDF, conditional on two intrusions, of number of waste parcel inventory equivalents discharged into backfill-buffer for low enriched uranium spent fuel (WPs 5, 13,17,23, and 25), salt disposal system.

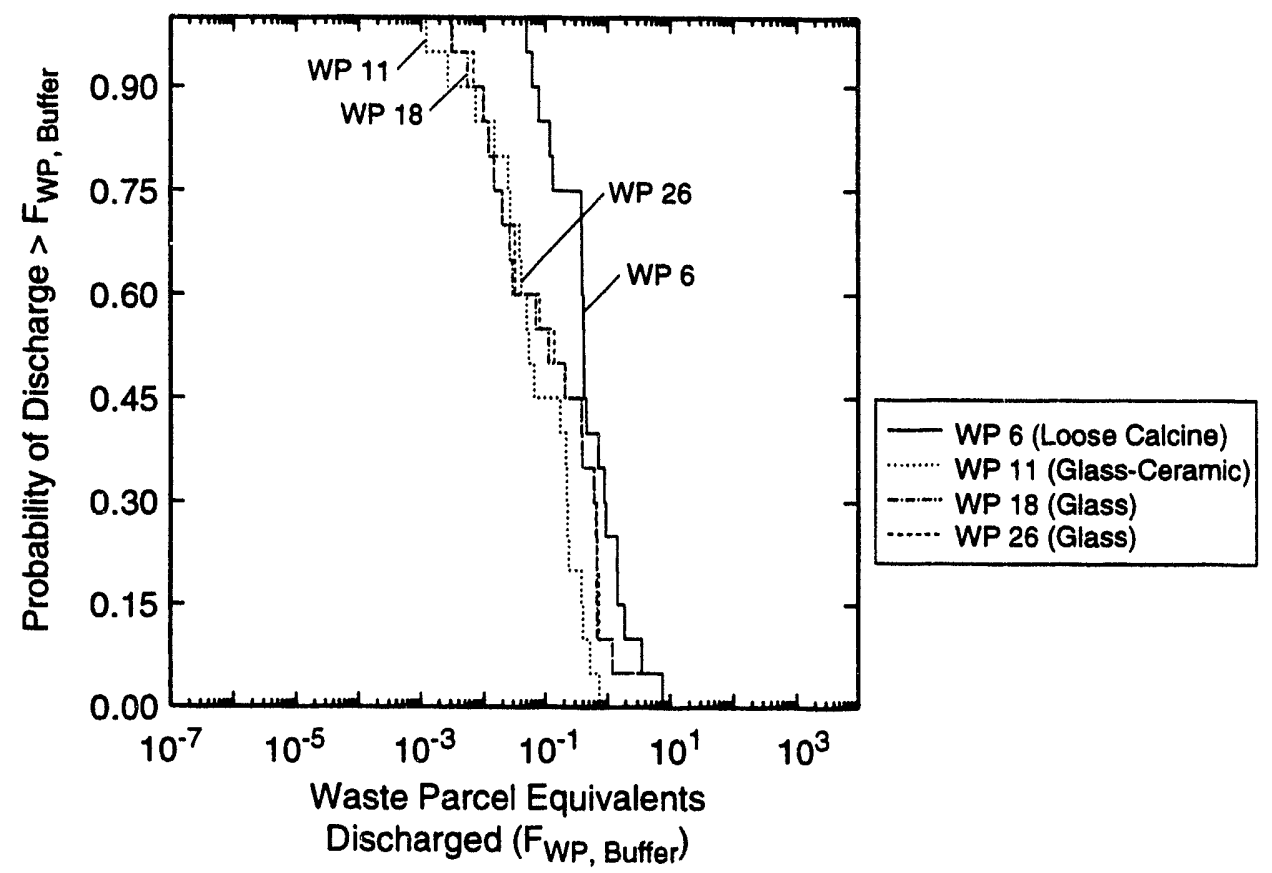

Figure 16.4-7. CCDF, conditional on two intrusions, fraction of initial waste parcel inventory by one waste parcel into backfill-buffer for calcine (WPs 6,11,18, and 26), salt disposal system. 


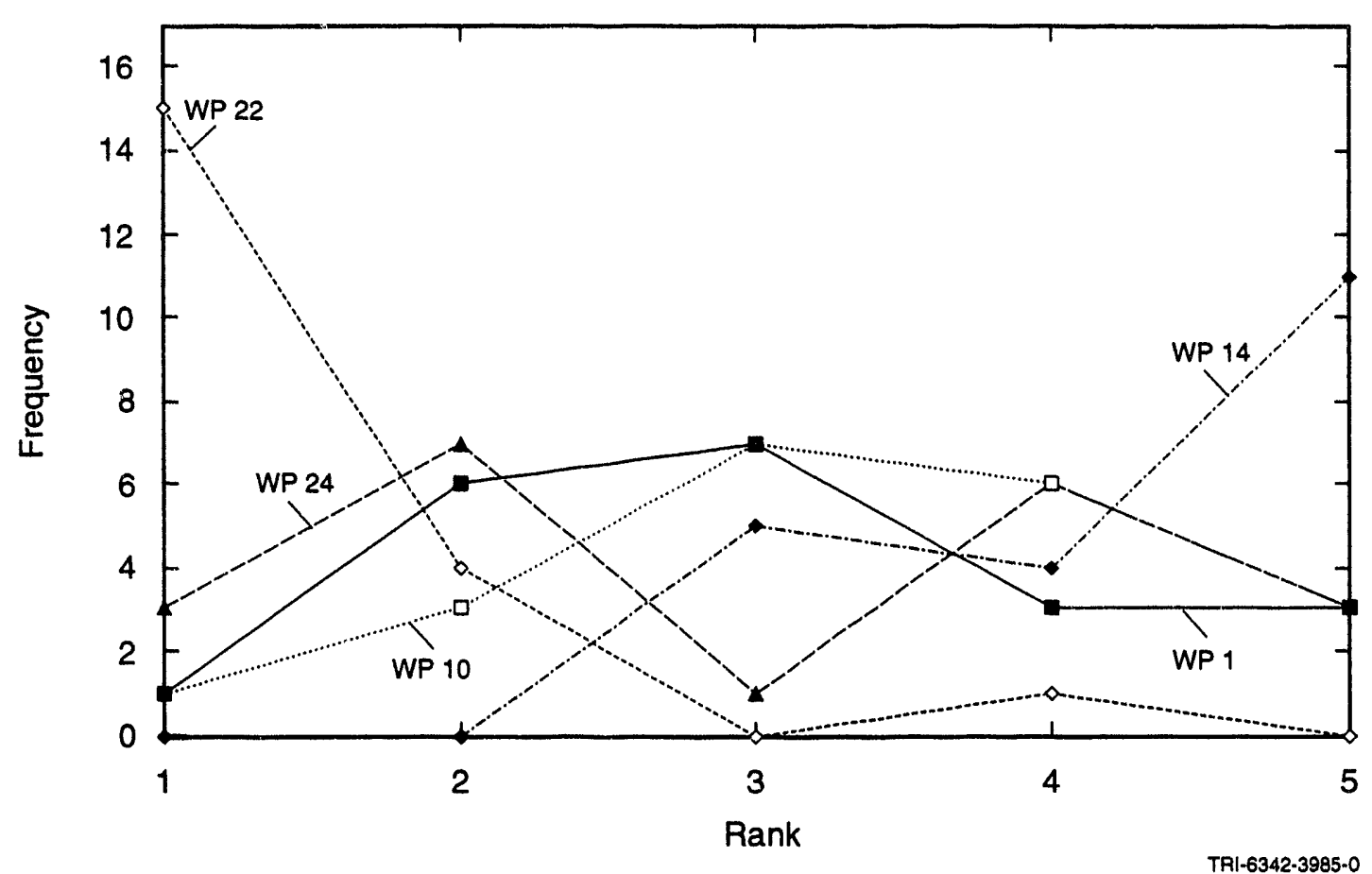

Figure 16.4-8. Frequency with which waste parcel fractional release for graphite spent fuel (WPs 1, 10, 14, 22, and 24) in salt disposal system was ranked with the least (rank 1) or most (rank 5) discharge.

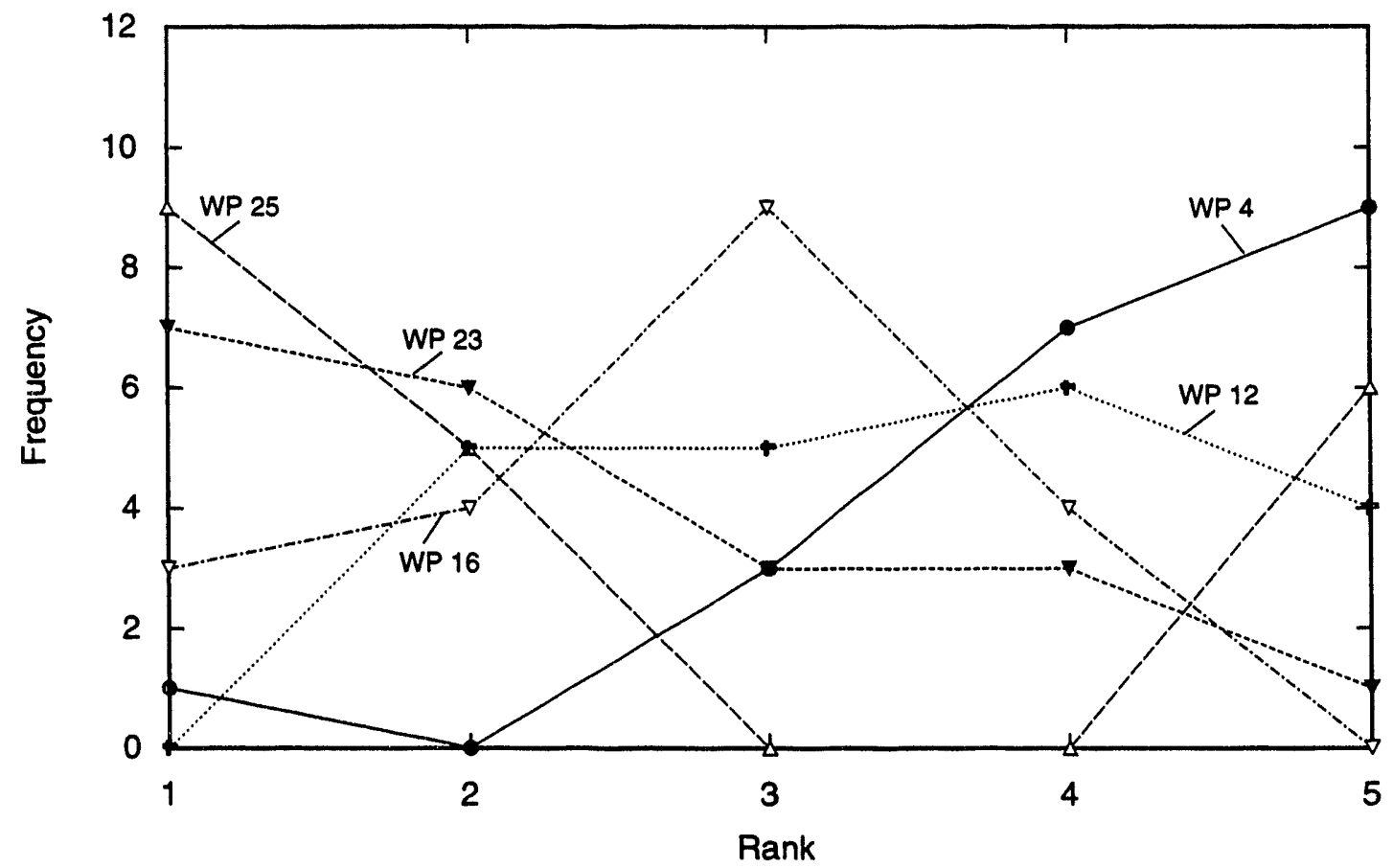

TRI-6342-3986-0

Figure 16.4-9. Frequency with which waste parcel fractional release for highly enriched uranium spent fuel (WPs $4,12,16,23$, and 25) in salt disposal system was ranked with the least (rank 1) or most (rank 5) discharge. Ranking of peaks is in the same order as the loading of radionuclides in the waste parcel. 


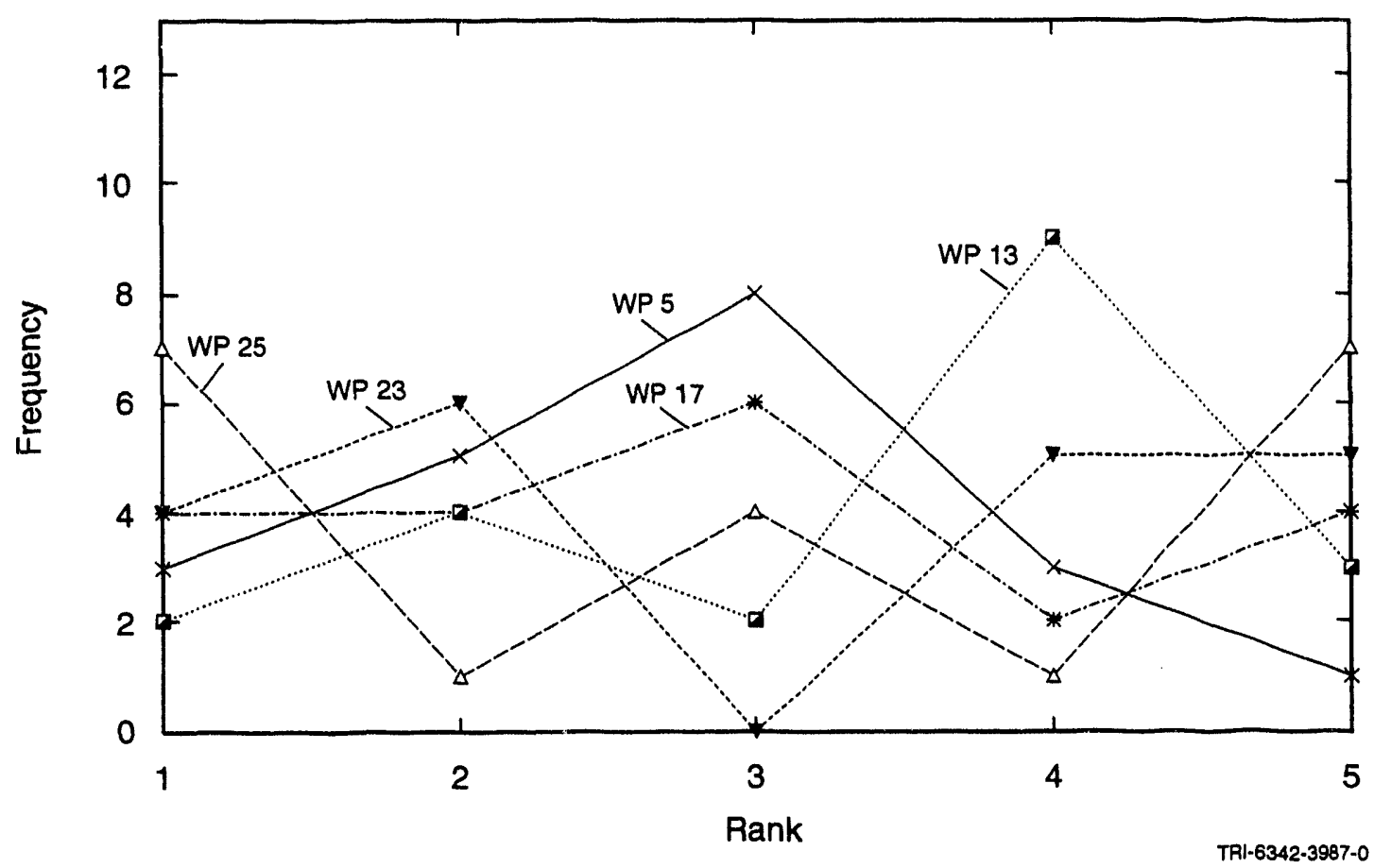

Figure 16.4-10. Frequency with which waste parcel fractional release for low enriched uranium spent fuel (WPs 5, $13,17,23$, and 25) in salt disposal system was ranked with the least (rank 1) or most (rank 5) discharge.

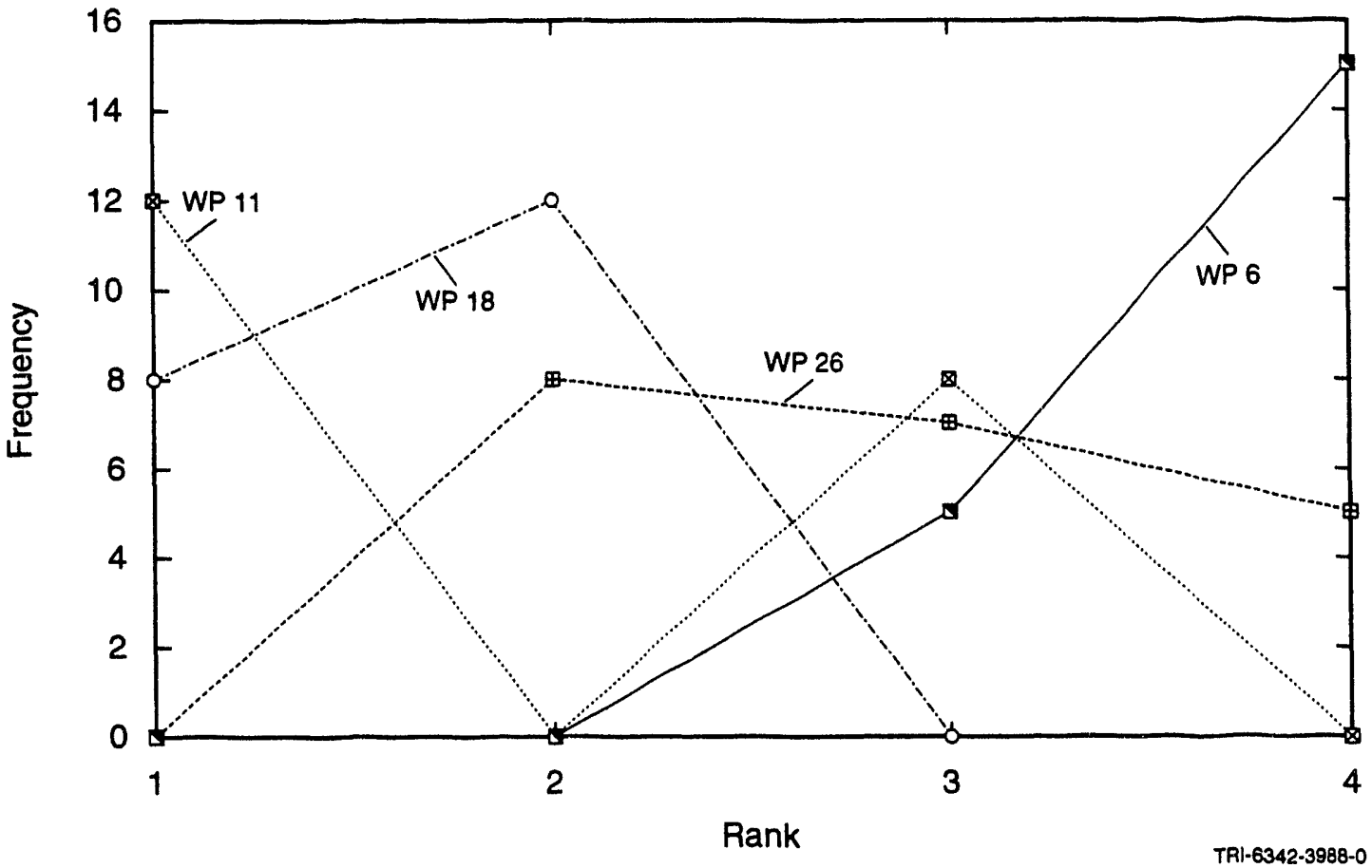

Figure 16.4-11. Frequency with which waste parcel fractional release for calcine (WPs 6,11,18, and 26) in salt disposal system was ranked with the least (rank 1) or most (rank 5) discharge. 
Figure 16.4-4 that WP 22 provides the minimum activity discharge for all 20 sample sets. This is not the case because, for a given exceedance probability (ordinate) on Figure 16.4-4, the sample sets giving the activity discharge (abscissa) at that probability may be different for each waste parcel.

Activity discharge from WP 14 is usually larger than activity discharge from other waste parcels, tending to lie to the right of other waste parcel activity discharge distributions in Figure 16.4-4 and providing the maximum activity discharge in 11 of the 20 sample sets (Figure 16.4-8). Based on the highest frequency of rank in Figure 16.4-8, the graphite spent fuel waste parcel activity discharge ranks (from lowest to highest) are WP 22, WP 24, WP 1, WP 10, and WP 14. Differences among the middle three waste parcels are not easy to see either in terms of activity discharge or rank, and are probably not meaningful. The order of the activity discharges from WPs 1,10 , and 14 are consistent with the fraction of graphite fuel with failed $\mathrm{SiC}$ coatings $\left(f_{\mathrm{wp}}\right.$, FAvail) for these WPs: $0.04,0.04$, and 0.07 , respectively. The fuel particles in WPs 22 and 24 will be broken open and all ${ }^{14} \mathrm{C}$ burned off prior to processing. In addition, WP 22 has uranium and plutonium removed and WP 22 will be immobilized in glass-ceramic, which degrades in a median time of $43,000 \mathrm{yr}$, while WP 24 has depleted uranium added and will be immobilized in glass which will take a median of $10,000 \mathrm{yr}$ to alter. From the observed order in the activity discharges from the waste parcels that contain graphite fuel in salt, it is clear that only extensive processing of the fuel will help reduce the activity discharges from that predicted for the intact fuel blocks.

Differences in activity discharge among waste parcels containing highly enriched uranium (HEU) spent fuel are not clear (Figures 16.4-5 and 16.4-9). Using the highest frequency of ranks in Figure 16.4-9, the waste parcel activity discharges from lowest to highest are WP 25, WP 23, WP 16, WP 12, and WP 4, but the rank distributions tend to be flat. Unlike the graphite spent fuel waste parcels, no HEU waste parcel occupies a given rank in a majority of the 20 sample sets. WP 25, with the largest number of minimum ranks, also provides the maximum activity discharge in 6 of the 20 sets. Figure 16.4-5 suggests that activity discharge from WP 25 tends to exceed activity discharge from alternative waste parcels when the magnitude of the activity discharge is large. WP 25 activity discharge shows a larger range (approximately 5 orders of magnitude) than activity discharge from the minimally treated WP 4 (approximately 3 orders of magnitude). The observed normalized activity discharges from the waste parcels are inversely correlated with the radionuclide content per waste parcel. This is consistent with the hypothesis that solubility limits are controlling the activity discharges so that adding more radionuclides per waste parcel does not increase the absolute activity discharge per waste parcel and decreases the fractional activity discharge per waste parcel. WP 23 not only contains a higher radionuclide content per waste parcel (reprocessed HEU and LEU and $99 \%$ of the calcine) but the radionuclides are immobilized in glass ceramic, which has a median alteration time of $42,846 \mathrm{yr}$. WP 25 contains no calcine but processed HEU, LEU, and depleted uranium immobilized in glass, which has a median alteration time of $10,694 \mathrm{yr}$. Because the uranium aluminum alloy fuel $\left(\mathrm{UAl}_{3}, \mathrm{Al}\right)$ is expected to react with groundwater in less than a year, the HEU inventory of WPs 4,12 and 16 will be available quickly while the inventory of WPs 23 and 25 is made available slowly as the matrix alters. The larger range of activity discharges from WP 25 indicates that the parameters that most greatly affect the activity discharge must have larger ranges for WP 25 than the other waste parcels. The matrix alteration rate may be one of these parameters: the glass corrosion rate has a larger range than that of glass-ceramic, and the rate of alteration of uranium-aluminum alloy fuel corrosion was not sampled.

As with the highly enriched uranium fuel, activity discharge from waste parcels containing low enriched fuel (Figures 16.4-6 and 16.4-10) is characterized by a higher variability of activity discharge for the extensively treated WP 25: activity discharge from the minimal treatment, WP 5, ranges over 2 orders of magnitude in contrast to the 5 order of magnitude variation in WP 25. The distribution of ranks of waste parcel activity discharge (Figurc 16.4-10) is ambiguous: no waste parcel occupies a given rank in a majority of sample sets; the WP 25 rank distribution is multimodal, with peaks at the minimum and maximum ranks reflecting the higher variability of WP 25 activity discharge seen in Figure 16.4-6. Once again the difference in the range in activity discharges may be explained by the matrix alteration rates of the waste parcels. The inventories of highly enriched uranium in WPs 23 and 25 are contained in glass-ceramic and glass, but the inventories of highly enriched uranium in WPs 5, 13, and 17 are contained in the $\mathrm{UO}_{2}$ grains, grain boundaries, and gap between the $\mathrm{UO}_{2}$ grains and zircalloy cladding of intact fuel rods. The alteration of glass and glass-ceramic matrices was modeled with alteration rates while the alteration of $\mathrm{UO}_{2}$ was modeled with a quick release fraction (the gap fraction plus the grain boundary fraction) and the solubility limited dissolution of $\mathrm{UO}_{2}$. Thus, the release rate of radionuclides from the matrices in WPs 23 and 25 depends only on the sampled matrix alteration rate while the release rate of radionuclides from WPs 5,13 and 17 depends on quick release 
fractions, the solubility of $\mathrm{UO}_{2}$, and the rate of diffusion of uranium out of the borehole. Because the quick release fractions and the effective diffusion coefficients were not sampled, we see less variability in the releases from the $\mathrm{UO}_{2}$ matrices.

The waste parcels containing calcine (Figures 16.4-7 and 16.4-11) show a clearer trend. Modes of the activity discharge rank distributions (Figure 16.4-11) occur in the following order from lowest to highest: WP 11, WP 18, WP 26, WP 6. The distribution for WP 26 is rather flat, while the remaining three waste parcels occur in their assigned rank for a majority of the 20 sample sets. Figure 16.4-7 is consistent with this ordering. WP 6 defines the largest activity discharge at all probability levels, while the lower limit is defined by either WP 11 or WP 18 . The difference in activity discharge between waste parcels is less than 2 orders of magnitude at all probability levels, and is typically 1 order of magnitude. The variability of activity discharge from a given waste parcel across sample sets is between 2 orders of magnitude (WP 6) and 3 orders of magnitude (WP 11). Once again these results can be explained by the difference in matrix alteration rates. The loose calcine in WP 6 is modeled to alter in less than a year and the alteration rate was not sampled in these calculations. Thus, WP 6 has the largest activity discharges but the least spread. The remaining waste parcels show a larger range in their activity discharges due to the sampling of the glass alteration rate for WPs 18 and 26 and glass-ceramic alteration rates in WP 11 . The order of the activity discharges of these remaining waste parcels is inversely correlated with their inventory per waste parcel (although the inventory per waste parcel differs by less than a factor of 2). For these waste parcels, the order is WP $11>$ WP $18>$ WP 26. The difference is consistent with solubility control causing the activity discharges expressed as numbers of equivalent waste parcels to be in the order of WP $11<$ WP $18<$ WP 26 but with very little difference between the activity discharges.

The effect of alternative waste parcel designs on discharge should be considered in light of the overall uncertainty in discharge created by uncertainty in system parameters. Some apparent differences in performance in Figures 16.4-2 through 16.4-7 may not be significant, given the small number of sample sets and the large variability of discharge from run to run.

\subsubsection{Normalized Activity Leaving Repository (W. E. Beyeler and C. T. Stockman)}

Figures 16.4-12 through 16.4-16 show normalized activity discharges from the repository boundary of the salt disposal system (rather than the radionuclide concentration in the backfill-buffer, as described in Section 16.4.2), for the 20 sampled parameter sets for the same four waste parcel groups. Figures 16.4-17 through 16.4-20 show the distribution of waste parcel ranks (by activity discharge) for the four waste parcel groupings.

For the graphite spent fuel (Figures $16.4-13$ and 16.4-17), the activity discharge at the repository boundary is qualitatively similar to the waste parcel activity discharge discussed previously: there is a 3 orders of magnitude difference between activity discharges from different waste parcels at all probability levels, and a consistent ordering of waste parcels across probability levels. The modes of the rank distributions occur in the following order: WP 22, WP 24, WP 10, WP 1, and WP 14 (Figure 16.4-7). The benefit of removing uranium and plutonium in WP 22 is still evident in the activity discharge at the repository boundary. The ordering of WP 10, WP 1 , and WP 14 is also consistent with the fraction of particles with failed $\mathrm{SiC}$ coatings $(0.04,0.04,0.07$, respectively), which controls the availability of radionuclides from the inner metal carbide matrix $\left(\mathrm{UC}_{2}\right.$ or $\left.\mathrm{ThC}_{2}\right)$.

Activity discharge at the repository boundary for waste parcels containing highly enriched uranium spent fuel (Figures 16.4-14 and 16.4-18) shows no clear ordering. No waste parcel occupies a given rank in a majority of sample sets. WP 25 has the largest number of minimum activity discharges (9), but also shows the maximum activity discharge in 4 of the 20 sets (Figure 16.4-18). In contrast to the activity discharge from the waste parcel into the backfill-buffer, these maxima do not correspond to high levels of activity discharge at the repository boundary (Figure 16.4-5). The variability is approximately 4 orders of magnitude (Figure 16.4-14).

For low enriched uranium spent fuel and for a given probability level, the difference in activity discharge at the repository boundary across waste parcels is typically 1 order of magnitude. The relative ranking of waste parcels in terms of activity discharge at the repository boundary (Figure 16.4-19) is somewhat less ambiguous than the ranking 


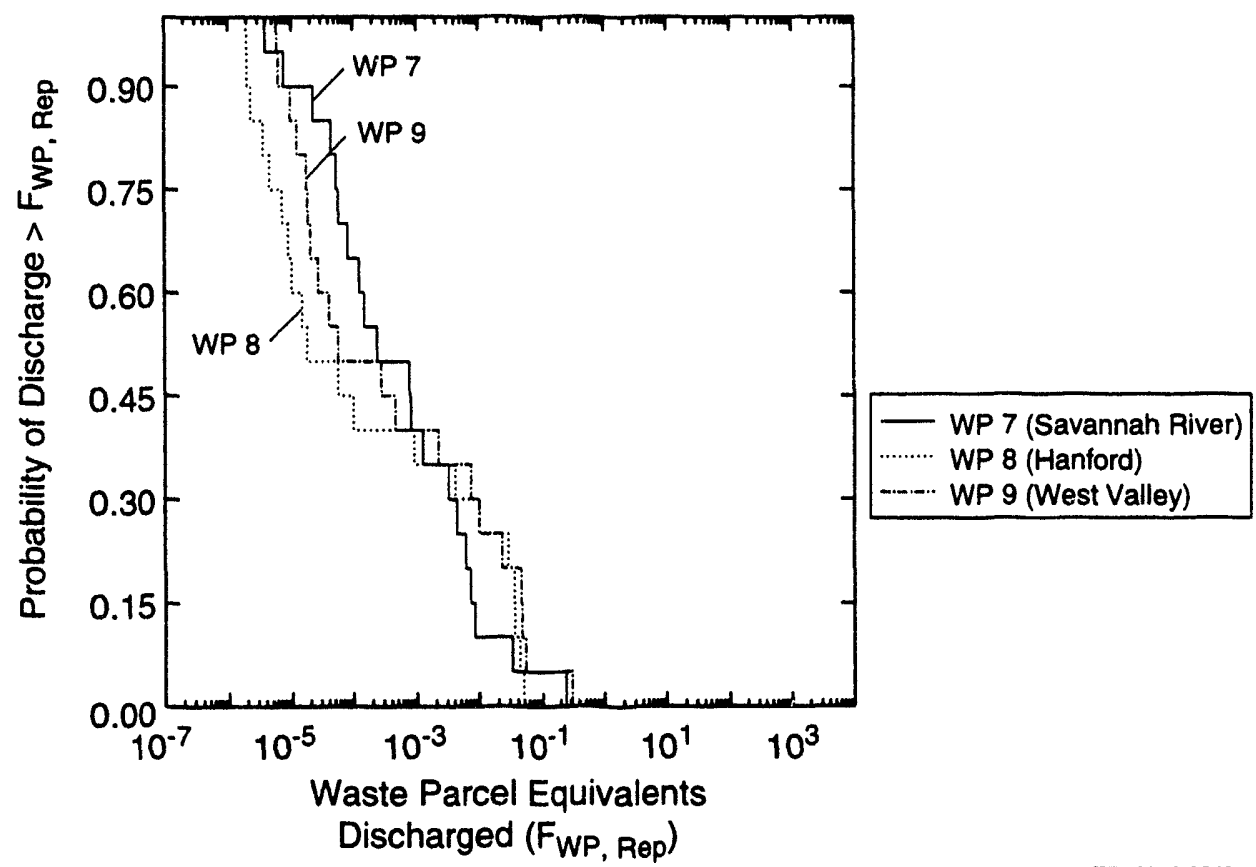

TR1.6342.3942.0

Figure 16.4-12. CCDF, conditional on two intrusions, of normalized activity discharge at repository boundary for high-level waste in borosilicate glass from DOE sites (WPs 7, 8, and 9), salt disposal system.

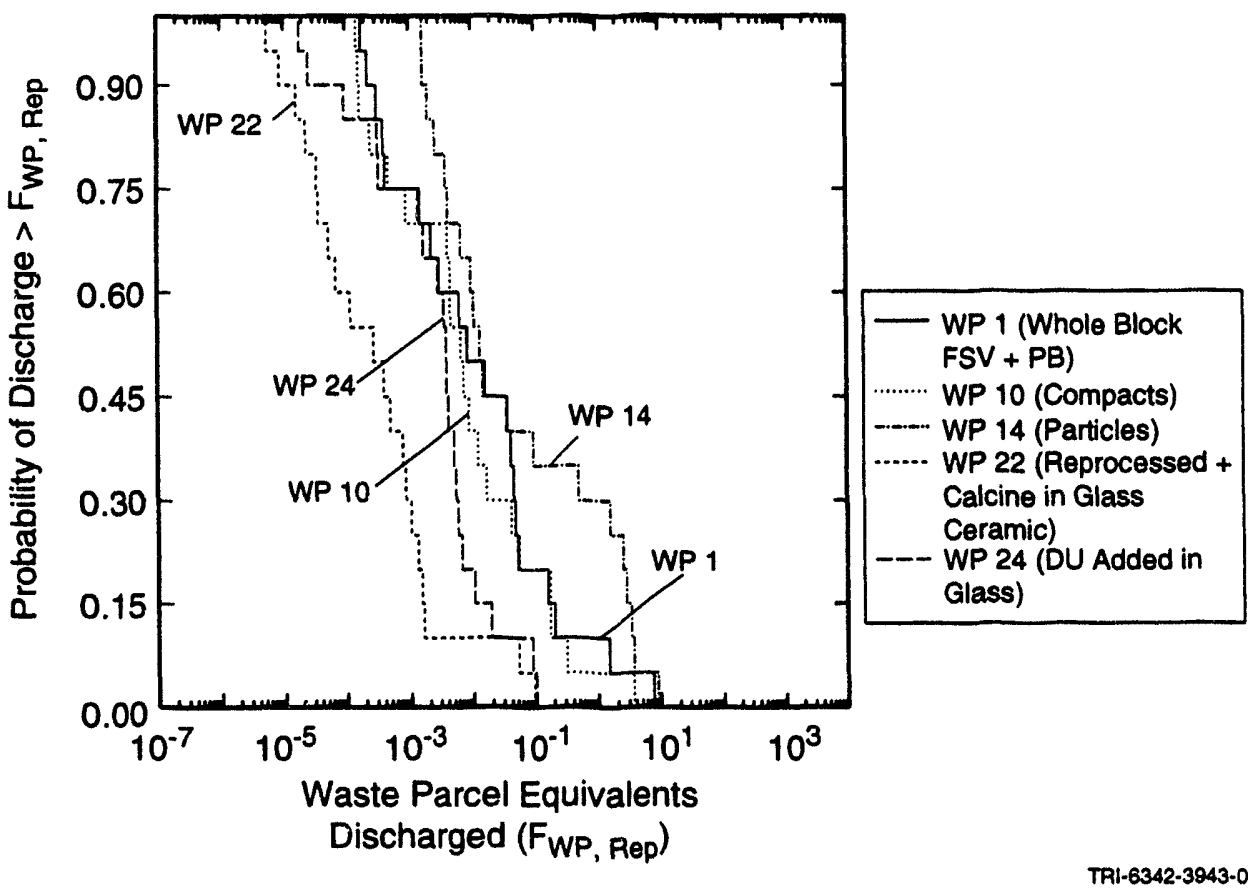

Figure 16.4-13. CCDF, conditional on two intrusions, of normalized activity discharge at repository boundary for graphite spent fuel (WPs 1, 10,14, 22, and 24), salt disposal system. 
16. Results and Conclusions

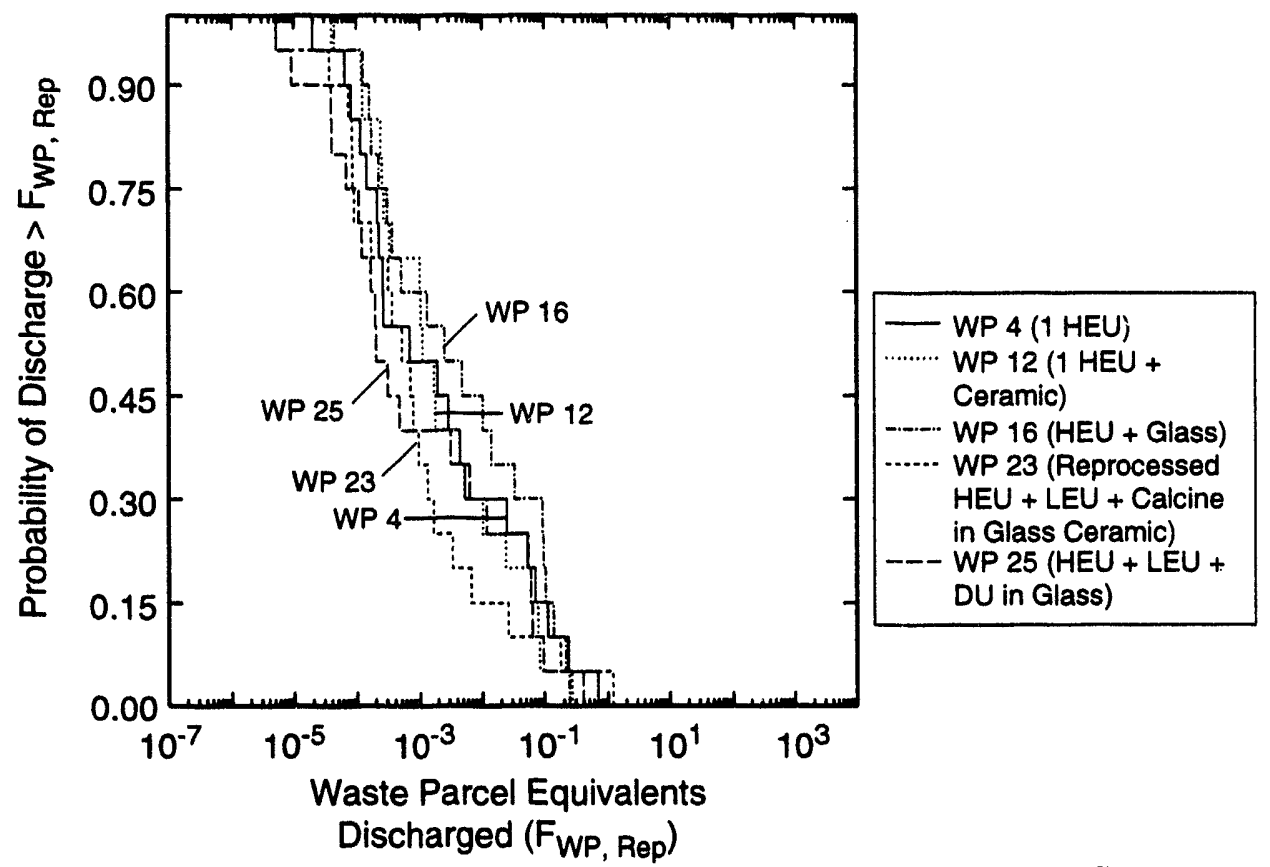

TR1-6342-3944-0

Figure 16.4-14. CCDF, conditional on two intrusions, of normalized activity discharge at repository boundary for highly enriched uranium spent fuel (WPs 4, 12,16,23, and 25), salt disposal system.
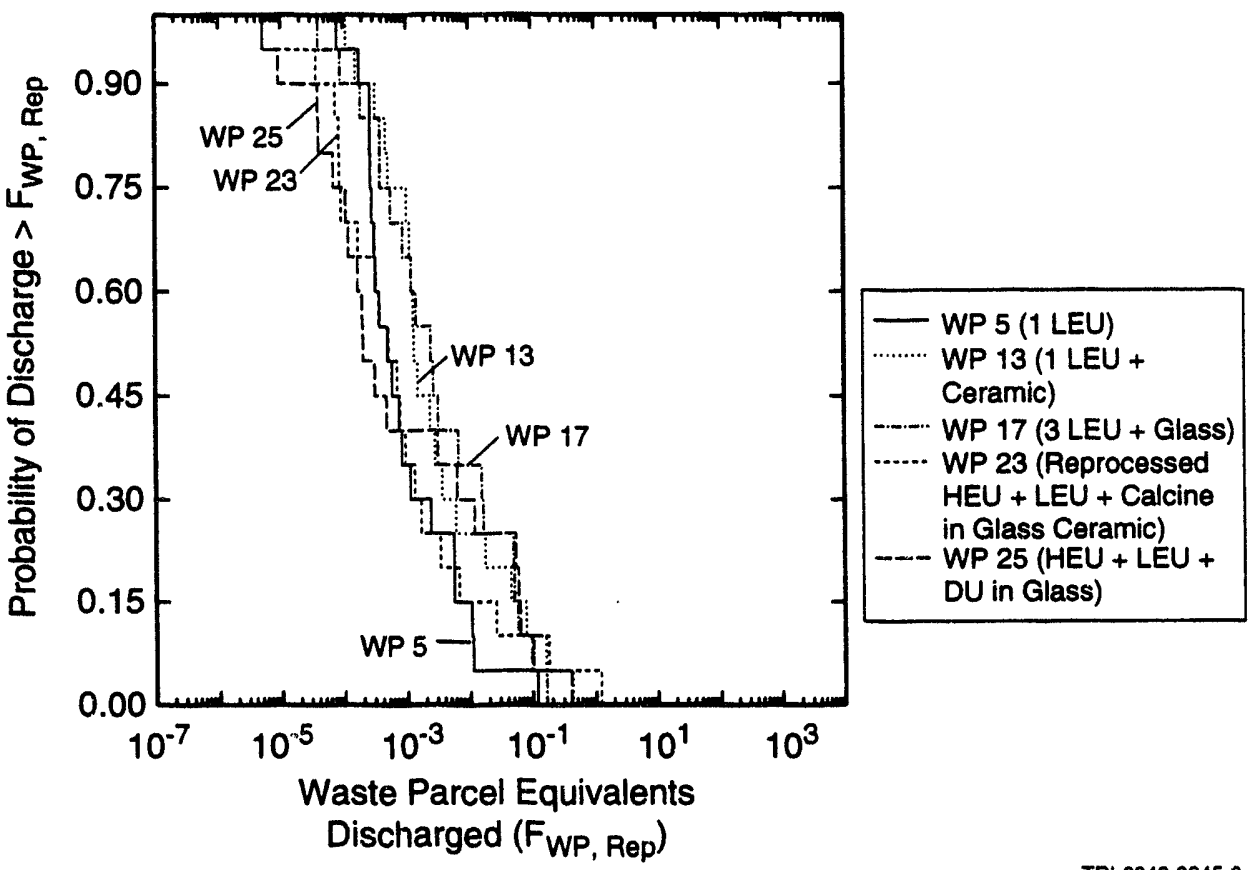

TRI-6342-3945-0

Figure 16.4-15. CCDF, conditional on two intrusions, of normalized activity discharge at repository boundary for low enriched uranium spent fuel (WPs 4, 12,16, 23, and 25), salt disposal system. 


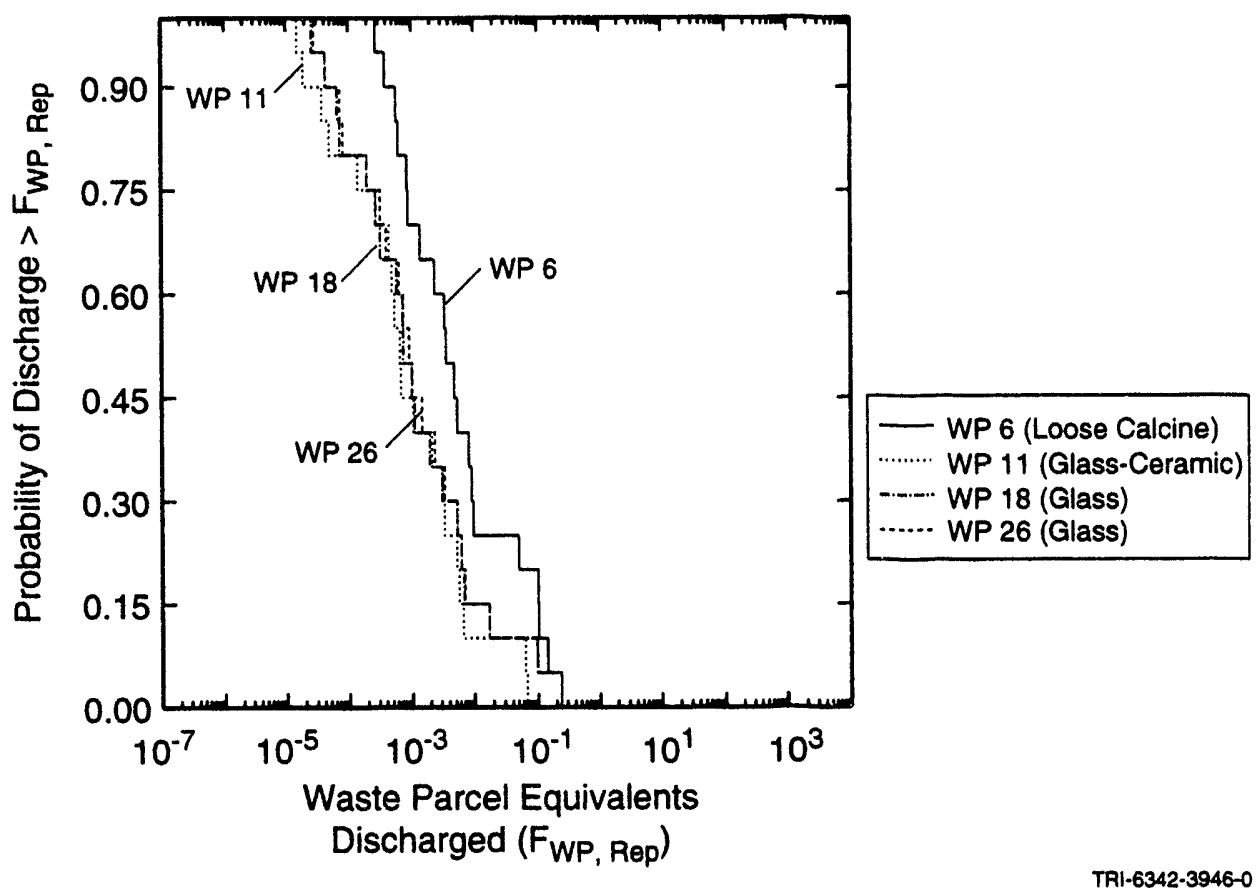

Figure 16.4-16. CCDF, conditional on two intrusions, of normalized activity discharge at repository boundary for calcine (WPs 6, 11, 18, and 26), salt disposal system.

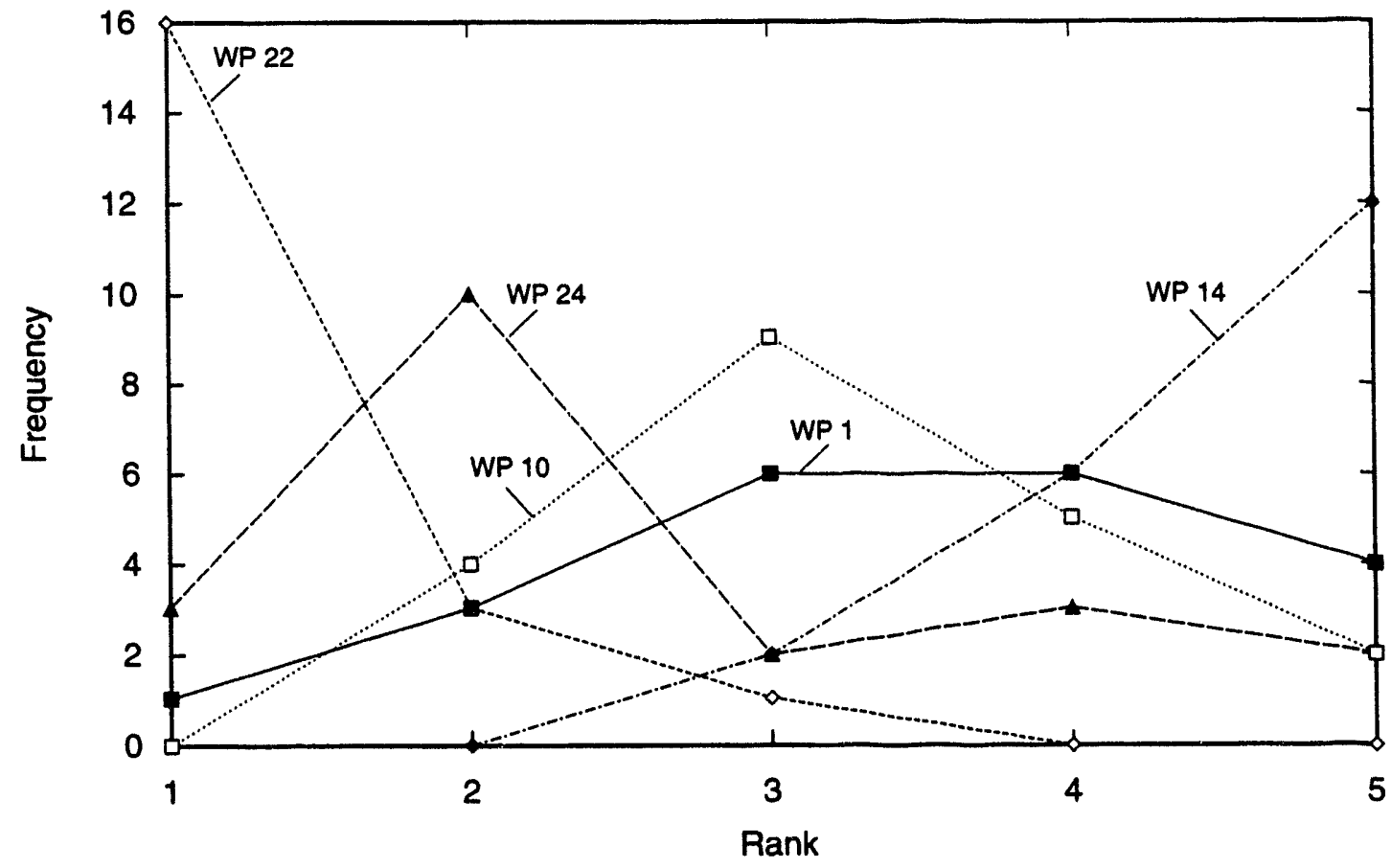

Figure 16.4-17. Frequency with which waste parcel fractional release at repository boundary for graphite spent fuel (WPs 1, 10,14, 22, and 24) in salt disposal system was ranked with the least (rank 1) or most (rank 5) discharge. 
16. Results and Conclusions

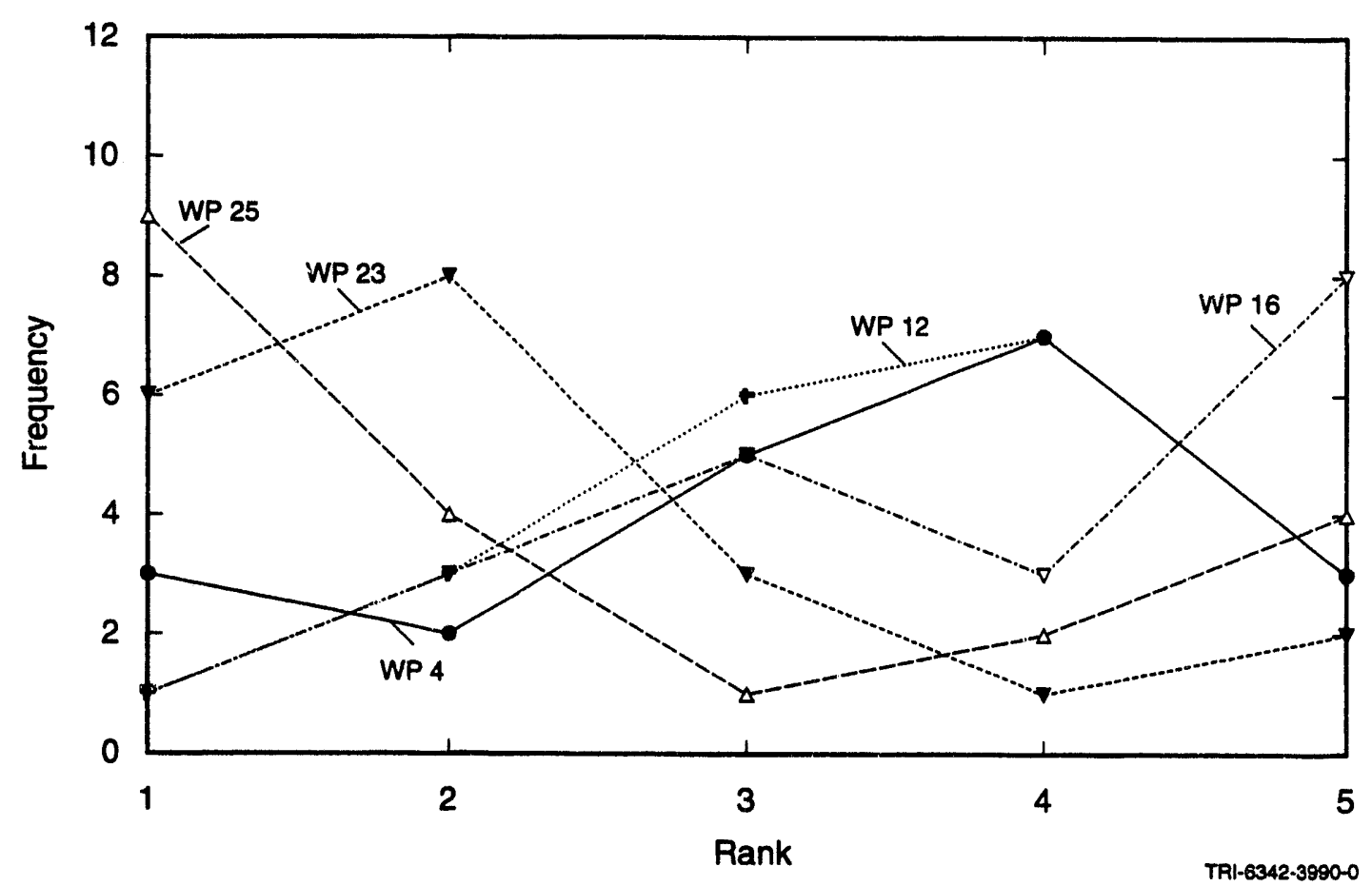

Figure 16.4-18. Frequency with which waste parcel fractional release at repository boundary for highly enriched uranium spent fuel (WPs 4,12,16,23, and 25) in salt disposal system was ranked with the least (rank 1) or most (rank 5) discharge.

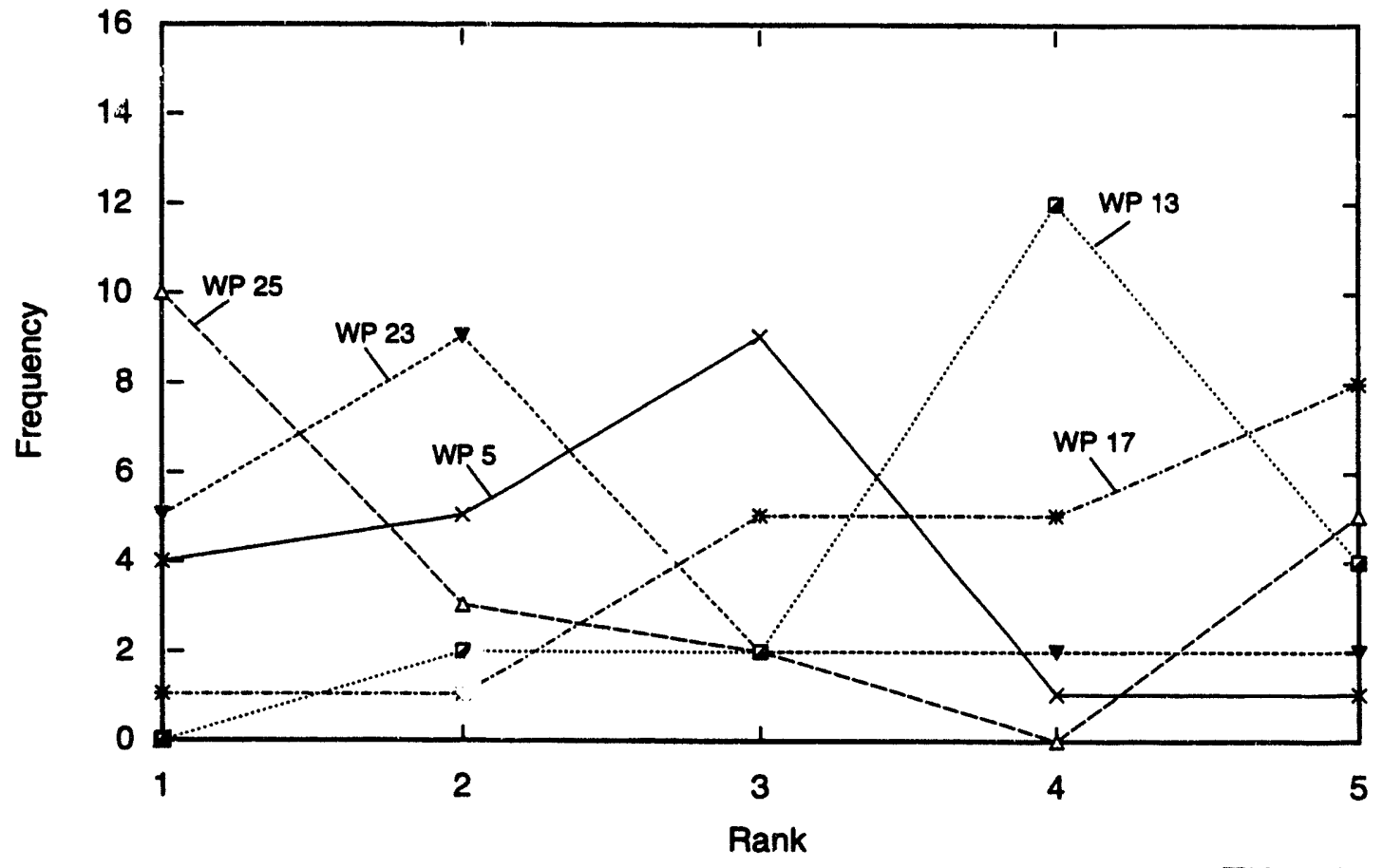

TR1-6342-3991-0

Figure 16.4-19. Frequency with which waste parcel fractional release at repository boundary for low enriched uranium spent fuel (WPs 5,13,17,23, and 25) in salt disposal system was ranked with the least (rank I) or most (rank 5) discharge. 


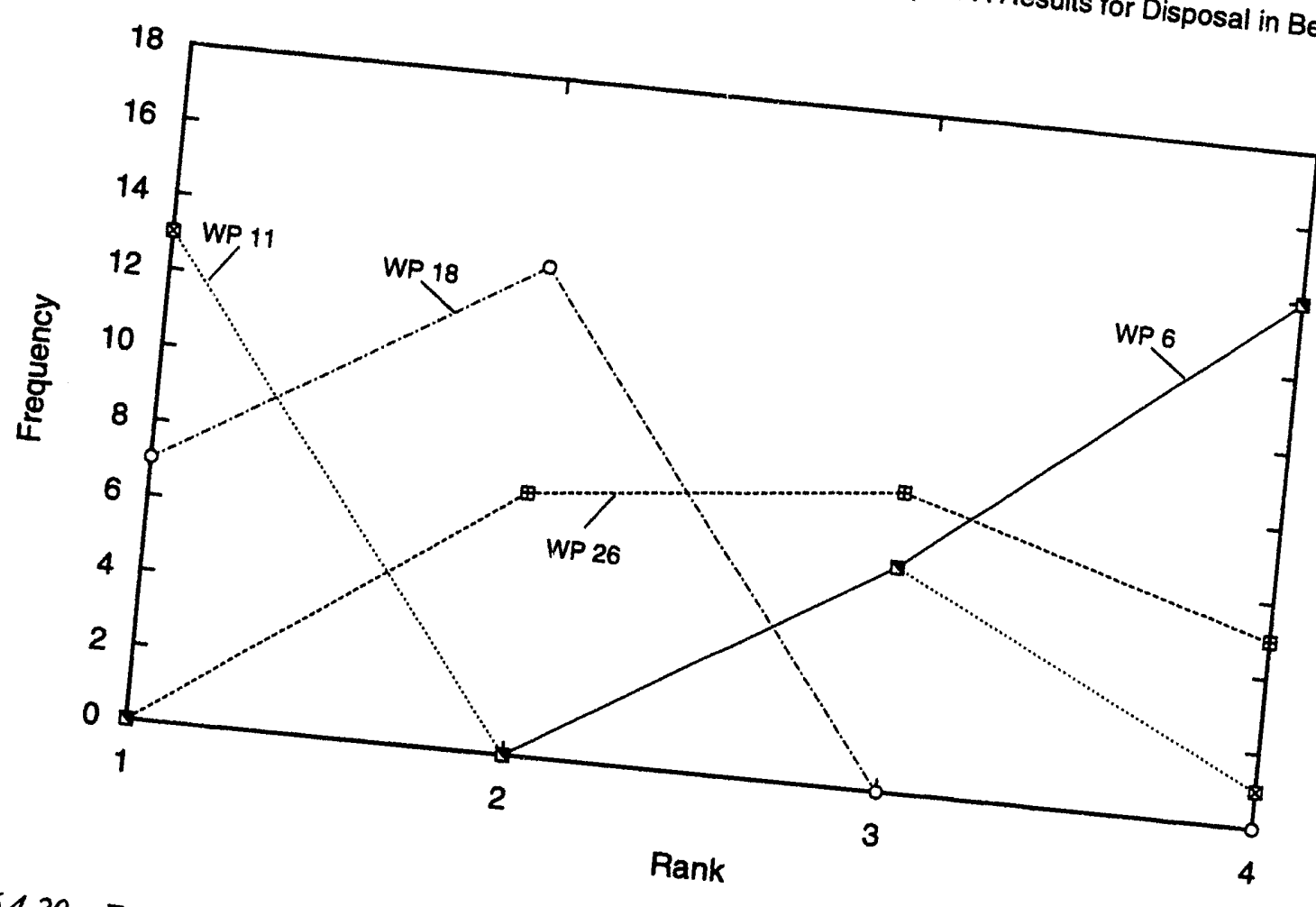

Figure 16.4-20.

Frequency with which waste parcel fractional release at reposithat 11,18 charge.$$
\text { samp }
$$

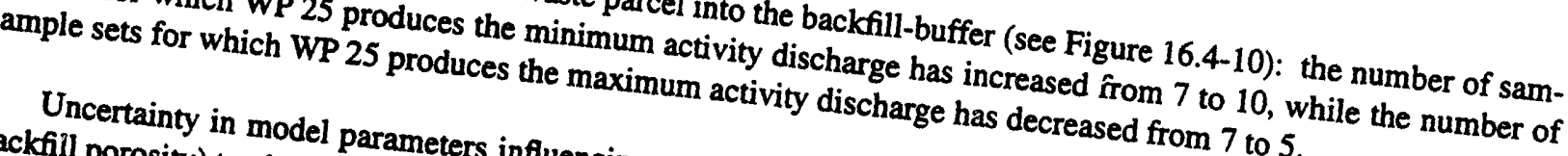
backfill porosity) tends to parameters influencing repository ligule from 7 to 5 .

waste parcel activity discharge. Activity uncertainty in repository activity (e.g., anhydrite permeability

(Figure 20 sample sets, while activity dity discharge from WP 4, for exascharge when compared with and repository liquid $16.4-14)$. If the range of waste parge from the repository fromple, has a range of 3 orders of lource flow model parameters to uncertaicel activity discharge is from WP 4 has a range of 4 orders of magnitude source activity discharges for Wr uncertainty in repository actige is already large, the contribut orders of magnitude cating that uncertainty in WP 25 rep both have a range of 5 ordity discharge is less pronounced: (e.g., glass alteration rate). 25 repository activity discharge is of magnitude (Figures $16.4-6$ waste parcel and

controlled by model parameters in the 50.15 ), indibetween the discharges from waste parcels conte is small, typically ance of WP 6 and the remaining calcine (Figures 16.4-16 and 16.4-20) for three of the four waste an order of magnitude. The activaste parcels. The magnitude show a clear distinction peaked.
(n) four waste parcels in the order WP 11, WP 18 discharge rank distributions have difference, however

As with a model param activity discharge from the waste parcels containing low 26 is less waste parcel activity discharge range of 2 orders of magnitude (see Figure 3 orders of magnitude, compared at reposi-
tory liquid flow parameters has a smaller impartich buffer is approximately 3 orders of magnert: the range activity disc 16.4-7). For WP 11, uncertainpared to the approximately 3.5 orders of magnitude. December 1993 


\begin{abstract}
Stepwise rank regression (see Section 16.1.3) was used to identify the parameters whose uncertainty strongly contributes to the uncertainty in activity discharge at the repository boundary. Table 16.4-2 lists for each sample flow parameter, the number of waste parcel designs for which the listed parameter uncertainty was a significant source of uncertainty in repository discharge. Among the parameters of the flow model, the permeability of the anhydrite marker bed (Figure 14-1) and the corrosion rate of stainless steel have the largest number of significant correlations. The importance of anhydrite permeability indicates that most waste-bearing fluid originates from or leaves through the anhydrite. Cross-flow between the intrusion boreholes is evidently of secondary importance. Given that the host rock was assumed to be impermeable in the Simple PA, this result is not unreasonable.
\end{abstract}

Table 16.4-2. Number of Waste Parcel Designs with Discharges from Repository having Significant Correlations with Sampled Flow Parameters in Bedded Salt for Simple PA

\begin{tabular}{lc}
\hline \multicolumn{1}{c}{ Parameter } & $\begin{array}{c}\text { Number of Waste Parcels } \\
\text { with Activity Discharges Having } \\
\text { Significant Correlations out of 20 }\end{array}$ \\
\hline $\begin{array}{l}\text { Stainless steel corrosion rate }\left(r_{3045}\right) \\
\text { Undisturbed permeability of anhydrite marker bed }\left(\mathrm{k}_{\mathrm{MB}}\right)\end{array}$ & 7 \\
\hline
\end{tabular}

Similarly, Table 16.4-3 shows the number of significant correlations of repository discharge with the sampled transport parameters. Among the transport parameters, the solubility of americium is the strongest contributor to uncertainty in activity discharge.

Table 16.4-3. Number of Waste Parcel Designs with Discharges from Repository having Significant Correlations with Sampled Transport Parameters in Bedded Salt for Simple PA

\begin{tabular}{lc}
\hline Parameter & $\begin{array}{c}\text { Number of Waste Parcels } \\
\text { with Activity Discharges Having } \\
\text { Significant Correlations out of 20 }\end{array}$ \\
\hline Solubility of americium $\left(\mathrm{S}_{\mathrm{Am}}\right)$ & 7 \\
Corrosion rate of glass $\left(\mathrm{r}_{\mathrm{g} \text { lass }}\right)$ & 3 \\
Solubility of uranium $\left(\mathrm{S}_{U}\right)$ & 3 \\
\hline
\end{tabular}




\subsection{Simple PA Results for Disposal in Granite Batholith}

\subsubsection{Fluid Flow through Granite Repository (L. Smith)}

Uncertainty/sensitivity analyses were performed to determine factors that affect gas generation and movement of gas and water away from the repository for all 21 waste parcels considered in the granite disposal system. Specific performance measures examined included cumulative gas and water flow out of the repository. Also examined were cumulative measures of the mass of water consumed and the mass of gas generated during chemical reactions.

Regression analyses were performed on results at the end of the 10,000-yr simulation. Table 16.5-1 identifies the independent and dependent variables used in the stepwise regression analysis.

Table 16.5-1. Number of Waste Parcel Designs with Discharge from Waste Parcel (out of a Total of 21) Showing Significant Correlation Between the Independent and Dependent Flow Variables in the Granite Disposal System for the Simple PA

\begin{tabular}{|c|c|c|c|c|c|}
\hline Variable & $\begin{array}{l}\text { Variable } \\
\text { No. in } \\
\text { LHS }\end{array}$ & Description & CUMMDH2O $^{1}$ & CUMMDGAS $^{2}$ & CMGENGAS $^{3}$ \\
\hline ALCORATE & 1 & Aluminum corrosion rate, $\left(\mathrm{r}_{\mathrm{Al}}\right)$ & 0 & 1 & 8 \\
\hline PBCORATE & 2 & Lead corrosion rate $\left(r_{\mathrm{Pb}}\right)$ & 0 & 6 & 14 \\
\hline SS304CRT & 3 & $\begin{array}{l}\text { Type } 304 \mathrm{~L} \text { stainless steel corro- } \\
\text { sion rate }\left(r_{304 S}\right)\end{array}$ & 0 & 11 & 21 \\
\hline PBPRFRAC & 4 & Lead perforation fraction $\left(f_{\mathrm{Pb}}\right)$ & 0 & 0 & 8 \\
\hline SS304PFR & 5 & $\begin{array}{l}\text { Type } 304 \mathrm{~L} \text { stainless steel perfora- } \\
\text { tion fraction }\left(\mathrm{f}_{304 \mathrm{~S}}\right)\end{array}$ & 0 & 5 & 12 \\
\hline CNFRFFLX & 6 & $\begin{array}{l}\text { Granite fracture liquid flow rate } \\
\text { into the repository, if the reposi- } \\
\text { tory were at atmospheric pres- } \\
\text { sure }\left(q_{\max }\right)\end{array}$ & 21 & 3 & 8 \\
\hline BHOLEPRM* & 7 & Borehole permeability ( $\left.k_{\text {borehole }}\right)$ & 21 & 0 & 8 \\
\hline $\begin{array}{ll}1 & \text { Cumulative } m \\
2 & \text { Cumulative } m \\
3 & \text { Cumulative } n \\
\text { * } & \text { Differ from sa }\end{array}$ & $\begin{array}{l}\mathrm{H}_{2} \mathrm{O} \mathrm{di} \\
\text { i gas di } \\
\text { f gas ge } \\
\text { osal sy }\end{array}$ & $\begin{array}{l}\text { charged from the repository. } \\
\text { charged from the repository. } \\
\text { erated during chemical reactions. } \\
\text { em, see Table 16.4-1. }\end{array}$ & & & \\
\hline
\end{tabular}

The scatterplot results show that the most important parameter affecting cumulative water flow out of the repository is the granite fracture flow rate, which yielded a strong correlation for each of the 21 waste parcels considered. Figure 16.5-1 shows a representative scatterplot of this particular independent variable; the plot is for Waste Parcel 6. Of secondary importance, but still of influence for all 21 waste parcels, was borehole permeability. Plots for this independent variable showed marked scatter. Independent variables affecting the cumulative gas flow out of the repository were the corrosion rates of lead and $304 \mathrm{~L}$ stainless steel $\left(\mathrm{r}_{\mathrm{Pb}}, \mathrm{r}_{304 \mathrm{~S}}\right)$ and the $304 \mathrm{~L}$ stainless steel perforation fraction $\left(f_{3045}\right)$. In addition, the cumulative gas flow was affected by the corrosion rate of aluminum and the granite fracture flow rate. Figure 16.5-2 shows a representative scatterplot of the 304L stainless steel corrosion rate for Waste Parcel 11. In contrast, variation in the cumulative gas flow for a given outcome was small, regardless of the magnitude of the corrosion rate of either the lead or stainless steel. 
16. Results and Conclusions

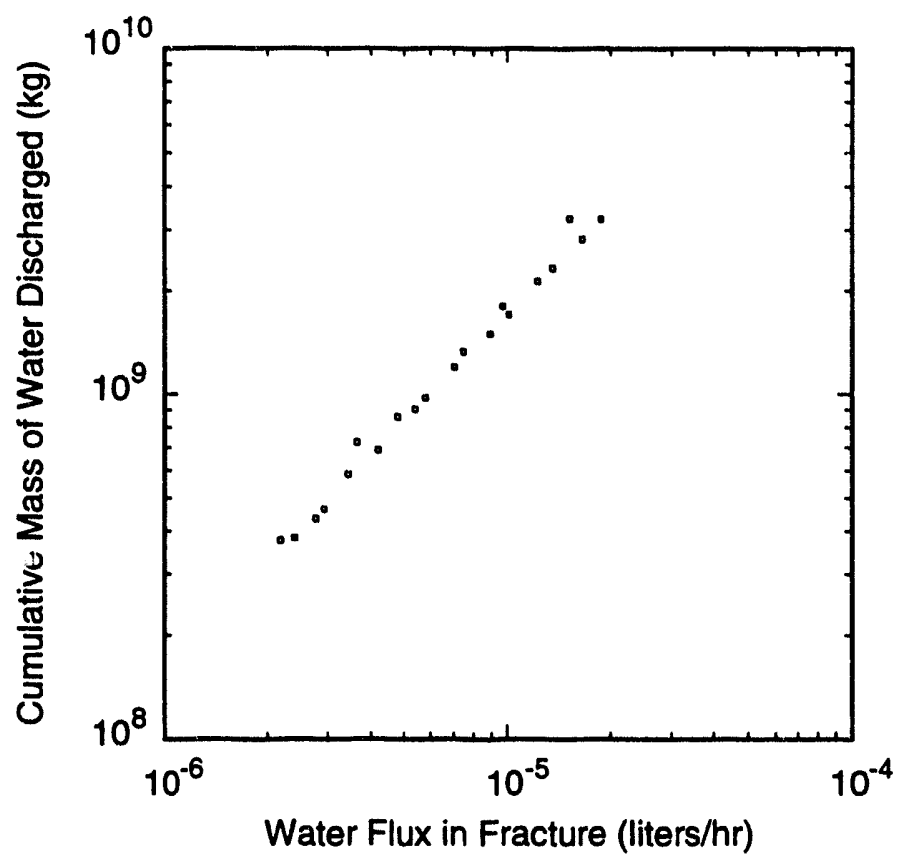

TRI-6342-4052-0

Figure 16.5-1. Representative scatterplot of fracture flow rate into repository for WP 6 in granite disposal system.

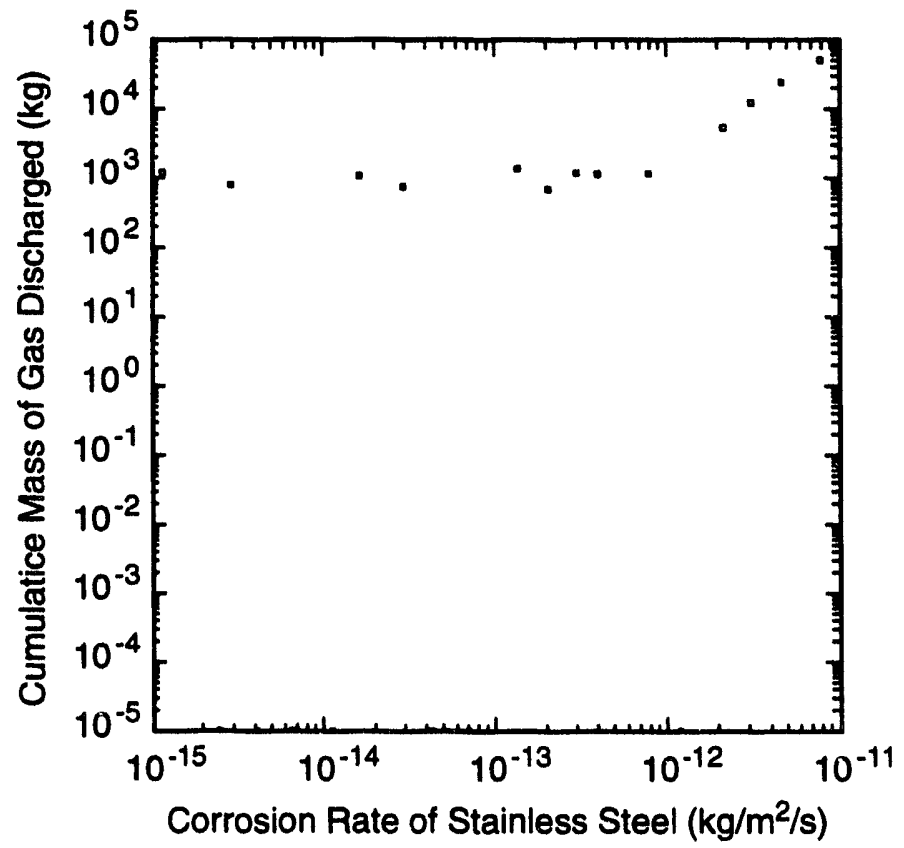

TRI-6342-4053-0

Figure 16.5-2. Scatterplot of the stainless steel corrosion rate for WP 11 in granite disposal system. 
The remaining two performance measures, the cumulative mass of gas generated during chemical reactions and the cumulative mass of water consumed during chemical reactions are functionally related and behaved virtually the same in the rank regression analysis. Thus, only the cumulative mass of gas generated is discussed. Each of the seven independent variables selected for regression showed contributions to cumulative gas generation, but often based on different individual waste parcels. Scatterplots for the corrosion rates of aluminum, lead, and type 304L stainless steel show a marked variation between the different materials and even between different waste parcels for a given material. In general, the scatterplots for lead and $304 \mathrm{~L}$ stainless steel were tightly grouped along a single line (similar to Figure 16.4-2). In contrast, the aluminum corrosion rate plots showed scatter over the entire corrosion rate range. Analysis of the other four independent variables, lead perforation fraction, stainless steel perforation fraction, granite fracture flow rate, and borehole permeability, resulted in scatterplots indicating even less effect on the cumulative mass of gas generated during chemical reactions. Table 16.5-1 tabulates the number of waste parcels (out of a total of 21) that showed significant correlation between the independent flow parameter and dependent flow results.

\subsubsection{Percentage of Radionuclides Leaving the Waste Parcel (W. E. Beyeler and C. T. Stockman)}

Discharges from the waste parcel and at the repository boundary were calculated for the 21 waste parcels considered for the granite disposal system using the models described in Chapter 15. As in the salt disposal system calculations, the purpose is to identify the effects of alternative processing options on discharge. Discharges for the granite disposal system are therefore presented in the same way as the salt disposal system discharges (Section 16.4.2).

Figures 16.5-3 through 16.5-7 are the CCDFs of discharge into the pore fluid surrounding the backfill-buffer. The integrated discharge is normalized by the normalized initial waste parcel inventory, as described in Section 16.4.2. The results of the separate waste parcel simulations are grouped by material type: high-level waste from DOE sites (WPs 7, 8, 9) in the first group; graphite (WPs 2, 3, 10, 15, 22, and 24) in the second; highly enriched uranium [HEU] (WPs 4, 12, 19, 23, and 25) in the third; low enriched uranium [LEU] (WPs 5, 13, 20, 23, 25) in the fourth, and calcine (WPs 6, 21, and 26) in the fifth.

Figures 16.5-8 through 16.5-11 show the distribution of rankings for waste parcel discharge for the four groups that include alternative waste parcel designs.

As in the salt disposal system, waste parcel activity discharges (in terms of number of equivalent parcels) for waste parcels containing graphite show large variability (approximately 4 orders of magnitude) from waste parcel to waste parcel (Figure 16.5-4). Activity discharge distributions for WP 3, WP 15, WP 10, and to a lesser extent WP 2, are characterized by two distinct clusters of release (Figure 16.5-8). WP 22 and WP 24 activity discharges tend to be more evenly distributed. As expected from Figure 16.5-4, activity discharge rank distributions (Figure 16.5-8) show that WP 3 and WP 15 consistently have the largest and second-largest activity discharge across the sample sets. The remaining waste parcels are in the order WP 22, WP 2, WP 10, and WP 24, although the ordering within this group is not well defined. The distributions for WP 2, WP 10, and WP 24 are bimodal.

As in the salt case, the activity discharges for all but the processed WPs 22 and 24 are in order of the fraction of graphite fuel with failed $\mathrm{SiC}$ coatings ( $f_{\mathrm{wp}}$ ) (FAvail, see Tables 9-2 and 9-3) (i.e., WP $3>$ WP $15>$ WP $10>$ WP 2 ). In the salt disposal system, FAvail ranged from 0.04 (for WP 1 and 10) to 0.07 (for WP 14). In granite this range is larger because the high-integrity Fort St. Vrain fuel is packaged separately from the damaged Peach Bottom fuel: FAvail ranged from 0.35 for WP 3 (Peach Bottom fuel) to 0.0016 for WP 2 (Fort St. Vrain fuel). The larger spread of FAvail causes the larger spread of activity discharges from these waste parcels.

${ }^{14} \mathrm{C}$ is the major contributor to discharge from WPs $2,3,10$ and 15 , because the solubility is assumed to be high. The large change in activity discharge at a probability of 0.7 , seen for WPs $2,3,10$, and 15 , is because of differences in breach time from run to run. The six lowest activity discharges for all the graphite waste parcels were from 6 sample runs $(1,6,8,10,12$, and 18$)$ in which only the waste containers with initial defects breached within $10,000 \mathrm{yr}$. In these defective waste parcels ( $0.5 \%$ of the total number of parcels), the spent fuel became exposed in a relatively short time (immediately for Fort St. Vrain fuels; between 0 and $1000 \mathrm{yr}$ for Peach Bottom fuels). Of the 14 sample 
16. Results and Conclusions

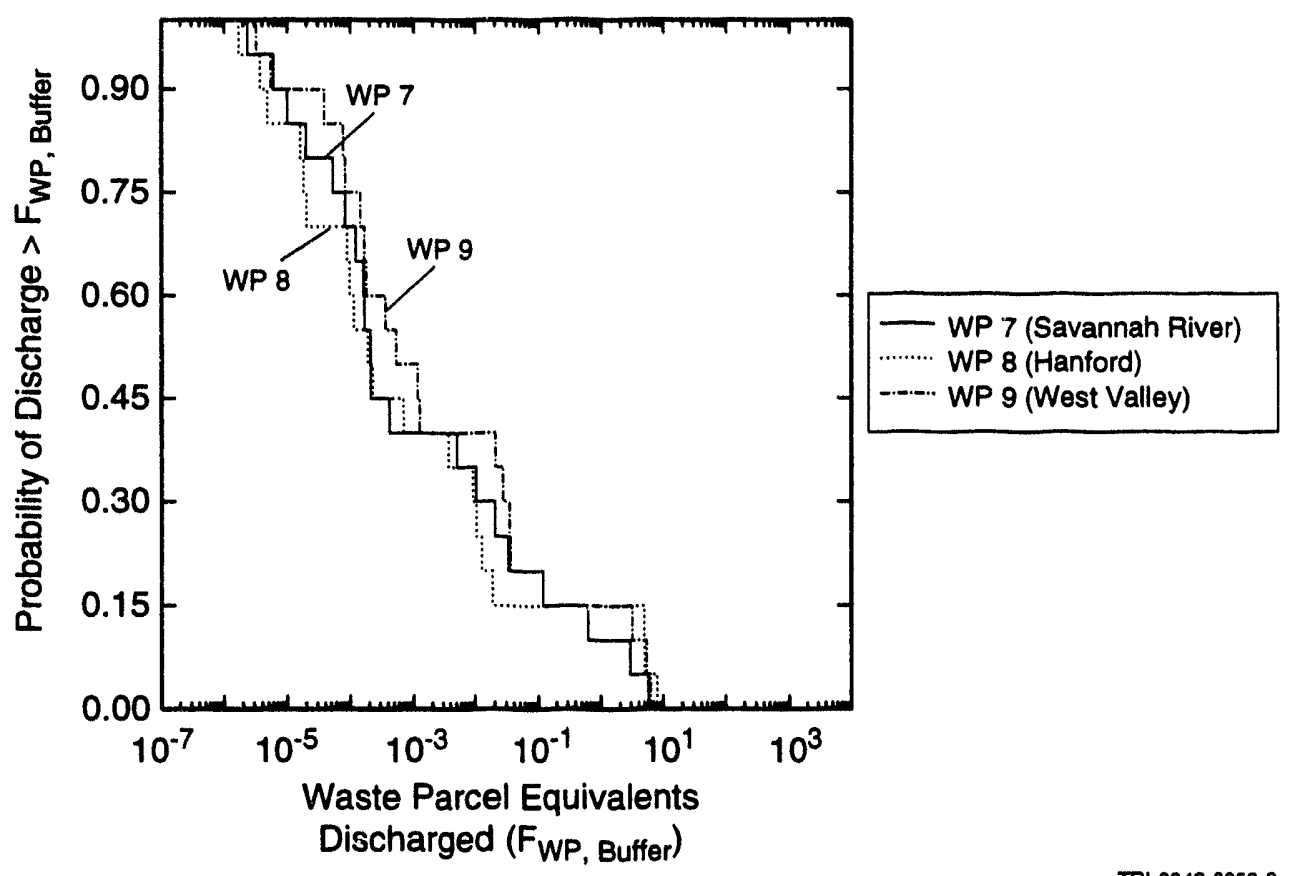

Figure 16.5-3. CCDF, conditional on one intrusion, of number of waste parcel inventory equivalents discharged into backfill-buffer for high-level waste in borosilicate glass from DOE sites (WPS 7, 8, and 9), granite disposal system.

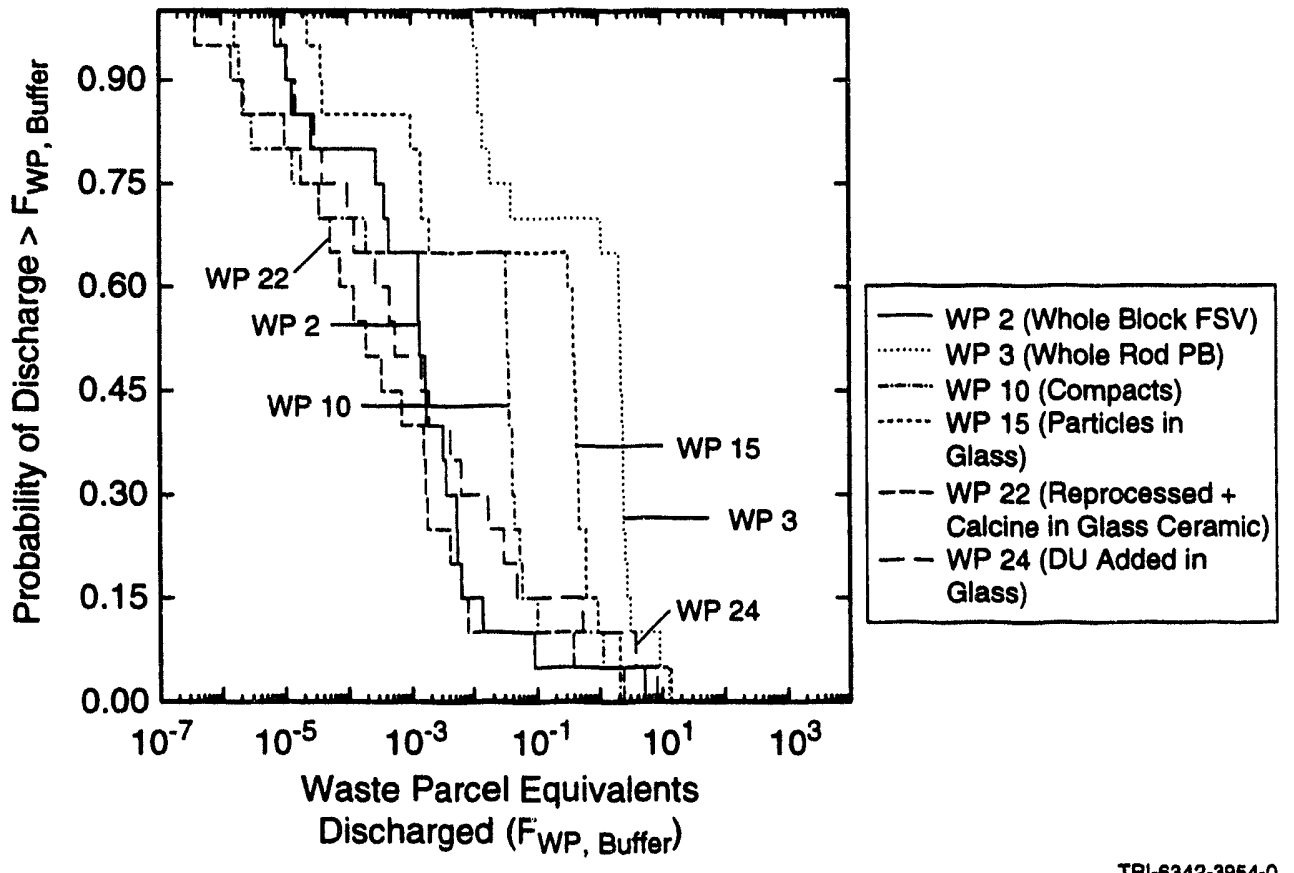

Figure 16.5-4. $\quad C C D F$, conditional on one intrusion, of number of waste parcel inventory equivalents discharged into backfill-buffer for graphite spent fuel (WPs 2, 3, 10,15, 22, and 24), granite disposal system. 


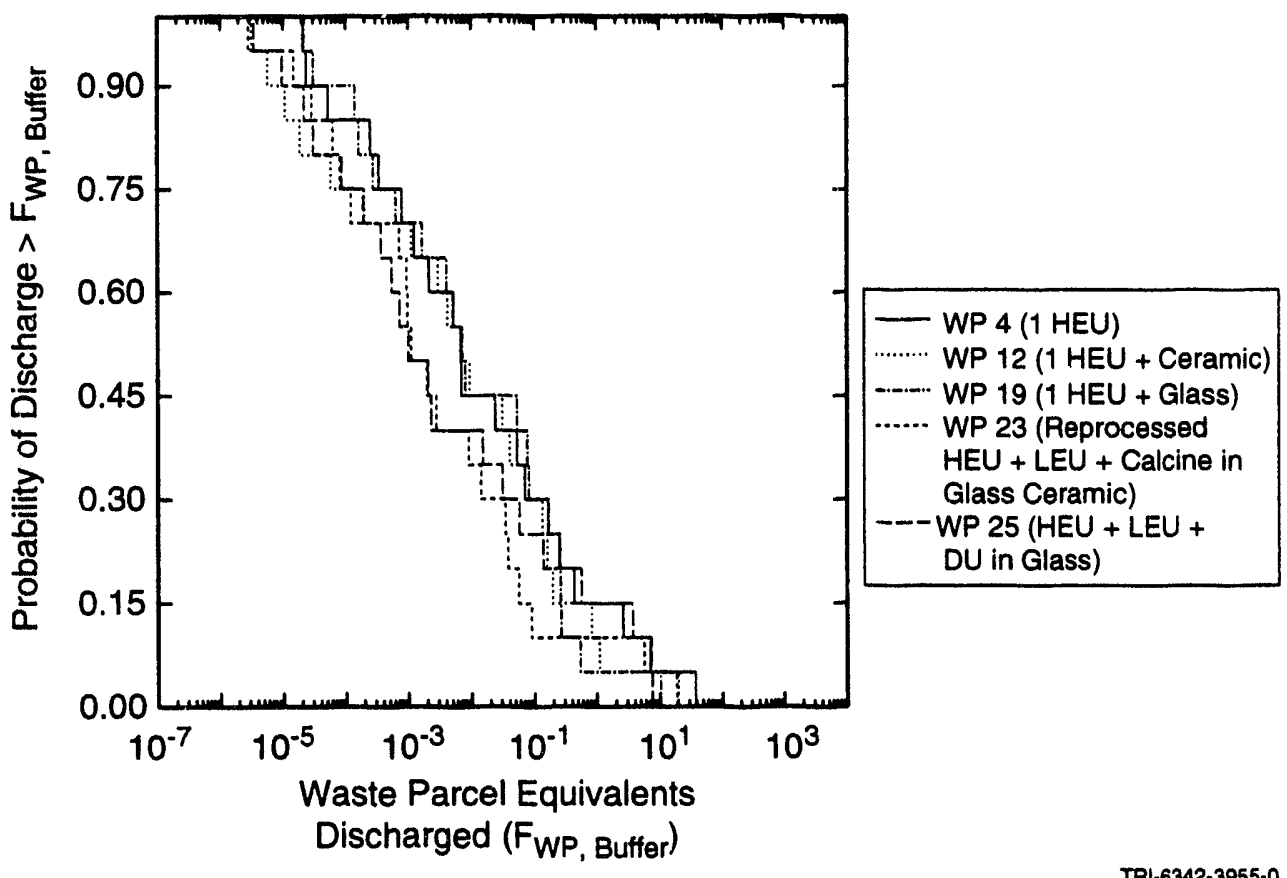

Figure 16.5-5. CCDF, conditional on one intrusion, of number of waste parcel inventory equivalents discharged into backfill-buffer for highly enriched uranium spent fuel (WPs 4, 12, 19, 23, and 25), granite disposal system.

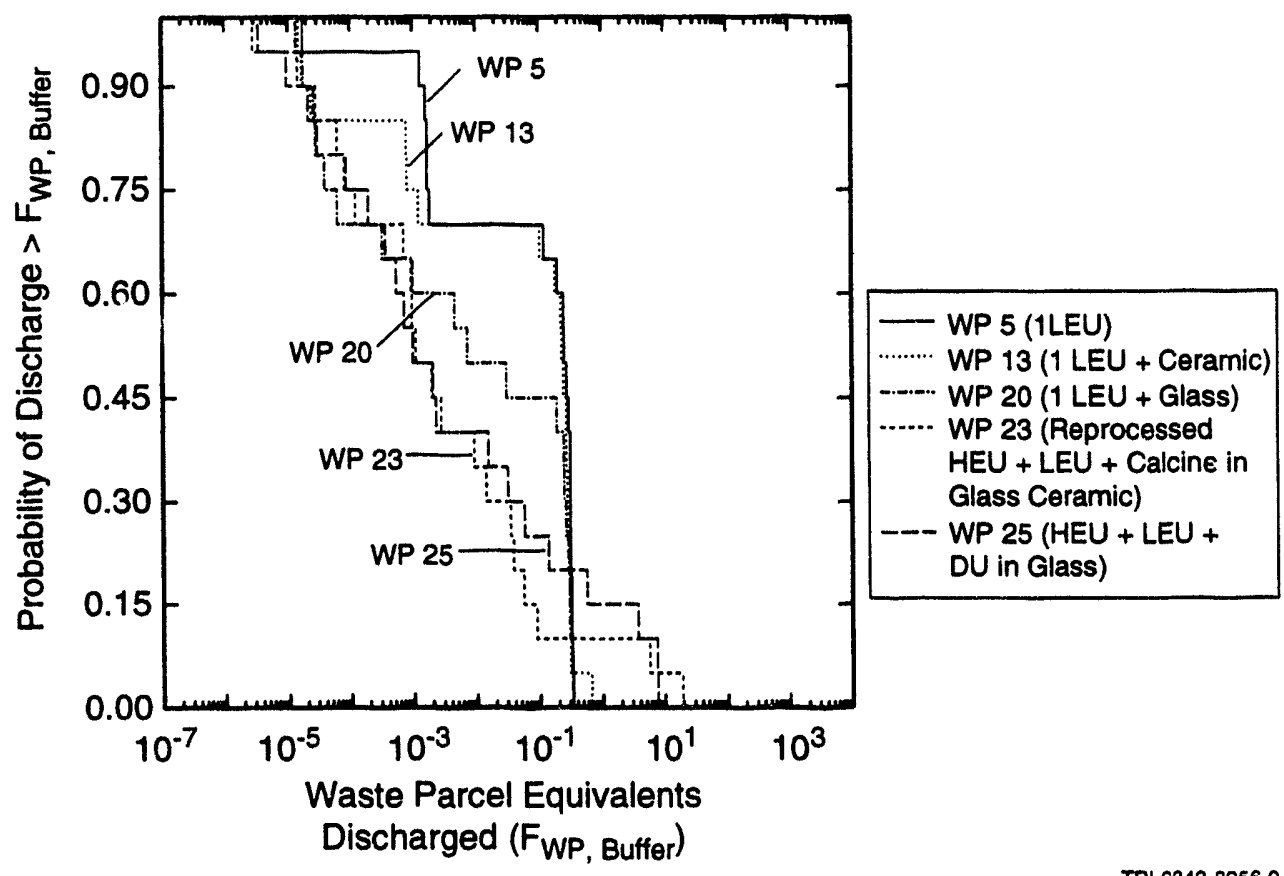

Figure 16.5-6. CCDF, conditional on one intrusion, of number of waste parcel inventory equivalents discharged into backfill-buffer for low enriched uranium spent fuel (WPs 5, 13,20,23, and 25), granite disposal system. 
16. Results and Conclusions

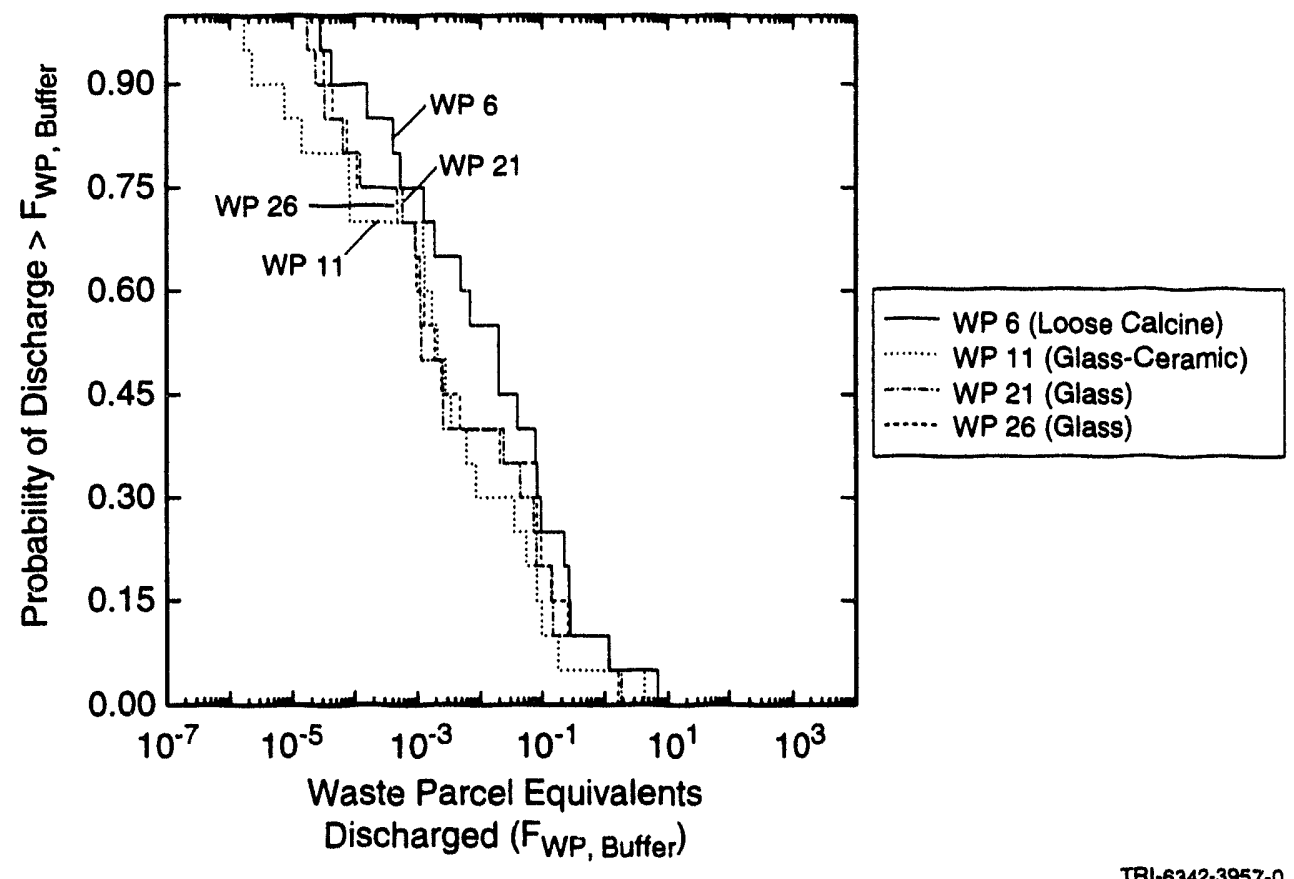

Figure 16.5-7. CCDF, conditional on one intrusion, of number of waste parcel inventory equivalents discharged into backfill-buffer for calcine (WPs 6,11,21 and 26), granite disposal system.

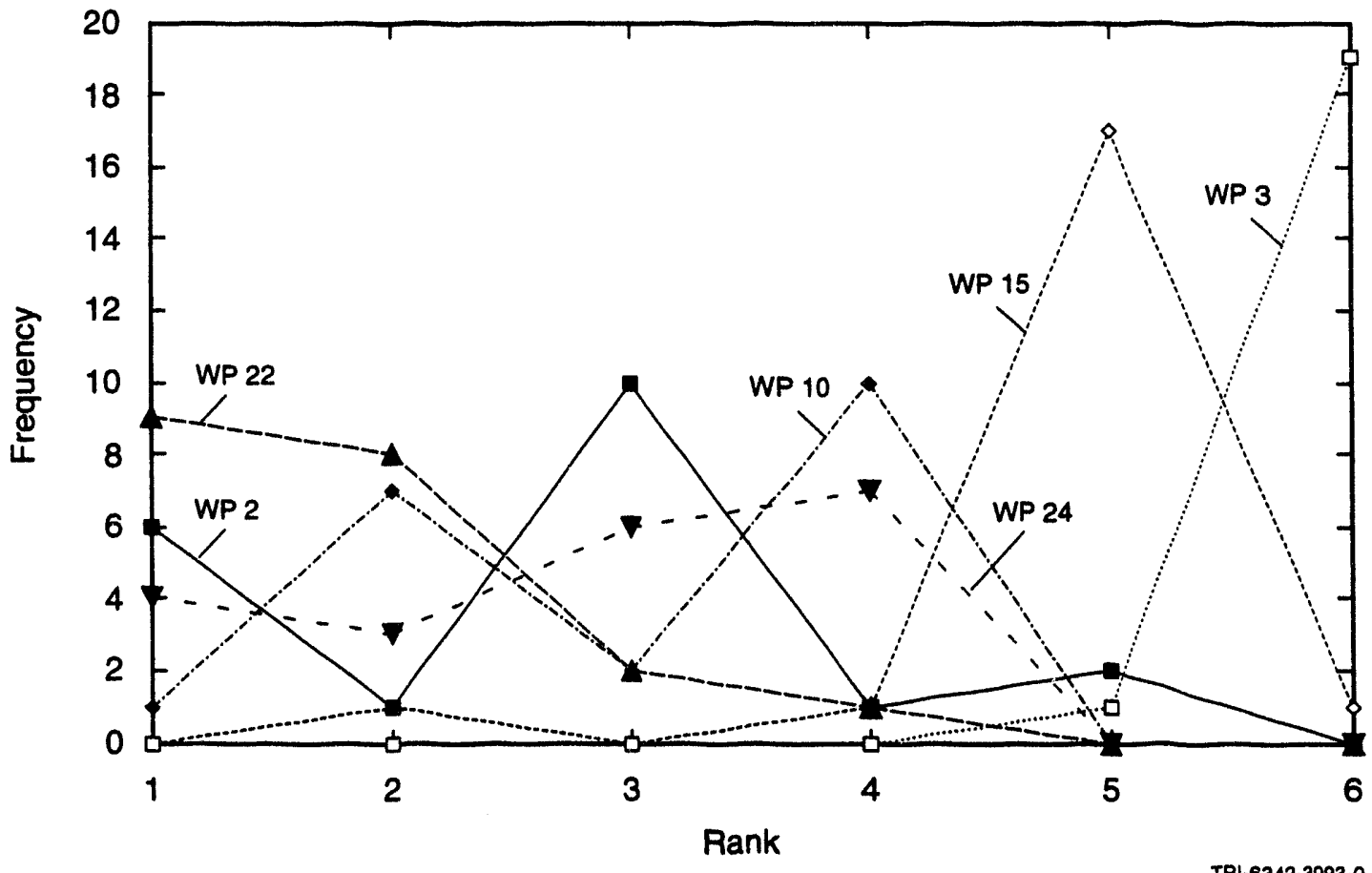

Figure 16.5-8. Frequency with which waste parcel fractional release for graphite spent fuel (WPs 2, 3, 10, 15, 22, and 24) in granite disposal system was ranked with the least (rank 1) or most (rank 5) discharge. 


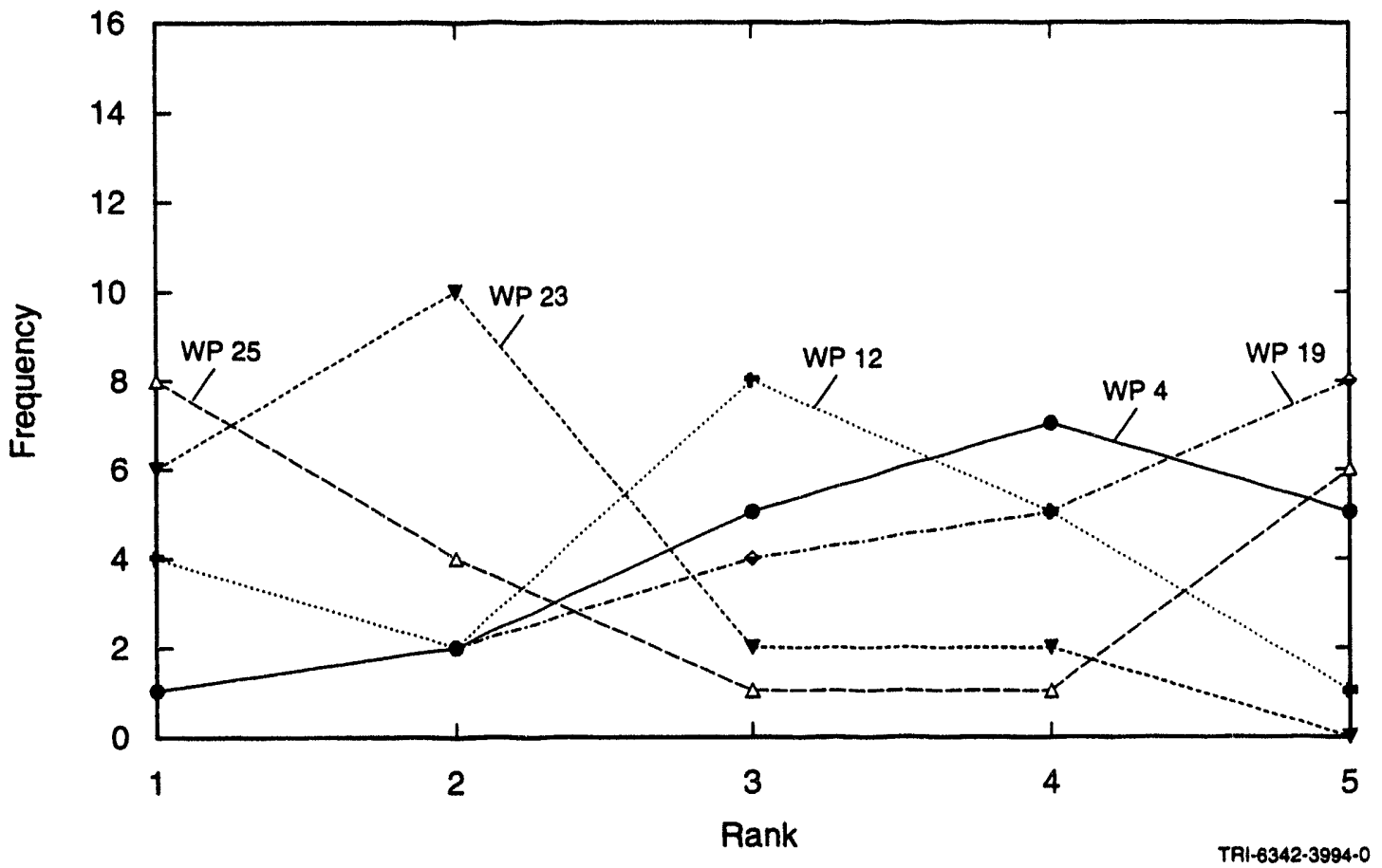

Figure 16.5-9. Frequency with which waste parcel fractional release for highly enriched uranium spent fuel (WPs $4,12,19,23$, and 25) in granite disposal system was ranked with the least (rank 1) or most (rank 5) discharge.

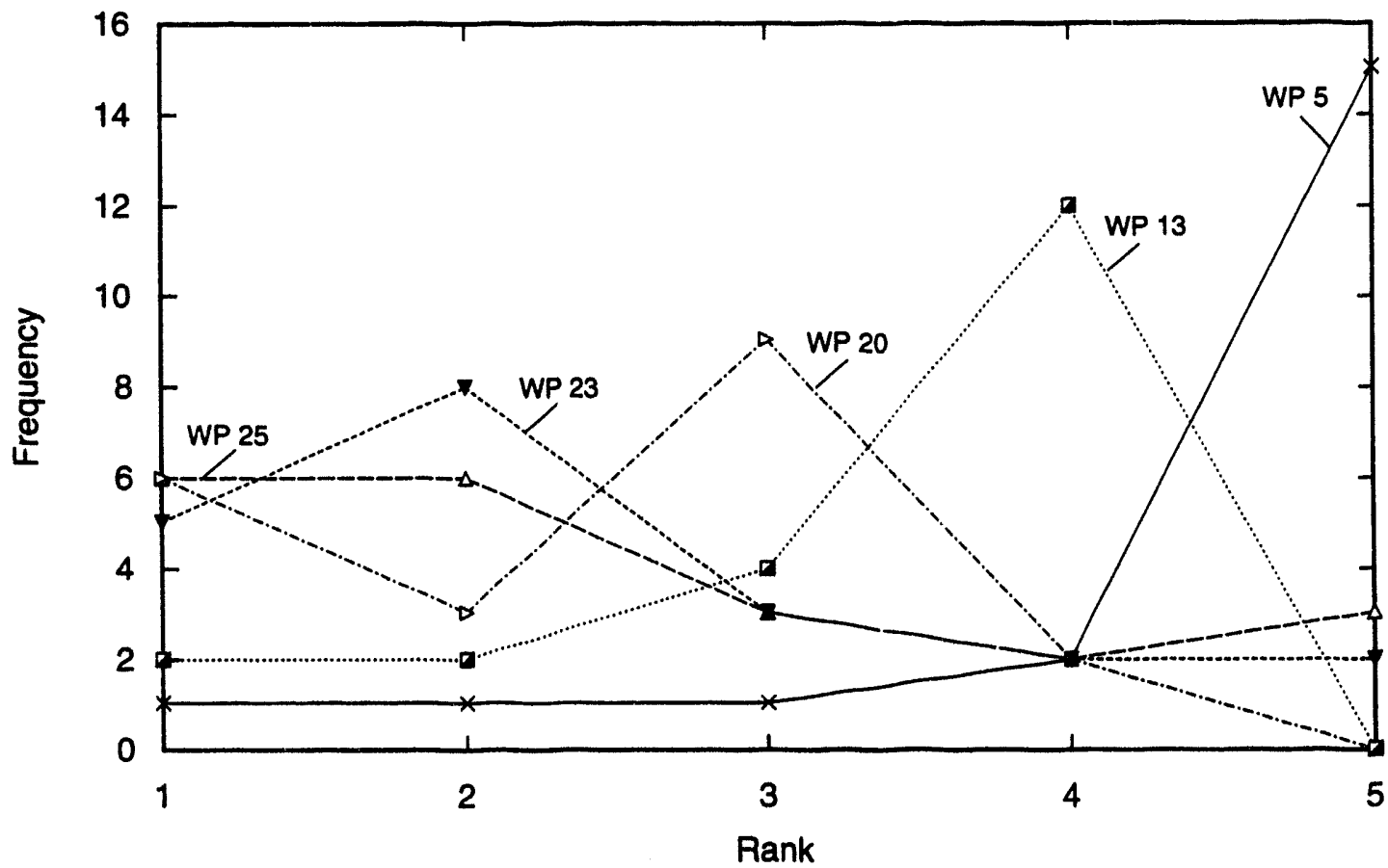

TRI-6342-3995-0

Figure 16.5-10. Frequency with which waste parcel fractional release for low enriched uranium spent fuel (WPs 5 , $13,20,23$, and 25) in granite disposal system was ranked with the least (rank 1) or most (rank 5) discharge. 


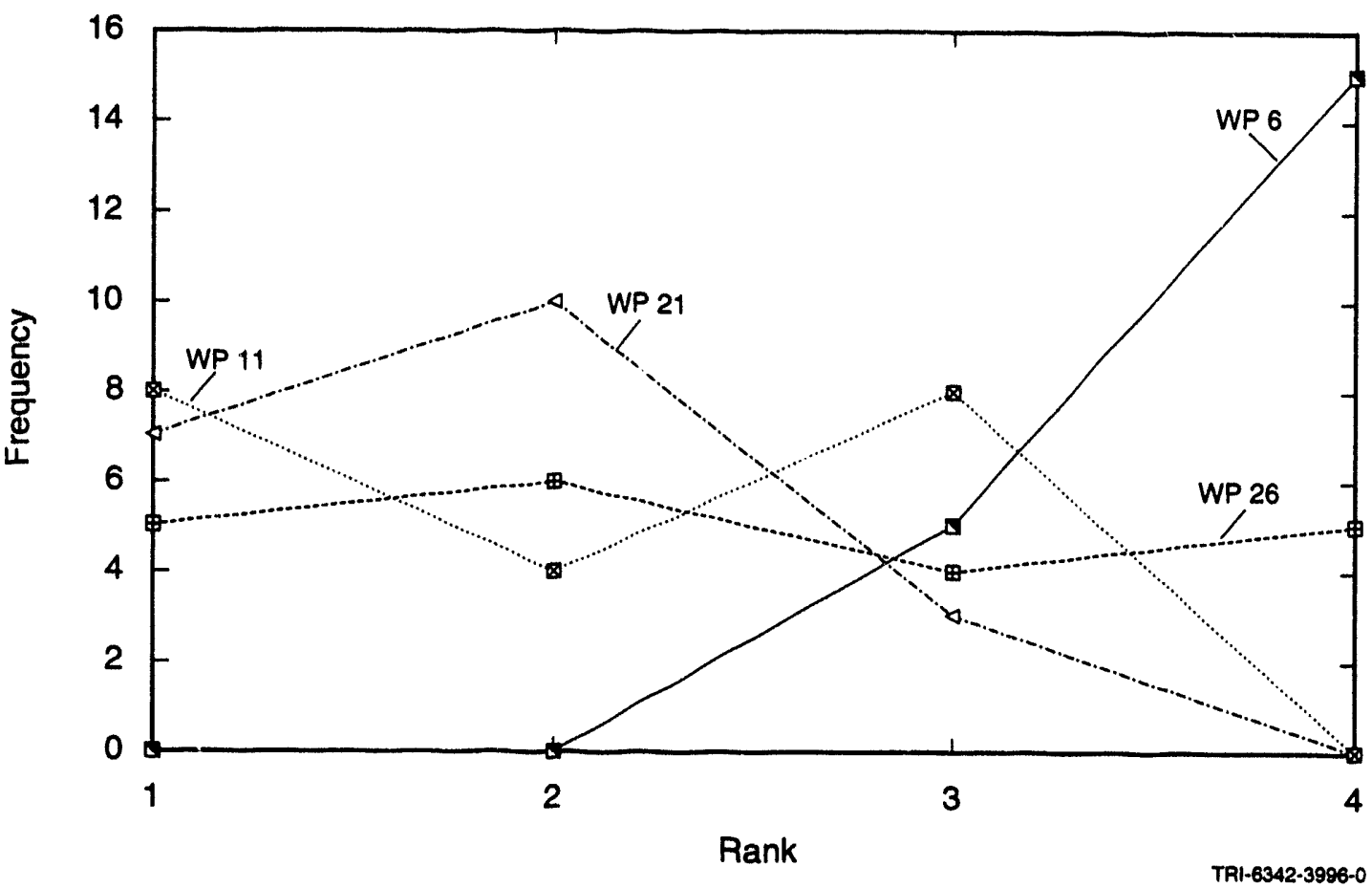

Figure 16.5-11. Frequency with which waste parcel fractional release for calcine (WPs 6, 11, 21, and 26) in granite disposal system was ranked with the least (rank 1) or most (rank 5) discharge.

sets in which non-defective parcels did breach within the 10,000-yr simulation period, the breach occurred between 0 and $3000 \mathrm{yr}$ in 13 sample sets. This relatively early breach (less than the ${ }^{14} \mathrm{C}$ half-life), combined with the fast matrix alteration rate of the metal carbide fuels $\left(\mathrm{UC}_{2}\right.$ and $\left.\mathrm{ThC}_{2}\right)$, implies that nearly all of the ${ }^{14} \mathrm{C}$ inventory contained in the metal carbide matrix is discharged when breach occurs within $10,000 \mathrm{yr}$. We expect to see a 2.5 order of magnitude difference between the activity discharges from the waste parcels whose non-defective parcels breach before $3000 \mathrm{yr}$ and those whose non-defective parcels do not breach before $10,000 \mathrm{yr}$. This difference is clearly seen for WPs 3,10 , and 15, but not for WPs 22 and 24 . WPs 22 and 24 behave differently because the inventory is immobilized in glassceramic and glass, whose alteration rates are sampled over a range of 4 and 6 orders of magnitude, respectively.

As in the salt disposal system, WP 22 (containing reprocessed graphite fuel with all the ${ }^{14} \mathrm{C}$ and most of the uranium and plutonium removed) shows the best performance. WP 24 shows intermediate performance. WP 24 contains the waste produced after graphite fuel is burned, dissolved, mixed with depleted uranium, and immobilized in glass. This treatment has two important effects. First, all of the inventory is made available, but can only be gradually discharged. FAvail is increased to 1 since all the particles are broken by the processing, but since the ash is then immobilized in glass, the full inventory becomes available only as the glass is altered (which takes a median time of $10,000 \mathrm{yr}$ ), rather then being available within $17 \mathrm{yr}$ of waste parcel breach, as in WPs $2,3,10$, and 15 . Second, the effective solubility of ${ }^{233} U$ and ${ }^{234} U$ is reduced through the "dilution" of the uranium inventory by depleted uranium. ${ }^{234} \mathrm{U}$ was one of the top contributing nuclides in the Simple PA for salt. Because the activity discharges in the salt disposal system were strongly solubility limited, addition of depleted uranium significantly improved performance. In the Simple PA for granite, the removal of ${ }^{14} \mathrm{C}$ during the processing of WP 24 again helps a great deal. However, uranium isotopes were not as important, consequently the addition of depleted uranium does not significantly help the performance of WP 24.

The reason ${ }^{14} \mathrm{C}$ is a much larger contributor to the waste parcel activity discharges in the granite than in the salt disposal system is because of the bentonite backfill-buffer used in the granite site. The "activity discharge from the waste parcel" reported is the amount of radionuclide injected into the pore fluid from the parcels after equilibration with the backfill-buffer. Thus elements that are strongly adsorbed will show an abnormally low parcel activity discharge until the backfill in the CONCSNF source-term control volume has adsorbed enough of that element to be in 
equilibrium with a saturated solution of that element. Correspondingly, the elements with very low partition coeffcients (Kds) (the $\mathrm{Kd}$ of carbon, ${ }^{14} \mathrm{C}$, is zero) will show a disproportionately large activity discharge. This effect is even larger in the activity discharge at the repository boundary (see Section 16.5.3) because the rooms are also filled with a mixture of $50 \%$ bentonite and gravel.

Waste parcel activity discharges for highly enriched uranium spent fuel (Figures 16.5-5 and 16.5-9) show little variability from waste parcel to waste parcel (approximately 1 order of magnitude) when compared to activity discharge variability over all sets (approximately 6 orders of magnitude), and are difficult to rank. WP 25 most frequently has the smallest activity discharge, but also has the largest activity discharge in 6 of the 20 sample sets (Figure 16.5-9). WP 12 is a bimodal with peaks at ranks of 1 and 3. The remaining waste parcels show consistent but poorly defined centers: the ranking of WP 25, WP 23, WP 12, WP 4, and WP 19 is not well supported, since no waste parcel is placed in a given rank in a majority of sets.

The mode ranking order is almost the inverse of the inventory-per-waste-parcel order: WP 4, WP 19, WP 12, WP 23, and WP 25. There are three matrices holding the inventory in these waste parcels: uranium-aluminum alloy $\left(\mathrm{UAl}_{3}, \mathrm{Al}\right)$ in the fuel elements in WPs 4, 12, and 19, glass in WPs 19 and 25, and glass-ceramic in WPs 12 and 22. Furthermore, the inventory is not exclusively the HEU inventory in WPs $12,19,23$, and 25 . Other inventories included in varying amounts are calcine, LEU, and depleted uranium. Once again a broader range of activity discharges is seen in the granite site than the salt site. Possibly 2 orders of magnitude of this increase are due to the later breach times in granite than salt. Like the graphite waste parcels, all the lowest activity discharges are from runs where the non-defective waste parcels do not breach in 10,000 yr. WP 19 is unusual in that it has five runs with breach times between 3000 and 10,000 yr. In all the other waste parcels, the breach times are clustered between 0 and $3000 \mathrm{yr}$, at $9000 \mathrm{yr}$, or above 10,000 yr. This more uniform distribution of breach times, however, is masked by other effects and does not manifest itself in the activity discharges.

Activity discharges from the waste parcels containing low enriched uranium spent fuel (Figures 16.5-6 and 16.5-10) show an interesting feature: activity discharges from WP 5 and WP 13 are apparently bimodal and quite similar, while activity discharges from the three remaining waste parcels are more uniformly distributed. The ranks of waste parcel activity discharges show a clear tendency for WP 5 and WP 13 to perform poorly. WPs 23 and 25 show similar rank distributions and are not clearly distinguished either by activity discharge or activity discharge rank.

As in the graphite waste parcels, the dramatic reduction in activity discharges due to the non-defective waste parcels lasting past 10,000 yr can be seen in the lower activity discharges. WP 20 is different in that it has 5 additional runs where the non-defective waste parcel never breaches (runs $5,3,11,13$, and 15). Consequently, the decrease in activity discharges is seen at the lower probability of 0.45 . When these lower activity discharges, which are due only to defective waste parcel breach, are put aside, we are left with the remaining activity discharges in a very narrow range $(0.1$ to 0.6$)$. This narrow range is because the major contributor to the activity discharges is ${ }^{14} \mathrm{C}$, which in turn occurs because a large fraction of the ${ }^{14} \mathrm{C}$ is held in the gap between the $\mathrm{UO}_{2}$ fuel and the zircaloy cladding. For WP 5 , in all but one sample set, ${ }^{14} \mathrm{C}$ accounted for more than $99.9 \%$ of the activity discharge. In tne largest activity discharge (run 16), the fraction of ${ }^{14} \mathrm{C}$ activity discharged is $12.4 \%$. In the smallest activity discharge of this group (run 11), the fraction of ${ }^{14} \mathrm{C}$ activity discharged is $10.1 \%$. When the amount in the pore liquids around the waste parcel are added to this, it accounts for $12.66 \%$ of the ${ }^{14} \mathrm{C}$. Since the gap fraction was set to $12.5 \%$, it is clear that very little of the $\mathrm{UO}_{2}$ has dissolved in $10,000 \mathrm{yr}$ and the gap fraction ${ }^{14} \mathrm{C}$ inventory, which does not vary from run to run, is the main contributor to activity discharge.

Activity discharge from waste parcels containing calcine (Figures 16.5-7 and 16.5-11) is similar across waste parcels with an upper limit established by WP 6 at most probability levels, and a lower limit defined by either WP 11 or WP 21. The rank distributions (Figure 16.5-11) are consistent, with WP 6 having the largest activity discharge in 15 of the 20 sample sets. WP 11 has a bimodal rank distribution. WP 26 occurs in each rank with nearly equal frequency.

The relative behavior of these waste parcels is the same as in salt, and for the same reasons. The high alteration rate for loose calcine in WP 6 makes the entire waste parcel inventory available for activity discharge shortly after the waste parcel is breached. In contrast, activity discharge from WPs 11,21 , and 26 can be limited by matrix alteration rate. The range of activity discharges from calcine waste parcels in the granite disposal system is noticeably larger 
than the range of activity discharges in the salt disposal system (compare with Figure 16.4-7). WP 6, which is common to both, has a waste parcel activity discharge range of 2 orders of magnitude in the salt disposal system but a range of more than 5 orders of magnitude in the granite disposal system (the maximum activity discharges are nearly identical). This behavior is consistent with the differences in breach times in the two disposal systems: nearly immediate breach in the salt disposal system allows the maximum time for activity discharge from the waste parcel, and low variability in activity discharge time from sample to sample; the wide distribution of breach times in the granite disposal system produces a lower average exposure time as well as variability of exposure time from run to run. Due to adsorption on the bentonite backfill, effective diffusion coefficients are much smaller in the granite disposal system than in the salt. To the extent that waste parcel activity discharge is diffusion limited, granite activity discharges will be smaller and more variable (due to the sampling of adsorption coefficients) than salt activity discharges.

Matrix Alteration Rate. In general, waste forms that release their radionuclides more slowly are better for compliance; however, in certain regimes the activity discharge rate is irrelevant because other factors such as solubility become dominant. This effect was seen in the correlation of the borosilicate glass alteration rate in WP 24 with the summed normalized activity discharges from the granite disposal system (Figure 16.5-12). There was a good linear correlation for rates less than $10^{-9} \mathrm{~kg} / \mathrm{m}^{2} \cdot \mathrm{s}$, but for rates greater than $10^{-9} \mathrm{~kg} / \mathrm{m}^{2} \cdot \mathrm{s}$, the total activity discharge is constant at the maximum value. This correlation was not seen, however, in any of the other waste parcels in granite.

\subsubsection{Normalized Activity Leaving Repository (W. E. Beyeler and C. T. Stockman)}

Figures 16.5-13 through 16.5-17 show normalized discharges from the granite repository for the 20 sampled parameter sets for the same waste parcel groups as described in Section 16.5.2. Figures 16.5-18 through 16.5-21 show the corresponding distribution of discharge ranks for the four waste parcel groupings that include design alternatives.

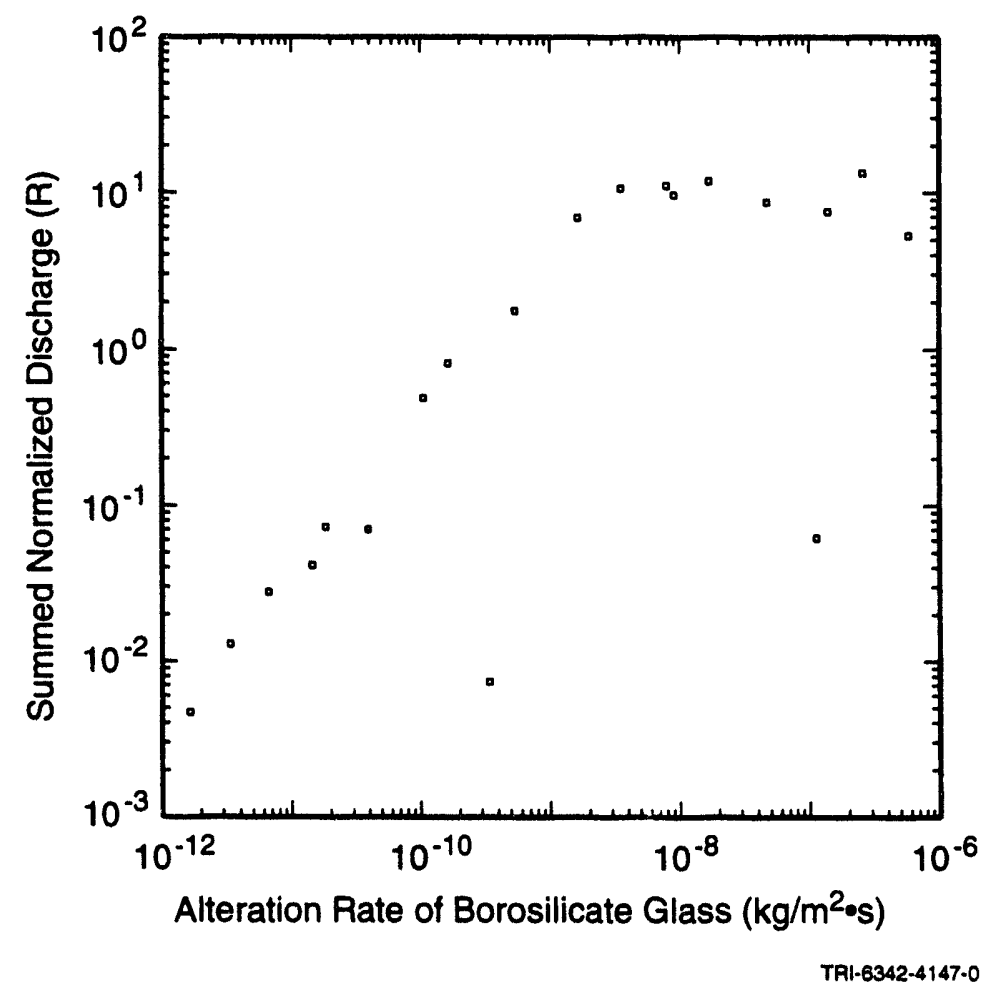

Figure 16.5-12. Scatterplot of the alteration rate for borosilicate glass and total activity discharge from the repository boundary for Waste Parcel 24 in granite disposal system. 


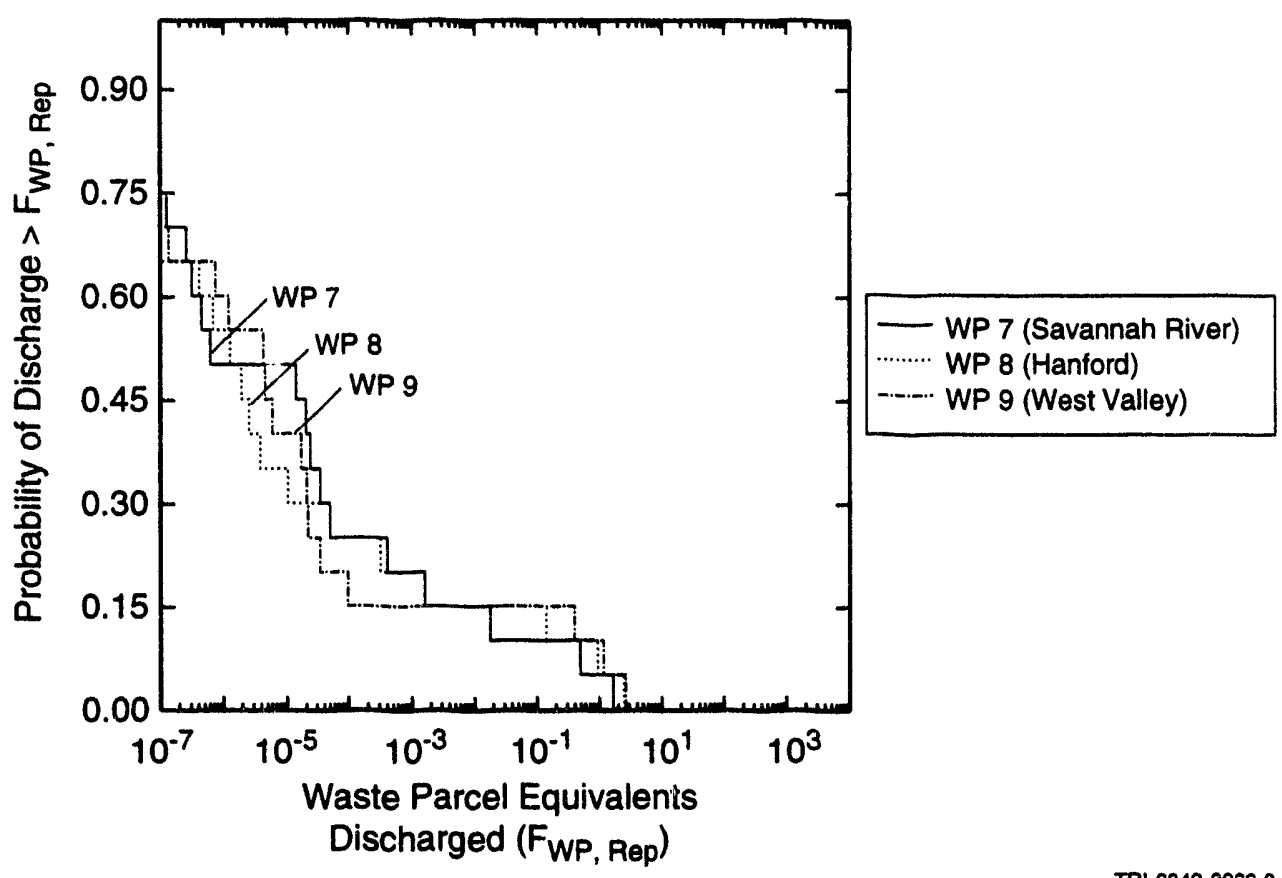

Figure 16.5-13. CCDF, conditional on one intrusion, of normalized activity discharge at repository boundary for high-level waste in borosilicate glass from DOE sites (WPs 7, 8, and 9), granite disposal system.

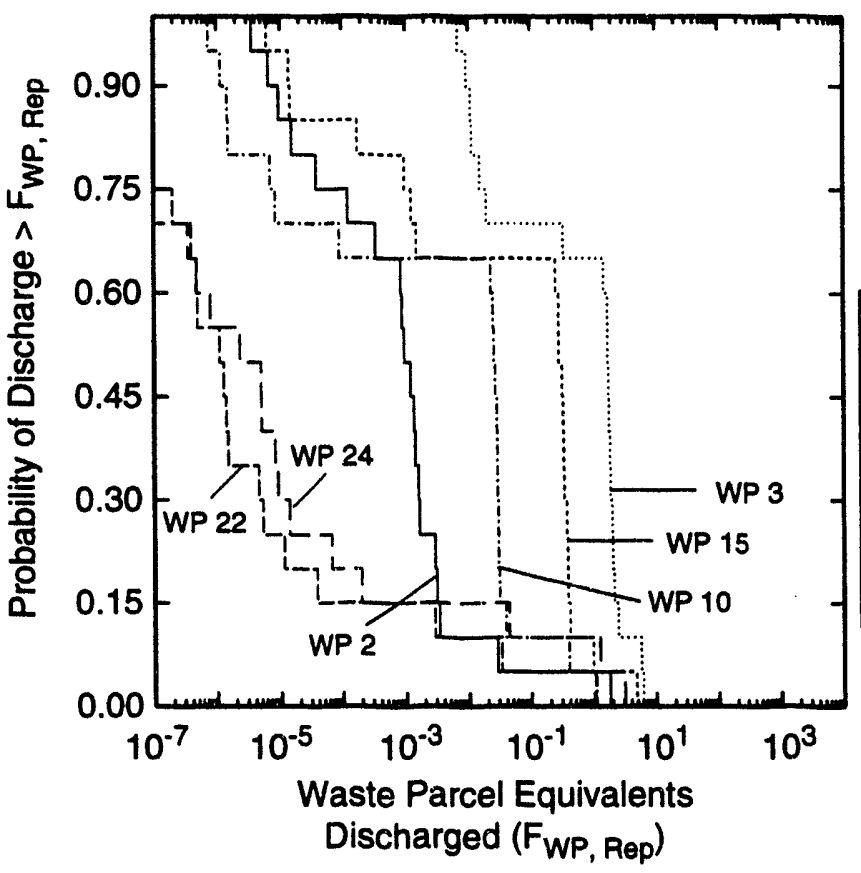

- WP 2 (Whole Block FSV) ........ WP 3 (Whole Rod PB)

-...-WP 10 (Compacts)

….. WP 15 (Particles in Glass)

-- WP 22 (Reprocessed + Calcine in Glass Ceramic) - WP 24 (DU Added in Glass)

TRI-6342-3963-0

Figure 16.5-14. CCDF, conditional on one intrusion, of normalized activity discharge at repository boundary for graphite spent fuel (WPs 2, 3,10,15,22, and 24), granite disposal system. 


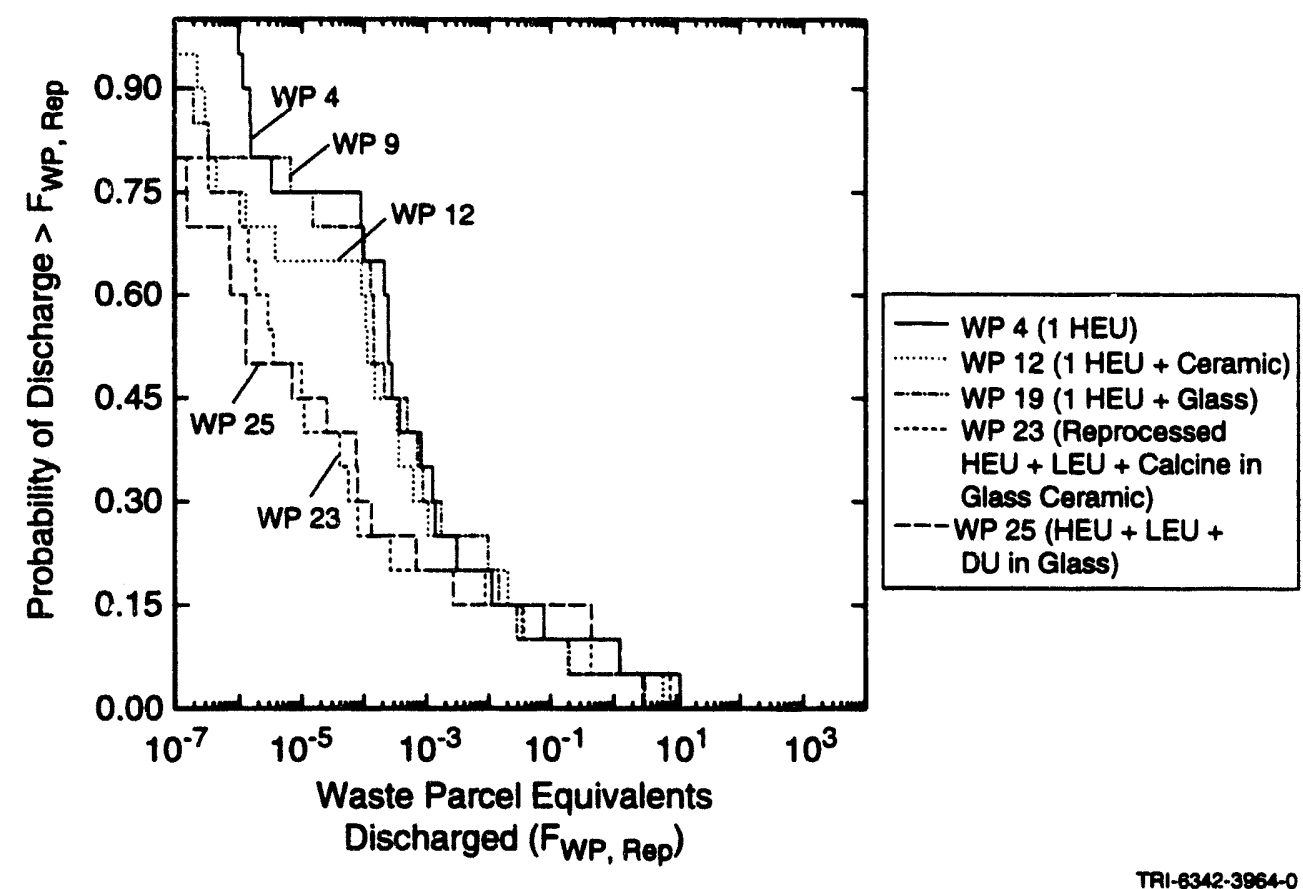

Figure 16.5-15. CCDF, conditional on one intrusion, of normalized activity discharge at repository boundany for highly enriched uranium spent fuel (WPs 4, 12, 19, 23, and 25), granite disposal system.

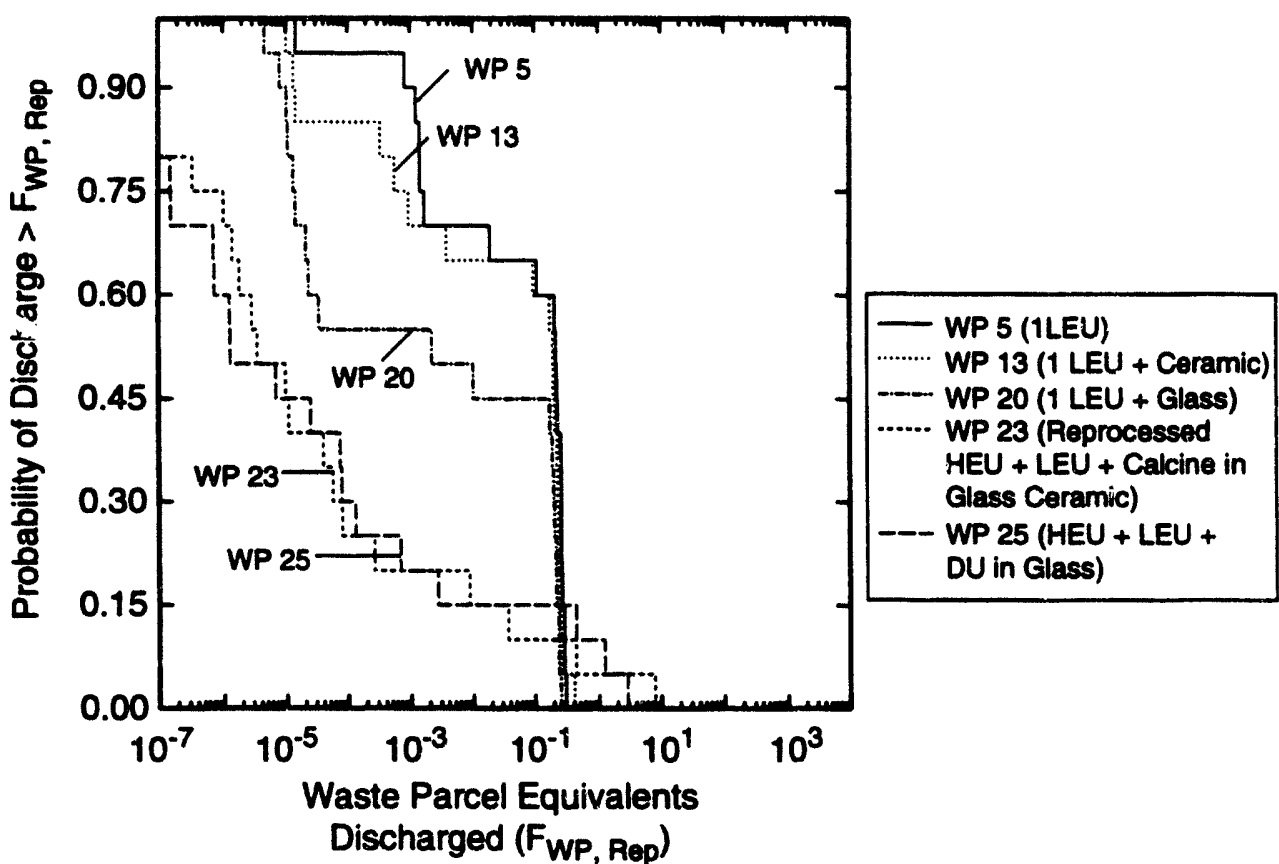

TRI-6342-3965-0

Figure 16.5-16. CCDF, conditional on one intrusion, of normalized activity discharge at repository boundany for low enriched uranium spent fuel (WPs 5, 13,20,23, and 25), granite disposal system. 


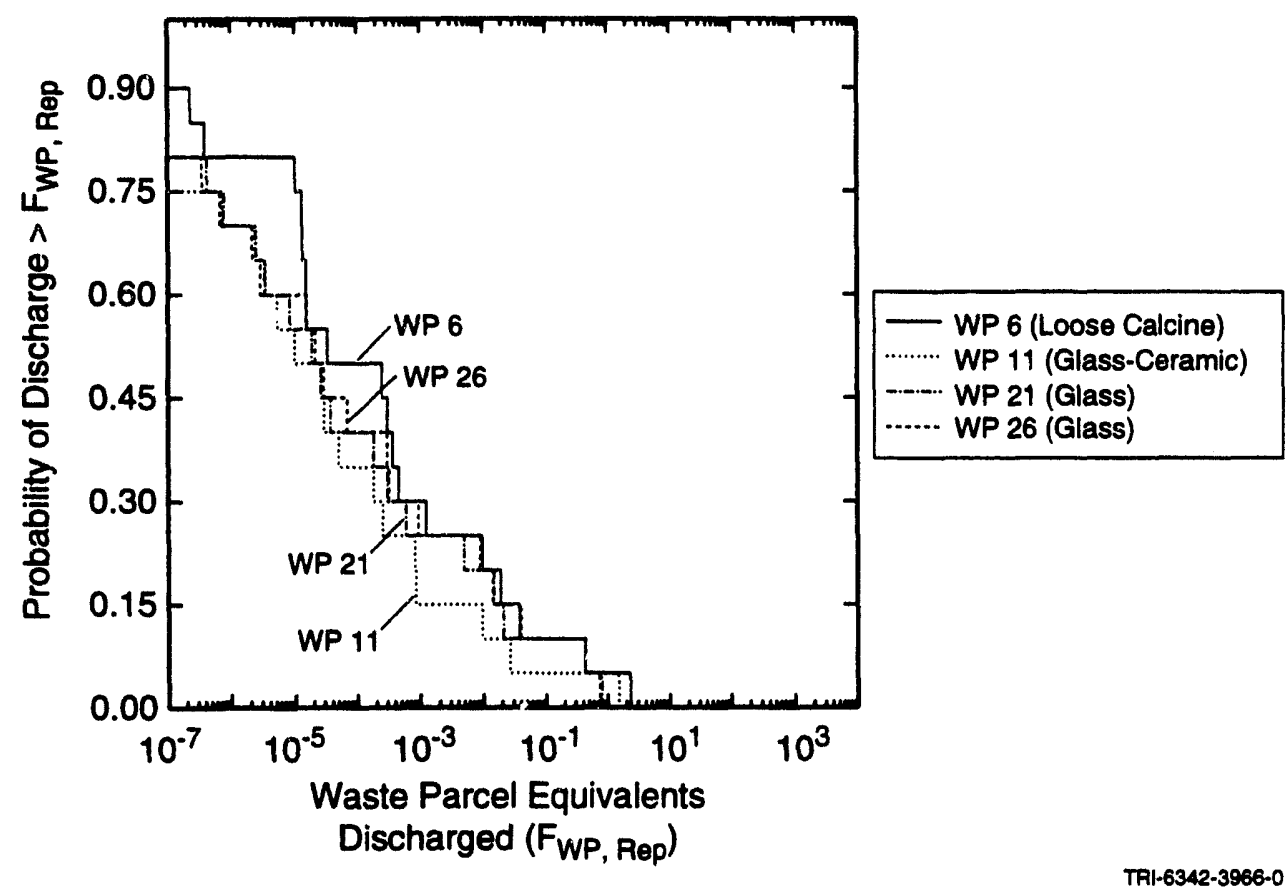

Figure 16.5-17. $C C D F$, conditional on one intrusion, of normalized activity discharge at repository boundary for calcine (WPs 6,11,21, and 26), granite disposal system.

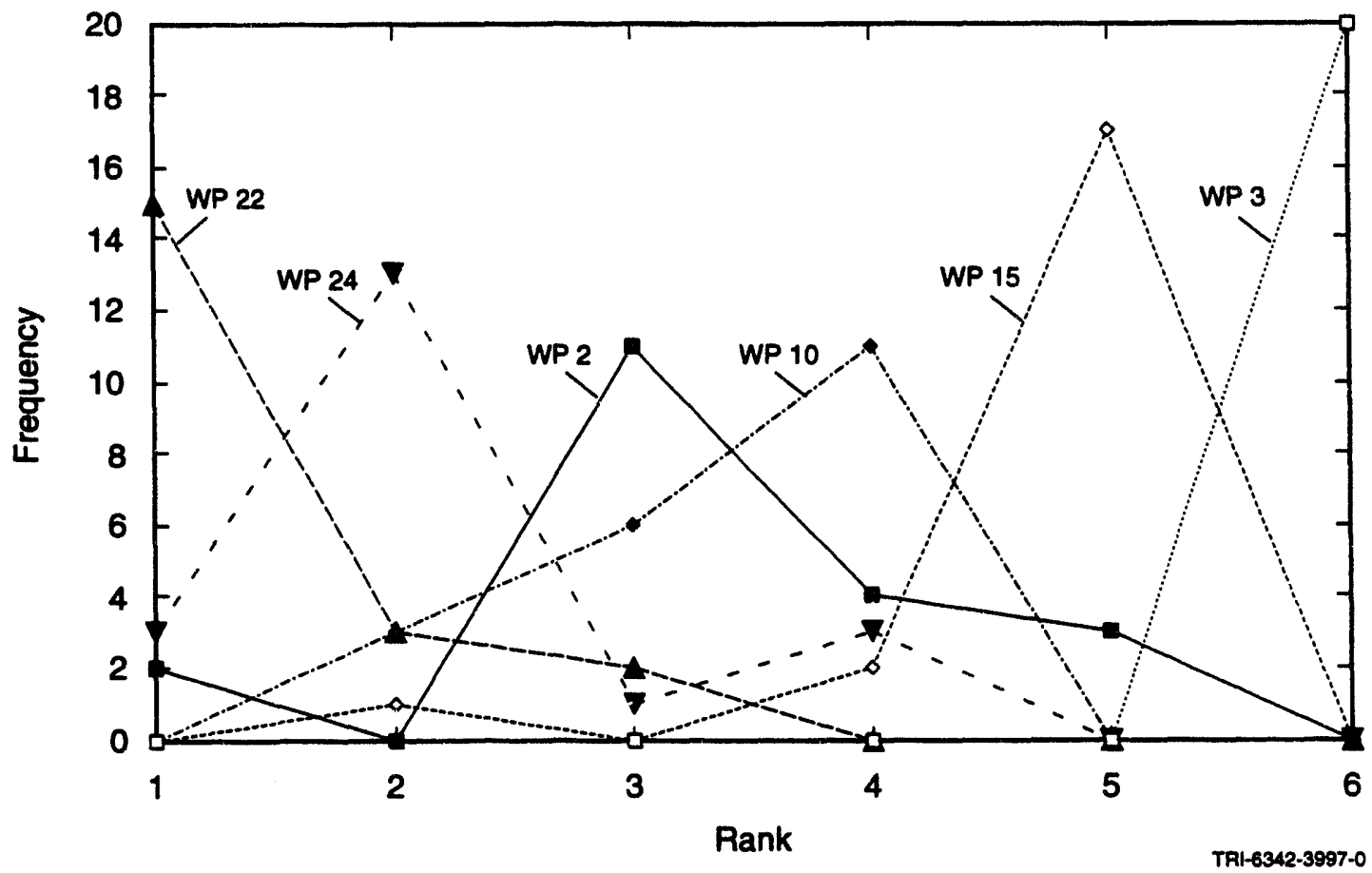

Figure 16.5-18. Frequercy with which waste parcel fractional release at repository boundary for graphite spent fuel (WPs 2,3,10,15,22, and 24) in granite disposal system was ranked with the least (rank 1) or most (rank 5) discharge. 
16. Results and Conclusions

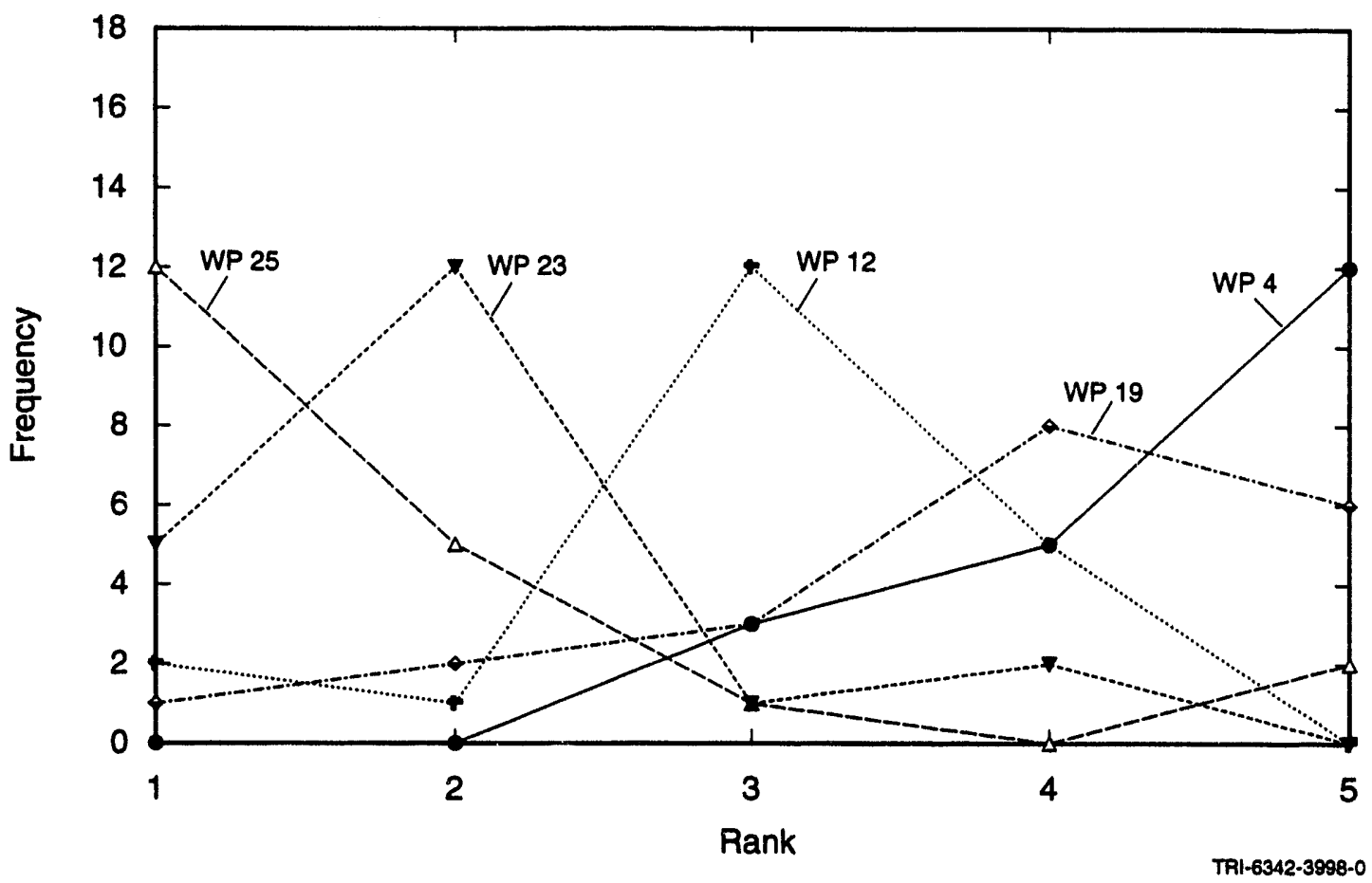

Figure 16.5-19. Frequency with which waste parcel fractional release at repository boundary for highly enriched uranium spent fuel (WPs 4, 12,19,23, and 25) in granite disposal system was ranked with the least (rank 1) or most (rank 5) discharge.

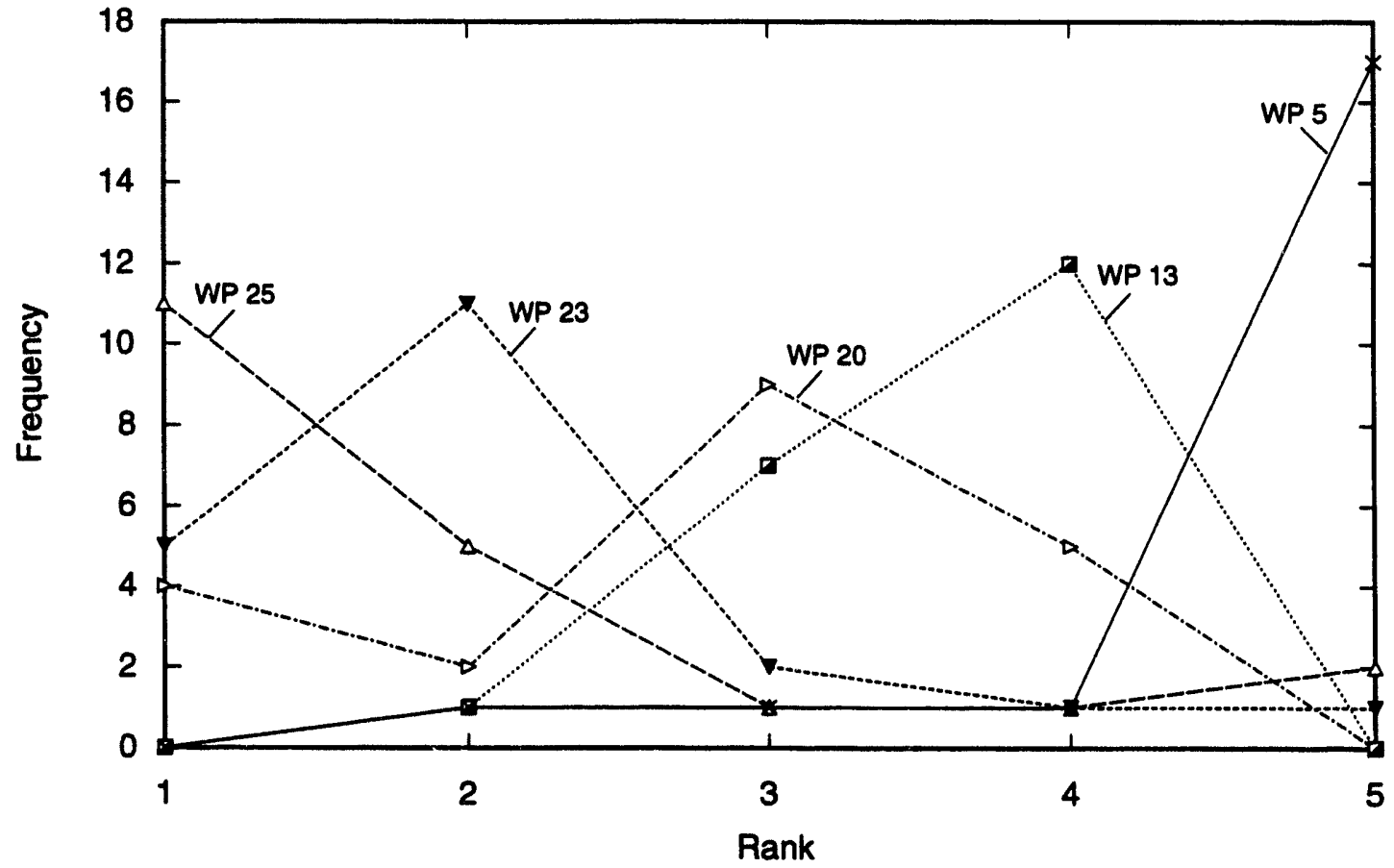

TR1-6342-3999-0

Figure 16.5-20. Frequency with which waste parcel fractional release at repositoru boundary for low enriched uranium spent fuel (WPs $5,13,20,23$, and 25) in granite disposal system was ranked with the least (rank 1) or most (rank 5) discharge. 


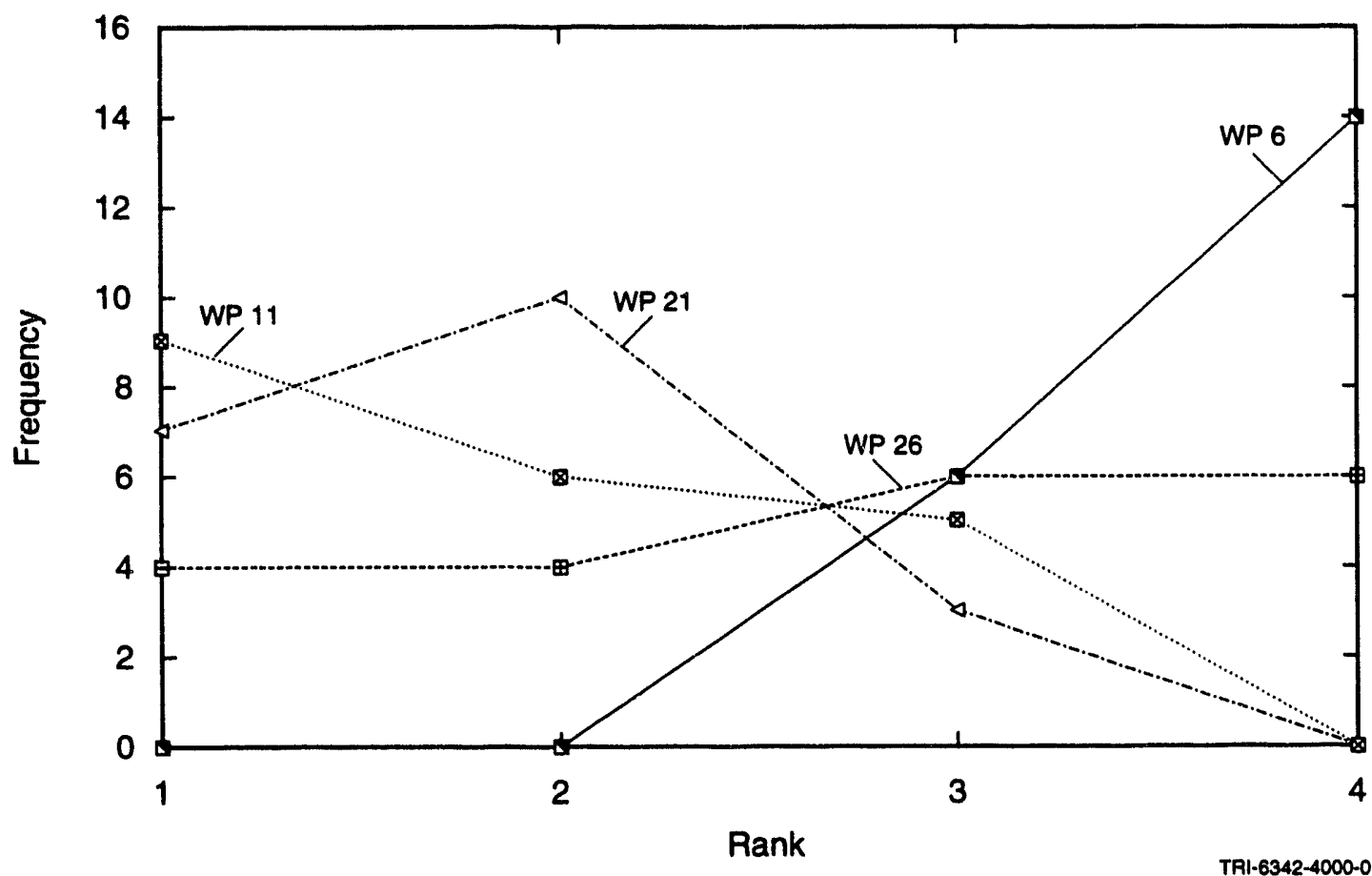

Figure 16.5-21. Frequency with which waste parcel fractional release at repository boundary for calcine (WPs 6 , 11,21 , and 26) in granite disposal system was ranked with the least (rank 1) or most (rank 5) discharge.

Activity discharge at the repository boundary of the granite disposal system for graphite spent fuel (Figures 16.5-14 and 16.5-18) shows large and consistent differences between waste parcels. Repository activity discharges are very similar to waste parcel activity discharges (Figure 16.5-4) for WPs 2, 3, 10, and 15, indicating little attenuation of activity discharge by the repository. Repository activity discharges from WPs 22 and 24 are similar to the waste parcel activity discharge at the high end of the distribution (below probabilities of 0.10 ), but are 2 to 3 orders of magnitude lower for the remaining sample sets indicating substantial attenuation of activity discharges by the repository for these waste parcels. This attenuation is reflected in the repository activity discharge rankings (Figure 16.5-18), with WP 22 providing the lowest activity discharge in 15 of the 20 sample vectors, and Waste Parcel 24 providing the second lowest activity discharge in 13 of the 20 sample sets. The mode-based ranks for the repository activity discharge are well defined for all waste parcels: from lowest to highest, WP 22, WP 24, WP 2, WP 10, WP 15 , and WP 3 . WP 3 produces the highest activity discharge in all of the 20 sample sets.

Activity discharges from WP $2,3,10$, and 15 are dominated by ${ }^{14} \mathrm{C} .{ }^{14} \mathrm{C}$ migration is not retarded by the bentonite backfill in the room. As discussed in Section 16.8.1, a large percentage of freely mobile radionuclides tends to be discharged from the granite disposal system because of the high flow rates calculated by GRFLOW. Nearly all of the ${ }^{14} \mathrm{C}$ discharged from a waste parcel is also discharged from the repository, therefore the integrated activity discharge from the repository for waste parcels with inventories dominated by ${ }^{14} \mathrm{C}$ approach the integrated activity discharge from the waste parcels themselves. ${ }^{14} \mathrm{C}$ has been removed from WPs 22 and 24 , and waste parcel activity discharges are dominated by americium, plutonium, thorium and neptunium. These elements are all retarded by the bentonite backfill-buffer, therefore the repository activity discharge is substantially lower than the waste parcel activity discharge for these waste parcels.

Activity discharge for highly enriched uranium spent fuel (Figures 16.5-15 and 16.5-19) shows greater attenuation due to repository transport (compare with Figure 16.5-5) than the graphite cases. Specifically, the maximum activity discharge for all waste parcels is approximately 10 equivalent waste parcels, while the minimum is less than $10^{-7}$ equivalent waste parcels, compared to the corresponding bounds of 40 and $2 \times 10^{-6}$ equivalent waste parcels 
1 (Figure 16.5-5). The separation between activity discharge distributions for HEU waste parcels is larger for repository activity discharge than for waste parcel activity discharge. Mode-based rankings from lowest activity discharge to highest activity discharge are WP 25, WP 23, WP 12, WP 19, and WP 4 (Figure 16.5-19). All but WP 19 occur in the most frequent rank in 12 of the 20 sample sets. The repository activity discharge ranking is better defined than the waste parcel activity discharge ranking (Figure 15.5-9), but differs in the order of WPs 4 and 19.

WPs 23 and 25 show greater attenuation of the waste parcel activity discharge by the repository than do the other three waste parcels. Waste Parcels 4,12 , and 19 contain small amounts of ${ }^{14} \mathrm{C}$, while WPs 23 and 25 do not. Because ${ }^{14} \mathrm{C}$ is not retarded by the repository backfill, ${ }^{14} \mathrm{C}$ is the dominant contributor to repository activity discharge in WPs 4,12 , and 19 for sample sets with low values of activity discharge, even though ${ }^{14} \mathrm{C}$ makes a negligible contribution to the waste parcel activity discharge. The ability of the repository to attenuate total integrated activity discharge from WPs 4,12 , and 19 is limited by the presence of trace amounts of ${ }^{14} \mathrm{C}$. In contrast, all radionuclides in WPs 23 and 25 are retarded by the repository backfill.

Activity discharges at the boundary of the repository for low enriched uranium spent fuel (Figures 16.5-16 and 16.5-20) are also more distinct than waste parcel activity discharges (refer to Figure 16.5-6). WPs 5 and 13 show virtually no attenuation of activity discharge between the waste parcel and repository. The repository activity discharge and waste parcel activity discharge distributions for WP 20 are very similar in the upper half of the distribution (probabilities below 0.5 ), while the repository activity discharge distribution for probabilities above 0.5 are shifted toward the minimum observed value. The repository activity discharges for WPs 23 and 25 are substantially lower than the waste parcel activity discharges, and generally much lower than repository activity discharges for the other waste parcels. The largest activity discharges for these two waste parcels, however, are greater than the largest activity discharges for the remaining waste parcels. The mode-based rankings for repository activity discharge are identical to the rankings for waste parcel activity discharge (WP 25, WP 23, WP 20, WP 13, and WP 5) (but are more clearly defined) and four of the five waste parcel ranks are consistently assigned in a majority of the sample sets.

As with the HEU waste parcels, the effect of repository transport on activity discharges from the LEU waste parcels is due to the presence of ${ }^{14} \mathrm{C}$. However, unlike the HEU waste parcels, ${ }^{14} \mathrm{C}$ is a major contributor to waste parcel activity discharge from WPs 5,13 , and 20 (waste parcels containing intact fuel assemblies). Of the waste parcels that contain intact fuel assemblies, there is 40,000 times as much ${ }^{14} \mathrm{C}$ in LEU waste parcels as in HEU waste parcels. The granite disposal system is therefore much less effective in attenuating total activity discharge from the LEU waste parcels containing intact fuel assemblies, than in attenuating activity discharge from HEU waste parcels containing intact fuel assemblies. Repository activity discharge from WPs 23 and 25 is substantially less than waste parcel activity discharge due to retardation of all contributing radionuclides in the repository backfill. The effects of extensive treatment on activity discharge are therefore larger for LEU than for HEU in the granite disposal system.

Repository activity discharges for waste parcels containing calcine (Figures 16.5-17 and 16.5-21) are more tightly grouped than activity discharge distributions for the other waste types. Large attenuation of activity discharge by the repository for all waste parcels is evident by comparing with Figure 16.5-7. Repository activity discharge ranks are similar to waste parcel activity discharge ranks: WP 6 has the largest activity discharge in a majority of the sample vectors; WPs 11 and 21 tend to have low activity discharge ranks, and WP 26 occurs in all ranks with nearly equal frequency.

Repository activity discharge distributions for all calcine waste parcels are also wider than the corresponding waste parcel activity discharge distributions. Waste parcel activity discharge for WP 6 varies over 5 orders of magnitude (Figure 16.5-7), while the WP 6 repository activity discharge range (Figure 16.5-17) is greater than 7 orders of magnitude. The overall reduction in repository activity discharge compared to waste parcel activity discharge is due to the retardation of the important radionuclides (isotopes of americium, plutonium, uranium, thorium, and neptunium) by the bentonite backfill-buffer, while the increase in activity discharge range is due to uncertainty in the corresponding adsorption coefficients. Because all calcine waste parcels have the same mix of radionuclides, repository transport has the same effect on all waste parcels. Differences in performance with respect to repository activity discharge are therefore due to differences evident in waste parcel activity discharge. 


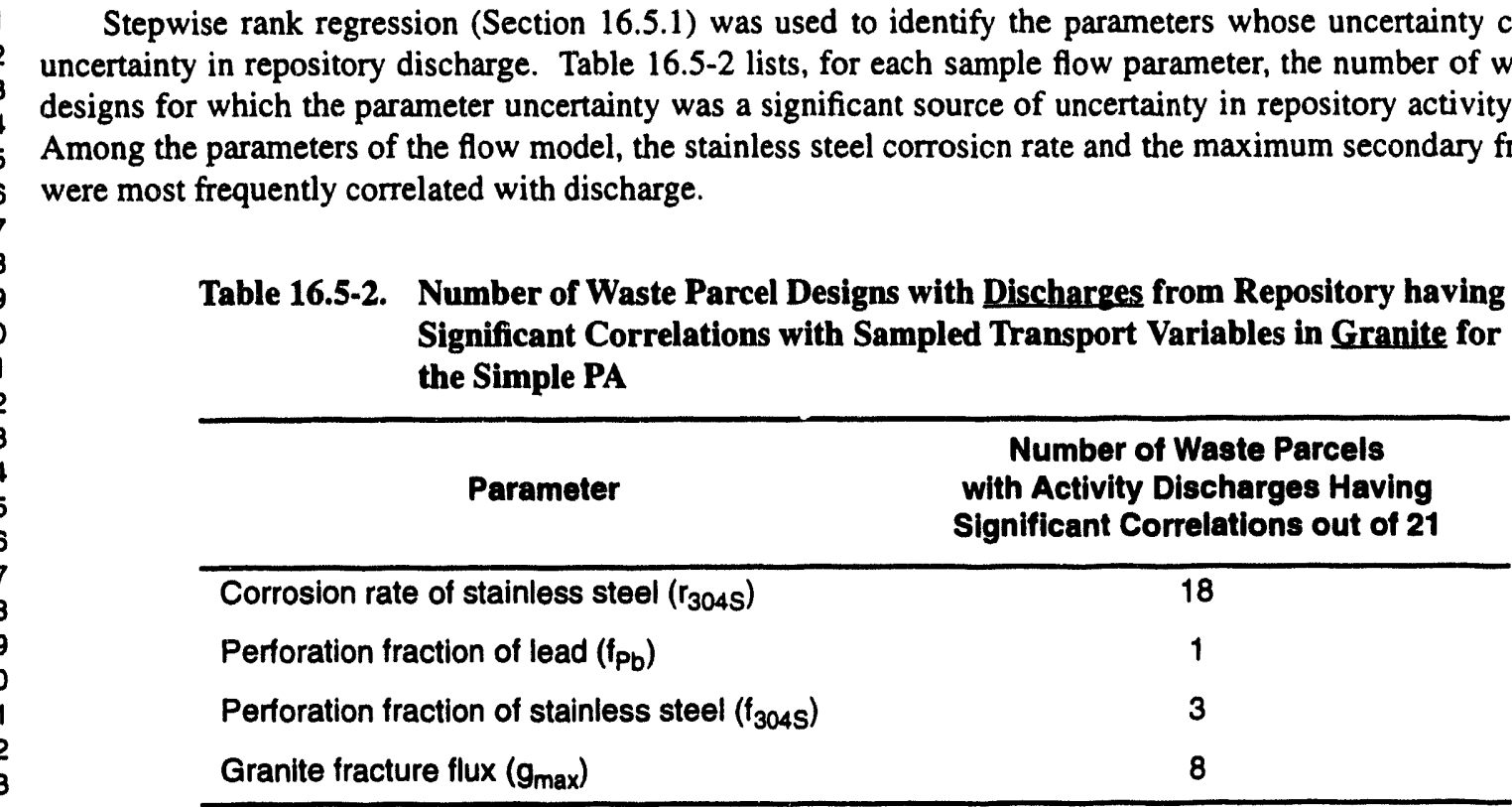

Similarly, Table 16.5-3 summarizes the regression of repository discharge against the sampled transport parameters. Americium retardation and graphite corrosion rate are most frequently correlated with total integrated discharge. The total activity discharge of individual radionuclides, however, was seen to be strongly controlled by the radionuclide adsorption coefficient $\left(\mathrm{Kd}_{\mathrm{Am}}\right)$.

Table 16.5-3. Number of Waste Parcel Designs with Discharges from Repository having Significant Correlations with Sampled Transport Variables in Granite for Simple PA

\begin{tabular}{lc}
\hline Parameter & $\begin{array}{c}\text { Number of Waste Parcels } \\
\text { with Activity Discharges Having } \\
\text { Significant Correlations out of 21 }\end{array}$ \\
\hline Alteration rate of graphite $\left(r_{\mathrm{c}}\right)$ & 3 \\
Alteration rate of glass $\left(\mathrm{r}_{\mathrm{glass}}\right)$ & 1 \\
Americium adsorption coefficient $\left(\mathrm{Kd}_{\mathrm{Am}}\right)$ & 5 \\
Solubility of uranium $\left(\mathrm{S}_{\mathrm{U}}\right)$ & 1 \\
\hline
\end{tabular}




\subsection{Breach Time of Containers (C. T. Stockman)}

The transport of radionuclides from a waste parcel requires that all of the layers surrounding the waste have been perforated and radionuclides are exposed to groundwater. The perforation of a waste parcel is dependent upon a number of factors including

1. Number of waste parcel layers.

2. The corrosion rate of material layers.

3. The perforation fraction of each layer.

4. The groundwater saturation.

We have chosen to downplay the role of the container by choosing stainless steel 304L, which is known to be susceptible to stress corrosion cracking along its welds. The use of lead filler, however, does give varying amounts of protection to a number of the different waste parcels.

Depending on the values of parameters sampled in this performance assessment, a range of breach times is observed for the waste parcels. Breach time is defined for each matrix within a waste parcel as the time when the last layer protecting the matrix is perforated (breached) and the matrix is exposed to groundwater. Breach times for the waste parcels in Waste Disposal Group 1 in salt and granite in both the Complex and Simple PAs are shown in Figures 16.6-1 through 16.6-4. In the Complex PA, BRAGFLO_T was used to calculate the saturation in each computational grid. In the Simple PA, the flow models, GRFLOW (for the granite repository) and SALFLOW (for the salt repository), assume that if the saturation in the drift is above $5 \%$, then the waste package boreholes are fully saturated. When the room/drift saturation is in the range of 0 to $5 \%$, the saturation in the waste package boreholes is linearly scaled from 0 to 1 . Consequently, the Simple PA uses a saturation of 1 in the waste package boreholes during most of the calculation and most of the runs.

The effect of the different methods of treating saturation is illustrated in the breach times calculated by the two methods. For the same waste group and the same repository type, the breach times calculated by the Simple PA fluid flow models are consistently earlier than those determined by the Complex PA fluid flow model, BRAGFLO_T (see Figures 16.6-1 through 16.6-4). This effect is much more pronounced for the salt disposal system where saturation tends to be lower, than in the granite disposal system. In fact, the times of matrix exposure for the two methods are nearly identical in granite, suggesting that the average saturation around the waste parcels in the Complex PA using BRAGFLO_T quickly exceeds residual saturation. Breach times also occur later when more than one layer surrounds the waste. For example, WPs $6,7,8$, and 9 all have a single layer of stainless steel, while WPs $1,2,3,4$, and 5 contain additional layers of aluminum and/or lead.

Figure 16.6-1, the exposure times from BRAGFLO_T in salt, shows some apparently contradictory results. For example, the exposure time for WP 1 is smaller than the exposure time for WP 7 in run 9. This result is an artifact of the way breach times are averaged over the entire repository for these plots. Figure 16.6-5 shows the exposure times for the waste parcels in a segment of a vertical column of the mesh for BRAGFLO_T. This column (i=11) passes through a side room with waste that is stored between rows $j=7$ and $j=10$ (see Figure 12-5). The cell where $j=7$ is the bottom cell, which is available only to the longest waste parcel. In the top cell, $j=10$, none of the waste parcels breaches in $10,000 \mathrm{yr}$, so these breach times are reported as 10,000. The average breach time for WP 1 is the average of two small numbers and 10,000 $\mathrm{yr}$ which is smaller than the average breach time for WP 7, which is the average of one small number and 10,000 yr. Hence, even though in any given cell, the breach time for WP 1 is greater than or equal to that for WP 7, WP 1 has a smaller average because the container is longer. It is clear that a straight average of the breach times gives misleading results when there is a large difference in lengths and breach times in the vertical direction as in the salt repository. Figures $16.6-6$ and 16.6-7 compare the average breach times with the minimum breach times in salt and granite. In the granite site, the minimum breach times are only slightly earlier than the average breach times, but in the salt site, the minimum breach times are significantly earlier than the average breach times, as expected.

The plots in Figures 16.6-1 and 16.6-2 show the runs sorted by the breach time using BRAGFLO_T for WP 7 (the Savannah River glass) in salt. Similarly, Figures $16.6-3$ and $16.6-4$ show the runs sorted by the breach times 


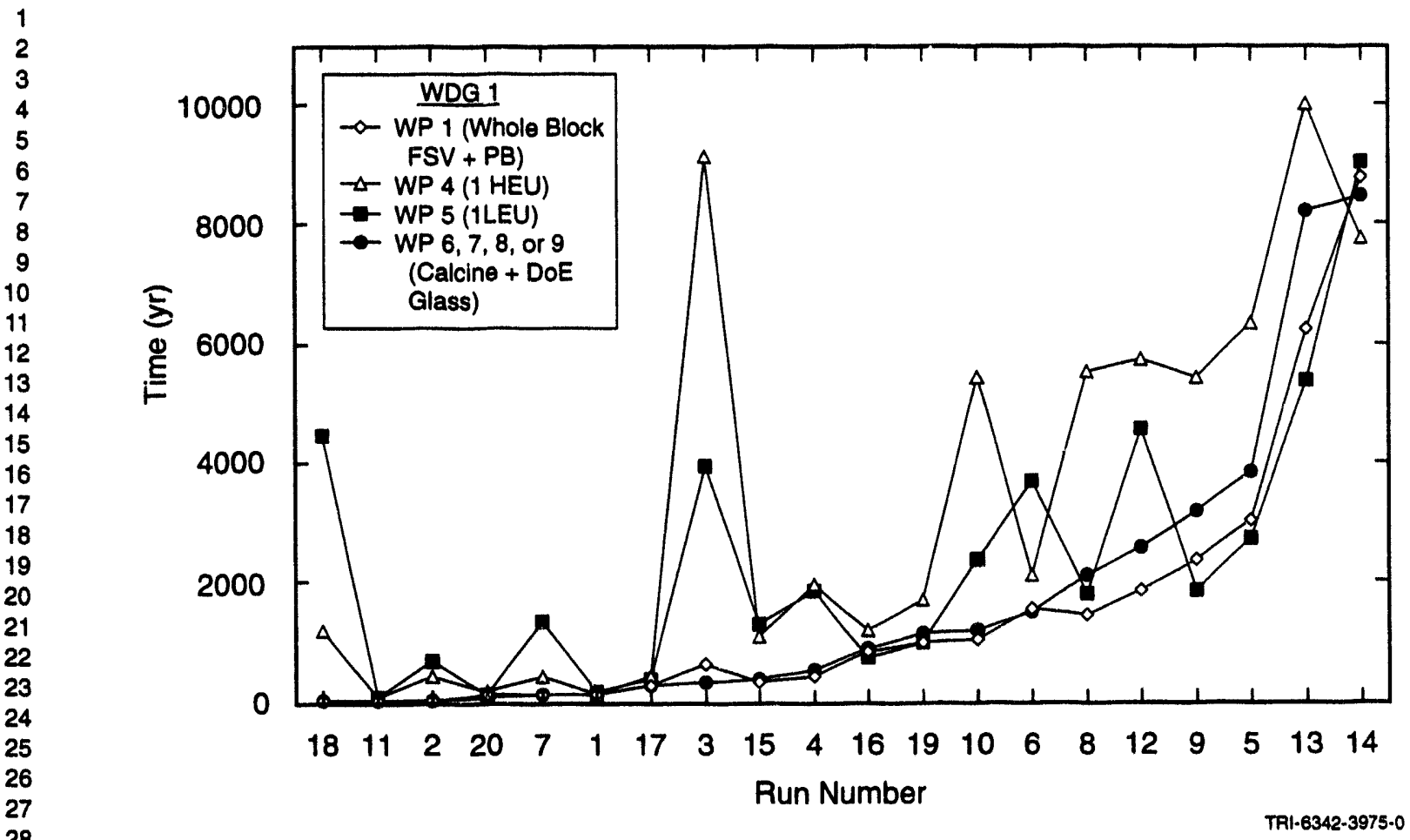

Figure 16.6-1. Mean time of perforation (breach) for all protective layers in all waste parcels in repository of Waste Disposal Group 1, in the Complex PA (BRAGFLO_T), salt disposal system. (Sorted by the BRAGFLO_T breach time of WP 7 in salt.)

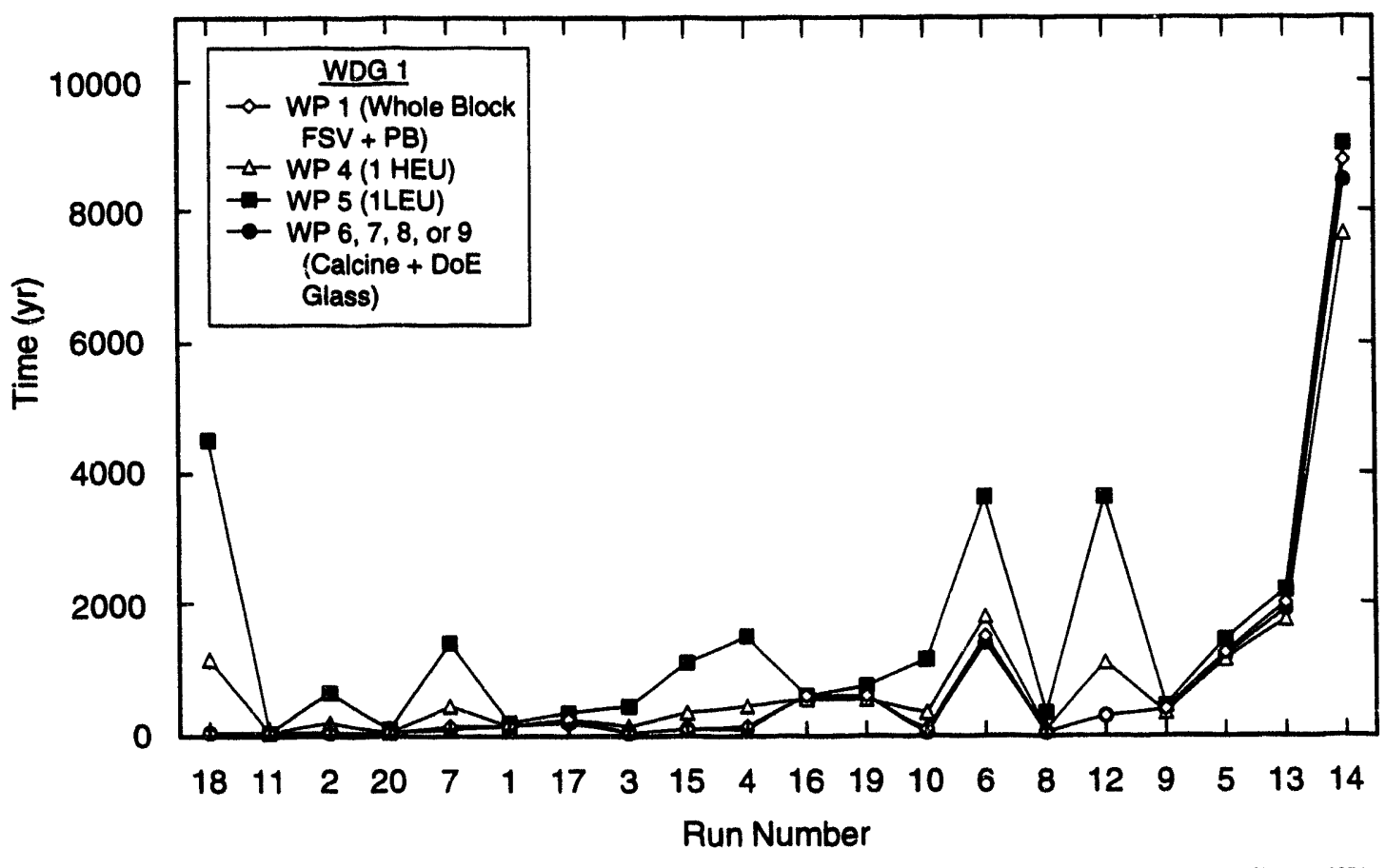

Figure 16.6-2. Mean time of perforation (breach) for all protective layers in all waste parcels in repository of Waste Disposal Group 1, in the Simple PA (SALFLOW), salt disposal system. (Sorted by the BRAGFLO_T breach time for WP 7 in salt.). 


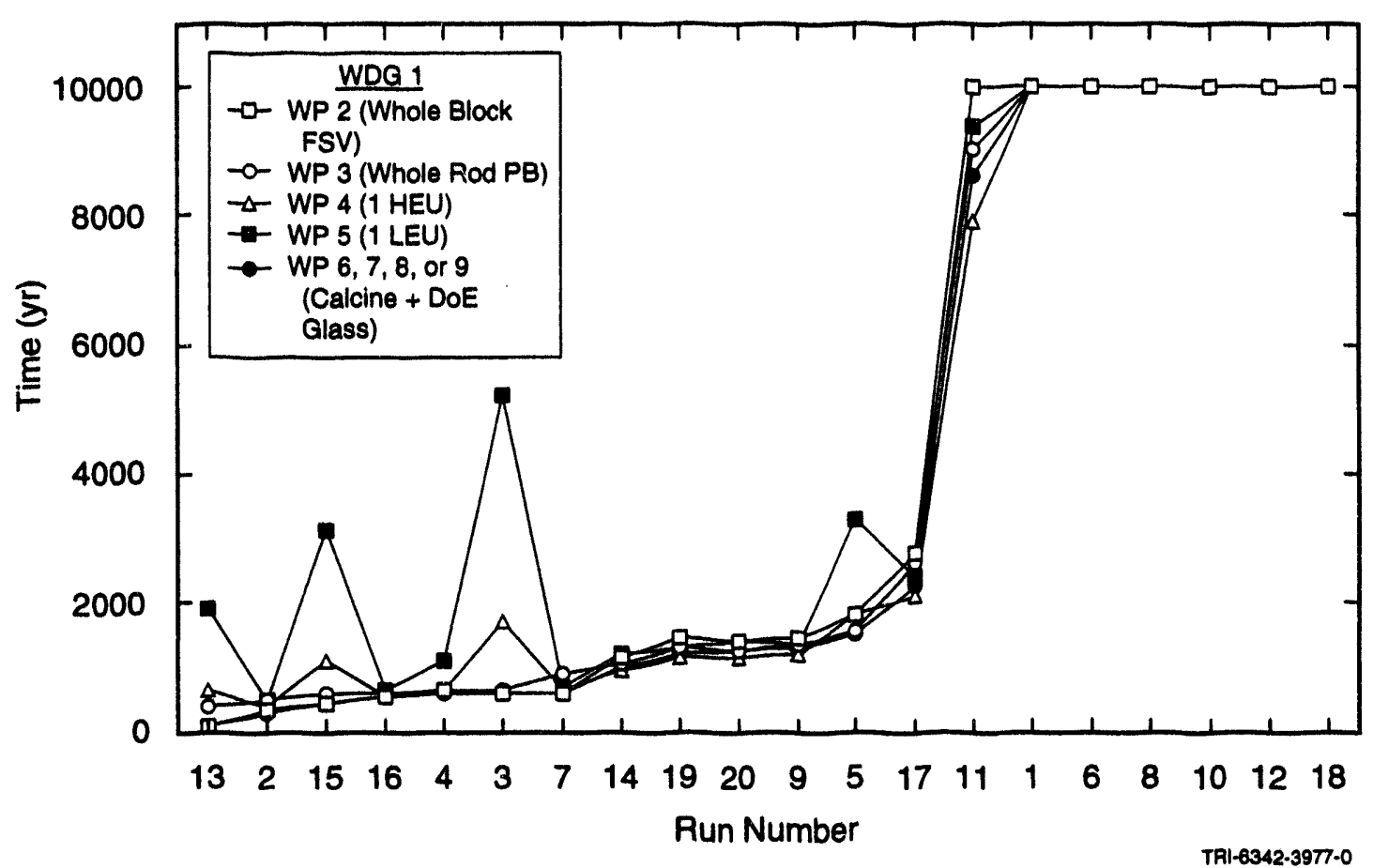

Figure 16.6-3. Mean time of perforation (breach) for all protective layers in all waste parcels in repository of Waste Disposal Group 1, in the Complex PA (BRAGFLO_T), granite disposal system. (Sorted by the BRAGFLO_T breach time for WP 7 in granite.).

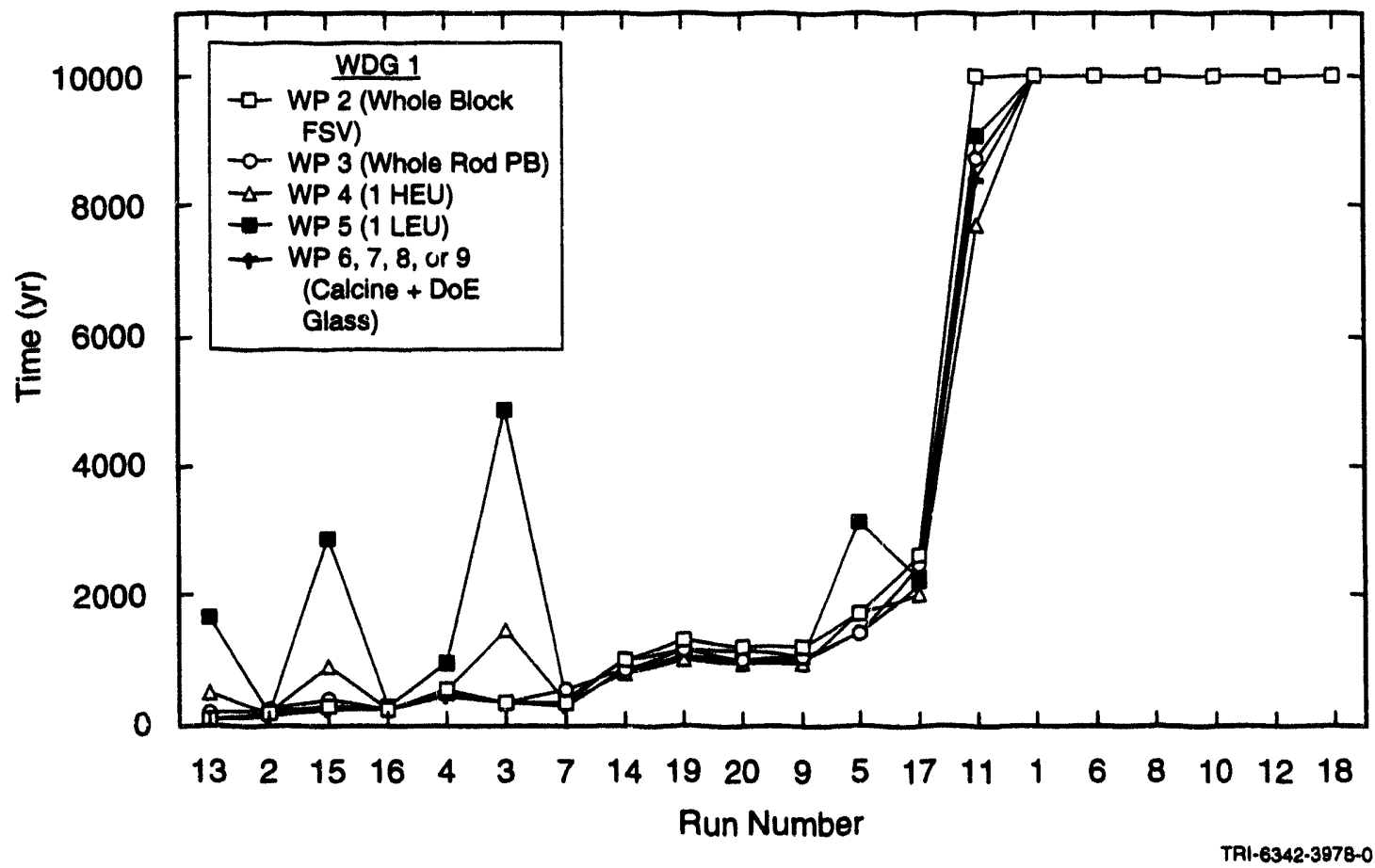

Figure 16.6-4. Mean time of perforation (breach) for all protective layers in all waste parcels in repository of Waste Disposal Group 1, in the Simple PA (GRFLOW), granite disposal system. (Sorted by the BRAGFLO_T breach time for WP 7 in granite.). 


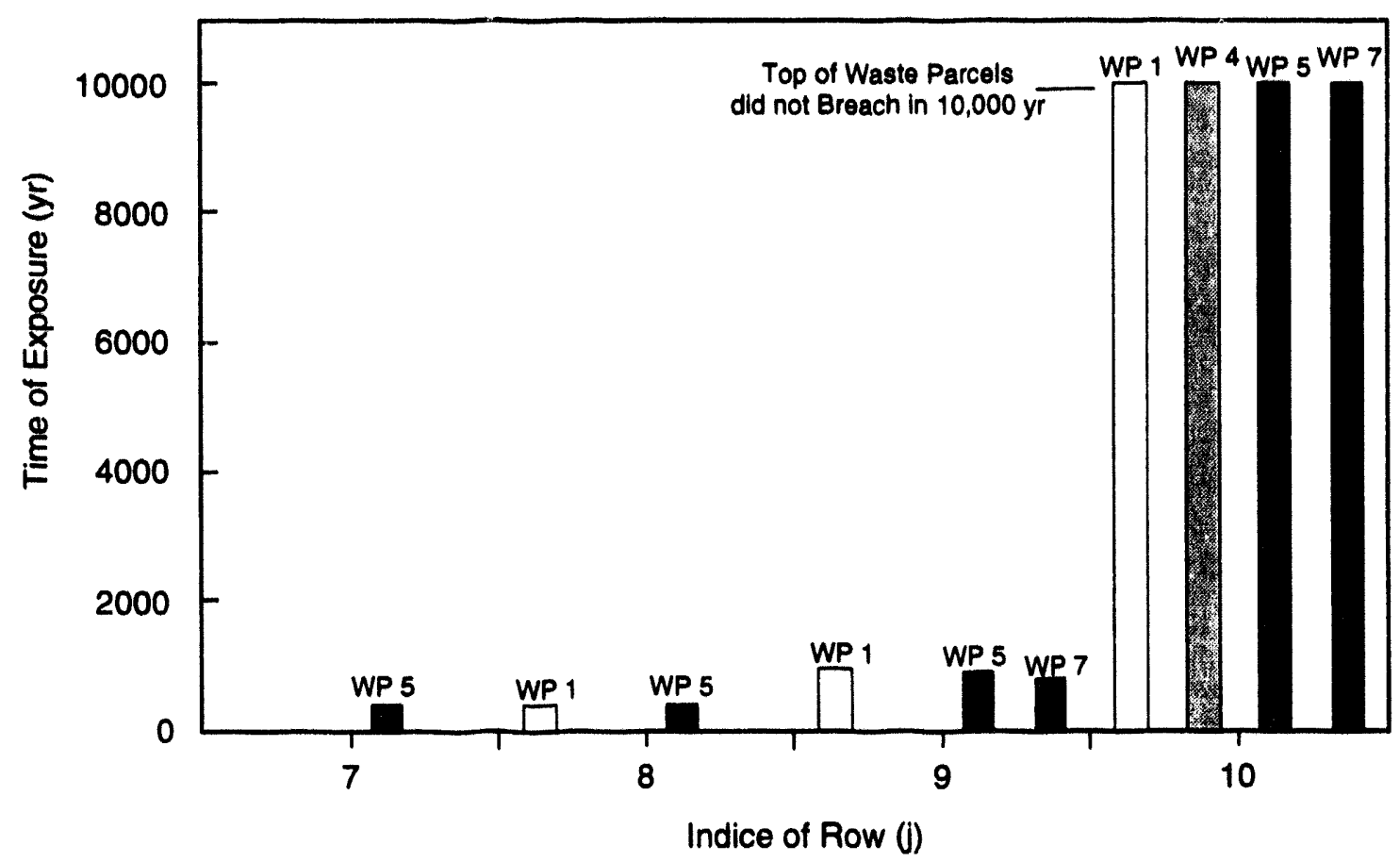

TR1-6342-3979-0

Figure 16.6-5. Exposure times in vertical cross-section (i=11) of Waste Disposal Group 1, in the Complex PA, for the salt disposal system. $(j=7$, bottom; $j=10$, top of waste parcel; refer to Figure 12-5.)

using BRAGFLO_T for WP 7 in granite. Since WP 7 has only a single layer of stainless steel, the sampled corrosion rates and perforation fractions for lead and aluminum do not affect the order. Because the fluid flow model parameters affect all the waste parcels equally, peaks in Figures 16.6-1 through 16.6-4 are due to increased breach time from slow penetration of lead, aluminum, or additional steel layers, as confirmed by examination of the sampled model parameters. The penetration rate when fully saturated for a layer is given by the fully saturated corrosion rate multiplied by the material density and divided by the perforation fraction. The fully saturated penetration rates were calculated from the sampled values for steel, lead, and aluminum, and the runs were ranked by the penetration rates (with Rank 1 having the fastest penetration). No correlation was found between breach time and aluminum penetration rate, but correlations were found for stainless steel and lead. Figures 16.6-8 and 16.6-9 show the penetration rate ranks for lead and steel in the same order as Figures 16.6-1 through 16.6-4. Figure 16.6-9 shows that the breach times of the single layer steel containers are well correlated with the rank of stainless steel penetration rate in the granite disposal system. The deviations from a linear plot for stainless steel are due to the sampling of the other parameters that affect saturation. The peaks in Figure 16.6-3 at runs 13, 15,3, and 5 correspond to slow lead penetration rates in Figure 16.6-9. The correlation between the breach time of the single layer steel containers and the rank of fully saturated stainless steel penetration rate is not as good in salt as it is in granite (see Figure 16.6-8), because saturation plays a much larger role in the calculated penetration rate for salt than for granite. Consequently, the correspondence between peaks in Figures 16.6-1 and 16.6-8 is not as good as the correspondence between the peaks in Figures 16.6-3 and 16.6-8. Still, runs 18, 2, 7, 6, and 12 show peaks on Figure 16.6-1 and correspond to slow lead penetration rates.

Figure 16.6-10a shows the breach times of WP 5 from the same run as Figure 16.6-5, but uses the distance from the bottom of WP 5 as the ordinate and includes the results from the main leg as well as the side leg of a room. In both cases, the bottoms breach relatively quickly, but the tops breach much later. This difference is due to brine flowing very slowly into the waste parcel holes from the bottom. The rate of filling the holes from the bottom is also slowed by the consumption of brine from the corrosion of the waste parcels. Because saturation near or below residual results in smaller calculated corrosion rates, the tops breach at much later times. The breach times of the top of the waste parcels are also quite different between the computational elements containing the side and main legs 


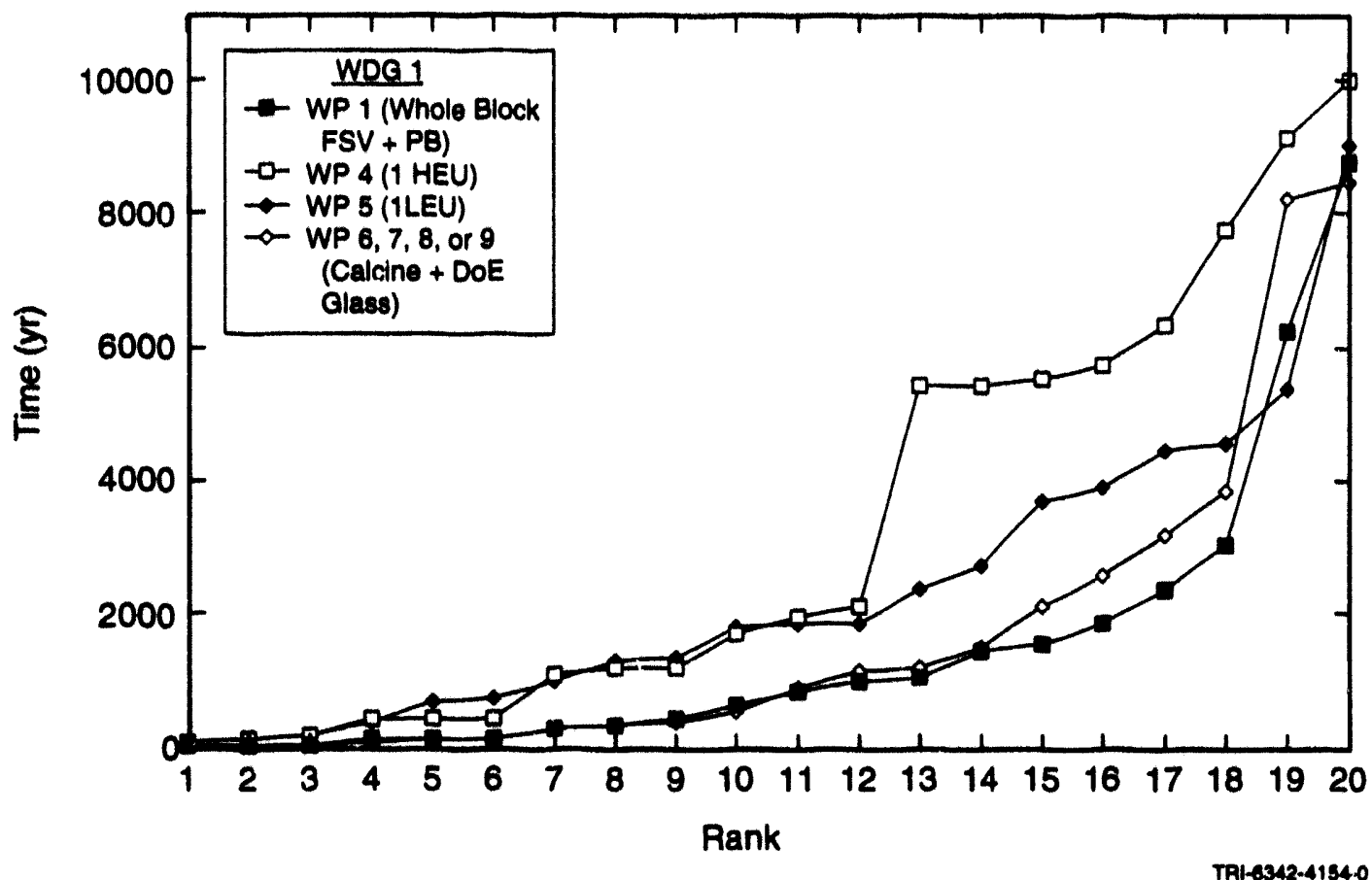

(a) Mean breach time

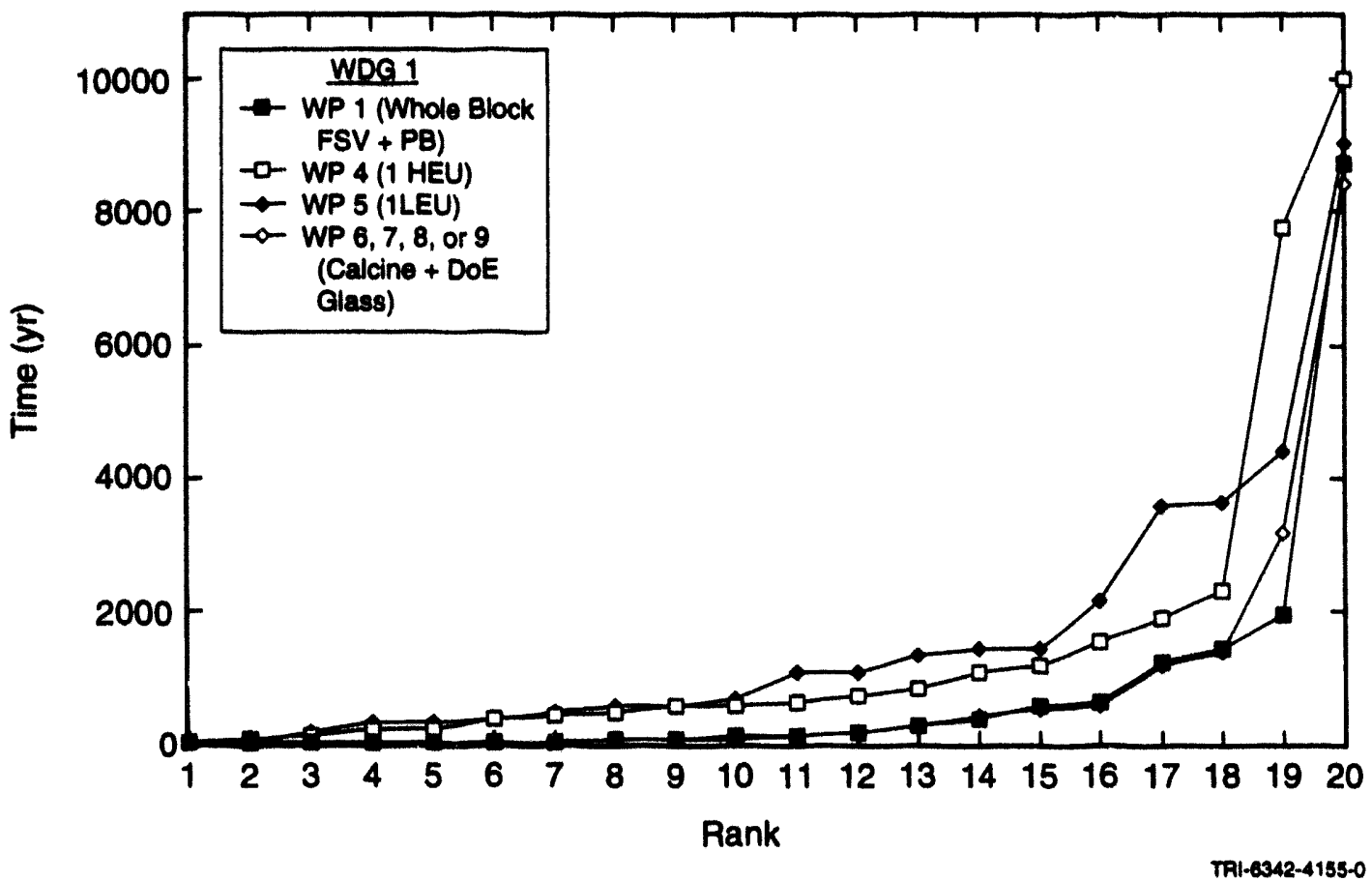

(b) Minimum breach time

Figure 16.6-6. Breach times for containers in Waste Disposal Group 1 in salt disposal system using Complex PA consequence models. Each waste parcel is sorted by ascending rank. (a) Mean breach times of all mesh elements for containers, and (b) minimum breach times predicted in any mesh element of container throughout the repository. 


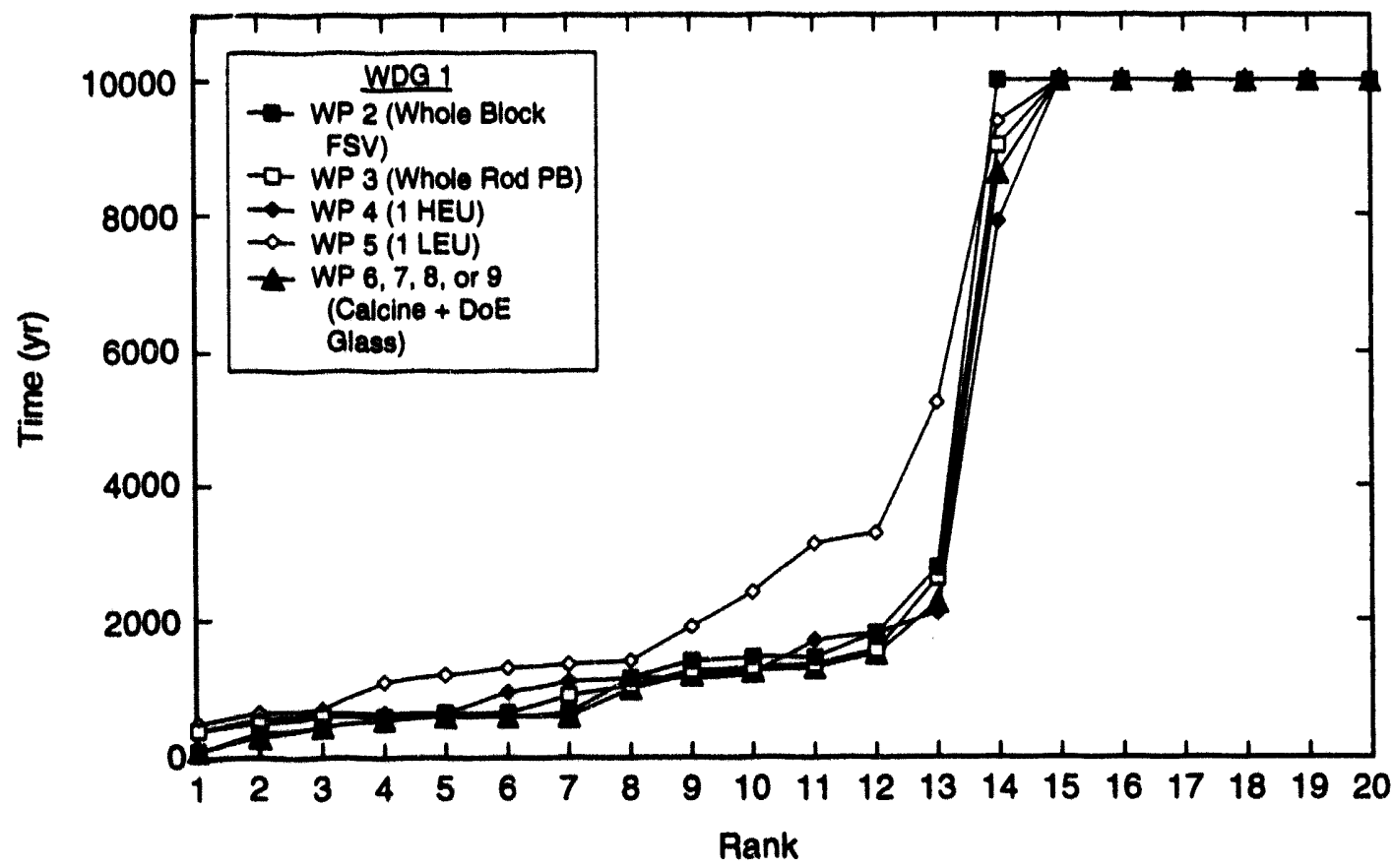

(a) Mean breach time

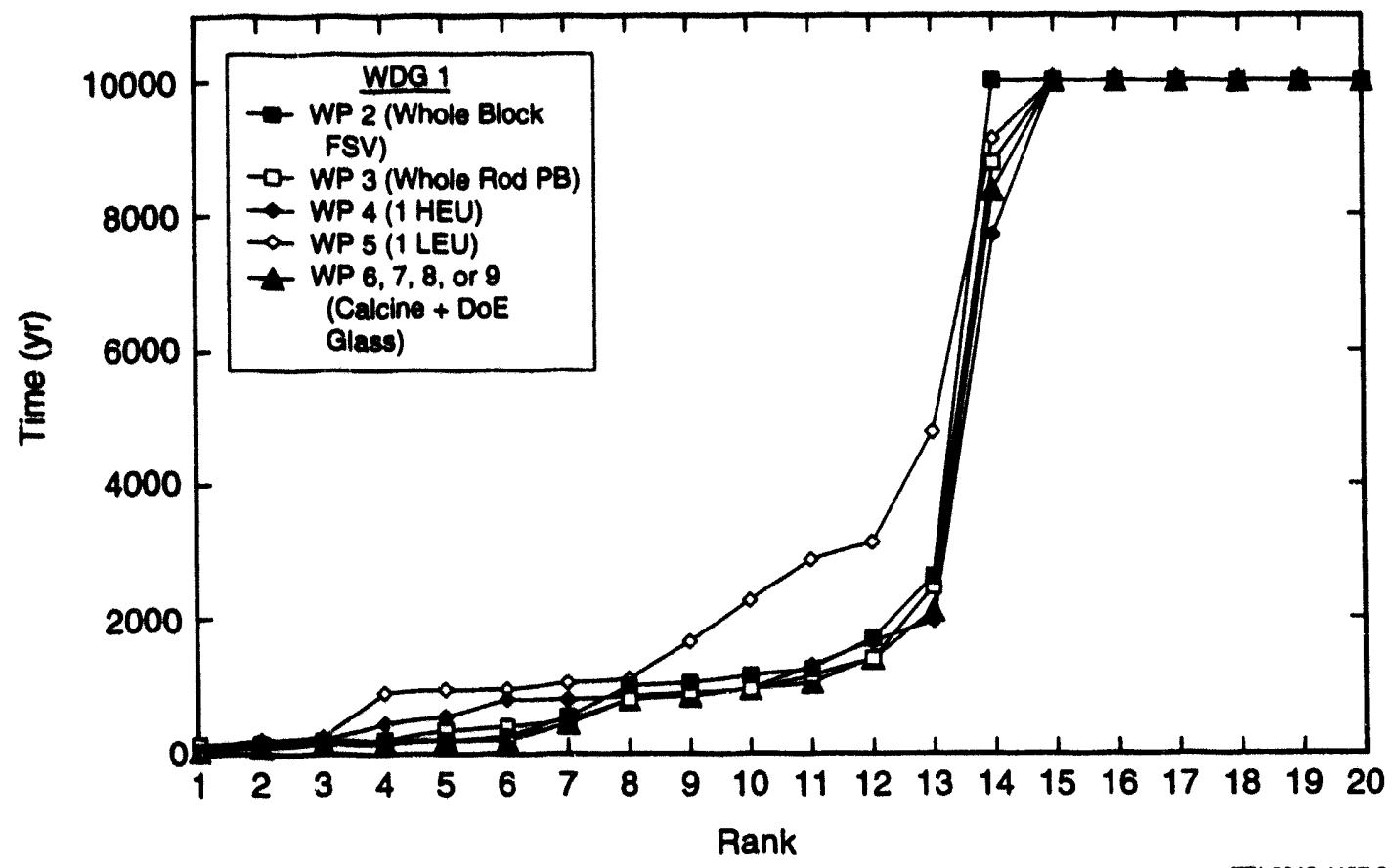

(b) Minimum breach time

Figure 16.6-7. Breach times for containers in Waste Disposal Group 1 in granite disposal system using Complex PA consequence models. Each waste parcel is sorted by ascending rank. (a) Mean breach times of all mesh elements for containers, and $(b)$ minimum breach times predicted in any mesh element of container throughout the repository. 
16. Results and Conclusions

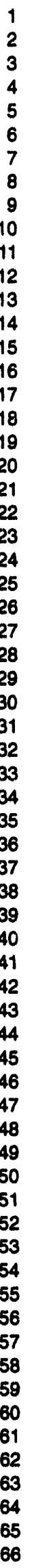

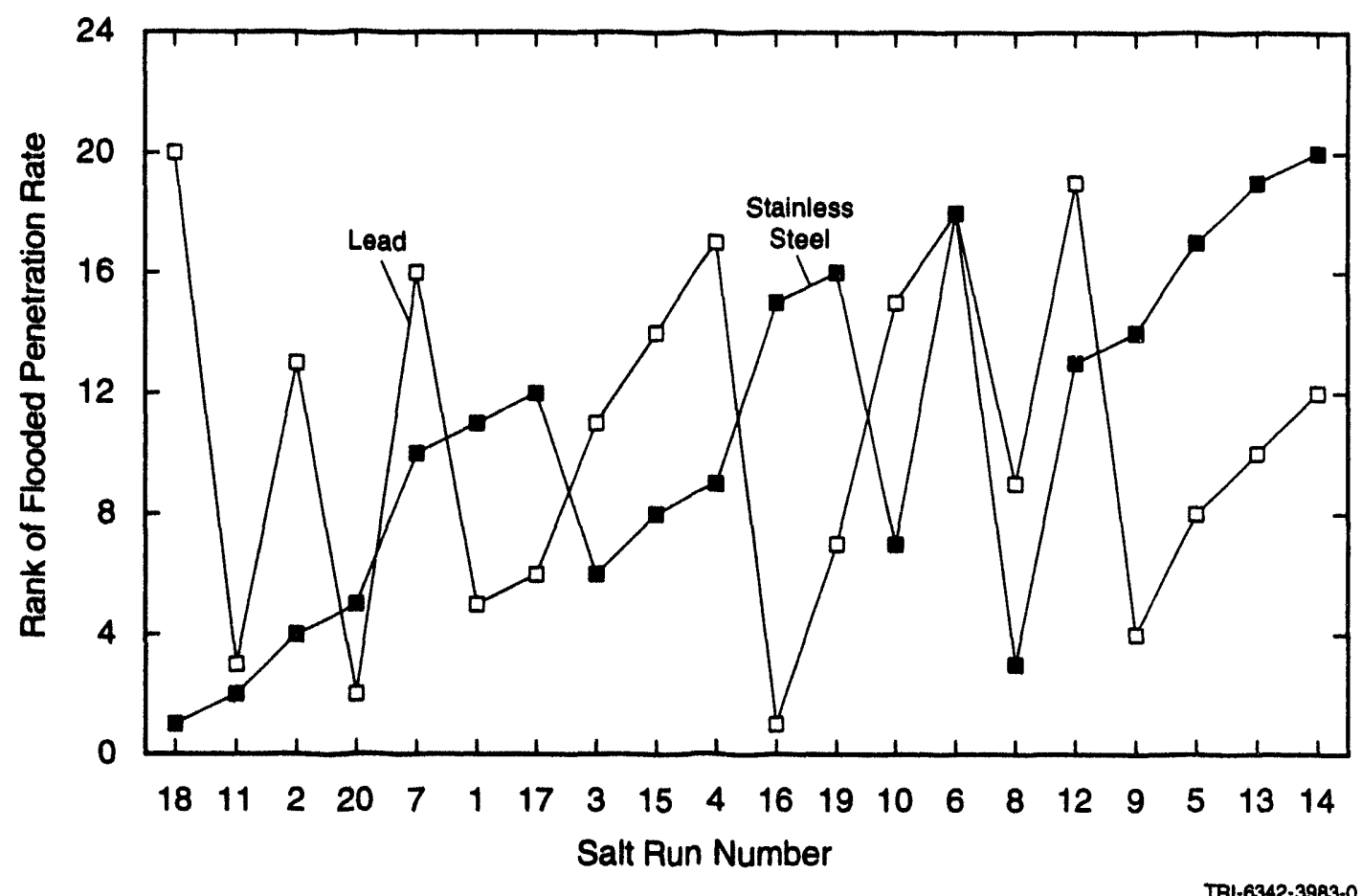

Figure 16.6-8. Rank of penetration rate assuming fully saturated conditions for stainless steel and lead for the salt disposal system. (Runs are shown in the same order as in Figures 16.6-1 and 16.6-2.)

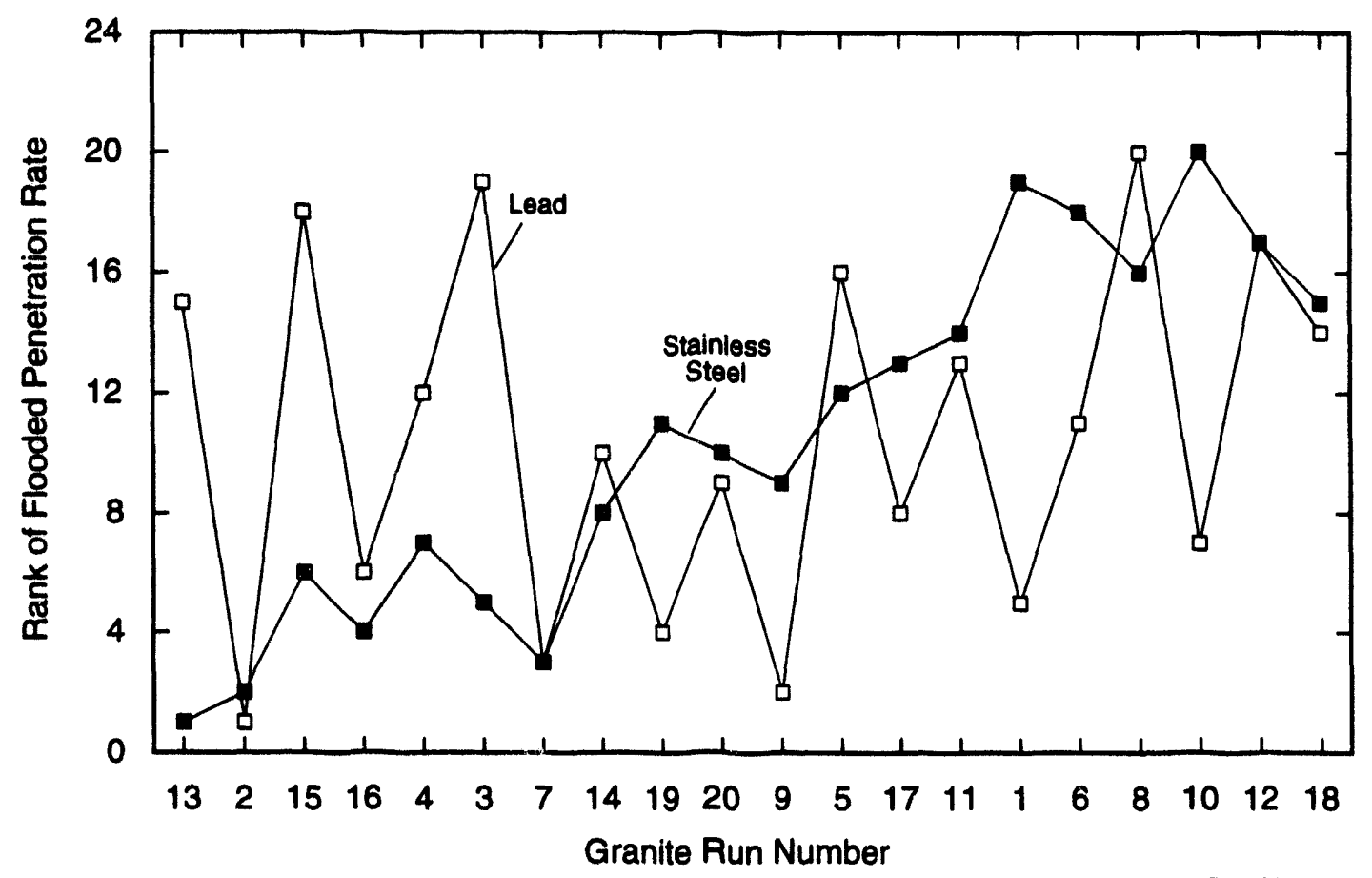

Figure 16.6-9. Rank of penetration rate assuming fully saturated conditions for stainless steel and lead for the granite disposal system. (Runs are shown in the same order as in Figures 16.6-3 and 16.6-4.) 


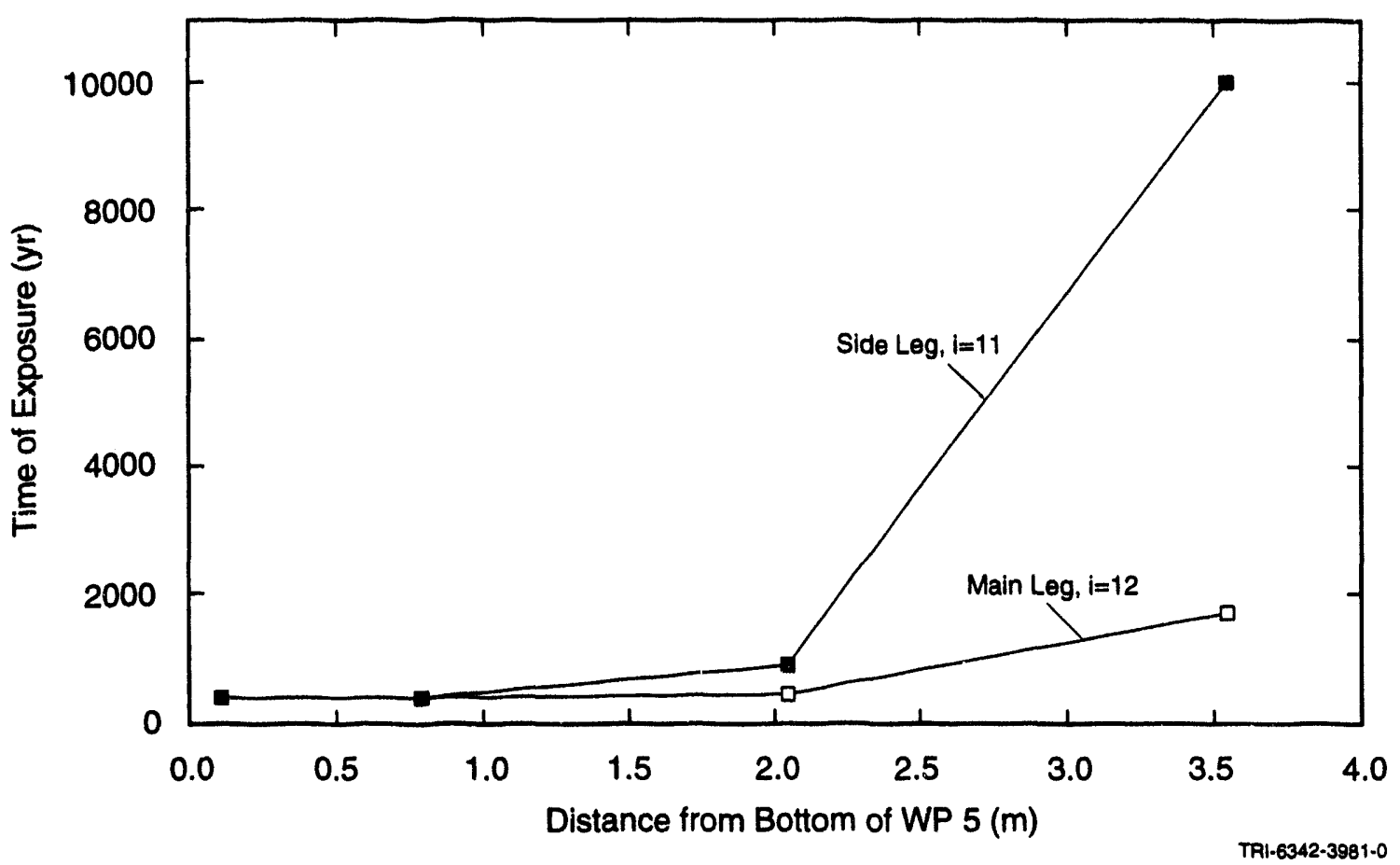

(a)

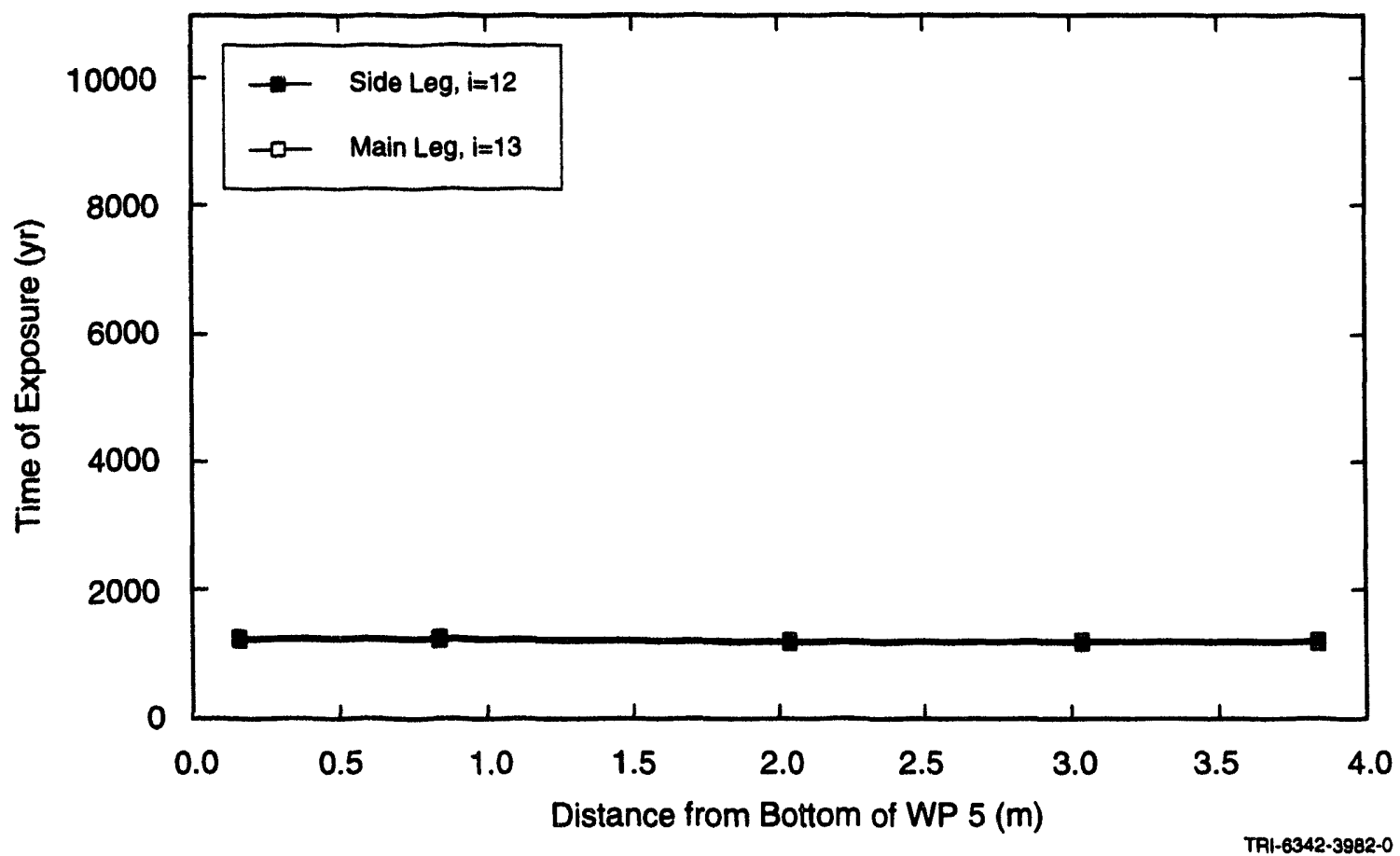

(b)

Figure 16.6-10. Exposure times for Waste Disposal Group 5, run 9, in the Complex PA (BRAGFLO_T), for (a) the salt disposal system and $(b)$ the granite disposal system. 
because of the difference in the element size and material properties. The main leg elements are 51 meters wide and include a cornbination of areas with waste package boreholes and solid salt pillars. The side leg elements are 9 meters wide and contain only areas with waste package boreholes. As brine flows in from the bottom, there is more flow per waste parcel in the main leg elements than the side legs, and thus the brine reaches the top cells in the main leg faster than in the side leg. Because the results from the side leg elements are not influenced by the averaging of properties between drifts and pillars, we expect the results from the side leg elements to be more representative of the real repository. However, when explaining results, the overall mean is still more representative of the release of radionuclides from the waste parcels in our model of the repository.

Figure 16.6-10b shows the breach times in the main and side rooms in the granite disposal system. As can be seen, the breach times are much more uniform vertically and between the side and main legs of the room because of fast resaturation in the granite site. Permeability within the drift is significantly larger in the granite site than in the salt site so resaturation is also a much more uniform process. The tops of the waste parcels breach at a slightly earlier time than the bottoms because the waste parcel holes are filled by flow from the room. The waste parcels in the side leg ( $i=12)$ breach slightly' before the waste parcels in the main leg ( $i=13)$ because they are closer to the shaft, which is the main source of water to refill the granite repository (Figure 13-3). 


\subsection{Calculations Using Corrosion-Resistant Waste Parcel (C. T. Stockman)}

Scoping calculations showed that the characteristics of multiple corrosion-resistant barriers dominated the resultant releases, masking the effects of waste form treatment (see Appendix C). The important characteristics included the generalized corrosion rate, the susceptibility to localized corrosion, and the probability of defects. Defects include manufacturing defects or mechanical damage occurring during transportation and emplacement. Because of this domination, calculations reported in other sections were performed on waste parcels that did not have corrosionresistant overpacks or canisters. However, two sets of calculations did use a corrosion-resistant waste parcel and the results are reported here.

The two corrosion-resistant materials chosen for the calculations were titanium Ti-Grade 12 and nickel alloy Inconel 625. Ti-Grade 12 was chosen for the overpack in the salt disposal system because of its superior performance in brine, Inconel 625 , which is less expensive and potentially available at INEL, was chosen for the inner layers of multiple resistant waste parcels and the overpack in the granite disposal system. Ti-Grade 12 has been ranked as the best material for the Yucca Mountain repository (Van Konynenburg et al., 1993), and Incoloy 825, which has properties that are similar but slightly better than Inconel 625, was ranked third. Both Inconel 625 and Ti-Grade 12 have very slow generalized corrosion rates; a $1-\mathrm{cm}$-thick container of either would not be breached by generalized corrosion in 10,000 yr. Because of these rates, other mechanisms of material penetration are extremely important. In these calculations, we have used conservative ranges for the perforation fractions for both Inconel 625 and Ti-Grade 12 (see Chapter 11). If, as reported, Ti-Grade 12 does not undergo crevice corrosion, and if other unknown mechanisms do not occur in $10,000 \mathrm{yr}$, then the only mechanism for breach in $10,000 \mathrm{yr}$ would be manufacturing defects or damage during transportation and emplacement.

The calculations used the consequence models from the Complex PA and Waste Disposal Group 1 for both salt and granite. In these calculations, the corrosion rates and perforation fractions were sampled for Ti-Grade 12, lead, Inconel 625 , and stainless steel $304 \mathrm{~L}$. The fraction of containers with defects in the outer layer (overpack) was fixed at $0.5 \%$, and the pour canister for the DOE glasses was changed to Inconel 625 in the granite disposal system. The materials used for layers of the individual waste parcels within the waste disposal groups are shown in order from the outside (see Table 16.7-1).

Figures 16.7-1 through 16.7-4 show the times of matrix exposure (breach time) for the waste parcels of Waste Disposal Group 1 in salt and granite for both the reference (i.e., stainless steel) and corrosion-resistant container. We have presented the breach times for each parcel in ascending order because, due to the necessary different sampling used in the corrosion-resistant container calculations, direct comparison of individual simulations between the reference and corrosion containers is not possible.

Figure 16.7-1 shows the exposure times for the waste parcels without any manufacturing or damage defects in salt. Adding corrosion-resistant materials greatly increases the performance of the parcels: in many runs, the matrixes are never exposed (shown as breach times of 10,000 yr). An additional feature of the corrosion-resistant results is that the DOE glasses are exposed significantly earlier than the INEL waste in 9 out of 18 runs. This earlier exposure is because of the lack of a second corrosion-resistant layer in the DOE glass waste parcels. This condition is especially significant for the three runs in which the DOE glass is the only matrix to be exposed in $10,000 \mathrm{yr}$. Looking at Figure 16-7-1 alone, one might conclude that there are no releases from the corrosion-resistant parcels from many of the simulations in salt; however, examination of the breach times for waste parcels that have a defect in their outermost layer shows that this is not the case (Figure 16.7-2). In all runs, the DOE glass matrices are exposed at early times when the only layer that is corrosion-resistant is defective.

Figure 16.7-5 shows the CCDFs for activity discharge from the salt disposal system for the corrosion-resistant and reference waste parcels of Waste Disposal Group 1. For most of the simulations with large discharges at the repository boundary, the corrosion resistance lowers the releases by 1.5 orders of magnitude, but for three of the simulations with small discharges, the corrosion-resistant parcels actually result in larger releases. This most likely is the result of the different combination of parameters in the two sample sets. Based on the matrix inventories and matrix exposed times alone, one would predict that the lower activity discharges would be up to three orders of magnitude lower for the corrosion-resistant parcels. However, the matrix alteration times and solubility also control the 
16. Results and Conclusions

Table 16.7-1. Materials Used in Layers for Individual Corrosion-Resistant and Reference Waste Parcels

\begin{tabular}{|c|c|c|c|}
\hline $\begin{array}{c}\text { Waste } \\
\text { Parcel } \\
\text { No. }\end{array}$ & Contents & $\begin{array}{l}\text { Layers for Corrosion-Resistant } \\
\text { Waste Parcel }\end{array}$ & $\begin{array}{l}\text { Layers for Reference } \\
\text { (Minimal) Waste Parcel }\end{array}$ \\
\hline \multicolumn{4}{|l|}{ Salt } \\
\hline WP 1 & Graphite spent fuel & $\begin{array}{l}\text { Ti-Grade 12, Lead, Inconel } 625 \text {, } \\
\text { Aluminum, Carbon }\end{array}$ & SS-304L, Aluminum, Carbon \\
\hline WP 4 & $\begin{array}{l}\text { Highly enriched special } \\
\text { spent fuel }\end{array}$ & $\begin{array}{l}\text { Ti-Grade 12, Lead, Inconel } 625 \text {, } \\
\text { Lead, Aluminum }\end{array}$ & SS-304L, Lead, Aluminum \\
\hline WP 5 & $\begin{array}{l}\text { Low enriched special } \\
\text { spent fuel }\end{array}$ & $\begin{array}{l}\text { Ti-Grade 12, Lead, Inconel } 625 \text {, } \\
\text { Lead, Zirconium }\end{array}$ & SS-304L, Lead, Zirconium \\
\hline WP 6 & Calcine & Ti-Grade 12, Lead, Inconel 625 & SS-304L \\
\hline WP 7 & Savannah River & Ti-Grade 12, Lead, SS-304L & SS-304L \\
\hline WP 8 & Hanford & Ti-Grade 12, Lead, SS-304L & SS-304L \\
\hline WP 9 & West Valley & Ti-Grade 12, Lead, SS-304L & SS-304L \\
\hline \multicolumn{4}{|l|}{ Granite } \\
\hline WP 2 & Fort St. Vrain & $\begin{array}{l}\text { Inconel 625, Lead, Inconel } 625 \text {, } \\
\text { Carbon }\end{array}$ & SS-304L, Carbon \\
\hline WP 3 & Peach Bottom & $\begin{array}{l}\text { Inconel 625, Lead, Inconel 625, } \\
\text { Aluminum, Carbon }\end{array}$ & SS-304L, Aluminism, Carbon \\
\hline WP 4 & $\begin{array}{l}\text { Highly enriched special } \\
\text { spent fuel }\end{array}$ & $\begin{array}{l}\text { Inconel 625, Lead, Inconel } 625 \text {, } \\
\text { Lead, Aluminum }\end{array}$ & SS-304L, Lead, Aluminum \\
\hline WP 5 & $\begin{array}{l}\text { Low enriched special } \\
\text { spent fuel }\end{array}$ & $\begin{array}{l}\text { Inconel 625, Lead, Inconel 625, } \\
\text { Lead, Zirconium }\end{array}$ & SS-304L, Lead, Zirconium \\
\hline WP 6 & Calcine & Inconel 625, Lead, Inconel 625 & SS-304L \\
\hline WP 7 & Savannah River & Inconel 625, Lead, Inconel 625 & SS-304L \\
\hline WP 8 & Hanford & Inconel 625, Lead, Inconel 625 & SS-304L \\
\hline WP 9 & West Valley & Inconel 625, Lead, Inconel 625 & SS-304L \\
\hline
\end{tabular}

discharge of many radionuclides and places a limit on the releases from the reference parcels, so this maximum spread is not realized.

Figure 16.7-3 shows the breach times for both the reference and corrosion-resistant containers from the granite disposal system. Here we see more improvement in the waste parcel performance than at the salt site. This improvement is mainly because of the use of two corrosion-resistant layers in all the DOE waste parcels, not just the INEL parcels. Thus, even in the waste parcels with defects in the outermost layers (Figure 16-7-4), there is still an intact corrosion-resistant inner layer that protects the matrix for over $10,000 \mathrm{yr}$ in 13 out of 20 simulations. This behavior can be seen in Figure 16.7-6 where only 7 simulations have a nonzero release from the corrosion-resistant parcels.

From the above results, it appears that multiple layers of corrosion-resistant materials can have an overriding influence on the releases from a disposal system in $10,000 \mathrm{yr}$. It is important to note, however, that unless defects can be reduced significantly below $0.5 \%$, or unless localized corrosion can be demonstrated to have a very low probability, two layers of corrosion resistant layers are necessary to ensure that most parcels will not breach within $10,000 \mathrm{yr}$. 


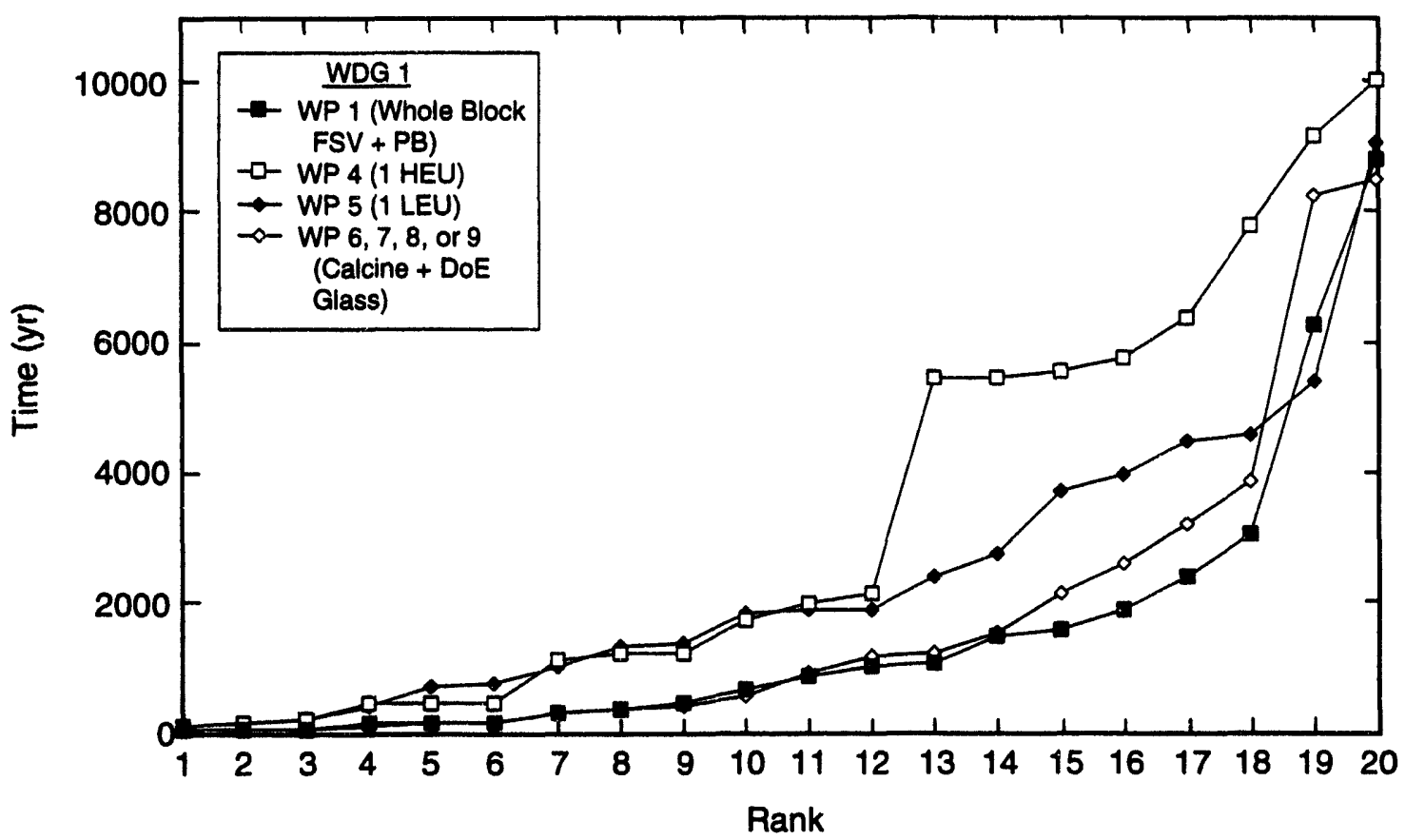

TR1-6342-4158-0

(a)

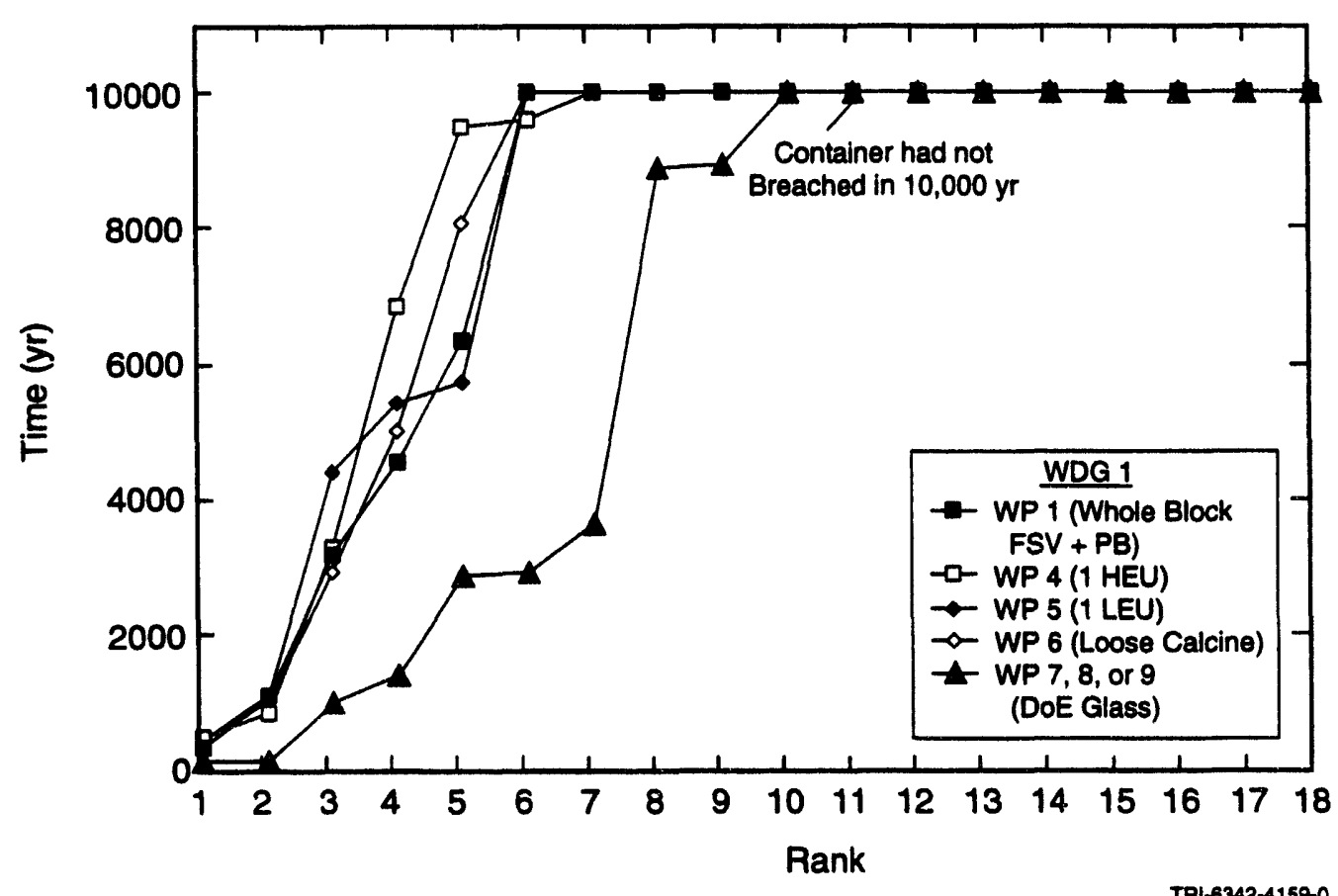

(b)

Figure 16.7-1. Mean breach times of all mesh elements for containers for waste parcels in Waste Disposal Group $I$ in salt disposal system using Complex PA consequence models. (a) Reference containers (repeat of Figure 16.6-6a) and (b) corrosion-resistant containers. Each waste parcel is sorted by ascending rank because comparisons by simulation number are not possible. 


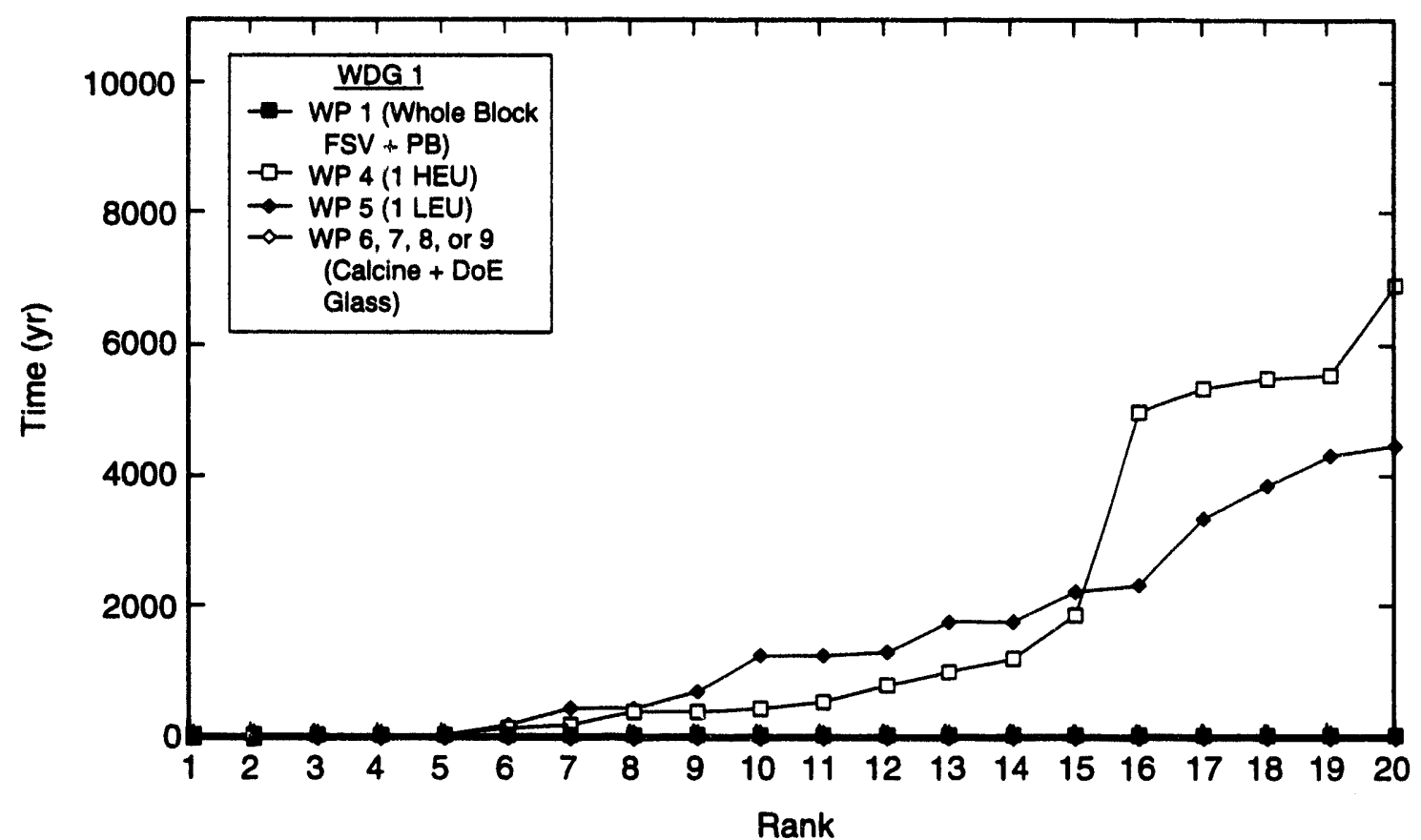

TRI-6342-4160-0

(a)

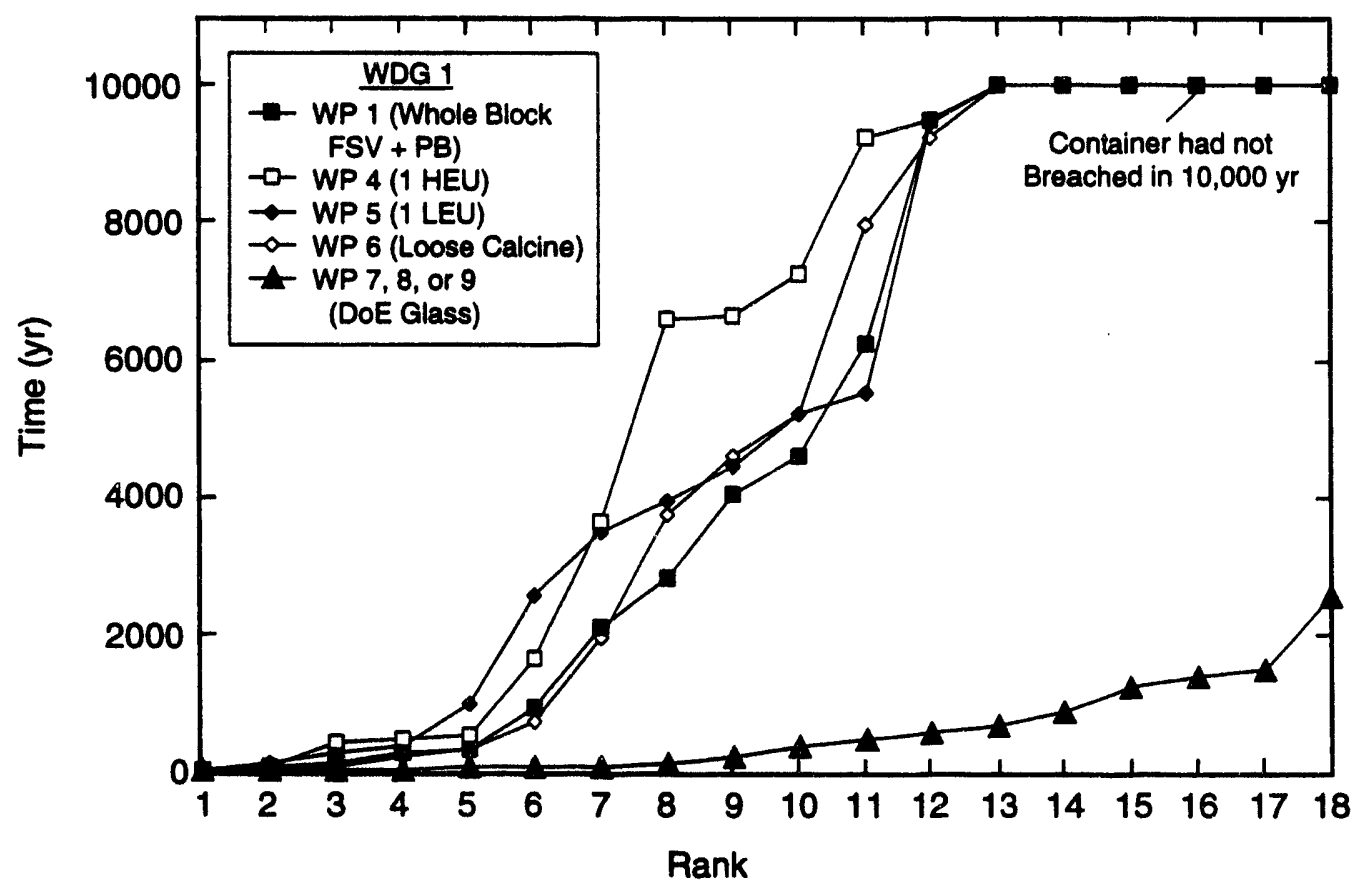

(b)

Figure 16.7-2. Mean breach times of all mesh elements for containers that have defects (median of $0.5 \%$ ) for waste parcels in Waste Disposal Group 1 in salt disposal system using Complex PA consequence models. (a) Reference containers (repeat of Figure 16.6-6a) and (b) corrosion-resistant containers. Each waste parcel is sorted by ascending rank because comparisons by simulation number are not possible. 


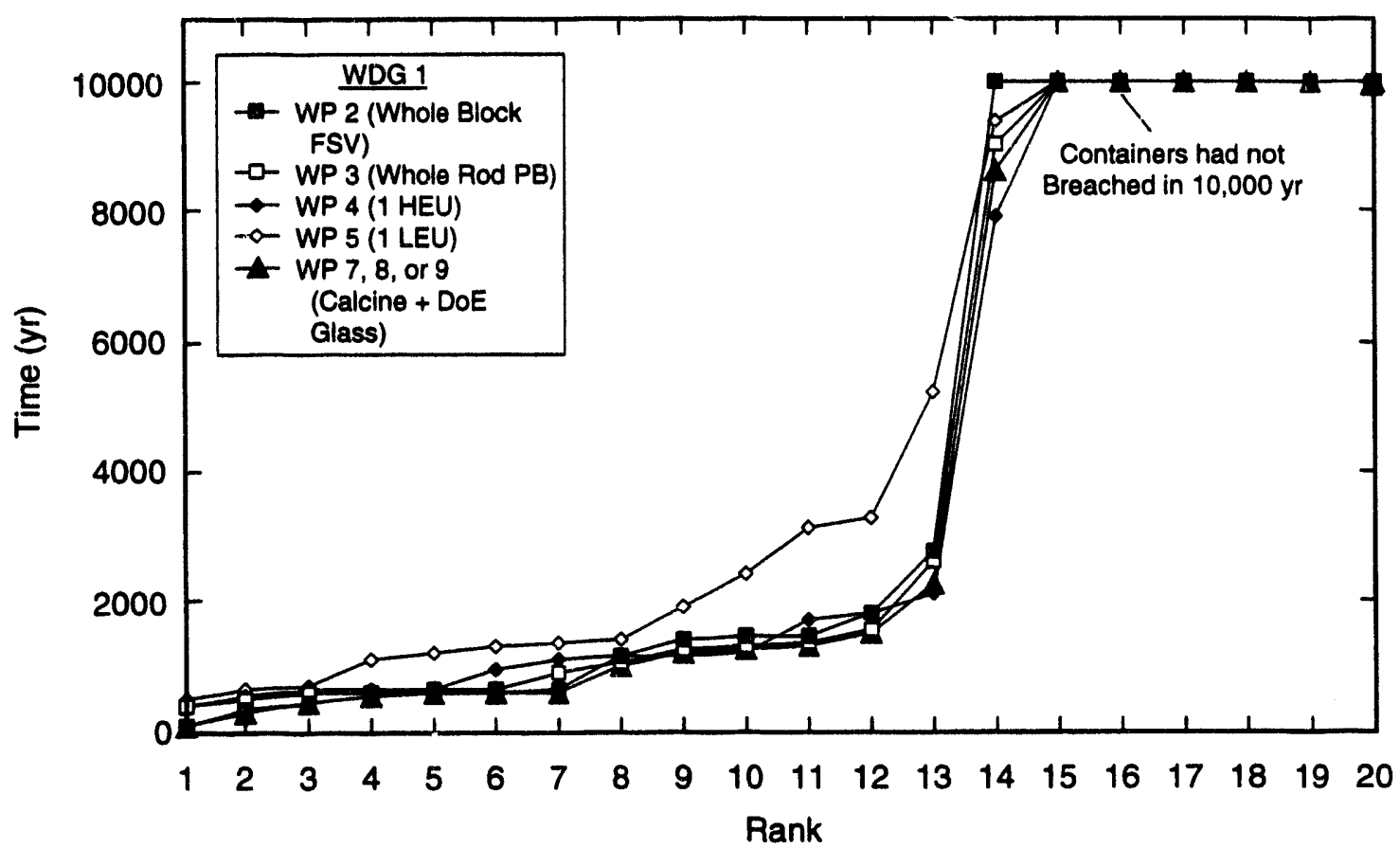

(a)

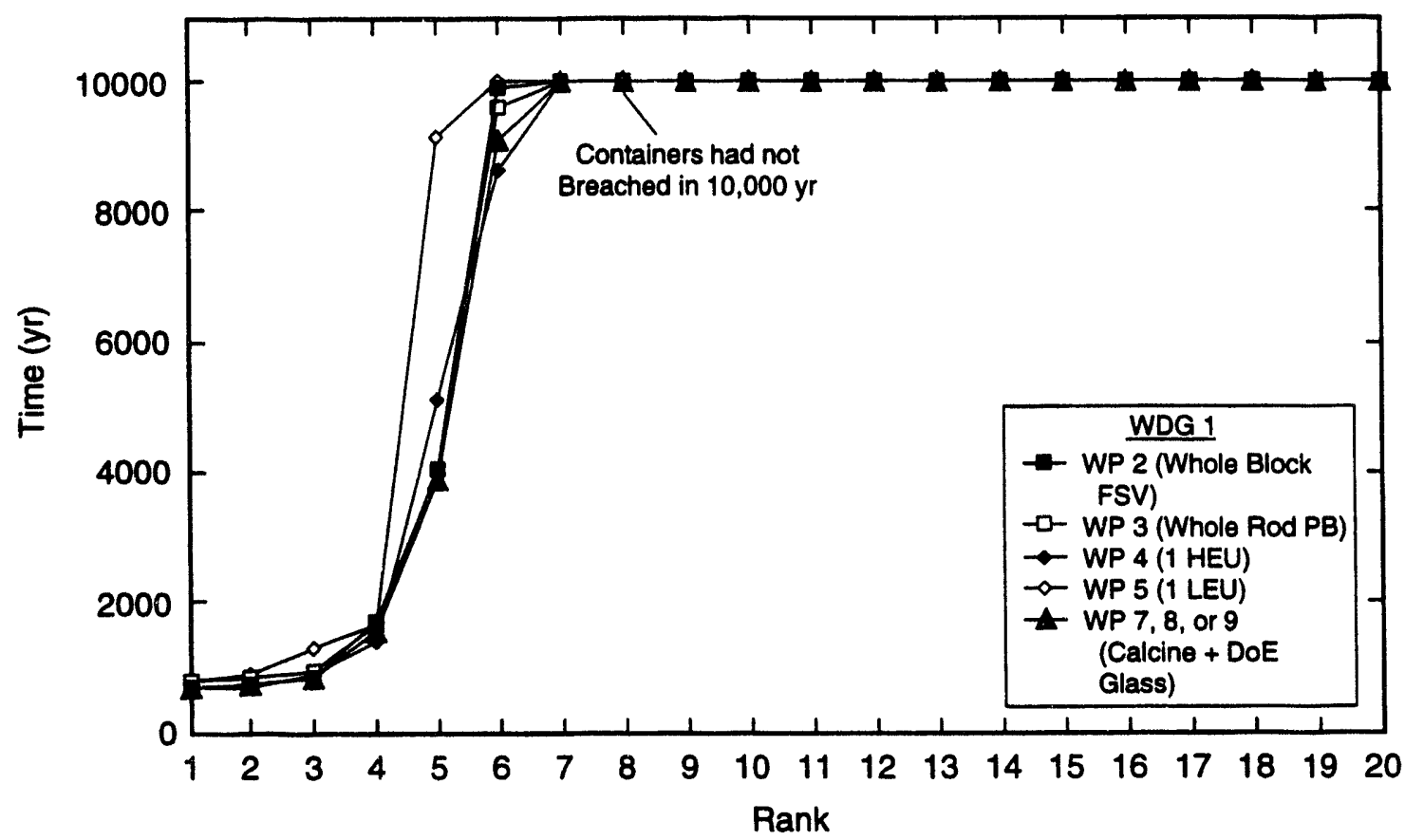

TRI-6342.4163.0

(b)

Figure 16.7-3. Mean breach times of all mesh elements for containers for waste parcels in Waste Disposal Group 1 in granite disposal system using Complex PA consequence models. (a) Reference containers (repeat of Figure 16.6-7a) and (b) corrosion-resistant containers. Each waste parcel is sorted by ascending rank because comparisons by simulation number are not possible. 
16. Results and Conclusions

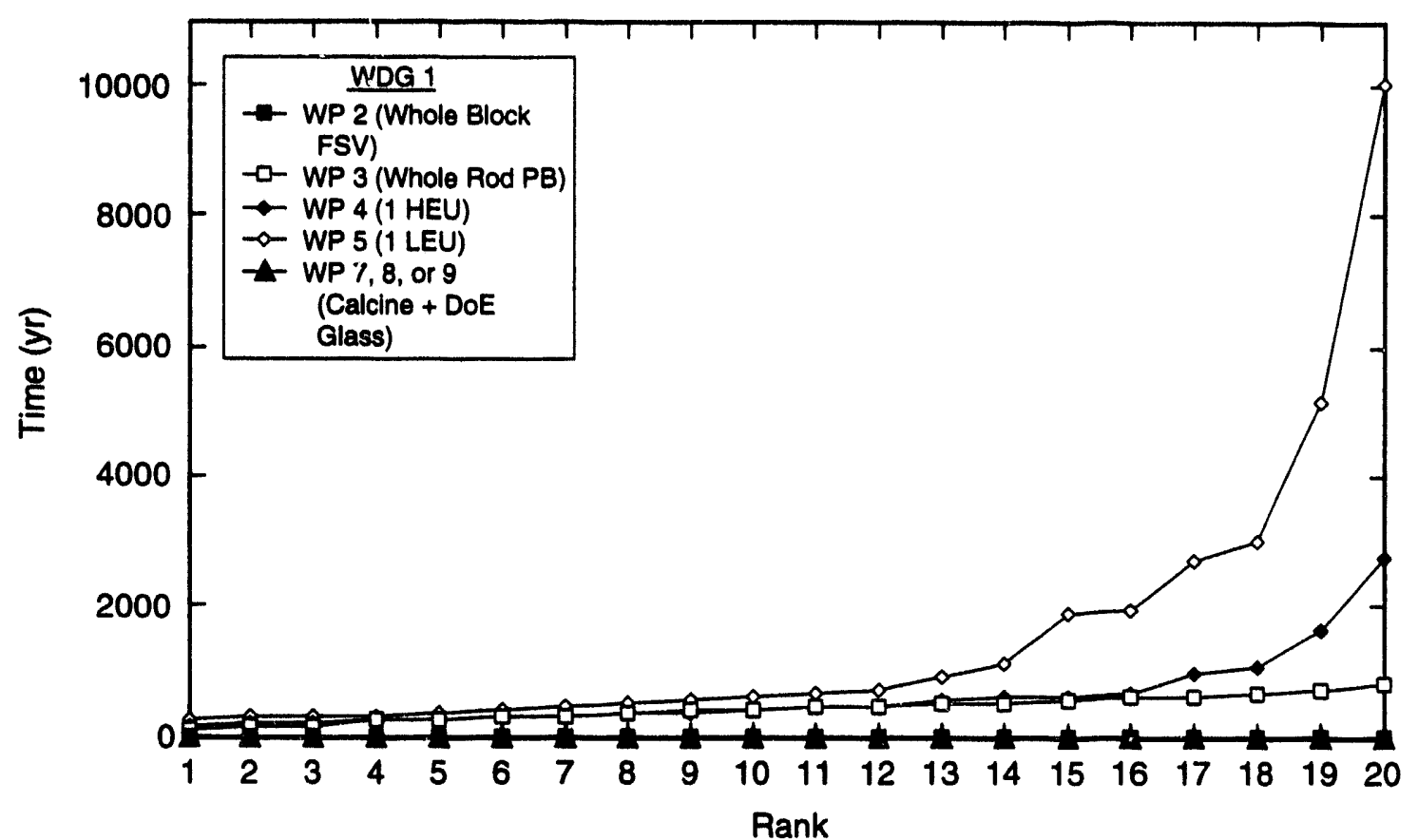

TRI-6342-4164-0

(a)

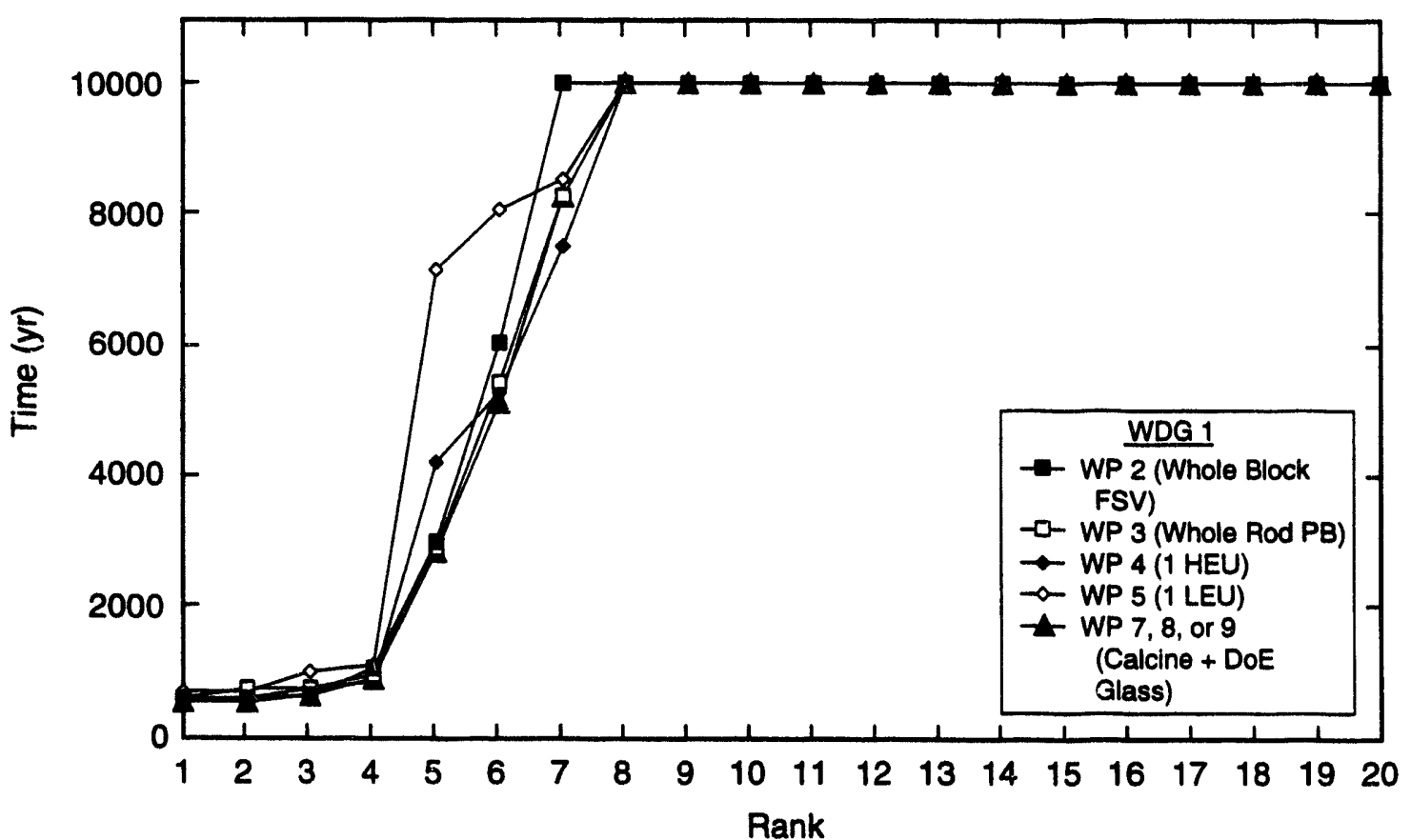

TRI-6342-4165-0

(b)

Figure 16.7-4. Mean breach times of all mesh elements for containers that have defects (median of 0.5\%) for waste parcels in Waste Disposal Group 1 in granite disposal system using Complex PA consequence models. (a) Reference containers (repeat of Figure 16.6-7a) and (b) corrosion-resistant containers. Each waste parcel is sorted by ascending rank because comparisons by simulation number are not possible. 


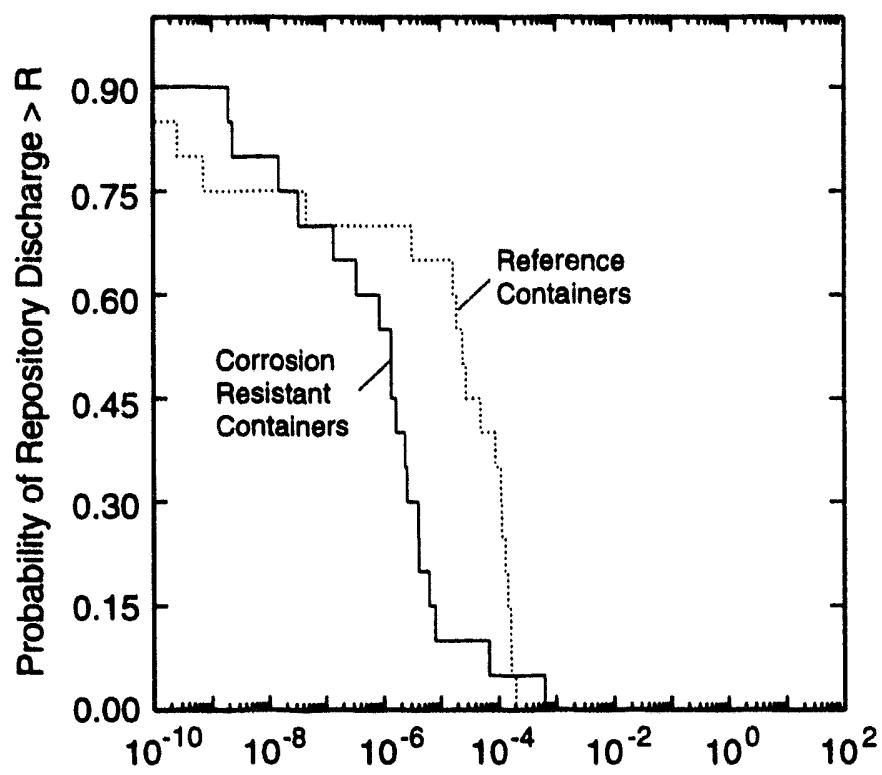

Summed Normalized Repository Discharge (R)

TR1-6342-3931-0

Figure 16.7-5. CCDFs, conditional on two intrusions, of summed normalized activity discharge at repository boundary for Waste Disposal Group 1 in reference containers (refer also to Figure 16.2-5) and corrosion-resistant containers for the salt disposal system.

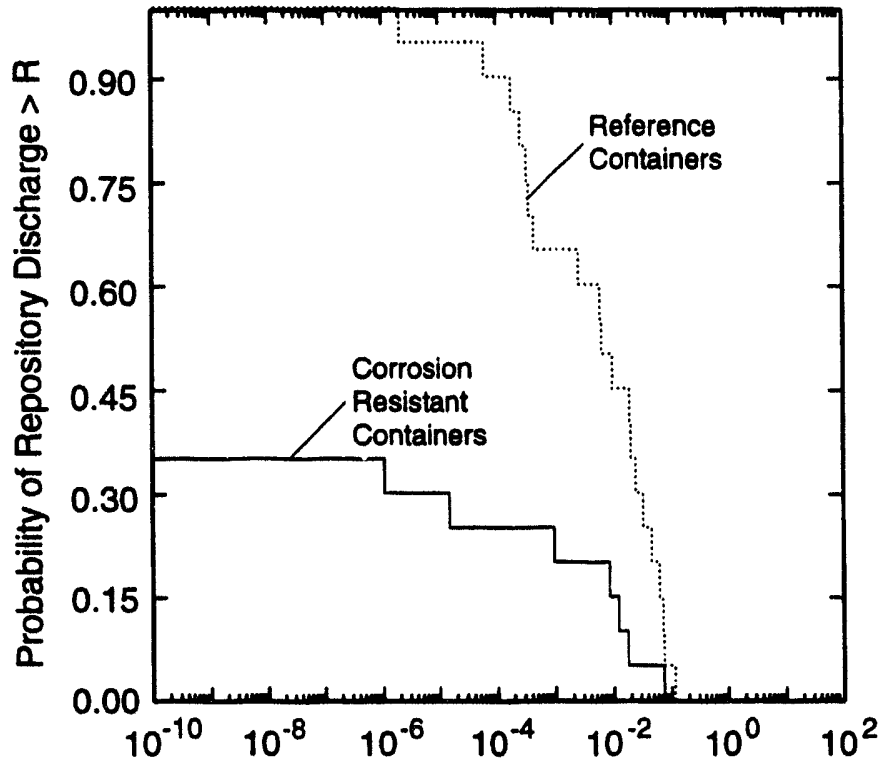

Summed Normalized Repository Discharge (R)

TR1-6342-3932-0

Figure 16.7-6. CCDFs, conditional on two intrusions, of summed normalized activity discharge at repository boundary for Waste Disposal Group 1 in reference containers (refer also to Figure 16.3-5) and corrosion-resistant containers for the granite disposal system. 


\subsection{Tracer and Other Radionuclide Transport in the Salt and Granite Disposal Systems (W. E. Beyeler, C. T. Stockman, and D. K. Rudeen)}

\subsubsection{Tracer Transport}

Integrated release from the salt and granite repositories is influenced by a number of processes, including source dissolution/alteration, adsorption, and advection. To isolate the effect of differences in groundwater flow in the two repositories, a tracer simulation described below was performed using the Simple PA consequence models.

A fixed mass of nonsorbing, non decaying tracer was added to the inventory of WP 7. The tracer was assigned a very high solubility. By removing the influence of dissolution, adsorption, and decay, tracer release from the repository is determined by the initial tracer mass and the repository flow field. The repository release fraction of the tracer (i.e., the ratio of released mass to initial mass) is a useful metric of the repository flow field that provides a basis for comparing the two repository designs (along with the stipulated parameters). In addition, the repository release fraction of the tracer is an upper bound on the repository release fraction of any radionuclide that is not produced by decay; the processes that are not included in tracer transport (dissolution, adsorption, and decay of the transported species) all tend to reduce the repository release fraction.

Figure 16.8-1 shows the distribution of tracer release fraction over the 20 sample vectors for the salt and granite disposal systems. The tracer fraction released from the salt disposal system ranges from $0.001 \%$ to more than $10 \%$, with a median of approximately $0.3 \%$; granite repository release ranges from less than $0.01 \%$ to $100 \%$, with a median of approximately $0.5 \%$. In roughly $33 \%$ of the simulations, effectively all of the initial tracer mass is released from the granite repository; in contrast to a maximum of $12 \%$ of the entire contents from the salt repository. In the granite disposal system, the large fracture connection permeability allows many repository pore volumes to pass through the repository in the $10,000 \mathrm{yr}$ simulation period. This result is greatly influenced by the omission of flow resistance in the repository backfill in the Simple PA (GRFLOW), however, the tendency of the granite repository to release a larger fraction of the inventory than the salt repository is also evident at low values of connection permeability.

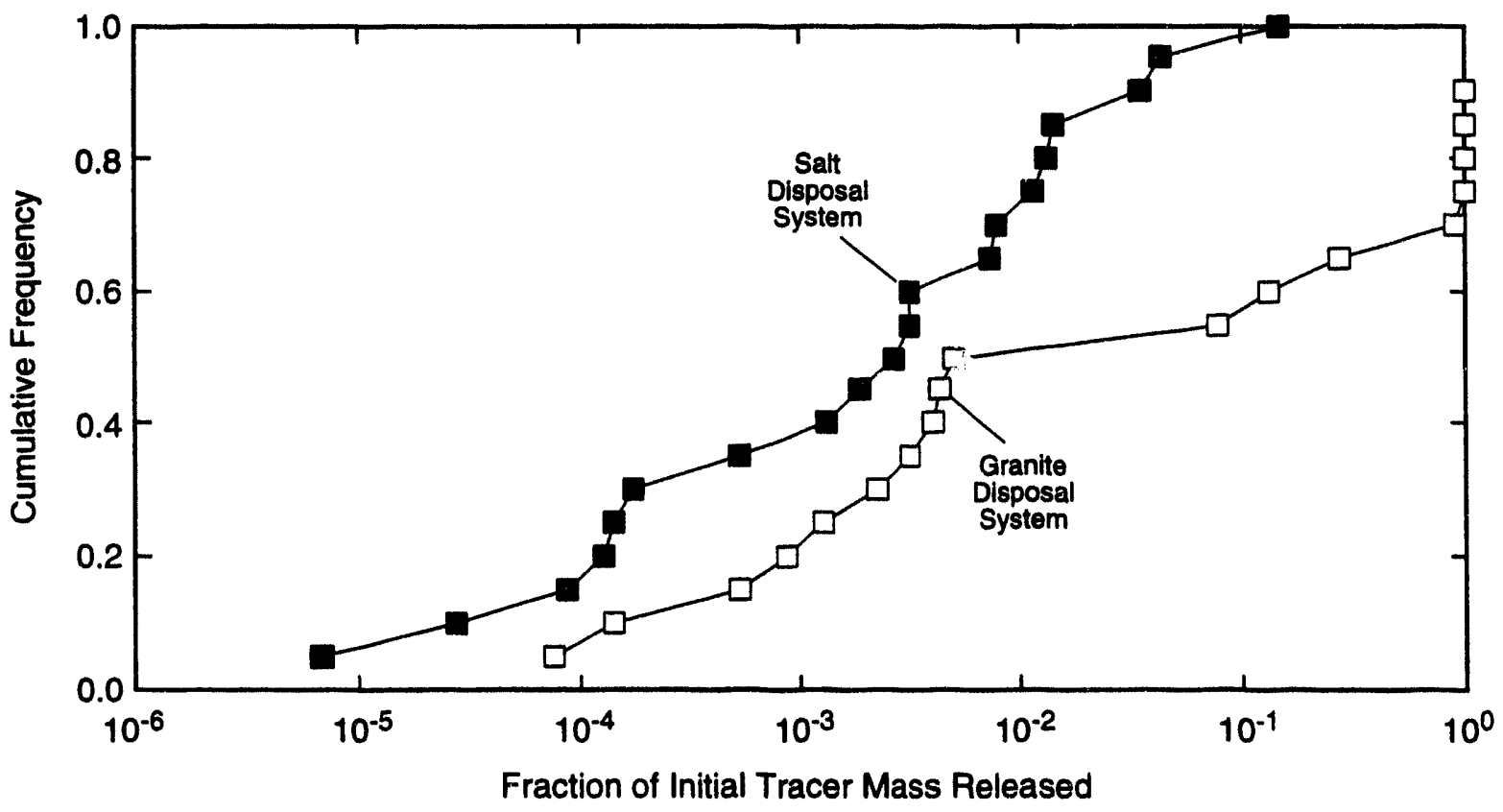

TRI-6342-4146-0

Figure 16.8-1. Cumulative distribution function (CDF) of fraction of initial tracer mass released. 


\subsubsection{Other Radionuclide Transport}

For the transport codes used in both the Simple and Complex PAs, execution time is approximately proportional to the number of radionuclides transported. Because of the large number of code executions required to evaluate the five disposal groups and 26 waste package designs in both repositories, all radionuclides initially present in the inventory could not be practically included in transport calculations. Radionuclides that can contribute significantly to integrated discharge over the repository lifetime must have a significant initial inventory, a moderately long half-life, and be mobile within the disposal system. The radionuclides included in the PA calculations were selected with these considerations in mind, informed by experience from other performance assessments (see Chapter 4).

Two simulations were made using the Simple PA models, one each for the salt and granite disposal systems, to gauge the error introduced by transporting a limited subset of radionuclides. The extended radionuclide list included all isotopes of any element in the inventory with an isotope whose EPA normalized activity exceeds 1 at any time.

The first column in Table 16.8-1 shows the complete list of radionuclides included in the two simulations. The second column lists the rank of the radionuclides as contributors to EPA normalized inventory at $10,000 \mathrm{yr}$. A blank entry in the second column means the radionuclide was not among the largest 30 contributors. Columns 3 and 4 indicate the radionuclides included in the primary Simple and Complex PA calculations.

These simulations used an entire repository filled with WP 7 in both the salt and granite disposal systems. These simulations used the same sampled parameter values as in the other PA calculations and the same model configuration as the Simple PA calculations. Solubilities and granite repository backfill retardations for elements not included in the other PA calculations were assigned a constant value (the median of the parameter distribution).

Figure 16.8-2 shows the conditional CCDF of waste parcel equivalents released from the waste parcels and repository for the salt disposal system. Releases calculated using the subset of radionuclides in the Simple PA (see Figures 16.4-3, 16.4-12, 16.5-3, and 16.5-13) are overlaid on each curve for comparison. The maximum difference observed in the waste parcel release (Figure 16.8-2a) is approximately 1 order of magnitude and occurs in the center of the distribution. The maximum difference created in the repository release (Figure 16.8-2b) by including the additional radionuclides is approximately $1 / 2$ order of magnitude. The error introduced in integrated release from the salt repository by using a subset of the radionuclides appears to be less than the error introduced in the integrated release from the waste parcels.

Figure 16.8-3 shows the CCDF of fractional release from the waste packages and repository for the granite disposal system. The maximum error in waste parcel release (Figure 16.8-3a) calculated using the radionuclide subset is approximately an order of magnitude, and is evident for probability levels above 0.15 . In contrast to the salt disposal system, release from the granite repository (Figure 16.8-3b) is less well represented than the waste package release using the radionuclide subset: the maximum error in granite repository release is approximately two orders of magnitude.

The last two columns of Table 16.8-1 summarize the importance of the radionuclides in integrated repository release for both the salt and granite disposal systems. For each simulation, the three radionuclides with the greatest contribution to integrated repository release were identified. The radionuclide contributing the largest amount received a rank of 1 , the radionuclide contributing the next largest a rank of 2 , the third largest a rank of 3 . Table 16.8-1 shows for each radionuclide in each disposal system the minimum rank observed for the radionuclide (e.g., ${ }^{59} \mathrm{Ni}$ was the second largest contributor to granite repository release in at least one simulation). Blank entries mean that the radionuclide was never among the top three contributors to integrated release. This does not necessarily mean that the radionuclide is unimportant, ${ }^{14} \mathrm{C}$ does not appear because it is not in the inventory of WP 7 .

Of the first-rank nuclides, ${ }^{151} \mathrm{Sm}$ and ${ }^{99} \mathrm{Tc}$, which were not included in the PA calculations, have low adsorption coefficients (Kds). Their relative contribution to repository release therefore tends to be greater than their relative contribution to waste parcel release. Five other radionuclides that were not included appeared as 2 nd or 3 rd rank contributors to repository release. This result is particular to WP 7 , which is especially rich in fission products relative to uranium, and does not include uncertainty in the solubilities and adsorption coefficients of radionuclides that are not 


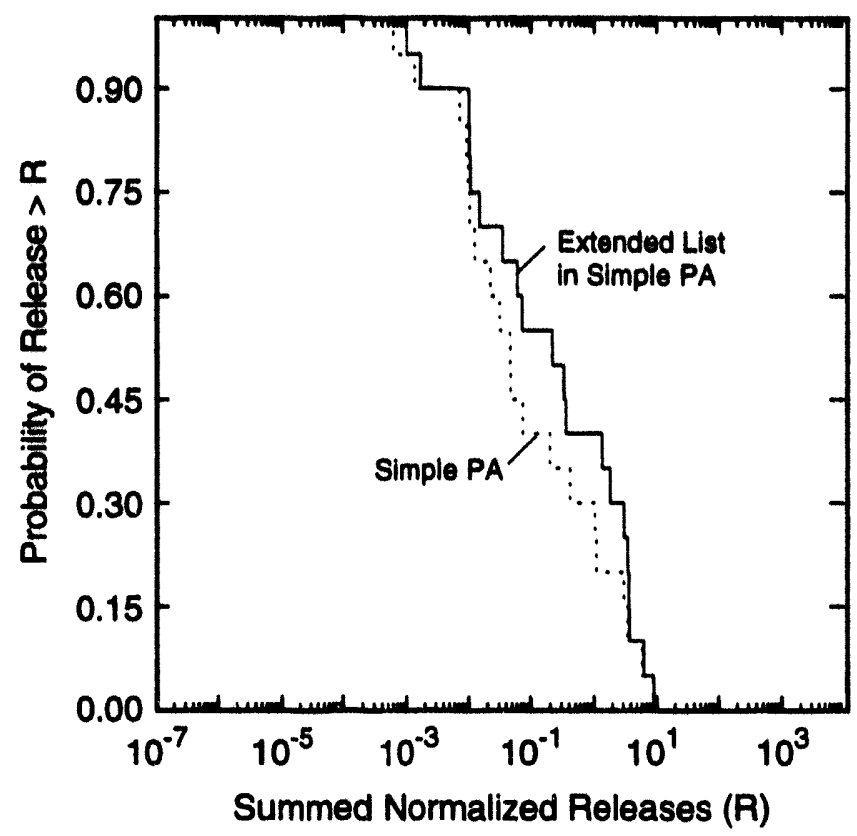

TRI-6342-4142-0

(a) Waste parcels

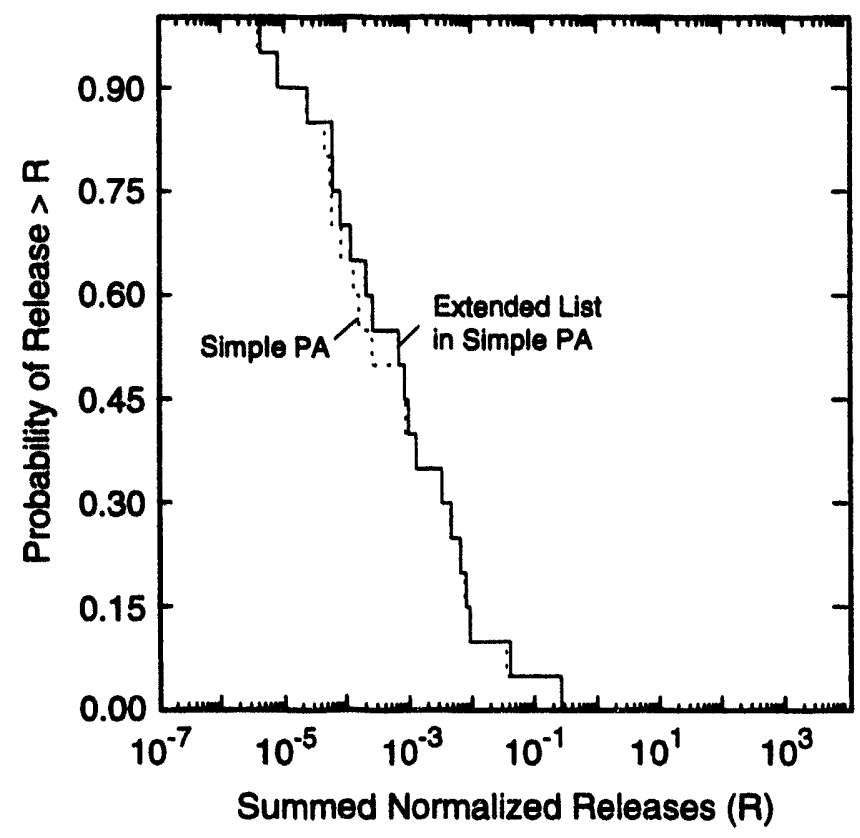

TR1-6342-4143-0

(b) Repository boundary

Figure 16.8-2. Comparison of CCDF, conditional on two intrusions, for transport of an extended list of radionuclides and the standard list of radionuclides in the Simple PA consequence models (see Figures 16.4-3 and 16.4-12) of the salt disposal system for (a) waste parcel equivalents discharged from waste parcels and (b) waste parcel equivalents discharged from repository boundary. 


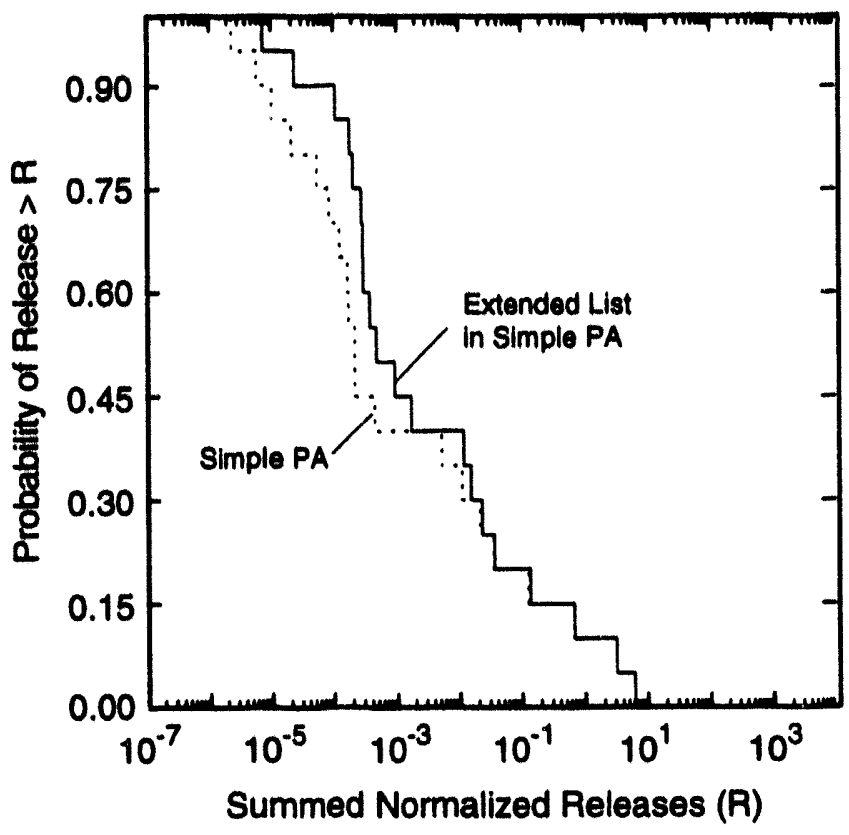

TRI-6342-4144.0

(a) Waste parcels

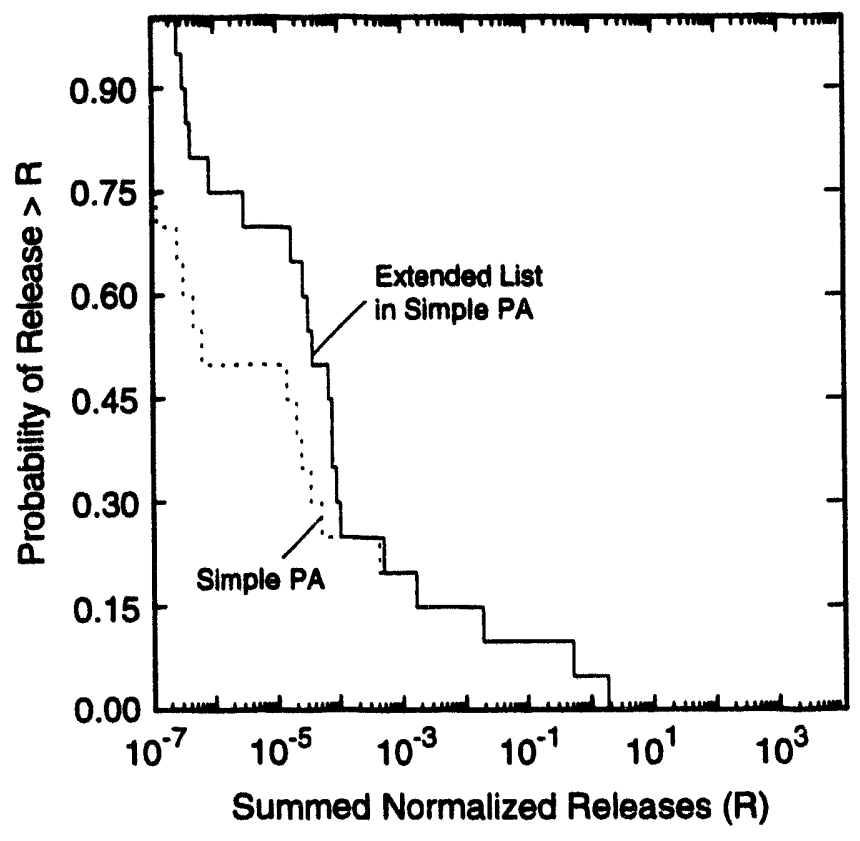

TRI-6342-4145-0

(b) Repository boundary

Figure 16.8-3. Comparison of CCDF, conditional on one intrusion, for transport of an extended list of radionuclides and the standard list of radionuclides in the Simple PA consequence models (see Figures 16.5-3 and 16.5-13) of the granite disposal system for (a) waste parcel equivalents discharged from waste parcels and (b) waste parcel equivalents discharged from repository boundary. 
16. Results and Conclusions

2

3

4

7

8

10

11
12

13

14

15

16

17

18

19

20

21

22

23

24

25

26

27

28

29

30

31

32

33

34

35

36

37

38

39

40

41

42

43

44

45

46

47

48

49

50

51

52

53

54

55

56

57

58

59

60

61

62

63

64

66

Table 16.8-1. Radionuclides Included in Extended Simple PA, Simple PA, and Complex PA

\begin{tabular}{|c|c|c|c|c|c|}
\hline $\begin{array}{l}\text { Radionuclides } \\
\text { Included in } \\
\text { Extended } \\
\text { Simple PA }\end{array}$ & $\begin{array}{l}\text { EPA Rank } \\
\text { at } 10,000 \mathrm{yr}^{*}\end{array}$ & $\begin{array}{l}\text { Included in } \\
\text { Complex PA }\end{array}$ & $\begin{array}{l}\text { Included in } \\
\text { Simple PA }\end{array}$ & $\begin{array}{l}\text { Importance } \\
\text { In Salt* } \\
\text { for WP } 7\end{array}$ & $\begin{array}{l}\text { Importance } \\
\text { in Granite* } \\
\text { for WP } 7\end{array}$ \\
\hline${ }^{241} \mathrm{Am}$ & 27 & $\cdot$ & $\cdot$ & 1 & 1 \\
\hline${ }^{242 m} \mathrm{Am}$ & & & - & & \\
\hline${ }^{243} \mathrm{Am}$ & 7 & & • & & 2 \\
\hline${ }^{14} \mathrm{C}$ & 16 & $\bullet$ & - & & \\
\hline${ }^{135} \mathrm{Cs}$ & 17 & & & 2 & \\
\hline${ }^{137} \mathrm{Cs}$ & & & & 2 & \\
\hline${ }^{59} \mathrm{Ni}$ & 15 & & & 3 & 2 \\
\hline${ }^{63} \mathrm{Ni}$ & 29 & & & & \\
\hline${ }^{237} \mathrm{~Np}$ & 6 & • & - & & \\
\hline${ }^{210} \mathrm{~Pb}$ & 20 & & & & \\
\hline${ }^{107} \mathrm{Pd}$ & 21 & & & & 3 \\
\hline${ }^{238} \mathrm{Pu}$ & 28 & & • & & \\
\hline${ }^{239} \mathrm{Pu}$ & 1 & - & - & 1 & 1 \\
\hline${ }^{240} \mathrm{Pu}$ & 2 & - & - & 2 & 3 \\
\hline${ }^{241} \mathrm{Pu}$ & & & - & & \\
\hline${ }^{242} \mathrm{Pu}$ & 10 & & • & & \\
\hline${ }^{244} \mathrm{Pu}$ & & & - & & \\
\hline${ }^{226} \mathrm{Ra}$ & 13 & - & - & 1 & \\
\hline${ }^{228} \mathrm{~Pa}$ & & & - & & \\
\hline${ }^{147} \mathrm{Sm}$ & 26 & & & & \\
\hline${ }^{151} \mathrm{Sm}$ & 30 & & & 1 & 3 \\
\hline${ }^{126} \mathrm{Sn}$ & 12 & & & & 2 \\
\hline${ }^{99} \mathrm{Tc}$ & 11 & & & & 1 \\
\hline${ }^{229} \mathrm{Th}$ & 8 & - & - & & \\
\hline${ }^{230} \mathrm{Th}$ & 4 & - & - & 1 & 1 \\
\hline${ }^{232} \mathrm{Th}$ & 19 & & - & & \\
\hline${ }^{232} \mathrm{U}$ & & & • & & \\
\hline${ }^{233} U$ & 5 & - & • & & \\
\hline${ }^{234} U$ & 3 & - & • & 1 & 2 \\
\hline${ }^{235} \mathrm{U}$ & 23 & - & - & & \\
\hline${ }^{236} U$ & 18 & . & - & & \\
\hline${ }^{238} \mathrm{U}$ & 14 & & - & & \\
\hline${ }^{93} \mathrm{Zr}$ & 9 & & & & \\
\hline
\end{tabular}

- A blank entry in this column means the radionuclide was not among the largest 30 contributors to the EPA summed normalized inventory at 10,000 yr.

- The minimum rank observed for the radionuclide in at least one simulation. 
present in the other PA calculations. Other radionuclides that have very low adsorption coefficients but were not considered in this analysis, such as ${ }^{129} \mathrm{I}$, may contribute to granite repository release in spite of their small contribution to initial inventory.

For both the salt and granite waste disposal systems, the high releases with low probability (on which compliance with the EPA containment requirement primarily depends) appears well defined using the selected subset of radionuclides. Lower releases (i.e., for probabilities greater than 0.1 ) calculated in the PA are evidently subject to some error as a result of the practical limitation on the number of radionuclides that may be transported. This error does not affect conclusions regarding compliance because radionuclides contributing to release when the release is large were included. 


\subsection{Comparison of Simple and Complex Consequence Models (D. K. Rudeen)}

The simple, one-dimensional network (SALFLOW, GRFLOW $\rightarrow$ CLAMVD) and complex, two-dimensional, finite-element (BRAGFLO_T $\rightarrow$ STAFF2D) consequence models can be verified to some extent by comparing the results presented in earlier sections. This comparison can be done because both PAs theoretically assumed the same conceptual and probability models and the same sampled property data sets. The performance assessment results for the Simp'e and Complex PAs are compared in Figure 16.9-1 for the salt disposal system and Figure 16.9-2 for the granite disposal system. The figures show CCDFs of summed, EPA normalized discharges leaving both the waste parcels and the repository for the five waste disposal groups. In both figures, the Complex PA results are shown with bold line types, and the Simple PA results with fine line types.

\subsubsection{Salt Disposal System}

The activity discharges from the waste parcel (Figure 16.9-1a) for the salt disposal system have a tight grouping of the five disposal groups for either the Simple and Complex PAs, and more important, are similar. The waste parcel results from the Complex PA (bold curves in Figure 16.9-1a) are slightly higher at the lower values of discharge. Although, the activity discharges from the repository (Figure 16.9-1b) also have a tight grouping for the Complex and Simple PAs, the results from the Complex PA (bold in Figure 16.9-1b) are much lower.

These differences are due to differences in the actual conceptual models chosen for the Simple and Complex PAs, because the simple models were developed in parallel with rather than based on the complex consequence modeling. For example, the differences for discharge from the waste parcel are likely due to diffusion into the surrounding host rock and radionuclide injection at the waste parcel/host rock interface (Figures 12-8 and 12-9) in the complex model.

The most significant difference in the salt conceptual model is the placement of the two intrusion boreholes and the resulting flow between them. In the complex conceptual model, the intrusion boreholes are located in opposite side rooms of the center panel (Figure 12-4) with one room or $110 \mathrm{~m}$ separating them. The complex flow simulations predicted the flow to be primarily up the intrusion borehole to ground surface with no circulation in the repository and very little $f$ i w through the repository to the second intrusion borehole. Also, flow in the repository was away from the brine reservoir borehole (Figure 12-10), which implies that the primary mechanism for nuclides to get to the borehole and out of the repository is via diffusion, resulting in very small discharges.

In contrast, the excavated disposal area, in the one-dimensional, simple conceptual model was set end-to-end into one equivalent, long panel. The intrusion boreholes were placed one third the distance from either end resulting in a separation of $320 \mathrm{~m}$ (see Figure 14-8). The simple flow simulations showed significant brine flow between the two intrusion boreholes, which results in advection of considerably more radionuclides to the boreholes and out of the repository. In general, flow through the salt repository was much greater for the simple consequence flow model.

\subsubsection{Granite Disposal System}

The activity discharges from the waste parcel for the Complex PA of the granite disposal system (bold curves, Figure 16.9-2a) are slightly larger and more tightly grouped than the waste parcel discharges from the Simple PA (fine curves, Figure 16.9-2a), which show a separation of Waste Disposal Groups 4 and 5 and Disposal Groups 2 and 3. The discharges from the granite repository for the Simple and Complex PAs (Figure 16.9-2b) are roughly similar, with slightly more separation between Disposal Groups 4 and 5 and Groups 1, 2, and 3 for the simple model (fine curves) and also more spread than the waste parcel discharges.

As with the salt repository, the differences are attributed to differences in the actual conceptual models used in the Simple and Complex PAs. In the simple model, the repository was bounded by two vertical fracture planes. That is, during excavation the fractures were detected, excavation was stopped, and waste placement near the fractures was minimized. In the complex model, two fracture planes sliced through the side rooms of the panels at either end of the 


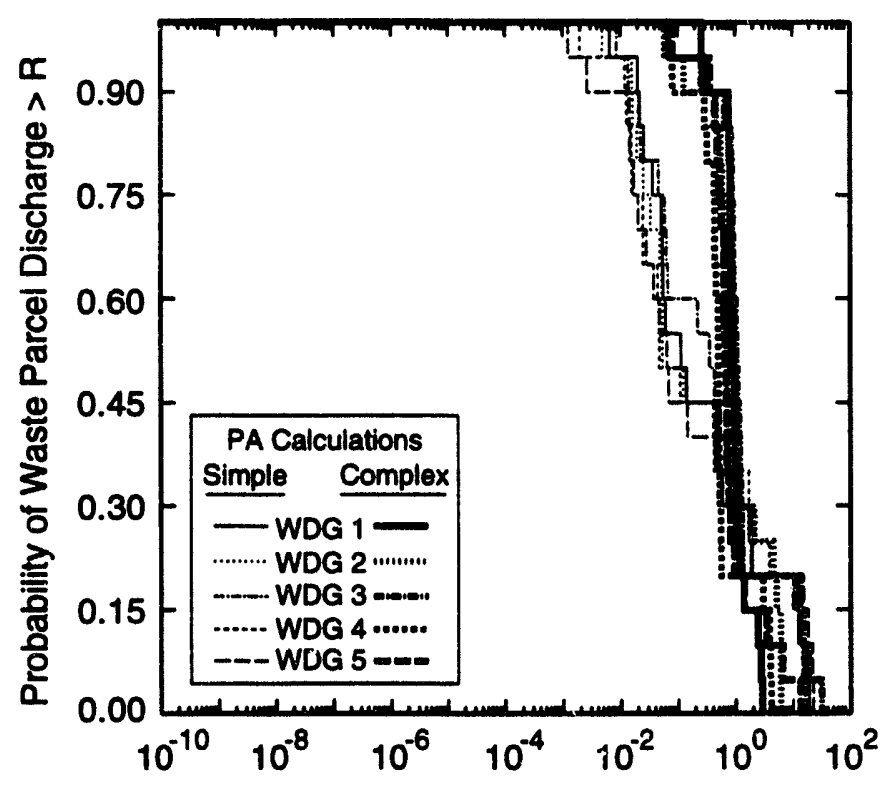

Summed Normalized Waste Parcel Discharge (R)

TRI-6342-3924-0

(a) Waste Parcel Discharge

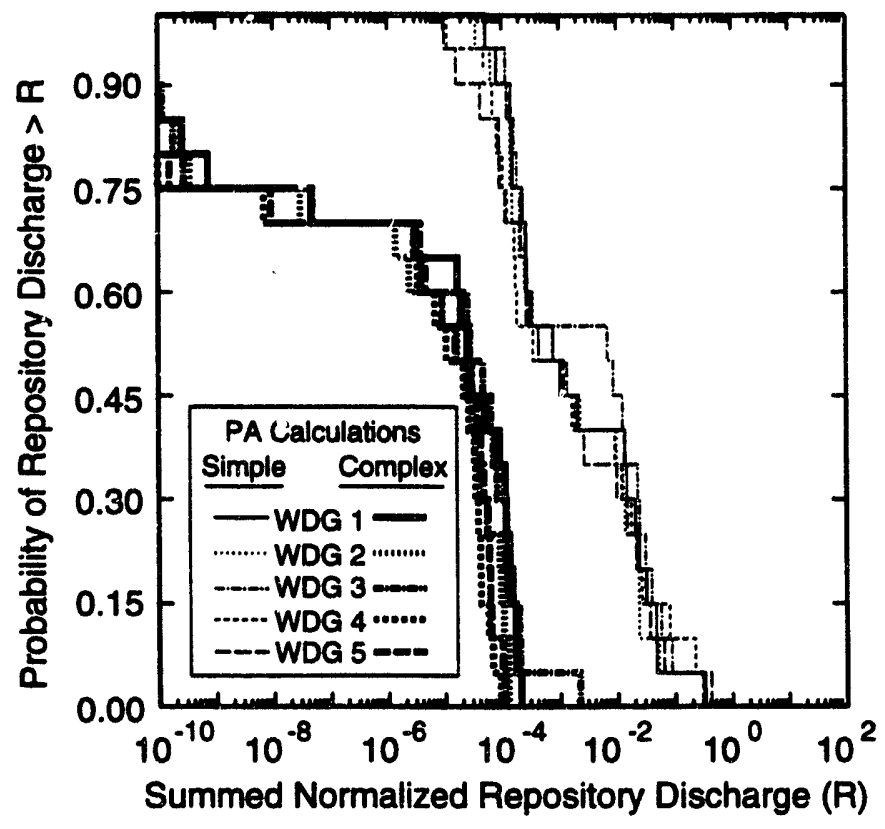

TA1-6342-3925-0

(b) Repository Discharge

Figure 16.9-1. Conditional CCDFs comparing simple and complex consequence model results for total EPA normalized for the five waste disposal groups, in salt disposal system, (a) activity discharge from waste parcels and $(b)$ defined discharge from repository boundary. 
16. Results and Conclusions

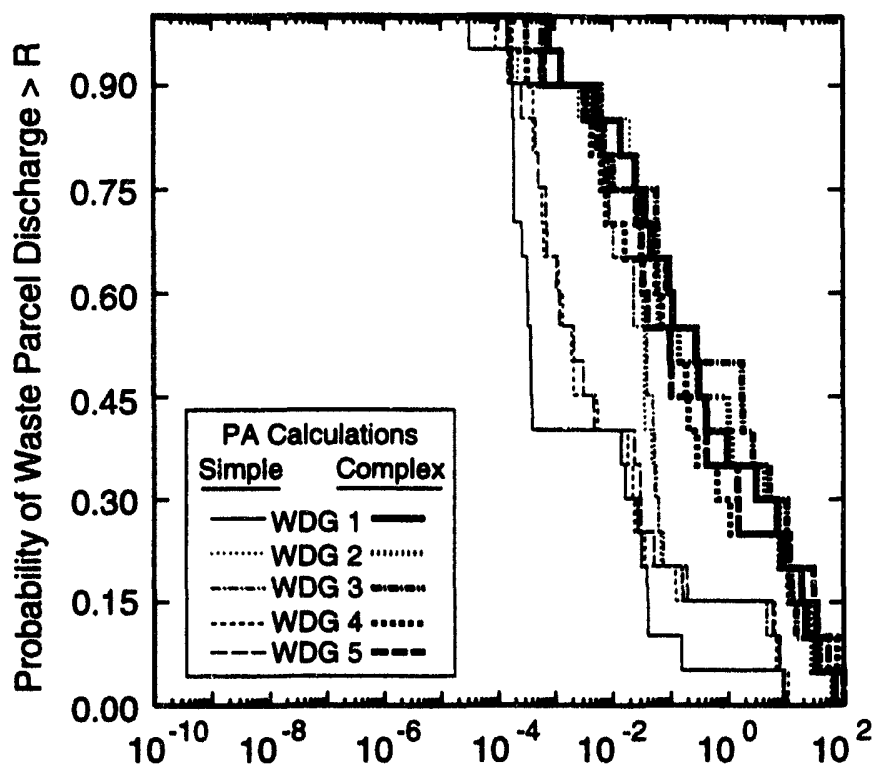

Summed Normalized Waste Parcel Discharge (R)

TRI-6342-3926-0

(a) Waste Parcel Discharge

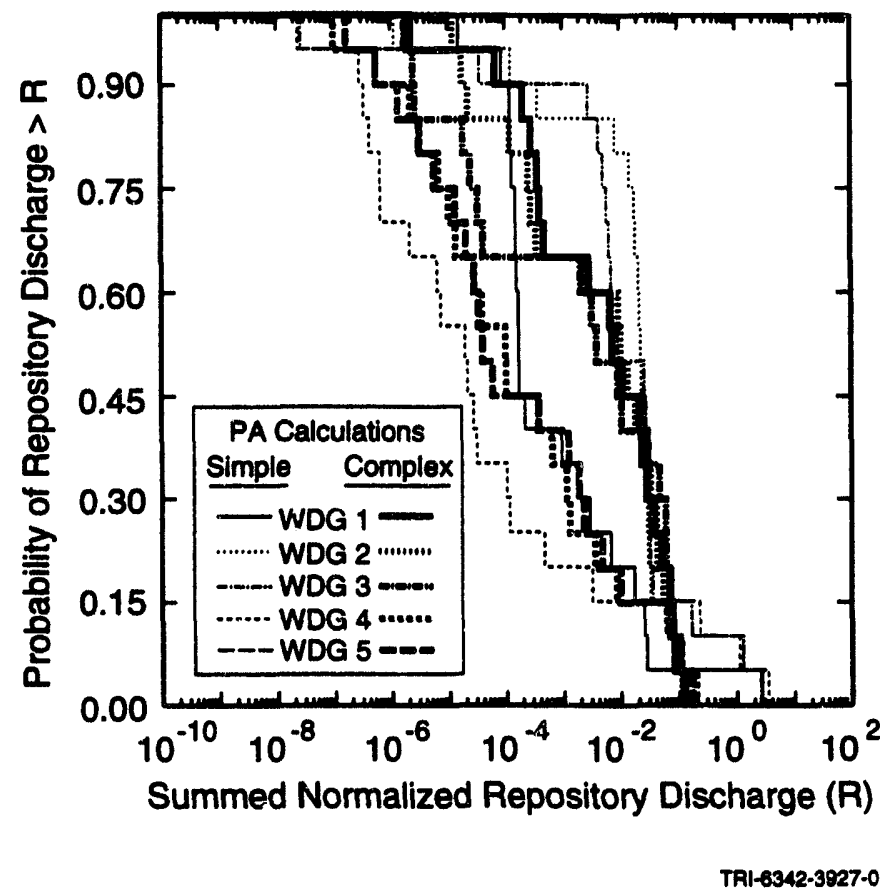

(b) Repository Discharge

Figure 16.9-2. Conditional CCDFs comparing simple and complex consequence model results for total EPA normalized for the five waste disposal groups, in granite disposal system, (a) activity discharge from waste parcels and $(b)$ defined discharge from repository boundary. 
repository. These fractures were either undetected or they formed after waste placement. The behavior of the waste parcels is different because in the complex model, radionuclides can diffuse into the surrounding host granite because the granite media is assigned a porosity even though permeability is set to zero. In addition, some radionuclide mass is actually injected directly into the granite at the granite/waste parcel material interface at the nodes of finite elements of STAFF2D. This latter injection is a modeling problem and not physical. This behavior is also true at the waste parcel/lower fracture interface, which allows more radionuclide mass to leave the waste parcels. Repository discharges are different because in the Simple PA, the head gradient, and therefore the flow, is from the lower fracture zone to the upper fracture zone. Also, the effect of the intrusion borehole was assumed insignificant and therefore was not included. For the two-dimensional complex model, where there is also a horizontal component to the head gradient, the head gradient and flow are from the top of the upper vertical fracture to the bottom of the lower vertical fracture; just the opposite of the simple model. Also, at the intrusion borehole fluid flows upward, providing a significant pathway out of the repository to the upper fracture zone.

\subsubsection{Comparison of Waste Disposal Group 1}

Because the Simple and Complex PAs were performed with different conceptual models, direct comparison of their consequence models (algorithms) was inappropriate. To improve comparisons of the simple and complex consequence models, a common conceptual model was required. ${ }^{* * *}$ In the granite disposal system, this requirement was partially accomplished by changing the conceptual model for the Complex PA to the simple model by removing the waste that was stored in the panel intersected by the vertical downward fracture as if the fracture had been detected (horizontal grid block indices 45 to 50 in Figure 13-2). The original flow simulations could be used since gas generation effects were negligible. In the original complex conceptual model, discharges out this lower fracture dominated discharges from the repository because of the downward flow through the fracture from the repository to the lower fracture zone (see Figure 13-6) and because the source term model injected some waste directly into this fracture. Waste was left alone at the other vertical fracture because flow was generally into the repository at this location and the fracture did not actually intersect the waste. In this new complex conceptual model, radionuclides must be transported to the fracture, as in the simple model, before the pathway becomes significant. This modification to the waste placement made the complex model much more similar, at least in geometry, to the simple model.

The results for Waste Disposal Group 1, in terms of conditional CCDFs, are compared with the original results from the Complex PA in Figure 16.9-3 for both the summed normalized discharges from the waste parcels and summed normalized discharges from the repository. Summed normalized discharges from both the waste parcels and the repository decreased by more than an order of magnitude even though the repository inventory decreased by only $20 \%$. This decrease is due to the isolation of the lower fracture zone. The primary pathway out of the repository is now up the intrusion borehole (which was omitted in the Simple PA) and could account for these changes in the flow pathway since the flow network was fixed. Also, discharges out of the repository are even more completely dominated by ${ }^{14} \mathrm{C}$ because retardation of all other radionuclides is occurring over a more significant part of the pathway (radionuclides are no longer dumped directly into the fracture flow). This difference implies that the granite repository performance is greatly affected by waste placement in proximity to fractures. Isolation of rooms and panels could also greatly improve performance.

The results of the new complex model for Waste Disposal Group 1, in terms of conditional CCDFs, are compared with the simple consequence model in Figure 16.9-4. Summed normalized discharges from both the waste parcels and the repository are shown in the figure. Although the summed normalized discharge from the repository for the simple and complex models is similar, it is probably serendipitous because the summed normalized discharges from the waste parcels differ by 2 orders of magnitude. This difference for the waste parcel discharge is likely occurring because in the complex model, radionuclides can diffuse into the surrounding host granite and some radionuclide mass is actually injected directly into the granite at the granite/waste parcel material interface, allowing more radionuclides to be released from the waste parcel, as explained at the end of the previous section.

\footnotetext{
***"Presumably the distributions of the model parameters for the Simple and Complex PAs would be nearly the same and so identical sample sets were again used in both cases, but the correctness of this assumption was not carefully examined.
} 
16. Results and Conclusions

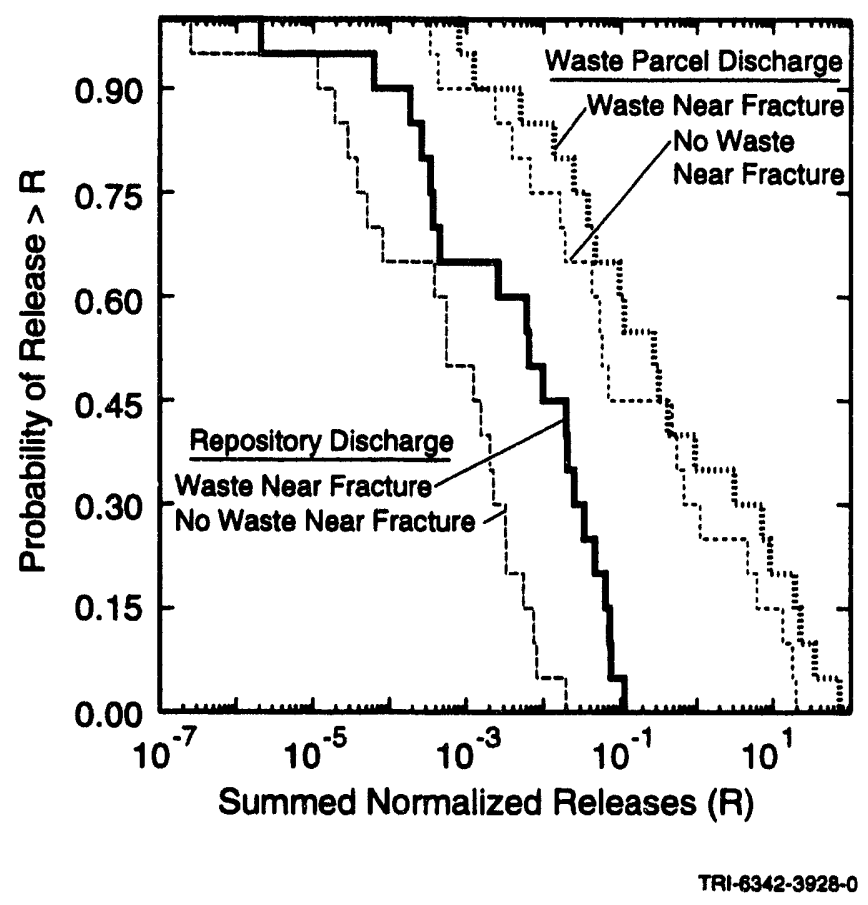

Figure 16.9-3. Comparison of conditional CCDFs of complex consequence model with and without waste in panel intersected by lower vertical secondary fracture, Waste Disposal Group 1, in granite disposal system.

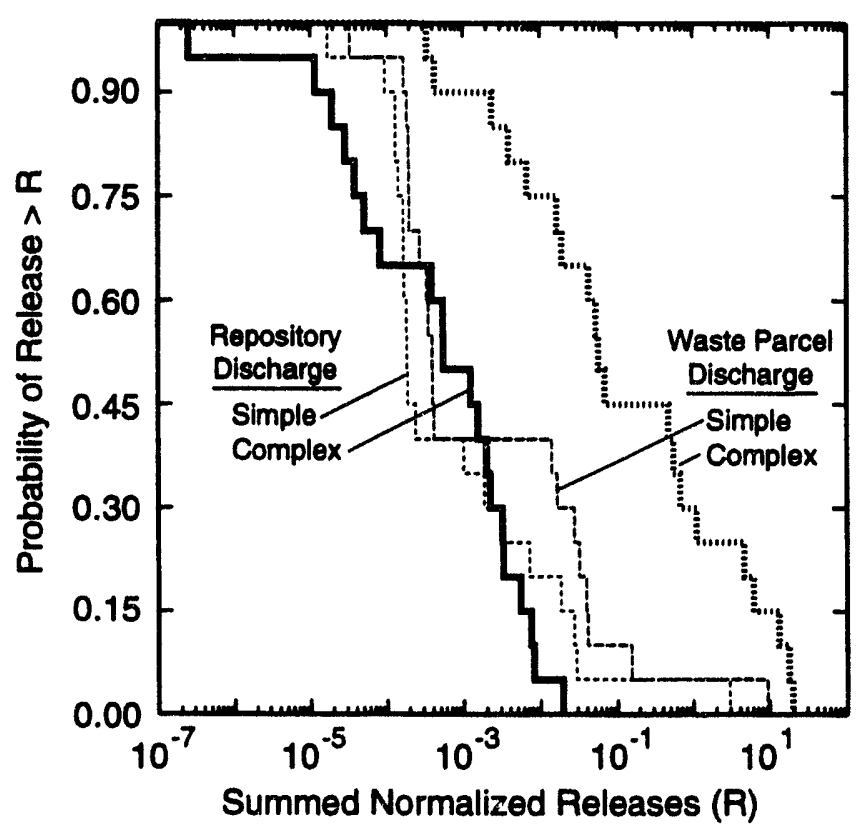

TRI-6342-3930-0

Figure 16.9-4. Comparison of conditional CCDFs of simple and modified complex consequence models for Waste Disposal Group 1, in granite disposal system. 
Further note that waste parcel discharges and repository discharges for the simple model are within an order of magnitude, except for a few simulations. The similarity in activity discharges from the waste parcel and repository in the Simple PA is likely due to the higher flow rates in the Simple PA. The higher flow rates in the simple consequence model decrease the effectiveness of the retardation of the bentonite backfill. The higher flow rates occur even though the geometry of the conceptual models is now similar, because the resulting flow and transport pathway is still quite different from the head gradient differences discussed above.

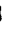




\subsection{Direct Release to Ground Surface During Drilling}

\section{(J. W. Berglund, J. S. Rath, and H. J. Iuzzolino)}

The direct release of radionuclides to the accessible environment because of a drilling intrusion is based on the assumption that any hit on a waste parcel transports the entire decayed contents of the waste parcel to the ground surface. For this portion of the calculation it is assumed that all waste parcels are intact and that no radionuclides have leaked into the region surrounding the waste parcels. Thus, only waste parcel hits remove radionuclides.

The likelihood of the drillbit hitting a waste parcel during an intrusion is governed by a number of factors, including the number of waste parcel types within each group, the individual waste parcel cross-sectional areas, the spacing of waste parcels, and the drillbit area. Also considered in the final analysis is the probability that a drillbit will pass through the projected area of the repository. Assuming that the drillbit does hit the repository, the probability of a waste parcel hit was tabulated in Table 9-4 as a function of waste parcel number and waste group. Assuming that the drillbit does penetrate the repository, the probability of striking any waste parcel within any waste group is generally less than $7 \%$ based on waste parcel numbers, dimensions, and drillbit area. ${ }^{\dagger \dagger \dagger}$ Assuming a waste parcel hit, the normalized waste removed, based on waste unit factor (Section 4.3.2) and time of intrusion, is also shown in Table 9-4.

Six times of intrusion were selected for consideration $(125,175,350,1000,3000$, and 7250 yr after emplacement) (see Section 7.5.2). For comparison, the decay in absolute activity for the 26 waste parcels based on these six intrusion times are shown in Figures 10-1 through 10-3. To compare the rankings of each waste disposal group with regard to direct (cuttings/cavings) removal, the probabilities and normalized releases for each waste disposal group are combined to form composite complementary cumulative distribution functions (CCDFs) as illustrated in Figures 16.8-1 and 16.8-2 for the salt and granite disposal systems, respectively. These types of plots, which total normalized releases, are used for comparison with the release limits specified in 40 CFR 191. Note, however, that the releases from fluid transport to the accessible environment are not included.

A comparison of the cuttings releases for the five waste groups and the release due to groundwater flow and transport can be made by overlaying Figures 16.10-1 and 16.10-2 on the figures from Sections 16.2.1 and 16.3.1, respectively, for the salt and granite disposal systems. For cuttings releases from the granite disposal system, the smaller number of assumed penetrations ( 3 holes $/ \mathrm{km}^{2} / 10,000 \mathrm{yr}$ for granite lithology, as opposed to $30 \mathrm{holes} / \mathrm{km}^{2} /$ $10,000 \mathrm{yr}$ for sedimentary bedded salt stratigraphy) generates releases with probabilities approximately one-tenth those for the salt disposal system (compare Figures 16.10-1 and 16.10-2). Consequently, the effect of cuttings on the CCDFs is considerably reduced with cuttings releases in granite becoming important only below probabilities of less than $2.5 \%$ (Figure 16.3-1). ${ }^{\ddagger \ddagger \ddagger}$

For cuttings-only, conditional on a drillbit hitting the repository, the waste groups can be ranked according to their release as shown in Table 16.10-1, where 1 corresponds to the lowest release at that time of intrusion.

Generally, Waste Disposal Groups 5, 1 and 3, generate the greatest cuttings release for both the salt and granite disposal systems. The least release occurs for Waste Disposal Group 4 in both the salt and granite disposal systems.

\footnotetext{
${ }^{++t+}$ The repository area for this computation was assumed to be $162,000 \mathrm{~m}^{2}$.

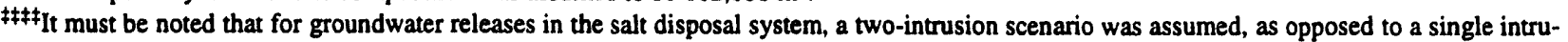
sion for the granite disposal system.
} 


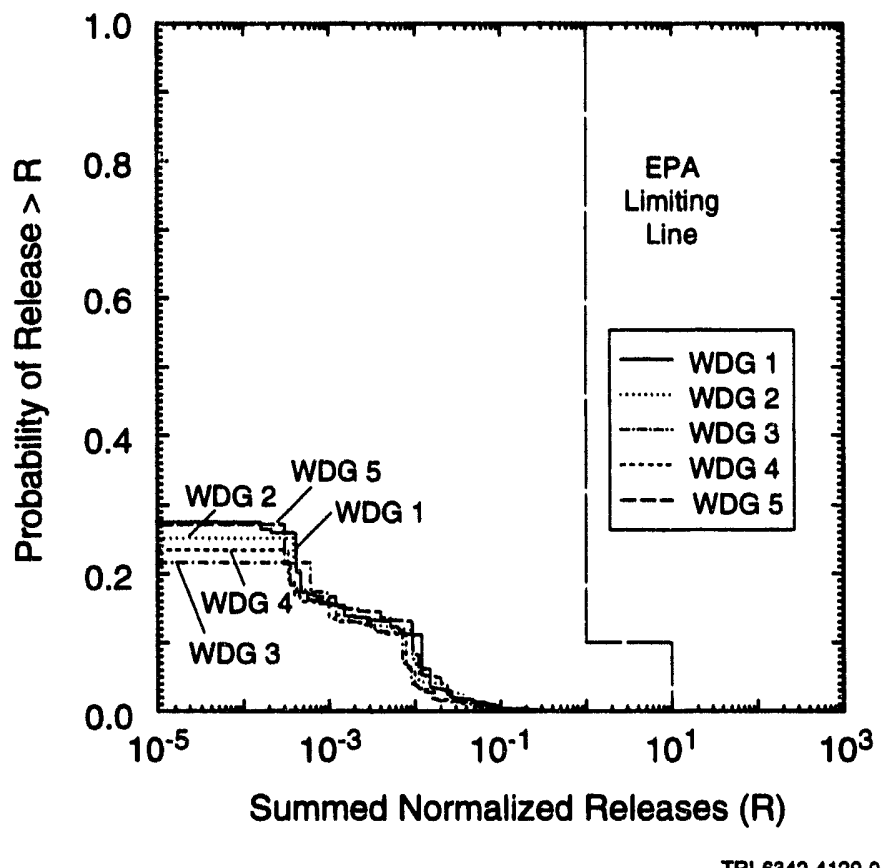

Figure 16.10-1. Composite CCDF of summed normalized releases from cuttings/cavings from the salt repository $\left(\lambda *=30\right.$ borehole $\left./ \mathrm{km}^{2} / 10,000 \mathrm{yr}\right)$ for all waste disposal groups.

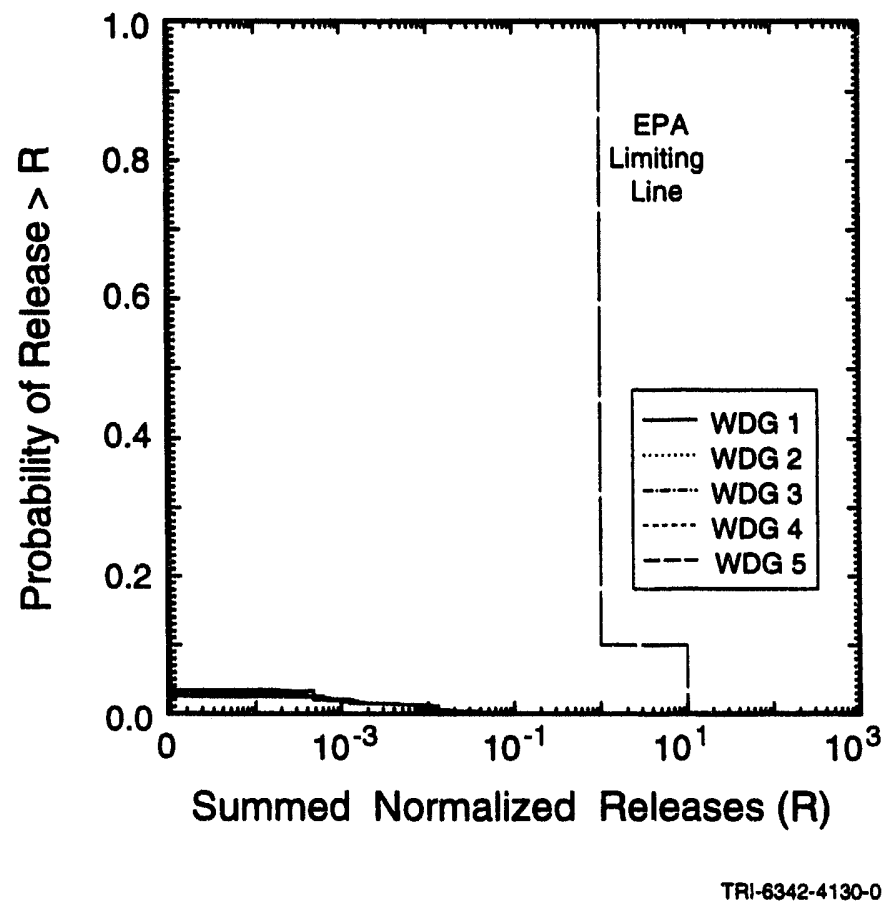

Figure 16.10-2. Composite CCDF of summed normalized releases from cuttings/cavings from the granite repository $\left(\lambda *=3\right.$ borehole $\left./ \mathrm{km}^{2} / 10,000 \mathrm{yr}\right)$ for all waste disposal groups. 
16. Results and Conclusions

Table 16.10-1. Waste Group Release Ranking Based on CUTTINGS Only-Conditional on Hitting the Repository (see Table 9-4)

\begin{tabular}{cc}
\hline Waste Disposal Group & Intrusion Time $=\mathbf{1 0 0 0} \mathbf{y r}^{*}$ \\
\hline Salt Disposal System & 4 \\
1 & 3 \\
2 & 5 \\
3 & 1 \\
4 & 2 \\
5 & \\
Granite Disposal System & 5 \\
1 & 3 \\
2 & 4 \\
3 & 1 \\
4 & 2 \\
5 & \\
\hline Rankings are identical for intrusion times of $125,175,350,3000$, \\
\hline
\end{tabular}




\begin{abstract}
16.11 Summary of Findings and Program Guidance as Related to 40 CFR 191 (R. P. Rechard)

The general findings of the performance assessment fall into two categories: those pertaining to the disposal system and those pertaining to the performance assessment modeling. A discussion of the findings in these two areas is followed by recommendations to be considered by the Waste Technology Development Program at INEL, based on the information gathered during this performance assessment. These recommendations are also summarized in tables.
\end{abstract}

\title{
16.11.1 Summary of Findings about the Disposal System
}

The findings about the disposal system are grouped into five areas: the waste form, the waste containers, the repository, the geologic barrier, and the agents acting on the disposal system. Conclusions drawn in each of these areas are presented in Tables 16.11-1 through 16.11-5.

\subsubsection{General Findings about the Performance Assessment Modeling}

How the disposal system is modeled changes as more information becomes available. Information gathered during this performance assessment that could influence the manner in which hypothetical deep geologic repositories are modeled in saturated salt and granite in the future are as follows:

- Future performance assessments could use Waste Disposal Groups 4 or 5 (as best case) and Waste Disposal Groups 1, 2, or 3 (as worst) to represent extremes of behavior in a saturated medium.

- Diffusive transport through the failed layers of the containers could be an important phenomenon to model in saturated media in the future.

- Possibly, the overall consequences of changing the waste form could be roughly predicted using only the gas generation and source term submodels.

- Provided the volume of gas that would potentially be generated remained small, the fluid-flow phenomenon around the repository in a saturated medium might be modeled as single-phase fluid flow, at least in granite, and thus only the corrosion portion of the gas generation model would be necessary.

In this performance assessment, an important strategy was the use of two different consequence modeling systems with two different levels of simplification. ${ }^{* * * *}$ The parallel development and use of two different consequence modeling systems allowed comparisons (even when one modeling system was simplified), helped provide a more thorough understanding of the disposal systems, and helped to uncover discrepancies in the analysis during the analysis process itself.

However, the use of two levels of simplification provided some sobering reminders of how difficult it is to correctly simplify a complex disposal system, especially without prior knowledge of which phenomena and features are important (see Section 16.9, "Comparison of Simple and Complex Consequence Models"). The result of developing the simplified model in parallel with the complex model (instead of in series, i.e., the simple model would be developed after results from the complex model had been analyzed), meant that some differences occurred between models that were not recognized until the results were compared.

For example, in the granite repository, a comparison of mean permeability data justified our neglecting flow resistance in the rooms and drifts for the Simple PA; and yet the behavior of pertinent model parameters, when extremes were sampled in the Complex PA, indicated that flow resistance should be considered. The difference in whether flow resistance was modeled meant that slightly different radionuclides contributed to the total releases because travel times through the repository differed. Another difference was whether the shaft was modeled. The

"***"In this case, a "simple" level of modeling means a lumped-parameter consequence model that can be quickly set up and is geared toward fast run times. 
Table 16.11-1. Summary of Information Gathered about Waste Form

\begin{tabular}{|c|c|c|}
\hline & ormation & $\begin{array}{l}\text { Supporting } \\
\text { Results } \\
\text { Found in } \\
\text { Section(s): }\end{array}$ \\
\hline 1. & $\begin{array}{l}\text { All proposed waste forms are acceptable for compliance with } 40 \text { CFR } 191 \text { in salt and granite } \\
\text { disposal systems. Specifically, } \\
\text { - In salt, all waste forms are acceptable without either a container or adsorptive, clay back- } \\
\text { fill. } \\
\text { - In granite, all waste forms are acceptable with a stainless steel container and clay backfill } \\
\text { to adsorb radionuclides, but could possibly require at least a moderately corrosion-resis- } \\
\text { tant container." (Note that acceptability is highly dependent on the modeling parameters } \\
\text { for the granite site, see Section 16.11.3.) }\end{array}$ & 16.2, C.1 \\
\hline 2. & $\begin{array}{l}\text { In general, higher levels of waste treatment monotonically improved performance. However, } \\
\text { - When waste forms were analyzed as a group (Complex PA), extensive treatment, as rep- } \\
\text { resented by Waste Disposal Groups } 4 \text { and } 5 \text {, was required to conclusively see improved } \\
\text { disposal system performance. } \\
\text { - An exception occurred for graphite spent fuel, which was composed of fuel particles that } \\
\text { were assumed to be generally intact. For graphite spent fuel, moderate treatment led to } \\
\text { degraded performance in comparison to low treatment, represented here by disposal of } \\
\text { the entire block or rod. Only extensive treatment improved performance. Hence, } \\
\text { - The durability of the graphite spent fuel can be an effective barrier against radionuclide } \\
\text { release (e.g., silicon carbide coating). } \\
\text { - The fraction of particles with failed silicon carbide coatings is an important modeling } \\
\text { parameter for the graphite spent fuel. }\end{array}$ & $\begin{array}{c}16.5 .3 \\
\text { C.1, } .2 .2 \\
\text { C. } 1, \text { c. } 2\end{array}$ \\
\hline 3. & $\begin{array}{l}\text { Waste Disposal Groups } 4 \text { and } 5 \text {, and the corresponding individual waste parcels, have the } \\
\text { best performance, followed by Waste Disposal Groups } 1,2 \text {, and } 3 \text {, and the corresponding } \\
\text { individual waste parcels. } \\
\text { - For salt, the improvement with the more extensive treatment options was primarily } \\
\text { caused by the increased radionuclide inventory per waste parcel, which lowered the frac- } \\
\text { tional release since many of the radionuclides were solubility limited. }\end{array}$ & 16.3 \\
\hline 4. & $\begin{array}{l}\text { The radionuclides that contribute the most to the normalized integrated activity at the bound- } \\
\text { ary of the repository differ between (a) the salt and granite disposal systems, (b) the level of } \\
\text { simplification of the consequence models (which affected the travel time through the reposi- } \\
\text { tory, and thus, the radionuclides present) (Complex PA versus Simple PA), and (c) Waste } \\
\text { Disposal Groups } 4 \text { and } 5 \text { (since Waste Disposal Group } 4 \text { eliminates radionuclides and Group } \\
5 \text { adds a radionuclide)." } \\
\text { - Complex PA: } \\
\text { - In salt, the top contributing radionuclides are }{ }^{234} \mathrm{U},{ }^{235} \mathrm{U},{ }^{226} \mathrm{Ra} \text {, and }{ }^{230} \mathrm{Th} \text {. } \\
\text { - In granite, the top contributing radionuclides are }{ }^{14} \mathrm{C} \text { (not in Waste Disposal Groups } 4 \\
\text { and 5), }{ }^{241} \mathrm{Am},{ }^{240} \mathrm{Pu},{ }^{239} \mathrm{Pu} \text {, and }{ }^{230} \mathrm{Th} \text {. } \\
\text { - Simple PA: } \\
\text { - In salt, the top contributing radionuclides are }{ }^{241} \mathrm{Am},{ }^{234} \mathrm{U},{ }^{230} \mathrm{Th},{ }^{243} \mathrm{Am} \text { (not tracked in } \\
\text { the Complex PA), and }{ }^{226} \mathrm{Ra} \text {. } \\
\text { - In granite, the top contributing radionuclides are }{ }^{14} \mathrm{C} \text { (not in Waste Disposal Groups } 4 \\
\text { and 5), }{ }^{230} \mathrm{Th},{ }^{241} \mathrm{Am},{ }^{239} \mathrm{Pu} \text { and }{ }^{237} \mathrm{~Np} \text {. }\end{array}$ & $\begin{array}{l}16.8 .2, C .1 \\
16.8 .2, C .2\end{array}$ \\
\hline \multicolumn{3}{|c|}{$\begin{array}{l}\text { - Stainless steel is not considered a corrosion-resistant material for disposal of spent fuel and high-level waste because of the } \\
\text { long time scale involved and stainless steel's susceptibility to several forms of failure besides generalized corrosion. } \\
\text {-" The contributing radionuclides are also sensitive to the regulatory period, the performance metric, and the location at which the } \\
\text { metric is measured. For instance, individual dose evaluated beyond } 10,000 \text { yr at a } 5-\mathrm{km} \text { boundary of the disposal system will } \\
\text { show different radionuclides contributing to the performance metric (human dose) than the normalized integrated activity } \\
\text { specified in } 40 \text { CFR 191. This sensitivity to and uncertainty as to the form of the final regulation makes it difficult to identify } \\
\text { radionuclides that should be immobilized or removed at this time. }\end{array}$} \\
\hline
\end{tabular}


Table 16.11-1. Summary of Information Gathered about Waste Form (Continued)

\begin{tabular}{|c|c|c|}
\hline \multicolumn{2}{|c|}{ Information } & $\begin{array}{l}\text { Supporting } \\
\text { Results } \\
\text { Found in } \\
\text { Section(s): }\end{array}$ \\
\hline 5. & $\begin{array}{l}\text { Element solubility in the assumed chemical environment of the repository is an important } \\
\text { parameter affecting release from the repository and to the surrounding accessible environ- } \\
\text { ment. }\end{array}$ & 16.3 \\
\hline 6. & $\begin{array}{l}\text { Extensive treatment of the relatively small amount ( } 700 \text { MTHM) of INEL waste only moder- } \\
\text { ately improved the performance (according to the Containment Requirement measure of } 40 \\
\text { CFR 191) of a moderately sized disposal system. Thus other considerations (e.g., other reg- } \\
\text { ulations or economics) can guide the selection of the treatment option. Specifically, } \\
\text { - In the salt disposal system, the horizontal separation between the CCDF in the most } \\
\text { extensive treatment options (Options } 4 \text { and } 5 \text { ) and the low or moderate treatment options } \\
\text { (Options } 1,2 \text {, or } 3 \text { ) was, at most, three orders of magnitude when individual waste forms } \\
\text { were compared. When the waste forms are combined as a disposal group with waste } \\
\text { from other DOE sites (vitrified high-level waste from Savannah River, Hanford, and West } \\
\text { Valley), the CCDF usually varies less than one order of magnitude. } \\
\text { - In the granite disposal system, separation in CCDFs along individual waste forms are } \\
\text { usually relatively large, varying by as much as seven orders or magnitude. When com- } \\
\text { bined as a disposal group with waste from other DOE sites, they vary by at most } 21 / 2 \\
\text { orders of magnitude. }\end{array}$ & $\begin{array}{c}16.2,16.4 \\
16.7 \\
\\
16.3,16.5 \\
16.7\end{array}$ \\
\hline
\end{tabular}


2

3

7

Table 16.11-2. Summary of Information Gathered about Waste Containers

- In granite, water fills the repository faster than in a salt repository. As a result, the entire waste parcel corrodes more uniformly at the fully saturated, inundated rate.

2. The amount of gas generated from corrosion in the granite repository is considerably less than that generated in the salt repository because the corrosion rate of stainless steel is slower in water than in brine.

$12.1 .4,13.1 .4$

3. For graphite spent fuel, corrosion-resistant containers are not essential for compliance with the Containment Requirements of 40 CFR 191 in either salt or granite.

4. Multilayer corrosion-resistant containers remain intact for such long periods of time that it was not useful to include them in most evaluations of the waste forms within a 10,000-yr regulatory period.

5. When the corrosion rate is fast enough to cause a breach within the 10,000 -yr regulatory period, the corrosion rate is an important parameter for determining releases for special spent nuclear fuel and calcine.

6. Variation of heat loading has little influence on time of complete corrosion of the container. ${ }^{* n}$

- Wicking of fluid to the container from lowered capillary pressure is also partially modeled in the detailed modeling of consequences of the disposal group (Complex PA).

* Some caveats exist. Although this condition was observed in this study, it may have occurred because (1) the low backfill permeabilities around the canisters prevent convection cells from forming, (2) vaporization was not modeled, (3) pH changes due to evaporation and precipitation were not explicitly modeled, and (4) time constraints and a lack of data for some materials determined that the activation energy be set to zero (corrosion rate independent of temperature); however, an expanded range for the corrosion rate between $293 \mathrm{~K}$ and $373 \mathrm{~K}$ was subjectively estimated. 
Table 16.11-3. Summary of Information Gathered about the Repository

\begin{tabular}{|c|c|c|}
\hline \multicolumn{2}{|c|}{ Information } & \multirow{2}{*}{$\begin{array}{c}\text { Supporting } \\
\text { Results } \\
\text { Found in } \\
\text { Section(8): } \\
16.3 .3\end{array}$} \\
\hline 1. & $\begin{array}{l}\text { The diffusive transport of radionuclides from the waste parcel holes into the rooms and } \\
\text { access drifts, followed by diffusive transport (salt repository) or advective iransport (granite } \\
\text { repository) through the rooms and drifts, were very important phenomena to model; their } \\
\text { importance to the position of the CCDF relative to the EPA release limits was comparable to } \\
\text { the effect of the waste treatment options. }\end{array}$ & \\
\hline 2. & $\begin{array}{l}\text { The waste form had little influence on the flow of liquid (which is the primary means of radio- } \\
\text { nuclide escape other than direct release through cuttings), in either the salt or granite dis- } \\
\text { posal system. }\end{array}$ & $12.1 .4,13.1 .4$ \\
\hline 3. & $\begin{array}{l}\text { Gas generation and thus gas flow was influenced by the waste form but the small volumes of } \\
\text { gas did not, in turn, greatly influence liquid flow. }\end{array}$ & $12.1 .4,13.1 .4$ \\
\hline 4. & $\begin{array}{l}\text { Heat generating capacity of the waste form were included in the detailed analysis (Complex } \\
\text { PA) of the disposal systems and resulted in a different maximum temperature, but the heat } \\
\text { had little influence on the overall fluid flow. }\end{array}$ & $12.1 .4,13.1 .4$ \\
\hline 5. & $\begin{array}{l}\text { For the Complex PA of the salt disposal system, the shaft had little influence. Therefore, it } \\
\text { was appropriately neglected in the Simple PA calculations. }\end{array}$ & $12.1 .4,13.1 .4$ \\
\hline 6. & In the granite repository, the shaft was an important source of liquid filling the repository. & $12.1 .4,13.1 .4$ \\
\hline 7. & $\begin{array}{l}\text { In the granite disposal system, the chemical adsorption (retardation) provided by the backfill- } \\
\text { buffer in the waste parcel hole and the backfill in the rooms and drifts was an important } \\
\text { parameter influencing releases at the repository boundary. }\end{array}$ & $16.3,16.5, C .2$ \\
\hline 8. & $\begin{array}{l}\text { The relative difference in the threshold pressure (a property of the rock used in the two- } \\
\text { phase fluid model for the Complex PA) between the backfill-buffer and the room backfill can } \\
\text { influence the availability of liquid around the waste parcel and consequently the time of com- } \\
\text { plete corrosion of the waste container. }\end{array}$ & C.4 \\
\hline 9. & $\begin{array}{l}\text { In the granite disposal system (for which the permeability of the host rock was zero), the final } \\
\text { permeability of backfill for the rooms and drifts influenced the liquid inflow and thus the time } \\
\text { of complete corrosion of the containers. }\end{array}$ & C.5 \\
\hline
\end{tabular}


16. Results and Conclusions

Table 16.11-4. Summary of Information Gathered about the Geologic Barrier

Supporting

Results

Information

Found in

Section(s):

1. Assuming the salt behaves as a porous medium, the permeability of the salt is an important parameter controlling the quantity of brine flowing into the repository.

2. In salt, the permeability of the intrusion borehole was an important parameter influencing radionuclide releases.

3. In salt, the initial pore pressure in the halite formation was an important model parameter influencing radionuclide releases.

C.1

4. For the parameters assumed in granite, the repository often filled rapidly (after which the chosen horizontal and vertical gradients assumed for the region determined the direction of water flow through the repository).

5. In granite, the permeability of the postulated connections between the repository and the fracture zones was an important model parameter that influenced the amount of water flowing into and out of the repository and, thus, radionuclide releases.

6. A noticeable difference occurred in the isolating capability of a nondecaying, nonabsorbing tracer in the inventory of the salt and granite disposal systems.

- In salt, a maximum of $12 \%$ of the contents of the tracer in a panel is removed.

- In granite, $33 \%$ of the runs removed the entire contents of the tracer in the repository. 
Table 16.11-5. Summary of Information Gathered about the Agents Acting on the Disposal System

Supporting

Results

Found in

Section(s):

1. Direct release of contaminants to the ground surface from exploratory drilling using presentday drilling technology dominates the release for the salt repositories. Hence,

- The direct release to the surface, as evaluated by EPA normalization factors, is very important."

$16.1,16.2$

- Drilling frequency from an inadvertent human intrusion (for which DOE cannot take credit for control after $100 \mathrm{yr}$, except through passive markers) is the most important parameter influencing radionuclide releases.

- The permeability of the material that eventually fills the intrusion borehole is an important parameter for regulatory compliance per Appendix B of 40 CFR 191. The permeability controls brine flow out of a borehole that intersects a brine reservoir under the repository (which was a feature selected for inclusion in the salt disposal system).

$16.10, C .1$

- Direct releases were sensitive to the waste form only because of the different radionuclide inventories that were assumed for the waste parcels; in this assessment, the entire contents of a waste parcel were assumed to have been removed if it was struck by an exploratory drill bit.

2. In the salt disposal system, crushed salt filled the rooms and drifts and was assumed to compress to a low but variable permeability, usually sufficient to limit circulation of brine between intrusion boreholes. Specifically,

- In only 1 case out of 10 was the permeability high enough to allow flow through the repository and up the other intrusion borehole; and even in this case, the volume of brine discharged was very small.

- Calculated doses from the direct release route, however, and possible adverse affects are likely much lower because the EPA normalized factors were originally based on drinking contaminated water (ingestion).

Also, it is conceivable that the domination of direct release would diminish for regulatory periods longer than $10,000 \mathrm{yr}$, especially if exploratory drilling were assumed to stop after some period of time. But this diminishing influence at longer periods is by no means certain since exploratory drilling is required to initiate any releases other than diffusion through salt.

shaft was ignored in the simple model, and yet showed up in the Complex PA as an important source of water to fill the repository when sampled values of the permeability for the borehole and the secondary fractures were low. Differences were also uncovered for the salt repository. Fluid flow from the halite was ignored in the Simple PA, but for many sampled values of halite permeability in the Complex PA, halite became the dominant source of brine flowing into the repository. The conclusion to be drawn from this experience is not that the simplified model was not useful (it was, in fact, extremely useful in its capability for performing numerous runs of the individual waste parcels), but that reliance should not be placed on the results from the simplified model in lieu of performing a detailed analysis.

\subsubsection{Program Guidance}

General Guidance for the Waste Treatment Program. The findings listed in the previous section have been used to provide the DOE with specific information about the potential impact of various waste treatment options for spent nuclear fuel and high-level waste to be disposed of in two saturated generic repositories: bedded salt and igneous rock (granite). In answer to the question of what decisions can be made by DOE about treatment of the waste stored at INEL before a repository site or type is identified, the findings suggest that most decisions should wait until a repository type is known. The findings differ somewhat, however, between high-level waste and spent nuclear fuel. 
For high-level waste, it would seem that only those decisions related to storage (as calcine) should be made before a repository site is known; this recommendation is based not only on the results of this performance assessment, which indicate that the current waste forms are acceptable for meeting the Containment Requirements of 40 CFR 191, but also in light of possible changes to the regulations. For example, policies may change from specifying generic regulatory criteria to developing site-specific criteria (e.g., as suggested in the Energy Policy Act of 1992, Section 801). The difficulty with this conclusion is (1) the obvious finding that a granite repository appears to require a moderately corrosion-resistant container and/or a robust waste form and (2) the length of time required to develop a robust container and/or waste form.

For spent fuel, the same recommendations as the high-level waste apply as long as critical conditions are avoided in the container. To be sure, this is an important assumption that must be carefully studied before general conclusions about the spent nuclear fuel waste forms can be drawn.

Guidance for Waste Acceptance Criteria. The general findings have also been analyzed to provide the DOE with information about minimum waste acceptance criteria for material to be placed in a generic, deep geologic repository.

Using the total system analysis approach consistent with 40 CFR 191, the results of the preliminary performance assessment indicate that the acceptability of the waste form shows a strong sensitivity to the specific geologic media (i.e., host rock) of the chosen repository. Therefore, any preliminary waste acceptance criteria must clearly show that acceptance depends upon the geologic media of the repository.

- For a repository in bedded salt, all the plausible waste forms (e.g., whole graphite block and rod spent nuclear fuel, low- and highly enriched uranium spent nuclear fuel, and calcined high-level waste) can be placed directly in the disposal region as a group or individually, without being placed in a corrosion-resistant container, or without using a backfill buffer such as bentonite clay. Thus, little treatment is expected to be necessary for compliance with 40 CFR 191 . To be consistent with the extremely conservative assumptions of the $\mathrm{PA}$ analysis to avoid critical conditions, up to $10 \mathrm{~kg}$ of ${ }^{235} \mathrm{U}$ can be placed in each container.

- For a repository in granite, when disposed of as a group or individually, all currently existing waste types can be placed in a repository without conditioning but do require a moderately corrosion-resistant container and a bentonite clay backfill-buffer around the waste and some bentonite clay in the rooms and drifts to adsorb radionuclides. To be consistent with the assumptions of the PA analysis to avoid critical conditions, only $0.7 \mathrm{~kg}$ of ${ }^{235} \mathrm{U}$ can be placed in each container (i.e., cut to keep the ${ }^{235} \mathrm{U}$ content below $0.7 \mathrm{~kg}$, if necessary).

Recommendations for Future Analysis and Data Acquisition. The Nuclear Waste Policy Act (NWPA) of 1982 mandates that the DOE develop a permanent disposal option. Although this option may not have to be fully implemented, its feasibility should be demonstrated.

A preliminary performance assessment is a valuable and low-cost tool (relative to implementing waste treatment and disposal) that responds to the spirit of the NWPA by developing and demonstrating the capability to dispose of spent nuclear fuel stored at the INEL. The performance assessment is also the primary means by which the DOE will demonstrate compliance with long-term environmental regulations, and therefore is an essential component of any development program concerned with conditioning spent fuel and radioactive waste for temporary storage and eventual permanent disposal in order to refine goals and priorities.

Because performance assessments are iterative, an initial performance assessment serves to inform the interdisciplinary team performing the study about (a) the scientific information and data that must be gathered (either through literature or investigative studies) and (b) the resolution required for converting available information and data into suitable forms for use in the model selected for the performance assessment. The performance assessment presented in this report was no exception. In addition to providing guidance on the waste treatment options available for the waste stored at the INEL, the study identified future goals for data collection and modeling development efforts. This information, which represents a valuable benefit to the program as a whole, is presented below (Table 16.11-6). The recommendations are grouped into two categories: those that can be treated through the performance assessment 
Table 16.11-6. Recommended Tasks of the Waste Treatment Program

Supporting
Results Found
in Section(s):

\section{Tasks for PA}

1. Pursue a performance assessment of a repository containing spent nuclear fuel and highlevel waste from INEL in unsaturated volcanic tuff. Also,

- Continue the practice of using several models to evaluate consequences.

2. Reexamine the salt and granite disposal options after the data, conceptual models, and computational models are improved so that more defensible (and possibly more confident) predictions can be made.

3. Review gas generation and source term models developed in other high-level waste repositories and compare with the models used in this performance assessment.

4. Examine performance criteria other than 40 CFR 191.

$16.9,16.11 .2$

Chs. 12, 13, 14,$15 ; 16.9$

$16.9,16.11 .2$

Ch. 2

5. Examine phenomena not currently modeled:

- Determine whether nuclear critical conditions in a repository are noncatastrophic and would be technically acceptable.

- Explore the possibility of reaching critical mass from migration of radionuclides to a central location.

- Attempt to model diffusion of contaminants through the failed layers of the containers.

- Perform calculations of detailed waste package models to determine the most effective annular thickness of the backfill-buffer, including a disturbed rock zone around the waste package in the granite disposal system.

- Perform scoping calculations to determine how long it takes to consume $\mathrm{O}_{2}$ in the repository since different corrosion rates could occur.

6. Develop versions of a corrosion submodel and source term submodel that could be run outside the fluid flow and transport codes.

\section{Tasks for Waste Treatment Program}

1. Seek to confirm alteration rates used in the performance assessment." For example, perform a more thorough literature search to obtain corrosion data. Specifically,

- For the gas generation submodel, generalized corrosion rates more specific to conditions expected in the salt and granite repositories are needed for container materials.

- Temperature-dependent corrosion data between $293 \mathrm{~K}$ and $373 \mathrm{~K}$ are needed.

- For the source term submodel, more reliable information about susceptibility and data on failure modes for the container materials are needed.

2. Seek data on alteration rate of glass-ceramic matrix and percentage of radionuclides trapped in glass and ceramic phases.

3. Pursue better characterization of the current fuel types. Specifically,

- Data on the fraction of particles with failed silicon carbide coatings and cladding on special nuclear fuel.

- A better understanding of element solubility.

- Estimates of the likelihood of the failure of silicon carbide coatings in graphite fuel in the environment of the repository during the 10,000-yr regulatory period.

4. Select a preferred (e.g., "base case" or "reference design") type of waste parcel container and filler to use for the performance assessment calculations.
11.1

11.2

11.2

11.1, C.1

A.2.7

11.1, C.1

4.5, D.1

- INEL may already possess a means of gathering information on alteration rates since some radionuclides have been detected in the basins used to store the fuel, which indicates that the protective layers on some fuel have failed.

December 1993 
portion of the waste treatment program, and those that may require input from throughout the DOE complex, possibly with consensus building.

Recommended Tasks for Future Performance Assessments. In the preliminary performance assessment reported here, the results hint that the waste form shows a strong sensitivity to the specific geologic media of the repository. In response to this finding and the mandate of the NWPA Amendments of 1987, which require an examination of unsaturated volcanic tuff, a performance assessment of a repository in unsaturated tuff should be pursued. This type of calculation should continue the practice of using several models to evaluate consequences (possibly at different levels of simplification) in order to provide checks on each other and thereby develop a deeper understanding of the disposal system, or an understanding that can be quickly achieved. This recommendation does not mean to imply that a performance assessment in unsaturated tuff is not being done (see, for example, Barnard et al., 1992), but rather is suggesting an assessment similar to the one performed here that focuses on INEL wastes and their incremental effects on a moderately sized repository. Nor does this recommendation imply that we view the examination of the salt and granite disposal systems as complete; on the contrary, we have spent much time and money developing tools to examine these disposal systems, which should be used to reexamine the salt and granite disposal systems with more defensible data and, just as important, more defensible conceptual and computational models (e.g., improved discretization) to provide more defensible understanding and thus possibly more confident predictions. Note, however, that numerous iterations are not necessary since the United States has no current plan to build a repository for spent fuel in salt or granite. Recall, however, that bedded salt and granite were selected for this phase of the performance assessment because the geologic media were thought different enough to highlight different aspects of the 40 CFR 191 regulation, but similar enough to be analyzed within the time and resources given.

Although most model results were compared between the Simple and Complex PAs, the gas generation and source term models were nearly identical. Because of the demonstrated benefit of model-to-model comparisons, we need to review gas generation and source term models developed elsewhere in other high-level waste repositories and compare them with the models used in this performance assessment.

Other performance criteria need to be examined, besides 40 CFR 191, to substantiate the initial guidance from this performance assessment, which indicates that the current waste forms are acceptable for long-term environmental compliance. Because of changes contemplated for regulations on required repository performance, the internationally recognized criterion of individual doses may be the only other criterion easily identified at tivis time for this purpose. (It is also the criterion stipulated by the Energy Policy Act of 1992 for Yucca Mountain.)

Because modeling analyses are conditional on what was modeled, phenomena not currently modeled should be examined in future work. Some phenomena were omitted because they were thought unimportant; this assumption may need to be substantiated. More important, some phenomena were omitted because of a lack of time and resources available for this initial performance assessment, and these should be explored. ${ }^{t+\dagger}+t$ For example, critical conditions in the repository were not examined in the initial performance assessment because the containers were limited to a mass of $10 \mathrm{~kg}$ and $0.7 \mathrm{~kg}$ of ${ }^{235} \mathrm{U}$ in the salt and granite repositories, respectively. The results of this assumption was that a large number of containers (especially for the highly enriched fuel) was required which, because of cost implications, suggest that future work should attempt to determine whether nuclear critical conditions in a repository are noncatastrophic and thus technically acceptable. Furthermore, a situation in which ${ }^{235} \mathrm{U}$ migrates from several containers to a central location was not simulated. Although this possibility is remote, a future performance assessment should perform supporting calculations to explore the possibility of reaching critical mass from migration of radionuclides to a central location.

Moreover, because movement of radionuclides at the waste package is almost exclusively by diffusion and will likely cause substantial delay, attempts should inade to model diffusion of radionuclides through failed layers of the containers. Finally, if emplacement within drillholes remains a preferred based case option to consider, then optimization of annular thickness of the backfill-buffer should be pursued and inclusion of a disturbed rock zone around the waste package when modeling the granite disposal system.

${ }^{++t++}$ Many of these tasks would not require entirely new performance assessment calculations, but instead would entail a more thorough examination of existing results, a search for information from other radionuclide waste disposal programs, or performing supporting side calculations. 
Scoping calculations using appropriate oxic corrosion rates need to be performed to determine how long it would take for the $\mathrm{O}_{2}$ in the repository to be consumed. If the time period for consuming all the $\mathrm{O}_{2}$ is not small compared to the regulatory period, the gas generation model should be modified to include two sets of stoichiometries and corrosion rates: one set for oxic conditions and one set for anoxic conditions.

Many of the results reported herein are directly related to the output of the gas generation and source term submodels, which presently run with the fluid flow and transport codes. To respond rapidly to future "what if" questions about the waste forms, it may be useful to develop a corrosion submodel (not necessarily the gas generation submodel) and source term submodel that can be run outside the fluid flow and transport codes.

Recommended Tasks for Waste Treatment Program. In general, an extensive need exists for defensible data for future iterations of evaluating the performance of INEL spent fuel and radioactive wastes in a geologic repository. Several specific data needs are mentioned below.

A more thorough literature search is needed to find corrosion data; specifically, temperature-dependent corrosion data between $293 \mathrm{~K}$ and $373 \mathrm{~K}$ are needed; for the gas generation submodel, generalized corrosion rates for container materials such as lead, stainless steel 304L, and Inconel-625 nickel-based alloy are needed at conditions expected in the salt and granite repositories. For the source term submodel (and, to some extent, the gas generation submodel), information on susceptibility and data on failure modes for the container materials are needed on stress-corrosion cracking, pitting, crevice corrosion, delayed hydrogen-induced failure, and aging reactions ("localized" corrosion) to adequately assess the longevity of the barriers of the container.

In this analysis, information was only available on experimental mixtures of glass-ceramic; specific alteration rates for calcined waste immobilized in a glass-ceramic or borosilicate glass matrix were not available (except for the $\sim 70 \%$ radionuclide loading possible with glass-ceramic, as opposed to $\sim 30 \%$ for glass). To distinguish these two waste treatment options, data on the alteration rate for a preferred glass-ceramic mixture and percentage of nuclides trapped in glass and ceramic phases are needed. For this need, the alteration rate refers to any change from one phase to another that liberates radionuclides.

In addition, scientifically agreed-upon characterization of many aspects of the current waste form is needed for the DOE to rely on many of the findings discussed here, for example, the general finding that the current graphite spent fuel (Waste Parcel 1) in a borehole already provides an effertive engineered barrier. Specific information needed includes data on the fraction of particles with failed silicon carbide coatings (an important parameter for the graphite spent fuel) and failed cladding on special spent nuclear fuel. Also, dependable estimates are needed of the likelihood of additional failure of silicon carbide coatings of the graphite fuel in the environment of the repository during the regulatory period. (As mentioned before, the regulatory period for $40 \mathrm{CFR} 191$ is $10,000 \mathrm{yr}$, but this time period could change with new regulations.)

Even when numerous corrosion layers are present in spent nuclear fuel, the ultimate limit to release from the source term submodel is element solubility. Although direct measurement may be infeasible because (1) equilibrium conditions are difficult to reach in short lab experiments and (2) the experiments are extremely costly and time consuming to prepare because of environmental, safety, and health issues, an understanding of elemental solubilities in proposed repositories is needed, if uncertainties in the PA predictions are to be reduced (or at least corroborated). As pointed out earlier, the particular radionuclides that are a significant percentage of the releases change with the performance metric and the location at which the metric is measured; hence, listing the most important solubilities to determine is difficult.

The Waste Treatment Program should select a preferred type (e.g., "base case" or "reference design") of the waste parcel container to use for the performance assessment calculations. Some containers, such as self-shielding containers, use significant amounts of metal that can affect the amount of gas produced through corrosion. Furthermore, highly corrosion-resistant containers would provide a redundant barrier for the waste form in addition to deep geologic disposal. Another decision that would be useful is to determine the type of filler (e.g., sand or metal) in the container to use for the performance assessment calculations. Ideally, the process would be as follows: (a) establish the containment requirements for container system, (b) select container configuration and material of 
construction, (c) determine whether a filler material is necessary, and (d) select the filler material, if it is necessary. Although this thorough process may be difficult to implement before the next phase of this initial performance assessment, it could be implemented over the long term. Furthermore, the first steps for establishing the minimum containment requirements that corrosion-resistant containers must meet could be discovered by further analysis of the results of this performance assessment.

7

8 
belin, H., L. Moreno, S. Tunbrant, J. Gidlund, and I. Neretnieks. 1985. "Flow and Tracer Movement in Some Natural Fractures in the Stripa Granite: Results from the Stripa Project Phase I," Proceedings of the Symposium on In Situ Experiments in Granite Associated with the Disposal of Radioactive Waste, International Stripa Project, Stockholm, Sweden, June 4-6, 1985. Paris, France: Organisation for Economic Co-Operation and Development. 67-81.

AECB (Atomic Energy Control Board). 1987. Regulatory Objectives, Requirements and Guidelines for the Disposal of Radioactive Wastes-Long-Term Aspects. Regulatory Policy Statement. INIS-MF-11334. Ottawa, Ontario: Atomic Energy Control Board.

Aikas, T. 1993. "Program and Results of Preliminary Site Characterization for Final Disposal of Spent Nuclear Fuel in Finland," Low and Intermediate Level Radioactive Waste Management, Proceedings of the 1993 International Conference on Nuclear Waste Management and Environmental Remediation, Prague, Czech Republic, September 5-11, 1993. Eds. D. Alexandre, R. Baker, R. Kohout, and J. Marek. New York, NY: The American Society of Mechanical Engineers. Vol. 1, 465-470.

American Heritage. 1975. The American Heritage Dictionary of the English Language. New York, NY: American Heritage Publishing Company.

Andersson, J., T. Carlsson, T. Eng, F. Kautsky, E. Söderman, and S. Wingefors. 1989. The Joint SKISKB Scenario Development Project. SKB TR-89-35. Stockholm, Sweden: Swedish Nuclear Fuel and Waste Management Co.

Asano, H., H. Wakamatsu, and M. Akashi. 1992. "Corrosion Lifetime Assessment for Candidate Materials of Geological Disposal Overpack for High-Level Nuclear Waste Canisters-Perspective of R\&D in Japan," High Level Radioactive Waste Management, Proceedings of the Third International Conference, Las Vegas, NV, April 12-16, 1992. La Grange Park, IL: American Nuclear Society, Inc.; New York, NY: American Society of Civil Engineers. Vol. 2, 1658-1669.

Barker, J.A. 1988. “A Generalized Radial Flow Model for Hydraulic Tests in Fractured Rock," Water Resources Research. Vol. 24, no. 10, 1796-1804.

Barnard, R.W. In preparation. "Source-Term Radionuclide Inventory," Chapter 4 of Total-System Performance Assessment for Yucca Mountain-SNL Second Iteration (TSPA-1993). SAND93-2675. Albuquerque, NM: Sandia National Laboratories.

Barnard, R.W., M.L. Wilson, H.A. Dockery, P.G. Kaplan, R.R. Eaton, F.W. Bingham, J.H. Gauthier, and T.H. Robey. 1992. TSPA 1991: An Initial Total-System Performance Assessment for Yucca Mountain, Yucca Mountain Site Characterization Project. SAND91-2795. Albuquerque, NM: Sandia National Laboratories.

Berglund, J.W. 1992. Mechanisms Governing the Direct Removal of Wastes from the Waste Isolation Pilot Plant Repository Caused by Exploratory Drilling. SAND92-7295. Albuquerque, NM: Sandia National Laboratories.

Berglund, J.W. In preparation. SUMQ_3D: A Heat Conduction Code That Computes a Temperature From a ThreeDimensiona: Layout of Heat Sources; (A computer program of the WIPP CAMCON system). Albuquerque, NM: Sandia National Laboratories.

Berreth, J.R. 1988. Inventories and Properties of ICPP Calcined High-Level Waste. WINCO-1050. Idaho Falls, ID: Westinghouse Idaho Nuclear Co., Inc. 
Bertozzi, G., and A. Saltelli. 1985. "LISA: A Performance Assessment Code for Geological Repositories of Radioactive Waste," High-Level Nuclear Waste Disposal, International Topical Meeting on High-Level Nuclear Waste Disposal-Technology and Engineering, Pasco, WA, September 24-26, 1985. Ed. H.C. Burkholder. Columbus, OH: Battelle Press. 607-615.

Bertram-Howery, S.G., and R.L. Hunter, Eds. 1989. Preliminary Plan for Disposal-System Characterization and Long-Term Performance Evaluation of the Waste Isolation Pilot Plant. SAND89-0178. Albuquerque, NM: Sandia National Laboratories.

Bertram-Howery, S.G., M.G. Marietta, R.P. Rechard, P.N. Swift, D.R. Anderson, B.L. Baker, J.E. Bean, Jr., W. Beyeler, K.F. Brinster, R.V. Guzowski, J.C. Helton, R.D. McCurley, D.K. Rudeen, J.D. Schreiber, and P. Vaughn. 1990. Preliminary Comparison with 40 CFR Part 191, Subpart B for the Waste Isolation Pilot Plant, December 1990. SAND90-2347. Albuquerque, NM: Sandia National Laboratories.

Betcher, R.N. 1983. Geology and Hydrogeology of Surficial Materials on the Underground Research Laboratory Lease Area. AECL-TR-219. Pinawa, Manitoba: Atomic Energy of Canada Limited, Applied Geoscience Branch, Whiteshell Nuclear Research Es'ablishment.

Bethke, C. 1992. The Geochemist's Workbench: A User's Guide to Rxn, Act2, Tact, React, and GtPlot. Urbana, IL: C. Bethke and Board of Trustees of the University of Illinois.

Billaux, D., J.P. Chiles, K. Hestir, and J. Long. 1989. "Three-Dimensional Statistical Modelling of a Fractured Rock Mass - An Example from the Fanay-Augères Mine," International Journal of Rock Mechanics and Mining Sciences \& Geomechanics Abstracts. Vol. 26, no. 3-4, 281-299.

Birgersson, L., and I. Neretnieks. 1990. "Diffusion in the Matrix of Granitic Rock: Field Test in the Stripa Mine," Water Resources Research. Vol. 26, no. 11, 2833-2842.

Blom, G. 1989. Probability and Statistics: Theory and Applications. New York, NY: Springer-Verlag.

Bonano, E.J., P.A. Davis, L.R. Shipers, K.F. Brinster, W.E. Beyeler, C.D. Updegraff, E.R. Shepherd, L.M. Tilton, and K.K. Wahi. 1988. Demonstration of a Performance Assessment Methodology for High-Level Radioactive Waste Disposal in Basalt Formations. SAND86-2325, NUREG/CR-4759. Albuquerque, NM: Sandia National Laboratories.

Bonano, E.J., S.C. Hora, R.L. Keeney, and D. von Winterfeldt. 1990. Elicitation and Use of Expert Judgment in Performance Assessment for High-Level Radioactive Waste Repositories. SAND89-1821, NUREG/CR-5411. Albuquerque, NM: Sandia National Laboratories.

Börgesson, L. 1993. "Mechanical Interaction Between Rock, Buffer and Canister in Repositories," Low and Intermediate Level Radioactive Waste Management, Proceedings of the 1993 International Conference on Nuclear Waste Management and Environmental Remediation, Prague, Czech Republic, September 5-11, 1993. Eds. D. Alexandre, R. Baker, R. Kohout, and J. Marek. New York, NY: The American Society of Mechanical Engineers. Vol. 1, 28-38.

Borns, D.J. 1987. Rates of Evaporite Deformation: The Role of Pressure Solution. SAND85-1599. Albuquerque, NM: · Sandia National Laboratories.

Brandshaug, T. 1986. A Thermomechanical Analysis of WIPP DHLW Experiments: Waste Container/Borehole Interactions. SAND85-7210, RSI-0287. Albuquerque, NM: Sandia National Laboratories.

Brinster, K.F. 1991. Preliminary Geohydrologic Conceptual Model of the Los Medaños Region Near the Waste Isolation Pilot Plant for the Purpose of Performance Assessment. SAND89-7147. Albuquerque, NM: Sandia National Laboratories. 

lication. F.A. Donath. 1978. Risk Methodology for Geologic Disposal of Radioactive Waste: Interim Report. NUREG/ CR-0458, SAND78-0029. Albuquerque, NM: Sandia National Laboratories.

Campbell, J.E., C.D. Leigh, and D.E. Longsine. 1991. NEFTRAN-S: A Network Flow and Contaminant Transport Model for Statistical and Deterministic Simulations Using Personal Computers. SAND90-1987. Albuquerque, NM: Sandia National Laboratories.

Carlsten, S., O. Olsson, O. Persson, and M. Sehlstedt. 1988. Site Characterization and Validation - Monitoring of Head in the Stripa Mine During 1987. Stripa Project 88-02. Stockholm, Sweden: Swedish Nuclear Fuel and Waste Management Co.

Carslaw, H.S., and J.C. Jaeger. 1959. Conduction of Heat in Solids. 2nd ed. Oxford, England: Clarendon Press.

Carter, L.J. 1987. Nuclear Imperatives and Public Trust: Dealing with Radioactive Waste. Washington, DC: Resources for the Future, Inc.; Baltimore, MD: Johns Hopkins University Press.

Castellan, G.W. 1971. Physical Chemistry, 2nd ed. Reading, MA: Addison-Wesley Publishing Company.

Chu, M.S.Y., N.R. Ortiz, K.K. Wahi, R.E. Pepping, and J.E. Campbell. 1983. An Assessment of the Proposed Rule (10CFR60) for Disposal of High-Level Radioactive Wastes in Geologic Repositories, Volume 1. SAND82-2969, NUREG/CR-3111. Albuquerque, NM: Sandia National Laboratories.

Clean Air Act. Modified 1991. 42 U.S.C.A 7401 et seq.

Corey, A.T. 1990. Mechanics of Immiscible Fluids in Porous Media. 2nd ed. Littleton, CO: Water Resources Pub-

Cranwell, R.M., J.E. Campbell, J.C. Helton, R.L. Iman, D.E. Longsine, N.R. Ortiz, G.E. Runkle, and M.J. Shortencarier. 1987. Risk Methodology for Geologic Disposal of Radioactive Waste: Final Report. NUREG/CR-2452, SAND81-2573. Albuquerque, NM: Sandia National Laboratories. 
Cranwell, R.M., R.V. Guzowski, J.E. Campbell, and N.R. Ortiz. 1990. Risk Methodology for Geologic Disposal of Radioactive Waste: Scenario Selection Procedure. NUREG/CR-1667, SAND80-1429. Albuquerque, NM: Sandia National Laboratories. (Original version of this report published in 1982 under the same title and report numbers.)

Crookston, R.B., W.E. Culham, and W.H. Chen. 1979. "Numerical Simulation Model for Thermal Recovery Processes," Society of Petroleum Engineers Journal. Vol. 19, no. 1, 37-58.

Davies, P.B. 1991. Evaluation of the Role of Threshold Pressure in Controlling the Flow of Waste-Generated Gas into Bedded Salt at the Waste Isolation Pilot Plant. SAND90-3246. Albuquerque, NM: Sandia National Laboratories.

Davies, P.B., and A.M. LaVenue. 1990. Appendix A: "Memo 11: Additional Data for Characterizing 2-Phase Flow Behavior in Waste-Generated Gas Simulations and Pilot Point Information for Final Culebra 2-D Model (SAND89-7068/1)," Data Used in Preliminary Performance Assessment of the Waste Isolation Pilot Plant (1990). R.P. Rechard, H. Iuzzolino, and J.S. Sandha. SAND89-2408. Albuquerque, NM: Sandia National Laboratories. A-139 through A-156.

Davies, P.B., R.L. Beauheim, and E.D. Gorham. 1992. Appendix A: "Additional Comments on Far-Field Anhydrite Permeability Distribution in 'PA Modeling Using BRAGFLO-1992' 7-8-92 Memo by J. Schreiber," Preliminary Performance Assessment for the Waste Isolation Pilot Plant, December 1992. Volume 3: Model Parameters. Sandia WIPP Project. SAND92-0700/3. Albuquerque, NM: Sandia National Laboratories. A-39 through A-45.

Davison, C.C. 1980. Physical Hydrogeology Measurements Conducted in Boreholes WN-1,WN-2 and WN-4 to Assess the Local Hydraulic Conductivity and Hydraulic Potential of a Granitic Rock Mass. AECL-TR-26. Pinawa, Manitoba: Atomic Energy of Canada Limited, Inland Waters Directorate.

de Marsily, G. 1986. Quantitative Hydrogeology: Groundwater Hydrology for Engineers. Orlando, FL: Academic Press.

de Marsily, G., V. Berhendt, D. Ensminger, C. Flebus, B. Hutchinson, P. Kane, A. Karpf, R. Klett, S. Mobbs, and M. Poulin. 1988. Feasibility of Disposal of High-Level Radioactive Waste into the Seabed. Vol. 2: Radiological Assessment. Paris, France: Nuclear Energy Agency, Organisation for Economic Co-Operation and Development.

Dershowitz, W., G. Lee, and J. Grier. 1991. User Documentation, FracMan (Beta) 2.3, Interactive Discrete Feature, Data Analysis, Geometric Modeling, and Exploration Simulation. Redmond, WA: Golder Associates, Inc.

Domenico, P.A., and F.W. Schwartz. 1990. Physical and Chemical Hydrogeology. New York, NY: John Wiley \& Sons.

Donovan, R.I. 1989. Letter to D. Gombert, "Complete Analysis of the Tank Farm Wastes." Letter RID-05-89, Dated August 15, 1989.

Dudley, A.L., R.R. Peters, J.H. Gauthier, M.L. Wilson, M.S. Tierney, and E.A. Klavetter. 1988. Total System Performance Assessment Code (TOSPAC): Volume 1, Physical and Mathematical Bases: Yucca Mountain Project. SAND85-0002. Albuquerque, NM: Sandia National Laboratories.

Durham, W.B., H.C. Heard, C.O. Boro, K.T. Keller, W.E. Ralph, and D.A. Trimmer. 1987. Thermal Properties of Permian Basin Evaporites to $493 \mathrm{~K}$ Temperature and $30 \mathrm{MPa}$ Confining Pressure. BMI/ONWI-633. Columbus, $\mathrm{OH}$ : Battelle Memorial Institute, Office of Nuclear Waste Isolation.

Dverstorp, B., and J. Andersson. 1989. "Application of the Discrete Fracture Network Concept with Field Data: Possibilities of Model Calibration and Validation," Water Resources Research. Vol. 25, no. 3, 540-550. 

Aspects," Risks Associated with Human Intrusion at Radioactive Waste Disposal Sites, NEA Workshop, Paris, France, June 5-7, 1989. Paris, France: Nuclear Energy Agency, Organisation for Economic Co-Operation and Development. 100-104.

EPA (Environmental Protection Agency). 1978. "Chapter V-Council on Environmental Quality," Code of Federal Regulations 40, Parts 1500-1508. Washington, DC: Superintendent of Documents, U.S. Government Printing Office. (As amended and published in the most recent Code of Federal Regulations.)

EPA (Environmental Protection Agency). 1984. Groundwater Protection Strategy. Washington, DC: Office of Groundwater Protection, Environmental Protection Agency.

EPA (Environmental Protection Agency). 1985. "40 CFR Part 191: Environmental Standards for the Management and Disposal of Spent Nuclear Fuel, High-Level and Transuranic Radioactive Wastes; Final Rule," Federal Register. Vol. 50, no. 182, 38066-38089.

EPA (Environmental Protection Agency). 1991. "Environmental Radiation Protection Standards for Management and Disposal of Spent Nuclear Fuel, High-Level and Transuranic Radioactive Wastes," Code of Federal Regulations 40, Part 191. Washington, DC: Superintendent of Documents, U.S. Government Printing Office.

EPA (Environmental Protection Agency). 1992a. Proposed Rule-40 CFR 191: Environmental Standards for the Management and Disposal of Spent Nuclear Fuel, High-Level and Transuranic Radioactive Wastes. Washington, DC: Environmental Protection Agency. (Also known as Working Draft 4-dated February 3, 1992.)

EPA (Environmental Protection Agency). 1992b. "Procedures for Decisionmaking," Code of Federal Regulations 40, Part 124. Washington, DC: Superintendent of Documents, U.S. Government Printing Office.

EPA (Environmental Protection Agency). 1992c. "Identification and Listing of Hazardous Waste," Code of Federal Regulations 40, Part 261. Washington, DC: Superintendent of Documents, U.S. Government Printing Office.

EPA (Environmental Protection Agency). 1992d. "Land Disposal Restrictions," Code of Federal Regulations 40, Part 268. Washington, DC: Superintendent of Documents, U.S. Government Printing Office.

EPA (Environmental Protection Agency). 1993a. "CFR Parts 144 and 191: Environmental Radiation Protection Standards for the Management and Disposal of Spent Nuclear Fuel, High-Level and Transuranic Radioactive Wastes," Federal Register. Vol. 58, no. 26, 7924-7936.

EPA (Environmental Protection Agency). 1993b. " 40 CFR 194: Criteria for the Certification of Compliance with Environmental Radiation Protection Standards for the Management and Disposal of Spent Nuclear Fuel, HighLevel and Transuranic Radioactive Wastes," Federal Register. Vol. 58, no. 53, 15320-15321.

EPRI (Electric Power Research Institute). 1992. Demonstration of a Risk-Based Approach to High-Level Waste Repository Evaluation: Phase 2. TR-100384. Palo Alto, CA: Electric Power Research Institute.

Ermold, L.F. 1992. "Production Department Monthly Report." Letter LFE-154-92.

Everitt, R.A., and R.S. Read. 1989. Geology of the 240 Level of the Underground Research Laboratory. Volume 1 General Geology. AECL-TR-4911. Pinawa, Manitoba: Whiteshell Nuclear Research Establishment, Atomic Energy of Canada Limited. 
Farmer, J.C., and R.D. McCright. 1989. "Localized Corrosion and Stress Corrosion Cracking of Candidate Materials for High-Level Radioactive Waste Disposal Containers in U.S.: A Critical Literature Review," Scientific Basis for Nuclear Waste Management XII, Materials Research Society Symposium Proceedings, Berlin, Germany, October 10-13, 1988. Eds. W. Lutze and R.C. Ewing. Pittsburgh, PA: Materials Research Society. Vol. 127, 359-371.

Feller, W. 1966. An Introduction to Probability Theory and Its Applications. New York, NY: John Wiley \& Sons, Inc. Vol. 1.

FFCA. 1992. Federal Facility Compliance Act of 1992. Public Law 102-386.

Fillmure, D.L. 1993. Private Communication to D.R. Evans, Dated January 5, 1993.

Finnish Centre for Radiation and Nuclear Safety. 1991. Decision of the Council of State on the General Regulations for the Safety of Nuclear Power Plants (395/91) and of a Disposal Facility for Reactor Waste (398/91). STUKB-YTO-87. Helsinki, Finland: Finnish Centre for Radiation and Nuclear Safety.

Freeze, R.A., and J.A. Cherry. 1979. Groundwater. Englewood Cliffs, NJ: Prentice-Hall.

Garisto, F., and N.C. Garisto. 1991. "Source Term Models for the Release of Radionuclides from Used Nuclear Fuel," Proceedings from the technical workshop on near-field performance assessment for high-level waste, Madrid, Spain, October 15-17, 1990. Eds. P. Sellin, M. Apted, and J. Gago. SKB-TR-91-59. Stockholm, Sweden: Swedish Nuclear Fuel and Waste Management Co. 51-65.

Garisto, N.C., and D.M. LeNeveu. 1989. Vault Model for the Disposal of Used CANDU Fuel. Documentation and Analysis of Scoping Calculations. AECL-9578. Pinawa, Manitoba: Atomic Energy of Canada Limited, Whiteshell Nuclear Research Establishment.

Gascoyne, M. 1986. SCSB - A Standard Canadian Shield Brine. AECL-TR-403. Pinawa, Manitoba: Atomic Energy of Canada Limited, Applied Geoscience Branch, Whiteshell Nuclear Research Establishment.

Gascoyne, M. 1988. Reference Groundwater Composition for a Depth of 500m in the Whiteshell Research AreaComparison with Synthetic Groundwater WN-1. AECL-TR-463. Pinawa, Manitoba: Atomic Energy of Canada Limited, Applied Geoscience Branch, Whiteshell Nuclear Research Establishment.

Gauthier, J.H., A.L. Dudley, L.H. Skinner, M.L. Wilson, and R.R. Peters. 1992. Total System Performance Assessment Code (TOSPAC): Volume 2, User's Guide: Yucca Mountain Site Characterization Project. SAND85-0004. Albuquerque, NM: Sandia National Laboratories.

Glimm, J. 1991. "Nonlinear and Stnchastic Phenomena: The Grand Challenge for Partial Differential Equations," SIAM Review. Vol. 33, no. 4, 626-643.

Goodwin, F.E., and R.E. Westerman. 1992. "Extended-Life Nuclear Waste Package Utilizing Redundant Corrosion/ Containment Barriers," High Level Radioactive Waste Management, Proceedings of the Third International Conference, Las Vegas, NV, April 12-16, 1992. La Grange Park, IL: American Nuclear Society, Inc.; New York, NY: American Society of Civil Engineers. Vol. 2, 1681-1686.

Green, D.H., and H.F. Wang. 1990. "Specific Storage as a Poroelastic Coefficient," Water Resources Research. Vol. 26, no. 7, 1631-1637.

Gustafsson, G., M. Liedholm, and I. Rhen. 1991. ASPO Hard Rock Laboratory Predictions Prior to Excavation and the Process of Their Validation. SKB-TR-91-23. Stockholm, Sweden: Swedish Nuclear Fuel and Waste Management Co. 
Guzowski, R.V. 1990. Preliminary Identification of Scenarios That May Affect the Escape and Transport of Radionuclides From the Waste Isolation Pilot Plant, Southeastern New Mexico. SAND89-7149. Albuquerque, NM: Sandia National Laboratories.

Guzowski, R.V., and J.C. Helton. 1991. "Chapter 4. Scenarios for Compliance Assessment," Preliminary Comparison with 40 CFR Part 191, Subpart B for the Waste Isolation Pilot Plant, December 1991. Volume 1: Methodology and Results. WIPP Performance Assessment Division. SAND91-0893/1. Albuquerque, NM: Sandia National Laboratories. 4-1 through 4-91.

Hammersley, J.M., and D.C. Handscomb. 1964. Monte Carlo Methods. New York, NY: John Wiley \& Sons, Inc.

Hara, K., T. Fujita, A. Saotome, M. Uchida, and N. Sasaki. 1991. "Conceptual Design Study of Geological Disposal System of High-Level Waste," High Level Radioactive Waste Management, Proceedings of the 2nd Annual International Conference, Las Vegas, NV, April 28-May 3, 1991. La Grange Park, IL: American Nuclear Society, Inc.; New York, NY: American Society of Civil Engineers. Vol. 1, 347-354.

Heinrich, W.F. 1984. Geosphere Submodel for the Second Interim Assessment of the Canadian Concept for Nuclear Fuel Waste Disposal - Post-Closure Phase. Technical Record TR-286. Pinawa, Manitoba: Atomic Energy of Canada Limited, Environmental Safety Assessment Branch, Whiteshell Nuclear Research Establishment.

Helton, J.C. 1991. "Performance Assessment Overview," Preliminary Comparison with 40 CFR Part 191, Subpart $B$ for the Waste Isolation Pilot Plant, December 1991. Volume 1: Methodology and Results. SAND91-0893/1. Albuquerque, NM: Sandia National Laboratories. 3-1 through 3-89.

Helton, J.C. 1993. "Risk, Uncertainty in Risk, and the EPA Release Limits for Radioactive Waste Disposal," Nuclear Technology. Vol. 101, no. 1, 18-39.

Helton, J.C., J.W. Garner, R.D. McCurley, and D.K. Rudeen. 1991. Sensitivity Analysis Techniques and Results for Performance Assessment at the Waste Isolation Pilot Plant. Contractor Report SAND90-7103. Albuquerque, NM: Sandia National Laboratories.

Helton, J.C., J.W. Garner, R.P. Rechard, D.K. Rudeen, and P.N. Swift. 1992. Preliminary Comparison with 40 CFR Part 191. Subpart B for the Waste Isolation Pilot Plant, December 1991. Volume 4: Uncertainty and Sensitivity Analysis Results. SAND91-0893/4. Albuquerque, NM: Sandia National Laboratories.

Henry, H.R. 1964. "Effects of Dispersion on Salt Encroachment in Coastal Aquifers," Sea Water in Coastal Aquifers. H.H. Cooper, Jr., F.A. Kohout, H.R. Henry, and R.E. Glover. Geological Survey Water-Supply Paper 1631C. Washington, DC: United States Government Printing Office. C-70 through C-84.

Hewlett-Packard. "Petroleum Fluids PAC"

Hickerson, J., T.J. Freeman, J.Y. Boisson, N. Murray, F. Gera, H. Nakamura, J.D. Nieuwenhuis, and K.H. Schaller. 1988. Feasibility of Disposal of High-Level Radioactive Waste into the Seabed. Vol. 4: Engineering. INIS-XN178, SAND87-2223. Paris, France: Nuclear Energy Agency, Organisation for Economic Co-Operation and Development.

Hirsekorn, R.P., A. Nies, H. Rausch, and R. Storck. 1991. Performance Assessment of Confinements for MediumLevel and $\alpha$-contaminated Waste, Pacoma Project, Rock Salt Option. EUR 13634 EN. Luxembourg: Commission of the European Communities.

HSWA. 1984. Hazardous and Solid Waste Amendments of 1984. Public Law 98-616.

Hunter, R.L. 1989. Events and Processes for Constructing Scenarios for the Release of Transuranic Waste From the Waste Isolation Pilot Plant, Southeastern New Mexico. SAND89-2546. Albuquerque, NM: Sandia National Laboratories. 
unter, R.L., R.M. Cranwell, and M.S.Y. Chu. 1986. Assessing Compliance With the EPA High-Level Waste Standard: An Overview. NUREG/CR-4510, SAND86-0121. Albuquerque, NM: Sandia National Laboratories.

Huyakorn, P.S., and G.F. Pinder. 1983. Computation Methods in Subsurface Flow. New York, NY: Academic Press.

Huyakorn, P.S., H.O. White, Jr., and S. Panday. 1991. STAFF2D Version 3.1: A Two-Dimensional Finite Element Code for Simulating Fluid Flow and Transport of Radionuclides in Fractured Porous Media with Water Table Boundary Conditions. Herndon, VA: HydroGeoLogic, Inc.

IAEA (International Atomic Energy Agency). 1989. Safety Principles and Technical Criteria for the Underground Disposal of High-Level Radioactive Wastes. Safety Series No. 99. Vienna, Austria: International Atomic Energy Agency.

Ibrahim, M.A., M.R. Tek, and D.L. Katz. 1970. Threshold Pressure in Gas Storage. Arlington, VA: American Gas Association, Inc.

ICRP (International Commission on Radiological Protection). 1983. "ICRP Publication 38: Radionuclide Transformations - Energy and Intensity of Emissions," Annals of the ICRP. Vols. 11-13. Elmsford, NY: Pergamon Press, Inc.

ICRP (International Commission on Radiological Protection). 1985. "ICRP Publication 46: Radiation Protection Principles for the Disposal of Solid Radioactive Waste," Annals of the ICRP. Vol. 15, no. 4. Elmsford, NY: Pergamon Press, Inc.

IDB (Integrated Data Base). 1992. Integrated Data Base for 1992: U.S. Spent Fuel and Radioactive Waste Inventories, Projections, and Characteristics. DOE/RW-0006-Rev. 8. Oak Ridge, TN: Oak Ridge National Laboratory.

Ikeda, B.M., M.G. Bailey, C.F. Clarke, and D.W. Shoesmith. 1989. Crevice Corrosion of Titanium Under Nuclear Fuel Waste Conditions. AECL-9568. Pinawa, Manitoha: Atomic Energy of Canada Limited, Whiteshell Nuclear Research Establishment.

Iman, R.L., and W.J. Conover. 1979. "The Use of the Rar. 4, 499-509.

Iman, R.L., and W.J. Conover. 1980. "Small Sample Sensitivity Analysis Techniques for Computer Models, With an Application to Risk Assessment," Communications in Statistics. Vol. A9, no. 17, 1749-1842. "Rejoinder to comments," pp. 1863-74.

Iman, R.L., and W.J. Conover. 1982. "A Distribution-Free Approach to Inducing Rank Correlation Among Input Variables," Communications in Statistics: Simulation and Computation. Vol. 11, no. 3, 311-334.

Iman, R.L., and J.C. Helton. 1985. A Comparison of Uncertainty and Sensitivity Analysis Techniques for Computer Models. SAND84-1461, NUREG/CR-3904. Albuquerque, NM: Sandia National Laboratories.

Iman, R.L., J.M. Davenport, E.L. Frost, and M.J. Shortencarier. 1980. Stepwise Regression with PRESS and Rank Regression (Program and User's Guide). SAND79-1472. Albuquezque, NM: Sandia National Laboratories.

Intera Technologies Ltd. 1985. Hydrogeologicnl Modeling of the Underground Research Laboratory Site: Characterization and Prediction. AECL-TR-345. Pinawa, Manitoba: Atomic Energy of Canada Limited, Environmental Safety Assessment Branch, Whiteshell Nuclear Research Establishment.

Isaacs, T.H., M.B. Blanchard, K. Baskin, T.A. Cotton, J. M. McGarry, J. Burns, and R. Waxman. 1993. A Proposed Alternative Strategy for the Department of Energy's Civilian Radioactive Waste Management Program. Prepared by Task Force on an Alternative Program Strategy. 
Isherwood, D.J. 1981. Geoscience Data Base Handbook for Modeling a Nuclear Waste Repository. Washington, DC: Office of Nuclear Material Safety and Safeguards, U.S. Nuclear Regulatory Commission.

IT Corporation. 1987. Laboratory Investigation of Crushed Salt Consolidation and Fracture Healing. BMI/ONWI631. Columbus, OH: Office of Nuclear Waste Isolation, Battelle Memorial Institute.

Johnson, N.L., and S. Kotz. 1970. Continuous Univariate Distributions. New York, NY: John Wiley \& Sons, Inc.

Kalos, M.H., and P.A. Whitlock. 1986. Monte Carlo Methods. New York, NY: John Wiley \& Sons, Inc.

Kaplan, S., and B.J. Garrick. 1981. “On the Quantitative Definition of Risk," Risk Analysis. Vol. 1, no. 1, 11-27.

Katsube, T.J., T.W. Melnyk, and J.P. Hume. 1986. Pore Structure from Diffusion in Granitic Rocks. Technical Record TR-381. Pinawa, Manitoba: Atomic Energy of Canada Limited, Whiteshell Nuclear Research Establishment.

KBS. 1983. Final Storage of Spent Nuclear Fuel. KBS-3. Stockholm, Sweden: SKBF/KBS, Swedish Nuclear Fuel Supply Co./ Division KBS.

Kelley, V.A., and G.J. Saulnier, Jr. 1990. Core Analyses for Selected Samples from the Culebra Dolomite at the Waste Isolation Pilot Plant Site. SAND90-7011. Albuquerque, NM: Sandia National Laboratories.

King, P.B., Comp. 1969. Tectonic Map of North America. Washington, DC: U.S. Geological Survey.

Kircher, J.F., and D.J. Bradley. 1983. "NWTS Waste Package Design and Materials Testing," Scientific Basis for Nuclear Waste Management VI, Materials Research Society Symposia Proceedings, Boston, MA, November 1-4, 1982. Ed. D.G. Brookins. New York, NY: North-Holland. Vol. 15, 383-390.

Kirkham, R., of Westinghouse Idaho Nuclear Company, personal communication by telephone. June, 1993.

Kirkham, R., and L. Taylor, of Westinghouse Idaho Nuclear Company, personal communication by telephone. June, 1993.

Krieg, R.D. 1984. Reference Stratigraphy and Rock Properties for the Waste Isolation Pilot Plant (WIPP) Project. SAND83-1908. Albuquerque, NM: Sandia National Laboratories.

Lanza, F. 1988. Feasibility of Disposal of High-Level Radioactive Waste into the Seabed. Vol. 8: Review of Processes Near a Buried Waste Canister. INIS-XN-174. Paris, France: Nuclear Energy Agency, Organisation for Economic Co-Operation and Development.

Lappin, A.R. 1988. Summary of Site-Characterization Studies Conducted From 1983 Through 1987 at the Waste Isolation Pilot Plant (WIPP) Site, Southeastern New Mexico. SAND88-0157. Albuquerque, NM: Sandia National Laboratories.

Lappin, A.R., R.L. Hunter, D.P. Garber, and P.B. Davies, Eds. 1989. Systems Analysis, Long-Term Radionuclide Transport, and Dose Assessments, Waste Isolation Pilot Plant (WIPP), Southeastern New Mexico; March 1989. SAND89-0462. Albuquerque, NM: Sandia National Laboratories.

LaQue, F.L. 1975. Marine Corrosion. New York, NY: John Wiley \& Sons, Inc.

LaVenue, A.M., and B.S. RamaRao. 1992. A Modeling Approach To Address Spatial Variability within the Culebra Dolomite Transmissivity Field. SAND92-7306. Albuquerque, NM: Sandia National Laboratories.

December 1993 
Lemire, R.J., and F. Garisto. 1989. The Solubility of $U, N p, P u, T$ Th and Tc in a Geological Disposal Vault for Used Nuclear Fuel. AECL-10009. Pinawa, Manitoba: Atomic Energy of Canada Limited, Whiteshell Nuclear Research Establishment.

LeNeveu, D.M. 1986. Vault Submodel for the Second Interim Assessment of the Canadian Concept for Nuclear Fuel Waste Disposal: Post-Closure Phase. AECL-8383. Pinawa, Manitoba: Atomic Energy of Canada Limited, Whiteshell Nuclear Research Establishment.

Lester, D.H., G. Jansen, and H.C. Burkholder. 1975. "Migration of Radionuclide Chains Through an Adsorbing Medium," Adsorption and Ion Exchange. Eds. I. Zwiebel and N.H. Sweed. AIChE Symposium Series Vol. 71, no. 152. New York, NY: American Institute of Chemical Engineers. 202-213.

Lide, D.R., Ed. 1991. CRC Handbook of Chemistry and Physics. 72nd ed. Boca Raton, FL: CRC Press.

Long, J.C.S. 1991. Construction of Equivalent Discontinuum Models for Fracture Hydrogeology. LBL-29159. Berkeley, CA: Lawrence Berkeley Laboratory. (Published in: Hudson, J.A., Ed. 1993. Comprehensive Rock Engineering: Principles, Practice, and Projects. 1st ed. New York, NY: Pergamon Press.)

Long, J.C.S., K. Karasaki, A. Davey, J. Peterson, M. Landsfeld, J. Kemeny, and S. Martel. 1991. “An Inverse Approach to the Construction of Fracture Hydrology Models Conditioned by Geophysical Data: An Example from the Validation Exercises at the Stripa Mine," International Journal of Rock Mechanics and Mining Sciences \& Geomechanics Abstracts. Vol. 28, no. 2/3, 121-142.

Lotts, A.L., W.D. Bond, C.W. Forsberg, R.W. Glass, F.E. Harrington, G.E. Michaels, K.J. Notz, and R.G. Wymer. 1992. Options for Treating High-Temperature Gas-Cooled Reactor Fuel for Repository Disposal. ORNL/TM12077. Oak Ridge, TN: Oak Ridge National Laboratory.

MacDougall, H.R., L.W. Scully, and J.R. Tillerson. 1987a. Site Characterization Plan Conceptual Design Report. Volume 2, Chapters 4-9. SAND84-2641. Albuquerque, NM: Sandia National Laboratories.

MacDougall, H.R., L.W. Scully, and J.R. Tillerson. 1987b. Site Characterization Plan Conceptual Design Report. Volume 3. SAND84-2641. Albuquerque, NM: Sandia National Laboratories.

Magnani, N.J. 1983. "Corrosion Resistant Canisters for Nuclear Waste Isolation," Scientific Basis for Nuclear Waste Management VI, Materials Research Society Symposia Proceedings, Boston, MA, November 1-4, 1982. Ed. D.G. Brookins. New York, NY: North-Holland. Vol. 15, 669-676.

Marietta, M.G., and W.F. Simmons. 1988. Feasibility of Disposal of High-Level Radioactive Waste Into the Seabed: Dispersal of Radionuclides in the Oceans: Models, Data Sets, and Regional Descriptions. SAND87-0753. Albuquerque, NM: Sandia National Laboratories.

Martz, H.F., and R.A. Waller. 1982. Bayesian Reliability Analysis. New York, NY: John Wiley \& Sons, Inc.

McKay, M.D., W.J. Conover, and R.J. Beckman. 1979. "A Comparison of Three Methods for Selecting Values of Input Variables in the Analysis of Output from a Computer Code," Technometrics. Vol. 21, no. 2, 239-245.

McKinley, I.G., P.A. Smith, and E. Curti. 1992. "Can the Kristallin-I Near-Field Model Be Considered Robust?", High Level Radioactive Waste Management, Proceedings of the 3ra' International Conference, Las Vegas, NV, April 12-16, 1992. La Grange Park, Il: American Nuclear Society, Inc.; New York, NY: American Society of Civil Engineers. Vol. 2, 1770-1776.

McLain, M.E., and D.W. Rhodes. 1958. Leaching of Fission Products from Calcined Process Waste. IDO-14440. Idaho Falls, ID: Phillips Petroleum Co., Atomic Energy Division. 

formance Assessment for High-Level Waste Madrid Spain October 15-17, 1990. Eds. P. Sellin, M Apted and J. Gago. SKB-TR-91-59. Stockholm, Sweden: Swedish Nuclear Fuel and Waste Management Co. 79-89.

Nilsson, L., L. Moreno, I. Neretnieks, and L. Romero. 1991. A Resistance Network Model for Radionuclide Transport into the Near Field Surrounding a Repository for Nuclear Waste (SKB, Near Field Model 91). SKB TR 9130. Stockholm, Sweden: Swedish Nuclear Fuel and Waste Management Co.

Nilsson, L.B., P. Sellin, F. Huertas, and R. Pusch. 1993. "A Spanish Reference Concept for a Repository in Granite The Role of the Barrier System," Low and Intermediate Level Radioactive Waste Management, Proceedings of the 1993 International Conference on Nuclear Waste Management and Environmental Remediation, Prague, Czech Republic, September 5-11, 1993. Eds. D. Alexandre, R. Baker, R. Kohout, and J. Marek. New York, NY: The American Society of Mechanical Engineers. Vol. 1, 1-4.

Nowak, E.J. 1989. Issues Pertinent to Sealing of Radioactive Waste Repositories in Bedded Salt Formations. SAND89-1257. Albuquerque, NM: Sandia National Laboratories.

NRC (Nuclear Regulatory Commission). 1983. "Disposal of High Level Radioactive Wastes in Geologic Repositories," Code of Federal Regulations 10, Part 60. Washington, DC: Superintendent of Documents, U.S. Government Printing Office.

NRC (Nuclear Regulatory Commission). 1993a. "Disposal of High-Level Radioactive Wastes in Geologic Repositories," Code of Federal Regulations 10, Part 60. Washington, DC: Superintendent of Documents, U.S. Government Printing Office.

NRC (Nuclear Regulatory Commission). 1993b. "Packaging and Transportation of Radioactive Material," Code of Federal Regulations 10, Part 71. Washington, DC: Superintendent of Documents, U.S. Government Printing Office.

NRDC (Natural Resources Defense Council) v. US EPA (United States Euvironmental Protection Agency). 1987. 824 Federal Reporter, $2 d$ Series (1st Circuit 1987). Vol. 824, 1258-1294.

NWPA. 1983. Nuclear Waste Policy Act of 1982. Public Law 97-425, 96 Stat. 2201.

NWPAA. 1987. Nuclear Waste Policy Amendments Act of 1987 as contained in the Omnibus Budget Reconciliation Act of 1987, Public Law 100-203, 101 Stat. 1330.

Olsson, O., J.H. Black, J.E. Gale, and D.C. Holmes. 1989. Site Characterization and Validation. Stage 2 - Preliminary Predictions. Stripa Project 89-03. Stockholm, Sweden: Swedish Nuclear Fuel and Waste Management Co. 
Ozisik, M.N. 1986. Boundary Value Problems of Heat Conduction. Scranton, PA: International Textbook Co.

Paige, B.E. 1966. Leachability of Alumina Calcine Produced in the Idaho Waste Calcining Facility. IN-1011. Idaho Falls, ID: Idaho Nuclear Corp.

Paktunc, A.D. 1993. "Site Selection and Characterization for Historic Low-Level Radioactive Waste in Ontario, Cooperative Siting Process," High Level Radioactive Waste and Spent Fuel Management, Proceedings of the 1993 International Conference on Nuclear Waste Management and Environmental Remediation, Prague, Czech Republic, September 5-11, 1993. Eds. P.-E. Ahlstroem, C.C. Chapman, R. Kohout, and J. Marek. New York, NY: The American Society of Mechanical Engineers. Vol. 2, 719-730.

Paxton, H.C., and N.L. Pruvost. 1987. Critical Dimensions of Systems Containing U-235, Pu-239, and U-233: 1986 Revision. LA-10860-MS. Los Alamos, NM: Los Alamos National Laboratory.

Pentreath, R.J., B.T. Hargrave, H.S. Roe, and M. Sibuet. 1988. Feasibility of Disposal of irigh-Level Radioactive Waste into the Seabed. Vol. 6: Deep Sea Biology. INIS-XN-176. Paris, France: Nuclear Energy Agency, Organisation for Economic Co-Operation and Development.

Pepping, R.E., M.S.Y. Chu, and M.D. Siegel. 1983a. "A Simplified Analysis of a Hypothetical Repository in a Basalt Formation," Technical Assistance for Regulatory Development: Review and Evaluation of the Draft EPA Standard 40CFR191 for Disposal of High-Level Waste. NUREG/CR-3235, SAND82-1557. Albuquerque, NM: Sandia National Laboratories. Vol. 2.

Pepping, R.E., M.S.Y. Chu, and M.D. Siegel. 1983b. "A Simplified Analysis of a Hypothetical Repository in a Bedded Salt Formation," Technical Assistance for Regulatory Development: Review and Evaluation of the Draft EPA Standard 40CFR191 for Disposal of High-Level Waste. NUREG/CR-3235, SAND82-1557. Albuquerque, NM: Sandia National Laboratories. Vol. 4.

Percival, J.B., M. Mirota, and J.P. Hume. 1984. Lithological and Density Data on URL-2 and URL-5 Standard Core Samples from the Underground Research Laboratory Site Near Lac du Bonnet, Manitoba. AECL-TR-277. Pinawa, Manitoba: Atomic Energy of Canada Limited, Whiteshell Nuclear Research Establishment.

Pereira, P., G.A. Somorjai, and H. Heinemann. 1992. "Catalytic Steam Gasification of Coals," Energy and Fuels. Vol. 6, no. 4, 407-410.

Perry, R.H., and C.H. Chilton, Eds. 1973. Chemical Engineers' Handbook. 5th ed. New York, NY: McGraw-Hill.

Pfeifle, T.W., K.D. Mellegard, and D.E. Munson. 1992. Determination of Probability Density Functions for Parameters in the Munson-Dawson Model for Creep Behavior of Salt. SAND90-2366. Albuquerque, NM: Sandia National Laborator:;s.

Pierce, W.G., and E.I. Rich. 1962. Summary of Rock Salt Deposits in the United States as Possible Storage Sites for Radioactive Waste Materials. Geological Survey Bulletin 1148. Washington, DC: Geological Survey.

Pitman, S.G., B. Griggs, and R.P. Elmore. 1981. "Evaluation of Metallic Materials for Use in Engineered Barrier Systems," Scientific Basis for Nuclear Waste Management, Proceedings of the Third International Symposium, Boston, MA, November 17-20, 1980. Ed. J.G. Moore. New York, NY: Plenum Press. Vol. 3, 523-530.

Popielak, R.S., R.L. Beauheim, S.R. Black, W.E. Coons, C.T. Ellingson, and R.L. Olsen. 1983. Brine Reservoirs in the Castile Formation, Waste Isolation Pilot Plant (WIPP) Project, Southeastern New Mexico. TME-3153. Albuquerque, NM: D'Appolonia Consulting Engineers.

Powers, D.W., S.J. Lambert, S-E. Shaffer, L.R. Hill, and W.D. Weart, Eds. 1978. Geological Characterization Report, Waste Isolation Pilot Plant (WIPP) Site, Southeastern New Mexico. SAND78-1596. Albuquerque, NM: Sandia National Laboratories. Vols. I-II. 
Public Law 96-164. 1979. Department of Energy National Security and Military Applications of Nuclear Energy Authorization Act of 1980.

Public Service of Colorado. 1991. Letter to A.A. Pitrolo, DOE-ID, Transmittal of Spent Fuel Shipping Data, Dated February 11, 1991.

Radhakrishna, H.S., and H.T. Chan. 1985. Strength and Hydraulic Conductivity of Clay-Based Buffers for a Deep Underground Nuclear Fuel Waste Disposal Vault. AECL-TR-327. Pinawa, Manitoba: Atomic Energy of Canada Limited, Whiteshell Nuclear Research Establishment.

Radiation Protection Ordinance. 1989. "Verordnung über den Schutz vor Schäden durch ionisierende Strahlen (Strahlenschutzverordnung - StrlSchV). Vom 30. Juni 1989," Bundesgesetzblatt. Part I, no. 34, 1321-1375.

Rasmussen, N.C. 1975. K'eactor Safety Study: An Assessment of Accident Risks in U.S. Commercial Nuclear Power Plants. NUREG-75/(114, WASH-1400. Washington, DC: U.S. Nuclear Regulatory Commission.

RCRA. 1976. Resource, Conservation, and Recovery Act of 1976. Public Law 94-580, 90 Stat. 2795.

Rechard, R.P. 1989. Review and Discussion of Code Linkage and Data Flow in Nuclear Waste Compliance Assessments. SAND87-2833. Albuquerque, NM: Sandia National Laboratories.

Rechard, R.P., Ed. 1992. User's Reference Manual for CAMCON: Compliance Assessment Methodology Controller, Version 3.0. SAND90-1983. Albuquerque, NM: Sandia National Laboratories.

Rechard, R.P., H.J. Iuzzolino, J.S. Rath, A.P. Gilkey, R.D. McCurley, and D.K. Rudeen. 1989. User's Manual for CAMCON: Compliance Assessment Methodology Controller. SAND88-1496. Albuquerque, NM: Sandia National Laboratories.

Rechard, R.P., H. Iuzzolino, and J.S. Sandha. 1990. Data Used in Preliminary Performance Assessment of the Wasie Isolation Pilot Plant (1990). SAND89-2408. Albuquerque, NM: Sandia National Laboratories.

Rechard, R.P., P.J. Roache, R.L. Blaine, A.P. Gilkey, and D.K. Rudeen. 1991. Quality Assurance Procedures for Computer Software Supporting Performance Assessments of the Waste Isolation Pilot Plant. SAND90-1240. Albuquerque, NM: Sandia National Laboratories.

Rechard, R.P., K.M. Trauth, and R.V. Guzowski. 1992a. Quality Assurance Procedures for Parameter Selection and Use of Expert Judgment Panels Supporting Performance Assessments of the Waste Isolation Pilot Plant. SAND91-0429. Albuquerque, NM: Sandia National Laboratories.

Rechard, R.P., D.K. Rudeen, and P.J. Roache. 1992b. Quality Assurance Procedures for Analyses and Report Reviews Supporting Performance Assessments of the Waste Isolation Pilot Plant. SAND91-0428. Albuquerque, NM: Sandia National Laboratories.

Rechard, R.P., K.M. Trauth, J.S. Rath, R.V. Guzowski, S.C. Hora, and M.S. Tierney. 1993. "The Use of Formal and Informal Expert Judgments When Interpreting Data for Performance Assessments," Scientific Basis for Nuclear Waste Management XVI, Materials Research Society Symposium Proceedings, Boston, MA, November 30December 4, 1992. Eds. C.G. Interrante and R.T. Pabalan. SAND92-1148C. Pittsburgh, PA: Materials Research Society. Vol. 294, 943-950.

Reeves, M., G.A. Freeze, V.A. Kelley, J.F. Pickens, D.T. Upton, and P.B. Davies. 1991. Regional Double-Porosity Solute Transport in the Culebra Dolomite Under Brine-Reservoir-Breach Release Conditions: An Analysis of Parameter Sensitivity and Importance. SAND89-7069. Albuquerque, NM: Sandia National Laboratories.

Regulatory Document R-21. 1980. Guidelines for Nuclear Facilities. R-21. HSK and KSA. 
Reid, R.C., J.M. Prausnitz, and B.E. Poling. 1987. The Properties of Gases and Liquids. 4th ed. New York, NY: McGraw-Hill.

Ripley, B.D. 1987. Stochastic Simulation. New York, NY: John Wiley \& Sons, Inc.

The Robbins Company. 1984. Final Report for Repository Drilled Hole Methods Study. SAND83-7085. Albuquerque, NM: Sandia National Laboratories.

Roberts, F.S. 1976. Discrete Mathematical Models. Englewood Cliffs, NJ: Prentice-Hall, Inc.

Romero, L., L. Moreno, and I. Neretnieks. 1991. A Compartment Model for Solute Transport in the Near Field of a Repository for Radioactive Waste (Calculations for Pu-239). SKB TR 91-48. Stockholm, Sweden: Swedish Nuclear Fuel and Waste Management Co.

Rose, W., and W.A. Bruce. 1949. "Evaluation of Capillary Character in Petroleum Reservoir Rock," Transactions of the American Institute of Mining and Metallurgical Engineers. Vol. 186, T.P. 2594, 127-142.

Ross, S.M. 1985. Introduction to Probability Models. 3rd ed. New York, NY: Academic Press, Inc.

Rubenstein, R. 1981. Simulation and the Monte Carlo Method. New York, NY: John Wiley \& Sons, Inc.

Safe Drinking Water Act. 42 U.S.C. $300 f$ et seq.

Saltelli, A., G. Bertozzi, and D.A. Stanners. 1984. LISA: A Code for Safety Assessment in Nuclear Waste Disposal Program Description and User Guide. Luxembourg: Commission of the European Communities.

Saltelli, A., T. Homma, P. Prado, and C. Torres. 1990. "Development of the LISA Code," Proceedings of the International Symposium on Safety Assessment of Radioactive Waste Repositories, Paris, France, October 9-13, 1989. Paris, France: Organisation for Economic Co-Operation and Development. 639-648.

Sandia WIPP Pruject. 1992. Preliminary Performance Assessment for the Waste Isolation Pilot Plant, December 1992. Volume 3: Model Parameters. SAND92-0700/3. Albuquerque, NM: Sandia National Laboratories.

Scheidegger, A.E. 1960. The Physics of Flow Through Porous Media. Toronto, Ontario: University of Toronto Press.

Shephard, L.E., G.A. Auffret, D.E. Buckley, R.T.E. Schuettenhelm, and R.C. Searle. 1988. Feasibility of Disposal of High-Level Radioactive Waste into the Seabed: Vol. 3, Geoscience Characterization Studies. INIS-XN-179, SAND87-1913. Paris, France: Nuclear Energy Agency, Organisation for Economic Co-Operation and Development.

Sjaardema, G.D., and R.D. Krieg. 1987. A Constitutive Model for the Consolidation of WIPP Crushed Salt and Its Use in Analyses of Backfilled Shaft and Drift Configurations. SAND87-1977. Albuquerque, NM: Sandia National Laboratories.

Skagius, K., and I. Neretnieks. 1986. "Porosities and Diffusivities of Some Nonsorbing Species in Crystalline Rocks," Water Resources Research. Vol. 22, no. 3, 389-398.

SKB (Swedish Nuclear Fuel and Waste Management Co.). 1992a. Final Disposal of Spent Nuclear Fuel. Importance of the Bedrock for Safety. SKB Technical Report 92-20. Stockholm, Sweden: Swedish Nuclear Fuel and Waste Management Co.

SKB (Swedish Nuclear Fuel and Waste Management Co.). 1992b. SKB Annual Report 1991 Including Summaries of Technical Reports Issued during 1991. SKB Technical Report 91-64. Stockholm, Sweden: Swedish Nuclear Fuel and Waste Management Co.

December 1993 
SNL (Sandia National Laboratories) and U.S. Geological Survey. 1983. Basic Data Report for Drillhole ERDA 9 (Waste Isolation Pilot Plant - WIPP). SAND79-0270. Albuquerque, NM: Sandia National Laboratories.

Sorenson, N.R., and M.A. Molecke. 1992. "Summary of the WIPP Materials Interface Interactions Test-Metal Corrosion," Workshop on In Situ Tests on Radioactive Waste Forms and Engineered Barriers, Corsendonk, Belgium, October 13-16, 1992. SAND92-1921C. Albuquerque, NM: Sandia National Laboratories.

Stahl, D., and D.J. Harrison. 1993. "Waste Package/Engineered Barrier System Design Concepts for the Direct Disposal of Spent Fuel in the Potential United States' Repository at Yucca Mountain, Nevada," Low and Intermediate Level Radioactive Waste Management, Proceedings of the 1993 International Conference on Nuclear Waste Management and Environmental Remediation, Prague, Czech Republic, September 5-11, 1993. Eds. D. Alexandre, R. Baker, R. Kohout, and J. Marek. New York, NY: The American Society of Mechanical Engineers. Vol. $1,9-15$.

Staples, B.A., G.S. Pomiak, and E.L. Wade. 1979. Properties of Radioactive Calcine Retrieved from the Second Calcined Solids Storage Facility at ICPP. ICP-1189. Idaho Falls, ID: Allied Chemical Corp.

Stephens, M.E., and B.W. Goodwin. 1989. "Scenario Analysis for the Postclosure Assessment of the Canadian Concept for Nuclear Fuel Waste Disposal," Proceedings of the Symposium on Safety Assessment of Radioactive Waste Repositories, Paris, France, October 9-13, 1989. Paris, France: Nuclear Energy Agency. 405-415.

Storck, R., J. Aschenbach, R.P. Hirsekorn, A. Nies, and N. Stelte. 1988. Performance Assessment of Geological Isolation Systems for Radioactive Waste, Disposal in Salt Formations. EUR 11778 EN. Luxembourg: Commission of the European Communities.

Strachan, D.M., B.P. McGrail, M.J. Apted, D.W. Engle, and P.W. Eslinger. 1990. Preliminary Assessment of the Controlled Release of Radionuclides from Waste Packages Containing Borosilicate Waste Glass. PNL-7591. Richland, WA: Pacific Northwest Laboratory.

Tang, Y.S., and J.H. Saling. 1990. Radioactive Waste Management. New York, NY: Hemisphere Publishing Corporation.

Thomas, L.K., D.L. Katz, and M.R. Tek. 1968. "Threshold Pressure Phenomena in Porous Media," Society of Petroleum Engineers Journal. Vol. 8, no. 2, 174-184.

Tien, P-L., F.B. Nimick, A.B. Muller, P.A. Davis, R.V. Guzowski, L.E. Duda, and R.L. Hunter. 1983. Repository Site Data and Information in Bedded Salt: Palo Duro Basin, Texas. NUREG/CR-3129, SAND82-2223. Albuquerque, NM: Sandia National Laboratories.

Tierney, M.S. 1990. Constructing Probability Distributions of Uncertain Variables in Models of the Performance of the Waste Isolation Pilot Plant: The 1990 Performance Simulations. SAND90-2510. Albuquerque, NM: Sandia National Laboratories.

Tierney, M.S. 1991. Combining Scenarios in a Calculation of the Overall Probability Distribution of Cumulative Releases of Radioactivity from the Waste Isolation Pilot Plant, Southeastern New Mexico. SAND90-0838. Albuquerque, NM: Sandia National Laboratories.

Toxic Substances Control Act. U.S.C. 2601 et seq.

Trabia, M.B., and M. Kiley. 1992. "ASME Pressure Vessel Code Application to Nuclear Waste Container Design," High Level Radioactive Waste Management, Proceedings of the Third International Conference, Las Vegas, NV, April 12-16, 1992. La Grange Park, IL: American Nuclear Society, Inc.; New York, NY: American Society of Civil Engineers. Vol. 2, 1244-1252. 
Trauth, K.M., S.C. Hora, R.P. Rechard, and D.R. Anderson. 1992. The Use of Expert Judgment to Quantify Uncertainty in Solubility and Sorption Parameters for Waste Isolation Pilot Plant Performance Assessment. SAND920479. Albuquerque, NM: Sandia National Laboratories.

Trauth, K.M., S.C. Hora, and R.V. Guzowski. 1993. Expert Judgment on Markers to Deter Inadvertent Human Intrusion into the Waste Isolation Pilot Plant. SAND92-1382. Albuquerque, NM: Sandia National Laboratories.

Tsang, C.F., J. Noorishad, and J.S.Y. Wang. 1983. "A Study of Coupled Thermomechanical, Thermohydrological and Hydromechanical Processes Associated with a Nuclear Waste Repository in a Fractured Rock Medium," Scientific Basis for Nuclear Waste Management VI, Materials Research Society Symposia Proceedings, Boston, MA, November 1-4, 1982. Ed. D.G. Brookins. New York, NY: North-Holland. Vol. 15, 515-522.

TSPA. In preparation. Total-System Performance Assessment for Yucca Mountain - SNL Second Iteration (TSPA1993). SAND93-2675. Albuquerque, NM: Sandia National Laboratories.

Unsworth, M.D. 1989. VANDAL Technical Overview. DOE/RW/89.107. London, England: Department of the Environment.

U.S. DOE (U.S. Department of Energy). 1979. Draft Environmental Impact Statement, Management of Commercially Generated Radioactive Waste. DOE/EIS-0046-D. Washington, DC: U.S. Department of Energy.

U.S. DOE (U.S. Department of Energy). 1980. Final Environmental Impact Statement: Waste Isolation Pilot Plant. DOE/EIS-0026. Washington, DC: U.S. Department of Energy.

U.S. DOE (U.S. Department of Energy). 1985a. Evaluation of Commercial Repository Capacity for the Disposal of Defense High-Level Waste. DOE/DP-0020/1. Washington, DC: Assistant Secretary for Defense Programs, U.S. Department of Energy.

U.S. DOE (U.S. Department of Energy). 1985b. An Evaluation of "Commercial Repository Capacity for the Disposal of Defense High-Level Waste." Response to Comments. DOE/DP-0027. Washington, DC: Assistant Secretary for Defense Programs, U.S. Department of Energy.

U.S. DOE (U.S. Department of Energy). 1986a. Environmental Assessment, Deaf Smith County Site, Texas. DOE/ RW-0069. Washington, DC: U.S. Department of Energy, Office of Civilian Radioactive Waste Management. Vol. 1.

U.S. DOE (U.S. Department of Energy). 1986b. Environmental Assessment, Yucca Mountain Site, Nevada Research and Development Area, Nevada. DOE/RW-0073. Washington, DC: U.S. Department of Energy, Office of Civilian Radioactive Waste Management.

U.S. DOE (U.S. Department of Energy). 1988a. Site Characterization Plan, Yucca Mountain Site, Nevada Research and Development Area, Nevada. Volumes I-IX. DOE/RW-0199. Washington, DC: U.S. Department of Energy, Office of Civilian Radioactive Waste Management.

U.S. DOE (U.S. Department of Energy). 1988b. Draft 1988 Mission Plan Amendment. DOE/RW-0187. Washington, DC: U.S. Department of Energy, Office of Civilian Radioactive Waste Management.

U.S. DOE (U.S. Department of Energy). 1989. Report to Congress on the Potential Use of Lead in the Waste Packages for a Geologir iepository at Yucca Mountain, Nevada. DOE/RW-0254. Washington, DC: U.S. Department of Energy, Office of Civilian Radioactive Waste Management.

U.S. DOE (U.S. Department of Energy). 1990. Final Supplement Environmental Impact Statement, Waste Isolation Pilot Plant. DOE/EIS-0026-FS. Washington, DC: Office of Environmental Restoration and Waste Management, U.S. Department of Energy. 
U.S. DOE (U.S. Department of Energy). 1992. Characteristics of Potential Repository Wastes. DOE/RW-0184-R1. Oak Ridge, TN: Oak Ridge National Laboratory. Vol. 2.

van Dorp, F., and J.O. Vigfusson. 1989. "Overview of Swiss Work Concerning Human Effect Scenarios," Risks Associated with Human Intrusion at Radioactive Waste Disposal Sites, NEA Workshop, Paris, France, June 5-7, 1989. Paris, France: Nuclear Energy Agency, Organisation for Economic Co-Operation and Development. 105111.

Van Genuchten, M. Th. 1980. "A Closed-form Equation for Predicting the Hydraulic Conductivity of Unsaturated Soils," Soil Jcience Society of America Journal. Vol. 44, no. 5, 892-898.

Van Konynenburg, R.A., W.G. Halsey, R.D. McCright, W.L. Clarke, Jr., and G.E. Gdowski. 1993. Selection of Candidate Container Materials for the Conceptual Waste Package Design for a Potential High Level Nuclear Waste Repository at Yucca Mountain. UCRL-ID-112058. Livermore, CA: Lawrence Livermore National Laboratory.

Veazey, W.R., and C.D. Hodgman. 1914. Handbook of Chemistry and Physics. 2nd ed. Cleveland, OH: The Chemical Rubber Company.

Vieno, T., A. Hantojèrvi, L. Koskinen, and H. Nordman. 1992. TVO-92 Safety Analysis of Spent Fuel Disposal. Report YJT-92-33E. Helsinki, Finland: Teclnical Research Centre of Finiand (VTT), Nuclear Engineering Laboratory.

Vinjamuri, K., S.V. Raman, D.A. Knecht, and J.D. Herzog. 1992. "Waste Form Development for Immobilization of High Level Waste Calcine at the Idaho Chemical Processing Plant," High Level Radioactive Waste Management, Proceedings of the Third International Conference, Las Vegas, NV, April 12-16, 1992. La Grange Park, IL: American Nuclear Society, Inc.; New York, NY: American Society of Civil Engincers. Vol. 2, 1261-1271.

Vogt, J. 1993. "Swedish Spent Fuel Management Systems, Facilities and Operating Experiences," Low and Intermediate Level Radioactive Waste Management, Proceedings of the 1993 International Conference on Nuclear Waste Management and Environmental Remediation, Prague, Czech Republic, September 5-11, 1993. Eds. D. Alexandre, R. Baker, R. Kohout, and J. Marek. New York, NY: The American Society of Mechanical Engineers. Vol. 1, 87-92.

Wai, R.S.C., and A. Tsai. 1985. Near-Field Thermomechanical Analyses Using Temperature-Dependent Rock Properties. AECL-TR-262. Pinawa, Manitoba: Atomic Energy of Canada Limited, Applied Geoscience Branch, Whiteshell Nuclear Research Establishment.

Weart, W.D. 1979. "WIPP: A Bedded Salt Repository for Defense Radioactive Waste in Southeastern New Mexico," Radioactive Waste in Geologic Storage, 176th Annual Meeting of the American Chemical Society, Miami Beach, FL, September 11-15, 1978. Ed. S. Fried. ACS Symposium Series No. 100. Washington, DC: American Chemical Society. 13-36.

Weast, R.C., Ed. 1976. Handbook of Chemistry and Physics. 56th ed. Cleveland, OH: CRC Press.

WEC (Westinghouse Electric Corporation). 1981. Engineered Waste Package Conceptual Design, Defense HighLevel Waste (Form 1) Disposal in Salt. AESD-TME-3086. Pittsburgh, PA: Westinghouse Electric Corporation, Advanced Energy Systems.

WEC (Westinghouse Electric Corporation). 1989. TRU Waste Acceptance Criteria for the Waste Isolation Pilot Plant, Revision 3. WIPP-DOE-069, Rev. 3. Carlsbad, NM: Westinghouse Electric Corporation for the U.S. Department of Energy.

Weitzman, M. 1985. Modeling of the Groundwater Flow System Around the URL Excavation Before and After Its Construction. AECL-TR-233. Pinawa, Manitoba: Atomic Energy of Canada Limited, Applied Geoscience Branch, Whiteshell Nuclear Research Establishment. 


\section{Glossary}

\section{Acronyms and Initialisms}

ALARA - As low as reasonably achievable; principle promulgated by the Nuclear Regulatory Commission (NRC) in 10 CFR 20 that organizations holding NRC licenses should make every reasonable effort to maintain radiation exposures and releases of radionuclides to as low as reasonably achievable.

BRAGFLO - A computational model (code) that evaluates the simultaneous flow of two phases through a porous heterogenous media.

BRAGFLO_T - A computational model (code) that evaluates the simultaneous flow of two phases through a porous heterogenous reservoir. BRAGFLO_T differs from BRAGFLO, its parent code, primarily by the addition of the energy equation for temperature.

CAMCON - Compliance Assessment Methodology CONtroller; computational system for assessing the performance of a disposal system (usually for nuclear wastes).

CCDF - complementary cumulative distribution function; see definition.

CDF - cumulative distribution function; see definition.

Chem Plant - The Idaho Chemical Processing Plant (ICPP); see ICPP.

CLAMVD - A computational model (code) to evaluate the transport of radionuclides in a single phase through a porous media. CLAMVD is used in the simple performance assessment calculations for both the salt and granite repositories.

CONCSNF - A source-term submodel that calculates the dissolution, sorption, and radioactive buildup and uniay of specified isotopes within the waste emplacement package. CONCSNF is designed as a subroutine to transport codes (i.e., CLAMVD and STAFF2D).

COND_1D - A one-dimensional thermal-conduction code that provides transient temperature data both inside and outside of a single waste parcel (i.e., both inside the waste parcel interior and in the host rock).

CUTTINGS - A computational model (code) that calculates the quantity of radioactive material (in curies) brought to the surface as cuttings/cavings by a drilling operation that penetrates the repository. The code determines the amount of cuttings removed from a borehole by drilling and decays radionuclides within the cuttings/cavings to the specified intrusion time.

DHLW - defense high-level waste, the primary high-level waste considered in this performance assessment.

DOE - U.S. Department of Energy; formed by DOE Organization Act (Public Law 95-91, 912 Stat 565), which replaced the Energy Research and Development Agency (ERDA). ERDA was formed by the 1974 Energy Reorganization Act (Public Law 93-438) and replaced the Atomic Energy Commission (AEC), which was formed in 1946 (August 1, 1946, 60 Stat. 755).

DU - depleted uranium

DWPF - Defense Waste Processing Facility (at Savannah River Plant, south of Aiken, Georgia); constructed to dissolve and solidify the high level waste sludge in borosilicate glass and encasing it in stainless steel canisters 


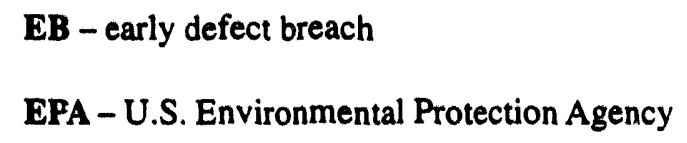

EPA of 1992 - Energy Policy Act of 1992; an act that requires the U.S. Environmental Protection Agency (EPA) to contract with the National Academy of Sciences (NAS) to recommend site-specific health-based (dose) standards to individuals for the Yucca Mountain Project and subsequent promulgation of new regulations by the EPA and revision of regulations by the Nuclear Regulatory Commission (NRC) consistent with NAS findings.

FFCA - Federal Facility Compliance Act of 1992; an act that removed sovereign immunity for federal facilities concerning hazardous waste regulations; the act requires a listing of mixed-waste inventory at U.S. DOE facilities, and the DOE chose to include spent fuel.

FSV - Fort St. Vrain (graphite moderated reactor in Colorado).

GASSNF - A submodel that calculates the mass rate of gas generation and rate of water consumption. GASSNF is designed as a subroutine if the fluid flow code (i.e., BRAGFLO_T, GRFLOW, and SALFLOW) and requires liquid saturation of the element as input.

GRFLOW - A computational model (code) that calculates the volume of gas and liquid present in the granite repository over time.

\section{Hanford - See HWVP}

HEU - highly enriched uranium; see definition.

HIP - Hot Isostatic Press; process of mixing radionuclides into glass ceramic and then compressing the container and mixture as it solidifies

HLW - high-level waste (see definition)

HWVP - Hanford Waste Vitrification Plant (Hanford, Washington), designed to immobilize liquid high-level waste stored in double-shelled and single-shelled tanks on the Hanford reservation.

IAEA - International Atomic Energy Agency, Vienna, Austria

ICPP - Idaho Chemical Processing Plant (Idaho Falls, Idaho); located at Idaho National Engineering Laboratory (INEL); also referred to as Chem Plant

INEL - Idaho National Engineering Laboratory (Idaho Falls, Idaho)

LEU - low enriched uranium; see definition.

MB - Marker bed, mapping unit for Sodium Springs Formation

$\mathrm{MC}_{2}$ - metal carbide $\left(\mathrm{ThC}_{2}, \mathrm{UC}_{2}\right)$; interior of the graphite spent fuel paricles.

MTHM - metric tons of heavy metal.

NEPA - National Environmental Policy Act of 1969, Public Law No. 91-190, 83 Stat. 852.

NRC - Nuclear Regulatory Commission; formed by the 1974 Energy Reorganization Act (Public Law 93-438) from the Atomic Energy Commission 
NWPA - Nuclear Waste Policy Act of 1982; provides a national policy for the interim storage, monitored retrievable storage, and eventual disposal of radioactive waste.

NWPAA - Nuclear Waste Policy Amendments Act of 1987; amendments to the Nuclear Waste Policy Act of 1982 specifying that only one repository site at Yucca Mountain was to be characterized by the DOE and placing renewed emphasis on the monitored retrievable storage option.

OCRWM - Office of Civilian Radioactive Waste Management within DOE; set up to comply with the directives within the NWPA of 1982.

PA-performance assessment (see definition)

PB - Peach Bottom (Reactor 1 and corresponding graphite Cores I and II in Pennsylvania)

PRA - probabilistic risk assessment (see definition for performance assessment)

QA - quality assurance

RCRA - Resource Conservation and Recovery Act of 1976 (Public Law 94-580) and, as used herein, subsequent amendments (e.g., HSWA, Hazardous and Solid Waste Amendments of 1984, Public Law 98-616)

SALFLOW - A computational model (code) that calculates the volume of gas and liquid present in the salt repository over time.

Sandia - See SNL.

Savannah River - See SRP.

SNF - spent nuclear fuel (see definition)

SNL - Sandia National Laboratories, a multiprogram laboratory located in Albuquerque, NM, and Livermore, CA. SNL was operated for the DOE from 1949 until October 1993 by AT\&T and is currently managed by Martin Marietta Corp.

SRC - standardized regression coefficient in the statistical regression model

SRP - Savannah River Plant (south of Aiken, Georgia); originally developed to produce plutonium, tritium, and heavy water for nuclear weapons; the facility of particular interest to this report is the Defense Waste Processing Facility (DWPF)

SS304L - stainless steel, type 304L

STAFF2D - As used in this performance assessment, STAFF2D evaluates radionuclide transport through dual porosity media using a finite-element solution technique.

SUMQ 3D - A three-dimensional code that calculates the time-dependent temperature at any point within an arbitrary array of linear heat sources of finite length; it is limited to providing reliable data outside the outside the backfill-buffer/host-rock interface.

$\mathrm{UAl}_{\mathbf{x}}$ - uranium aluminum alloy matrix in special highly enriched spent fuel

$\mathrm{UO}_{2}$ - uranium dioxide in low enriched fuel from pressurized water reactor

WDG - Waste Disposal Group. 
WEP - waste emplacement package (see definition)

West Valley - See WVDP.

WINCO - Westinghouse Idaho Nuclear Company; wholly owned subsidiary of Westinghouse Incorporated; manages the Idaho Chemical Processing Plant (ICPP) at Idaho National Engineering Laboratory (INEL)

WIPP - Waste Isolation Pilot Plant; a potential repository near Carlsbad, NM, authorized in 1979 for the management, storage and eventual disposal of waste generated by DOE defense programs that is contaminated with transuranic radionuclides and some RCRA hazardous chemicals.

WP - Waste parcel (see definition)

WVDP - West Valley Demonstration Project (West Valley, New York)

YMP - Yucca Mountain Project; a potential repository, to be located near the Nevada Test Site, for spent nuclear fuel and high-level waste; under study by the DOE as mandated by U.S. Congress (NWPAA, 1987)

10 CFR 60 - Code of Federal Regulations, Title 10, Part 60, Licensing Requirements for Land Disposal of Radioactive Waste

40 CFR 191 - Code of Federal Regulations, Title 40, Part 191, Environmental Radiation Protection Standards for Management and Disposal of Spent Nuclear Fuel, High-Level and Transuranic Radioactive Wastes

\section{Definitions}

accessible environment - “... (1) the atmosphere, (2) land surfaces, (3) surface water, (4) oceans, and (5) all of the lithosphere that is beyond the controlled area." (40 CFR 191.12[k]); the overall performance of the disposal system is calculated at this boundary.

activity - Disintegration (decay) of radionuclei per unit time (e.g., 37 billion disintegrations per second is one curve [Ci]).

alpha particle (from alpha emitter) - A type of radiation; the helium nucleus (two protons and two neutrons)

alternative conceptual models - Multiple working sets of hypotheses and assumptions of a system that are all scientifically acceptable (i.e., consistent with the purpose of the model, logically consistent with one another, in agreement with existing facts and observed data, and able to be tested).

alternative models - Alternatives in model form that may exist at each stage of model development. Alternatives at the first stage of model development (alternative conceptual models) are often of the most concern.

assembly - A bundle of fuel rods (typically of low enriched uranium fuel).

Assurance Requirements - Qualitative requirements in Subpart B of 40 CFR 191 ( $\$ 191.14)$ that specify actions and procedures to increase confidence that the probabilistic release limits in the Containment Requirements will be met.

backfill - Material used to fill the shafts, access drifts, and other excavated openings. Special types of backfill include concrete and clay plugs for sealing (seals) and bentonite clay around the waste parcel (backfill-buffer). 
backfill-buffer - The earthen backfill between the waste parcel and host rock; because some emplacement designs envision using the backfill material to moderate (or buffer) the release of contaminants to the host rock (e.g., bentonite clay in the granite repository), this backfill is also referred to as "backfill-buffer." The term "backfillbuffer" is used in this report even when the crushed-salt backfill around the waste parcel is discussed, although it does not technically provide a moderating function. The term "buffer" is also frequently found in the literature but is not used here to avoid confusion with the many other uses of the term (e.g., in chemistry, a solistion containing a weak acid and conjugate base that resists changes in $\mathrm{pH}$ ).

barrier - As defined in 40 CFR 191, a barrier "means any material or structure that prevents or substantially delays movement of water or radionuclides toward the accessible environment. For example, a barrier may be a geologic structure, a canister, a waste form with physical and chemical characteristics that significantly decrease the mobility of radionuclides, or a material placed over and around water, provided that the material or structure substantially delays movement of water or radionuclides" (Section 191.12).

batholith - a shield or stock-shaped body of plutonic igneous rock at least $100 \mathrm{~km}^{2}\left(40 \mathrm{mi}^{2}\right)$ in area

bentonite - A common term applied to clay containing montmorillonite (smectite) as the predominate mineral.

beta particle - A type of radiation; a high energy electron.

buffer - See backfill-buffer.

calcine - Oxidized high-level waste produced by heating in a fluidized bed.

canister - The first container placed around the waste form; it usually serves as structural reinforcement for subsequent containers (e.g., corrosion-resistant overpack container); sometimes a canister is the sole container for storage, handling, and transportation prior to disposal preparation or occasionally the sole container for disposal.

canister overpack - The container(s) enclosing the canister that generally serves to provide one barrier function (e.g., shielding or corrosion resistance).

cavings - Particulates brought to the ground surface in the drilling fluid of a rotary drill from erosion of the borehole wall by the circulating drilling fluid.

cladding - The outer coating of nuclear fuel, for example, a tube or a plate.

complementary cumulative distribution function - One minus the cumulative distribution function.

Complex PA - A performance assessment that uses consequence models based on a mathematical model with partial differential equations and spatially varying model parameters.

computational model - The solution and implementation of the mathematical model. The solution may be either analytical, numerical, or empirical (where an empirical solution uses observed data directly by means of lookup tables and/or interpolation on statistical relationships). Conceivably, the implementation may be evaluated either by hand or on a computer. However, because the latter is more frequent, computational model is often synonymous with computer model. The computational model is generic until system-specific data are used.

computational scenario - A member of a class of subsets of parameter space that is obtained by subdividing the subspace $S$ spanned by parameters of a summary scenario. 
conceptual model - The set of hypotheses and assumptions that postulates the description and behavior of a system. To be scientifically acceptable, the set of hypotheses must be (1) consistent with the purpose of the model, (2) logically consistent with one another, (3) in agreement with existing information and observed data, and (4) able to be tested (i.e., capable of being falsified by the collection of more observed data). At a minimum, these hypotheses i is assumptions describe the following about the system, including whether time dependent: (1) the simplified physical arrangement of system components, (2) the initial and boundary condition types, and (3) the nature of the relevant chemical, physical, cultural, or biological phenomena.

Containment Requirements - Requirements in Subpart B of 40 CFR 191 ( $\$ 191.13)$ that set probabilistic limits on cumulative releases of radionuclides beyond the "controlled area" (defined in the regulation as a surface location that encompasses no more than $100 \mathrm{~km}^{2}$ and extends horizontally no more than a $5-\mathrm{km}$ radius from the outer boundary of a radioactive-waste disposal system, plus the underlying subsurface $[\$ 191.12(\mathrm{~g})])$ for 10,000 years.

controlled area - As defined in 40 CFR 191, Subpart B, "(1) a surface location, to be identified by passive institutional controls, that encompasses no more than 100 square kilometers and extends horizontally no more than five kilometers in any direction from the outer boundary of the original location of the radioactive wastes in a disposal system; and (2) the subsurface underlying such a surface location" (Section 191.12).

coordinate grid or mesh - The grid or mesh on the properly sized modeled object. The coordinate grid is usually called simply the "grid" or "mesh," except when it is important to distinguish it from the logical grid.

critical condition - A condition in which fission (splitting of nuclei by neutrons) is self sustaining by producing on average one slow neutron for every one absorbed in the fission (subcritical conditions produce less than one; supercritical conditions produce more).

cumulative distribution function (CDF) - The sum (or integral as appropriate) of the probability of those values of a random variable that are less than or equal to a specified value.

cuttings - See drill cuttings.

data - Information that is purposely collected, organized, and used in preparing values for parameters of computational models in an analysis or used as the basis of a decision. As used here, data is a subset of general information.

disposal system - Any combination of engineered and natural barriers that isolate spent nuclear fuel or radioactive waste after disposal (40 CFR 191.12(a)). The natural barriers extend to the accessible environment.

dose - The quantity of radiation absorbed accounting for biologic effect; measured in sieverts (Sv) or rem.

drift or access drift - Approximately horizontal underground excavated passageway from the shaft(s) to the mined panels and room(s); in this report, the drift is $9 \mathrm{~m}$ wide and $6 \mathrm{~m}$ high.

drill cuttings - Particulates brought to the surface in the drilling fluid of a rotary drill either from directly cutting through a material with the drill bit or from erosion of the borehole wall by the circulating drilling fluid.

Eh - A measure of the oxidizing or reducing tendency of a solution and defined by the Nernst equation, Eh (volts) = $\mathrm{Eh}^{0}+\mathrm{RT} / \mathrm{nF} \ln ([\mathrm{oxidant}] /[$ reductant]) (e.g., see Freeze and Cheery, 1979, p. 121). 
element flaring - The modeling technique of increasing the width (third dimension) of two-dimensional elements according to the radial distance times the desired fraction of a circle, usually a $180^{\circ}$ arc. This technique differs from a fully axisymmetric modeling technique in that not all elements are automatically increased in thickness nor are they always increased by the radial distance from one axis times the full $360^{\circ}$ circle. Instead, several axes may be used with only a fraction of a circular arc times the radial distance. This technique has been frequently used by the PA Department (since 1990, see Rechard et al., 1990, p. 37) to account for the effective volume (not just area) increase of elements as one moves away from the point of study in a system that is not fully symmetric (e.g., disposal region of the repository with shafts at one end); in contrast, portions of the study domain with, for example, uniform flow fields do not have the width region increased but instead are set at a constant (e.g., the maximum width of a plume contour).

engineered barrier - Human-designed (engineered) barriers of the waste disposal system. This definition, which is used herein and in 40 CFR 191, includes shafts, boreholes, and their seals. Note that the 10 CFR 60 definition is narrower and omits shafts, boreholes and their seals in its definition.

enriched - An increased percentage of a desired isotope, such as ${ }^{235} \mathrm{U}$.

EPA release limits - The normalizing factors for various radionuclides listed in Table 1 of Appendix A of the EPA regulation, 40 CFR 191.

EPA limiting line - The set of lines in CCDF space that connect two limiting points specified in the Containment Requirements (Section 13) of 40 CFR 191. According to the guidance of Appendix B of 40 CFR 191, the EPA can consider a disposal system to be in compliance if the CCDF (predicted by the appropriate probability and consequence models) meets or is less than the two limiting points.

event - A natural or anthropogenic phenomenon that occurs either instantaneously or over a small portion of the time frame of interest.

feature - An object or condition of the disposal system that influences release of contaminants.

fugacity - An "invented" parameter ( $f$ ) that measures the chemical potential $(\mu)$ of a real gas in the same way that pressure (P) measures the free energy of an ideal gas, $f=\gamma(P) * P$, where $\gamma$ is the fugacity coefficient that varies with pressure for a real gas (e.g., see Castellan, 1971, p. 222).

general environment - Used in 40 CFR 191, Subpart A, as the "total terrestrial, atmospheric, and aquatic environments outside sites within which any activity, operation, or process associated with the management and storage of... radioactive waste is conducted (Section 191.02).

granite - In this report, granite refers to coarse-grained rock formed at great depth (plutonic) such as occurs at the Canadian site (Lac due Bonnet, Manitoba) (Everitt and Read, 1989). When modified by a descriptive term, such as "massive grey," we are referring to a specific granite, essentially of alkali feldspar and quartz.

Groundwater Protection Requirements - Requirements in Subpart B of 40 CFR 191 ( $\$ 191.16)$ that set limits on radioactive contamination of certain sources of groundwater within or near the controlled area for 1000 years after disposal.

half-life - The time required for half the atoms of a radioactive substance to decay (see also activity).

high level (radioactive) waste (HLW) - “ ... the highly radioactive material [fission products and some actinides, atomic number 89-103] resulting from the reprocessing of spent nuclear fuel, including liquid waste produced directly in reprocessing and any solid maierial derived from such liquid waste that contains fission products in sufficient concentrations ..." (NWPA, :982). Although not used in this manner in this report, general articles regarding radioactive waste use the term high level waste to imply any combination of spent nuclear fuel and HLW (and sometimes transuranic [TRU] vaste) that requires disposal in a deep, geologic repository. 10 CFR 60, which was promulgated by the NRC, also iricludes spent nuclear fuel in its definition of high-level waste. 
highly enriched uranium (HEU) - fuel with a content of ${ }^{235} U$ that is larger than $20 \%$ of the total uranium mass; typically used in U.S. Navy propulsion reactors and some experimental reactors.

implementing agency - The NRC for facilities licensed by the NRC, the EPA for those implementation responsibilities given to the EPA by the WIPP Land Withdrawal Act, and the Department of Energy for any other disposal facility and for implementation responsibilities for the Waste Isolation Pilot Plant not given to the EPA.

Individual Protection Requirements - Requirements in Subpart B of 40 CFR 191 (\$191.15) that set limits on radiation doses to members of the public in the accessible environment (the environment beyond the controlled area $[\$ 191.12(\mathrm{k})]$ ) for 1000 years of undisturbed performance. (The repromulgation of 40 CFR 191 will likely extend this requirement to $10,000 \mathrm{yr}$.)

internal backfill - Filler placed in the canister or between the canister and overpack to transfer structural and heat loads.

Latin hypercube sampling - A Monte Carlo sampling technique that divides the cumulative distribution function into intervals of equal probability and then samples from each interval.

logical grid - The scaled finite-difference grid of the modeled object such that each cell has unit dimensions, i.e., 1 by 1 by 1 . The logical grid is useful for displaying spatial results when cells have greatly varying coordinate dimensions. Because of the irregular connectivity of a finite-element mesh, a logical finite-element mesh is impossible to draw.

low enriched uranium (LEU) - fuel with a higher content of ${ }^{235} U$ than ore $(\sim 0.77)$ but less than or equal to $20 \%$ of the total uranium mass; typically used in commercial reactors.

low level waste (LLW) - All radioactive waste other than spent nuclear fuel, high level waste, transuranic waste, and mill tailings. (Mill tailings are the residue from the physical and chemical processing of uranium ore to obtain uranium.) In the United States, LLW is divided into three categories: A, B, and C. Category A has the lowest activity, and Category $\mathrm{C}$ has the highest. Some countries create a category called "Intermediate Level Waste (ILW)" by grouping together the U.S.'s Category C LLW, which requires shielding during handling, and transuranic waste.

mast wasting - A general term for the process whereby loosened soil and rock material are transported downslope under the direct influence of gravity either slowly or quickly (e.g., landslide).

mathematical model - The mathematical representation of a conceptual model (i.e., the algebraic, differential, or integral equations that predict quantities of interest of a system and any constitutive equations of the physical material that appropriately approximate system phenomena in a specified domain of the conceptual model. Mathematical models can be categorized as either deterministic or stochastic models.

metric tons of heavy metal (MTHM) - metric tons $(1000 \mathrm{~kg}$ ) of heavy metal. (Metric mass, all isotopes of uranium, plutonium, and thorium) before placed into a reactor; the measurement is for initial mass.

model form uncertainty - Uncertainty in the most appropriate model form for a system. The uncertainty results from sparse observed data and lack of information able to corroborate or refute alternative models. Developing alternative models is a way to explicitly acknowledge model form uncertainty; however, in some cases it may be appropriate to combine the alternative models into a megamodel and represent the model-form uncertainty as uncertainty in a parameter.

Monte Carlo - The use of random sampling (and usually computer simulation) to approximately integrate mathematical models.

neutron - The basic particle of an atom; electronically neutral and weighing nearly the same as the proton. 
nuclear fuel - Fissionable material (material able to be split by a low energy neutron) for a nuclear reactor.

overpack or overpack container - Container around the inner containers of the waste form (e.g., a canister) that provides some specialized function(s) (e.g., corrosion allowance, corrosion resistance, or structural support).

panel - A grouping of pillars and rooms; in this report, there are 10 rooms per panel.

parameters - The underlying fundamental entities $\left(x=x_{1}, \ldots, x_{n}, \ldots, x_{n V}\right)$ of a computational model. As $x$ changes, so does the model result. The individual parameters, $x_{n}$, are usually scalar quantities, but they may also be vectors or functions. Furthermore, the individual parameters are usually coefficients of the mathematical model, but they may also relate to scenario uncertainty or model form uncertainty.

parameter uncertainty - Uncertainty in the most appropriate parameter value for a computational model. The uncertainty results from sparse observed data and lack of information able to corroborate or refute alternative parameter values. Developing distributions of the parameter values is a way to explicitly acknowledge parameter uncertainty; however, in some cases it may be more appropriate to evaluate the computational model at a few possible values of the parameter.

performance assessment (PA) - The process of assessing whether a system meets a set of performance criteria. For INEL, the system is a deep geologic repository disposal system (salt and granite) for graphite spent nuclear fuel, special spent nuclear fuel, and calcine high-level waste. The performance criteria are various long-term environmental metrics in U.S. government regulations (not short-term operational safety issues).

pH - A measure of the proton activity of a solution and equal to the negative logarithm of concentration of hydrogen ions in a solution, $\mathrm{pH}=-\log \left(\mathrm{CH}^{+}\right)$

physical process - A natural or anthropogenic phenomenon that occurs continuously or over a significant portion of the time frame of interest.

pillar - A block of rock left intact to support the overlying strata of the underground excavations.

repository - The portion of a waste disposal facility that includes the waste panels, access drifts, and access shafts. The repository does not include either the above-ground facilities or the undisturbed host rock.

room - An excavated cavity underground for disposal of waste and part of a panel; in this report, the room is $9 \mathrm{~m}$ wide and $6 \mathrm{~m}$ high.

salt - In this report, salt refers to evaporite deposits that are predominantly sodium chloride $(\mathrm{NaCl})$. Mineralogic names such as halite $(\mathrm{NaCl})$, sylvite $(\mathrm{KCL})$, gypsum $\left(\mathrm{CaSo} \bullet \mathrm{H}_{2} \mathrm{O}\right)$, and anhydrite $\left(\mathrm{CaSO}_{4}\right)$ are used when referring to specific evaporite minerals. The term evaporite includes all of the above minerals; evaporites are formed by the evaporation of a saline solution such as sea water.

scenario - In PA work, a compound statement, $A(\mathbf{x})$, that is built by linking with the conjunction "and" any series of simple statements about values taken by elements of the parameter vector, $\mathbf{x}$, and/or the vector of consequences, $f(x)$.

scenario screening - A necessary step in the construction of any model of a natural or engineered system in which the phenomena identified through scenario selection are incorporated in a mathematical model of the system.

scenario selection - A necessary step in the construction of any model of a natural or engineered system in which the physical, chemical, and anthropogenic phenomena that might play a significant role in determining the performance of the system are identified. 
scenario uncertainty - Uncertainty in the most appropriate scenarios for a system. The uncertainty results from omission of discrete, short-term, natural, or anthropogenic phenomena (events), continuous natural or anthropogenic phenomena (processes), and objects and conditions (features) of a system (completeness errors) and the imperfect aggregation of histories of a system (aggregation errors).

seiche - An oscillation of the surface of a lake or landlocked sea that varies in period from a few minutes to several hours.

sensitivity analysis - An analytic or numerical technique for examining the effects of parameters being varied in a model; specifically, an analysis of the effects that parameter changes have on model predictions.

shaft - An approximately vertical or steeply inclined passageway from the ground surface to the underground level of the disposal region; in this report, the shaft has a 9-m diameter in salt and a 6-m diameter in granite.

Simple PA - A performance assessment that uses consequence models based on a mathematical model with ordinary differential equations and lumped-model parameters; the Simple PA provides a faster model setup and run times compared to a Complex PA, which uses consequence models with spatially varying parameters.

site - As used in this report, the "site" is the general location of the controlled area (the disposal system, including the land surface directly above it). The term "site" also includes any important features surrounding the controlled area (e.g., "site characterization"). Except for the latter addition, this report's definition of site is most similar to the regulatory definition in 10 CFR 60.2: "the location of the controlled area." It is roughly equivalent to the rigulatory definition of "site" in 40 CFR 191.02, provided "effective control" is loosely defined (e.g., as simply land use or ownership records): "an area contained within the boundary of a location under the effective control of persons possessing or using ... radioactive waste that are involved in any activity, operation, or process covered by this Subpart."

special spent nuclear fuel (special SNF) - Experimental fuel that originates from many government research and test reactors and non-government research reactors.

spent nuclear fuel (SNF) or spent fuel - “... fuel that has been withdrawn from a nuclear reactor following irradiation, the constituent elements of which have not been separated by reprocessing" (NWPA, 1982). Although spent nuclear fuel has fissionable ${ }^{235} \mathrm{U}$, it contains too many radionuclides (primarily short-lived) that adsorb neutrons from the fission process for it to be usefully left in the reactor. Because of spent nuclear fuel's high value, some countries choose to recycle it (recycling becomes more attractive after the short-lived fission products have decayed away). It is also designated separately from other high level and transuranic wastes in the U.S. Environmental Protection Agency's standard on disposal of radioactive wastes, 40 CFR 191. In this report, its status as "spent" nuclear fuel, rather than as a "waste," is maintained when the source of the material is described. However, in the description of the treatment options that specify disposal, it is treated as a "waste" and therefore becomes part of a "waste form."

stock - A body of plutonic igneous rock less than $100 \mathrm{~km}^{2}\left(40 \mathrm{mi}^{2}\right)$ in area.

summary scenario - A subset of plausible histories (or fractures) of the disposal system.

threshold pressure - In this performance assessment, threshold pressure is defined as the capillary pressure associated with the incipient development of a continuous connection of the nonwetting (gas) phase through a porous medium, providing gas pathways not only through relatively large pore spaces, but also through necks between pores. Thus, threshold pressure $\left(P_{t}\right)$ is the capillary pressure $\left(P_{c}\right)$ at the residual gas saturation $\left(S_{g r}\right)$.

transuranic (TRU) - Transuranic waste is also termed Alpha-Bearing Waste in some countries. Transuranic waste is contaminated with transuranic radionuclides (atomic number greater than 92) emitting $\alpha$ radiation with a halflife greater than 20 years and an activity greater than $100 \mathrm{nCi}$. 
tsunamis - A great sea wave produced by subinarine earth movement or volcanic eruption.

uncertainty analysis - An analytic or numerical technique to evaluate how important the uncertainties of a parameter are (i.e., how likely it is to vary) in explaining uncertainty in the predictions. For example, the results of a model may be very sensitive to varying a parameter, but if the parameter's value is precisely known, the parameter is not important to producing any uncertainty in the prediction. (Uncertainty analysis is sometimes called importance analysis.)

uncertainty propagation - Methods for propagating the uncertainty in parameters of a model, through the model, to calculate the uncertainty in the predictions. Uncertainty propagation is an important aspect of stochastic simulation.

underground facility - The underground structure including openings and backfill materials, but excluding shafts, boreholes, and their seals (10 CFR 60.2).

validation - The process of making valid by confirming, corroborating, substantiating, or supporting, where valid means of good authority, well founded, sound and to the point, and applicable to the subject or circumstances against which few objections can be fairly brought.

validation of an (applied) model - The process of validating through sufficient testing (subjective) using systemspecific observed data that a conceptual model and the corresponding mathematical and computational models explain a system with sufficient accuracy (subjective), consistent with the purpose of the model. In other words, model validation is the process of confirming that the applied model is solving the appropriate idealization of the system (correct applied model produced). Verification of a computational model precedes validation of an applied model. Model validation is an ongoing process.

verification of a (computational) model - The process of verifying that a computational model appropriately solves and implements the mathematical model. In other words, model verification is the process of illustrating that the mathematical model is being solved appropriately (computational model correctly produced). Often the computational model is implemented as a code, and verification of the computational model is closely connected to verification of software. Given that the computational model is verified, the assumptions of the conceptual model underlying the mathematical and computational model using system-specific data (the applied model) should be validated.

verification of software - The process of illustrating through sufficient testing (subjective) that the software satisfactorily performs its stated capabilities (subjective) and providing a point of reference for future modifications. The extent to which the software is tested is determined by the complexity of the software.

waste - The contents of a nuclear waste canister, except any internal backfill or neutron poisons packed inside the container, i.e., high-level waste from reprocessing spent fuel; nuclear spent fuel, brackets and hardware highly contarninated with radionuclides; and constituents that have been defined as hazardous by the Resource Conservation ard Recovery Act (RCRA, 1976).

waste disposal group - A grouping by similar treatment option of the waste emplacement packages that contain the waste types being considered for disposal. For this performance assessment, five treatment options are studied per three INEL waste types and so five waste disposal groups exist. Each waste disposal group consists of a number of waste parcels that are representative of the three INEL waste types (graphite spent fuel, special spent fuel, and calcine) and the two potential repositories (salt and/or granite).

waste form - The waste type in combination with a specific treatment option; the form of the waste just before placement in a canister. The waste form can often be succinctly described by an adjective summarizing the treatment option (e.g., shredded graphite spent nuclear fuel). 
waste package or waste emplacement package (WEP) - All components that enclose the waste form out to the host rock (10 CFR 60). This grouping is defined as a unit as a convenience for subsystem modelers; also 10 CFR 60 places release limits on the components in this grouping.

waste package hole backfill - See backfill-buffer.

waste parcel (WP) - The waste form, internal backfill, canister, and overpack. This definition describes a unit that could be transported; it does not include the waste package hole backfill placed in the annulus between the canister and host rock.

waste source - The source of spent nuclear fuel, e.g., graphite or experimental.

waste type - A description of the waste that indicates either its spent nuclear fuel source or its type of reprocessing. For this report, three waste types are discussed: Graphite spent fuel, special spent fuel, and calcine waste. 


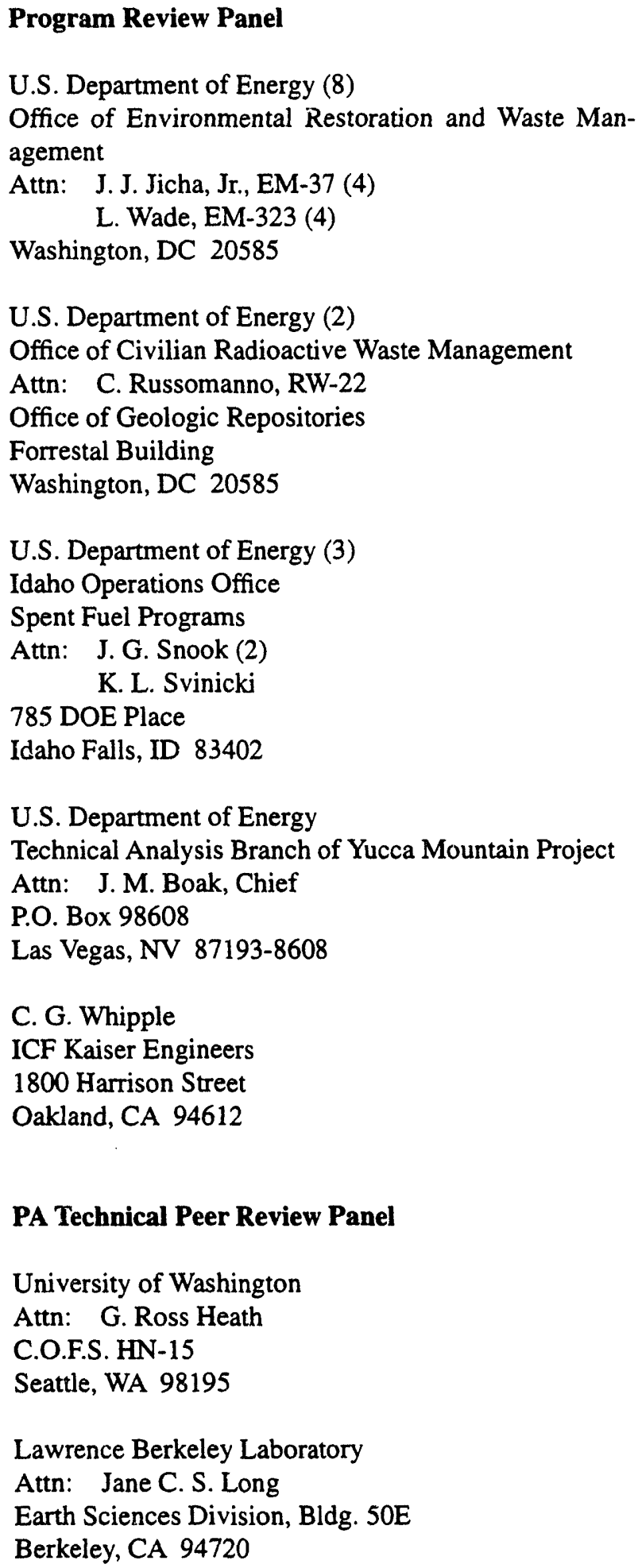

Program Review Panel

U.S. Department of Energy (8)

Office of Environmental Restoration and Waste Management

Attn: J. J. Jicha, Jr., EM-37 (4)

L. Wade, EM-323 (4)

Washington, DC 20585

U.S. Department of Energy (2)

Office of Civilian Radioactive Waste Management

Attn: C. Russomanno, RW-22

Office of Geologic Repositories

Forrestal Building

Washington, DC 20585

U.S. Department of Energy (3)

Idaho Operations Office

Spent Fuel Programs

Attn: J. G. Snook (2)

K. L. Svinicki

785 DOE Place

Idaho Falls, ID 83402

U.S. Department of Energy

Technical Analysis Branch of Yucca Mountain Project

Attn: J. M. Boak, Chief

P.O. Box 98608

Las Vegas, NV 87193-8608

C. G. Whipple

ICF Kaiser Engineers

1800 Harrison Street

Oakland, CA 94612

\section{PA Technical Peer Review Panel}

University of Washington

Attn: G. Ross Heath

C.O.F.S. $\mathrm{HN}-15$

Seattle, WA 98195

Lawrence Berkeley Laboratory

Attn: Jane C. S. Long

Earth Sciences Division, Bldg. 50E

Berkeley, CA 94720

Los Alamos National Laboratory

Attn: Thomas McLaughlin

Group HSE-S, MS-F691

P.O. Box 1663

Los Alamos, NM 87545

Donald W. Peaceman

4807 Glenmeadow Drive

Houston, TX 77096

University of California

Attn: Thomas H. Pigford

Department of Nuclear Engineering

4159 Etcheverry Hall

Berkeley, CA 94270

Battelle Pacific Northwest Labs

Attn: Richard Westerman

Richland, WA 99352

\section{Federal Agencies}

U.S. Department of Energy (5)

Office of Environmental Restoration and Waste

Management

Attn: T. Grumbly, Assistant Secretary, EM-1

G. Sjoblom, EM-1

C. W. Frank, EM-50

J. E. Lytle, Deputy Assistant Secretary, EM-30 (2)

Washington, DC 20585

U.S. Department of Energy (2)

Office of Waste Management Projects

Attn: M. W. Frei, Director, EM-34

J. Rhoderick

12800 Middlebrook Rd.

Suite 400

Germantown, MD 20874

U.S. Department of Energy

Office of Environmental, Safety and Health

Attn: R. P. Berube, Director, EH-20

1000 Independence Ave SW

Washington, DC 20585 
U.S. Department of Energy

Research \& Waste Management Division

Attn: Director

P.O. Box E

Oak Ridge, TN 37831

U.S. Department of Energy (4)

Office of Civilian Radioactive Waste Management

Attn: D. Dreifus, Director, RW-1

L. Barrett, Deputy, RW-2

R. Nelson, Associate Director, RW-20

Office of Geologic Disposal

A. Berusch, RW-22

Forrestal Building

Washington, DC 20585

U.S. Department of Energy

Idaho Operations Office

Attn: A. A. Pitrolo, Manager

785 DOE Place

Idaho Falls, ID 83402

U.S. Department of Energy (2)

Idaho Operations Office

Manager, Spent Fuel Programs

Attn: M. J. Bonkoski

785 DOE Place

Idaho Falls, ID 83402

U.S. Department of Energy (4)

Idaho Operations Office

Spent Fuel Programs

Attn: B. G. Edgerton (2)

W. J. Mings (2)

785 DOE Place

Idaho Falls, ID 83402

U.S. Department of Energy

Idaho Operations Office

Energy Programs

Attn: W. A. Owca

785 DOE Place

Idaho Falls, ID 83402

U.S. Department of Energy

Idaho Operations Office

Assistant Manager for Energy Programs

Attn: W. H. Thielbahr

785 DOE Place

Idaho Falls, ID 83402
U.S. Department of Energy

Idaho Operations Office

Office of Environmental Safety \& Health Oversight

Attn: G. C. Bowman

785 DOE Place

Idaho Falls, ID 83402

U.S. Department of Energy

Savannah River Operations Office

Defense Wastes Processing Facility Project Office

Attn: W. D. Pearson

P.O. Box A

Aiken, SC 29802

U.S. Department of Energy (2)

Richland Operations Office

Nuclear Fuel Cycle \& Production Division

Attn: R. E. Gerton

825 Jadwin Ave.

P.O. Box 500

Richland, WA 99352

U.S. Department of Energy (3)

Nevada Operations Office

Attn: J. R. Boland

D. Livingston

P. K. Fitzsimmons

2753 S. Highland Drive

Las Vegas, NV 87183-8518

U.S. Department of Energy (2)

Regulatory and Site Evaluation Division

Attn: J. R. Dyer, Director

P.O. Box 98608

Las Vegas, NV 87193-8608

U.S. Department of Energy (2)

WIPP Project Office (Carlsbad)

Attn: G. E. Dials, Director

P.O. Box 3090

Carlsbad, NM 88221-3090

U.S. Environmental Protection Agency (2)

Office of Radiation and Indoor Air (ANR-460)

Attn: M. Oge

Washington, DC 20460

U.S. Nuclear Regulatory Commission

Division of Waste Management

Attn: H. Marson

Mail Stop 4-H-3

Washington, DC 20555 
U.S. Nuclear Regulatory Commission (4) Advisory Committee on Nuclear Waste

Attn: D. Moeller M. J. Steindler

P. W. Pomeroy

W. J. Hinze

7920 Norfolk Avenue

Bethesda, MD 20814

Defense Nuclear Facilities Safety Board

Attn: D. Winters

625 Indiana Avenue NW

Suite 700

Washington, DC 20004

Nuciear V'aste Technical Review Board (3)

Attr: J. Cathlon, Chairman

W. North

L. Reiter

Suite 910

1100 Wilson Blvd.

Arlington, VA 222l $;-2297$

Office of Management and Budget

Energy and Science Division

Attn: K. Yuracko

725 17th Street NW

Washington, DC 20503

U.S. Department of Energy

Albuquerque Operations Office

Attn: National Atomic Museum Library

P.O. Box 5400

Albuquerque, NM 87185

Westinghouse Idaho Nuclear Company (30)

Attn: H. H. Loo

1955 Fremont Avenue

Idaho Falls, ID 83415

G. E. Bingham

H. V. Chamberlain

H. D. Christiansen

L. F. Ermold

D. R. Evans

R. N. Henry

G. W. Hogg

A. P. Hoskins

R. D. Klingler (14)

D. A. Knecht

H. H. Loo

V. C. Maio

A. L. Olson

B. M. Palmer
W. B. Palmer

J. I. Pruitt

L. L. Taylor

National Research Council's Board

on Radioactive Waste Management

National Research Council (3)

Board on Radioactive Waste Management

RM HA456

Attn: C. Anderson, Staff Director (2)

I. B. Alterman

2101 Constitution Ave.

Washington, DC 20418

WIPP Performance Assessment Peer Review Panel

JK Research Associates, Inc.

Attn: Thomas A. Cotton

442.9 Butterworth Place NW

Washington, DC 20016

Future Resources Associates, Inc.

Attn: Robert J. Budnitz, President

2000 Center Street, Suite 418

Berkeley, CA 94704

Department of Geology

Attn: C. John Mann

245 Natural History Bldg.

1301 West Green Street

University of Illinois

Urbana, Il 61801

\section{Corporations/Members of the Public}

INTERA, Inc. (2)

Attn: J. F. Pickens

$$
\text { S. Pahwa }
$$

6850 Austin Center Blvd.

Suite 300

Austin, TX 78731

Roy Weston, Inc.

Attn: Christopher Charles

955 L'Erdant Plaza SW

Suite 800

Washington, DC 20024 
Southwest Research Institute

Center for Nuclear Waste Regulatory Analysis (2)

Attn: B. Sagar

P. K. Nair

6220 Culebra Road

San Antonio, TX 78228-0510

Tech Reps, Inc. (3)

Attn: J. Chapman

D. Pulliam

C. Crawford

5000 Marble NE, Suite 222

Albuquerque, NM 87110

Western Water Consultants

Attn: P. A. Rechard

P.O. Box 4128

Laramie, WY 82071

Western Water Consultants

Attn: D. Fritz

1949 Sugarland Drive \#134

Sheridan, WY 82801-5720

\section{Universities}

University of Wyoming

Department of Civil Engineering

Attn: V. R. Hasfurther

Laramie, WY 82071

\section{NEA/Performance Assessment Advisory Group}

(PAAG)

Daniel A. Galson

Galson Sciences Ltd.

35, Market Place

Oakham

Leicestershire LE15 6DT

UNITED KINGDOM

Brian G. J. Thompson

Department of the Environment, Her

Majesty's Inspectorate of Pollution

Room A5.33, Romney House

43 Marsham Street

London SWIP 2PY,

UNITED KINGDOM
U.S. Nuclear Regulatory Commission (2)

Attn: R. Codell N. Eisenberg

Mail Stop 4-H-3

Washington, DC 20555

Geostatistics Expert Working Group (GXG)

Dennis McLaughlin

Parsons Laboratory

Room 48-209

Department of Civil Engineering

Massachusetts Institute of Technology

Cambridge, MA 02139

Ghislain de Marsily (GXG Chairman)

University Pierre et Marie Curie

Laboratorie de Geologie Applique

4, Place Jussieu

T.26 - $5^{\mathrm{e}}$ etage

75252 Paris Cedex 05, FRANCE

\section{Sandia Internal}

$\begin{array}{lll}\text { MS } & \text { Org. } & \\ 0724 & 6000 & \text { D. L. Hartley } \\ 1337 & 6300 & \text { D. E. Ellis } \\ 1335 & 6301 & \text { F. Binghan } \\ 1333 & 6302 & \text { L. E. Shephard } \\ 1335 & 6303 & \text { W. D. Weart } \\ 1335 & 6305 & \text { S. A. Goldstein } \\ 1326 & 6312 & \text { H. A. Dockery } \\ 1328 & 6342 & \text { D. R. Anderson } \\ 1328 & 6342 & \text { J. W. Berglund } \\ 1328 & 6342 & \text { W. E. Beyeler } \\ 1328 & 6342 & \text { K. F. Brinster } \\ 1328 & 6342 & \text { M. M. Gruebel } \\ 1328 & 6342 & \text { M. E. Lord } \\ 1328 & 6342 & \text { H. J. Iuzzolino } \\ 1328 & 6342 & \text { A. Johnson } \\ 1328 & 6342 & \text { M. G. Marietta } \\ 1328 & 6342 & \text { J. L. Ramsey } \\ 1328 & 6342 & \text { J. S. Rath } \\ 1328 & 6342 & \text { R. P. Rechard (5) } \\ 1328 & 6342 & \text { D. K. Rudeen } \\ 1328 & 6342 & \text { C. T. Stockman } \\ 1328 & 6342 & \text { M. S. Tierney } \\ 1328 & 6342 & \text { K. M. Trauth } \\ 1328 & 6342 & \text { P. Vaughn }\end{array}$




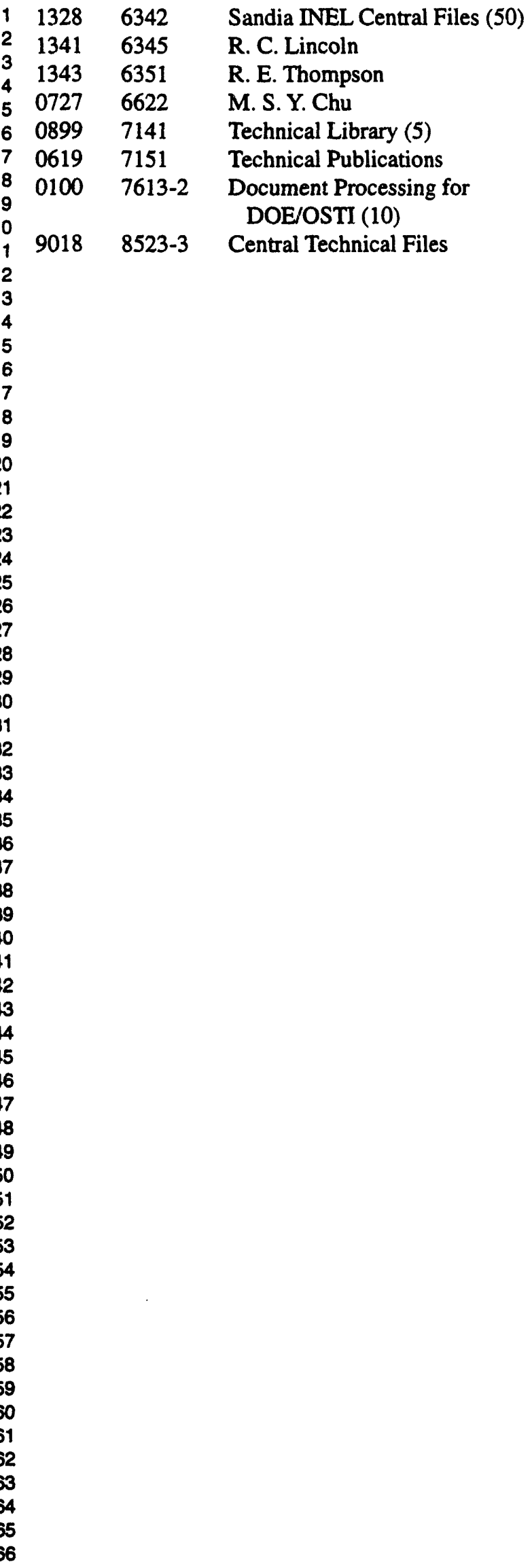



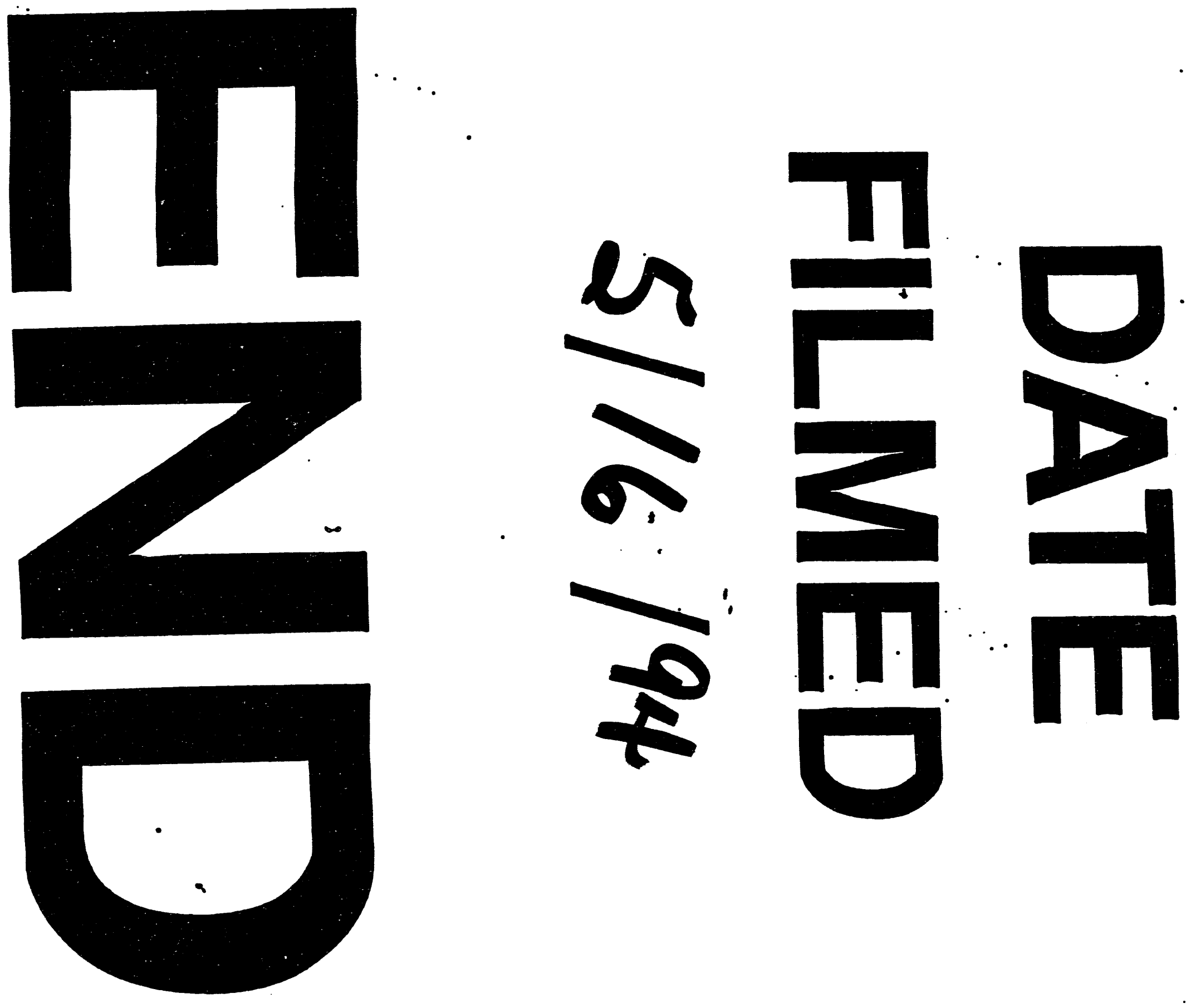
